\title{
Abstracts and Programme
}

\section{EUROANAESTHESIA 2007}

Annual Meeting of the European Society of Anaesthesiology

Munich, Germany,

June 9-12, 2007 


\section{European Journal of Anaesthesiology}

\author{
Editor-in-Chief \\ B. J. Pollard Manchester, UK
}

\section{Deputy Editor-in-Chief and Editor of Commissioned Reviews}

T. A. Crozier Göttingen, Germany

\author{
Editors \\ J.-P. Haberer Paris, France \\ $\mathrm{M}$. Tramèr Genève, Switzerland \\ G. Capogna Roma, Italy \\ H. Tydén Uppsala, Sweden \\ M. Leuwer Liverpool, UK \\ A. W. Gelb London, Canada \\ L. Bogar Pécs, Hungary \\ B. Matta Cambridge, UK \\ C. Bailey London, UK \\ P. Rosenberg Helsinki, Finland \\ M. Columb Manchester, UK
}

\author{
Editor of Supplements \\ B. Matta Cambridge, UK
}

\section{Editor of EACTA Supplements}

R. Feneck London, UK

Statistical Adviser

C. J. D. Pomfrett Manchester, UK

Associate Editors

C. J. D. Pomfrett Manchester, UK

N. J. N. Harper Manchester, UK

\section{Editorial Board}

Chairman A. R. Aitkenhead Nottingham, UK

\section{Members}

J. N. Cashman London, UK

A. Hoeft Bonn, Germany

B. Matta Cambridge, UK

H. Van Aken Münster, Germany

Editor-in-Chief

Deputy Editor-in-Chief

*P. Simpson Bristol, UK, President of the European Society of Anaesthesiology

*E. Kochs Munich, Germany, Secretary of the European Society of Anaesthesiology

*M. Tramèr Geneva, Switzerland, Treasurer of the European Society of Anaesthesiology

*C.-J. Jakobsen Aarbus, Denmark, President of the European Association of Cardiothoracic Anaesthesiologists

*J. T. A. Knape Utrecht, Netherlands, President of the Union Européenne des Médecins Spécialistes, Section of Anaesthesiology

*P. Scherpereel Lille, France, President of the Fondation Européenne d'Enseignement en Anesthésiologie

[*Ex-officio]

\section{Commissioning Editor}

Dan Edwards (E-mail: dedwards@cambridge.org)

\begin{abstract}
European Journal of Anaesthesiology (EJA) is the official publication of the European Society of Anaesthesiology. The EJA is also the official journal of the following organizations: EACTA (European Association of Cardiothoracic Anaesthesiologists), EFAAD (European Federation for the Advancement of Anaesthesia in Dentistry), ESCTAIC (European Society for Computing and Technology in Anaesthesia and Intensive Care), the FEEA (Fondation Européenne d'Enseignement en Anesthésiologie), SESAM (Society in Europe for Simulation Applied to Medicine) and of the Section Anaesthesiology of the UEMS (Union Européenne des Médecins Spécialistes). The Journal publishes original scientific work. Preference is given to experimental work or clinical observations in man, and to laboratory work of clinical relevance. There will be regular commissioned reviews by an authority in a field of interest to those working in anaesthesiology or intensive care.
\end{abstract}

Subscription information. European Journal of Anaesthesiology is published monthly (one volume per annum) and the subscription prices for 2007 are as follows. Institutional: $€ 1014$ (Europe), $\$ 1173$ (USA and Canada) and $£ 744$ (Rest of the World); Personal: €244 (Europe), \$249 (USA and Canada), $£ 160$ (Rest of the World), in all cases post-free. Subscribers in Canada must add $7 \%$ to the subscription price, to allow for GST. Subscribers in Europe must quote their VAT registration number or state that they are not registered. Subscribers in the following countries who are not VAT-registered must add VAT at the appropriate rate: Belgium (6\% TVA/BTW), France (2.1\% TVA), Germany (7\% MWST), Spain (4\% IVA), The Netherlands (6\% BTW). Orders for current subscriptions and back issues should be sent to EJA Subscription Orders, Cambridge University Press, Edinburgh Building, Shaftesbury Road, Cambridge CB2 8RU, UK. E-mail: ejaeditorial@cambridge.org.

The Publishers' Policy is to use acid-free permanent paper, to the draft standard ISO/DIS 9706, made from sustainable forests using chlorine-free pulp.
Despatch. European Journal of Anaesthesiology is dispatched within the UK by 2 nd class post, within Europe by airmail, and to other countries by various forms of air-speeded delivery. Add to the cost of regular subscription $£ 12 / \$ 18$ for airmail delivery outside Europe.

Editorial material should be sent to the Editor, European Journal of Anaesthesiology, Cambridge University Press, Edinburgh Building, Shaftesbury Road, Cambridge CB2 8RU, UK. E-mail: journals_societies@ cambridge.org.

Business correspondence, including orders for offprints and advertising, should be sent to the publishers: European Journal of Anaesthesiology, Advertising Department, Cambridge University Press, Edinburgh Building, Shaftesbury Road, Cambridge CB2 8RU, UK. E-mail: ejaeditorial@ cambridge.org. Information on this journal is available on the website: www.journals.cambridge.org.

Copyright and photocopying: (C) 2007 European Society of Anaesthesiology. Authorization to photocopy items for internal or personal use, or the internal or personal use of specific clients, is granted by the European Society of Anaesthesiology for libraries and other users registered with their national Reproduction Rights Organisations - such as the the Copyright Clearance Center (USA), Copyright Licensing Agency (UK), and the Copyright Agency Limited (Australia) - provided that payments are made to the relevant Organisation at the base rates currently prevailing. This consent does not extend to other kinds of copying, such as copying for general distribution for advertising or promotional purposes, for creating new collective works or for resale. Special requests should be addressed to the Editor.

The views expressed in this journal represent those of the authors only. In no way can they be construed necessarily to reflect the view of either the Editors or the Publishers. 


\title{
EUROANAESTHESIA 2007
}

\author{
Annual Meeting of the European Society of Anaesthesiology \\ Munich, Germany, June 9-12, 2007
}

\section{ABSTRACT PRESENTATION PROGRAMME}

For the first time at Euroanaesthesia meetings, abstract presenters will NOT be required to make a formal presentation of their abstract in a separate room, using audiovisual aids (except for the Best Abstract Prize Competition). Instead, two chairpersons will conduct, in front of each poster, a short discussion of each abstract with the presenter and the audience, for every abstract in that session. Poster presenters will be required to stand by their poster for 45 minutes before and 30 minutes after their session, to address further questions.

Note that all posters of regular abstract sessions will be displayed in Hall B0. The Best Abstract Prize Competition, however, takes place in Room 03.

As of this year, the accepted abstract number has a new format, consisting of the session reference, followed by a number denoting the order of the abstract within this session (e.g. 6AP1, which would be the first (1) Abstract Presentation (AP) session for subcommittee 6). The first abstract to be presented in session 6AP1 will thus be called 6AP1-1, the second one 6AP1-2 and so on.

The locate abstract 6AP1-1 or other abstracts for session 6AP1 in this supplement, look for the session reference (6AP1) in the schedule below, then browse to the appropriate page number, which always refers to the page number of the first abstract within the specified session.

Date Time

Reference

Page

ESA Best Abstract Prize Competition (BAPC1)

Sunday June 10

12:15-13:45

BAPC1

1

Subcommittee 1 - Evidence based practice and quality assurance

Saturday June 09

15:15-16:45

10:30-12:00

Monday June 11

14:00-15:30

1AP1

$1 \mathrm{AP} 2$

$1 \mathrm{AP} 3$

Subcommittee 2 - Ambulatory anaesthesia

Saturday June 09

15:15-16:45

2AP1

Monday June 11

10:30-12:00

2AP2

Subcommittee 3 - Monitoring: equipment and computers

Saturday June 09

13:15-14:45

3AP1

$13: 15-14: 45$

Saturday June 09

10:15-11:45

nday June 10

12:15-13:45

14:00-15:30

Sunday June 10

$14: 00-15: 30$

16:00-17:30

14:00-15:30

Monday June 11

16:00-17:30

3AP2

3AP3

3AP4

3AP5

3AP6

3AP7

3AP8

Monday June 11

3AP9

Subcommittee 4 - Clinical and experimental circulation

Saturday June 09

13:15-14:45

15:15-16:45

10:15-11:45

Sunday June 10

$10: 15-11: 45$
$12: 15-13: 45$

Sunday June 10

14:00-15:30

Sunday June 10

16:00-17:30

Sunday June 10

16:00-17:30

Monday June 11

16:00-17:30

Monday June 11

10:30-12:00

Tuesday June 12

4AP9

Subcommittee 5 - Respiration

Sunday June 10

Sunday June 10

10:15-11:45

14:00-15:30

5AP1

16:00-17:30

5AP2

16:00-17:30

$5 \mathrm{AP3}$

5AP4

Monday June 11

\section{tasis}

Saturday June 09

Sunday June 10

15:15-16:45

10:15-11:45 
Subcommittee 7 - Neurosciences

Saturday June 09

Sunday June 10

15:15-16:45

$10: 15-11: 45$

$7 \mathrm{AP} 3$

12:15-13:45

Sunday June 10

14:00-15:30

$7 A P 4$

Monday June 11

10:30-12:00

Monday June 11

14:00-15:30

7 AP5

7AP6

Subcommittee 8 - Local and regional anaesthesia

Saturday June 09

$15: 15-16: 45$

8AP1

8AP2

$15: 15-16: 45$

Sunday June 10

10:15-11:45

$8 A P 3$

$12: 15-13: 45$

Sunday June 10

$12: 15-13: 45$

Sunday June 10

14:00-15:30

14:00-15:30

Monday June 11

$16: 00-17: 30$

16:00-17:30

Monday June 11

$10: 30-12: 00$

Tuesday June 12

10:15-11:45

12:15-13:45

12:15-13:45

14:00-15:30

16:00-17:30

16:00-17:30

10:30-12:00

10:30-12:00

$8 A P 4$

8AP5

8AP6

8AP7

8AP8

8AP9

8 AP10

Subcommittee 9 - Pharmacology

Sunday June 10

9AP1

9AP2

9AP3

9AP4

9AP5

9AP6

9AP7

Monday June 11

9AP8

Sunday June 09

$12: 15-13: 45$

Sunday June 10

16:00-17:30

10AP4

14:00-15:30

Monday June 11

14:00-15:30

Subcommittee 11 - Obstetric anaesthesia

Sunday June 10

Subcommittee 12 - Intensive care medicine

Sunday June 10

Sunday June 10

Sunday June 10

Sunday June 10

Sunday June 10

$16: 00-17: 30$

$12 \mathrm{AP} 4$

$12 \mathrm{AP5}$

$16: 00-17: 30 \quad 12 \mathrm{AP} 6$

Sunday June 10

10:30-12:00

12AP7

16:00-17:30 12AP8

Monday June 11

$12 \mathrm{AP} 8$

Subcommittee 13 - Resuscitation and emergency medicine

Sunday June 10

Subcommittee 14 - Acute and chronic pain management

Sunday June 10

Subcommittee 15 - Education, research and presentation 
Subcommittee 17 - Patient safety

Sunday June 10

$10: 15-11: 45$

17AP1

186

Sunday June 10

$14: 00-15: 30$

17AP2

187

Monday June 11

14:00-15:30

17AP3

189

Subcommittee 19 - EAMS (European Airway Management Society) - Airway management

Saturday June 09

13:15-14:45

12:15-13:45

19AP1

19AP2

191

Sunday June 10

10:30-12:00

19AP3

193

onday June 11

14:00-15:30

19AP4

195

14:00-15:30

19AP5

197

Monday June 11

16:00-17:30

19AP6

200

Monday June 11

The abstracts published in this Supplement have been typeset from electronic submissions and camera-ready copies prepared by the authors. Every effort has been made to reproduce faithfully the abstracts as submitted. These abstracts have been prepared in accordance with the requirements of the European Society of Anaesthesiology and have not been subjected to review nor editing by the European Journal of Anaesthesiology. However, no responsibility is assumed by the organizers or publisher for any injury and/or damage to persons or property as a matter of products liability, negligence or otherwise, or from any use or operation of methods, products, instructions or ideas contained in the material herein. Because of the rapid advances in medical sciences, we recommend that independent verification of diagnoses and drug doses should be made. 
https://doi.org/10.1017/S0265021507000798 Published online by Cambridge University Press 


\section{BAPC1-1}

Reduced propofol sensitivity in $\mathbf{S 2 6 7}$ mutant glycine receptors

J. Ahrens, M. Leuwer, J. Lambert, G. Haeseler

Anaesthesiology, Hannover Medical School, Hannover, Germany

Background and Goal of study: The aromatic alcohol propofol (2,6 diisopropylphenol) positively modulates $\mathrm{GABA}_{A}$ and glycine receptors (1). While molecular determinants for binding of propofol to $\mathrm{GABA}_{A}$ receptors have been extensively studied (2), little is known about the binding site of propofol on glycine receptors.

Material and Methods: We investigated the effects of propofol on chloride inward currents via two specific mutations within the $\alpha_{1}$ subunit of the rat glycine receptor ( $\alpha_{1}$ S267I and $\alpha_{1}$ S267M) with standard whole-cell experiments. Glycine receptor $\alpha_{1}$ subunits (wild type or mutants) were heterologously expressed in human embryonic kidney cells.

Results: Both mutants (a) decreased sensitivity to the natural agonist glycine (glycine $\mathrm{EC}_{50} 12.8 \pm 2.3 \mu \mathrm{M}$ in wild type (WT), $81.5 \pm 21.2 \mu \mathrm{M}$ in $\alpha_{1}$ S267I and $122.8 \pm 18.5 \mu \mathrm{M}$ in $\alpha_{1}$ S267M); (b) decreased sensitivity to propofol with respect to co- activation of an $\mathrm{EC}_{20}$ glycine response (propofol $\mathrm{EC}_{50} 4.8 \pm 1.2 \mu \mathrm{M}$ in WT, $36.3 \pm 17.5 \mu \mathrm{M}$ at the $\alpha_{1}$ S267I and $9.5 \pm 3.2 \mu \mathrm{M}$ at the $\alpha_{1}$ S267M receptor); and (c) abolished direct receptor activation by propofol.

Conclusions: These results suggest that the serine residue at the position 267 in the transmembrane domain 2 of the $\alpha_{1}$ glycine receptor is crucial for receptor activation by the natural agonist glycine and by the anaesthetic propofol in the absence of glycine. The fact that the exchange of serine for the bulkier isoleucine had a stronger impact on the co- activating actions of propofol than the exchange for the polar methionine suggests that larger hydrophobic side chains inhibit access to the co- activating binding site.

\section{References:}

1 Krasowski MD, Harrison NL Cell Mol Life Sci 1999; 55: 1278-1303.

2 Mihic SJ, Ye Q, Wick MJ et al. Nature 1997; 389: 385-389.

\section{BAPC1-2}

Maintaining normoglycemia and not glycemia-independent actions of insulin preserves myocardial performance by protecting mitochondrial function

B. Ellger, I. Vanhorebeek, Y. Debaveye, R. De Voss, G. Van den Berghe Department of Intensive Care Medicine, Catholic University of Leuven, Leuven, Belgium

Background and Goal of Study: Intensive insulin therapy (IIT) reduces mortality and morbidity of critically ill patients. Several investigations suggest that insulin might ameliorate myocardial contractility independently of its actions on blood glucose.

Materials and Methods: In a rabbit model of prolonged critical illness using a 4-arm design ( $n=8$ per group), blood glucose (normal NG-high HG) and plasma insulin levels (normal NI-high $\mathrm{HI}$ ) were independently manipulated over 7 days, to elucidate relative impact of maintaining normoglycemia and glycemia-independent actions of insulin on left ventricular contractility in an open chest preparation, cellular ultra-structure by electron microscopy, the activities of the respiratory chain complexes in biopsies from the left ventricle, and plasma levels of serum heart-fatty-acid-binding-protein.

Results and Discussions: Contractility increased in HI/NG animals and deteriorated in $\mathrm{HI} / \mathrm{HG}$ animals compared to other groups and healthy controls. Cardiac output and surrogate parameters of preload and afterload did not differ among groups. Electron microscopy revealed severely damaged mitochondria in cardiac myocytes in particular in $\mathrm{HI} / \mathrm{HG}$ rabbits. Concomitantly, the activities of complex I, III and V were compromised in the left ventricle biopsies of both hyperglycemic groups, in particular in the HI/HG group. Both normoglycemic groups revealed no changes in ultra-structure and complex activity compared to healthy controls. Compromised mitochondrial enzyme activities correlated with cardiac damage assessed by plasma levels of heart-fatty-acid-binding-protein, suggesting that mitochondrial protection mediated part of the prevention of organ failure.

Conclusions: In our animal model of prolonged critical illness, insulin ameliorated myocardial contractility but only when normoglycemia was maintained concomitantly. Maintaining normoglycemia and not glycemia-independent actions of insulin appear crucial for preserving mitochondrial function in the myocardium.

\section{BAPC1-3}

The protective role of carbon monoxide in ventilator induced lung injury

A. Hoetzel, Y. Zhang, A.M.K. Choi

Pulmonary, Allergy, and Critical Care Medicine, University of Pittsburgh Medical Center, Pittsburgh, USA

Background and Goal of Study: Ventilator induced lung injury (VILI) still remains a major cause of death in intensive care medicine. Carbon monoxide (CO) has been shown to exert anti-inflammatory, anti-oxidative, and antiproliferative effects. Here, we hypothesized that $\mathrm{CO}$ might reduce lung injury during mechanical ventilation.

Materials and Methods: C57/BL6 mice ( $n=5 /$ group) were ventilated with $12 \mathrm{ml} / \mathrm{kg}$ tidal volume for 1,4 , and $8 \mathrm{~h}$, with room air or air $+250 \mathrm{ppm} \mathrm{CO}$. Control mice were sham operated. Blood samples were taken to measure blood gases. Bronchoalveolar lavage (BAL) and lung tissue were analyzed to determine total cell and neutrophil count, cytokine release, and heat shock response, respectively.

Results and Discussions: Mechanical ventilation without $\mathrm{CO}$ led to an increase in neutrophil and total cell count in BAL after 4 and $8 \mathrm{~h}(\mathrm{p}<.05)$ of ventilation as compared to sham. Furthermore, heat shock protein 70 and heme oxygenase- 1 were up regulated in lung tissue. In contrast, additional CO-application attenuated the augmentation of neutrophil and total cell count $(p<.05)$ in BAL as well as the up regulation of heat shock proteins during ventilation. These findings show that $\mathrm{CO}$ reduces lung injury that results from mechanical ventilation. Most interestingly, while the ventilationinduced IL-6 release was decreased in the presence of $\mathrm{CO}$ in the BAL, tissue levels of IL-6 were further increased by CO treatment as compared to ventilation alone. This data indicates that $\mathrm{CO}$ might disturb neutrophil migration and cytokine release into the alveolar space.

Conclusion(s): 1) VILI occurs even with tidal volumes as low as $12 \mathrm{ml} / \mathrm{kg}$ that are used in clinical routine. 2) $\mathrm{CO}$ in low dose decreases lung injury that is observed with ventilation alone. 3) We suggest that the protective mechanism involves the inhibition of neutrophil migration and cytokine release by $\mathrm{CO}$.

\section{BAPC1-4}

Indirect markers of pulmonary endothelial dysfunction correlate with high-altitude induced pulmonary hypertension M.M. Berger, P. Bärtsch, H. Mairbäurl, E.R. Swenson, C. Dehnert Department of Anesthesiology, University of Heidelberg, Heidelberg, Germany

Background and Goal of Study: Hypoxia-induced pulmonary hypertension plays a major role in the pathophysiology of hypoxic pulmonary edema formation (e.g. high-altitude pulmonary edema; HAPE). We hypothesized that the rise in pulmonary artery pressure (PAP) during hypoxic exposure is related to impaired pulmonary endothelial function with reduced nitric oxide and enhanced endothelin-1 (ET-1) bioavailability.

Materials and Methods: 34 participants were investigated at low altitude ( $110 \mathrm{~m}$ ) and after rapid ascent to high altitude ( $<24$ hours to $4559 \mathrm{~m})$. 3-4 hours after arrival at high-altitude blood samples were drawn via a central venuous and a radial artery catheter, respectively, for determination of plasma nitrite (measured by flow injection analysis technique), plasma ET-1 (measured by radioimmunoassay), and plasma catecholamines (noradrenaline, adrenaline measured by HPLC). Systolic PAP (PASP) was estimated by transthoracic doppler-echocardiography, and chest radiography was used to diagnose pulmonary edema. In subjects developing HAPE the presented data were collected before the onset of edema formation. Data are expressed as Mean \pm SEM, a p-value $<0.05$ indicates statistical significance.

Results and Discussion: After ascent to high altitude arterial $\mathrm{pO}_{2}$ decreased to $38 \pm 0.5 \mathrm{mmHg}(p<0.001)$ and PASP increased from $23 \pm 0.7 \mathrm{mmHg}$ to $37 \pm 2 \mathrm{mmHg}(p<0.001)$. HAPE developed in 4 participants. Central venuous ET-1 plasma levels increased about 3 -fold $(p<0.001)$ while plasma nitrite remained stable $(p=0.331)$. At low altitude arterial-central venous (ACV) plasma gradients were negative for ET-1 and positive for nitrite $(p<0.001)$. They reversed after ascent to high-altitude $(p<0.05)$ and significantly correlated with PASP (ET-1: $R=0.49, p<0.001$; nitrite: $R=-0.21, p<0.05)$. ACV plasma gradients of ET-1 and plasma nitrite showed an inverse correlation $(R=-0.48 ; p<0.001)$, indicating a reciprocal regulation. Central venuous plasma levels of noradrenaline and adrenaline increased about 2-fold $(p=0.001)$ and 1.6 -fold $(p<0.05)$, respectively, but showed no correlation with PASP $(p=0.118$ and $p=0.594)$. 
Conclusion: These reults indicate that an impairment of pulmonary endothelial function with a shift in the balance between plasma ET-1 and nitric oxide is of major significance for hypoxia-induced pulmonary hypertension.

\section{BAPC1-5}

Protective effects of sevoflurane preconditioning on Oxygen-Glucose Deprivation injury. Role of reactive oxygen species and adenosine triphosphate-regulated potassium channels

L. Velly, B. Guillet, P. Canas, P. Pisano, N. Bruder

Bouches du Rhone, DAR Adultes, CHU Timone, Marseille, France

Background and Goal of Study: The purpose of the present study was first to compare the extent of sevoflurane-induced neuroprotection on primary cortical cells cultures subjected to transient oxygen-glucose deprivation (OGD) either when applied during or before (preconditioning) the insult. Secondly, the possible involvement of intracellular reactive oxygen species (ROS) levels and $\mathrm{K}_{\text {ATP }}$ channels in the mechanism of the preconditioning effect of sevoflurane (SEVO) was explored.

Materials and Methods: Mature primary cortical neuronal-glial cultures were exposed to 90 min OGD followed by reoxygenation. Culture were randomly exposed to SEVO either during OGD without preconditioning or during a 90 min preconditioning period followed by a $60 \mathrm{~min}$ wash-out period before OGD. 2-mercaptopropionyl glycine (2MPG) a free radical scavenger or glibenclamide (GLB) a blocker of $\mathrm{K}_{\text {ATP }}$ channels were added during the preconditioning. Twenty-four hours after the injury, neuronal death was quantified by lactate dehydrogenase $(\mathrm{LDH})$ release into the media. Free radical generation in cells was assessed after preconditionning period using $2^{\prime}, 7^{\prime}$-dichlorofluorescin diacetate (DCFH-DA). Data are presented as mean \pm SD. Statistical analysis used ANOVA.

Results and Discussions: Twenty-four hours after our cell cultures were subjected to $90-\min$ OGD, $78.5 \pm 4.4 \%$ of the neurons died. SEVO added at the start of the injury, elicited a potent and dose-dependent neuroprotective effect. SEVO preconditioning elicited a threshold neuroprotective effect at concentrations higher than $0.03 \mathrm{mM}$. In the presence of $2 \mathrm{MPG}(100 \mu \mathrm{M})$ or GLB $(0.3 \mu \mathrm{M})$ sevoflurane lost its preconditioning effect as assessed by LDH test. In normoxic cultures preconditioned by SEVO (3.4 mM), DCFH-DA fluorescence intensity increased in a significant manner compared to sham wash cultures. The SEVO preconditioning-induced increase in ROS levels was inhibited by $2 M P G$ and GLB.
Conclusions: Sevoflurane preconditions neuronal-glial cell cultures against OGD by mechanisms that apparently involve release of ROS and $\mathrm{K}_{\text {ATP }}$ channels.

\section{BAPC1-6}

Caffeic acid phenethyl ester reduces mortality and sepsis induced injury in rats

H. Fidan, O. Sahin, Y. Yavuz, A. Kilbas, Z. Cetinkaya

Department of Anesthesiology, Afyon Kocatepe University, Afyon, Turkey

Background and Goal of study: Sepsis and ensuing multi organ failure continue to be the major causes of morbidity and mortality in the intensive care units. Nuclear factor-Kappa beta (NFKB) activation is supposed to be one of the targets in the treatment of sepsis and ensuing mortality. We studied the effectiveness of caffeic phenethyl ester (CAPE), a known NFKB inhibitor, in cecal ligation and puncture (CLP) induced sepsis and lung injury. Materials and Methods: 80 rats are randomized to 5 groups that included 8 rats for the mortality study and the rest 8 rats for histopathological and biochemical study. All rats were operated to induce sepsis with cecal ligation and puncture (CLP) except control and CAPE groups that were operated just with laparotomy. CAPE $(50 \mu \mathrm{g} / \mathrm{kg})$ was administered to rats intramuscularly at the time of operation in CAPE and CAPE+Sepsis(0) groups. CAPE was administered to rats in CAPE+Sepsis(12) group 12 hours after CLP. Rats were observed for mortality. 8 rats from each group were sacrificed 24 hours after CLP; blood was taken for interleukin 1, IL-6, IL-10 and TNF- $\alpha$ study and right lung was taken out for histopathological, and left lung was taken out for oxidative stress parameters. Apoptosis was examined with Tunnel staining. Induced nitric oxide synthase(iNOS) and heat schock protein(HSP70) were examined with immunohistochemistry. Malonlydialdehyde (MDA), catalase (CAT), superoxide dismutase (SOD) and glutathione peroxidase (GSH-Px) were studied for oxidative stress evaluation.

Results and Discussion: Mortality was significantly decreased in CAPE+ sepsis(0) (3deaths/8rats) and CAPE+sepsis(12) (3deaths/8rats) groups compared to the sepsis group (8deaths/8rats). IL-1, IL-6, and IL-10 increased except TNF- $\alpha$ levels in sepsis group compared to control group. All cytokine levels were similar to control levels only in CAPE+sepsis(12) group. Apoptosis, iNOS and HSP70 evaluation were significantly changed between all groups in following order; control $<$ CAPE $<$ CAPE + sepsis(12) $<$ CAPE + sepsis(0) $<$ sepsis. SOD and GSH-Px levels were not different among groups. MDA, CAT were increased sepsis.

Conclusion: CAPE reduced mortality in sepsis and histopathological changes best when it was administered after sepsis formation.

\section{Evidence based practice and quality assurance}

\section{AP1-1}

\author{
Can we predict those patients who require anaesthetic \\ assessment for major colorectal surgery? \\ B. Langham, R. Elliott, J. Low \\ Department of Anaesthesia, Derby Hospitals NHS Foundation Trust \\ Derby, United Kingdom
}

Background and Goal of Audit: Major colorectal surgery can have significant morbidity and mortality in the peri-operative period. We feel that it is important to identify high-risk patients before a date for surgery is confirmed to allow time for informed consent, investigations and optimization. The aim of the audit was to look at current practice and identify from this patients who should be assessed by a Consultant Anaesthetist prior to listing for surgery compared to the number who were seen.

Materials and Methods: We audited 102 consecutive patients undergoing colo-rectal surgery. A questionnaire was completed by the Consultant Anaesthetist at operation and consisted of a number of patient demographic questions and whether the Anaesthetist had seen the patient before listing for surgery, and if not would they have found it of benefit.

Results and Discussions: Results were analyzed by dividing the patients into 2 groups. Group $1(n=45)$ : all patients who had been seen by an anaesthetist or those we would have liked to have seen prior to listing for surgery (44\% of total patients seen). Group $2(n=57)$ : all other patients. Data was analyzed using the Mann Whitney $U$ test which compares Group 1 versus Group 2 for ASA grade, age, number of chronic diseases, number of drugs taken and chronic health score. $\mathrm{P}<0.001$ for all categories. Further analysis suggested that by looking at 2 categories and assessing all those with an
ASA category of 3 or higher, or all above 79 years of age, we would ensure that we captured $98 \%$ of the patients we wished to see.

Conclusion(s): We would suggest that in our hospital best practice could be achieved by requesting to see all patients (for major colorectal surgery) who were ASA 3 or above or over 79 years of age. Within our institution this would average out at 2 patients per week involving 1 to 1.5 hours Consultant time. This may be useful for other centers who wish to assess the cost and time implications of offering anaesthetic pre-assessment to colorectal patients.

\section{AP1-2}

\section{Anaesthesiologist view by future nurses and pharmacists}

S. Parente, R. Loureiro

Anaesthesiology, S. Francisco Xavier Hospital, Lisboa, Portugal

Background and Goal of Study: Knowledge of the activities and responsibilities along with communication between health care professionals represent one of the key factors for the improvement of health care quality.

How are anaesthesiologists and anaesthesia understood by future non medical health care professionals.

Materials and Methods: Last year students of pharmacy and nursing filled in 256 valid questionnaires. The answers were analyzed according to professional group and age.

Results and Discussions: It is clear the influence of the academic background of future professionals with future pharmacists focusing mainly on the interface between the anaesthetists and the drugs and to a lesser degree with the patient (e.g. pain management $<5 \%$ ). Future nurses are more focused 
on monitoring and management of patients by the anaesthetists in operating room and post anaesthesia care.

Anaesthesiologist activities in emergency medicine, intensive care and even pain management are in general not recognized or minimized.

Conclusion(s): From the data gathered it is clear the need to make more visible the scope of knowledge and activities of anaesthesiologists. This is in our view even more crucial if one consider that the results were obtained from future professionals and the importance of implicit and explicit knowledge of one's skills inside a team

Reference:

To Err Is Human: Building a Safer Health System, Institute of Medicine, 2000

\section{AP1-3}

\section{Efficient operating room management: an empirical} analysis of german hospitals

M. Berry, T. Berry-Stoelzle, A. Schleppers

Institute for Anesthesiology and Operative Intensive Medicine, Klinikum Mannheim, Mannheim, Germany

Background and Goal of Study: The goal of this study is to evaluate the cost efficiency of different OR management forms empirically.

Materials and Methods: We use cross-sectional data from a survey of all Anesthesiology departments in German hospitals for the year 2002. We develop hypotheses about the effect of structural, organizational and workflow components of the OR management process on OR efficiency. These components are taken out of existing management literature as well as suggestions from practitioners. To test our hypotheses, we develop a new proxy for operating room efficiency and regress variables which describe the different OR management forms on this proxy.

Results and Discussions: While the size of the hospital is the most important factor for increasing OR efficiency, the enforcement capacity of the OR manager is the single influential factor which is independent of external constraints. A hospital cannot change its size easily, but can equip its OR manager with the power necessary for this position.

Conclusion(s): The analysis shows that while structural characteristics have an important influence on OR efficiency, the implementation of the elementary management functions planning and controlling are critical to efficient OR performance.

\section{AP1-4}

\section{The ratio between anaesthesia and surgical procedure} time as a controlling tool in the operating room

T. Kaufmann, C. Cheah, G. Schuepfer

Department of Anesthesiology, Kantonsspital Lucerne, Lucerne, Switzerland

Background and Goal of Study: Anaesthesia time (AT) and incision-to-suture time (IST) are important variables representing the production process in the Operating room (OR). The Ratio ( $R=A T / I S T)$ between these two times is dependant on different variables and may also reflect the productivity in an OR. Therefore these times were analysed and assessed for simple influencing factors such as emergency versus elective situations and the surgical disciplines. Materials and Methods: 40024 consecutive anaesthesia records of a general hospital were included in this study representing a 2 year period. 435 records had to be excluded because of inconsistent data. At is defined as the time from the first contact with the patient till its transfer to the PACU or ICU. The Ratio (R) of AT divided by IST was calculated.

Results and Discussions: The mean surgical duration of a procedure (IST) was 66 minutes $(\mathrm{min}) \pm 69$ standard deviation (S.D.) min for all 39589 cases (median: $45 \mathrm{~min}$ ). The mean $\mathrm{R}$ was 2,62, the median 2,27 and the mode $2,0 \pm 6,25$ S.D. 30698 (77,54\%) procedures were elective and 8891 emergency cases. Mean ART, IST and R were statistically different between elective and surgical cases $(p<0.01, R$ elective mean $2,9 \pm 3.2$ S.D. and $R$ emergency mean $3,2 \pm 4,0$ S.D.). The following surgical disciplines were included (mean R): General Surgery: $20,83 \%$ (R 2,97), plastic surgery $6,87 \%$ (R 3,5), obstetrics and gynaecology $11,72 \%(R 3,2)$, vascular $3,08 \%(R 2,8)$, orthopaedics 5,5\% (R 2,73), urology $4 \%(R 2,97)$, ophthalmology $16,18 \%$ (R $2,64)$, paediatrics $14,23 \%$ (R 3,4$)$, ENT $8,92 \%$ (R 2,9), mandible-facial surgery $2,28 \%(R 2,51)$, adult heart surgery $2,51 \%(R 2,12)$ and neurosurgery $1,29 \%(R 2,4)$. In a regression model the surgical discipline ( $p$ 0.03) and case urgency (p0.02) influenced R statistically significant.

Conclusion(s) and discussion: $R$ is dependant of the surgical discipline and the urgency of the case. It is a variable tool together with other data to analyze productivity in the OR, but $\mathrm{R}$ is clearly dependant on the definition of AT, which may also influenced by local settings.

\section{AP1-6}

Measurements of psychomotor performance of anesthesiologists during the 24-hours in-hospital call

M. Carev, N. Karanovic, A. Ujevic, G. Kardum, Z. Dogas

Department of Anesthesiology and Intensive Care, University of Split School of Medicine, Split, Croatia

Background and Goal of Study: Anesthesiologists' work along with extended duty shifts is combined with intensive stress (1). Moreover, anesthesia practice requires sustained vigilance, parallel decision-making, and fine motor skills. The aim of this study was to find out the impact of sleep deprivation, fatigue and stress on anesthesiologists' psychomotor performances, measured by the CRD (Complex Reactionmeter Device), a computer based psychometric system, able to detect even discrete psychological and mental changes (2). Materials and Methods: After the Medical Ethics Committee approval and informed consent, 27 staff anesthesiologists (35-55 yrs) were tested. A battery of 4 computer-generated cognitive psychological tests was used to record 2 parameters of cognitive performance: total test solving time (TTST - describing speed of reactions), and variability of reaction time (VRT-attention, alertness). Two tests were made during on call (8 am and 4 pm i.e. D8, D16), and two during the ordinary working day ( $8 \mathrm{am}, 4 \mathrm{pm}$, i.e. WD8, WD16). ANOVA for repeated measures and LSD post-hoc test were used.

Results and Discussions:

\begin{tabular}{lllcclcc}
\hline Tests & Tasks & & D8 & D16 & WD8 & WD16 & p \\
\hline AO & 35 & TTST & $141 \pm 37$ & $141 \pm 35$ & $118 \pm 28$ & $113 \pm 21$ & $<0,001$ \\
& & VRT & $53 \pm 18$ & $65 \pm 17$ & $49 \pm 11$ & $43 \pm 9$ & 0,001 \\
SV & 35 & TTST & $49 \pm 10$ & $50 \pm 11$ & $45 \pm 7$ & $44 \pm 5$ & $<0,001$ \\
& & VRT & $17 \pm 4$ & $17 \pm 4$ & $14 \pm 4$ & $15 \pm 4$ & 0,049 \\
DLP & 60 & TTST & $37 \pm 3$ & $38 \pm 4$ & $35 \pm 5$ & $36 \pm 6$ & 0,117 \\
& & VRT & $10 \pm 3$ & $11 \pm 2$ & $10 \pm 3$ & $12 \pm 4$ & 0,078 \\
LAC & \multirow{2}{*}{35} & TTST & $50 \pm 31$ & $45 \pm 12$ & $37 \pm 11$ & $36 \pm 11$ & 0,059 \\
& & VRT & $25 \pm 16$ & $25 \pm 10$ & $20 \pm 9$ & $19 \pm 11$ & 0,069 \\
\hline
\end{tabular}

$\mathrm{AO}=$ arithmetic operation, $\mathrm{SV}=$ spatial visualization, $\mathrm{DLP}=$ discrimination of light position, LAC = leg and arm coordination; results times given in seconds.

Conclusions: Comparing TTST and VRT, the test results were worse while on duty, implicating reduced speed of reaction, as well as attention and alertness. Anesthesiologists have impaired performance in cognitive complex reaction time tests during on call day, suggesting increased stress for an on call anesthesiologist, and this deserves further research. We consider $\mathrm{CRD}$ as a valuable tool for precise psychomotor testing.

References:

1 Anaesthesia 2006; 61: 856-66.

2 http://www.crd.hr/ (accessed on December 14, 2006).

\section{AP1-7 \\ Fast track rehabilitation of patients undergoing major abdominal surgery}

K. Oremus, V. Majeric-Kogler, D. Tonkovic, D. Korolija, M. Skegro

Anaesthesiology and intensive care medicine, University Hospital Center Zagreb, Zagreb, Croatia

Background and Goal of Study: It has been proposed that a fast track (FT) programme omitting preoperative fasting, bowel preparation, routine abdominal drainage and NG tube insertion, together with epidural analgesia, infusion volume restriction, early enteral nutrition and mobilization, can reduce perioperative stress and enhance recovery without compromising patient safety(1). Our aim was to implement such a protocol and verify its safety.

Materials and Methods: During a 6 month period 30 patients undergoing major abdominal surgery were treated according to FT principles. Median age 63 yrs (40-88), 18/30 female, 77\% (23) ASA I\&II, 23\% (7) ASAlll. Continuous 24 hour perioperative epidural infusion of levobupivacaine $0.125 \%$ \& tramadol $5 \mathrm{mg} / \mathrm{ml}$ commenced preoperatively, supplemented by NSAIDs postoperatively. All admitted for $24 \mathrm{hr}$ ICU observation, where enteral nutrition and mobilization started $5 \mathrm{hrs}$ postoperatively. VAS pain score assessed 4 hourly during $24 \mathrm{hrs}$. A matched control group (group C) of 30 patients operated during the same period received "traditional" care. Independent samples t-test and ANOVA were used for result analysis and group comparison.

Results and Discussions:

\begin{tabular}{llll}
\hline & Mean (FT/C) & Range & $\mathrm{p}$ \\
\hline First stool passage(day) & $2,6 / 4,28$ & $1-5 / 2-7$ & $<0.001$ \\
Discharge (days) & $7,64 / 10,44$ & $6-18 / 7-16$ & $<0.001$
\end{tabular}

No significant differences in VAS scores and complication rates between groups. No complications needing surgical revision in FT group. Three FT patients had complications prolonging hospitalization (wound infection, urinary 
infection, central venous catheter related bacteriaemia), none of which directly related to any FT procedure.

Conclusion(s): Implementation of a fast track protocol resulted in improved patient recovery and a shorter hospital stay without increasing the complication rate compared to a traditional approach to perioperative care for patients undergoing major abdominal surgery ranging from oesophagojejunal to low colorectal anastomoses.

Reference:

1 Kehlet H. Br J Anaesth 1997; 78: 606-617.

\section{AP1-8}

\section{Factors influencing the length of time for induction of anesthesia}

R. Gonzalez-Rodriguez, E. Soler-Mándoli, J. Vallès, LI. Gallart,

J. Fernandez-Candil

Anesthesiology, Hospital del Mar, Barcelona, Spain

Background and Goal of Study: The time needed for induction of anesthesia $(T I A)$ is influenced by many factors. The aim of the study was to analyze variables described as significant in a recent observed-based study (1) and include a new set.

Materials and Methods: A retrospective observational study was performed with 14.342 charts from patients scheduled in the last 3 years. Data were directly collected from the automated Anesthesia record and Hospital databases. Variables analyzed were those previously described as significant (1): surgical service, ASA physical status (ps), resident teaching, anesthesiologists, techniques used (anesthetic, monitoring), and we also included the use of airway devices. ANOVA was performed to evaluate these factors.

Results and Discussions: The time of anesthesia induction was significantly increased by ASAps, anesthetic techniques, monitoring, airway devices, anesthesiologists, surgical service and resident teaching.

Table 1. TIA as a function of ASAps and primary anesthetic technique

\begin{tabular}{llllll}
\hline & ASA I & ASA II & ASA III & ASA IV & P value \\
\hline General & $26 \pm 0.4$ & $32 \pm 0.4$ & $37 \pm 0,6$ & $36 \pm 2.3$ & $<0.0001$ \\
$\mathrm{n}$ & 1642 & 3464 & 1829 & 162 & \\
Spinal & $20 \pm 0.5$ & $29 \pm 0.4$ & $33 \pm 0,6$ & $38 \pm 2.1$ & $<0.0001$ \\
$\mathrm{n}$ & 973 & 2188 & 1279 & 137 & \\
\hline
\end{tabular}

$\mathrm{TIA}$ is presented as mean \pm Std. Error; ASA $=$ American Society of Anesthesiologists

Table 2. TIA in cases with invasive monitoring

\begin{tabular}{lccc}
\hline & No & Yes & P value \\
\hline AC & $37 \pm 1,0$ & $50 \pm 0.9$ & $<0.0001$ \\
& 716 & 1255 & \\
CVP & $40 \pm 1,2$ & $47 \pm 0.8$ & $<0.0001$ \\
& 481 & 1490 & $<0.0001$ \\
PAC & $44 \pm 0,7$ & $70 \pm 5.0$ & \\
& 1888 & 83 &
\end{tabular}

TIA is presented as mean \pm Std. Error

$\mathrm{AC}=$ Arterial Catheter; CVP = Central venous pressure;

$\mathrm{PAC}=$ Pulmonary Artery Catheter

Table 3. TIA in cases with different airway devices

\begin{tabular}{lc}
\hline LM & $23 \pm 0.7$ \\
& 505 \\
OTI & $33 \pm 0.3$ \\
& 6455 \\
DLT & $57 \pm 3.0$ \\
& 183 \\
\hline
\end{tabular}

$\mathrm{TIA}$ is presented as mean \pm Std. Error

$\mathrm{LM}=$ Laryngeal Mask;

$\mathrm{OTI}=$ Orotracheal intubation

DLT $=$ Double Lumen Tube

Conclusion(s): Our study demonstrates than there are several variables than extend time of anesthesia induction. Added to a recent study (1) the use of airway devices were predictors of prolonged TIA. These factors should be taken in the account to optimize operating room schedule.

Reference:

1 Escobar A. Anesth Analg 2006; 103: 922-927.

\section{AP1-9}

Effects of an OR Manager on Workflow Effectiveness and Organizational Hierarchy in the OR Suite

M. Berry, A. Schleppers

Institute for Anesthesiology and Operative Intensive Medicine, Klinikum

Mannheim, Mannheim, Germany
Background and Goal of Study: The goal of this study is to identify how the existence of and the perceived effectives of an OR manager changes workflow and hierarchical relationships in the OR suite.

Materials and Methods: We use data from a survey of all Anesthesiology departments in German hospitals for the year 2005. This survey includes not only questions to the status quo of the OR management process but also on how anesthesiologists would rate this process at their institution, and how they would improve it. We then analyze for each component of the OR management process separately whether hospitals with a satisfying OR management are different from the others, and whether anesthesiologists which are unsatisfied with the OR management recommend improvements already in place in satisfying hospitals. We are particularly interested in how the OR manager is integrated into the existing personnel structure.

Results and Discussions: We found a number of OR-management significant correlations. For example, the presence of an effective OR manager correlates significantly ( $p=0.001$, Spearman's rho) with a more effective management of OR schedule changes. On the hierarchy side, the presence of a stronger OR manager correlates significantly ( $p=0.001$, Spearman's rho) with a decreased influence in the OR of the Chief of Surgery.

Conclusion(s): We find that the appointment of an OR manager is associated with a number of workflow effects and changes personnel hierarchy. These effects are overwhelming positive; however, some do not reach significance.

\section{AP2-1}

\section{Auditing our audits. Are we completing the cycle?}

\section{E. Duff}

Anaesthetic department, University Hospital of Wales, Cardiff, United Kingdom Background and Goal of study: There is great emphasis on a comprehensive programme of clinical audit with it lying at the heart of clinical governance[1]. The audit cycle consists of choosing a topic, observing practice, setting standards, comparing practice with standards, implementing change and observing new practice (six stages). An assessment of the standard of clinical audit was completed in three directorates (medicine, surgery groups, women and children).

Materials and Methods: A quantitative retrospective assessment of clinical audit in three directorates between April 2003 and April 2005 was performed. Levels of audit completed were defined as full audit (FA, 5 out of 6 stages), partial audit (PA, 3 stages), potential audit (PO, 2 stages), planning audit (PL, topic chosen, intentions outlined), no audit (NA, not considered to be audit)[2]. The target standard set was that $100 \%$ of audits should fulfill criteria for being full audits.

Results and Discussions: Findings are presented in the table as number performed (percent). The results for anaesthesia are included within the surgery group

\begin{tabular}{lrlllrr}
\hline & \multicolumn{1}{l}{ FA } & PA & PO & PL & \multicolumn{1}{l}{ NA } & Total \\
\hline Med & $78(31)$ & $30(12)$ & $20(9)$ & $84(34)$ & $34(14)$ & 248 \\
Surg & $92(33)$ & $54(19)$ & $28(11)$ & $68(25)$ & $30(12)$ & 272 \\
Anaes & $7(12)$ & $10(17)$ & $18(32)$ & $16(28)$ & $6(11)$ & 57 \\
W\&C & $106(44)$ & $62(26)$ & $12(5)$ & $46(19)$ & $16(6)$ & 236 \\
\hline
\end{tabular}

Implementing change: $11 \%$ of the audits in anaesthesia led to change in practice. Re-audit: $1.7 \%$ of all audits in anaesthesia were re-audited. Conclusions: Re-education on the basic principles of clinical audit and regularly "auditing the audits" will help derive maximum benefit from good quality audits. Re-auditing after implementing recommendations is essential to ensure clinical practice has changed and to establish improvements are sustained. References:

1 NICE. 2002.

2 Derry J, Lawrence M, Griew K et al. BMJ 1991; 303: 1247-9.

\section{AP2-2}

Does preoperative ECG testing offer any advantage over clinical risk scoring in the prediction of postoperative cardiovascular morbidity and mortality

G. Howard-Alpe, J.W. Sear, J. Giles, K. Anderson, P. Foex

Nuffield Department of Anaesthetics, University of Oxford, Oxford, United Kingdom

Background \& Goals: Patients undergoing vascular surgery have a high incidence of postoperative (postop) complications[1]; in order to predict and potentially prevent these, investigations as well as scoring systems based on clinical information may be used. We have compared the utility of the Revised Cardiac Risk Index (RCRI) with 3 simple, non-invasive tests using 
electrocardiography (ECG) for detecting pre-existing cardiac disease to ask whether these tests offer greater predictive ability.

Materials \& Methods: 222 vascular pts were recruited after informed consent and ethics committee approval. Full clinical assessment was made, together with routine clinical biochemistry, allowing determination of the RCRI.[2] A preoperative (preop) resting 12-lead ECG was performed and the presence of abnormalities noted (rhythm other than sinus, LVH, left axis deviation, ST seg $\downarrow$, or $\geqslant 5$ VEs). QTc dispersion was calculated from the resting ECG (abnor$\mathrm{mal} \geqslant 60 \mathrm{msec}$ ). Preop ambulatory ECG (AECG) monitoring was performed for silent myocardial ischaemia (SMI). The follow-up period was $12 \mathrm{mths}$ after surgery and the timing of all adverse cardiovascular events was noted (cardiac death, MI, unstable angina, CCF, CVA or significant arrhythmia). The association between an abnormal clinical test and adverse outcome were made by $\mathrm{Chi}^{2}$ analysis; calculation of relative odds ratio, and the positive and negative predictive values of the 3 different investigations.

Results \& Discussion: 63 pts were excluded from the final analysis (equipment failure, non-analyzable ECG), leaving 159. The preop ECG showed abnormalities in 70 pts; while SMI occurred in 30. Abnormal QTc dispersion was seen in 129 subjects. There were 24 events in the $1^{\text {st }} 30$ days, with a further 12 in the next 11 mths. There were no associations between any of the 3 preop investigations and postop outcomes. However, there was an association between the RCRI and outcome at $12 \mathrm{mths}\left(X^{2}=6.068 ; p=0.0482\right)$. Conclusions: Preop 12-lead ECG and AECG monitoring give a poor indication of the likelihood of complications in vascular surgery pts. The RCRI has a role in identifying pts who may benefit from preop cardiological referral. References:

1 Mangano DT, Goldman L. N Engl J Med 1995; 333: 1750-6.

2 Lee TH, Marcantonio ER, Mangione CM, et al. Circulation 1999; 100: 1043-9.

\section{AP2-3}

\section{Cause of failure of cannulation the internal jugular vein}

I. Asouhidou, K. Natsis, Th. Asteri, P. Sountoulides, P. Tsikaras

Cardioanesthesia, G.Papanikolaou, Thessaloniki, Greece

Background and Goal of Study: Internal jugular vein (IJV) catheterization is a common procedure in anesthesiology and critical care everyday practice, however anatomical variations of the IJV might prevent cannulation while repeated efforts might lead to complications such as arterial puncture, hematoma formation, pneumothorax, injury to the trachea, vein thrombosis. Cannulation is not always successful and uneventful mainly due to anatomical variations of the size and/or location of the IJV.

Materials and Methods: Autopsy findings in 93 human cadavers revealed the presence of a hypoplastic left IJV in 3 cases (3/93, 3.2\%).

Results and Discussions: In first case the diameter of the left IJV was $3 \mathrm{~mm}$, while in the other two cases the diameter was about $5-6 \mathrm{~mm}$. In both cases the external jugular veins were larger than average. In the first case there was a local hematoma formation due to unsuccessful cannulation. Alderson et al. studied a group of children under the age of 6 and found $4 \%$ to have very small diameters of right IJV. ${ }^{1}$ Small size $(<5 \mathrm{~mm})$ located in the normal position were found in $8,7 \%$ of both the right and the left IJV. ${ }^{2}$ Denys and Uretsky also reported in their adult series that unusually small veins ( $\leqslant 5 \mathrm{~mm}$ ) were seen in six $(3 \%)$ patients. ${ }^{3}$

Conclusion(s): These findings suggest that anatomical variation may partly account for the inability to cannulate the internal jugular vein in certain patients. Taking into consideration the frequency of anatomical variations of IJV, one should consider to attempt an ultrasound-guided puncture for safe and effective cannulation of these veins.

\section{References:}

1 Alderson PJ, Burrows FA, Stemp LI, et al. Use of ultrasound to evaluate internal jugular vein anatomy and to facilitate central venous cannulation in paediatric patients. $\mathrm{Br}$ J Anaesth 1993; 70: 145-8.

2 Lin B.S, Kong C.W, Tarng D.C et al. Anatomical variation of the internal jugular vein and its impact on temporary haemodialysis vascular access: an ultrasonographic survey in uraemic patients. Nephrol Dial Transplant 1998; 13: 134-8.

3 Denys B.G., Uretsky B.F Anatomical variations of internal jugular vein location: impact on central venous access. Crit Care Med 1991; 19(12): 1516-9.

\section{AP2-4}

Quality indicators for anesthesia, the need for nationwide comparison of health care

A. Schuurhuis, J.B.M. Harbers, T.A. van Barneveld, Ch. Keijzer, P.L. Houweling

Anesthesiology, University Medical Center Utrecht, Utrecht, The Netherlands

Objective: To develop a nationwide quality control program for anesthesia based on indicators for anesthesia, as required by national health authorities for all medical specialties.
Materials and methods: The National Society of Anaesthesiology has selected 18 indicators out of an original set of 65 to be put on trial for this year. First focus is on reliable registration of structure and proces indicators, the next step will be registration and implementation of outcome indicators.

Results: The selected indicators are listed below.

Preoperative care:

Preoperative clinic. \% patients seen by anesthesiologist.

Fasting: \% that had clear fluids 2 hours before anesthesia.

$\beta$ blockers: $\%$ high-risk patients that had $\beta$-blockers preop. Intraoperative care:

Thermoregulation: $\%<35^{\circ}$ core-temperature postop Epidural for vascular and colorectal surgery: \% used

Epidural for lateral thoracotomy: \% used

Immediate availability of anesthesiologist.

Postoperative care:

Urinary retention: \% spinal with bladder volume measured

Recovery facilities: is 24 hour recovery available?

Recovery discharge criteria: \% with PAR-score determined

Pain treatment:

Severe postoperative pain: $\%$ VAS $>7$ (of 10), < first 72 hours

Acute pain service: availability and \% treated for postop pain

Epidural for delivery: availability 24 hours, 7 days

Life support:

Code for full/no resuscitation: \% recorded in medical records

Resuscitation committee: \% evaluated resuscitations

Advanced Life Support: available ALS team 24 hours $<5^{\prime}$

Basic Life Support: \% BLS trained hospital personnel

Conclusion: The feasability of the set is piloted in 2007 in 14 hospitals. Usefull indicators will be implemented nationwide and, in the future, can be used by health authorities and health insurance companies for evaluation of quality.

\section{AP2-5}

\section{Beta error free riskfactor identification based on SPELA, a neuro-evolution method}

T. Kaufmann, K. Wassermann, G. Schuepfer

Medical staff office, Kantonsspital Luzern, Luzern, Switzerland

Background and Goal of Study: Preoperative risk identification is important for resource allocation in perioperative medicine. A beta error free identification of risk factors assessed by anaesthetists would help to steer the perioperative process more efficiently.

Materials and Methods: Data for predictive modeling: 18788 patients for elective surgery has been selected in order to exclude a bias due to different baseline risk. 68 patients $(0.37 \%)$ died within 30 days. Using ASA risk classification, age, gender and 48 binary variables a risk stratification for postoperative mortality was made. The generated records were linked with the administrative database of the hospital. Therefore, mortality within 24 hours and 30 days after surgery was linkable with the two different classifications strategies. For predictive modeling with integrated validation a machine learning techniques has been applied. SPELA, a neuro-evolution method able to create models without type-II-errors and to identify interactions between variables, was used to simultaneously identify sub-sets of risk factors and typical risk profiles.

Results and Discussions: For $74.8 \%$ of all patients perioperative risk ( $t \leqslant 30$ days) could be excluded with a very low risk for a false positive statement (prob. $<10^{-5}$, AUC-ROC 0.82). The ,value of information' [Vol] for preaop-ASA is $78.8 \%$. For the remaining $25.2 \%$ risk is not zero. For $29.4 \%$ of all patients who died after 30 days it is possible to determine the risk elevation as 31.4fold (AUC-ROC 0.74, Vol for ASA <28.1\%). The initial models has been tested successfully by out-of-sample validation on a smaller sub population.

Conclusion(s): Determination of risk being free of type-ll-error is a new and important approach to predict postoperative moratlity in patients with destinct subset of risk factors identified preoperatively. The new methode may guide perioperative ressource allocation such as ICU or PACU care. Reference:

Yao X. (1999). Evolving Artificial Neural Networks. Proc. of the IEEE, Vol. 8, No. 9, 1423-1447 Sept.1999.

\section{AP2-6}

Beyond Medline: how much do other trial search strategies contribute to a systematic review in anaesthesia?

K. Walker, K. McGrattan, K. Aas-Eng, A.F. Smith

Anaesthesia, Royal Lancaster Infirmary, Lancaster, United Kingdom

Background and Goal of Study: Exhaustive multi-method searching is recommended [1] to locate all relevant randomised controlled trials (RCTs) for systematic reviews, but there are few empirical data to support the additional 
effort involved [2]. We used our data from a Cochrane review of ultrasound guidance of regional blockade to explore this question.

Method: We used expert, database-specific search strategies for Medline (1966 on), EMBASE (1974 on) and CENTRAL (the Cochrane Collaboration register of controlled trials). We handsearched 6 major anaesthesic journals and the annual meeting supplements of the European, American and Canadian anaesthetic societies, all from 2004 on. We also contacted researchers in the field. Results and Discussion: The Medline search missed none of the RCTs that were finally included. EMBASE and CENTRAL both found possibly relevant material missed by the Medline search. The eventual yields of the 3 databases are shown in the Table.

\begin{tabular}{lccc}
\hline & Medline & EMBASE & CENTRAL \\
\hline 'Hits' & 803 & 892 & 106 \\
Possibly relevant & 10 & 9 & 10 \\
In final review & 4 & 3 & 3 \\
Yield (\%) & 0.005 & 0.003 & 0.03 \\
\hline
\end{tabular}

Handsearching journals yielded no extra RCTs. Searching the meeting abstracts found 7 abstracts of otherwise unpublished RCTs. Personal contact yielded a further trial not yet indexed in the above databases, and a further RCT just submitted for publication.

Conclusions: Addition of a second database to Medline helped find more material. CENTRAL may be preferable as it contains only RCTs. Handsearching meeting supplements and contact with researchers is clearly of benefit when new technologies are still undergoing evaluation.

References:

1 www.cochrane.org/resources/handbook.

2 Dickersin K JAMA 2003: 290: 516-23.

\section{AP2-7}

Systematic review of effectiveness and safety of ultrasound guidance in regional neural blockade: preliminary findings

K. McGrattan, K. Walker, K. Aas-Eng, A.F. Smith

Anaesthesia, Royal Lancaster Infirmary, Lancaster, United Kingdom

Background and Goal of Study: Ultrasound (US) has been introduced into anaesthetic practice for the insertion of regional blocks. There have been many publications on the subject but few rigorous evaluations. We aimed to identify and collate all randomized controlled trials (RCTs) on the effectiveness and safety of this technique.

Method: We searched for RCTs studying the use of US, in comparison with another method of nerve location, in adults undergoing regional blockade as sole anaesthetic technique for surgery. We searched Medline (1996 on), EMBASE (1974 on) and CENTRAL, handsearched 6 journals from 2004 to October 2006 and abstract supplements of 3 major international anaesthesia conference from the last 3 years.

Results and Discussion: We identified 5 full reports of RCTs, including 338 patients. All studied the technique in brachial plexus blockade (axillary alone 2 trials, supraclavicular alone 1, infraclavicular alone 1, axillary/interscalene 1). Two compared US with electrical nerve stimulation, one with surface landmarks, one with a transarterial technique and one with US plus nerve stimulation. Outcomes assessed included time taken for insertion, onset time, quality/extent of block, need for supplementary injections, conversion to general anaesthesia (GA) and 'adverse events' (inadvertent arterial puncture, painful paraesthesiae). No single trial reported on all outcomes. In view of the diversity of techniques, outcomes and comparisons, we have not applied statistical meta-analysis.

The effect of US on the quality of sensory block is inconsistent, the effect size apparently being dependent on the control technique. However, US appears to reduce insertion time by between 2 and 5 minutes, reduce the need for GA and reduce the incidence of adverse events. The 8 trials we found published as abstracts showed similar findings. Their results will be incorporated when the full trial data are available.

Conclusion: Ultrasound guidance of brachial plexus blocks appears to offer some advantages, especially in terms of insertion time and complications. We will quantify this benefit when further full data appear.

\section{AP2-8}

Use of Personal Digital Assitant for managing artificial nutrition in intensive care unit after cardiovascular and thoracic surgery

Y. Attof, M. Hachemi, M. Cannesson, C. Chambrier, J.J. Lehot Service danesthesie reanimation, Hopital cardiovasculaire et pneumologique L.Pradel, Lyon, France
Background and Goal of Study: The aim of our study was to assess the effect of NutriPDA, a personal digital assistant (PDA)-based clinical decision-support system (CDSS) for the management of artificial nutrition. A CDSS was developed and implemented on a handheld computer for use in the ICU after cardiovascular and thoracic surgery. System impact was assessed in a prospective "before/after" cohort trial.

Materials and Methods: After informed consent we studied 61 patients in the postcardiovascular and thoracic surgery ICU (length of stay $>8$ days). Patients were divided into two groups (before and after the use of NutriPDA: Group A: 32 patients (4-month period in 2005); group B: 29 patients (4month period in 2006). Analysis of variance $(p<0.05)$ was performed to test difference between groups.

Results and Discussions: There were no significant differences in anthropometric and clinical parameters between the 2 groups. Energetic intakes were $<80 \%$ of basal energetic expenditures in $21 \%$ and $1 \%$ of patient, respectively $(p<0.001)$. Caloric and nitrogen intakes were beyond international recommendation in the Group A: $20 \pm 7 \mathrm{kcal} / \mathrm{kg} / \mathrm{d}$ (mean $\pm \mathrm{SD}$ ), $104 \pm 33 \mathrm{mg} / \mathrm{kg} / \mathrm{d}$, but not in the Group B: $26 \pm 4 \mathrm{kcal} / \mathrm{kg} / \mathrm{d}, 196 \pm 61 \mathrm{mg} / \mathrm{kg} / \mathrm{d}$ $(\mathrm{p}<0.001)$.

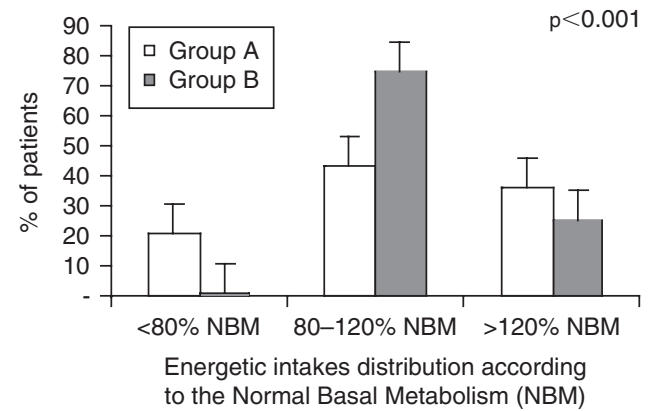

Conclusion(s): NutriPDA was found to be able to optimize artificial nutrition by improving caloric intake in ICU.

This new software has potential clinical applications.

Reference:

Heyland, et al. Canadian clinical practice guidelines for nutrition support in mechanically ventilated, critically ill adult patients. J Parenter Enteral Nutr 27(5): 355-73.

\section{AP2-9}

Renal function after laparoscopic donor nephrectomy

D. Vernetta, P. Sierra, J.C. Ortiz, D. Hernando, P. Baxarias

Anesthesia, Fundació Puigvert, Barcelona, Spain

Background and Goal of Study: Living donation in the field of renal transplantation has increased over time as well as the use of laparoscopic nephrectomy. Pneumoperitoneum, as used in laparoscopic surgery, may result in negative effects on renal function. This study was performed to evaluate renal function after laparoscopic donor nephrectomy (LDN).

Patients and Methods: A retrospective analysis of LDN was performed from March 2002 to December 2006. During laparoscopic kidney donation we adopted an strategies for "renal protection" to preserve kidneys from possible injuries associated with abdominal insufflation and adequate intravascular fluid volume. Serum creatinine was recorded and analyzed pre and post nephrectomy and we evaluated renal function by estimed GFR by mean of MDRD standardized IDMS. Associated variables were studied: age, weight, ASA, duration of procedure, blood loss, complications and re-operations. Results: During the study 100 LDN were performed (37\% males, $63 \%$ females and mean age $50.67 \mathrm{ys}$ ). The average duration of surgery was 227.5 minutes. Six laparoscopic procedure required conversion to laparotomy.

All de donors presented an alteration of the renal statistically significant function that was kept for one month.
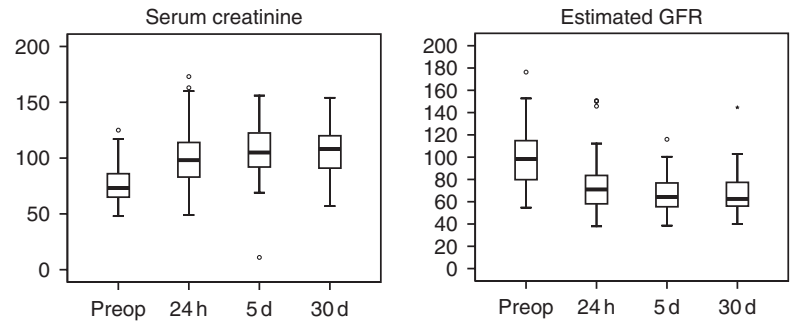
Conclusion: Our results confirmed that LDN is a safe procedure with a low morbidity. Despite that we adopted an strategy for "renal protection", there was an alteration of the renal function without clinical repercussions.

\section{AP2-10}

\section{Are SF-36 and QoR feasible tools to assess quality of} recovery after major abdominal surgery?

M. Arnberger, D. Inderbitzin, S. Jakob, R. Greif, A. Vogt

Department of Anesthesiology, University Hospital Bern, Bern, Switzerland

Background and Goal of Study: Besides morbidity and mortality quality of recovery has benn recommended as an important outcome after anaesthesia [1]. The SF-36 - a validated quality of life score - was used to show advantages of epidural anesthesia [2]. The quality of recovery score (QoR) was validated in English and German [3]. Quality of recovery after hepatic resection is unknown. We assessed the feasibility of the SF-36 and QoR (German versions) to determine the time course of postoperative quality of life and recovery. Methods: In 13 patients scheduled for hepatic resection a thoracic epidural was used for pain management and general anaesthesia was standardized. The SF-36 (7-day recall) was recorded preoperatively and at postoperative day 7 (pod7). The QoR was performed preoperatively and at pod1, pod3, pod5 and pod7. In addition the duration of interviews was recorded.

Results and Discussions: Physical and mental health decreased at pod7. QoR-duration of interview was shorter than SF-36 preop (by 8min) and at pod 7 (by $5.6 \mathrm{~min})(\mathrm{p}<0.001)$.
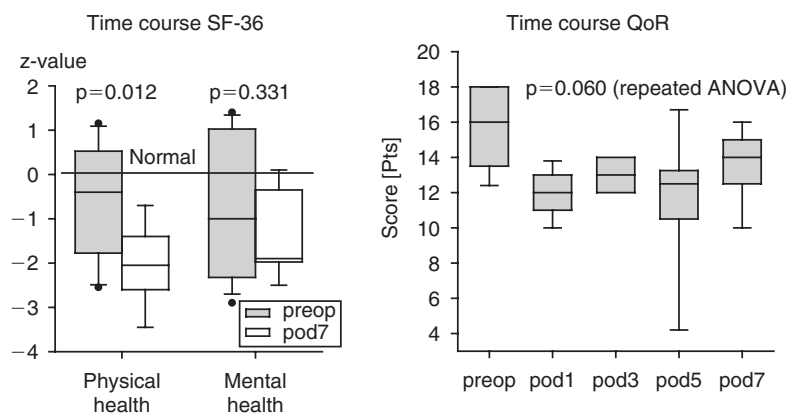

Conclusions: Major abdominal surgery has a significant impact on physical health $>7$ days. SF-36 and the QoR are feasible and valuable tools to evaluate this. The QoR Score may be superior in terms of short term quality of recovery because of shorter recall time of the items and time needed for assessment.

References:

1 Kehlet, H. and J.B. Dahl, Lancet, 2003: 362: 1921-8.

2 Carli, F., et al., Anesthesiology, 2002: 97: 540-9.

3 Eberhart, L.H., et al., Anaesthesist, 2002: 51: 463-6.

\section{AP3-1}

Practical Implementation of a peri-operative smoking cessation package. Experiences in a scottish DGH

C. Hawe, C. Cairns, A. Longmate

Anaesthesia and Intensive Care, Stirling Royal Infirmary, Stirling, United Kingdom

Background and Goals: Smoking is the biggest cause of preventable morbidity and mortality in the UK. Millions of smokers requiring surgery are at increased risk of respiratory, cardiovascular and wound related complications (1). According to Scottish national guidelines this risk should be presented to the patient, alongside the offer of cessation support (2). However, this may not be achieved because of lack of time, staff training, and perceived effectiveness. Planned elective surgery represents a window of opportunity for successful smoking cessation interventions as demonstrated by a recent Cochrane Collaboration Review (3). This study aimed to assess our existing pre-operative cessation advice and success rates, to develop an intervention package to support cessation and then to assess its impact.

Materials and Methods: Initially data was collected from 57 smokers attending for elective surgery, prior to introduction of the cessation package. The package included "brief advice training" for nurses involved in pre-operative care, improved written information for patients, and a publicity campaign, which involved written advice and encouragement from the GP and surgeon involved. After the package was implemented a further 30 smokers were assessed.
Results and Discussion: Post-intervention those who remembered receiving peri-operative smoking cessation advice increased from $37 \%(21 / 57)$ to $90 \%(27 / 30)$. This resulted in an increased cutting down rate from $21 \%$ $(12 / 57)$ to $87 \%(26 / 30)$. Recall of risks and available support also improved. Conclusions: This study demonstrates that brief advice training increases the likelihood of smokers receiving cessation advice and consequently, a reduction in peri operative smoking rate.

References:

1 Pearce A. Anaesthesiology 1984; 61: 576-584.

2 Smoking Cessation Guidelines for Scotland (2004); http://www.hebs.scot.nhs.uk/ services/pubs/pdf/SmokingCes2004.pdf.

3 Moller A. Interventions for preoperative smoking cessation. The Cochrane Database of Systematic Reviews 2005, Issue 3. Art. No.: CD002294. pub2.

\section{AP3-2}

Incidence of transurethral resection of the prostate syndrome in a regional hospital

F. Peramo, Jesus. Maldonado-Contreras, A. Maldonado-Campos, R. Escudero, E. Belinchon

Anestesiologia, Reanimacion y Tratamiento del Dolor, Anestesiologia, Granada, Spain

Background and Goal of study: Transurethral resection of the prostate syndrome (TURPS) may be a cause of death [1]. We conducted a study to know the incidence of TURPS among patients undergoing TURP with intradural bupivacaine. We aimed to identify those patients at greater risk and determine wich factors can predict outcome.

Materials and methods: For a period of 1 year (Nov. 2005 to Nov. 2006) all patients at Hospital San Cecilio scheduled for TURP entered the study. Every patient received a crystalloid preload (Hartmann's solution $8-10 \mathrm{~mL} / \mathrm{Kg}$ ) before spinal bupivacaine was administered $(0.5 \%, 12 \mathrm{mg}, \mathrm{L} 2-3 / \mathrm{L} 3-4,27 \mathrm{G}$ needle) and afterwards patients received Hartmann's solution $100-150 \mathrm{~mL} / \mathrm{h}$. Urologists used destiled water for TURP. Patient's management was not altered by being in the study and we prospectively recorded several variables of preoperative, intraoperative and postoperative period that would explain outcome. Every variable was binary and we tried to determine both variables associated with TURPS (chi square) and those that could predict TURPS appearing (multiple logistic regression model, stepwise method of variable selection). We used SPSS for windows 12.0 ( $p<0.05$ significant). Results: 104 patients entered the study. Patient's characteristics were (mean $\pm \mathrm{sd}$ ): age $72.38 \pm 2.3$ years, weight $76 \pm 4.8 \mathrm{~kg}$, height $1.70 \pm 5.36 \mathrm{~m}$, length of operation $51.3 \pm 9.4 \mathrm{~min}$ and prostate size $67.26 \pm 14.64 \mathrm{gr}$. TURPS appeared in 2 patients (1.9\%): 1 patient complaint of visual disturbances and agitation $\left(\left[\mathrm{Na}^{+}\right]: 123 \mathrm{mEq} / \mathrm{L}\right)$ while the other patient had hypotension, bradycardia $(45 \mathrm{bpm})$ and $\left[\mathrm{Na}^{+}\right]$was $132 \mathrm{mEq} / \mathrm{L}$. The variable associated with TURPS was length of operation greather than 55 min and we didn't find variables that could predict TURPS $(p<0.05)$.

Conclusions: In our study TURPS incidence is similar to other studies and this syndrome was associated with the surgery duration. No variable could predict TURPS appearing.

Reference:

1 Gyomber D. BJU Int 2006; 97: 758-761.

\section{AP3-3}

Preoperative carbohydrate improves postoperative patient recovery after thyroidectomy

E. Monfort, S. Lauwick, A. Kaba, M. Lamy, J. Joris

Anesthesia and Intensive Care Medicine, CHU Liège, Liège, Belgium

Background and Goal of Study: Preoperative oral administration of carbohydrate $(\mathrm{CHO})$ improves preop patient comfort. ${ }^{1}$ Its benefits on early postop recovery remain controversial. ${ }^{2,3}$ We investigated the effect of preop $\mathrm{CHO}$ on patient recovery after thyroidectomy.

Material and Methods: After IRB approval and informed consent, 200 ASA III women scheduled for thyroidectomy were enrolled in this randomized double-blind study. Patients were assigned to preoperative ( $2-3 \mathrm{~h}$ before surgery) oral administration of $50 \mathrm{~g}$ glucose in $400 \mathrm{~mL} \mathrm{H}_{2} \mathrm{O}$ or $0.5 \mathrm{~g}$ aspartam in $100 \mathrm{~mL}$ $\mathrm{H}_{2} \mathrm{O}$. Anesthesia was maintained with sevoflurane in $50 \% \mathrm{O}_{2}$ :air. Analgesia consisted of $2 \mathrm{mg} / \mathrm{kg}$ tramadol and $1 \mathrm{~g}$ paracetamol given iv $30 \mathrm{~min}$ before the end of surgery. Nausea, vomiting, antiemetics and analgesics requests, pain, thirst, hunger, fatigue, and anxiety ( $100 \mathrm{~mm}$ VAS) were recorded during 3 postop epochs: $0-2 \mathrm{~h}, 2-6 \mathrm{~h}$, and 6-24h. Data were analyzed using ANOVA, chi $^{2}$ or Students' $t$ test when appropriate; $P<0.05=$ statistically significant. Results: Patients data were similar in the two groups. Incidence and severity of PONV, and request for antiemetic did not differ between the two groups during each of the three epochs. Preop $\mathrm{CHO}$ significantly improved postop thirst $(P=0.01)$, hunger $(P=0.02)$, and fatigue $(P=0.02)$. Postop $(24 h)$ 
paracetamol consumption (median: $2 \mathrm{~g}$ vs $3 \mathrm{~g} ; P=0.008$ ) was also significantly less in the $\mathrm{CHO}$ group. No effect on anxiety was detected.
Thirst (100 mm VAS)

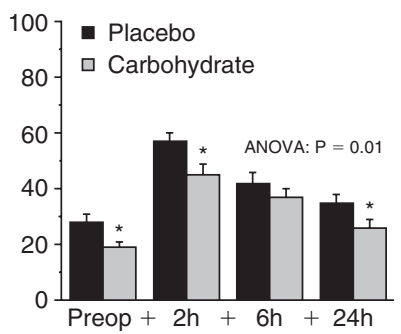

Fatigue (100 mm VAS)

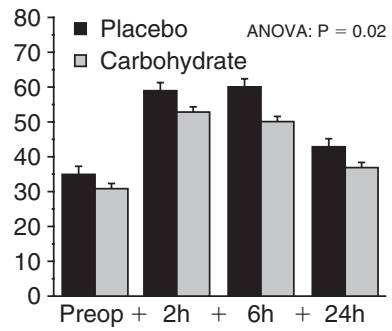

Conclusions: Oral preoperative carbohydrate improves postoperative patient recovery but does not reduce the risk of PONV after thyroidectomy References:

1 Hausel et al., Anesth Analg 2001: 93: 1344-50.

2 Hausel et al., BJS 2005: 92: 415-21.

3 Bisgaard T et al., BJS 2004: 91: 151-8.

\section{AP3-4}

Perioperative hyperglycaemia is similar in nondiabetic morbidly obese and nonobese patients undergoing prolonged laparoscopy

J. Pahaut, A. Kaba, A. DeRoover, M. Lamy, J. Joris Anesthesia and Intensive Care Medicine, CHU Liège, Liège, Belgium

Background and Goal of Study: Surgical stress results in perioperative hyperglycemia. ${ }^{1}$ Insuline resistance frequently develops in morbidly obese patients. ${ }^{2}$ We tested the hypothesis that perioperative hyperglycemia is greater in nondiabetic obese patients than in nonobese patients.

Materials and Methods: After IRB approval, 30 ASA I-III nondiabetic (normal $\mathrm{HbA} 1 \mathrm{c}$ and fasting glycemia) morbidly obese patients (BMl: $43.1 \pm 7.9 \mathrm{~kg} / \mathrm{m}^{2}$ ) scheduled for laparoscopic gastric bypass were compared to 20 nondiabetic nonobese patients (BMI: $26.4 \pm 3.1 \mathrm{~kg} / \mathrm{m}^{2}$ ) scheduled for laparoscopic colectomy. Anesthetic technique (sevoflurane in $\mathrm{O}_{2}$ :air) was standardized in all patients. After surgery, all patients were administered Glucose $10 \%$. $80 \mathrm{ml} / \mathrm{h}$. Blood glucose was measured before premedication, at induction of anesthesia, after tracheal intubation, every $30 \mathrm{~min}$ intraop, and then every $6 \mathrm{~h}$ postop for $24 \mathrm{~h}$. Data (mean $\pm \mathrm{SD}$ ) were analyzed using Students' $t$ test or ANOVA for repeated measures when appropriate; $P<0.05=$ statistically significant.

Results and Discussions: $\mathrm{HbA} 1 \mathrm{c}$ plasma concentrations were similar in nonobese $(5.5 \pm 0.5 \%)$ and obese $(5.6 \pm 0.6 \%)$ patients. Intra- and postop glycemia were not significantly different in morbidly obese and nonobese patients (Fi.).

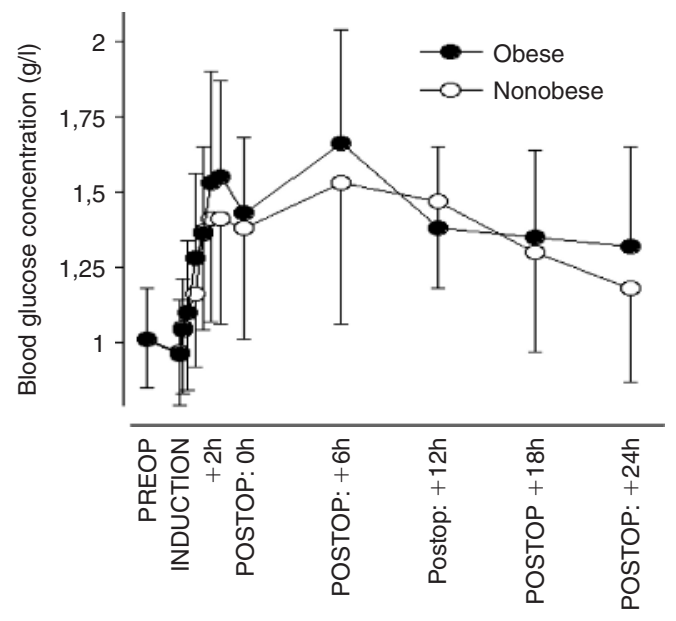

Conclusion(s): Despite well-documented insuline resistance in obese patients, surgical stress during prolonged laparoscopy results in similar hyperglycaemia in nondiabetic obese and nonobese patients

1 Weissman C, Anesthesiology 1990: 73: 308-27.

2 Shenkman Z et al., BJA 1993: 70: 349-59.

\section{AP3-5}

The use of remifentanil sedation in interventional radiology S. Uddin, I. Renfrew, F.M.L. Dancey, G. Mandersloot

The Royal London Hospital Anaesthetics Department, Barts and the London NHS Trust, London, United Kingdom

Background and Goal of Study: Advances in interventional radiology have expanded the type of procedures performed in the radiology suite. While this allows patients to avoid open surgery, the procedures can be prolonged and painful, rendering 'conventional' sedation used by radiologists inadequate to ensure patient comfort. Yet the increase in demand for anaesthesia has not been matched by an increase in access to general anaesthesia and theatre time ${ }^{1}$.

We developed a protocol for the use of remifentanil as an alternative analgesic/sedative administered as an infusion by a solo consultant anaesthetist (no additional theatre staff are required). All procedures are performed in the radiology department.

Materials and Methods: Patients are referred by the radiologists and assessed by the anaesthetist for suitability. Monitoring, including capnography, is established. The dose of remifentanil required depended on patient physiology and procedure, was given as a continuous infusion and ranged from 0.01 to $1 \mathrm{mcg} / \mathrm{kg} / \mathrm{min}$.

Results and Discussions: From August 2004 to December 2006, we performed a total of 47 procedures under remifentanil sedation (26 male, 21 female, age range 24 to 86 years, ASA grades $2-5$ ).

Procedures performed included: radiofrequency ablation of liver, renal or lung tumours (20), percutaneous transhepatic cholangiography, dilatation \& stent insertion (18), occlusion of vascular supply (5), superior vena cava stenting (3), insertion of percutaneous entrogastrostomy(1) Patient satisfaction was high and no patient required conversion to general anaesthesia. Conclusion(s): Whilst Remifentanil may not replace general anaesthesia for painful interventional radiology procedures, it is safe and effective and allows reduction in the demand on theatre resources.

Reference:

1 Martin ML, PH Lennox. Journal of Vascular and Interventional Radiology 14: 1119-1128.

\section{AP3-6}

\section{Complications and mortality in older surgical patients in} Catalonia, Spain

I. Martí, E. Vilà, Z. Briones, J. Vallès, J. Canet

Anesthesiology, Hospital Universitari Germans Trias i Pujol, Badalona, Spain Background and Goal of Study: Ageing has been shown to bear a relation to increased risk of perioperative morbidity and mortality. We aimed to describe the characteristics of older surgical patients in Catalonia and to assess the rates of perioperative complications and mortality in this population.

Materials and Methods: We analysed data for the population $>60 \mathrm{yr}$ old extracted from a cross-sectional multicentre study that gathered pre-, intra- and postop. information on a random sample of patients $>18$ years old undergoing surgery (excluding obstetric) under general or regional anaesthesia. The software SPSS.14 was used to compile descriptive statistics and compare qualitative variables with a $\chi^{2}$ test and quantitative variables with a Student $t$ test. Results and Discussions: Of a total population of 1966 patients, data were extracted for 912 patients $>60$ yr. Complications arose during surgery in $28.5 \%$ of these older patients. Postop. complications developed in $18.7 \%$. The rates of intraop. and postop. complications were significantly lower in the overall population, at $14.7 \%$ and $6.6 \%$, respectively $(p<0.001)$. The complication rates were significantly higher in the subpopulation of surgical patients $>80 \mathrm{yr}$ (intraop $39.9 \%$; postop, $31.9 \%$ ) ( $p<0.01$ vs. the patients $60-80 y$ old). The hospital mortality rate was $1.5 \%$ and the 3 -month mortality rate was $2.4 \%$ in the overall surgical population, whereas in patients $>60 \mathrm{yr}$ the rates were $2.9 \%(p=0.06)$ and $4.8 \%(p=0.01)$, respectively. In patients aged over $80 \mathrm{yr}$, hospital mortality was $6.5 \%(\mathrm{p}=0.06 \mathrm{vs}$ patients $60-80 \mathrm{yr}$ old $)$ and $3-$ month mortality was $10.1 \%$ ( $p=0.02$ vs the patients $60-80 \mathrm{yr}$ old). Conclusion(s): This study assessed the increased risk of perioperative complications and mortality for surgical patients aged over $60 \mathrm{yr}$ in Catalonia. We observed that the incidence of complications and mortality in patients over $80 \mathrm{yr}$ rises sharply. Our elderly surgical patients require diligent monitoring and preventive measures.

References:

Jin F. et al. Br J Anaesth 2001; 87: 608-24.

Levine WC. Curr Opin Anaesthesiol 2006; 19: 320-324.

\section{AP3-7}

Potential to optimize preoperative medication

J. Brugger, J. Broscheit, S. Hoefer, T. Winklmaier, N. Roewer

Anesthesiology, University Clinic, Wuerzburg, Germany 
Background and Goal of Study: Anxiety is an important determinant of perioperative stress that can be reduced by an appropriate procedure. Thus a questionnaire was sent to 21 German university hospitals to elucidate the current procedure.

Materials and Methods: The questionnaire was structured into 3 parts: Part 1 included the kind of preoperative medication of ambulatory and stationary patients. The latter group was differentiated into adult patients and children for cardiac surgery and non-cardiac surgery. Part 2 contained details of the procedure. Part 3 dealt with subjective satisfaction of different aspects of the premedication by the operator using a pseudodichtome fourpoint-scale.

Results and Discussions: In the 21 interviewed German university hospitals benzodiazepines were preferred. Mostly Midazolam was administered orally for stationary and ambulatory patients. Patients for cardiac surgery received prolonged effective benzodiazepines. Midazolam $(0,5 \mathrm{mg} / \mathrm{kg})$ is mostly used for children and the oral way is preferred against the rectal. Most patients received premedication by nurse on the peripheral station. The subjective estimation of the success of premedication was $73 \%$ (SD $+-10.25 \%)$. In $90 \%$ it was stated that an improvement of premedication is desirable. Especially a high rating was attached to the importance of the anaesthesist making premedication and narcosis in personal union, improvement of time management and a stronger anxiolytic effect.

Conclusion(s): Benzodiazepines are the standard medicament for premedication. Other medication as the intramuscular premedication has no more rank in Germany and was left in favour of the oral application.

Patients of all age groups get Benzodiazepines with short half-life. Benzodiazepines with long half-life are preferred for patients undergoing a cardiac surgery. In spite of a contentment of over $70 \%$ there is a great necessity of optimising anxiolysis and time management. The physician making premedication and narcosis in personal union is desirable.

\section{Reference:}

Tolksdorf W: Preoperative stress. Research approach and methods of treatment. AINS; 1997 Oct: 32(3 Suppl): S318-24. Review.

\section{AP3-8}

\section{Attitudes of anaesthetists and surgeons to informed consent}

A. Jamjoom, JG. Hardman, IK. Moppett

Anaesthesia, University of Nottingham, Nottingham, United Kingdom

Background and Goal of Study: The attitudes of patients' to informed consent have changed over the years ${ }^{1}$, but there has been little systematic study of the attitudes (and possible differences) of anaesthetists and surgeons in this process. We aimed to describe the attitudes of medical professionals in our local hospital to issues surrounding informed consent.

Materials and Methods: A custom designed questionnaire was administered to a randomly selected cohort of 75 anaesthetists and surgeons. All answers were selected from a 5-point scale: 1 - strongly disagree to 5 - strongly agree. Results and Discussions: Surgeons and anaesthetists generally agreed in their attitudes towards informed consent. Although the clear majority were happy with the provision of informed consent in their practice, less than $25 \%$ had had formal training. Respondents were more inclined towards consent as an ethical and legal obligation than being of benefit to the doctorpatient relationship; anaesthetists were more likely to feel that informed consent may reduce anxiety. Around $50 \%$ of both groups felt that informed consent was inappropriate since most patients do not usually remember all the information given to them. Although, in the UK consent can be taken by any appropriately trained person, both groups were strongly opposed to non-physician consent, anaesthetists more so. The majority of both groups felt that major risks more common that 1:1000 should be disclosed; a minority felt this for risks $>1: 10,000$. For minor risks, the majority favoured disclosure $>1: 100$, the minority for risk $>1: 1000$. UK case law does not set a particular frequency of risk for disclosure. The level of the patient's education, inquisitiveness and seriousness of co-morbidity were more likely to influence anaesthetists than surgeons. Neither group was particularly influenced by patient's sex or publicly versus privately funded healthcare settings. Both groups favoured the provision of written information.

Conclusion: Surgeons and anaesthetists in the UK have similar attitudes to informed consent, though anaesthetists may be more influenced by individual circumstances than surgeons. Both groups tend towards informed consent as an obligation rather than being of benefit to the patient.

Reference:

1 AAGBI. Consent for Anesthesia. London 2006.

\section{AP3-9}

\section{National Institute for clinical excellence preoperative tests:} Is the consensus hard to get?

L. Cabral, T. Rodrigues, L. Nicolau, L. Ormonde, J. Figueiredo Lima Anaesthesiology Department and Biomathematic Laboratory, Hospital de Santa Maria and University of Lisbon Medical School, Lisboa, Portugal

Background and Goal of Study: It is routine in our preoperative consultation the evaluation of chest X-ray, lab tests and ECG, ordered previously by the patient surgeon. In June 2003, the National Institute for Clinical Excellence (NICE) published Guidelines defining the preoperative tests needed. However the consensus was not obtained in some tests. The goal of our study was to quantify the number of tests with alterations for each NICE indication, in order to verify if there were significant differences between the group "don't do" and the group "without consensus".

Materials and Methods: 268 records, two months of the Urology and Gynaecology Anaesthesiology consultation were included. To guarantee homogeneity in the classifying criteria of the tests, any examination with values out of the reference intervals, or any examination with anomalies in the report was defined as altered. To test the equality proportions the Z-test and Fisher exact tests were applied.

Results: Of the 268 records (ASA I-III), 110 were M and 158 were F, with 57,07 years as the average age. The tests with alterations by type of NICE indication are presented in the following table:

\begin{tabular}{|c|c|c|c|c|c|}
\hline $\begin{array}{l}\text { NICE } \\
\text { indication }\end{array}$ & $\begin{array}{l}\text { Chest } \\
\text { X-ray }\end{array}$ & ECG & $\begin{array}{l}\text { Full blood } \\
\text { count }\end{array}$ & Haemostasis & $\begin{array}{l}\text { Renal } \\
\text { function }\end{array}$ \\
\hline Do the test & - & $\begin{array}{l}40(28.4 \%) \\
\mathrm{n}=141\end{array}$ & $\begin{array}{l}18(13,1 \%) \\
n=137\end{array}$ & - & $\begin{array}{l}19(19.4 \%) \\
\mathrm{n}=98\end{array}$ \\
\hline \multirow{4}{*}{$\begin{array}{l}\text { Without } \\
\text { consensus } \\
\text { Don't do } \\
\text { the test } \\
\text { Tests with } \\
\text { alterations }\end{array}$} & $\begin{array}{l}10(9.3 \%) \\
\mathrm{n}=107\end{array}$ & $\begin{array}{l}1(12.5 \%) * 2 \\
\mathrm{n}=8\end{array}$ & $\begin{array}{l}2(5.3 \%) * 3 \\
n=38\end{array}$ & - & $\begin{array}{l}2(4.3 \%) * 4 \\
\mathrm{n}=47\end{array}$ \\
\hline & $\begin{array}{l}8(5.4 \%) * 1 \\
n=148\end{array}$ & $\begin{array}{l}9(8.0 \%) * 2 \\
n=113\end{array}$ & $\begin{array}{l}11(13.1 \%) * 3 \\
n=84\end{array}$ & $\begin{array}{l}10(100 \%) \\
n=246\end{array}$ & $\begin{array}{l}3(2.8 \%){ }^{*} 4 \\
n=106\end{array}$ \\
\hline & $18(7.1 \%)$ & $50(19.1 \%)$ & $31(12.0 \%)$ & $10(4.1 \%)$ & $24(9.6 \%)$ \\
\hline & $n=255$ & $n=262$ & $n=259$ & $\mathrm{n}=246$ & $\mathrm{n}=251$ \\
\hline
\end{tabular}

Equal proportions tests, p-value: *1-0.2295; *2-0.2, (one-sided test 0.014); *3-0.341; *4-0.643

Conclusion: The guidelines "don't do" and "without consensus" seems to have the same risk of not detecting alterations in all tests, except the ECG. In those tests we may state that the two indications can be merged in to "don't do the test" indication. To complete this study it is important to evaluate the alterations found in the tests, determining its importance in per operative period. Reference:

1 National Institute for Clinical Excellence (2003) Preoperative tests: The use of routine preoperative tests for elective surgery. London: Oaktree Press.

\section{Ambulatory anaesthesia}

\section{AP1-1 \\ VIMA with sevoflurane versus balanced anesthesia in pediatrics outpatient's surgery. Is premedication really worth? I.G. Cucereanu Bãdicã, A.A. Brãdi', I.M. Grinpescu \\ Department of Anesthesia and Intensive Care, Emergency Clinical Hospital, Bucharest, Romania \\ Background and Goal of Study: "The simplest the best" seems to work in the operating theater where children that receive only sevoflurane spend less time in hospital than those premedicated or those that received balanced anes- thesia. The aim of the study is to evaluate the perioperative evolution, inci- dence of side effects and time spent in hospital for those children.}

Materials and Methods: After Hospital Ethics Committee approval 128 children 2-10 years of age scheduled for tonsillectomy, adenoidectomy and circumcision were randomly assigned in 3 groups: the first group (B-41 pts) was premedicated with $0,5 \mathrm{mg} / \mathrm{kgc}$ Midazolam given per os in $5 \mathrm{~mL}$ lbuprofen suspension and received propofol, opioids and muscle relaxants (balanced anesthesia technique with intravenous induction), the second group ( $\mathrm{P}-42 \mathrm{pts})$ received just premedication and Sevoflurane and the third group (S-45 pts) just Sevoflurane. All the children had an intravenous line and received $5 \mathrm{mg} / \mathrm{kgc}$ hemisuccinate hydrocortisone preoperative and $15 \mathrm{mg} / \mathrm{kgc}$ iv. Paracetamol. We assess the time to spontaneous breathing, extubation, Aldrete score $\geqslant 9$, the incidence of PONV and agitation and the hospitalization time. Statistics used ANOVA, Mann-Whitney U-test and $\chi^{2}$ test $\left({ }^{*} p<0,05\right)$. 
Results and Discussions: The incidence of PONV was significantly statistic lower in Groups II and III (VIMA groups), recovery was faster in III group compared with group I. Agitation was practically present in all patients.

\begin{tabular}{lllc}
\hline Time $(\mathrm{min})$ & Group I & Group II & Group III \\
\hline Spont breathing & $4,2 \pm 2,3$ & $3,1 \pm 1,7$ & $2,8 \pm 1,8$ \\
Extubation & $8,9 \pm 4,1$ & $5,7 \pm 2,9$ & $5,6 \pm 2,7$ \\
Aldrete $\geqslant 9$ & $25 \pm 11$ & $20 \pm 9$ & $18 \pm 7^{\star}$ \\
PONV & $11(27 \%)$ & $4(9,5 \%)$ & $4(9 \%)^{\star}$ \\
Agitation $(\%)$ & $\approx 100 \%$ & $\approx 100 \%$ & $\approx 100 \%$ \\
hospitalization & 8,3 hours & 5,6 hours & 5,2 hours \\
\hline
\end{tabular}

Conclusion(s): The incidence of PONV was lower and recovery was faster for VIMA with Sevoflurane than for balanced anesthesia. Premedication had no advantages but it was time consuming. Children have a strong fear of needles and they prefer an anesthetic technique without needles. As long as we can satisfy this preference and this have only advantages we see no reason not to do it.

\section{AP1-2}

\section{Reaction time monitored patient maintained propofol} sedation: a volunteer safety study

S. Allam, C. Obrien, KJ. Anderson, A. Macpherson, GN. Kenny

Department of Anaesthesia, Glasgow Royal Infirmary, Glasgow, United Kingdom

Background and Goal of Study: Effect-site controlled patient maintained propofol sedation (ePMS) was found to be safe and effective in patients having dental surgery ${ }^{1}$, but when healthy volunteers deliberately attempted to over sedate themselves, potentially unsafe levels of sedation were seen in a few volunteers ${ }^{2}$. As a patient's effect-site propofol concentration $(\mathrm{Ce})$ increases, their reaction time increases. We have now incorporated a reaction time monitor into our current ePMS system's handset. The aim of this study was to assess whether this modification would reduce the risk of over sedation. Materials and Methods: 20 healthy volunteers (ASA 1-2) were recruited and an average baseline reaction time (RT) was recorded (handset button pressed in response to its vibration). Propofol sedation was commenced at Ce of $1 \mathrm{mcg} / \mathrm{ml}$ and the handset vibrated at one minutely intervals during sedation to monitor RT. The volunteer could increase target Ce in increments of $0.2 \mathrm{mcg} / \mathrm{ml}$ by double pressing the demand button, provided calculated $\mathrm{Cp}$ (plasma concentration) and Ce had equilibrated within 10\%. In addition, the patient's RT compared to baseline was used in an algorithm that would prevent further increases or actually reduce target $\mathrm{Ce}$ if their RT was becoming too slow. Volunteers were encouraged to use the button to make themselves as sedated as possible. The study would end if verbal contact was lost, if $\mathrm{SaO}_{2}$ fell below $90 \%$, if airway intervention was required, after 3 consecutive reductions in target Ce due to RT slowing or after 30 minutes if none of the above. Results and Discussions: All 20 volunteers maintained verbal contact throughout, and did not reach any unsafe end point. The average maximum propofol $\mathrm{Ce}$ (range) was $1.7(1.2-2.4) \mathrm{mcg} / \mathrm{ml}$. The mean lowest $\mathrm{SaO}_{2}$ (range) was $97 \%$ (93-99\%). No airway intervention was required in any volunteer.

Conclusions: This study indicates that the addition of reaction time monitoring has improved the safety of propofol ePMS. It may be possible to use this system in the absence of an anaesthetist.

References:

1 Chapman RM, Anderson K, Kenny GN et al. Anaesthesia 2006; 61: 345-349.

2 Anderson KJ, Leitch JA, Kenny GN et al. Anaesthesia 2005; 60: 235-8.

\section{AP1-3}

\section{Anesthetic management in a case of melkersson-rosenthal} syndrome

\section{A.M. Castro E Melo, T. Pereira, N. Lages, J. Tavares}

Anesthesiology, Hospital de São João, Porto, Portugal

Background and Goals: The Melkersson Rosenthal Syndrome (MRS) is a disorder of unknown etiology, with an incidence of $0.08 \%$ (1), clinically characterized by the triad of chronic or recurrent orofacial edema, with edema of one or both lips, recurrent unilateral or bilateral facial palsy and lingua plicata. We are not aware of any reports on the anesthetic management of a case of MRS. Material and Methods: A 64-year-old woman, $60 \mathrm{Kg}$, with MRS confirmed by lip biopsy was submitted to shoulder arthroscopic procedure. She presented mild facial edema and volumous tongue (Mallampati 3). After intravenous midazolam ( $2 \mathrm{mg}$ ), an interscalene brachial plexus block was performed with $30 \mathrm{~mL}$ of $0.5 \%$ ropivacaine. A $22 \mathrm{G} / 35 \mathrm{~mm}$ needle with a neurostimulator
$\mathrm{NH} 12$ Braun $\AA$ was used to elicit a biceps contraction at a $0.4 \mathrm{~mA}$ current. The block was complemented with a superficial cervical plexus block with $10 \mathrm{~mL}$ of $1 \%$ lidocaine ( $23 \mathrm{G} / 25 \mathrm{~mm}$ needle).

Results: Regional anesthesia (RA) avoided the manipulation of the airway, providing a proper sensitive block throughout the surgery with no unexpected events. The association of MRS with edema of the airway poses problems with airway management. The orofacial features of MRS include edema of the face, lips, gingivae and buccal mucosa, anesthesia around the mouth and facial palsy. Intraoral involvement may appear as palatal mucosa, sublingual area, larynx and pharynx swelling (2). The facial and lip edema may evolve rapidly (1). As to what relates to anesthesiology, there is one emergency case report of a 21-year-old woman with MRS presenting with sudden upper airway obstruction and cardiopulmonary arrest. Anesthesiologists must be aware of the MRS as a possible, although rare, cause of edema of the larynx (1). Conclusions: To the best of our knowledge this is the first report of the anesthetic management of a patient with MRS. Anesthesiologists must be aware of MRS as a cause of problematic airway management and RA should be preferred to minimize the risk of larynx edema.

References:

1 Jayamaha J, Anesth Analg 1993; 77: 95-7.

2 Wall R, Schullen E, Scheur MR et al, J Eur Acad Dermatol Venereol 2001; 15: 519-523.

\section{AP1-4}

\section{Unilateral spinal anesthesia versus conventional spinal} anesthesia in ambulatory lower abdominal surgery

\section{Cindea, A. Balcan, V. Gherghina, G. Nicolae}

Anesthesiology, Intensive Care, Emergency Clinical Hospital of Constanta, Constanta, Romania

Background and Goal of Study: Unilateral spinal anesthesia offers analgesia and operating conditions as good as standard spinal technique in outpatient scheduled for lower abdominal surgery (1).

The objective of the prospective randomized current study is to evaluate the two spinal anesthesia techniques with respect to recovery times, level of patient postoperative comfort and anesthetic-related costs.

Materials and Methods: One hundred ASA I-II, premedicated subjects scheduled for hernioplasty were randomly allocated into two groups to receive conventional $(n=50)$ and unilateral $(n=50)$ spinal anesthesia with $10 \mathrm{mg}$ hyperbaric bupivacaine. In unilateral spinal anesthesia group a lateral decubitus position with the operative side down was maintained for $20 \mathrm{~min}$, after spinal injection. For both groups recovery times, postoperative effects profile during a 5 days follow-up period, as well as costs were registered. 5 days after the procedure, the patients were interviewed by telephone about analgesia medication requirements after discharge, as well as occurence of headache or backache.

Results and Discussions: Compared to bilateral spinal anesthesia, the subjects receiving unilateral spinal technique had shorter recovery times $(120 \pm 56 \mathrm{~min}$ vs $240 \pm 70 \mathrm{~min}, \mathrm{p}<0.001)$ and lower pain scores at discharge $(14 \pm 13 \mathrm{~mm}$ vs $32 \pm 30 \mathrm{~mm}, \mathrm{p}<0.001)$. These patients had less requirements for analgesic medication at home $(22 / 50$ vs $40 / 50, p<0.001)$. Frequency of headache (2/50 vs $9 / 50, p<0.05)$ and backache $(1 / 50$ vs $6 / 50$, $\mathrm{p}<0.05)$ registered lower values in unilateral spinal anesthesia group, too. The anesthetic-related costs were also less for unilateral spinal technique $(102.14 \pm 30.82 \$$ vs $134.93 \pm 31.03 \$)$.

Conclusion(s): The unilateral spinal anesthesia technique is more costeffective than traditional spinal anesthesia for lower abdominal surgical procedures in ambulatory setting, as it is associated with an earlier recovery, high postoperative patient satisfaction and decreased costs.

Reference:

1 Song D. Anesth Analg 2000; 91: 876-881.

\section{AP1-5}

Comparison of propofol-remifentanyl anesthesia versus desflurane-remifentanyl anesthesia in obese patients whom laparoscopic cholecystectomy planned

N. Karahan, K.U. Altunbay, T. Adanir, U. Ozgurbuz, G. Aran

Anaesthesiology and Reanimation, Izmir Ataturk Training and Research Hospital, Izmir, Turkey

Background and Goal: In obese patients, due to increased fat tissue, changes are seen in metabolism of anesthetic drugs. In outpatient surgery this situation forms a severe problem. In our study, we aimed to compare propofolremifentanyl and desflurane-remifentanyl anesthesia methods in terms of 
preoperative hemodynamic and recovery properties in obese patients whom laparoscopic cholecystectomy planned.

Material and Methods: After permission was obtained from ethical council of our hospital, 60 patients who were between 18-65 years of age, matching ASA I-II classification, having BMI greater than 30 and undergoing laparoscopic cholecystectomy were included in this study. Patients were randomly subdivided into two groups and maintenance of anesthesia was achieved with remifentanyl and propofol infusion in Group $P(n=30)$, remifentanyl infusion and desflurane (1 MAC) in Group $D(n=30)$. During operation, dose of propofol infusion and MAC value of desflurane were kept constant. According to requirements opioid dose was increased or decreased. At the end of the operation, quality of recovery was evaluated by Aldrete Recovery Scale. In PACU, patients were followed for 2 hours in terms of hemodynamics, recovery and pain.

Results and Discussion: It is understood that group $D$ is more effective to prevent tachycardia during intra-operative and post-extubation period than group P. In each group when remifentanyl values during intra-operative period is compared, it's found that desflurane decreases intra-operative remifentanyl requirement $(p=0,014)$. In each group, when $\mathrm{SPO}_{2}$ values in $1^{\text {st }}(P=0,000)$ and $5^{\text {th }}(P=0,020)$ minutes after extubation are compared; a statistically significant difference is found in group $D$ than in group $P$. In each group, when postoperative MAP values are compared, it is understood that desflurane is more effective to decrease MAP values according to basal values than propofol $(p=0,001)$. In group $D$, VAS values and post-op analgesic requirement is found to be less $(p<0,05)$. In our study, when Aldrete Recovery Scale is compared in each group, no statistically significant difference was found $(p=0,110)$.

Conclusion: In our study, we determined that inhalation agent desflurane protects hemodynamic stability better, provides more rapid and qualified recovery.

\section{AP1-6}

Quality of care in elderly patients for short urologic procedure E. Antonopoulou, K. Papaioannou, K. Tataraki, E. Nikouli, G. Konstantinou Anaesthesiology, General Hospital of Xanthi, Xanthi, Greece

Background and Goal of Study: The aim of the study was to evaluate the incidence of side effects and patient's satisfaction after short urologic procedures on a day case basis.

Materials and Methods: 60 geriatric patients were scheduled for short elective transurethral surgical procedures. Participants were men, $>65 \mathrm{yrs}$ (Mean $74 \pm 5$ ), ASA I-III. All patients were asked to follow pre-op and post-op instructions. Anaesthesia was induced and maintained with fentanyl, propofol and $\mathrm{N} 2 \mathrm{O} / \mathrm{O} 2$ and a laryngeal mask. Anesthesia time was $17 \pm 9 \mathrm{~min}$, time spending in the PACU was $23 \pm 11 \mathrm{~min}$. Patients were discharged from the unit according to the departmental criteria. A telephone interview followed 24 hours later. Patients were asked to evaluate their pain (VAS scale), nausea and vomiting dizziness, drowsiness, headache, pruritus, allergies, continence problems. They were also asked if they followed the given instructions, if they preferred staying in hospital overnight, if they contacted their doctor or if they were readmitted to the hospital, and finally their overall satisfaction (scale 1-5). Results and Discussions: $92 \%$ of the patients were very satisfied (scale 1-5). Pain was not the main problem in the participants (VAS score $0-3$ ) but $52 \%$ of them had discomfort during micturition. Nausea and vomiting had a very low incidence of $1 \% .23 \%$ had dizziness during the first six hours and $18 \%$ drowsiness. 1 patient stated that he didn't follow the instructions. 3 preferred to stay overnight in hospital, although they didn't have any complications. 1 patient was readmitted to the hospital because of haematuria. There were no other side effects. Finally 6 patients refused to be interviewed.

Conclusion(s): Ambulatory surgery seems to be safe in geriatric patients for short urological procedures.

\section{AP1-7}

Regional anaesthesia in ambulatory care - a Welsh survey C. Janakiraman, D. McPherson, A.R. Wilkes, O. Adekanye Anaesthetics, Cardiff University and University Hospital of Wales, Cardiff, United Kingdom

Background: Regional anaesthesia (RA) is an attractive alternative for many ambulatory cases due to excellent postoperative analgesia and rapid recovery profile with excellent patient satisfaction [1]. In view of the early ambulation and potential cost reduction associated with RA use, we carried out a survey to determine the extent to which RA is practiced in Wales, UK. Methods: Questionnaires were sent to 266 consultant anaesthetists in Wales between August and October 2006. Information regarding the proportion of anaesthetic practice involving day case and the use of peripheral neuraxial blocks were solicited. Data analysis included valid responses expressed as a percentage of the total. The relationship between hospital type (University and District General Hospital (DGH)) and the frequency of use of nerve blocks were compared using Fisher's exact test.

Results and Discussions: The response rate was $62 \%$. The median (IQR) experience of the consultants was 9 years (5-15). 59\% of responding consultants were from DGH, $28 \%$ from a University Hospital, $11 \%$ and $2 \%$ from affiliated and specialist hospitals respectively. $15 \%$ of respondents had $\geqslant 50 \%$ of ambulatory practice and $67 \%$ had $<25 \%$ practice. There is no difference in the practice of RA in ambulatory care between hospital types $(p=0.75)$.

\begin{tabular}{|c|c|c|c|}
\hline \multirow[t]{2}{*}{ Use of block } & \multicolumn{3}{|c|}{ Type of block } \\
\hline & $\begin{array}{l}\mathrm{UL} \\
\mathrm{n}=153\end{array}$ & $\begin{array}{l}\mathrm{LL} \\
\mathrm{n}=149\end{array}$ & $\begin{array}{l}\text { CNB } \\
\mathrm{n}=157\end{array}$ \\
\hline Never & $67(44)$ & $97(65)$ & $2(1)$ \\
\hline Occasionally & $69(45)$ & $43(29)$ & $62(40)$ \\
\hline Frequently & $16(10)$ & $8(5)$ & $87(55)$ \\
\hline Always & $1(1)$ & $1(1)$ & $6(4)$ \\
\hline
\end{tabular}

UL: upper limb block; LL: lower limb block;

CNB: central neuraxial block. Data $=$ number $(\%)$.

Conclusion(s): CNB use in ambulatory care is more common than UL and LL in Wales; this is comparable to the American practice. However lower limb neuraxial blocks are more commonly performed than upper limb neuraxial blocks which is in contrast to the American survey [2].

References:

1 Raeder J C. Can J Anaesth 2001; 48: 6 R1-5.

2 Hadzic A. et al. Anesthesiology 1997; 3A: A22.

\section{AP1-8}

\section{Minimal anaesthesia care a cost effective alternative for elective day surgery of the foot; A follow up of 162 consecutive patients}

J. Jakobsson, Z. Turan, I. Turan

Department of Anaesthesia, Foot \& Ankle Surgical Centre, Stockholm, Taiwan

Background: Day surgery is increasing and simple, safe and effective anaesthetic techniques are sought providing rapid recovery with a minimum of side effects enabling a early discharge and high turnover of patients. Material and Methods: We studied 162 consecutive patients undergoing elective day surgery of the foot. All patients followed a simple and standardised perioperative protocol; sedation dose of propofol and $0.3-0.4 \mathrm{mg}$ alfentanil, local anaesthesia in the operating area, washing and dressing while patients' were sedated, "light general sevoflurane anaesthesia". Sevofluane was introduced in a fresh gas flow of $2 \mathrm{l} / \mathrm{min} 2 \%$ per 2 breath up to $8 \%$ and maintained until the patient was a sleep, did not respond to verbal command or light painful stimuli OAAS 0. Sevoflurane was titrated in accordance to clinical needs during the procedure. All patients were spontaneously breathing through a face mask or laryngeal mask airway and breathing was assisted only when necessary.

Results: One hundred and sixty two ASA 1-2 consecutive patients operated during June through November 2006 were studied, median age 46 (18-80) years, weight $71(47-114) \mathrm{kg}$ and length $171(154-195) \mathrm{cm}$. Surgical prrocedure were Hallux Valgus 65 (40\%), ligament reconstruction $30(18 \%)$, dorsal kilektomi $22(14 \%)$, Morton $14(9 \%)$, ankle arthroscopy $10(6 \%)$ and miscellaneous 21 (13\%). Median duration of the procedures was 15 (7-48) minutes. All patients had an uncomplicated intraoperative course.

All but 2 patients walk out of the operating theatre escorted right after the procedure and all patients were alert and drinking and taking oral analgesics within median 16 (5-55) minutes and discharged within 2 hours median 42 minutes. Four patients experience emesis requiring intervention antiemetics during the stay in hospital.

Conclusion: Sevoflurane is a feasible option for light general anaesthesia in combination with local anaesthesia during elective foot surgery providing good intraoperative conditions and fast emergence allowing for fast tracking and early discharge.

\section{AP1-9}

Disposable laryngeal mask airway better or worse than the classical ima? A clinical feasibility study

J. Jakobsson, Z. Turan, A. Doolke, GB. Saros

Department of Anaesthesia, Foot \& Ankle Surgical Centre, Stockholm, Sweden 
Background: The laryngeal mask airway has become Gold Standard for airway management during Day Surgery. The Classical mask has recently been challenged by a variety of different dispoable masks. The aim of the present survey was to evaluate in clinical routine practice the usefulness of two different disposable Lma; AMBU mask and Intersurgical mask.

Method: 189 ASA 1-2 patients undergoing elective day surgery in general anaesthesia was studied. The patients were randomised to one of the three Lma's Classic, AMBU or Intersurgical.

All patients had an uncomplicated perioperative course no major complications or complaints were noticed during anaesthesia or recovery.

The nurse anaesthetists' subjective comments are given in the tabe. Conclusion: There are no major clinical differences between different laryngeal mask air ways. The AMBU mask seems reassuringly easy to use.

\begin{tabular}{llll}
\hline & $\begin{array}{l}\text { Classic } \\
\mathrm{N}=63\end{array}$ & $\begin{array}{l}\text { AMBU } \\
\mathrm{N}=63\end{array}$ & $\begin{array}{l}\text { Intersurgical } \\
\mathrm{N}=63\end{array}$ \\
\hline $\begin{array}{l}\text { In place 1 } \\
\text { attempt }\end{array}$ & 57 & 63 & 53 \\
$\begin{array}{l}\text { Subjective } \\
\text { Ok }\end{array}$ & & & \\
Leakage no & 59 & 57 & 44 \\
Any & 63 & 62 & 61 \\
complication & 2 & 1 & 1 \\
& change & change & change \\
$\begin{array}{l}\text { Taking out } \\
\text { Blood stained }\end{array}$ & mask & mask & mask \\
$\begin{array}{l}\text { During } \\
\text { Recovery }\end{array}$ & 5 & & \\
Any complaints & & 0 & 6 \\
No & & & \\
Small & & & 54 \\
Some & 59 & 59 & 6 \\
\hline
\end{tabular}

\section{AP2-1}

\section{Postoperative hyperalgesia after ambulatory surgeries using} remifentanil

K. Hwang, S. Lee

anesthesiology, Wooridul Spine Hospital, Seoul, Republic of Korea

Background and Goal of Study: The aim of this study was to investigate whether remifentanil had any effect on post-operative pain and analgesic consumption after ambulatory surgery like percutaneous endoscopic lumbar discectomy (PELD).

Materials and Methods: Eighty ASA patients with physical status 1 or 2 patients who underwent a PELD were enrolled in this study. They were allocated randomly to receive one of two treatments; a fentanyl bolus of $0.7 \mu \mathrm{g} / \mathrm{kg} 5 \mathrm{~min}$ before the procedure and of $0.7 \mu \mathrm{g} / \mathrm{kg}$ during the procedure ( $n=40$, group $F$ ), or remifentanil, titration of the target effect-site concentration $(0.3-2.5 \mathrm{ng} / \mathrm{ml})$ available throughout the procedure according to the appeal of pain, level of sedation and side-effects ( $n=40$, group $R$ ). The visual analogue scale of pain (VAS), the time and frequency of requiring analgesia and the amount of requiring analgesics by patients and adverse drug reactions such as nausea, vomit and hallucination were recorded for 2 hours and 24 hours after surgery.

Results and Discussions: No significant differences were found in the demographic characteristics. There were no significant differences in terms of the recovery characteristics, incidence of complications. The study didn't find significant differences between the two groups on the cumulative morphine requirement during the 2 hours and the 24 hours $(p>0.05)$. There were no significant difference in terms of the postoperative VAS score between remifentanil group $(2.45 \pm 1.53,2.46 \pm 1.43)$ and fentanyl group $(2.33 \pm 1.48$, $2.5 \pm 1.39$ ) during the 2 hours and the 24 hours $(p>0.05)$

Conclusion(s): We conclude that analgesia using remifentanil for the ambulatory surgery such as PELD does not significant effect on the postoperative pain.

\section{AP2-2}

Use of elastomeric pumps in day surgery - assessment of patient satisfaction

D. Factor, A. Koo, K. Raghavan, R. Blanco

Anaesthesia, University Hospital Lewisham, London, United Kingdom

Background and Goal of Study: In Orthopaedic cases performed in the Day Surgery setting, the two commonest reasons for hospital admission are poor postoperative pain control, and nausea and vomiting associated with the use of opioids.
Elastomeric pumps are a safe way of delivering a preset infusion of local anaesthestic. They are associated with minimal complications, as well as being simple to use, portable, economical, and disposable(1).

With the aim of decreasing re-admission rates due to poor pain control, elastomeric pumps were introduced into our day surgery service(2). We were interested in the level of patient satisfaction going home with this device. Materials and Methods: We performed ultrasound guided regional anaesthesia on 51 consecutive patients undergoing shoulder keyhole surgery. $100 \%$ of the blocks were considered successful and additional analgesia was not required. The elastomeric pump was connected to an indwelling catheter at the end of the procedure, and commenced on a background infusion of Ropivicaine $0.2 \%$ at $5 \mathrm{mls} / \mathrm{hr}(3)$, with a PCA component of $5 \mathrm{mls}$, max every 25mins.

Over a 72 hour period we assesssed pain control, nausea and vomiting, time to mobility, and patient satisfaction using a telephone questionnaire. Results and Discussions: Patient satisfaction was excellent overall, with the main complaint being lack of sensation. The average time to pump removal was 49.3 hours, and $92.5 \%$ of patients were happy to use this technique again.

Conclusion(s): In Orthopaedic shoulder keyhole surgery, elastomeric pumps provide a safe alternative for analgesia in day surgery patients, and are associated with a high level of patient satisfaction.

References:

1 Skryabina EA,Dunn T Am J Health Syst Pharm 2006; 63(13): 1260-8.

2 Hoenecke HR Jr, Pulido PA, Morris BA, Fronek J Arthroscopy. 2002; 18(8): 854-8.

3 Zaric D, Boysen K, Christiansen J, Haastrup U, Kofoed H, Rawal N Acta Anaesthesiol Scand. 2004; 48(3): 337-41.

\section{AP2-3}

Postoperative pain management for shoulder arthroscopy in ambulatory surgery: comparation between single injection or continuous perineural infusion of local anesthetic

C. Botana, G. Agreda, I. Salgado, I. Vives, P. Cabre

Anestesiología y Reanimación, Hospital Vall d'Hebron, Barcelona, Spain

Background and Goal of Study: Postoperative pain after shoulder arthroscopy is a major concern, and management in an ambulatory setting even more important. We compare postoperative analgesia after shoulder arthroscopy in patients with single injection and patients with an elastomeric device and perineural perfusion of local anesthetic.

Materials and Methods: 73 patients ASA I-II, without respiratory compromise, aged between 18-65 years, programmed for shoulder arthroscopy in an ambulatory setting, were divided into two groups: I single injection $(n=41)$, and II continuous perineural infusion $(n=35)$. After preoperative interescalene braquial plexus block (group I: single injection after neurostimulated needle location; group II: location, stimulated catheter placement, and administration of local anesthetic) a general anesthesia was induced. Postoperative pain was registered the $1 \mathrm{st}$ and $2 \mathrm{nd}$ nights, and at $24 \mathrm{~h}, 48 \mathrm{~h}$, and a week. Rescue analgesia consumption and complications were also registered.

Results and Discussions: No statistical differences were found in demographical parameters in either group. Stadistical differences $(p<0.05)$ in pain control according to verbal numeric scale were found the first night, at 24 and 48 hours at rest and during movement, with a lower pain score in group II. Oral rescue analgesic consumption was lower in group II. Amount of minor complications was larger in group II, but all of them were selflimited and of no clinical significance (Horner's Syndrome, recurrent nerve palsy.

Conclusion(s): Characteristics in the use of peripheral nerve blocks with elastomeric pumps are better than single injection administration for outpatient pain control: prolonged postoperative analgesia with fewer rescue analgesia consumption, and a similar porcentage of complications was found.

References:

1 Boezaart, Anesthesiology 2006; 104: 872-80.

2 Ilfeld Anesth Analg 2005; 100: 1822-33.

\section{AP2-4}

\section{Patient satisfaction: are we singing from the same} songsheet?

\section{A.M. Denner, S.A. Townley}

Department of Anaesthetics, Royal Hampshire County Hospital, Winchester, United Kingdom

Background and Goal of Study: Patient satisfaction constitutes one of the four domains of effective healthcare governance as defined by the WHO (1). The aim of the survey was to assess patient satisfaction with respect to the anaesthetist-patient relationship. 
Materials and Methods: Patient satisfaction was assessed in a cohort of 80 adult day-case patients using the CARE questionnaire (2). The questionnaire contains 10 items exploring the non-technical aspects of the anaesthetistpatient relationship which may be broadly divided into 3 areas: 1) emotional support 2) respect for patient values 3) information-giving and education. Patients were asked to rate the 10 items on a scale ranging from poor to excellent. In addition, patients were asked to indicate how important the 10 items were to them and free comments were invited. Reponses were obtained by post within 2 weeks of discharge.

Results and Discussion: The overall response rate was $70 \% .91 \%$ of the responders rated the items in the emotional support category as either very good or excellent. Regarding the respect for patient values and informationgiving and education items, they were rated as very good or excellent in $72 \%$ and $74 \%$ of the responses respectively. $89 \%$ considered the items on the questionnaire to be either moderately $(30 \%)$ or very $(59 \%)$ important. Conclusions: An important indicator of quality of care is patient satisfaction and we have demonstrated a high level through our survey. This study has enabled us to implement changes to address the shortfalls that have been identified. The free comments have been invaluable in this process of tailoring our service to the patients' specific needs.

References:

1 Scally G, Donaldson LJ. Clinical governance and the drive for quality improvement in the new NHS in England. BMJ. 1998; 317: 61-5

2 Mercer SW, Reynolds W. Empathy and quality of care. BJGP. 2002; S2 (supplement): S1-S12.

\section{AP2-5}

\section{The patient experiences with the preoperative assessment} clinic (PEPAC): validation of a questionnaire

G.M. Edward, L.C.J.M. Lemaire, F. Oort, B. Preckel, H. de Haes

Anaesthesiology, Academic Medical Centre, Amsterdam, The Netherlands

Background and Goal of Study: No comprehensive and validated questionnaire to measure patient experiences with the preoperative assessment clinic (PAC) is available so far. We developed and validated the Patient Experiences with the Preoperative Assessment Clinic (PEPAC) questionnaire, which can be used for quantitative measurements of patient experiences to establish the quality level of care within one PAC or nationwide.

Materials and Methods: The NHS outpatient questionnaire was adapted for the PAC, incorporating questions specific for anesthesiology. Both patients and health professionals judged its content validity. To make the PEPAC appropriate for quantitative measurements, dimensions were constructed fit for statistical analysis along with single items. Each dimension consists of multiple items that measure the same care aspect. Reliability was established by computing Cronbach's alpha coefficients. Construct validity was assessed by correlating the dimensions with the patient's overall care appraisal (Pearson r). Also, these dimensions should explain a substantial level of variance of the patients' overall appraisal; therefore regression analysis was performed.

Results and Discussions: After a pilot phase, the questionnaire was sent to 700 consecutive patients (response $74 \%$ ). Five scales measuring five dimensions of patient experiences were constructed: reception ( 3 items), waiting ( 6 items), the nurse (5 items), the anesthetist (20 items), and other questions ( 15 items). Cronbach's alpha ranged from 0.56 to 0.84 , supporting reliability of the PEPAC. Correlations between the dimensions and the patients' overall appraisal ranged from 0.22 to 0.56 . Collectively, the five scales explained $51 \%$ of patients' overall appraisal.

Conclusion(s): The PEPAC is a comprehensive, reliable and valid instrument to measure patient experiences with the PAC. It can determine the areas of the PAC that require improvement in a single institute or be used to compare the quality of care across institutions.

\section{AP2-6}

Is postdischarge nausea and vomiting very frequent after day surgery?

M. Sá Monteiro, D. Moreira, F. Barros, A. Castro, P. Lemos

Anaesthesiology, Hospital Geral Santo António, Porto, Portugal

Background and Goal of Study: Postdischarge nausea and vomiting (PDNV) may have an important impact on patient recovery after ambulatory surgery. PDNV should be quantified to evaluate quality of health care after ambulatory surgery. Our goal is to evaluate the patient complains at home, quantify the incidence of NV, identify the risks and the possibility of reducing that incidence.

Materials and Methods: We planned a prospective study with 592 ambulatory surgery patients, collecting data from hospital stay and telephone interviews $24 \mathrm{~h}$ after surgery. All patients made intraoperative prophylaxis (dexamethasone $5 \mathrm{mg} \mathrm{ev}+$ droperidol 0,625 mg ev) for PONV. Several variables were analysed in order to find possible relations with NV incidence. We used Pearson correlation, t-test, Kruskal-Wallis test and Chi-square test in this analysis.

Results and Discussions: Our sample included 320 females (54\%), ASA I-IV, $46 \pm 17$ years, surgery time $40 \pm 27 \mathrm{~min}$. The incident of PDNV was $6,6 \%$. Independent variables associated with incidence of NV were: female gender; pain, disturbed sleep, insufficient therapy and limiting activity at $24 \mathrm{~h}$ $(P<0,01)$. We did not find any relation between age, anaesthesia time and technique, surgery type, PONV at day surgery unit (DSU) or PONV therapy at DSU (ondansetron) and PDNV.

Conclusion(s): The lower incidence of PDNV found suggests the need to collected more data in order to identify more reliable conclusions. Nevertheless, these results allows us to provide a better PDNV strategy, such as a more aggressive control of pain. The authorswould also recommended on female patients a rescue treatment for PDNV at home.

\section{AP2-7}

\section{Are our patients suffering too much after day surgery?}

D. Moreira, M. Sá Monteiro, F. Barros, A. Castro, P. Lemos

Anaesthesiology, Hospital Geral Santo António, Porto, Portugal

Background and Goal of Study: Pain following hospital discharge may have an important role on patient recovery after day surgery procedures. Postdischarge pain should be assessed to evaluate morbidity and quality of health care after ambulatory surgery. We determined the most painful procedures and evaluated pain $24 \mathrm{~h}$ after ambulatory surgery, quantifying the incidence, intensity and associated risks.

Materials and Methods: We designed a prospective study with 592 ambulatory surgery patients, collecting data on hospital stay and by telephone calls $24 \mathrm{~h}$ after surgery. All patients were provided with take-home analgesia. Pain scores, in numerical scale $0-3$, were collected at $24 \mathrm{~h}$ postoperatively. Data were analysed in two groups, those with moderate to severe pain (pain score $2-3$ ) and those with no or mild pain (0-1). Several variables were analysed in order to find possible relations with pain incidence. We used Pearson correlation, t-test, Kruskal-Wallis test and Chi-square test in this analysis.

Results and Discussions: From our sample 320 patients females (54\%), ASA $1-4,46 \pm 17$ years, surgery time $40 \pm 27 \mathrm{~min}$. The most painful procedures included inguinal hernia repair, varicocele surgery and hemorrhoidectomy $(P<0,01)$. The incidence of moderate to severe pain was $4,1 \%$. Independent variables associated with moderate and severe pain at $24 \mathrm{~h}$ were: higher level pain at PACU, disturbed sleep, insufficient therapy and limiting activity at $24 \mathrm{~h}(\mathrm{P}<0,01)$. We did not find relation between age, sex, anaesthesia technique, surgery time and PONV at day surgery unit (DSU) or analgesic therapy at DSU.

Conclusion(s): Improvements in pain management after discharge appear justified to provide a better and more comfortable recovery and accelerate return to normal activity. The patients submitted to most painful procedures and those with higher pain in PACU could benefit with a better rescue treatment for pain at home.

\section{Monitoring: Equipment and Computers}

\section{AP1-1}

Is it possible to measure the abdominal pressure volume relation before surgery?

J.P. Mulier, B. Dillemans, T. Declercq, T. Sablon

Anaesthesiology, AZ St Jan AV Brugge, Brugge, Belgium

Background and Goal of Study: The abdominal pressure volume relation (APVR) is measured during a pneumoperitoneum.(1) If the APVR is known earlier therapeutic actions can differ. Goal of this study was to measure the APVR direct after induction and to compare it with the APVR during the pneumoperitoneum.

Materials and Methods: Approval from the ethical committee was given. 10 Patients scheduled for a gastric banding who need a gastric tube with a balloon are investigated After anesthesia induction the gastric tube is introduced in the stomach and used to measure the gastric pressure during end inspiration and expiration. The airway pressure is measured at the same 
time. Patient is volume ventilated with a tidal volume of 10 and $6 \mathrm{ml} / \mathrm{kg}$. The abdominal movement is free during a first measurement and is blocked by an abdominal corset during a second measurement. After both measurements ventilation is proceeded as clinical required.

The total compliance is calculated during abdominal fixation with two tidal volumes. Without abdominal fixation part of the tidal volume pushes the diaphragm down giving a rise in gastric pressure. The airway pressure without abdominal fixation is lower and allows to calculate the comparable tidal volume that should be used with abdominal fixation. The difference is the volume blown in the abdomen. The concomitant intra-gastric pressure rise is used to calculate the abdominal elastance. The intra gastric pressure at end expiration without abdominal fixation is used as PVO calculated. At the start of the pneumoperitoneum the abdominal pressure volume relation with E and PVO are measured as previously described. TOF is used to assures no change in muscle relaxation. Both values of $E$ and $P V O$ are compared with a paired t test. Results and Discussions:

\begin{tabular}{lllll}
\hline & E calc & E meas & PV0 calc & PV0 meas \\
\hline Mean & 4.76 & 3.23 & 7.2 & 7 \\
st. dev. & 1.52 & 1.34 & 1.3 & 0.7 \\
Paired t test & 0.362 & & 0.044 & \\
\hline
\end{tabular}

Conclusion: PVO is correctly estimated while $\mathrm{E}$ is significant different. This technique is not suitable to measure the $E$ before the pneumoperitoneum. Reference:

1 JPMulier Eur J Anesth 2006, vol23 s37. A124.

\section{AP1-2}

Evaluation of a mechanical build model of the abdominal pressure volume relation

J.P. Mulier, B. Dillemans, L. Heremans, A. Vanlander

Anaesthesiology, az St Jan AV Brugge, Brugge, Belgium

Background and Goal of Study: We calculated the pressure volume relation (PVR) of several theoretical mechanical models. We found that a half rigid sphere with one elastic membrane behaves initial linear and probably comparable to the abdominal PVR. We have build such a mechanical model and wanted to test its PVR.

Materials and Methods: No approval from the ethical committee was needed as no patients or animals were investigated in this study. A half rigid sphere of $25 \mathrm{~mm}$ radius is covered with an elastic membrane. Water is injected with an increment of $2 \mathrm{ml}$ till an elevation of $40 \mathrm{~mm}$ is reached. The pressure is measured with a pressure transducer in $\mathrm{mmHg}$ and the elevation above the sphere is measured in $\mathrm{mm}$. A linear fit is made using the least square with $\mathrm{R}^{2}$ as fit quality. The maximum elevation up to the radius with a fit quality of 0.99 is calculated. Results and Discussions: Graph 1 shows the measured pressure volume relation and the measured elevation volume relation. The fitted line using the data points up to an elevation of the radius gives an $R^{2}$ of 0.999 and is given on the same graph.

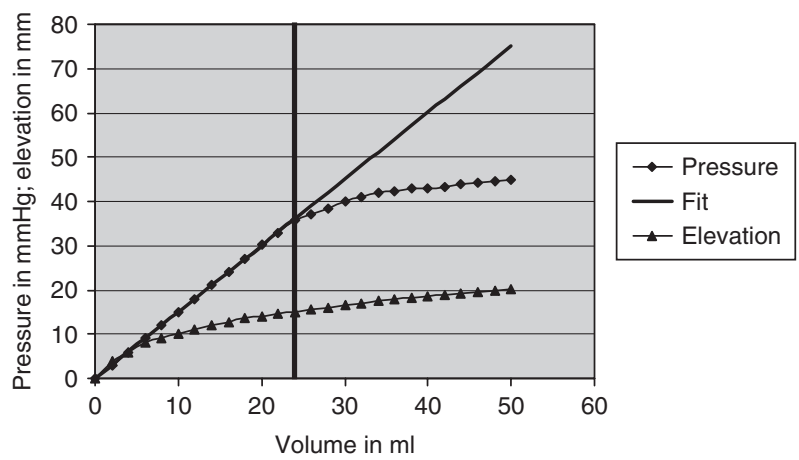

Conclusion: A half rigid sphere covered with an elastic membrane behaves linear with an elevation up to the radius. This model is a possible explanation for the linear abdominal elastance behavior (1).

Reference:

1 J.P. Mulier Eur J Anesth 2006, vol 23 s37. A124.

\section{AP1-3}

Is $\mathrm{CO}_{2}$ leakage or absorption important during measurement of the abdominal pressure volume relation in a pneumoperitoneum?

J.P. Mulier, B. Dillemans, A. Luijten, R. Nieuwenhuizen

Anaesthesiology, az St Jan AV Brugge, Brugge, Belgium
Background and Goal of Study: We measure the abdominal pressure inflated volume relation at the beginning of a pneumoperitoneum. We assume that $\mathrm{CO}_{2}$ absorption and leakage during the initial measurements is minimal. We wanted to test if absorption or leakage during a period of 5 minutes disturbs the measurements.

Materials and Methods: Approval from the ethical committee was given. 10 Patients scheduled for a laparoscopic operation are included in this study. One trocar is placed and inflation is stopped when abdominal pressure reaches $15 \mathrm{mmHg}$. We measured the abdominal pressure at 1 and at 5 minutes after the insufflation stop and analysed with the paired $t$ test. No medication was given in bolus, no inhalation concentration was changed, no surgeon was allowed to touch the patient and table position or ventilation remained unchanged. Results and Discussions: The insufflator stops when $15 \mathrm{mmHg}$ is reached during inflation. The insufflator is closed and the abdominal pressure stabilises in the first minute around $14 \mathrm{mmHg}$ and stays constant. Table 1 gives the mean and standard deviation together with the paired $t$ test.

\begin{tabular}{lll}
\hline & After 1 minute & After 5 minutes \\
\hline mean & 14,01 & 13,94 \\
st dev & 0,5858517 & 0,6040603 \\
$t$ test & 0,132303 & \\
\hline
\end{tabular}

No statistical difference with the paired t test was found between both measurements suggesting that absorption and leakage do not play an important role during the measurement of the abdominal pressure volume relation.

Conclusion: Leakage is minimal and absorption is small in the first 5 minutes during a pneumoperitoneum.

Reference:

1 J. P. Mulier Eur J Anesth 2006, vol 23 s37. A124.

\section{AP1-4}

\section{Effect of desflurane on the abdominal pressure volume} relation without muscle relaxants

J.P. Mulier, B. Dillemans, K. Verbeke, F. Akin

Anaesthesiology, Az St Jan AV Brugge, Brugge, Belgium

Background and Goal of Study: Relaxes desflurane the abdominal muscles without muscle relaxants? The use of the abdominal pressure volume relation (1) allows the evaluation of the effects of desflurane on the abdominal muscles. We found in previous studies that only the pressure at zero volume (PV) changes with muscle relaxation. Goal of this study was to test if elastance (E) or $\mathrm{PV}^{0}$ changes with 1,5 Mac versus 0,5 MAC desflurane in air/oxygen.

Materials and Methods: 20 patients, ASA class I, II or III between 21 and 75 years old and scheduled for a laparoscopic surgery were included in this study with approval from the hospital ethical committee.

Anaesthesia was induced with Propofol 200 mg, Sufentanil 20 ug, and succinylcholine $100 \mathrm{mg}$. Anaesthesia was maintained with a remifentanyl infusion of $0,5 \mathrm{ug} / \mathrm{kg} / \mathrm{minute}$. and patients were ventilated with $50 \% \mathrm{O}_{2} / \mathrm{N}_{2} \mathrm{O}$. Desflurane was given randomly at 0,5 or at $1,5 \mathrm{Mac}$ and followed by 1,5 or $0,5 \mathrm{Mac}$ in a $50 \% \mathrm{O}_{2}$ /air concentration. When end tidal concentration was stable the abdomen was inflated with calculation of $\mathrm{E}$ and $\mathrm{PV}^{0}$. Patients were asked to empty the bladder before surgery. The stomach was emptied by suction through a gastric tube. All the $\mathrm{CO}_{2}$ was allowed to escape between two measurements. A paired t test was used to analyse the difference between 0,5 and 1,5 mac for $E$ and $\mathrm{PV}^{0}$.

Results and Discussions:

\begin{tabular}{lllll}
\hline & $\mathrm{E} \mathrm{0,5} \mathrm{mac}$ & $\mathrm{E} \mathrm{1,5} \mathrm{mac}$ & $\mathrm{PV}^{0} 0,5 \mathrm{mac}$ & $\mathrm{PV}^{0} 1,5 \mathrm{mac}$ \\
\hline mean & 2,76 & 2,81 & 6,2 & 5,4 \\
st dev & 0,87 & 0,94 & 0,4 & 0,7 \\
paired t test & 0,262 & & 0,045 & \\
\hline
\end{tabular}

Table 1 gives the mean and standard deviation for $E$ and $\mathrm{PV}^{0}$ at 0,5 and 1,5 mac with the paired t test. $\mathrm{PV}^{0}$ did fall significantly with increasing concentration of desflurane while $E$ remained unchanged.

Conclusion: Desflurane has some independent muscle relaxant effect lowering $\mathrm{PV}^{0}$ without affecting $\mathrm{E}$.

Reference:

1 J. P. Mulier Eur J Anesth 2006, vol 23 s37. A124.

\section{AP1-5}

Online monitoring of propofol in expiratory air in patients undergoing total intravenous anaesthesia

C. Hornuss, S. Praun, J. Villinger, M. Dolch, G. Schelling

Department of Anaesthesiology, University of Munich, Munich, Germany 
Background and Goal of Study: Propofol is an aromatic compound with low water solubility and low vapour pressure $\left(0.142 \mathrm{mmHg}\right.$ at $\left.20^{\circ} \mathrm{C}\right)$. These properties could allow diffusion of propofol through the alveolocapillary membrane and detection of volatile propofol in exhaled breath of patients undergoing total intravenous propofol anaesthesia with this substance.

The aim of this study was to detect propofol in exhaled breath and to examine the relationship between propofol in exhaled breath and propofol whole blood levels in patients undergoing total intravenous anaesthesia.

Materials and Methods: Eleven patients received target controlled infusion of propofol during neurosurgical procedures.

For detection of volatile propofol a gas analysing system based on ion molecule reactions coupled with quadrupole mass spectrometry ${ }^{1}$ was used. The system was directly connected to the endotracheal tube by a T-piece and $50 \mathrm{ml} / \mathrm{min}$ of breathing gas were continuously analysed for propofol concentrations. Propofol whole blood levels were determined by liquid chromatography tandem mass spectrometry.

Results and Discussion: Propofol could be detected in exhaled breath of patients undergoing propofol anaesthesia. A close interindividual correlation between propofol levels in exhaled breath and propofol whole blood levels was found ( $\mathrm{r}^{2}=0.722$ of 49 measurements in eleven patients). Correlation between expiratory breath and blood propofol levels within individual patients ranged from $r^{2}=0.615$ to $r^{2}=0.970$. Expiratory propofol levels followed changes in propofol whole blood levels within less than 60s.

Conclusions: Analysis of propofol in exhaled breath by ion molecule reaction mass spectrometry allows monitoring of relative changes in propofol whole blood concentrations. This could permit non-invasive routine estimation of propofol blood levels in patients undergoing total intravenous anaesthesia similar to monitoring of MAC with volatile anaesthetics.

\section{AP1-6}

Performance of a tactile adductor pollicis close-loop control for managing stable paralysis levels in abdominal surgery.

A. Dhollander, E. Schiffer, I. Madzar, C-E. Klopfenstein

Anesthesiology, CHU-Geneva, geneva, Switzerland

Background and Goal of the Study: Performance of many automatic muscle relaxants close-loop [C-L] control systems are generally excellent. As measuring the performance of a clinician closing the loop with a simple tactile monitoring remains poorly documented (1), the goal of the present trial was to measure the performance of clinicians managing a full manual $\mathrm{C}$ - $\mathrm{L}$ control with tactile adductor pollicis TOF counting [TOFc].

Methods: After IEC approval, twenty ASA I-II written consenting adult patients scheduled for elective lower abdominal laparotomies were anesthetized (sufentanil/propofol/ mivacurium or cis-atracurium), intubated and mecanically normoventilated. The muscle relaxant perfusion was adjusted to target two TOFc levels: either 1 or 2 . TOFc and muscle relaxant perfusion were concomitantly assessed every 5 min during the first $60 \mathrm{~min}$ and, thereafter, at 15 min intervals until the end of the surgery. Thenar and oro-pharyngeal temperatures were controlled and maintained, by different warming devices, above 32.5 and $35.9^{\circ} \mathrm{C}$, respectively. For each patient, the manual C-L control performance was defined by the ratio: total number of TOFc 1 or 2 episodes/total number of control periods.

Results: The data collected, expressed as [median]-p10/p90] were: age (years)[68] 49/80, weight (kg)[73] 46/82, height (cm) [171]-160/177; muscle relaxant infusion durations (min) [207-111/365]. Descriptive statistics the TOFc based C-L control observed are detailed below:

\begin{tabular}{lr}
\hline Total number of TOFc 1 or 2 episodes & {$[19]-10 / 30$} \\
Total number of control periods & {$[21]-15 / 33$} \\
Performance of manual C-L control & {$[0.89]-0.72 / 1$} \\
\hline
\end{tabular}

Conclusion: The performance level obtained with the present manual C-L control should appear quite attractive for all the clinicians concerned for producing stable paralysis levels by such a simple, robust but reliable metho-dology.

Reference:

1 Pedersen NA al. Ugeskr Laeger 2000; 162: 6532-5.

\section{AP1-7}

The effect of volatile anaesthetic agents on filtration efficiency

C. Cann, A.R. Wilkes, J.E. Hall, D. Chethan, M.A. Hampson

Anaesthetics and Intensive Care Medicine, Cardiff University, Cardiff, United Kingdom

Background and Goal of Study: The European standard for breathing systems filters (BSF) tests filtration performance by challenging filters with an aerosol of sodium chloride particles (1). Volatile anaesthetic vapours are organic molecules and may affect the filtration performance of certain BSF during anaesthesia (2). Therefore, the filtration performance of the Filta-Therm BSF (Intersurgical Limited, Wokingham, UK) was measured following exposure to sevoflurane and isoflurane.

Materials and Methods: The pressure drop across all samples was measured at a flow of $30 \mathrm{Lmin}^{-1}$. Five samples were then exposed to sevoflurane at the following: $1.8 \%$ for $1 \mathrm{~h}, 3.6 \%$ for $1 \mathrm{~h}$ and $3.6 \%$ for $4 \mathrm{~h}$. This was repeated for isoflurane at $1.2 \%$ for $1 \mathrm{~h}, 2.4 \%$ for $1 \mathrm{~h}, 2.4 \%$ for $4 \mathrm{~h}$. Five samples were exposed to air only for $1 \mathrm{~h}$ and five were not exposed. The filtration performance was then measured using a Moore's test rig (SFP Services, Christchurch, $\mathrm{UK}$ ) at a flow of $30 \mathrm{Lmin}^{-1}$. One-way analysis of variance (SPSS 14, SPSS Inc. Chicago, II) was used to analyse the penetration results with anaesthetic (sevoflurane (3 groups), isoflurane (3 groups), air or no exposure) added as a factor.

Results and Discussion:

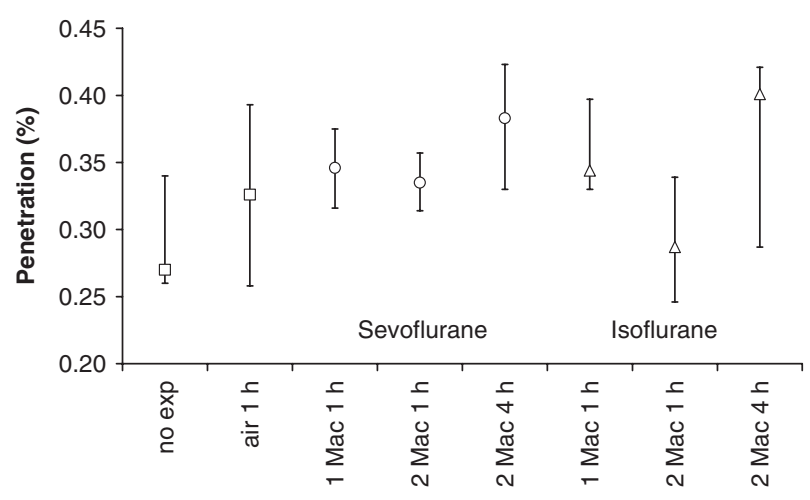

Conclusion: Neither volatile anaesthetic has a clinically significant affect on filtration efficiency on this BSF $(p>0.05)$.

References:

1 British Standards Institution (BSI). BS EN 13328-1: 2001. London: BSI, 2001.

2 Wilkes AR. Br J Anaesth 2005; 95: 577P.

\section{AP1-8}

The validity of esophageal temperature during open chest surgery

S.K. Liu, R.SC. Wu, B.C. Lin

China Medical University Hospita, Departments of Anesthesia, Pain Service and Critical Care Medicine, Taichung, Taiwan

Background and Goal of Study: Accurate monitoring of core body temperature is vitally important in patients during major surgery under general anesthesia. In the operation room, core temperature can be measured at various sites, such as the lower esophagus, nasopharynx, pulmonary artery and tympanic membrane. ${ }^{1}$

Liver transplantation ${ }^{2}$ with procedure for esophageal temperature monitoring closed to the measurement sites of esophageal temperature recording may cause faulty readings. We hypothesize that esophageal temperature would be affected by ambient environment and surgical manipulations during the procedure. This study investigated the validity of esophageal temperature in open chest surgery with full lateral position.

Materials and Methods: We studied 32 adult patients undergoing thoracic surgery placing in the full lateral position. Patients with hypothermia $\left(<36^{\circ}\right)$ or already being hyperthermic $\left(>38^{\circ}\right)$ before entrancing the operating room were excluded. The operating room was kept at a temperature of $22 \pm 1{ }^{\circ} \mathrm{C}$. General anesthesia was maintained with sevoflurane, and the fresh gas flow was $2 \mathrm{~L} / \mathrm{min}$ constantly throughout the surgery. Tympanic and esophageal temperatures were measured by temperature probes. All core temperatures were recorded at 15-minute intervals following induction of anesthesia and throughout the surgery.

Results and Discussions: Esophageal temperature (Te) correlated well with tympanic temperature ( $\mathrm{Tt}$ ) before chest opening but significant difference was observed after chest opening ( $p<0.05$, paired t-test). The difference of Te at different time after chest opening was also significant difference with $\mathrm{Tt}$ by using mixed model analysis. Te is influenced by both dependent lung, warmer part, and nondependent lung, cooler part. The mixed effects of perfusing blood temperature of the esophagus and cooler surroundings adjacent to the esophagus lower Te. 
Conclusion(s): Te is affected during open chest surgery and the validity is not reliable. Tt is still a proper site for monitoring during open chest surgery. References:

1 Sessler DI Anesthesiology 1998; 89: 1298-1300.

2 Russell SH \& Freeman JW. British Journal of Anaesthesia 1995; 74: 415-418.

\section{AP1-9}

The liver-vein catheter: a feasible tool to assess hepatic oxygenation and metabolism in partial hepatectomy

A. Vogt, C. Pulver, V. Hartwich, D. Inderbitzin, S. Jakob

Department of Anesthesiology, University Hospital Bern, Bern, Switzerland

Background: Hepatic resection is associated with high morbidity [1] Reported complications include: hemorrhage, biliary leakage and transient liver failure [2]. To reduce intraoperative blood loss low central venous pressure [3] and non-selective intermittent hepatic pedicle clamping has been suggested [2]. The effects of these manoeuvres on liver oxygenation are unknown. We assessed the feasibility of perioperative liver-vein catheterization for the measurement of hepatic oxygenation.

Methods: 9 patients scheduled for partial hepatectomy were included. A CCO/SVO2 pulmonary artery catheter was inserted in a hepatic vein using fluoroscopy and the correct position confirmed with a small amount of contrast medium. Time from start insertion to the correct positionning, length of catheter insertion and complications were recorded. Pressure and hepaticvein saturation were continuously recorded.

Results: Mean placement time was 16 minutes and correct position was achieved at $35-40 \mathrm{~cm}$. No complications occured. In the table data are presented as mean (SD).

\begin{tabular}{llllll}
\hline & $\begin{array}{l}\text { Invasive art. } \\
\text { pressure } \\
{[\mathrm{mmHg}]}\end{array}$ & $\begin{array}{l}\text { CVP } \\
{[\mathrm{mmHg}]}\end{array}$ & $\begin{array}{l}\text { Liver-vein } \\
\text { pressure } \\
{[\mathrm{mmHg}]}\end{array}$ & $\begin{array}{l}\text { Liver-vein- } \\
\text { saturation [\%] }\end{array}$ & $\begin{array}{l}\text { Inspired } \\
\text { oxygen } \\
\text { fraction [\%] }\end{array}$ \\
\hline $\begin{array}{l}\text { Before } \\
\text { resection }\end{array}$ & $70(6)$ & $8(3)$ & $12(9)$ & $75(10)$ & $38(4)$ \\
$\begin{array}{l}\text { During } \\
\text { resection }\end{array}$ & $70(10)$ & $5(2)$ & $6(4)$ & $67(18)$ & $36(2)$ \\
$\begin{array}{l}\text { After } \\
\text { resection }\end{array}$ & $70(6)$ & $6(2)$ & $8(8)$. & $67(22)$ & $36(2)$ \\
ANOVA RM & $\mathrm{p}=0.967$ & $\mathrm{p}=0.009$ & $\mathrm{p}=0.476$ & $\mathrm{p}=0.784$ & $\mathrm{p}=0.165$ \\
\hline
\end{tabular}

Conclusion: Insertion of a liver-vein catheter for monitoring during partial hepatectomy is feasible and valuable to assess liver oxygenation and metabolism.

References:

1 Jarnagin, W.R., et al., Ann Surg, 2002. 236: 397-406.

2 Aldrighetti, L., et al., J Surg Oncol, 2006. 931: 86-93.

3 Wang, W.D., et al., World J Gastroenterol, 2006. 12: 935-9.

\section{AP2-1}

Monitoring with Narcotrend (NT) or standard anaesthesiologists technique (SAT) during anesthesia with desflurane-remifentanil in major gynaecological surgery.

\section{Our experience}

S. Pirri, L. Scimeca, M. Farbo, G. Alagna, F. Giambartino

Anesthesia and Intensive Care D.A.R.E., Policlinico Universitario, Palermo (PA), Italy

Background and Goal of Study: To compare the influence of the monitoring with NT or SAT (based on the classic clinical parameters), during anaesthesia with desflurane (D) and remifentanil (R).

Materials and Methods: 20 patients (ASA I-II) candidate to elective major gynaecological surgery have been randomly assigned in 2 groups that received D-R under the guide of NT monitoring (group A) or SAT (group B). Premedication in both groups: atropine $0,01 \mathrm{mg} / \mathrm{kg}$, ranitidine $50 \mathrm{mg}$ i.v. At the induction of anaesthesia all the patients received propofol $2 \mathrm{mg} / \mathrm{kg}$ and remifentanil $0,25 \mathrm{mcg} / \mathrm{kg} / \mathrm{min}$. After muscle paralysis (cisatracurium $0,2 \mathrm{mg} / \mathrm{kg}$ ) and endotracheal intubation, D has been administered (at MAC 1) with FGF $4 \mathrm{~L} / \mathrm{min}$ in $\mathrm{O}_{2} 50 \%$ mixture for $3 \mathrm{~min}$ that it came then reduced to $0.5 \mathrm{~L} / \mathrm{min}$. The $\mathrm{D}$ came therefore titrated (up and down technique) in order to obtain a NT index score of "D0-E1" during maintenance (group A). In the group B the D titration has been guided by the observation of clinical parameters which cardiac frequency, arterial pressure, spontaneous movements. The times of recovery were recorded. The vaporiser of the $D$ has been weighed at the beginning and the end of every participation and the gas consumption for every patient has been calculated.
Results and Discussions: The 2 groups were similar for demographic data, medium dosage of $R$ and duration of anaesthesia. The group $A$ has received a smaller amount of $D(391,7 \mathrm{mg} / \mathrm{min})$ regarding the group $B(444,6 \mathrm{mg} / \mathrm{min})$ $(P=0.003)$. The times of recovery not deferred meaningfully between the 2 groups: time eyes opening group $\mathrm{A}: 4.1 \pm 1.8 \mathrm{~min}$, group $\mathrm{B}: 4.5 \pm 2.1 \mathrm{~min}$. The mean end tidal\% D to obtain a NT index of D0-E1 was 4.8.

Conclusion(s): Our data suggest that during anaesthesia with $\mathrm{D}-\mathrm{R}$ in closed circuit $0,5 \mathrm{~L} / \mathrm{min}$ in $\mathrm{O} 250 \%$ mixture, in major gynaecological surgery, NT monitoring has concurred a reduction in the consumption of $D$, while minimal differences in the reduction of the times of recovery regarding the monitoring with SAT have been observed.

Reference:

Anesth Analg 2005; 101: 427-434.

\section{AP2-2}

Changes of electroencephalographic bicoherence induced by hypothermia

S. Hagihira, M. Takashina, T. Mori, T. Mashimo

Anesthesiology, Osaka University Graduate School of Medicine, Suita City, Japan

Background and Goal of Study: We previously investigated the changes of EEG bicoherence, the degree of phase coupling among the frequency components of a signal, during anesthesia. We found two dominant peaks were emerged in bi-frequency space during anesthesia. We defined those peak heights as pBIC_low and pBIC_high, and their frequencies as $f B I C \_l o w$, fBIC_high. Here we investigated the effect of hypothermia on those peaks. Materials and Methods: After IRB approval and obtained informed consent from the participants, we enrolled 10 patients (either gender; 51-79 yr) who underwent elective cardiac surgery using cardio-pulmonary bypass (CPB). Anesthesia was induced with propofol using target controlled infusion (TCI) system followed by fentanyl and vecuronium. Besides the monitors for cardiac surgery, BIS-Plus probe was attached and raw EEG wave data as well as BIS and other EEG derived parameters were continuously recorded on a computer and processed using our original software BSA for BIS. Anesthesia was maintained with propofol ( $\mathrm{TCl}$ was set at $1.5-2.0 \mu \mathrm{g} / \mathrm{ml}$ ) and fentanyl. Just before starting CPB, target of propofol was set at $1.0 \mu \mathrm{g} / \mathrm{ml}$ and was kept until body temperature (BT) was returned to $35.0^{\circ} \mathrm{C}$. We measured those parameters at 3 points; (1) when $\mathrm{BT}$ was decreased to $35.0^{\circ} \mathrm{C}$, (2) during $\mathrm{CPB}$ at $28.0^{\circ} \mathrm{C}$ and (3) when $\mathrm{BT}$ was returned to $35.0^{\circ} \mathrm{C}$.

Results and Discussions:

\begin{tabular}{lccc}
\hline & Pre $\left(35.0^{\circ} \mathrm{C}\right)$ & CPB $\left(28.0^{\circ} \mathrm{C}\right)$ & Post $\left(35.0^{\circ} \mathrm{C}\right)$ \\
\hline pBIC_low & $35.9 \pm 9.0(\%)$ & $24.6 \pm 7.0(\%)$ & $39.1 \pm 8.6(\%)$ \\
pBIC_high & $35.1 \pm 8.9(\%)$ & $28.2 \pm 5.5(\%)$ & $34.3 \pm 5.6(\%)$ \\
fBIC_low & $4.1 \pm 0.4(\mathrm{~Hz})$ & $2.3 \pm 0.3(\mathrm{~Hz})^{\star}$ & $4.5 \pm 0.3(\mathrm{~Hz})$ \\
fBIC_high & $9.1 \pm 0.8(\mathrm{~Hz})$ & $5.3 \pm 0.7(\mathrm{~Hz})^{\star}$ & $9.5 \pm 0.9(\mathrm{~Hz})$ \\
\hline
\end{tabular}

${ }^{*} \mathrm{p}<0.05$

We previously showed that fBIC_high was decreased while fBIC_low remained around $4.0 \mathrm{~Hz}$ when the concentration of anesthetic was increased. Thus changes of EEG bicoherence during hypothermia was quite different from those when the concentration of anesthetic was increased.

Conclusions: Hypothermia altered the phase coupling of EEG in different way of the anesthetics.

Reference:

1 Hagihira S et al. Anesthesiology 2002; 97: 1409-15.

\section{AP2-3}

Does AEP monitoring reveal changes in anesthetic depth during one lung ventilation in sevoflurane anesthesia?

J. Tyczka, P. Ciszewski, M. Skrzypczak, A. Mikstacki, J. Nadolski

Intensive Care Department, Pulmonary Diseases and Thoracic Surgery Center, Poznan, Poland

Background and Goal of Study: One lung ventilation (OLV) during thoracic sugery is associated with rapid and significant changes in alveolar ventilation and blood perfusion in the dependent lung. The inhalation anesthesia with sevoflurane, an agent with the low blood solubility is regarded as a save method that protects patients from the intraoperative awareness and ensures quick recovery. In our prospective study we evaluated changes in the depth of anesthesia measured by the AEP Monitor/2 (Danmeter) during two lungs ventilation (TLV) and OLV in patients under combined anesthesia with sevoflurane. Materials and Methods: 24 patients, aged $52.8 \pm 17.4$ yrs undergoing lung resection due to primary lung cancer or metastasectomy were enrolled. Thoracic epidural analgesia (catheter T5-6, bupivacaine $0.25 \%$, fentanyl 
$100 \mathrm{mcg})$ and induction with propofol $\left(2 \mathrm{mg} \cdot \mathrm{kg}^{-1}\right)$, fentanyl $\left(3 \mathrm{mcg} \cdot \mathrm{kg}^{-1}\right)$ and rocuronium $\left(0.6 \mathrm{mg} \cdot \mathrm{kg}^{-1}\right)$ were used. After intubation with double-lumen endobronchial tube both lungs were ventilated with sevoflurane in oxygen with end-tidal concentration adjusted to achieve AAI 15-25. The adjusted concentration of sevoflurane was maintained during OLV. Average AAI during TLV, initial $10 \mathrm{~min}$ of OLV and remaining period of OLV were analyzed and compared. Patients were observed for signs of inadequate anesthesia and reviewed for the intraoperative awareness.

Results and Discussions: Average AAl during initial $10 \mathrm{~min}$ of OLV was higher than during TLV $(18.8 \pm 4.9$ vs $16.9 \pm 3.9, \mathrm{p}<0.005)$ and higher than AAl in the remaining period of OLV $(18.8 \pm 4.9$ vs $17 \pm 4.2, p<0.05)$, with the maximal increase to $27.1 \pm 7.6(18-40)$. The peak AAl value appeared at $4.3 \pm 2$ $\mathrm{min}$ and returned to baseline at $7.9 \pm 2.4 \mathrm{~min}$ after OLV had been started. The initial sevoflurane concentration had to be increased in 4 pts $(16.7 \%)$ with $\mathrm{AAl}>35$ and signs of inadequate anesthesia. Mean sevoflurane concentration was $0.97 \pm 0.2 \%$ and showed a negative correlation with the age $(r=-0.67$, $\mathrm{p}<0.05$ ). No incidents of intraoperative awareness were observed.

Conclusion: The depth of sevoflurane anesthesia during OLV may change rapidly. Monitoring the depth of anesthesia helps to avoid unsuspected awareness.

Reference:

Janshon GP, Anesthesist 1998; 47: 52-7.

\section{AP2-4}

BIS as predictor of anterograde amnesia in nasal midazolam administration

M.J. Estruch-Pérez, J. Sánchez, M.T. Richart, J. Soliveres, C. Solaz

Anesthesiology and Critical Care, Dr. Peset University Hospital, Valencia, Spain

Background and Goal of Study: Surgery room memories or venopuncture may be unpleasant. Nasal midazolam has a good pharmacokinetic profile and can be used for premedication ${ }^{1}$. The objective is to assess the sensitivity and specificity of BIS level under 90 to determine anterograd amnesia after $10 \mathrm{mg}$ of nasal midazolam administration.

Materials and Methods: After ethics committee approval, written informed consent was obtained from 42 ASA I-III consecutive patients scheduled for any surgery and included in a prospective study. Patients with a body mass index more than 35 , possible pregnancy, benzodiazepine sensitivity or allergy, psychiatric disease or medication, drug or ethanol abuse, myasthenia gravis, rhinitis and nasal obstruction were excluded. BIS levels (Aspect A2000, software rev3) and standard monitoring was started and recorded at basal, after administration of $10 \mathrm{mg}$ midazolam ( $1 \mathrm{ml}, 5 \mathrm{mg}$ into each nostril). Time to BIS under 90 (BIS $<90$ ) was defined as the time until BIS values reached 90 or less for at least 4 minutes. When BIS $<90$ was reached (test positive) or after 15 minutes (BIS $>90$, test negative) an intravenous line was placed with an 18G standard canula. After surgery, the patients were asked for venopuncture recall. BIS $<90$ was defined as true positive (TP) without recall or false positive (FP) with recall. BIS $>90$ was defined as true negative (TN) without recall or false negative ( $F N$ ) with recall. Sensitivity (S), specificity (E), positive predictive value (PPV) and negative predictive value (NPV) were analysed. Results are expressed as number (percent) or mean $\pm \mathrm{SD}$. Results and Discussion: Results are shown in the table.

\begin{tabular}{llll}
\hline & Amnesia & No amnesia & Total \\
\hline BIS $<90$ & $33(\mathrm{TP})$ & $3(\mathrm{FP})$ & $\mathrm{S}=97,1 \%$ \\
BIS $>90$ & $1(\mathrm{FN})$ & $5(\mathrm{TN})$ & $\mathrm{E}=62,5 \%$ \\
Total & $34(81 \%)$ & $8(19 \%)$ & \\
\hline
\end{tabular}

Time to reach BIS $<90$ was $5,57 \pm 2,17$ min. PPV was $91,7 \%$ and NPV was $83,3 \%$. Benefits of midazolam include anterograde amnesia and reduction in reported undesirable event recalls.

Conclusion: These results suggest that BIS $<90$ is a good test to predict anterograde amnesia after $10 \mathrm{mg}$ midazolam nasal administration.

Reference:

1 Malinovsky J.-M. et al. BJA 1993; 70: 617-20.

\section{AP2-5}

The difference between BIS guided vs. BIS unguided anesthesia in off pump coronary artery bypass grafting surgery

N. Karanovic, I. Husedzinovic, N. Nikic, M. Carev, Z. Dogas

Anesthesiology and Intensive Care, University of Split School of Medicine, Split, Croatia

Background and Goal of Study: Clinical evaluation of BIS is still controversial (1). The aim was to find out the difference regarding amount of used anesthetic agents between BIS guided vs. BIS unguided anesthesia, during off pump coronary artery bypass grafting surgery (OPCABG).

Materials and Methods: After Medical Ethics Committee approval and written consent, patients scheduled for OPCABG surgery were randomly assigned into BIS (B) and control (C) groups. Excluding criteria: diabetes, liver and renal impairment, carotid circulatory insufficiency. In B group anesthesia was maintained according to BIS values at $40-45$, whereas in $C$ group according to anesthesiologist's estimation and experience. The total amounts of midazolam and fentanyl were compared at the end of anesthesia. End tidal sevoflurane (ETsevo) values were compared at 5 time points (S1-S5) according to particular surgery procedures. Ventilation was maintained with $50 \%$ oxygen in air, mean arterial pressure (MAP) between 70 and $85 \mathrm{~mm} \mathrm{Hg}$, heart rate between 60 and 90 . The usage of other agents was registered. GLM for repeated measures, $\chi^{2}$ and $t$ tests were used for statistical analysis; $\mathrm{p}<0.05$ was considered statistically significant.

Results and Discussions: There was no difference between the groups regarding gender $(p=0.31)$, age $(p=0.77)$, body mass $(p=0.34)$, body height $(p=0.13)$, duration of anesthesia $(p=0.70)$ and surgery $(p=0.78)$.

\begin{tabular}{lccl}
\hline Agent & B group $(\mathrm{n}=23)$ & C group $(\mathrm{n}=25)$ & $\mathrm{p}$ \\
\hline Fentanyl $(\mathrm{mg})$ & $1.66 \pm 0.34$ & $1.49 \pm 0.26$ & 0.060 \\
Midazolam (mg) & $38.04 \pm 7.15$ & $35.60 \pm 9.50$ & 0.320 \\
ETsevo 1 (S1) & $1.23 \pm 0.34$ & $1.06 \pm 0.36$ & 0.090 \\
ETsevo 2 (S2) & $1.31 \pm 0.22$ & $1.08 \pm 0.25$ & 0.001 \\
ETsevo 3 (S3) & $1.17 \pm 0.19$ & $1.25 \pm 0.41$ & 0.380 \\
ETsevo 4 (S4) & $1.10 \pm 0.26$ & $0.94 \pm 0.24$ & 0.030 \\
ETsevo 5 (S5) & $0.90 \pm 0.18$ & $0.80 \pm 0.21$ & 0.070 \\
\hline
\end{tabular}

The significant difference existed among ETsevo values $(F=21.6$, $p<0.001$ ), as well as between $B$ and $C$ groups regarding repeated measures of ETsevo $(F=3.3, p=0.012)$.

Conclusion: There was a significant difference in anesthesia for OPCABG surgery regarding ETsevo values guided by BIS vs. BIS unguided. Fentanyl appeared to be used more in group B than in group C.

Reference:

1 Anaesth Analg 2003; 96: 336-43.

\section{AP2-7}

Quantative EEG monitored anaesthesia; cost comparison of three anaesthetic techniques management

B. Berkel, Z. Alanoglu, Y. Ates, O. SelviCan, F. Tuzuner

Anaesthesiology and ICM, Medical School of Ankara University, Ankara,

Turkey

Background and Goal of Study: Monitoring the central nervous system effects of anaesthesia in an objective, reliable fashion has been an ultimate goal (1). Objective criteria for the depth of anaesthesia are the most challenging and missing part for cost and recovery studies (2). The purpose of this study was to compare quantitative EEG (QEEG), haemodynamic changes and cost analysis of a propofol/remifentanil (TIVA), desflurane, sevoflurane anaesthesia in major abdominal surgery.

Material and Methods: With IRB approval and written informed consent 60 ASA I-III patients undergoing elective major abdominal surgery were allocated randomly to groups to receive propofol/remifentanil (Group P $n=20$ ), desflurane (Group D $n=20$ ) or sevoflurane (Group $S n=20$ ) based anaesthesia. ECG, NIBP, $\mathrm{SpO}_{2}$, oesophagus temperature, TOF, QEEG monitoring were done and data were collected at before induction, at induction, at intubation; during anaesthesia management, before and after skin suturing and at complete recovery. The minute cost of anaesthesia agent was calculated. Area under (AUC) haemodynamic variables, QEEG values and time curves were calculated and assessed with one way ANOVA. Repeated measures for ANOVA and Chi-square tests were also used and $p<0.05$ was considered as significant. Values expressed as mean and SD.

Results and Discussion: The area under haemodynamic variables vs time curves were higher in Group P compared to other groups $(p<0.05)$. Similarly, SEF 95 and alpha wave value were higher and values of power and theta waves were lower in Group P compared to other groups during noxious stimuli $(p<0,05)$. Calculated minute cost of anaesthetic agent was lower in Group $S$ $(0,13 \pm 0,02$ Euro) then both Group $D(0,17 \pm 0,02$ Euro, $p=0.001)$ and Group $P(0,16 \pm 0,04$ Euro, $p=0.015)$. The recovery and postoperative variables were similar among groups.

Conclusions: QEEG demonstrated lighter plains of anaesthesia with propofol/ remifentanil based maintenance; however the use of sevoflurane as the anaesthetic agent not only provided efficient anaesthesia depth, but also the cheapest one. 
References:

1 Rosow C. et al. Anesthesiol Clin North America 2001; 19: 947-66.

2 Rohm KD. et al. Acta Anaesthesiol Scand 2006; 50: 14-8.

\section{AP2-8}

Spectral entropy reduces propofol consumption during long lasting $\mathrm{TCl}$ anaesthesia

N. Van Rompaey, E. Engelman, O. Caelen, A. Verleije, B. Ickx

Anesthesiology, CUB Hopital Erasme, Bruxelles, Belgium

Background and Goal of Study: Spectral entropy monitoring has already been shown to decrease anaesthetic drug consumption during short to medium term anaesthesia (1). Target controlled infusion (TCl) anaesthesia using propofol/remifentanil is associated with the highest intraoperative cost but the fewest postoperative side effects (2). In our study, the entropy sparing effects on propofol/remifentanil TCl anaesthesia was evaluated.

Materials and Methods: Eighty patients scheduled for abdominal surgery lasting more than 2 hours were prospectively randomized to receive propofol/remifentanil effect-site $\mathrm{TCl}$ anaesthesia guided by Entropy or not (entropy values hidden but recorded). $0.3 \mathrm{mg}$ intrathecal morphine was injected before the start of the anaesthesia. Effect concentration (Ce) of propofol was adjusted to maintain entropy values between 40 and 60 in the Open Group (OG), or according to the usual practice in the Blind Group (BG). Remifentanil Ce was adjusted to keep haemodynamic variables stable in both groups.

Results: Groups were similar for demographic data. Perioperative data are presented in the table as mean \pm SD and compared by a Mann-Whitney test:

\begin{tabular}{lrrl}
\hline & $\mathrm{OG}(\mathrm{n}=37)$ & $\mathrm{BG}(\mathrm{n}=43)$ & $\mathrm{P}$ Value \\
\hline Anaesthesia, min & $376 \pm 237$ & $311 \pm 154$ & 0.478 \\
Propofol, mg & $1704 \pm 948$ & $2050 \pm 808$ & 0.009 \\
Remifentanil, mg & $3.48 \pm 1,61$ & $3.58 \pm 1.42$ & 0.569 \\
Open eyes, min & $5.65 \pm 4.91$ & $8.07 \pm 4.68$ & 0.009 \\
Extubation, min & $7.73 \pm 5.02$ & $9.64 \pm 5.12$ & 0.085 \\
\hline
\end{tabular}

When normalized to time, propofol consumption was reduced by $22 \%$ in OG $(5.52 \mathrm{mg} / \mathrm{min}$ versus $7.08 \mathrm{mg} / \mathrm{min}$ in $B G ; p=0.010)$. 2 -way analysis of variance showed no differences between groups, nor any interaction $(p=0.89)$ for the distribution of entropy values in the 3 ranges [0-39], [40-60] and [60-95]. Postoperative hospital stay was the same in both groups (12 \pm 10 days in OG versus $11 \pm 10$ days in $B G p=0.911$ ).

Conclusion: Spectral entropy monitoring reduces propofol consumption during propofol/remifentanil effect-site $\mathrm{TCl}$ anaesthesia.

References:

1 Vakkuri A. Anesthesiology 2005; 103(2): 274-9.

2 Suttner S. Anesthesia Analgesia 1999; 88: 77-82.

\section{AP2-9}

Propofol versus sevoflurane general anaesthesia: influence on the state entropy variability

A. Castro, E. Amadeu, F. Martins, P. Sa Couto, P. Amorim

Matematica Aplicada, Faculdade de Ciencias da Universidade do Porto, Porto, Portugal

Background and Goal of Study: The goal of this study was to determine if there are differences in SE variability for different anaesthetic techniques: with sevoflurane (Sevo Group) and with propofol (Prop Group).

Materials and Methods: Data collected during 32 urology procedures every 5 s from Datex S/5 monitors and infusion pumps with RuglooplI ${ }^{\circ} \mathrm{TCl}$ software. Schnider [1] model was used for propofol TCl. Induction with propofol 1\% infusion at $200 \mathrm{ml} / \mathrm{h}$ until loss of consciousness, and effect-site concentration (Ce) target maintained until intubation. In Prop Group propofol Ce was adjusted regarding patient stability and BIS target [40-60]. In the Sevo Group, propofol was stopped after intubation and sevoflurane started at $1.5 \%$ (in $\mathrm{O}_{2}$ and air), also adjusted to maintain BIS in the target interval [40-60]. Remifentanil was used in all cases. SE variability during maintenance phase was analyzed using the variability sequences: difference between original and smoothed filtered signal (Butterworth). (Data: mean $\pm \mathrm{SD}$ )

Results and Discussions: 16 patients in Sevo Group: $57 \pm 14$ years, $72 \pm 12 \mathrm{~kg} 164 \pm 7 \mathrm{~cm}, 7$ female, procedure time $223 \pm 127 \mathrm{~min}$ and average SE $44 \pm 9 ; 16$ patients in Prop Group: $55 \pm 14$ years, $70 \pm 15 \mathrm{~kg}, 164 \pm 9 \mathrm{~cm}$, 7 female, procedure time $192 \pm 66 \mathrm{~min}$ and average SE $45 \pm 6$, with no statistical difference between groups (t-test). Variability sequences had logistic distribution characterized by the shape parameter $b$, which gives information about signal variability (greater $b$, greater variability). The $b$ parameter was correlated to mean SE values in both groups (although not statistically significant). There was no statistical difference between groups for the $b$ parameter (t-test).
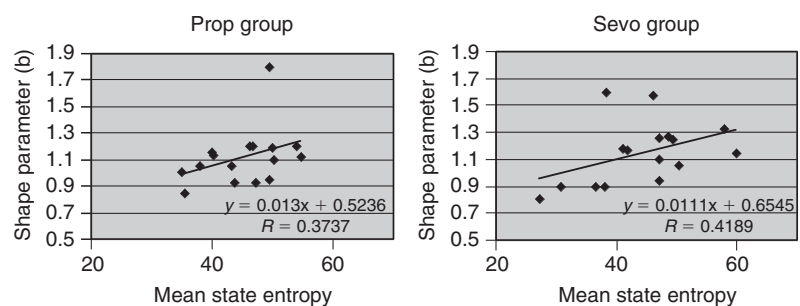

- Logistic shape parameter Linear (logistic shape parameter)

Fig 1. Mean SE level for each patient and corresponding $b$

Conclusion(s): SE variability was similar in both groups. For both propofol and sevoflurano groups the higher the SE the higher its variability.

Reference:

1 Anesthesiology, 1998, 88: 1170-82.

\section{AP2-10}

BIS and CSM fail to asses the clinical evolution of children during inducction with sevoflurane

M. García, J.C. Álvarez, I. González, E. Soler, B. Rodríguez

Anesthesia, IMAS, Barcelona, Spain

Background and Goal of Study: The EEG waveform in children and the evolution in time under anesthesia with sevoflurane represent a challenge for the monitorage of hypnosis. The aim of the study was to correlationate the evolution of BIS ${ }^{\mathrm{TM}}$ and the new hypnosis monitor $\mathrm{CSM}^{\mathrm{TM}}$ with depth of anaesthesia. Materials and Methods: A prospective observational study was performed in 20 pediatric patients (ages: 2 to $9 \mathrm{yrs}$ ). BIS and CSM monitors were attached in all the patients before anesthesia. Penile or caudal block was completed after sevoflurane induction and laryngeal mask insertion.Anesthesia was uniquely maintained with sevoflurane. BIS and CSI values, University of Michigan Sedation Scale (UMSS) score, and non steady state end-tidal sevoflurane were register in different moments of anesthesia. The anesthesist guided the administration of sevoflurane by clinical signs and was blinded to the hypnosis monitors. Pearson correlation analysis was used.

Results and Discussions: A great interindividual varability of BIS and CSI for the same clinical event was found. The hypnosis scores correlate with UMSS except for the induction period until the insertion of laringeal mask (LM). Correlation coefficient between BIS and CSI for all the anesthesia period is weak (0.506). The correlation improves regarding to the period from LM insertion until the recovery of conscience $(0.726)$.

\section{$\mathrm{CSI} / \mathrm{BIS}$ evolution in patients}

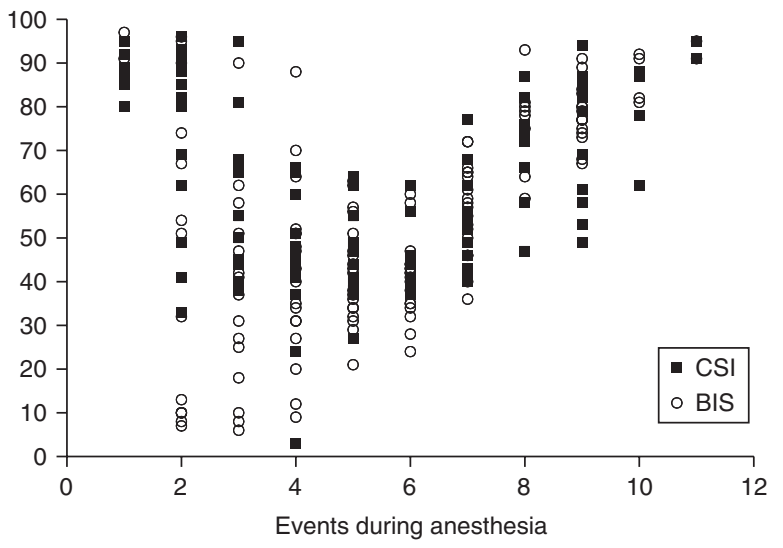

Conclusion(s): Our study demostrated that the BIS ${ }^{\mathrm{TM}}$ and $\mathrm{CSM}^{\mathrm{TM}}$ may not reflect the clinically assessed depth of anesthesia during sevoflurane induction in children. The epileptogenic potential of sevoflurane could be the cause of this effect(1).

Reference:

1 Constant I. Pediatric Anesth 2005; 15: 266-274. 


\section{AP3-2}

Effects of remifentanil and rocuronium on RE, SE, BIS values with and without stimuli

J. Liu, Y. Yue

Department of Anesthesiology, Beijing Chaoyang Hospital, Capital Medical University, Beijing, China

Background and Goal of Study: We evaluated effects of remifentanil and rocuronium on RE (response entropy), SE (state entropy), BIS (bispectral index) with and without noxious stimuli.

Materials and Methods: 50 patients were randomly divided into two groups (group A,B. $n=25$ each).Both groups were received an effect-site controlled infusion starting at $2.0 \mathrm{ug} \mathrm{ml}^{-1}$ and increased in steps of $0.3 \mathrm{ug} \mathrm{ml}^{-1}$ till loss of consciousness (loss of response to verbal commands and eyelash reflex). The effect-site concentration of loss of consciousness of propofol ( $\left.\mathrm{Ce}_{\text {prop.loc }}\right)$ was maintained, then effect-site controlled infusion of remifentanil (A $0 \mathrm{ng} \mathrm{ml}^{-1}$, B $2 \mathrm{ng} \mathrm{ml}^{-1}$ ) was maintained, 2 minutes late an electric stimulus $\left(50 \mathrm{~Hz}, 80 \mathrm{~mA}, 4\right.$ seconds) was given. After that, $\mathrm{Ce}_{\text {remi }}$ (effect-site concentration of remifentanil) was increased (A $2 \mathrm{ng} \mathrm{ml}^{-1}, \mathrm{~B} 4 \mathrm{ng} \mathrm{ml}^{-1}$ ), and rocuronium $0.6 \mathrm{mg} \mathrm{kg}^{-1}$ was given to facilitate tracheal intubation 2 minutes late. Finally the same electric stimulus was given $6 \sim 8$ minutes after intubation. The base values before noxious stimuli and values at $10 \mathrm{~s}, 20 \mathrm{~s}, 30$ s after stimuli of $R E$, $\mathrm{SE}, \mathrm{BIS}$ and MAP (mean arterial pressure), HR were recorded. Independent sample $T$ test was used to describe inter-group difference.

Results and Discussions: There is no significant difference in gender, age and weight between two groups. The base values and values at $10 \mathrm{~s}, 20 \mathrm{~s}, 30$ s of RE,SE, BIS before and after these three stimuli (intubation included) between group $A$ and $B$ also have no significant difference $(p>0.05)$. However, the MAP of group $A$ were significantly higher than group $B$ in all the same time-points $(p<0.05)$. The RE, SE, BIS values before and after the second electric stimulus of group $\mathrm{A}\left(\mathrm{Ce}_{\text {prop.loc }}, \mathrm{Ce}_{\text {remi }}=2 \mathrm{ng} \mathrm{ml}^{-1}\right.$, rocuronium $\left.06 \mathrm{mg} \mathrm{kg}^{-1}\right)$ is significantly lower than those values before and after the first electric stimulus of group $\mathrm{B}\left(\mathrm{Ce}_{\text {prop.loc }}, \mathrm{Ce}_{\text {remi }}=2 \mathrm{ng} \mathrm{ml}^{-1}\right.$, no rocuronium $)$.

Conclusion(s): Increase of $\mathrm{Ce}_{\text {remi }}\left(2 \mathrm{ng} \mathrm{ml}^{-1}\right)$ has no effect on RE,SE and BIS values regardless of stimulus, even though it can significantly decrease MAP. However, the use of rocuronium can decrease RE, SE, BIS values.

\section{AP3-3}

\section{Spectral Entropy and bispectral index as guidance for} propofol-remifentanil anaesthesia in combination with regional anaesthesia compared with a standard clinical practice group

R.K. Ellerkmann, G. Riese, M. Söhle, A. Hoeft, J. Bruhn

Department of Anaesthesiology and Intensive Care Medicine, University of Bonn, Bonn, Germany

Background and Goal of Study: This study was designed to investigate the impact of Spectral Entropy and bispectral index (BIS) monitoring on drug consumption and recovery times when compared with standard anaesthetic practice in patients undergoing orthopaedic surgery in a combination of regional and general anaesthesia. Anaesthesia was performed by an experienced anaesthesiologist.

Materials and Methods: After having obtained approval from the institutional review board and written informed consent, 80 adult patients undergoing surgery to the upper or lower extremity received regional anaesthesia for postand intra-operative pain control and were randomized to receive general anaesthesia by propofol/remifentanil infusion controlled either solely by clinical parameters or by targeting Entropy or BIS values of 50 . Recovery times and drug consumption were recorded.

Results and Discussions: Compared with standard practice, patients with Entropy or BIS monitoring needed similar propofol concentrations (standard practice $101 \pm 22 \mu \mathrm{g} / \mathrm{kg} / \mathrm{min}$, Entropy $106 \pm 24 \mu \mathrm{g} / \mathrm{kg} / \mathrm{min}$, BIS $104 \pm 20 \mu \mathrm{g} / \mathrm{kg} / \mathrm{min}$ ), and had similar recovery times and profiles (extubation: $7.3 \pm 2.9 \mathrm{~min}, 9.2 \pm 3.9 \mathrm{~min}$, and $6.8 \pm 2.9 \mathrm{~min}$; Aldrete score $(10 / 10)$ at extubation: $8.8 \pm 0.4,8.4 \pm 0.6$, and $8.6 \pm 0.5$ ).

Conclusion(s): Compared with standard anaesthetic practice Entropy and BIS targeting a value of 50 did not result in a reduction of propofol consumption or recovery times during general anaesthesia combined with regional anaesthesia performed by an experienced anaesthesiologist in orthopaedic patients.

\section{AP3-4}

The role of neuromuscular block monitoring during anaesthesia in the optimization and cost reduction of cisatracurium usage

E. Musiol, B. Seifert, E. Karpel, K. Grzybowska, I. Duda

Anaesthesiology and Intensive Care, Central Clinic Hospital, Katowice, Poland
Background and Goal of Study: Cisatracurium is a neuromuscular (n-m) blocking agent commonly used in general anaesthesia. Due to the higher price of this drug - as compared to its analogue - the authors attempted to determine the results of the $n-m$ block monitoring on the optimization of its usage. Materials and Methods: We have analyzed of anaesthesia protocols in 688 women who underwent gynecological operations comparing the use of cisatracurium and neostygmine in patients who were monitored during $n-m$ block (TOF group, $\mathrm{n}=378$ ) and patients who had the mentioned drug administered without monitoring ( $\mathrm{K}$ group, $\mathrm{n}=310$ ). The authors documented: age, weight and BMI of the patients, duration of anesthesia, total, induction and conduction doses of cisatracurium, time elapsed from the last drug dose to the end of surgery and the percentage of patients who needed neostygmine to reverse the $n-m$ block. The use of cistracurium and neostygmine was compared additionally in the sub-groups who underwent short duration operational procedures ( $\leqslant 90 \mathrm{~min}-\mathrm{TOF}_{\mathrm{S}}-\mathrm{K}_{\mathrm{S}}$ ) and long duration ones ( $>90 \mathrm{~min}-\mathrm{TOF}_{\mathrm{L}}-\mathrm{K}_{\mathrm{L}}$ ).

Results and Discussions: In group TOF compared to group $\mathrm{K}$ authors observed that slightly older age and higher weight (BMI) of patients prevailed, lower conduction $(0,3$ vs $0,41 \mu \mathrm{g} / \mathrm{kg} / \mathrm{min})$ and total $(1,59 \mathrm{vs}$ $1,68 \mu \mathrm{g} / \mathrm{kg} / \mathrm{min}$ ) cisatracurium doses were administered, shorter time elapsed from the last dose of the drug to the termination of operation (39 vs $46 \mathrm{~min}$ ), and a more frequent administration of neostygmine (53\% vs $65 \%$ ). The comparison in paired subgroups defined according to the duration of procedure showed parallel differences, with exception of the lack of change in the frequency of administration of neostygmine in the $\mathrm{TOF}_{\mathrm{L}}$ and $\mathrm{K}_{\mathrm{L}}$ subgroups. Conclusion(s): The neuro-muscular transmission monitoring provides an optimal muscle relaxation with regard to the duration of the procedure, furthermore ensuring an objective control over the $n-m$ blocker action and its cessation after the termination of the procedure (especially in short duration procedures with a high risk of residual block) leading to reduction of the total dose and subsequent costs.

\section{AP3-5}

Number of skin conductance fluctuations increased differently from BIS during tetanic stimuli. Increasing doses of remifentanil attenuated the skin conductance response

H. Storm, I. Røeggen, R. Støen, P. Klepstad, J. Ræder

University Administration, The Skills training Center, UIO, Os/o, Norway

Background and goal: Number of skin conductance fluctuations per sec (NSCF) in the palmar surface correlates well with sympathetic nerve activity. NSCF has been proposed to measure pain responses. The BIS index measures disorders in the EEG signal and is associated with awakening. The purpose of the study was to examine if NSCF and BIS could detect the pain response from tetanic stimuli, and to further examine if the tetanic stimuli response was stronger in a situation without analgesic infusion compared to a situation with ongoing analgesic target control infusion (TCl).

Materials and methods: 28 patients in ASA 1 or 2 were studied after induction of general anaesthesia with propofol (BIS between 40-50), but before intubation and start of laparoscopic surgery. The patients were given 3 series of tetanic stimulus of $50 \mathrm{~mA}$ that lasted for $30 \mathrm{sec}$ : Tetanic 1 (T1) without ongoing remifentanil analgesic infusion, Tetanic 2 (T2) after $4 \mathrm{~min}$ with $\mathrm{TCl} 4 \mathrm{ng} / \mathrm{ml}$ remifentanil and Tetanic 3 (T3) after $4 \mathrm{~min}$ with $\mathrm{TCl} 10 \mathrm{ng} / \mathrm{ml}$ remifentanil. The NSCF and BIS responses were registered continuously, starting $30 \mathrm{sec}$ before stimuli and ending $30 \mathrm{sec}$ after the stimuli started. The maximum values for NSCF and BIS during the tetanic pre stimuli periods were compared with the maximum values of the tetanic post stimuli periods. Moreover, NSCF and BIS responses during T1 were compared with the responses during T2 and T3. The Wilcoxon non-parametric test was used. Result and discussion:

\begin{tabular}{lllllll}
\hline & pre-post & pre-post & pre-post & pre-post & pre-post & pre-post \\
& T1 NSCF & T2 NSCF & T3 NSCF & T1 BIS & T2 BIS & T3 BIS \\
\hline Mean(SD) & $0.00(0.01)-$ & $0.00(0.00)-$ & $0.00(0.00)-$ & $43(9)-$ & $42(9)-$ & $42(6)-$ \\
& $0.07(0.07)$ & $0.02(0.04)$ & $0.01(0.06)$ & $44(13)$ & $44(12)$ & $44(7)$ \\
$P$ value & 0.000 & 0.027 & 0.180 & 0.272 & 0.393 & 0.227 \\
\hline
\end{tabular}

\begin{tabular}{lllll}
\hline & Response T1- & Response & Response T1- & Response \\
& T2: NSCF & T1-T3: NSCF & T2: BIS & T1-T3: BIS \\
\hline Mean (SD) & $0.07(0.07)-$ & $0.07(0.07)-$ & $44(13)-44(12)$ & $44(13)-44(7)$ \\
& $0.02(0.04)$ & $0.01(0.06)$ & & \\
$P$ value & 0.000 & 0.001 & 0.873 & 0.882 \\
\hline
\end{tabular}

The NSCF post stimulus level was higher than the pre stimulus level during T1 and T2, contrasting BIS, which did not change significantly. After 4 min with $\mathrm{TCl} 10 \mathrm{ng} / \mathrm{ml}$ remifentanil, no differences between post stimulus and pre stimulus levels during T3 was observed for NSCF and BIS. The NSCF response during tetanic stimuli was reduced when the remifentanil doses was increased different from BIS. 
Conclusion: In contrast to BIS, this study showed that NSCF is sensitive to tetanic noxious stimuli during sleep, and the measured response is attenuated when an ongoing analgesic infusion is given.

\section{AP3-6}

In-vitro comparison of three different tracheal wall pressure measurement techniques

T. Horisberger, S. Gerber, V. Bernet, A. Schmitz, M. Weiss

Dept. of Paediatrics, University Children's Hospital Zurich, Zurich, Switzerland

Background and Goal of the Study: Measurement of pharyngeal and tracheal wall pressure exerted by artificial airways is of great interest, because of pressure-induced reduction of mucosal perfusion und related morbidity. The aim of this study was to compare three different wall pressure measuring techniques using an in vitro model.

Materials and Methods: Using a high volume low pressure (HVLP) Portex Soft Seal cuffed tracheal tube with internal diameter (ID) $7.5 \mathrm{~mm}$ and a tracheal model the pressure difference technique (1), the wall membrane transducer technique (2) and the microchip Codman probe technique with and without lubrication (3) was studied. Wall pressures were noted every $0.5 \mathrm{ml}$ aliquots of air inflated in to the cuff from 0 to $50 \mathrm{mmHg}$ cuff pressure. All experiments were performed $\left(\mathrm{T} 20^{\circ} \mathrm{C}\right.$, ambient pressure) twice with four cuffed tracheal tubes. Measured mean pressure values were plotted against volume of air inflated. The coefficient of variance (CV) was calculated.

Results: Median CV was largest for pressure probe technique without lubrication (214\%) and with lubrication (29\%) and was lowest for the wall membrane transducer (22\%) and the pressure difference technique (19\%).

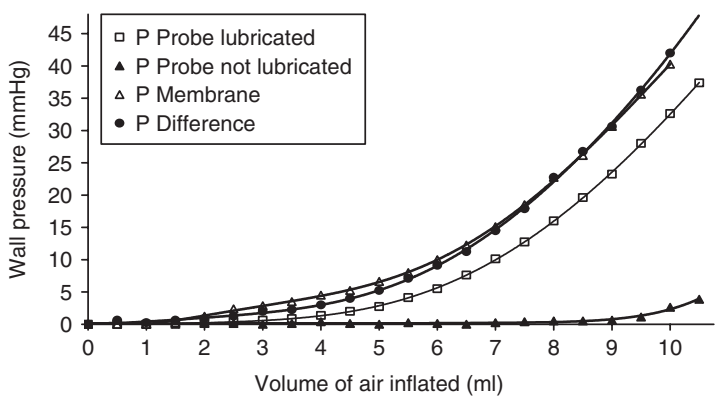

Conclusions: Membrane transducer technique and pressure difference technique provided comparable results. The microchip Codman probe technique underestimates wall pressure due to recessed pressure sensor at the probe. This can partially overcome by lubrication of the sensor probe.

\section{References:}

1 MacKenzie CF. Br J Anaesth 1976; 48: 105-10.

2 Tonnesen AS. Anesthesiology 1981; 55: 680-3.

3 Ulrich-Pur H. Anesthesiology 2006; 104: 933-8.

\section{AP3-7}

Electroencephalogram-based anaesthesia monitors: correlation between the BIS and the Narcotrend index?

S. Paprotny, G. Schneider, M. Kreuzer, B. Horn, E.F. Kochs

Anaesthesiology, Klinikum rechts der Isar der Technischen Univerität München, München, Germany

Background and Goal of Study: The bispectral index (BIS) and the Narcotrend (NCT) are indices of the hypnotic component of anaesthesia. Both produce a number between 0 and 100 with deeper values indicating a deeper hypnotic level, but they are based on different algorithms. Reflecting the same clinical endpoint, one may expect a high correlation between the indices. [1] Materials and Methods: EEG was recorded from 40 adult patients, receiving different anaesthetic regimes. Group 1: sevoflourane, remifentanil $<0.1 \mu \mathrm{g}$ $\mathrm{kg}^{-1} \min ^{-1}$ ("remi low"); Group 2: sevoflourane, remifentanil $>0.2 \mu \mathrm{g} \mathrm{kg}^{-1}$ $\mathrm{min}^{-1}$ ("remi high"); Group 3: propofol, "remi low"; Group 4: propofol, "remi high". EEG was recorded with a sample rate of $256 \mathrm{~Hz}$ and played back to the monitors simultaneously via an EEG player. The index values were stored every 5 s and Pearsons Correlation was calculated.

Results and Discussion: The overall correlation coefficient is 0.5678 . The figure shows BIS versus NCT index values. The level for general anaesthesia is represented by index values 40-60 (BIS) or 13-64 (NCT) (see figure, lines). White background indicates that both monitors display the same level of anaesthesia. In grey areas, BIS and NCT indicate different levels of anaesthesia, i.e. BIS and NCT are inconsistent with one another.

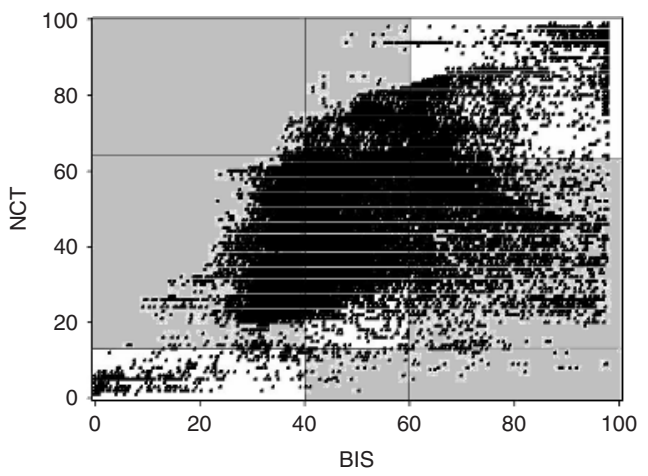

Conclusion: There is only weak correlation between BIS and NCT. Designed for the same purpose, one may expect that the indices should generally agree. But BIS and NCT often show no agreement in attribution to the level of anaesthesia. The correlation between BIS and NCT may not be linear, possibly due to differences in scaling. This is in contrast to previous findings suggesting a high concordance of both indices. Further studies are required to identify mechanisms behind conflicting index values.

Reference

1 Kreuer et al., Anesth Analg; 2004; 98: 692-697.

\section{AP3-8}

Performance of surgical stress index during sevofluranefentanyl and isoflurane-fentanyl anaesthesia

S. Mustola, T. Parkkari, K. Uutela, M. Huiku, M. Kymalainen Anaesthesia, South Karelia Central Hospital, Lappeenranta, Finland

Background and Goal of Study: Surgical Stress Index (SSI, GE Healthcare, Helsinki, Finland), based on heart rate and photopletysmography, has been recently introduced for the assessment of surgical stress (1). We recorded SSI values during sevoflurane-fentanyl and isoflurane-fentanyl anaesthesia. Materials and Methods: Thirty patients, ASA I-III, aged 27-80 yr, gender (F/M) 19/11, scheduled for surgical procedure, were studied. Patients received fentanyl $2 \mathrm{microg} / \mathrm{kg}$ and thiopentone for induction of anaesthesia and were paralyzed with rocuronium. Tracheal intubation was performed $5 \mathrm{~min}$ after fentanyl. Patients were randomly assigned to receive fentanyl $1.5 \mathrm{microg} / \mathrm{kg}$ five minutes before (FB) or after (FA) skin incision. 20 patients were anesthetized with sevoflurane and 10 with isoflurane. State entropy was maintained at 40-60. Reactivity of SSI to surgery and fentanyl boluses was recorded. Twotailed M-U-test was used for statistics.

Results and Discussion: SSI increased significantly after tracheal intubation and skin incision in both groups $(P<0.05)$. The increase of SSI after skin incision was lower in FB than in FA group but it was statistically insignificant (fig. 1). A reason for this can be that the surgical procedures were very diverse and stimuli could be very different or fentanyl boluses were not high enough.

Conclusions: Fentanyl given five minutes before tracheal intubation or skin incision did not block totally the increase of SSI. The increase of SSI in FB group compared to FA group after skin incision was statistically insignificant.

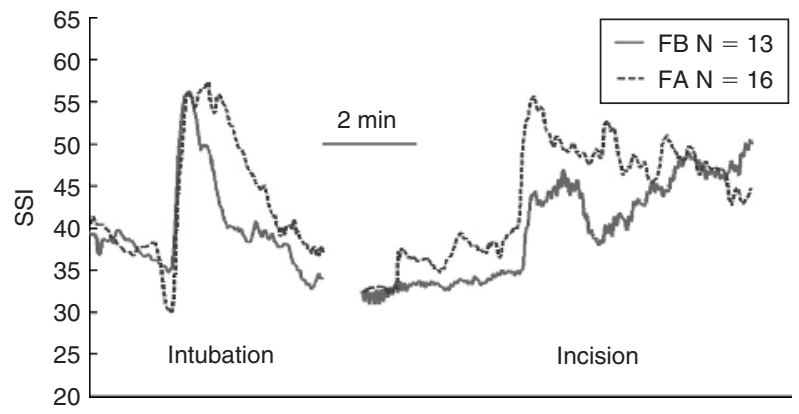

Fig. 1. SSI values after intubation and skin incision.

Reference:

1 Huiku M, Kymalainen M, Uutela K, et al. Anesthesiology 2005; 103: A67. 


\section{AP3-9}

Correlation dimension, an EEG complexity measure, reflects increasing and decreasing anaesthetia using data of a multicenter study

D. Jordan, G. Schneider, G. Stockmanns, A. Hock, E. F. Kochs ${ }^{\star}$ *Department of Anaesthesiology, Technische Universität München, München, Germany

Background and Goal of Study: Correlation Dimension (CD) was introduced to quantify the order/disorder of signals generated by low dimensional chaotic systems [1]. The present investigation was performed to evaluate the capability of the EEG parameter CD in separating consciousness from unconsciousness and the monotonic behaviour of $C D$ in phases of increasing and decreasing anaesthesia.

Materials and Methods: EEG data from a study performed in 6 European centres involving 263 adult patients undergoing surgery under general anaesthesia was used. Patients were randomly assigned to one of 11 anaesthetic combinations. The capability of $C D$ in separating consciousness from unconsciousness was evaluated using prediction probability $\left(P_{K}\right)$ [2]. For this purpose, parameter values were calculated immediately before and after loss and return of consciousness. The parameter values in phases of anaesthetic increase (until EEG burst suppression) and anaesthetic decrease were analysed computing Spearman correlation coefficients as a measure of monotonic behaviour. A perfect correlation of the parameter and the monotonic decreasing (anaesthetic increase) respectively increasing (anaesthetic decrease) function will result in a correlation coefficient of 1.

Results and Discussions: Analysis of $P_{K}$ revealed a value of 0.65 (transition between consciousness and unconsciousness). Spearman correlation coefficients were 0.30 ("deepening" of anaesthesia) and 0.18 ("lightening" of anaesthesia), where positive values denote a monotone relationship of CD to "depth of anaesthesia". It may be explained by more "order" and less "randomness" in EEG signals with increasing anaesthesia. The results of $\mathrm{P}_{\mathrm{K}}$ indicate that $C D$ is less adapted to separate consciousness and unconsciousness, because the parameter may be affected by high dimensional EEG signals.

Conclusion(s): Based on the challenging data selection involving numerous anaesthetic regimes, CD shows a monotone behavior in phases of anaesthetic increase and decrease but is less useful for separating consciousness from unconsciousness.

References:

1 Lai YC et al., Phys Rev E 2002; 65: 1-5.

2 Smith WD et al., Anesthesiology 1996; 84: 38-51.

* European Multicenter EEG/AEP Anaesthesia Monitoring Study Group

\section{AP3-10}

\section{Factors associated with the occurrence of BIS values} below 45

A. Castro, C. Anfiloquio, P. Azevedo, D. Oliveira, C.S. Nunes

Matematica Aplicada, Faculdade de Ciencias da Universidade do Porto, Porto, Portugal

Background and Goal of Study: It is accepted that BIS should be maintained between 45 and 60 . We did a retrospective study to analyze the incidence of BIS below 45 and identify possible causative factors.

Materials and Methods: Data records from neurosurgeries were collected every 5 s from Aspect XP, Datex S/5 and IV pumps with RuglooplI ${ }^{\circ} \mathrm{TCl}$. Anaesthesia was with TCl of Propofol and Remifentanil. The only defined goal that the anaesthetists had, was avoiding BIS above 60. BIS was analyzed from BIS $<70$ at induction, until BIS $>70$ at recovery - anaesthesia time (AT). For poor neuro grade cases, AT was from induction until end of surgery. An algorithm (in Matlab7®) extracted the time periods with BIS in different levels $(<30,30-35,35-40$ and $40-45)$. Several variables were analyzed in relation to the occurrence of BIS below 45 and 30. (Data: mean \pm SD)

Results and Discussions: 211 patients, ASA 1-4, $51 \pm 17$ years, $69 \pm 13 \mathrm{~kg}, 163 \pm 9 \mathrm{~cm}, 92$ male, 166 head surgeries and 45 spinal, Glasgow score 3-8 in 8 patients and 9-12 in 11. Factors associated with increased time at BIS $<45$ were: cranial surgery $(P<0.05$, t-test), higher ASA grade ( $P<0.05$, Kruskal-Wallis and t-test), supine position $(P<0.01$, t-test $)$ and the anaesthetist $(P<0.01$, Kruskal-Wallis test). Factors associated with more time at $B I S<30$ were: cranial surgery $(P<0.05)$, supine position $(P<0.05$, t-test) and anaesthetist $(\mathrm{P}<0.01, \mathrm{~K}$.-Wallis). The amount of drugs had no relation.

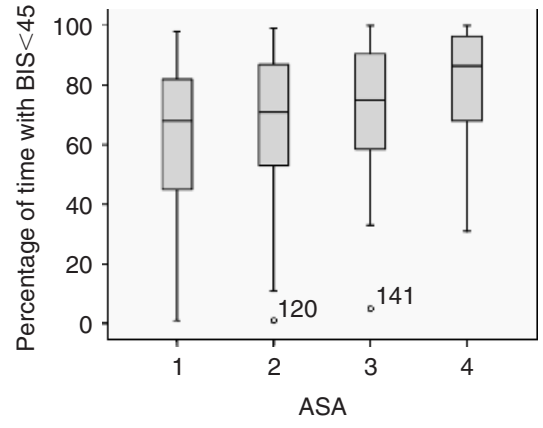

Fig. 1 - Boxplot of $\%$ of time with BIS $<45$ vs ASA

Conclusion(s): We identified variables associated with low BIS in neurosurgery: cranial surgery, supine positioning, higher ASA and the anaesthetist. This may be useful information in preventing the occurrence of low BIS values, and its possible consequences: the human factor should be addressed and may be easy to correct.

\section{AP4-1}

\section{Computer generated pathophysiological diagnoses during} anaesthesia

M. Harrison, B. Gohil, H. Gholam-Hossieni, A. Lowe, A. Al-Jumaily Department of Anaesthesiology, University of Auckland, Auckland, New Zealand

Background and Goal of Study: Display of trend data during anaesthesia is commonplace; however, data integration to automate diagnoses is not. The purpose of this 'proof of concept' study was to examine a system that could, potentially, recognise absolute hypovolaemia. We report the efficacy of the software when applied, retrospectively, to data collected during major surgery where blood loss and hypovolaemia was likely.

Materials and Methods: The software uses probabilistic statistics, fuzzy logic (1) and respiratory related systolic-pressure-variation (2). Data collected from the analogue port of the GE/Ohmeda Datex monitor was filtered extensively to reduce artefacts, processed using MATLAB and LabVIEW. The data were analysed in 15-minute epochs, the agreement between clinician and computer assessed using Kappa.

Results and Discussions: There were 54 15-minute epochs. The positive agreement was 0.84 , negative agreement 0.72 , Kappa $=0.56(95 \% \mathrm{Cl}$ 0.33-0.79) which indicates moderate agreement. Neither the clinician nor the software was considered a gold-standard and therefore in this preliminary study only agreement could be assessed. It is hoped that a redundancy of diagnostic information may reduce false alarms. On-line testing is imminent. Conclusion: Hypovolaemia can be diagnosed using variables routinely monitored during anaesthesia, with a moderate degree of agreement with a clinical assessment.

References:

1 Lowe A, Harrison MJ. Anaesth Intensive Care 1999; 27: 41-44.

2 Stoneham MD. Br J Anaesth 1999; 83: 550-551.

\section{AP4-2}

Interim results of a study to evaluate the effectiveness of a new Electrocardiography (ECG) monitor in helping doctors improve their diagnostic proficiency and certainty in detecting acute ischaemia

A. Al-Hassani, C. Zizzo, D. Turner

\section{Anaesthetics, Broomfield Hospital, Chelmsford, United Kingdom}

Background and Goal of Study: It is crucial to have accurate and prompt interpretation of signs of acute ischaemia during Electrocardiograph (ECG) monitoring. Yet, competency in reading ECGs among 120 residents in Medicine was shown to be low ${ }^{1}$. Cardiac monitors with automated ST segment analysis are available for ischaemia detection. However, the use of such monitors has been limited for several reasons ${ }^{2}$. A new ECG monitor with automated ST analysis capability has been developed to assist professionals identify changes of acute ischaemia with the site and severity of the changes. The monitor projects the changes as a 3D image of the heart showing progression of acute changes in real time. We conducted a study to evaluate the performance of the new ECG monitor in aiding doctors improve their diagnostic proficiency and certainty. 
Material and methods: 50 resident doctors took part in a 2-phase evaluation. In Phase-1 we asked participants to make the diagnosis of acute ischaemia using a set of 8 ECG printouts on the basis of ST elevation or depression. They also had to indicate their diagnostic certainty on a scale of $0-4$ ( $0=$ lowest $4=$ highest). This methodology was similar to that used by previous investigators $^{1}$. In Phase-2 they reviewed a second set of 8 ECGs, but they also had access to the new imaging monitor to help them reach a diagnosis. The new monitor could recall any of the 8 ECG's which were stored as electronic records. Two specialists marked the diagnosis made by participants in a scale of $0-2$, and the aggregated score for all ECGs was labelled Diagnostic Proficiency score. The time to complete the test was also noted in both phases. Results: Diagnostic Proficiency scores and Certainty scores (Median value with 25th-75th percentile) were calculated for both Phases. We compared data from the two phases to establish effectiveness of the new ECG monitor as an aid to doctors. Using the new imaging ECG monitor, doctors increased their median Diagnostic Proficiency from $50 \%$ to $100 \%$ $(P<0.001)$; while their Certainty increased from $65 \%$ to $80 \%(P<0.001)$. The average time taken to reach diagnosis dropped from 15 minutes to 9 minutes $(P<0.001)$.

References:

1 Berger JS. Am J Med 2005; 118: 873-80.

2 AHA Statement. Circulation 2004; 110: 2721-2746.

\section{AP4-3}

Validation of the loC-view device for monitoring the level of consciousness during propofol anaesthesia

H. Litvan, M. Revuelta, ML. Maestre, JM. Campos, V. Moral

Cardiac Anaesthesia and ICU, Hospital Santa Creu i Sant Pau, Barcelona,

Spain

Background and Goal of Study: The purpose of this study was to validate a new device for monitoring the level of consciousness, the loC-view (Morpheus Medical Inc, Spain)(1) during propofol anaesthesia. The Index of Consciousness (loC) was compared with the Bispectral Index (BIS-XP, Aspect Medical Systems, USA) and the Observers Assessment of Alertness and Sedation Scale (OAAS)(2) during the induction of anaesthesia. The loC is based on a chaos mathematical analysis, termed Symbolic Dynamics (SD), of the frontal $\mathrm{EEG}(3)$. The basic principle of SD is to transform a time-series (here the EEG) into a symbol sequence.

Materials and Methods: After Ethical Committee approval, data was collected from 20 patients scheduled for cardiac surgery. In all patients the standard departmental procedure for propofol induction was applied: TCI reaching an effect site concentration of $5 \mathrm{ug} / \mathrm{ml}$ in $5 \mathrm{~min}$ or until loss of consciousness was reached, defined as loss of response to mild shaking and prodding (OAAS 1). Five minutes after the patient had reached OAAS 1 , atracurium $0.6 \mathrm{mg} \cdot \mathrm{kg}^{-1}$ and continuous remifentanil infusion $0.3 \mu \mathrm{g} \cdot \mathrm{kg}^{-1} \cdot \min$ (after a bolus of $1 \mu \mathrm{g} \mathrm{kg}^{-1}$ in $1 \mathrm{~min}$ ) were administered and the trachea was intubated. Data was collected until the surgery was ended. The $\mathrm{IOC}$ and the BIS were recorded simultaneously. The prediction probability $(\mathrm{Pk})$ of the two indices ability to predict the OAAS was assessed along with the Spearmans rank correlation.

Results and Discussions: The $\mathrm{Pk}(\mathrm{SE})$ values for loC and BIS were 0.93(0.03) and $0.91(0.04)$, respectively. The Spearmans rank correlation of the loC vs. BIS was 0.88 . Both monitors presented a similar evolution during the whole procedure.

Conclusion(s): The loC seems to be a promising alternative for monitoring the level of consciousness during propofol anaesthesia.

References:

1 Litvan et al. Anesthesiology 2006; 105: A1029.

2 Chernik et al. Clin Psychopharmacol 1990; 10: 244-51.

3 Pincus et al. J Clin Monit 1991; 7: 335-45.

\section{AP4-4}

\section{Improving of Preoperative Evaluation by electronic form}

J. Mata, B. Tolksdorf, V.M. Estrada, I. Valldeperas, J.L. Aguilar Anesthesiology, Son Llàtzer Hospital, Palma de Mallorca, Spain

Background and Objectives: Each patient, even more those with elevated risk factors who require an anesthetic for surgical treatment have to be evaluated by an anesthesiologist prior to surgery to prevent complications and to treat accompanying diseases. Each detail has to be documented as important information for the anesthesiologist involved in the patient's care. For several reasons (human errors, technical difficulties...) data may get lost. Computer programs might minimize the risk of data loss.

Methods: By 2002 an electronic form for preoperative documentation according to the Spanish Society of Anesthesiology was introduced into daily practice. Since 2005 it was decided to insert spaces that need to be filled in. No reprint of the document can be obtained if those spaces are not completed with the aim to prevent loss of data. Specifically:ASA, CMA, blood transfusions, results of cardiac and pulmonary auscultation, difficult intubation, and allergy require documentation. Retrospectively, we compared the amount of data losses before the introduction of detailed data documentation between 2002 - 02/2005 and after between 03/2005-12/2006.

Results:

\begin{tabular}{lll}
\hline & $2002-02 / 2005$ & $03 / 2005-12 / 2006$ \\
& Not completed $(\mathrm{n}=9444)$ & Not completed $(\mathrm{n}=8774)$ \\
\hline Blood transf & $81(0,8 \%)$ & 0 \\
CMA & $3212(60 \%)$ & 0 \\
ASA & $219(2,3 \%)$ & 0 \\
Auscultation & & \\
Cardiac & $836(8,8 \%)$ & 0 \\
Pulmonary & $648(6,9 \%)$ & 0 \\
Difficult int & $3212(60 \%)$ & 0 \\
Allergy & $184(2 \%)$ & 0 \\
\hline
\end{tabular}

The loss of data before meticulous documentation resulted in additional time to recover those data. Calculating $10^{\prime}$ for the recovery of each loss of data resulted in a loss of time up to seven hours/week.

Conclusions: The electronic form of preoperative documentation improves the preoperative evaluation. Additionally, filling out a prepared form prevented the loss of data and shortened the time to recover them.

\section{AP4-5}

SSI is sensitive to both sevoflurane and alfentanil during general anaesthesia

J. Vieri, A. Yli-Hankala, P. Kärkäs, M. Kymäläinen, K. Uutela

Department of Anaesthesia, Tampere University Hospital, Tampere, Finland

Background and Goal of Study: During general anaesthesia (GA), neuromuscular blockade and hypnosis are routinely monitored, whereas measurement of nociception still remains a challenge. Surgical stress index (SSI) is a new numerical indicator of patient's state, designed to indicate the nociceptive - anti-nociceptive balance. We hypotized that a change in alfentanil (A) concentration would cause the SSI value to change.

Materials and Methods: 39 adult ASA I-III females scheduled for abdominal surgery under GA were randomized in two groups. After anaesthetic induction with propofol, A and rocuronium, 20 patients received $A$ at $50 \mathrm{ng} / \mathrm{ml}$ target site concentration ( $\mathrm{Ce}$ ), while sevoflurane (S) MAC was changed between 0.7 and 1.5 every 15 minutes. In other 19 patients S-MAC was kept constant at 0,8 whereas $A-C e$ was changed between 25 and $75 \mathrm{ng} / \mathrm{ml}$ every $25 \mathrm{~min}$ utes. SSI was calculated on-line by utilizing ECG and plethysmographic pulse wave data (1). SSI changes during 51 S-MAC increases and $25 \mathrm{~A}-\mathrm{Ce}$ increases were analyzed. Wilcoxon signed rank test was used. $P$ value $<0.05$ was considered significant.

Results: Results are mean \pm SD (Figure).
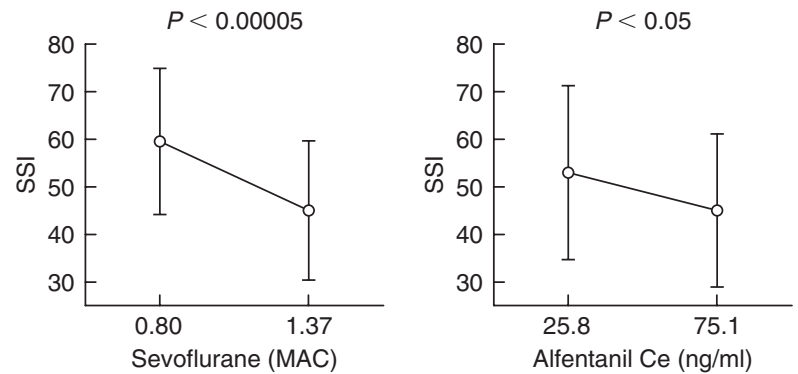

Conclusions: With both $\mathrm{S}$ and $\mathrm{A}$, the increase in drug concentration is associated with a decrease in SSI. Both drugs possess anti-nociceptive properties and SSI behaves as a reliable indicator of nociceptive - anti-nociceptive balance.

Reference:

1 Huiku M, Kymäläinen M, Uutela K, et al. Anesthesiology 2005; 103: A67.

\section{AP4-6}

Implementation of an emergency department infomation system resulted in significantly reduced waiting times B. Bohy, C. De Deyne, G. Vundelinckx, M. Vander Laenen, R. Heylen Anesthesia and Critical Care Medicine, Ziekenhuis Oost-Limburg, Genk, Belgium 
Introduction: Patient satisfaction at emergency departments (EDs) can be improved by reductions in waiting time. In the present paper, we evaluated the impact of an ED information system, available for all ED (medical and nursing) staff and displaying a visual tracking system of all pts admitted to the ED. Patients and Methods: We retrospectively analysed all different waiting times for all patients admitted to the ED in a 3months periods before and after the installation of the visual tracking system. Different waiting times were : first, pts have a wait for registration (wR), then a wait for the initial triage (wX), a separate wait for the consulting room $(w C)$, a wait for - if necessary - technical investigations (wl), and finally a further wait for treatment (wT) and discharge (wD). Results: A total of 4.720 pts were included in the first 3 months period, compared to 4.910 pts for the second period. We observed a significant decrease in all, except one (wR), waiting times after the installation of the visual tracking system. Analysis of different waiting times revealed that largest reductions were obtained in the wait for initial triage and consult ( $w X: m 28 \mathrm{~min}$ reduced to $\mathrm{m} 19 \mathrm{~min}$ and $\mathrm{wC}: \mathrm{m} 43 \mathrm{~min}$ reduced to $\mathrm{m} 27 \mathrm{~min}$ ). We also observed a significant reduction in outliers, i.e. extremely long waiting times, mostly occurring for technical investigations.

Conclusion: The implementation of an ED information system, with the use of a visual tracking screen including all ED pts, resulted in signicificantly reduced waiting times.

\section{AP4-7}

Ability of anaesthesiologists to assess the incidence of deep anaesthesia (BIS below 45) in their own cases

A. Castro, F. Lobo, I. Santos, L. Ferreira, M. Casal

Matematica Aplicada, Faculdade de Ciencias da Universidade do Porto, Porto, Portugal

Background and Goal of Study: Individual anaesthetists may deal differently with the information obtained from BIS monitoring. We studied the occurrence of low BIS in neurosurgeries and the ability of the anaesthetists who conducted the cases to self-assess its incidence.

Materials and Methods: Data records of neurosurgeries were collected from Aspect XP with RuglooplI® TCI software. Anaesthesia was with propofol and remifentanil. The only defined goal of the anaesthetists was to avoid BIS $>60$. BIS was analyzed from after induction up to recovery - anaesthesia time (AT). An algorithm developed in Matlab7 $®$ extracted the time periods with $\mathrm{BIS}<45$. All anaesthetists involved did an auto-evaluation indicating the expected percentage of their patients with $0-10 \%, 10-25 \%, 25-50 \%, 50-75 \%$ and $75-100 \%$ of AT with BIS $<45$.

Results and Discussions: There were 5 anaesthetists and 194 cases. BIS was below 45 for any time period in all records. Estimations by the anaesthetists were below the real incidence of low BIS (Table):individual anaesthetists' underestimation of the \% of patients who had $75-100 \%$ of AT below 45 was: $-45,-21,-22,-75$ and $-59 \%$.

Table 1 - Expected and real patient's division for each anaesthetist in each percentage stage of AT with BIS $<45$

\begin{tabular}{|c|c|c|c|c|c|}
\hline & Anesth. 1 & Anesth. 2 & Anesth. 3 & Anesth. 4 & Anesth. 5 \\
\hline \multicolumn{6}{|c|}{ Expected Patient's Division (\%) } \\
\hline $0-10 \%$ of $A T$ & 5 & 50 & 80 & 5 & 10 \\
\hline $10-25 \%$ of AT & 25 & 20 & 15 & 5 & 15 \\
\hline $25-50 \%$ of AT & 50 & 20 & 1 & 10 & 25 \\
\hline $50-75 \%$ of $\mathrm{AT}$ & 15 & 8 & 1 & 70 & 35 \\
\hline $75-100 \%$ of AT & 5 & 2 & 3 & 10 & 15 \\
\hline \multicolumn{6}{|c|}{ Real Patient's Division (\%) } \\
\hline $0-10 \%$ of AT & 0 & 3 & 0 & 0 & 4 \\
\hline $10-25 \%$ of AT & 0 & 9 & 0 & 0 & 0 \\
\hline $25-50 \%$ of AT & 11 & 26 & 17 & 0 & 4 \\
\hline $50-75 \%$ of AT & 34 & 40 & 58 & 17 & 29 \\
\hline $75-100 \%$ of AT & 55 & 23 & 25 & 83 & 64 \\
\hline
\end{tabular}

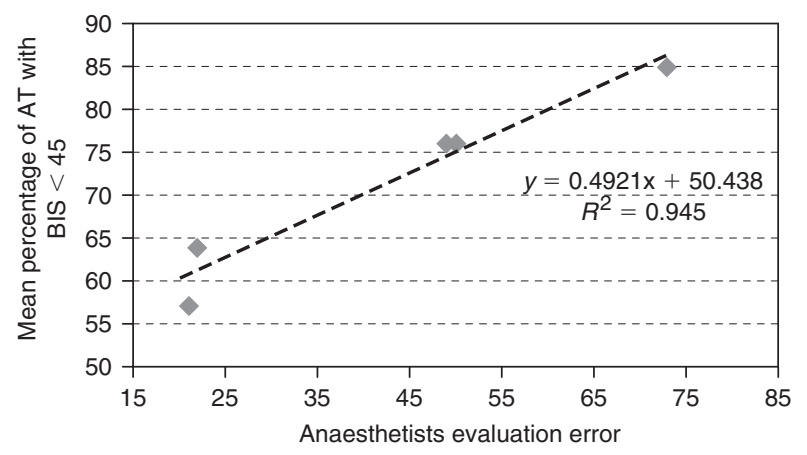

There was a high correlation between anaesthetists' evaluation error (75-100\% of AT) and the correspondent mean \% of AT with BIS $<45(R=0.972$, $\mathrm{P}=0.005$ ) (Fig. 1: Regression line between the anaesthetists evaluation error (Real - Expected Patients' Division $75-100 \%$ AT) and mean \% of AT with BIS $<45$ for each anaesthetist).

Conclusion(s): Our data shows that BIS guided anaesthesia aiming at preventing awareness may result in a high incidence of low BIS and that the anaesthetists who conducted the anaesthetics underestimate its occurrence. We also show that anaesthetists whose cases have lower BIS have a higher auto-evaluation error. Our results may be important if one wants to avoid occurrence of BIS $<45$.

\section{AP4-8}

Computer-guided versus paper-based protocol for Insulin administration in diabetic patients undergoing cardiac surgery

L. Saager, G. Collins, H. Tymkew, E. Jacobsohn, M. Avidan

Department of Anesthesiology, Washington University, St. Louis, USA

Background and Goal of Study: Patients with diabetes mellitus have increased morbidity and mortality following cardiac surgery ${ }^{1}$. Tight blood glucose control in patients with diabetes seems to improve outcome following myocardial infarction or cardiac surgery ${ }^{2}$. Established protocols aim for a blood glucose level between 90 and $150 \mathrm{mg} / \mathrm{dl}$. Usually the adjustment to insulin dosage is based only on single blood sugar readings. Our goal was to investigate if by analyzing a trend of blood sugar results and mathematical modeling to predict the extent and rapidity with which blood sugar is likely to change, we might facilitate a tighter blood glucose control.

Materials and Methods: 40 diabetic patients undergoing cardiac surgery with cardiopulmonary bypass were assigned to one of two groups (computerguided and paper-based). The Endotool Glucose Management System was used to guide Insulin dosage for the computer group, whereas insulin administration in the paper-based group followed the local sliding scale protocol. The study started with induction of anesthesia and continued until the patients were in the ICU for up to 12 hours.

Results and Discussions: Patients in the computer guided group spent significantly more time in the targeted glucose range during surgery $(p=.02)$ and ICU-stay $(p=.01)$. Following admission on the ICU the first glucose reading below $150 \mathrm{mg} / \mathrm{dl}$ was achieved much faster in the computer-group $(p=.02)$. There was one blood glucose level of $48 \mathrm{mg} / \mathrm{dl}$ in the Computer group.

Conclusion(s): Computer-guided insulin administration achieved tighter blood sugar control than a standard sliding scale protocol in diabetic patients undergoing cardiac surgery in both the intra-operative and in the early postoperative periods. Levels of blood glucose $<150 \mathrm{mg} / \mathrm{dl}$ could be achieved much more rapidly than previously described. One hypoglycemic episode demonstrates the potential risks of attempting aggressive glucose control. This risk may be outweighed by the beneficial effects of tight blood glucose control.

References:

1 Ann Thorac Surg 2002; 74: 2712 Eur Heart J 1996; 17: 1337.

\section{AP4-9}

\section{Analyzing human plasma and liquor on multi electrode arrays (MEA)}

F. Kletzin, A. Gramowski, R. Hofmockel, D.G. Weiß, G.F.E. Noeldge-Schomburg Department of Anaesthesia and Intensive Therapy, University of Rostock, Rostock, Germany

Background and Goal of Study: MEA-technique allows real time analysis of drug effects of up to 128 neurons. The analysis of human plasma was already used in patients with hepatic encephalopathy. The method was registered for international patent. We used primary murine frontal cortex networks on MEAs to study effects of human plasma and liquor of anaesthetized patients $(\mathrm{BIS}=40)$.

Materials and Methods: Animal protection regularities were respected. Murine frontal cortex cells were incubated on coated MEAs (Center for Network Neuroscience, Denton, Texas) at $37^{\circ} \mathrm{C}$, pH at 7.4 , in a $10 \% \mathrm{CO}_{2}$ atmosphere until ready for use. After establishing stable activity with bursts and spike train patterns networks were used for analyses. Spike (SR) and burst rate (BR) data were analyzed offline with NeuroEXplorer (NEX, Plexon, Inc.). Effects of plasma ultrafiltrate $(n=3)$ and liquor $(n=4)$ of anaesthetized patients to native activity were recorded after $20 \%$ or $60 \%$ medium exchange, respectively (BIS $=40$, Propofol $6-8 \mathrm{mg} / \mathrm{kg} / \mathrm{h}, 0.1-0.4 \mu \mathrm{g} / \mathrm{kg} / \mathrm{min}$ 
Remifentanil (plasma). Sufentanil $30 \mu \mathrm{g}$ bolus (liquor)), Effects of plasma of awake volunteers $(n=4)$ were recorded as a control group.

Results and Discussions: Mean SR significantly decreased to $78 \pm 5 \%$ $(p=0.04)$ and $45 \pm 2 \%(p=0.001)$ with 20 or $60 \%$ medium exchange against plasma of anaesthetized patients, whereas SR significantly increased to $121 \pm 1 \%(p=0.0004)$ and $174 \pm 23 \%(p=0.05)$ with 20 or $60 \%$ medium exchange against plasma of awake volunteers. Medium exchange against liquor of anaesthetized patients caused a significant decrease of SR to $79 \pm 6 \%(p=0.03)$ and $58 \pm 11 \%(p=0.03)$ with 20 or $60 \%$ medium exchange. Plasmaultrafiltrates and liquor of BIS40 anaesthetized patients caused a similar decrease of spontaneous neuronal activity of murine frontal cortex networks, whereas plasma of wake volunteers caused the opposite effect. These effects were pronounced with $60 \%$ medium exchange.

Conclusion(s): These results show that the method is useful to analyze quantitative effects of plasma and liquor of anaesthetized patients using neuronal networks of murine frontal cortex cells.

\section{AP5-1}

Effect of epidural opioids on postoperative transcutaneous carbon dioxide tensions and peripheral oxygen saturations in spontaneously breathing patients with the TOSCA monitor

E. Wallace, A. Kopka, S. Binning

Anaesthetics, Western Infirmary, Glasgow, United Kingdom

Background and Goal of Study: Hypoventilation is associated with opioid analgesics, independent of route of administration. In previous research we found significant hypercapnia, but no hypoxaemia, in post-operative patients on oxygen $4 \mathrm{~L} \cdot \mathrm{min}^{-1}$ with PCA-morphine. ${ }^{2}$ This observational, prospective study evaluates the effect of commonly administered epidural opioids on transcutaneous carbon dioxide partial pressures $\left(\mathrm{PtcCO}_{2}\right)$ and peripheral oxygen saturations $\left(\mathrm{SpO}_{2}\right)$ with a non-invasive earlobe monitor. (TOSCA) The processed readings correlate well with arterial blood gas results.

Materials and Methods: 14 patients (ASA1\&2; $m=10, f=4$ ) scheduled for laparotomy participated. Post-operatively, patients received oxygen $\left(4 \mathrm{~L} \cdot \min ^{-1}\right)$. Pain management was with continuous epidural bupivacaine $(0.1 \%)$ and diamorphine $\left(40 \mathrm{mcg} \cdot \mathrm{ml}^{-1} ; \mathrm{n}=2\right)$, or fentanyl $\left(2 \mathrm{mcg} \cdot \mathrm{ml}^{-1} ; \mathrm{n}=8\right)$. Data was collected for 8 hours on both pre- and post-operative nights and analysed with TOSCA.

Results and Discussion: Descriptive data are shown $(\Delta M=$ post-pre-operative median change; $\mathrm{t}=$ time spent)

\begin{tabular}{lrrrrr}
\hline Variable & Med & \multicolumn{1}{c}{ LQ } & UQ & \multicolumn{1}{c}{ Min } & Max \\
\hline$\Delta \mathrm{MPtcCO}_{2}$ & 0.2 & -0.1 & 0.9 & -1.2 & 1.8 \\
$\Delta \mathrm{MSpO}_{2}$ & 3.6 & 2.7 & 3.9 & 1.0 & 6.6 \\
$\Delta \mathrm{Mt}>6 \mathrm{kPa}$ & -0.1 & -0.6 & 2.3 & -5.8 & 3.9 \\
$\Delta \mathrm{Mt}<94 \%$ & -1.4 & -3.8 & -0.8 & -6.1 & -0.2 \\
\hline
\end{tabular}

Hypercapnia-time: mean change $-0.1 ; 95 \% \mathrm{Cl}(-1.9$ to 1.7$),(\mathrm{P}=0.92)$.

Desaturation-time: mean change $-2.2 ; 95 \% \mathrm{Cl}(-3.4$ to -1.1$),(\mathrm{P}=0.0009)$.

Conclusions: We found no evidence of significant post-operative hypercapnia or hypoxaemia in patients receiving epidural bupivacaine containing opioids at commonly used doses. There was also significant evidence to show a decrease in de-saturation time with epidural analgesia.

References:

1 Rathmell JP, Lair TR, Nauman B. Anesth Analg 2005; 101: S40-43.

2 Kopka A, Wallace E, Binning A. Eur J Anaesthesiol 2005; 22 (Suppl 34): A60

\section{AP5-2}

Infrared tissue oximetry and metabolic oxygen consumption. New anaesthetic applications

A. Abad-Gurumeta, J.M. Calvo-Vecino, T. Lopez-Quesada, M. Ortega-Urbaneja Anesthesia, Gregorio Marañon General University Hospital, Madrid, Spain

Background and Goal of Study: The use of infrared spectra is applied every time with more success in monitoring of cerebral tissue oximetry. It is still unknow if these systems can show additional information about metabolic state and flow in other organs like liver, kidney or asplenic system. Our intention is to analyze during paediatric anaesthesia, modifications in oximetry at hepatic and asplenic flow level, correspond with modifications in the measured values of metabolic oxygen consumption by indirect calorimetry $\left(\mathrm{VO}_{2}\right)$.

Materials and Methods: We studied 21 patients, 1-5 years, ASA I-II, under balanced general anaesthesia, during one hour of surgery time. $8 \%$ Sevoflurane for anesthesia induction and $2 \%$ sevoflurane in $50 \% \mathrm{O}_{2} / \mathrm{Air}$, in spontaneous ventilation, for maintenance, were used. Hemodynamic and respiratory pattern; temperature; hypnosis level by Entropy of EEG; inspiration and expiration gases pattern; and $\mathrm{VO}_{2}$ were studied. Data were gathered by Datex-Ohmeda ${ }^{\circledR}$ monitoring system S/5TM and CAiOVX module, and information was processed by " $\mathrm{S} / 5$ Collect $^{\circledR}$ " Software. Oximetry values were registered in a separate system, measured by "Somanetics ${ }^{\circledR}$ "sensor of INVOX ${ }^{\circledR}$ oximetry. Results and Discussions: After induction regular differences $-p<0.05-$ between the average values from oximetry in liver $(\chi=84 \pm 9)$ and asplenic territory $(x=92 \pm 6)$, was found. These values stayed stables in both and show a reduction that correlated $\left(\mathrm{Cl}\right.$ statistically 0.93 ) with $\mathrm{VO}_{2}$ increase. Reductions of Median Arterial Tension, and/or increase of cardiac frequency, superior to $15 \%$ of basal value; greater increase of respiratory frequency $(20 \%$ of basal value); pain or intraoperatory awakening episodes; produced a significant elevation of $\mathrm{VO}_{2}(\mathrm{p}<0.01)$. Each degree of Temperature loss correspond to $\mathrm{VO}_{2}$ elevation $>10 \%(\mathrm{p}<0.01)$. These situations that alter $\mathrm{VO}_{2}$, correlates with significant reduction values of asplenic and hepatic oximetry $(p<0.05)-C l$ statistically $> \pm 0.9 ; z>4.500$-, staying differences of oximetry average values between both territories.

Conclusion(s): Cerebral oximetry by infrared monitoring systems can be used to the asplenic and hepatic territory. They could be systems of visceral sanguineous indirect measurement flow and/or tissue metabolic stress during anaesthesia.

\section{AP5-3}

Comparison of blood glucose estimated by continuous blood glucose monitor (STG-22 ${ }^{\mathrm{TM}}$ ) and $\mathrm{ABL}^{\mathrm{TM}} \mathbf{8 0 0 F L E X}$

T. Yatabe, K. Yamashita, T. Yokoyama, H. Tokoroyama, M. Manabe

Anesthsiology and Critical Care Medicine, Kochi Medical School, Nankoku, Japan

Background and Goal of Study: Intensive insulin therapy has been reported to reduce the mortality in critically ill patients (1). However, it has been remained a concern that target blood glucose exceeded in $45 \%$ of ICU patients (2), although the conventional insulin infusion nomogram requires frequent blood glucose testing. Therefore, a continuous blood glucose monitoring might be necessary. In the present study, we compared blood glucose measured by STG-22 ${ }^{\mathrm{TM}}$ (Nikkiso Inc, Tokyo, Japan), a continuous blood glucose monitor, with that measured by ABL TM800FLEX which is widely used in the world (Radiometer Medical Aps, Brønshøj, Denmark) to know the reliability and the accuracy during surgery.

Materials and Methods: Twenty-nine patients who underwent scheduled operation (hepatectomy, pancreoduodenectomy, vascular surgery, off-pump coronary artery bypass grafting and others) were enrolled in this study. After anesthesia induction, $20 \mathrm{G}$ intravenous catheter was inserted in the peripheral vein and connected to STG-22 ${ }^{\mathrm{TM}}$. And then a radial arterial catheter was inserted. Blood glucose samples were obtained from an arterial line by an anesthesiologist following an established protocol of discarding three milliliters prior to the actual blood sample. Blood glucose was measured by ABL ${ }^{\text {TM } 800 F L E X ~ i m m e d i a t e l y . ~ T o t a l ~} 100$ points of paired blood glucose values were obtained. Values were compared using correction analysis and Blant and Altman analysis.

Results: Correlation coefficient between STG-22 ${ }^{\mathrm{TM}}$ and ABL $\mathrm{TM} 800 \mathrm{FLEX}$ was 0.95 . Bias and upper/lower limit of agreement were -2.6 and $23 /-28$ respectively.

Conclusion(s): The precision of blood glucose measurements between STG$22^{\mathrm{TM}}$ and $A B L^{\mathrm{TM}} 800 \mathrm{FLEX}$ was very similar. STG-22 ${ }^{\mathrm{TM}}$ is a reliable device for measuring blood glucose continuously. Therefore, we could use the device routinely for intensive insulin therapy.

References:

1 Van den Berghe G. N Engl J Med. 2001; 345: 1359-1367.

2 Kee CA. Can J Cardiovascular Nursing 2006; 16: 20-27.

\section{AP5-4}

\section{Clinical observation of perfusion index for noxious stimuli detection}

X. Zhu, X. Zhu, L. Chen, Q. Xue, B Yu

Anesthesiology, Ruijin Hospital, Shanghai Jiaotong University, School of Medicine, Shanghai, China

Background and Goal of Study: It is quite important to find a useful indicator for noxious stimuli during operation. Previous researches showed that perfusion index(PI), obtained from the area of plethysmography, could detect the reaction of nociception, so we hypothesized that the PI would be a potential indicator for noxious stimuli during general anesthesia. 
Materials and Methods: Thirty-six patients undergoing gynecological celiotomy were randomly assigned to three groups ( $n=12$ ): Group 1 (G1), fentanyl $1 \mu \mathrm{g} / \mathrm{kg}$; Group 2 (G2), fentanyl $3 \mu \mathrm{g} / \mathrm{kg}$; Group 3 (G3), fentanyl $5 \mu \mathrm{g} / \mathrm{kg}$. $8 \mathrm{ml} / \mathrm{kg}$ of Lactated Ringer's solution was given before induction, and continued at $10 \mathrm{ml} / \mathrm{kg} / \mathrm{h}$. Anesthesia was induced by TCl of propofol, different doses of fentanyl and $0.1 \mathrm{mg} / \mathrm{kg}$ of vecuronium. Tracheal intubation was performed when Ce reached at $3 \mu \mathrm{g} / \mathrm{ml}$ (the beginning of tracheal intubation) while skin incision at $3.9 \mu \mathrm{g} / \mathrm{ml}$ (the beginning of incision). HR, MAP, BIS and Perfusion Index were recorded at time points of baseline, $1 \mu \mathrm{g} / \mathrm{ml}, 2 \mu \mathrm{g} / \mathrm{ml}$ and $3 \mu \mathrm{g} / \mathrm{ml}$ Ce of propofol, process of induction ( $10 \mathrm{~s}, 30 \mathrm{~s}, 60 \mathrm{~s}), \mathrm{TCl}$ induction $(3.9 \mu \mathrm{g} / \mathrm{ml})$ and process of skin incision (10 s, $30 \mathrm{~s}, 60 \mathrm{~s}, 120 \mathrm{~s})$.

Results and Discussions: When the noxious stimuli appeared, the values of PI had remarkable and immediate decreases, which were less than $30 \%$ of corresponding beginning values. The time points of its lowest value were $42.6 \pm 6.4 \mathrm{~s}$ during tracheal intubation and $16.6 \pm 4.6 \mathrm{~s}$ during skin incision. $\mathrm{PI}$ also had the highest sensitivity than HR and MAP. During tracheal intubation, the sensitivity was $89 \%$ of $\mathrm{PI}, 56 \%$ of $\mathrm{HR}$, and $28 \%$ of MAP $(\mathrm{P}<0.001)$, during skin Incision, the sensitivity was $100 \%$ of $\mathrm{PI}, 11 \%$ of $\mathrm{HR}$, and $36 \%$ of MAP $(P<0.001)$. At all time points during the process of noxious stimuli, there was a slight but not significant difference of $\mathrm{PI}$ among three groups (lowest values, intubation: $\mathrm{G} 1=24.9 \pm 10.6, \mathrm{G} 2=26.1 \pm 6.3, \mathrm{G} 3=33.3 \pm 11.6, \mathrm{P}=0.107$; Skin Incision: $\mathrm{G} 1=23.4 \pm 13.0, \mathrm{G} 2=24.3 \pm 9.5, \mathrm{G} 3=24.8 \pm 7.1, \mathrm{P}=0.945$, respectively) while the values of HR, MAP and BIS changed very little.

Conclusion: During the induction and the beginning of anesthesia by $\mathrm{TCl}$, $\mathrm{Pl}$ indicates the noxious stimuli effectively and immediately, but it shows no correlation with the different doses of fentanyl.

\section{AP5-5}

The interference of electrical appliances with SCS and the effectiveness of shielding clothing for the patient with SCS

Y. Kume, H. Igarashi, M. Matsumoto, H. Matsui, S. Sato

Anesthesiology and Intensive Care, Hamamatsu University School of

Medicine, Hamamatsu, Japan

Background and Goal of Study: Spinal cord stimulation system (SCS) is widely used for the patients with chronic pain. Recently electrical appliances which make strong magnetic field, such as an induction heating $(\mathrm{IH})$ cooking plate, have been widely used. In spite of many reports of electrical interference in pace-maker (PM), no report has been described about the SCS. We investigated electromagnetic interference of $\mathrm{IH}$ to SCS.

Materials and Methods: At first, we assessed electromagnetic interference of $\mathrm{IH}$ to SCS with a human body model and the shielding effectiveness of shielding clothing by using oscilloscope. Then we adapted the shielding clothing for the patients with PM which is commercially available in Japan for use with SCS, and we assessed the shielding effectiveness for a patient with SCS, who had complained of discomfort in the back when using $\mathrm{IH}$.

Results and Discussions: With a human body model, although the generator of SCS was not affected by induction heater, we observed that the slight alternating current $(60 \mathrm{~Hz}, 0.3 \mathrm{~mA})$ was inducted when $\mathrm{IH}$ was close to SCS. And we found that the shielding clothing removed both the inducted alternating current and the symptom of the patient. We supposed that the part of coiled electrode of SCS might be affected by $\mathrm{IH}$ to produce electromagnetic interference.

Conclusion(s): IH gives electromagnetic interference with SCS not in the generator but at the part of coiled electrode. Appropriate shielding clothing can prevent the interference and protect the patient discomfort and safety.

\section{AP5-6}

Metabolic monitoring at reduced flow inhalation anesthesia with sevoflurane

E. Shifman, A. Smorodnikov

Intensive Care Department, Republican Perinatal Center, Petrozavodsk, Russian Federation

Background and Goal of Study: General anesthesia with inhalation anesthetics combined with drugs for neuroleptanalgesia and mechanical ventilation is easier controlled and less toxic than total intravenous anesthesia (TIVA). Prospective randomized study was performed to assess safety of anesthesia with sevoflurane by respiratory and metabolic monitoring.

Materials and Methods: Total of 218 patients operated on for the spine and extremities fractures, and joint replacement were included in the study. All of them received $1 \%$ propofol and fentanil for induction anesthesia (high flow anesthesia). Out of them 100 patients received TIVA (sedation with 1\% propofol, myoplegia with atracurium), 80 - low flow (1.0-1.2 l/min) anesthesia and myoplegia with atracurium, 38 - minimal flow $(0.5 \mathrm{l} / \mathrm{min})$ anesthesia and myoplegia with rocuronium bromide.
Results and Discussions: Collected data is presented in the following table:

\begin{tabular}{lccc}
\hline $\begin{array}{l}\text { Data of reduced } \\
\text { flow anesthesia }\end{array}$ & Initial data & $\begin{array}{l}\text { Immediately after } \\
\text { surgery }\end{array}$ & In 24 hours \\
\hline $\mathrm{pH}$ & $7.43 \pm 0.06$ & $7.39 \pm 0.008^{\star}$ & $7.4 \pm 0.042$ \\
$\mathrm{pCO}_{2} \mathrm{mmHg}$ & $42.72 \pm 0.8$ & $39.68 \pm 0.81$ & $43.32 \pm 0.4$ \\
$\mathrm{pO}_{2} \mathrm{mmHg}$ & $97.01 \pm 6.21$ & $141.62 \pm 9.97^{\star}$ & $95.67 \pm 6.4$ \\
$\mathrm{HCO}_{3}{ }^{-} \mathrm{mM} / \mathrm{I}$ & $25.07 \pm 0.23$ & $24.09 \pm 0.21$ & $24.7 \pm 0.4$ \\
Bilirubin mcM/I & $14.18 \pm 0.88$ & $12.81 \pm 0.91$ & $11.83 \pm 1.15$ \\
Conjugated & $7.53 \pm 0.44$ & $7.34 \pm 0.46$ & $6.17 \pm 0.35^{\star}$ \\
bilirubin mcM/I & & & \\
Creatinine mM/I & $0.11 \pm 0.004$ & $0.095 \pm 0.004$ & $0.1 \pm 0.004$ \\
ALT mM/l & $0.72 \pm 0.07$ & $0.6 \pm 0.04^{\star}$ & $0.64 \pm 0.05$ \\
AST mM/l & $0.42 \pm 0.04$ & $0.35 \pm 0.03$ & $0.44 \pm 0.03$ \\
Uurea mM/l & $6.01 \pm 0.37$ & $5.54 \pm 0.34$ & $5.58 \pm 0.39^{\star}$ \\
Compliance & $37.7 \pm 0.45$ & $45.6 \pm 0.5$ & $36.5 \pm 0.65$ \\
$\mathrm{ml} / \mathrm{mmHg}$ & & & \\
Utilization $\mathrm{O}_{2}(\%)$ & $3.7 \pm 0.2$ & $9.21 \pm 0.38^{\star}$ & $3.5 \pm 0.25$ \\
\hline
\end{tabular}

* $-\mathrm{p}<0.01$

Conclusion(s): Characteristics of oxygenation, gas exchange, respiratory function and hemodynamics were within the range of physiologic norm during anesthesia with reduced flow. Use of sevoflurane to support anesthesia reduces pharmacological homeostasis loading in a patient, and synergism reduces stress-induced response to surgical intervention. Reliable improvement in $\mathrm{O}_{2}$ utilization and compliance is an additional advantage of reduced flow anesthesia as compared to high flow anesthesia.

\section{AP5-7}

Detection of cerebral ischemia during carotid endarterectomy in patients under general or regional anesthesia: evaluation of the new monitor Invos 5100B Y. Leteurnier, H. Lagadec, Y. Goueffic, B. Rozec, Y. Blanloeil Anesthesia and surgical intensive care, University hospital chu Nantes, Nantes Saint Herblain, France

Background and Goals: Detection of cerebral ischemia during carotid endarterectomy (CEA) may be improved by cerebral oximetry $\left(\mathrm{rSO}_{2}\right)$ as evidenced in patients awaked under regional anesthesia (LRA) (1). We conducted a prospective evaluation of a new version of INVOS (5100B) comparing this monitoring in patients with general anesthesia (GA) and LRA.

Patients and Methods: 25 patients undergoing CEA with GA and 28 with LRA were monitored with INVOS $5100 \mathrm{~B}$ allowing bilateral $\mathrm{rSO}_{2}$ along with radial arterial pressure and standard monitoring with a continuous computerized recording (Fusion Pegase ${ }^{\mathrm{TM}}$ ). Variations in $\mathrm{rSO}_{2}$ from preclamp values, and values at 3,5,10 min after internal carotid clamping were compared between patients with and without neurologic symptoms. Median [min-max]; Mann-Whitney; $\mathrm{p}<0.05$.

Results: Stroke occurred in 1 patient with GA and 2 with LRA. During LRA neurologic symptoms appeared after 3 and $8 \mathrm{~min}$ of clamping. There was a significant ipsilateral $\mathrm{rSO} 2$ decrease in patients with $(-24,-30,-49 \%)$ in comparison to patients without neurologic symptoms ( $-7.5 \%[+10,-27 \%])$ $(p<0.004)$ between preclamp values and values at 5 min after clamping. One patient during $\mathrm{LRA}$ had a $27 \%$ decrease in $\mathrm{rSO}_{2}$ without neurologic symptoms. The cutoff value for prediction of neurologic deterioration was $-24 \%$ at 5 min. No significant controlateral $\mathrm{rSO}_{2}$ variations were observed in all patients. Conclusions: This study confirms the cutoff value of $20-25 \%$ of ipsilateral $\mathrm{rSO}_{2}$ decrease with INVOS 5100B (1). Further study must evaluate whether a systematic shunt use when this decrease occurred at 5 min after clamping will decrease the incidence of neurologic accidents.

Reference:

1 Samra SK, Dy EA, Dorge P et al. Anesthesiology 2000; 93: 964-70.

\section{AP5-8}

\section{A prospective study to validate continuous non-invasive measurement of hemoglobin via pulse CO-oximetry M. Macknet, P. Kimball-Jones, R. Applegate II, R. Martin, M. Allard Anesthesiology, Loma Linda University, Loma Linda, USA}

Background and Goal of Study: New advances in pulse oximetry technology have led to the development of multi-wavelength pulse $\mathrm{CO}$-oximeters designed to measure multiple physiologic parameters. This study utilizes a prototype pulse $\mathrm{CO}$-oximeter that allows for measurement of continuous hemoglobin concentration $(\mathrm{SpHb})$.

Materials and Methods: After Review Board approval and informed consent 19 patients scheduled to undergo surgery were enrolled in this ongoing study. Each subject was monitored with ASA standard monitors and radial artery 
cannulas. Three prototype $\mathrm{SpHb}$ sensors, optically isolated from each other, were attached to a data collection system (Masimo Inc., Irvine, CA). In addition to $\mathrm{SpHb}$, the $\mathrm{SpO}_{2}$, pulse rate and perfusion index $(\mathrm{PI})$ values were recorded. Routine surgical care of these patients was not altered. Data was collected throughout the course of each case. $\mathrm{SpHb} / \mathrm{Hb}$ data pairs were collected at baseline, throughout the course of surgery and at completion of the case. Arterial blood samples were analyzed by laboratory CO-oximeter (Radiometer $\mathrm{ABL} 735$ ), and the resulting $\mathrm{Hb}$ measurements were compared with the data collected from the corresponding $\mathrm{SpHb}$ readings. Regression analysis, bias, precision and $A_{R M S}$ were calculated.

Results and Discussions: Nineteen patients ( 5 males, 14 females) ranged in age from 10 to 87 years. Mean (SD) $\mathrm{SpO}_{2}$ was $99.1 \%(2.4 \%) .303 \mathrm{SpHb} / \mathrm{Hb}$ data pairs were collected and analyzed. $\mathrm{Hb}$ concentration ranged from 5.8 to $14.1 \mathrm{~g} / \mathrm{dL}$ (mean $=9.5 \mathrm{~g} / \mathrm{dL}, \mathrm{SD}=1.8 \mathrm{~g} / \mathrm{dL}$ ). Regression analysis between $\mathrm{Hb}$ and $\mathrm{SpHb}$ yielded a correlation coefficient of 0.822 and the S.E.E $=1.02$. Bias, precision and $A_{\mathrm{RMS}}$ were $0.186,1.06$ and 1.07 respectively.

Conclusion(s): This device is the first device developed that can noninvasively continuously measure hemoglobin concentration in addition to the other common hemoglobin species, and therefore provides a significant expansion of existing physiologic monitoring technology. Rapid measurement of hemoglobin would be an extremely useful tool in many clinical scenarios.

\section{AP5-9}

\section{Tromboelastography during liver transplantation}

N. Martella, F. Vitale, MT. Cazzato, C. Modesti, L. Sollazzi

Anesthesia and Intensive Care, Catholic University of Sacred Heart, Rome, Italy

Background and goal of study: To date a major problem in liver transplantation (OLT) is to maintain an adequate haemocoagulative homeostasis Conventional tests provide only quantitative and punctual informations on haemostatic process whereas tromboelastography allows a dynamic analysis of coagulation and a functional interpretation of blood components involved.

Aim of the study was to compare tromboelastographic data and standard laboratory tests (PT, aPTT, INR, fibrinogen, ATIII, d-dimer, PLT, Hb, Hct). Materials and methods: Twenty consecutive patients undergone OLT for end stage liver disease (ESLD) were included in the study. In all patients conventional and tromboelastographic tests were simultaneously collected at standard times: post anesthesia induction, during pre-anhepatic phase, during anhepatic phase, after venous and arterial graft reperfusion and the end of surgery.

Statistics were performed by ANOVA and regression analysis using SPSS 6.0.

Results: According to literature a statistically significant correlation $(p<0.05)$ between the analysed data was found as follow: the reaction time $(R)$ relates well to aPTT and, as the last, is prolonged at baseline and at anhepatic phase; the clot kinetic time $(\mathrm{K})$ directly relates to INR and aPTT and inversely relates to fibrinogen; it is prolonged in case of platelet dysfunction; $\alpha$-angle inversely relates to aPTT; maximum amplitude negatively relates to INR and aPTT. Particularly significant is the lack of correlation between tromboelastographic parameter Lys 30 -direct index of fibrinolysis- and d-dimer, in postreperfusive phase too.

Conclusions: According to clinical evidence of poor bleeding, tromboelastography suggests that postreperfusive d-dimer increase reflects a graft release and not a host hyperfibrinolitic state.

Tromboelastography reliably detects actual hyperfibrinolysis allowing to avoid a potentially dangerous antifibrinolitic

Reference:

Di Benedetto P. et al Minerva Anestesiol 2003 Jun; 69(6): 501-15.

\section{AP6-1}

Induction dose of atracrium does not interfere intra-operative neuromonitoring of the recurrent laryngeal nerve during thyroidectomy

I. Lu, K.S. Chu, C.J. Tseng

Anesthesiology, Kaohsiung Medical University Hospital, Kaohsiung, Taiwan

Background and Goals: Though muscle relaxants are suggested to avoid in intraoperative neuromonitoring (IONM), the laryngeal muscles were reported to exhibit a quicker recovery than the abductor pollicis (1). The purpose of this study is to investigate whether induction dose of atracurium interfere IONM during total thyroidectomy.
Material and Methods: In a prospective study involving 27 patients undergoing elective total thyroidectomy, the influence of induction dose of atracrium on neuromonitoring of the recurrent laryngeal and vagus nerve was investigated. The patients received general anesthesia with oxygensevoflurane and atracurium was used to facilitate tracheal intubation. The degree of relaxation was monitored continuously by acclerometry (twitch\%). Evoked potentials obtained from an insitu Nemed endotracheal tube connected to EMG monitor expressed in $\mu \mathrm{V}$.

Results: First response signals were recorded for 45 vagus and recurrent laryngeal nerves. For vagus nerve, the time of first response signal occurred at $56.8 \pm 15.6$ minutes and evoked potential was $122 \pm 106.9 \mu \mathrm{V}$. First response twitch was $0.8 \pm 1.5 \%$. For recurrent laryngeal nerve, the time of first response signal occurred at $102.8 \pm 28.3$ minutes and evoked potential was $389.9 \pm 377.6 \mu \mathrm{V}$. First response twitch was $31.6 \pm 27 \%$.(data were expressed as mean $\pm \mathrm{SD}$ )

Conclusions: 1. Relaxant-free anaesthesia may not be mandatory for intraoperative neuromonitoring (IONM) of the vagus and recurrent laryngeal nerve. 2 . Giving only induction dose of atracrium does not interfere with intraoperative neuromonitoring and can facilitate intubation.

Reference:

1 Marusch F, Hussock J, Haring G, et al. Br J Anaesth 2005; 94: 596-600.

\section{AP6-2}

Accuracy of forehead $\mathrm{SpO}_{2}$ monitoring in ICU patients with acute respiratory disease: a clinical comparison with conventional finger sensors

A. Casati, G. Squicciarini, E. Spadini, C. Giovanardi, G. Fanelli

Anesthesiology and Pain Therapy, University of Parma, Parma, Italy

Background: New forehead reflectance sensors represent an excellend alternative site to monitor $\mathrm{SpO}_{2}$ when finger pulse size is reduced. The aim of this prospective study was to compare the accuracy of $\mathrm{SpO}_{2}$ reading with forehead and digit sensors in ICU patients with acute respiratory failure.

Methods: $12 \mathrm{ICU}$ patients with acute respiratory failure requiring mechanical ventilation were prospectively studied. Blood oxygen saturation was simultaneously measured with forehead sensor (Max-FAST, Tyco, Italy) and a new generation digit sensor (Max-A, Tyco, Italy), and compared to corresponding CO-oximetry-measured arterial oxygen saturation values $\left(\mathrm{SaO}_{2}\right)$ taken at the same times. The Bland-Altman analysis was used to calculate the bias and precision of the forehead and finger sensors relative to $\mathrm{SaO}_{2}$ values.

Results: We obtained a total of 40 sample sets from 12 patients. The $\mathrm{SaO}_{2}$ values ranged from $80 \%$ to $100 \%$. The bias and precision of the forehead-to$\mathrm{SaO}_{2}$ difference was $0.8 \%$ (Confidence Intervals: $-3.5 \%-3.6 \%$ ) and $1.9 \%$, respectively, versus $1.8 \%$ (Confidence Intervals: $-3.7 \%-5.2 \%$ ) and $2.3 \%$ for the digit-to- $\mathrm{SaO}_{2}$ difference $(\mathrm{P}=0.11)$.

Conclusions: Estimation of arterial oxygen saturation provided by the new reflectance forehead sensor as accurate as a new generation conventional digit sensor in ICU patients with acute respiratory failure, with a smaller bias and narrower confidence intervals.

\section{AP6-3}

Validity of the modified observer's assessment of alertness/sedation scale (MOAA/S) during low dose propofol sedation

R. Kowalski, P. Mahon, G. Boylan, B. McNamara, G. Shorten

Medical School, University College Cork, Cork, Ireland

Background and Goal: The MOAA/S scale a measure of alertness/sedation is used widely in clinical research. It is derived from the original Observer's Assessment of Alertness/Sedation scale. This scale was originally validated for use with midazolam. The observer rates patient responsiveness, speech and facial expression/eye movements. The modified form uses only the responsiveness component of the original scale [Awake (5) - Unresponsive (1)]. We set out to determine the sensitivity and specificity of the MOAA/S scale to detect characteristic EEG changes that occur during conscious propofol sedation. These changes are: decreased alpha amplitude and drop out, enhanced beta activity and/or the presence of theta waves.

Materials and Methods: 12 unpremedicated ASA I patients were recruited. Propofol was administered using a target effect site controlled infusion. The starting concentration was $0.5 \mu \mathrm{g} / \mathrm{ml}$, this increased in $0.5 \mu \mathrm{g} / \mathrm{ml}$ increments at four minute intervals. The study duration was 16 minutes. Multi-channel (19) EEG was recorded continuously. MOAA/S score was recorded at one minute intervals. The EEG was later graded by a blinded clinical neurophysiologist. A grade representing evidence of sedation was assigned to each four minute period corresponding to a fixed propofol concentration. 
Results and Discussions: There were 48 study periods each of four minutes duration. Characteristic EEG changes associated with sedation were present in 29 (Table 1).

Table 1:

\begin{tabular}{lll}
\hline MOASS Score & Sedated $(\mathrm{EEG}) \mathrm{n}=29$ & Not Sedated $(\mathrm{EEG}) \mathrm{n}=19$ \\
\hline 5 & 12 & 19 \\
$<5$ & 17 & 0
\end{tabular}

MOAA/S score was $100 \%$ specific and $59 \%$ sensitive in identifying patients with EEG evidence of sedation. MOAA/S failed to identify sedation in $41 \%$ of patients (negative prediction value $61 \%$ ).

Conclusion: MOAAVS failed to identify sedation (as defined by EEG) in $41 \%$ of ASA I patients receiving low dose propofol sedation.

Reference:

J Clin Psychopharmacol 1990; 10: 244-51.

\section{AP6-4}

Effects of epidural administration of lidocaine andropivacaine on myogenic motor evoked potentials

T. Horiuchi, M. Kawaguchi, H. Hayashi, S. Inoue, H. Furuya Department of Anesthesiology, Nara Medical University, Kashihara, Japan Background and Goal of Study: Motor evoked potentials (MEP) to transcranial electrical stimulation are used for the patients undergoing thoracoabdominal aortic surgery to prevent postoperative neurological deficits (1). During such surgery, epidural anesthesia may be applied, however, the effects of epidural anesthesia on myogenic MEPs remained undetermined. We investigated the effects of epidural administration of lidocaine and ropivacaine on myogenic MEPs.

Materials and Methods: This study is a prospective randomized control trial. After institutional approval and written informed concent, twenty-four patients who underwent elective abdominal aortic aneurysm surgery were studied. After the recording of control MEPs in response to transcranial multi-pulse stimulation under propofol and fentanyl anesthesia, $5 \mathrm{ml}$ of $2 \%$ lidocaine $(n=8)$ or $0.75 \%$ ropivacaine $(n=8)$ or saline $(n=8)$ was epidurally administered and then MEPs were recorded every 5 to 15 minutes. Percent changes of MEP amplitudes were analyzed by repeated measures analysis of variance. Results and Discussions: Control MEP amplitudes were similar among three groups. In all groups MEP amplitudes remained unchanged during the study period until 45 minutes after epidural administration.

Conclusion(s): The results indicated that relatively small dose of lidocaine and ropivacaine had little influences on myogenic MEPs in patients under propofol and fentanyl anesthesia.

Reference:

1 Jacobs MJ, Meylaerts SA, de Haan P, et al. J Vasc Surg 1999; 29: 48-59.

\section{AP6-5}

\section{Photoplethysmogram amplitude provides information on the} adequacy of analgesia of post surgical patients

K. Uutela, P. Kärkäs, M. Huiku, T. Savolainen, A. Yli-Hankala

Clinical Systems, GE Healthcare, Helsinki, Finland

Background and Goal of Study: Analgesia is an important part of the care of post surgical patients. The aim of this study was to compare the information obtained from the photoplethysmographic waveform with the post surgical patients' self-report of their pain intensity.

Materials and Methods: Physiological parameters were recorded from 15 female ASA I-II patients recovering from a major laparotomy. The analgesia was provided with patient controlled oxycodone. The patients' pain intensity was asked at 15-minute interval with the numeric rating scale (0-10); total of 93 pain reports were obtained. Photoplethysmographic wave amplitude (PPGA) time series was extracted off line and the power ratio of high frequency power $(0.15-0.4 \mathrm{~Hz})$ and sub high frequency power $(0.003-0.15 \mathrm{~Hz})$ was calculated. Prediction probability $(\mathrm{Pk})$ values were calculated in respect to the patients' pain reports. Additionally, the Pk values of heart rate (HR) and non-invasive mean blood pressure (MBP) were calculated as references. The standard errors of the Pk values were estimated with the jackknife method. Statistical significance was tested with two-tailed t-test.

Results and Discussions: MBP, PPGA, and PPGA ratio predicted pain intensity in the sense of $\mathrm{Pk}$ (see figure). PPGA ratio had the highest $\mathrm{Pk}$ value $(0.72+-0.03)$ and it was significantly higher than the $P k$ values of $H R$ and BP.

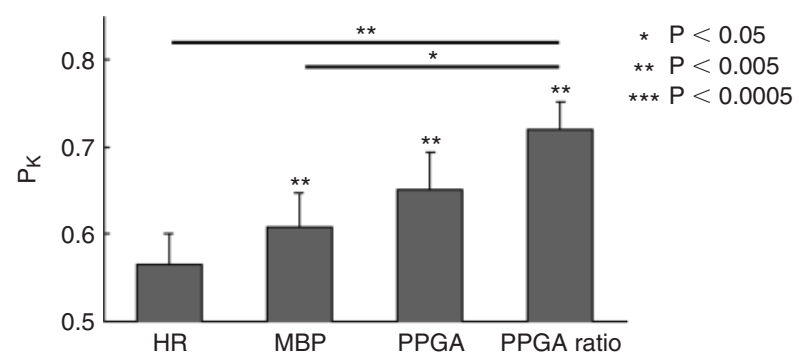

Conclusion(s): Photoplethysmogram amplitude provides information on the pain intensity of post surgical patients that could be used to help the administration of analgesics. In addition, PPGA based parameters perform significantly better than HR and BP that are commonly used to monitor the patient's state.

\section{AP6-6}

Effect of neuromuscular block reversal on bispectral index in anesthetized patients

F. Romero, F. Martins, C. Martins, R. Franca, M. Tardeli

Surgery, Sao Paulo Federal University, Sao Paulo, Brazil

Background and Goal of Study: Intraoperative memory leads to psychological disorders in anesthetized patients. Anesthesia depth can be assessed by Bispectral Index (BIS). BIS values above 60 present risk of conscious ${ }^{1}$. Glicopirrolate and neostigmine can alter anesthesia depth in patients that received propofol anesthesia ${ }^{2}$. There are no research on neuromuscular block reversal and anesthesia depth during inhalatory anesthesia. The goal of this study was evaluate BIS changes after atropine and neostigmine administration in propofol or sevoflurane anesthetized patients.

Materials and Methods: Eleven patients ASA 1 or 2 were randomized to propofol $(n=6)$ or sevoflurane $(n=5)$ groups. Anesthesia induction: remifentanil, propofol, and rocuronium. Manutention: continuous remifentanil and propofol (propofol group - P) or sevoflurane (sevoflurane group -S). Titrated doses of propofol and sevoflurane were used to maintain BIS values \pm 40 . Atropine and neostigmine were administrated when TOF $=3$. BIS variation between initial and maximum values was calculated. $T$ test was used to compare BIS change between the groups (significant $p<0,05$ ).

Results and Discussions: BIS change (mean \pm SD) was 22,8 \pm 9,2 (group $P$ ) and $8,2 \pm 1,8$ (group $S$ ) and $p=0,009$. BIS values can be affected by central nervous system (SNC) muscular tonus perception. SNC inhibition by sevoflurane is due to GABA and glicine spinal receptors that modulate muscular tonus, while propofol inhibits only GABA receptors ${ }^{3}$. Diverse spinal mechanism of action could lead to lowest BIS change in sevoflurane anesthetized patients.

Conclusion(s): Atropine and neostigmine administration increases BIS values of propofol anesthetized patients deeper than sevoflurane anesthetized patients.

References:

1. Sigalovsky N. AANA J, 2003; 71: 373-9.

2. Vasella FC, Frascarolo P, Sphan DR et al. Br J Anaesth, 2005; 94: 742-7.

3. Grasshoff C, Antkowiak B. Anesthesiology, 2004; 101: 1167-76.

\section{AP6-7}

Indocyanine green used for PDE imaging system increases mixed venous oxygen saturation $\left(\mathrm{SvO}_{2}\right)$ reading

H. Sakamoto, K. Harasawa, Y. Morimoto, T. Mayumi, H. Wakisaka

Department of Anesthesia, Cardiovascular Center Hokkaido Ohno Hospital, Sapporo, Japan

Background and Goal of Study: Laser light causes indocyanine green (ICG) to fluoresce and near infrared sensitive videocamera can capture this fluorescence image. PDE Imaging System (Hamamatsu Photonics, Hamamatsu, Japan) is such device and can use intraoperatively. This is a study to clarify whether ICG used for PDE Imaging System affects mixed venous oxygen saturation $\left(\mathrm{SvO}_{2}\right)$ readings, which also measured by using infrared light. Materials and Methods: After obtaining IRB approval and written informed consent, we studied 64 consecutive patients scheduled for OPCAB. After completion of anastomoses, 56 patients received assessment of grafts and anastomoses using PDE Imaging System with $0.3125 \mathrm{mg}, 0.625 \mathrm{mg}$, $1.25 \mathrm{mg}$ or $2.5 \mathrm{mg}$ of ICG $(1 \mathrm{ml})$ injection with $5 \mathrm{ml}$ of normal saline. Eight patients were injected $5 \mathrm{ml}$ of normal saline. $\mathrm{SvO}_{2}$ was measured with SwanGanz Continuous Cardiac Output/End Diastolic Volume Thermodilution Catheter with CEDV/Vigilance system (Edwards Lifesciences, Irvine, USA) 
and recorded every 2 seconds from 20 seconds before ICG injection till 90 seconds after ICG injection. Paired t-test was performed and $P<0.05$ was considered significant.

Results and Discussions: Immediately after ICG injection, $\mathrm{SvO}_{2}$ showed spiky increase and baseline increase except ICG $0.3125 \mathrm{mg}$ and normal saline group. In both groups, only slight peak was observed. Since hemodynamics is extremely stable during image capturing with PDE Imaging System, increase of $\mathrm{SvO}_{2}$ reading does not result from hemodynamic change. Flushing with normal saline may concerns the spiky increase to some extent but it does not explain baseline increase. It is known that ICG decreases pulse oximetry readings dose-dependently. Because pulse oximetry is two-wavelength system and $\mathrm{SvO}_{2}$ monitoring is three-wavelength system, this difference may cause the significant increase in $\mathrm{SvO}_{2}$ after ICG injection unlike pulse oximetry.

Conclusion: ICG based PDE Imaging System is a prominent intraoperative graft and anastomosis assessment device. Although very low dose of ICG is injected, ICG increases $\mathrm{SvO}_{2}$ reading significantly.

\section{AP6-8}

Usefulness of BIS monitoring to manage adequate depth of anaesthesia without muscle relaxants for cerebellopontine angle surgery with evoked potential monitoring and

\section{electromyography}

A. Skudre, B. Mamaya, I. Vanags, J. Stepanovs, J. Ozolins

Anaesthesiology, Hospital, Riga, Latvia

Background and Goal of Study: To asses the usefulness of BIS monitoring to manage adequate depth of anaesthesia without muscle relaxants at Cerebellopontine Angle surgery with evoked potential monitoring $\left(E_{S}\right)$ and electromyography (EM) to preserve the functional integrity of nerve tissue placed at risk Materials and Methods: The ethical committee of the hospital approved the study. 45 patients ASA II-IV aged 15-78 from 2000-2006 undergoing elective neurosurgery with tumours of Cerebellopontine Angle - neurinomas of the Vestibulocochlear nerve, Facial nerve, Trigeminal nerve, Glossopharyngeal nerve and meningeomas of the Cerebellopontine Angle. Patients were divided in two groups: I group 2000-2002 without BIS monitoring $(\mathrm{n}=20)$; II group 2003-2006 BIS monitoring was used. All patients were asked after surgery about consciousness and comfort during operation. Intra-operative $\mathrm{EP}_{\mathrm{S}}$ and EM were used for avoiding acute intra-operative nerve injury. Induction technique was standardised with Midazolam $5 \mathrm{mg}$, Propofol (Pr) $2 \mathrm{mg} / \mathrm{kg}$, Fentanyl (F) $3 \mu \mathrm{g} / \mathrm{kg}$ and Nimbex $0.15 \mathrm{mg} / \mathrm{kg}$ during opening and closure of the skull. Anaesthesia was maintained with Sevofluran (Sevo) and mixture $50 \% \mathrm{O}_{2}$ in air. Sevo MAC 0.5-0.7 was titrated to maintain BIS among 30-45 during the surgery. Cerebral perfusion pressure was maintained above $70-80 \mathrm{mmHg}$. F and Pr were added in requirement perfusion.

Results: In the I group, use of F was $4 \mu \mathrm{g} / \mathrm{kg} / \mathrm{h}, \operatorname{Pr} 3-4 \mathrm{mg} / \mathrm{kg} / \mathrm{h}$ and patients extubation time was $1 \mathrm{~h} 30^{\prime}-6 \mathrm{~h}$. $50 \%$ of patients were extubated at the operating room. In the II group use of $F$ was $3 \mu \mathrm{g} / \mathrm{kg} / \mathrm{h}$, $\operatorname{Pr} 3-4 \mathrm{mg} / \mathrm{kg} / \mathrm{h}$ and patients extubation time was $30^{\prime}-4 \mathrm{~h}$. $85 \%$ of patients were extubated at the operating room. Operation time was $2 \mathrm{~h} 20^{\prime}-11 \mathrm{~h} 45^{\prime}$. Nerve tumour microsurgical removal time was $1 \mathrm{~h} 30^{\prime}-10 \mathrm{~h} 25^{\prime}$. In nerve tumour microsurgical removal time, haemodynamic parameters were supported stable. Surgical procedures didn't create a significant impairment of neurological status. We haven't seen any episodes of awakening and moving during surgery. BIS during EPM was 35-45.

Conclusion: BIS monitoring is a very useful method to manage adequate depth of anaesthesia without muscle relaxants at Cerebellopontine angle surgery with $\mathrm{EP}_{\mathrm{S}}$ and $\mathrm{EM}$ and patients safety.

\section{AP6-9}

A survey of current practice of peri-operative neuromuscular transmission monitoring in the United Kingdom

Y. Kharkar, O. Adekanye, I. Hodzovic, A.R. Wilkes, J.E. Hall Anaesthetics, Cardiff University, Cardiff, United Kingdom

Background and Goal of Study: Neuromuscular function monitoring (NFM) in patients having neuromuscular blocking drugs is a minimal monitoring standard recommended by the Association of Anaesthetists of Great Britain and Ireland (AAGBI) [1]. Objective NFM assesses neuromuscular transmission avoiding residual paralysis [2]. Surveys of NFM practice of anesthetists are published [3], with practices differing internationally. We surveyed NFM practice in the United Kingdom (UK).

Materials and Methods: A questionnaire was conducted of $702(20 \%)$ randomly selected consultant anesthetists on the 2005/2006 UK medical directory database. We asked if NFM was routinely used with muscle relaxants (Q4), how routinely reversal agents were used (Q11) and whether they thought NFM should be a minimal monitoring requirement (Q12). Data are number and percentage and compared by $\chi^{2}$ using SPSS 12 .

Results and Discussions: 571 questionnaires (81\%) were returned. 29(5\%), $131(23 \%)$ and $401(70 \%)$ had experience of $<5,5-10$ and $>10$ y respectively. Years of experience did not affect routine NFM use (Q4) $(p=0.091$ and 0.29$)$ or whether reversal is routinely used (Q11) $(p=0.48$ and 0.09$)$. Whether NFM should be part of minimal monitoring varied significantly with experience $(p=0.046)$.

Table 1 Responses to questions: Q4, Q12 and Q11.

\begin{tabular}{lcclc}
\hline & Q4 & Q12 & Q11 & \\
\hline Yes & $150(26 \%)$ & $192(34 \%)$ & Never & $13(2 \%)$ \\
No & $416(73 \%)$ & $366(64 \%)$ & Occasionally & $144(25 \%)$ \\
Blank & $5(1 \%)$ & $12(2 \%)$ & Frequently & $220(39 \%)$ \\
& & & Always & $12(2 \%)$ \\
\hline
\end{tabular}

Conclusion(s): The majority of consultant anesthetists in the UK do not follow the AAGBI recommendations on NFM use. Despite evidence that clinical evaluation alone will not prevent residual blockade, $64 \%$ do not think that NFM should be part of minimal monitoring. However $6 \%$ gave an unsolicited response stating that it should be available instead of being made mandatory. References:

1. AAGBI 2000; http:// www.aagbi.org.

2. Viby-Mogensen J. Curr Op Anaesth 2001; 14: 655-9.

3. Shorten GD et al. Can J Anaes 1995; 42: 711-5.

\section{AP6-10}

Development of new ankle compression type foot pump with bio-impedance evaluations

H. Watanabe, T. Kawae, N. Kanaya, A. Namiki

Anesthesiology, Sapporo Medical University, Sapporo, Japan

Background and Goal of Study: We had recommended the bio-impedance measurement for anti-DVT (deep vein thrombosis) foot pump's evaluations, instead of an ultrasonic Doppler flow meter, at ESA 2005 Vienna. 1) Impedance changes are good blood volume shift parameter.It is possible to cary out multiple measurements in a short period. Using this method we have produced new ankle compression type foot pump.

Materials and Methods: We evaluated four different designed trial products of foot pumps (including with/without ankle compression) in each 4 male healthy volunteers aged 29 to 59 . Each product had 4 chamber bladders. Three different compression pressures $(80,100,130 \mathrm{mmHg})$ were regulated with a pressure generator. After electrodes fixation on dorsal foot and frontal thigh, we measured bio-impedance with a bio-impedance amplifier (Nihon-kohden, Japan) under $50 \mathrm{kHz} 20 \mathrm{~mA}$ transmit current Output signals were stored in a computer and analyzed.

Results and Discussions: Totally 48 times measurements were carried out. Control leg impedance value was $205.6 \pm 45.2 \Omega$ (mean \pm SD). The most prominent impedance changes were derived only by ankle compression (figure 1) and it showed same pattern with ultrasonic Doppler flow meter. This means that not only plantar compression but also ankle compression is very important in foot pump function. An ankle may be more vascular/blood rich than we thought. This new foot pump with ankle compression was commercialized lately in Japan.

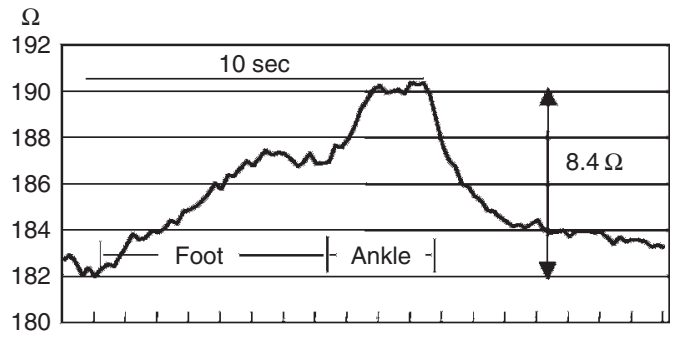

Conclusion(s): Bio-impedance measurement is also good evaluation method for producing anti-DVT foot and/or leg compression devices. Reference:

Watanabe H, et al. ESA 2005 Vienna.

\section{AP7-1}

Respiratory variations in pulse oxymetry plethysmographic waveform amplitude to predict fluid responsiveness in the operating room

M. Cannesson, Y. Attof, P. Rosamel, O. Desebbe, JJ. Lehot

Anesthesiology, Hospices civils de Lyon, Hospital Louis Pradel, Bron, France 
Background and Goal of Study: Respiratory variations in pulse oximetry plethysmographic waveform amplitude $(\triangle \mathrm{POP})$ are related to respiratory variations in pulse pressure $(\Delta \mathrm{PP})$ and are sensitive to changes in preload. However, nothing is known about its ability to predict fluid responsiveness. We hypothesized that $\triangle \mathrm{POP}$ can predict fluid responsiveness in mechanically ventilated patients under general anesthesia.

Materials and Methods: Twenty-five patients referred for cardiac surgery were studied after induction of general anesthesia. Hemodynamic data (cardiac index $(\mathrm{Cl})$, central venous pressure (CVP), pulmonary capillary wedge pressure (PCWP), $\triangle \mathrm{PP}$, and $\triangle \mathrm{POP}$ ) were recorded before and after volume expansion (VE) $(500 \mathrm{ml}$ of hetastarch $6 \%)$. Fluid responsiveness was defined as an increase in cardiac index $(\mathrm{Cl}) \geqslant 15 \%$.

Results and Discussions: VE induced changes in $\mathrm{Cl}(2.0 \pm 0.4$ to $2.3 \pm 0.5 \mathrm{mmHg} ; \mathrm{p}<0.05), \Delta \mathrm{PP}(11 \pm 7$ to $6 \pm 5 \% ; \mathrm{p}<0.05)$, and $\triangle P O P$ (12 \pm 9 to $7 \pm 5 \% ; p<0.05$ ). $\Delta \mathrm{POP}$ and $\Delta \mathrm{PP}$ were higher in responders than in non-responders ( $17 \pm 8$ vs. $6 \pm 4 \%$ and $14 \pm 7$ vs. $6 \pm 4 \%$ respectively; $\mathrm{p}<0.05$ for both). A $\triangle \mathrm{POP}>13 \%$ before VE allowed discrimination between responders and non-responders with $80 \%$ sensitivity and $90 \%$ specificity. There was a significant relationship between $\triangle P O P$ before VE and percent change in $\mathrm{Cl}$ after $\mathrm{VE}(r=0.62 ; \mathrm{p}<0.05)$.

Conclusion(s): $\triangle \mathrm{POP}$ can predict fluid responsiveness non-invasively in mechanically ventilated patients under general anesthesia. This index has potential clinical applications.

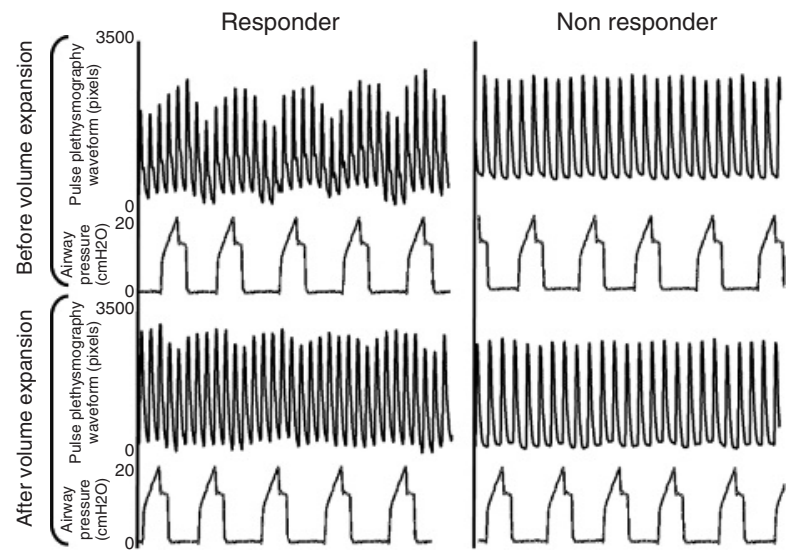

\section{AP7-2}

Haemodynamic variations and indices of cardiac performance assessed with PiCCO system during bariatric laparoscopic surgery

M. Sanfilippo, A. Guerricchio, A. Piccardo, P. Coppola, R. Guerricchio

Department of Anesthesiology, Intensive Care and Pain Medicine, University "La Sapienza" Roma, Italy, Roma, Italy

Background and Goal of Study: The haemodynamic parameters and the index of cardiac contractility often change in obese patients undergoing surgical laparoscopic procedures. We have employed the PiCCO System Pulsion in order to evaluate the variations of these parameters ${ }^{(1)}$.

Materials and Methods: In 27 consecutive obese patients (BMI 50,2 \pm 5 ) undergoing bariatric procedures (gastric by-pass and sleeve resection) have been studied the following parameters: cardiac output (CO), central venous pressure (PVC), global end-diastolic volume (GEDV), systemic vascular resistance (SVR), stroke volume variation (SVv), cardiac function index (CFI), mean arterial pressure (MAP), left ventricular contractility $(\mathrm{dP} / \mathrm{dt}$ ), intrathoracic blood volume (ITBV) and extra-vascular lung water (EVLW) in 5 different surgical steps: after induction of general anesthesia in supine position, 5 mins after reverse Trendelemburg position (TP), at 90 mins and 120 mins after the beginning of pneumoperitoneum (PP) and finally 5 mins after abdominal decompression. A standardized TIVA anesthesia was used and based on the ideal body weight. The Wilcoxon test was been applied for statistical analysis and $\mathrm{p}<0,05$ as significant.

Results and Discussions: All the parameters evaluated were greatly influenced by PP: $d P / d t$ and CFI significantly decreased $(p<0,01)$, while MAP and SVR increased at 90 mins $(p<0,05)$; GEDV and CO showed a significant decrease with PP $(p<0,01)$; PVC, ITBV, EVLW and SVv variations were not significant. All these indices dramatically return to the basic values after the end of PP.
Conclusions: The decrease of the main haemodynamic parameters is a clear consequence of $\mathrm{PP}(2)$. The reverse TP does not influence the results. $\mathrm{dP} / \mathrm{dt}$, $\mathrm{CFI}, \mathrm{PVC}$ a GEDV are mainly correlated to the PP and have to be considered to optimize the anesthetic and surgical procedures.

References:

1 Buhre W, Weyland A, Kazmaier S et al. J Cardiothorac Vasc Anesth 1999; 13: 437-440.

2 Perilli V, Sollazzi L, Modesti C et al. Obesity surgery 2003; 13: 605-609.

\section{AP7-3}

Accuracy of stroke volume variation (SVV) and pulse pressure variation (PPV) measured automatically with pulse contour analysis (PICCO) for evaluation of preload dependency during aortic surgery

S. Perbet, F. Wallet, J.P. Goarin, O. Ducombs, P. Coriat

Departement of Anesthesiology and Critical Care, Centre Hospitalier universitaire Pitié-Salpétrière, PARIS, France

Background and Goal of Study: Stroke volume and pulse pressure respiratory variation are generally considered as accurate indices of preload dependency. Those indices have been mostly validated in septic patients or during postoperative period. The goal of this study was to evaluate the accuracy of automatic measurement of SVV and PPV during aortic surgery characterized by dramatic changes of volemia and aorto-ventricular coupling factors. Materials and Methods: SVV and PPV are measured with PiCCO system (Pulsion, Munich) with long radial artery and central venous catheters. Fluid challenges (BMI . $10 \mathrm{ml}$ ) of hydroxyethylstarch were performed with the infusion rate of $100 \mathrm{ml} / \mathrm{min}$ and at different times of the surgical procedure during period of hemodynamic stability. An increase of stroke index measured by Transpulmonary thermodilution superior to $10 \%$ was considered as a positive sign of preload dependency.

Results and Discussions: One hundred and thirty fluid challenges $(5+2$ per patient) have been performed in 26 patients. A positive response to fluid loading (increase of $\mathrm{SI} \geqslant 10 \%$ ) was present in $46 \%$ of patients. A correlation between initial SV or PPV was observed with $\mathrm{SI}$ changes after volume loading ( $p<0,0001 ; R=0,605$ and $p<0,0001 ; R=0,521$ respectively) (Fig 1). The area under the curve (AUC) of the receiving operating curve for SVV $(>9 \%)$ and PPV(>7\%) was respectively 0,884 (95\% Cl: 0,82-0,93) Vs 0,832 (95\% Cl: 0,73-0,91) (Fig 2). There were no significant difference between SVV and PPV for sensibility (respectively $87 \%$ Vs $85 \%$ ) and specificity (respectively $84 \%$ Vs $71 \%$ ) for diagnosis of fluid responsiveness.

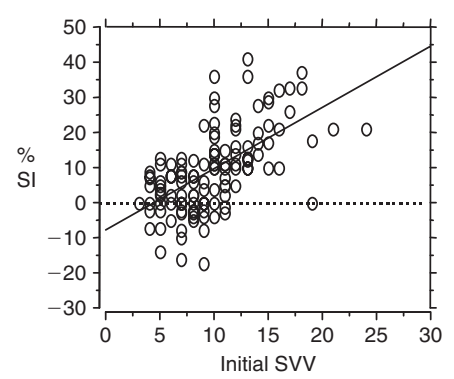

Fig. 1

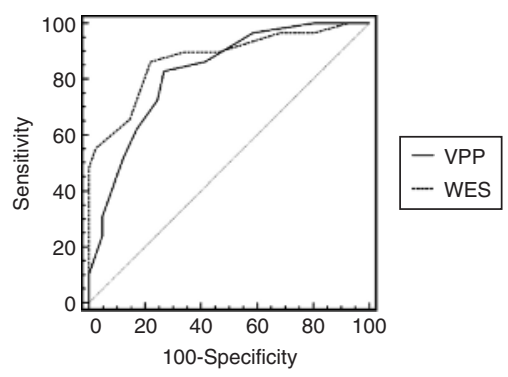

Fig. 2

Conclusion: Automatic measurement of SVV and PPV with PiCCO system can be used intraoperatively to guide fluid therapy and for optimization of cardiac output. 


\section{AP7-4}

Usefulness of intraoperative three-dimensional transesophageal echocardiography in the identification of individual segment/scallop of the mitral valve during mitral valve repair

C. Ho, M. Yang, C. Chen, C. Lee, J. Chu

Anesthesia, Chang Gung Memorial Hospital, Kweishan, Taiwan

Background and Goal of study: Intraoperative three-dimensional transesophageal echocardiography (3D-TEE) offers great promise for improving the understanding of the mitral valve anatomy, function, and pathology. It may have important implications for medical or surgical management of different mitral valve disease. The study aim was to evaluate the feasibility, accuracy and potential usefulness of intraoperative 3D-TEE, coupled with two-dimensional TEE (2D-TEE) in evaluating mitral valve structure, before and after mitral valve repair. Material and Methods: Between August 2004 and December 2006, preoperative 2D and 3D-TEE were assessed in 65 patients (mean age $65 \pm 10$ years) in whom surgical intervention was undertaken for severe mitral insufficiency due to different mitral valve disease. The location and severity extent of mitral valve prolapse, flail, and regurgitation were demonstrated preoperatively by three-dimensional dynamic sequences of the reconstructed mitral valve to the surgeon and later compared with the intraoperative finding. Leaflet scallops and commissures were displayed in short axis en-face and long-axis views. Echocardiographic results were surgically validated.

Results and Discussion: There was an excellent correspondence and closely agreed between the echocardiographic localization of the mitral valve pathology and surgical inspection. The quality of the 3D TEE was graded good in 35 patients $(54 \%)$, fair in 19 patients $(29 \%)$, and poor in 11 patients $(17 \%)$ where atrial fibrillation did not allow ECG gating. Intraoperative 3D-TEE correctly identified the location and scallops/segment prolapse in 54 of 65 patients (83\%). Compare with 2D-TEE, 3D-TEE was higher sensitivity in commissural and multiple leaflet defects $(40.9 \%$ vs. $92.5 \%, p<0.05)$. 11 patients areas of prolapse seen by surgeon were missed by 3D-TEE because some of those scallops/segments could not be well imaged due to poor "drop out" and artifacts. Thus, perfect correlation between 3D-TEE and surgery was noted in 54 of $65(83 \%)$ patients.

Conclusions: 3D-TEE is useful in identifying the location of mitral valve prolapse, allowed a precise localization and an accurate recognition of prolapsing portion of the leaflets. It may also potentially useful in assessing the extent of individual scallop/segment prolapse and beneficial for the evaluation and classification of the specific pathology before mitral valve repair. The more complex the lesion, the more valuable 3D-TEE is compared with 2D-TEE.

\section{AP7-5}

\section{Changes in renal function in laparoscopic versus retropubic} prostatectomy

S. Sabaté, JC. Ortiz, D. Vernetta, P. Sierra, P. Baxarias

Anaesthesiology, Fundació Puigvert (IUNA), Barcelona, Spain

Background and Goal of Study: Pneumoperitoneum of laparoscopic surgery is a complex physiologic event associated with neuroendocrine, respiratory, cardiovascular, and renal disturbances, as well as compromised organ blood flow. The changes in renal function of laparoscopic surgery of prostate in comparison to open surgery are unknown. This historical cohort study was performed to compare the outcome of laparoscopic versus open surgery in terms of glomerular filtration rate (GFR).

Patients and Methods: The study group comprised all patients undergoing radical laparoscopic prostatectomy from July 2003 to July 2006 at our institution. These patients were compared with patients undergoing retropubic prostatectomy. We evaluated renal function by estimated GFR through Modification of Diet in Renal Disease formula (MDRD-4 standardized IDMS) before and two days after surgery. Associated variables were studied: age, BMI, ASA status, duration of procedure and blood loss. T test and $\chi^{2}$ test were used to compare means or percentages.

Results: 432 patients were scheduled for radical prostatectomy. Patients' characteristics are shown below:

\begin{tabular}{llll}
\hline & Laparoscopic & Retropubic & $\mathrm{P}$ \\
\hline $\mathrm{N}$ & $224(51.9 \%)$ & $208(48.1 \%)$ & 0.487 \\
AGE $^{\star}$ & $63(55-69)$ & $65(55.5-69)$ & 0.118 \\
BMI $^{\star \star}$ & $26.6(26.3-27.0)$ & $26.8(26.4-27.2)$ & 0.508 \\
ASA physical status (I/II/III) $^{\star}$ & $13.3 \% / 75.2 \% / 11.5 \%$ & $4.7 \% / 71 \% / 24.3 \%$ & 0.007 \\
GFR pre & $77.5(75.2-79.8)$ & $80.1(77.3-82.8)$ & 0.633 \\
GFR post $^{\star \star}$ & $77.4(74.6-80.2)$ & $77.1(74.1-80.1)$ & 0.769 \\
Haemoglobin pre $^{\star \star}$ & $153.8(152.1-155.6)$ & $153.6(151.5-155.7)$ & 0.561 \\
Haemoglobin post $^{\star \star}$ & $124.2(121.5-126.8)$ & $109.5(106.8-112.2)$ & $<0.001$ \\
Duration of Procedure $^{\star}$ & $300(230-390)$ & $220(174-290)$ & $<0.001$ \\
\hline
\end{tabular}

* Median (10-90th percentile); ** Mean (95\% Cl)
Conclusion: The glomerular filtration rate didn't change significantly in laparoscopic prostatectomy compared to open surgery, despite an important pneumoperitoneum in Trendelenburg position was performed. Haemoglobin decreased more in open than laparoscopic surgery, and the duration of procedure was longer in laparoscopic surgery.

References:

1 Crozier TA. Anaesthesia for minimally invasive surgery. Cambridge University Press. United Kingdom 2003

2 Conacher ID et al. Br J Anaesth 2004; 93: 859-64.

3 Koivusalo AM et al. Anesth Analg 1997; 85: 886-91.

\section{AP7-6}

\section{Portal ligation monitored by ICG clearance}

A. Szijarto, B. Hargitai, S. Fischer, K. Darvas, P. Kupcsulik

1st Department of Surgery, Semmelweis University, Budapest, Hungary

Background and Goal of study: To predict and estimate the optimal time of resection following portal vein ligation $(P V L)$ with the aid of 3D CT volumetry and indocyanine green (ICG) clearance in healthy liver. Following right portal vein ligation, hypertrophy of the left liver lobe was induced prior to an extended right hepatectomy.

Materials and Methods: Fourteen patients with huge right lobe colorectal liver metastases underwent right PVL. Cut off points of ICG clearance test were: $\mathrm{R} 15<14 \%$ or PDR $>15 \% / \mathrm{min}$. The criteria for resection also required a remnant liver volume of $25 \%$ of the whole liver volume. The latter was assessed by CT scan prior to the procedures. Liver function was measured with routine biochemical tests and ICG clearance. Postoperatively, repeated ICG clearance and 3D CT volumetry tests were used to estimate the liver's regeneration. Liver resections were performed as a second stage.

Results and Discussion: After portal ligation, ICG clearance increased significantly in some patients, while in the rest, the ICG clearance remained unchanged with borderline low or normal clearance values. Between the two operations, the patients with high clearance had less complications and a better regeneration rate of the left lobe with a shorter waiting period in contrast to the 'low ICG group'.

Conclusion: ICG clearance has a significant prognostic value. Patients with an apparently inoperable right lobe liver tumor can be successfully treated using a two-stage hepatectomy. The 3D CT volumetry and ICG clearance test were essential monitoring tools in these liver resections.

\section{AP7-7}

\section{Very early hemodynamic optimization on $\mathrm{ScvO}_{2}$ in high risk patients undergoing major abdominal surgery}

L. Pompei, S. Tomasino, A. Cristofaro, C. Schember, G. Della Rocca

Anesthesia and Intensive Care, University of Udine, Udine, Italy

Background and Goal: Many strategies have been proposed to improve the outcome of patients undergoing major abdominal surgery (1). The aim of the study was to evaluate the effect of perioperative EGDT on $\mathrm{ScvO}_{2}$ (2) on the number of complications and length of stay (LOS) in high risk patients undergoing major abdominal surgery.

Material and methods: This was a randomised, prospective, double blind trial. 22 adult high risk patients (ASA III-IV) undergoing major surgery were enrolled and divided into two groups: continuous $\mathrm{ScvO}_{2}$ (PreSep, Edwards, Irvine CA,USA) (10 patients) and CRTL (12 patients). In the study group patients followed an $\mathrm{ScvO}_{2}(>70 \%$, according to the Rivers protocol) (2) guided fluid, vasoactive drugs, blood and inotropes protocol. CTRL group patients were managed using routine cardiovascular monitoring aimed for CVP between 8-12 $\mathrm{mmHg}$, mAP between $65-95 \mathrm{mmHg}$ and Hct more than $30 \%$ according to the Rivers protocol. $\mathrm{ScvO}_{2}, \mathrm{CVP}, \mathrm{mAP}$, Hct data were managed and recorded since the beginning of the anesthesia (OR) and for 48 hours after admission in ICU. Changes in these parameters occurring during the study period were corrected to reach the hemodynamic goals. Complications and deaths occurring during the hospital stay were included in the data analysis until the end hospital stay. Student $T$ test and $\chi^{2}$ were used to analyze parametric and non parametric data.

Results: are shown in the following table $\left({ }^{*} p<0.05\right)$.

\begin{tabular}{lcc}
\hline & ScvO2(\#10) & CTRL (\#12) \\
\hline Complications (\#pts) & 8 & 10 \\
Episodes for pts & $1.2 \pm 0.92$ & $1.83 \pm 1.4$ \\
Complications tot & 12 & $22^{*}$ \\
LOHS & $13.8 \pm 9.2$ & $14 \pm 7.7$ \\
Deaths & 3 & 2 \\
\hline
\end{tabular}

Conclusion: Very early perioperative optimization of $\mathrm{ScvO}_{2}$ according to Rivers (2) since the OR in high-risk patients reduced the number of postoperative complications and shows a tendency to reduce the postoperative LOS. 
References:

1 Pearse R. et al. Critical Care 2005, 9: 694-699.

2 Rivers E. et al. N Engl J Med. 2001; 345(19): 1368-77.

\section{AP7-8}

Minimal invasive cardiac output monitoring, and fluid guidance: intraoperative comparison of the new Vigileo with the trial tested CardioQ device

A. Vogt, P. Schumacher, D. Inderbitzin, S. Jakob, R. Greif Department of Anesthesiology, University Hospital Bern, Bern, Switzerland Background and Goal of Study: Stroke volume guided fluid therapy using esophageal doppler (CardioQ, Deltex Medical Systems) can reduce the length of hospital stay after colorectal surgery [1]. In another trial, fluid challenges were performed with a corrected flow time (Ftc) $<0.35 \mathrm{sec}$ and a stroke volume increase $>10 \%$ [2]. Recently, pulse pressure contour analysis without need for calibration has been brought on the market (Vigileo, Edwards Lifesciences) [3]. The aim of this study was to compare the two methods for the assessment of the response to fluid administration.

Methods: In 9 patients scheduled for partial hepatectomy we compared the change in cardiac output and stroke volume variation of the Vigileo with the simultaneously CardioQ- recorded cardiac output and Ftc after a volume bolus. At the end of surgery (abdominal fascia closed) and after a ten minute baseline measurement Hetastarch $130 / 0.4$ (Voluven) $7 \mathrm{mlkg}^{-1}$ was rapidly infused to replace intraoperative deficits and measurements were continued for ten minutes. All data were recorded online.

Results: In contrast with CardioQ, Vigileo readings were very stable and artifact free.

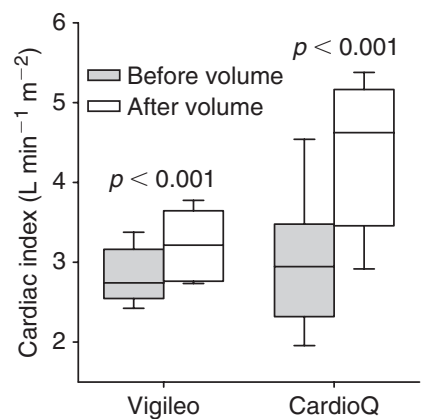

Fig. $1 \Delta$ Vigileo $\mathrm{Cl}=0.40 \mathrm{~L} \mathrm{~m} \mathrm{in}^{-1} \mathrm{~m}^{-2} ; \Delta$ CardioQ $\mathrm{Cl}=1.32 \mathrm{~L} \mathrm{~m} \mathrm{in}^{-1} \mathrm{~m}^{-2}$

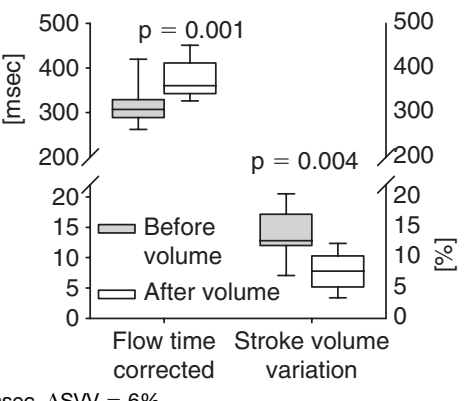

Fig. $2 \Delta$ Ftc $=58 \mathrm{msec}, \Delta \mathrm{SVV}=6 \%$

Conclusion: Vigileo and CardioQ provided satisfactory intraoperative information about changes in cardiac index and response to volume load in major abdominal surgery.

References

1 Noblett, S.E., et al., Br J Surg, 2006. 93: 1069-76.

2 Gan, T.J., et al., Anesthesiology, 2002. 97: 820-6.

3 Manecke, G.R., Expert Rev Med Devices, 2005. 2: 523-7.

\section{AP7-9}

\section{Cardiovascular monitoring by pulse dye densitometry or arterial ICG dilution}

M. Reekers, M.J.G. Simon, F. Boer, R.A.G. Mooren, J. Vuyk

Anesthesiology, Leiden University Medical Centre, Leiden, the Netherlands Background and Goal of Study: Indocyanine Green (ICG) dilution measured by Pulse Dye Densitometry (PDD) allows noninvasive cardiac output (CO) measurement. So far, PDD has been validated poorly (1). We compared hemodynamic parameters derived from PDD (DDG-2001, Nihon Kohden, Japan) to those derived from simultaneously taken arterial blood ICG concentrations.
Materials and Methods: In 20 patients (6M/14F), ASA I or II, 36 experiments were performed $(n=26$ with the PDD-finger probe and $n=10$ with the PDDnose probe). After iv administration of $10 \mathrm{mg}$ ICG, 32 arterial blood samples were taken in each session, of which 20 in the first $2 \mathrm{~min}$. Comparison of $\mathrm{CO}$, central blood volume (CBV) and total blood volume (TBV) between methods was done by Bland-Altman analysis, reporting mean difference (bias) and limits of agreement (LOA $= \pm 2 \mathrm{SD})$.

Results and Discussions: PDD overestimated CO by $8-30 \%$ for the finger or nose probe, and CBV by $46-48 \%$. The LOA for CO varied from $-67 \%$ to $+127 \%$ for the nose probe. The LOA for CBV were from $-98 \%$ to $+193 \%$ as compared to intravascular measurements. TBV agreed better: $-10 \%$ to $-15 \%$ with LOA of $-47 \%$ to $27 \%$.

Conclusion(s): The significant bias and large limits of agreement of PDD compared to invasive measurement suggest that PDD is unsuitable for evaluation of cardiovascular parameters in the individual patient. In contrast to other studies, the nose probe did not perform better than the finger probe for $\mathrm{CO}$ measurement (2).

References:

1 Hofer CK, Bühlmann S, Klaghofer R et al. Acta Anaesthesiol Scand 2004; 48: 653-57. 2 Imai T, Takahashi K, Goto F et al. J Clin Monit Comput 1998; 14: 477-84.

\section{AP7-10}

\section{Cardiac output monitoring: evaluation of the FloTrac $^{\mathrm{TM}}$} system

M. Biais, K. Nouette-Gaulain, V. Cottenceau, P. Revel, F. Sztark Réanimation chirurgicale, Pellegrin, Bordeaux, France

Background and Goal of Study: The aim of the study was to compare cardiac output (CO) measurement by FloTrac ${ }^{\mathrm{TM}}$ system (APCO) versus static (ICO) and continuous $\mathrm{CO}(\mathrm{CCO})$ obtained with automatically thermodilution by a pulmonary artery catheter (PAC) in patients undergoing liver transplantation (LT). Materials and Methods: After obtaining approval from the Ethics committee and written informed consent, we studied 20 patients, undergoing liver transplantation for acute or chronic liver failure. Patients were monitored with PAC and FloTrac $^{\mathrm{TM}}$. 20 sets of measurement were made in steady-state periods in operating room $(n=15)$ and in intensive care unit $(n=5)$. Comparisons between APCO-ICO and between APCO-CCO were performed using linear regression and Bland-Altman method. Data were expressed as mean \pm SD. Results and Discussions: Twenty patients (14 males/6 females; age $51 \pm 9$ years) were enrolled and 400 simultaneous measurements performed between ICO and APCO were obtained. No data were rejected. The range for ICO, CCO and APCO were respectively $2.5-12.3$ litre $\cdot \mathrm{min}^{-1}, 2.3-12.8$ litre $\cdot \mathrm{min}^{-1}$ and 2.1-9.5 litre $\cdot \mathrm{min}^{-1} . \mathrm{ICO}_{\mathrm{SM}}$ and APCO were correlated $\left(r^{2}=0.73\right)$. Bias between $\mathrm{ICO}_{\mathrm{SM}}$ and APCO was 0.8 litre $\mathrm{min}^{-1}, 95 \%$ limits of agreement was large $\left(-1.8\right.$ to 3.5 litre $\left.\cdot \mathrm{min}^{-1}\right)$. CCO and APCO were bad correlated $\left(r^{2}=0.66\right)$ and bias between $\mathrm{CCO}$ and APCO was 1.0 litre $\cdot \mathrm{min}^{-1}, 95 \%$ limits of agreement was large $\left(-1.8\right.$ to 3.9 litre. $\left.\mathrm{min}^{-1}\right)$. Bias and $95 \%$ limits of agreement between $\mathrm{ICO}_{\mathrm{SM}}$ and APCO were rather acceptable for $\mathrm{CO}<6$ litre $\cdot \mathrm{min}^{-1}$ whereas they were not for $\mathrm{CO} \geqslant 6$ litre $\cdot \mathrm{min}^{-1}$.

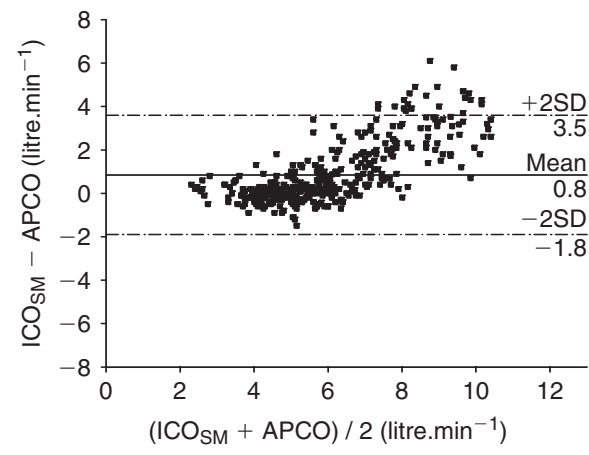

Conclusion(s): In summary, bias and precision between ICO and APCO were acceptable in liver transplanted patients as long as CO was $<6$ litre $\cdot \mathrm{min}^{-1}$. Our results indicate that $\mathrm{CO}$ monitoring by the Flotrac ${ }^{\mathrm{TM}}$ system during LT may be employed with precaution.

\section{AP8-1}

Temperature and humidity alterations in the course of low flow anaesthesia

I. Kanus, S.S. Gratchev, S.J. Gratchev

Anaesthesiology, Belorussian Medical Academy for Postgraduate Training, Minsk, Belarus 
Introduction: The study was conducted to examine the temperature and humidity alterations in the course of Low Flow Anesthesia (LFA) and to compare it to parameters of high gas flow.

Patients and methods: 60 patients: age 21-80; male - 22; female - 38; ASA I-II; body weight - 56-84 kg (average 72,4 $\pm 3,1$ ), who undergoing abdominal and gynecological surgery, were divided into two groups - by 30 patients each. Average anesthesia duration was equal in the both groups - $156 \mathrm{~min}$ (from 120 to $215 \mathrm{~min}$, average $156 \pm 4,3$ ). General anesthesia with fentanyl $2,0-2,2 \mu \mathrm{g} / \mathrm{kg} / \mathrm{hour}$, halothane $0,8-1,0 \mathrm{vol} . \%$ (1-1,25 MAC) and pancuronium $0,04-0,05 \mathrm{mg} / \mathrm{kg} / \mathrm{hour}$ or atracurium $0,5-0,6 \mathrm{mg} / \mathrm{kg} / \mathrm{hour}$ was performed in both groups. Fresh gas flow (FGF) at the 1-st group was $6 \mathrm{~L} / \mathrm{min}$, at the $2-n d-2 \mathrm{~L} / \mathrm{min}$. Two thermoelectric sensors were incorporated directly to the patients circuit, in case of the temperature measuring - at the inspiration and expiration lines; in case of humidity measuring - at the inspiration line successively. The 1-st sensor was dry, and the 2-nd - wet. Ambient air temperature at the operating theatre was $18-20^{\circ} \mathrm{C}$. Absolute humidity was calculated by the following formula: $K=f-a\left(t_{d r y}-t_{\text {wet }}\right) B$, where $K-a b s o l u t e$ humidity; $f$ - maximal water vapors pressure under the temperature of wet sensor; $\mathrm{a}$ - psychrometric coefficient $=0,0011$; $\mathrm{B}$ - barometric pressure.

Results and discussion: Gas mixture temperature at 1-st group (FGF $=6 \mathrm{~L} / \mathrm{min}$ ) came to $20,2 \pm 0,5^{\circ} \mathrm{C}$ in the inspiration line, and to $21,08 \pm 0,4^{\circ} \mathrm{C}$ in the expiration line. Temperature alterations were insignificant during anesthesia maintenance, temperature increase in the expiration line was not more than $4,5 \%(p>0,05)$. Absolute humidity in the expiration line was unchanged $-15 \mathrm{~mm} . \mathrm{Hg}$.

Initial inspiration gas mixture temperature at 2-nd group (FGF $=2 \mathrm{~L} / \mathrm{min}$ ) was $20,6 \pm 0,5^{\circ} \mathrm{C}$, in a $30 \mathrm{~min}$ became $23^{\circ} \mathrm{C}$, in a $90 \mathrm{~min}-27,0 \pm 0,6^{\circ} \mathrm{C}$. Expiration temperature came in a $90 \mathrm{~min}$ to $28,1 \pm 0,5^{\circ} \mathrm{C}$, while increase was $0,05-0,1^{\circ} \mathrm{C}$ per min, or $29,5 \%$. Absolute humidity in the expiration line became $21,8 \mathrm{~mm} . \mathrm{Hg}$, or humidity has increased up to $25 \%$.

Conclusion: Low Flow Anesthesia is the optimal method for the physiological temperature and humidity value maintenance at the prolonged anesthesia.

\section{AP8-2}

Clinical usefulness of a novel algorithm for automatic estimation of the respiratory variations in arterial pulse pressure

M. Cannesson, O. Desebbe, J. Slieker, O. Bastien, JJ. Lehot

Department of Anaesthesiology, Hospices Civils de Lyon, Hopital Louis Pradel, Bron, France

Background and Goal of Study: Respiratory variations in arterial pulse pressure $\left(\Delta \mathrm{PP}_{\mathrm{man}}\right)$ are accurate predictors of fluid responsiveness in mechanically ventilated patients. However, they have to be calculated manually and can not be continuously monitored. The aim of our study was to assess the clinical utility of a novel algorithm for automatic estimation of $\Delta \mathrm{PP}(\Delta \mathrm{PP}$ auto $)$. Materials and Methods: We studied 23 patients referred for coronary artery bypass grafting. $\triangle \mathrm{PP}_{\text {auto }}$ was continuously displayed using a method based on automatic detection algorithms, kernel smoothing, and rank-order filters(1). All patients were under general anesthesia, mechanical ventilation, and were equipped with a pulmonary artery catheter. $\Delta \mathrm{PP}_{\operatorname{man}}$ and $\Delta \mathrm{PP}_{\text {auto }}$ were recorded simultaneously at eight steps during surgery including before and after a volume expansion (VE) (500 ml hetastarch). Responders to VE were defined as patients presenting $>15 \%$ increase in cardiac output following VE. Agreement between $\triangle \mathrm{PP}_{\operatorname{man}}$ and $\triangle \mathrm{PP}_{\text {auto }}$ was determined using Bland-Altman analysis. Results and Discussions: There was a strong and significant relationship between $\Delta \mathrm{PP}_{\text {man }}$ and $\Delta \mathrm{PP}_{\text {auto }}$ over the 184 pairs of collected data $(r=0.90$; $\mathrm{p}<0.05)$. We observed a good agreement between $\Delta \mathrm{PP}_{\text {man }}$ and $\Delta \mathrm{PP}_{\text {auto }}$ (bias $=-1.3 \pm 3.3 \%)$. Fifteen $(65 \%)$ patients were responders to VE. A threshold $\Delta \mathrm{PP}_{\operatorname{man}}$ value of $12 \%$ allowed discrimination of responders to VE with a sensitivity of $80 \%$ and a specificity of $100 \%$. A threshold $\Delta \mathrm{PP}_{\text {auto }}$ value of $9 \%$ allowed discrimination of responders to VE with a sensitivity of $80 \%$ and a specificity of $88 \%$. There was no statistically significant difference between area under the ROC curve for $\Delta \mathrm{PP}_{\operatorname{man}}$ and $\Delta \mathrm{PP}_{\text {auto }}(0.908 \pm 0.068$ vs $0.908 \pm 0.065$ respectively; $p<0.05)$.

Conclusion(s): $\Delta \mathrm{PP}_{\text {auto }}$ is strongly correlated to $\Delta \mathrm{PP}_{\mathrm{man}}$, is an accurate predictor of fluid responsiveness, and allows continuous monitoring of $\Delta \mathrm{PP}$. This novel algorithm has potential clinical applications.

Reference:

1 Aboy M, McNames J, Thong T, et al. IEEE Trans Biomed 2004; 51: 2198-203.

\section{AP8-3}

Reclaiming waste anesthetic gas: initial clinical trials

J. Berry, J. Barwise, L. Lancaster

Anesthesiology, Vanderbilt University Medical Center, Nashville, USA
Background and Goal of Study: There are potentially both economic and environmental benefits in reclaiming and recycling waste volatile anesthetics. Of the technologies available, only condensation of waste anesthetic gas (WAG) provides both economy and ease of implementation. We evaluated a new technology which combines efficient anesthetic gas scavenging with cold-trap condensation of WAG.

Materials and Methods: A suite of 4 operating rooms was equipped with new waste gas scavenging interface valves using demand valves rather than the traditional open "active" scavenging systems. These were connected to a proprietary vacuum unit, water trap, and cold-trap condenser operating at -90 degrees $\mathrm{C}$ and $200 \mathrm{kPa}$. Efficiency, anesthetic yields, and cost of operation were evaluated.

Results and Discussions: The units were easily installed in operating suites with existing, dedicated piping for WAG disposal. Efficiencies of extraction approached $99 \%$ under most conditions. The cost per operating room was US $\$ 5500$; this would be lower in the case of a larger operating suite. The widespread implementation of this technology could reduce WAG emissions in the US by as much as 1500 tonnes/year (equivalent to $3.5 \mathrm{Tg}$ of $\mathrm{CO}_{2}$ ). Conclusion(s): The use of condensation as a tool for capture of WAG is both economical and practical in the hospital setting. The recycled product may be able to reduce the cost and increase the availability of modern volatile anesthetics worldwide.

\section{Reference:}

Berry JM: Volatile anesthetic recycling: It's about time (and temperature!) Society for Technology in Anesthesia, San Diego USA, January 2005 (abstract)

\section{AP8-4}

\section{Airway pressures monitoring in anesthesia: intra and extratracheal differences}

A. Abad-Gurumeta, J.M. Calvo-Vecino, M. Ortega-Urbaneja, T. LopezQuesada

Anesthesia, Gregorio Marañon General University Hospital, Madrid, Spain

Background and Goal of Study: We designed an endotracheal sensor for monitoring intra and extratracheal pressures. Our hypothesis try to demonstrate airways pressures obtained inside and outside trachea, are different.

Materials and Methods: We studied average, peak and plateau pressures of the respiratory ways, during general anaesthesia in 71 children. The study was managed by Datex-Ohmeda ${ }^{\circledR}$ Cardiocap 5 spirometer. Values were obtained by means of a sensor placed inside the anaesthetic circuit before the endotracheal tube (connected to the paediatric D-lite system), and by means of another sensor - specifically designed - placed in the trachea, immediately before the carina and after the end of the endotracheal tube. This sensor has two elements: first is a capnography measuring cannula which has been sectioned to be introduced through the second element. This one consists of a paediatric urinary fixer that was introduced in an opening of respiratory circuit. The cannula was introduced through out, which lets the compartment stay watertight. Before orotracheal intubation, we measured and marked the sensor length with regard to the endotracheal tube for its correct location. The veracity of the obtained intratracheal pressure values was checked by means of a pressure manometer and using the correction factor (1.36) of $\mathrm{Hg} \mathrm{mm}$ to $\mathrm{H}_{2} \mathrm{O} \mathrm{cm}$. Every variable was analysed in the following times: starting, $5,10,15,20,30,40,50$ and 60 minutes. Chi ${ }^{2}$ was used.

Results and Discussions: Globally the pressures (obtained in the airways intra and extratracheal) was present a differential of $3.5 \pm 0.35$ points $(p<0.01)$. There was not peak pressure in the endotracheal measurement and it was practically the same than the Plateau pressure.

Conclusion(s): Pressure measurements differences are due to the different places where the measurement is carried out. In the trachea, the peak pressure disappears and is the same as the Plateau pressure due to the fact that the trachea is not a rigid conduct, as the tubing. By this reason, we obtain a more physiological airway pressure result (1).

Reference:

1 Kawati R, et al. Anesth Analg. 2005; 100(3): 889-893.

\section{AP8-5}

Intraoperative transesophageal echocardiography in refractary hypotension

C. Cabrera Schulmeyer, R. Vega, S. Schmied, R. Hernández, J. De la Maza Anesthesiology, Hospital Clínico FACH, University of Valparaíso, Santiago, Chile

Background and goal of the study: According to the practice guidelines for perioperative transesophageal echocardiography (TEE), the evaluation 
of acute and persistent hypotension is a category 1 indication. This is an unfrecuent perioperative situation but determining the etiology is essential for a successful therapy. The utility of TEE during hypotension is not well explored during non cardiac surgery and the aim of this study was to evaluate if TEE was useful for the hemodynamic management of these patients. Material and Methods: Adults were prospectively enrolled. Refractary hypotension was arbitrary defined as sistolic blood pressure lower than $85 \mathrm{mmHg}$, have not responded to adecuate volume administration and to three bolus doses of ephedrine. Then a multiplanar TEE probe was inserted and a preestablish protocol of 2-D images and color, continuous and pulsed doppler was done.

Results and Discussion: 38 adults ( $71 \pm 12$ years) were studied, $82 \%$ during elective non cardiac surgery. The most frequentely surgeries done were abdominal (open and laparoscopic), thoracic and orthopedics. In all patients TEE was useful for determinig the etiology of persistent hypotension. Hipovolemia was diagnosed in 14 patients $(41 \%)$. Low ejection fraction $(E F<30 \%)$ was detected in 5 cases and was treated with inotropic drugs successfully. Systolic anterior motion (SAM) of the mitral valve was the diagnosis in 5 patients and in another 6 patients severe embolism was detected during hip and knee surgery. Myocardial ischemia causing persistent hypotension was detected in only 5 patients and in 3 patients cardiac tamponade was the etiology of refractory hypotension. Two patients died and both had severe myocardial ischemia.

Conclusion: Perioperative TEE is useful can be quickly inserted with few complications index and heart and great vessels can be directly seen and functionally evaluated. Hypovolemia may be frequentely underdiagnose because wrong estimations of volumen. TEE not only permits to assess volemia it also allows to manage volume reposition. TEE is a minimally invasive image tool and it is the only monitor that permits to diagnose ischemia, cardiac tamponade, embolic phenomena and SAM in the operating room.

\section{AP8-6}

\section{Filtration performance of breathing system filters after 24 hours pre-conditioning}

\section{Chethan, A. Wilkes, C. Cann, J. Hall}

Anaesthetics and Intensive Care Medicine, Cardiff University, Cardiff, United Kingdom

Background and Goal of Study: The European standard for breathing system filters specifies pre-conditioning each filter for the manufacturer's maximum recommended time of use for the filter (usually 24 hours) before testing the filtration performance (1). However, in a previous study, the filtration performance of filters was determined after only three hours of pre-conditioning (2). This study measured the filtration performances of six different types of filters after 24 hours of pre-conditioning.

Materials and Methods: Three samples of six different filters (three pleated and three electrostatic) intended for adult use were pre-conditioned for 24 hours before testing by connecting each filter to a patient model breathing with a tidal volume of $0.5 \mathrm{~L}$, a frequency of 12 breaths per minute and an I:E ratio of $1: 2$ and expiring fully saturated air at $34^{\circ} \mathrm{C}$. The filtration performance of each sample was measured by determining the penetration of sodium chloride particles using a sodium flame photometer Type No. 1100 (SFP Services, Christchurch, $\mathrm{UK}$ ) at a flow of $30 \mathrm{~L} \mathrm{~min}^{-1}$. Five samples of each filter were also tested without any pre-conditioning. Analysis was by general linear modeling to allow for the effect of pressure drop.

Results and Discussions:

\begin{tabular}{lll}
\hline Model & \multicolumn{2}{l}{ Mean (range) penetration \% } \\
\cline { 2 - 3 } & Unused & Pre-conditioned \\
\hline Electrostatic & & \\
Barrierbac S & $.40(2.8-8.95)$ & $3.61(2.24-4.92)$ \\
Filta-Therm & $0.28(0.26-0.34)$ & $0.27(0.21-0.37)$ \\
Inter-Guard & $3.40(2.61-4.11)$ & $3.60(2.62-4.60)$ \\
Pleated & & \\
BB25 & $0.04(0.03-0.09)$ & $0.03(0.03-0.04)$ \\
Hydro-Guard & $0.02(0.01-0.07)$ & $0.01(0.01-0.01)$ \\
Sterivent Mini & $0.51(0.49-0.54)$ & $0.52(0.51-0.56)$ \\
\hline
\end{tabular}

Conclusion(s): Pre-conditioning, and hence exposure to humidification, did not have a significant effect $(p>0.05)$ on the filtration performance of any of the filters.

References:

1 British Standards Institution (BSI). BS EN 13328-1: 2001. London: BSI, 2001.

2 MHRA Evaluation 04005. London: MHRA, 2004.

\section{AP8-7}

\section{Changes in regional cerebral oximetry during off-pump} coronary artery bypass graft surgery

C. Verborgh, S. Hasan, R. Lauwers, N. Rooselaer, F. Camu

Anesthesie, UZ Brussel, Brussel, Belgium

Background and Goal of Study: Neurocognitive performance following offpump coronary artery bypass (OPCAB) surgery remains impaired after surgery as compared to before (1). Haemodynamic disturbance resulting from optimal surgical exposure (2) might be a causal factor. We studied changes in regional cerebral oxygen saturation measurement $\left(\mathrm{SrO}_{2}\right)$ by near infrared optical spectroscopy (3) as a non-invasive monitoring endpoint during the different positions of the heart needed to perform anastomoses on specific coronary arteries. Materials and Methods: Thirty-five patients scheduled for off-pump CABG agreed to take part in this open observational study. $\mathrm{SrO}_{2}\left(\mathrm{Invos}^{\circledR}\right.$, Somanetics Corporation) was recorded via sensors placed left and right on the frontoparietal area. Cardiac output (CO) and mixed venous oxygen saturation $\left(\mathrm{SvO}_{2}\right)$ and other haemodynamic variables were obtained with the heart in neutral position (PRE), and after stabilisation and exteriorisation for anastomosis on the left anterior descending artery (LAD), the first diagonal branch (D1), the obtuse marginal artery (OM) and the right circumflex artery (RCX). Wilcoxon test was performed with Statistica ${ }^{\circledR}$, data expressed as means (S.E.M.)

Results and Discussions: The patients were 71 (2) years old, with an ejection fraction of 57 (3)\% (ventriculography). Ten patients had previous myocardial infarction. Results are shown below:

\begin{tabular}{llllll}
\hline & PRE & LAD & D1 & OM & RCX \\
\hline $\mathrm{Cl}\left(\mathrm{L} . \mathrm{M}^{-2}\right)$ & $2.43(0.1)$ & $2.01(0.09)$ & $1.69(0.24)^{\star}$ & $1.64(0.17)^{\star}$ & $1.8(0.13)$ \\
$\mathrm{SV}\left(\mathrm{mL}^{-} \mathrm{M}^{-2}\right)$ & $37(2)$ & $29(2)$ & $22(3)^{\star}$ & $24(2)^{\star}$ & $25(2)^{\star}$ \\
$\mathrm{SrO}_{2}(\mathrm{~L})(\%)$ & $62(2)$ & $58(2)$ & $55(5)^{\star}$ & $57(3)$ & $51(3)^{\star}$ \\
$\mathrm{SrO}_{2}(\mathrm{R})(\%)$ & $62(2)$ & $58(2)$ & $52(5)^{\star}$ & $58(4)$ & $52(3)^{\star}$ \\
$\mathrm{SvO}_{2}(\%)$ & $82(1)$ & $77(2)$ & $73(4)^{\star}$ & $73(3)^{\star}$ & $75(2)^{\star}$ \\
\hline
\end{tabular}

*: significantly different from PRE with $\mathrm{p}<0.05$

Conclusion(s): The Invos ${ }^{\circledR}$ regional cerebral oximetry seems to accurately reflect the consequences of haemodynamic disturbance during OPCAB surgery, in a noninvasive way.

References:

1 Chernov V et al. Eur J Cardiothorac Surg 2006; 29: 74-81.

2 Nierich AP et al. Ann Thorac Surg 2000; 70: 466-72.

3 Murkin JM. Semin Cardiothor Vasc Anes 2005; 9: 139-42.

\section{AP8-8}

\section{Flow through intravenous cannulae: neither easily} predictable nor laminar

D. McPherson, O. Adekanye, A.R. Wilkes, J.E. Hall

Department of Anaesthesia and Intensive Care Medicine, University Hospital of Wales, Cardiff, United Kingdom

Background and Goal of Study: Predicting flow through an intravenous cannula (IVC) is useful to clinicians if changes in flow are required and to guide selection of IVC. We set out to find the usefulness of manufacturers' quoted flows according to standard tests (1) in predicting actual flow and to characterise that flow.

Materials and Methods: We built a model of a vein and inserted IVCs of varying gauges into it. The height of the fluid bag above the vein was varied to set the filling pressure and the flow through the IVC was measured.

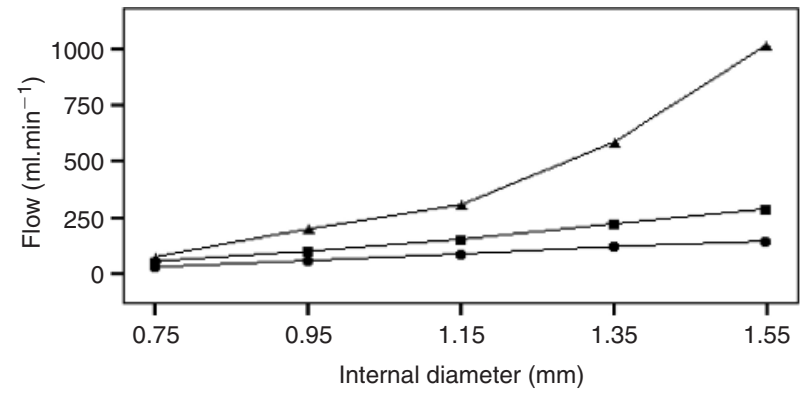

Results and Discussions: The graph shows measured $(\bullet)$ and theoretical (४) flow at a pressure of $10 \mathrm{cmH}_{2} \mathrm{O}$ and the quoted ( $\bullet$ ) flow. The flow through an IVC is not a consistent ratio of the quoted maximum flow: at $100 \mathrm{cmH}_{2} \mathrm{O}$ it is 0.83 [0.06] (mean[sd]) for $0.75 \mathrm{~mm}$ ID IVCs compared with 0.72[0.04] for $1.55 \mathrm{~mm}$. Flow at the manufacturer's quoted rate is turbulent (best curve fit flow $=113.3[1.41]^{*} \mid D^{2.21[0.05]}$ (mean [standard error]), as is the flow at rates above $12 \mathrm{ml} \cdot \mathrm{min}^{-1}$ (flow $=2.87[0.13]^{\star}$ pressure ${ }^{0.62[0.01]}$ in $20 \mathrm{G} \mathrm{IVCs}$ ). This is despite Reynolds' number being as low as 328 . 
Conclusion(s): Flow through IVCs is turbulent at the upper range of clinically used flows, therefore Poiseuille's law is not useful in predicting flow and the effect of changing radius is less than commonly believed. Neither are the quoted maximum flows easily useful. There are many determinants of laminar flow apart from Reynolds' number. Further work would determine useful correlates of flow and find if laminar flow ever occurs in clinical situations.

Reference:

1 BS-EN-ISO-10555-5: 1997. Sterile, single-use intravascular catheters. Over-needle peripheral catheters. London: British Standards Institute, 1997.

\section{AP8-9}

Monitoring of propofol concentration in breathing gas: effect of the lung on the course

M. Grossherr, A. Hengstenberg, L. Dibbelt, B.W. Igl, H. Gehring

Anaesthesiology, University Clinic of Schleswig-Holstein Campus Luebeck, Luebeck, Germany

Background and Goal of Study: The part of the lung in propofol monitoring has to be defined more precisely as measurements of breathing gas may be used for the non-invasive monitoring of propofol concentration in plasma. The following study should investigate how the course of propofol concentration in breathing gas is related to the course of propofol in plasma concentration.

Materials and Methods: After approval of the local ethic board we determined the propofol concentrations in plasma and breathing gas before and during an anaesthesia. Propofol was constantly infused $(6 \mathrm{mg} / \mathrm{kg}$ bodyweight $\times$ h). Samples of propofol and breathing gas were drawn at four points of time (T1-T4) after 10, 20, 30 and 40 minutes in 6 cardiac surgery patients. To describe the course of propofol concentration in plasma and in breathing gas as a function of time alone the values of each point of time (T1-T4) were compared to point of time 4 (T4) for each individual and were calculated as a ratio. Non-parametric tests were used to analyze the data.

Results: Propofol concentration ranged between 2,2 to $3,3 \mu \mathrm{g} / \mathrm{ml}$ in plasma and 2,4 to $17,7 \mathrm{ppb}$ in breathing gas. Data of the calculated ratios are shown in the table as mean and standard deviation 0.

\begin{tabular}{lllll}
\hline & $\mathrm{T} 1$ & $\mathrm{~T} 2$ & $\mathrm{~T} 3$ & $\mathrm{~T} 4$ \\
\hline $\mathrm{RaCprPL}$ & $69,2(24)$ & $99,9(17) \#$ & $93,9(15) \#$ & $100(0) \#$ \\
$\mathrm{RaCprBr}$ & $38,0(10)$ & $65,9(12) \#$ & $96,9(9) \#,{ }^{*}$ & $100(0) \#,{ }^{*}$ \\
\hline
\end{tabular}

RaCprPL rsp. RaCprBr: ratio of propofol concentration in plasma rsp. breathing gas at the points of time $1-4$ to point of time 4 .

\# $\mathrm{P}<0.05$ vs $\mathrm{T} 1 ;{ }^{*} \mathrm{P}<0.05$ vs $\mathrm{T} 2$.

Conclusion: Using a constant infusion propofol concentration in breathing gas reached a steady state after 30 minutes, which was reached earlier in plasma. The lung may delay the appearance of propofol in breathing gas. This has to be acknowledged for using propofol monitoring in breathing gas.

\section{AP8-10}

\section{Accuracy of a novel bioacoustic sensor for monitoring respiratory rate}

M. Macknet, P. Kimball-Jones, R. Applegate II, R. Martin, M. Allard

Anesthesiology, Loma Linda University, Loma Linda, USA

Background and Goal of Study: Monitoring respiration of spontaneously breathing patients is a concern in the operating room, post anesthesia care unit (PACU), and on general care wards. Currently available devices require either a cannula system positioned in line with airflow to detect respiration or utilize impedance pneumography. These devices have multiple limitations which have precluded widespread implementation in a general care setting. A novel bioacoustic sensor for continuously monitoring respiration has been developed. We evaluated the accuracy of the prototype sensor in the post anesthesia care unit.

Materials and Methods: Following institutional IRB approval and informed consent, 10 patients, upon arrival to the PACU, were monitored in the standard fashion. In addition, a nasal cannula was placed, secured with tape, and connected to a capnometer. An adhesive bioacoustic sensor connected to a breathing frequency monitor prototype (Masimo Corp, Irvine $\mathrm{Ca}$ ) was applied to the patient's neck just lateral to the cricoid cartilage. Both the capnometer and the bioacoustic monitor were connected to a computer for continuous data recording and subsequent data analysis. The accuracy of the new acoustic sensor and the capnometer were compared to a reference respiratory rate from a manual scoring system. Bias, precision and $A_{R M S}$ were calculated as either bioacoustic sensor - reference or capnometer-reference.

Results and Discussions: All data is expressed as mean ( \pm SD). 10 patients (age $=26$ to 88 years, weight $=55$ to $135 \mathrm{~kg}$ ) were enrolled. Duration of monitoring time in PACU was $55.2 \pm 38.9 \mathrm{~min}$. Respiratory rate varied 3 to $28 \mathrm{bpm}$ during this time. The resultant bias, precision and $\mathrm{A}_{\mathrm{RMS}}$ for the capnometer was $-0.53,2.11$, and 2.23 respectively. The bias, precision and $A_{R M S}$ for the bioacoustic sensor was $-0.15,2.23$, and 2.36 respectively.

Conclusion(s): The new prototype bioacoustic respiratory sensor demonstrates accuracy for respiratory rate monitoring as good as capnometry, in this population of postanesthesia patients in the PACU. This device offers multiple benefits over existing devices and has a potential to improve monitoring in a general care setting.

\section{AP8-11}

\section{Cerebral state index (CSI) monitoring in dogs during} induction of anaesthesia with propofol

L. Ribeiro, D. Ferreira, N. Bressan, P. Amorim, L. Antunes

Anestesia, Hospital Veterinário Porto, Porto, Portugal

Background and Goal of Study: The aim of the study is to evaluate the correlation between $\mathrm{CSI}$ and the predicted propofol plasma concentrations (PropCp) in dogs during induction of anaesthesia.

Materials and Methods: Fifteen healthy dogs undergoing scheduled routine surgical procedures were enrolled in this study. All dogs were premedicated with $0.5 \mathrm{mg} \mathrm{kg}^{-1}$ morphine sulphate and with $0.03 \mathrm{mg} \mathrm{kg}^{-1}$ acepromazine IM, thirty minutes prior to the beginning of the induction of anaesthesia. Three electrodes placed in the middle of forehead, in the left side of forehead and in the mastoid left side were used to collect the EEG signal converted by the Cerebral State Monitor into the CSI. Anesthesia was induced with a propofol $1 \%$ bolus dose of $6 \mathrm{mg} \mathrm{kg}^{-1}$ using a syringe pump programmed to allow a maximum infusion rate of $600 \mathrm{ml} \mathrm{h}^{-1}$. A Datex $\mathrm{S} / 5$ monitor was used to collect the haemodynamic data (heart rate (HR) and mean arterial pressure (MAP)). Rugloop software based in the pharmacokinetic model for propofol for dogs* was used to control the syringe pump, to predict propofol concentrations and to store Prop $\mathrm{cp}$, haemodynamic and electroencephalographic data every five seconds. Spearman rank correlation analysis was used to compare PropCp and CSI data. Data are mean \pm sd.

Results and Discussions: Before induction of anaesthesia, HR was $103.6 \pm 20.3 \mathrm{bpm}$ and MAP was $83.1 \pm 14.9 \mathrm{mmHg}$. CSI was $90.7 \pm 4$ with an EMG of $100 \%$ and a SQI of $48.6 \%$. During the induction of anaesthesia, the maximum PropCp was $7.18 \pm 0.39 \mu \mathrm{g} \mathrm{ml}^{-1}$, with a CSI of $67 \pm 12$, SQI $78.0 \pm 12.4 \%$ and to an EMG of $32.9 \pm 30.8 \%$; HR was $122 \pm 33.3 \mathrm{bpm}$ and MAP was $78.4 \pm 19.4 \mathrm{mmHg}$. The minimum CSI values were $52.3 \pm 9.6$ with a PropCp of $5.2 \pm 0.96 \mu \mathrm{g} \mathrm{ml}^{-1}, 1.8 \pm 1.5$ minutes after the maximum PropCp observed; SQI was $83 \pm 12.1 \%$, EMG was $30.7 \pm 26.5 \%$, HR was $117.4 \pm 31.9 \mathrm{bpm}$ and MAP was $73.3 \pm 16.2 \mathrm{mmHg}$. There was a negative correlation between PropCp and CSI $(P<0.0001)$.

Conclusion(s): The cerebral electric changes induced by increasing propofol concentrations appear to be reflected by CSI values during induction. CSI monitoring may be a useful tool to access depth of anaesthesia and to titrate propofol predicted plasma concentrations during induction of anaesthesia with propofol in dogs.

Reference:

*Vet Record, 2001; 148: 198-203.

\section{AP9-1}

\section{Comparison of the cerebral state index with clinical} assessment of the level of sedation during lower digestive tract endoscopy

E. Cardenas, R. Aparicio, J.A. Casellas, J. Cano

Anesthesiology and Pain Clinic. Digestive Endoscopy Unit., USP Hospital San Jaime, Torrevieja, Spain

Background and Goal of Study: The aim of our study is to assess the correlation between a clinical sedation scale and the cerebral state index (CSI) during digestive endoscopy that required mild to moderate sedation.

Materials and Methods: 80 patients scheduled for either diagnostic or therapeutic colonoscopy participated in this observational study approved by the institutional ethics committee. The level of sedation was determined clinically at regular intervals using the Observer's Assessment of Alertness/ Sedation Scale (OASS) and the CSI was registered by a cerebral state monitor (CSM) in all patients. The attending anaesthesiologist was blinded for the CSI values. All the information stored in the monitor was recovered afterwards using the capture software provided by the manufacturer. The statistical correlation between these two measures of sedation was analyzed using the Spearman's test.

Results and Discussion: Although a positive correlation was obtained for the tested OASS-CSI paired episodes $(n=393 ; r=0,601)$, the scattering of the CSI values particularly at deep sedation levels should be considered the main finding of the present study. 


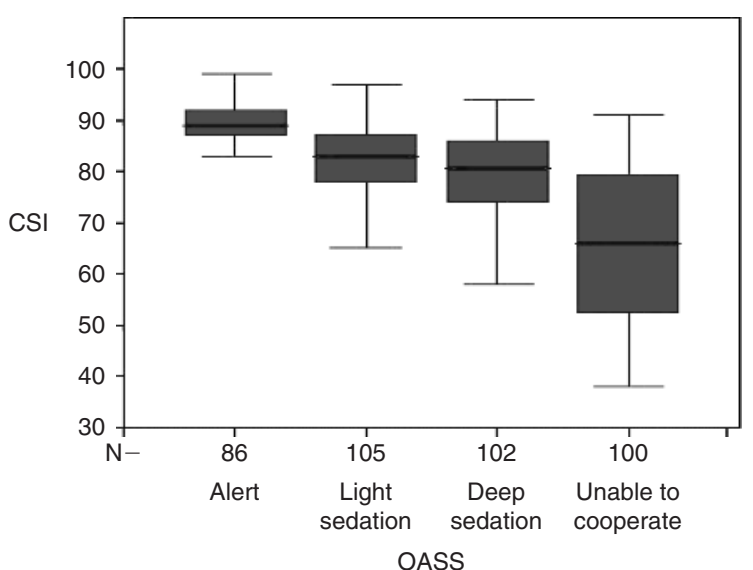

Conclusion: The scattering of the CSI values according to the different levels of sedation limits the clinical usefulness of the CSM for lower digestive tract endoscopy. The scale provided by the manufacturer may not be reliable to optimize sedation for such procedures.

References:

1 Chisholm CJ, et al. Mayo Clin Proc. 2006; 81: 46-52.

2 Jensen EW, et al. Anesthesiology. 2006; 105: 28-36.

\section{AP9-2}

Comparison between spectral edge frequency, fractal dimension and Hurst coefficient as measures of the electroencephalographic effects of sevoflurane

M. Soehle, R. Ellerkmann, C. Boehm, A. Hoeft, J. Bruhn

Klinik für Anästhesiologie und Operative Intensivmedizin, Universitätsklinikum Bonn, Bonn, Germany

Background and Goal of Study: Various attempts have been made to quantify depth of anaesthesia based on the electroencephalogram (EEG). We compared spectral edge frequency (SEF95) - a traditional, frequencydomain based parameter - with fractal dimension (FD, Higuchi's method [1]) and Hurst coefficient $(H)[2]$ - two parameters derived from advanced nonlinear EEG analysis - to investigate their dose response relationship during sevoflurane anaesthesia.

Materials and Methods: Twenty one patients were studied without surgical stimulus. Sevoflurane anaesthesia was induced via face mask and maintained via laryngeal mask. Sevoflurane concentration was increased until burst suppression occurred and subsequently decreased until a state of light anaesthesia was achieved. This procedure was repeated twice until patients were intubated for subsequent surgery. A single-channel EEG was recorded from the right frontal scalp (Fp2-Fz), sampled at $250 \mathrm{~Hz}$ and stored - along with entidal sevoflurane concentration - for later offline analysis. SEF95, FD and $\mathrm{H}$ were calculated using MATLAB R13 and compared with the estimated effect side concentration as derived from simultaneous pharmacokinetic and - dynamic modelling.

Results and Discussions: Sevoflurane pharmacodynamic was in close agreement with a sigmoid dose response relationship for SEF95 (correlation coefficient $\left.r^{2}=0.62 \pm 0.17\right), F D\left(r^{2}=0.73 \pm 0.11\right)$ and $H\left(r^{2}=0.78 \pm 0.12\right)$. The probability of $\mathrm{H}$ to predict sevoflurane effect side concentration $\left(P_{k}=0.86 \pm 0.05\right)$ was significantly higher $(p<0.05)$ as compared with FD $\left(P_{k}=0.81 \pm 0.06\right)$ and SEF95 $\left(P_{k}=0.76 \pm 0.08\right)$.

Conclusion(s): The Hurst coefficient is superior to fractal dimension and spectral edge frequency in predicting depth of sevoflurane anaesthesia. Nonlinear EEG analysis seems to be a promising tool to improve monitoring of anaesthetic depth.

References:

1 Higuchi T, Physica D 1988; 31: 277-283.

2 Cannon MJ, Physica A 1997; 241: 606-626.

\section{AP9-3}

Efficacy of an interaction model to predict State Entropy during propofol/remifentanil anaesthesia

C.S. Nunes, A. Castro, H. Alonso, T. Mendonca, P. Amorim

Dep Matematica Aplicada, Faculdade de Ciencias da Universidade do Porto, Porto, Portugal
Background and Goal of Study: The aim of this study was to inspect if a published interaction model(1), could be used under induction/surgery to predict state entropy.

Materials and Methods: Data was collected during 7 urological surgeries with propofol/remifentanil (Prop/Remi) anesthesia, using RugLooplI ${ }^{\circledR}$ from Datex Entropy module every 5s. TCl was used with Marsh(2) for Prop and Minto(3) for Remi. Anesthesia started with a Remi infusion to reach an effect concentration of $2.5 \mathrm{ng} / \mathrm{ml}$, followed by a constant Prop $200 \mathrm{ml} / \mathrm{h}$ infusion until loss of consciousness, thereafter Prop and Remi were changed according to patient stability. The SE signal was prefiltered with a Butherworth filter. The interaction model(1) was fitted to the data of each patient in the induction phase (first $15 \mathrm{~min}$ ), parameters optimized using nonlinear least squares and a hybrid identification method. The individual patient models were then used to predict SE considering the drugs concentrations (data: mean $\pm S D)$.

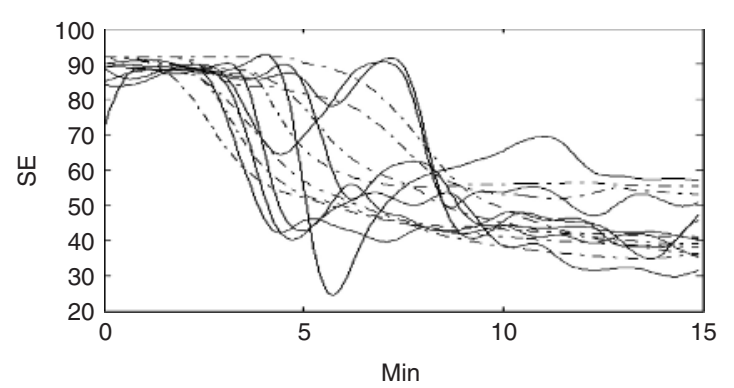

Fig. 1 Results of the interaction model on the 7 patients. Induction (optimization) phase. (solid line - patients SE data; dash dot line - model results)

Results and Discussions: 7 patients, ASA 1/2, $42 \pm 15$ years, $67 \pm 15 \mathrm{~kg}$, $166 \pm 5 \mathrm{~cm}, 4$ female. The individual models had good performance in the induction phase (optimization) with statistical zero errors $(P<0.05)$ in 5 patients. The average of absolute errors (AAE) was $5.07 \pm 1.14$, capturing SE trend in all patients. When the individual models were used for prediction ( $15 \mathrm{~min}$ until the end) the AAE was $7.26 \pm 2.52$. In 5 patients the model was able to predict recovery.

Conclusion(s): The model can capture the individual patient response during induction (non-steady state conditions) identifying unique characteristics. This result leads to the idea that the PK/PD of the drugs maybe different during induction/recovery and maintenance.

References:

1 Anesthesiology, 2000, 92: 1603-16.

2 Br J Anaesth $199167: 41-8$.

3 Anesthesiology, 1997, 86: 24-33.

\section{AP9-4}

\section{Bispectral Index and State Entropy variability under propofol anaesthesia}

A. Castro, E. Amadeu, F. Martins, P. Sa Couto, C.S. Nunes

Matematica Aplicada, Faculdade de Ciencias da Universidade do Porto, Porto, Portugal

Background and Goal of Study: BIS and State Entropy (SE) indices are used to guide anaesthesia. The variability of these signals may affect the easiness of achieving a desired target. The aim of this study was to compare BIS and SE variability under general anaesthesia.

Materials and Methods: Data was collected during urology surgeries. Data recorded every $5 \mathrm{~s}$ from Aspect $\mathrm{XP}$, Datex $\mathrm{S} / 5$ monitors and infusion pumps with Rugloop II ${ }^{\circledR} \mathrm{TCl}$ software. Schnider [1] and Minto [2] PK models were used for propofol and remifentanil TCl, respectively. Drugs Ce were adjusted regarding patient stability and BIS target [40-60]. BIS and SE variability during maintenance were analyzed using their variability sequences: the difference between the original and the smoothed filtered signal (Butterworth). (Data: mean \pm SD) Results and Discussions: 20 patients, $55 \pm 16$ years, $69 \pm 14 \mathrm{~kg}$, $164 \pm 9 \mathrm{~cm}$, ASA 1/2/3, 9 female. Procedure time was $202 \pm 77 \mathrm{~min}$, mean propofol and remifentanil Ce were $2.7 \pm 0.6 \mu \mathrm{g} / \mathrm{ml}$ and $3.9 \pm 1.8 \mathrm{ng} / \mathrm{ml}$. Mean BIS and SE (38 \pm 3 and $45 \pm 6$ ) were statistically different $(P<0.01$, paired sample t-test). The variability sequence follows a logistic distribution characterized by the shape parameter - the higher the shape parameter the higher the signal variability. BIS and SE shape parameters were different $(\mathrm{P}<0.01$, paired t-test). 
Logistic distribution shape parameter for each patient

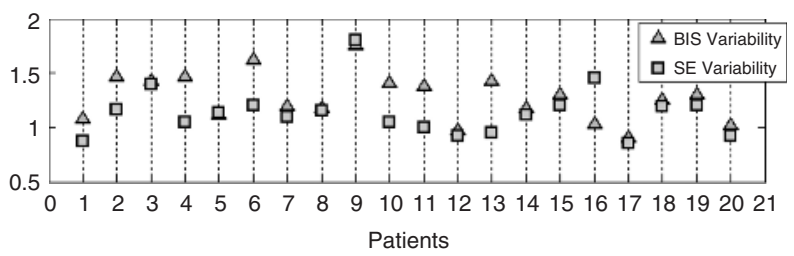

Fig. 1 BIS and SE logistic distribution shape parameters

Conclusion(s): For the same amount of anaesthetics, BIS had a higher variability than SE. Average BIS was below the desired target and lower than SE. The higher variability of BIS may account for the lower BIS values.

References:

1 Anesthesiology, 1998, 88: 1170-82.

2 Anesthesiology, 1997, 86: 24-33.

\section{AP9-5}

\section{Differences between BIS and State Entropy during general} anaesthesia: blind study

A. Castro, E. Amadeu, F. Martins, P. Sa Couto, C.S. Nunes

Matematica Aplicada, Faculdade de Ciencias da Universidade do Porto, Porto, Portugal

Background and Goal of Study: Based on clinical observations we hypothesized that average BIS and State Entropy (SE) during maintenance may be different. In the present work we performed a blind study (clinician blind to SE) to compare BIS and SE levels.

Materials and Methods: Data was collected during urology surgeries under general anaesthesia. Data recorded every $5 \mathrm{~s}$ from Aspect XP, Datex S/5 monitors and infusion pumps with Rugloop $I^{\circledR} \mathrm{TCl}$ software. Schnider [1] and Minto [2] PK models were used for propofol and remifentanil TCI. Anaesthesia was induced with $1 \%$ propofol at $200 \mathrm{ml} / \mathrm{h}$ and a remifentantil effect-site concentration (Ce) target of $2.5 \mathrm{ng} / \mathrm{ml}$. After loss of consciousness (LOC) propofol $\mathrm{Ce} \mathrm{TCl}$ was started, the target being $\mathrm{Ce}$ at LOC. Following intubation, drugs $\mathrm{Ce}$ were adjusted regarding patient requirements and BIS target [40-60]. During the procedure the anaesthetist was blinded to SE values. (Data:Mean \pm SD) Results and Discussions: 13 patients, ASA 1/2/3, $64 \pm 10$ years, $70 \pm 13 \mathrm{~kg}$, $164 \pm 11 \mathrm{~cm}, 4$ female. Procedure time was $180 \pm 55 \mathrm{~min}$, mean propofol and remifentanil Ce were $2.5 \pm 0.6 \mu \mathrm{g} / \mathrm{ml}$ and $3.7 \pm 1.2 \mathrm{ng} / \mathrm{ml}$. BIS and SE mean values were $39 \pm 2$ and $46 \pm 6$, respectively, during the maintenance phase. The BIS and SE presented different mean values $(P<0.01$, paired t-test). The average difference between BIS and SE was $-7 \pm 6$, and only four patients had average BIS $>40$.

BIS and SE mean values - maintenance phase

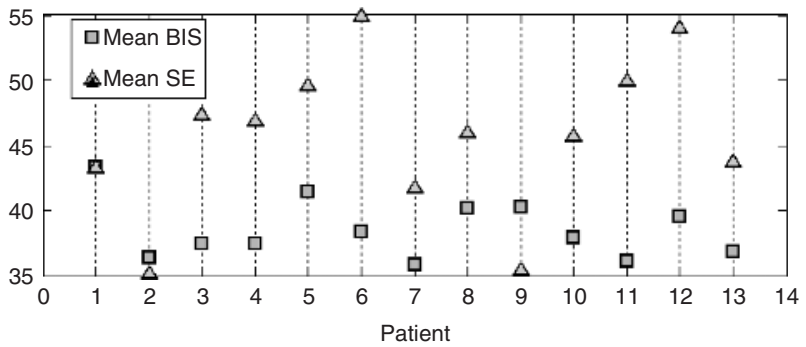

Fig. 1 BIS and SE mean values for each patient

Conclusion(s): For the same amount of anaesthesia BIS was significantly lower than SE. Also BIS was below target. This should not invalidate the existence of a difference between BIS and SE.

References:

1 Anesthesiology, 1998, 88: 1170-82.

2 Anesthesiology, 1997, 86: 24-33.

\section{AP9-6}

Intraoperative low doses of ketamine increase BIS-guided sevoflurane requirements during combined general and thoracic epidural anesthesia for major abdominal surgery K. Messada, P. Janin, A. Kaba, S. Lauwick, J. Joris Anesthesia and Intensive Care Medicine, CHU Liège, Liège, Belgium Background and Goal of Study: BIS monitoring can be used to titrate sevoflurane (SEVO) administration during combined general-epidural anesthesia. ${ }^{1}$ Intraoperative ketamine (KET) improves postop analgesia provided by thoracic epidural analgesia (TEA), ${ }^{2}$ but can increase BIS scores. ${ }^{3}$ We therefore tested the hypothesis that intraop KET increases BIS-guided SEVO requirements during major surgery under combined general-TEA anesthesia. Materials and Methods: After IRB approval and informed consent, 40 patients scheduled for abdominal surgery under combined general-TEA were included in this randomized double-blind study. TEA was standardized in all patients. After induction of anesthesia patients were allocated in two groups: iv KET $(0.5 \mathrm{mg} / \mathrm{kg}$ then infusion $0.25 \mathrm{mg} / \mathrm{kg} / \mathrm{h})$ or saline (SAL). Anesthesia was maintained with SEVO in $\mathrm{O}_{2}$ /air. SEVO was adjusted to keep BIS scores around 50. Arterial pressure, heart rate, BIS scores, and end-tidal SEVO were recorded every 15 min. Data (mean \pm SD) were analysed using ANOVA or Students' $t$ test; $P<0.05=$ statistical significance.

Results and Discussions: Patient data were similar in the two groups. Endtidal SEVO were $20 \%$ greater $(P<0.001$, Fig) in the KET group although BIS scores were greater in the KET group $(55 \pm 16$ vs $50 \pm 16, P=0.005)$. MAP was also lower in the KET group $(70 \pm 15$ vs $78 \pm 17, \mathrm{P}<0.0001)$.

Conclusion: Low intraoperative doses of KET increase BIS guided SEVO requirements during combined general-TEA. These results raise the question of monitoring the depth of general anesthesia when combined with TEA and KET. References:

1 Hodgson P et al., Anesthesiology 2001, 94: 799-803.

2 Suzuki $M$ et al., Anesthesiology 2006, 105: 111-9.

3 Hans P et al., BJA 2005, 94: 336-40.

\section{Sevoflurane end-tidal concentration (\%)}

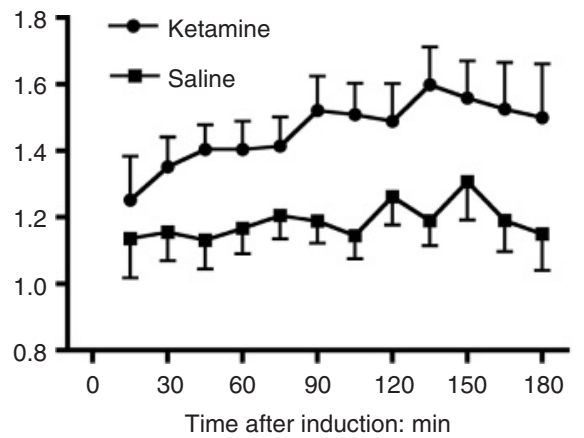

\section{AP9-8}

Remifentanil bolus and entropy values in awake and anaesthetized patients. Are there any gender differences?

M. Vassiliadis, D. Geros, M. Karta, V. Siakavaras, I. Paschalidis Anesthesiology, General Hospital of Giannitsa, Giannitsa, Greece

Background and Goal of Study: Remifentanil bolus decreases bispectral index during anaesthesia ${ }^{1}$. Aim of this study is to estimate its effect in spectral and response entropy values, both at awake and anaesthetized patients. Also, we seek for gender differences for this effect.

Materials and Methods: In forty surgical patients (21 female, 19 male), spectral (SE) and response (RE) entropy were monitored (Datex-Ohmeda $\mathrm{S} / 5$ Entropy Module). While awake, remifentanil bolus $1 \mu \mathrm{g} / \mathrm{kg}$ was administered and SE-RE were recorded before and after 2 minutes. Propofol $2 \mathrm{mg} / \mathrm{kg}$ was then infused and for intubation cis-atracurium $0.15 \mathrm{mg} / \mathrm{kg}$. Maintenance was provided with continuous propofol and remifentanil infusions. During operation and in a pause of surgical stimuli, another remifentanil bolus was given. SE and RE, pre- and post bolus, were also recorded. For statistical comparison, two-sample t-test was applied. $\mathrm{P}<0.05$ was considered statistically significant.

Results and Discussions: In female and male awake patients, SE and RE were similar before and after bolus (Table 1). While anaesthetized, the difference in SE and RE (pre minus post value) was very significant, compared to awake state ( $P<0.0001$, power: $99 \%)$, in both sexes. The reduction in both SE and $\mathrm{RE}$, was more significant in females (For SE: $\mathrm{P}=0.009$, power $78 \%$, for $\mathrm{RE}$ : $\mathrm{P}=0.002$, power $91 \%$ ).

Table 1. Mean values $\pm S D$

\begin{tabular}{llllll}
\hline & AWAKE & & & \multicolumn{2}{l}{ ANAESTHESIA } \\
\cline { 2 - 3 } \cline { 5 - 6 } & Males & Females & & Males & Females \\
\hline Pre bolus SE & $89 \pm 2$ & $89 \pm 1$ & & $44 \pm 10$ & $50 \pm 12$ \\
Post bolus SE & $88 \pm 3$ & $88 \pm 3$ & & $39 \pm 13$ & $40 \pm 10$ \\
Pre bolus RE & $98 \pm 1$ & $98 \pm 1$ & & $49 \pm 11$ & $54 \pm 12$ \\
Post bolus RE & $97 \pm 2$ & $95 \pm 5$ & $41 \pm 13$ & $40 \pm 9$ \\
\hline
\end{tabular}


Conclusion(s): Remifentanil, given as a bolus, significantly reduces spectral and response entropy value in anaesthetised, but not in awake patients, male or female. The effect is more pronounced in female patients.

Reference:

1 Ferreira DA, Nunes CS, Antunes LM, et al. Eur J Anaesthesiol 2006; 23: 305-10.

\section{AP9-9}

The application of response entropy to monitoring propofol sedation depth

P. Mahon, R. Kowalski, E. Khan, B. McNamara, G. Shorten

Anaesthesia and Intensive Care, Cork University Hospital, Cork, Ireland

Background and Goal: The aim of this prospective observational study was to investigate the associations between; (1) Response / State Entropy (RE/SE), (2) defined EEG parameters and (3) clinical assessment [Modified Observer's Assessment of Alertness /Sedation scale (MOAA/S)] in patients receiving lowdose propofol infusion.

Materials and Methods: A target-controlled infusion of propofol was administered (using Schneider's pharmacokinetic model) to 12 ASA I patients. The concentration of the target-effect site infusion was increased in $0.5 \mu \mathrm{g} \mathrm{mL}^{-1}$ increments at four minute intervals to a maximum of $2.0 \mu \mathrm{g} \mathrm{mL}^{-1}$. SE/RE indices (Entropy ${ }^{\mathrm{TM}}$ Monitor, GE Healthcare) and clinical sedation score (MOAA/S) were recorded. $\mathrm{RE}$ is calculated over the frequency range $0.8-47 \mathrm{~Hz}$ and includes muscle activity. SE is confined to the EEG frequency range. ${ }^{1}$ Each patient had concurrent multi-channel (19) EEG recording. EEG recordings were later assessed by a neurophysiologist blinded to the propofol concentration. A grade was assigned according to predefined EEG criteria of sedation, for each four-minute time period or "patient/time unit" analyzed. Each unit corresponded to a fixed concentration of propofol $(n=48)$. Robust analysis (linear mixed model) did not demonstrate an intra-patient effect $(p=0.016$ ). Results and Discussion: RE but not SE was less in patient/time units with EEG evidence of sedation (Table 1).

Table 1. State/Response entropy (SE/RE) and EEG evidence of sedation [data are median (range)]

\begin{tabular}{lllll}
\hline $\begin{array}{l}\text { Measure } \\
(\mathrm{n}=48)\end{array}$ & $\begin{array}{l}\text { EEG evidence } \\
\text { of sedation }(\mathrm{n}=29)\end{array}$ & $\begin{array}{l}\text { No EEG evidence } \\
\text { of sedation }(\mathrm{n}=19)\end{array}$ & $\mathrm{P}$ value & $\mathrm{P}_{\mathbf{K}}$ value \\
\hline SE & $89(60-91)$ & $89(80-91)$ & 0.324 & 0.58 \\
RE & $96(62-99)$ & $98(90-100)$ & 0.003 & 0.75 \\
\hline
\end{tabular}

RE but not SE values were less in patient/time units in which clinical evidence of sedation was present (MOAA/S $<5)$ [ $p=0.007]$.

Conclusion: At low levels of propofol induced sedation, RE offers potential as a monitor of sedation depth.
Reference:

1 Acta Anaesthesiol Scand 2004; 48: 154-161.

\section{AP9-10}

Pain detection during anesthesia with different EEG derived monitoring systems (Power Spectrum, BIS, Entropy)

J.M. Calvo-Vecino, A. Abad-Gurumeta, M. Ortega-Urbaneja, T. LopezQuesada, A. Pérez-Gallardo

Anesthesia, Niño Jesus Children University Hospital, Madrid, Spain

Background and Goal of Study: Prospective Study to compare different systems of hypnosis monitoring during general anaesthesia (1 hour long approximately) in 102 ASA I patients (2-12 years old). We realized EEG power spectrum analysis by spectral edge frequency (SEF) and delta wave \% $(\delta \%)$; BIS and, state and response entropy (SE \& RE). Values were collected by modular $\mathrm{S} / 5^{\mathrm{TM}}$ Datex-Ohmeda system; information was processed off-line by "DatexOhmeda S/5 Collect ${ }^{\circledR}$ " software. Continuous monitoring of haemodynamic and respiratory profiles, and inspired and expired gases and vapours fractions, was performed.

Materials and Methods: Anaesthesia was maintained, in spontaneous ventilation, with $2 \%$ Sevoflurane in $\mathrm{O}_{2} / \mathrm{N}_{2} \mathrm{O}(50 \%)$ and continuous remifentanil perfusion $\left(0.2 \mu \mathrm{g} \mathrm{kg}^{-1}\right)$, without neuromuscular blockade.

Sample homogeneity was checked. Temporal course curves among the systems and absolute punctual values, when specific events appear, were compared. Multivariant general linear model and correlations were studied. Cases with specific events were isolated and curves correlations among themselves and with total sample were performed. Tridimensional surface model was obtained.

Results and Discussions: BIS, SE and RE global temporal average curves don't present significant differences, and there are good correlations, but induction and eduction are detected more rapidly by entropy system.

We have detected significant $(p<0,05)$ specific events consist an elevation of RE, SE and more slowly BIS range (more than 1 minute or, in certain cases, not detected), coincident with supramaximal surgical stimuli, heart rate ( $>15 \%$ than basal) and SEF ( $\geqslant 25 \mathrm{~Hz}$ ) elevations. $\delta \%$ remained cuasi invariable when events $(\geqslant 65)$.

If fentanil $\left(1 \mu \mathrm{g} \mathrm{kg}^{-1}\right)$ or sevoflurane $(8 \%)$ bolus are administered immediately when events, SEF, SE and RE curves return to previous values, and BIS don't detect event. If event remain for more than 1 minute, BIS detect too it. Curves of isolated cases do not present correlation with global (excluded isolated cases) sample curve.

Conclusion(s): Entropy system, similar to BIS, detect more quickly specific events, nevertheless we need complementary systems to explain these events. $\delta \%$ could inform if hypnosis remain, and SEF elevation (without $\delta \%$ lose) could indicate pain events.

\section{Clinical and Experimental Circulation}

\section{AP1-2}

\section{Relevance of elevated Serum Troponin I after elective major abdominal surgery}

R. Sundaram, JS. Noble, C. Triantyfyllou, AW. Reid, M. Murray

Anaesthetics, Victoria Infirmary, Glasgow, United Kingdom

Background and Goal of Study: The aim of this study was to identify preoperative risk factors, intraoperative events and postoperative events that can be linked to myocardial injury as evidenced by increase in serum troponin levels during major surgery.

Materials and Methods: After obtaining local ethics approval and written consent, fifty six consecutive patients undergoing elective major surgery were recruited. Preoperative risk factors, intraoperative events such as hypotension and tachycardia and postoperative outcomes such as adverse cardiac events, pain scores and outcomes at 30 days were recorded. ECGs and serum troponin assays were done at baseline and on postoperative days 1,2 and 3 .

Results and Discussions:

\begin{tabular}{lll}
\hline & No increase in troponin & Trop 0.04-0.4ng/l \\
\hline number & 16 & 35 \\
Age & Median 66 (35-86) & Median 69 (37-83) \\
Preop risk factors & $7(43 \%)$ & $14(40 \%)$ (chi square 1.88; p 0.215) \\
Postop Tachycardia & $1(6 \%)$ & $9(25 \%)$ (chi square 3.4; p 0.06) \\
Postop pain & 0 & $7(20 \%)$ (chi square 2.2; p 0.13) \\
ECG Changes & $4(25 \%)$ & $12(34 \%)$ \\
30 day Mortality & 0 & 0 \\
\hline
\end{tabular}

Conclusion(s): Sixty eight percent of patients undergoing elective abdominal surgery have positive cardiac Troponin I levels postoperatively, consistent with myocardial damage. We conclude that it would be prudent to repeat this study with larger numbers statistically powered to detect significant difference before drawing any definite conclusions.

Reference:

1 Noble et al. BJA.1999; 82; 41-46.

\section{AP1-3}

Acute renal failure in patients undergoing mitral valve surgery E. Dedola, O. Fochi, C. Marchetti, G. Bruno, G. Crescenzi

Department of Cardiothoracic Anesthesia and Intensive Care, San Raffaele Scientific Institute, Milano, Italy

Background and Goal of Study: The aim of this study was to define the incidence, predictors and mortality related to ARF after different types of mitral valve (MV) surgery.

Materials and Methods: We studied 1276 consecutive adult patients undergoing isolated MV surgery with cardiopulmonary bypass. Mitral valve repair was mostly performed with the edge to edge technique with the positioning of a rigid ring while patient who had mitral replacement mostly had a mechanical valve implanted.

Results and Discussions: 32 patients (2.5\%) developed postoperative ARF. The incidence of ARF for MV replacement and MV repair was $8 \%$ $(25 / 312)$ and $0.7 \%(7 / 964)$ respectively $(p<0.001)$. At a multivariate analysis, MV replacement was an independent risk factor for the development of 
postoperative ARF (OR 4.0, 95\%, confidence interval 1.49-10.59, $\mathrm{p}<0.01$ ) together with low-output syndrome (OR 13.7, 5.4-34.9, $p<0.01)$, emergency surgery (OR 8.53.98, 1.4-52.2, $p=0.02$, creatinine $>124$ umol L $^{-1}$ (OR 7.9, 2.8-22, $p<0.01$ ), reopening for bleeding (OR 4.5, 1.4-14.3, $p=0.01$ ), diabetes (OR 4.4, 1.08-18.2, $\mathrm{p}=0.04$ ) and age (OR 1.048 per year, 1.002-1.096, $\mathrm{p}=0.04)$. Hospital death occurred in 23 patients: those who developed ARF had a $46.9 \%$ incidence of death versus $0.6 \%$ in the patients without ARF. ARF requiring renal replacement therapy occurred in 20 patients: $0.3 \%$ in the MV repair group and $5.4 \%$ in the MV replacement group $(p<0.001)$. Patients who developed ARF requiring renal replacement therapy had $65 \%$ incidence of death. Death occurred in $6.1 \% \mathrm{MV}$ replacement and in $0.4 \%$ $M V$ repair patients $(p<0.0001)$. Our main result is that MV valve replacement is an independent risk factor for the development of ARF after MV valve surgery (OR 4.0; 95\% $\mathrm{Cl} 1.5-10.6)$. The other risk factors for in our study population were perioperative low-output syndrome, emergency operation, preoperative renal impairment, re-operation for bleeding, diabetes and age.

Conclusions: In conclusion, our study identifies risk factors for the development of ARF in MV surgery and, for the first time, shows that MV replacement is an independent risk factor for this complication.

\section{AP1-4}

Postoperative risk of patients with previous coronary stenting undergoing a non cardiac surgery

F. Le Saché, S. Perbet, Y. Le Manach, G. Godet

Anesthesiology and Intensive Care, La Pitié-Salpetrière Hospital, Paris, France

Background and Goal of Study: Percutaneous cardiac interventions with bare metal stent (BMS) or drug eluting stent (DES) implantation is a routine treatment for coronary artery disease. Anaesthesiologists have to cope with management of antiplatelet therapy. We tried to evaluate the incidence of perioperative cardiac and hemorrhagic complications in non cardiac surgery. Materials and Methods: From January 1st, 2006, we conduct a prospective observational study in our institution. To be eligible, patients had to have coronary artery disease treated by previous surgery stenting and need to undergo a non cardiac surgical procedure. All urgent or elective procedures, be it minor, intermediate or major surgery, were included. Data were collected from the patients' coronarography reports. Troponin Ic (cTni) level measures and electrocardiograms were conducted daily to detect possible in-stent thrombosis, myocardial damage or myocardial infarction during 3 days postoperatively.

Results and Discussions: During 11 months, 6816 patients were admitted to the ICU. We included 115 patients (1,7\%) (Median Revised Cardiac Risk Index: 2[1-3]). Among these patients, 31 were carrying DES (26\%). The cardiovascular post-operative complications are marked by 14 myocardial damages (abnormal cTnl $<1.5 \mathrm{ng} / \mathrm{ml}$ ), 6 myocardial infarction (cTnl $\geqslant 1.5 \mathrm{ng} / \mathrm{ml}$ ) including 4 in-stent thrombosis. 4 in-stent thrombosis occured in BMS, in 2 cases with discontinuation of antiplatelet therapy more than 5 days. The hemorrhagic complications are found among 6 patients of which 2 had a preoperative disruption of the antiplatelet more than 5 days.A 22,6\% cardiac and hemorrhagic complication rate is reported. 3 patients died before leaving the hospital because of myocardial infarction (2,6\%).

Conclusion(s): During perioperative period, in patients with previous coronary stenting, myocardial damage is the most frequent complication (12\%) without any mortality associated. It seems that in-stent thrombosis is an unfrequent event $(3,4 \%)$ even if it is a high lethal complication $(50 \%)$.

\section{AP1-5}

\section{Predictors and outcome of renal replacement therapy in} patients undergoing cardiac surgery

O. Fochi, E. Dedola, G. Bruno, T. Bove, G. Landoni

Department of Cardiothoracic Anesthesia and Intensive Care, San Raffaele Institute, Milano, Italy

Background and Goal of Study: Acute renal failure is a serious complication of cardiac surgery. We studied the long-term survival and quality of life of patients requiring renal replacement therapy after cardiac surgery, since they represent a heavy burden on hospital resources and their outcome has never been adequately evaluated.

Materials and Methods: Out of 7846 consecutive cardiac surgical patients, $126(1.6 \%)$ required postoperative renal replacement therapy: their preoperative status and hospital course was compared with patients who had no need of postoperative renal replacement therapy. Patients who were on preoperative dialysis were excluded from this study. A multivariate forward stepwise analysis was used to identify predictors of renal replacement therapy.
Long-term (34 \pm 18 months) follow-up information was collected and quality of life and presence of hearing impairment assessed.

Results and Discussions: Hospital mortality in the study group was $84 / 126(66.6 \%)$ versus $118 / 7720(1.5 \%)$ in the control population $(p<0.001)$. Predictors of renal replacement therapy were: emergency surgery, preoperative renal impairment, intra-aortic balloon pump positioning, reoperation for bleeding, previous cardiac surgery, female gender, low ejection fraction, bleeding $>1000 \mathrm{ml}$, chronic obstructive pulmonary disease and age. Patients who underwent renal replacement therapy and were discharged from the hospital had an excellent long-term outcome: survival at $34 \pm 18$ months was $70 \%$, with $8.3 \%$ of survivors on dialysis and $29.1 \%$ complaining of hearing impairment; no other limitations in daily living were reported.

Conclusions: In conclusion, this study confirms that the in-hospital hospital mortality of patients requiring renal replacement therapy is high and shows an excellent long-term survival and good quality of life in patients discharged from hospital alive.

\section{AP1-6}

Postoperative statin withdrawal is associated with increased cardiac morbidity

Y. Le Manach, G. Godet, JP. Goarin, MH. Fleron, P. Coriat

Anesthésie Réanimation, Pitié Salpétrière, Paris, France

Background and Goal of Study: Statins reduce cardiac morbidity in nonsurgical populations and they may provide benefit for surgical patients (1). We assess cardiac outcome in patients who continued compared with those who discontinue statin therapy after major vascular surgery.

Materials and Methods: Between January 2001 and December 2003, there were no guidelines for perioperative continuation of statins (Discont., $n=491$ ). From January 2004, guidelines were instituted whereby statin therapy was continued starting as soon as possible after surgery (Cont., $n=178$ ). The occurrence of cardiac myonecrosis (defined as cardiac troponin I $>0.2 \mathrm{ng} / \mathrm{mL}$ ) was analyzed. Intra-cohort (propensity score) and extra-cohort (Lee score) (2) adjustments of the risk were performed. Pooled analysis was then conducted to detect the predictors of cardiac myonecrosis in the all population. All analyses were done with SPSS 13.0.

Results and Discussions: The median delay between surgery and resumption of statin therapy was 4 days and 1 day in Discont. and Cont. Groups $(P<0.001)$, respectively. Using propensity score matching for likelihood of preoperative treatment, the odds ratio associated with chronic statin treatment to predict myonecrosis for patients with vs without early postoperative statin resumption (Cont. vs Discont. Groups) was 0.38 and 2.1 (relative risk reduction of $5.4 ; 95 \% \mathrm{Cl}: 1.2-25.3$ ), respectively. The odds ratio after adjustment for the Lee score was 0.38 in the Cont.Group and 2.1 in the Discont. Group (relative reduction of 5.5 ; $95 \% \mathrm{Cl}: 1.2-26.0$ ). A postoperative withdrawal ( $>4$ days) was an independent predictor of postoperative myonecrosis (OR 2.9; 95\% Cl: 1.6-5.5).

Conclusion(s): Discontinuation of statin therapy after major vascular surgery is associated with an increased postoperative cardiac risk, suggesting that statin therapy should be resumed early after major vascular surgery.

References:

1 Hindler et al., Anesthesiology, 2006.

2 Lee et al., Circulation, 1999.

\section{AP1-7 \\ Short-term neurocognitive outcome following off-pump coronary revascularization}

G. Tsaousi, A. Pitsis, D. Deliaslani, D. Vasilakos, M. Giala

AHEPA University Hospital, Anaesthesiology and ICU, Thessaloniki, Greece Background and Goal of Study: Cardiopulmonary bypass has long been considered to be a major factor in the development of post coronary artery bypass grafting (CABG) cerebral morbidity. Cerebral dysfunction can manifest as a spectrum, from stroke to cognitive decline, resulting in excess of medical resource utilization. Goal of the study was to validate the impact of off-pump CABG (OPCAB) procedures on short-term cognitive outcome.

Materials and Methods: Prospective study enrolling 103 candidates (92 M/11 F, aged 63,7 $\pm 8,9$ ) scheduled for their first elective OPCAB surgery. Exclusion criteria included prior stroke history, carotid artery occlusion, poor left ventricular function or hemodynamic performance, renal failure and low educational status. Neurocognitive status was assessed by Mini Mental State Examination (MMSE) at baseline (2 days before CABG) and at ICU and hospital discharge ( 2 and 6 days post CABG, respectively). Demographic and clinical parameters were also validated as prognostic indices of cognitive outcome. Data were analyzed by ANOVA and stepwise logistic regression. 
Results: No frank neurologic event was recorded. Changes of total MMSE values and their sub-divisions are presented in the table. Advanced age, diabetes and longstanding hypertension predicted $42 \%$ of the variance $(p<0,001)$ of the early cognitive derangement.

\begin{tabular}{|c|c|c|c|c|c|}
\hline \multicolumn{2}{|c|}{ MMSE } & $\begin{array}{l}\text { Baseline } \\
\text { discharge }\end{array}$ & $\begin{array}{l}\text { ICU } \\
\text { discharge }\end{array}$ & $\begin{array}{l}\text { Hospital } \\
\text { discharge }\end{array}$ & $p$-value \\
\hline \multicolumn{2}{|c|}{ Total (mean) } & 26,2 & 22,2 & 25,4 & $<0.001$ \\
\hline \multirow{5}{*}{ 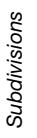 } & Orientation & $9,3 \pm 1,4$ & $8,2 \pm 1,8$ & $9,1 \pm 1,1$ & $<0.001$ \\
\hline & Registration & $3,02 \pm 0,3$ & $2,8 \pm 0,5$ & $2,9 \pm 0,3$ & $<0.01$ \\
\hline & Attention & $3,2 \pm 0,8$ & $2,3 \pm 1,1$ & $3,1 \pm 1,1$ & $<0.01$ \\
\hline & Recall & $2,8 \pm 0,4$ & $1,6 \pm 0,9$ & $2,7 \pm 0,5$ & $<0.001$ \\
\hline & Language & $7,8 \pm 1,1$ & $7,3 \pm 1,3$ & $7,6 \pm 0,7$ & ns \\
\hline
\end{tabular}

Conclusion: OPCAB procedures might affect short-term neurocognitive outcome in a positive manner, although a detectable intellectual impairment (involving mainly the functions of orientation and recall), presents in the early postoperative period. Advanced age, history of diabetes and hypertension seem to be predisposing factors to this cognitive decline, which can possibly be attributed to derogation of functioning, depressive state and lack of motivation as consequences of major surgical procedures.

\section{AP1-8}

Incidence, risk factors and outcome of renal dysfunction after coronary surgery in patients with preoperative normal renal function

A. Flo, A. Escudero, M. Sariñena, E. Massó, E. Moret

Anaesthesia, Hospital Universitari Germans Trias i Pujol, Badalona, Spain

Background and Goal of Study: To evaluate the incidence and risk factors of a decrease in creatinine clearance $(\mathrm{CrCl})$ early after coronary surgery and its impact on the postoperative outcome in patients with preoperative normal renal function.

Materials and Methods: We prospectively followed up all patients undergoing coronary surgery under CPB in a 2 years period. Patients with a preoperative renal dysfunction were excluded. Renal function was assessed by $\mathrm{CrCl}$, and renal impairment was defined as $\mathrm{CrCl}<50 \mathrm{ml} / \mathrm{min}$. In order to identify independent predictors of renal impairment, demographic, preoperative, intraoperative, $\mathrm{CPB}$ and postoperative variables were recorded.

Results and Discussion: A total of 312 patients were studied (age $62,8 \pm 8,7$, male gender $81,1 \%$, preoperative $\mathrm{CrCl} 84,6 \pm 23,7)$. Postoperative $\mathrm{CrCl}<50 \mathrm{ml} / \mathrm{min}$ occurred in $16 \%$ patients.

Multivariated analysis showed 4 independent risk factors for postoperative renal impairment:

\begin{tabular}{llll}
\hline & OR & IC 95\% & $\mathrm{p}$ \\
\hline Preoperative $\mathrm{CrCl}$ & 0,92 & $0,89-0,95$ & 0,000 \\
Blood transfusion & 2,84 & $1,29-6,25$ & 0,009 \\
Age (per year) & 1,07 & $1,02-1,14$ & 0,012 \\
Inotropic durgs & 2,66 & $1,22-5,81$ & 0,014 \\
\hline
\end{tabular}

Postoperative outcome was as follow:

\begin{tabular}{llll}
\hline & PostopCrCl $>50$ & PostopCrCl $<50$ & $\mathrm{p}$ \\
\hline Hemofiltration & $0 \%$ & $4 \%$ & 0,025 \\
days in ICU & 2,62 & 3,47 & 0,047 \\
Mortality & $0 \%$ & $2 \%$ & 0,162 \\
\hline
\end{tabular}

Conclusions: In our study renal impairment in patients with previous normal renal function presented in $16 \%$ after CABG surgery. Preoperative low $\mathrm{CrCl}$, advanced age, blood transfusion and need of inotropic drugs in ICU were found to be predictive for renal dysfunction.

Postoperative renal dysfunction was associated with a longer stay in ICU and increased need of hemofiltration, but not in an higher mortality rate.

\section{Reference:}

"Estimated creatinine clearance instead of plasma creatinine level as prognostic test for postoperative renal function in patients undergoing coronary artery bypass surgery". Lnoyez et al. Eur J Cardio thor Surg 2006; 29: 461-465.

\section{AP2-1}

Transesophageal echocardiographic automated border detection to predict fluid responsiveness in mechanically ventilated patients

M. Cannesson, J. Slieker, O. Desebbe, M. Hachemi, JJ. Lehot

Department of Anesthesiology, Hospices Civils de Lyon, Hopital Louis Pradel, Bron, France
Background and Goal of Study: Left ventricular (LV) stroke area by transesophageal echocardiographic automated border detection (ABD) is related to LV stroke volume. Respiratory variations in LV stroke volume or its surrogates are good predictors of fluid responsiveness in mechanically ventilated patients. We hypothesized that respiratory variations in LV stroke area $(\Delta \mathrm{SA})$ can predict fluid responsiveness.

Materials and Methods: Eighteen mechanically ventilated patients undergoing coronary artery bypass grafting were studied. Stroke area was measured on a beat-to-beat basis using transesophageal ABD. Haemodynamic and echocardiographic data were measured at baseline and after volume expansion induced by a passive leg raising manoeuvre (PLR). Responders to PLR were defined as patients presenting more than $15 \%$ increase in cardiac output. Data are presented as mean \pm SD.

Results and Discussions: Cardiac output increased significantly in response to PLR (from $2.16 \pm 0.79$ to $2.78 \pm 1.08 \mathrm{~L} / \mathrm{min} ; \mathrm{p}<0.01$ ). $\Delta S A$ decreased significantly in response to PLR, from $17 \pm 7$ to $8 \pm 6 \%, p<0.01 . \Delta S A$ was higher in responders than in non-responders ( $20 \pm 5$ vs. $10 \pm 5 \% ; p<0.01)$. A cut-off $\Delta S A$ value of $16 \%$ allowed fluid responsiveness prediction with a sensitivity of $92 \%$ and a specificity of $83 \% . \Delta S A$ at baseline was related to the percent increase in cardiac output in response to volume expansion $(r=0.53, p<0.01)$.
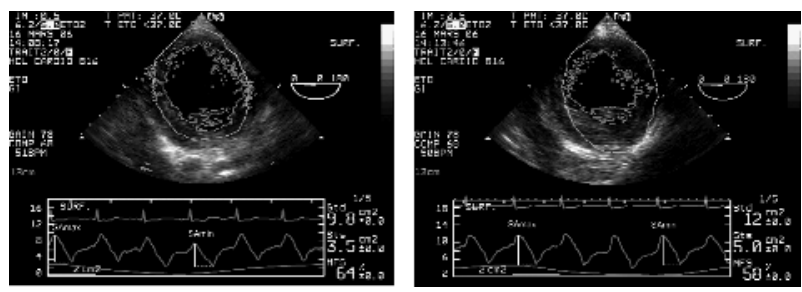

Fig. ABD recording before (left) and after (right) PLR.

Conclusion(s): Respiratory variations in LV stroke area ( $\triangle S A)$ by transesophageal echocardiographic ABD are sensitive to changes in preload, can predict fluid responsiveness, and can quantify the effects of volume expansion on cardiac output. It has potential clinical applications.

\section{AP2-2}

Hepatic resection surgery under low CVP anaesthesia - can FTc be used as an indicator of cardiac preload?

A. Patel, A. Mohabir, W.C. Fawcett, N.F. Quiney

Anaesthetics and Critical Care Medicine, Royal Surrey County Hospital, Guildford, United Kingdom

Background and Goal of Study: Hepatic resection surgery is commonly performed under low central venous pressure (CVP) anaesthesia (1). Given the significant haemodynamic changes encountered we have looked at the use of corrected flow time (FTc) as an indicator of cardiac preload using Trans Esophageal Doppler (CardioQ®).

Materials and Methods: We studied 10 consecutive patients undergoing elective hepatic resection surgery for isolated colorectal metastases under low CVP anaesthesia. All patients received a standardized general anaesthetic technique, combined with intraoperative reduction of CVP. Trans-Esophageal Doppler was used intraoperatively to obtain FTc. The same surgeon operated on all patients and an ultrasonic dissection method was utilized for the resection. Results and Discussion: Data presented as mean values $(95 \% \mathrm{Cl}$ as error bars). Mean fall in CVP was $5 \mathrm{cmH}_{2} \mathrm{O}$. Blood loss in all patients was in the range $100-600 \mathrm{mls}$.
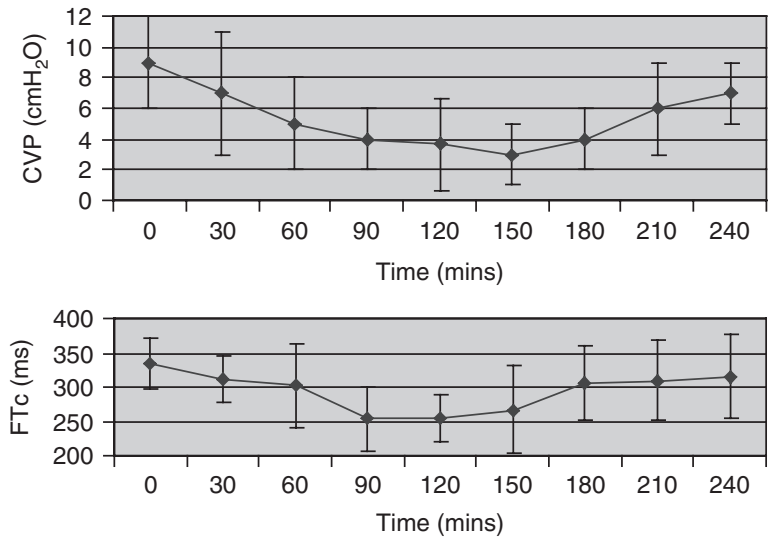
Conclusion: In our study, trends in corrected flow time (FTc) obtained using Trans-Esophageal Doppler accurately reflected parallel changes in CVP. FTc may therefore be used as a guide to ensure adequate volume resuscitation following completion of the resection phase of surgery.

Reference:

1 Eid A et al. Low CVP anesthesia in major hepatic resection. Middle East $\mathrm{J}$ Anesthesiol. 2005; 18(2): 367-77

\section{AP2-3}

A new diagnostic method for visualizing the ascending aorta in patients undergoing cardiac surgery

B. van Zaane, A.P. Nierich, W.F. Buhre, G.J. Brandon Bravo Bruinsma, K.G.M. Moons

Division of Perioperative Care and Emergency Medicine, University Medical Center Utrecht, Utrecht, The Netherlands

Background: Atherosclerosis of the ascending aorta (AA) and emboli-related complications after cardiac surgery are related. Knowledge on presence of AA-atherosclerosis before sternotomy allows changes in surgical strategy that avoid manipulation of the AA. The "Gold"-standard for assessment of AAatherosclerosis is epiaortic ultrasound scanning (EUS), but this can only be performed after sternotomy. Transesophageal echocardiography (TEE) is unable to detect atherosclerosis in distal AA due to the "blind" spot. A new method (A-View ${ }^{\circledR}$ method, an extension of TEE) enables assessment, preoperatively, of AA-atherosclerosis using a intra-tracheal fluid-filled balloon catheter. The aim of this diagnostic study was to evaluate if the A-View ${ }^{\circledR}$ method enables visualization of distal $A A$ and safety of the diagnostic.

Methods: In a cross-sectional diagnostic trial; patients undergoing cardiac surgery by sternotomy underwent TEE, the A-View ${ }^{\circledR}$ method, EUS, and routine operative monitoring. Bronchoscopy was performed before and after the A-view ${ }^{\circledR}$ method in order to detect possible damage to the bronchial or tracheal wall.

Results: Study population consisted of 41 consecutive patients, 28 (68.3\%) males and 13 females (31.7\%), mean age 67 years ( \pm 11 years). With TEE the distal AA was visible in 4 (9.8\%) patients whereas with A-View method the distal AA was visible in all (100\%) patients. There were no clinical significant sideeffects associated with the use of the A-View ${ }^{\circledR}$ catheter. In 7 (18\%) patients bronchoscopy revealed insignificant mucosal bleeding, this required no additional interventions nor delayed planned surgery. Severity of atherosclerosis visualized with $A$-view ${ }^{\circledR}$ method compared to EUS results showed a Kappa of 0.69 (0.50-0.88)

Conclusion(s): The A-View ${ }^{\circledR}$ method offers an easy, safe, and minimally invasive approach of visualizing the distal AA. Compared to EUS, the A-View method yielded adequate results in the detection of $A A$ atherosclerosis. The A-View ${ }^{\circledR}$ method can be used prior to sternotomy. Therefore, surgical strategy can still be adjusted to reduce manipulation of the $A A$ in case of aortic atherosclerosis.

\section{AP2-4}

A diagnostic systematic review using a bivariate metaregression model of transesophageal echocardiography for the assessment of atherosclerosis in the ascending aorta in cardiac surgery patients

B. van Zaane, N.P.A. Zuithoff, J.B. Reitsma, A.P. Nierich, K.G.M. Moons

Division of Perioperative Care and Emergency Medicine, University Medical Center Utrecht, Utrecht, The Netherlands

Background: Post-operative stroke in cardiac surgery is caused by emboli merging from the atherosclerotic ascending aorta (AA) after manipulation. It has been shown that the use of epiaortic scanning (EUS) combined with changes in surgical technique when atherosclerosis is present reduces the incidence of stroke. EUS has not gained widespread use, mainly because it is performed after sternotomy when changes in strategy are hardly feasible. Transesophageal echocardiography (TEE) can be performed before sternotomy to timely allow changes in surgical strategy. Hence, if accurate enough, TEE could replace EUS for the assessment of $A A$ atherosclerosis and guide medical decisions in cardiac surgery patients. Aim of the study was to systematically review the diagnostic accuracy of TEE for the assessment of atherosclerosis in the $A A$ in patients undergoing cardiac surgery, with EUS as reference. Methods: We searched Medline, Embase, Cochrane library, Medion, Dare for studies comparing TEE versus EUS for detection of clinical relevant atherosclerosis. Studies were selected, data abstracted and validity assessed, according to QUADAS criteria, by independent reviewers. A random-effects bivariate meta-regression model was used to obtain summary estimates of sensitivity, specificity, and diagnostic odds ratio (DOR). Pairs of logit transformed sensitivity and specificity from each study were analysed to account for possible correlations due to different thresholds.

Results: We extracted 6 studies with a total of 346 patients of whom 419 aortic segments were analysed, including 100 segments with atherosclerosis (median prevalence 25\% (range 17-62\%)). Summary estimates of sensitivity, specificity and of the DOR were $21 \%$ (95\% Cl 13-32\%), 99\% (96-99\%), and 18.1 (5.8-56.3) respectively.

Conclusion: Because of the low sensitivity of TEE for the detection of AA atherosclerosis a negative test result requires verification by additional testing using epiaortic scanning. In case of a positive test result, AA atherosclerosis can be considered as present, and less manipulative strategies might be indicated.

\section{AP2-5}

\section{Haemodynamic monitoring during orthotopic xenogenic heart transplantation}

A. Bauer, V. Renz, U. Brandl, M. Schmoeckel, F. Christ

Clinic for Anaesthesiology, Ludwig-Maximilians University, Munich, Germany Background and Goal of Study: Xenotransplantation offers a potential solution to the world-wide shortage of organ donors. The orthotopic xenogenic transplantation of pig hearts into baboons (oXHTx) is the only accepted preclinical animal model for cardiac xenotransplantation. Femoral artery thermodilution (FATD) is an invasive method to monitor haemodynamic and volumetric parameters; however, FATD reference values for baboon do not exist so far. Thus we investigated haemodynamic parameters before and after oXHTx.

Materials and Methods: oHXTx from six hCD46 transgeneic piglets to baboons (body weight. 10 to $26 \mathrm{~kg}$ ) were performed. We used FATD (PiCCO, Pulsion, Munich, Germany) for the invasive assessment of cardiac index (Cl), stroke volume index (SVI), global end-diastolic volume index (GEDI), intrathoracic blood volume index (ITBI), cardiac function index (CFI) and global ejection fraction (GEF) in baboons.

Results and Discussions: Haemodynamic data of the baboons before and after oXHTx are given as mean \pm SD (table)

\begin{tabular}{llcc}
\hline & & Baseline & after oXHTx \\
\hline HR & beats min & \\
MAP & $\mathrm{mm} \mathrm{Hg}^{-1}$ & $86 \pm 15$ & $122 \pm 19^{\star}$ \\
Cl & $\mathrm{I} \mathrm{min}^{-1} \mathrm{~m}^{-2}$ & $75.7 \pm 16.6$ & $68.3 \pm 12.9$ \\
SVI & $\mathrm{ml} \mathrm{m}^{-2}$ & $2.26 \pm 0.56$ & $2.09 \pm 0.32$ \\
GEDI & $\mathrm{ml} \mathrm{m}^{-2}$ & $28.4 \pm 4.7$ & $17.3 \pm 2.2^{\star}$ \\
ITBI & $\mathrm{ml} \mathrm{m}^{-2}$ & $506 \pm 125$ & $399 \pm 42$ \\
CFI & $\mathrm{min}^{-1}$ & $584 \pm 100$ & $499 \pm 52$ \\
GEF & $\%$ & $4.77 \pm 0.91$ & $5.20 \pm 0.72$ \\
SVRI & Dyne sec cm & $19.3 \pm 5.4$ \\
\hline
\end{tabular}

HR: heart rate; MAP: mean arterial pressure; SVRI: systemic vascular resistance index; ${ }^{*} P<0.05$ paired t-test.

Volumetric FATD parameters differ from the normal range of adult and infant humans. Porcine xenograft maintained $\mathrm{Cl}$ with elevated $\mathrm{HR}$ and reduced SV. Conclusion(s): We present FATD reference values for baboons using FATD and could show that porcine xenografts are capable to maintain cardiac function in the perioperative period after oXHTx.

\section{AP2-6}

\section{Global enddiastolic volume as a variable of fluid responsiveness in a paediatric animal model}

J. Renner, M. Gruenewald, E. Cavus, J. Scholz, B. Bein

Anaesthesiology and Intensive Care Medicine, University Hospital Schleswig-Holstein, Campus Kiel, Kiel, Germany

Background and Goal of Study: Pulse pressure variation (PPV) and stroke volume variation (SVV) have been shown to predict fluid responsiveness in adults, albeit they depend on mechanical ventilation and therefore are only applicable in selected patients (1). Global enddiastolic volume (GEDV) determined by transpulmonary thermodilution accurately reflects changes in preload in adults (2). The present study was designed to evaluate whether GEDV is a suitable parameter of preload and fluid responsiveness during changing loading conditions in a paediatric animal model.

Materials and Methods: The study protocol was approved by the institutional animal research committee. 19 anaesthetized piglets $6.5 \mathrm{~kg}( \pm 0.9 \mathrm{~kg})$, ventilated with a tidal volume of $10 \mathrm{ml} / \mathrm{kg}$ were studied during normovolemia and after infusion of $20 \mathrm{ml} / \mathrm{kg}$ of $6 \%$ HES. GEDV was obtained by transpulmonary thermodilution. PPV and SVV were monitored continuously (PiCCO Plus ${ }^{\circledR}$, Version 6.0), and central venous pressure (CVP), pulmonary capillary wedge pressure (PCWP), bolus $\mathrm{CO}$ and stroke volume index (SVI) were measured using a paediatric thermodilution pulmonary artery catheter. 
Results and Discussions: All hemodynamic variables changed significantly comparing the different experimental stages. PPV and SVV, as well as CVP and PCWP showed a close correlation during changing loading conditions, whereas there was no correlation with the percentage change of SVI ( $\Delta \mathrm{SVI})$ after volume loading. GEDV significantly correlated with SVI, $\triangle$ GEDV and with $\Delta S V I(r=0.66, r=0.80, r=-0.45)$.

Conclusion(s): In this experimental paediatric model, GEDV was the only variable to accurately reflect cardiac preload, suggesting that GEDV might be superior in guiding fluid therapy in children.

References:

1 Kumar et al.; 2004, Crit Care Med 8:128-136.

2 Michard et al.; 2003, Chest 124:1900-1908.

\section{AP2-7}

Continuous central venous and pulmonary artery oxygen saturation monitoring in cardiac surgery after cardiopulmonary bypass

A. Ducart, M. Dejonckheere, M. Koch, L. Perrin, D. Schmartz

Anesthesiology, Erasme ULB Hospital, Brussels, Belgium

Background and Goal of Study: Continuous measurement of central venous oxygen saturation (Scvo2) is now available and could be an attractive alternative to continuous monitoring of mixed venous oxygen saturation (Svo2). The accuracy of both fiberoptic measurements was assessed by comparison to reference co-oximeter results in cardiac surgery after cardiopulmonary bypass (CPB).

Materials and Methods: In 20 patients, after informed consent, we performed the placement of a Continuous Cardiac Output (CCO) catheter with Svo2, 7.5F (Edwards Lifesciences ${ }^{\circledR}$, Irvine, USA) in the pulmonary artery and of a $2 \mathrm{~F}$ CeVOX fiberoptic probe (Pulsion ${ }^{\circledR}$ Medical Systems, Munich, Germany) placed trough a $7 \mathrm{~F}$ triple lumen, Arrow $^{\circledR}$, in the superior vena cava. Blood samples were analysed for oxygen saturation by Instrumentation Laboratory $682 \mathrm{co}-$ oximeter. Data were compared using a Pearson correlation and by the Bland and Altman method.

Results and Discussions: Both fiberoptic oximetry catheters correlated significantly after CPB with standard blood gas oximetry (SvcO2: $r=0.95$, $p<0.001$; Svo2: $r=0.98, p<0.001$ ). For Svco2, measurements by CeVOX were on average $0.65 \%( \pm 2.44 \mathrm{SD})$ lower than blood gas oximetry measurements (limits of agreement: -4.13 to 5.43 ) and the error range was $0.9 \%$. For Svo2, measurements by CCO were on average $1.00 \%$ ( \pm 1.57 SD) higher than blood gas oximetry measurements (limits of agreement: -4.1 to 2.1 ) and the error range was $1.39 \%$.

Conclusion: In cardiac surgery, after CPB, in spite of haemodilution, the results of both fiberoptic oximetry (Scvo2 and Svo2) are nearly identical with standard blood gas oximetry.

Reference:

Reinhart K et al. Intensive Care Med 2004; 30 (8): 1572-78.

\section{AP2-8}

Comparsion of FloTrac ${ }^{\mathrm{TM}}$ cardiac output monitoring system in patients undergoing coronary artery bypass grafting with pulmonary artery cardiac output measurements

M. Cannesson, Y. Attof, P. Rosamel, P. Chiari, JJ. Lehot

Department of Anaesthesiology, Hospices Civils de Lyon, Hopital Louis Pradel, Bron, France, Hospices Civils de Lyon, Bron, France

Background and Goal of Study: Recently, arterial pulse waveform analysis has been proposed for cardiac output $(\mathrm{CO})$ determination and monitoring without calibration or thermodilution (FloTrac ${ }^{\mathrm{TM}} /$ Vigileo $^{\mathrm{TM}}$, Edwards Lifescience, Irvine, CA, USA). However, the accuracy and clinical applicability of this new technology has not been fully evaluated. We designed this prospective study to compare the accuracy of the FloTrac ${ }^{\mathrm{TM}}$ system $\left(\mathrm{CO}_{\mathrm{FT}}\right)$ versus pulmonary artery catheter standard bolus thermodilution $\left(\mathrm{CO}_{\mathrm{PAC}}\right)$ in patients undergoing coronary artery bypass grafting (CABG).
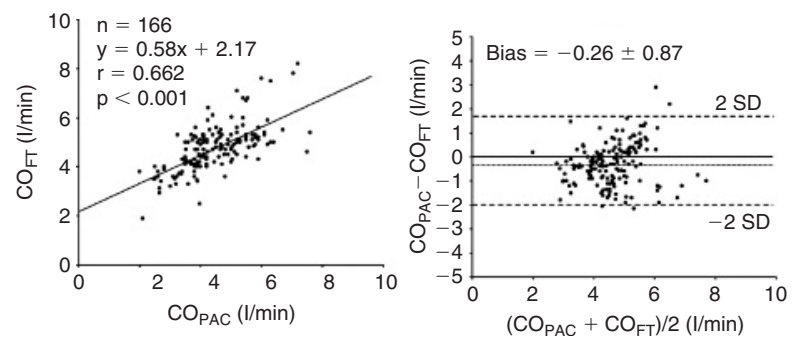

Materials and Methods: After informed consent, we studied 11 patients referred for $\mathrm{CABG}$. $\mathrm{CO}_{\mathrm{FT}}$ and $\mathrm{CO}_{\mathrm{PAC}}$ were determined at 6 time points in the operating room including before and 5 minutes after volume expansion (VE) (500 $\mathrm{ml} \mathrm{6 \%} \mathrm{hetastarch).} \mathrm{Following} \mathrm{measurements} \mathrm{were} \mathrm{performed} \mathrm{at} \mathrm{the}$ arrival in the intensive care unit and every four hours. Bland-Altman analysis was used to assess the agreement between $\mathrm{CO}_{\mathrm{FT}}$ and $\mathrm{CO}_{\mathrm{PAC}}$.

Results and Discussions: $\mathrm{CO}_{\mathrm{PAC}}$ ranged from 2.0 to $7.6 \mathrm{I} / \mathrm{min}$ and $\mathrm{CO}_{\mathrm{FT}}$ ranged from 1.9 to $8.2 \mathrm{l} / \mathrm{min}$. There was a significant relationship between $\mathrm{CO}_{\mathrm{PAC}}$ and $\mathrm{CO}_{\mathrm{FT}}(\mathrm{r}=0.662 ; \mathrm{p}<0.001)$ over the 166 pairs of data. Agreement between $\mathrm{CO}_{\mathrm{PAC}}$ and $\mathrm{CO}_{\mathrm{FT}}$ was $-0.26 \pm 0.87 \mathrm{l} / \mathrm{min}$. VE induced a significant increase in both $\mathrm{CO}_{\mathrm{PAC}}$ and $\mathrm{CO}_{\mathrm{FT}}$ (from $3.4 \pm 0.8 \mathrm{l} / \mathrm{min}$ to $4.4 \pm 1.0 \mathrm{l} / \mathrm{min}$; $\mathrm{p}<0.001$ and from $3.9 \pm 1.2$ to $5.0 \pm 1.1 \mathrm{l} / \mathrm{min} ; \mathrm{p}<0.001$, respectively) and there was a significant relationship between percent change in $\mathrm{CO}_{\mathrm{PAC}}$ and $\mathrm{CO}_{\mathrm{FT}}$ following VE $(r=0.722 ; \mathrm{p}=0.01)$.

Conclusion(s): We found clinically acceptable agreement between $\mathrm{CO}_{\mathrm{FT}}$ and $\mathrm{CO}_{\mathrm{PAC}}$ in this setting. This new device has potential clinical applications.

\section{AP2-9}

\section{Heart rate variability predicts cardiovascular events during} general anaesthesia

R. Hanss, M. Kaufmann, R. Gaupp, J. Scholz, B. Bein

Anaesthesiology and Intensive Care Medicine, University Hospital Schleswig-Holstein, Campus Kiel, Kiel, Germany

Background: Heart rate variability (HRV) has been shown to predict hypotension after spinal anaesthesia. ${ }^{1,2}$ Preoperative differences of HRV may be present in patients with underlying cardiovascular (CV) diseases scheduled for general anaesthesia. Goal: The predictive value of HRV for cardiovascular events (CVE) was analysed in 50 patients with cardiovascular risk factors scheduled for general anaesthesia.

Materials and Methods: After IRB approval and written informed consent total intravenous anaesthesia was performed in standardized fashion. CV risk was evaluated based on Lee's Revised Cardiac Risk Index. ${ }^{3}$ Patients were included if they had a Lee score $>2$. CVE were defined as ST-segment depression $(>0.1 \mathrm{~mm})$ or elevation $(>0.1 \mathrm{~mm})$ during 24 hours Holter-ECG recording. HRV of patients without events ("NO-CVE") were compared to patients with CVE. Total Power (TP), low frequency (LF), high frequency (HF), LF/HF ratio, and standard deviation of mean $\mathrm{R}-\mathrm{R}$ interval (SD-RR) were studied. LF and HF are given as percentage part of TP. Statistics: Baseline HRV was compared with Mann Whitney U test, $\mathrm{p}<0.05$.

Results: ST segment depression or elevation was found in 18 patients (median duration: 52 (min: 5 / max: 274) minutes). 32 patients did not demonstrate Holter-ECG abnormalities. Demographic data did not differ between groups. LF/HF, reflecting autonomic balance (NO-CVE:0.9 \pm 1.4 vs. CVE:2.8 $\pm 2.3, \quad p<0.05), \quad L F$, reflecting sympathetic activity (NOCVE: $25 \pm 18 \%$ Vs. CVE: $32 \pm 24 \%, p<0.05$ ), SD-RR (NO-CVE: $83 \pm 18 \mathrm{~ms}$ vs. CVE: $44 \pm 29 \mathrm{~ms}, \mathrm{p}<0.05$ ), and TP (NO-CVE: $1310 \pm 770 \mathrm{~ms}^{2}$ vs. CVE: $\left.310 \pm 620 \mathrm{~ms}^{2}, \mathrm{p}<0.05\right)$ demonstrated significant differentces. HF, reflecting parasympathetic activity did not differ.

Discussions: Baseline HRV of patients with and without CVE differed significantly. Increased sympathetic activity and depressed TP predicted CVE. Conclusion: These data suggest that HRV analysis of patients at high cardiovascular risk prior to general anaesthesia may detect those individuals at high risk for perioperative CVE.

References:

1 Hanss R et al. (2005) Anesthesiology 102: 1086-1093.

2 Hanss R et al. (2006) Anesthesiology 104: 635-43.

3 Lee TH et al. (1999) Circulation 100: 1043-9.

\section{AP3-1}

Electrophysiologic mechanism of sevoflurane on action potential prolongation in rat ventricular myocytes

W. Park, J. Chae, D. Ahn, M. Kim, C. Lynch III

Anesthesiology and Pain Medicine, Yonsei University College of Medicine, Seoul, Republic of Korea

Background and Goal of Study: Despite prolongation of the $\mathrm{QT}_{\mathrm{C}}$ interval in humans during sevoflurane anesthesia, little is known about the mechanisms that underlie these actions. In rat ventricular myocytes, the effect of sevoflurane on action potential (AP) duration and underlying elctrophysiological mechanisms were investigated.

Materials and Methods: The AP was measured using a current clamp technique. The transient outward $\mathrm{K}^{+}$current was recorded during depolarizing steps from $-80 \mathrm{mV}$, followed by short pulses to $-40 \mathrm{mV}$ and then depolarization up to $+60 \mathrm{mV}$. The voltage-dependence of steady-state inactivation 
was determined using a standard double-pulse protocol. The sustained outward current was obtained by addition of $5 \mathrm{mM} 4$-aminopyridine. The inward rectifier $\mathrm{K}^{+}$current was recorded from a holding potential of $-40 \mathrm{mV}$ before their membrane potential was changed from -130 to $0 \mathrm{mV}$. Sevoflurane actions on L-type $\mathrm{Ca}^{2+}$ current were obtained.

Results and Discussions: Sevoflurane prolonged AP duration, while the amplitude and resting membrane potential remained unchanged. The peak transient outward $\mathrm{K}^{+}$current was significantly reduced by $18 \pm 2 \%$ and $24 \pm 2 \%$ by $0.35 \mathrm{mM}$ and $0.7 \mathrm{mM}$ sevoflurane, respectively, at $+60 \mathrm{mV}$. There was no effect on the sustained outward current by sevoflurane. Whereas $0.7 \mathrm{mM}$ sevoflurane did not shift the steady-state inactivation curve, it significantly accelerated the decay of current inactivation $(P<0.05)$. The inward rectifier $\mathrm{K}^{+}$current at $-130 \mathrm{mV}$ was little altered by $0.7 \mathrm{mM}$ sevoflurane. The L-type $\mathrm{Ca}^{2+}$ current was reduced by $28 \pm 3 \%(\mathrm{P}<0.05)$ and $33 \pm 1 \%$ $(P<0.05)$ by $0.35 \mathrm{mM}$ and $0.7 \mathrm{mM}$ sevoflurane, respectively.

Conclusion(s): Prolongation of AP duration by clinically relevant concentrations of sevoflurane appears due to the suppression of transient outward $\mathrm{K}^{+}$current in rat ventricular myocytes.

References:

1 Kleinsasser A et al. Anesth Analg 2000; 90: 25-27.

2 Kang J et al. Anesthesiology 2006; 104: 1015-1022.

\section{AP3-2}

\section{Cardiac surgery anesthesia with new volatile agents} (desflurane or sevoflurane): a meta-analysis

E.Dedola, O.Fochi, E.Bignami, D.Poli, G.Landoni

Department of Cardiothoracic Anesthesia and Intensive Care, San Raffaele Scientific Institute, Milano, Italy

Background and Goal of Study: We performed a meta-analysis to investigate whether the cardioprotective effects of volatile anesthetics translate into decreased morbidity and mortality in patients undergoing cardiac surgery.

It is commonly believed that the choice of the primary anesthetic agent does not result in different outcomes after cardiac surgery. Recent evidence however has indicated that volatile anesthetics improve post-ischemic recovery at cellular level, in isolated hearts, in animals, and in humans.

Materials and Methods: Four investigators independently searched BioMedCentral and PubMed. Inclusion criteria were random allocation to treatment, comparison of a total intravenous anesthesia regimen vs an anesthesia plan including new volatile anesthetics (desflurane or sevoflurane), performed on cardiosurgical patients. Exclusion criteria were duplicate publications, non-human experimental studies, no outcome data. The primary endpoint was the incidence of perioperative myocardial infarction, while the co-primary endpoint was the rate of hospital mortality.

Results and Discussions: The search yielded 22 studies, involving 1922 patients. Volatile anesthetics were associated with significant reductions of myocardial infarctions $(24 / 979$ [2.4\%] in the volatile anesthetics group vs $45 / 874$ [5.1\%] in the control arm, odds ratio [OR] $=0.51[0.32-0.84], p$ for effect $=0.008, p$ for heterogeneity $=0.77)$, and mortality $(4 / 977[0.4 \%]$ vs $14 / 872[1.6 \%]$, OR $=0.31$ [0.12-0.80], $p$ for effect $=0.02, p$ for heterogeneity $=0.88)$.

Conclusions: The new anesthetics desflurane and sevoflurane have cardioprotective effects which result in decreased morbidity and mortality. Our data show for the first time that the choice of an anesthetic regimen based on administration of halogenated anesthetics is associated with a better outcome after cardiac surgery.

\section{AP3-3 \\ Desflurane - but not Sevoflurane - preserves microvascular oxygenation of the gastric mucosa in dogs}

I.Schwartges, M.Stephan, A.Fournell, L. A.Schwarte, O.Picker

Department of Anaesthesiology, University Hospital Duesseldorf, Duesseldorf, Germany

Background and Goal of Study: Maintenance of adequate microcirculatory haemoglobin oxygenation $\left[\mu \mathrm{HbO}_{2}\right]$ is crucial for the integrity of the gastric mucosa [1]. Although the depressing effects of volatile anaesthetics on systemic haemodynamics are well known their effects on microcirculatory oxygenation of splanchnic mucosa remain unclear.

Materials and Methods: Chronically instrumented dogs (Foxhounds, $30 \pm 1 \mathrm{~kg}, \mathrm{n}=5$ ) were repeatedly anaesthetized either with sevoflurane [SEV] or with desflurane [DES]. Dogs were mechanically ventilated $\left(\mathrm{FiO}_{2} \quad 0.3\right.$, $\mathrm{EtCO}_{2} 35 \mathrm{mmHg}$ ). The concentration of the respective anaesthetic agent was increased stepwise from 1.0, to 1.5 and finally to $2.0 \mathrm{MAC}$, each step maintained for 30 minutes. The $\mu \mathrm{HbO}_{2}$ was measured by reflectance spectrophotometry [2]. Systemic haemodynamics (cardiac output [CO], mean arterial pressure [MAP]) were continuously recorded. Oxygen delivery [DO $\left.{ }_{2}\right]$ was calculated from intermittently obtained arterial blood samples (e.g., for $\mathrm{O}_{2}$-content determination) and $\mathrm{CO}$.

Statistics: Means \pm SEM, ANOVA, $p<0.05$.

Results and Discussions: During anaesthesia with DES, $\mu \mathrm{HbO}_{2}$ was significantly higher than with SEV already at $1 \mathrm{MAC}$ (67 \pm 3 vs $57 \pm 1 \%$ ) while $\mathrm{DO}_{2}$ $\left(19.5 \pm 3.2 \mathrm{vs} 14.4 \pm 1.3 \mathrm{ml} \cdot \mathrm{kg}^{-1} \cdot \mathrm{min}^{-1}\right)$ did not differ significantly. $\mathrm{DO}_{2}$ decreased significantly with both agents (2.0 MAC: DES $15.5 \pm 1.4$; SEV $\left.8.2 \pm 1.0 \mathrm{ml} \cdot \mathrm{kg}^{-1} \cdot \mathrm{min}^{-1}\right) \cdot \mu \mathrm{HbO}_{2}$ however remained virtually unchanged with DES (63 $\pm 2 \%$ ) while SEV led to a significant depression of $\mu \mathrm{HbO}_{2}(41 \pm 5 \%)$. Conclusion(s): DES preserves $\mu \mathrm{HbO}_{2}$ despite a significant depression of systemic oxygen delivery and circulation whereas SEV exerts its depressing effects on both systemic oxygen delivery and regional microvascular oxygenation. Thus the use of DES could be favourable in patients at risk for splanchnic hypoxygenation.

References:

1 Sato, N et al. (1988) J Clin Gastroentrol 10: 13-8.

2 Scheeren, TWL et al. (2002) Crit Care Med 30: 881-7.

\section{AP3-4}

A comparison of the influence of propofol based TIVA and sevoflurane based anesthesia on hemodynamic function during laparoscopy in morbidly obese

T. Gaszynski, T. Szewczyk, W. Gaszynski

Anaesthesiology and Intensive Therapy, Medical University of Lodz, Lodz, Poland

Background and Goal of Study: Pneumoperitoneum has important impact on haemodynamic function during general anaesthesia. The aim of the study was to estimate influence of type of anaesthesia during laparoscopic procedures on cardiac function in morbidly obese during gastric banding surgery. Materials and Methods: Haemodynamic function was measured by transaesophageal Doppler probe using HemoSonic 100 device (Arrow, USA). Measurements time points: T1- after induction to anaesthesia, T2- insuflation of abdomen, T3- anti-Trendellenburg (Fowler) position and pneumoperitoneum. 28 pts were randomly divided into two groups: anaesthetised using sevoflurane/ $\mathrm{O}_{2}$ /air (group Sevo) - 13 pts or TIVA with propofol (group Prop) 15 pts. Patients also received FNT, midazolam and atracurium. Pneumoperitoneum pressure was $15 \mathrm{mmHg}$.

Results: Demographic data: Age: $38.15 \pm 11.31$ vs $36.13 \pm 9.75$ yrs; Weight: $138.77 \pm 26.67$ vs $122.69 \pm 14.43 \mathrm{~kg}$; High: $173.54 \pm 8.29$ vs $169.47 \pm 9.26 \mathrm{~cm}$; BMl: $45.98 \pm 7.7$ vs $43.23 \pm 4.14 \mathrm{~kg} / \mathrm{m}^{2}$ in groups Sevo vs Prop respectively. Values are Mean \pm SD. No difference were recorded. Changes in measured parameters. Results are Mean \pm SD:

\begin{tabular}{lllll}
\hline Parameter & Group & T1 & T2 & T3 \\
\hline CO & Sevo & $6.88 \pm 1,13$ & $5.15 \pm 0.91^{*}$ & $5.55 \pm 1.12$ \\
(I/min) & Prop & $7.07 \pm 1.4$ & $5.55 \pm 1.44^{*}$ & $5.03 \pm 1.27$ \\
TSVR & Sevo & $1089.2 \pm$ & $1370.47 \pm$ & $1672.87 \pm$ \\
(dyn.s.cm - 5) & & 359.52 & 515.79 & 651.47 \\
& Prop & $974.8 \pm$ & $1365.07 \pm$ & $1652.2 \pm$ \\
& & 243.21 & $535.18^{*}$ & 378.22 \\
\hline
\end{tabular}

${ }^{*} \mathrm{p}<0.05$ compared with $\mathrm{T} 1$

In both groups $\mathrm{CO}$ decreased significantly after insuflation of abdomen. The drop in CO was higher in group Sevo but when compared with group Prop the difference was not significant $(p=0.18)$. In Time point T3 CO increased in group Sevo but in group Prop decreased further more. However, the comparison revealed no significant difference between groups $(p=0.12)$. Changes in MAP and HR were not important $(p=0.13, p=0.15)$.

Conclusion: There was no important difference between studied types of anesthesia regarding influence on hemodynamic function during laparoscopy in $\mathrm{MO}$ pts.

\section{AP3-5 \\ The efficacy of sevoflurane-induced cardioprotection is not affected by bupivacaine in rat hearts}

R.R. Lamberts, M.J.A. Vreden, L.E.J. Wassenaar, S.A. Loer, R.A. Bouwman Department of Anesthesiology, Institute for Cardiovascular Research (ICaRVU), VU University medical center (VUmc), Amsterdam, The Netherlands

Background and Goal of Study: Plasma levels of bupivacaine may induce cardiotoxic effects, which could alter signalling pathways and thereby influence sevoflurane-induced cardioprotection against ischaemia-reperfusion injury. Therefore, we assessed the effect of bupivacaine on the efficacy of sevoflurane-induced cardioprotection. 
Materials and Methods: Isolated Langendorff-perfused rat hearts received 35 minutes of global ischaemia followed by 60 minutes of reperfusion (I/R). The hearts were randomly divided into 4 groups: (1) control (CON, $n=8$ ); (2) sevoflurane preconditioning (SEVO, $n=8$ ) receiving three times 5 -minute episodes of sevoflurane (2,5 vol\%) before I/R; (3) pre-treatment $1 \mu \mathrm{g} / \mathrm{ml}$ bupivacaine during 40 minutes before $\mathrm{I} / \mathrm{R}$ combined with sevoflurane preconditioning (BUPI-SEVO, $n=8$ ); and (4) bupivacaine pre-treatment alone (BUPI, $n=4)$. After $I / R$, cardiac function was determined as recovery of left ventricular pressures (LVP, expressed as percentage to values before I/R) and cellular injury was determined by infarct size with triphenyltetrazolium chloride (TTC) staining.

Results: After $1 / R$, the SEVO group showed increased recovery of LVP ( $53 \pm 3 \%$ SEVO vs. $46 \pm 3 \%$ CON, $n=8, p<0.05$ ), as well as a reduced infarct size $(25 \pm 8 \%$ SEVO vs. $59 \pm 6 \% \mathrm{CON}, \mathrm{n}=6, \mathrm{p}<0.01)$. Interestingly, in the BUPI-SEVO and BUPI group infarct size was reduced to a similar extent as the SEVO group ( $24 \pm 7 \%$ BUPI-SEVO $n=8$ and $34 \pm 3 \%$ BUPI $n=4$ vs. CON, $p<0.05$ ), whereas LVP were unaltered compared to controls. Conclusion: The efficacy of the volatile anaesthetic sevoflurane-induced cardioprotection against ischaemia-reperfusion injury was not affected by the local anaesthetic bupivacaine. Therefore, we suggest that both anaesthetics might induce similar cardioprotective signalling pathways.

\section{AP3-6}

Sevoflurane-induced cardioprotection is mediated by protein kinase $\mathbf{C}$-alfa via production of reactive oxygen species

R.A. Bouwman, J.J. de Lange, C. Boer, S.A. Loer, R.R. Lamberts Anesthesiology, VU University Medical Center, Amsterdam, The Netherlands

Background and Goal of the Study: Recently we demonstrated that sevoflurane-induced cardioprotection is mediated via the $\mathrm{Ca}^{2+}$-insensitive protein kinase $C$ (PKC)- $\delta$ isoform, reactive oxygen species (ROS) and ATPsensitive mitochondrial $\mathrm{K}^{+}$(mitoK ${ }^{+}{ }_{\text {ATP }}$ ) channels (1). Since sevoflurane modulates cardiac calcium handling, this study focuses specifically on the role of the $\mathrm{Ca}^{2+}$-sensitive PKC- $\alpha$ isoform in sevoflurane-induced cardioprotection and the relation with PKC- $\delta$, ROS and mitoK ${ }_{\text {ATP }}^{+}$channels.

Materials and Methods: Isolated rat trabeculae were preconditioned with $3.8 \%$ sevoflurane (15 minutes) and subsequently subjected to an ischemic protocol by superfusion of trabeculae with hypoxic, glucose-free buffer ( 40 minutes) followed by reperfusion (60 minutes). The functional role of PKC- $\alpha$, PKC- $\delta$, ROS and mitoK ${ }^{+}$ATP channels was investigated by using the inhibitors Go6976, rottlerin, n-(2-mercaptopropionyl)-glycine and 5-hydroxydecanoic acid sodium. PKC- $\alpha$ activation in trabeculae exposed to sevoflurane was studied with immunofluorescent co-localization analysis.

Results: Ischemia and reperfusion $(I / R)$ reduced the contractile recovery $\left(F_{\text {rec; }}\right.$ expressed as percentage of the initial contractile force) from (Mean $\pm \mathrm{SE}$ ) $79 \pm 7 \%$ [Time control] to $47 \pm 3 \%[\mathrm{l} / \mathrm{R}](\mathrm{n}=9 ; P<0.05)$. Sevoflurane preconditioning improved $\mathrm{F}_{\text {rec }}$ to $65 \pm 3 \%[\mathrm{l} / \mathrm{R}+\operatorname{Sevo}](\mathrm{n}=8 ; P<0.05$ vs. $[\mathrm{l} / \mathrm{R}])$. Inhibition of PKC- $\alpha$ by Go6976 during preconditioning reduced the $F_{\text {rec }}$ to $42 \pm 4 \%[\mathrm{l} / \mathrm{R}+$ Sevo $+\mathrm{Go}](\mathrm{n}=10 ; P<0.05$ vs. [l/R + Sevo]). Finally, sevoflurane preconditioning resulted in translocation of $\mathrm{PKC}-\alpha$ to the mitochondria. The translocation of PKC- $\alpha$ was reduced by ROS scavenging, but not by inhibition of PKC- $\delta$ or mitoK ${ }^{+}$ATP channels.

Conclusion: We showed that sevoflurane-induced activation of $\mathrm{Ca}^{2+}$-sensitive PKC- $\alpha$, via production of ROS, is essential for cardioprotective signaling. In addition, $\mathrm{Ca}^{2+}$-insensitive PKC- $\delta$ activation and mitoK ${ }^{+}$ATP channel opening are not involved in sevoflurane-induced PKC- $\alpha$ activation. Reference:

1 Bouwman RA. Anesthesiology 2004; 100: 506-514.

\section{AP3-7}

\section{Antioxidant effect of sevoflurane and desflurane anesthesia during coronary artery bypass graft surgery}

B.Tezcan, P.Durak, N.Genel, M.Taslýpýnar, O.Erdemli

Anesthesiology, Býochemistry, Turkýye yuksek ýhtýsas hospital, Gazi University, Ankara, Turkey

Background and Goal of the Study: Cardiac surgery induces oxidative stress which may lead to impairment of cardiac function. In this study we evaluated the circulating lipid peroxidation product (MDA) and markers of blood antioxidant status superoxide dismutase (SOD) and glutathione peroxidase(GPx) during balanced anesthesia established by sevoflurane and desflurane. Material and Methods: After getting hospital ethics committee approval and patient consent 40 patients undergoing elective coronary artery bypass graft surgery were randomly assigned to receive Desflurane in Group I ( $n=20)$ and Sevoflurane in Group II $(n=20)$. Blood samples were drawn t1-before anesthesia induction,t 2-before surgical incision,t 3-before perfusion, t4-after perfusion,t 5- at the end of the operation. Statistical analysis was performed by Mann-Whitney $U$ and Friedman test.

Results and Discussion: Serum levels of MDA were significantly decreased after the operation $(\% 40)$ when compared with preoperative measurements in sevoflurane group where it was only decreased (\%23) in desflurane group. SOD measurements were significantly increased after perfusion (\%55) in sevoflurane group where it was only $\% 6$ in desflurane group with respect to measurements before anesthesia. GPx measurements were decreased $\% 7$ after the operation in sevoflurane group where it was decreased $\% 35$ in desflurane group. It is resulted that sevoflurane anesthesia significantly decreased lipid peroxidation product (MDA) together with an increase in antioxidant marker SOD which was accompanied by an antioxidant enzyme GPx consumption when compared with desflurane .

Conclusion: We concluded that sevoflurane anesthesia demonstrated a better oxidative status and defensed the antioxidant pool better than desflurane anesthesia.

\section{AP3-8}

The evaluation of QTc Interval, Qtc dispersion, dysrhythmia and heart rate variability undergoing sevoflurane and desflurane anesthesia

\section{Kaymak, T. Dogru, H. Basar}

Department of Anaesthesiology, University of Kirikkale, Faculty of Medicine, Kirikkale, Turkey

Background and Goal of Study: The aim of the present study was to asses the effects of sevoflurane and desflurane as inhalational anaesthetics on heart rate variability, dysrhythmias, QT interval and QT dispersion in patients who will undergo laporoscopic cholecystectomy under general anaesthesia. Material and Methods: 34 patients underwent elective laparoscopic cholecystectomy were enrolled in the study. The patients were monitored with Holter device 15 minutes before the anaesthesia induction till 30 minutes in recovery room. Anaesthesia was induced using $4-7 \mathrm{mg} \mathrm{kg}^{-1}$ thiopental sodium, $1 \mu \mathrm{g} \mathrm{kg}^{-1}$ fentanyl and $1 \mathrm{mg} \mathrm{kg}^{-1}$ vecuronium for muscle relaxation. Cases were randomized into 2 groups as Group 1 Sevoflurane and Group 2 Desflurane. End tidal $\mathrm{CO}_{2}, \mathrm{O}_{2}, \mathrm{~N}_{2} \mathrm{O}$ were monitorized and ventilation were maintained to keep $\mathrm{EtCO}_{2}$ between $30-40 \mathrm{mmHg}$. Minimum, maximum, mean heart rates and heart rate variability parameters included low frequency (LF), high frequency (HF) values. LF/HF ratio, Global Sympathetic Index (GSI), R-R intervals were measured in preoperative, intraoperative and 2nd, 10th and 30th minutes after extubation. QT interval was corrected using Bazett's formula (QTc $=\mathrm{QT} \sqrt{\mathrm{RR}}$ ). QT dispersion was calculated by finding shortest and longest QT interval. Mann Whitney- $U$ test and Spearman Correlation analysis were used for statistical analysis. If $p$ value was less than 0.05 , it was accepted statistically significant.

Results: Assessing datas from Holter Monitor maximum heart rates values were significantly higher in Desflurane group $(p=0.049)$. LF/HF ratio, GSI values were higher in Desflurane group in peroperative period except in induction-extubation events. Maximum R-R intervals in Sevoflurane group in peroperative period were significantly higher. In desflurane group, QT intervals were significantly longer and QT dispersion was significantly more frequent. Positive correlation between sympathetic tonus increase and VES was recognized in both groups.

Conclusion: In this study, sympathetic activation was frequently seen in desflurane group. However severe dysrhythmias did not occur in both groups. Although both desflurane and sevoflurane increased the rate of QT, the increased of QTc and QTd in desflurane anesthesia were significantly higher than sevoflurane anesthesia.

Reference:

Acta Anaesth Scand 2004; 48: 93-101.

\section{AP4-1}

Does morphine induce preconditioning in the isolated rat heart? A. Heinen, R. Huhn, B. Preckel, CJ. Zuurbier, NC. Hauck

Laboratory of Experimental Intensive Care and Anesthesiology, Academic Medical Center, Amsterdam, The Netherlands

Background and Goal of Study: Morphine induces cardioprotection against ischemia-reperfusion (I/R) injury in vivo ${ }^{1}$ and in rabbit hearts in vitro. ${ }^{2}$ While aiming to investigate the underlying signal transduction cascade of morphine preconditioning in isolated rat hearts we failed to detect a cardioprotective effect. Thus, we investigated the protective effects of different preconditioning protocols in this experimental model.

Materials and Methods: Langendorff perfused rat hearts were assigned to one of seven groups. All hearts underwent $35 \mathrm{~min}$ global ischemia and $60 \mathrm{~min}$ reperfusion. Morphine PC was initiated by administration of $3^{\star} 5 \min 1 \mu \mathrm{M}$ 
morphine with either 5 min washout (3PC5-WO5, $\mathrm{n}=5$ ) or 15 min washout (3PC5-WO15, $\mathrm{n}=6$ ) before $\mathrm{l} / \mathrm{R}$; by $15 \mathrm{~min}$ morphine with $15 \mathrm{~min}$ washout before I/R (PC15-WO15, $\mathrm{n}=6)$; or by $15 \mathrm{~min} 10 \mu \mathrm{M}$ morphine with $15 \mathrm{~min}$ washout (H-PC15-WO15, $\mathrm{n}=6)$. Control hearts were not subject to morphine PC $(n=8)$. Ischemic preconditioning (IPC, $n=5)$ was initiated by 3 cycles of ischemia ( $3 \mathrm{~min}$ ) prior to $\mathrm{I} / \mathrm{R}$; and hearts from group 7 (MORcon, $\mathrm{n}=6$ ) received $1 \mu \mathrm{M}$ morphine continuously for $10 \mathrm{~min}$ before ischemia until the end of reperfusion. Left ventricular pressure was measured and infarct size (IS) was determined by triphenyltetrazolium staining. Statistic: One way ANOVA followed by Dunnett's post hoc test. Data are mean \pm SD.

Results and Discussions: IPC increased left ventricular developed pressure (LVDP) at the end of reperfusion ( $78 \pm 11 \mathrm{mmHg}$ vs. $51 \pm 17 \mathrm{mmHg}, \mathrm{P}<0.05)$. All other treatments had no effect on LVDP compared to control group. IPC and MORcon reduced IS by $80 \%(5.2 \pm 3.3 \%$ vs. $25.5 \pm 5.7 \%, \mathrm{P}<0.05)$ and $44 \%(14.3 \pm 7.5 \%$ vs. $25.5 \pm 5.7 \%, P<0.05)$, respectively. None of the morphine PC pretreatments had an effect on IS.

Conclusion(s): In this Langendorff rat heart model, morphine administration followed by a washout period did not protect against I/R injury. The underlying reason for the difference to in vivo studies and in vitro results from rabbits need further study.

References:

1 Schultz et al, Circ Res. 1996; 78: 1100-4.

2 Cohen et al, Circ Res. 2001; 89: 273-8.

\section{AP4-2}

Hyperhomocysteinemia, oxidative stress and arrhythmia-risk factors for cardiac events

D. Rosenberger, N. Tyagi, W. Rodriguez, M. Steed

Physiology and Biophysics, University of Louisville, Louisville, USA

Background and Goal: Hyperhomocysteinemia (HHcy) is associated with atrial fibrillation and stroke in humans (1). Homocysteine (Hcy) is an amino acid byproduct in the metabolism of methionine. Methionine intake is increased in western diets with a typically high amount of meat, milk and egg products. HHcy occurs in renal insufficiency and with specific enzyme defects in hereditary diseases. The role of Hcy in the cardiac conduction system in an in vivo rodent model has not yet been investigated. The goal of the study was to show that $\mathrm{Hcy}$ has a major influence in changes of the left ventricular geometry and conduction system with rhythm disturbances. We hypothesized that the remodeling of the extra cellular matrix (ECM) is due to Hcy induced oxidative stress (2). We wanted to show that HHcy is a risk factor for cardiac events and should be considered in the preoperative evaluation of high risk patients.

Material and Methods: Hcy was supplemented to drinking water of male C57BL6J mice to achieve moderate to high Hcy levels. ECG was monitored in freely moving mice with a subcutaneously implanted telemetric ECG probe. $2 \mathrm{D}$ transthoracic echocardiography was performed to assess regional wall motion abnormalities (RWMA) and left ventricular diameter. Immunoblotting was used to evaluate oxidative stress and ECM changes.

Results: RWMA with left ventricular diameter changes was seen in more than $60 \%$. The changes in the conduction system are documented as arrhythmia. Hcy induced the expression of matrix metalloproteinases (MMP) and marker of oxidative stress. With a downregulation of thioredoxin and upregulation of free oxygen radicals. This leads to the assumption that Hcy plays a major role in oxidative stress induced remodeling of ECM in the myocardium and endothelium.

Conclusion: HHcy should be considered as a risk factor in the preoperative evaluation to screen patients with a high risk for perioperative arrhythmia or thrombembolic events. HHcy should be included in the criteria for the cardiometabolic syndrome.

References:

1 Golstein LB and the AHA/ASA Stroke Council. Circulation 2006; 113(24): e873-923.

2 Tyagi N et al. Am J Physiol Heart Circ Physiol 2005; 289(6): H2649-56.

\section{AP4-4}

Effects of leukocyte-depleted blood on the anti-lipid peroxidation ability during canine myocardial ichemia/reperfusion

H. Fang, Q Y. Wang, C X. Li, J. Liu, Y X. Zuo

Department of Anesthesiology, West China Hospital, Sichuan University, Chengdu, China

Background and Goal of Study: Myocardial ischemia reperfusion during cardiopulmonary bypass (CPB) causes migration of neutrophils, peroxidation of lipids, and depletion of free radical scavengers(1). The invading neutrophils may attenuate the myocardium endogenous antioxidant defenses by generating oxygen free radicals during $\mathrm{CPB}(2)$.
Materials and Methods: Eighteen adult Mongolian dogs undergoing CPB, were randomized into 3 groups: the control group, whole blood group and experimental group with it use of the leukocyte depletion filter (LDF) on the bypass circuit. In experimental group, the hearts, which were declamping after 60 minutes aortic cross-clamping, were continuously reperfused for 20 minutes with leukocyte-depleted artery blood $2 \mathrm{ml} / \mathrm{kg}$ by LDF connected with bypass circuit. Control group and whole blood group were treated as experimental group but employing respectively physiological saline and artery blood as a substitute for leukocyte-depleted blood. The level of superoxide dismutase(SOD), glutathioneperoxidase (GSH-PX), myeloperoxidase(MPO), malondialdldehyde (MDA) and mitochondrial swelling degree were determined before CPB, 60 minutes after cross-clamping, 30 minutes and 60 minutes after declamping.

Results and Discussions: The white blood cell account decreased significantly after the filtration. Reperfused with leukocyte-depleted blood by LDF connected with bypass circuit, the hearts of experimental group at 60 minutes after cross-clamping,30 minutes and 60 minutes after declamping were much better in the recovery of SOD and GSH-PX than those in control group and whole blood group $(P<0.01)$. MPO,MDA levels and mitochondrial swelling degree at 60 minutes after cross-clamping,30 minutes and 60 minutes after declamping were distinctly lower in experimental group than those in control group and whole blood group $(P<0.01)$.

Conclusion(s): Myocardial lipid peroxidation was restrained and ability of antioxidation in myocardium was improved by infusion of leukocyte-depleted blood to the heart before declamping, which could distinctly attenuate myocardial ischemia/reperfusion injury.

References:

1 Chen YF. Ann Thorac Surg 2004; 78: 634-642

2 Mallet RT. Mol Cell Biochem 2003; 253: 103-111.

\section{AP4-5}

Changes of pro-inflammatory mediators in dog heart

reperfused with leukocyte-depleted blood during cardiopulmonary bypass

H. Fang, Q Y. Wang, C X. Li, J. Liu, Y X. Zuo

Department of Anesthesiology, West China Hospital, Sichuan University, Chengdu, China

Background and Goal of Study: The reperfusion injury to the myocardium will develop during the process of aortic cross-clamping and declamping(1). All of these harmful substances might come from leukocytes and/or cytokines(2). Materials and Methods: Eighteen adult Mongolian dogs undergoing CPB, were randomized into 3 groups: the control group $(n=6)$, whole blood group $(n=6)$ and the experimental group $(n=6)$ with it use of the leukocyte depletion filter (LDF) on the bypass circuit. In experimental group,the hearts, which were declamping after 60 minutes aortic cross-clamping, were continuously reperfused for 20 minutes with leukocyte-depleted artery blood $2 \mathrm{ml} / \mathrm{kg}$ by LDF connected with bypass circuit. Control group and whole blood group were treated as experimental group but employing respectively physiological saline and artery blood as a substitute for leukocyte-depleted blood. The levels of serum TNF-a, IL-1 $\beta$ and IL-8 in the coronary sinus venous blood were determined before CPB, 5 minutes after CPB, 60 minutes after cross-clamping, and 30 minutes, 60 minutes after declamping, respectively. The samples from the heart tissues were taken for determination of the activity of myeloperoxidase (MPO) and the levels of malondialdehyde(MDA) before CPB, 60 minutes after cross-clamping, and 60 minutes after declamping.

Results and Discussions: The white blood cell count decreased significantly after the filtration $(P<0.01)$. The concentrations of pro-inflammatory cytokines (TNF-a, IL-1 $\beta$ and IL-8) increased significantly after declamping in control group and whole blood group but were significantly lower in experimental group than in control group $(P<0.01)$ and whole blood group $(P<0.01)$. The MPO activity and the MDA levels 60 minutes after cross-clamping and 60 minutes after declamping were significantly lower in experimental group than in control group $(P<0.01)$ and whole blood group $(P<0.01)$.

Conclusion(s): Pro-inflammatory cytokin (TNF-a, IL-1 $\beta$ and IL-8) and leukocytes may participate in the inflammation-mediated myocardial ischemiareperfusion injury, which can be significantly attenuated by the infusion of the leukocyte-depleted blood to the heart before declamping.

References:

1 Civelek A. Thorac Cardiovasc Surg 2003; 51: 249-254.

2 Okubo N. Ann Thorac Cardiovasc Surg 2003; 9: 43-49.

\section{AP4-6}

Opioid receptor expression in the rat heart during late ischemic preconditioning

J. Fräßdorf, I. Stegmann, N.C. Weber, B. Preckel, W. Schlack Department of Anaesthesiology, University Hospital of Düsseldorf, Düsseldorf, Germany 
Background and Goal of Study: Opioid receptors $(\mathrm{OR})$ are trigger and mediator of ischemic late preconditioning (LPC) (1). Leu-Enkephalin plasma levels are increased after a short term myocardial ischemia in rats (2). Whether OR Expression in the myocardium after LPC is altered is unknown. We investigated by Western Blot the expression of the three OR subtypes $\left(\delta-, \kappa^{-}, \mu-\mathrm{OR}\right)$ in the rat heart up to $24 \mathrm{~h}$ after 5 min of coronary artery occlusion (CAO) in the conscious rat.

Materials and Methods: After approval by the local animal care committee, 28 male Wistar rats were in a first operation chronically instrumented with a coronary artery occluder. After a recovery period of 7 days, the animals were ischemic preconditioned by a 5 min coronary occlusion in the awake animal. The SHAM animals were not preconditioned. Immediately $(0 \mathrm{~h})$, or 2, 4, 8, 12 or $24 \mathrm{~h}$ later the hearts were excised. Myocardium of the area at risk was proceeded for Western Blot detection of the three OR subtypes. All data are mean \pm SD, Statistics: Student's t-test and Bonferroni's correction for multiple comparisons, $\mathrm{P}<0.05$ was regarded as significant.

Results and Discussions: We could detect all three OR subtypes in the rat heart. $\delta$-OR were increased $8-24 \mathrm{~h}$ after LPC $\left({ }^{*} \mathrm{P}<0.05\right)$.
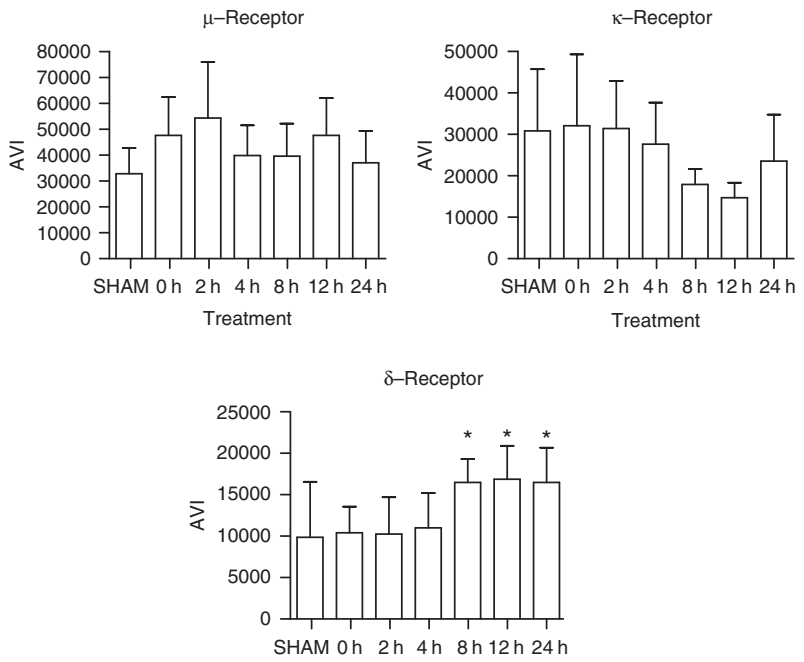

Treatment

Conclusion(s): Starting $8 \mathrm{~h}$ after LPC $\delta-\mathrm{OR}$ protein levels are increased after $5 \mathrm{~min}$ of CAO. LPC increases the $\delta$-OR expression in the rat heart. Therefore $\delta$-OR could be involved in ischemic LPC in rat hearts in vivo.

References:

1 Fräßdorf J. et al. Anesthesiology 2005; 103:A555.

2 Stegmann I. et al. Abstract DAC 2006, P02.1.10.

\section{AP4-7}

Carbon monoxide inhalation attenuates inflammatory cytokine response after aortic ischemia and reperfusion

P. Schober, A. Koch, J. Weimann, K. Zacharowski, S.A. Loer

Department of Anaesthesiology, VU University Medical Center, Amsterdam, The Netherlands

Background and Goal of Study: Carbon monoxide inhalation has been shown to exert anti-inflammatory effects and to confer protection against ischemia-reperfusion injury in various organs [1]. Ischemia and subsequent reperfusion of the aorta is known to induce a systemic inflammatory response with elevated plasma cytokine levels [2]. Therefore, we tested the hypothesis that the inflammatory cytokine response due to aortic clamping can be attenuated by carbon monoxide inhalation.

Materials and Methods: With approval of the local Animal Investigation Committee, anaesthetized, intubated and mechanically ventilated $\left(\mathrm{FiO}_{2} 1.0\right.$, normocapnia) male Wistar rats $(n=26)$ underwent midline laparotomie and dissection of the retroperitoneum to expose the aorta. The rats were randomly assigned to three groups: the infrarenal aorta of animals assigned to the control-goup ( $n=10$ ) was clamped for 2 hours, followed by a 3 hour reperfusion period. In the intervention-group $(n=10)$, the same protocol was applied but 250 parts per million carbon monoxide was added to the inspiratory gas, beginning from 1 hour prior to clamping until the end of reperfusion. Sham animals $(n=6)$ underwent no clamping but were mechanically ventilated for the same period of time as in the other groups. At the end of the reperfusion period, blood plasma was collected and the levels of interleukins 4, 6, 10 and 12 were determined by microsphere array technique (Luminex 100 system, Luminex, Austin, TX, USA). Statistics: mean \pm SD, ANOVA + Bonferroni
Results and Discussions: Interleukin plasma levels were significantly $(p<0.05)$ lower in CO treated animals than in controls (IL-4: $0.05 \pm 0.04$ vs $0.12 \pm 0.10 \mathrm{pg} / \mathrm{ml} ; \quad$ IL-6: $1842 \pm 1396$ vs $3959 \pm 1962 \mathrm{pg} / \mathrm{ml} ; \quad$ IL-10: $221.2 \pm 126.9$ vs $393.6 \pm 169.4 \mathrm{pg} / \mathrm{ml}: \quad$ IL-12: $194.4 \pm 136.5$ vs $332.3 \pm 120.0 \mathrm{pg} / \mathrm{ml})$.

Conclusion: Carbon monoxide inhalation attenuated the cytokine response, suggesting an anti-inflammatory effect in this model of aortic ischemia and reperfusion.

References:

1 Wu L and Wang R. Pharmacol Rev 2005; 57: 585-630.

2 Holzheimer RG et al. Shock 1999; 11: 305-310.

\section{AP4-8}

Rat chromosome 18 substitution-dependant differences in mitochondrial function are associated with anesthetic preconditioning

J. Novalija, V. Frkovic, E. Novalija

Anesthesiology, Medical College of Wisconsin, Milwaukee, USA

Background and Goals: Isoflurane produces a delayed preconditioning (APC) against myocardial ischemia and reperfusion injury (1). In this study we tested if APC mediates protection in isolated mitochondria by preserving mitochondrial respiratory function after anoxia and reoxygenation in two genotypically distinct parental strains of rats (Dahl Salt Sensitive [SS] and Brown Norway [BN]) and in a consomic (chromosomal transfer) strain (SS-18BN) (2). Materials and Methods: APC trigger was achieved in vivo by 120 min exposure to isoflurane (1.0 minimum alveolar concentration) in three male adult (9-12 weeks-old) animals of each strain. Respective control groups were not subjected to above treatment. Twenty-four hours later mitochondria $\left(0.5 \mathrm{mg} \cdot \mathrm{mL}^{-1}\right)$ were isolated by differential centrifugation and basal rates of ADP-dependent (state 3) and ADP-independent (state 4) respiration were measured using a Clark-type oxygen electrode in a sealed, stirred chamber. Anoxia was induced via consumption of available oxygen and maintained for $15 \mathrm{~min}$. The chamber was then exposed to room air and respiratory rates in the presence of ADP $(250 \mu \mathrm{M})$ were calculated again. Data are means \pm SEM $\left(p<0.05 ;{ }^{*}\right.$ vs. BN).

Results: There were no differences in basal mitochondrial respiratory rates between strains. Rates of state 3 and state 4 respiration declined as a result of anoxia and reoxygenation and were significantly lower in SS group compared to $\mathrm{BN}$ and SS-18BN groups. Respiratory rates between three control groups were not different.

Conclusions: This study demonstrates significant difference in preservation of the coupling between respiration and phosphorylation afforded by APC between two inbred strains that can be changed by a single chromosomal substitution. This knowledge provides a basis for selectively studying genetic factors associated with mechanisms of APC related to this chromosomal substitution.

References:

1 Tanaka K. Anesthesiology 2004; 100: 525-31.

2 Cowley AW. Acta Physiol Scand 2004; 181: 585-92.

\section{AP5-1}

Cardiac surgery with cardiopulmonary bypass: does aprotinin affect outcome

D. Van Doninck, A. Daper, A. Trenchant, Ph. Van Der Linden, S. de Hert

Anesthesiology, Universital Hospital Antwerp, Edegem, Belgium

Background: Aprotinin therapy is mostly confined to a population at increased risk for perioperative blood transfusion. This population is also at risk for postoperative adverse events. This study evaluated the effects of aprotinin on outcome (mortality, cardiac events, renal failure, and cerebrovascular events) in patients undergoing cardiac surgery with cardiopulmonary bypass.

Materials and methods: Data were obtained in patients who received a strict blood conservation protocol: no antifibrinolytic therapy when at low risk $(n=854)$, and aprotinin $(n=1210)$ when at high risk for blood transfusion. Relative risk of different pre- and intra-operative variables was calculated for the different outcome variables. Backward stepwise regression analysis was used to identify the independent risk factors associated with the different outcome variables.

Results: Postoperative mortality and morbidity were higher in the aprotinin group but this was related to an increased incidence of peri-operative risk factors. Mortality was similar to what was predicted by the Euroscore. Complex surgery was the only independent variable associated with postoperative cardiac events. Preoperative heart failure and renal dysfunction, urgency and redo surgery were the independent variables associated with postoperative 
haemodialysis. Age $>70$ years was identified as the only independent variable associated with neurologic dysfunction.

Conclusions: In the present study population the increased postoperative morbidity in patients receiving aprotinin as part of a blood conservation strategy was related to their higher risk profile. For none of the outcome variables studied was aprotinin administration identified as an independent risk factor.

\section{AP5-2}

\section{Effect of preoperative medication on postoperative outcome} in coronary surgery

E. De Wit, P. verdonck, J. Wens, Ph. Van Der Linden, S. De Hert Anesthesiology, Universital Hospital Antwerp, Edegem, Belgium

Introduction: Several studies have assessed the potential beneficial effects of chronic pre-operative medication on postoperative outcome. Results of these studies however are conflicting. The present study investigated the effects of pre-operative medication on outcome variables after coronary surgery.(1) Material and methods: In a retrospective study on 1670 coronary surgery (CABG) patients, preoperative risk-factors and medication were related to the following postoperative outcome variables: 30 -day mortality, myocardia infarction, low cardiac output, and hemodialysis. Relative risks were calculated for the individual pre-operative data. All significant variables were entered in a backward stepwise regression analysis to identify the independent risk factors. Statistical significance was accepted at $p<0.01$.

Results: Age $>70$ years, diuretic therapy and low molecular weight heparin therapy were identified as the significant independent predictors of mortality. Unstable angina and clopidogrel therapy were identified as the significant independent predictor for postoperative myocardial infarction. Age $>70$ years, diuretic therapy, sex, unstable angina and pre-operative myocardial infarction were identified as the significant independent predictors for postoperative low cardiac output. Diuretic and clopidogrel therapy were identified as the significant independent predictors for the need for postoperative hemodialysis. Conclusions: Only pre-operative diuretic, low molecular weight heparin and clopidogrel therapy were identified as independent risk factors for outcome after coronary surgery. Other chronic pre-operative medication such as $\beta$-blocking therapy, calcium channel blockers, angiotensin converting enzyme inhibitors, angiotensin II antagonists, acetylsalicylic acid and nitrates did not affect outcome in this particular patient population.

\section{Reference:}

1 Wijeysundera DN et al: J Thorac Cardiovasc Surg 2004; 127: 755-62.

\section{AP5-3}

\section{Effect of preoperative medication on postoperative outcome} in cardiac valve surgery

E. De Wit, J. Wens, P. Verdonck, Ph. Van Der Linden, S. De Hert Anesthesiology, Universital Hospital Antwerp, Edegem, Belgium

Introduction: Several studies have assessed the potential beneficial effects of chronic pre-operative medication on postoperative outcome. The present study investigated the effects of pre-operative medication on outcome variables after cardiac valve surgery. ${ }^{(1)}$

Material and methods: In a retrospective study on 477 cardiac valve surgery patients, preoperative risk-factors and medication were related to the following postoperative outcome variables: 30 -day mortality, myocardial infarction, low cardiac output, and hemodialysis. Relative risks were calculated for the individual pre-operative data. All significant variables were entered in a backward stepwise regression analysis to identify the independent risk factors. Statistical significance was accepted at $\mathrm{p}<0.01$.

Results: Nitrates and low molecular weight heparin therapy were identified as the significant independent predictors for postoperative low cardiac output. The only significant independent predictor identified for postoperative creatinine $>2$ was age $>70$ years. Postoperative mortality, need for haemodialysis and postoperative occurrence of AMI were not significantly influenced by any preoperative medication ( $\beta$-blockers, calcium channel blockers, nitrates, angiotensin converting enzyme inhibitors, diuretics, LMWH, acetylsalicylic acid or clopidogrel) or other, general preoperative factors (urgency, sex, age $>70$ years, unstable angina, myocardial infarction, diabetes, hypercholesterolemia or peripheral arterial disease) in this particular population.

Conclusions: Only pre-operative nitrates and low molecular weight heparin therapy were identified as independent risk factors for outcome after cardiac valve surgery. Other chronic pre-operative medication did not affect outcome in this particular patient population.

Reference:

1 Wijeysundera DN et al: J Thorac Cardiovasc Surg 2004; 127: 755-62.

\section{AP5-4 \\ Eliminating cardiopulmonary bypass for CABG: a propensity score analysis}

O. Fochi, E. Dedola, M.G. Calabrò, E. Cristallo, G. Marino

Department of Cardiothoracic Anesthesia and Intensive Care, San Raffaele Scientific Institute, Milano, Italy

Background and Goal of Study: To investigate if the off-pump technique could reduce the hospital mortality after coronary artery bypass grafting when compared to the standard cardiopulmonary bypass technique.

Materials and Methods: An observational study with propensity score matching analysis was performed in a university teaching hospital in 2899 consecutive patients undergoing elective coronary artery bypass grafting. No intervention was performed. Major perioperative complications and hospital mortality were noted.

Results and Discussions: The overall hospital mortality was 1.3\% (39/2899) with no difference between the off-pump (16/802, 2.0\%) and the CPB group $(23 / 2097,1.1 \%) p=0.09$. Since the off-pump group included patients at high risk, a propensity score analysis was then performed and off-pump patients matched 1:1 to CPB patients in order to have the same preoperative variables identified by a multivariate analysis as associated to surgeon propensity to operate off-pump: (age, chronic renal failure and low ejection fraction) and the same number of graft performed. The results of the propensity matching still show no difference in hospital mortality between off-pump and CPB group $(1.6 \% \vee 1.1 \% p=0.6)$. The off-pump technique showed advantages in terms of transfusion of blood products $(p<0.001)$ and reduction of surgical re-exploration $(p=0.04)$.

Conclusions: No difference in hospital mortality in coronary artery bypass grafting patients could be observed between patients operated off-pump or with the classic cardiopulmonary bypass technique.

\section{AP5-5}

Aprotinin and perioperative complications in cardiac surgery M. Kertai, K. Varga, D. Royston, C. Grebenik, J. Gal

Cardiovascular Surgery, Semmelweis University, Budapest, Hungary

Background: Recently, the clinical significance of aprotinin-induced renal dysfunction and other end-organ ischemic events in patients undergoing cardiac surgery has engendered substantial controversy. Therefore, we assessed the effect of aprotinin on perioperative complications in cardiac surgery patients. Methods: We prospectively evaluated 674 patients (mean age $65.4 \pm 11.0$ years, 457 males) undergoing cardiac surgery between January 1 and December 31, 2005 at Semmelweis University. Preoperative patient characteristics and intraoperative and postoperative clinical and surgical variables were recorded. Patients administered aprotinin received the drug either as a loading dose of 1 million kallikrein-inhibitor units (KIU), with a total dose of more than 2 million KIU (a low-dose regimen); or a loading dose of 2 million KIU, with a total dose of more than 4 million KIU (a high-dose regimen). The outcomes were renal complications defined as a $25 \%$ reduction in postoperative calculated creatinine clearance compared to the preoperative baseline or renal failure requiring dialysis; and the composite of renal, cardiovascular and cerebrovascular complications and all-cause mortality.

Results: Patients underwent coronary artery bypass surgery (63\%), valvular $(27 \%)$ or a combination $(5 \%)$ and surgery on the ascending aorta $(5 \%)$. There were 550 patients $(81.6 \%)$ who received aprotinin treatment. In multivariate regression analyses when the relation between high- or low dose aprotinin compared to no aprotinin was evaluated, the likelihood of renal complications (high dose: Odds Ratio [OR] = 1.4, 95\% confidence interval [Cl], 0.6-3.0, $\mathrm{p}=0.4$; low dose: $\mathrm{OR}=1.2,95 \% \mathrm{Cl}, 0.7-2.3, \mathrm{p}=0.5)$, and the composite outcome variable (high dose: $\mathrm{OR}=1.6,95 \% \mathrm{Cl}, 0.8-3.4, \mathrm{p}=0.2$; low dose: $\mathrm{OR}=1.3,95 \% \mathrm{Cl}, 0.7-2.3, \mathrm{p}=0.4$ ) were not significantly increased. Although aprotinin use (either group) was ineffective in reducing the requirement for transfusion of blood products it was associated with a significant reduction in mediastinal drainage in the first 12 hours after surgery (high dose, $400 \mathrm{ml}$, interquartile range [IQR], 300-650 ml; low-dose, $400 \mathrm{ml}, 300-500 \mathrm{ml}$; no use, $700 \mathrm{ml}, 500-800 \mathrm{ml}, \mathrm{p}<0.001)$.

Conclusion: Our analysis suggests that aprotinin use in either a high or low dose regimen was not associated with an increase in adverse ischemic outcomes.

\section{AP5-6}

Intraoperative blood glucose concentration: relevance for postoperative outcome

R.M. Basciani, F.F. Immer, C. Zobrist, T. Carrel, B. Eberle

Department of Anaesthesiology, University of Bern, Bern, Switzerland 
Background and Goal of Study: Blood glucose (BG) and its indices (e.g. variability) are important variables in critically ill patients, myocardial infarction and neuro-logical complications $(1,2,3)$. However, most studies were conducted in intensive care units. Goal of this study was to evaluate the significance of intraoperative BG indices for perioperative outcome.

Materials and Methods: 212 consecutive anaesthesia records and hospital charts were analysed from patients undergoing surgery for acute type $A$ aortic dissection with deep hypothermic circulatory arrest. Intraoperative BG values, demographics and outcome data were extracted. SD and coefficient of variability (SD*100/mean BG) were calculated. Statistics: Correlations between BG indices and outcome (mortality, persistent neurological deficit, myocardial infarction, and length of stay in the intensive care unit (LOS ICU)) were analysed. Results and Discussion: There were 434 BG measure-ments, yielding up to $\mathrm{n}=212$ independent BG index values (mean, SD, coefficient of variability, and $B G$ on admission). Demographic data, duration of deep hypothermic circulatory arrest, and BG indices were not significantly different between the following outcome categories: survivors/nonsurvivors; persistent neurological deficit yes $/ \mathrm{no}$, myocardial infarction yes/no. Coefficient of BG variability was significantly but very weakly correlated to mortality and LOS ICU (Spearman correlation coefficient $\mathrm{r} 0.10$ and $-0.21 ; p=0.04$ and 0.03 , respectively). No other significant association between $B G$ indices and outcome parameters was found.

Conclusion: In patients undergoing surgery for acute type A aortic dissection, there is no clinically relevant correlation between intraoperative BG indices and mortality, persistent neurological deficit, myocardial infarction, and length of stay in the intensive care unit.

References:

1 Egi M. Anesthesiology 2006; 105: 244-252.

2 Foo K. Heart 2003; 89(5); 512-516.

3 Capes SE. Stroke 2001; 32(10): 2426-2432.

\section{AP5-7}

Predictors of intra-operative regional cerebral oxygen saturation decrease in patients with risk factors for cerebral hypoxia undergoing cardiac surgery

J. Viterbo, A.P. Lourenço, P. Maia, C. Soares, F. Vieira

Serviço de Anestesiologia, Hospital de São João, Porto, Portugal

Background and Goal of Study: Monitoring regional cerebral oxygen saturation $\left(\mathrm{rSO}_{2}\right)$ identifies cerebral hypoxia during cardiac surgery, probably improving outcome and reducing ICU and hospital stay. Cost restrictions may limit its use to high-risk patients. Our goal was to test the adequacy of a set of criteria based on age, carotid stenosis and previous stroke.

Materials and Methods: We continuously monitored $\mathrm{rSO}_{2}$ (INVOS 4100) in 26 elective cardiac surgery patients. Inclusion criteria were: age above 70 years, carotid stenosis superior to $50 \%$ or previous stroke. Medical history and per-operative information were obtained. All patients received general anesthesia with propofol, remifentanil, and vecuronium under bispectral index monitoring. Statistical analysis used Mann-Whitney, chi-square and Fisher's exact test. Results are mean \pm SEM for quantitative variables.

Results and Discussions: Of the 26 patients $(73.5 \pm 0.9 \mathrm{yrs}$ old, $46 \%$ female, all ASA III/IV, EuroSCORE $=7.8 \pm 0.5$ points) $73 \%$ were older than 70 yrs, $42 \%$ presented carotid stenosis, and $33 \%$ had previous stroke; $38 \%$ underwent valve replacement, $42 \%$ aortocoronary bypass, and $15 \%$ both, with synchronous carotid endarterectomy in $12 \%$; one patient underwent left atrial myxoma exeresis. During surgery, 14 patients (54\%) presented $\mathrm{rSO}_{2}$ decrease superior to $20 \%$ from baseline or absolute decrease below $50 \%$ that lasted $17.6 \pm 27.2 \mathrm{mins}$. These patients showed a longer time to extubation (18.8 \pm 7.0 vs $6.7 \pm 1.5 \mathrm{~h}, \mathrm{P}=0.035$ ), but no difference in ICU or hospital stay. They were not different regarding age $(74.3 \pm 1.2$ vs $72.6 \pm 1.6 \mathrm{yrs})$, stroke history (50/50\%), EuroSCORE ( $8.4 \pm 0.6$ vs $7.1 \pm 0.7$ points), type of surgery (79 vs $58 \%$ open-heart), duration of surgery, associated medical conditions or usual medication, but had higher ASA status (86 vs $25 \%$ ASA $\mathrm{IV}, \mathrm{P}=0.0064)$ and greater frequency of carotid stenosis above $60 \%$ (29 vs $8 \%, \mathrm{P}=0.036$ ). Only the latter two predicted intra-operative $\mathrm{rSO}_{2}$ decrease in multivariate stepwise regression.

Conclusion(s): Our preliminary results in aged high-risk patients undergoing cardiac surgery suggest that ASA status and carotid stenosis above $60 \%$, but not age, previous stroke or EuroSCORE, may predict intra-operative $\mathrm{rSO}_{2}$ decrease.

\section{AP5-8}

Postoperative hyperglycaemia - a marker of increased risk of complication or death in non-diabetic patients following coronary artery surgery

P. Nadziakiewicz, P. Knapik, E. Urbanska, E. Kucewicz-Czech, J. Foremny

Cardioanesthesia, Silesian Centre for Heart Diseases, Zabrze, Poland
Background and Goal of Study: Control of hyperglycaemia in patients after coronary artery surgery is known to be vital [1]. The aim of this study was to find out whether difficult glycaemia control in the early postoperative period is linked to postoperative complications.

Materials and Methods: 585 consecutive non-diabetic patients who underwent first-time coronary artery surgery (412 CABG, 173 OPCAB) in a period of one year had their blood glucose levels (BGL) assessed postoperatively every hour during in the first postoperative day. Group A had all BGL $<12 \mathrm{mmol} / \mathrm{l}$ (334 CABG, 160 OPCAB), while group $B$ had at least one $B G L \geqslant 12 \mathrm{mmol} / \mathrm{l}$ (78 CABG, 13 OPCAB). Complications were recorded in our database and compared between groups. Descriptive statistics and t-test were used. $\mathrm{p}<0.05$ was considered significant.

Results and Discussions: Patients in group $B$ were older, with higher EuroSCORE and more often operated in CABG mode. In group $B$ we have observed significantly more deaths $(0.41 \%$ vs. $4.4 \%, p<0.01)$, low $\mathrm{CO}$ syndromes $(14.1 \%$ vs. $36.3 \%, p<0.001)$, perioperative $\mathrm{Ml}(1.02 \%$ vs. $9.9 \%$, $\mathrm{p}<0.001)$, neurological $(4.3 \%$ vs. $12.1 \%, \mathrm{p}<0.01)$ and renal $(2.1 \%$ vs. $6.6 \%, p<0.05)$ complications.

Conclusion(s): Elevated blood glucose levels in the postoperative period are linked to the increased risk of complications and death in non-diabetic patients after coronary artery surgery.

Reference:

1 Furnary AP; J Thorac Cardiovasc Surg 2003, 125, 1007.

\section{AP6-1}

Effect of acute normovolemic hemodilution on left ventricular function in coronary surgery patients

S. Cromheecke, S. Lorsomradee, P. Ten Broecke, Ph. Van Der Linden, S. De Hert

Anesthesiology, Universital Hospital Antwerp, Edegem, Belgium

Background: Acute normovolemic hemodilution (ANH) may induce profound hemodynamic alterations which may have deleterious effects on left ventricular function. Previous echocardiographic studies have indicated that $\mathrm{ANH}$ could be safely performed in coronary surgery patients ${ }^{(1)}$. The present study analyzed the effects of ANH using LV pressure measurements in coronary surgery patients.

Materials and methods: 82 elective coronary surgery patients were included in this study. A pressure micromanometer was inserted in the left ventricle. The measurements consisted of recordings of electrocardiographic and LV pressure tracings during an increase in systolic and diastolic pressure, obtained by leg elevation. Measurements were obtained before and after ANH. Data were compared using paired t-test. All data were expressed as mean $\pm S D$. Data were considered significant if $p<0.05$.

Results: $\mathrm{ANH}$ resulted in a decrease in hematocrit from $39.8 \pm 3.1$ to $30.1 \pm 1.7 \%$.

In all patients cardiac output (CO) increased significantly from $5.6 \pm 1.3$ to $6.8 \pm 1.7 \mathrm{l} / \mathrm{min}$ after ANH. This was associated with a significant decrease in systemic vascular resistance (SVR) from $977 \pm 214$ to $782 \pm 153$ dynes.sec.cm ${ }^{-5}$. However, in 12 of the patients ANH was associated with a significant decrease in $\mathrm{dP} / \mathrm{dt}_{\max }$ (from $873 \pm 216$ before $\mathrm{ANH}$ to $680 \pm 204 \mathrm{mmHg} / \mathrm{s}$ after $\mathrm{ANH}$ ) and a significant increase in time constant of isovolumic relaxation $\tau(65 \pm 7$ before $\mathrm{ANH}$ to $77 \pm 12 \mathrm{~ms}$ after $\mathrm{ANH})$, while in the remaining 70 patients, this depression did not appear. $\left(\mathrm{dP} / \mathrm{dt}_{\text {max }}\right.$ : $868 \pm 160$ before ANH and $854 \pm 152 \mathrm{mmHg} / \mathrm{s}$ after ANH, $\tau: 64 \pm 9$ before $\mathrm{ANH}$ and $67 \pm 9 \mathrm{~ms}$ after $\mathrm{ANH})$.

Conclusion: Although ANH appeared mostly well tolerated in patients with coronary artery disease, it may be associated with a depression of myocardial function not reflected in the global variables of the hemodynamics. Reference:

1 Licker et al: Crit Care Med 2005; 33: 591-597.

\section{AP6-2}

The effects of microcirculatory responses to hypovolemic shock following resuscitation with Ringer's acetate and Ringer's lactate solutions

M. Mielniczuk, L. Krokowicz, S. Gibson, K. Kusza, M. Siemionow

Plastic surgery, Cleveland Clinic, Cleveland, USA

Background and Goals: There is no clear indication which volume replacement therapy may have the best prognosis on hemorrhagic resuscitation. Aim of the study was comparison of volume replacement with Ringer's acetate $(R A)$ versus Ringer's lactate $(R L)$ solutions following induction of hypovolemic shock (HS).

Material and Methods: Rat cremaster model was used to evaluate in vivo microcirculatory changes after induction of HS by withdrawal of $15 \%$ of total 
blood volume corresponding to decreasing MAP from basal $82.9( \pm 6.76)$ $\mathrm{mmHg}$ to $47.8( \pm 3.6) \mathrm{mmHg}$ after HS. Rats were randomized into 4 groups of 10 each: 1) control (CTRL) and had microcirculation hemodynamic parameters taken for four consecutive hours; 2) hemorrhagic shock (HSG) had blood withdrawn; 3) Ringer's acetate (RAG), had blood taken and replaced with $\mathrm{RA}$; 4) Ringer's lactate (RLG), had blood withdrawn and replaced with RL. During 4 hrs microcirculatory measurements of RBC velocity, vessel diameter, functional capillary perfusion (FCP) and leukocyte-endothelial interactions where recorded. Endothelial micropermeability (EMP) was evaluated after $4 \mathrm{hrs}$ by FITC-albumin extravasation and immunofluorescence image analysis. The vital parameters (MAP, CVP, HR), as well as tissue oxygenation measurements were performed continuously.

RESULTS: At 4 hrs after volume resuscitation with RAG and RLG $66 \%$ return of FCP was recorded compared to $33 \%$ in HSG. There were no significant differences between RAG and RLG (after four hr. $8.48 \pm 0.36$ vs. $8.64 \pm 0.22$ ). RA resuscitation significantly reduced $(P<0.001)$ level of sticking and transmigrated leukocytes (PMN) compared to HSG. There were no significant differences between the HSG and RLG. The animals treated with volume replacement presented higher tissue oxygenation than HSG (RAG - $9.5 \pm 0.25$ and RLG $9.2 \pm 0.22$ vs. HSG $8.1 \pm 0.15 \mathrm{mmHg}$ ).

CONCLUSIONS: After HS induction Ringers resuscitation significantly improved microcirculatory hemodynamics, however (RAG and RLG) hemodynamics never returned to pre-shock values. Ringer's acetate administration decreased level of sticking and transmigrating PMN's. Histological assessment showed degree of systemic inflammatory responses caused by HS in case of each group.

\section{AP6-3}

\section{Differences between a conventional intraoperative fluid} management and a restrictive fluid regime in abdominal surgery F. Rocha, D. Diaz, M. Barreiro Anesthesiology, Complexo Hospitalario de Ourense, Ourense, Spain

Background: The correct perioperative fluid management is still an unresolved discussion. Underneath we report a study that evaluate the impact of two different types of fluid regimes on postoperative outcome.

Material and Methods: Sixty patients who were undergoing elective intraabdominal surgery were assigned to two cohorts. Surgical procedures included colon resection, gastric resection and rectum resection. One cohort, $n=30$, recived a conventional fluid intraoperative regime of $10 \mathrm{ml} / \mathrm{kg} / \mathrm{h}$ of lactated Ringer's solution [CFC]. The other cohort, $n=30$, recived a restrictive fluid management of $5 \mathrm{ml} / \mathrm{kg} / \mathrm{h}$ of lactated Ringer's solution [RFC]. We excluded from the study pregnant patients, patients aged younger than 18 years, and those with coagulophaty, hepatic or renal disfunction, ASA V-VI, and congestive heart failure. Primary endpoint was the number of patients who died or experienced complications. The secondary endpoints included : Time to initial passage of flatus. Hemoglobin, hematocrit, creatinine, $\mathrm{Na}$ and $\mathrm{K}$ serum concentration in the postoperative. Central Venous Presure (CVP) during the surgery.

Results and Discussion: None of the patients died during the perioperative and postoperative period. The number of patients with complications was smaller in the $[\mathrm{RFC}]$ compared with [CFC] $(p<0.05)$. The pneumonia was the most common complication and it was higher in the [ CFC] than in the [RFC] $(p<0.05)$. Patients in the RFC passed flatus significantly earlier than CFC $(p<0.05)$. There were no significant differences between the cohorts in hematocrit, haemoglobin, creatinine, $\mathrm{Na}$ and $\mathrm{K}$ serum concentrations in the immediate postoperative period $(p=0.75)$. CVP was significantly higher in CFC compared with RFC; however, at discharge there were no significant differences between the cohorts $(p=0.98)$.

The current study included patients ASA III, and were performed in relatively homogeneus group of type of surgical procedures. Other major difference between other studies includes the intraoperative use of epidural catheter and the CVP intraoperative measurement.

Conclusions: The intraoperative fluid restriction in patients who are undergoing major intraabdominal surgery reduces the number of complications and shortens the time to recovery of gastrointestinal function.

\section{AP6-4}

\section{Hemodynamic changes at the end of prolonged gynecological laparoscopy (GL)}

W. Skorzynski, K. Przesmycki

II Dept of Anaesthesiology and Intensive Care, F. Skubiszewski Medical University in Lublin and PWSZ in Biala Podlaska, Poland, Lublin, Poland

Background and Goal of Study: More prolonged GL are being performed in last years. The recent experimental study suggests that a significant decrease in cardiac index could occur after prolonged pneumoperitoneum (PP) [1]. The goal of this study was to compare aortic blood flow (ABF), total systemic vascular resistance referenced to ABF (TSVRa) measured by transesophageal Doppler device (TED) with classical hemodynamic data of mean arterial pressure (MAP) and heart rate (HR) obtained at the end of different lasting GL.

Materials and Methods: 57 patients ASA I/II undergoing GL were enrolled after an informed consent was obtained. Propofol-fentanyl anaesthesia and noninvasive monitoring were performed according to institutional standard. The Doppler probe (Hemosonic-Arrow) (TED) was inserted after tracheal intubation. The mean values $( \pm S D)$ of ABF, TSVRa, MAP and HR at the end of GL 5 min before vs. 5 min after CO2-desufflation (PP-DES) were analyzed and compared as a percentage change of initial values $5 \mathrm{~min}$ before PP in Trendelenburg position in 3 groups of patients according to the duration of PP: $<30 \mathrm{~min}, 30-60 \mathrm{~min},>60 \mathrm{~min}$. Results were analyzed using Student's ttest with statistical significance $p<0.05$.

Results and Discussion: Significant increase and decrease of MAP \& TSVRa respectively were observed, before and after PP-DES in all groups. TSVRa but not MAP, was significantly increased at the end of prolonged GL (before PP$D E S), P P>60$ min vs. $P P<60$ min. HR did not change significantly after PPDES in all groups. Significant decrease of ABF was observed before PP-DES in GL lasting 30-60 min and $>60$ min vs. PP $<30 \mathrm{~min}$. PP-DES produced significant increase of $A B F$ in all groups.

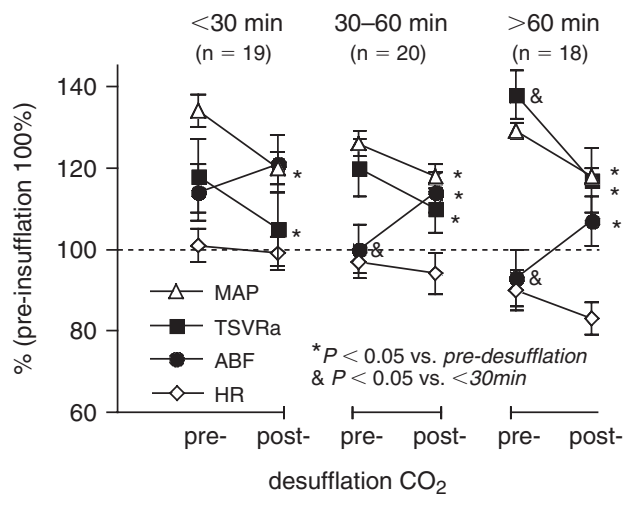

Conclusion: Continuous hemodynamic monitoring with noninvasive TED but not classical hemodynamic data (MAP, HR) allows to recognize the increase of TSVR and decrease of ABF in prolonged GL.

Reference:

1 Sümpelmann R. et al. Br J Anaesth 2006; 96: 563-8.

\section{AP6-5 \\ High thoracic epidural anesthesia attenuates left ventricular dysfunction causing aortic clamp during abdominal aortic aneurysm repair}

J. Ariyama, H. Imanishi, Y. Sugimoto, Y. To-oyama, A. Kitamura Anesthesiology, Tonami General Hospital, Tonami, Japan

Background and Goal of Study: Several studies have demonstrated myocardial dysfunction partially due to afterload increase and diastolic dysfunction by infrarenal aortic cross-clamping during infrarenal abdominal aortic aneurysm (AAA) repair under general anesthesia. At the same time high thoracic epidural anesthesia (HTEA) is reported to improve cardiac function due to afterload and diastolic characteristics. Our objective was to investigate whether HTEA attenuated hemodynamic response with aortic cross clamp.

Material and Methods: We studied 25 patients who underwent elective repair of AAA. Twelve patients received high thoracic anesthesia (THE) and thirteen patients did not (non-THE). Transesophageal echocardiography and hemodynamic measures were recorded before and after aortic cross clamp.

Result: Significant difference ( $p$ less than 0.05) were observed between HTEA group and non-HTEA group at aortic clamping in the left ventricular ejection fraction (from 0.56 to 0.45 versus from 0.58 to 0.53 ), end-diastolic volume (from 171 to $219 \mathrm{ml}$ versus from 168 to 200), end-systolic volume (from 85 to $124 \mathrm{ml}$ versus from 83 to 93), mean blood pressure (from 83 to $95 \mathrm{mmHg}$ versus from 78 to 83 ), and end-systolic wall stress (from 53 to $7010^{(3)} \mathrm{dyne} / \mathrm{cm}^{2}$ versus from 50 to 55$)$.

Conclusion: HTEA attenuates hemodynamic dysfunction due to aortic cross clamp during AAA repair. 


\section{AP6-6}

Evidence of differential renal dysfunction secondary to ischaemia reperfusion injury following liver transplantation and major vascular surgery

S. Turner, M. Bosomworth, M. Bellamy, S. Homer-Vanniasinkam, S. Howell Academic Unit of Anaesthesia, The General Infirmary at Leeds, Leeds, United Kingdom

Background and Goal of Study: Renal damage secondary to ischaemia reperfusion injury (IRI) is a recognised feature complicating vascular and transplant surgery. Urinary markers of tubular dysfunction may allow detection of renal injury. $\mathrm{N}$-acetyl- $\beta$-D glucosaminidase $(\beta-\mathrm{NAG})$ is a marker of proximal convoluted tubule (PCT) dysfunction, occurring as a consequence of an increased tubular protein load [1]. Albuminuria reflects an increase in glomerular permeability. Alpha-1 microglobulinuria $(\alpha-1 \mathrm{M})$ represents damage to the PCT. We present data on the magnitude and nature of renal IRI in orthotopic liver transplantation (OLT) and elective abdominal aortic aneurysm (AAA) surgery. Materials and Methods: Randomised controlled trials were performed to study the effects of IRI on renal tubule dysfunction following OLT $(n=34)$ and AAA repair $(n=20)$. Urine samples were taken to detect changes in $\beta$-NAG, albumin and $\alpha-1 \mathrm{M}$.

Results and Discussions: Following reperfusion there was a significant increase in albuminuria in both groups. Both groups exhibited changes in $\alpha-1 \mathrm{M}$ and $\beta$-NAG consistent with altered function within the PCT. However the magnitude of the $\beta$-NAG changes was five-fold greater in the OLT group.
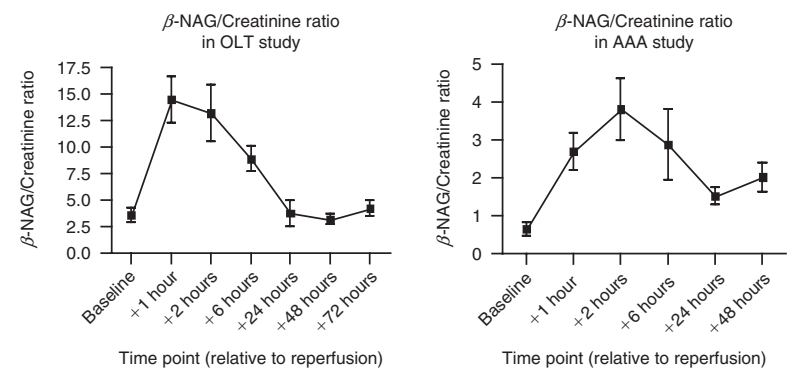

Conclusions: Reperfusion results in subclinical renal dysfunction. The reduced elimination of $\beta$-NAG in the AAA group suggests less PCT lysosomal turnover as compared to the OLT group. This may be due to a reduced renal tubular protein load due to collateralisation in the AAA patients.

Reference:

1 Bosomworth MP. Nephrology Dialysis Transplantation. 1999; 14: 620-6.

\section{AP6-7}

The effects of pneumoperitoneum and head-up positioning on the activity of the autonomic nervous system and QT dispersion during laparoscopic surgery

T. Cafiero, R.M. Di Minno, C. Di lorio

Anaesthesia, Postoperative Intensive Care, Burn Center and Hyperbaric Center, "Antonio Cardarelli" Hospital, Napoli, Italy

Background and Goals: The effects of pneumoperitoneum (PP) and headup positioning (HUP) on the activity of the autonomic nervous system (ANS) and QT dispersion (QTD) can lead to adverse cardiocirculatory events during laparoscopic surgery $(1,2)$. The aim of this study was to compare the effect of PP and HUP on ANS and QTD during two different anaesthetic techniques.

Material and Methods: 30 patients without cardiorespiratory disease, undergoing elective laparoscopic cholecystectomy were randomly assigned to receive either total intravenous anaesthesia with propofol (TIVA, $n=15$ ) or inhalational anaesthesia with sevoflurane $(I A, n=15)$. For analgesia remifentanil was given as continuous infusion in both groups. ANS, by heart rate variability (HRV) analysis, and QT interval were monitored using computerized measurement with a 12-lead ECG system (Norav Medical, Israel). The low and high frequency bands ratio of HRV (LF/HF), QTmax, and rate-corrected QTD (QTDC) were recorded in baseline (T1), during $\mathrm{CO}_{2} \mathrm{PP}(12 \mathrm{mmHg})$ (T2) and PP + HUP (60 degrees) (T3), and at the end of procedure (T4).

Results: Data (mean) are shown in the table.

\begin{tabular}{lccc}
\hline IA & QTmax & QTDc & LF/HF \\
\hline T1 & 360 & 33 & 0.8 \\
T2 & 380 & 45 & 1.2 \\
T3 & 420 & $66^{\star}$ & $3.6^{\star}$ \\
T4 & 380 & 60 & 1.6 \\
\hline TIVA & & & \\
\hline T1 & 390 & 49 & 1.4 \\
T2 & 410 & 60 & 1.0 \\
T3 & 400 & $68^{\star}$ & 2.2 \\
T4 & 390 & 58 & 1.1 \\
\hline
\end{tabular}

Conclusions: Statistically significant increases of QTDc and sympathetic activity, which are associated with an increased risk of arrhythmias, occur during PP + HUP, regardless of the anaesthetic techniques used.

References:

1 Sato N. Surg Endosc. 2000; 14(4): 362-366.

2 Egawa H. Surg Laparosc Endosc Percutan Tech. 2006; 16(2): 78-81.

\section{AP6-8}

Haemodynamic variables during acute subclinical hypovolaemia in anaesthetized patients

D. Bystritski, A. Eden, M. Kligman, R. Pizov

\section{Anaesthesia and CCM, Carmel Medical Center, Haifa, Israel}

Background and Goal of Study: Arterial waveform variables (systolic pressure variation, SPV, and pulse pressure variation, PPV) are predictive of fluid loading responsiveness. Less is known about their response to hypovolaemia. The aim of this study was to evaluate standard hemodynamic and arterial waveform variables during acute gradual subclinical hypovolemia.

Materials and Methods: After IRB approval and informed consent 62 patients were included in this prospective study. Under general anesthesia and positive pressure ventilation they underwent gradual autodonation by steps of $2 \%$ of circulating blood volume (CBV) each, up to $20 \%$ or until a blood pressure drop by $20 \%$. SPV and PPV were measured after each step. Patients who tolerated all 10 steps of autodonation are included in this report. Data are presented as mean $\pm S D$ and were analyzed using ANOVA with Bonferroni correction $(p<0.005)$.

Results and Discussions: 20 patients tolerated acute hypovolaemia of $20 \%$ of CBV. Males/females 12/8, age $56 \pm 13$. Heart rate (HR) and systolic blood pressure (Syst.BP) changed significantly from base line only after $16 \%$ reduction of $\mathrm{CBV}$, while arterial waveform variables changed significantly after $4 \%$ (figure).

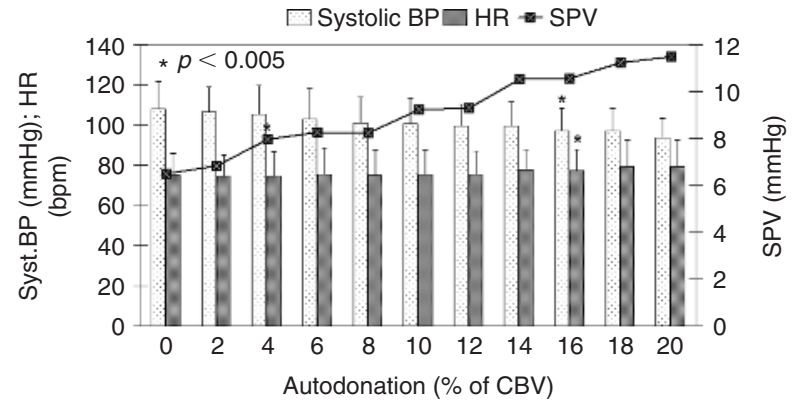

Conclusions: In patients who tolerated an acute $20 \%$ reduction of CBV under general anaesthesia there was no change in systolic blood pressure or heart rate until $16 \%$ reduction in CBV. Arterial waveform variables, however, changed gradually and significantly after $4 \%$ reduction.

\section{AP6-9}

\section{Prolonged hypervolemic hemodilution impairs functional} capillary density of ileal mucosa in pigs Z. Turek, V. Cerny, M. Oberreiter, R. Parizkova, P. Dostal

Department of Anesthesiology and Intensive Care Medicine, University Hospital Hradec Kralove, Hradec Kralove, Czech Republic

Background and Goal of Study: Hypervolemic hemodilution (HHD) is a commonly used anesthetic technique with evident effects on macrohemodynamics, but the effects on the microcirculation are not well described. Sidestream darkfield (SDF) imaging is a new method to assess microcirculation (1). SDF imaging was used to show the changes in functional capillary density (FCD) of small intestine during HHD with Hartmann's solution. 
Materials and Methods: Microcirculation of terminal ileum was assessed by SDF imaging via an ileostomy in 5 anesthetized pigs. Four sequences of microcirculatory status were recorded on-line at baseline conditions and after first, second and third hour of HHD with Hartmann's solution $(20 \mathrm{~mL} / \mathrm{kg} / \mathrm{h})$ iv and analyzed off-line. Systolic (SBP), diastolic (DBP) and mean arterial pressures were monitored continuously. One-way ANOVA on ranks was applied to compare changes in FCD during HHD.

Results: Data are presented in the table:

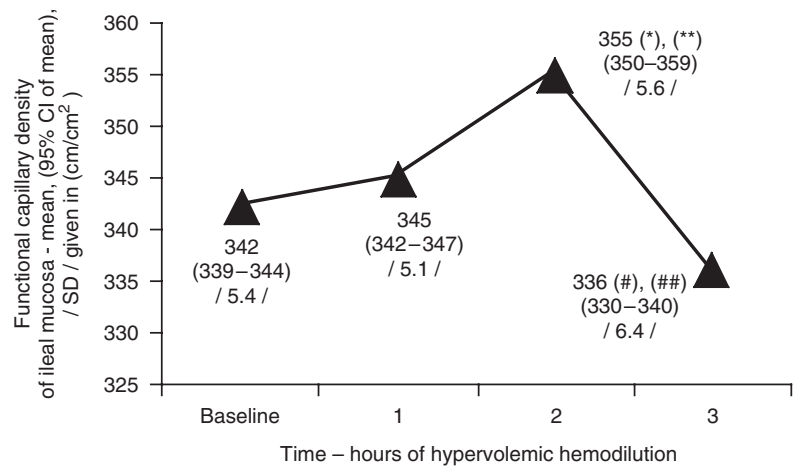

$\left.{ }^{*}\right) \mathrm{P}<0.05$ vs baseline; (\#) $\mathrm{P}<0.05$ vs baseline;

(*) $\mathrm{P}<0.05 \uparrow S B P ;(\# \#) \mathrm{P}<0.05 \uparrow S B P$ and $\uparrow D B P$

Conclusions: Prolonged HHD impairs functional capillary density of ileal mucosa in pigs when using SDF imaging, interpretation of macrohemodynamics during fluid therapy deserves caution.

Reference:

1 Ince C. Crit Care 2005; 8(suppl): P72.

\section{AP7-1}

\section{Randomized study comparing iNO and inhaled lloprost in} cardiac surgery

M. Winterhalter, S. Simon, N. Rahe-Meyer, S. Piepenbrock, M. Strüber Anesthesiology, Hannover Medical School, Hannover, Germany

Background and Goal of Study: The objective of this study was to compare the efficacy of inhaled iloprost (ILO) and nitric oxide (iNO) in reducing pulmonary hypertension (PHT) during cardiac surgery immediately following weaning from cardiopulmonary bypass (CPB).

Materials and Methods: Forty-six patients with PHT (mean pulmonary artery pressure $(\mathrm{mPAP}) \geqslant 26 \mathrm{mmHg}$ preoperatively at rest, after induction of anesthesia and at the end of CPB) scheduled to undergo cardiac surgery were enrolled in this prospective randomized study. Patients were randomly allocated to receive iloprost (group $A, n=23$ ) or iNO (group $B, n=23$ ) during weaning from cardiopulmonary bypass.

Results and Discussions: Both substances caused a significant reduction in mPAP and pulmonary vascular resistance (PVR) 30 minutes post administration $(p<0.001)$.

However, in a direct comparison of the two substances, iloprost caused a significantly greater reduction in PVR (PVR before ILO $455 \pm 373$, after ILO $161 \pm 131$ dyn.s.cm ${ }^{-5}, p=0.036$ ) and mPAP (mPAP before ILO $31 \pm 5$, after ILO $22 \pm 4 \mathrm{mmHg}, \mathrm{p}=0.048$ ) than iNO (PVR before iNO $450 \pm 254$, after iNO $239 \pm 118$ dyn.s.cm ${ }^{-5}$ ); (mPAP before iNO $29 \pm 4$, after iNO $25 \pm 5 \mathrm{mmHg}$ ). At the same time, patients in the iloprost group exhibited a significant increase in cardiac output (CO before ILO $3.9 \pm 1.5$, after ILO $6.5 \pm 1.8 \mathrm{l} / \mathrm{min}$, $\mathrm{p}=0.005)$ (CO before iNO $3.2 \pm 1.2$, after iNO $4.9 \pm 1.7 \mathrm{l} / \mathrm{min}$ ).

Conclusion(s): PHT following weaning from $\mathrm{CPB}$ was significantly reduced by the selective pulmonary vasodilators iNO and iloprost. However, in a direct comparison of the two substances, iloprost proved to be significantly more effective.

\section{AP7-2}

Effects of levosimendan in cardiac surgery patients with poor left ventricular function

H. Vanden Eede, S. Lorsomradee, S. Cromheecke, P.J. Van der Linden, S. De Hert

Anesthesiology, Universital hospital of Antwerp, Edegem, Belgium

Background and Goal of Study: Patients with poor left ventricular function frequently require inotropic drug support immediately after cardiopulmonary bypass. Levosimendan ${ }^{1}$ improves cardiac function by a novel mechanism of action compared to currently available agents ${ }^{2}$. We hypothesized that in patients with severely compromised ventricular function the use of levosimendan would be associated with better postoperative cardiac function than with inotropic drugs that increase myocardial oxygen consumption.

Material and Methods: Thirthy patients with a pre-operative ejection fraction $\leqslant 30 \%$ scheduled for elective cardiac surgery with cardiopulmonary bypass were randomized to two different inotropic protocols: milrinone $0.5 \mu \mathrm{g} \cdot \mathrm{kg}^{-1} \cdot \mathrm{min}^{-1}$ or levosimendan $0.1 \mu \mathrm{g} \cdot \mathrm{kg}^{-1} \cdot \mathrm{min}^{-1}$, started immediately after the release of the aortic cross-clamp. The treatment was masked to the observers. All patients received dobutamine $5 \mu \mathrm{g} \cdot \mathrm{kg}^{-1} \cdot \mathrm{min}^{-1}$.

Results and Discussion: Stroke volume was similar between groups initially after surgery, but it declined 12 hours after surgery in the milrinone group but not in the levosimendan group ( $p<0.05$ between groups), despite similar filling pressures. Total dose, duration of inotropic drug administration and norepinephrine dose were lower in the levosimendan group than in the milrinone group $(p<0.05)$ The duration of tracheal intubation was shorter in the former group compared with the milrinone group $(p=0.008)$. Three patients in the milrinone group but none in the levosimendan group died within 30 days of surgery.

Conclusion: In cardiac surgery patients with a low preoperative ejection fraction, stroke volume was better maintained with the combination of dobutamine with levosimendan than with the combination of dobutamine with milrinone. References:

1 Toller W, Stranz C.: Anesthesiology 2006; 104: 556-69.

2 Raya SG, Rayen B: Ann Thorac Surg 2006; 81: 1536-46.

\section{AP7-4}

Target-controlled infusion of remifentanil during propofol induction: a comparison between hypertensive and normotensive patients

S.H. Lee, J.I. Han

Department of Anesthesiology and Pain Medicine, Ewha Womans University Hospital, Seoul, Republic of Korea

Background and Goal of Study: Hypertensive patients are more prone to exhibit extensive cardiovascular changes during induction (1) and may differ in dosing requirement from normotensive patients. We compared between normotensive and hypertensive patients the effect of target-controlled infusion of remifentanil $\left(4,5\right.$, or $\left.6 \mathrm{ng} \mathrm{ml}^{-1}\right)$ during propofol induction. We also hypothesized that increasing remifentanil doses may reduce propofol consumption at loss of consciousness (2).

Materials and Methods: Forty five ASA 1 or 2 patients without hypertension and forty five ASA 2 patients with hypertension were randomly allocated according to the remifentanil target effect-site concentration $(\mathrm{C} 4, \mathrm{C} 5, \mathrm{C} 6$, $\mathrm{H} 4, \mathrm{H} 5, \mathrm{H} 6$ groups respectively). $5 \mathrm{ml} \mathrm{kg}^{-1}$ of crystalloid solution and $0.2 \mathrm{mg}$ of glycopyrrolate were given at start of induction. Target-controlled Infusion (TCl) of propofol $\left(4 \mathrm{ng} \mathrm{ml}^{-1}\right)$ was started when target effect-site concentration of remifentanil (TCe remi) was reached, and effect-site concentration of propofol was recorded at loss of consciousness (LOC). When target effect-site concentration of propofol (TCe ppf) was reached, $0.6 \mathrm{mg} \mathrm{kg}^{-1}$ of rocuronium was administered and endotracheal intubation was carried out after 2 minutes. Noninvasive blood pressure, heart rate (HR), bispectral index (BIS) and infused dose of remifentanil and propofol were recorded before induction, on reaching TCe remi, at LOC, at TCe ppf, before intubation, and 1, 2 and 5 minutes after intubation.

Results and Discussions: In hypertensive patients, remifentanil effect-site concentration as low as $4 \mathrm{ng} \mathrm{ml}^{-1}$ may attenuate cardiovascular responses to endotracheal intubation. Increasing remifentanil concentration in moderate range (from 4 to $6 \mathrm{ng} \mathrm{ml}^{-1}$ ) is devoid of sparing propofol requirements for hypnosis.

Conclusion(s): In hypertensive patients, lower effect-site concentration of remifentanil may be adequate during propofol induction.

References:

1 Maguire AM, Kumar N, Parker JL et al. Br J Anaesth 2001; 86: 90-3.

2 Milne SE, Kenny GN, Schraag S. Br J Anaesth 2003; 90: 623-9.

\section{AP7-5}

\section{Does nitroglycerin really act as a selective venous} vasodilator?

K. Lebedinskiy, S. Parvanian, I. Puzanova

Anaesthesiology and Reanimatology, Medical Academy of Postgraduate Studies, St. Petersburg, Russian Federation

Background and Goals: It is generally accepted, that nitroglycerin (NG) acts in dose-dependent manner, and arteriolar dilation requires higher i.v. infusion rates than venous dilation (1). Our aim was to determine the exact NG infusion rate to achieve selective venous dilation. 
Material and Methods: On institutional approval and informed consent, 10 healthy volunteers (group A), $23 \mathrm{ICU}$ patients, receiving NG for common indications (group B), and 7 ICU patients recovering after elective CABG (group C) were included in prospective study. All the patients had stable circulation and received neither inotropic nor vasoactive medication. Groups $A$ and $B$ received i.v. NG infusion in ascending rates (0.01 to $\left.0.1 \mathrm{mkg} \mathrm{kg}^{-1} \mathrm{~min}^{-1}\right)$. Hemodynamic variables were measured using impedance cardiography (Diamant, Russia). In group C patients NG i.v. infusion (0.05 and $0.1 \mathrm{mkg} \mathrm{kg}^{-1}$ $\mathrm{min}^{-1}$ ) was readministered for the short time just before Swan-Ganz pulmonary artery catheter removal (S/5 monitor, GE) and ICU discharge.

Results: In A and B groups the earliest hemodynamic change was significant systemic vascular resistance decline leading to stroke volume rise without shift of heart rate. This result prompted us to compare preload and afterload dynamics during NG infusion using invasive monitoring in group $C$. The latter data are shown in the table (Mean $\pm S D,{ }^{*}-p<0.01$ vs base variable level):

\begin{tabular}{lccc}
\hline & Base level & \multicolumn{2}{c}{$\mathrm{NG}, \mathrm{mkg} \mathrm{kg}^{-1} \mathrm{~min}^{-1}$} \\
\cline { 2 - 4 } & & 0.05 & 0.1 \\
\hline $\mathrm{SVI}, \mathrm{ml} \cdot \mathrm{m}^{-2}$ & $31.8 \pm 2.2$ & $34.6 \pm 3.3^{\star}$ & $9.8 \pm 5.7^{\star}$ \\
$\mathrm{HR}, \mathrm{min}{ }^{-1}$ & $82 \pm 14$ & $79 \pm 14$ & $81 \pm 15$ \\
$\mathrm{SVRI}, \mathrm{dyn} \cdot \mathrm{s} \cdot \mathrm{cm}^{-5} \cdot \mathrm{m}^{2}$ & $2799 \pm 457$ & $2608 \pm 492^{\star}$ & $2181 \pm 444^{\star}$ \\
$\mathrm{PVRI}, \mathrm{dyn} \cdot \mathrm{s} \cdot \mathrm{cm}^{-5} \cdot \mathrm{m}^{2}$ & $269 \pm 83$ & $227 \pm 80$ & $174 \pm 76^{\star}$ \\
$\mathrm{CVP}, \mathrm{mm} \mathrm{Hg}$ & $7.8 \pm 2.1$ & $7.8 \pm 2.7$ & $5.5 \pm 1.4^{\star}$ \\
PAOP, $\mathrm{mm} \mathrm{Hg}$ & $13.8 \pm 1.2$ & $14.4 \pm 2.9$ & $11.0 \pm 0.6^{\star}$ \\
\hline
\end{tabular}

Conclusion: In all the groups, afterload decrease followed by stroke volume rise surprisingly left behind preload decline. Since even low-rate NG i.v. infusion causes circulatory changes typical for arterial dilation, despite undoubted venodilator properties, the selectivity of $\mathrm{NG}$ as a venous dilator in clinical settings may be revised.

Reference:

1 Abrams J. Am J Cardiol 1992; 70: 30-42

\section{AP7-6}

Cardiovascular effects of normobaric hyperoxia in patients with heart rate fixed by permanent pacemaker

K. Anderson, J. Harten, C. Berry, A. McConnachie, J. Kinsella

University Department of Anaesthesia, Glasgow Royal Infirmary, Glasgow, United Kingdom

Background and Goal of Study: Hyperoxia has significant effects on hemodynamics in humans [1]. It is unclear whether the reduction in heart rate and cardiac output are the primary effect or occur secondary to the increase in systemic vascular resistance. In this study we studied the cardiovascular effects of oxygen on patients with a fixed heart rate on their permanent pacemaker. Materials and Methods: Following ethical approval, nine patients with permanent pacemakers and no other co-morbidity, gave informed consent. Patients were paced at a fixed rate and then exposed to medical air $\left(\mathrm{F}_{\mathrm{i}} \mathrm{O}_{2}=0.21\right)$ and oxygen $\left(\mathrm{F}_{\mathrm{i}} \mathrm{O}_{2}=0.8\right)$ in a randomised and double-blinded fashion. We used a thoracic bio-impedance machine (BioZ System 1.52, Cardiodynamics San Diego, USA) to determine heart rate, stroke volume, cardiac index, non-invasive blood pressure and systemic vascular resistance index.

Results and Discussions: When increasing the $\mathrm{F}_{1} \mathrm{O}_{2}$ from 0.21 to the higher level the delivered $\mathrm{F}_{1} \mathrm{O}_{2}$ was (median 0.85 (range 0.70-0.93)). On increasing $\mathrm{F}_{1} \mathrm{O}_{2}$ there were significant differences compared to low $\mathrm{F}_{\mathrm{i}} \mathrm{O}_{2}$ for $\mathrm{SpO}_{2}$ (an increase of $0.85 \%$ (95\% Cl: $0.01,1.68), p=0.045)$, ETCO2 (a decrease of $0.18 \mathrm{kPa}(0.06,0.30), \mathrm{p}=0.01)$ and systemic vascular resistance index (an increase of $172(19,325)$ dyne $\left.\cdot \mathrm{s} \cdot \mathrm{cm}^{-5} \cdot \mathrm{m}^{-2}, \mathrm{p}=0.03\right)$. There was no evidence of difference in heart rate $(p=0.51)$, stroke index $(p=0.44)$, cardiac index $(p=0.18)$ or mean arterial pressure $(p=0.52)$

Conclusion(s): This suggests that the increase in systemic vascular resistance seen in previous studies is the primary effect, and that the reduction in heart rate and cardiac index are secondary to changes in vascular tone.

Reference:

1 Harten J. Anaesthesia 2003,58: 885-888.

\section{AP7-7}

Reliability of buccal mucosal visible light spectrometry and laser Doppler flowmetry

V. Gath, Y. Sakr, S. Klinzing, T. Simon, G. Marx

Department of Anaesthesiology and Intensive Care, Friedrich Schiller University Hospital, Jena, Germany

Background and Goal of Study: There are insatiable demands for new technologies to advance basic biological investigation at the microcirculatory level. Oxygen to see $(\mathrm{O} 2 \mathrm{C}) \AA$ is a newly developed system that combines laser
Doppler flow (LDF) and visible light spectroscopy (VLS) technology. The aim of our study was to assess the reliability of $\mathrm{O} 2 \mathrm{C} \otimes$ measurements applied to the buccal mucosa in healthy volunteers.

Materials and Methods: Microcirculatory hemoglobin oxygen saturation $\left(\mu \mathrm{HbO}_{2}, \%\right)$ and blood flow (flow, $\mathrm{AU}$ ) were measured using O2C $\AA$ (Lea Medizintechnik $\mathrm{GmbH}$, Giessen, Germany) probe applied to the buccal mucosa. Measurements were obtained simultaneously at two depths; superficial $(2 \mathrm{~mm})$ and deep $(6 \mathrm{~mm})$ every 2 seconds for 5 minutes and recorded for later analysis. The procedure was repeated in another occasion at least one week apart.

Results: We studied 20 healthy subjects; 10 males and 10 females (mean age $=38 \pm 18$, range $21-74$ years). Both $\mu \mathrm{HbO}_{2}$ and flow measurements were consistently higher when measured from the deep tissue layers $(6 \mathrm{~mm})$ than those measured from the superficial layers. Buccal mucosal $\mu \mathrm{HbO}_{2}$ ranged from 78 to $96 \%$ and varied only minimally (CV: 4-7.5\%), whereas, there was a marked variability in flow measurements (CV: 29-63.9\%). The reproducibility of buccal mucosal $\mu \mathrm{HbO}_{2}$ and flow measurements were moderate to good (i.e intra-individual reliability, ICC: range $0.7-0.87, \mathrm{p}<0.05$ ). However, only measurements from the superficial mucosal layers showed a moderate to good degree of inter-individual agreement (i.e intra-individual reliability, ICC: range 0.68-85, $\mathrm{p}<0.001)$.

Conclusion: $\mathrm{O} 2 \mathrm{C} \otimes$ provides a reliable measurements of buccal $\mu \mathrm{HbO}_{2}$ and microvascular flow.

\section{AP7-8}

The association of erythrocyte aggregation index and acute phase proteins after cardiac surgery with CPB

H. Mungroop, B.G. Loef, W. van Oeveren, L.P.H.J. Aarts, A.H. Epema Department of Anesthesiology, University Medical Center Groningen, Groningen, The Netherlands

Background and Goal of Study: Erythrocyte aggregation affects blood viscosity and micro vascular flow dynamics. Aggregation is influenced by the concentration of acute phase proteins, which changes during and after cardiac surgery with cardiopulmonary bypass (CPB). We studied the acute phase response by measuring acute phase proteins and erythrocyte aggregation during a six day perioperative period.

Materials and Methods: After IRB approval and informed consent 41 patients scheduled for cardiac surgery with CPB were prospectively studied. Blood samples were collected before surgery (T1), at the end of surgery (T2), on the first (T3), third (T4) and fifth (T5) postoperative day. Erythrocyte aggregation index was measured using a laser assisted optical rotational cell analyser (LORCA, Mechatronics Instruments, Hoorn, Netherlands). Fibrinogen, C-reactive protein, albumin, ceruloplasmin, haptoglobulin and malondialdehyde were determined. For statistical analyses Student $t$ test, Mann-Withney $\mathrm{U}$ test and ANOVA were performed when appropriate, and significance accepted at $P<0,05$.

Results and Discussions: Erythrocyte aggregation index decreased at the end of surgery and significantly increased above baseline (Fig) in the study period. Haptoglobuline (Fig), and the other acute phase proteins showed a similar pattern. The changes in aggregation index and acute phase proteins were associated.

Conclusions: Changes of acute phase protein concentrations affect aggregation index after cardiac surgery with CPB. The acute phase respons is still ongoing on the fifth postoperative day after cardiac surgery.
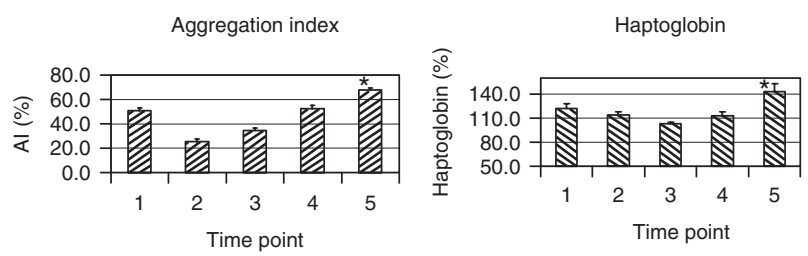

Reference:

1 Gu et al. Clinical Hemorheology and Microcirculation 2005; 33: 95-107.

\section{AP7-9}

Comparison of serum lipids and glucose levels during propofol or midazolam infusions in coronary artery bypass surgery

A. Aygün, I. Oztekin, D. Oztekin, G. Topcu, S. Canik

Anesthesiology, Siyami Ersek Thoracic and Cardiovascular Surgery Hospital, Istanbul, Turkey 
Background and Goal of study: Plasma triglyceride (TRG) levels are preserved with propofol $(P) 1 \%$ infusion whereas it is decreased compared to start level with midazolam $(M)$ infusion during cardiac surgery $(1,2,3)$. The aim was to document changes in serum lipids and glucose (GLU) levels during P $2 \%$ or $\mathrm{M}$ infusions for cardiac surgery in normolipidaemic patients.

Materials and Methods: 30 normolipidaemic patients undergoing elective coronary artery bypass graft surgery were randomly assigned into two groups. The $P$ group of 15 patients received a continous $P 2 \%$ infusion and the $M$ group of 15 patients received an $M$ infusion for induction and maintenance of anaesthesia. Serum total cholesterol (CHO), TRG, high-density lipoprotein (HDL), low-density lipoprotein (LDL), very low-density lipoprotein (VLDL) and GLU levels were measured at five periods until postoperative 24th hour. Variance analysis in repeated measures" and "t test for coupled series" were used. $\mathrm{p}<0.05$ was accepted as statistically significant.

\section{Results and Discussion:}

\begin{tabular}{|c|c|c|c|c|c|}
\hline & Grp & Pre-induction & Post-induction & End CPB & Postop. $24^{\text {th }} \mathrm{hr}$ \\
\hline \multirow[t]{2}{*}{ TRG } & $\mathrm{P}$ & $112 \pm 29.5$ & $106 \pm 36.1$ & $69 \pm 21.3^{\star}$ & $101 \pm 26.4$ \\
\hline & $M$ & $115 \pm 29$ & $101 \pm 23.6$ & $54 \pm 16^{*}$ & $104 \pm 23.1$ \\
\hline \multirow[t]{2}{*}{$\mathrm{CHO}$} & $\mathrm{P}$ & $147 \pm 16.7$ & $133 \pm 20.1$ & $84 \pm 14.6^{\star}$ & $119 \pm 36.9^{*}$ \\
\hline & $\mathrm{M}$ & $160 \pm 26.9$ & $148 \pm 20.3$ & $89 \pm 12.8^{\star}$ & $127 \pm 17.2^{\star}$ \\
\hline \multirow[t]{2}{*}{ HDL } & $\mathrm{P}$ & $39 \pm 11.1$ & $35 \pm 9.3$ & $23 \pm 7^{\star}$ & $33 \pm 14.7^{\star} \#$ \\
\hline & $M$ & $41 \pm 7.6$ & $37 \pm 7.8$ & $24 \pm 6.1^{*}$ & $34 \pm 4.6^{\star}$ \\
\hline \multirow[t]{2}{*}{ LDL } & $\mathrm{P}$ & $86 \pm 10.9$ & $78 \pm 17.2$ & $49 \pm 10.3^{*}$ & $70 \pm 25.8^{\star} \#$ \\
\hline & $M$ & $96 \pm 23.9$ & $91 \pm 18.8^{*}$ & $54 \pm 8.3^{\star}$ & $72 \pm 15.1^{*}$ \\
\hline \multirow[t]{2}{*}{ VLDL } & $\mathrm{P}$ & $23 \pm 6.9$ & $21 \pm 6.2$ & $13 \pm 3.9^{\star} \#$ & $20 \pm 5.2 \#$ \\
\hline & $M$ & $23 \pm 5.9$ & $20 \pm 4.7$ & $11 \pm 2.4^{*}$ & $26 \pm 20.8$ \\
\hline \multirow[t]{2}{*}{ GLU } & $\mathrm{P}$ & $98 \pm 6.9$ & $124 \pm 23.6^{\star} \#$ & $149 \pm 23.7^{*}$ & $148 \pm 25^{\star}$ \\
\hline & $\mathrm{M}$ & $95 \pm 10.5$ & $119 \pm 12.2^{\star}$ & $144 \pm 33.3^{*}$ & $148 \pm 18.6^{*}$ \\
\hline
\end{tabular}

${ }^{*} \mathrm{p}<0.05$ (in the same group); \#p $<0.05$ (between the groups); CPB, cardiopulmonary bypass

Conclusion: Use of P $2 \%$ infusion does not increase significantly the risk of hyperlipidaemia when used in cardiac surgery.

References:

1 Inoue S, Takauchi Y, Kayamori Y, et al. Eur J Anaesthesiol 2001; 18: 113-117.

2 Barrientos-Vega R, Sanchez-Soria MM, Morales-Garcia C, et al. Crit Care Med 2001; 29: 317-322.

3 Myles MB, DipR, Acog DA et al. J Cardiothorac Vasc Anesth 1995; 9: 373-378.

\section{AP8-1 \\ Glyceryl trinitrate markedly influences haemodynamic multiscale sample entropy \\ M. Stephan, L.A. Schwarte, I. Schwartges, O. Picker \\ Department of Anaesthesiology, University Hospital of Duesseldorf, Duesseldorf, Germany}

Background and Goal of Study: Cardiovascular dynamics in health and disease appear to reflect complex regulatory mechanisms [1], which can be assessed by analysing haemodynamic sample entropy. Therefore, we studied the effects of Glyceryl trinitrate (GTN) on haemodynamic sample entropy. Materials and Methods: With approval of the District Governmental Animal Research Committee, six mechanically ventilated foxhounds received GTN at $1.5 \mathrm{mg} \mathrm{kg}^{-1} \mathrm{~h}^{-1}$. Systolic arterial pressure (SAP) and RR interval (RRI) time series (1000 cardiac cycles) were analysed using a multiscale sample entropy (MSE) algorithm [2, 3].

Statistics: Medians (Range), RM ANOVA on Ranks, Dunn's post test, $\mathrm{p}<0.05$.

Results and Discussions: SAP was reduced with GTN from 106 (98-136) $\mathrm{mmHg}$ to $87(75-108) \mathrm{mmHg}$ together with a reduction in RRI from 734 (607-901) ms to 542 (485-636) ms. SAP and RRI recovered during a 30 minutes washout period. In contrast, RRI MSE decreased from 1.5 (0.9-1.6) to $0.6(0.4-1.0)$ during GTN and did not recover after GTN washout. In parallel, MSE of SAP tended to decrease from $0.8(0.5-1.0)$ to $0.6(0.3-1.2)(p=0.18)$ and did likewise not recover with $0.5(0.4-1.0)$. The course of MSE was almost independent of the selected scale.

Conclusion(s): Glyceryl trinitrate has a marked and persistent influence on haemodynamic sample entropy which is independent of the blood pressure level. Thus, the use of glyceryl trinitrate is one factor crucially influencing haemodynamic sample entropy.

References:

1 Glass L. Nature 2001; 410: 277-284.

2 Lake DE, Richman JS, Griffin MP, et al. Am J Physiol Regul Integr Comp Physiol 2002; 283: R789-797.

3 Costa M, Goldberger AL, Peng CK. Phys Rev E Stat Nonlin Soft Matter Phys 2005; 71: 021906.

\section{AP8-2}

Measurement of volatile organic compounds in expiratory air generated during liver ischemia-reperfusion injury

E. Li, S. Liu, P. Li, G. Xu, Y.H

Anesthesiology, First Affiliated Hospital, Harbin Medical University, Harbin, China

Background and Goal of Study: Analysis of exhaled air is of particular interest as an indicator of health as well as a tool for the diagnosis of diseases. This kind of analysis presents numerous advantages over other methods, the most important being that it is not an invasive procedure. Pentane is known as a marker of lipid peroxidation [1] .The aim of this study was to investigate the correlation of pentane and liver damage caused by ischemia-reperfusion. Materials and Methods: Thirty rabbits were randomly divided into two groups: (I) sham group; (II) liver ischemia-reperfusion group, clamped for 25 minutes. Exhaled breath samples $(10 \mathrm{ml})$ and blood samples $(4 \mathrm{ml})$ were taken from the animals at baseline; reperfusion $0 \mathrm{~min}$; $60 \mathrm{~min}$; $120 \mathrm{~min}$; $180 \mathrm{~min}$. Pentane was determined by SPME[2] in GC-MS. At the end of the experiment liver tissue samples were obtained. AST and ALT plasma levels were measured in the blood samples.

Results: Data (Mean $\pm \mathrm{SD}$ ) are shown in the table:

\begin{tabular}{lllcccc}
\hline & & B & I25 min & R60 min & R120 min & R180min \\
\hline $\begin{array}{l}\text { Pentane } \\
\text { (ppb) }\end{array}$ & I & $10.6 \pm 1.6$ & $10.0 \pm 0.9$ & $9.6 \pm 1.4$ & $10.0 \pm 2.4$ & $9.4 \pm 0.7$ \\
& II & $9.1 \pm 2.5$ & $7.7 \pm 1.5^{\star}$ & $11.2 \pm 2.0^{\#}$ & $9.0 \pm 1.7$ & $8.6 \pm 1.4$ \\
ALT(u/l) & I & $55 \pm 32$ & $55 \pm 27$ & $54 \pm 28$ & $48 \pm 25$ & $45 \pm 23$ \\
& II & $47 \pm 15$ & $45 \pm 18$ & $85 \pm 20$ & $111 \pm 27^{\square}$ & $128 \pm 30^{\#}$ \\
AST(u/l) & I & $38 \pm 8$ & $31 \pm 8$ & $30 \pm 9$ & $32 \pm 12$ & $37 \pm 13$ \\
& II & $40 \pm 18$ & $46 \pm 14$ & $241 \pm 36^{\#}$ & $268 \pm 56^{\#}$ & $327 \pm 77^{\square}$ \\
\hline
\end{tabular}

B: baseline ; I: ischemia ; R: reperfusion

Compare with $\mathrm{B} * \mathrm{P}<0.05 ; \square \mathrm{P}<0.01$

Conclusion(s): 1) Pentane can indicate the level of the liver injury during ischemia-reperfusion; 2) Pentane was prior to serum enzyme elevation.

References:

1 Phillips M.Eur Respir J 2003; 21: 48-51.

2 Prado C. Journal of Chromatography A.2003; 1011; 125-134.

\section{AP8-3}

\section{Effect of nutritional status on hepatocyte integrity and} function in an ex vivo perfused rat liver model

L. Van Der Goot, V. Nuyens, M. Stadler, J. Boogaerts

Anaesthesiology, CHU Charleroi, Charleroi, Belgium

Background: The pre-existing nutritional status of the liver might contribute to the extent of tissue injury occurring during surgical procedures, ischaemic state and multiple organ failure $(1,2)$. The aim of this study was to determine the role of starvation on hepatic integrity and function measured by the kinetics of Indocyanine Green (ICG) in an ex vivo perfused rat liver model. Materials and Methods: Wistar rats were divided into 2 groups $(n=5)$ : one had free access to food, the other was fasted for $16 \mathrm{~h}$. The portal vein was cannulated, the liver removed and perfused in a closed ex vivo system during $135 \mathrm{~min}$ (2). Five $\mathrm{mg} \mathrm{ICG}$ were added to $125 \mathrm{ml}$ perfusate at the start of experiment. Glucose and lactate (mg/dl), enzymes ALT, AST, LDH (IU/l), potassium $(\mathrm{mEq} / \mathrm{l})$, free radicals, i.e. dienes and trienes (\% Oxidative Index: O.I.), cytochrome $c$ (Cyt $c$; $\mathrm{ng} / \mathrm{ml}$ ) and ICG (spectrophotometry) were measured in perfusate samples at different times. The proportion (\%) of glycogen in hepatocytes was determined in biopsies. ICG kinetic was summarized as $\mathrm{k}_{\mathrm{p}}$, rate constant for flux of ICG from perfusate to liver and $\mathrm{k}_{\mathrm{lp}}$, from liver to perfusate $\left(\mathrm{min}^{-1}\right)$ (3). Mean $\pm \mathrm{SD}$. Student $t$ test with Bonferroni corrections. Results: Values at $135 \mathrm{~min}$ are displayed in table 1. Enzymes, potassium, cyt $c$ and dienes levels were higher in fasting rats. Glucose, lactate and glycogen levels were greater in the fed group. The kinetics of ICG was similar in the two groups.

\begin{tabular}{llcl}
\hline & Fasting & \multicolumn{1}{l}{ Fed } & $\mathrm{P}$ \\
\hline Glucose $(\mathrm{mg} / \mathrm{dl})$ & $88 \pm 55$ & $156 \pm 102$ & 0.036 \\
Lactate $(\mathrm{mg} / \mathrm{dl})$ & $9.0 \pm 13.5$ & $119.7 \pm 106.6$ & $<0.001$ \\
AST $(\mathrm{IU} / \mathrm{l})$ & $1,618 \pm 1,533$ & $55 \pm 23$ & 0.003 \\
ALT $(\mathrm{IU} / \mathrm{l})$ & $1,620 \pm 505$ & $19 \pm 24$ & 0.002 \\
$\mathrm{LDH}(\mathrm{IU} / \mathrm{l})$ & $24,099 \pm 15,258$ & $348 \pm 284$ & $<0.001$ \\
$\mathrm{~K}^{+}(\mathrm{mEq} / \mathrm{l})$ & $10.1 \pm 1.4$ & $7.0 \pm 0.7$ & $<0.001$ \\
Glycogen (\%) & $4.5 \pm 2.8$ & $44.3 \pm 19.3$ & 0.013 \\
Dienes (\%) & $43.2 \pm 7.5$ & $27.7 \pm 8.5$ & 0.027 \\
Trienes $(\%)$ & $20.7 \pm 7.5$ & $14.5 \pm 5.7$ & 0.205 \\
Cyt c (ng/ml) & $24.1 \pm 10.2$ & $16.3 \pm 7.3$ & 0.312 \\
$\mathrm{k}_{\mathrm{pl}}\left(\mathrm{min}^{-1}\right)$ & $0.0070 \pm 0.0074$ & $0.0089 \pm 0.0062$ & 0.192 \\
$\mathrm{k}_{\mathrm{lp}}\left(\mathrm{min}^{-1}\right)$ & $-0.0011 \pm 0.0021$ & $-0.0017 \pm 0.0019$ & 0.309 \\
\hline
\end{tabular}


Conclusion: In the present experimental conditions, fasting impaired hepatocytes integrity but did not seem to alter liver function.

Reference:

1 Adam R, Reynes M, Johann M et al. Transplant Proc 1991; 23: 1538-40.

2 Stadler M, Nuyens V, Seidel L et al. Anesthesiology 2005; 103: 978-86.

3 Lund M, Kang L, Tygstrup N et al. Am J Physiol Gastrointest Liver Physiol 1999; 277 : 91-100.

\section{AP8-4}

Effect of increase in blood pressure on plasma volume loss under normal microvascular permeability

M. Dubniks, P.O. Grande

Anaesthesiology and Intensive Care, Lund University Hospital, Lund, Sweden

Background and Goal of Study: Adequate intravascular volume is one of the most important prerequisites for hemodynamic stability in the perioperative and intensive care settings. In a previous study (1) we showed that at a state of increased microvascular permeability, the transcapillary loss of plasma fluid is increased when blood pressure is increased. The present study evaluated the effect of an increase in blood pressure on plasma fluid loss, but now at normal permeability.

Materials and Methods: In 3 groups of adult male rats ( $N=11$ each group), plasma volume (PV) was determined by a ${ }^{125} \mathrm{I}$-albumin tracer technique at baseline condition and at the end of experiment after $2.5 \mathrm{~h}$ period of noradrenalin (NA) infusion. Before NA was started, $5 \%$ albumin in the dose of $15 \mathrm{ml} / \mathrm{kg}$ was infused for $15 \mathrm{~min}$ in group 1 . In group 2, $15 \mathrm{ml} / \mathrm{kg}$ of $5 \%$ albumin was given after the animals were bled by $15 \mathrm{ml} / \mathrm{kg}$. In group 3 , the animals received NA infusion only. The rate of NA infusion was adjusted to keep mean arterial pressure slightly above the baseline value. PV loss was calculated as the difference between PV before and after NA infusion.

Results and Discussions: PV loss was $12.4 \pm 3.5 \mathrm{ml} / \mathrm{kg}$ in group 1 ( $P<0.001), 1.6 \pm 3.6 \mathrm{ml} / \mathrm{kg}$ in group 2 , and $2.0 \pm 3.6 \mathrm{ml} / \mathrm{kg}$ in group 3 , to be compared with PV losses from the previous study (1) of $3.4 \mathrm{ml} / \mathrm{kg}$ and $13.4 \mathrm{ml} / \mathrm{kg}$ without and with increased blood pressure, respectively, at increased permeability and normovolaemia.

Conclusion(s): 1. In normovolaemia, transcapillary plasma fluid loss associated with NA-induced increase in blood pressure is larger at increased than at normal permeability. 2. Excess of circulating plasma volume escapes circulation even at normal permeability.

Reference:

1 Dubniks M, Persson J, Grände P-O Intensive Care Med 2006; 32: S289. (Abstract 1121)

\section{AP8-5}

Non heart beating donors porcine livers: effect of three different pump flow rate during cardiopulmonary bypass

R. Valero, P. Santos-Cidón, M. Net, LI. Capdevila, JC. García-Valdecasas Anesthesiology, Hospital Clinic, Barcelona, Spain

Background and Goal of Study: Normothermic Recirculation [NR] before Total Body Cooling [TBC] is an effective method to improve graft viability in porcine livers obtained from non heart beating donors [NHBD][1]. Optimization of the hepatic flow perfusion during NR using a non pulsatile roll pump was evaluated at three different pump flow rate [PFR].

Materials and Methods: pigs were transplanted with an hepatic allograft obtained from NHBD after 60 min of warm ischemia, $30 \mathrm{~min}$ of NR and TBC up to liver temperature was $15^{\circ} \mathrm{C}$, divided in three groups: Control (n(10) PFR at maximum flow rate depending on venous blood return, High constant (n(8): PFR at 2,4 L/min/ $/ \mathrm{m}^{2}$, Low constant $\left(\mathrm{n}(8)\right.$ : PFR at $1,2 \mathrm{~L} / \mathrm{min} / \mathrm{m}^{2}$ ], hepatic artery flow (AF) and portal flow (PF) and blood gas analysis (artery, porta suprahepatic vein) were taken at baseline, $5,15,30 \mathrm{~min}$ and $15^{\circ} \mathrm{C}$. Oxygen hepatic extraction (OER\%) was calculated as $\mathrm{HVO}_{2} / \mathrm{HDO}_{2}$. Survival was evaluated at fifth day posttransplant.

Results and Discussions: Data (Mean $\pm \mathrm{SD}$ ) are shown:

\begin{tabular}{llll}
\hline & Control & High Constant & Low Constant \\
\hline $\mathrm{AF}_{\mathrm{B}} P F_{B}$ & $176 \pm 80639 \pm 138$ & $140 \pm 34533 \pm 146$ & $184 \pm 172712 \pm 299$ \\
$\mathrm{AF}_{5} P F_{5}$ & $159 \pm 66242 \pm 108$ & $152 \pm 48300 \pm 133$ & $55 \pm 37^{*} i 187 \pm 91$ \\
$\mathrm{AF}_{15} P F_{15}$ & $176 \pm 66287 \pm 169$ & $174 \pm 52378 \pm 215$ & $73 \pm 26^{*} ; 206 \pm 101$ \\
$\mathrm{AF}_{30} P F_{30}$ & $156 \pm 93327 \pm 212$ & $178 \pm 62362 \pm 196$ & $72 \pm 26 ; 194 \pm 91$ \\
$\mathrm{AF}_{\mathrm{TBC}} P F_{\mathrm{TBC}}$ & $132 \pm 75231 \pm 133$ & $136 \pm 44326 \pm 116$ & $64 \pm 33 ; 180 \pm 56^{*} i$ \\
$\mathrm{OER}_{\mathrm{B}}$ & $23 \pm 3,0$ & $22,7 \pm 12,8$ & $20,6 \pm 12,4$ \\
$\mathrm{OER}_{5}$ & $35,2 \pm 20,7$ & $44,8 \pm 19,4$ & $63,5 \pm 10,8^{\star}$ \\
$\mathrm{OER}_{15}$ & $28,1 \pm 11,2$ & $47,1 \pm 10,7^{\star}$ & $62,5 \pm 11,6$ \\
$\mathrm{OER}_{30}$ & $44,7 \pm 19,0$ & $42,1 \pm 11,9$ & $51,0 \pm 20,3$ \\
OER $_{\mathrm{TBC}}$ & $12,2 \pm 0,7$ & $11,7 \pm 6,8$ & $26,2 \pm 21,7$ \\
Survival & $80 \%(8 / 2)$ & $62,5 \%(5 / 3)$ & $37,5 \%(3 / 5)$ \\
\hline
\end{tabular}

$\mathrm{P}<0.05 \mathrm{GH}$ o $\mathrm{GL}$ vs $\mathrm{G}$ Control; $\mathrm{i} \mathrm{P}<0.05 \mathrm{GL}$ vs $\mathrm{GH}$
Conclusion(s): Low pump flow rate decreases significantly arterial and portal blood flow during NR and TBC. OER was higher in constant flow rate groups ( $\mathrm{LG}>\mathrm{HG}>\mathrm{CG})$. The best survival was achieved in group control

Reference:

1 Valero R. Transplantation 1998; 66: 170-176.

\section{AP8-6}

Dobutamine addition during cardiopulmonary bypass decreases hepatocellular and endothelial damage in porcine liver grafts from non heart beating donors

P. Santos-Cidón, R. Valero, M. Net, G. Rodriguez-Laiz, JC. GarcíaValdecasas

Anaesthesiology, Hospital Clinic Barcelona, Barcelona, Spain

Background and Goal of Study: Normothermic Recirculation (NR) protects livers from non heart beating donors [NHBD] by a preconditioning-like action [1]. Dobutamine (Dbt) protects against ischemia reperfusion liver injury by induction of heme-oxygenase1[2]. We evaluated the effect of Dbt administration during NR in pig livers from NHBD over hepatic artery and portal flows, makers of hepatocellular and endothelial damage.

Materials and Methods: pigs were transplantated with an hepatic allograft from NHBD. After 60 min of cardiac arrest, NR was performed during 30 min at maximum pump flow rate and finally total body cooling until liver temperature of $15^{\circ} \mathrm{C}$. Animals were randomly treated with Dbt $5 \mu \mathrm{g} / \mathrm{Kg} / \mathrm{min}$ (Dbt G; $\mathrm{n}=8$ ) or glucose $5 \%$ (Control $\mathrm{G} ; \mathrm{n}=10$ ). Hepatic flows were recorded at baseline, 5, 15, $30 \mathrm{~min}$ and at the end of TBC. Blood samples for transaminases (AST, ALT) and hyaluronic acid (HA) were collected from suprahepatic and hepatic artery at baseline (T1), the end of $\mathrm{NR}(\mathrm{T} 2), 60 \mathrm{~min}$ post-reperfusion (T3), at second (T4) and fifth day (T5) post-transplantation. HA extraction $(\mathrm{AHE})=$ arterial $\mathrm{AH}$-suprahepatic vein $\mathrm{AH}$ ).

Results and Discussions: Data (Mean $\pm S D$ ) are shown:

\begin{tabular}{|c|c|c|}
\hline & Control Group & Dbt Group \\
\hline$\overline{\mathrm{AF}_{\mathrm{B}} ; P F_{B}}$ & $176 \pm 80639 \pm 138$ & $137 \pm 36576 \pm 240$ \\
\hline $\mathrm{AF}_{5} ; P F_{5}$ & $159 \pm 66242 \pm 108$ & $149 \pm 49380 \pm 211$ \\
\hline $\mathrm{AF}_{15} ; P F_{15}$ & $176 \pm 6687 \pm 169$ & $161 \pm 57538 \pm 291$ \\
\hline $\mathrm{AF}_{30} ; P F_{30}$ & $156 \pm 93327 \pm 212$ & $140 \pm 55450 \pm 202$ \\
\hline $\mathrm{AF}_{\mathrm{TBC}} P F$ & $132 \pm 75231 \pm 133$ & $133 \pm 103373 \pm 203$ \\
\hline $\mathrm{AST} \mathrm{ALT}_{T 1}$ & $50 \pm 1438 \pm 10$ & $50 \pm 13734 \pm 7$ \\
\hline $\mathrm{AST} / \mathrm{ALT}_{\mathrm{T} 2}$ & $93 \pm 85 \pm 729 \pm 11$ & $132 \pm 7522 \pm 9$ \\
\hline$A S T / A_{T} T_{T 3}$ & $581 \pm 32957 \pm 19$ & $829 \pm 80754 \pm 36$ \\
\hline $\mathrm{AST} / \mathrm{ALT}_{\mathrm{T} 4}$ & $2803 \pm 144107 \pm 46$ & $1253 \pm 567^{*} 64 \pm 18^{\star}$ \\
\hline $\mathrm{AST} / \mathrm{ALT}_{\mathrm{T} 5}$ & $669 \pm 59964 \pm 15$ & $255 \pm 12937 \pm 12^{*}$ \\
\hline $\mathrm{AHE}_{\mathrm{T} 1}$ & $32,7 \pm 10,1$ & $29,3 \pm 10,2$ \\
\hline $\mathrm{AHE}_{\mathrm{T} 2}$ & $-4,6 \pm 19,5$ & $-1,2 \pm 7,7$ \\
\hline $\mathrm{AHE}_{\mathrm{T} 3}$ & $-1,0 \pm 11,4$ & $12,8 \pm 26,6$ \\
\hline
\end{tabular}

${ }^{*} \mathrm{p}<0.05$ vs Control Group.

Conclusion(s): Dobutamine addition during NR and TBC increases portal blood flow, significantly decreases AST ( $\left.2^{\text {nd }} d a y\right)$ and ALT $\left(2^{\text {nd }}-5^{\text {th }} d a y\right)$. AHE showed a positive value $60 \mathrm{~min}$ after reperfusion.

References:

1 Net M.Am J Transplant 2005; 5(10): 2385-92.

2 Raddatz A.Am J Respir Crit CareMed 2006; 174: 198-207.

\section{AP8-7}

\section{Plasma BNP and diastolic dysfunction in patients with} preserved systolic function

\section{F. Guarracino, B. De Lorenzo, C. Vullo, C. Pasquini, A. Boldrini}

Cardiothoracic Department, Cardiothoracic Anaesthesia and ICU, Pisa, Italy Background and Goals of the Study: Plasma BNP levels have utility for diagnosing and managing heart failure. ${ }^{(1,2)}$ The aim of our study was to evaluate if BNP levels can detect diastolic dysfunction, and if the new echo indexes of diastolic dysfunction add new information when compared with standard tools.

Materials and Methods: Sixty patients with preserved left ventricular ejection fraction [LVEF] $>0.45$ ), were enrolled in this study. Thirty-five were scheduled for CABG, twenty-five for aortic valve replacement (AVR). Basal plasma BNP levels were measured preoperatively on day of surgery. After induction of anaesthesia we performed comprehensive TEE with assessment of left ventricular diastolic function with transmitral flow velocity and pulmonary vein flow interrogation, and by measuring flow propagation velocity and mitral annular velocities with color $\mathrm{M}$-mode (CMM) and tissue Doppler imaging (TDI). 
Results and Discussion: In CABG group we found $71 \%$ of pts with grade I of diastolic dysfunction, while in AVR group we found $75 \%$ of grade I of diastolic dysfunction. Median plasma BNP levels were elevated in both groups compared with pts without diastolic dysfunction. Using mitral annular velocities with CMM and TDI, we could observe that pts with grade I of diastolic dysfunction in CABG group were $88 \%$ and in AVR group $92 \%$. In $17 \%$ of CABG group and in $17 \%$ of AVR group (grey zone), diastolic dysfunction was detected only by mitral annular velocities with CMM and TDI. Basal BNP in these subgroups were respectively $134 \pm 74$ and $145 \pm 80$.

\begin{tabular}{lcc}
\hline & BNP Group CABG & BNP Group AVR \\
\hline Diastolic dysfunction & $161 \pm 43$ & $178 \pm 58$ \\
Normal pattern & $26 \pm 11$ & $20 \pm 8$ \\
Grey zone & $134 \pm 74$ & $145 \pm 80$ \\
\hline
\end{tabular}

Conclusion: Plasma BNP levels and newer diastolic indexes measured from TDI and CMM both detect diastolic dysfunction. The present study shows that plasma BNP levels reflect diastolic dysfunction, not only in patients with systolic dysfunction as demonstrated in other studies ${ }^{(3)}$, but also in patients with preserved left ventricular function. It also demonstrates that standard echo evaluation can miss diastolic dysfunction in $17 \%$ of cases.

References:

1 Troughton RW, et al: Treatment of heart failure guided by plasma aminoterminal brain natriuretic peptide (N-BNP) concentrations. Lancet 2000; 355: 126-30.

2 Wei T. et al: Systolic and diastolic heart failure are associated with different plasma levels of B-type natriuretic peptide" Int J Clin Pract, August 2005; 59, 8: 891-894.

3 Valle R. et al: B-Type Natriuretic Peptide Can Predict the Medium-Term Risk in Patients With Acute Heart Failure and Preserved Systolic Function. Journal of Cardiac Failure, 2005; Vol. 11, No. 7 498-503.

\section{AP8-8 \\ Effects of activated protein $\mathrm{C}$ on leukocyte adherence and mesenteric plasma extravasation during experimental endotoxemia}

C. Lehmann, R. Scheibe, M. Schade, M. Wendt, D. Pavlovic

Department of Anesthesiology and Intensive Care Medicine, Ernst-MoritzArndt-University, Greifswald, Germany

Background and Goal of Study: To evaluate the effects of activated protein C (APC) on mesenteric microcirculation (leukocyte-endothelial interactions, plasma extravasation) during experimental endotoxemia in rats.

Materials and Methods: Prospective, randomized, controlled animal study in 40 male Lewis rats. Group 1 (CON) served as healthy control group. Group 2 (LPS) and group 4 (LPS + APC) received an endotoxin bolus i.v. (15 mg/kg lipopolysaccharide, LPS). In group 3 (APC) and group 4 (LPS + APC) 2 mg/kg APC were administered before endotoxin or placebo. Leukocyte-endothelial interactions and mesenteric plasma extravasation were determined at 0,1 and 2 hours during the experiment by intravital microscopy (IVM).

Results and Discussions: During endotoxemia, a significant increase of leukocyte rolling behaviour, leukocyte adherence and plasma extravasation in the LPS group ( $p<0,05$ versus control group) was observed. APC treatment (group 4) reduced leukocyte adherence and plasma extravasation significantly compared to untreated LPS animals (group 2). Also in group 3 (APC) a significant decrease of the mesenteric plasma extravasation was to be seen ( $p<0,05$ vs. control group).

Conclusion(s): APC treatment prevented the detrimental impact of endotoxemia on parameters of the mesenteric microcirculation in rats.

\section{References:}

1 Iba T, Kidokoro A, Fukunaga M, Nagakari K, Shirahama A, Ida Y. Activated protein C improves the visceral microcirculation by attenuating the leukocyte-endothelial interaction in a rat lipopolysaccharide model.

2 Crit Care Med. 2005 Feb; 33(2): 368-72.

\section{AP8-9}

Inhibition of neuronal nitric oxide syhnthase with 7-nitroindazole attenuates oxidative stress after combined burn and smoke injury in sheep

F. Saunders, M. Lange, C. Szabo, M. Westphal, D.L. Traber

Investigational Intensive Care Unit, University of Texas Medical Branch, Galveston, USA

Background and Goal of Study: Excessive production of nitric oxide (NO) by the neuronal NO synthase (nNOS) is believed to play a pivotal role in the pathogenesis of the inflammatory response to smoke inhalation and burn injury. We tested the effects of 7-nitroindazole (7-NI), a selective nNOS inhibitor, on crucial molecular mechanisms in an established model of acute lung injury in sheep.
Materials and Methods: Eleven healthy ewes were chronically instrumented for the measurement of cardiopulmonary hemodynamics. After one week of recovery, injury was induced by a total burn of $40 \%$ of the body surface and 48 breath of cotton smoke. Afterwards, the animals were randomly allocated to either receive a continuous infusion of $7-\mathrm{NI}(1 \mathrm{mg} / \mathrm{kg} / \mathrm{h})(\mathrm{n}=5)$ or the equivalent amount of placebo $(n=6)$.

Results and Discussions: The combination injury of burn and smoke resulted in an inflammatory response associated with oxidative stress, as indicated by significant increases in plasma nitrite/nitrate (NOx) levels, myeloperoxidase (MPO) and poly (ADP-ribose) polymerase (PARP) in the placebo group. In sheep treated with 7-NI, NOx plasma levels ( $8.4 \pm 1$ vs. $26 \pm 10 \mu \mathrm{mol} / \mathrm{L})$, MPO (3.9 \pm 0.2 vs. $5.9 \pm 0.7 \mathrm{U} / g$ tissue) and PARP were significantly reduced ( $p<0.05$ each).

Conclusion(s): Selective eNOS inhibition with 7-NI reduced important markers of systemic inflammation and oxidative burst in sheep. Therefore, administration of 7-NI may represent a beneficial approach to treat patients with smoke inhalation associated lung injury.

\section{AP9-1}

Influence of the anesthetic on the left ventricular function in rats assessed by $99 \mathrm{mTc}$ MIBI pinhole gated SPECT

R. Lauwers, P. Franken, C. Verborgh, F. Camu, T. Lahoute

Anesthesiology, Academisch Ziekenhuis Vrije Universiteit Brussel, Brussels, Belgium

Background and Goal of Study: Pinhole gated SPECT is used for serial follow up of myocardial perfusion and function of cardiac disease in rat models. The anesthetic used is critical for the correct assessment of cardiac function. The aim of our study was to compare the effect of different intraperitoneal (IP) and inhalation anesthetics on cardiac function.

Materials and Methods: Wistar rats $(n=48)$ were scanned 2 times with one week interval. Once under Pentobarbital $(60 \mathrm{mg} / \mathrm{kg}$ IP) anesthesia for the control study and a second time using a different anesthetic: (a) Isoflurane induction (5\%) followed by Pentobarbital $(60 \mathrm{mg} / \mathrm{kg} \mathrm{IP})$, (b) Ketamine-Xylazine (100-2 mg/kg)IP, (c) propofol $(200 \mathrm{mg} / \mathrm{kg}) I \mathrm{P}$, (d) midazolam $(70 \mathrm{mg} / \mathrm{kg}) I \mathrm{P}$, (e) Isoflurane (2,5\%), (f) Halothane (4\%), (g) sevoflurane $(2 \%)$ or (h) desflurane(7\%). Pinhole gated SPECT was performed 1 hour after intravenous injection of $330 \mathrm{MBq}{ }^{99 \mathrm{~m}} \mathrm{Tc}-\mathrm{MIBI}$ Reconstruction was performed iteratively. Parameters of cardiac function (EDV: end-diastolic volume, ESV: end-systolic volume) were derived using Quantitative gated SPECT. Left ventricular ejection fraction (EF), stroke volume (SV) and cardiac output (CO) were calculated. Results and Discussions: The \% change in comparison with the control are shown in the Table below. $\left({ }^{*} p<0.05,{ }^{\circ} p<0.01\right)$.

\begin{tabular}{lcccrrr}
\hline Group & EDV & ESV & HR & EF & SV & CO \\
\hline a & 2.2 & -4.5 & 1.1 & 1.4 & 3.8 & 3.8 \\
b & $33.6^{\star}$ & 25.6 & $-29.7^{\circ}$ & 3.6 & 38.3 & -2.6 \\
c & -3.6 & $-19.6^{\circ}$ & -2 & $8.3^{\circ}$ & 4.4 & 2.3 \\
d & $-20.8^{\star}$ & $-56.8^{\circ}$ & 8.4 & $21.3^{\circ}$ & -5.1 & 0.9 \\
e & $23.3^{\circ}$ & 0.0 & 3.8 & $7.0^{\star}$ & $32.9^{\circ}$ & $38^{\circ}$ \\
f & $43.2^{\circ}$ & $120^{\circ}$ & $11.8^{\star}$ & $-34.4^{\circ}$ & -4.8 & 5.9 \\
g & $20^{\circ}$ & -5.8 & -3.4 & $9.3^{\circ}$ & $26.9^{\circ}$ & $33^{\circ}$ \\
h & $19.6^{\star}$ & 4.3 & 0.2 & 5.3 & $25.5^{\circ}$ & $23^{\circ}$ \\
\hline
\end{tabular}

Conclusion: Depending on the anesthetic used, cardiac function alters dramatically. Our results suggest that Sevoflurane is the inhalation anesthetic of choice because of the better EF and CO. Midazolam is the intraperitoneal drug to use, because of the best EF, in rat heart studies.

\section{AP9-2}

Systolic arterial pressure variability reflects circulating blood volume alterations in endotoxin induced hypotension in rabbits A. Egi, M. Kawamoto, S. Kurita, O. Yuge

Anesthesiology and Critical Care, Division of Clinical Medical Science, Graduate School of Biomedical Sciences, Hiroshima University, Hiroshima, Japan

Background and Goal of Study: The high frequency component of power spectrum analysis of systolic arterial pressure variability (PSSAPV) has been shown to be comparable with systolic pressure variation (SPV) as an indicator of graded hemorrhaging (1), however, it was compared only in hemorrhagic shock induced animals. We attempted to determine the best means reflecting circulating blood volume (CBV) by using PSSAPV, SPV, pulse pressure variation (PPV), central venous pressure, and mean arterial pressure during endotoxin induced hypotension and fluid resuscitation.

Materials and Methods: Under isoflurane general anesthesia and mechanical ventilation, the rabbits in group $N(n=6)$ had LPS (lipopolysaccharide) induced hypotension only and those in group $R(n=6)$ had LPS induced hypotension followed by fluid resuscitation. After collecting baseline data, $1.5 \mathrm{mg} / \mathrm{kg}$ of LPS 
was injected intravenously in both groups and then data collection was performed. In group $\mathrm{R}$, hydroxyethyl-starch was continuously infused at a rate of $1 \mathrm{ml} / \mathrm{kg} / \mathrm{min}$ for $25 \mathrm{~min}$. Data were obtained at 5 and $60 \mathrm{~min}$ after fluid resuscitation in group R and at same timing in group N. Correlations between CBV and the parameters were analyzed using linear regression analysis. $P<0.05$ was considered statistically significant.

Results and Discussions: The correlation between CBV and total power $\left(R^{2}=0.241, p=0.0004\right)$ of PSSAPV was more significant as compared to high frequency $\left(R^{2}=0.172, p=0.003\right)$, low frequency $\left(R^{2}=0.105, p=0.025\right)$ of PSSAPV, and central venous pressure $\left(R^{2}=0.113, p=0.020\right)$, while no correlations were noted between CBV and SPV $\left(R^{2}=0.065, p=0.080\right)$, PPV $\left(R^{2}=0.025, p=0.283\right)$, and mean arterial pressure $\left(R^{2}=0.003, p=0.692\right)$. Conclusion(s): Total power of PSSAPV best reflected the changes of CBV even in endotoxin induced hypotension as compared to conventional circulatory parameters.

Reference:

1 Lai HY, Yang CCH, Huang FY, et al. Clin. Sci. 2003; 105: 491-497.

\section{AP9-3}

Anaesthetic techniques affect vascular responsiveness in experimental hemorrhagic shock

I. Samarska, A.H. Epema, H. Buikema, L.P.H.J. Aarts, R.H. Henning

Departments of Anesthesiology and Clinical Pharmacology, University Medical Center Groningen, Groningen, The Netherlands

Background and Goal of Study: Altered vascular reactivity after severe hemorrhagic shock may be influenced by the choice of anaesthetic technique and interfere with the therapeutic effectiveness of vasoactive agents. The aim of the present study was to explore the changes in vascular reactivity following hemorrhagic shock using two types of general anaesthesia.

Materials and Methods: After institutional approval, mice $(n=24)$ were randomized to undergo hemorrhagic shock $(90 \mathrm{~min}, 30 \mathrm{mmHg}$ ) by withdrawal of blood, or a sham-procedure. Half of the animals were anaesthetized with isoflurane (ISO, 1.4\%), the other half received additional nitrous oxide $\left(\mathrm{N}_{2} \mathrm{O}\right.$, $66 \%$ ). Vasomotor responses were studied in 2-mm rings of aorta mounted in a wired myograph.

Results and Discussions: Contraction to phenylephrine $(\mathrm{PE})$ was increased in shock-mice anaesthetized with ISO compared to SHAM $(P<0.05$; fig). In contrast, addition of $\mathrm{N}_{2} \mathrm{O}$ to ISO resulted in similar PE-evoked contractions in mice with and without shock (fig). In SHAM, acetylcholine (ACh) caused a biphasic curve consisting of an initial relaxation followed by a contractile response sensitive to the COX-inhibitor indomethacin $(1 \mu \mathrm{M})$. In contrast, in ISO animals undergoing shock, $\mathrm{ACh}$ evoked only relaxing responses.
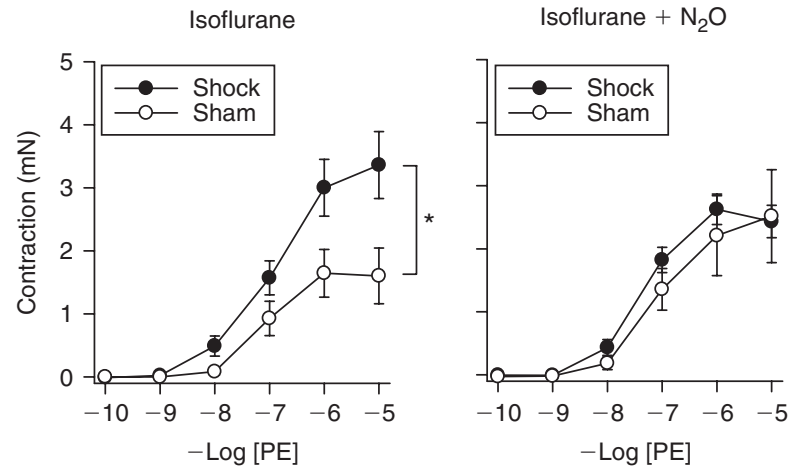

Conclusion(s): Short-term hemorrhagic shock affects both contractile and relaxing vasomotor responses in aortic rings of mice anaesthetized with ISO. Addition of nitrous oxide attenuates shock-dependent changes.

References:

Savoye G. Shock 2005; 23 (5): 411-416.

Baumert J.-H. BJA 2005; 94 (6): 727-32.

\section{AP9-4}

Diabetes inhibits coronary collateral development in a canine model of repetitive coronary occlusion

W. Gu, B. Yu, J. Tessmer, D. Warltier, J. Kersten

Department of Anesthesiology, Ruijin Hospital, Shanghai, China

Background and Goal: Diabetic individuals with acute coronary syndrome have an increased risk of adverse cardiovascular events (1). Recent studies demonstrated that a well-developed collateral circulation is associated with higher survival rate, smaller infarct size as compared to poor collaterals (2). We tested the hypothesis that diabetes inhibits the coronary collateral development in a canine model of repetitive coronary occlusion.

Materials and Methods: Dogs were subjected to brief (2 min), repetitive coronary artery occlusions (1/h, 8/day, 21 day duration) in the absence (control) or presence of diabetes (diabetes). A sham group was instrumented identically but received no occlusion. Myocardial blood flow was determined in the myocardium perfused by the LAD and the LCCA. Peak reactive hyperemia response $(\mathrm{PRH})$ was recorded. Collateral blood flow was expressed as the percentage of normal zone blood flow.

Results and Discussions: Coronary collateral blood flow was shown in the figure. $\mathrm{PRH}$ in the control group was significantly lower than that on day 1. PRH remained unchanged in the diabetes and the sham group.

Conclusion(s): Diabetes inhibits the development of coronary collateral blood flow.

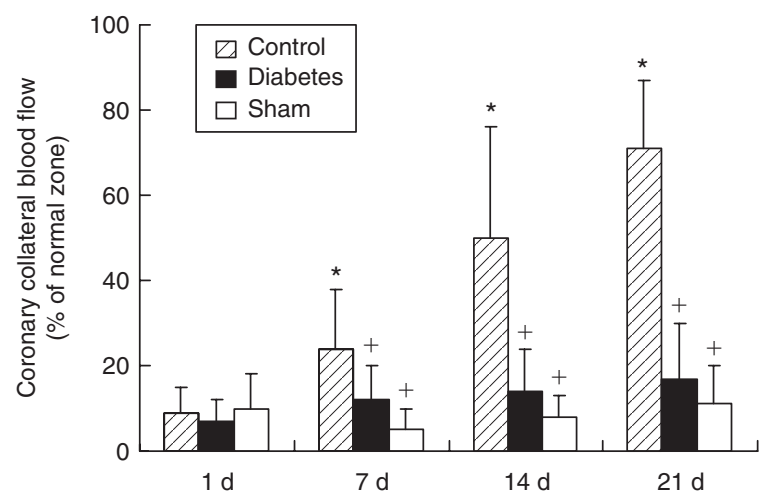

Fig. ${ }^{*} \mathrm{P}<0.05$ vs day $1 ; \mathrm{\dagger P}<0.05$ vs the respective value in the control group.

\section{References:}

1 Fergus TS, Fazel R, Fang J, et al. Heart 2004; 90: 1051-1052.

2 Perez-Castellano N, Garcia EJ, Abeytua M, et al. J Am Coll Cardiol 1998; 31: 512-518.

\section{AP9-5 \\ Alterations of mitochondrial apoptotic pathways in load- induced right ventricular failure}

C. Dewachter, B. Rondelet, L. Dewachter, S. Brimioulle, F. Kerbaul

Service d'Anesthésiologie Réanimation, Hopital ERASME, Bruxelles, Belgium

Background and Goals: We previously reported an experimental model of persistent right ventricular (RV) failure consecutive to a transient, 90-min increase in afterload induced by pulmonary arterial banding (1). We hypothesized that this particular type of ventricular stunning might be related to an early activation of apoptotic pathways.

Material and Methods: Pulmonary arterial elastance (Ea) and RV end-systolic elastance (Ees) were determined by a single beat method (2) in 7 anaesthetized dogs before and 30 min after the release of a transient PA banding. The animals were killed by an anaesthetic overdose and RV and left ventricular (LV) myocardial tissue sampled for the measurements of mitochondrial mRNA of Bax (proapoptotic) and Bcl-2 (antiapoptotic) by real time polymerase chain reaction (RTQPCR). The Bax/Bcl-2 ratio was taken as an index of apoptototic activity. Results and Discussion: Values are reported as mean \pm SEM. The transient increase in PA pressure persistently increased right atrial pressure from $8.0 \pm 0.4$ to $10.0 \pm 0.8 \mathrm{mmHg}(P<0.05)$, Ea from $0.99 \pm 0.05$ to $2.86 \pm 0.15 \mathrm{mmHg} / \mathrm{ml}(P<0.05)$, and decreased Ees from $1.12 \pm 0.05$ to $0.55 \pm 0.03 \mathrm{mmHg} / \mathrm{ml}(P<0.05)$, Ees/Ea from $1.14 \pm 0.06$ to $0.20 \pm 0.02$ $(P<0.05)$, and cardiac output from $4.5 \pm 0.1$ to $2.3 \pm 0.1 \mathrm{~L} / \mathrm{min}(P<0.05)$, indicating RV failure with altered RV-arterial coupling. As compared to 8 normal control dogs, RV Bax/Bcl-2 mRNA was increased (1.86 \pm 0.35 vs. $1.00 \pm 0.30 ; \mathrm{P}<0.05)$, while LV Bax/Bcl-2 mRNA was not different (1.61 \pm 0.41 vs. $1.00 \pm 0.30 ; P>0.05)$.

Conclusion: An early induction of apoptotic pathways may be involved in persistent RV failure induced by a transient increase in pulmonary artery pressure.

References:

1 Kerbaul et al. Crit Care Med. 2004; 32: 1035-40.

2 Brimioulle et al. Am J Physiol. 2003; 284: H1625-30. 


\section{AP9-6 \\ Influence of temperature on the positive inotropic effect of Levosimendan and Dobutamine}

A. Rieg, S. Schroth, R. Autschbach, R. Rossaint, G. Schälte

Anesthesilogy, University Hospital Aachen, Aachen, Germany

Background and Goal of the study: Levosimendan is a new calcium sensitizing agent with rising clinical use in heart surgery. Due to accumulation of adverse factors patients during open heart surgery are at high risk to develop dysregulation of body temperature. We performed this study to investigate the effect of temperature on the contractile response of myocardial trabeculae to levosimendan in comparison to dobutamine.

Materials and Methods: Guinea pig ventricular trabeculae were placed in an oxygenated HEPES-Buffer and stimulated at a frequency of $1.5 \mathrm{~Hz}$. Trabeculae were randomized to a temperature of $40^{\circ} \mathrm{C}, 37^{\circ} \mathrm{C}, 34^{\circ} \mathrm{C}$ and $31^{\circ} \mathrm{C}$, and levosimendan and dobutamine were stepwise increased from $10^{-9} \mathrm{M}$ to $10^{-5} \mathrm{M}$. Maximum developed force was continuously recorded. At the end of each experiment all trabeculaes were tested for functional integrity.

Results and Discussion: Results after 26 experiments for levosimendan and 16 for dobutamine revealed a dose dependent positive inotropic effect for both agents. But, the positive inotropic effect of levosimendan proved to be independent of temperature; in contrast, the positive inotropic effect of dobutamine is suppressed by hypothermia. Hypothermia per se induced a positive inotropic effect. Possibly, hypothermia which per se interacts with intracellular calcium homeostasis inhibits the cAMP dependent dobutamine effect, but not the calcium sensitizer levosimendan.

\begin{tabular}{lll}
\hline & Levosimendan & Dobutamine \\
\hline $37^{\circ} \mathrm{C}$ & $120.7 \pm 8.5$ & $134.3 \pm 7.3$ \\
$31^{\circ} \mathrm{C}$ & $124.1 \pm 17.6$ & $112.4 \pm 4$ \\
\hline
\end{tabular}

Increase of developed force of contraction by levosimendan and dobutamine 10-6 $\mathrm{M}$ in percent of baseline $\pm \mathrm{SD}$.

Conclusion: Our results suggest no modulation of the positive inotropic effect of levosimendan by the experimental temperature. Acting as a calcium sensitizer instead of cAMP coupled dobutamine, levosimendan might prove to be a favourable positive inotropic substance after cardiopulmonary bypass, if patients show a low-output syndrom during hypothermia.

\section{AP9-7}

Involvement of NOS1 in the Beta3-adrenoceptor signaling pathway in diabetic cardiomyopathy

A. Birenbaum, J. Amour, P. Michelet, P. Coriat, B. Riou

lle de France, Hopital Pitié-Salpêtrière, Paris, France

Background: In the diabetic heart, the positive inotropic response to betaadrenergic stimulation is markedly altered owing to the negative inotropic effect of beta3-adrenoceptor(1). The nitric oxide (NO) involved in the beta3adrenoceptor signalling pathway is depending on the NO synthase (NOS). The specific NOS isoform of this pathway (NOS1, NOS2 or NOS3), which could be a new therapeutic target, is still unknown in diabetic cardiomyopathy. Material and Methods: Left ventricular papillary muscles were provided from healthy and streptozotocin-induced diabetic rats. The effect of beta3adrenergic signaling pathway inhibition on the inotropic response following beta-adrenergic stimulation was investigated using S-cyanopindolol (direct antagonist of beta3-adrenoceptor), L-NAME (a non-specific NOS antagonist) or L-VNIO (a specific antagonist of NOS1) at $29^{\circ} \mathrm{C}$ with 12 pulses $/ \mathrm{min}$. Immunoblots experiments were performed within cardiomyocytes. The data are mean percentages of baseline \pm SD.
Results and Discussion: In healthy rats, the positive inotropic effect (179 $\pm 15 \%)$ was unchanged by S-cyanopindolol (174 $\pm 20 \%)$ or L-NAME $(183 \pm 19 \%)$ or LVNIO $(179 \pm 22 \%)$. In contrast, in diabetic rats, the impaired positive inotropic effect (112 $\pm 5 \%$ ) was partially restored by S-cyanopindolol $(137 \pm 8 \%, P<0.05)$, L-NAME $(133 \pm 11 \%, P<0.05)$ or LVNIO $(129 \pm 12 \%$, $\mathrm{P}<0.05)$. NOS isoform expression was exclusively NOS1 in diabetic cardiomyocyte. NOS2 and NOS3 proteins were not expressed in diabetic cardiomyocyte.

Conclusion: The beta3-adrenoreceptor effect which plays an important role in the diabetic beta-adrenergic dysfunction is exclusively mediated by NOS1-derived NO in the diabetic cardiomyocyte. These findings could lead to new therapeutic targets.

Reference:

1 Amour et al. EJA 2006; 23: suppl S37.

\section{AP9-8}

Does clonidine improves post-hypoxic vascular reactivity? Evaluation on rats isolated aorta

M. Gourdin, J. Jamart, V. Collet, M. DE Kock

Anesthesiology, University Hospital UCL -Mont-Godinne, Yvoir, Belgium

Background and Goal of Study: Clonidine (CL), an $\alpha 2$-adrenoreceptor agonist, reduces perioperative myocardial ischemia in-patients undergoing surgery. ${ }^{1}$ In isolated heart model, this class of drugs protects against ischemia. Our experimental study intends to demonstrate that improvement of posthypoxic vasomotricity is the mechanism underlying this beneficial effect. Materials and Methods: After animal ethic committee approval, 60 rings aorta $(3 \times 20)$ from 15 different rats were studied according to a validated methodology. ${ }^{3} \mathrm{CL}\left(10^{-4} \mathrm{M}\right)$ was added in two baths (CL Group). Two were used as the control group (CTL Group). After fifteen minutes, all baths were washed and 25 minutes of hypoxia $\left(\mathrm{PpO}_{2}<10 \mathrm{mmHg}\right)$ was applied. After 40 minutes re-oxygenation $\left(\mathrm{PpO}_{2}>400 \mathrm{mmHg}\right)$, post-hypoxic vaso-constriction was evaluated by cumulative Phenylephrine (PE) concentrations $\left(10^{-10}-10^{-4} \mathrm{M}\right)$. Post-hypoxic endothelium-dependent and independent vasodilatations were investigated respectively by cumulative acetylcholine and nitroprusside concentrations $\left(10^{-10}-10^{-4} \mathrm{M}\right)$ on pre-contracted aorta. The statistical analysis used GEE regression, $p<0.05$ significant.

Results and Discussions: In CL group, post-hypoxic endothelium-dependent vasodilatation and vaso-constriction were significantly different from the CTL group ( $p<0.002$ and $p<0.018$, respectively fig 1 and 2 ). When considering post-hypoxic endothelium-independent no significant difference was found.
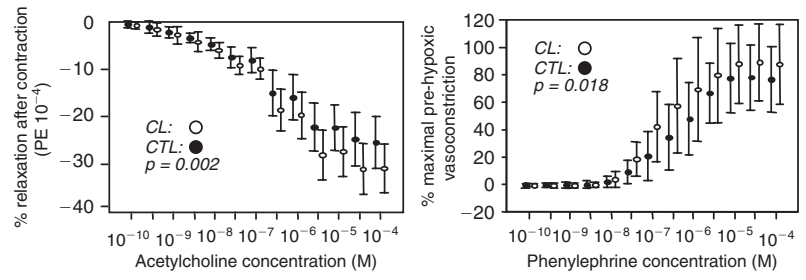

Fig. 1 Dose response relationship of post-hypoxic of endothelium depnedent vasodilatation Fig. 2 Dose response relationship of post-hypoxic vasoconstriction

Conclusion(s): CL enhances endothelium-dependent vasodilatation and posthypoxic vasoconstriction, a phenomenon involved in myocardial ischemic protection.

References:

1 Wallace A. Cur Opinion Anaesth. 2006; 19(4): 411-17.

2 Guo H. Anesth Analg. 2005; 100(3): 629-35

3 Besse S. Eur J Pharmacol. 2006; 531(1-3): 187-93.

\section{Respiration}

\section{AP1-1}

Development and test of a new mode of assisted spontaneous ventilation: Chaotic variable pressure support ventilation (noisy PSV)

M. Gama De Abreu, P.M. Spieth, P. Pelosi, P. Aikele, T. Koch

Clinic of Anesthesiology and ICU, University Clinic Carl Gustav Carus,

Dresden, Germany
Background and Goal of Study: Pressure support ventilation (PSV) combined with intermittent sighs (PSV + Sighs) is widely used when resuming spontaneous breathing, but the resulting respiratory pattern shows low variability if the inspiratory effort is constant. We aimed at implementing and testing a new mechanical ventilation mode that is able to enhance the variability of the respiratory pattern independently of the inspiratory effort.

Materials and Methods: A mechanical ventilator EVITA XL 4 Lab (Dräger Medical AG, Lübeck, Germany) was controlled by an external laptop to vary 
pressure support chaotically (noisy PSV). During noisy PSV, pressure support values followed a normal distribution, with mean comparable to traditional PSV and standard deviation set at $1 / 3$ of mean. During PSV + Sighs, sighs were applied with a frequency of $1 / \mathrm{min}$. Tests were performed in 8 pigs with acute lung injury induced by surfactant lavage. Gas exchange, work of breathing, breathing comfort (visual analogue scale), and lung aeration (helicoidal computer tomography -CT- scans at end-expiration, CHRISTIAN Software) were assessed during controlled ventilation (baseline + injury) and after resuming of spontaneous breathing, with both modes (1 hour duration each, random sequence).

Results and Discussions: Surfactant washout resulted in significant deterioration of the pulmonary function. After resuming of spontaneous breathing, pulmonary function improved with both modes, but noisy PSV led to higher $\mathrm{PaO}_{2} / \mathrm{FIO}_{2}$, lower $\mathrm{AaDO}_{2}$ and higher breathing comfort than PSV + Sighs (median values: $296.8 \mathrm{mmHg}\left(\mathrm{Q}_{1-3} 174.0-395.2\right)$ vs. $216.8 \mathrm{mmHg}\left(\mathrm{Q}_{1-3}\right.$ $152.2-321.2) ; \quad 309.9 \mathrm{mmHg}\left(\mathrm{Q}_{1-3} 272.6-371.2\right)$ vs. $350.9 \mathrm{mmHg}\left(\mathrm{Q}_{1-3}\right.$ 301.9-389.9); $53.5\left(\mathrm{Q}_{1-3} 49-55\right)$ vs. 50 ( $\left.\mathrm{Q}_{1-3} 47-51.8\right)$, respectively, $\left.\mathrm{p}<0.05\right)$. Both modes were associated with comparable distributions of hyper-, normal, hypo- and non-aerated compartments in CT scan analysis, as well as inspiratory pressure time product and $\mathrm{P} 0.1$ values.

Conclusion(s): Noisy PSV restored the variability of the respiratory pattern during assisted spontaneous breathing and was superior to PSV + Sighs with regard to oxygenation and comfort of breathing in this model of acute lung injury. This new mode may prove valuable.

\section{AP1-2}

\section{Volume-controlled versus pressure-controlled ventilation in morbid obesity}

A. Parera, R. Hoffmann, J.I. Casas, M.V. Moral

Anaesthesiology, Hospital de la Santa Creu i Sant Pau, Barcelona, Spain

Background and Goal of Study: Comparison of two different mechanical ventilation modes: volume controlled (VCV) vs. pressure controlled ventilation (PCV) and influence on oxygenation in morbidly obese patients during open bariatric surgery.

Materials and Methods: Prospective, randomized cross-over clinical trial in 50 with $\mathrm{BMI}>40 \mathrm{~kg} / \mathrm{m}^{2}$ admitted to open bariatric surgery. After randomization of the initial ventilatory mode, V(25): VCV and P(25): PCV, we performed induction of general anaesthesia. Ventilatory parameters: $12 \mathrm{bpm}, \mathrm{FiO}_{2} 0.5$, and group-related tidal volume or pressure level were set to achieve normoventilation. We analyzed arterial blood gas every $20 \mathrm{~min}$ and then changed the ventilatory mode completing $2 \mathrm{VCV}$ and $2 \mathrm{PCV}$ phases. Main variable $\mathrm{PO}_{2}$ and secondary variables: $\mathrm{PCO}_{2}, \mathrm{EtCO}_{2}$, parameters of ventilation and hemodynamics were assessed. The results of both techniques in each patient and between groups were compared through an ANOVA. Alpha error of $5 \%$ and statistical power of $80 \%$.

Results and Discussions: We observed an increase of $\mathrm{PO}_{2}$ during surgery, an increase of the compliance and a decrease of the airway endurance in both cases. This change could be due to a FRC recovery doing the laparotomy ${ }^{1} . \mathrm{V}$ group: initial $\mathrm{PO}_{2}(105.4 \pm 34.5)$ and final $\mathrm{PO}_{2}(135.4 \pm 36.5)$, and $\mathrm{P}$ group: initial $\mathrm{PO}_{2}(125.4 \pm 32.7)$ and final $\mathrm{PO}_{2}(147.6 \pm 43.1)$. $\mathrm{PCO}_{2}: 34-38 \mathrm{mmHg}$ and $\mathrm{EtCO}_{2} 30-34 \mathrm{mmHg}$. Airway pressures, Ppl in $\mathrm{VCV}(20.5 \pm 3.8 \mathrm{mmHg})$ was inferior to Pmax in PCV $(23.5 \pm 4 \mathrm{mmHg})$ and homogeneous Pmed in both groups $(8.4 \pm 1.45 \mathrm{mmHg})$. No significant statistical differences $(p=0.07)$ were observed among the two ventilation modes according to oxygenation, mechanic ventilation and hemodynamics, neither on each patient nor in the total research group.

Conclusions: We found no evidence that any of the two ventilation modes (PCV or VCV) provide a better oxygenation and ventilation to the patients. Nevertheless, we cannot conclude if any of these ventilatory modes may have a more protective effect on the lung than the other in case of extended mechanical ventilation.

Reference:

1 Auler JO Jr et al. Anesth Analg.2002; 94(3): 741-748.

\section{AP1-3}

\section{Randomised controlled trial comparing application of PEEP} and ZEEP during laparoscopic surgery by electrical impedance tomography (EIT)

J. Karsten, A. Jedmowski, M. Grossherr, S. Leonhardt, T. Meier

Department of Anaesthesiology, University Medical Center SchleswigHolstein, Luebeck, Germany

Background and Goal of Study: Pneumoperitoneum (PP) influences respiratory mechanics. This leads to formation of atelectasis and impairment of oxygenation. Application of positive-end-expiratory pressure (PEEP) can maintain pulmonary gas exchange. We investigated the effect of anaesthesia, mechanical ventilation and PP on arterial oxygenation and regional ventilation monitored by EIT with and without PEEP.

Materials and Methods: We prospectively randomised 32 consecutive patients (ASA I/II) scheduled to undergo elective laparoscopic cholecystectomy. The patients were randomly assigned to PEEP $\left(10 \mathrm{cmH}_{2} \mathrm{O}\right)$ or ZEEP group $\left(0 \mathrm{cmH}_{2} \mathrm{O}\right)$. The patients were ventilated with constant tidal volume. EIT (Dräger Medical) was performed before the induction of anaesthesia [T0], after induction of anaesthesia and tracheal intubation [T1], after application of the PP $(15 \mathrm{mmHg})$ [T2] and after exsufflation of the PP [T3] during a one minute time interval. We calculated the impedance ratio (IR) (1) to investigate the differences in distribution and homogeneity of ventilation. $\mathrm{PaO}_{2}$ and regional ventilation were compared between both groups. $\mathrm{p}<0.05$ was considered as statistically significant.

Results and Discussions: Both groups did not differ regarding age, sex, BMI, ASA score, length of surgery and anaesthesia. No significant difference in IR was found between the two groups at TO, whereas the PEEP group showed a more homogenous ventilation. At $\mathrm{T} 1$ there was a significant improvement in $\mathrm{PaO}_{2}(p=0.003)$ and a more homogenous ventilation $(p=0.001)$ in the PEEP group. At T2 no significant difference in $\mathrm{PaO}_{2}(p=0.06)$ was found between both groups, whereas there was a trend towards a better oxygenation in the PEEP group. At T3 we found a more homogenous distribution of ventilation $(p=0.001)$ in the PEEP group. There were no significant differences in $\mathrm{PaO}_{2}$ between both groups.

Conclusions: EIT is capable providing a continuous monitoring of the distribution of regional lung volumes under clinical circumstances. The effect of intraoperative PEEP application can be evaluated by dynamic EIT monitoring. PEEP compared to ZEEP preserves arterial oxygenation only after induction of anaesthesia and beginning of mechanical ventilation.

References:

1 Kunst PW et al. Chest 1999; 115(4): 1102-6.

\section{AP1-4}

\section{Effect of continuous positive airway pressure on} haemodynamic variables in human volunteers

\section{I.L. Dorairaj, J. Bingham, F. Shivji, R.P. Mahajan}

University Department of Anaesthesia and Intensive Care, Queens Medical Centre, Nottingham, United Kingdom

Background and Goal of Study: The reported effects of continuous positive airway pressure (CPAP) on systemic haemodynamics are confounded by disease and/or drug therapy. Using the recently introduced Finometer ${ }^{1}$, we investigated the effects of two levels of CPAP on haemodynamic variables, including heart rate (HR), mean arterial pressure (MAP), cardiac output (CO), stroke volume (SV), total peripheral resistance (TPR) and central arterial compliance (CAC) in healthy awake volunteers.

Materials and Methods: Haemodynamic variables were recorded on beatto-beat basis using Finometer and beatscope software in 15 healthy male volunteers, aged 19-21 years. In a predetermined random order, CPAP of 5 and $10 \mathrm{~cm}$ was applied.

Results and Discussion:

\begin{tabular}{|c|c|c|c|c|}
\hline & Baseline & $\begin{array}{l}\text { CPAP } \\
5 \mathrm{~cm} \\
\mathrm{H}_{2} \mathrm{O}\end{array}$ & $\begin{array}{l}\text { CPAP } \\
10 \mathrm{~cm} \\
\mathrm{H}_{2} \mathrm{O}\end{array}$ & Fried-man \\
\hline HR (bpm) & $75[72,81]$ & $70[66,76]$ & $76[72,80]$ & $\mathrm{P}<.05$ \\
\hline $\begin{array}{l}\text { MAP } \\
(\mathrm{mm} \mathrm{Hg})\end{array}$ & $89[79,103]$ & $89[85,110]$ & $95[85,109]$ & \\
\hline $\mathrm{CO}\left(\mathrm{L} \mathrm{min}^{-1}\right)$ & $6.4[5.9,7.3]$ & $6.1[5.3,6.9]$ & $6.0[4.8,6.4]$ & $P<0.01$ \\
\hline $\mathrm{SV}(\mathrm{ml})$ & $87[77,95]$ & $86[72,98]$ & $72[70,88]$ & $P<0.01$ \\
\hline TPR (AU) & $0.78[0.71,0.98]$ & $0.89[0.75,1.05]$ & $0.96[0.79,1.44]$ & $P<0.01$ \\
\hline $\mathrm{CAC}(\mathrm{AU})$ & $2.7[2.6,2.9]$ & $2.7[2.6,3.0]$ & $2.6[2.4,2.9]$ & \\
\hline
\end{tabular}

Table: Median [IQR], AU - arbitrary units

The CO decreased and TPR increased significantly with each step change in CPAP. The decrease in SV was significant at CPAP of $10 \mathrm{~cm} \mathrm{H}_{2} \mathrm{O}$.

Conclusions: 1) This is the first study using non-invasive Finometer measurements to evaluate changes in haemodynamic variables with CPAP. 2) The consistency of change in CO and TPR with CPAP can potentially be developed into a test for integrity of autonomic responses.

Reference:

1 Elvan-Taspinar A et al. J Hypertens 2003; 21: 2053-60

\section{AP1-5}

Evaluation of fixed performance oxygen masks in simulated chronic obstructive airways disease

A. Shah, Q. Siddiqui, JG. Hardman, IK. Moppett

Anaesthesia, University of Nottingham, Nottingham, United Kingdom 
Background and Goal of Study: Previous studies have evaluated the performance of Venturi masks in volunteers ${ }^{1}$ and face models ${ }^{2}$ with simulated normal respiration but not with abnormal respiration as may occur in chronic obstructive pulmonary disease (COPD). We assessed the effects of simulated COPD breathing patterns on Venturi mask performance.

Materials and Methods: The breathing patterns of normal adult subjects and patients with acute exacerbations of $\mathrm{COPD}^{3}$ were simulated in our Human Patient Simulator (METI Inc.). Spirometric and end-tidal $\mathrm{CO}_{2}$ values were matched to clinical data $^{3}$, all measured using a calibrated DatexOhmeda Capnomac. Three different fixed-performance masks (Flexicare Venturi (F1), Flexicare Ventimask (F2) and Intersurgical Venturi (I)) were applied to the face and oxygen was supplied with the manufacturers' recommended flow rates. $\mathrm{FiO}_{2}$ was measured at the lips and in the larynx for each of the masks. All measurements were made 3 times after 3 minutes of steady conditions.

Results and Discussions:

\begin{tabular}{|c|c|c|c|c|c|c|}
\hline \multirow[b]{2}{*}{ Set $\mathrm{FiO}_{2}$} & \multicolumn{3}{|c|}{ Normal $\mathrm{FiO}_{2}$ (lips/larynx) } & \multicolumn{3}{|c|}{ COPD $\mathrm{FiO}_{2}$ (lips/larynx) } \\
\hline & $\mathrm{F} 1$ & 1 & F2 & $\mathrm{F} 1$ & 1 & F2 \\
\hline $24 \%$ & $24 / 25$ & $24 / 23$ & $26 / 26$ & $24 / 24$ & $22 / 23$ & $27 / 26$ \\
\hline $28 \%$ & $27 / 28$ & $28 / 28$ & $28 / 30$ & $26 / 27$ & $25 / 27$ & $30 / 29$ \\
\hline $35 \%$ & $34 / 34$ & $34 / 36$ & $34 / 36$ & $27 / 32$ & $31 / 33$ & $37 / 36$ \\
\hline $40 \%$ & $39 / 39$ & $39 / 40$ & $44 / 42$ & $37 / 35$ & $29 / 35$ & $43 / 41$ \\
\hline $60 \%$ & $48 / 52$ & $48 / 52$ & $58 / 58$ & $41 / 43$ & $42 / 41$ & $56 / 53$ \\
\hline
\end{tabular}

Variation of $\mathrm{FiO}_{2}$ was trivial between experiments (<1\%). Measured $\mathrm{FiO}_{2}$ was close to desired values up to $40 \%$ for each mask, but only one (F2) was close at $60 \%$. Simulated COPD respiration caused a clinically significant reduction in $\mathrm{FiO}_{2}$ in two of the masks, whereas $\mathrm{F} 2$ was relatively unaffected. Conclusion: Even if Venturi masks perform acceptably under normal conditions, abnormal ventilation may cause clinically significant changes in performance.

References:

1 Hill SL Barnes PK, Hollway T et al. Br Med J 1984; 288: 1261-63.

2 Cox D, Gillbe C. Anaesthesia 1981; 36: 958-64.

3 Tobin MJ Chadha TS, Jenouri G et al. Chest 1983; 84: 286-94.

\section{AP1-7}

Diferences in the gas distribution in volume controlled ventilation compared to pressure controlled ventilation in a multialveolar lung model

J. Navarro-Martinez, R. Company, J. Del Fresno, R. Roca

Anestesiología y Reanimación, Hospital General de Alicante, Alicante,

Spain

Background and Goal of Study: Exists quite a controversy as to whether ventilate a patient with pressure controlled ventilation (PCV) over volume controlled ventilation (VCV). The goal was to determinate if PCV offers advantages in terms of pressure and alveolar distribution in a multialveolar compartment lung model compared to a VCV.

Materials and Methods: The lung model was a passive simulator with 3 compliances of 25 or $12,5 \mathrm{ml} / \mathrm{cmH} 20$ and 4 resistances of $5 \pm 10 \%$ and $20 \pm 10 \% \mathrm{cmH}_{2} \mathrm{O} / \mathrm{l} / \mathrm{seg}$. A total of 64 situations were simulated. We used as respirator the TEMEL Supra -GA,. The respirator setting was: open circuit, of

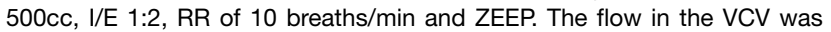
$60 \mathrm{l} / \mathrm{min}$. The pressure in the VCP was the lower to reach the tidal volume fixed. We used a endtracheal tube of 7,5 ID.

Results and Discussions:

Table 1:

\begin{tabular}{llcc}
\hline & & VCV & VCP \\
\hline MOUTH & Paw peak & $18 \pm 4$ & $11 \pm 2^{\star \star \star}$ \\
& Paw plateau & $10.2 \pm 1.5$ & $10.6 \pm 1.5$ \\
& Paw mean & $5.6 \pm 0.6$ & $4.4 \pm 0.5^{\star \star \star}$ \\
& t Flow $=0$ & $0.7 \pm 0.6$ & $0.1 \pm 0.1^{\star \star \star}$ \\
ALVEOL 1 & IPF & $1.3 \pm 0.1$ & $0.75 \pm 0.1^{\star \star \star}$ \\
& $P_{\mathrm{A}}$ initial & $11 \pm 3$ & $12 \pm 2^{\star}$ \\
& IPFinitial & $0.5 \pm 0.01$ & $0.3 \pm 0.01^{\star \star \star}$ \\
ALVEOLI 2 & t Flow $=0$ real & $0.3 \pm 0.1$ & $0.08 \pm 0.01^{\star \star \star}$ \\
& P inicial & $8.3 \pm 2.7$ & $9.6 \pm 2^{\star}$ \\
ALVEOLI 3 & IPFincial & $0.3 \pm 0.01$ & $0.17 \pm 0.04^{\star \star \star}$ \\
& t Flow $=0$ real & $0.3 \pm 0.02$ & $0.08 \pm 0.02^{\star \star \star}$ \\
& P initial & $10 \pm 2$ & $10 \pm 2$ \\
& IPFincial & $0.37 \pm 0.02$ & $0.22 \pm 0.02^{\star \star \star}$ \\
& t Flow $=0$ real & $0.39 \pm 0.02$ & $0.09 \pm 0.02^{\star \star \star}$ \\
\hline
\end{tabular}

* $p<0.05^{* \star *} p<0.01$

We also calculated the flowback and the gas distribution parameters based on the studies made by Hedenstierna calculating the initial and final distribution. The PCV group obtained worse results compared to the VCV group.

Conclusion(s): PCV does not offer advantages over the VCV in terms of alveolar pressure, which is the harmful to the lung; obtaining worse results in the redistribution parameters. The VCV guarantees a tidal volume fixed, whereas the PCV maintains a constant pressure, but the tidal volume turns on the compliance and resistance and may create a situation of hypoventilation.

\section{AP1-8}

Analysis of the dynamic airway pressure concavity and distribution of lung aeration in piglets

A. Carvalho, F. Jandre, M. Melo, M. Abreu, A. Giannella-Neto

Biomedical Engineering Program, Federal University of Rio de Janeiro, Rio de Janeiro, Brazil

Background and Goal of Study: The institution of a protective ventilatory strategy in anaesthetic conditions seems to be difficult, since the compromise between the best oxygenation with less hemodynamical and ventilatory effects, is not commonly satisfied. This work aims to evaluate the usefulness of the shape of the dynamic airway pressure (Paw dyn $)$ concavity as a guide to set positive end-expiratory pressure (PEEP) in order to institute a ventilatory strategy to minimize the lung mechanical stress.

Materials and Methods: Twelve healthy piglets $(17-23 \mathrm{Kg})$, laying supine position were anesthetized and mechanically ventilated with a square flow waveform. A PEEP titration from 16 to $0 \mathrm{cmH}_{2} \mathrm{O}$, in steps of $4 \mathrm{cmH}_{2} \mathrm{O}$, was performed and the airway pressure (Paw) and flow $(\mathrm{F})$ signals were recorded. In six animals, helical CT-scans were obtained during end-expiratory (EEP) and end-inspiratory pauses (EIP) at each PEEP. Two mechanical models were fitted to the data: $P a w=E_{1} V+E_{2} V^{2}+R . F+P_{0}\left(V\right.$ is volume, $P_{0}, E_{1}$, $E_{2}$ and $R$ are constants) and $P a w=a \cdot t^{b}+c(t$, is the inspiratory time, $\boldsymbol{a}, \boldsymbol{b}$ and $c$ are constants). The $\% E_{2}\left(\% E_{2}=100 .\left[E_{2} V_{T} /\left(E_{1}+E_{2} V_{T}\right)\right]\right.$, where $V_{T}$ is the tidal volume) and $\boldsymbol{b}$ were employed to assess the concavity of the $\mathrm{Paw}_{\mathrm{dyn}}$ and compared to the radiological evidences of tidal recruitment and overdistension. A Wilcoxon signed rank test for paired samples was applied to compare changes in $\% \mathrm{E}_{2}$ and $\boldsymbol{b}$ and lung aeration between end-expiration and end-inspiration at each PEEP value. A $P<0.05$ was considered significant.

Results and Discussions: Hyperinflated areas decreased as PEEP was reduced from $16 \mathrm{cmH}_{2} \mathrm{O}$ to $0 \mathrm{cmH}_{2} \mathrm{O}(24-62 \%$ to $1-7 \%$ at EEP and $44-73 \%$ to $4-17 \%$ at EIP) whereas normally aerated areas increased $(30-66 \%$ to $72-83 \%$ at EEP and $19-48 \%$ to $73-77 \%$ at EIP). From 16 to $8 \mathrm{cmH}_{2} \mathrm{O}, \% \mathrm{E}_{2}$ and $\boldsymbol{b}$ were positive (upward $\mathrm{Paw}_{\mathrm{dyn}}$ concavity) suggesting tidal overdistension and decreased concurrently with tidal hyperinflation. A straight $\mathrm{Paw}_{\text {dyn }}$ was obtained at PEEP of $8 \mathrm{cmH}_{2} \mathrm{O}$. At PEEP $<4 \mathrm{cmH}_{2} \mathrm{O}, \% \mathrm{E}_{2}$ became negative and $\boldsymbol{b}<0.9$ (downward $P V d y n$ concavity), suggesting tidal recruitment with an increase of tidal re-aeration.

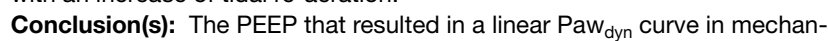
ically ventilated healthy piglet maximized normally aerated areas and minimized tidal hyperinflation and re-aeration. This PEEP value is likely associated with minimal mechanical stress.

\section{AP2-1}

Inhaled carbon monoxide (iCO) prevents LPS-induced impairment of hypoxic pulmonary vasoconstriction (HPV) in mice

D. Oswald, C.J. Busch, C. Reich, W.M. Kuebler, J. Weimann

Department of Anesthesiology, VU University medical center, Amsterdam, The Netherlands

Background and Goal of Study: Pulmonary vasoconstriction in response to alveolar hypoxia (HPV) is frequently impaired in patients with sepsis or the acute respiratory distress syndrome (ARDS) or in animal models of endotoxemia. Recently, inhaled carbon monoxide (iCO) has been shown to elicit antiinflammatory effects and to be beneficial in animal models of lung injury. Therefore, we hypothesized that iCO may prevent the development of impaired HPV during sepsis.

Materials and Methods: The pulmonary vasoconstrictor response to hypoxia $(\triangle \mathrm{PAP})$ was quantified in an isolated, perfused and ventilated mouse lung model (1). Groups of LPS-pretreated and untreated control mice were exposed to 21 hours of $0,50,125,250$, and $500 \mathrm{ppm}$ iCO in air ( $n=5-8$ each), respectively. Moreover, $\mathrm{CO}-\mathrm{Hb}$ blood levels were measured before lung perfusion experiments. To further study the effects of increased $\mathrm{CO}-\mathrm{Hb}$ levels on HPV responsiveness following $\mathrm{CO}$ exposure, additional groups of mice were exposed to $500 \mathrm{ppm}$ iCO in $50 \% \mathrm{O}_{2}$. Groups were compared by ANOVA followed by a LSD post hoc analysis. 
Results and Discussions: LPS pretreatment caused a significant reduction in $\triangle$ PAP ([mean \pm SD] LPS: $7 \pm 5 \%$ vs. control: $55 \pm 5 \%$; $<<0.05)$. There was no effect of iCO exposure on $\triangle \mathrm{PAP}$ in untreated control animals. Exposure of LPS-pretreated mice to $50 \mathrm{ppm}$ iCO completely prevented the development of impaired HPV ( $\triangle$ PAP $49 \pm 21 \% ; p<0.05$ vs. LPS). However, this effect vanished with increasing iCO doses and was absent at $500 \mathrm{ppm}$ iCO $(\triangle \mathrm{PAP}$ $12 \pm 8 \%)$. This was associated with increasing $\mathrm{CO}-\mathrm{Hb}$ blood levels, but could be overcome by reducing these by exposure with $500 \mathrm{ppm}$ iCO in $50 \% \mathrm{O}_{2}$ ( $\triangle$ PAP $60 \pm 18 \%$ ).

Conclusions: Here we showed that low dose CO inhalation may prevent LPS-induced impairment of HPV in mice. Of interest, this effect vanished with increasing doses of iCO. Our data further suggest, that high $\mathrm{CO}-\mathrm{Hb}$ levels associated with $\mathrm{CO}$ exposure may counteract the beneficial effects of iCO during sepsis.

Reference:

1 Spöhr F, Cornelissen AJM, Busch C, et al. Am J Physiol Heart Circ Physiol 2005; 289 : 823-831.

\section{AP2-2}

Inhibition of $\mathrm{Kv}$ channels by 4-aminopyridine restores impaired hypoxic pulmonary vasoconstriction (HPV) in endotoxemic mice

F. Spoehr, C.J. Busch, W.M. Kuebler, K.D. Bloch, J. Weimann

Department of Anaesthesiolgy, University of Heidelberg, Heidelberg,

Germany

Background and Goal of Study: Voltage-gated potassium (Kv) channels play a central role in mediating hypoxic pulmonary vasoconstriction (HPV)(1). HPV is impaired during inflammatory lung processes such as pneumonia or the acute respiratory distress syndrome. The aim of this study was to determine if inhibition $\mathrm{Kv}$ channels by 4-aminopyridine (4-AP) may restore this impairment of HPV during sepsis.

Materials and Methods: The effects of $0.01,0.1$, and $1.0 \mathrm{mM}$ of the $\mathrm{Kv}$ channel inhibitor 4-AP on HPV responsiveness were assessed in isolated lungs of untreated mice and of mice 18 hours after LPS injection $(20 \mathrm{mg} / \mathrm{kg} \mathrm{E}$. coli 0111:B4 LPS i.p.). HPV was quantified as the increase in perfusion pressure in response to hypoxic ventilation in percent of baseline perfusion pressure ( $\triangle$ PAP) (2). Moreover, intrinsic pulmonary vascular resistance $\left(R_{0}\right)$ and pulmonary vascular distensibility $(\alpha)$ were determined by non-linear regression analysis of pulmonary vascular pressure-flow $(\mathrm{P} / \mathrm{Q})$ curves generated during normoxic and hypoxic ventilation, respectively.

Results and Discussions: HPV was impaired in lungs isolated from LPSchallenged mice. Addition of 4-AP to the perfusate had no effect on baseline PAP and the HPV response in untreated mice, but dose-dependently restored HPV in LPS-treated animals. Analysis of pulmonary vascular P/Q curves revealed that 4-AP (i) counteracted the observed LPS-induced changes in vascular distensibility $(\alpha)$ and intrinsic vascular resistance $\left(R_{0}\right)$ under normoxic conditions, and (ii) augmented the hypoxia-induced increase in intrinsic vascular resistance $\left(\mathrm{R}_{0}\right)$.

Conclusions: These results suggest that $\mathrm{Kv}$ channels play a critical role in LPS-induced pulmonary vascular hyporesponsiveness to hypoxia, and that this impaired HPV can be restored by the $\mathrm{Kv}$ channel inhibitor 4-AP in murine endotoxemia.

References:

1 Archer S, Michelakis E. News Physiol Sci 2002; 17: 131-137.

2 Spöhr F, Cornelissen AJM, Busch C, et al. Am J Physiol Heart Circ Physiol 2005; 289 : 823-831.

\section{AP2-3}

The protective role of carbon monoxide in ventilator induced lung injury

A. Hoetzel, Y. Zhang, A.M.K. Choi

Pulmonary, Allergy, and Critical Care Medicine, University of Pittsburgh Medical Center, Pittsburgh, USA

Background and Goal of Study: Ventilator induced lung injury (VILI) still remains a major cause of death in intensive care medicine. Carbon monoxide (CO) has been shown to exert anti-inflammatory, anti-oxidative, and anti-proliferative effects. Here, we hypothesized that $\mathrm{CO}$ might reduce lung injury during mechanical ventilation.

Materials and Methods: C57/BL6 mice ( $n=5 /$ group) were ventilated with $12 \mathrm{ml} / \mathrm{kg}$ tidal volume for 1,4 , and $8 \mathrm{~h}$, with room air or air $+250 \mathrm{ppm} \mathrm{CO}$. Control mice were sham operated. Blood samples were taken to measure blood gases. Bronchoalveolar lavage (BAL) and lung tissue were analyzed to determine total cell and neutrophil count, cytokine release, and heat shock response, respectively.
Results and Discussions: Mechanical ventilation without $\mathrm{CO}$ led to an increase in neutrophil and total cell count in BAL after 4 and $8 \mathrm{~h}(\mathrm{p}<.05)$ of ventilation as compared to sham. Furthermore, heat shock protein 70 and heme oxygenase-1 were up regulated in lung tissue. In contrast, additional COapplication attenuated the augmentation of neutrophil and total cell count $(p<.05)$ in BAL as well as the up regulation of heat shock proteins during ventilation. These findings show that $\mathrm{CO}$ reduces lung injury that results from mechanical ventilation. Most interestingly, while the ventilation-induced IL-6 release was decreased in the presence of $\mathrm{CO}$ in the BAL, tissue levels of IL6 were further increased by $\mathrm{CO}$ treatment as compared to ventilation alone. This data indicates that CO might disturb neutrophil migration and cytokine release into the alveolar space.

Conclusion(s): 1) VILI occurs even with tidal volumes as low as $12 \mathrm{ml} / \mathrm{kg}$ that are used in clinical routine. 2) $\mathrm{CO}$ in low dose decreases lung injury that is observed with ventilation alone. 3 ) We suggest that the protective mechanism involves the inhibition of neutrophil migration and cytokine release by $\mathrm{CO}$.

\section{AP2-4}

Indirect markers of pulmonary endothelial dysfunction correlate with high-altitude induced pulmonary hypertension M.M. Berger, P. Bärtsch, H. Mairbäurl, E.R. Swenson, C. Dehnert Department of Anesthesiology, University of Heidelberg, Heidelberg, Germany

Background and Goal of Study: Hypoxia-induced pulmonary hypertension plays a major role in the pathophysiology of hypoxic pulmonary edema formation (e.g. high-altitude pulmonary edema; HAPE). We hypothesized that the rise in pulmonary artery pressure (PAP) during hypoxic exposure is related to impaired pulmonary endothelial function with reduced nitric oxide and enhanced endothelin-1 (ET-1) bioavailability.

Materials and Methods: 34 participants were investigated at low altitude $(110 \mathrm{~m})$ and after rapid ascent to high altitude ( $<24$ hours to $4559 \mathrm{~m}) .3-4$ hours after arrival at high-altitude blood samples were drawn via a central venuous and a radial artery catheter, respectively, for determination of plasma nitrite (measured by flow injection analysis technique), plasma ET-1 (measured by radioimmunoassay), and plasma catecholamines (noradrenaline, adrenaline measured by HPLC). Systolic PAP (PASP) was estimated by transthoracic doppler-echocardiography, and chest radiography was used to diagnose pulmonary edema. In subjects developing HAPE the presented data were collected before the onset of edema formation. Data are expressed as Mean \pm SEM, a p-value $<0.05$ indicates statistical significance.

Results and Discussion: After ascent to high altitude arterial $\mathrm{pO}_{2}$ decreased to $38 \pm 0.5 \mathrm{mmHg}(\mathrm{p}<0.001)$ and PASP increased from $23 \pm 0.7 \mathrm{mmHg}$ to $37 \pm 2 \mathrm{mmHg}(p<0.001)$. HAPE developed in 4 participants. Central venuous ET-1 plasma levels increased about 3 -fold $(p<0.001)$ while plasma nitrite remained stable $(p=0.331)$. At low altitude arterial-central venous (ACV) plasma gradients were negative for ET-1 and positive for nitrite $(p<0.001)$. They reversed after ascent to high-altitude $(p<0.05)$ and significantly correlated with PASP (ET-1: $R=0.49, p<0.001$; nitrite: $R=-0.21, p<0.05$ ). ACV plasma gradients of ET-1 and plasma nitrite showed an inverse correlation $(R=-0.48 ; p<0.001)$, indicating a reciprocal regulation. Central venuous plasma levels of noradrenaline and adrenaline increased about 2-fold $(p=0.001)$ and 1.6-fold $(p<0.05)$, respectively, but showed no correlation with PASP $(p=0.118$ and $p=0.594)$.

Conclusion: These reults indicate that an impairment of pulmonary endothelial function with a shift in the balance between plasma ET-1 and nitric oxide is of major significance for hypoxia-induced pulmonary hypertension.

\section{AP2-5}

\section{A development of a warm lung ischemia-reperfusion} experimental study model in pigs

K. Kalimeris, G. Kostopanagiotou, T. Chamogeorgakis, G. Kampouroglou, P. Matsota

2nd Department of Anaesthesia, Attikon Hospital, University of Athens, Athens, Greece

Background and Goal of Study: Lung ischemia-reperfusion injury (LIRI) is a severe pathophysiological consequence of lung transplantation, cardiopulmonary bypass and pulmonary sleeve resection, leading to increased morbidity and mortality ${ }^{(1)}$. The exact pathways leading to LIRI are not fully elucidated while efforts are made for new therapies ${ }^{(2)}$. The scope of this study is the description of an experimental model of warm lung ischemia-reperfusion injury in pigs for further investigation of the pathophysiology and potential therapeutic strategies in LIRI.

Materials and Methods: Seven domestic pigs were undergone general anaesthesia with conventional mechanical ventilation (tidal volume: $8-10 \mathrm{~mL} / \mathrm{kg}$, zero positive end-expiratory pressure), followed by left thoracotomy, occlusion of the 
left pulmonary artery for 90 min and subsequent reperfusion for 3 hours. During observation period haemodynamic parameters, respiratory mechanics and blood gases were monitored. Statistical analysis was made by Student's $t$-test or Mann-Whitney test. Data are expressed as mean \pm standard deviation. Results and Discussions: Mean arterial, central venous, and pulmonary capillary wedge pressures decreased both after ischemia and after reperfusion. No differences were found in $\mathrm{PaCO}_{2}$, mean pulmonary artery pressure and cardiac output after ischemia or after reperfusion compared to baseline. The ratio $\mathrm{PaO}_{2} / \mathrm{FiO}_{2}$ decreased at the end of reperfusion $(297 \pm 98 \mathrm{~mm} \mathrm{Hg}$ after reperfusion, vs. $609 \pm 197 \mathrm{~mm} \mathrm{Hg}$ baseline, $P<.05)$. Significant increase in pulmonary vascular resistance (dynes sec/cm ${ }^{5}$ ) both after ischemia $(254 \pm 157$ baseline vs. $330 \pm 155$ after ischemia, $P<.05)$ and after reperfusion $(344 \pm 156, P<.05$ compared to baseline) was observed. Dynamic pulmonary compliance $(\mathrm{mL} / \mathrm{cm}$ $\mathrm{H}_{2} \mathrm{O}$ ) reduced after ischemia ( $22 \pm 4$ baseline vs. $18 \pm 2$ after ischemia, $P<.05)$ and after reperfusion (17 $\pm 2, P<.05$ compared to baseline). Conclusion(s): This experimental model provides a fairly reproducible experimental model of lung warm ischemia-reperfusion injury in pigs for further investigation in the era of LIRI.

Reference:

1 de Perrot M, Liu M, Waddell T, et al. Am. J. Respir. Crit. Care. Med. 2003; 167: 490-511.

2 Waldow T, Alexiou K, Witt W, et al. Nitric Oxide 2004; 10: 195-201.

\section{AP2-6}

\section{Effects of metabolic or hypercapnic acidosis on contractile} and energetic properties of diaphragm muscle

P. Michelet, S. Jaber, G. Orliaguet, B. Riou, O. Langeron Département dAnesthésie Réanimation, Hôpital de la Pitié Salpêtrière, Paris, France

Background and Goal of Study: Acidosis, a common feature in several clinical diseases, adversely affects respiratory muscle function. Nevertheless, metabolic or respiratory (i.e. hypercapnic) acidosis could induce different effects on diaphragmatic contractility. The aim of this study was to compare the effects of a metabolic and a hypercapnic acidosis on cross-bridge (CB) and energetic properties of rat diaphragm.

Materials and Methods: In vitro contractile and energetic properties were measured from ventral diaphragm strips obtained from 4 mo old male Wistar rats submitted to acute metabolic (reduced bicarbonate bath, $\mathrm{n}=12$, $\mathrm{pH}=7.00 \pm 0.02)$ or hypercapnic acidosis $(\mathrm{n}=12, \mathrm{pH}=7.00 \pm 0.03)$ for a 20 min period. After stabilization of contractility properties, the diaphragm strips were submitted to fatigue conditions (up to $60 \%$ of the maximum active force in tetanus), then a recovery period of 20 mins was observed. Calculations of muscle energetic and characteristics of cross-bridge properties were determined from standard Huxley equations. Data were expressed as mean $\pm \mathrm{SD}$ and comparison between groups was performed by $t$-test. Results: Compared to baseline values, hypercapnic acidosis rats were characterized by a decrease in active force $\left(110 \pm 25\right.$ vs $172 \pm 13 \mathrm{mN} \cdot \mathrm{mm}^{-2}$, $\mathrm{p}<0.05)$ associated with a decrease in the total number of CBs per sectional area (13 \pm 3 vs $\left.20 \pm 210^{9} \cdot \mathrm{mm}^{-2}, \mathrm{p}<0.05\right)$. In contrast, no significant changes were observed in the metabolic group. This impairment was associated with a decrease in maximal unloaded shortening velocity in the hypercapnic group $\left(3.9 \pm 0.8\right.$ vs $\left.5.8 \pm 0.9 \mathrm{Lmax} \cdot \mathrm{s}^{-1}, \mathrm{p}<0.05\right)$. After fatigue, hypercapnic group showed a lower recovery in active force compared with metabolic group ( $61 \pm 11$ vs $97 \pm 5 \%$ of pre-fatigue values, $p<0.05$ ).

Conclusion(s): At the same external $\mathrm{pH}$ value, compared with a metabolic acidosis, a hypercapnic acidosis is associated with a greater impairment in contractility properties and a reduced recovery capacity.

\section{AP2-7}

Effect of ageing on the contractilities properties and recovery of fatigue in the rat diaphram

P. Michelet, N. Salvy, G. Orliaguet, B. Riou, O. Langeron

Département dAnesthésie Réanimation, Hôpital de la Pitié Salpetrière, Paris, France

Background and Goal of Study: Ageing is associated with an alteration of contractile force in the senescent rat. The aim of this study was to evaluate the effect of ageing on cross-bridge (CB) and energetic properties before and after fatigue recovery.

Materials and Methods: In vitro contractile and energetic properties were measured with ventral diaphragm strips obtained from young (4 mo old, $\mathrm{n}=12$ ) and old (24 mo old, $\mathrm{n}=12$ ) male Wistar rats. The strips were submitted to fatigue conditions (to $60 \%$ of the tetanic tension) after what a recovery period of 20 mins was observed. Data are expressed as mean \pm SD. Results: Ageing was associated with a decrease in active force $(98 \pm 43$ vs $131 \pm 27 \mathrm{mN} \cdot \mathrm{mm}^{-2}, \mathrm{p}<0.05$ by $t$-test) associated with a decrease in the total number of CBs per sectional area $\left(10.3 \pm 5\right.$ vs $14.5 \pm 3.210^{9} \cdot \mathrm{mm}^{-2}$, $\mathrm{p}<0.05$ by $t$-test). An increase in maximal unloaded shortening velocity was observed between old and young rats $\left(7.9 \pm 2.1 \mathrm{vs} 6.0 \pm 1.6 \mathrm{Lmax} \cdot \mathrm{s}^{-1}\right.$, $\mathrm{p}<0.05$ by $t$-test). After fatigue, senescent rats were characterized by an incomplete recovery $(R)$ of active force compared with young's (Figure) while both groups have recovered the maximal unloaded shortening velocity (98 \pm 8 vs $95 \pm 7 \%$ of baseline values, NS).

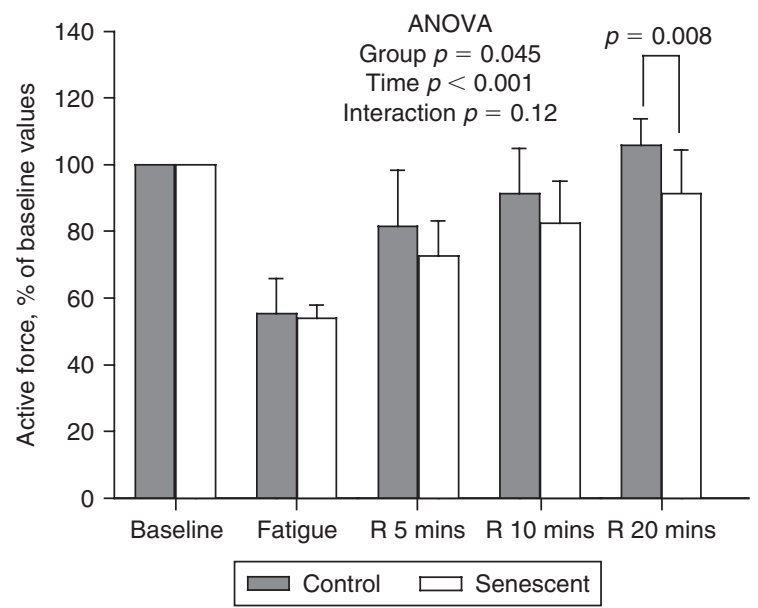

Conclusion(s): These results demonstrate that ageing is associated with an impairment of diaphragmatic contractile function associated with impairment in fatigue recovery.

\section{AP2-8}

\section{Effect of beta2-adrenoceptor stimulation on contractile properties and fatigue recovery in senescent rats}

P. Michelet, J. Amour, G. Orliaguet, B. Riou, O. Langeron

Département dAnesthésie Réanimation, Hôpital de la Pitié Salpétrière,

\section{Paris, France}

Background and Goal of Study: Ageing is associated with an alteration of contractile force in the senescent rat. The $\beta_{2}$-adrenoceptor agonists are widely used in the treatment of chronic respiratory disease. The aim of this study was to investigate whether $\beta_{2}$-adrenoceptor stimulation improves contractile properties of senescent rat diaphragm during tetanic stimulation and on fatigue recovery.

Materials and Methods: Effect of $\beta_{2}$-adrenoceptor stimulation by Terbutaline $\left(10^{-6} \mathrm{M}\right)$ on contractile function of ventral diaphragm strips obtained from 24 mo old male Wistar rats were studied, in vitro (Krebs-Henseleit solution, $\left.29^{\circ} \mathrm{C}, \mathrm{pH} 7.40, \mathrm{Ca}^{++} 2.5 \mathrm{mmol}\right)$. After stabilization of contractility properties (T0), the diaphragm strips were submitted or not ( $n=10$ by group) to $\beta_{2}$-stimulation (T1), to fatigue conditions (to $60 \%$ of the tetanic tension, F) after what a recovery period of 15 mins was observed $(R 1, R 2$ and $R 3$, respectively after 5,10 and 15 mins of recovery). Data were expressed as mean \pm SD and comparison between groups was performed using ANOVA.

Results: $\beta_{2}$-adrenoceptor stimulation resulted in an improvement of active force (AF) during tetanic stimulation and a greater recovery after fatigue (Figure).

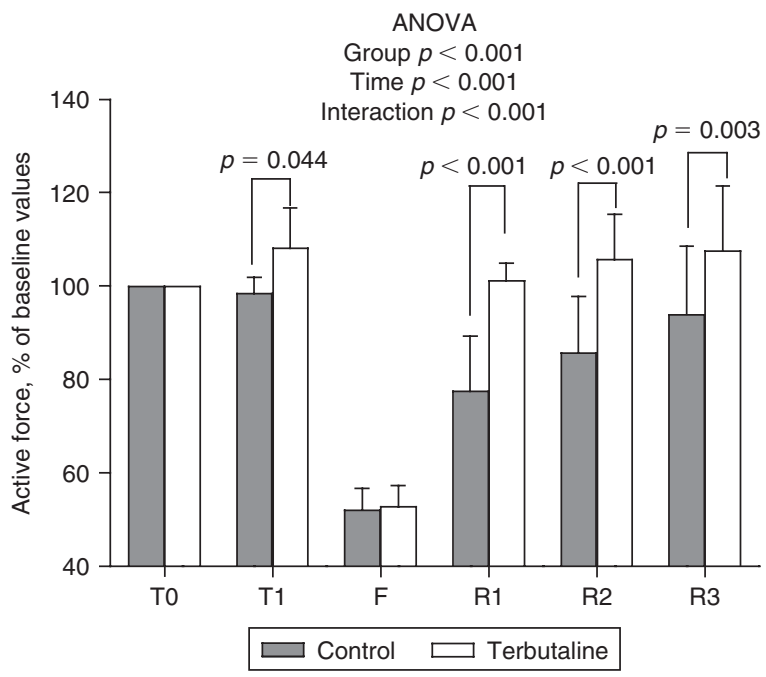


Conclusion(s): The $\beta_{2}$-adrenoceptor stimulation partially reverse the impairment of contractile force associated with ageing and improve the fatigue recovery in senescent rat diaphragm.

\section{AP3-1}

Evaluation of respiratory resistance, central drive and muscle capacity in early postoperative period after spinal and general anaesthesia

A. Bogdanets, O. Shishkin

Anesthesiology, Republican hospital, Petrozavodsk State University, Petrozavodsk, Russian Federation

Background and Goal of Study: Considering influence of anesthesia on respiratory system there is a lack of data regarding simultaneous evaluation of ventilation determinants - respiratory resistance, central nerve output and capacity of muscle pump. Aim of the study was to analyze differences of mechanisms by which lung ventilation may be compromised in early postoperative period after spinal and general anesthesia.

Materials and Methods: Forced oscillation technique (IOS, Jaeger) provided assessment of respiratory impedance whereas measurement of both maximal muscle efforts (PmaxEx, PmaxIn) and central output $\left(P_{0,1}\right)$ were obtained by occlusion technique (Masterscreen, Jaeger). Examination of respiratory function was performed in patients undergoing general $(n=12)$ or spinal $(n=11)$ anesthesia for open abdominal surgery once prior operation and again within the first hour following one. In all calculations we used parameters expressed as percent of predicted value. $95 \%$ confidence interval for means of differences $(\Delta)$ was calculated.

Results and Discussions: By the end of general anesthesia respiratory impedance increased by $47,4 \%(16,8 ; 77,9)$ versus $19,8 \%(-4,3 ; 44,0)$ when spinal anesthesia was accomplished. In group of general anesthesia there was a substantial reduction of muscle strength: $\Delta P \operatorname{maxln}=-21,4 \%$ $(-32,4 ;-10,3), \Delta \mathrm{PmaxEx}-49,7 \%(-70,2 ;-29,1)$. Spinal anesthesia didn't effect inspiratory efforts $(\Delta \mathrm{Pmax} / \mathrm{n}=-4,7 \%[-21,4 ; 12,0])$ and central output but expiratory muscle dysfunction was observed: $\triangle P \max E x=-21,7 \%$ $(-37,7 ;-5,8)$. There was a weak tendency of increasing of $P_{0,1}$ in group of general anesthesia: $\Delta \mathrm{P}_{0,1}=7,1 \%(-9,4 ; 23,7)$.

Conclusion(s): Abdominal surgery combined with general anesthesia produces profound effect on respiratory system in particular disturbing respiratory mechanics and muscle efforts while combination of spinal anesthesia with similar surgical settings has lesser impact limited by expiratory muscle dysfunction.

\section{AP3-2}

Hyperoxic ventilation reduces oxygen consumption of healthy volunteers and anesthetized patients

J. Meier, S. Huismann, P. Enselmann, B. Zwissler, O. Habler Department of Anesthesiology, J.W. Goethe-University Hospital Frankfurt, Germany, Frankfurt, Germany

Background and Goal of Study: Ventilation with pure oxygen (hyperoxic ventilation; $\mathrm{HV} ; \mathrm{FiO}_{2}$ 1.0) has been described to decrease whole body oxygen consumption $\left(\mathrm{VO}_{2}\right)$ in unanesthetized patients ${ }^{1,2}$. This decrease of $\mathrm{VO}_{2}$ has been determined by the reverse Fick method based on data obtained by means of a pulmonary artery catheter $\left(\mathrm{CO}, \mathrm{CaO}_{2}, \mathrm{CvO}_{2}\right)$. There are several methodological weaknesses inherent to this indirect calculation of $\mathrm{VO}_{2}$, however during $\mathrm{HV}$ direct measurement of $\mathrm{VO}_{2}$ with a metabolic monitor (indirect calorimetry) was impossible so far, due to technical problems. The goal of the study presented was to determine $\mathrm{VO}_{2}$ during normoxic and hyperoxic ventilation with a modified metabolic monitor designed for this purpose (Oxycon Pro, Viasys, Würzburg).

Materials and Methods: After government approval 14 healthy volunteers and 14 anesthetized patients (ASA I) sequentially breathed room air $\left(\mathrm{FiO}_{2} 0.21\right)$, pure oxygen $\left(\mathrm{FiO}_{2} 1.0\right)$, and room air $\left(\mathrm{FiO}_{2} 0.21\right)$ again. Oxygen consumption $\left(\mathrm{VO}_{2}\right)$, the respiratory quotient $(\mathrm{RQ})$, and energy expenditure $(\mathrm{EE})$ were determined for each time point by indirect calorimetry using a modified metabolic monitor.

Results and Discussions: $\mathrm{HV}$ reduced $\mathrm{VO}_{2}$ from $3.4(3.0 / 4.0) \mathrm{ml} / \mathrm{kg} / \mathrm{min}$ to 2.8 $(2.5 / 3.6) \mathrm{ml} / \mathrm{kg} / \mathrm{min}$ in volunteers $(p<0.05)$, and from $2.1(1.7 / 2.7) \mathrm{ml} / \mathrm{kg} / \mathrm{min}$ to $1.8(1.7 / 2.7) \mathrm{ml} / \mathrm{kg} / \mathrm{min}$ in anesthetized patients $(\mathrm{p}<0.05)$. After onset of HV RQ increased from $0.9(0.8 / 0.9)$ to $1.1(1.0 / 1.1)$ in volunteers $(p<0.05)$, whereas it remained unchanged in anesthetized patients. Calculated EE decreased in volunteers $(23.4(21.1 / 27.2) \mathrm{kcal} / \mathrm{kg} /$ day vs. $20.6(18.5 / 28.0)$ $\mathrm{kcal} / \mathrm{kg} /$ day, $\mathrm{p}<0.05)$ as well as in patients $(14.4(11.1 / 17.5) \mathrm{kcal} / \mathrm{kg} / \mathrm{day}$ vs. $12.5(10.5 / 14.5) \mathrm{kcal} / \mathrm{kg} / \mathrm{day}, \mathrm{p}<0.05)$ after onset of hyperoxic ventilation. All changes observed were reversible.

Conclusion(s): $\mathrm{HV}$ reduces $\mathrm{VO}_{2}$ in healthy volunteers and anesthetized patients. Determination of the underlying mechanisms and the clinical impact of this phenomenon requires further studies.

References:

1 Reinhart K et al. Chest 1991; 99(3): 690-694.

2 Reinhart K et al. Anaesthesist 1989; 38(5): 233-237.

\section{AP3-3}

preoxygenation in healthy volunteers: a randomised crossover study comparing supine and $45^{\circ}$ seated position

S. Smith, J. Harten, E. Jack, R. Carter, J. Kinsella

\section{Dept of Anaesthesia, University of Glasgow, Glasgow, United Kingdom}

Background and Goal of the study: Pre-oxygenation in the sitting up position has been associated with better oxygenation [1]. The aim of this study was to compare oxygenation in the supine position with the $45^{\circ}$ seated position in young, healthy volunteers.

Materials and Methods: Research ethics approval and informed consent was obtained for this controlled cross-over study. Inclusion criteria were age $18-30$ years, BMI $<30$ and an ASA score $\leqslant 2$. Oxygen was administered through a circle system and a tight fitting face mask. Volunteers were randomised to order of positions. Transcutaneous $\mathrm{PO}_{2}\left(t c \mathrm{PO}_{2}\right)$ levels were recorded by a TCM400 (Radiometer, Copenhagen) at $10 \mathrm{~s}$ intervals from 2 measurement points. $\mathrm{TcPO}_{2}$ measurements were recorded after $5 \mathrm{~min}$ of oxygenation in supine and seated position. Values are mean (SD). Paired $T$ tests were performed. Sample size was based on difference of $40 \mathrm{mmHg}$ (SD 83), alpha error 0.05 and beta error 0.83 .

Results and Discussion: There was balance of gender (20/20), age $21.5 \mathrm{yrs}$ (1.3), BMI $23.4 \mathrm{~kg} / \mathrm{m}^{2}$ (2.4), peak expiratory flow rate $584.5 \mathrm{l} / \mathrm{min}$ (101.8) and $\mathrm{SpO}_{2} 98.1$ (0.5).

\begin{tabular}{|c|c|c|c|}
\hline & \multicolumn{2}{|c|}{ Position, mmHg (SD) } & \multirow[t]{2}{*}{ P-Value } \\
\hline & Seated & Supine & \\
\hline Baseline tcPO & $73.8(10.9)$ & $72.5(12.1)$ & 0.174 \\
\hline $\begin{array}{l}\mathrm{tcPO}_{2} \\
\text { (3-4 min average) }\end{array}$ & $321.8(58.5)$ & $321.2(64.1)$ & 0.902 \\
\hline $\begin{array}{l}\text { Peak tcPO } \\
\text { (3-4 min average) }\end{array}$ & $336.6(58.0)$ & $332.7(61.8)$ & 0.394 \\
\hline
\end{tabular}

Conclusions: Pre-oxygenation in the $45^{\circ}$ sitting up position does not improve levels of tissue oxygenation in young healthy non-obese volunteers. Reference:

1 Lane. Anaesthesia 2005; 60(11): 1064

\section{AP3-4}

Measurements of diaphragmatic strength using different abdominal and thoracic pressures

L. Gallart, L.G. Aguilera, I. Ramos, J.C. Alvarez, J. Vallès

Servei dAnestesiologia, Hospital del Mar-IMAS, Barcelona, Spain

Background and Goal of Study: Maximal Transdiaphragmatic pressure (Pdimax) is used to measure diaphragmatic strength. It is determined with oesophageal and gastric balloons, often with a sniff manoeuvre (1), but these are not handy methods and other options are needed $(2,3)$. The goal of study was to determine the validity of alternative pressures to measure Psniff.

Materials and Methods: Preoperatively, 8 patients scheduled for major abdominal surgery were asked to perform a maximal sniff manoeuvre (Psniff) from FRC in supine position. The manoeuvre was performed 3 times, always with the same command. Central venous (Pcv), oesophageal (Poes), gastric (Pga), vesical (Pves) and rectal (Prect) pressures were recorded. Psniff was determined as (absolute values): 1/Pes + Pga; 2/Pcv + Prect; 3/ Pcv + Pves. Intraclass Correlation Coefficient (ICC) was used to compare Pdimax measurements.

Results and Discussion: Psniff values were (mean $\pm \mathrm{SD}, \mathrm{cmH}_{2} \mathrm{O}$ ): Pes + Pga, $64 \pm 20 ;$ Pcv + Prect $58 \pm 25 ;$ Pcv + Pves, $58 \pm 26$. ICC showed agreement among methods $(p<0.01)$. 
Figure shows an example of Psniff pattern recorded.

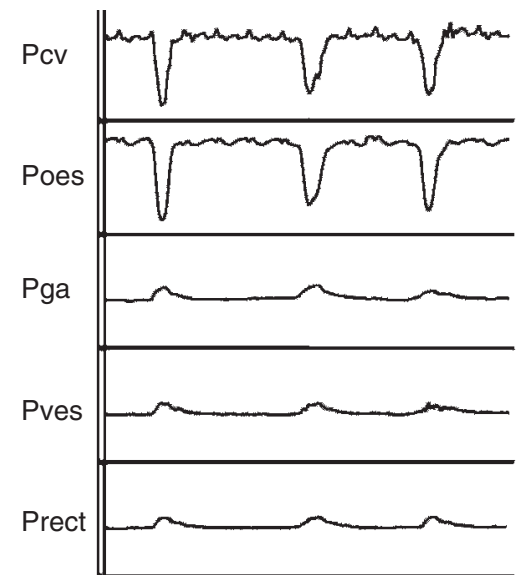

Conclusions: Measurement of diaphragmatic strength can be performed with central venous, vesical and rectal catheters as alternatives to gastric and oesophageal balloons.

References:

1 Gallart L et al. Anesthesiology 1995; 83: 48-55.

2 Aguilera LG et al. Eur J Anaesth 2006; 23: A-257.

3 Chieveley-Williams $S$ et al. Chest 2002; 121: 533-8.

\section{AP3-5}

The effect of step-changes in inspired partial pressure and ventilation-perfusion mismatch on end-tidal, arterial and brain partial pressures of sevoflurane

J. Mamatha, I.L. Dorairaj, J.G. Hardman

University Department of Anaesthesia, Queens Medical Centre, Nottingham, United Kingdom

Background and Goal of Study: The end-expiratory partial pressure of inhaled anaesthetic agent is used as a surrogate for effect-site partial pressure to monitor depth of anaesthesia. Previous clinical work has shown that an endexpired to arterial partial pressure difference exists [1, 2]; this risks under- or overdosage. The end-expiratory to effect-site gradient has not been studied and quantified for different patterns of ventilation-perfusion (VQ) mismatch and with changes in inspired partial pressure.

Materials and Methods: Three virtual patients with distinct VQ distributions but otherwise identical were configured within the Nottingham Physiology Simulator. The measured physiological deadspace and venous admixture of the 3 patients were mild $(11.6 \% \& 1.6 \%$, respectively), moderate $(19.5 \%$ \& $7.8 \%)$ and severe $(32.9 \%$ \& $36 \%)$. Each patient underwent the following clinically-based protocol: $8 \%$ inspired sevoflurane (Pi) for $1 \mathrm{~min}, 3 \%$ for $4 \mathrm{~min}$, $2 \%$ for $15 \mathrm{~min}, 1 \%$ for $2 \mathrm{~min}, 3 \%$ for $2 \mathrm{~min}$ and $0 \%$ for $10 \mathrm{~min}$. During this $45 \mathrm{~min}$ period, inspired, end-expired, arterial and brain partial pressures of sevoflurane were recorded every 5 seconds.

Results: Substantial differences (up to $1.4 \mathrm{kPa}$ ) between arterial and end-tidal sevoflurane partial pressure were observed, and these were largest soon after step changes in $\mathrm{Pi}$. Significant differences (up to $3.3 \mathrm{kPa}$ ) were observed between brain and end-tidal partial pressures; again, these were largest soon (but not immediately) after step changes in Pi. The severe VQ defect caused larger differences between arterial and end-tidal partial pressures than the mild defect (up to 7.6 times larger). The severe VQ defect also magnified the brain to end-tidal gradient, but this effect was less marked (up to 1.8 times larger). Following a step change in $\mathrm{Pi}$, the difference between brain and end-tidal partial pressure was slower to return to zero in the presence of the severe defect. Conclusions: End-tidal sevoflurane partial pressure variably represents brain partial pressure. Step changes in inspired sevoflurane and VQ mismatch increase this gradient, risking overdosage of inhaled anaesthetic and awareness.

References:

1 Frie FJ, et al. Br J Anaes 1991; 66: 331-9

2 Landon MJ, et al. Br J Anaes 1993; 70: 605-11

\section{AP3-6}

Monitoring FRC by the oxygen washout technique during alveolar De- and Re-recruitment

H. Heinze, M. Grossherr, P. Schmucker, W. Eichler

Clinic for Anaesthesiology, University of Luebeck, Luebeck, Germany
Background and Goal of Study: Assessing alveolar recruitment may help in guiding respiratory therapy in intensive care medicine ${ }^{1}$. The method of functional residual capacity (FRC) evaluation by oxygen washout is applicable routinely at bedside ${ }^{2}$. We studied the course of FRC after alveolar Derecruitment and Re-recruitment.

Materials and Methods: The LUFU system (Draeger Medical, Luebeck, Germany) estimates FRC by oxygen washout, a variant of multiple breath nitrogen washout. A sidestream $\mathrm{O}_{2}$-analyser calculates $\mathrm{FRC}$ from the endinspired- and end-exspired $\mathrm{O}_{2}$ concentrations during fast changes of $\mathrm{FiO}_{2}$. After approval of the local ethics committee and written informed consent we measured FRC nine times (measuring interval 4-6 min) in postoperative cardiac surgery patients during 6 hours after surgery; 3 times before (the mean is defined as basal value (pre)), 3 times after ( $t 4, \mathrm{t} 5, \mathrm{t} 6)$ a standard suctioning procedure with disconnection of the ventilator $(20 \mathrm{sec}, 14 \mathrm{~F}$ catheter, $200 \mathrm{mmHg}$ negative pressure), and 3 times (t7, t8, t9) after a standard recruitment maneuver (RM) (PEEP 15 mbar, PIP 35-40 mbar for $30 \mathrm{sec}$ ). Patients were ventilated with biphasic airway pressure (PEEP $=10 \mathrm{mbar}$ ). Arterial blood gases were analysed before (pre) and after suctioning ( $t 6)$, and after RM (t9). Results and Discussions: 17 patients were studied. FRC decreased after suctioning (pre: $3.4 \pm 1.1 \mathrm{~L}) ; \mathrm{t} 4: 3.0 \pm 1.1 \mathrm{~L}(p=0.010) ; \mathrm{t} 5: 3.1 \pm 1.2 \mathrm{~L}$ $(p=0.047)$. No differences compared to baseline values could be detected after RM (t7: $3.4 \pm 1.2 \mathrm{~L}(\mathrm{p}=0.957)$; $\mathrm{t} 8: 3.3 \pm 1 \mathrm{~L}(\mathrm{p}=0.332)$. Ratio of $\mathrm{paO}_{2}$ and $\mathrm{FiO}_{2}$ increased after RM (pre: $373 \pm$ 102; t6: $359 \pm 98$; t9: $408 \pm 109$; $\mathrm{p}<0.0001$ ) and arterial $\mathrm{pCO}_{2}$ decreased after $\mathrm{RM}$ (pre: $38.3 \pm 5.6 \mathrm{mmHg}$; t6: $39.2 \pm 5.6 \mathrm{mmHg}$; t9: $38.3 \pm 5.0 \mathrm{mmHg} ; \mathrm{p}=0.013$ ).

Conclusion(s): Alveolar De-recruitment and Re-recruitment could be detected in postoperative patients by monitoring FRC. Routine measurements of FRC might be of considerable interest in ventilated patients.

References:

1 Calzia E, Radermacher P, Bein: Intensive Care Med 2006; 32: 1686-8

2 Heinze H, Schaaf B, Grefer J, Klotz K, Eichler: Anesth Analg 2007; in press

\section{AP3-7}

\section{Is surgery a chance to really quit smoking?}

M. Sariñena, A. Martí, M. Rodríguez, G. Paluzie, J. Canet

Anesthesiology, Hospital Universitari Germans Trias i Pujol, Badalona, Spain Background and Goal of Study: Scheduling elective surgery favours smoking cessation. Within a prospective cohort study of risk factors for postoperative complications related to smoking in Catalonia (ARISCAT) we investigated the characteristics of patients who had quit smoking spontaneously three months after surgery.

Materials and Methods: We conducted a cross-sectional multicentre study collecting pre-, intra- and postoperative information on patients aged over 18 years undergoing surgery (excluding obstetrics) under general or regional anaesthesia. Information on smoking habits was recorded before surgery. Three months after surgery, information on changes in smoking habits was obtained by phone. Statistical analyses were performed with a $\chi^{2}$ test for qualitative variables and a $t$ test for quantitative ones. Data are presented as mean \pm standard deviation and percentage.

Results and Discussions: From 1966 patients analysed we selected persons who were smokers at the time of surgery $(422,21.4 \%$ of all participants) There were 263 men (62.3\%) and 159 women (37.7\%) with a mean \pm standard deviation age of $44.3 \pm 15.02$ years; $89.8 \%$ were ASA $1-2$ and $9.9 \%$ had been diagnosed with chronic lung disease before surgery. The majority underwent elective surgery (86.5\%) with hospital admission (77.5\%). Three months after surgery $13.6 \%$ of the patients had quit smoking and $29.9 \%$ had reduced daily consumption. Variables that were significantly related to smoking cessation were: mean age (non-smokers, 49.6 years; smokers, 43.6 years; $p=0.007$ ); $A S A \geqslant 3(21.6 \%$ vs. $8 \%, p=0.003)$; mean pack-years $(29.7 \pm 16.58$ vs. $24.7 \pm 14.57 ; p=0.025)$; chronic lung disease (19.6\% vs. $8.4 \% ; p=0.012$ ); hospitalization ( $88.2 \%$ vs. $75.5 \%$; $p=0.048)$; duration of surgery $(2.1 \pm 1.48$ vs. $1.6 \pm 1.38$ hours; $p=0.020)$; intra- and postoperative complications (29.4 [20\%] vs. 12.4 [4.9\%]; $p=0.001)$; and duration of hospital stay $(6.4 \pm 7.47$ vs. $3.0 \pm 6.06$ days; $p=0.003$ ).

Conclusion(s): The perioperative period is a good moment to encourage a patient to quit smoking. Certain variables are related to success: older age and higher ASA score, heavier smoking, previous lung disease, intra- and postoperative complications, and longer postoperative hospital stay.

\section{AP3-8}

Is it applicable to use the FEV1/FEV6 ratio for the preoperative assessment severity of COPD in elderly patients M.K. Moustafa, M. Salem

Anaesthesia, ICU, Alain Hospital, Alain, AbuDhabi, United Arab Emirates 
Background and Goal of Study: Spirometry can be used to determine agreement between $\mathrm{FEV} / \mathrm{FEV}_{6}$ ratio and $\mathrm{FEV} / \mathrm{FVC}$ ratio by using $\mathrm{ROC}$-curve analysis and Kappa-statistics during the preoperative assessment in COPD elderly patients.

Materials and Methods: 387 participants in Alain Hospital, UAE aged 60 years or more with acceptable spirometry. Mean differences between $\mathrm{FEV}_{1} / \mathrm{FEV}_{6}$ ratio (\%) and the $\mathrm{FEV}_{1} / \mathrm{FVC}$ ratio (\%) were calculated according to age, sex, smoking habit, and the degree of airflow limitation. The mean difference between $\mathrm{FEV}_{1} / \mathrm{FEV}_{6} \%$ and $\mathrm{FEV}_{1} / \mathrm{FVC} \%$ was $2.7 \%$ in both men and women.

Results and Discussions: The value for the $\mathrm{FEV}_{1} / \mathrm{FEV}_{6}$ ratio which best predicted an $\mathrm{FEV}_{1} / \mathrm{FVC}$ ratio of $70 \%$, was $73 \%$, and two cut-off values (kappa $=0.86$ ). As Vandevoorde ${ }^{1}$ \& coauthors, we found $\mathrm{FEV}_{1} / \mathrm{FEV}_{6}$ cut-off of $73 \%$ to be the substitution for the used $\mathrm{FEV}_{1} / \mathrm{FVC}$ threshold of $70 \%$.

Table 1. Prevalence, sensitivities, and specificities of thresholds of the $\mathrm{FEV}_{1} / \mathrm{FEV}_{6}$ ratio in predicting $\mathrm{FEV}_{1} / \mathrm{FVC}<70 \%$

\begin{tabular}{|c|c|c|c|}
\hline $\mathrm{FEV}_{1} / \mathrm{FEV}_{6}$ ratio & \multicolumn{2}{|c|}{ Sensitivity } & Specificity \\
\hline$<72 \%$ & \multicolumn{2}{|l|}{0.82} & 0.99 \\
\hline$<73 \%$ & \multicolumn{2}{|l|}{0.89} & 0.97 \\
\hline$<74 \%$ & \multicolumn{2}{|l|}{0.93} & 0.92 \\
\hline \multirow[t]{2}{*}{$<75 \%$} & \multicolumn{2}{|l|}{0.96} & 0.86 \\
\hline & $\begin{array}{l}\text { Daily smoker } \\
(\mathrm{n}=71)\end{array}$ & $\begin{array}{l}\text { Previous smoker } \\
(\mathrm{n}=147)\end{array}$ & $\begin{array}{l}\text { Non smoker } \\
(\mathrm{n}=169) \\
\end{array}$ \\
\hline $\begin{array}{l}\text { Prevalence of } \mathrm{FEV}_{1} / \\
\mathrm{FVC}<70 \%(\%)\end{array}$ & $43.1 \%$ & $25.1 \%$ & $12.2 \%$ \\
\hline $\begin{array}{l}\text { Positive predictive value } \\
\text { of } \mathrm{FEV}_{1} / \mathrm{FEV}_{6}<73 \% \text { (\%) }\end{array}$ & 92.6 & 87.8 & 83.8 \\
\hline $\begin{array}{l}\text { Negative predictive value } \\
\text { of } \mathrm{FEV}_{1} / \mathrm{FEV}_{6}<73 \%(\%)\end{array}$ & 93.7 & 96.5 & 97.5 \\
\hline
\end{tabular}

Conclusion: The $\mathrm{FEV}_{1} / \mathrm{FEV}_{6}$ ratio appears to be a good substitute for the $\mathrm{FEV}_{1} / \mathrm{FVC}$ ratio in COPD patients.

Reference:

1 Vandevoorde J. Eur Respir J 27(2006)(2), pp. 378-383.

\section{AP4-1}

Changes in extravascular lung water determined with single transpulmonary thermodilution after pneumonectomy and lung resection

V. Kuzkov, D. Uvarov, M. Kirov, L. Bjertnaes, E. Nedashkovsky

Anesthesiology Department, Northern State Medical University, Arkhangelsk, Russian Federation

Background and Goal of Study: Lung reduction surgery, particularly, pneumonectomy (PE) can result in postoperative acute lung injury. Postpneumonectomy pulmonary edema remains a poorly understood clinical entity, which results in mortality rate of up to $50-100 \%$ [1]. We have shown that after PE, single transpulmonary thermodilution (STD) is acceptably accurate for quantification of extravascular lung water (EVLW). Moreover, volutrauma might play a crucial role in the pathophysiology of this complication [2, 3]. The goal of the present study was to investigate the changes in EVLW that occurs after pneumonectomy in human.

Materials and Methods: We studied 27 patients who underwent elective $\mathrm{PE}(\mathrm{PE}, n=16)$ or lung resection (LR, $n=11)$. After induction of total intravenous anesthesia (ketamine, midazolam, and fentanyl), a $5 \mathrm{~F}$ thermodilution catheter was inserted into the femoral artery and a $7 \mathrm{~F}$ flotation catheter into the pulmonary artery. Cardiac index $(\mathrm{Cl})$, pulmonary artery pressure (PAP), pulmonary artery occlusion pressure (PAOP), central venous pressure (CVP), intrathoracic blood volume (ITBVI), and EVLW index (EVLWI) were assessed by STD (PiCCOplus, Pulsion). The measurements were performed after induction of anesthesia, after thoracotomy, lung reduction and at the end of surgery as well as at $6,12,18,24,36$, and $48 \mathrm{hrs}$ postoperatively.

Results and Discussion: Pneumonectomy resulted in an immediate decline in EVLWI from $7.7 \pm 2.4 \mathrm{ml} / \mathrm{kg}$ to $5.4 \pm 1.5 \mathrm{ml} / \mathrm{kg}(p<0.05)$. Postoperatively, EVLWI increased significantly by $24 \%$ compared with immediately after pneumonectomy, peaking at $36 \mathrm{hrs}$ following transfer to the ICU. This was not accompanied by changes in PAP, PAOP, and $\mathrm{PaO}_{2} / \mathrm{FiO}_{2}$. The LR group did not demonstrate any significant changes in EVLWI.

Conclusion: In major thoracic surgery, STD appears to be a valuable tool for assessment of extravascular lung water. The postoperative increase in EVLWI may be explained by a redistribution and accumulation of pulmonary interstitial fluid due to augmented perfusion of the residual lung.
References:

1 Fuentes PA. Eur J Cardiothor Surg 2003; 23: 439-445.

2 Roch A et al. Chest 2005; 128: 927-933.

3 Kuzkov V et al. Crit Care Med 2006; Accepted.

\section{AP4-2}

Early prophilactic cpap after lung resection surgery: a prospective, randomized, controlled study

A. Ramelli, A. Casati, A. Bobbio, E. Masoni, M. Barbagallo

Department of Anesthesiology and Thoracic Surgery, University of Parma, Parma, Italy

Background: The aim of this prospective, randomized, controlled study was to assess the efficacy of prophilactic treatment with continuous positive airway pressure (CPAP) on gas exchange after lung resection surgery. Methods: With IRB approval and written consent 40 consecutive patients receiving lung resection surgery with general anesthesia and thoracic epidural analgesia were randomly allocated to receive two cycles of two hours of CPAP (PEEP: $8 \mathrm{~cm} \mathrm{H} \mathrm{H}_{2} \mathrm{O} ; \mathrm{FiO}_{2}: 0.4$ ) during the first $12 \mathrm{~h}$ after surgery (group CPAP, $\mathrm{n}=21)$ or conventional oxygen therapy with ventimask $\left(\mathrm{FiO}_{2}\right.$ : 0.4 ) (group Control, $n=19$ ). Blood gas analyses were performed after 6,12 , 24,48 hours. Occurrence of pulmonary complications during hospital stay (sputum retention, atelectasis, pneumonia, broncopleural fistula, need for broncoscopy and minitrach placement) as well as duration of hospital stay were also recorded.

Results: No differences in anthropometric parameters were observed between the two groups. The Table shows effects of therapy on patients' oxygenation (median [range]).

\begin{tabular}{lll}
\hline & group CPAP $(n=19)$ & $\begin{array}{l}\text { group Control } \\
(\mathrm{n}=21)\end{array}$ \\
\hline $\mathrm{PO} / \mathrm{FiO} 20 \mathrm{~h}$ & $283[160-600]$ & $360[175-657]$ \\
$\mathrm{PO2} / \mathrm{FiO} 26 \mathrm{~h}$ & $372[200-578]$ & $332[168-430]$ \\
$\mathrm{PO} / \mathrm{FiO} 212 \mathrm{~h}$ & $370[158-613]$ & $248[173-375]^{\S}$ \\
$\mathrm{PO2} / \mathrm{FiO} 2$ 24 h & $223[168-423]$ & $255[163-422]^{\S}$ \\
$\mathrm{PO2} / \mathrm{FiO} 248 \mathrm{~h}$ & $202[153-530]$ & $210[158-36])$ \\
\hline
\end{tabular}

$\S \mathrm{P}<0.05$ vs group CPAP

No differences were recorded in hospital stay (CPAP $=7$ [6-10], Control $=8$ [7-16], $p=0,06$ ) and in incidence of pulmonary complications (CPAP $=14 \%$ vs Control $=16 \%, p=0,99$ ).

Conclusions: Early prophylactic CPAP produced minor improvements in oxygenation during first $24 \mathrm{~h}$ but did not reduce the incidence of pulmonary complications.

\section{AP4-4}

Remote lung injury after experimental thoracic aortic occlusion and reperfusion: a comparison of sevoflurane vs. propofol anaesthesia

T. Annecke, K. Langer, J. Hilberath, S. Kahr, P. Conzen

Clinic of Anaesthesiology, LMU Munich, Munich, Germany

Background and Goals: Respiratory failure after major vascular surgery is quite common. Systemic inflammatory response following reperfusion, mechanical ventilation, and direct and indirect effects of anaesthetic techniques contribute to observed pulmonary dysfunction. We compared propofol and sevoflurane with respect to their influence on remote lung injury after thoracic aortic occlusion and reperfusion.

Materials and Methods: Experiments had been approved by local committees. After surgical preparation in midazolam-anaesthesia, pigs were randomised to receive either propofol or sevoflurane $(n=9$, each) in an investigator blinded fashion. The thoracic aorta was occluded for 90min. Study medication was continued for $120 \mathrm{~min}$ after reperfusion, than midazolam was restarted for the remaining 180min of the experiment. Fentanyl was given throughout the experiment for analgesia. Pulmonary injury was assessed by oxygenation index, broncho-alveolar lavage analysis and histological damage score. Oxidative burst of alveolar macrophages was measured by DCF-FACS analysis. 5 animals each without aortic occlusion served as time-controls.

Results and Discussion: Oxygenation index decreased, and intrapulmonary shunt fraction increased significantly in both study groups following reperfusion. No differences in functional or morphological lung injury, or alveolar macrophage oxidative burst activity could be detected between both anaesthetics. Neither oxygenation nor other parameters were changed in non-occlusion animals. 
Conclusion: Pulmonary function was only moderately impaired during the observation period in our model of severe porcine abdominal-ischaemia reperfusion injury. Remote lung injury was not different between a propofol or sevoflurane based anaesthetic regimen.

\section{AP4-5}

\section{Comparison of the effect of the double lumen endotracheal} tube and Arndt bronchial blocker on respiratory mechanical properties

E. vila, R. Garcia-Guasch, Z. Briones, A. Escudero, M. Monerris Anesthesia, Hospital Universitari Germans Trias i Pujol, Badalona, Spain

Goal of Study: To compare airway pressures, compliance and resistance during one- and two-lung ventilation between techniques based on a double-lumen endotracheal tube (DLT) and an Arndt $₫$ bronchial blocker (ABB). Materials and Methods: Twenty-five patients scheduled for lung surgery in lateral decubitus position were included. Patients were allocated randomly to two groups: ABB $(n=13)$ or DLT $(n=12)$. A 37 or 39 French tube was placed in the DLT group and a 7.5-to-8.5-mm internal diameter single-lumen endotracheal tube with a 9 French blocker was used in the ABB group. The variables recorded were peak and plateau inspiratory pressures, compliance and resistance at three surgical times: during two-lung ventilation in latera decubitus (T1), five minutes after initiation of one-lung ventilation (T2) and five minutes after lung resection (T3). Ventilation was accomplished with flow volume control. Tidal volume, respiratory rate and minute volume were kept constant at each time. We used a $33 \%$ inspiratory time and a $20 \%$ inspiratory pause. Statistical analyses were performed with two-way ANOVA considering intragroup (three times) and intergroup factors. Statistical significance was assumed at $p=0.05$

Results: All groups were similar with regard to sex, age, weight, height, ASA, tidal and minute volumes. Data are presented as mean \pm SD.

\begin{tabular}{|c|c|c|c|c|}
\hline \multirow[t]{2}{*}{ Values } & \multirow[t]{2}{*}{ Device } & \multicolumn{2}{|l|}{ Ventilation } & \multirow{2}{*}{$\begin{array}{l}\text { TLV- } \\
\text { postresection }\end{array}$} \\
\hline & & 2 lungs & 1 lung & \\
\hline P.peak & ABB & $21 \pm 3$ & $29.8 \pm 5$ & $22.5 \pm 3$ \\
\hline$\left(\mathrm{cmH}_{2} \mathrm{O}\right)$ & DLT & $20.2 \pm 4$ & $29.3 \pm 3$ & $22.2 \pm 3$ \\
\hline P.plateau & $A B B$ & $18 \pm 3$ & $26.9 \pm 5$ & $19.6 \pm 3$ \\
\hline$\left(\mathrm{cmH}_{2} \mathrm{O}\right)$ & DLT & $17.5 \pm 5$ & $26.1 \pm 3$ & $19 \pm 4$ \\
\hline Compliance & $A B B$ & $40.4 \pm 9$ & $25 \pm 6$ & $38.5 \pm 7$ \\
\hline$\left(\mathrm{ml} / \mathrm{cmH}_{2} \mathrm{O}\right)$ & DLT & $40 \pm 12$ & $25.6 \pm 9$ & $39 \pm 20$ \\
\hline Resistance ${ }^{*}$ & $A B B$ & $16.3 \pm 4$ & $23.5 \pm 8$ & $18.6 \pm 5$ \\
\hline$\left(\mathrm{cmH}_{2} \mathrm{O} / \mathrm{L} / \mathrm{s}\right)$ & DLT & $14 \pm 4$ & $26.3 \pm 8$ & $16.9 \pm 6$ \\
\hline
\end{tabular}

*Interaction between inter- and intragroup factors $p=0.067$

Conclusions. Our results show a significant trend toward a higher decrease in airway resistance during one-lung ventilation with the $A B B$ compared to DLT althought there were no differences in mechanical properties between the devices used.

\section{AP4-6}

\section{Effects of Trendelenburg position on intraloulmonary shunt and oxygenation during one-lung ventilation}

Y. Choi, J. Shim, Y. Kwak, Y. Hong, B. Shin

Department Anesthesiology and Pain Medicine, Yonsei University Medical College, Seoul, Republic of Korea

Background and Goal of Study: Surgical posture considerably influences the deterioration speed and the nadir value of $\mathrm{PaO}_{2}$ during one lung ventilation (OLV) (1). In thoracic surgery with OLV in the lateral position, Trendelenburg position is often required. However, Trendelenburg position causes more compression of the dependent ventilated lung and may significantly affect the ratio of ventilation/perfusion and oxygenation. Therefore, we evaluated the effect of Trendelenburg position on intrapulmonary shunt (Qs/Qt) and oxygenation during OLV in a prospective, randomized, controlled trial.
Materials and Methods: With IRB approval, 34 patients requiring OLV for thoracic surgery were randomly allocated to either control $(n=17, C G)$ or Trendelenburg group $(n=17, T G)$. After anesthesia induction, all patients were turned to the lateral horizontal position and OLV was initiated for $15 \mathrm{~min}$ ( $\mathrm{T} 1$, baseline). Then, patients were $10^{\circ}$ head-down tilted for $10 \mathrm{~min}$ and returned to horizontal position in the TG, while the patients remained horizontal throughout the study period in the CG. Hemodynamic and respiratory variables were measured at T1, 5 and 10 min after head-down tilt (T2 and T3 respectively), $10 \mathrm{~min}$ after the patients were returned to horizontal position (T4). Results: There were no significant differences in baseline hemodynamic and respiratory variables between the groups. Central venous pressure (CVP) at T2 and pulmonary vascular resistance index and peak airway pressure at T2 and T3 were significantly greater in the TG than in the CG. In the TG, CVP at T2 and T3, and pulmonary capillary wedge pressure and Qs/Qt at T3 were significantly increased compared to baseline values and returned to baseline values at $\mathrm{T} 4$.

Conclusion(s): Trendelenburg position during OLV in the lateral position caused significant increase in Qs/Qt without affecting oxygenation. These results were accompanied by distinct increase in cardiac filling pressures without any increase in cardiac output.

References:

1 Hakim TS, Dean GW, Lisbona R. J Appl Physiol 1988; 64: 1160-70.

\section{AP4-7}

Comparative effects of sevoflurane, desflurane and propofol on bronchoalveolar lavage fluid

K. Kalimeris, G. Kostopanagiotou, N. Papoutsidakis, K. Christodoulaki, G. Nakos

2nd Department of Anaesthesiology, Attikon Hospital, University of Athens, Athens, Greece

Background and Goal of Study: Volatile anaesthetics may amplify the injurious effects of mechanical ventilation on the lung ${ }^{(1)}$, while propofol reduces lipid peroxidation and enhances antioxidant defenses. We hypothesized that anaesthetics could induce inflammatory alterations in the healthy lung. To this point, we measured biochemical indices in bronchoalveolar lavage fluid (BALF) during anaesthesia with volatile anaesthetics, propofol or ketamine and midazolam.

Materials and Methods: In our study, 36 domestic pigs were randomly assigned to 5 groups and were subjected to intubation only (control group, $\mathrm{n}=4$ ), $4 \mathrm{~h}$ sedation with ketamine and midazolam with maintenance of spontaneous breathing (sham group, $\mathrm{n}=4$ ) or $4 \mathrm{~h}$ general anesthesia with propofol $(1 \mathrm{mg} / \mathrm{kg} / \mathrm{h}, \mathrm{n}=8)$, sevoflurane $(1,0 \mathrm{MAC}, \mathrm{n}=11)$ or desflurane $(1,0 \mathrm{MAC}, \mathrm{n}=9)$ using mechanical ventilation with tidal volume of $10 \mathrm{~mL} / \mathrm{kg}$ and zero positive end-expiratory pressure. At the end of the experiment BALF was obtained for measurement of protein, phospholipase $A_{2}\left(P L A_{2}\right)$ and platelet activating factor acetylhydrolase (PAF-AH). For statistical analysis one-way Anova or Kruskal-Wallis tests were used. Data are expressed as mean \pm SD. Results and Discussions: In sevoflurane, propofol, desflurane and sham groups PAF-AH ( $\mathrm{nmol} \mathrm{C} \mathrm{C}_{6}-\mathrm{NBD}-\mathrm{FA} / \mathrm{mL} / \mathrm{h}$ ) was reduced compared to controls $(12,6 \pm 1,3$ in control vs. $3,86 \pm 0,8$ in sham group, $P<.001)$; however, levels of PAF-AH were higher in propofol compared to sevoflurane group $(6,98 \pm 4,1$ vs. $3,18 \pm 0,5$, respectively, $P<.05)$. PAF-AH in desflurane group $(5,21 \pm 1,9)$ did not differ from other anaesthetics groups. Levels of protein $(\mu \mathrm{g} / \mathrm{mL}$ BALF) did not differ between groups (control, $160 \pm 402$; sham, $221 \pm 28$; propofol, $159 \pm 80$; sevoflurane, $210 \pm 124$; desflurane, $190 \pm 114$ ). No differences were detected in $\mathrm{PLA}_{2}$ activity (nmol $\mathrm{C}_{12}-\mathrm{NBD}-\mathrm{FA} / \mathrm{mL} / \mathrm{h}$ ) (control, 3,43 $\pm 1,2$; sham, 3,72 $\pm 1,2$; propofol, $3,15 \pm 1,9$; sevoflurane, $4,37 \pm 2,2$; desflurane, $4,28 \pm 2,6$ ) or wet-to-dry ratio.

Conclusion: Anaesthesia with propofol, sevoflurane or desflurane under mechanical ventilation or with ketamine and midazolam with maintenance of spontaneous breathing induces a mild inflammatory response in the lung; propofol may exert partial protective effects on lung inflammation compared to sevoflurane.

References:

1 Gunaydin B, Karadenizli Y, Babacan A et al. Respir. Med. 1997; 91: 351-360.

\section{Transfusion and Haemostasis}

\section{AP1-1}

\section{Intravenous iron to treat anaemia in orthopaedic surgery}

O.M. Theusinger, P-F. Leyvraz, D.R. Spahn

Anesthesiology, CHUV, Lausanne, Switzerland
Background and Goal of Study: Preoperative anaemia is frequent in patients undergoing orthopaedic surgery. (1) The purpose of this study was to determine the increase of haemoglobin $(\mathrm{Hb})$ preoperativley in patients with iron deficiency anaemia (IDA) when treated with iv iron. 
Materials and Methods: After obtaining written informed consent, 20 patients with IDA received $900 \mathrm{mg}$ iv iron sucrose (Venofer) over 2 weeks starting 4 weeks prior to surgery. Changes of $\mathrm{Hb}$ were measured over 4 weeks and at discharge. In the last 11 patients endogenous erythropoietin (EPO) was measured. Data were analysed using ANOVA for repeated measures.

Results and Discussions: Changes in $\mathrm{Hb}$ were significant $(\mathrm{p}<0.02)$ at week 2,3 and 4 of treatment. Overall, the mean maximum increase was $1.0 \pm 0.6 \mathrm{~g} / \mathrm{dL}$ (range: $0.2-2.2 \mathrm{~g} / \mathrm{dL}$ ). Ferritin levels also increased significantly $(p<0.01)$, where as transferrin saturation, serum iron, soluble transferrin receptor (sTfr) did not change significantly due to iv iron treatment. Endogenous EPO decreased due to iv iron treatment in week $3(p=0.049)$. No adverse events related to iv iron were observed.

\begin{tabular}{lclc}
\hline & Baseline & Week 3 & Week 4 \\
\hline $\mathrm{Hb}[\mathrm{g} / \mathrm{dL}]$ & $11.9 \pm 0.8$ & $12.8 \pm 0.8^{*}$ & $12.4 \pm 0.7^{\star}$ \\
Ferritin $[\mathrm{mg} / \mathrm{L}]$ & $78 \pm 70$ & & $428 \pm 191^{\star}$ \\
$\mathrm{sTfr}[\mu \mathrm{g} / \mathrm{ml}]$ & $4.1 \pm 2.3$ & & $3.7 \pm 2.3$ \\
Epo $[\mathrm{mg} / \mathrm{L}]$ & $261 \pm 130$ & $190 \pm 49^{\star}$ & $189 \pm 75$ \\
Serum Fe $[\mu \mathrm{mol} / \mathrm{l}]$ & $13.3 \pm 4.6$ & & $13.1 \pm 4.5$ \\
\hline
\end{tabular}

$p<0.05$ vs. Baseline

The highest increase of $\mathrm{Hb}$ was at week 3 , indicating that administration of iv iron 2 to 3 weeks prior to surgery may be optimal.

Conclusion(s): Treatment with iv iron allows to correct IDA prior to elective surgery and may reduce perioperative transfusion needs.

Reference:

1 Shander A., Knight K. Thurer R. et al. Am. J. Med. 2004; 116 Suppl 7A: 58-69.

\section{AP1-2}

\section{Liposome encapsulated hemoglobin (TRM-645) increases} survival time of critical normovolemic anemia

O. Horn, A. Pape, H. Kertscho, B. Zwissler, O. Habler

Clinic of Anesthesiology, Intens. Care Med. and Pain Management, J.W. Goethe-University Hospital, Frankfurt/Main, Germany

Background and Goal of Study: Hemoglobin-based $\mathrm{O}_{2}$ carriers (HBOC) increase arterial $\mathrm{O}_{2}$-content $\left(\mathrm{CaO}_{2}\right)$ and systemic $\mathrm{O}_{2}$-delivery $\left(\mathrm{DO}_{2}\right)$. TRM-645 (Terumo Inc., Kanagawa, Japan) is a liposome encapsulated $\mathrm{Hb}$ formulation ( $\mathrm{LEH}$ ) with low $\mathrm{O}_{2}$-affinity and facilitated $\mathrm{O}_{2}$-offloading to the tissues ${ }^{1}$. Due to its specific $\mathrm{O}_{2}$-transport properties, this LEH should represent an attractive alternative to the transfusion of allogeneic red blood cells. The goal of the present study was to investigate the efficacy of TRM-645 in critical normovolemic anemia.

Materials and Methods: 14 anesthetized and mechanically ventilated beagle dogs were splenectomized and hemodiluted to their individual critical hemoglobin concentration $\left(\mathrm{Hb}_{\text {crit }}\right.$, i.e. onset of $\mathrm{O}_{2}$-supply-dependency of total body $\mathrm{O}_{2}$-consumption ${ }^{2}$ ) by exchange of whole blood for hetastarch (HES). At $\mathrm{Hb}_{\text {crit }}$, animals were randomized to receive a bolus infusion $(20 \mathrm{ml} / \mathrm{Kg})$ of either TRM645 (LEH-group, $n=7$ ) or normal saline (N.S.-group, $n=7$ ). Subsequently, animals were observed without further intervention. The primary endpoint was survival-time after the completion of treatment, secondary endpoints were parameters of central hemodynamics, $\mathrm{O}_{2}$-transport and tissue oxygenation. Results: Animals of the LEH-group survived $98 \pm 92 \mathrm{~min}$ (vs. $49 \pm 31 \mathrm{~min}$, N.S.-group, $\mathrm{p}<0.05)$. 30 minutes after treatment, tissue $\mathrm{O}_{2}$ tension $\left(\mathrm{tpo}_{2}\right)$ on the surface of a skeletal muscle was significantly higher in the LEH-group (24 $\pm 7 \mathrm{mmHg}$ vs. $9 \pm 2 \mathrm{mmHg}$ ). Regarding central hemodynamics, no significant differences were observed between the groups.

Conclusion(s): TRM-645 provides sufficient tissue oxygenation in critical normovolemic anemia and may be used for bridging a period of acute blood loss until allogeneic red blood cells are available.

Disclosure: The study was sponsored by a research grant from Terumo Inc., Kanagawa, Japan.

References:

1 Meier J, Woelkhammer S, Habler O, Comp. Biol. Med. 2003; 33: 395-405.

2 Ogata Y, Polymers for Advanced Technologies 2000; 11: 205-209.

\section{AP1-4}

The tolerance of acute normovolemic anemia depends on the choice of the plasma substitute

S. Kutschker, A. Pape, H. Kertscho, B. Zwissler, O. Habler

Clinic of Anesthesiology, Intens. Care Med. and Pain Management,

JW Goethe University Hospital, Frankfurt, Germany
Background and Goal of Study: During initial treatment of acute blood losses, the infusion of cristalloid and/or colloidal solutions implies the dilution of the red cell mass remaining in the vascular system (acute normovolemic anemia). The microcirculatory effects of particular plasma substitutes on tissue oxygenation have not been elucidated so far. The goal of the present study was to compare the effects of four different plasma substitutes on the physiological limit of anemia tolerance.

Materials and Methods: Thirty-six anaesthesized and mechanically ventilated pigs were hemodiluted to their individual critical hemoglobin concentration $\left(\mathrm{Hb}_{\text {crit }}\right) . \mathrm{Hb}_{\text {crit }}$ was defined as the $\mathrm{Hb}$-concentration corresponding with a critical limitation of $\mathrm{O}_{2}$-delivery to the tissues $\left(\mathrm{DO}_{2}\right)$ and a sudden decrease of total body $\mathrm{O}_{2}$-consumption $\left(\mathrm{VO}_{2}\right)^{1}$. The hemodilution protocol was randomly performed with either 6\% HES 130/0.4 (Group 1, $n=9$ ), 3.5\% Gelatin (Group 2, $n=9$, infusion ratio 1:1.2), 6\% HES 450/0.7 (Group 3, $n=9$ ) or Ringer's solution (Group 4, $n=9$, infusion ratio 1:3). Primary endpoint was the dimension of $\mathrm{Hb}_{\text {crit }}$, secondary endpoints were parameters of central hemodynamics, $\mathrm{O}_{2}$-transport und tissue oxygenation.

Results: In each animal, normovolemia was maintained at any timepoint of the protocol. $\mathrm{Hb}_{\text {crit }}$ was met at $2.1 \pm 0.4 \mathrm{~g} / \mathrm{dl}$ (group 1), $2.7 \pm 0.6 \mathrm{~g} / \mathrm{dl}$ (group $2, p<0.05$ vs. group 1), $3.0 \pm 0.6 \mathrm{~g} / \mathrm{dl}$ (group $3, \mathrm{p}<0.05$ vs. group 1 ) and $3.7 \pm 0.6 \mathrm{~g} / \mathrm{dl}$ (group $4, \mathrm{p}<0.05 \mathrm{vs}$. group 1 ). Regarding secondary endpoints no significant differences between the groups were observed.

Conclusion(s): During acellular volume replacement, the choice of the plasma substitute is a factor which significantly influences anemia tolerance. Low molecular HES preparations (MW $130 \mathrm{kDa}$ ) seem to be most advantagous for the treatment of acute blood losses ${ }^{2}$. The underlying mechanism has to be elucidated so far.

Disclosure: The project was sponsored by a research grant from the Else Kröner Fresenius - Stiftung, Bad Homburg, Germany.

References:

1 Meier J, Woelkhammer S, Habler O, Comp. Biol. Med. 2003; 33:395-405.

2 Standl T, Burmeister MA, Schroeder F, et al. Anesth. Analg 2003; 96:936-943.

\section{AP1-5}

Colloids as intraoperative fluid management provide better blood oxygenation than crystalloids

I. Soumpasis, S. Marinou, E. Antypa, Th. Georgiadou, F. Kanakoudis Anaesthesia, G. Gennimatas General Hospital of Thessaloniki, Thessaloniki, Greece

Background and Goal of Study: Colloids stay intra-vascularly longer than crystalloids, which are rapidly diffused into interstitial space ${ }^{1}$. The aim of the study was to investigate possible alteration in blood oxygenation, as it is affected by the blood-gas barrier thickness.

Materials and Methods: Forty ASA I-II patients aged $58 \pm 14$ years, who underwent abdominal operations under general anaesthesia, were randomly allocated in two groups (Group COL and Group CRS). Intraoperative fluid management consisted of colloids in group COL and crystalloids in group CRS. Infusion rate was guided by blood pressure and heart rate, in order to maintain $\pm 20 \%$ of baseline values, obtained before anaesthesia. The oxygenation index $\left(\mathrm{pO}_{2} / \mathrm{FiO}_{2}\right)$ was recorded before induction of anaesthesia and before extubation, and the difference of these values $\left(\Delta \mathrm{pO}_{2} / \mathrm{FiO}_{2}\right)$ was calculated in both groups. Student's t-test was used for statistical analysis of the data, with $p<0.05$ as level of significance.

Results and Discussions: Demographic data were similar in both groups, as well as the duration of operations (152 $\pm 40 \mathrm{~min}$ vs $145 \pm 34 \mathrm{~min})$. A total amount of $43.4 \pm 20.6 \mathrm{ml}$ and $18.9 \pm 8.3 \mathrm{ml}$ of fluid was administered in groups COL and CRS respectively. The $\Delta \mathrm{pO}_{2} / \mathrm{FiO}_{2}$ values were significantly higher $(p<0.001)$ in the COL group $(123.3 \pm 118.4 v s-33.5 \pm 91.4)$ as it is shown in the figure.

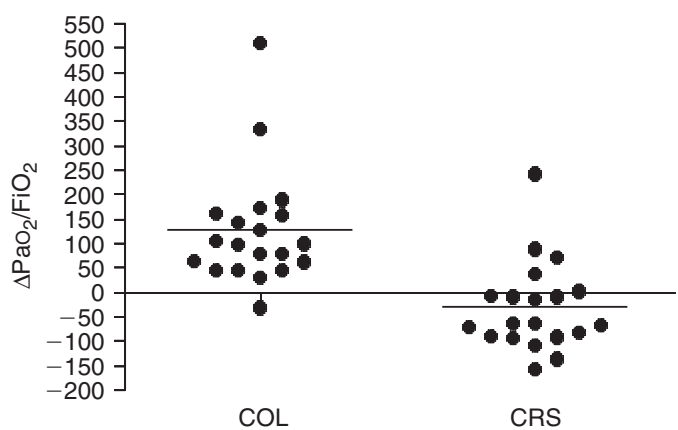


Conclusion(s): According to the results of this study, the use of colloids as a sole intraoperative fluid management could lead to better blood oxygenation during abdominal operations under general anaesthesia compared to the use of crystalloids.

Reference:

1 Boldt J. Eur J Anaesthesiol 2006; 23: 631-40.

\section{AP1-6}

The effect of hydroxyethyl starch $(670 / 0.75)$ on hemostasis during acute normovolemic hemodilution

O. Kim, Kye-Min. Kim, Sang-Seok. Lee, Jun-Heum. Yon, Ki-Hyuk. Hong

Department of Anesthesia and Pain Medicine, Sanggye Paik Hospital, Inje University, Seoul, Republic of Korea

Background and Goal of Study: Hextend ${ }^{\circledR}$ is a plasma volume expander containing $6 \%$ hydroxyethyl starch (HES) in a physiologically balanced medium of electrolytes, lactate and glucose (Molecular weight $670 \mathrm{kDa}$, molar substitution 0.75). HES has been associated with platelet dysfunction and inhibition of coagulation. The aim of this study is to evaluate the effect of Hextend ${ }^{\circledR}$ on hemostasis during acute normovolemic hemodilution ( $\mathrm{ANH})$.

Materials and Methods: Eighteen healthy adult male patients scheduled for spine surgery were enrolled in this study. Before general anesthesia, patients underwent $\mathrm{ANH}$ with $20 \mathrm{ml} / \mathrm{kg}$ of Hextend ${ }^{\circledR}$. Hemoglobin, platelet count, plasma fibrinogen concentration, factor VIII activity, PT, PTT and thromboelastography (TEG) were measured before and after ANH. Paired t-test was used.

Results: Age, weight and height of patients were $32.6 \pm 7.5$ years, $70.5 \pm 8.1 \mathrm{~kg}$ and $172.9 \pm 8.0 \mathrm{~cm}$, respectively (mean $\pm \mathrm{SD}$ ). Data are shown in the following table.

\begin{tabular}{lcc}
\hline & Pre-ANH & Post-ANH \\
\hline Hemoglobin $(\mathrm{g} / \mathrm{dL})$ & $14.9 \pm 0.9$ & $11.1 \pm 0.9^{*}$ \\
Platelet $\left(10^{3} / \mu \mathrm{L}\right)$ & $245.8 \pm 47.1$ & $205.8 \pm 43.3^{*}$ \\
Fibrinogen $(\mathrm{mg} / \mathrm{dL})$ & $273.9 \pm 182.0$ & $182.0 \pm 50.1^{*}$ \\
factor VIII activity(\%) & $71.1 \pm 43.4$ & $25.1 \pm 15.0^{*}$ \\
PT (INR) & $1.00 \pm 0.01$ & $1.08 \pm 0.06^{*}$ \\
PTT $(\mathrm{sec})$ & $26.7 \pm 2.3$ & $35.5 \pm 4.6^{*}$ \\
$\alpha\left(22-38^{\circ}\right)$ & $26.8 \pm 6.8$ & $27.6 \pm 6.7$ \\
K $(330-630 \mathrm{sec})$ & $518.5 \pm 129.6$ & $498.4 \pm 193.1$ \\
$\mathrm{R}(930-1380 \mathrm{sec})$ & $909.8 \pm 334.4$ & $993.1 \pm 497.0$ \\
$\mathrm{MA}(47-58 \mathrm{~mm})$ & $41.8 \pm 10.5$ & $40.7 \pm 8.5$ \\
$\mathrm{Cl}(-3-+3)$ & $-2.2 \pm 1.9$ & $-2.5 \pm 1.9$ \\
\hline
\end{tabular}

: $\mathrm{P}<0.05$ compared to the values of pre-ANH.

Post-ANH: 120 minutes after completion of ANH.

Data are mean \pm SD.

Conclusions: In spite of the decrease in the platelet count, fibrinogen concentration and factor VIII activity, ANH with $20 \mathrm{ml} / \mathrm{kg}$ of Hextend ${ }^{\circledR}$ didn't cause impairment of TEG parameters.

\section{AP1-7}

Quantitative and qualitative changes of von Willebrand factor during cardiac surgery

N. Rahe-Meyer, C. Solomon, E. Czaja, S. Schneppenheim, M.v. Depka Department of Anaesthesiology, Hannover Medical School, Hannover, Germany

Background and Goal of Study: Von Willebrand factor (vWf) consists of large multimeric molecules which interact with the GP1b receptor on the surface of the platelets leading to aggregation and activation of platelets at the lesion site. A decrease of the highest molecular weight multimers - as described in aortic valve defects - is reported to be associated with increased peri-operative bleeding (acquired von Willebrand syndrome avWs). The main question was whether cardio-pulmonary bypass changes the multimeric structure of vWf.

Materials and Methods: 25 patients with a negative standardized bleeding anamnesis which underwent heart surgery were analyzed. Operation types: coronary artery bypass grafting (10), double valve surgery (4), thoracic aorta surgery (10), pulmonary artery surgery (1). 14 patients had aortic valve disease. We investigated the changes of $\mathrm{vWf}$ levels, collagen binding activity (CBA) and the multimeric structure at two time points: directly before the operation and at the end of the operation after the weaning from cardio-pulmonary bypass. We monitored the intra- and post-operative consumption of blood products and the drainage volume.
Results and Discussions: From the 14 patients with aortic valve disease 8 patients $(57 \%)$ had preoperatively a loss of the high molecular weight multimers but all had normal vWf levels and CBA. From the 11 other patients 3 (27\%) had avWS and one additionally a pathologic CBA. There was no correlation between the pre-operative state of $\mathrm{vWf}$ and the consumption of blood products or the drainage volume. Only 3 patients had still pathologic multimers after the weaning from cardio-pulmonary bypass, none had pathologic vWf levels or CBA. The normalisation of the vWF after the extracorporeal circulation can be attributed to a high release of $\mathrm{vWf}$ from the endothelial storage pools.

Conclusions: (1) The cardiac surgery patients - even those with no aortic valve disease - had a high percentage of pre-operative avWs, which was not detected by a bleeding anamnesis. (2) A pre-operative avWs was intraoperatively corrected by the release of intact vWf from endogenous stores. (3) The consumption of blood products and the drainage volume was not correlated with the pre-operative state of the vWf.

\section{AP1-8}

Perioperative management of a patient with Bernard - Soulier Syndrome undergoing major abdominal surgery

R.M. Groessle, C.F. Hoffmann, U. Schuler, R.J. Litz, T. Koch

Department of Anaesthesiology and Intensive Therapy, University Hospital TU Dresden, Dresden, Germany

Background and Goal of Study: Perioperative management of coagulationdisorders remains a challenge in anaesthesia and can lead to high treatment costs. In 1948 Bernard and Soulier described a severe hemorrhagic thrombocytodystrophy showing a prolonged bleeding time, a low platelet count as well as enlarged platelets. These features are the hallmark of the Bernard - Soulier - Syndrome, characterized by a missing or dysfunctional expression of the glycoprotein $\mathrm{lb}-\mathrm{V}-\mathrm{IX}$ complex, a receptor for the von - Willebrand factor in the platelets membrane. Therefore von Willebrand factor mediated adhesion to the subendothelial wall and aggregation at high shear forces are reduced or even impossible. We report the perioperative management of a 39-year-old male with Bernard-Soulier-Syndrome, who was admitted for elective duodenotomy for chronic intestinal bleeding.

Materials and Methods: The Patient was presented for surgery with a preoperative platelet count of $12 \mathrm{GPt} / \mathrm{l}$ despite repeated preoperative substitution. Administration of recombinant factor VII and factor XIII perioperatively as well as further substitution of platelets 96 hours perioperatively was suggested by haematologists.

Results and Discussions: On day of surgery the patient presented with anaemia (haemoglobin $3.8 \mathrm{mmol} / \mathrm{l}$ ) and was therefore substituted with two units of packed red cells. He was perioperatively monitored with thrombelastography using the ROTEM ${ }^{\circledR}$-analyzer. Soon after incision the patient received two units of platelets and $4 \mathrm{~g}$ of fibrinogen according to ROTEM analysis. Estimated intraoperative blood loss was $200 \mathrm{ml}$ and surgery was performed uneventfully. With clinical demand responding administration of fibrinogen no relevant bleeding occurred though perioperative platelet-count was as low as $7 \mathrm{GPt} / \mathrm{l}$. Postoperatively the patient recovered from the procedure without complication.

Conclusion(s): Even in severe coagulation disorders major surgery can be performed without bleeding complications. The patient described was effectively monitored with the ROTEM ${ }^{\circledR}$-analyzer perioperatively and sufficiently substituted with fibrinogen according to clinical demand. The widely established and recommended cost consuming recombinant factor VII and XIII substitution could be avoided.

\section{AP1-9}

Absence of hypercoagulable state, defined by endogenous thrombin potential measurement, after radical mastectomy for cancer

B. Richez, Y. Kabbani, G. Freyburger, J.P. Gekiere, D. Monnin Département Danesthésie-Réanimation IV, Hôpital Pellegrin, Bordeaux, France Background: Cancer and surgery are known to produce hypercoagulability. The aim of this study was to investigate the modification of the coagulability after radical mastectomy for cancer.

Methods: after local ethics committee approval and written consent, 50 patients undergoing radical mastectomy and control group of 20 persons were included in this prospective, observational study. Specific markers for coagulation (D-dimer (DD), thrombin-antithrombin III complex (TAT), endogenous thrombin potential measurement by calibrated automated thrombography (CAT) were examined at T0 (before operation), T1 (end of surgery) and T2 (24 h after surgery). 
Résults and discussion:

\begin{tabular}{lllll}
\hline & Control & Cancer group T1 & Cancer group T2 & Cancer group T3 \\
\hline DD $(\mu \mathrm{g} / \mathrm{ml})$ & 0,20 & 0,32 & 0,33 & 0,37 \\
& $0,12-0,25$ & $0,22-0,54$ & $0,21-0,55$ & $0,23-0,66$ \\
p vs T1 & $\mathbf{0 , 0 1 9}$ & & NS & $\mathbf{0 , 0 0 3}$ \\
TAT $(\mathrm{ng} / \mathrm{ml})$ & 2,25 & 3,46 & 4,52 & 3,95 \\
& $1,75-2,52$ & $2,23-7$ & $3,15-15,49$ & $2,89-5,99$ \\
p vs T1 & NS & & $\mathbf{0 , 0 2 2}$ & NS \\
CAT $(\mathrm{ng} / \mathrm{ml})$ & 1703 & 1667 & 1737 & 1708 \\
& $1442-1961$ & $1522-1922$ & $1498-1941$ & $1589-1984$ \\
p vs T1 & NS & & NS & NS \\
\hline
\end{tabular}

Results are median and $25-75$ percentiles.

Conclusion: Only a significant higher plasmatic concentration of DD in cancer group before surgery was recorded. There is a minimal activation of coagulation in breast cancer patients without hypercoagulability. Radical mastectomy for cancer was associated with significant transient increase in plasma concentrations of DD and TAT without significant modification of CAT. There is an activation of thrombin generation without hypercoagulability. Nor breast cancer neither surgery is responsible of a hypercoagulable state. These results are different of previous study ${ }^{1}$.

Reference:

1 J.F Payen. British Journal of Anaesthesia 1998;80:464-6.

\section{AP1-10}

\section{Detection of aspirin and clopidogrel using multiple electrode aggregometry}

A. Calatzis, N. Rahe-Meyer, F. Theisen, M. Spannagl

Haemostasis and Transfusion Medicine, Munich University Clinic, Munich Germany

Background and Goal of Study: During invasive procedures aspirin and clopidogrel can induce bleeding. The detection of these drugs may therefore be necessary. We evaluated multiple electrode aggregometry, a new platelet analysis technique based on whole blood.

Methods: Venous blood was drawn from 212 patients treated with either aspirin (88) or clopidogrel (13) or both (57) or none of the two drugs (54) and anticoagulated using heparin (sarstedt blood collection tubes) or hirudin (collection tubes provided by Dynabyte, Munich). Blood was analyzed on the Multiplate analyzer (Dynabyte) using the following tests: ASPItest (arachidonic acid induced aggregation), ADPtest (ADP induced aggregation) or ADPtest HS (high sensitivity = addition of prostanglandin E1). Aggregation was quantified by the area under the curve ( $1 \mathrm{U}=10 \mathrm{AU}^{*} \mathrm{~min} ; \mathrm{AU}=$ aggregation units). Results:

\begin{tabular}{llc}
\hline & Patients on Aspirin (145) & Patients without aspirin (67) \\
\hline ASPItest (heparin blood) & $35+-31$ & $100+-36$ \\
ASPItest (hirudin blood) & $23+-27$ & $90+-37$ \\
\hline
\end{tabular}

\begin{tabular}{lcc}
\hline & Patients on Clopidogrel (70) & $\begin{array}{c}\text { Patients without } \\
\text { Clopidogrel (142) }\end{array}$ \\
\hline ADPtest (heparin blood) & $65+-30$ & $95+-28$ \\
ADPtest (hirudin blood) & $39+-26$ & $81+-33$ \\
ADPtest HS (heparin blood) & $34+-24$ & $80+-31$ \\
ADPtest HS (hirudin blood) & $22+-16$ & $66+-35$ \\
\hline
\end{tabular}

Conclusion: Multiple electrode aggregometry allows for a sensitive detection of Aspirin and Clopidogrel. For the detection of clopidogrel in heparin blood ADPtest HS is preferable to the use of ADPtest.

\section{AP2-1}

Tranexamic acid reduces blood transfusion requirements in scoliosis surgery

O. Kaabachi, W. Koubaa, D. Chafii, R. Ouezzini

Anesthesiology and Intensive Care, Institut Kassab d-orthopédie, Tunis, Tunisia

Background and Goal of Study: Pediatric patients undergoing posterior spinal fusion surgery for scoliosis often require multiple blood transfusions. Antifibinolytics drugs have been used with controversial results. In a previous study, administration of high-dose tranexamic acid reduced bleeding by $41 \%$ but did not lead to significant reduction in the blood transfusion requirements (1). We evaluated the efficacy of prophylactic high-dose tranexamic acid to reduce perioperative blood transfusion requirements in a prospective, double-blinded, placebo control study.

Materials and Methods: Following ethical committee approval and informed parental consent, 40 patients, $11-20$ years of age, scheduled to undergo elective spinal posterior fusion were randomly assigned to receive either $100 \mathrm{mg} / \mathrm{kg}$ tranexamic acid before incision followed by an infusion of $10 \mathrm{mg} / \mathrm{kg} / \mathrm{h}$ during surgery (tranexamic acid group) or $0.9 \%$ saline (placebo group). General anesthesia was administered according to a standard protocol. Blood loss, transfusion requirements, coagulation parameters, and complications were assessed. Continuous parameters were tested by MannWithney test and non-parametric data were tested with fisher exact test. $\mathrm{P}<0.05$ was significant.

Results and Discussions: Demographic data and surgical characteristics (etiologies, number of fused vertebrae) were similar in both groups. In the tranexamic acid group, blood loss was reduced by $35 \%$ compared with placebo $(1529 \pm 683 \mathrm{ml}$ vs. $2346 \pm 1255 \mathrm{ml} ; \mathrm{p}=0.016)$. The total amount of blood transfused in the perioperative period was significantly reduced in the tranexamic group $(1.75 \pm 1.55$ units vs. $3.60 \pm 2.06$ units; $P=0.03)$. No thrombotic complications were detected. Adverse drug effects occurred in the tranexamic acid group (vomiting in $55 \%$ of patients).

Conclusion(s): Intraoperative administration of high-dose tranexamic acid reduces significantly blood loss and blood transfusion requirements during spinal posterior fusion in adolescents with scoliosis.

Reference:

1 Navil F. Anesthesiology 2005; 102: 727-32.

\section{AP2-3}

Recombinant FVIla in critical patients with massive bleeding L. Mora, MJ. Colomina, E. Guerrero, M. Martorell, S. González Anesthesia, Hospital Vall dHebron. Área de Traumatología, Barcelona, Spain Background and Goal of Study: Uncontrolled bleeding after traumatic injury is a result of surgical and coagulopathic bleeding and it is the leading cause of early in-hospital mortality in trauma victims. Massive transfusion is also very high-risk. Recombinant activated factor VII ( $r F V I l a)$ can be effective. Materials and Methods: We describe seven patients who between 2004-2005 received rFVIla in cases of shock for massive bleeding: 2 women subjected to major orthopaedic surgery (78-81 years) and 5 men with severe traumatic injuries (18-78 years, mean 36 ). We reviewed the processes of resuscitation, the emergency surgical indications, the levels of haemoglobin and coagulation, the administered doses of rFVIla, the amount of packed red blood cells used as well as the survival.

Results and Discussion: A surgical damage control was performed in all patients. The average of total packed red blood cells was 21 (12-31). Before rFVIla administration the mean was 17 units (10-25), Haemoglobin levels were 7.58 (4.3-11.5) and INR 1.87 (1.05-2.81). After rFVIla administration the mean was 5 units (4-9), Haemoglobin levels 10.5 (8.3-12) and INR 1.34 (0.85-2.17). The mean doses of rFVIla administered were $113 \mu \mathrm{g} / \mathrm{kg}$ $(70-180 \mu \mathrm{g} / \mathrm{kg})$ in $1-3$ times. All polytrauma patients survived and only one patient who had major orthopaedic surgery died.

Conclusions: Our experience demonstrates that rFVIla can reduce or stop coagulopathic bleeding in polytrauma patients when vascular or surgical control fails. It is necessary to establish a protocol treatment to optimize the resources while we are waiting for the different studies results about clinical indications.

References:

1 Bowles KM, et al. Br J Anaesth 2006; 97(4): 476-81.

2 Levy JH, et al. Transfusion 2006; 46(6): 919-33.

\section{AP2-4}

Aprotinin causes fewer complications after cardiac surgery than tranexamic acid

T. Breuer, K. Martin, R. Bauernschmitt, P. Tassani

Institute of Anaesthesiology, Deutsches Herzzentrum München, Münich, Germany

Background and Goal of Study: Recently, the routine application of aprotinin, a serine protease inhibitor, during open-heart surgery has been debated (1). Our aim was to investigate the antifibrinolytic effect and the side-effects of aprotinin comparing with tranexamic acid, another antifibrinolytic drug.

Materials and Methods: We retrospectively analysed the data of patients undergoing open heart surgery in our Institute from September 2005 to June 2006. According to the institutional protocol, every patient operated on using cardiopulmonary bypass was treated with full-dose aprotinin according to the Hammersmith protocol until January 2006. In February 2006, this regimen was changed due to ethical considerations (1), and thereafter every 
patient received tranexamic acid (4-6g) as antifibrinolytic therapy. In all other aspects, the anaesthesia protocol has remained unchanged.

Results and Discussions: 596 patients were treated with aprotinin (group A) and 592 with tranexamic acid (group T). The descriptive parameters and the comorbidities were well comparable in the two groups. The blood loss was significantly lower in group A comparing with group T at 6, 12 and 24 hours after operation ( $24 \mathrm{~h}$ : $555 \pm 419$ vs. $686 \pm 589 \mathrm{ml}, \mathrm{p}<0.001$ ). Similarly, the frequency ( $58.9 \%$ vs. $66.6 \%, p=0.006)$ and the amount of blood transfusion $(2.8 \pm 5.0$ vs. $3.1 \pm 4.5$ units, $p=0.001)$ were also significantly lower in group $A$. There was no difference in the incidence of postoperative renal dysfunction, renal failure and other organ dysfunctions between the two groups, except that there were significantly less postoperative neurological complications, in particularly seizures, in group $\mathrm{A}(2.7 \%$ vs. $5.4 \%, \mathrm{p}=0.017$ and $0.8 \%$ vs. $4.4 \%, p<0.001$, respectively).

Conclusions: According to our results, the efficacy of aprotinin regarding the reduction of postoperative bleeding and blood transfusion is superior to that of tranexamic acid. Comparing possible side effects, including renal and other organ dysfunctions, we found significantly more neurological complications after administration of tranexamic acid.

Reference:

1 Mangano DT, Tudor IC, Dietzel C. The risk associated with aprotinin in cardiac surgery. N Engl J Med 2006;354:353-65.

\section{AP2-5}

Are antifibrinolytics useful in a global blood sparing strategy after Revision Total Hip Arthroplasty?

F. Remerand, C. Couvret, A. Baud, F. Narcisse, J. Fusciardi

SAR 2, CHU Trousseau, Chambray les Tours, France

Background and Goal of Study: Patients undergoing revision total hip arthroplasty (RTHA) are at high risk of allogenic blood transfusion. Studies dealing with antifrinolytics (AF) in RTHA are scarse and tested AF alone, without consideration of a global blood sparing strategy (preoperative autologous donations, EPO, intra and post-operative autologous blood reinfusion) (1). This is the aim of our present study.

Materials and Methods: Between 2001 and 2006, we prospectively collected data on 101 consecutive patients scheduled for aseptic non tumoral RTHA during their first postoperative week. A global perioperative blood sparing strategy included preoperative autologous blood donations and recombinant human erythropoietin (2), and intra and postoperative autotransfusion replacement systems (CATS ${ }^{\circ}$, Fresenius and Constavac ${ }^{\circ}$, Gamida). When hemoglobin concentration was below $8 \mathrm{~g} / \mathrm{dL}$, patients received either autologous transfusion if available, or an allogenic one in other cases. Indication and choice of AF (tranexamic acid $15 \mathrm{mg} / \mathrm{kg}$ before incision followed by $10 \mathrm{mg} / \mathrm{kg} / \mathrm{h}$ intraoperatively, or aprotinin $2 \mathrm{MKIU}$ followed by $0.5 \mathrm{MKIU} / \mathrm{h}$ intraoperatively) was left to each physician's preferences. Chi2 or Student t tests were used to compare the 2 groups of patients (with and without AF). Results and Discussions: Patients' characteristics, hemoglobin concentration before surgery and 7 days latter, and duration of surgery were similar in both groups. None of the 22 patients who received antifibrinolytics (17 aprotinin, 5 tranexamic acid) had allogenic transfusion, versus 18 of the 79 patients who did not $(23 \%, p=0.03)$. Patients with AF had less intra operative autologous blood volume reinfused than the ones without AF $(206 \pm 93 \mathrm{~mL}$ versus $343 \pm 264 \mathrm{~mL}, p=0.0687$ ), and less preoperative autologous blood transfused $(21 \%$ of units versus $56 \%, p=0.0004)$.

Conclusion(s): Adding antifibrinolytics in patients undergoing aseptic non tumoral RTHA significantly decreased allogenic and autologous blood transfusion, even in the presence of a global blood sparing strategy.

References:

1 Murkin JM. Anesthesia Analgesia 1995; 80: 343-8.

2 Couvret C. Anesthesia Analgesia 2004; 99: 262-71.

\section{AP2-6}

\section{Clinical experience with recombinant activacted factor VIla} (2003-2006)

S. Beltrán De Heredia, A. Rojo, E. Bisbe, M. López, F. Escolano

Anaesthesiology, Hospital del Mar, Barcelona, Spain

Background and Goal of Study: Although treatment with recombinant activated factor VII ( $r F V I l a)$ is only accepted in haemophilia A or B with inhibitors and Glanzmann's thrombasthenia), several case reports and small series suggest that rFVIla could be also useful in patients with massive bleeding. The aim of the study was to review the off-label use of rFVIla in our hospital.
Materials and Methods: Four years retrospective review of massive bleeding patients treated with rFVIla in both, surgical and non surgical environments. The variables analysed included: demographics, indications for the treatment, dose of rFVIla, blood products used, laboratory data, mortality, blood products used and factors that could alter rFVIla function ( $\mathrm{pH}$ and temperature). Results: 24 patients received $\mathrm{rFV}$ Ila for compassive treatment, mean age of 55 (26-88) years being 9 women and 15 men. Indications were: traumatic patients (2), obstetric bleeding (2), drug-related coagulation disorders (4), surgical bleeding (9) and medical indications, including respiratory disorders (3), digestive bleeding (2) and CID-sepsis (2) . Mean dose of rFVIla was $6.8 \mathrm{mg}$.

Pre-rFVIla administration laboratory data were: mean haemoglobin of $7.5 \mathrm{~g} / \mathrm{dL}$, platelet count 98.000/uL, arterial pH 7.25 (6.98-7.42). Mean axillary temperature was $35.2^{\circ} \mathrm{C}(32.8-37.6)$. Severity of bleeding was estimated by blood products administered (mean [range]): packed red blood cells 15 [9-24], fresh frozen plasma 8 [3-15], platelet units 3 [0-26]. Fibrinogen was administered on $4 / 24$ patients (16.6\%), tranexamic acid in $3 / 24(12 \%)$ and desmopresin in 4/24 (16.6\%).

$59 \%$ patients achieved complete or partial cessation of bleeding, $42 \%$ patients died (10/24), being only $16 \%$ of them bleeding related. No thromboembolic events were reported. Prevalence rFVIla prescription: 1:5200 hospitalised patients, 1:3000 surgical patients.

Conclusion: In our experience, rFVlla was safely used in a very selected truly massive bleeding patients. Probably, our results could be improved with temperature and $\mathrm{pH}$ pre-treatment optimisation. Although more data are needed we do believe that treatment with rFVIla should be take into account on the hospital protocols of massive bleeding patients.

Reference:

1 U. Martinowitz et al. J Thromb Haemost. 2005; 3: 640-648.

\section{AP2-7}

Antifibrinolytic agents in lumbar arthrodesis surgery Randomized controlled trial preliminary results

S. Ramirez, JM. Marzal, L. Fuentes, D. Arnal, Jl. Gomez-Arnau Anesthesia and Reanimation, Fundacion Hospital Alcorcon, Alcorcon, Spain

Background and Goal of Study: To establish if the antifibrinolytic agents (aprotinin and tranexamic acid) are a safe and effective blood saving method during the intraoperative and postoperative period of the lumbar arthrodesis surgery. To compare the tranexamic acid vs. Aprotinin in this setting.

Materials and Methods: Prospective, randomized, double blind study of 62 patients undergoing lumbar arthrodesis surgery. Patients where assigned to one of the three groups (aprotinin, tranexamic acid and placebo) and perioperative bleeding, transfusion and secondary effects were recorded. Patients were restrictively transfused according to the institutional policy. Data were analyzed with the Kruskal-Wallis test.

Results and Discussions: We did not find significant differences in perioperative bleeding between the placebo and aprotinin group: In contrast we found less blood loss in the tranexamic acid group in the six-hour postoperative period $(p<0,05)$. We could not find significant differences of the transfusion requirements among the three groups. One patient of the aprotinin group suffered an allergic reaction. No other secondary effects due to the antifibrinolytic therapy were recorded.

\begin{tabular}{llll}
\hline Group & Intraop BL & 6 h PO BL & PTP \\
\hline Placebo & 739 & 214 & $28 \%$ \\
Aprotinin & 765 & 166 & $23 \%$ \\
Tranex. Acid & 865 & 64 & $15 \%$ \\
\hline
\end{tabular}

BL: Blood loss (ml). PO: Postoperative time. PTP: \% of transfused patients.

Conclusion: Tranexamic acid may be an effective, efficient and safe antifibrinolytic agent to decrease the postoperative bleeding of lumbar arthrodesis surgery.

\section{AP2-8}

Risk associated with aprotinin in acute type $A$ aortic dissection surgery

A. Morand, M. Cannesson, Y. Attof, M. Hachemi, JJ. Lehot Department of Anaesthesiology, Hospices Civils de Lyon, Hopital Louis Pradel, Bron, France, Hospices, Hospices Civils de Lyon, Bron, France Background and Goal of Study: Aprotinin is commonly used during cardiac surgery to limit blood loss. Recent studies (1) have suggested that aprotinin may increase post-operative morbidity following coronary artery bypass grafting. However, its clinical utility is still debated. The aim of our study was to 
investigate the effects of aprotinin on the postoperative course of patients with acute type $\mathrm{A}$ aortic dissection.

Materials and Methods: We reviewed all acute type $A$ aortic dissections that were surgically managed in our institution from $1 / 1 / 1991$ to $12 / 31 / 2001$. Patients were divided into three groups: A: aprotinin $(n=153)$, TA: tranexamic acid $(n=89)$, and NA: no antifibrinolytic agent $(n=68)$. Analysis of variance (ANOVA) was used to test differences between groups. In all cases, two-tailed $p$-values $<0.05$ were considered statistically significant.

Results and Discussions: $A$ total of 317 acute type $A$ aortic dissections were analyzed. Mean age was $60 \pm 12$ years. Overall mortality was $22.5 \%$. We observed no statistically significant differences between the three groups regarding postoperative morbidity and mortality. Especially, postoperative renal failure was not significantly increased in patients receiving aprotinin (see Table).

Conclusion(s): In our experience, the use of aprotinin was not associated with increased postoperative morbidity and mortality in the management of patients with acute type A aortic dissection.

\begin{tabular}{lcccl}
\hline & $\mathrm{A}$ & $\mathrm{TA}$ & $\mathrm{NA}$ & P value \\
\hline Mortality & $19 \%$ & $29 \%$ & $23 \%$ & 0.26 \\
Renal failure & $9 \%$ & $4 \%$ & $6 \%$ & 0.76 \\
Cardiovascular events & $12 \%$ & $18 \%$ & $13 \%$ & 0.48 \\
Hemiplegia & $9 \%$ & $10 \%$ & $7 \%$ & 0.82 \\
ICU stay (days) & $13 \pm 24$ & $21 \pm 65$ & $12 \pm 14$ & 0.29 \\
\hline
\end{tabular}

Reference:

1 Mangano DT, Tudor IC, Dietzel C, et al. The risk associated with aprotinin in cardiac surgery. N Engl J Med 2006; 354: 353-65.

\section{AP2-9}

Effectiveness and safety of tranexamic acid administration during total knee arthroplasty

M. Basora, M. Lozano, L. Peidro, I. Merino, F. Macule

Anestesiologia, Hospital Clinic Barcelona, Barcelona, Spain

Background and Goal of Study: The administration of tranexamic acid (TA), an antifibrinolytic drug, has been associated with a reduction in perioperative blood loss and a decrease in the number of red blood cells (RBC) units transfused in several surgical settings (1). However the fear of thrombotic complications has hindered its generalization. We have evaluated the effect of introducing TA treatment in total knee arthroplasty (TKA) in red blood transfusion and investigated its effect in deep venous thrombosis (DVT).

Materials and Methods: We studied 416 patients who underwent TKA in our institution, 217 before TA administration and 199 after its introduction. Anaesthetic and surgical techniques were the same in both groups. Transfusion received and haemoglobin concentrations at day 5 were also recorded. In 37 patients a lower extremities phlebography between the 6th and 10th postoperative day was performed.

Results and Discussions: $53 \%$ of patients who did not receive TA were transfused with RBC while in those receiving TA only $18 \%$ were transfused $(p<0.0005)$. Furthermore the number of units given were significantly lower in TA treated patients (2.8 vs. $1.9 ; p<0.0005)$. In $43 \%$ of patients receiving TA and $40 \%$ of the control an asymptomatic distal DVT was detected.

Conclusions: The administration of TA during TKA procedures reduced significantly the need of RBC transfusion and the number of units used. TA treatment was not associated to an increase in DVT incidence.

Reference:

1 Cid J. Transfusion 2005; 45: 1302-1307.

\section{AP2-10}

\section{Aprotinin in liver transplantation is not associated with} adverse outcome

O. Collange, G. Freys, C. Favel, P. Wolf, T. Pottecher

Anesthesiology and Critical Care, Strasbourg University Hospital, Strasbourg, France

Background and Goal: Hyperfibrinolysis contributes to bleeding and increases red blood cells (RBCs) requirements during orthotopic liver transplantation (OLT). Aprotinin is a well-known antifibrinolytic drug decreasing RBCs transfusion during major surgery such as OLT or cardiac surgery. However, a recent study has shown aprotinin negative impact in cardiac surgery [1]. The goal of our study was to evaluate aprotinin on OLT postoperative outcome.

Materials and Methods: We retrospectively screened all OLT performed in our institution from 2002 to 2006 . We compared OLT in which aprotinin was used (Apro group) to those in which aprotinin was not used (NoApro group). We recorded basic peroperative data (operation duration, RBCs units requirements), hepatic function at day3 (SGOT and factor V) and postoperative outcome (mortality, graft rejection, ICU and hospital length of stay).

Results and Discussions: 349 OLT were performed during the 5 years period. 283 (81\%) presented exploitable data. Apro group included 155 OLT and NoApro group 128. The Apro group was characterized by higher RBCs units requirements $(4+/-2$ vs $2+/-2.5, \mathrm{p}=0.003)$ and longer operation duration (339+/- 86 vs $305+/-64 \mathrm{~min}, \mathrm{p}=0.002)$. At day3, hepatic tests were not different across groups (SGOT $=243+/-326$ vs $180+/-157$, $\mathrm{p}=0.09$ and factor $\mathrm{V}=90+/-33$ vs $93+/-31, \mathrm{p}=0.49)$. ICU and hospital length of stay were not different (ICU $=6.3+/-8.2$ vs $5.5+/-6.8$ days, $\mathrm{p}=0.44$, hospital $=22.8+/-13.2$ vs $24.2+/-15.2$ days, $\mathrm{p}=0.48)$. Graft rejections (21/155 vs $13 / 128, p>0.4)$ and mortality $(5 / 155$ vs $4 / 128, p<0.75)$ were similar within the two groups.

Conclusions: Although aprotinin was associated with OLT's requiring more blood transfusion, it was not associated with early hepatic dysfunction or adverse outcome. Aprotinin seems to be safe during OLT.

Reference:

1 Mangano, D.T., The risk associated with aprotinin in cardiac surgery. N Engl J Med, 2006; 354(4): p. 353-65.

\section{AP3-1}

\section{Blood transfusion in radical retropubic prostatectomy}

F. Peramo, Jesus. Maldonado-Contreras, Antonio. Maldonado-Campos, Elisa. Belinchon, Raimundo. Escudero

Anestesiologia, Reanimacion y Tratamiento del Dolor, Anestesiologia, Granada, Spain

Background and Goal of Study: Blood transfusion is associated with the risk of immunosupression and infections[1]. We conducted a study to know the incidence of hypotension and blood transfusion (BT) in patients who underwent radical retropubic prostatectomy.We aimed to identify those patients at greater risk and determine wich factors can predict outcome.

Materials and Methods: For a period of 1 year (Nov. 2005 to Nov. 2006) all patients at Hospital San Cecilio scheduled for radical retropubic prostatectomy entered the study. Their management was not altered by being in the study and we prospectively recorded several variables of preoperative, intraoperative and postoperative period that would explain outcome. Specially, hypotension appearing, hemoglobin level $(\mathrm{Hb})$, blood loss and others variables related to BT were evaluated. Every variable was binary and we tried to determine both variables associated with hypotension or BT (chi square) and those that could predict BT (multiple logistic regression model, stepwise method of variable selection). We used SPSS for windows 12.0 ( $p<0.05$ significant).

Results: 47 patients entered the study. Patient's characteristics were (mean $\pm \mathrm{sd}$ ): age $72.38 \pm 2.3$ years, weight $76 \pm 4.8 \mathrm{~kg}$, height $1.73 \pm 6.24 \mathrm{~m}$, $\mathrm{Hb}$ level before surgery $13.5 \pm 2.3 \mathrm{gr} / \mathrm{dL}$, length of operation $129.3 \pm 35.2 \mathrm{~min}$ and prostate size $73.35 \pm 12.54 \mathrm{gr}$. Hypotension (SBP $<90 \mathrm{mmHg}$ ) appeared in 25 patients $(53,19 \%), 18$ needed 2 or more blood units $(\mathrm{Hb}<8.0 \mathrm{gr} / \mathrm{dL}$, $72 \%$ of hypotensive patients) and we required $2.1 \pm 0.54$ blood units ( 1 patient needed 5 blood units). Variables associated with hypotension were: blood loss $>1000 \mathrm{~mL}$, surgery time $>2$ hours and previous HTA $(p<0.05)$. Variables associates with BT were: hypotension, blood loss $>1500 \mathrm{~mL}$ and surgery time $>2.5$ hours. Variables that could predict BT were length of operation $>$ 3 hours, surgeon experience (less than 15 prostatectomies) and blood loss $>1500 \mathrm{~mL}(\mathrm{p}<0.05)$.

Conclusions: In our study surgical team experience, length of operation and blood loss greater than $1500 \mathrm{~mL}$ can predict the need of 2 or more blood units in patients undergoing radical retropubic prostatectomy. Reference:

1 Dash A, et al. Urology, 2004; 64: 117-22.

\section{AP3-2}

Prospective, randomized comparison between spinal and general anesthesia on haematological factors affecting outcome during Radical Retropubic Prostatectomy (RRP)

E. Dedola, G. Bruno, A. Gandolfi, A. Albertin, D. Poli

Department of Anesthesia, San Raffaele Scientific Institute, Milano, Italy

Background and Goal of Study: Radical prostatectomy can be performed using two very different anesthetic techniques. The objective of the current study was to identify factors associated with haematological changes as a function of anesthetic approach.

Materials and Methods: 112 patients undergoing RRP for prostate cancer were randomly assigned to receive either spinal anesthesia (SA, $n=56$ ) or general anesthesia $(G A, n=56)$. Patients of both groups were further 
divided into two groups as a function of the analgesic drug used for postoperative pain (ketorolac group, $K$ group, $n=23$ and paracetamol group, $P$ group, $n=23$ ). The intraoperative and postoperative anesthetic and surgical variables were evaluated as well as postoperative risk factors, including $d$ dimer increase and haemostatic dysfunction. Statistical analysis was performed using the program Statistica 5.0 (StatSoft Italia, Vigonza, Padova, Italy). Analysis of variance for repeated measures was used to analyze changes over time, while the Student's "t"-test for unpaired data was used for inter-group comparison. Ordinal data were analyzed using the contingency table analysis with the Pearson Chi Square.

Results and Discussions: No differences in age and height were observed between the four groups. Weight and body mass index (BMI) resulted significantly higher in GA-P group compared to SA-P group. All groups were similar with respect to haematological and haemostatic arrangement as well as to intra-postoperative blood losses and intra-postoperative blood transfusion requirements.

Conclusions: These results suggest that spinal and general anesthesia offer a similar anesthetic and surgical success on outcome in patients undergoing radical retropubic prostatectomy.

\section{AP3-3}

\section{Determining the true cost of RBC transfusion from a health care providers perspective - a preliminary report \\ O.M. Theusinger, A. Hofmann, H. Gombotz, D.R. Spahn \\ Anesthesiology, CHUV, Lausanne, Switzerland}

Background and Goal of Study: RBC-transfusions necessitate an integrated system of diagnostic, therapeutic, technical, lab, logistic, administrative, information, education, quality and legal processes. This study is an activity-based cost analysis, flow-charting these processes, capturing their frequencies and total resource consumptions, uncovering hidden cost factors and aiming to calculate the true cost of transfusion therapies in hospitals.

Materials and Methods: In 2003, a panel of experts convened a multidisciplinary cost-of-blood consensus conference and agreed upon activity-based costing as the appropriate method to account for the total cost of transfusion. Accordingly, software modules were developed to capture labour time, materials, $3^{\text {rd }}$ party services, use of equipment and the frequency of each process step in RBC transfusion. With these data and locally applicable cost rates total process cost per RBC unit transfused were calculated.

Results and Discussions: Preliminary results show 14 main processes beginning with pre-transfusion medical and clerical routines and ending with managing cases of transfusion transmitted disease. Blood bank clerks, couriers, medical technicians, nurses, nursing assistants, anesthesiologists, surgeons, hematologists, risk managers, administrators and other personnel are involved. More than 250 serial and parallel resource consuming process steps in this intra hospital transfusion chain have been identified. Numerous process steps occur with a multiple frequency compared to the frequency of actual transfusions, because many more transfusions are prepared than given.

Conclusion(s): Former cost analyses are incomplete because significant amounts of resources being used have not been calculated. Therefore existing cost-effectiveness analyses comparing transfusion to other treatments may be deficient and should be re-evaluated. Current cost of transfusion may be grossly underestimated.

Reference:

Shander A. et al., COBCON I, Transfus Med Rev (2005) 19: pp 66-78.

\section{AP3-4}

Blood loss and transfusion requirements during hepatic resection surgery under low CVP conditions - is routine cross-matching essential?

N. Quiney, L. Langford, A. Patel, W.C. Fawcett

Anaesthetics and Critical Care Medicine, Royal Surrey County Hospital, Guildford, United Kingdom

Background and Goal of Study: Patients undergoing hepatic resection surgery routinely have blood cross-matched pre-operatively. However, with advances in both anaesthesia and surgery, blood loss and consequent transfusion requirements have reduced substantially. With rapid on site crossmatching available, we question whether routine cross matching of blood is still indicated.

Materials and Methods: We performed a retrospective audit on 71 consecutive patients undergoing elective liver resection in our unit during 2005 . All patients received a standardized low CVP anaesthetic technique (1). Blood loss, number of units of blood cross-matched, and the number of units administered was recorded.
Results and Discussion: Data was collected from 71 patients (30 males/ 41 females). Mean (range) age was 62.4 (25-81) years. Mean (range) measured blood loss was $415(50-800) \mathrm{mls}$. Mean preoperative haemoglobin was $13.1 \mathrm{~g} / \mathrm{dL}$, and the mean postoperative haemoglobin was $10.5 \mathrm{~g} / \mathrm{dL}$.

\begin{tabular}{lcc}
\hline $\begin{array}{l}\text { Segments } \\
\text { Resected }\end{array}$ & $\begin{array}{c}\text { No. of } \\
\text { Patients }\end{array}$ & $\begin{array}{c}\text { Mean Blood } \\
\text { Loss (mls) }\end{array}$ \\
\hline 1 & 3 & 83 \\
2 & 13 & 264 \\
3 & 9 & 384 \\
4 & 35 & 498 \\
5 & 16 & 522 \\
6 & 5 & 420 \\
\hline
\end{tabular}

Only 4 patients (5.6\%) required blood transfusion, receiving between them 9 units of the 209 units cross-matched prior to surgery. No patient who had 3 or fewer segments resected required blood. Less than $5 \%$ of cross-matched blood was transfused. Given that rapid transfusion can be arranged at short notice if a group and save sample is available, we question whether routine cross matching is warranted. The cost of transfusion per unit is currently $£ 131.80$, and the cost of cross matching $£ 11.43$ therefore significant cost savings can be achieved.

Conclusion: Routine cross matching for our liver resection patients is not necessary, particularly for those undergoing smaller ( $<3$ segments) resections. Group and save of blood pre-operatively appears to be adequate.

Reference:

1 Fawcett WJ, Quiney NF, Karanjia ND. Liver resection and hypovolaemia: a technique vindicated. Anaesthesia 2006; 61: 82-3.

\section{AP3-5}

\section{Transfusion practice in lower limb joint arthroplasty}

S. Quasim, M. Kyi

Anaesthetics, Good Hope Hospital, Sutton Coldfield, United Kingdom

Background and Goal of Study: The use of blood conservation techniques reduces complications from infection and transfusion reaction that may result from using allogenic blood components. This is a study looking at transfusion practice for elective orthopaedic lower limb joint arthroplasty operations. Our aim was to assess whether changes could be made in order to reduce the costs of crossmatch and transfusion of homologous blood components.

Materials and Methods: This is a retrospective analysis of data on 124 patients undergoing elective lower limb joint arthroplasty at one centre. Results are presented relating to age, sex, operation, pre-operative and post-operative haemoglobin concentrations. We have also looked at whether the patients have been transfused.

Results and Discussions: The overall male to female ratio was 1:2.1. The mean age of those transfused was $70.7+/-9.5$ years and the mean age of those not transfused was $70.2+/-8.3$ years (not significant, $p=0.76$ ). Of those transfused, the median number of units given was 2 . Transfusion led to a significantly increased length of stay $(9.16+/-4.8$ days in those not transfused; $12.91+/-5.2$ days in those transfused; unpaired t-test, $p=0.0001$ ). The table shows transfusion rates for different procedures.

\begin{tabular}{lcc}
\hline Procedure & Number of patients & \\
\cline { 2 - 3 } & Transfused & Not transfused \\
\hline Total hip replacement (THR) & 39 & 7 \\
Conversion to THR & 5 & 0 \\
THR Revision & 10 & 2 \\
THR Resurfacing & 1 & 1 \\
Total knee replacement (TKR) & 2 & 53 \\
TKR Revision & 5 & 0 \\
\hline
\end{tabular}

Unwashed autologous blood collected in wound drains after TKR has reduced the need for allogenic transufusion. $85 \%$ of total hip replacements received blood transfusion.

Conclusion(s): Allogenic transfusion of blood components may cause complications and increase length of stay. Further consideration towards cell salvage techniques should be given, particularly in total hip replacement.

\section{AP3-6}

Impact of leukocyte-depleted red blood cells transfusion on postoperative infections in chronic alcoholics with major abdominal surgery

I. Cindea, A. Balcan, G. Nicolae, V. Gherghina

Anesthesiology, Intensive Care, Emergency Clinical Hospital of Constanta, Constanta, Romania 
Background and Goal of Study: Major abdominal surgery involves important intra- and postoperative blood losses (1). On the other hand, chronic alcoholic candidates for such procedures recognize an increased risk for bleeding complications. The simultaneity of these two independent factors requires adequate hemoglobin correction by transfusions that are well known for their risk of postoperative infections due to immunosuppressive effect of leukocytes (2).

The goal of this retrospective observational study is to evaluate the impact of leukocyte-depleted red blood cells transfusions on incidence of postoperative infections in chronic alcoholics with elective major abdominal procedures. Materials and Methods: We have analyzed 134 patients with documented chronic alcohol misuse that underwent major abdominal procedures during a period of one year.

These subjects were allocated into two groups: group $A(n=65)$ with leukocyte-depleted red blood cells transfusion therapy, respectivelly group $B(n=69)$ that has received non-leukocyte-depleted red blood cells products.

We have retrospectivelly investigated the incidence of postoperative infections and length of hospital stay in both groups in order to comparatively analyse these finding.

'Student $\mathrm{t}$ ' test was used for statistical analysis.

Results and Discussions: Demographic and surgical variables were not different between groups. The level of perioperative coagulation parameters and transfusion requirements were similar, too.

No significant difference was recorded neither in postoperative infections incidence (12 meaning $18.5 \%$ vs 13 meaning $18.8 \%, p=0.9$ ), nor in hospital stay length $(17.3 \pm 0.9$ versus $19.1 \pm 0.6$ days, $p=0.9)$ between groups.

Conclusion(s): These data did not conclusively demonstrate any possible advantage of leukocyte-depleted red blood cells products concerning the rate of postoperative infectious complications, as well as the hospitalization duration for alcoholics undergoing elective abdominal major surgery.

References:

1 Mynster T. Br J Surg 2000; 87: 1553-62.

2 Jensen LS.Br J Surg 1996; 83: 973-7.

\section{AP3-7}

\section{Preoperative transfusion strategies in a sub-group of} population undergoing cardiac surgery

F. Mangia, S. Casalino, E. Stelian, E. Novelli, G. Lanzillo

\section{Cardiac Anaesthesia and Intensive Care, Clinica San Gaudenzio, Gruppo} Policlinico di Monza, Novara, Italy

Background and Goal of Study: In the last years the tendency is to avoid blood transfusions due to several factors: the decrease of blood donation, the immuno-modulating effects that may increase the risk of nosocomial infection and cancer recurrence (1). The blood prestorage and the use of recombinant erythropoietin (EPO) are accepted therapies, but with high costs and not without risks. The aim of the study was to identify a sub-group of population who underwent elective cardiac surgery that could have the best benefit from the above mentioned technique and avoiding thus blood transfusion.

Materials and Methods: We analyzed retrospectively 350 patients who underwent elective cardiac surgery with cardiopulmonary bypass (CPB) in 2006. Red blood cells (RBC) concentrates were transfused to maintain a haematocrit $(\mathrm{Ht})$ above $24 \%$ during CPB and above $27 \%$ during the first $48 \mathrm{~h}$ after $\mathrm{CPB}$. For each patient we recorded the number of RBC units required. Then we tested a logistic regression model which identified as risk factors for transfusion age, preoperative $\mathrm{Ht}$ and body surface area (BSA).

Results and Discussions: Among the patients with a preoperative $\mathrm{Ht}<36 \%$ received transfusion $89 \%$ of them, those with a $\mathrm{Ht}$ between 36 and $42 \%$ received transfusion $31.5 \%$ and those with a $\mathrm{Ht}>42 \%$ received transfusion $6 \%$. The average number of transfused RBC units among the patients with preoperative $\mathrm{Ht}$ between $36 \%$ and $42 \%$ was $2,67 \pm 0.85$. In order to identify a sub-group of low risk for receiving transfusion we used median reference values of age and BSA as cut-off values. Among the patients $(n=49)$ with an age $<70$ years and a $B S A>1.8 \mathrm{~m}^{2}$ received transfusion $7(14,28 \%)$, with an average of 1.85 units of RBC per patient.

Conclusion: Due to the fact that the above described sub-group (age $<70$, $\mathrm{BSA}>1.8 \mathrm{~m}^{2}$ ) had a low risk of receiving transfusion and the usual requirements are under two units of RBC for a patient, we believe that can be fair to put in balance the risks of prestorage, the costs and the risks of EPO with all the risks of transfusion. In this sub-group transfusion probably could be avoided with the above mentioned technique.
Reference:

1 Raghavan M, Marik PE. Anemia, Allogenic Blood Transfusion, and Immunomodulation in the Critically III. Chest, Jan 2005; 127: 295-307.

\section{AP3-9}

\section{Influence of bowel preparation and fluid management on blood loss and transfusion requirement in patients undergoing radical cystectomy}

D. Wiessner, S. Dingler, O.W. Hakenberg, T. Koch, R.J. Litz

Urology, University of Technology, Dresden, Germany

Background and Goal of Study: Preoperative dehydration in patients undergoing major surgery is primarily attributed to preoperative fasting and bowel preparation. Therefore large amounts of fluids are administered intraoperatively to maintain circulation and kidney function, often exceeding measured fluid and blood loss. After changing our practice of fasting and bowel preparation in two steps, we examined our cohort of radical cystectomy (RCE) patients regarding intraoperative fluid requirement, blood loss and transfusion requirements.

Materials and Methods: Up to 12/2004 patients received bowel preparation with $4 \mathrm{I}$ of polyethylene glycol (PEG) and were NPO from the start of bowel preparation (group $1 ; n=71$ ). As of $1 / 2005$ patients received clear oral fluids until two hours prior to surgery (group $2 ; n=54$ ). Since $1 / 2006$ patients additionally received only $2-3$ I PEG orally (group $3 ; n=49$ ). Intraoperative fluids were administrated to maintain a central venous pressure between 7 and $10 \mathrm{mmHg}$. Estimated blood loss was replaced by colloids. Transfusion of packed red cells $(\mathrm{PRC})$ was triggered by a haemoglobin $(\mathrm{Hb})$ value of $8 \mathrm{~g} / \mathrm{dl}$. Data were collected prospectively by a computer database.

Results and Discussions: There were no difference between groups regarding age, gender, weight, height, preoperative $\mathrm{Hb}$ value, duration of surgery and the type of urinary diversion. The amount of intraoperative fluids decreased over time $(3500 \mathrm{ml}$ [3000-4000] vs. $3500 \mathrm{ml}$ [3000-4000] vs. $2500 \mathrm{ml}$ [2000-3000]; $p<0.001)$. Estimated blood loss was likewise highest in group 1 as compared to both other groups (1400 ml [800-2000] vs. $1200 \mathrm{ml}$ [600-1675] vs. $800 \mathrm{ml}$ [600-1400]; $\mathrm{p}<0.01)$. The incidence of transfusions in group 1 and 2 was higher than in group $3(39 \%$ vs. $39 \%$ vs. $16 \% ; p<0.05)$. There were no differences in the overall complication rate, however, the incidence of minor pulmonary and cardiac complications tended to be lower in group 3 without reaching significance.

Conclusion(s): Performing moderate bowel preparation in addition to liberal preoperative oral intake of clear fluids until $2 \mathrm{~h}$ before surgery led to a reduction of intraoperative fluid administration, thus resulting in reduced blood loss and decreased transfusion requirement in patients undergoing RCE.

\section{AP3-10}

\section{Red blood cell transfusion is associated with organ injury after cardiac surgery}

F. Hoekstra, H.E. Mungroop, B.G. Loef, L.P. Aarts, A.H. Epema

Department of Anesthesiology and Biomaterials, University Medical Center Groningen, Groningen, The Netherlands

Background and Goal of Study: Several retrospective studies in cardiac surgical patients reported an association between red blood cell (RBC) transfusion and adverse outcome (ref). We studied the effects of RBC transfusion on markers of myocardial, renal and intestinal injury after cardiac surgery with CPB.

Materials and Methods: After IRB approval and informed consent 41 patient scheduled for cardiac surgery with CPB were prospectively studied. Blood and urine samples were obtained at the start of surgery (T1), at the end of surgery (T2) and on the first (T3), third (T4) and fifth (T5) postoperative day. Cardiac troponin I was measured in the blood. IFAB (intestinal type fatty acid binding proteins) and NAG ( $\mathrm{N}$-acetyl- glucosaminidase) were determined in the urine. Data were expressed as mean \pm SEM and analysed using t-test, MannWhitney test and ANOVA where appropriate. Statistical significance was accepted at $\mathrm{p}<0,05$.

Results and Discussions During the 6 day study period 16 patients received RBC transfusions $(1225 \pm 237 \mathrm{ml})$. At the end of surgery (T2) cardiac troponin T, NAG as a marker of kidney damage and IFAB as a marker of intestinal injury were significantly higher in the transfusion group (Fig). This is the first study evaluating effects of blood transfusion on these markers of organ injury in cardiac surgery patients. 
IFABP

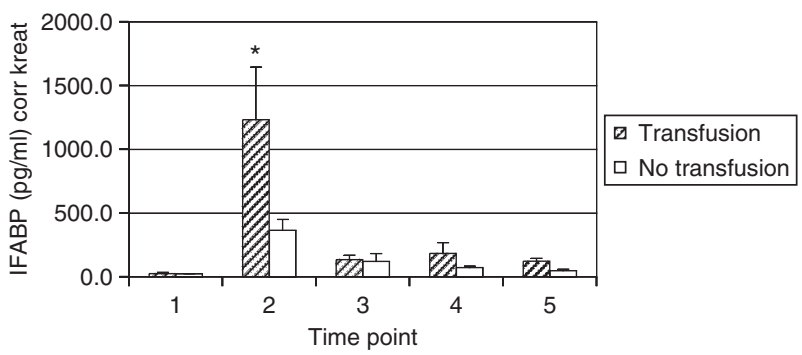

Conclusion: Perioperative RBC transfusion is associated with cardiac, intestinal and renal injury after cardiac surgery.

References:

Koch et al. Crit Care Med 2006; 34: 1608-16.

\section{Neurosciences}

\section{AP1-1}

\section{Critical events during interventional neuroradiology} procedures performed under general anaesthesia

H. Krovvidi, C. Moody, N. Huggins

Anaesthetics, Queen Elizabeth Hospital, Birmingham, United Kingdom

Background and Goal of Study: Most interventional neuroradiology procedures can be undertaken with intravenous sedation but general anaesthesia is used frequently for more complex and prolonged procedures. We studied the critical events occurring during interventional neuroradiology under genera anaesthesia over a period of 1 year.

Materials and Method: We collected the data prospectively over a period of 12 months. Emergency and elective procedures in adults were audited using a standardized form.

Results and Discussion: A total of 184 interventional neuroradiology procedures were performed in 12 months period. Among them 70.6\% $(n=138)$ cases were intracranial aneurysm coiling. $47(25.4 \%)$ patients had one or more complications during these interventional procedures. A total of 80 critical events occurred during this period of 12 months. Cardiac events were most commonly occurring events and hypertension and tachycardia were most frequently occurring complications among all the critical events. Our audit had few procedure related complications such as intraprocedural vasospasm $1.08 \%(n=2)$ and intracerebral bleed $0.54 \%(n=1)$.

The severity of complications was classified according to Salisbury grading of critical events ${ }^{1}$ and among the patients having critical events, $95.74 \%$ $(n=45)$ belonged to mild grade (grade1 \& 2) of severity. Emergence from anaesthesia was the most critical period during which maximum number of events occurred ( $n=52$ ).

Conclusion(s): Major incidents in our series were only $0.54 \%(n=1)$, majority of events that occurred were minor, of transient nature with minimal prolonged effects and included cardiac events (hypertension and tachycardia). Better preoperative blood pressure control, more adequate analgesia and graded and careful extubation with an intravenous beta-blocker cover were recommended to improve the complications in our institute after presenting this data.

Reference:

1 Lack JA. Preoperative anaesthetic audit. In: Automated Anaesthetic Records. Kenny GNC. Ed. Bailliere Tindall, London, 1990; 171-184.

\section{AP1-2}

\section{The inflammatory response in acute ischemic stroke}

M. Lonardo, S. Cotena, G. Scarpati, O. Piazza, E. De Robertis

Anesthesia and Intensive Care, Università Degli Studi di Napoli Federico II, Napoli, Italy

Background and Goal of the Study: IL-18, a pro-inflammatory cytokine of IL-1 family, has got a crucial role in neuroinflammation and neurodegeneration (1). Serum protein $\mathrm{S} 100 \mathrm{~B}$ has been suggested as a marker of brain injury and it is involved in cytokine cascade (2).

The aim of our study was to evaluate IL-18 and S100B serum levels in acute ischemic stroke patients.

Materials and Methods: We enrolled 10 acute ischemic stroke patients. Patients with signs of inflammation were excluded.

S100B and IL-18 serum levels were measured within 24 hours of the ischemic insult; IL-18 measurement was repeated at day 5. S100B and IL-18 serum levels were quantified by ELISA.

The volume of early CT signs of brain infarction was evaluated at admission.
Results: IL-18 serum levels in the control group (10 healthy voluntaries) were $168,9 \pm 40 \mathrm{pg} / \mathrm{mL}$

Data (mean $\pm \mathrm{SD}$ ) are shown in the table:

\begin{tabular}{lllll}
\hline $\begin{array}{l}\mathrm{S} 100 \mathrm{~B}(\mu \mathrm{g} / \mathrm{mL}) \\
\text { day } 1\end{array}$ & $\begin{array}{l}\mathrm{IL}-18(\mathrm{pg} / \mathrm{mL}) \\
\text { day } 1\end{array}$ & $\begin{array}{l}\mathrm{IL}-18(\mathrm{pg} / \mathrm{mL}) \\
\text { day } 5\end{array}$ & GCS day 1 & $\begin{array}{l}\text { Ischemic volume } \\
\left(\mathrm{cm}^{3}\right)\end{array}$ \\
\hline $0,99 \pm 1,18$ & $309,4 \pm 58,4$ & $246,7 \pm 73,6$ & $6,37 \pm 2,32$ & $42 \pm 45$ \\
\hline
\end{tabular}

Conclusions: S100B serum levels were significantly higher in stroke patients (normal values $<0,2 \mu \mathrm{g} / \mathrm{mL}$ ). Compared to controls ischemic stroke patients had statistically significant higher serum levels of IL-18 at day $1(p<0.001)$ and at day $5(\mathrm{p}<0.016)$.

IL-18 is an important but aspecific marker in monitoring severe inflammatory conditions. High serum levels of IL-18 are not associated with adverse neurological outcome and with the volume of CT hypodense areas.

High serum levels of IL-18 and S100B in ischemic stroke patients suggest a massive activation of the inflammatory cascade in acute ischemic stroke. References:

1 Felderhoff-Mueser U. Trends in Neurosciences 2005; 28: 487-493.

2 Cotena S. J Neurosurg 2006 Jun; 104: 435-6.

\section{AP1-3}

\section{Capnometry changes due to posturing patient in sitting position}

A. Hoxha, K. Pilika, M. Demneri, M. Saraci, M. Kerci

Department of Anesthesiology \& Critical Care, University Hospital Center "Mother Theresa" Tirana, Albania, Titana, Albania

Background and Goal of Study: Sitting position is frequently used for neurosurgery. Posturing patient in this position frequently causes change of capnometry. The aim of this study is to analyze capnometric changes during this kind of positioning.

Materials and Methods: There were enrolled 257 patients, without significant heart and respiratory problems, operated in sitting position ( $F: M=108: 149$, age $48.24 \pm 16.14)$. After standard induction, mechanical ventilation was started (VT $=6 \mathrm{ml} / \mathrm{kg}, \mathrm{RR}=12 / \mathrm{min}$ ) and after $10 \mathrm{ml} / \mathrm{kg}$ of saline given in 15 $\mathrm{min}$, the patients were postured in sitting position. Standard ECG, IBP, EtCO $\mathrm{SpO}_{2}$, VT, and RR were monitored, as well as CVP in 138 patients. Data are presented as mean $\pm \mathrm{SD}$, T-test and correlation $(r)$ are used for statistical evaluation and $p<0.05$ is considered as statistically significant.

Results: Decrease of $\mathrm{EtCO}_{2}$ was observed instantly after positioning the patients in sitting position. Drop of $\mathrm{EtCO}_{2}$ was $4.36 \pm 2.56 \mathrm{mmHg}(p<0.01)$ and it paralleled the decrease of MAP $(12.25 \pm 8.13 \mathrm{mmHg}(p<0.05)$, increase of HR $11.69 \pm 8.48 \mathrm{bpm}(\mathrm{p}<0.01)$ and CVP drop of $5.12 \pm 1.04 \mathrm{mmHg}$ $(p<0.001)$. Such changes were present for $5-8$ minutes and usually were corrected with administration of $5-10 \mathrm{ml} / \mathrm{kg}$ saline. 49 patients $(19.6 \%)$ developed pronounced haemodynamic instability (MAP $<20 \%$ of baseline) and showed significantly larger decrease of $\mathrm{EtCO}_{2}(7.54 \pm 3.42 \mathrm{mmHg}, \mathrm{p}<0.05)$ compared with others. Haemodynamic in this group of patients was corrected with ephedrine in perfusion. Correlation between $\mathrm{EtCO}_{2}$ changes and haemodynamic instability shows strong relationship $(r=0.87)$.

Conclusion: Changes of capnography are significant in sitting position. $\mathrm{EtCO}_{2}$ changes parallels the haemodynamic and are an early warning of its change due to positioning of patient.

Reference:

Schwarz G, Fuchs G, Weihs W et al: Sitting position for neurosurgery:Experience with preoperative contrast echocardiography in 301 patients. J Neurosurg Anesthesiol 6: 83, 1994. 


\section{AP1-4}

Inflammatory cytokines and clinical manifestations during the first week after subarachnoid bleeding

A. Hoxha, A. Bulo-Kasneci, K. Pilika, M. Demneri, M. Saraci

Department of Anesthesia \& Critical Care, University Hospital Centre "Mother Theresa", Tirana, Albania

Background and Goal of the Study: Release of inflammatory factors after subarachnoid bleeding (SAH) is actually considered as a major factor in induction of brain vasospasm, the main factor of brain damage. Such pathologic vascular changes are supposed to be induced by immunological mechanisms mediated by inflammatory factors. The aim of the study is to describe the release of IL- 6, IL- 8, TNF- $\alpha$ and hs-CRP parallel to clinical signs in patients during the first week after SAH outbreak.

Materials and Methods: Blood concentrations of inflammatory cytokines (interleukin IL-6, IL-8 and tumor necrosis factor -TNF- $\alpha$ ) and C-reactive protein (CRP) were measured in 45 patients (aged $51.32 \pm 8.73, \mathrm{~F}: \mathrm{M}=19: 26$ ) suffering from subarachnoid bleeding. Aneurismal bleeding was confirmed in 42 patients. Measurements were performed 1-3 days and 5-7 days after bleeding. Clinical criteria considered were Hunt\&Hess scale, Fisher scale and GOS 14 days (Glasgow outcome scale).

Results and Discussion: Blood concentration of inflammatory factors and CRP in our patients were higher than in norm. No significant difference was observed $(p>0.05)$ between patients with aneurismal bleeding and others. Relation of Hunt\&Hess grade of the patients to blood level of the inflammatory factors showed a significant relation (during days 1-3 after bleeding for TNF- $\alpha p=0.03$, IL- $6 p=0.01$, CRP $p=0.003$ and during days $5-7$ after bleeding CRP $p=0.001$ ). But we did not observe such a close relation toward Fisher scale: only CRP levels during days $5-7$ showed $p<0.05$. GOS as well as Fisher scale showed significant relation only to IL-8 during days $5-7 \mathrm{p}=0.012$. IL-6 as well as hs-CRP showed a strong correlation to GOS in days $5-7(p<0.001)$.

Conclusion: In our patients we have observed the raise of blood concentration for all inflammatory factors. We did not observe any significant difference in their values between patients with aneurismal bleeding and others. Most close relation was observed between Hunt\&Hess scale and raise of inflammatory factors especially 1-3 days after the bleeding. Relation of inflammatory factors blood level to GOS was less expressed. and inverse correlation was evident at the end of the week after SAH attack.

Reference:

Kassbender K, Hodapp B, et al. Inflammatory cytokines in subarachnoid haemorrhage: association with abnormal blood flow velocities in basal cerebral arteries. J. of Neurol. Neurosurg.Psychiatry 2001; 70: 534-537.

\section{AP1-5}

\section{Role of BIS monitoring in propofol dose adjustment for intracranial tumor surgery}

\section{L.D. Mishra, N. Rajkumar, A. Tiwari, R. Gairola}

Anaesthesiology, Institute of Medical Sciences, Varanasi, India

Background and Goal of Study: Use of propofol anaesthesia in neurosurgery is increasing world over. Though its dose can be rapidly adjusted, appropriate titration is required to allow for inter patient variability. Traditionally, we have been utilizing the clinical responses to maintain an adequate depth of anaesthesia, yet many technology intensive techniques, including Bispectral Index (BIS) monitoring, have become available in recent years. However, fewer studies using BIS monitoring in neurosurgical patients are reported in literature. Information about appropriate BIS values required in various operations is also lacking. We aimed to compare the propofol dose requirement between the conventional clinical responses monitoring and BIS monitoring techniques, keeping BIS at a pre-determined value.

Materials and Methods: A prospective randomized study was conducted on 120 ASA grade I and II patients undergoing intracranial tumour operations. All patients were given identical general anaesthesia induced and maintained on propofol. $\mathrm{N}_{2} \mathrm{O}$ in $\mathrm{O}_{2}$ was supplemented. Dose requirement was titrated by monitoring the conventional clinical responses in one and by monitoring BIS values in the other group of patients. In the BIS group we aimed at achieving a BIS value of 40 or less at the time of intubation and at 45-50 (40-60 range) for maintenance of anaesthesia. The total propofol dose, awareness incidence and recovery profile including sedation grade, PONV etc. were recorded.

Results and Discussions: Propofol requirement was similar in both the groups $(81.71 \pm 19.09 \mu \mathrm{g} / \mathrm{kg} / \mathrm{min}$ Vs $87.09 \pm 16.59 \mu \mathrm{g} / \mathrm{kg} / \mathrm{min} ; \mathrm{p}>0.05)$ but the BIS monitoring (range $=45.83 \pm 6.71$ ) helped to ensure adequate depth of anaesthesia associated with absence of awareness and a relatively earlier recovery and lower sedation grades.
Conclusion: The propofol dose requirement is similar in BIS monitored patients (keeping above BIS values) to the requirement in conventionally monitored patients. However, we need to learn the dose requirements at different BIS values and the optimal BIS value required for intracranial surgeries.

\section{AP1-6}

\section{Role of clinical decision support systems in quality of postoperative neuro-intensive care}

L. Schouteten, C. De Deyne, J. Wuyts, D. Peuskens, R. Heylen

Anesthesia and Critical Care Medicine, Ziekenhuis Oost-Limburg, Genk, Belgium

Introduction: The introduction of clinical decision support systems in electronic medical records (EMRs) could prevent diagnostic and medication errors and could results in a better quality of patient care. In this paper, we want to illustrate the introduction of decision support in the EMR considering the postoperative instructions for neurosurgical patients.

Materials and Methods: Since jan 2006, we implemented a clinical decision support system in our EMR for all postoperative neurosurgical pts. Supported by the type of neurosurgical intervention and by patient history, all postoperative instructions were prefetched and made electronically available for the physician. At end of surgery, both anesthesiologist and neurosurgeon confirmed and/or (if necessary) changed the postoperative instructions, concerning all aspects of IC management : technical investigations, monitoring, medication etc.... For the purpose of this paper, we compared at random all postoperative instructions for 20 pts after the introduction of the clinical decision support system to 20 control pts. For these control pts, all postoperative instructions were handwritten by the physician without any decision support.

Results: In 18 of 20 handwritten instructions, essential medical information and/or instructions were considered as missing, while in only 2 of 20 instructions (build on decision support) essential information was missing (one concerning specific instructions for intracranial pressure monitoring, another concerning the unusual prescription of anti-epileptic drugs). Missing handwritten information concerned in 12 cases the prescription of medication (antibiotics, anti-epileptic drugs, corticosteroids... or missing dosages of medication), while in 6 cases specific instructions concerning management of blood pressure and intracranial pressure were missing. In 3 of 20 handwritten instructions, errors were observed in dosages of medication

Conclusion: The introduction of clinical decision support for the postoperative IC instructions for neurosurgical patients resulted in a significant decrease of missing essential information.

\section{AP1-7}

Problems of intraoperative blood loss and application of modern blood-saving techniques in neuroanesthesiology

V. Gromova, A. Imaev, A. Lubnin, A. Belov, V. Cherekaev

Anesthesiology, Burdenko Neurosurgery Institute, Moscow, Russian Federation

Background and Goal of Study: The human brain receives about $20 \%$ of stroke volume. However the volume of the operative hemorrhage in neurosurgical patients may exceed 20 liters of blood. Our general transfusion strategy in neurosurgical interventions consists in decreasing the amount of allogenic blood products using the maximal number of blood-saving techniques.

Materials and Methods: We studied patients with intracranial neoplasms operated in our clinic The average intraoperative blood loss was 4,9l (1,119,5I). Autologic blood components have been received at use of the following techniques: a preoperative autodonor plasmapheresis (PAP); isovolemic hemodilution with separation (IVGDS) and intraoperative cell savage (ICS).

\begin{tabular}{lr}
\hline Total number of studies & 672 \\
Preoperative plasmaferesis & 159 \\
Isovolaemic haemodilution & 120 \\
Blood salvage & 258 \\
Autologic fibrin glue & 135 \\
\hline
\end{tabular}

Results and Discussions: The average amount of auto-blood components used in operation are shown below:

\begin{tabular}{ll}
\hline Autoblood components & $\mathrm{ml}$ \\
AutoRBC (IVHDS) & $750-900$ \\
AutoRBC (ICS) & $800-3000$ \\
Autoplasma (PAP) & $600-1700$ \\
Autoplasma (IVHDS) & $750-900$ \\
Autoplatelets (IVHDS) & $100-150$ \\
\hline
\end{tabular}


The components of homologous blood were used minimally. Postoperative hemoglobin, hematocrit levels and parameters of hemostasis were within normal range.

Conclusions: In a combination with IVGDS PAP allows to refuse transfusion of donor blood at blood loss up to $3000 \mathrm{ml}$. For patients with massive blood loss (up to $8000 \mathrm{ml}$ and more) the combination of PAP, IVGDS and ICS is optimum.

References:

Jimenez DF, Barone CM. Neurosurgery. 1995 Dec; 37(6): 1075-9.

Di Rocco C, Tamburrini G, Pietrini D. Semin Pediatr Neurol. 2004 Dec; 11(4): 278-87.

\section{AP1-8}

Changes in brainstem autonomic function following induction of anaesthesia

M. Staber, S. Hansen, POO. Julu

Anaesthesia, Inverclyde Royal Hospital, Greenock, United Kingdom

Background and Goal of Study: The interaction between anaesthesia and autonomic function is not fully understood. The aim of the study was to determine the effect of anaesthesia on the autonomic nerve system as measured by the heart rate, cardiac vagal tone, blood pressure and cardiac sensitivity to baroreflex.

Materials and Methods: Seventeen patients (median age 65 years, range 31-81) undergoing major surgery were included in this prospective study. An arterial line was inserted prior to induction of anaesthesia for which Propofol and Morphine or Fentanyl was used. The effect of anaesthesia on the brain and depth of anaesthesia was measured using the Bispectral Index (BIS). The autonomic nervous system activity in the brainstem was assessed with the Neuroscope ${ }^{T M}$ system, which processes the ECG and BP signals to produce continuous real-time indices of parasympathetic activity, the cardiac vagal tone (CVT) and cardiac sensitivity to baroreflex (CSB). CVT is measured in an arbitrary scale, the Linear Vagal Scale (LVS) (Julu, 1992). Continuous recording of all parameters was started in theatre prior to induction of anaesthesia and continued during the whole operation. Statistical significance was assessed by paired t-test.

Results and Discussions: Mean values of recordings just before and after induction of anaesthesia were calculated. The following results are presented as the mean value before induction and the $95 \%$ confidence interval for the mean difference. There were significant decreases in BIS: $94.7(58.0-66.9) \%$, CVT: $2.9(0.1-2.7)$ LVS, CSB: $1.7(0.3-1.8) \mathrm{ms}^{-} \mathrm{mmHg}^{-1}$ and SBP: 154 $(27-56) \mathrm{mmHg}$. There was no change in HR $\left(76.3[-6.2-6.2]\right.$ beats $\cdot \mathrm{min}^{-1}$. These results suggest that both sympathetic and parasympathetic activity in the brainstem is reduced by anaesthesia. The decreased parasympathetic drive, as shown by reduced CVT and CSB, would alone increase the heart rate. However this is counteracted by the concurrent decreased sympathetic activity, which also causes the decrease in BP.

Conclusion: Measuring heart rate and BP alone during anaesthesia does not provide enough information about brainstem function. We therefore advocate that a direct measure of parasympathetic activity (CVT) would be valuable in monitoring the patient's cardiovascular status.

\section{AP1-9}

Traumatic intracranial aneurysms after gunshot injuries. Anaesthetic and endovascular management may be a prompt safe-to-use technique and a valuable option: about two cases reports

M. Hachemi, C. Jourdan, C. Di Roio, F. Dailler, F. Turjman

Departement of Anaesthesia, Groupement Hospitalier Est, Hôpital Neurologique P. Wertheimer, Bron, France

Background and Goal of Study: There are few published large series on civilian craniocerebral gunshot injuries in children. Traumatic intracranial aneurysms (TICAs) are rare and highly unstable lesions. They represent less than $1 \%$ of all aneurysms and can either rupture within minutes after formation or remain quiescent for several weeks or years, manifesting with delayed hemorrhage and neurologic deterioration.

Materials and Methods: We report two cases ot TICAs after gunshot injuries in civilian practice. We discuss the pathogenesis and the management of TICA after civilian craniocerebral gunshot injuries

Results and Discussions: Case 1: A 10-year-old girl who was referred for coma after high-velocity craniocerebral gunshot wound and neurological deterioration 7 days after the initial injury. A massive right posterior occipital hematoma caused by the rupture of an unsuspected right posterior cerebral artery TICA was discovered, and was treated by coil embolization, with a good neurological recovery at 6-month follow-up.
Case 2: A 34-year-old women was referred for a lead shot gun injury after suicide and deteriorated neurologically secondary after the initial injury with an initial massive right sylvian valley haematoma. An unsuspected right sylvian artery TICA was discovered, and was treated by coil embolization at day two with a good neurological recovery at 8-month follow-up.

Conclusion(s): TICAs should be suspected in patients with civilian craniocerebral gunshot injuries, presenting with secondary neurological deterioration, to carry out emergent CT scan and angiographic exploration before contemplating definitive endovascular treatment. Endovascular management may be a prompt safe-to-use technique and a valuable option, especially when surgery is highly risky.

\section{AP2-1}

\section{Complications alter lumbar posterior spinal fusion} instrumentation: influence of patient characteristics, surgical procedure and learning curve

M.J. Colomina, L. Mora, F. Pellisé, E. Guerrero, J. Bagó

Anesthesia, Hospital Universitario Vall dHebron. Área de Traumatología,

Barcelona, Spain

Background and Goal of Study: To evaluate the association between early major complications in lumbar posterior spinal fusion instrumentation (PSFI) and patient baseline characteristics, the surgical procedure and learning curve. Materials and Methods: Between 1997 and 2002 the same surgical team performed PSFI in 118 patients, 70 women and 48 men with a mean age of 49.25 years (range 13-83). The patient's baseline characteristics (age, gender, body-mass-index, ASA and associated co-morbidities), surgical data (fused levels, blood loss, duration and date of surgery) and early complications (EMC) were recorded. Major complications were defined as potentially life-threatening or cause of considerable suffering. Logistic regression analysis, non-parametric tests for comparison and Chi-Square were used for statistics.

Results and Discussion: 23 patients (19.4\%) developed 32 EMC: 8 had radicular symptoms, 3 an infection, 8 pulmonary, 7 cardiovascular and 6 other complications (hematoma requiring reoperation, disorientation and confusion, impotence, pancreatitis). Bivariate Analysis: Age, body mass index, ASA, cardiac, pulmonary and vascular co-morbidity increased the rate of EMC. Duration and date of surgery modified significantly the rate of major complications. Blood loss and number of fused levels did not modify the rate of EMC. Logistic Regression: vascular (OR 5.05, $\mathrm{p}=.16$ ) and pulmonary disease (OR $2.34, p=.26$ ) were the co-morbid conditions most closely associated with complications. Procedures lasting 4 to 6 hours had more complications (OR $3.61, p=.15)$ than those lasting less than 4 hours (OR 7.90, $p=.02)$. The experience of the surgical team was the parameter most strongly associated with complications.

Conclusions: When performing PSFI, the experience of the surgical team and the length of the surgical procedure seem to have more influence on the complication rate than the patient's baseline characteristics.

References:

1 Deyo RA, et al. N Engl J Med 2004; 350(7): 722-6.

2 Fritzell P, et al. Eur Spine J 2003; 12(2): 178-89.

\section{AP2-3}

Impact of surgery-related factors and anaesthesia-related variables on cerebral infarction after aneurysmal subarachnoid hemorrhage (Pre-embolization Era)

D. Kim, J. Shim, K. Kim, J. Shu, H. Yi

Department of Anesthesiology, Neurosurgery, Hanyang University Medical Center, Seoul, Republic of Korea

Background and Goal of Study: To investigate surgery-related or anesthesiarelated predictors of cerebral infarction after early aneurysmal surgery(1). Materials and Methods: A retrospective study was conducted on consecutive 382 patients who undertook early microsurgery for the anterior circulation aneurysms. We collected quantitative information about surgery-related factors (intraoperative rupture, temporary clipping, clipping- and retraction time) and anesthesia-related variables (blood pressure, oxygen saturation, hematocrit) (2). Postoperative occurrence of vasospasm or cerebral infarction was chosen as a reference event. Patients were dichotomized according to $\mathrm{H}-\mathrm{H}$ grade. Results and Discussions: In total patients, close relation existed between intraoperative rupture, prolonged clipping and retraction time, intraoperative hypotension, low hematocrit and occurrence of cerebral infarction. However, statistical significance in 2 separate groups of corresponding clinical grade was not found. On multiple logistic regression analyses, intraoperative rupture, hypotension and low hematocrit showed Odds ratio of $2.123,3.016$ and 1.628 , respectively. 
Conclusion(s): Early surgery for poor grade SAH patients carries significant risk of ongoing ischemic complication, and thus endovascular embolization should be considered as a strong alternative. Additionally, cardiovascular and pulmonary dysfunction hampers successful postoperative recovery in some cases.

References:

1 Fridriksson, et al. J Neurosurgery 2002; 96: 515-522.

2 Ferch R, et al. J Neurosurgery 2002; 97: 836-842.

\section{AP2-4}

What is the actions mechanism of lumbar epidural blood patch in the treatment of the headache by spontaneous carvical CSF leak?

I. Arpino, E. Ferrante, C. Guarnerio, A. Citterio, R. Sterzi

$I^{\circ}$ Anesthesiology Department, Niguarda Ca Granda Hospital, Milan, Italy

Background and Goal of Study: To evaluate the efficacy and to identify the action's mechanism of lumbar epidural blood patch (LEBP) in the treatment of headache by spontaneous cervical CSF leak (SCCSFL).

Materials and Methods: We observed 46 patients with spontaneous intracranial hypotension (SIH) between 1992 and 2006. 16 pts received LEBP of these 5 pts ( 2 women and 3 men; age range $34-51$ years) showed SCCSFL. LEBP was performed using 15 to $34 \mathrm{ml}$ of autologous blood mixed with $1 \mathrm{ml}$ of gadolinium. All patients manteined a 30 degree Trendelemburg position during the procedure and for 24 hours after. All pts performed spinal RMl about $1 \mathrm{~h}$ post- LEBP. Follow-up ranged from 6 to 18 months.

Results and Discussions: All patients had orthostatic headaches $(\mathrm{OH})$. Other manifestations were nausea, vomiting, tinnitus, diplopia and bilateral upper limb numbness. All pts failed an initial conservative treatment (bed rest and rehydration) over a period of 1 to 18 months. Brain RMI showed diffuse pachymeningeal gadolinium enhancement (neuroimaging of intracranial hypotension) and spinal MRI showed cervical CSF leak in all pts. Spinal MRI post-LEBP showed blood mixed with gadolinium in the epidural space from lumbar to cervical level in $2 \mathrm{pts}$ and only at lumbar level in $3 \mathrm{pts}$. $\mathrm{OH}$ recoveries within $24 \mathrm{~h}$ in all pts, when they were upright position. Untill now none has had a relapse. Conclusion(s): Our data confirm the efficacy of LEBP in SCCSFL and identify the two action mechanism of LEBP in these cases. The first is the plug effect that is showed when the blood arrives at the CSF cervical leak. The blood cloting closes the leak and stops liquoral cervical leakage, this is showed in only two pts. The second is the mass effect: the high quantity of blood injected in the epidural space shifts the dura madre to the spinal marrow and cauda equine to cause one reduction on the volume in the subaracnoide space, and determine the increase of liquor pressure. The liquor that is pushed up determines the increase of cranial pressure. This mechanism is showed in all our patients and is the only action mechanism in three of them.

\section{AP2-5}

\section{Dexmedetomidine-ketamine-midazolam combination for} sedation in endovascular embolizations of cerebral arteriovenous malformations and carotid stenting

\section{J. Schreiberova, L. Hess, A. Krajina}

Department of Anesthesiology and Intensive Care Medicine, Charles University in Prague, Faculty of Medicine and University Hospital Hradec Kralove, Hradec Kralove, Czech Republic

Background and Goal of Study: Sedation is indicated in endovascular embolizations of cerebral arteriovenous malformations and carotid stenting to secure immobility, analgesia, anxiolysis, and amnesia of the patient during these procedures. Hemodynamic stability, absence of respiratory depression, preserved patient responsiveness and cooperation are required. We used theoretically promising combination of dexmedetomidine with low doses of ketamine and midazolam for sedation, and compared it with routine sufentanilmidazolam sedation.

Materials and Methods: After local ethic committee approval, 34 ASA 2-3 adults scheduled for endovascular interventions, were randomly allocated into 2 groups for sedation. Group A: bolus application of dexmedetomidine $0,5 \mu \mathrm{g} \mathrm{kg}^{-1}$, ketamine $0,25 \mathrm{mg} \mathrm{kg}^{-1}$, midazolam $0,02 \mathrm{mg} \mathrm{kg}^{-1}$ i.v. over $5 \mathrm{~min}-$ utes, followed by infusion of dexmedetomidine $0,25 \mu \mathrm{g} \mathrm{kg}^{-1} \mathrm{~h}^{-1}$ and ketamine $0,25 \mathrm{mg} \mathrm{kg}^{-1} \mathrm{~h}^{-1}$. Infusion rate was altered to keep the patient sedated, but responsive and cooperative. Group B: bolus of sufentanil $10 \mu \mathrm{g}$ and midazolam $0,02 \mathrm{mg} \mathrm{kg}^{-1}$, followed by further bolus increments as needed. Blood pressure (BP), ECG, sat $\mathrm{O}_{2}, \mathrm{p}_{2} \mathrm{CO}_{2}$, Ramsay sedation score, complications, rescue medication, amnesia, and recovery times were recorded. Patient cooperation was assessed by the radiologist (blinded to the sedation used) as excellentsufficient-poor at the end of each procedure. Statistical analysis: t-test, $\chi^{2}$ test, ANOVA for repeated measures.
Results and Discussions: Decrease of BP was significantly deeper in A group, than in B group (systolic BP: $p<0,05$, diastolic BP: $p<0,01$ ), as well as the decrease in heart rate $(p<0,001)$. Cooperation was excellent in $94 \%$ in A group, vs. $30 \%$ in B group ( $p<0,001)$. No other statistically significant differences were found. No clinically significant complications were recorded. Conclusion: Dexmedetomidin-ketamine-midazolam combination seems to be safe and advantageous method of sedation in endovascular interventions. Improved patient cooperation and lower blood pressure can potentially contribute to decreased risk of severe complications, as intracranial haemorrhage and hyperperfusion syndrom (1).

Reference:

1 Buhl JH, Cepek L, Knauth M. Am J Neuroradiol 2006; 27: 1508-13.

\section{AP2-6}

The effect of low dose propofol on cortical memory networks - a functional MRI study

S. Berg, S. Zysset, W. Heinke

Anesthesiology, University of Leipzig, Leipzig, Germany

Background and Goals: Blood oxygenation level dependent (BOLD) based $\mathrm{fMRl}$ is an effective tool to investigate central drug actions (1). Recent studies showed that even low doses of propofol have significant amnesic effects (2). Whether these amnesic actions are caused by a selective or a global reduction of memory network activity is yet not clear. Thus, $\mathrm{AMRI}$ has been used to investigate the effects of propofol on the BOLD signal changes while subjects performed different memory tasks.

Materials and Methods: 26 male volunteers (ASA 1) were double blind randomised on three study branches: placebo (normal saline), propofol $0.6 \mu \mathrm{g} / \mathrm{ml}$ and $1.2 \mu \mathrm{g} / \mathrm{ml}$ (TCl infusion). After 10 minutes of propofol equilibration subjects performed several tasks (episodic, semantic, and working memory tasks, hyper- and hypoventilation tasks) within the scanner. 45 minutes after cessation of propofol subjects had to perform an unexpected recognition task of items (faces) presented during the working memory task.

Results and Discussions: Propofol did not affect the BOLD signal during the equilibration period. Moreover, ventilation-induced BOLD signal changes (cerebral vasoreactivity) did not differ between propofol and placebo. Reaction time and error rates of the different tasks increased dose dependently $(p<0.05$ ). Subjects of the placebo group recalled $69 \pm 7 \%$ (mean $\pm S D$ ) of the faces correctly. $0.6 \mu \mathrm{g} / \mathrm{ml}$ propofol decreased the recognition rate by $17 \%$ ( $p<0.05), 1.2 \mu \mathrm{g} / \mathrm{ml}$ by $39 \%$ ( $<<0.01$ ) respectively. Significant BOLD signal reductions $(z>3.1, p<0.001)$ caused by increasing propofol concentrations during the different memory tasks were found in circumscribed memory network areas. There was no global reduction of cerebral network activity under propofol during the different memory tasks.

Conclusion(s): Low dose propofol does neither influence the baseline BOLD signal nor the cerebral vasoreactivity. Therefore, the observed signal changes under propofol reflect real changes of neuronal activity. As a result attention and memory deficits induced by amnesic propofol concentration can be interpreted as a highly selective impairment of cerebral networks.

References:

1 Heinke W, Schwarzbauer C Br J Anaesth. 2002; 89:112-22.

2 Veselis RA, Reinsel RA, Feshchenko VA, Johnson R Jr. Anesthesiology. 2004; 101:831-41.

\section{AP2-7}

Does the presence of a subarachnoid haemorrhage (SAH) influence analgesic requirements after treatment with surgery or endovascular coiling in patients with intracranial aneurysms?

D. Wijayatilake, Suwimon. Tangwiwat, Atul. Prabhu, Hossam. El-Beheiry, Pirjo. Manninen

Anesthesia, Toronto Western Hospital, Toronto, Canada

Background and Goal of Study: Analgesia requirements after aneurysm repair remain controversial(1)(2). The purpose of this study is to assess if the presence of a sub arachnoid haemorrhage $(\mathrm{SAH})$ influences the post procedure opiate use in patients treated with open craniotomy compared to endovascular coiling.

Materials and Methods: Data was collected prospectively on 134 patients undergoing treatment of intracranial aneurysm. Patients were divided into 4 groups: unruptured (no $\mathrm{SAH}$ ) endovascular repair (EU), ruptured $(\mathrm{SAH})$ endovascular (ER), unruptured craniotomy (CU), and ruptured craniotomy (CR). Total opioid pain medications given in the 48 hours post procedure were recorded (fentanyl, codeine, morphine). These doses given were converted to morphine equivalents for comparison. 
Continuous data was analyzed using a one way ANOVA. Ordinal data was analyzed using Mann Whitney test.

Results and Discussions: (Values are mean $+/-\mathrm{SD}$ )

\begin{tabular}{lcccc}
\hline${ }^{*} \mathrm{p}=$ values $\left.<0.05\right)$ & $\mathrm{EU}$ & $\mathrm{ER}$ & $\mathrm{CU}$ & $\mathrm{CR}$ \\
& $\mathrm{N}=43$ & $\mathrm{~N}=16$ & $\mathrm{~N}=28$ & $\mathrm{~N}=47$ \\
\hline Age $(\mathrm{yr})$ & $48 \pm 14$ & $55 \pm 12$ & $52 \pm 10$ & $51 \pm 10$ \\
$\begin{array}{l}\text { Intra operative } \\
\text { fentanyl (mcg) }\end{array}$ & $213 \pm 98$ & $241 \pm 102$ & $330 \pm 147$ & $376 \pm 123$ \\
$\begin{array}{l}\text { Morphine equivalent } \\
\text { Postop(mg) }\end{array}$ & $11^{*} \pm 15$ & $27 \pm 19$ & $31 \pm 20$ & $29 \pm 21$
\end{tabular}

Patients in the EU group required statistically less postoperative opioids than the other 3 groups.

Conclusion(s): We conclude if a patient has had a SAH from the aneurysm. They will experience similar levels of pain and require similar levels of opioids regardless of the method of repair within the first 48 hours post procedure. Thus it is important to administer adequate analgesia to all patients with $\mathrm{SAH}$, and not reduce the dose if the repair has been performed by an endovascular technique.

References:

1 Roberts GC. EJA 2005; 22: 328-32.

2 Dubar PJ. Anesth Analg 1999;88:335-40.

\section{AP2-8}

Magnesium sulphate used for prevention of cerebral vasospasm

A. Hoxha, K. Pilika, M. Kerci, M. Demneri, M. Saraci

Anesthesia \& critical care, University hospital centre, Tirana, Albania

Background: Last years clinical researchers show an increasing interest in use of magnesium sulphate $\left(\mathrm{MgSO}_{4}\right)$ in prevention of delayed ischemic neuronal damage (DIND) caused by vasospasm after $\mathrm{SAH}(1,2)$. The aim of the study is to reveal the protective effect of $\mathrm{MgSO}_{4}$ on neurological damage after $\mathrm{SAH}$ in our patients.

Materials and Methods: 35 randomly enrolled patients $(42.23 \pm 12.56$ years old) after aneurismal $\mathrm{SAH}$, which had received $\mathrm{MgSO}_{4} 60 \mathrm{mmol} / \mathrm{L} /$ day from day 4 to 10 after $\mathrm{SAH}$, were compared for their neurological follow up (for 14 days) with other 96 analogous patients treated in our clinic in the previous 1.5 year period. All patients had surgery for clipping of aneurism. The difference in treatment consists only in administration of $\mathrm{MgSO}_{4}$. Neurological status at 14-th day was expressed through GOS. There were assessed SIRS and MODS, too. For statistics T-test was used and $p<0.05$ was considered as significant.

Results and Discussion: Patients who received $\mathrm{MgSO}_{4}$ showed a much lower (very near to significant) incidence of clinically assessed DIND (31.42\%10 from 35 to $42.67 \%-41$ from $96, p=0.06)$. GOS at 14 -th day did not result in significant difference $(3.86 \pm 1.19$ pts to $3.34 \pm 0.96 \mathrm{pts})$. Mortality rate was $22.86 \%$ in magnesium group and $26.05 \%$ in other $(p>0.05)$. Frequency of SIRS on the first day after operation was slightly lower in magnesium group $(91.42 \%$ to $94.78 \%)$. In 14 days period MODS was presented in $34.28 \%$ of patients in $\mathrm{MgSO}_{4}$ group and $39.58 \%$ in others $(\mathrm{p}>0.05)$.

Conclusion: Although statistic significance was not achieved at any of indexes we observe a lower incidence of DIND, MODS and SIRS in patients treated with $\mathrm{MgSO}_{4}$. Mortality rate and GOS showed lower values in magnesium group. Lack to achieve statistic significances may be to the little number of patients in our study.

Reference:

1 van den Berg WM, et al. magnesium sulfate in aneurismal subarachnoid hemorrhage: a randomized controlled trial. Stroke 33: 1011-1015, 2005.

\section{AP2-9}

Survive and mortality in patients with severe brain trauma M. Kërçi, T. Zhurda, A. Hoxha, D. Muzha, N. Baftiu

Anesthesia \& ICU Department, National Trauma Center, Tirana, Albania

Background and Goal of the Study: Brain injury is an important part of overall injuries. The goal of the study was to assess the dynamic of head injury of $\leqslant 8$ GCS during 2005 in our department.

Materials and Methods: 112 patients were attended to our Critical Care Department for brain injury during the year 2005. F/M ratio was 30/82, age $33.12 \pm 8.42$. Related to their GCS assessment they were presented as GCS 3-4 pts 36 patients, as GCS $5-6$ pts 28 patients and as $7-8$ pts 48 patients.
Results and Discussion: Most of them were road casualties (90 patients). 38 of them had other important damages. 17 of the patients were operated for traumatic brain haematomas. Immediately after the attendance the patients were intubated, sedated and mechanically ventilated with Tidal volume $6-8 \mathrm{ml} /$ body weight. CT-scans performed at the attendance showed $69 \%$ haemorrhagic contusion, $26 \%$ were massive haematomas and $5 \%$ only pronounced hypoxemic brain swelling. $26 \%$ of the patients were presented to the hospital in shock. In 32 patients Diclofenac was used as antiflogistic agent. Overall mortality was $54 \%$ (61 from 112). Mortality rate in-patients with GCS 3-4 pts was $94 \%$, in-patients with 5-6 GCS was $53 \%$ and in the group with 7-8 GCS $25 \%$.

Conclusion: The most frequent cause of brain injury in our patients was road casualties. The overall mortality was over $50 \%$. Use of Diclofenac showed better recovery to other patients.

Reference:

John J. Marini, Arthur P. Wheeler, Critical care Medicine (the essentials-third edition), 2006; 568-573, 576-578.

\section{AP2-10}

The effects of 7,2 hypertonic saline in $6 \%$ hydroxyethyl starch $200 / 0,5$ on intracranial pressure and cerebral regional oxygen saturation at acute normovolemic haemodilution in patients undergoing supratentorial tumor surgery

L. Israelyan, A. Lubnin

Neuroanesthesiology, Burdenko Neurosurgery Institute, Moscow, Russian Federation

Background and Goal of Study: Hypertonic/colloid fluid resuscitation has not been studied in cases of brain tumor. The goal of this study was to evaluate the effects of 7,2\% hypertonic saline in 6\% hydroxyethyl starch 200/0,5 (HyperHAES, Fresenius Kabi, Germany) on intracranial pressure (ICP) and cerebral $\mathrm{rSO}_{2}$ at acute normovolemic haemodilution (ANH) in patients undergoing supratentorial brain tumor surgery.

Materials and Methods: $250 \mathrm{ml}$ HyperHAES $(3,8 \mathrm{ml} / \mathrm{kg})$ has been infused during $13,7 \pm 3,1 \mathrm{~min}$ after $1200 \mathrm{ml}$ blood withdrawal at $\mathrm{ANH}$ in 21 patients undergoing supratentorial brain tumor resection under invasive haemodynamic and laboratory control. ICP and cerebral $\mathrm{rSO}_{2}$ continuously were measured during and $15 \mathrm{~min}$ after HyperHaes infusion. We used paired Student $t$-test or Mann Whitney test.

Results and Discussions: Changes in ICP and cerebral $\mathrm{rSO}_{2}$ are shown below on Figures. Standard deviations have been shown at beginning of study, on peak of transitory changes and in the end of infusion and study.
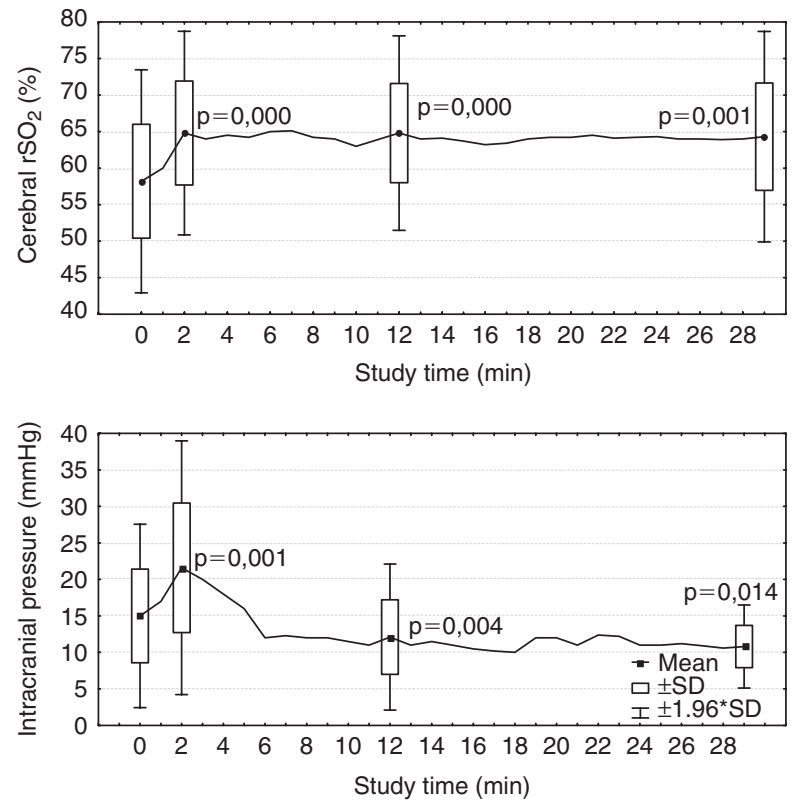

Conclusion: Revealed reduction of ICP with 7,2\% hypertonic saline $6 \%$ hydroxyethyl starch $200 / 0,5$ at $\mathrm{ANH}$ in patients with supratentorial brain tumors has biphasic character with initial increase of ICP which is accompanied by increase of cerebral $\mathrm{rSO}_{2}$ parameters. 


\section{AP3-2}

"Overnight intensive recovery" after neurosurgical procedures. A survey results

N. Fabregas, J. Carazo, R. Valero, E.J. Carrero, F.J. Tercero

Anaesthesiology, Hospital Clínic, Barcelona, Spain

Background and Goal of Study: Close observation in an ICU setting for $>12-24 \mathrm{~h}$ following craniotomy is generally recommended (1). Lack of intensive care beds availability for postoperative care lead to develop shortterm surgical critical care, to a well-defined patient population, in the post anaesthesia recovery room (2). We investigated this "overnight intensive recovery" (OIR) actual use in neurosurgical postoperative care setting.

Materials and Methods: A survey was done to the attendants of a neuroanesthesia workshop at ESA 2005 Meeting; to all attendants of EuroNeuro 2005 Meeting and to the members of "Spanish Anaesthesia Society (SEDAR) Neuroanaesthesia Section". Postoperative care options were: neurosurgical ward, OIR or Intensive Care Unit (ICU).

Results and Discussions: 80 anaesthesiologists from 68 Hospitals all over Europe filled up the survey.

\begin{tabular}{lccr}
\hline Intervention & ward & OIR & ICU \\
\hline Small supratentorial tumor & $26.5 \%$ & $32.3 \%$ & $32.3 \%$ \\
Large supratentorial tumor & $10.3 \%$ & $19.1 \%$ & $61.8 \%$ \\
Infratentorial tumor & $7.3 \%$ & $14.7 \%$ & $69.1 \%$ \\
Epilepsy surgery & $19.1 \%$ & $11.8 \%$ & $29.4 \%$ \\
Craniosynostosis & $5.9 \%$ & $16.2 \%$ & $42.6 \%$ \\
"Awake" craniotomy & $22 \%$ & $19.2 \%$ & $17.6 \%$ \\
Neuroendoscopic tumour resection & $17.6 \%$ & $32.4 \%$ & $17.6 \%$ \\
Neuroendoscopic ventriculostomy & $41.2 \%$ & $25 \%$ & $10.3 \%$ \\
Stereotactic brain biopsy & $57.3 \%$ & $14.7 \%$ & $10.3 \%$ \\
Frameless brain biopsy & $47 \%$ & $8.8 \%$ & $8.8 \%$ \\
Neuroendoscopic brain biopsy & $41.2 \%$ & $23.5 \%$ & $8.8 \%$ \\
Transphenoidal hypohysectomy & $25 \%$ & $30.9 \%$ & $29.4 \%$ \\
Neuroendoscopic hypophysectomy & $26.5 \%$ & $20.6 \%$ & $13.2 \%$ \\
Intracerebral electrode implantation & $19.1 \%$ & $14.7 \%$ & $16.2 \%$ \\
Cerebral aneurysm surgery & $4.4 \%$ & $7.4 \%$ & $70.6 \%$ \\
Arterio-venous malf. surgery & $4.4 \%$ & $13.2 \%$ & $67.6 \%$ \\
Cerebral aneurysm coiling & $19.1 \%$ & $26.4 \%$ & $30.9 \%$ \\
Aterio-venous malf. embolisation & $19.1 \%$ & $23.5 \%$ & $26.5 \%$ \\
\hline
\end{tabular}

Conclusion(s): OIR is a real alternative to ICU postoperative neurosurgical care, its use ranges between $7.4 \%$ following cerebral aneurysm surgery to $32.4 \%$ following neuroendoscopic tumour resection.

References:

1 Neurosurg Clin N Am 1994; 5: 789-810.

$2 \mathrm{Br} J$ Anaesth 2004; 92(2): 164-166.

\section{AP3-3}

\section{Correlation dimension of field potential activity in vitro and} EEG at increasing concentrations of sevoflurane

M. Bretschneider, B. Drexler, G. Schneider, B. Antkowiak, E.F. Kochs

Klinik für Anaesthesiologie, Klinikum Rechts der Isar der TU München, München, Germany

Background and Goal of Study: Correlation dimension (CD) [1] was used to analyse down-state sequences of field potential activity in cultured rat neocortical slices and in human EEG data. In both cases the influence of increasing concentrations of sevoflurane on the CD was evaluated.

Materials and Methods: Field potential activity of neocortical slices was recorded at increasing levels of sevoflurane $[0,0.25,0.5,0.75,1.5,2.5,5$ $M A C] .213$ down state sequences were analysed with CD. Standard human EEG was monitored from volunteers at five distinct levels of anaesthesia [awake, loss of consciousness (LOC), burst suppression (BSUPP) and two intermediate levels inter1 and inter2]. Sequences of 120 s length without artefacts were selected and CD was calculated.

The used parameter, the correlation dimension is a measure of dimensionality with a lower CD indicating a less complex signal.

Results and Discussions: The CD values of the in vitro data increase with higher concentrations of sevoflurane indicating higher randomness in the signal at higher concentrations. This is explained by a less organised communication within the cortical network, resulting from a severe anaesthetic-induced depression of neuronal activity. Surprisingly, the CD showed an inverse behaviour in the EEG data, strongly suggesting the involvement of anaesthetic effects mediated by subcortical areas. The concentration-dependent increase of CD for the in vitro data appeared to be almost linear. In the EEG data an obvious threshold of CD was detected between signals from awake and anesthetized volunteers. No significant differences between different levels of anaesthesia were observed.
Conclusion(s): CD can be used to detect and evaluate sevoflurane-induced changes in the field potential activity of cortical tissue slices in vitro. Anaesthetic actions mediated by cortical and subcortical brain areas may underlie the inverse course of CD in vitro and in the EEG. Because of the "threshold" in EEG data, CD might be a parameter to separate consciousness from unconsciousness during anaesthesia. This has to be evaluated in i. shorter ii. artefact containing signals from iii. dynamic phases of anaesthesia.

Reference:

1 Grassberger et al., Physica D: Nonlinear Phenomena, 1983, 9, Issue 1-2, 189-208.

\section{AP3-4}

\section{Use of cerebral microdialysis during wake-up craniotomy to} monitor local cerebral metabolism

J. Biesemans, C. De Deyne, J. Wuyts, F. Weyns, R. Heylen

Anesthesia, Ziekenhuis Oost-Limburg, Genk, Belgium

Introduction: Wake-up craniotomy has been advocated as thé solution for patients undergoing epilepsy surgery or tumor surgery in eloquent areas. The anesthetic challenge is to provide sedation, analgesia, hemodynamic stability, a safe airway as well as optimal neurosurgical conditions. In the present paper, we monitored local cerebral metabolic changes with cerebral microdialysis (MD) during wake-up procedures.

Materials and Methods: In this paper, we report on 9 adult pts scheduled for brain tumor surgery in eloquent areas of the brain. Induction was accomplished with propofol TCl $(3 \mu \mathrm{g} / \mathrm{ml})$, remifentanil TCl $(8 \mathrm{ng} / \mathrm{ml})$ and rocuronium $(0.6 \mathrm{mg} \cdot \mathrm{kg})$. After insertion of a laryngeal mask airway (LMA), anesthesia was maintained with propofol TCI (titrated to BIS between 40 and 60) and remifentanil TCI (titrated to hemodynamic responses). Surgical field was infiltrated with up to $30 \mathrm{ml}$ bupivacaine $0.5 \%$. For wake-up, $\mathrm{TCl}$ propofol-remifentanil was gradually reduced until pt became responsive and LMA could be removed After opening of the dura, a MD catheter was inserted by the surgeon in the at-risk cortex This monitoring was sustained throughout the operation, during which intra-operative events, as lobe retraction and especially awakening, were documented. The MD catheter was perfused at $5 \mu \mathrm{l} / \mathrm{min}$ enabling analysis (for glucose, lactate, pyruvate and glycerol) of the dialysates every $5 \mathrm{~min}$. Results: In all 9 pts, wake-up was successfully managed. In a mean of 23 min (range 12-39 min) after start of the wake-up procedure, pts became responsive to stimulation and LMA was removed. Hemodynamic parameters remained stable, respiratory rate and arterial $\mathrm{PCO}_{2}$ remained within normal limits. $\mathrm{MD}$ dialysates revealed decreases in local glucose, most probably induced by brain retraction. In 6 pts, wake-up procedure went along with further decreases in local glucose, in 4 of these pts significant increases in local lactate were observed. In none of them, an increase in lacate/pyruvate ratio or an increase in local glycerol occurred during the wake-up procedure.

Conclusions: Wake-up procedures, assuring stable hemodynamic and respiratory conditions, seem not to induce an increased risk for local cerebral metabolic (ischemic) changes, as monitored by cerebral microdialysis.

\section{AP3-5}

Local cerebral metabolic changes, induced during minimally invasive supraciliary craniotomy for cerebral aneurysm surgery, monitored by cerebral microdialysis

\section{E. Voets, C. De Deyne, J. Wuyts, K. Engelborghs, R. Heylen}

Anesthesia and Neurosurgery, Ziekenhuis Oost-Limburg, Genk, Belgium

Introduction: An alternative approach for surgical treatment of anterior/middle cranial fossa lesions is frontolateral craniotomy through supraciliary skin incision. Brain retractors applied during brain surgery may induce local changes in cerebral perfusion. Cerebral microdialysis (MD) is a tool for monitoring local cerebral metabolism. In this study, we evaluated whether MD revealed any changes in local metabolism in the brain area under the retractor and in the vascular territory of aneurysm surgery during supraciliary craniotomy.

Patients and Methods: With IRB approval, 14 pts scheduled for anterior/ middle cerebral artery aneurysms, received peroperative MD. A MD catheter was inserted under direct vision into at-risk cortex at start of surgery. This monitoring was sustained throughout operation, during which intra-operative events, as cerebral aneurysm manipulation and/or clipping or lobe retraction, were precisely documented. The MD catheter was perfused at $5 \mu \mathrm{l} / \mathrm{min}$ enabling analysis (for glucose, lactate, pyruvate and glycerol) of dialysates every $5 \mathrm{~min}$.

Results: In all pts, MD catheter was successfully inserted into cerebral cortex and dialysates could be obtained throughout whole operation. Insertion of brain retractors resulted in a overall decrease in local glucose, most probably reflecting a decrease in local perfusion. In all patients, we observed a shortlasting increase in local glucose after retractor removal or repositioning. In 12 of 14 pts, local lactate increased, mostly related to retraction. In 4 pts, we 
observed an increase in lactate/pyruvate ratio. Only 2 of these episodes went along with a local increase in glycerol, a sign of ongoing cerebral cell death. We observed no local metabolic changes induced by cerebral aneurysm manipulation. Postoperative outcome was uneventfull for all pts.

Conclusion(s): This minimally invasive technique, a $2.5 \times 3.0 \mathrm{~cm}$ craniotomy, through supraciliary incision, is a simple approach to the anterior/middle cranial fossa. These first results seem not to reveal an increased risk of local metabolic changes induced by brain retraction.

\section{AP3-6}

\section{Incidence of low BIS values during general anaesthesia in neurosurgical patients}

A. Castro, P. Amorim, G. Costa, S. Oliveira, C.S. Nunes

Matematica Aplicada, Faculdade de Ciencias da Universidade do Porto, Porto, Portugal

Background and Goal of Study: BIS below 45 has been associated with worse outcome following anaesthesia and surgery [1]. We decided to investigate the incidence of low BIS in our neuroanaesthesia practice.

Materials and Methods: Data records from neurosurgical surgeries were collected every 5s from Aspect XP with RuglooplI ${ }^{\circ}$. Anaesthesia was with $\mathrm{TCl}$ of propofol and remifentanil. The only defined goal that the anaesthetists had, was avoiding BIS above 60. BIS was analyzed from the moment when BIS dropped below 70 (induction) until the first moment at recovery when BIS increased above 70 - anaesthesia time (AT). For poor neurological grade patients, AT was from the beginning of induction until end of surgery. An algorithm that we developed in Matlab7 $®$ extracted the time periods with BIS in different levels $(<30,30-35,35-40$ and $40-45)$. (Data: mean \pm SD) Results and Discussions: 211 patients, ASA 1-4, $51 \pm 17$ years, $69 \pm 13 \mathrm{~kg}$, $163 \pm 9 \mathrm{~cm}, 92$ male, 166 head surgeries and 45 spinal, Glasgow score 3-8 in 8 patients and $9-12$ in 11. BIS was bellow 45 for any period of time in all records: for $25-50 \%$ of AT in 31 patients, for $50-75 \%$ in 77 patients and for $75-100 \%$ of the time in 90 patients. BIS below 30 occurred for $75-100 \%$ of AT in 3 patients, for $50-75 \%$ in 11 , for $25-50 \%$ in 28 and for $10-25 \%$ in 45 .

Patient's distribution: BIS levels

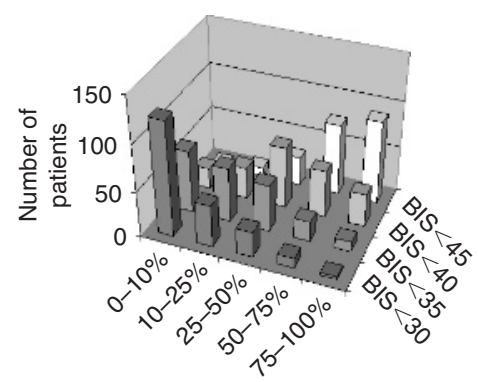

Fig. 1 Patient's distribution: time percentage at each level

Conclusion(s): We found, in our neuroanaesthesia practice, an incidence of low BIS that we think was too high: $79 \%$ of the patients had BIS below 45 more than $50 \%$ of the time. These results suggest that maybe, worrying mostly about awareness may result in too deep anaesthesia. It may be desirable to implement measures to achieve less deep anaesthesia.

Reference:

1 Anesthesia Analgesia 2005, 100: 4-10.

\section{AP3-7}

\section{Acute changes in cerebral autoregulation following carotid} endarterectomy under general anaesthesia

G. Chapman, D. Dellagrammaticas, M. Gough, S. Howell

Academic Unit of Anaesthesia, University of Leeds, Leeds, United Kingdom Background and Goal of Study: Carotid endarterectomy (CEA) may be associated with postoperative haemodynamic instability requiring treatment with vasoactive drugs(1). If autoregulation is disordered, treatment to raise or lower the blood pressure may lead to dramatic changes in cerebral blood flow. Furthermore, some patients may exhibit postoperative blood pressures outside their normal limits of autoregulation. This study examines the change in the cerebral autoregulation index following CEA and the association between perioperative blood pressure change and autoregulation.
Materials and Methods: Fifteen patients who underwent CEA under general anaesthesia were studied. Pre and postoperative arterial pressure (MAP) were recorded. Patients underwent tilt-testing on the day before surgery and on the first postoperative day (POD1). The static autoregulation index (Al) was derived as the gradient of the relationship between CPP and MCAv $\left(\mathrm{cm} \mathrm{sec}^{-1}\right.$ $\mathrm{mmHg}^{-1}$ ). Data are reported as medians and ranges.

Results and Discussions: Ten of the patients were male. The median(range) age of patients was $67(53-83)$ years. The median preoperative MAP was $97(82-131) \mathrm{mmHg}$. Median postoperative MAP was $89(73-140) \mathrm{mmHg}$. The median preoperative Al was $0.21(-0.16-0.68)$ and the median postoperative Al 0.14(-0.61-0.54). The median change in Al was 0.072(-0.46-.039) and was not statistically significant $(p=0.61)$. There was no significant correlation between the change in blood pressure and the postoperative $\mathrm{Al}(\mathrm{r}=-0.3)$ Conclusion(s): Our data do not support a significant change in cerebral autoregulation following CEA. There was no association between large changes in MAP and poor cerebral autoregulation. These data suggest that patients who have undergone CEA under general anaesthesia and do not have clinically evident hyperperfusion can be given vasoactive drugs for hypo or hypertension without undue concern.

\section{Reference:}

1 McKevitt FM et al. Stroke 2003; 34: 2581-2.

\section{AP3-8}

Assessment of cerebral autoregulation with the Transient Hyperemic Response test. Comparison with $\mathrm{CO}_{2}$ Reactivity in head-injury patients

L. Velly, N. Bruder, F. Gouin

DAR Adultes, CHU Timone, Marseille, France

Background and Goal of Study: Impaired cerebral autoregulation is associated with an unfavorable outcome after head-injury. Recently, a noninvasive method using transcranial Doppler (TCD), only validated in volunteers, has been described: the Transient Hyperemic Response (THR) test. The aim of this study was to assess the reproducibility of the method in head-injury patients and to compare derived autoregulation indices with those of a standard test of autoregulation, $\mathrm{CO}_{2}$ Reactivity $\left(\mathrm{RCO}_{2}\right)$.

Materials and Methods: Seventeen artificially ventilated patients with intracranial pressure monitoring were examined. Middle cerebral artery (MCA) was insonated with a 2-MHz TCD probe (Looki; Atys Medical). The probe was fixed in position by using a headband to maintain a constant angle of insonation during the study. At least 2 THR tests were performed by using a 10 -s compression of the ipsiplateral common carotid artery (CC). For analysis, two wave forms were chosen: F1, the wave form immediately preceding the compression; F2, the second wave form after the release of compression. THR ratio $=F 2 / F 1$. Normal value of THR ratio was defined as $>1.10 . R_{2}$ was evaluated by a hyperventilation trial to lower $\mathrm{ETCO}_{2}$ by $5 \mathrm{mmHg}$. Normal range for $\mathrm{RCO}_{2}$ was defined as $3.5 \pm 1 \% \Delta \mathrm{V}_{\mathrm{MCA}} / \mathrm{mmHg}$. Data were analyzed using linear regression and Bland-Altman analysis.

Results and Discussion: Thirty-four THR test was obtained from 17 patients. Bland-Altman analysis for THR test showed a bias $-6.10^{-3}$ and a precision \pm 0.03 . THR test $<1.10$ was found for 5 patients. Loss of $\mathrm{RCO}_{2}$ was found for 10 patients. Linear regression analysis did not found any correlation between $\mathrm{THR}$ test and $\mathrm{RCO}_{2}$ (figure).

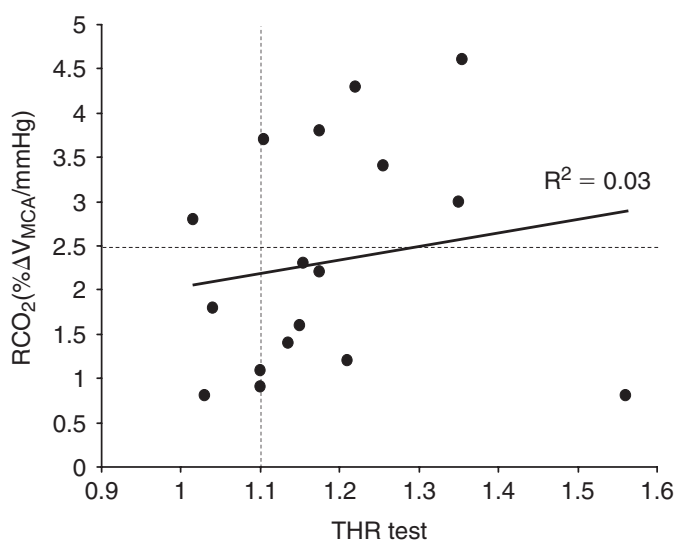

Conclusions: THR test is reproducible in clinical arena. But it does not allow prediction of cerebral circulation $\mathrm{CO}_{2}$ reactivity. Its clinical usefulness has thus to be determined. 


\section{AP4-1}

Bilateral measurement of Bispectral Index (BIS) and Middle Latency Acoustic Evoked Potentials (MLAEP) in sedated patients with unilateral supratentorial intracranial haemorrhage

A.Fudickar, J.H.Jacobsen, N.Weiler, J.Scholz, B.Bein

Klinik für Anästhesiologie und operative Intensivmedizin, Universitätsklinikum Schleswig-Holstein, Campus Kiel, Kiel, Germany

Background and Goal of Study: The impact of intracranial lesions on the results of Bispectral Index (BIS) and Middle Latency Acoustic Evoked Potentials (MLAEP) remains unclear. If an EEG-based measurement is influenced by intracranial lesions, bilateral application in patients with unilateral brain lesions should result in different values obtained from both sides. We studied if a significant side difference exists in MLAEP $(\mathrm{Pa}, \mathrm{Nb})$ and $\mathrm{BIS}$ obtained bilaterally from patients with unilateral brain lesions.

Materials and Methods: BIS and MLAEP were measured bilaterally in eight sedated patients with unilateral supratentorial intracranial haemorrhage using the BIS- and AEP-module of the (S/5) monitor (GE, Helsinki, Finland). The mean difference (bias) of the measurements of both sides was calculated (Bland-Altman-Statistic). Significance of differences was calculated by paired t-Test ( $p<0.05$ was regarded as significant).

Results and Discussions: Nb-Latency was not measurable in two patients. A significant difference of mean values of BIS existed between the side of the lesion (32 \pm 5$)$ and the contrary side $(40 \pm 7, \mathrm{p}=0.011)$. Also, $\mathrm{Nb}-$ latency showed lower values on the side of the lesion $(55 \pm 18$ vs. $58 \pm 17$, $\mathrm{p}=0.045$ ). There was no significant difference between values of $\mathrm{Pa}$ $(37 \pm 18, p=0.32)$. Mean bias of values was $1.5 \%$ (SD 3.6) for $\mathrm{Pa}$ latency, $-7 \%$ (SD 5.2) for Nb-latency and $-18 \%$ (SD 17.0) for BIS, suggesting that $\mathrm{Pb}$ and $\mathrm{Nb}$ are less affected by intracranial lesions than BIS.

Conclusions: If BIS is used for measurement of depth of sedation in patients with unilateral intracranial lesions, the unaffected side should be preferred. BIS values regarded as guidelines for estimation of depth of sedation in patients without brain pathology cannot be used for patients with intracranial lesions.

Reference:

Newton DEF. Electrophysiological monitoring of general intensive care patients. Intensive Care Med. 1999;25:350-352.

\section{AP4-2}

Stress measured by biopyrrin, an oxidative substance of bilirubin, in two groups who underwent different neurosurgical procedures

H.Dohgomori, S.Nagata, R.Kanemaru, A.Kawanishi, K.Tanioka

\section{Division of Emergency Medicine, Shinshu University Hospital, Matsumoto,} Japan

Background and Goal of Study: Urine biopyrrin (BYP) reveals the degrees of oxidant stress and various kinds of stress. The purpose in the present study was to evaluate the stress between two groups of patients who underwent different neurosurgical procedures by measuring BYP values.

Patients and Methods: After obtaining institutional approval and informed consent, 15 patients were enrolled in the present study. Patients were divided into two groups: patients who underwent cranial surgical procedures (G-A) and those who underwent interventional radiology (IVR) to treat their cranial diseases (G-B). BYP values were measured three times in each patient: 1 ) on the morning before surgery $(\mathrm{T}-1)$; 2) the first postoperative day (T-2); and 3) the third or fourth postoperative day ( $\mathrm{T}-3)$. The urine samples were frozen and measured later, using the Biopyrrin EIA Kit produced by Shino-test Ltd. Urine creatinine was measured at the same time. The BYP data (micromol/gCre) were calculated as the BYP values divided by the urine creatinine values. All values were expressed as means. Statistical analysis was performed using repeated measurements analysis (ANOVA) with Scheff's test.

Results and Discussion: In G-A, nine patients (6 males) were enrolled with a mean age of $63.9 \mathrm{yr}$ (range 39-74 yr). Six patients formed the G-B group (5 males), with a mean age of $72.9 \mathrm{yr}$ (range 55-78 yr). BYP values at times T1, T2 and T3 were as follows for groups $A$ and $B$, respectively: 3.1, 6.2, 5.4 $(P=0.02)$; and 3.1, 4.2, $3.8(P=0.195)$. There was no significant difference between the two groups $(P=0.06)$. Biopyrrin is formed from the oxidation of bilirubin. It reveals the cascade condition of the bilirubin metabolism. Some studies reported that it also revealed the degree of stressed condition and increased even with surgical stress. Generally, craniotomy is thought to be more a more invasive and stressful procedure compared with less invasive surgical maneuvers, such as IVR. In the study, we could not find any difference between the two groups, but a change was noted in more stressful G-A subjects.
Conclusions: BYP levels might reveal different levels of surgical stress and be useful in evaluating various kinds of surgical stress. Further studies are warranted.

\section{AP4-3}

Metabolic acidosis induced by topiramate in neurosurgery

L.Rodriguez, S.Fernández, R.Valero, J.Tercero, N.Fàbregas

Anesthesiology, Hospital Clinic de Barcelona, Barcelona, Spain

Background and Goal of Study: Topiramate, a new generation antiepileptic drug, can produce asymptomatic metabolic acidosis ${ }^{1}$ through carbonic anhydrase isoenzymes inhibition. ${ }^{2}$ Intraoperative decompensation could be missed without blood gas control.

Materials and Methods: Intraoperative blood gas analysis after anesthesia induction was performed on every patient operated on brain surgery between 2004 and 2006. Patients with renal disease were excluded. Finally 320 patients were included. Surgery was performed under propofol/remifentanyl (TCl) and rocuronium, with standard monitoring plus invasive arterial pressure. Results and Discussions: 5 patients had severe intraoperative metabolic acidosis not previously known.

\begin{tabular}{lllllr}
\hline & $\mathrm{pH}$ & $\mathrm{pO}_{2}$ & $\mathrm{pCO}_{2}$ & $\mathrm{HCO}_{3}{ }^{-}$ & $\mathrm{BD}$ \\
\hline Case 1 & 7,26 & 190 & 42 & 19 & 7,5 \\
Case 2 & 7,25 & 328 & 34,9 & 15,6 & 11,2 \\
Case 3 & 7,29 & 296 & 39 & 18 & 7,4 \\
Case 4 & 7,31 & 346 & 37,1 & 18,6 & 7,3 \\
Case 5 & 7,32 & 348 & 32,7 & 16,6 & 8,3 \\
mean & 7,286 & 301,6 & 37,14 & 17,56 & 8,34 \\
SD & 0,030 & 65,778 & 3,599 & 1,424 & 1,647 \\
\hline
\end{tabular}

2 male 3 female, aged 33,6 $\pm 4,6$ years, 3 of them scheduled for surgery of epilepsy, one for pituitary gland's adenoma resection, and one for resection of arteriovenous malformation. All of them were receiving topiramate preoperatively. We couldn't found any other cause of acidosis. All were treated with sodium bicarbonate iv, with partially correction of metabolic disorder. In case 1, differential diagnose with propofol infusion syndrome was made (no CK or lactate serum levels elevation). Postoperatively low bicarbonate persisted but $\mathrm{pH}$ normalized on regaining spontaneous ventilation.

Conclusion(s): Chronic Topiramate intake can produce compensated metabolic acidosis. Acute decompensation can be triggered by mechanical ventilation. We have to be aware of this side effect, and routinely perform blood gas analysis in these patients undergoing surgical procedures in order to normalize intraoperative homeostasis.

References:

1 Paediatr Anaesth 15(2):167-70, 2005.

2 Epilepsia 41 (suppl 1) S35-39, 2000

\section{AP4-4}

Influence of the sevoflurane on spontaneous bioelectric brain activity in neurosurgical patients

D.Moshchev, O.Sazonova, A.Ogurtsova, A.Lubnin

Neuroanesthesiology, Burdenko Neurosurgery Institute, Moscow, Russian Federation

Background and Goal of Study: The electrophysiologic effects of sevoflurane (S) are not well characterized in patients with intracranial pathology. There is no unequivocal opinion on safety of the use of $S$ in neurosurgical patients with epileptic seizures: $S$ can exert both anti - and proconvulsant's properties. The expiratory concentration of the $S$ is important determinator of this influence. The aim of the our research was the investigation of influence of $S$ on bioelectrical brain activity in neurosurgical patients with focal cerebral lesions.

Materials and Methods: We examined 41 patients: 36 with supratentorial hemispheric tumors ( 26 with seizure activity on preoperative EEG and 10 without) and 5 with brain arteriovenous malformations (AVM) with epileptic activity on EEG.

Results and Discussions: $S$ causes a dose dependent depression pattern on EEG. EEG spike frequency increased in all patients during $S$ anesthesia compared with awake recordings. Compared with 1 MAC S, spike frequency was higher in all patients during 1.5 MAC S.

$S$ provoke high spike activity with 2 MAC especially in patients and AVM with epileptic seizures.

Conclusions: 1 . S causes a dose-dependent depression pattern on EEG. 2. $\mathrm{S}$ can be used in the patients with epilepsy caused by organic brain lesions 
without risk of development of seizures during and after surgery. 3. Careful attention should be paid to the concentration of $S$ in patients with AVMinduced epileptic seizures.

\section{AP4-5}

Quantitative evaluation of cerebrovascular reactivity by brain arterial and internal-jugular vein differences: a comparison with Doppler sonography

K.McGrattan, P.Ainslie, L.Celi, C.Murrell, K.Peebles

Physiology, School of Medical Science, University of Otago, Dunedin, New Zealand

Background and Goal of Study: To verify whether $\mathrm{CO}_{2}$ reactivity in the middle cerebral artery (MCA), as assessed with Transcranial Doppler ultrasound, adequately reflects cerebral blood flow (CBF) reactivity in healthy humans.

Materials and Methods: Twelve healthy individuals [aged $27 \pm 4$ (mean $\pm \mathrm{SD})$ ] volunteered for this study; all were non-smokers without history of disease. Changes in velocity in the MCA (MCAv) were compared with changes in CBF as assessed by measurements of the difference in the reciprocal of the arterial (radial) and venous (internal jugular vein) content in oxygen (1/AVDO ${ }_{2}$ ) during acute changes in $\mathrm{PaCO}_{2}$. Incremental hypercapnic steps (4-min) were made during inhalations of $4 \%$ and $8 \% \mathrm{CO}_{2}$. Participants were then instructed to hyperventilate, to generate two levels (4-min each level) of incremental hypocapnia to match, in an equal and opposite direction, the rise in end-tidal $\mathrm{CO}_{2}$ incurred during the hypercapnia steps. Cerebrovascular reactivity to $\mathrm{CO}_{2}$ was expressed as the percentage (\%) change in MCAv or CBF (i.e., 1/AVDO ${ }_{2}$ ) per $\mathrm{mmHg}$ change in $\mathrm{PaCO}_{2}$. Results: Over the range of $\mathrm{PaCO}_{2}$, the change in $\mathrm{MCAv}$ and $1 / \mathrm{AVDO}_{2}$ were highlight related $(r=0.80 ; P<0.05) . \mathrm{CO}_{2}$ reactivity was not different when calculated using the change in MCAv or change in $1 / \mathrm{AVDO}_{2}$ $(3.8 \pm 1.2 \% / \mathrm{mmHg}$ vs. $3.2 \pm 1.8 \% / \mathrm{mmHg}$, respectively, $\mathrm{P}>0.05)$

Conclusion(s): MCAv variations adequately reflect $\mathrm{CBF}$ and $\mathrm{CO}_{2}$ reactivity changes as compared with $\mathrm{AVDO}_{2}$ measurements, indicating that marked variations in $\mathrm{PaCO}_{2}$ and $\mathrm{CBF}$ do not act noticeably on the diameter of the MCA. These results may serve as the point of reference for 'normal' responses for comparison against patients with neurological disorders.

\section{AP4-6}

\section{Bispectral index monitoring in traumatic brain injury patients}

R.Badenes, ML.García-Pérez, A.Maruenda, G.Aguilar, FJ.Belda

Anesthesiology and Intensive Care, Hospital Clínico Universitario de Valencia., Valencia, Spain

Background and Goal of Study: Maintaining an optimal level of comfort and safety for critically ill patients is an universal goal for critical practitioners. The assessment of sedation level remains a challenge for the intensivist in order to avoid over - or under-sedation phenomena. The introduction of the bispectral index (an EEG parameter) could bring potential advantages in monitoring sedation. According to the reports, the Richmond Agitation-Sedation Scale (RASS) has been shown to be highly reliable among multiple types of health care professionals. The RASS has an expanded set of scores at pivotal levels of sedation that are determinated by patients' response to verbal vs physical stimulation, which will help the clinician in titrating medication. Materials and Methods: This is a prospective, nonrandomized, observational study in a surgical and trauma tertiary intensive care of an universitary hospital. Twenty-six consecutive traumatic brain injury patients (age range 17-68 yrs, mean age $42 \mathrm{yrs}$ ) were included. They were sedated (with propofol by continuous infusion at an initial dose of $2 \mathrm{mg} / \mathrm{kg} / \mathrm{h}$, which could be modulated with steps of $0.5 \mathrm{mg} / \mathrm{kg} / \mathrm{h}$ ), in order to mantain an adequated RASS score. BIS value was continuously recorded, and manually calculated on a mean average of a minute during the measuring of RASS score, and every $10 \mathrm{~min}$ utes for 6 hours on par with RASS score. ECG, $\mathrm{SpO}_{2}$, invasive arterial pressure, ventilatory module, $\mathrm{ET}_{\mathrm{CO}}, \mathrm{FiO}_{2}$, temperature were also recorded. For the statistic analysis, Friedman test and Spearman coefficient were utilized. Results and Discussions: Nine hundred and thirty-six observations were carried out. The variation range of RASS score was between 0 and -5 . BIS range varied from 27 to 96 . Statistics analysis of the data obtained pointed out a significative correlation between RASS score and BIS $(p<0,01)$.

Conclusion(s): According to the reports, bispectral index correlates with levels of sedation on the sedation scales. In our personal experience, this study demonstrates the utility of BIS and RASS score to track levels of sedation in traumatic brain injury patients.

References:

Am J Crit Care 12(4): 343-8 2003.

Curr Opinion Crit Care 10(1): 40-46 2004

\section{AP4-7}

Monitoring of anaesthesia depth with entropy modul allows reduction of sevofluran consumption in patient undergoing craniotomy for intracranial tumor surgery

G.Banevicius, D.Bilskiene, A.Karbonskiene

Anaesthesiology clinic, Kaunas university of medicine, Kaunas, Lithuania

Background and Goal of Study: Balanced anaesthesia with sevofluranfentanyl has been the most frequently used for intracranial surgery. The study was to determine the role of anaesthesia depth monitoring to requirement of sevoflurane for maintaining of anaesthesia in patient undergoing craniotomy for intracranial tumors.

Materials and Methods: 80 patients with ASA I-III undergoing scheduled surgery for intracranial tumors, were enrolled into the study and randomly divided into equal groups: group $\mathrm{E}$ with entropy monitoring and group $\mathrm{O}-$ without it. Anaesthesia was maintained using sevofluran with Et-Sevo of $1,5-2,0 \%$, maintaining MAP within $20 \%$ of preinduction baseline in group $O$ and sensory entropy of 40-60 in group E. Standard monitoring of vital signs was performed. Et-Sevo, state entropy (SE) and response entropy (RE) data were recorded after induction (1), craniotomy (2), tumor removal (3) and at the end of operation (4) (Table 1).

Results: There were no differences between the groups with respect to demographic characteristics. Mean anaesthesia time in group $E$ was $241,6 \pm 74,2 \mathrm{~min}$ (mean $\pm \mathrm{SD}$ ) and $263 \pm 63,1 \mathrm{~min}$ in group O. Data (Mean \pm SD) are shown in the table 1 :

Table 1

\begin{tabular}{lrrrr}
\hline & \multicolumn{1}{l}{1} & \multicolumn{1}{l}{ 2 } & \multicolumn{1}{l}{4} \\
\hline Et-Sevo O & $0.81 \pm 0.16$ & $1.43 \pm 0.17$ & $1.41 \pm 0.15$ & $1.40 \pm 0.14$ \\
Et-Sevo E & $0.48 \pm 0.21$ & $0.75 \pm 0.25$ & $0.79 \pm 0.28$ & $0.70 \pm 0.27$ \\
RE E & $43.48 \pm 8.15$ & $42.45 \pm 7.26$ & $42.53 \pm 7.47$ & $46.53 \pm 10.43$ \\
SE E & $41.15 \pm 8.87$ & $40.48 \pm 6.87$ & $40.20 \pm 6.54$ & $43.43 \pm 9.31$ \\
\hline
\end{tabular}

In the group without monitoring of entropy dose of sevofluran was significant higher $(p<0,05)$.

Conclusions: Monitoring of anaesthesia depth with entropy allows reduce requirement of sevoflurane for maintaining of anaesthesia in patient undergoing craniotomy for intracranial tumor surgery without risk of awareness.

\section{AP4-8}

Monitoring of regional cerebral oxygen saturation: a supplementary tool for management of ventilation in patients with chronic obstructive pulmonary diseases

P.Delgado, G.Schwarz, G.E.Klein

Anesthesiology and Critical Care, Medical University of Graz, Graz, Austria Background and Goal of Study: In specific patients with chronic obstructive pulmonary disease (COPD) the cerebrovascular response to partial carbon dioxide pressure $\left(\mathrm{PaCO}_{2}\right)$ may be altered in unknown extent. Therefore the mechanical ventilation for these patients is not easy to adapt adequately and shows a wide interindividual variation. Regional cerebral oxygenation $\left(\mathrm{rSO}_{2}\right)-$ monitoring was used for detection of cerebral oxygen desaturation events. Materials and Methods: We present 3 patients with COPD (GOLD-criteria(1)) receiving total intravenous anesthesia for neurointerventional procedures. $\mathrm{rSO}_{2}$ - monitoring was performed with an INVOS 5100 cerebral oximeter (Somanetics). A decrease of $\mathrm{rSO}_{2}(>19 \%)$ compared with the baseline values before induction of anesthesia was defined as significant. After adjusting all conventional vital parameters as recommended in our NIRS matrix(2) the mechanical ventilation was reduced and $\mathrm{PaCO}_{2}$ increased in the initial normocapnic patients (Table) until $\mathrm{rSO}_{2}$ values returned near to the baseline. Results and Discussions: Table: Parameters at the time of minimal $\mathrm{rSO}_{2}$ values $\left(T_{0}\right)$ and at return of $\mathrm{rSO}_{2}$ close to baseline values $\left(T_{1}\right)$

\begin{tabular}{|c|c|c|c|c|c|}
\hline & & P 1 & $\mathrm{P} 2$ & P 3 & Abbreviations \\
\hline \multirow[t]{5}{*}{$\mathrm{T}_{0}$} & $\Delta \mathrm{rSO}_{2}$ & -25 & -22 & -19 & P: patient; \\
\hline & etCO ${ }_{2}$ & 36 & 41 & 37 & $\begin{array}{l}\Delta \mathrm{rSO}_{2} \text { : difference of } \\
\mathrm{rSO}_{2} \text { related to } \\
\text { baseline (\%); }\end{array}$ \\
\hline & MAP & 90 & 73 & 80 & $\begin{array}{l}\text { etCO }{ }_{2} \text { : end expiratory } \\
\text { carbon dioxide; }\end{array}$ \\
\hline & $\mathrm{SaO}_{2}$ & 100 & 89 & 100 & $\begin{array}{l}\text { MAP: mean arterial } \\
\text { pressure; }\end{array}$ \\
\hline & $\mathrm{FiO}_{2}$ & 80 & 80 & 60 & $\begin{array}{l}\mathrm{SaO}_{2} \text { : peripheral } \\
\text { oxygen saturation; }\end{array}$ \\
\hline \multirow[t]{5}{*}{$\mathrm{T}_{1}$} & $\Delta \mathrm{rSO}_{2}$ & +3 & -2 & 0 & $\begin{array}{l}\mathrm{FiO}_{2} \text { : inspiratory } \\
\text { oxygen fraction }\end{array}$ \\
\hline & etCO $_{2}$ & 44 & 46 & 42 & \\
\hline & MAP & 80 & 84 & 80 & \\
\hline & $\mathrm{SaO}_{2}$ & 98 & 91 & 99 & \\
\hline & $\mathrm{FiO}_{2}$ & 55 & 90 & 50 & \\
\hline
\end{tabular}


Conclusion(s): In specific COPD-patients the NIRS monitoring appears to be a very helpful tool for correction of the mechanical ventilation in order to avoid cerebral oxygen desaturation events.

References:

1 GOLD Update 2006 (www.goldcopd.org)

2 Schwarz et al. Neurol Res 2005; 27: 423-428

\section{AP5-1}

Changes of somatosensory evoked potentials during graded spinal cord ischemia/reperfusion injury in rabbits

H. Fang, Q Y. Wang, C X. Li, J. Liu, Y X. Zuo

Department of Anesthesiology, West China Hospital, Sichuan University, Chengdu, China

Background and Goal of Study: Ischemia and reperfusion of the spinal cord induce one of the most common complications after surgery for paraplegia(1). Investigation on the etiology and the intraoperative monitoring and prevention of the spinal cord injuries will be important(2).

Materials and Methods: Thirty rabbits were randomized into spinal cord ischemia-reperfusion groups by inflating the balloon of a $4 \mathrm{~F} \mathrm{Swan-Ganz}$ catheter positioned in the infrarenal abdominal aortic for 30 minutes, 45 minutes and 60 minutes. SEP was monitored before ischemia, 5 minutes, 10 minutes after ischemia, 15 minutes, 30 minutes, 1 hour, 2 hours after reperfusion. Neurologic function score(NFS)was evaluated at 6 hours, 12 hours, 24 hours and 48 hours after reperfusion. The pathological changes of spinal cord were observed after reperfusion 48 hours.

Results and Discussions: The pathological characters with mild,moderate and severe spinal cord ischemia/reperfusion injury could be simulated by deflating after 30 minutes, 45 minutes and 60 minutes infrarenal abdominal aortic blockading. SEP amplitude returned to normal after reperfusion 15 minutes $(P>0.05)$ and SEP latency returned to normal after reperfusion 30 minutes $(P>0.05)$ in ischemia 30 minutes group. SEP amplitude returned to normal after reperfusion 30 minutes $(P>0.05)$ and SEP latency returned to normal after reperfusion 60 minutes $(P>0.05)$ in ischemia 45 minutes group. SEP latency increased and SEP amplitude decreased in ischemia 60 minutes group, compared with other groups, there were significant differences in SEP latency and SEP amplitude $(P<0.01)$. With graded spinal cord ischemia/ reperfusion injury,compared with ischemia 30min group, spinal cord ischemiareperfusion groups had significant differences in NFS $(P<0.01)$.

Conclusion(s): 1) SEP was much quicker in the recovery of interpeak amplitude than onset latency during spinal cord ischemia/reperfusion. 2)SEP is a sensitive and accurate index for spinal cord function during ischemia/reperfusion injury. 3) SEP monitoring spinal cord ischemia/reperfusion injury during operation provides experimental basis for clinical application.

References:

1 Gloviczki P.Cardiovasc Surg 2002; 10: 434-441.

2 Wan IY. Eur J Cardiothorac Surg 2001; 19: 203-213.

\section{AP5-2}

\section{Xenon reduces synaptic transmission in the mouse} amygdala, prefrontal and somatosensory cortex but does not affect synaptic transmission in the motor cortex R. Haseneder, S. Kratzer, W. Zieglgaensberger, E. Kochs, G. Rammes Klinik für Anaesthesiologie, Klinikum rechts der Isar der Technischen Universitaet Muenchen, Munich, Germany

Background and Goal of Study: Xenon (Xe) displays anaesthetic and analgesic properties. The neuronal and molecular mechanisms are not yet fully understood (1). Our current knowledge is largely based on studies using neurons in culture (2) or heterologous expression systems (3). We compared the effect of $\mathrm{Xe}$ on the synaptic transmission in different brain areas in brain slice preparations of the mouse.

Materials and Methods: Coronar and sagital brain slices were obtained from male mice (BI6; $d 28-35)$. Neurons in the basolateral amygdala, laminae II/III and $\mathrm{V}$ of prefrontal cortex, lamina II/III of somatosensory cortex and lamina II/III of motor cortex were identified by infrared-phase contrast-enhanced videomicroscopy. Patch clamp technique was used to record postsynaptic currents (PSC). Under control conditions, the slices were kept in artificial cerebro-spinal fluid (ACSF) gassed with $65 \% \mathrm{~N}_{2} / 30 \% \mathrm{O}_{2} / 5 \% \mathrm{CO}_{2}$. For xenon application, the ASCF was gassed with $65 \% \mathrm{Xe} / 30 \% \mathrm{O}_{2} / 5 \% \mathrm{CO}_{2}$.

Results: Xe reduced PSC recorded in the basolateral amygdala, laminae II/III and V of prefrontal cortex, and lamina II/III of somatosensory cortex to $80.8 \pm 5.3 \%, 70.5 \pm 12.6 \%, 70.1 \pm 10.2 \%$, and $86.1 \pm 7.2 \%$ of control, respectively ( $p<0.05 ; n=3-6$ for each brain region). Upon washout of Xe, the responses recovered to control level. Xe had no distinct effect on PSC recorded in lamina II/III of the motor cortex (99.2 \pm 9.8 of control; $p>0.05$; $\mathrm{n}=4$ ).

Conclusion(s): Xenon differentially affected synaptic transmission in the tested brain areas. Xenon had no effect in neurons of the motor cortex. In contrast, synaptic transmission was markedly reduced in various brain areas processing sensory information. This site-dependent effect might contribute to the anaesthetic mechanisms of xenon.

References:

1 Preckel B, Weber NC, Sanders RD, et al. Anesthesiology 2006; 105: 187-97.

2 De Sousa S, Dickinson R, Lieb WR, et al. Anesthesiology 2000; 92: 1055-66.

3 Yamakura T, Harris RA Anesthesiology 2000; 93: 1095-1101.

\section{AP5-4}

Effect of propofol on excitatory amino acid accumulation for ischemia-reperfusion injury to spinal cord in rabbits

Q. Wang, Y. I. Zhang, H. Fang, Y. X. Zuo, J. Liu

Department of Anesthesiology, West China Hospital, Sichuan University, Chengdu, China

Background and Goal of Study: The spinal cord ischemia-reperfusion injury is the principal reason of secondary spinal cord injury which could lead to neuron damage and extremity disfunction(1).Propofol has neuroprotective effects against ischemia and that this neuroprotective effects has great relationship with excitatory amino acids in rat brain(2).

Materials and Methods: Seventy healthy adult New Zealand white rabbits were randomly divided into 7 groups (Groups N, A to F). Under general anesthesia, the infrarenal circumaortic clamping model was used.Normal saline, $10 \%$ intralipid, propofol $30 \mathrm{mg} / \mathrm{kg}$, propofol $40 \mathrm{mg} / \mathrm{kg}$, propofol $50 \mathrm{mg} / \mathrm{kg}$, and propofol $60 \mathrm{mg} / \mathrm{kg}$ were given in Group A to Group F, respectively. Ischemia lasted for $30 \mathrm{~min}$. Normal saline,10\% intralipid and propofol were continuously infused through a catheter into the aorta distal to the clamping site at a speed of $12 \mathrm{ml} / \mathrm{kg} / \mathrm{h}$ during the ischemia period.Concentrations of excitatory amino acids (aspartate and glutamate) of the lumbar spinal cord were measured after $48 \mathrm{~h}$ of reperfusion with the high performance liquid chromatography.

Results and Discussions: Data (Mean \pm SD, $n=10$ ) are shown in the table:

\begin{tabular}{|c|c|c|}
\hline Group & Glutamate $\left({ }^{*} 10^{-6} \mathrm{mmol} / \mathrm{g}\right)$ & Aspartate $\left({ }^{*} 10^{-6} \mathrm{mmol} / \mathrm{g}\right)$ \\
\hline $\mathrm{N}$ & $1962.5695 \pm 122.2719$ & $791.4751 \pm 75.7955$ \\
\hline A & $6489.2905 \pm 382.6102 \boldsymbol{\Delta}$ & $3358.7695 \pm 263.1474 \boldsymbol{\Lambda}$ \\
\hline B & $6294.6118 \pm 249.5627 \boldsymbol{\Delta}$ & $3190.8310 \pm 347.0592 \boldsymbol{\Delta}$ \\
\hline C & $4810.8660 \pm 711.6919 \boldsymbol{\Delta} \#^{\star}$ & $2493.7772 \pm 237.9328 \boldsymbol{\Delta} \#^{\star}$ \\
\hline D & $3945.2200 \pm 417.7513 \boldsymbol{\Delta} \#^{*}$ & $1922.1510 \pm 239.1342 \mathbf{\Delta} \#^{*}$ \\
\hline $\mathrm{E}$ & $3026.2863 \pm 246.5196 \boldsymbol{\Delta} \#^{*}$ & $1365.5351 \pm 120.6491 \mathbf{\Delta} \#^{*}$ \\
\hline $\mathrm{F}$ & $3930.1598 \pm 332.3011 \mathbf{\Delta} \#^{*}$ & $1908.1174 \pm 140.4767 \boldsymbol{\Delta} \#^{*}$ \\
\hline
\end{tabular}

$\Delta P<0.05$ vs group $\mathrm{N}$; $\# P<0.05$ vs group $\mathrm{A}$; ${ }^{*} P<0.05$ vs group $\mathrm{B}$

Conclusion(s): Propofol can provide a protective effect against an ischemiareperfusion injury to the spinal cord in rabbits: 1) This neurological protection may be due to the acceleration of excitatory amino acid elimination in the reperfusion; 2) The protective effect is parallel with the dose of propofol.

References:

1 Back MR.Ann Vasc Surg 2005; 19: 648-656.

2 Hans P.J Neurosurg Anesthesiol 1994; 6: 249-253.

\section{AP5-5}

Cross-approximate-entropy as a measure of isofluraneinduced changes in recordings of neuronal population signals in vivo

M. Kreuzer, C. Schwarz, G. Schneider, H. Hentschke, E.F. Kochs

Department of Anaesthesiology, Technische Universitä München, Munich, Germany

Background and Goal of Study: Cross-Approximate-Entropy (XApEn) is a parameter designed to analyse multivariate data sets. It was used to evaluate changes in field potential activity, recorded from multi-channel electrode arrays placed in the barrel cortex of rats, at increasing levels of isoflurane. Materials and Methods: Electrode arrays were implanted through a craniectomy over the right hemisphere into the somatosensory ('barrel') neocortex of 3 Sprague Dawley rats. Field potential activity was recorded at 3, 8 or 14 channels at increasing levels of isoflurane [0, 0.3, 0.53, 0.75, 1.45 MAC]. For every combination of channels, a XApEn value was calculated. 
The Cross-Approximate-Entropy is a modification of the Approximate Entropy and can be used to quantify asynchrony between different channels [1] i.e. signals from different sites within the neural network. A decrease in the XApEn value represents a lower degree of "chaos in the signal".

Results and Discussions: The XApEn decreased with increasing concentrations of the volatile anaesthetic, indicating that the signals were more synchronised across all recording sites. The pattern of XApEn was inhomogeneous, featuring a number of channel combinations with low values. This pattern of inhomogenities, pointing to particularly synchronous activities of the neural populations at the corresponding recording sites, did not change significantly with isoflurane.

Conclusion: Our results indicate that isoflurane fosters synchronous activity between neuronal populations in somatosensory cortex without causing major disturbances of inter-site relationships.

Reference:

1 Pincus et al, Proc. Natl. Acad. Sci., 1996, 93, 14100-14105.

\section{AP5-6}

Protective effects of sevoflurane preconditioning on OxygenGlucose Deprivation injury. Role of reactive oxygen species and adenosine triphosphate-regulated potassium channels

L. Velly, B. Guillet, P. Canas, P. Pisano, N. Bruder

Bouches du Rhone, DAR Adultes, CHU Timone, Marseille, France

Background and Goal of Study: The purpose of the present study was first to compare the extent of sevoflurane-induced neuroprotection on primary cortical cells cultures subjected to transient oxygen-glucose deprivation (OGD) either when applied during or before (preconditioning) the insult. Secondly, the possible involvement of intracellular reactive oxygen species (ROS) levels and $K_{\text {ATP }}$ channels in the mechanism of the preconditioning effect of sevoflurane (SEVO) was explored.

Materials and Methods: Mature primary cortical neuronal-glial cultures were exposed to $90 \mathrm{~min} O G D$ followed by reoxygenation. Culture were randomly exposed to SEVO either during OGD without preconditioning or during a $90 \mathrm{~min}$ preconditioning period followed by a $60 \mathrm{~min}$ wash-out period before OGD. 2-mercaptopropionyl glycine (2MPG) a free radical scavenger or glibenclamide (GLB) a blocker of $\mathrm{K}_{\text {ATP }}$ channels were added during the preconditioning. Twenty-four hours after the injury, neuronal death was quantified by lactate dehydrogenase (LDH) release into the media. Free radical generation in cells was assessed after preconditionning period using $2^{\prime}, 7^{\prime}$-dichlorofluorescin diacetate (DCFH-DA). Data are presented as mean \pm SD. Statistical analysis used ANOVA.

Results and Discussions: Twenty-four hours after our cell cultures were subjected to $90-\min$ OGD, $78.5 \pm 4.4 \%$ of the neurons died. SEVO added at the start of the injury, elicited a potent and dose-dependent neuroprotective effect. SEVO preconditioning elicited a threshold neuroprotective effect at concentrations higher than $0.03 \mathrm{mM}$. In the presence of $2 \mathrm{MPG}(100 \mu \mathrm{M})$ or GLB $(0.3$ $\mu \mathrm{M})$ sevoflurane lost its preconditioning effect as assessed by LDH test. In normoxic cultures preconditioned by SEVO $(3.4 \mathrm{mM})$, DCFH-DA fluorescence intensity increased in a significant manner compared to sham wash cultures. The SEVO preconditioning-induced increase in ROS levels was inhibited by 2MPG and GLB.

Conclusions: Sevoflurane preconditions neuronal-glial cell cultures against OGD by mechanisms that apparently involve release of ROS and $\mathrm{K}_{\text {ATP }}$ channels.

\section{AP5-7}

Protective effect of sevoflurane-induced preconditioning on focal cerebral ischemia in rats

L. Velly, JL. Codaccioni, B. Guillet, P. Pisano, N. Bruder

Bouches du Rhone, DAR Adultes, CHU Timone, Marseille, France

Background and Goal of Study: A number of investigations have shown that volatile anesthetics possess direct neuroprotective effects during ischemic cerebral injury and can mimic ischemic preconditioning in myocardium. However, direct neuroprotective effects have been shown to be transient. The aim of this study was to demonstrate sevoflurane-induced preconditioning effect on in vivo focal cerebral ischemia in rats and whether any preconditioning effect is evident after long term survival.

Materials and Methods: 69 male Sprague-Dawley rats $(250 \mathrm{~g})$ were randomly allocated in 2 groups: Control group $(\mathrm{C} ; \mathrm{n}=35)$ non-preconditioned and a preconditioning group ( $\mathrm{P} ; \mathrm{n}=34$ ) exposed to 2.7 vol\% sevoflurane ( $45 \mathrm{~min}$ ) $60 \mathrm{~min}$ before surgery. Animals in both groups were anesthetized with 3.0 vol\% sevoflurane and subjected to transient middle cerebral artery occlusion (MCAO) by use of the intraluminal filament model. Sevoflurane administration was discontinued as soon as the MCAO was initiated and the animals were allowed to awaken. After $60 \mathrm{~min}$ of focal ischemia the filament was removed. Neurologic function (Longa's score) and infarct volumes (Nissl-staining) were determined at 3 days $(C, n=22 ; P, n=18), 7$ days $(C, n=9 ; P, n=10)$, and 14 days $(C, n=8 ; P, n=10)$ after ischemia. Data are presented as mean \pm SD. Statistical analysis used ANOVA.

Results and Discussions: Tree days after ischemic insult, we observed that the percentage of rats with severely impaired neurological score (C: $22.7 \%$ vs $\mathrm{P}: 0 \%, P<0.05)$ and infarct volumes (C: $137 \pm 66 \mathrm{~mm} 3$ vs $\mathrm{P}: 84 \pm 47 \mathrm{~mm}$, $P<0.05)$ were significantly higher in the control group. A mean infarct volume decrease of $39 \%$ was measured in the preconditioned group. However, these differences were absent after a recovery period of 7 or 14 days. Infarct volumes in control and preconditioning groups were not significantly different (Day 7: C: $81 \pm 85$ vs P: $79 \pm 64 \mathrm{~mm}^{3}$; Day 14, C: $138 \pm 24$ vs P: $127 \pm 71 \mathrm{~mm}^{3}$ ). Conclusions: These results suggested that sevoflurane-induced preconditioning in brain. However, protection afforded by sevoflurane preconditioning seems to be attenuated in late recovery periods suggesting that sevoflurane preconditioning delays but does not prevent cerebral infarction cause by focal ischemia.

\section{AP5-8}

\section{Pentobarbital inhibits the release of nitric oxide and the effect is antagonized by the application of neostigmine and magnesium-free perfusion in the rat striatum: an in vivo microdialysis study}

Y. Adachi, S. Mimuro, M. Doi, S. Sato

Intensive Care Unit of University Hospital, Hamamatsu University School of Medicine, Hamamatsu, Japan

Background and Goal of Study: Previously, we demonstrated that pentobarbital (PB) inhibits I-DOPA-induced dopamine (DA) increases in the rat striatum using in vivo microdialysis study (1). Barbiturates have possibility to modify DA feedback system. Recently, the important role of nitric oxide (NO) on the homeostasis of neurotransmitters, including DA, was suggested (2). In the current investigation, we studied the effect of PB on the extracellular concentration of $\mathrm{NO}$ and the relationship between $\mathrm{NO}$ and acetylcholine in the rat striatum.

Materials and Methods: Male Sprangue-Dawley rats, weighing 280-320 g, were used. Microdialysis probe was inserted into the right striatum and perfused with modified Ringer solution after recovering from the surgery. Samples were collected every $15 \mathrm{~min}$ and directly injected into an online analytical HPLC system. $\mathrm{NO}_{2}^{-}$and $\mathrm{NO}_{3}^{-}(\mathrm{NOX})$ were mixed with a Griess reagent to form a purple azo dye and the absorbance was measured by a flow-through spectrophotometer. The rats were freely moving and PB was administered intraperitoneally. Neostigmine was applied with perfusate.

Results and Discussions: Pentobarbital decreased $\mathrm{NO}_{3}^{-}$and NOX in a dose dependent manner. Perfusion with neostigmine both 1 and 10 microM showed no effect on NOX release, however, diminished PB-induced NOX decrease in low concentration and abolished it in high concentration. Magnesium-free perfusate showed no effect on NOX release, whereas, the perfusate antagonized pentobarbital-induced NOX reduction.
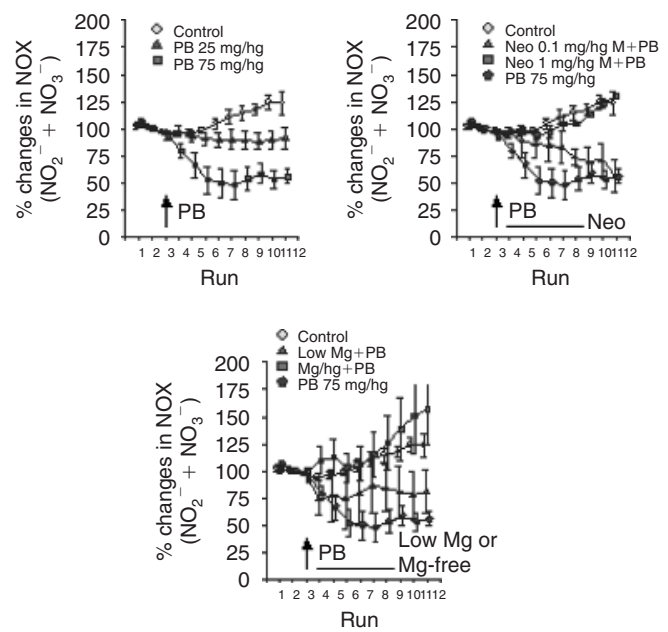

Fig. 1 The inhibitory effect of pentobarbital on nitric oxide release (left, PB: pentobarbital ip). The antagonistic effect of neostigmine (center, Neo: neostigmine perfusion) and of low magnesium perfusion (right, Low Mg: magnesium concentration in perfusate was a half of control, Mg-free: magnesium free perfusate). Data are expressed as Mean $\pm \mathrm{SE}$. 
Conclusion(s): The results of current investigation demonstrated that PB might decrease NO release by the reduction of acetylcholine concentration. In central nervous system, acetylcholine has an important role for NO and DA homeostasis.

References:

1 Adachi YU et al. Brain Res Bull 2006; 69: 593-6.

2 Kiss JP et al. Neurochem Int 2004; 45: 485-9.

\section{AP5-9}

Hypertonic-hyperoncotic solutionsand healthy brain cells

S. Himmelseher, Ch. Ament, C. Schaedeli, E. Pfenninger, E. Kochs Anesthesiology, Techn. Universitaet Muenchen, Munich, Germany

Background and Goal of Study: Hypertonic-hyperoncotic salinehydroxyethylstarch solutions (HHS) may help to control intracranial hypertension; however, no clinical study has so far shown an improved outcome after HHS (1). Because increased interstitial osmotic/oncotic pressures may jeopardize brain cells, long-term effects of HHS on healthy neurons and astroglia were studied.

Materials and Methods: Adult rat hippocampal neurons or astroglia were exposed to hypertonic, hyperoncotic or hypertonic / hyperoncotic medium (concentrations: 317,336 , or $377 \mathrm{mOsm}$, and $0.05 \%, 0.1 \%$, or $0.25 \% \mathrm{HES}$ ) for $15 \mathrm{~min}$ and studied over 1 week. The Mann-Whitney U-test with Bonferroni's correction compared control versus HHS-exposed cultures at a significance level of $p<.05$.

Results and Discussions: Viability of astroglia remained unchanged, but neuronal viability was reduced by $20 \%$ at 1 week after exposure to media with 377 mOsm. Water space of astroglia was decreased by approx. $80 \%$ at $1 \mathrm{~h}$ and $24 \mathrm{~h}$ after exposure to media with $377 \mathrm{mOsm}$. Water space of neurons was reduced by approx. $50 \%$ directly after exposure, increased by $180 \%$ at $1 \mathrm{~h}$, decreased by $50 \%$ at $24 \mathrm{~h}$, and re-increased by $140 \%$ at 7 days postexposure to media with the highest concentrations of HHS. Glucose uptake of astroglia decreased by approx. $60 \%$ at $1 \mathrm{~h}$ after exposure to media with $377 \mathrm{mOsm}$, whereas neuronal glucose uptake decreased by $60 \%$ at $1 \mathrm{~h}$, increased by $120 \%$ at $24 \mathrm{~h}$, and remained elevated by $80 \%$ at 7 days after exposure.

Conclusions: Hypertonic-hyperoncotic solutions (osmolarity $>350 \mathrm{mOsm}$ ) may reduce the viability of healthy neurons. Exposure to HHS induces volume regulatory processes in neurons and astroglia; volume perturbations in hippocampal neurons are more pronounced than in glia, and a long-lasting increase in glucose uptake predispose neurons to metabolic disturbances. Our data indicate that HHS may have a harmful potential for healthy neurons.

References:

1 Ogden AT et al. Neurosurgery 2005; 57: 207-215.

2 Strange K. Adv Physiol Educ 2004; 28: 155-159.

\section{AP5-10}

Hypertonic-hyperoncotic solutions and injured brain cells

S. Himmelseher, Ch. Ament, C. Schaedeli, E. Pfenninger, E. Kochs

Anesthesiology, Techn Universitaet Muenchen, Munich, Germany

Background and Goal of Study: Hypertonic-hyperoncotic salinehydroxyethylstarch solutions (HHS) have been used for rapid small-volume resuscitation in hypovolemia and hemorrhagic shock associated with brain trauma (BT) (1); however, prospective trials have failed to show better outcome after HHS. Because the induction of intracellular dehydration by HHS may especially endanger injured brain cells, the long-term response of traumatized neurons and astroglia to HHS were examined over 1 week.

Materials and Methods: To simulate traumatic forces of brain injury, adult rat hippocampal neurons or astroglia were first subjected to damage by ultrasound, and then exposed to hypertonic, hyperoncotic or hypertonic/hyperoncotic medium (concentrations: 317,336 , or $377 \mathrm{mOsm}$, and $0.05 \%, 0.1 \%$, or $0.25 \% \mathrm{HES}$ ) for $15 \mathrm{~min}$. The Mann-Whitney U-test corrected with Bonferroni's factor was used to compare injured controls versus injured and HHS-exposed cultures at a significance level of $p<.05$.

Results and Discussions: Seven days after exposure to media with $377 \mathrm{mOsm}$, astroglial viability decreased by approx. $20 \%$ and neuronal viability by approx. $35 \%$. Water space of glia was enhanced by approx. $90 \%$ at $24 \mathrm{~h}$ after exposure to media with the highest concentrations, and neuronal water space was increased by approx. $60 \%$ directly after the 15 -min exposure to the same media. Astroglial glucose uptake was reduced by approx. 50\% directly after exposure to media with $377 \mathrm{mOsm}$ and increased by approx. $140 \%$ at $1 \mathrm{~h}$ later; neuronal glucose uptake was enhanced by approx. $150 \%$ at $24 \mathrm{~h}$ after exposure to media with $377 \mathrm{mOsm}$.

Conclusions: Hypertonic-hyperoncotic solutions (osmolarity $>350 \mathrm{mOsm}$ ) may exacerbate the reduction of survival of traumatized astroglia and neurons.
Injured cells respond to HHS with impaired volume regulation capabilities, and especially neurons indicate disturbed metabolism. In addition to possibly damaging effects on injured brain, HHS may only cause cerebral stabilization when a substantial amount of functional astroglia is preserved. Our data agree with a recent study showing opposed effects of hypertonic saline on contusions and non-contused brain tissue in patients with BT (2).

References:

1 White $\mathrm{H}$ et al. Anesth Analg 2006; 102: 1836-1846.

2 Lescot T et al. Crit Care Med 2006; 34: 1-5.

\section{AP6-1}

\section{Selective brain lesions in aortic denervated vs. sham operated rats}

E. Amaniti, D. Kouvelas, C. Pourzitaki, D. Kapoukranidou, D. Vasilakos

Anaesthesiology, Ahepa University Hospital, Thessaloniki, Greece

Background and Goal of Study: Deep hypothermic circulatory arrest (DHCA) is used for the correction of heart abnormalities, although is often associated with neurologic morbidity. Apart from the ischemia, brain is also exposed to temporary inhibition of baroreceptor input during DHCA. It is possible that complications are partly related to baroreceptor input inhibition and glutamate excitotoxicity. Aim of the present study was the evaluation of potential brain damage after aortic denervation in rats.

Materials and Methods: 30 wistar rats were assigned into two groups to undergo either aortic denervation (AoDN group) or sham operation (SHAM group) under continuous blood pressure monitoring. Two hours after completion of the procedure, the rats were sacrificed and the brains were sectioned in specific levels corresponding to motor cortex, somatosensory cortex, dentate gyrus and cerebellar Purkinje cells. Rats were evaluated regarding blood pressure, blood pressure variability and numerical density of dead neurons. Differences between groups were evaluated using ANOVA, unpaired t-test and Mann Whitney $U$ test. Level of significance was set at $p<0.05$.

Results and Discussions: Groups were comparable regarding blood pressure and pressure variability. Percentages of dead neurons were significantly greater in AoDN group in motor cortex ( $p=0.003$ for layer MIII, $p=0.000$ for layer MV) somatosensory cortex ( $p=0.03$ for SV) but not in dentate gyrus and Purkinje cells $(p>0.05)$.

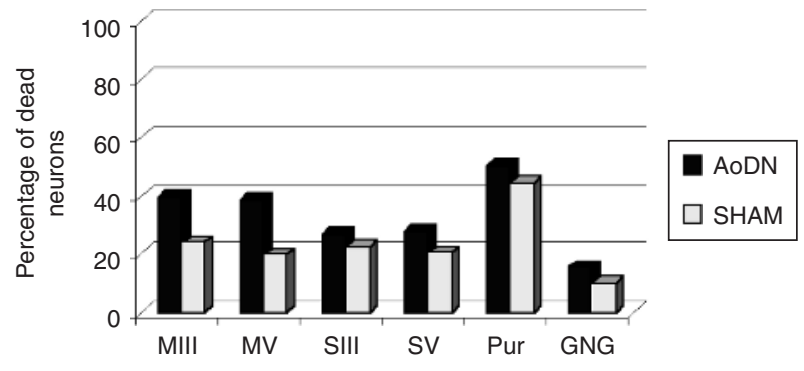

Fig 1. Percentages of dead neurons

Conclusion(s): Aortic denervation leads to neuronal damage in specific brain regions, possibly ascribed to excitotoxic phenomena induced by altered glutamate and GABA release.

Reference:

1 Singewald N et al. Trends Pharmacol Sci. 1996; 10: 356-363.

\section{AP6-2}

Effect of ketorolac in Spinal Cord Ischemic injury in rats: the role of Glutamate Transporters and inducible Nitric Oxide Synthase

Y. Hsieh, Y. Wang, K. Chan, C. Wong

Nociceptive Signal Transduction Laboratory, Department of Anesth, Tri-Service General Hospital and National Defense Medical Center, Taipei, Taiwan

Background and Goal of Study: Our previous study found that intrathecal (i.t.) ketorolac pretreatment protected rats against ischemic spinal cord injury in lower limbs motor function and histopathological changes and excitatory amino acids (EAAs) and nitric oxide NO might play a role. This study was examined the possible role of glutamate transporters (GTs) and inducible NO synthase (iNOS) in the spinal ischemic injury (SCI). 
Materials and Methods: $\mathrm{SCl}$ was induced in male Wistar rats by balloon inflation of $2 \mathrm{~F}$ Forgathy catheter in thoracic aorta and controlled proximal arterial blood pressure at $40 \mathrm{mmHg}$ for 10 minutes. Lumbar spinal cord was removed at various time points to examine the expression of GTs (GLAST, GLT-1 and EAAC1), COX-2 and NOS isoforms (endothelial, neuronal and inducible NOS) by real time polymerase chain reaction and immunohistochemistry. Ketorolac pretreatment $(60 \mu \mathrm{g}$, i.t.) was used to determine its neuroprotective effect.

Results and Discussions: Real time PCR showed increasing of gene expression of GLAST, GLT-1 and EAAC1, peaked at 3 hours after SCI. The maximal change on COX-2 was also observed at 3 hours after SCl, and the maximal increase of iNOS was reached later. I.t. ketorolac suppressed these increases of gene expression. In the immunohistochemistry experiment, motor neurons and perivascular regions at both ventral and dorsal horn showed positive COX-2 staining. I.t. ketorolac also suppressed this change.

Conclusions: The expression of GTs, COX-2 and iNOS play a role in SCl. Ketorolac (i.t.) pretreatment, via inhibiting the GTs, COX-2 and iNOS expression, could ameliorate the neurological injury, it is suggested that ketorolac i.t. administration can be used for ischemic neuroprotection in clinical.

\section{AP6-4}

\section{Evidence for implicit memory of automatic learning such} as Fear Conditioning under deep anesthesia in rats

L. Pain, JC. Lleu, P. Oberling

Faculty of Medicine, INSERM U666/GRERCA; CHRU de Strasbourg, Strasbourg, France

Background and Goal of Study: Formation of some form of memory under anesthesia is still a matter of debate. We examined whether or not automatic learning as Fear Conditioning, FC (a learning mechanism sustaining phobias in humans) is processed under deep anesthesia in rats.

Materials and Methods: 80 male rats were randomized in 8 groups. All rats received either propofol ("anesthesia") or intralipids ("awake"). Twenty minutes later, they received either 5 no painful electric shocks, ES $(0.5 \mathrm{mAmp})$ or no shock in a specific context (with olfactory cue).

Electroencephalogramm (fronto-parietal) was recorded in rats under general anesthesia. At Day 15, all rats was tested for any residual anxiety in an elevated plus maze (1). Then half of the rats of each group were tested (day 16a) for explicit FC for the context (log latency to enter the context) whereas the other half was submitted to two ES in the initial context (reinstatement) to be tested (day 16b) for implicit memory for FC to the context.

Results and Discussions: Two-way ANOVA revealed no effect of anesthesia nor shocks on anxiety at Day 15 . Two-way ANOVA revealed a significant effect of anesthesia and shocks on the log latency to enter the context in rats tested on day 16a. Post-hoc analyses showed a significant fear to the context only in rats submitted to shocks when "awake". Two way ANOVA revealed a significant effect of initial shocks but no more effect of anesthesia in rats tested on day $16 b$.

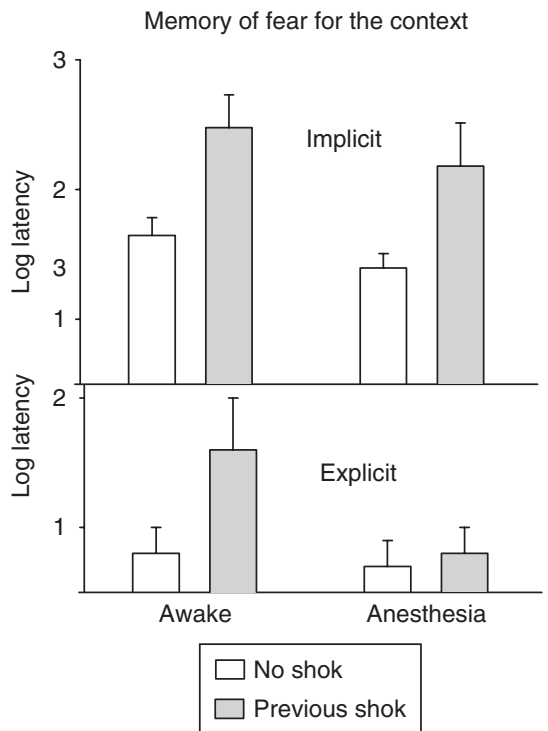

Conclusion(s): Despites no explicit memory of it, FC is preserved under anesthesia and lead to long lasting "savings" (two weeks in the current study) that might be expressed in an implicit way.

\section{AP6-5}

Anaesthesia and brain ageing: the Cholinergic Septo Hippocampal system participates to general (Propofol) anaesthesia

F. Laalou, JC. Cassel, L. Jouffroy, L. Pain Faculty of Medicine, INSERM U666/GRERCA;CHRU de Strasbourg, Strasbourg, France

Background and Goal of Study: Recent study pointed out the participation of the Septo Hippocampal (SH) GABAergic system in mediating the effects of general anesthetics (1). As brain ageing is associated with a dysfunction of cholinergic Septo Hippocampal pathway, we examined how such a dysfunction may interact with the potency of general anesthesia, using propofol in rats.

Materials and Methods: The rats were randomly allocated to one of the two following groups: rats with $\mathrm{SH}$ cholinergic depletion induced by the selective immunotoxine 192 lgG-saporin (SAPO; $n=14$ ) and sham-operated controls $(\mathrm{SHAM} ; n=6)$. During mini-craniotomy under anaesthesia, the rats received stereotaxically guided injections of a total of $0.8 \mu \mathrm{g} 192 \mathrm{lgG}$-saporin into the medial septum and the vertical limb of diagonal band of Broca. One month later, animals in each group received propofol by steps of $50 \mathrm{mg} / \mathrm{kg}$ intraperitoneally to set up an "anaesthesia state" (at least loss of righting reflex, i.e. "anaesthetic score" $\geqslant 0.6$ ). The comparison of the anaesthetic scores in rats with saporin lesions with those of sham-operated controls allowed to assess the effect of the cholinergic lesion on the propofol-induced anaesthesia. Postmortem histochemical staining of acetylcholinesterase was used as a marker for assessing the extent of cholinergic dysfunction.

Results and Discussions: After the repetitive injection of $50 \mathrm{mg} / \mathrm{kg}$ ("anaesthesia state"), the anaesthetic score increased as the cumulative dose of propofol was increased. This effect was more marked in lesioned animals compared to the control ones. Analysis of variance for repeated measures showed a significant effect of the Group factor $(F(1,17)=18.20, p<0.05)$ and of the Cumulative Dose of propofol $(F(4,68)=1.19, p<0.05)$, but there was no significant interaction between both factors $(F(4,68)=1.19)$. Thus, a previous cholinergic depletion of the septo hippocampal system increased the anesthetic potency of propofol.

Conclusion(s): These results extent to the cholinergic neurotransmission, the participation of the Septo Hippocampal system in mediating the effects of general anesthetics.

\section{Reference:}

1 Ma et al. Neuropsychopharmacology 2006: 1177-1192.

\section{AP6-6}

A free radical scavenger, edaravone ( $\mathrm{MCl}-186)$ protects nearly pure neuronal culture against hypoxic or glutamate insults through the different process

Y. Morimoto, K. Hisano

Anesthesia and preoperative Med., Hokkaido Univ. Grad. Sch. of Med., Sapporo, Japan

Background and Goal of Study: Edaravone is a free radical scavenger, which is available in Japan. It has been reported that edaravone has neuroprotective effect in the rodent model of forebrain and focal ischemia(1).

However, the mechanism of it has been well unknown. In this study, we utilized nearly pure neuronal culture and evaluated whether the protective effect against hypoxic or glutamate insults was related to apoptotic or necrotic pathways.

Materials and Methods: With the institutional approval of animal care and use, neurons were collected from 18 days embryo-fetus rat brains and almost pure neurons were obtained after 14 day-culture in Neurobasal medium without serums. The neurons were exposed to 2 different insults: 50 microM glutamate exposure for $10 \mathrm{~min}$ (glutamate insult) followed by normal culture for $24 \mathrm{hrs}$ and $1 \%$ below atmosphere for $24 \mathrm{hrs}$ (hypoxic insult). First, three different doses of edarvaone (10, 100, 500 microM) were applied into the medium from thirty $\mathrm{min}$ before the exposures until the end of protocol. Cell survival rate was measured by using staining technique with trypan blue. Second, the cells were stained by C-DCDHF-DA after the insults and relative amount of reactive oxygen species (ROS) was measured by flow cytometry. Third, the cells were stained by Hochest33342 and propidium iodide after the insults and the number of apoptotic or necrotic cells were counted.

Results and Discussions: Dose-dependent protective effect was observed and 500 microM of edaravone has significant effect compared to no treatment in glutamate and hypoxic insults. The amount of ROS was significant lower under 500 microM of edaravone in both insults. Apoptosis was significantly inhibited after hypoxic insult, while necrosis was significantly inhibited after glutamate insult. 
Conclusions: Edaravone could protect neurons against glutamate or hypoxic insults via inhibition of the ROS production. However, intracellular pathway may be different between these two insults.

Reference:

1 Stroke 36(10):2220-5, 2005.

\section{AP6-7}

\section{Effects of remifentanil on the NMDA receptor activation} injury of rats cortical neurons

X. Wu, Q. Xue, B. Yu

Anesthesiology, Ruijin Hospital, Shanghai Jiaotong University, School of Medicine, Shanghai, China

Background and Goal of Study: Remifentanil, an ultra-short-acting opioids, is wildly used in clinical practice, recent study shows that clinically relevant concentrations of remifentanil can stimulate human NMDA receptor expressed in xenopus laevis oocytes[1], so it may enhance brain injury related to NMDA receptor activation, but there is no report about this hypothesis, so we designed this experiment to evaluate the effects of remifentail on the NMDA receptor activation injury in cultured rats' cerebral cortical neurons.

Materials and Methods: Primary cultured rat cortical neurons were established from the 18-day-old embryos of SD rats and cultured for 7 days, then 500 microM of glutamate was added into the culture medium for 30 minutes to produce the NMDA receptor activation injury, different concentrations of remifentanil as $0.1,1,10$ micro $M$ were added into the culture medium with glutamate to evaluate its effects, and 50 micro $\mathrm{M}$ of ketamine was applied $30 \mathrm{~min}$ before glutamate and remifentanil co-application to find the mechanism of remifentanil's effects. After $24 \mathrm{~h}$ of drug application, neuron viability was assessed by the methods of MTT assay and LDH release measurement.

Results and Discussions: After 7 days' culture, the proportion of neurons was about $70 \% .500$ microM of glutamate produced a significant injury of cultured neurons indicated by the decreasing of MTT values and the increasing of LDH values, remifentanil enhanced the glutamate injury in a concentrationdependent manner, while, 50 microM of ketamine inhibited the decreasing of MTT and increasing of LDH values induced by glutamate and remifentanil co-application.

Conclusion: Remifentanil enhances the glutamate induced injury in rat cultured cortical neurons, and its mechanism may relate to its NMDA receptor activation property.

Reference:

1 Klaus H, Joke N, Hugo VA, et al. Remifentanil Directly Activates Human N-methyld-aspartate Receptors Expressed in Xenopus laevis Oocytes. Anesthesiology, 2004, 100: 1531-1537.

\section{AP6-8}

Regional and local brain oxygenation in haemorrhagic shock: effects of different therapy strategies

E. Cavus, P. Meybohm, V. Doerges, M. Steinfath, B. Bein

Anaesthesiology and Intensive Care Medicine, University Hospital Schleswig-Holstein, Campus Kiel, Kiel, Germany

Background and Goals: Patients with uncontrollable bleeding may benefit if resuscitation with large amounts of fluids is replaced by a small volume (HHS) or vasopressor until surgery $(1,2)$. Noradrenaline $(N A)$ is commonly used as a vasopressor to control hypotension. Despite cerebral autoregulation, haemorrhagic hypotension and shock may result in cerebral ischaemia (3). The purpose of this study was to compare the effects of HHS alone, or combined with $\mathrm{NA}$ on local brain tissue oxygen $\left(\mathrm{PbtO}_{2}\right)$ and regional brain oxygen saturation $\left(\mathrm{rSO}_{2}\right)$ in a model of uncontrolled haemorrhage.

Materials and Methods: After approval of the Animal Investigational Committee, 22 anaesthetised pigs underwent a penetrating liver trauma. At haemodynamic decompensation, animals were randomly assigned to receive either HHS (Hyperhaes ${ }^{\circledR} ; 4 \mathrm{ml} / \mathrm{kg} ; \mathrm{n}=8$ ) alone, or combined with low-dose NA (low NA: $500 \mu \mathrm{g}$, and $1 \mu \mathrm{g} / \mathrm{kg} / \mathrm{min} ; \mathrm{n}=7$ ), or high-dose NA (high NA; $1000 \mu \mathrm{g}$, and $1 \mu \mathrm{g} / \mathrm{kg} / \mathrm{min} ; \mathrm{n}=7$ ), respectively. Bleeding was controlled manually 30 mins after drug administration.

Results: Cerebral perfusion pressure (CPP), $\mathrm{PbtO}_{2}$, and $\mathrm{rSO}_{2}$ decreased with haemorrhage in all groups (Baseline vs. decompensation, mean $\pm \mathrm{SEM}$; CPP: HHS: $83 \pm 5$ vs. $9 \pm 1 \mathrm{mmHg}$; low NA: $67 \pm 6$ vs. $16 \pm 2 \mathrm{mmHg}$; high $\mathrm{NA}: 77 \pm 7$ vs. $15 \pm 1 \mathrm{mmHg}$. PbtO $_{2}$ : HHS: $13 \pm 2$ vs. $4 \pm 1 \mathrm{mmHg}$; low NA: $16 \pm 3$ vs. $6 \pm 1 \mathrm{mmHg}$; high NA: $26 \pm 6$ vs. $7 \pm 2 \mathrm{mmHg} . r \mathrm{SO}_{2}: \mathrm{HHS}$ : $61 \pm 1$ vs. $42 \pm 2 \%$; low NA: $63 \pm 1$ vs. $48 \pm 2 \%$; high NA: $69 \pm 2$ vs. $44 \pm 5 \%)$. Therapy with HHS, low NA, and high NA resulted in a comparable increase of $\mathrm{CPP}, \mathrm{PbtO}_{2}$, and $\mathrm{rSO}_{2}$, respectively $(5 \mathrm{~min}$ after therapy, mean \pm SEM; CPP: HHS: $29 \pm 3 \mathrm{mmHg}$; low NA: $27 \pm 3 \mathrm{mmHg}$; high NA: $28 \pm 3 \mathrm{mmHg}$. $\mathrm{PbtO}_{2}$ : HHS: $23 \pm 7 \mathrm{mmHg}$; low NA: $21 \pm 7 \mathrm{mmHg}$; high $\mathrm{NA}: 45 \pm 7 \mathrm{mmHg}$. $r \mathrm{SO}_{2}$ : HHS: $57 \pm 2 \%$; low NA: $52 \pm 2 \%$; high NA: $60 \pm 4 \%$. Overall survival was $6 / 8,3 / 7$, and $4 / 7$, respectively.

Conclusion: Following haemorrhagic shock in this model, additional use of $\mathrm{NA}$, independently of the dosage used, showed no beneficial effect on either $\mathrm{CPP}, \mathrm{PbtO}_{2}, \mathrm{rSO}_{2}$.

Reference:

1 Chiara O, Pelosi P, Brazzi L, et al. Crit Care Med 2003; 31: 1915-1922.

2 Voelckel WG, Wenzel V. Crit Care Med 2003; 31: 2552-2553.

3 Kovach AG, Szabo C, Farago M, et al. Stroke 22: 1541-1547, 1991.

\section{AP6-9}

\section{Circadian rhythms of rat temperature and locomotor activity} are altered by propofol anesthesia

\section{G. Dispersyn, L. Pain, Y. Touitou}

INSERM U713 Laboratoire de Biochimie médicale et Biologie moléculaire, Faculté de Médecine Pitié-Salpêtrière, Paris, France

Background and Goal of Study: It has been previously shown ${ }^{1}$ that when propofol is injected at the sleep-wake transition of rats in constant darkness it involves a 1-h phase advance of the rest-activity rhythm. The aim of the study was to examine the effects in rats of propofol anaesthesia performed during the activity period in dark-light conditions on markers of the temporal structure i.e temperature and locomotor activity.

Materials and Methods: Fourteen male Wistar rats (6-weeks-old at the beginning of experiment) were housed in individual cages (food and water available ad libitum) and maintained in a chronobiologic animal facility (temperature-controlled $21 \pm 1^{\circ} \mathrm{C}$ ). Rats were synchronized under a LD $12: 12$ cycle ( $12 \mathrm{~h}$ light, $12 \mathrm{~h}$ dark). Propofol $(10 \mathrm{mg} / \mathrm{ml})$ was injected at the dose of $120 \mathrm{mg} / \mathrm{kg}$ and intralipids $10 \%(10 \mathrm{ml} / \mathrm{kg})$ was used as control solution. The intraperitoneal injection was made in the middle of rat activity phase. Each rat was instrumented with an intra-abdominal radiotelemetric implant (Model TA10TA-F40, Data Sciences Int., St. Paul, Mn, USA) to permit automatic recording of motor activity and body temperature every $10 \mathrm{~min}$ throughout the $24 \mathrm{~h}$. Temperature and locomotor activity were continuously recorded for 29 days. On day 15, rats received a propofol injection or intralipids.

Results and Discussions: Our results show that propofol anesthesia modifies greatly the circadian rhythms of temperature and locomotor activity. A shortduration propofol anaesthesia resulted in a 5-h phase advance of locomotor activity rhythm (Student test $p<0.001$ ) and a phase advance in the acrophase of core body temperature $(02: 42 \mathrm{~h} \pm 16 \mathrm{~min}, \mathrm{p}<0.0004)$.

Conclusion(s): Our data clearly show that propofol anesthesia is responsible for potent desynchronizing effects on the circadian time structure. These results emphasize and extent the potent chronobiotic properties of propofol anesthesia, resetting the biological clock.

Reference:

1 Challet et al. Neuropsychopharmacology 2006.

\section{Local and Regional Anaesthesia}

\section{AP1-1}

The construction of learning curve and recommended case load for training of epidural anaesthesia in Taiwanese patient I. Chien, I. Chien, I-Cheng. Lu, Chien-Jen. Wang, Chao-Shun Tang Anesthesiology, Yuans General Hospital, Kaohsiung, Taiwan

Background and Goal of Study: Epidural anaesthesia was reported to be the most difficult manual anaesthetic procedures $(1,2)$. We designed this study to construct learning curve for epidural anaesthesia in an university hospital epidural where anaesthesia was predominate than spinal anaesthesia.
Materials and Methods: Our study was designed to evaluate 400 lumbar epidurals performed by 4 first-year residents. The study enrolled 400 cases classified as grade 1 and 2 by grades of spinal process landmarks (3). We documented the first 100 performed epidural anaesthesia for each resident. For each procedure, following data were recorded and analyzed to construct learning curve and evaluate case load for training, including first-level successful rate for catheter indwelling, trying attempts and consumption time. Results and Discussions: First level success rate maintained around than $90 \%$ after thirty procedures of epidural anaesthesia. Average success rate before and after 30 procedures of epidural anaesthesia were $72.57 \%$ and 
91.1\% respectively $(\mathrm{P}<0.001)$. The mean trying attempts were $1.26(95 \%$ confidence interval: $1.18 \sim 1.34)$ and $1.14(95 \%$ confidence interval: 1.09 1.17) for epidural anaesthesia before and after 30 procedures respectively $(\mathrm{P}<0.05)$. The mean (standard deviation) consumption time were 8.98 $(0.89)$ and 6.12 (1.21) minutes for epidural anaesthesia before and after 30 procedures respectively $(\mathrm{P}<0.001)$

Conclusions: We concluded that after practice more than 30 cases of epidural anaesthesia residents can achieve maturity of the most difficult manual anaesthetic skill in our institution. Our recommended case load for training of epidural anaesthesia was at least thirty.

References:

1 Konrad C, Schupfer G, Wietlisbach M, et al. Anesth Analg 1998; 86: 635-39.

2 Kestin IG. Br J Anaesth 1995; 75: 805-9.

3 Chien I, Lu IC, Wang FY, et al. Kaohsiung J Med Sci 2003; 19: 563-8.

\section{AP1-2}

\section{Complication analysis of epidural space identification - a meta-analysis of air vs. saline technique for loss of resistance}

R. Schier, D. Guerra, G. Pratt, K. Boddu, B. Riedel

Department of Anesthesiology and Pain Medicine Research, The University of Texas M.D. Anderson Cancer Center, Houston, USA

Background and Goals: Controversy exists as to the best method for identifying the epidural space. The choice among anesthesiologists of whether to use saline or air during the loss of resistance (LOR) technique to identify the epidural space has largely been a matter of personal preference. In this metaanalysis we compared the incidence of side effects and complications depending on the medium used to identify the epidural space during the LOR technique.

Materials and Methods: We searched Medline (Ovid, 1966-present), Cochrane Central Register of Controlled Trials (Ovid), and Embase (Dialog, 1974-present). Two authors independently abstracted data from 6 prospective, randomised trials ( $n=4,422$ patients). A meta-analysis was performed to compare outcomes reported using LOR with air vs. saline/lidocaine. Outcomes measures were difficult catheter insertion, paraesthesia, intravascular catheter insertion, accidental dural puncture (ADP), postdural puncture headache (PDPH), and partial block.

Results: LOR using saline vs. air was associated with a $65 \%$ reduction in the risk of difficult catheter insertion ( $4.3 \%$ vs. $11.0 \% ; P=0.002)$, a $53 \%$ reduction in the risk of intravascular catheter insertion (5.6\% vs. $11.7 \%$; $P=0.010)$, a $81 \%$ reduction in the risk of PDPH $(0.3 \%$ vs. $1.6 \% ; \mathrm{P}<0.0001)$, and a $57 \%$ reduction in the risk of partial block $(7.6 \%$ vs. $15.5 \%$; $P=0.001)$. There were no statistically significant differences in the risk of ADP and paraesthesia between the air and saline groups.

Conclusion: Using the LOR technique with saline may reduce the incidence of certain complications in patients undergoing epidural analgesia. However, large, good quality randomised controlled trials are needed to study the differences between these LOR techniques regarding analgesic onset, analgesic distribution, and quality of pain relief.

References:

1 Norman D et al. AANA Journal 2006; 74: 301-8.

2 Evron S et al. Anesthesia \& Analgesia 2004; 99: 245-50.

3 Beilin Y et al. Regional Anesthesia \& Pain Medicine 2000; 25: 596-9.

\section{AP1-3}

Comparison of the four different epidural catheter types during placement in the lower thoracic region

A. Ogura, J. Ogura, T. Saito, T. Inoue, A. Sakamoto

Anesthesiology, Shizuoka Red Cross Hospital, Shizuoka, Japan

Background and Goal of Study: We compared the incidence of transient paresthesia and other complications for four different epidural catheter types (closed-end), two types of polyamide catheters (PLA A and PLA B), polyethylene catheter (PLE) and polyamide soft tip catheter (PLA Soft Tip), during epidural catheterization in the lower thoracic region.

Materials and Methods: After obtaining IRB approval and informed consent, adult patients were randomly divided into four groups according to the type of epidural catheter; i.e. Group A: PLA A $(n=302)$ and Group B: PLA B $(n=306)$, Group C: PLE $(n=300)$ and Group D: PLA Soft Tip $(n=303)$. The patient was positioned in a moderate chest-knee position. Epidural puncture was performed at T11-12 interspace via paraemdian approach. The epidural space was identified by the loss-of-resistance technique to saline. The catheter was inserted to leave $5 \mathrm{~cm}$ in the epidural space. Transient paresthesia and other complications during epidural catheterization were recorded. Data were analyzed by ANOVA test followed by Scheffe's post-test or Chi-square analysis as appropriate. $\mathrm{P}<0.05$ was considered significant.

Results and Discussions: Patient characteristics, number of attempts and blood aspiration were comparable among the groups. There were no major complications noted in all groups. The incidence of transient paresthesia was different among the groups, 24 (7.9\%) in Group A, 19 (6.2\%) in Group B, $26(8.7 \%)$ in Group $C$ and $3(1.0 \%)$ in Group D $(P<0.01)$. Occurrence ratio of resistance to introduction of the catheter was also different among the groups, 33 (10.9\%), 18 (5.9\%), $6(2.0 \%)$ and $1(0.3 \%)$, respectively $(P<0.01)$. It has been reported that the relatively high incidence of complications associated with epidural catheterization was observed in the lower thoracic region. In this study, transient paresthesia was observed in all groups during epidural catheterization. However, in Group D, PLA Soft Tip group, paresthesia was rarely recognized.

Conclusion: The path and the ultimate position of PLA Soft Tip catheter might not be affected by the epidural structure during catheterization at the lower thoracic level.

References:

1 Giebler, Reiner M, et al. Anesthesiology 1997; 86: 55-63.

2 Akira Ogura, et al. Anesthesiology 2006; 105: A874.

\section{AP1-4}

\section{Advantages of preoperative epidural infusion of $0.2 \%$} ropivacaine in laparoscopic colectomy

\section{K. Panteleou, P. Mavridou, O. Igropoulou, K. Daflou, A. Manataki}

Department of Anesthesiology, Hatzikosta General Hospital, loannina, Greece

Background and Goal of Study: It is a fact that sympathetic proganglionic fibres from $T_{5}$ through $L_{1}$ inhibit gut action and that epidural blockade to the midthoracic levels remove this inhibition, yielding a contracted small intestine. Goal of our study was to assess the effect of preoperative epidural infusion of ropivacaine in laparoscopic colectomy.

Materials and Methods: 30 patients (16 women, 14 men ASA I-III), scheduled for laparoscopic resection of colon cancer (colectomy), were randomized into two groups of 15 patients each. An epidural catheter was inserted at the $T_{12}-L_{1}$ space in both groups.

- Group A: the epidural catheter was inserted the day before surgery and a continuous infusion of $0.2 \%$ ropivacaine started at a rate of $4 \mathrm{ml} / \mathrm{h}$ for 1262 hours before surgery.

- Group B: the epidural catheter was placed just before surgery and no preoperative infusion was used.

The epidural catheters were also used for postoperative analgesia.

General anaesthesia was induced with propofol, remifentanil, cis-atracurium and was maintained with a mixture of oxygen/air $\left(\mathrm{FiO}_{2}: 0.4\right)$, sevoflurane, remifentanil, cis-atracurium as needed.

Intraabdominal pressure in both groups were $12 \mathrm{mmHg}$

The quality of surgical conditions and the duration of pneumoperitoneum were recorded

Unpaired t-test was used with $p<0.05$ considered statistically significant. Results and Discussions: Patients had similar demographic data. Preoperative epidural ropivacaine yielded a small and contracted intestine in group A versus a non-contracted and large in volume intestine in group B. This fact created optimal surgical conditions in group $A$ that led to better control of laparoscopic instruments with statistically significant reduction of surgical time and reduction of the duration of pneumoperitoneum $(p<0.05)$. Conclusion: Preoperative epidural infusion of ropivacaine, proved to be beneficial in laparoscopic colectomy, as it improved the quality of surgical conditions, reduced the duration of pneumoperitoneum and eliminated its adverse patophysiological effects during anesthesia.

\section{AP1-5}

Documentary evidence of appropriate consent for combined spinal epidural, epidural and spinal in anaesthetic practice S. Bell, T. Sheraton Department of Anaesthetics and Intensive Care Medicine, Royal Gwent Hospital, Newport, United Kingdom

Background and Goal of Study: Lack of informed consent is an issue in approximately $30 \%$ of claims against anaesthetists (1). The AAGBI, the RCA and the GMC have produced guidance on consent. Current guidance advises that adequate documentation of the discussion of risks and benefits is mandatory and that anaesthetists should warn patients of common or serious risks but should avoid providing a bewildering quantity of information. This audit aims to assess documentary evidence of consent for epidural, spinal and combined spinal epidural (CSE) anaesthesia. 
Materials and Methods: A review of the notes provided by the anaesthetist was undertaken in the post-operative period for forty-four patients who had received CSE, epidural or spinal anaesthetic techniques.

Results: In thirty cases (68.2\%) some documented evidence of a discussion of the elements of the regional technique and its associated risks and benefits was seen. The following discussions of risks were documented: headache $17(39 \%)$, paraesthesia 0, nerve damage $15(34 \%)$, failure $12(27 \%)$, shivering $1(2 \%)$, infection $2(5 \%)$, haematoma $1(2 \%)$, hypotension $4(9 \%)$, urinary retention $5(11 \%)$, nausea $4(9 \%)$ and reason for lack of discussion 0. Conclusion(s): In nearly a third of cases anaesthetists failed to document the discussion of risks. The particular risks discussed varied considerably. The anaesthetist will always be required to exercise clinical judgement in discussion of risks but the authors feel that reasons for not discussing risks (which can be perfectly valid) should also be documented. Documentation should be improved to avoid legal complications. This might be achieved by improving training of anaesthetists on issues of consent. Evidence of understanding consent might constitute part of the forthcoming re-validation process.

Reference:

1. Royal College of Anaesthetists Raising the Standard 2000 Dr E James Consent to anaesthesia 1.2

\section{AP1-6}

\section{Confirmation of epidural puncture by change in epidural} pressure using Queckenstedt-test procedure in patients with cervical spinal canal stenosis

R. Yokoyama, T. Yokoyama, T. Ushida, A. Imoto, K.A. Sluka

Department of Anesthesiology and Critical Care Medicine, Kochi Medical School, Nankoku, Japan

Background and Goal of study: The loss-of-resistance test is the most popular method for identifying the epidural space, but it cannot confirm epidural puncture. Therefore, we developed a new method to confirm epidural puncture by assessing indirect changes in epidural pressure (EP) using the Queckenstedt-test ${ }^{1}$ procedure $(\mathrm{E}-\mathrm{QST})^{2}$, which increases subarachnoid pressure by compressing the internal jugular veins. This method depends on the dynamics of cerebrospinal fluid (CSF), hence blockade of CSF flow, occuring with severe spinal stenosis, is predicted to reduce changes in EP using E-QST. Thus, we examined the effect of spinal stenosis on the E-QST. Material and Methods: After institutional approval and informed consent, patients undergoing cervical spine surgery were enrolled for this study. Epidural puncture using the loss-of-resistance test was utilized to insert an electrode after anesthetic induction. EP was monitored with E-QST through a Tuohy needle to confirm epidural puncture. The insertion of the electrode into the epidural space was confirmed by observation of muscle twitch evoked after electric stimulation using the electrode.

Results and Discussion: In 60 patients, epidural puncture was performed with the loss-of-resistance test; a second trial was required for 13 patients due to less catheter advance. Increased EP during E-QST was observed in $57 / 73$ trials. When increased EP was observed, epidural puncture was always successful. The sensitivity and specificity of this E-QST method was $91.9 \%$ $(57 / 62)$ and $100 \%(11 / 11)$. The positive and negative predictive values were $100 \%(57 / 57)$ and $68.8 \%(11 / 16)$ respectively.

Conclusion: EP monitoring combined with E-QST offers a reliable method for confirming epidural puncture in combination with the loss-of-resistance test, even if patients have spinal canal stenosis.

References:

1 Queckenstedt H. Deuche Zeitshaft fur Nervenheilkunde 1916; 55: 325-33.

2 Yokoyama T, et al. J Clin Anesth (Japan) 2005; 29: 1815-8.

\section{AP1-7}

The effect of ketamine on combination of bupivacaine and fentanyl for quality of epidural block

L. Ozdogan, M. Erol, V. Taspinar, S. Barcin, F. Sahin

Anaesthesiology and Reanimation, Numune education and research hospital, Ankara, Turkey

Background and Goal of Study: Opioid and local anesthetic combination has been used to enhance the quality of epidural anesthesia. It has reported that epidural ketamine tends to antagonize the antinociceptive activity of fentanyl in rat(1). The aim of this study was to evaluate the effect of ketamine on combination of bupivacaine and fentanyl for quality of epidural block.

Materials and Methods: After ethic committee approval and informed consent, forty patients undergoing hip arthroplasty were studied. Epidural catheter was inserted $\mathrm{L}_{3-4}$ or $\mathrm{L}_{4-5}$ interspaces by lost of resistance with air in sitting position. Patients randomly divided to two groups. Group BF received $75 \mathrm{mg}$ bupivacaine and $100 \mu \mathrm{cg}$ fentanyl, Group BFK received $30 \mathrm{mg}$ additional ketamine to this combination of same dose bupivacaine and fentanyl. Onset time of sensory and motor blockage, maximum level of sensory block, degree of motor block, regression of two segments, duration of sensory and motor blockade were evaluated. Statistical analyses were performed by Kruskall Wallis and Chi Square tests.

Results and Discussions: Demographical and haemodynamic parameters, onset of sensory and motor blockade, and the degree of motor block were similar. Maximum level of sensory block was higher in Group BFK. Regression time of two segments and duration of sensory block were lower in Group BFK.

Table

\begin{tabular}{llll}
\hline & BF & BFK & P \\
\hline Onset of sensory block (minute) & $2,9 \pm 1$ & $2,3 \pm 0,5$ & 0,068 \\
Two segment regression time (minute) & $197,7 \pm 28$ & $121 \pm 42$ & $<0,001$ \\
Duration of sensorial block (minute) & $252,5 \pm 25$ & $213,5 \pm 23$ & $<0,001$ \\
Maximum level of sensory block & T6(T4-T6) & T2(T2-T4) & $<0,001$
\end{tabular}

Conclusions: Preoperative use of epidural ketamine antagonizes the beneficial effects of epidural fentanyl.

Reference:

1 Hoffmann VLH, et al. European Journal of Pain 2003; 7: 121-130.

\section{AP1-8}

Epidural analgesia decreases intraabdominal pressure in postoperative patients with intraabdominal hypertension

A. Varosyan, G. Mkhoyan, G. Harutyunyan, R. Hakobyan

Anaesthesiology and Intensive Care, Yerevan State Medical University, Yerevan, Armenia

Background and Goal of Study: Intraabdominal hypertension (IAH) causes significant morbidity and mortality in critically ill surgical patients. Pain relief may hypothetically decrease the secondary muscle spasm and increase the anterolateral abdominal wall compliance, therefore decreasing intraabdominal pressure (IAP). To rule out this hypothesis the effect of epidural analgesia on IAP in postoperative patients with IAH was investigated.

Materials and Methods: In a prospective double-blinded study 58 postoperative critically ill surgical patients with $\mathrm{IAH}$ receiving postoperative epidural analgesia were investigated. IAH defined as a sustained IAP $\geqslant 12 \mathrm{mmHg}$ or an abdominal perfusion pressure (APP) $\leqslant 60 \mathrm{mmHg}$ recorded by a minimum of 3 standardized measurements conducted 6 hours apart. Epidural catheterization was performed at $\mathrm{Th}_{8}-\mathrm{Th}_{10}$ level. After test dose and correct placement of the catheter patients received $10 \mathrm{ml}$ of $0,2 \%$ ropivacaine, followed by its continuous infusion at a rate of $5 \mathrm{ml} / \mathrm{h}$ for maximum 96 hours. IAP was measured transvesically after instillation of $50 \mathrm{ml}$ saline in supine position immediately before and 1 hour after initiation of epidural analgesia and every 6 hours consequently. Mean arterial pressure (MAP) was measured invasively in all patients. APP was calculated for each IAP measurement as APP $=$ MAP-IAP. A repeated measure ANOVA was used to analyze repeated measurements of IAP, MAP and APP.

Results and Discussions: ANOVA for repeated measures showed significant within subject decrease in IAP ( $p<0.0001)$, but failed to show any significant differences for repeated measurements of MAP $(p=0.147)$. Mean and standard deviation values of IAP, APP and MAP in all observations immediately before and 1 hour after initiation of epidural analgesia were $13.909 \pm 3.006 \mathrm{mmHg}$ vs. $7.727 \pm 3.439 \mathrm{mmHg}(\mathrm{p}<0.0001) ; 63.732 \pm 16.244 \mathrm{mmHg}$ vs. $75.658 \pm$ $18.986 \mathrm{mmHg} \quad(p=0.005)$ and $77.641 \pm 17.398 \mathrm{mmHg}$ vs. $83.385 \pm$ $17.781 \mathrm{mmHg}(p=0.071)$ respectively. Measurements show a significant decrease of IAP with no significant change in MAP, which maintained APP stable or even elevated during epidural analgesia: APP 1 hour $<1$ day $(p<0.001), 1$ day $<2$ day $(p=0.006)$.

Conclusion: Continuous thoracic epidural analgesia significantly decreases IAP and improves APP with no hemodynamic compromise in critically ill postoperative patients with IAH.

\section{AP1-9}

Selective segmental epidural anesthesia for ambulatory pilonidal sinus surgery

Y. Pala, S. Okur, V. Taspinar, F. Donmez, S. Barcýn

II. Anesthesiology and Reanimation, Ankara Numune Education and Research Hospital, Ankara, Turkey

Background and Goal of Study: Epidural anesthesia (EA) can provide sensorial block with no motor blockade and the level of block can be easily controlled (1). Our aim was to compare low dose segmental EA with conventional EA for ambulatory pilonidal sinus (PS) surgery. 
Materials and Methods: ASA I-II forty patients undergoing PS surgery were included. In the sitting position Tuohy needle was inserted L4-5 or L5-S1 intervertebral spaces and the bevel of needle oriented caudally. Patients were randomly divided in to two groups. In Group I $15 \mathrm{~mL}$ bupivacaine (\%0.5) and in Group II $6 \mathrm{~mL}$ bupivacaine (\%0.5) were given over 180 seconds for EA. Then epidural catheter was advanced $3-4 \mathrm{~cm}$ in the same direction. Patients were repositioned in the prone position. Epidural block characteristics, hemodynamic parameters, first analgesic requirement time, time to PACU discharge, time to first voiding and time to home readiness were recorded. Mann Whitney- $U$, students $T$ and chi-square tests were used for statistical analysis and $p<0.05$ was considered as significant.

Results and Discussions: Demographic data, hemodynamic changes were similar. Maximum levels of sensorial and motor block degree were lower in group II. Epidural block was limited in sacral region in group II but cephalic spread was manifest in group I. Other significant parameters were showed table $(p<0.001)$

\begin{tabular}{llr}
\hline & Group I & Group II \\
\hline Onset of sensory block (sec) & 13,5 & 18 \\
Duration of sensory block (min) & 195 & 120 \\
First analgesic time (hr) & 540 & 382 \\
PACU discharge (hr) & 127 & 52 \\
First voiding (hr) & 397 & 190 \\
Home readiness (hr) & 420 & 200 \\
\hline
\end{tabular}

Conclusion: We think that the low dose selective segmental epidural anesgery.

\section{Reference:}

1 Aaron J: Anesth analg 1982; 61: 570-5. thesia may be preferred as an alternative technique for ambulatory PS sur-

\section{AP2-1}

Ultrasonographic assessment of the influence of head rotation on the interscalene brachial plexus block

I. Lu, I. Lu, Yi-Wei. Kuo, Fu-Yuan. Wang, Koung-Shin Chu

Anesthesiology, Kaohsiung Medical University Hospital, Kaohsiung, Taiwan

Background and Goal of Study: Head rotation can affect neck vessels orientation and may affect brachial plexus possibly (1). The goal of this study was to evaluate whether landmark-based precision of interscalene brachial plexus block and depth of brachial plexus could be altered based solely on different head rotation.

Materials and Methods: We simulated a needle insertion via interscalene approach to brachial plexus using an ultrasonographic probe in 53 volunteers (2). Ultrasonographic measurements were recorded to evaluate the influence of head rotation. First, we documented the measured deviation (d) between the simulated needle path and actual center of the brachial plexus by using a perpendicular line between them. Second, distance from skin to center of brachial plexus(s-p) was recorded. Finally, precise and venous hit were recorded.

Results and Discussions: Increased head rotation from $0^{\circ}, 15^{\circ}, 30^{\circ}, 45^{\circ}$ and $60^{\circ}$ to the right of midline associated with more medial deviation of a simulated needle path to the center of brachial plexus and lower precise rate $(p<0.01)$. The risk of stimulated needle path intersecting internal jugular was lower than $5 \%$ for head rotation within $30^{\circ}$ and significantly higher for head rotations over $45^{\circ}(p<0.01)$.

\begin{tabular}{lllllr}
\hline & \multicolumn{1}{l}{$0^{\circ}$} & \multicolumn{1}{l}{$15^{\circ}$} & \multicolumn{1}{l}{$30^{\circ}$} & \multicolumn{1}{l}{$45^{\circ}$} & \multicolumn{1}{l}{$60^{\circ}$} \\
\hline $\mathrm{d}(\mathrm{mm})$ & $-5.6 \pm 5.9$ & $-6.8 \pm 5.9$ & $-7.8 \pm 6.5$ & $-10.8 \pm 4.9$ & $-11.5 \pm 5.4$ \\
$\mathrm{~s}-\mathrm{p}(\mathrm{mm})$ & $12.2 \pm 1.8$ & $11.8 \pm 1.6$ & $10.8 \pm 1.7$ & $11.2 \pm 1.6$ & $11.1 \pm 1.9$ \\
\hline
\end{tabular}

Conclusions: Whenever we perform the interscalene brachial plexus block, the head rotation angle should within $30^{\circ}$. The measured medial deviation should be considered when the surface anatomic landmarks are used to approach interscalene brachial plexus.

References:

1 Liberman JA, Williams KA, Rosenberg AL, et al. Anesth Analg 2004; 99: 982-8.

2 Marhofer P, Greher M, Kapral S, et al. Br J Anaesth 2005; 94: 7-17.

X. Souvatzis, P. Katonis, V. Nyktari, N. Tzanakis, H. Askitopoulou

Anaesthesiology, University Hospital of Heraklion, Heraklion, Greece

Background and Goal: In only $29 \%$ of patients anaesthesiologists were correct in identifying lumbar vertebral interspaces (LVIs) in patients with the lumbar spine flexed as much as possible (1). The purpose of the present study was to assess the accuracy of residents and consultants anaesthesiologists in identifying the LVI in patients positioned with their lumbar spine straightened or in the physiological lordosis.

Materials and Methods: The study was approved by the hospital research and ethics committee. Patients scheduled for spine surgery were examined after induction of general anaesthesia and placement in the prone position. Both a resident (group R) and a consultant (group C) did select blindly one of the five LVIs. After the identification of the requested interspace by palpation, each anaesthesiologist placed on the skin a needle at the assumed level. The needle's position was confirmed with a lumbar radiograph. The requested and the actually found vertebral interspace by both the resident and the consultant were recorded. Additional recorded data included: Patients' age, weight, height, gender. Tuffier's line (line joining the iliac crests) level (TLL) was determined with x-rays.

Results: 77 consecutive patients were included in the study, 29 male and 48 female, resulting in a total of 154 examinations (by groups $R$ and $C$ ). Patients' data [age: $58( \pm 17)$ years, BMI: $26,5( \pm 4,6)]$ were normally distributed and expressed as mean \pm SD. Group R identified the vertebral interspace correctly in 25 patients compared to 32 accurate localizations by group $\mathrm{C}$. The actually found vertebral interspace ranged from 3 levels below to 2 levels above the requested interspace for the group $R$ and from 1 level below to 2 levels above for the group $C$. The differences between groups $R$ and $C$ did not reach statistical significance. The TLL ranged from $L_{3-4}$ to $L_{5}$. The higher the TLL was found, the higher was the actually identified vertebral interspaces for both groups.

Conclusions: In the present study, the anaesthesiologists presented a poor rate in identifying correctly a LVI. The study also confirmed the poor value of Tuffier's line in the correct identification of the intervertebral space.

Anaesthesiologists performing spinal anaesthesia have to be aware of the inaccuracy in identifying a given LVI.

Reference:

1 Broadbent CR, Maxwell WB, Ferrie R, et al. Anaesthesia 2000; 55(11): 1122-1126.

\section{AP2-2}

Ultrasound guidance for axillary block: a prospective, randomized, observer-blinded comparison with nerve stimulation

A. Casati, S. Leone, M. Baciarello, G. Danelli, S. Di Cianni

Anesthesiology and Pain Therapy, University of Parma, Parma, Italy

Background and Goal of Study: The aim of this prospective, randomized, observer-blinded study was to test the hypothesis that ultrasound guidance can shorten the onset time of axillary brachial plexus block as compared to nerve stimulation guidance when using a multiple injection technique.

Materials and Methods: 60 ASA physical status I-III patients, receiving axillary brachial plexus with $20 \mathrm{~mL}$ of $0.75 \%$ ropivacaine using a multiple injection technique were randomly allocated to receive either nerve stimulation (group NS, $n=30$ ), or ultrasound guidance (group US, $n=30$ ) for nerve location. A blinded observer recorded the onset of sensory and motor blocks, procedure-related pain, success rate, and patient satisfaction.

Results and Discussions: The median (range) number of skin punctures was $2(1-2)$ in group US and $2(2-3)$ in group NS $(P=0.94)$; however, group US required less needle passes [4 (3-8)] than group NS [8 $(5-13)](P=0.002)$. The onset of sensory block was shorter in group US (14 $\pm 6 \mathrm{~min}$ ) than in group NS $(18 \pm 6 \mathrm{~min})(P=0.01)$; while no differences were observed in onset of motor block ( $24 \pm 8 \mathrm{~min}$ in group US and $25 \pm 8 \mathrm{~min}$ in group $\mathrm{NS}$; $\mathrm{P}=0.33$ ). No failed block was reported in either group. Insufficient block was observed in 1 patients of group US (3\%) and 2 patients of group NS $(6 \%)(P=0.61)$. Procedure-related pain was reported in 6 patients (20\%) in group US and 14 patients $(48 \%)$ in group NS $(P=0.028)$; patient acceptance was similarly good in the two groups.

Conclusion: Multiple injection axillary block with ultrasound guidance provided a slightly faster sensory block, fewer needle redirections, and less anesthesiarelated pain as compared to nerve stimulation guidance. 


\section{AP2-3}

Complications of interscalene brachial plexus block following shoulder surgery in the awake sitting position

U. Panchagnula, S. Sagadai, M. Columb

Department of Anaesthesia, University Hospital of South Manchester, Manchester, United Kingdom

Background: Interscalene brachial plexus block (ISB) is commonly used for shoulder surgery in the awake, sitting position (1). Aim of the study was to determine the complications following ISB, especially the incidence of syncopal episodes and neurological sequelae.

Methods: After ethical review, perioperative data from consecutive patients presenting for shoulder surgery under ISB was collected over 5 years. A chart review was done to identify postoperative complications. Patients received a standardised ISB with superficial cervical plexus block using levo-bupivacaine and lidocaine (200 mg each) diluted to $60 \mathrm{~mL}$ with 1:400,000 epinephrine. Syncopal episodes requiring treatment were classified as symptomatic or haemodynamic. Neural sequelae were classified as major or minor, definitely, possibly or not attributable to ISB. Data are presented as mean (SD), median [interquartiles] and frequency. Student's $t$-, Mann-Whitney U-, Fisher exact and chi-square tests were used and results are presented with $95 \%$ confidence intervals $(\mathrm{Cl})$ with $P<0.05$ (two-sided) considered as significant. Results: 321 patients were studied (Table). Postoperative follow-up was for 20 [10-32] weeks. The success rate for ISB was $0.96(95 \% \mathrm{Cl} 0.93-0.98)$. Syncopal incidence was $0.43(95 \% \mathrm{Cl} 0.38-0.49)$, (symptomatic 0.14 ; haemodynamic 0.29 ) and was significantly associated with Horner's syndrome (relative risk $1.3295 \% \mathrm{Cl} 1.02-1.71$ ) and reduced use of midazolam. One patient had accidental intravascular injection of a test dose (transient myclonus) with successful ISB. Four patients developed neurological sequelae: one major - surgical damage to radial nerve and three minors - two due to coincidental pathology and one possibly attributable to ISB.

\begin{tabular}{llll}
\hline Variable & Asymptomatic $(\mathrm{N}=183)$ & Syncopal $(\mathrm{N}=138)$ & $\mathrm{P}$ \\
\hline Age (year) & $50.4(17.1)$ & $49.4(17.6)$ & 0.62 \\
Weight $(\mathrm{kg})$ & $78.6(15.1)$ & $82.4(14.3)$ & 0.17 \\
Horner's syndrome & $87: 96$ & $82: 56$ & 0.042 \\
Midazolam $(\mathrm{mg})$ & $1(0,2)$ & $0(0,1)$ & 0.045 \\
\hline
\end{tabular}

Conclusion: Syncopal episodes are common and are significantly associated with Horner's Syndrome and absence of sedation. Neurological sequelae attributable to ISB are minor and rare $0.3 \%(95 \% \mathrm{Cl} 0.008-1.7)$.

Reference:

1 Maguire SL, Columb MO. European Journal of Anaesthesiology 2002; 19 (suppl 24): A401.

\section{AP2-4}

Evaluation of the analgesic effects of 2 doses of verapamil with bupivacaine compared with bupivacaine alone in supraclavicular brachial plexus block

F. Mosaffa, A.R. Salimi, F. Lahiji, M. Kazemi, A.R. Mirkheshti

Anesthesiology, Akhtar Hospital, Shahid Beheshti University of Medical Sciences, Tehran, Iran

Background and Goal of study: Different adjuvant drugs has been used with local anesthetics in order to decrease the time of onset and elongate the duration and quality of regional blocks (1). In this study we used Verapamil(V) locally combined with Bupivacaine(B), in supraclavicular block(SB)(2).

Material and Methods: In this randomised double blinded clinical trial, 60 18-40 years old patients divided randomly into 2 groups (Gl:B $30 \mathrm{ml} 0.5 \%$,GIl:B $30 \mathrm{ml} 0.5 \%+\mathrm{V} 2.5 \mathrm{mg}$ ). Onset time (OT) of sensory,motor,complete blocks (SB) (MB) (CB), blood pressure (BP), heart rate (HR) changes were taken into consideration. We used SPSS 11.5 software.

Results: Data(Mean $\pm \mathrm{SD})$ are shown in the table. $(\mathrm{P}<0.05)$

\begin{tabular}{lcr}
\hline Mean OT & G I & \multicolumn{1}{c}{ G II } \\
\hline SB & $12.8 \pm 3.41$ & $9.95 \pm 0.88$ \\
MB & $20.44 \pm 8.11$ & $11.65 \pm 2.71$ \\
CB & $25.1 \pm 5.3$ & $12.75 \pm 3.09$ \\
\hline
\end{tabular}

There are no significant changes in $\mathrm{BP}$ and HR. $(P=0.236)$

Conclusion: Applying $\mathrm{V}$ combined with $\mathrm{B}$ in $\mathrm{SB}$ can decrease the OT of SB,

$\mathrm{MB}$ and $\mathrm{CB}$ which confirm the role of calcium channel blockers in analgesia(3).

References:

1 Saied E.L., J.M. Can J Anesth, 2000; 47: 962-7.

2 Choe, H., Anesth Analg, 1998; 86: 786-90.

3 Hasegawa, A.E., Anesth Analg, 1997; 85: 633-8.

\section{AP2-5}

Effects of the addition of fentanyl to levobupivacaine during axillary brachial plexus anesthesia

S. González, M.I. Rochera, A. Lacasta, L. Mora, J. Roigé

Anaesthesiology, Hospital Vall d'Hebron, barcelona, Spain

Background and aim: Peripheral co-administration of local anaesthetic and narcotic drugs has been reported to improve the nerve block characteristics $(1,2)$. The aim of this prospective, randomized, double-blind study was to evaluate the effects of adding $100 \mu \mathrm{g}$ fentanyl to levobupivacaine $0.33 \%$ for axillary brachial plexus anesthesia.

Material and Methods: The Protocol was approved by the Human Ethics Committee of the Institution, and all patients gave informed consent. Sixty four ASA I-II patients, scheduled for orthopaedic hand and forearm procedures were randomly allocated to receive an axillary brachial plexus block with $30 \mathrm{~mL}$ of levobupivacaine $0.33 \%$ (L) or levobupivacaine $0.33 \%+100 \mu \mathrm{g}$ fentanyl $(L+F)$, using a selective multiple nerve stimulation technique. Sensory and motor block were tested for the main terminal nerves of the arm at 2-5-10-1520-25 and 30 minutes after completion of the local anesthetic injection. Onset time, the time for the patients to be ready for surgery, the need for intraoperative analgesics, the duration of the block and adverse effects were recorded. Results: No differences in anthropometric parameters and hemodynamic variables were observed throughout the study, and no signs of central nervous system and cardiovascular toxicity.

\begin{tabular}{llll}
\hline Block characteristics & $\mathrm{L}$ & $\mathrm{L}+\mathrm{F}$ & \\
\hline mean $\pm \mathrm{SD}$ & mean $\pm \mathrm{SD}$ & $\mathrm{t}$-Student \\
Onset monsory $(\mathrm{min})$ & $11.78 \pm 5.20$ & $12.73 \pm 5.90$ & $\mathrm{p}=0.463$ \\
Ready to surgery $(\mathrm{min})$ & $12.02 \pm 5.31$ & $13.58 \pm 7.48$ & $\mathrm{p}=0.223$ \\
Duration analgesia $(\mathrm{h})$ & $23.98 \pm 5.88$ & $24.68 \pm 6.19$ & $\mathrm{p}=0.535$ \\
Duration sensory $(\mathrm{h})$ & $14.10 \pm 3.74$ & $13.64 \pm 5.00$ & $\mathrm{p}=0.492$ \\
Duration motor $(\mathrm{h})$ & $11.24 \pm 3.12$ & $11.36 \pm 4.09$ & $\mathrm{p}=0.613$ \\
\hline
\end{tabular}

Conclusions: Levobupivacaine $0.33 \% 30 \mathrm{~mL}$, produces axillary brachial plexus block of similar characteristics than the addition of $100 \mu \mathrm{g}$ fentanyl to levobupivacaine.

References:

1 Karakaya D. Reg Anesth Pain Med 2001; 26: 434-8.

2 Nishikawa K. Anesth Analg 2000; 91: 384-7.

\section{AP2-6}

Contributing factors for vasovagal event during shoulder arthroscopy in the sitting position after interscalene block W. Roh, J. Chung, C. Park, B. Kim, S. Cho

Department of Anesthesiology and Pain Medicine, Daegu Catholic University Medical Center, Daegu, Republic of Korea

Background and Goal of Study: Vasovagal event has been reported in $15-25 \%$ of patients undergoing shoulder arthroscopy in the sitting position after interscalene block (ISB). This study was conducted to assess underlying contributing factors for the vasovagal events in these patients.

Materials and Methods: A retrospective analysis of 63 patients who underwent shoulder arthroscopy in the sitting position with ISB revealed 13 patients who experienced potentially dangerous vasovagal event characterized by sudden severe hypotension and bradycardia (Group 1). Fifty patients (Group 2) did not experience a vasovagal event.

Results and Discussions: There were no significant demographic or baseline hemodynamic differences between groups.

\begin{tabular}{llll}
\hline & Group 1 $(\mathrm{n}=13)$ & Group 2 $(\mathrm{n}=50)$ & P value \\
\hline ASA PS (I/II/III) & $8 / 4 / 1$ & $39 / 10 / 1$ & 0.374 \\
Block Site (Rt./Lt.) & $12 / 1$ & $32 / 18$ & 0.048 \\
Epinephrine in LA (yes/no) & $4 / 9$ & $26 / 24$ & 0.264 \\
Maximal Decrease of MAP from & $30.1 \pm 3.4$ & $9.2 \pm 0.7$ & 0.000 \\
$\quad$ Baseline (\%) & & & \\
Time of Maximal Decrease of & $28.5 \pm 3.5$ & $33.8 \pm 2.0$ & 0.236 \\
$\quad$ MAP from Baseline (min) & & & \\
Sensory Blockade (none; 0, moderate; 1, complete; 2) & & \\
C3 dermatome & $1.7 \pm 0.2$ & $1.4 \pm 0.1$ & 0.293 \\
C4 dermatome & $2.0 \pm 0.0$ & $1.9 \pm 0.5$ & 0.403 \\
C5 dermatome & $1.9 \pm 0.8$ & $2.0 \pm 0.0$ & 0.305 \\
C6 dermatome & $1.7 \pm 0.2$ & $1.6 \pm 0.8$ & 0.782 \\
C7 dermatome & $1.3 \pm 0.2$ & $1.3 \pm 0.1$ & 0.976 \\
C8 dermatome & $0.6 \pm 0.2$ & $1.1 \pm 0.1$ & 0.060 \\
T1 dermatome & $0.5 \pm 0.2$ & $0.8 \pm 0.1$ & 0.286 \\
T2 dermatome & $0.5 \pm 0.2$ & $0.3 \pm 0.1$ & 0.488 \\
Use of Fentanyl before Events & $7 / 6$ & $2 / 48$ & 0.000 \\
\hline
\end{tabular}

Values are mean \pm SEM. 
Conclusion(s): These results indicate that the site of ISB and incomplete block or perioperative fentanyl are possible contributing factors for vasovagal events in shoulder arthroscopy in the sitting position under ISB.

References:

D'Alessio JG et al. Anesth Analg 1995; 80: 1158-62.

Kinsella SM et al. Br J Anaesth 2001; 86: 859-68.

\section{AP2-7}

The effects of adding tramadol to ropivacine on axillary brachial plexus blockade in uremic patients

B. Dikmen, M. Gamli, E. Horasanli, D. Ornek, M. Pekel

II. Anesthesiology and Reanimation, Ankara Numune Training and Research Hospital, Ankara, Turkey

Background and Goal of Study: Clinical studies to determine the effects of tramadol added to local anesthetics for regional anesthesia have yielded contradictory results $(1,2)$. The aim of this study was to evaluate the effect of tramadol added to ropivacaine for axillary brachial plexus block.

Materials and Methods: Thirty uremic patients scheduled for arteriovenous fistula operation were included in the study. Patients were randomly allocated into two groups. We performed axillary block with peripheral nerve stimulator by multiple injection technique. Patients in Group I received ropivacaine $(3.75 \mathrm{mg} / \mathrm{mL}) 38 \mathrm{~mL}$ and $2 \mathrm{~mL}$ of normal saline and patients in Group II received ropivacaine $(3.75 \mathrm{mg} / \mathrm{mL}) 38 \mathrm{~mL}$ and $2 \mathrm{~mL}$ of tramadol $(50 \mathrm{mg} / \mathrm{mL})$. The onset and the duration of sensory blockade were assessed by pinprick. Motor block was tested by the movements of the fingers and the wrist. Mann Whitney-U and chi-square tests were used for statistical analysis and $p<0.05$ was considered as significant.

Results and Discussions: Demographic data, onset of sensory and motor block, duration of sensory and motor block, quality of anesthesia and first analgesic requirement times were similar between two groups.

Conclusion: The results of our study showed that adding tramadol to ropivacaine may have no beneficial effects for the motor and sensorial blockade quality on axillary brachial plexus blockade.

References:

1 Mannion S. Br J Anaesth 2005; 94: 352-356.

2 Robaux S. Anesth Analg 2004; 98: 1172-1177.

\section{AP2-8}

Infraclavicular brachial plexus block with lateral approach: Single injection versus double injection

L. Bouattour, M. Smaoui, M. Dabbabi, M. Salhi, A. Karoui

Anesthesiology, University Habib Bourguiba Hospital, Sfax, Tunisia

Background and Goal of Study: Single-injection coracoid infraclavicular brachial plexus block produces inconsistent anesthesia of the upper limb $(1,2)$. Can Single-injection lateral infraclavicular brachial plexus block with stimulation of the Posterior Cord, provide both sensory and motor block enough for upper limb surgery?

Material and Methods: After institutional ethics approval and informed consent, 80 patients undergoing upper limb surgery, using infraclavicular block were included in a double-blinded, prospective and comparative study. Patients with conditions precluding brachial plexus block were excluded. Patients were randomly assigned to receive a lateral infraclavicular block guided by nerve stimulator: Group 1: single injection, radial response; $30 \mathrm{ml}$ of local anesthetic ( $\left.L A^{*}\right)$, Group 2: dual-injection, radial response; $15 \mathrm{ml}$ of $L A^{*}$ and median response; $15 \mathrm{ml}$ of $L A^{*}$. (LA*: mixture of $2 \%$ lidocaine and $0.5 \%$ bupivacaine). The patient was supine with the head turned to the contralateral side with the operated arm in addiction, the elbow was fixed to the body and the palm of the hand was on the belly.The puncture site was located in the middle of infraclavicular fossa; the needle was perpendicular to the skin. Sensory and motor block were assessed 10 and 20 min after the end of the injection of LA. Statistical analysis used Chi-square test and student's t test. $p<0.01$ was significant.

Results and Discussion: Data are shown in the table:

\begin{tabular}{llll}
\hline & $\mathrm{G} 1(\mathrm{n}=40)$ & $\mathrm{G} 2(\mathrm{n}=40)$ & $P$ value \\
\hline Success rate (\%) & 92.5 & 95 & 0.64 \\
Sensory block score at 20 mn (mean $\pm \mathrm{sd})$ & $12 \pm 1,5$ & $12,6 \pm 1,2$ & 0,054 \\
Motor block score at 20 mn (mean $\pm \mathrm{sd})$ & $7,2 \pm 1,6$ & $8 \pm 1,6$ & 0,028 \\
Duration to perform the block (mn) & $4 \pm 2,4$ & $6,2 \pm 2,7$ & $<0,001^{*}$ \\
EVA when block was performed & $36 \pm 13,5$ & $44 \pm 15,1$ & $0,008^{*}$ \\
Vascular puncture (\%) & 2.5 & 10 & 0.17 \\
\hline
\end{tabular}

Conclusion: Single injection with radial response appears not only as efficient as dual injection in vertical paracoracoid approach, but also less painful and quickly to perform.

References:

1 Fuzier R. Eur J Anaesthesiol. 2006; 23:271-5.

2 Rodrïguez J. Anesth Analg 2004; 99:1225-30.

\section{AP3-1}

Spread of single and multiple injections in the paravertebral space

R. Malchow, S. Aikele

Department of Anesthesia and Operative Svc, Brooke Army Medical Center, Ft Sam Houston, USA

Background and Goal of Study: Significant controversy exists regarding whether multiple injections are required for adequate spread within the paravertebral space. We compared single versus multiple paravertebral injections to evaluate adequacy and extent of spread.

Materials and Methods: As an observational study, ten volunteers were randomly assigned to receive either a single 30-ml injection (T4) or two separate $15-\mathrm{ml}$ injections (T2, T5). Under fluoroscopy, a 22-gu needle was advanced $2.5 \mathrm{~cm}$ lateral from midline to the transverse process, then advanced caudally an additional $1.25 \mathrm{~cm}$. With the bevel caudad, injections of diluted Isovue $200 \mathrm{M}$ were performed at a rate of $0.5-1 \mathrm{ml} / \mathrm{sec}$. Within 30 minutes, thoracic MRI scans were performed in the coronal, saggital, and axial planes. A blinded neuroradiologist documented the extent of spread within the paravertebral, epidural, contralateral, subpleural, paraspinal, and the intercostal spaces.

Results and Discussions: Results are summarized in table.

\begin{tabular}{|c|c|c|c|c|c|}
\hline Volunteer & $\begin{array}{l}\text { \# of } \\
\text { Levels }\end{array}$ & Lateral & Epidural & Paraspinal & Subpleural \\
\hline \multicolumn{6}{|c|}{ Single Injection } \\
\hline 2 & 6 & Yes & None & Yes & No \\
\hline 4 & 4 & Yes & Yes & Min & Min \\
\hline 8 & 4 & Yes & Min & Min & Yes \\
\hline 9 & 5 & Yes & Min & Min & Yes \\
\hline 10 & 6 & Yes & Yes & Yes & Min \\
\hline Ave & 5 & $100 \%$ & Varies & Varies & Varies \\
\hline \multicolumn{6}{|c|}{ Multiple Injections } \\
\hline 1 & 9 & Min & None & Yes & Yes \\
\hline 3 & 10 & Yes & Yes & Yes & Yes \\
\hline 5 & 10 & Yes & Yes & Yes & Yes \\
\hline 6 & 8 & Yes & Min & Yes & Yes \\
\hline 7 & 10 & Yes & Yes & Yes & Yes \\
\hline Ave & 9.4 & $80 \%$ & Varies & $100 \%$ & $100 \%$ \\
\hline
\end{tabular}

Conclusion(s): A multiple injection technique may more reliably block up to 9 contiguous levels. Both techniques result in significant spread into intercostal, epidural, paraspinal, subpleural, and contralateral spaces.

\section{AP3-2}

\section{5-year-course of infections in 5,500 continuous peripheral nerve blocks}

F. Reisig, M. Neuburger, J. Breitbarth, J. Buettner

Anesthesiology and Intensive care medicine, Traumacenter Murnau, Murnau, Germany

Background and Goal of Study: During the years 2002-2004 data of 3941 cPNB was analyzed. The results showed that the most common complications in continuous peripheral nerve blocks (cPNB) were infections, with a rate of medium and severe infections of $3,2 \%(1,2)$. Which factor could play a leading role in improving the existing standard operating procedures to reduce that enormous rate?

Materials and Methods: Over a 5 year period we investigated in a prospective study design all cPNB in a level one trauma centre (until 10/2006: $n=5536$ ). The type of catheter, type of operation, the basic demographic data of the patient, duration of catheters and emerging complication were recorded in a computer based datasheet. It was available $24 \mathrm{~h}$ in each operation theatre and on each surgical ward. At that time we took part in the development of the German recommendations for the hygienic handling of PNB (2), which were carried out in our institution since 2002. In the beginning of 2005 we additionally reinforced our disinfection regime before nerve puncture. The "classical" five-swab-disinfection was prolonged to a combined disinfection using spraying and swamp cleaning lasting a minimum period of ten minutes. Results and Discussions: In the time from 01/2005 to 10/2006 we carried out the intensified regime on further 2045 cPNB. In 2005 we found an infection 
rate of $1,4 \%$ for medium and serve infections $(p<0,05$, compared to 2002-2004), and even lower in 2006 (until 10/06) with 0,9\% ( $p<0,05)$. The type of CPNB (data not shown), representing the location as a risk factor, as well as the medium duration of the catheter (2002-2004: 4,03 d[0-36]; 2005: 4,69d[0-34]) did not differ significantly during 2002-2006.

Conclusion: By simply spending extra time on disinfection by combining spraying- and swab-disinfection, one can even improve an established, strict hygienic regime. With this small alteration in procedure, it seems to be possible to reach a reduction of more than $50 \%$ of the medium and severe infections in cPNB.

References:

1 Neuburger M. Anaesthesist 2006 Jan; 55(1): 33-40.

2 Neuburger M. Acta Anaesthesiol Scand. 2006 Nov 1 [Epub ahead of print].

3 Morin et al. Anaesthesiologie und Intensivmedizin 2006, 6: 372-379.

\section{AP3-3}

A survey of resident training in peripheral nerve blocks

A. Belbachir, L. Eyrolle, N. Rosencher, F. Bonnet, Y. Ozier

Anesthesie-Reanimation Chirurgicale, Hopital Cochin, Paris, France

Background and Goal of Study: Peripheral nerve blocks (PNBs) are increasingly used. Data on PNBs teaching methods, training and learning process of anesthesiology residents are scarce. As a preliminary approach, we have performed a survey of residents experience in this field in teaching hospitals. Materials and Methods: A questionnaire was sent by regular mail to the residents in anesthesiology of Paris teaching hospitals in March 2004, followed 2 months later by a second mail to non-respondents. The questions focused on several items including duration of training, which PNB techniques were performed and total number of attempts for each one, level of self-confidence for each one, theoretical and practical courses, additional sources of training.

Results and Discussions: 174 questionnaires were sent, with a reply rate of $60.4 \% .71 \%$ of the residents underwent 1 or 2 periods of PNB practical training periods occurring mainly between the 3rd and the 6th semester of education. Duration of training was: $<1 \mathrm{mo}: 33 \%$; $1-3 \mathrm{mo}: 39 \%$; $>3 \mathrm{mo}$ : $27 \%$. The most commonly performed PNBs were: femoral, axillary, humeral, sciatic ( $70 \%$ cases) and interscalene brachial plexus block (43\%); PNBs performed $>20$ times were: femoral (35\% (of the respondents ?)); humeral canal (23\%). The rate of residents feeling self-confident with a technique was: $48 \%$ after $\geqslant 15$ cases; $35 \%$ after $10-14$ cases; $12 \%$ after $<10$ cases:. Only $56 \%$ of residents followed up the patients in postoperative period. $52.5 \%$ of residents rated their PNB training as insufficient.

Available published data on the minimal number of PNB cases to be performed suggest that 40 cases are necessary to achieve proficiency in each procedure (1). For some PNBs, such as the interscalene brachial plexus block, over 20 cases are deemed necessary to obtain at least a $70 \%$ success rate (2). Conclusion(s): Despite a preset program, residents training in PNBs appears insufficient. Anesthetic education may include more complete training in PNBs to guarantee the expertise of practitioners.

References:

1 Anesth Analg 2002; 95: 1423-7.

2 Anesth Analg 1998; 86: 635-9.

\section{AP3-4}

The use of ultrasound in thoracic paravertebral blockade

K. Hara, S. Sakura, T. Nomura, K. Doi, J. Ota

Anesthesiology, Shimane University School of Medicine, Izumo City, Japan

Background and Goal: Thoracic paravertebral block is the technique of injecting local anesthetic to the paravertebral space, which results in unilateral somatic and sympathetic nerve blockade. Previous studies have reported its effectiveness for thoracic surgery including breast surgery and pain of unilateral origin from the chest and abdomen. Its clinical advantages include the inhibition of stress and pressor response to surgical stimuli, maintenance of hemodynamic stability, low incidence of complication, and long duration of analgesia. Recent ultrasound technological advances would further increase the effectiveness and the safety of thoracic paravertebral block, although the identification of the nerve and needle is not still possible. The purpose of this study was to examine if thoracic paravertebral block is reliably performed with ultrasound.

Materials and Methods: With IRB approval and informed consent, 20 patients undergoing unilateral breast surgery received thoracic paravertebral block using $0.5 \%$ ropivacaine combined with general anaesthesia. Patients were placed in the lateral decubitus position with the side to be blocked upper. A $3-11 \mathrm{MHz}$ linear array transducer was applied to the paravertebral area $2.5 \mathrm{~cm}$ from the midline at T1 and T4 in a longitudinal fashion. The transverse process (TP) was located and its distance from the skin and that of the parietal pleura (PP) from the skin were measured. Paravertebral blocks at $\mathrm{T} 1$ and $\mathrm{T} 4$ were then performed using an 18G Touhy needle with the lossof-resistance technique. The distances of the needle from the skin to TP and to the loss of resistance (LOR) were measured. A single injection of 5 and $15 \mathrm{ml}$ of the anesthetic solution was performed at $\mathrm{T} 1$ and $\mathrm{T} 4$, respectively. Results and Discussion: Ultrasound imaging of TP and PP was always successful. The distance measured with ultrasound was always less than the needle distance. The spread of local anesthetic solution was visualized at T4. Conclusion: Thoracic paravertebral block is reliably performed with ultrasound. Ultrasound imaging of TP and PP provides a prior knowledge of the depth to the paravertebral space and may increase the safety of paravertebral blocks. The visualization of the anesthetic spread may increase the success rate.

\section{AP3-5}

Paravertebral thoracic adrenaline and hemodynamic effects during open thoracic surgery

I. Garutti, P. Quesada, E. de la Fuente, B. Gutierrez, P. Piñeiro

Anesthesiology, Hospital Gregorio Maranon, Madrid, Spain

Background and Goal of Study: Adrenaline (Ad) added to the anesthetic local solutions may produce cardiovascular effects. Systemic absorption of small amounts of Ad in regional blocks is related with hemodynamic effects attributed to beta adrenergic stimulation. We want to compare hemodynamic effects of paravertebral block (PVB) using a bolus doses of plain Lidocaine $(L)$ or $L$ with $A d(L+A d)$.

Materials and Methods: Prospectively, forty patients undergoing thoracic surgery were randomly in two groups Group L, L $2 \%\left(5 \mathrm{mg} \cdot \mathrm{kg}^{-1}\right)$ or Group $\mathrm{L}+\mathrm{Ad}, \mathrm{L} 2 \%\left(5 \mathrm{mg} \cdot \mathrm{kg}^{-1}\right)$ with $\mathrm{Ad}\left(5 \mu \mathrm{g} \mathrm{mL}{ }^{-1}\right)$. Cardiac index (Cl), derivate pressure (dP max), cardiac function index (CFI) heart rate (HR) and mean arterial pressure (MAP) were measured with an aortic transpulmonary thermodilution technique (PICCO system monitor) during one-lung ventilation. Concentrations of $L$ were measured in arterial samples obtained after 15 and 30 minutes of paravertebral injection. Hemodynamic data were analyzed using Student's t test. Plasma concentration data were analyzed with oneway ANOVA for repeated measures.

Results and Discussions:

\begin{tabular}{llclc}
\hline & Group & Before PVB & After 15 min & After 30 min \\
\hline $\mathrm{Dpmax}$ & $\mathrm{L}$ & $963(301)$ & $795(351)^{\star}$ & $667(278)$ \\
$\mathrm{mmHg} \cdot \mathrm{s}^{-1}$ & $\mathrm{~L}+$ Ad & $1014(311)$ & $723(265)$ & $782(244)$ \\
$\mathrm{Cl}$ & $\mathrm{L}$ & $3.12(.3)$ & $2.7(.2)^{\star}$ & $2.6(.3)^{\star}$ \\
$\mathrm{L} / \mathrm{m} / \mathrm{m}^{-2}$ & $\mathrm{~L}+$ Ad & $2.8(.2)$ & $2.28(.2)$ & $2.25(.3)$ \\
$\mathrm{MAP}$ & $\mathrm{L}$ & $81,7(12)$ & $74,6(13)$ & $72,4(11)$ \\
$\mathrm{mmHg}$ & $\mathrm{L}+$ Ad & 86.8 & 75,4 & $78,6(17)$ \\
$\mathrm{CFI}$ & $\mathrm{L}$ & $4.45(1.4)$ & $4.41(1.2)^{\star}$ & $4.36(1.2)$ \\
& $\mathrm{L}+$ Ad & $4.7(1.4)$ & $4.22(0.9)$ & $4.21(1)$ \\
$\mathrm{HR}$ & $\mathrm{L}$ & $71,3(13)$ & $70,4(13)$ & $70,0(13)$ \\
& $\mathrm{L}+$ Ad & $70,9(16)$ & $67,6(13)$ & $69,5(13)$ \\
\hline
\end{tabular}

Mean (Standard deviation). ( $\left.{ }^{\star}\right) \mathrm{p}<0.05$ between groups Lidocaine plasmatic was $53 \%$ and $34 \%$ lower in the L+Ad group at 15 and 30 minutes respectively.

Conclusion(s): Addition of Ad to $\mathrm{L}$ solutions reduces both the plasma concentrations of $\mathrm{L}$ and myocardial contractility depression observed after injection in the thoracic paravertebral space.

\section{AP3-6}

\section{Thoracic paravertebral block for breast surgery: A randomised, double blind study}

P. Carlsson, L. Nikolajsen, J.F. Moeller, S.A. Rodt, H. Roenning

Anaesthesiology, Aarhus University Hospital, Aarhus C, Denmark

Background and Goal: Breast surgery is often associated with postoperative pain, nausea and vomiting.

We examined the effect of multiple level paravertebral block in a randomised, double blind study on postoperative pain, opioid requirement and POVN. Material and Methods: 194 patients were eligible, 36 met exclusion criteria, 70 declined and 10 were excluded during and after the study. Paracetamol $1 \mathrm{gr}$. was given preop. Immediately before the induction of anaesthesia paravertebral block was performed (1) from C7 to Th4-5 with either Ropivacaine 
$5 \mathrm{mg} / \mathrm{ml} 5 \mathrm{ml}$ at each level (R) or Saline $5 \mathrm{ml}$ at each level (S). Anaesthesia was induced and maintained with propofol infusion and fentanyl. The airway was maintained with a laryngeal mask.

The anaesthetist performing the block, the anaesthetising nurse, the recovery nurse, the interviewing anaesthetist and the patient were all blinded to the type of treatment.

Postoperatively fentanyl was administered as PCA in doses of $50 \mu \mathrm{g}$ Pain was assessed on a numeric scale $(0-10)$ and the occurrence of POVN noted on days 0 to 2 of surgery.

Results and Discussion: The two groups were comparable with respect to age (mean 57 years), BMI (mean 24) and type of surgery. One half of the patients had lumpectomy and axillary dissection and the other half had mastectomy and axillary dissection.

During anaesthesia the $\mathrm{R}$ group received $200 \mu \mathrm{g}$ of fentanyl (median, range $100-400 \mu \mathrm{g})$ and the $S$ group $350 \mu \mathrm{g}(100-550 \mu \mathrm{g})$. In the recovery room 23 of 38 patients in the R group did NOT receive opioids; in the Saline group only 10 of 40 did not use opioid.

\begin{tabular}{lcc}
\hline & Ropivacaine & Saline \\
\hline VAS $\geqslant 3$ in PACU & 13 & 31 \\
10 p.m. at rest & 12 & 11 \\
10 p.m. movement & 12 & 17 \\
8 a.m. day 1, at rest & 4 & 9 \\
8 a.m. day 1, movement & 12 & 16 \\
Nausea day 0 to 2: & 7 & 9 \\
Vomiting day 0 to 2: & 2 & 1 \\
\hline
\end{tabular}

Conclusion: Paravertebral block provided good pain relief until the evening and fewer patients needed opioid in the PACU. A surprisingly low incidence of POVN was seen in this study.

Reference:

1 Klein S. et al. Anesth Analg 2000; 90; 1402-5.

\section{AP3-7}

Techniques of peripheral nerve location during loco-regional anaesthesia

C. Janakiraman, D. McPherson, A.R. Wilkes, O. Adekanye

Anaesthetics, Cardiff University and University Hospital of Wales, Cardiff, United Kingdom

Background: There is an increasing interest in loco-regional anaesthesia [1] and a number of new approaches to the traditional block methods have been published recently. However, the success of peripheral nerve blocks is still influenced by the method of localization of a nerve plexus. The aim of this survey is to define the methods used to locate peripheral nerves by anaesthetists in Wales, UK.

Methods: Questionnaires were sent to 266 consultant anaesthetists in Wales between August and October 2006. Information was sought on the methods used to locate peripheral nerves and the magnitude of current used when a peripheral nerve stimulator (PNS) was utilized. The number of nerve blocks performed per month was also solicited.

Results and Discussions: Data expressed as percentage; $n=164$

\begin{tabular}{lrc}
\hline Methods & Use & Do not use \\
\hline Landmark & 63 & 37 \\
Paraesthesia & 8 & 92 \\
PNS & 67 & 33 \\
2D USG & 13 & 8 \\
\hline
\end{tabular}

2D USG: 2 dimensional ultrasound guidance

$67 \%$ of consultants performed $<10$ blocks/month, $25 \%$ between 11 and 50 and $8 \%$ more than 50 blocks. A majority of anaesthetists $(55 \%)$ used a minimum current range of $0.3-0.5 \mathrm{~mA}$ and $67 \%$ used that as the injecting current. $11 \%$ were injecting at $0.6-0.9 \mathrm{~mA}$ and another $11 \%$ at $\leqslant 0.2 \mathrm{~mA}$. $8 \%$ injected at $1 \mathrm{~mA}$. Lowest current was almost always sought by $84 \%$ of anaesthetists and occasionally by $11 \% .5 \%$ never sought the lowest current. Conclusion(s): Landmarks and PNS were the most commonly used techniques of nerve location in Wales, paraesthesia is rarely used. The use of 2D USG is probably not yet a routine practice globally. The magnitude of current chosen in our survey is comparable to the American practice [2].

References:

1 Klein MS et al. Anesth Analg 2002; 94: 71-6.

2 Hadzic A. et al. Anesthesiology 1997; 3A: A22.

\section{AP3-8}

The feasibility of teaching ultrasound for peripheral nerve identification within a residency program - learning curves in brachial plexus block

M. Frank, C. Hoffmann, D. Wiessner, T. Koch, R.J. Litz

Anaesthesiology, University of Technology, Dresden, Germany

Background and Goal of Study: High resolution ultrasound (US) in experienced hands allows real-time visualization of peripheral nerves, needle insertion, and the spread of the local anaesthetic solution. However, due to the suspected simplicity of the procedure most residents were educated in identification of peripheral nerves by means of peripheral neurostimulation at first rather than by using ultrasound. We evaluated the feasibility of teaching US for nerve identification at first by analyzing the learning curves of residents without experience in performing peripheral nerve blocks nor in applying US in axillary brachial plexus block (BPB) according to the residency teaching program.

Materials and Methods: Six residents underwent a standardized training program for US-guided BPB performance. In a first step sonoanatomy of the upper arm and the axilla was examined in each other under supervision of an experienced staff anesthetist. Thereafter residents watched 5 BPB performed by the staff with special regards to nerve identification, anatomical variations, needle visualization and local anesthetic spread. Then, each resident performed 30 BPBs under supervision. Technique was corrected if appropriate. A maximum of $40 \mathrm{ml}$ of local anesthetic solution was allowed. According to a standardized protocol sensory and motor block of 5 nerves were recorded in 5 min intervals. Likewise duration of the BPB performance was recorded.

Results and Discussions: Within 30 BPB residents reached a mean success rate of $89.4 \%$ [85-96] which was not significantly different between residents $(p=0.3)$. However, duration of procedure significantly differed between residents $(10.9 \pm 5.2 \mathrm{~min}$ vs. $16.9 \pm 5.2 \mathrm{~min} ; \mathrm{p}<0.001)$ and decreased over time in all residents. During performance of the first 10 blocks the identification of the radial and the musculocutaneous nerve was the most commonly seen problem for all residents. In all patients no complication related to block performance occured.

Conclusion(s): Teaching US-guided performance of BPB to unexperienced residents was appropriate in all 6 candidates and resulted in a rapid increase in the individual learning curve, thus providing a high success rate after a short period of training.

\section{AP4-1}

Prevention of spinal-induced hypotention by bolus injection of Lactate Ringers solution and Hydroxyethyl Starch

R. Martinez, X. Santiveri, A. Arxer, B. Pardina, J. Castillo

Anesthesiology, Hospital Mar-Esperança IMAS, Barcelona, Spain

Background and Goal of Study: Several studies have questioned the advantage of preloading with crystalloid in the prevention of spinal-induced hypotension. The aim of this study was to determine the efficacy of crystalloids administered 30 min before spinal anaesthesia compared with a 'bolus' administration of crystalloids or colloids at the time of spinal block, in preventing spinal-induced hypotension $(\mathrm{SIH})$.

Materials and Methods: We studied 72 patients ASA I-III scheduled for surgical interventions without tourniquet or, important blood loss and with the patient placed in supine position. Monitoring included non-invasive arterial pressure (NIAP), electrocardiogram and pulse oximetry. NIAP, heart rate and peripheral oxygen saturation measurements were recorded every 5-min the first $30 \mathrm{~min}$ after the induction of anaesthesia and every $15 \mathrm{~min}$. The sensory level was assessed $20 \mathrm{~min}$ after the intrathecal injection. Patients were randomized into three groups: Group $1(n=24)$ received $350 \mathrm{~mL}$ of lactate Ringer's (LR) over 30 min prior to spinal anaesthesia, Group 2 ( $n=24)$ received a bolus injection of $170 \mathrm{~mL}$ of hydroxyethyl starch (HS) over $3 \mathrm{~min}$ after spinal anaesthesia and, Group $3(n=24)$ received a bolus injection of $350 \mathrm{~mL}$ of LR over $3 \mathrm{~min}$ after spinal anaesthesia.

Results and Discussions: The LR preload and postload group, and the HS postload group were comparable in sex, height, weight, level sensory block, ASA, type of surgery and operation time. The incidence SIH was significantly higher $(P=0.003)$ in the LR preload group (37.5\%) than in the HS postload (3.8\%) and in LR postload group (8\%).

However, no differences in the incidence of hypotension were found between the LR postload and HS postload group.

Conclusion: It is recommendable to use a fast and small postload of volume immediately after spinal anaesthesia in order to prevent $\mathrm{SIH}$.

Reference:

Ewaldsson CA, Hahn RG. Volume kinetics of Ringer's solution during induction of spinal and general anaesthesia. Br J Anaesth. 2001; 87: 406-14. 


\section{AP4-2}

The prognostic value of baroreflex sensitivity for severe bradycardia during spinal anaesthesia

A. Chatzimichali, A. Zoumprouli, E. Astyrakaki, M. Maniadakis, H. Askitopoulou

Anaesthesiology, University Hospital of Heraklion, Heraklion, Greece

Background and goal of study: During spinal anaesthesia the incidence of severe bradycardia is reported to be $13 \%{ }^{1}$ while the incidence of cardiac arrest is reported as $6.4 \pm 1.2 / 10.000$ patients $^{2}$. The purpose of the present study was to investigate the prognostic value of baroreflex sensitivity in predicting which patients will develop severe bradycardia during spinal anaesthesia. To test autonomic function the reflex heart rate responses to blood pressure changes induced by vasoactive drugs were used.

Materials and Methods: Thirteen ASA I-II patients, 20-50 years of age, subjected to elective lower abdominal or limb surgery under spinal anaesthesia were studied. Patients with a history of cardiovascular disease and disorders of the autonomic nervous system were excluded. All patients received $10-15 \mathrm{mg}$ of $0.5 \%$ plain bupivacaine through a 25 gauge spinal needle at L3-L4 level. The level of spinal block was at T5-T12 dermatome level. The baroreflex sensitivity was assessed before the induction of the spinal block using a rapid bolus injection of phenylephrine $(200 \mu \gamma)$. The values of blood pressure were plotted versus RR intervals and a regression analysis was performed. The relation was linear between the two variables over a limited pressure range and the slope of the regression line allows a qualification of the sensitivity of the reflex. Statistical analysis was performed with student t-test after the homogeneity of variances.

Results and Discussions: The analysis of baroreflex slopes in the six patients with severe bradycardia $(<45 \mathrm{bpm})$ showed steep slope of the regression line, indicating strong vagal reflexes. In contrast, the pattern of response in patients who did not develop bradycardia was flat, indicating weak vagal reflexes. Using Levene and Brown-Forsythe tests for homogeneity of variances, the confidence interval was $98.7 \%$.

Conclusions: The assessment of baroreflex sensitivity before spinal anaesthesia can predict patients prone to develop severe bradycardia. However, to assess more accurately its prognostic value probably are needed more patients. References:

1 Carpenter R.L, et al. Incidence and risk factors for side effects of spinal anaesthesia. Anesthesiology, 1992; 76: 906-16.

2 Auroy. E et al. Serious complications related to regional anaesthesia. Anesthesiology 1997; 87: 479-86.

\section{AP4-3}

Intrathecal fentanyl added to 0.5 bupivacaine in spinal anesthesia for urologic surgery may impair haemodynamic stability

S. Fontes, D. Chalo, M.E. Amadeu, B. Pinto, G. Cardoso

Serviço de Anestesiologia, Hospital Geral de Santo Antonio, Porto, Portugal

Background and Goal of Study: Adding an intrathecal opioid to a local anesthetic results in synergism, but the hemodynamic effets have not been studied. Our study evaluated the haemodynamic stability of the association of bupivacaine and fentanyl in spinal anaesthesia.

Materials and Methods: Prospective, randomized, double-blind study. Patients (ASA I-III), had prostate surgery (TURP): groups A/B; or vesical surgery (TUR-VT): groups C/D. Spinal anaesthesia was with hyperbaric bupivacaine (Bupi) $0.5 \%$ with fentanyl (Fenta) $(15 \mu \mathrm{g})$ or saline. There were 4 groups: $A(n=10)$ : Bupi $10 m g$ + Fenta; $B(n=12)$ : Bupi $10 m g$ + saline; $C(n=18)$ : Bupi $7 \mathrm{mg}+$ Fenta; $\mathrm{D}(\mathrm{n}=10)$ : Bupi $7 \mathrm{mg}+$ saline. Mean arterial pressure (MAP) was recorded at T0 (baseline) and at 5', 10', 15', 30', 60' after spinal, before legs down and last measure in OR. The difference between each recorded MAP and MAP at TO was calculated. Average MAP differences were compared between Groups A/B and between Groups C/D using MannWhitney test $(p<0.05)$. Results are mean \pm SD.

Results and Discussions: Fifty patients were studied (46 men), 76\% ASA II, age $67.7 \pm 8.7$ years. Demographics did not differ. The table shows MAP differences at each time point for each group. A statistically significant difference was found for (T5-T0) between $A$ and $B\left(p=0.029^{\star}\right)$.

\begin{tabular}{lcllll}
\hline Group & T5-T0 & T10-T0 & T15-T0 & T60-T0 & Tfinal-T0 \\
\hline A & -14.5 & -11.9 & -13.4 & -15.14 & -17.2 \\
& $\pm 11.2^{*}$ & \pm 17.5 & \pm 10.8 & \pm 13.6 & \pm 11.4 \\
B & 0.42 & -10.9 & -11.7 & -5.6 & -9.3 \\
& $\pm 17.8^{*}$ & \pm 17.9 & \pm 18.1 & \pm 16.4 & \pm 16.2 \\
C & -1.89 & -1.22 & -3.28 & -1.43 & -4.8 \\
& \pm 9.1 & \pm 10.3 & \pm 9.1 & \pm 7.6 & \pm 7.3 \\
D & -7.2 & -12 & -7.5 & -11.4 & -9.9 \\
& \pm 16.0 & \pm 31.6 & \pm 16.5 & \pm 21.5 & \pm 21.7 \\
\hline
\end{tabular}

Conclusions: Adding fentanyl to spinal bupivacaine resulted in significant MAP reduction at $5 \mathrm{~min}$. This was not observed when a lower dose of bupivacaine was used. Further studies will be required.

References:

1 Kararmaz A. Anaesthesia. 2003 Jun; 58(6): 526-30.

2 Bano F. J Coll Physicians Surg Pak. 2006 Feb; 16(2): 87-90.

\section{AP4-4}

The role of carbohydrate - rich drink on perioperative discomfort, hemodynamic changes, and insulin responses in spinal anesthesia patients

H. Yagmurdur, S. Gunal, H. Yildiz, H. Gulec, C. Topkaya

Clinic of Anaesthesiology and Reanimation, The Ministry of Health Ankara Research and Training Hospital, Ankara, Turkey

Background and Goal of Study: The fasting period before elective surgery is often long enough to deplete carbohydrate reserves and also uncomfortable for the patient(1). The aim of this study was to investigate the role of carbohydrate-rich drink on perioperative discomfort, haemodynamic changes, and insulin response in elective lower abdominal surgery patients with spinal anaesthesia.

Materials and Methods: Fourty-four consecutive ASA I-II adult patients were randomly assigned to one of two groups: preparation with carbohydrate-rich drink ( $\mathrm{CHO}=22$ patients) or fasting from midnight (Fasted $=22$ patients). During the evening before surgery, patients in the $\mathrm{CHO}$ group ingested $800 \mathrm{~mL}$ of carbohydrate-rich drink. Also another $400 \mathrm{~mL}$ of morning drink at least $90 \mathrm{~min}$ before spinal anaesthesia was allowed in the $\mathrm{CHO}$ group. The patients in the Fasted group underwent spinal anaesthesia after the routine fast from midnight. Visual analogue scales were used to score 10 different discomfort variables. Also blood glucose and insulin concentrations and haemodynamic changes were recorded during perioperative period. Results and Discussion: The visual analogue scale scores in a control situation and before intake of morning drink not different between groups. The $\mathrm{CHO}$ group was less hungry, less thirsty and experienced less malaise and unfitness during the period before and after the spinal anaesthesia $(p<0.05)$. Also the $\mathrm{CHO}$ group was less anxious than the Fasted group before spinal anaesthesia $(p<0.05)$. Trend analysis showed decreasing hunger, thirst, malaise, and unfitness during perioperative period and decreasing anxiety during the waiting period before surgery $(p<0.05)$. In the Fasted group, hunger and thirst increased during perioperative period $(p<0.05)$. Plasma glucose and insulin concentrations were increased in the $\mathrm{CHO}$ group before spinal anaesthesia $(p<0.05)$. Plasma glucose increased and insulin decreased in the Fasted group at $60 \mathrm{~min}$ after the spinal anaesthesia $(p<0.05)$. In the Fasted group, mean arterial pressure was lower at before and 10 and 20 min after the spinal anaesthesia compared to the $\mathrm{CHO}$ group ( $p<0.05)$.

Conclusion: Preparation with oral carbohydrate before spinal anaesthesia had advantages over overnight fasting by reducing perioperative discomfort, improving insulin response and stabilizing mean arterial pressure in lower abdominal surgery patients(2).

References:

1 Søreide E, Eriksson LI, Hirlekar G et al. Acta Anaesthesiol Scand 2005; 49: 1041-7. 2 Hausel J, Nygren J, Lagerkranser M et al. Anesth Analg 2001; 93: 1344-50.

\section{AP4-5}

\section{Unilateral spinal anesthesia for total knee replacement surgery: comparison of low dose hyperbaric and hypobaric ropivacaine}

Y. Pala, M. Canturk, M. Arslan, O. Kilci, L. Ozdogan

II. Anesthesiology and Reanimation, Ankara Numune Education and Research Hospital, Ankara, Turkey

Background and Goal of Study: The baricity of local anesthetic solutions can effect quality of subarachnoid block (1). We aimed to determine the efficiency of hyperbaric and hypobaric ropivacaine solutions with unilateral spinal anesthesia applied for total knee replacement (TKR) surgery. Materials and Methods: 60 patients with ASA risk status I-III scheduled for TKR was included in the study. To maintain spinal anesthesia, in Group I (hyperbaric group) the operated extremity of the patient was lowermost with $15^{\circ}$ fowler position, in Group II (hypobaric group) the operated extremity was uppermost with $15^{\circ}$ trendelenburg position. The study solution containing $11.25 \mathrm{mg}$ ropivacaine in $3 \mathrm{ml}$ was introduced to cerebrospinal fluid through $\mathrm{L}_{3-4}$ interspace with combined spinal epidural anesthesia (CSEA) in $180 \mathrm{sec}-$ onds. Hemodynamic parameters, sensory and motor block data were 
recorded. Statistical analysis was performed using Mann Whitney-U, Wilcoxon and chi-square tests and $p<0.05$ was considered as significant.

Results and Discussions: Demographical data were similar. The time to reach $T_{10}$ level of the sensory block was significantly shorter in Group I $(p=0.003)$. The regression time of the sensory block was shorter in Group II (0.046). The incidence of unilateral spinal anesthesia was higher in Group II (\%73.3 vs \%66.6). The hemodynamic parameters were well preserved in both groups.

Conclusion: We think that the hypobaric technique may provide higher incidence of unilateral block and shorter recovery time of sensory block.

Reference:

1 Kaya M: Reg Anesth Pain Med 2004; 29(1): 17-22.

\section{AP4-6}

\section{Determination of factors affecting the success rate of spinal} anaesthesia

B. Cevik, S. Colakoglu, A. Orskiran, Y. Ekti, S. Karaman

Department of II. Anesthesiology and Intensive Care, Dr.Lutfi Kirdar Kartal Training and Research Hospital, Istanbul, Turkey

Background and Goal of Study: Spinal anaesthesia is widely used for many surgical procedures and multiple factors can influence the chance of a successful block(1). This study aimed to identify the factors affecting the success rate of our spinal anaesthesia managements.

Material and Methods: After approval by Institutional Ethics Committee and informed consent, all spinal anaesthesia procedures were recorded over a period of 4 months. Before attempting, the attending anaesthesiologist recorded the patient's characteristics, anatomical landmarks and previous history of spinal anaesthesia. We recorded the training level of the provider as 1-2 years (Group A), 2-4 years (Group B) anaesthesia resident or staff (Group C) having more than 3 yrs' experience. Needle gauge and spinal level used, was also recorded. All spinal blocks were performed in the sitting position through a midline approach. Redirection of the needle and each new skin puncture was considered as a new attempt. Logistic regression was used to test the association between recorded data and success.

Results: We studied 295 patients (Table-1). Totally 445 attempts were recorded. Successful anaesthesia following one or more attempts at the first or second interspace was attained in $94.77 \%$ of patients, while the overall success rate at the first attempt was $62.71 \%$. First- level success was not affected by age, sex, body mass index (BMI),anatomic landmarks, previous history of spinal anaesthesia, spinal needle gauge and level $(p>0.05)$. First attempt success rates of Group A,B and C were $44.23,60.67$ and $68.24 \%$, respectively. Anaesthesiologist having more than 3yrs' experience had a success rate significantly higher than the other groups $(p=0.013)$. The incidence of postdural puncture headache was $1.02 \%$. The most frequently encountered adverse events were hypotension (3.39\%), nausea (3.05\%) and bradycardia $(2.03 \%)$. No neurologic complications were observed.

Table-1. Patients' demographics $(n=295)$

Age(yr)

$\operatorname{BMI}\left(\mathrm{kg} / \mathrm{m}^{2}\right)$

Gender (M/F)

ASA(I/II/III)

$25.76 \pm 3.38$

$276 / 19$

$78 / 161 / 56$

Conclusion: Our results suggest that more experienced anaesthesiologists would have greater chances of reaching the subarachnoid space.

Reference:

1 EJA 2002; 19: 447-451.

\section{AP4-7}

Comparison between hypobaric unilateral spinal anaesthesia and conventional spinal anesthesia in elderly patients for hip trauma

Z. Triki, K. Krid, F. Amouri, M. Ayedi, A. Karoui

\section{Anesthesiologie, CHU Habib Bourguiba, Sfax, Tunisia}

Background: Hip fracture is common in the geriatric population. All anaesthetic techniques aim to avoid deleterious fall in arterial blood pressure. Hypobaric unilateral spinal anaesthesia can be associated with hypotension but has less hemodynamics effects compared to conventional spinal anesthesia (1). This study aims to do that in a prospective manner.

Material and Methods: After ethical committee approval and written informed consent, 40 elderly patients (>65 yr) were randomized in two groups: conventional group and a unilateral group. Following a preload with (hemacell囚) $(7 \mathrm{ml} / \mathrm{kg})$ and after an iliofascial block, patients were placed in the lateral decubitus position, operative side upper most Both groups received spinal anesthesia as a combination of hyporbaric bupivacaine $7.5 \mathrm{mg}$ and fentanyl 10 ug.

Patients were kept in position for 15 minutes in the unilateral group and immediately tourned on the dorsal side in the conventional group. Systolic, mean and diastolic arterial blood pressures, heart rate, $\mathrm{SaO} 2 \%$, ephedrine consumption, duration of sensory and motor block were recorded.

Results: Unilateral group had higher mean values of systolic pressure from the 20th minute to the 80 th minutes after spinal ponction than conventional group ( $P<0.05)$. Hypotension occurred in $60 \%$ of patients in the conventional group and $15 \%$ in the unilateral group $(P=0.003)$. The hypotension was deeper in conventional group $(30,71 \%)$ than in unilateral group $(18,57 \%)(\mathrm{P}<0.0001)$. Ephedrine was required to control hypotension in six unilateral patients $(30 \%)$ and twelve conventional patients $(60 \%)(P=0.05)$. The ephedrine consumption was $1.35 \pm 3.7 \mathrm{mg}$ for unilateral group and $11.25 \pm 15.10 \mathrm{mg}$ for conventional group $(p=0.007)$. Mean duration of sensory and motor block on the operative side were comparable in the both groups.

Conclusion: In elderly patients undergoing hip surgery, hypobaric unilateral spinal anaesthesia induces less haemodynamic change than the conventional spinal anesthesia and more satisfactory condition.

Reference:

1 M. Khatouf. Annales Françaises d'Anesthésie et de Réanimation 2005; 24: 249-254.

\section{AP4-8}

\section{Laparoscopic cholecystectomy under spinal anaesthesia:} intraoperative findings from a pilot study

K. Pratsas, D. Bagka, S. Georgopoulou, F. Fafoulakis, G. Tzovaras

Anesthesiology, University Hospital of Larissa, Larissa, Greece

Background and Goal of Study: Regional anaesthesia alone or combined with general anaesthesia has been used for laparoscopic cholecystectomy for high risk patients (1). We conducted a pilot study to assess the feasibility and safety of the use of spinal anaesthesia for laparoscopic cholecystectomy in fit patients, with a view to later compare the two methods of anaesthesia in a prospective randomized trial.

Materials and Methods: We studied 15 patients, ASA I or II (12 women, age 27-67). All patients received $500 \mathrm{ml}$ of Ringer's Lactate solution preoperative and a nasogastric tube was inserted before the introduction of anaesthesia. With the patient at the right decubitus position a $25 \mathrm{G}$ pencil point spinal needle was introduced into the subarachnoid space at the L2-L3 intervertebral space and $3 \mathrm{ml}$ hyperbaric plain bupivacaine $0.5 \%, 0.25 \mathrm{mg}$ morphine and $20 \mu \mathrm{g}$ fentanyl were injected intrathecaly.

Results and Discussions: All operations were completed laparoscopicaly and conversion from spinal to general anaesthesia was not required in any of the cases. No patient reported intra-abdominal complaints. Serial arterial blood gas assessment revealed that $\mathrm{CO}_{2}$ loading from the pneumoperitoneum was compensated $\left(\mathrm{pH} \geqslant 7.34\right.$ and $\left.\mathrm{PCO}_{2} \leqslant 47\right)$. Intraoperative incidents are present on table.

\begin{tabular}{lc}
\hline Intraoperative incidents & $\mathrm{N}(\%)$ \\
\hline Mild right shoulder pain (no medication given) & $2(13.3)$ \\
Severe shoulder pain (midazolame-fentanyl iv) & $2(13.3)$ \\
Nausea & $1(6.6)$ \\
Vomiting & $0(0)$ \\
Bradycardia $(<40 \mathrm{p} / \mathrm{min})$ & $2(13.3)$ \\
Hypotension $(<20 \%$ pre-anaesthetic value) & $10(66)$ \\
\hline
\end{tabular}

Conclusions: Laparoscopic cholecystectomy can be successfully and safely performed under spinal anaesthesia. Intraoperative incidents related to either the pneumoperitoneum (shoulder pain) or the high level of sympathetic blockage (hypotension, bradycardia) are easily manageable and do not preclude completion of the procedure.

Reference:

1 Pursnani KG. Surg Endosc 1998; 12: 1082-1084.

\section{AP4-9}

Postoperative course after laparoscopic cholecystectomy under spinal anaesthesia: a pilot study

S. Georgopoulou, D. Bagka, K. Pratsas, G. Stamatiou, G. Tzovaras

Anaesthesiology, University Hospital of Larissa, Larissa, Greece

Background and Goal of Study: Regional anaesthesia alone or combined with general anaesthesia has been used for laparoscopic cholecystectomy for high risk patients (1). We conducted a pilot study to assess the postoperative pain, recovery and side effects of spinal anaesthesia for ASA I-II patients, with a view to later compare the two methods of anaesthesia. 
Materials and Methods: Fifteen ASA I-II patients (12 women, age 27-67) underwent laparoscopic cholecystectomy after informed consent was obtained. With the patient at the lateral decubitus position a 25 gauge pencil point spinal needle was introduced into the subarachnoid space at the L2-L3 intervertebral space and $3 \mathrm{ml}$ hyperbaric plain bupivacaine $0.5 \%$, $0.25 \mathrm{mg}$ morphine and $20 \mu \mathrm{g}$ fentanyl were injected intrathecaly. Postoperative analgesia was standardized (rofecoxibe $25 \mathrm{mg}$ every $12 \mathrm{hrs}$ and paracetamol $500 \mathrm{mg}$ every $6 \mathrm{hrs}$ orally). Postoperative pain was assessed using the Visual Analogue Scale (VAS). Patients were followed up as outpatients at 10-15 days postoperatively and were asked to complete a standardized questionnaire regarding the quality of their recovery.

Results and Discussions: All operations were completed laparoscopicaly and conversion from spinal to general anaesthesia was not required. Cardiovascular, respiratory or neurologic complications were not detected in any case during the immediate postoperative course. Nausea or vomiting occurred in 4 patients, while 1 patient developed urinary retention requiring catheterization. Median VAS score at mobilization was 0 at the recovery room and at $4 \mathrm{hrs}$ postoperatively was 1.5 (range $0-5$ ), at $8 \mathrm{hrs}$ was 1 (range 0-6) and at $24 \mathrm{hrs}$ was 1 (range $0-4$ ). All patients were mobilized the evening after the operation and discharged at $24 \mathrm{hrs}$ postoperatively. No patient required readmission. At 2 weeks follow up 12 patients reported highly satisfied, 2 fairly satisfied and 1 dissatisfied from the procedure.

Conclusion: Laparoscopic cholecystectomy under spinal anaesthesia appears to offer adequate postoperative pain control without limiting recovery. Reference:

1 Pursnani KG. Surg Endosc 1998; 12: 1082-1084.

\section{AP5-1}

Application of cold to the skin: does it effectively attenuate the pain associated with the infiltration of local anaesthetic?

C. Burchman, K. Judy

Department of Anesthesiology, Dartmouth-Hitchcock Medical Center, Lebanon, USA

Background and Goal of Study: Ice has been employed for thousands of years as an adjunct to analgesia. Many studies have demonstrated that ice is effective in attenuating perioperative pain (1). This study evaluated the efficacy of ice in reducing the amount of pain associated with the infiltration of local anesthetic.

Materials and Methods: Forty-eight patients underwent interventional pain management procedures of the lumbar spine which required preliminary skin and soft tissue injections of local anesthetic (1\% lidocaine.) Approximately half of the injections were preceded by a 20 -second application of sterile ice, directly to the skin. Patients were immediately asked to rate the pain associated with the infiltration on a verbal scale from 0-10.

Results and Discussions: Pain intensity measurements were made for procedures pretreated with and without ice. Results were compared for each subject. A Wilcoxen-Signed Ranks Test demonstrated that $77 \%$ of the subjects noted use of the ice conferred significant analgesia during the local anesthetic infiltration $(p<.0001)$. Cooling reaches superficial nerves via conduction through the skin and subcutaneous tissue. Decreased temperature results in a decrease in the conduction velocity of neuronal conduction (2). The Gate Control Theory of Pain, introduced by Melzack and Wall in 1965 may provide an additional basis for why diminished temperature alters the perception of pain. Afferent input from A fibers "closes the gate," modulating pain transmission at the level of the cord (3). Thermal alteration may provide the afferent barrage necessary to modulate the pain by "closing the gate" to further transmission of pain impulses.

Conclusion: Ice, when applied to the skin, was statistically significant in its ability to diminish the amount of pain associated with local anesthetic injection. Topical cooling reaches superficial nerves via conduction, which may result in a decrease in the velocity of neuronal conduction. This simple, low cost technique may be useful in a wide range of clinical settings where brief injection procedures are performed

\section{References:}

1 Bierman W.Therapeutic JAMA 1955; 14: 1189-92.

2 Fox RH. BR Med Bull 1961; 17: 14-18.

3 Melzack R and Wall PD. Science 1965; 150: 971-979.

\section{AP5-2}

Phrenic nerve infiltration with $0.2 \%$ ropivacaine reduces the incidence of shoulder pain after thoracotomy surgery

G. Danelli, M. Berti, R. Mele, D. Ghisi, A. Casati

Anesthesiology and Pain Therapy, University of Parma, Parma, Italy

Background and Goal of Study: The aim of this prospective, randomised, double blind, placebo controlled study was to test the efficacy of phrenic nerve infiltration with ropivacaine $0.2 \% \mathrm{wt} / \mathrm{vol}$ on the incidence and severity of ipsilateral shoulder pain after thoracotomy in patients receiving continuous thoracic epidural analgesia.

Materials and Methods: 50 ASA physical status II-III patients, receiving thoracic epidural analgesia for post-thoracotomy pain, were randomly allocated to receive the infiltration of the ipsilateral phrenic nerve with $10 \mathrm{~mL}$ of either $0.2 \%$ ropivacaine (group Ropivacaine, $n=25$ ), or normal saline (group Control, $n=25$ ) just before lung expansion and chest closure. A blind observer recorded the incidence and severity of ipsilateral shoulder pain 6 , $12,24,36$, and $48 \mathrm{~h}$ after surgery. Postoperative respiratory function was also evaluated with blood gas analyse.

Results and Discussions: The cumulative incidence of ipsilateral shoulder pain during first $24 \mathrm{~h}$ after surgery was $8 / 25$ in group Ropivacaine and 16/25 in group Control $(\mathrm{P}=0.047)$; with a median $\left(\mathrm{Cl}_{95 \%}\right)$ onset time of shoulder pain of $36(2-48) \mathrm{h}$ in group Ropivacaine and $16(0.5-48) \mathrm{h}$ in group Control $(P=0.01)$. No differences were reported on the second postoperative day. The area under the curve of the degree of pain over time was $0(0-2760) \mathrm{mm}$ $h$ in group Ropivacaine and $350(0-1900) \mathrm{mm} h$ in group Control $(P=0.06)$. Postoperatively, the indices of respiratory function worsened in both groups, without differences between the groups.

Conclusion: Phrenic nerve infiltration with $10 \mathrm{ml}$ of $0.2 \% \mathrm{wt} / \mathrm{vol}$ ropivacaine reduced the incidence and delayed the onset of ipsilateral shoulder pain during first $24 \mathrm{~h}$ after open lung resection, with no clinically relevant effects on respiratory function.

\section{AP5-3}

Tramadol added to $1.5 \%$ lidocaine enhances postoperative analgesia dose dependently

O. Kaabachi, R. ouezzini, B. Ghrab, W. Koubaa, A. Zarguouni

Anesthesiology and Intensive Care, Institut Kassab Dorthpédie, Tunis, Tunisia

Background and Goal of Study: Adjuncts to local anesthetics for peripheral plexus blockade have been proposed to enhance the quality and duration of anesthesia and postoperative analgesia. Tramadol, has a unique mechanism of action that suggests efficacy as such an adjunct $(1,2)$. We designed a prospective, randomized, controlled and double-blind clinical trial to assess the effect of Tramadol added to brachial plexus anesthesia.

Materials and Methods: After institutional ethical committee approval and consent from patient, 102 patients scheduled for either hand or forearm surgeries were randomized into three groups. All patients received $1.5 \%$ lidocaine $34 \mathrm{~mL}(+1 / 200000$ epinephrine) plus $4 \mathrm{ml}$ of either: saline solution (placebo group), $100 \mathrm{mg}$ Tramadol (TL group) or $200 \mathrm{mg}$ Tramadol (TH group). We evaluated the time of onset of anesthesia, duration of sensory (SB) and motor blockade (MB), duration and quality of postoperative analgesia and occurrence of adverse effects.

Results and Discussions: demographic data were similar in all groups. The main results are in the table below.

\begin{tabular}{lcccl}
\hline & $\begin{array}{l}\text { Placebo } \\
\mathrm{n}=33\end{array}$ & $\begin{array}{l}\text { TL group } \\
\mathrm{n}=34\end{array}$ & $\begin{array}{l}\text { TH group } \\
\mathrm{n}=35\end{array}$ & $\mathrm{P}$ \\
\hline Onset time(min) & \multicolumn{1}{c}{$8,9 \pm 3.6$} & $11,1 \pm 4$ & $16,1 \pm 6.8 \ddagger$ & 0.01 \\
Duration of SB (min) & $126,4 \pm 48$ & $189,8 \pm 87^{\star}$ & $265,5 \pm 119^{\star} \dagger$ & 0.018 \\
Duration of MB (min) & $142,7 \pm 53$ & $180 \pm 76^{*}$ & $231,2 \pm 82^{*}$ & 0.02 \\
Rescue analgesia $(\mathrm{min})$ & $371,6 \pm 316$ & $572,9 \pm 516$ & $733,8 \pm 434 \ddagger$ & 0.02 \\
Rescue analgesia(\%) & $94 \%$ & $76 \%^{*}$ & $77 \%^{*}$ & 0.04 \\
\hline
\end{tabular}

$\left({ }^{\star}\right)$ : significance between placebo and both tramadol groups; $(\dagger)$ : significance between

TL group and TH group. (†): significance between TH group and placebo and TL groups.

Conclusion(s): In our study, tramadol enhanced anesthesia and postoperative analgesia in dose dependant way when used as a perineural adjunct of lidocaine $1.5 \%$ for axillary nerve block

References:

1 Robaux S. Anesth Analg 2004; 98: 1172-77.

2 Kapral S. Anesth Analg 1999; 88: 853-6.

\section{AP5-4}

Elastomeric pumps: clinical evaluation differs from in vitro reliability

F. Remerand, M. Palud, S. Buchet, B. Dabo, J. Fusciardi

SAR2, CHU Trousseau, Chambray les Tours, France

Background and Goal of Study: In postoperative regional analgesia, perineural infusion of local anesthetic with an continuous elastomeric pump (CEP) increases portability and patient satisfaction (1). In vitro, CEP infusion remains within $\pm 15 \%$ of their set rates during 76 to $90 \%$ of their duration, but infusion rate is higher than expected during the first hours of duration (2). No in vivo data are available. The aim of our work was to assess in vivo CEP infusion rate consistency and accuracy in regional analgesia after orthopedic surgery. 
Materials and Methods: We prospectively studied 117 consecutive CEP connected to 78 perineural catheters (49 femoral, 25 popliteal and 3 infraclavicular ones) in 73 patients for postoperative analgesia (knee, foot or elbow surgery). Two CEP were indifferently used in the unit : Infusor ${ }^{\circ}$ LV5 (Baxter, France) or Easypump ${ }^{\circ}$ (Braun, Germany), both with a $5 \mathrm{~mL} / \mathrm{h}$ set rate. As a routine procedure, CEP were weighed by nurses at bedside using a portable electronic scale, several time a day, until catheter was removed. A constant mass of CEP during time traduced an obstructed catheter. Masses and hours of measures allowed accurate deflation profile assessment and flow rate calculation: (first measure - last measure) / duration. We used Fisher exact tests for statistical analysis.

Results and Discussions: After being connected to the catheter, 28 CEP did not deflate (27 Braun and 1 Baxter, $p=0.04$ ): 9 catheters were removed 13 to $40 \mathrm{H}$ latter, 9 were injected 5 to $39 \mathrm{H}$ latter with $5 \mathrm{~mL}$ lidocain $1 \%$ and then deflated correctly, and 10 spontaneously deflated 10 to $35 \mathrm{H}$ latter. Deflation defect was less frequent when a CEP was connected for the first time to a catheter (12 out of 78 ) than when CEP renewed a previous one (16 out of $39, p=0.005)$.

Flow rates differed from $5 \mathrm{~mL} / \mathrm{h} \pm 15 \%$ in 45 cases out of 69 (30 in excess and 15 by default).

Conclusion(s): Physicians should be aware of frequent deflation defect of elastomeric pump (a possible cause of early postoperative analgesia failure). Moreover, in vivo measures of their infusion rate yielded greater variability than in vitro ones.

References

1 Capdevila X. Anesth Analg 2003; 96: 414-417

2 Ilfeld BM. Reg Anesth Pain Med 2003; 28: 424-432

\section{AP5-5}

Postoperative analgesia with intraarticular bupivacaine and a2 - agonist clonidine after arthroscopic knee surgery: a prospective, randomized, double - blinded clinical comparison

R. Tamosiunas, A. Karbonskiene, A. Augutiene, G. Keraitiene, A. Gelmanas Department of Anaesthesiology, Kaunas Medical University Hospital, Kaunas, Lithuania

Background and Goal of Study: The aim of the study was to compare analgesic effectiveness of intraarticular $0.5 \% 20 \mathrm{ml}$ bupivacaine and clonidine, used separately and in combination for postoperative analgesia after arthroscopic knee surgery. We hypothesized that $0.5 \% 20 \mathrm{ml}$ of bupivacaine with $1 \mu \mathrm{g} / \mathrm{kg}$ of clonidine provides best control of postoperative pain.

Materials and Methods: 48 patients, ASA 1 to 2 , scheduled for elective knee arthroscopy under spinal anaesthesia were prospectively randomized to receive intraarticularly, in a double - blind manner, either $0.5 \% 20 \mathrm{ml}$ of bupivacaine (group I); $0.5 \% 20 \mathrm{ml}$ of bupivacaine with $1 \mu \mathrm{g} / \mathrm{kg}$ of clonidine (group II); or normal saline (group III - placebo group). Rescue treatment consisted of ketorolac $30 \mathrm{mg} \mathrm{i} / \mathrm{v}$. VAS pain scores at rest and with motion every $2 \mathrm{~h}$, postoperative ketorolac consumption and patient satisfaction (VAS) with postoperative analgesia were assessed. Statistical analysis was performed using tests of Mann - Whitney, Kolmogorov - Smirnov, Chi ${ }^{2}$ and Student t, $\mathrm{p}$ value $<0,05$ was considered significant.

Results and Discussions: Pain scores at rest and with motion were similar in group I and II during the whole study period, except 6 ( $2.5 \pm 0.4$ vs $0.9 \pm 0.2)$ and $8 \mathrm{~h}(2 \pm 0.3$ vs $0.7 \pm 0.2)(\mathrm{p}<0.05)$ after intraarticular administration of study drugs. Pain relief was significantly better controlled in these groups at rest and with motion when compared with the group III and number of demanded doses of rescue analgetic was also less (median (min-max) was as follows: group I - $0(0-1)$, group II - $0(0-1)$, group III - $2(2-3))$. Patient satisfaction with postoperative analgesia did not differ in groups I and II and there was a significant difference between groups I and III, II and III (median (min-max) of satisfaction VAS score in groups: I - 8(7-9), II - 10 (8-10), III - 7 (6-7)).

Conclusion(s): The administration of intraarticular bupivacaine and clonidine after arthroscopic knee surgery provides good and comparable control of postoperative pain with a high degree of patient satisfaction.

\section{AP5-6}

\section{Ropivacaïn mandibular nerve block decreases bleeding} during sagittal mandibular osteotomy

F. Espitalier, AF. Dubost, S. Ehrmann, M. Laffon, J. Fusciardi

Anesthesiology, C.H.U Trousseau, Tours Cedex, France

Background and Goals: In bilateral mandibular osteotomy (MO), percutaneous mandibular nerve blocks (MNB) are performed in our institution for postoperative analgesia. A surgical bleeding decrease has been noted, but no publishing data is available in head and neck surgery. A surgical bleeding decrease following regional anaesthesia has been noted only in orthopaedic limb surgery (1). This study's goal was to asses the effect of MNB on surgical bleeding.

Material and Methods: After informed consent, 22 patients (14-65 years), $A S A \leqslant 2$, scheduled for bilateral $\mathrm{MO}$ were prospectively included. They were randomized in two groups, BLOCK in which a bilateral MNB $(5 \mathrm{ml}$ ropivacaïn $0,375 \% \times 2$ ) was performed before the surgery and CONTROL in which a bilateral cutaneous puncture without injection was performed before the surgery. The surgery was performed under standardized general anaesthesia (propofol + remifentanil + atracurium) with objective to control common bleeding factors. During the MO the surgeon (blinded to group allocation) assessed the surgical bleeding using a continuous scale (0: no bleeding, perfect surgical comfort to 5: heavy bleeding needing to stop the surgery). Moreover, we noted peroperative remifentanil doses used $(\mathrm{mcg})$ and postoperative visual analogical scale (VAS) in the recovery room (mm). Mann Whitney U-test and Fischer's exact test were performed, $p<0.05$ was considered significant.

Results: No significant differences were noted between the groups in demographic data and in anaesthesia's objectives. Other data (mean $\pm \mathrm{SD}$ ) are shown in the table.

\begin{tabular}{lccc}
\hline & Block & Control & $\mathrm{p}$ \\
\hline Surgical bleeding score & $1.89 \pm 2.78$ & $3.62 \pm 2.85$ & 0.044 \\
Peroperative Remifentanil doses & $1277 \pm 890$ & $3625 \pm 1563$ & 0.003 \\
VAS in recovery room & $15.0 \pm 22.7$ & $24.5 \pm 26.9$ & 0.398
\end{tabular}

Conclusion: In this preliminary study, ropivacaïn MNB decreases: 1) the surgical bleeding in bilateral MO following a better surgical comfort; 2) the peroperative remifentanil doses.

Reference:

1 Modig J. Acta Chir Scand Suppl. 1989;550:95-100.

\section{AP5-7}

Endovascular aortic aneurysm repair under local anaesthesia and conscious sedation

H. Bojahr, C. Kyriakides

Department of Anaesthesia, The Royal London Hospital, London, United Kingdom

Background and Goal: Endovascular aortic aneurysm repair (EVAR) is gaining widespread acceptance and can be offered to patients not amenable to traditional open repair. We wish to report our experience of performing EVARs under local anaesthesia with conscious sedation.

Materials and Methods: We performed 15 consecutive EVARs under local anaesthesia; cases were abdominal and thoracic, elective or leaking. ASA grades were all 3 and over, 1 patient being classified as $5 \mathrm{E}$. The range of comorbidities included aortic stenosis (gradient $>100 \mathrm{mmHg}$ ), severe ischaemic heart disease, chronic obstructive pulmonary disease and severe rheumatoid arthritis with atlantoaxial subluxation. 2 patients were non-English speakers and verbal communication was not possible during the procedure. All patients were prepared as for open repair except for a CVP line, and blood pressure was manipulated as necessary. Remifentanil sedation was commenced through a dedicated intravenous line and maintained at a rate of $0.01-0.05 \mathrm{mcg} / \mathrm{kg} / \mathrm{min}$. Local anaesthesia was provided by the surgeon using Lidocaine $1 \%$ with Epinephrine. Mean operating times were 90 minutes. Patients were discharged either to the vascular ward or the surgical high dependency unit.

Results and Discussions: Operating conditions were judged good to excellent in all cases. No patient required conversion to general anaesthesia or an open repair. 1 patient developed a tachycardia with $>140$ beats per minute; this settled with correcting the potassium level and intravascular filling. 1 patient experienced discomfort during the procedure, however, this was an obese patient (BMI 35) and the procedure lasted over 4 hours. The only patient who died was in extremis with an aorto-duodenal fistula and had refused surgery earlier. In all other patients satisfaction was high.

Conclusion(s): Judicious local anaesthesia with Remifentanil sedation is a safe and effective way of providing anaesthesia for EVAR, producing consistently good operating conditions. This technique also reduces pressure on critical care facilities by allowing the patients to be fast-tracked to the ward. The ease of this procedure must not be underestimated as there is still potential for major disaster.

\section{AP5-8}

Quantitative evaluation of the efficacy of different components of topical anaesthetic creams. Prospective, randomized, double-blinded, placebo controlled study N. Rahe-Meyer, C. Hoppe, C. Weilbach, S. Piepenbrock, M. Winterhalter Anaesthesiology, Hannover Medical School, Hannover, Germany 
Background and Goal of Study: Our goal was to test the effect of different ingredients of topical local anaesthetics that might influence their efficacy and thus to find the composition with the fastest onset, the best intensity and the lowest toxic potential.

Materials and Methods: 8 different compositions were tested on 29 healthy volunteers. Approval was given by the regional Ethics Committee. They were randomly applied on the forearm in $1,77 \mathrm{~cm}^{2}$ skin areas for 20,40 and 60 minutes. After removal of the anaesthetics we gave definite, reproducible pain stimuli with a special test-stamp. This contained a $0,55 \mathrm{~mm}$ disposable hypodermic needle (Sterican) which was put with $0,2,0,4$ and 0,6 Newton pressure on the skin. The pain experience of the test subjects were quantitatively, subjectively measured on a visual analog scale (VAS) and quantitatively, objectively by skin impedance (ESG 1001, Dr. Janitzki, Altenbeken Germany). All together we got 12528 values from all tests.

Results and Discussions: It showed that the alkaline creams make a deeper skin analgesia than the neutral ones. Propylenglycol - as a skin infiltration accelerator - had no significant effect. There was no difference in the efficacy of lidocaine $5 \%, 10 \%$ or $20 \%$. The eutectic mixture of lidocaine and prilocaine (EMLA ${ }^{T M}$ ) was insignificantly better than lidocaine as a mono substance. After 20 minutes all creams were still ineffective. After 40 minutes we observed a good dermal analgesia in all cases which got even better after 60 minutes. VAS and ESG both showed the effects but the ESG had smaller standard deviations.

Conclusion(s): This study shows that the application time is the most important parameter in topical analgesia. Even a so called skin infiltration accelerator cannot reduce this time. At least $\mathbf{4 0}$ minutes are necessary for a good anaesthetic effect. Topical anaesthetics work better as alkaline than as neutral cremes. Lidocaine even in the low concentration (5\%) is highly effective. $\mathrm{EMLA}^{\mathrm{TM}}$ is moderately more effective (not significant) but it could potentially produce methaemoglobinaemia by prilocaine which might be relevant in sensitive patients like neonates.

\section{AP6-1}

$1 \%$ Chloroprocaine. A very promising short acting local anaesthetic drug in spinal anaesthesia. Clinical experience with 2000 patients since 2001

T. Palas

Anesthesia, Klinik Linde, Biel, Switzerland

Background and Goal of Study: Chloroprocaine (cp) is a very short acting local anesthetic drug with the lowest potential toxicity. It would be an ideal replacement for lidocaine in spinal anesthesia for short surgical procedures. We have evaluated patients in clinical settings since year 2001 with special interest in possible postoperative TNS.

Materials and Methods: 2000 patients have been evaluated. Both inpatients and ambulatory cases. $40 \mathrm{mg}$ of an additive free, plain 1\% chloroprocaine was injected intrathecally mostly at the L3/4 level in a sitting position. Types of surgery were: general surgery, orthopedic surgery, gyn/ob and urologic surgery. Time of surgery was planned not to take longer than 50 minutes.

Results and Discussions: Good surgical anesthesia was achieved in every case. There was never a burning sensation during or after the $\mathrm{cp}$ injection. No postopreative urine retention, no allergic reactions and no signs of TNS. The mean time for mobilization after the spinal local anesthetic application was 90 minutes. Patient satisfaction was excellent in every case.

Conclusion: $1 \%$ chlorprocaine seems to be a very good substitute for lidocaine as a very short acting spinal local anesthetic without having TNS as a side effect.

\section{AP6-2}

\section{Is Unilateral spinal anaesthesia worth all the troubles}

B. Osinaike, S. Amanor-Boadu, A. Sanusi, R. Eyelade

Anaesthesia, Lautech Teaching Hospital, Osogbo, Nigeria

Background and Goals: Unilateral spinal anaesthesia has been suggested as a valuable technique in reducing the incidence of hypotension, fastening the resolution of surgical block and enhancing the quality of surgical block ${ }^{1,2}$. We compared clinical profile of unilateral spinal and conventional spinal anaesthesia.

Material and Methods: We studied 74 ASA 1 and 2 patients, randomized into two groups. In the lateral position with operative side down, patients received $10 \mathrm{mg}(2 \mathrm{mls})$ of $0.5 \%$ hyperbaric bupivacaine slowly through a 25 -gauge Whitacre needle without barbotage. The unilateral group (USA) was maintained in the lateral position for 15 minutes following spinal injection while those in the conventional group (CSA) were turned supine immediately after injection. Results and Discussion: Hypotension occurred in 3 and 5 patients in the USA and CSA groups respectively $(P=0.71)$, with 1 patient in the USA group requiring vasopressor compared to 4 in the CSA group $(P=0.17)$. Block was lower than $\mathrm{T} 12 /$ no sensory block observed in the non-operated limb at 5,10 and 20 minutes in 24, 22 and 12 patients in the unilateral group compared to 10,9 and 0 in the conventional group $(P<0.05)$ respectively. Maximum sensory level on the operated side was T8 (T12-T2) in the unilateral group and T6 $(\mathrm{T} 11-\mathrm{T} 2)$ in the conventional group $(P=0.07)$, attained at 20 minutes in both groups. Maximum level on the non-operated side was T8 (no sensory block-T2) at 30 minutes in unilateral group and T6 (T12-T2) at 20 minutes in the conventional group $(P=0.04)$. At 1 hour, motor blockade (bromage scale) of the operated limb in patients in the USA group was $0 / 1 / 2 / 3: 0 / 0 / 0 / 37$ and 0/1/2/3:0/0/2/35 $(\mathrm{P}<0.05)$ in the CSA group. In the non-operated limb, it was $0 / 1 / 2 / 3: 5 / 10 / 5 / 17$ in the USA group, and $0 / 1 / 2 / 3: 0 / 0 / 4 / 33$ in the CSA group $(\mathrm{P}<0.05)$.

Conclusion: Unilateral spinal anaesthesia was associated with less cardiovascular perturbations and also provided a denser block in the dependent limb when using hyperbaric bupivacaine solution.

References:

1 Fanelli G. BJA 1996; 76: A 242.

2 Casati A. Reg Anesth Pain Med 1999; 24: 214-9.

\section{AP6-3}

Levobupivacaine-fentanyl spinal anaesthesia for transurethral urologic surgery

\section{R. Krobot, I. Bacak, J. Premuzic}

Department of Anesthesiology and Intensive Care, General Hospital Varazdin, Varazdin, Croatia

Background and Goal of Study: Spinal anaesthesia is commonly associated with high incindence of hypotension. Intrathecal coadministrations of opioids intensify sensory block without increasing motor or sympathetic block (1). In this study we compared the effect of intrathecal levobupivacaine with or without fentanyl in patients undergoing transurethral urologic surgery.

Materials and Methods: A total of 40 patients were allocated to 2 groups in this prospective, randomized, double-blind study: Group $L(n=20)$ intrathecally received levobupivacaine $10 \mathrm{mg}$ and Group LF $(n=20)$ levobupivacaine $7.5 \mathrm{mg}+$ fentanyl $25 \mu \mathrm{g}$. Sensory (pin-prick test) and motor block (modified Bromage scale), hemodynamic data and side-effects were recorded. Statistical analyses were done using t-test, Mann-Whitney and Fisher's exact test.

Results and Discussions: Demographic data, duration of operation and baseline values of systolic arterial pressure (SAP) and heart rate (HR) were comparable between the groups. Anaesthesia was adequate in all patients and pick sensory level was T9 (T10-T6) in Group L and T10 (T10-T8) in Group LF, $P=0.11$. Complete motor block (Bromage score 3) had 18 (90\%) Group L and 6 (30\%) Group LF patients, $P<0.001$. The mean duration of motor block was $137 \pm 43 \mathrm{~min}$ in Group $\mathrm{L}$ and $95 \pm 37 \mathrm{~min}$ in Group LF, P $=0.01$. Decrease of SAP for more than $25 \%$ from baseline had 12 (60\%) Group L and $2(10 \%)$ Group LF patients, $P<0.01$. Maximum decrease of SAP from start value was $24 \pm 12 \%$ in Group $L$ and $11 \pm 7 \%$ in Group LF, P $=0.02$, and of $\mathrm{HR} 24 \pm 9 \%$ and $18 \pm 9 \%, \mathrm{P}=0.29$, respectively. Bradycardia (HR $<50 / \mathrm{min})$ had $3(15 \%)$ Group L patients. Postoperative rescue analgesic administration needed 15 (75\%) Group L and 6 (30\%) Group LF patients, $\mathrm{P}=0.01$. Pruritus had $5(25 \%)$ Group LF patients. No postdural puncture headache, vomiting, respiratory depression or neurological complications were recorded.

Conclusion(s): Levobupivacaine $7.5 \mathrm{mg}$ plus fentanyl $25 \mu \mathrm{g}$ provide adequate spinal anaesthesia, faster motor recovery, better postoperative analgesia and less cardivascular effects than levobupivacaine $10 \mathrm{mg}$ in patients undergoing transurethral urologic surgery.

Reference:

Lee YY, Mucchal K, Chan CK et al. Eur J Anaesthesiol 2005; 22: 899-903.

\section{AP6-4}

\section{Which drugs are used for spinal anaesthesia in the Netherlands?}

S. Schiere, A. Krul-Sterk, K. Kuizenga, L.P.H.J. Aarts

Anesthesiology, Tjongerschans Hospital, Heerenveen, The Netherlands

Background and Goal of Study: A Cochrane metanalysis pointed out that one out of seven patients who received lidocaine intrathecally developed transient neurological symptoms (TNS) ${ }^{1}$. To avoid TNS alternatives are used in clinical practice and also published, often without extensive scientific background $^{2}$.

Materials and Methods: To investigate common practice in the Netherlands all members of the Dutch association of anesthesiology were questioned on 
paper about their preferences to regional anesthetic technique (spinal vs. peripheral) and drug choice when confronted with a patient admitted for ambulatory knee arthroscopy. Neither recommendations about TNS nor other specific comments were made in the cover letter or in the presented case. Results and Discussion: The response rate to the questionnaire was 42, 4 percent. Spinal anesthesia appeared to be the preferred technique for $96 \%$ of the respondents. For spinal anesthesia lidocaine was most popular $(50 \%)$, followed by articaine $(28 \%)$, bupivacaine $(13 \%)$, prilocaine $(5 \%)$, mepivacaine $(3 \%)$ and lidocaine mixed with bupivacaïne (1\%). Seven percent would use additives like fentanyl, sufentanil and clonidine. Furthermore, the dosage range was wide. A favourable time course of action, the potential occurrence of TNS and drug registration issues were reported as most important criteria for drug selection.

Conclusions: According to our study, despite the high incidence of TNS, lidocaine is still the most often used drug for spinal anesthesia. To reduce TNS incidence more research has to be performed on alternative drugs or drug combinations.

References:

1 Zaric D, et al. Cochrane. Database. Syst. Rev. 2005; CD003006.

2 Hodgson PS, et al. Anesth. Analg. 1999; 88: 797-809.

\section{AP6-5}

\section{Comparison of plain ropivacaine and hyperbaric bupivacaine} in spinal anesthesia for endovascular surgery

M. Sanfilippo, R. Drago, A. Piccardo, P. Coppola, L. Pasquarelli

Department of Anesthesiology, Intensive Care and Pain Medicine,

University "La Sapienza" Roma, Italy, Roma, Italy

Background and Goal of Study: The aim of this perspective and randomized study was to compare clinical efficacy and safety of $15 \mathrm{mg}$ plain ropivacaine (group 1) and $15 \mathrm{mg}$ of hyperbaric bupivacaine (group 2) given intratecally in patients scheduled for endovascular repair of abdominal aortic aneurysms. Materials and Methods: 64 ASA II-III patients (mean age $69.1 \pm 7.0$ years) were scheduled after obtaining written informed consent. All patients had a history of arterial hypertension and other cardiovascular disorders pharmacologically treated.

Profile of spinal block (onset and recovery times) and quality of intraoperative anesthesia were recorded.

Pulse rate (HR), mean arterial pressure (MAP), cardiac output (CO), left ventricular contractility (dP/dt), systemic vascular resistance (SVR) and global end-diastolic volume (GEDV) were monitored by the PiCCO System Pulsion in order to evaluate their variations ${ }^{(1)}$

Results and Discussions: The median times to onset of sensory block at $\mathrm{T} 10$ were similar for both anesthetics. Median times to complete regression of both sensory (144 vs $174 \mathrm{~min} ; \mathrm{p}<0.05$ ) and motor block (189 vs $126 \mathrm{~min}$; $p<0.05)$ were longer in the group 2. Patients therefore were mobilized sooner in the group 1 (195 vs $255 \mathrm{~min} ; \mathrm{p}<0.05$ ). $\mathrm{CO}$ and SVR were sligthly decreased in the group 2, MAP decreased in the group $2(p<0.05)$. The other parameters were not influenced by the technique or by anesthetics. None of the studied patients required supplementary analgesia or general anesthesia to complete surgery.

Conclusions: Spinal anesthesia produced with $15 \mathrm{mg}$ plain ropivacaine is effective and safe in ASA II-III patients undergoing endovascular major surgery. Ropivacaine gives an earlier recovery of sensory and motor functions after surgery compared to hyperbaric bupivacaine and also offers a greater advantage for the cardiovascular stability in high-risk patients.

Reference:

1 Buhre W, Weyland A, Kazmaier S et al. J Cardiothorac Vasc Anesth 1999; 13: 437-440.

\section{AP6-6}

\section{Effect of preoperative feeding and midazolam sedation on} gastric emptying rate following intratechal anaesthesia

A. Protic, A. Sustic, D. Turina

Anaesthesiology and ICU, University Hospital Rijeka, Rijeka, Croatia

Background and Goal of study: Overnight fasting as a standard of elective surgery may have influence on the insulin resistance. ${ }^{1}$ Some studies point out advantages of preoperative feeding with carbohydrate-rich drink two hours before surgery with no risk of aspiration of gastric contents. ${ }^{2}$ The goal of our study was to find out the influence of preoperative feeding and sedation with midazolam on gastric emptying during the orthopaedic surgery in intratechal anaesthesia.

Materials and Methods: Forty-five patients scheduled for elective orthopaedic surgery were randomly divided into four groups. Group 1 (G1); the patients were given $200 \mathrm{ml}$ of enteral formula PreOp $®$ before surgery and midazolam $(0,3 \mathrm{mg} / \mathrm{kg}$ i.v.) during the surgery. Group 2 (G2); the patients were given $200 \mathrm{ml}$ of enteral formula PreOp $®$ before surgery with no sedation during the surgery. Group 3 (G3); the patients were sedated only with midazolam $(0,3 \mathrm{mg} / \mathrm{kg}$ i.v.) during the surgery. The control group (Gc) was without preoperative feeding and sedation. Intratechal anaesthesia, using 25-27 Gauge needle with local anaesthetic (bupivacaine 0,5\% + glucose 50\%) was used on all patients. Gastric emptying was measured by the paracetamol test at six different times $(0,15,30,60,90$ and 120 min after administration of paracetamol). The statistical analysis of paracetamol plasma concentration was performed by K-W ANOVA test.

Results: Plasma concentrations (Pc) 0,15,30,60,90 and $120 \mathrm{~min}$ after administration of paracetamol showed no statistical differences among the all four groups (all values were presented as the median): $(t+15) P c G 1=5,79$, PcG2 = 2,90, $\quad$ PcG3 =11,38, $\quad$ PcGc $=2,62 \quad(P=0,271), \quad(t+30)$ PcG1 $=10,85$, PcG2 = 7,12, PcG3 = 14,23, PcGc = 9,38 $(P=0,454) ;(t+60)$ PcG1 $=11,86$, PcG2 = 13,22, PcG3 = 15,55, PcGc = 11,25 $(P=0,309)$; $(\mathrm{t}+90) \quad \mathrm{PcG} 1=11,97, \quad \operatorname{PcG} 2=16,08, \quad \operatorname{PcG} 3=13,09, \quad \operatorname{PcG} \quad 10,50$ $(P=0,379) ; \quad(t+120) \quad P c G 1=9,80, \quad P c G 2=14,63, \quad P c G 3=12,55$, $\mathrm{PcGc}=7,97(\mathrm{P}=0,268)$.

Conclusion: Sedation with midazolam during surgery in intratechal anaesthesia has no influence on postoperative gastric emptying. The preoperative feeding of patients in intratechal anaesthesia before surgery has no influence on gastric emptying of patients sedated with midazolam, neither of patients without sedation.

References:

1 Nygren J, et al. O.Clin Nutr.1999; 18: 117

2 Phillips S, et al. Br J Anesth 1993; 70: 6.

\section{AP6-7}

Effect of diamorphine and dilutents on baricity when added to hyperbaric bupivacaine

S. Sagadai, W. DeMello, MO. Columb, SL. Maguire

Anaesthesia, South Manchester University Hospital, Manchester, United Kingdom

Background: Changes in baricity of injected intrathecal solutions is one determinant of final block height. Density differences as small as $0.0006 \mathrm{~g} \cdot \mathrm{mL}^{-1}$ may influence the distribution of local anaesthetic (1). We assessed the change in baricity of hyperbaric bupivacaine $0.5 \% \mathrm{w} / \mathrm{v}$ by the addition of various proportions of diamorphine in different diluents.

Materials and Methods: Densities of undiluted bupivacaine $0.5 \% \mathrm{w} / \mathrm{v}$ (+glucose $\left.80 \mathrm{mg} \cdot \mathrm{mL}^{-1}\right)$, diamorphine $\left(1 \mathrm{mg} \cdot \mathrm{mL}^{-1}\right)$ in saline $0.9 \% \mathrm{w} / \mathrm{v}$ or water for injection and their different combinations were measured using a density meter (DMA 5000 - Anton Paar, UK) with accuracy to the 6th decimal at $37 \pm 0.001^{\circ} \mathrm{C}$. Data were analyzed using GLM ANOVA and linear regression analysis. $P<0.05$ was defined as significant.

Results and Discussion: The repeatability $S D$ was $\leqslant 0.00012 \mathrm{~g} \cdot \mathrm{mL}^{-1}$. Tests for both main effects (diluent, combination) were highly significant $(P<0.0001)$. The baricity relationships were linear $\left(r^{2}>0.999, P<0.0001\right)$ over the range of combinations tested.

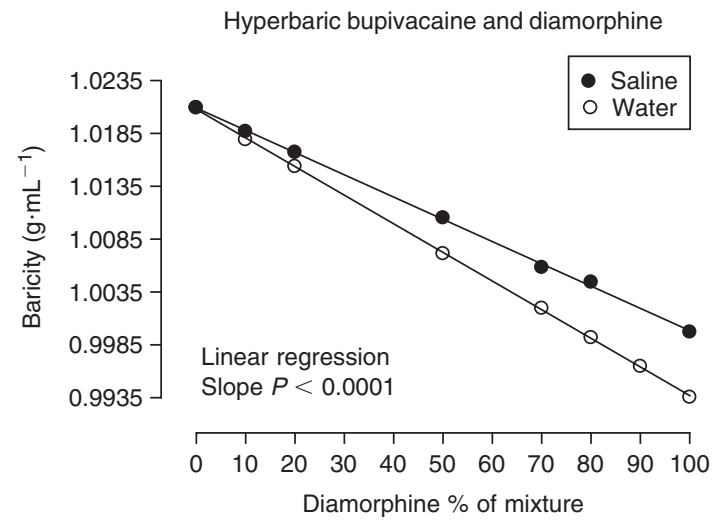

Conclusion: Addition of diamorphine (dissolved in either saline or water) to hyperbaric bupivacaine predictably reduces the baricity of the resultant mixture, with water having a significantly more pronounced effect. These data may facilitate further explanation of the mechanisms underlying the behaviour of intrathecal local anaesthetic-opioid mixtures.

Reference:

1 Horlocker TT, Wedel DJ. AnesthAnalg 1993; 76: 1015-8. 


\section{AP6-8}

Experiences of spinal anesthesia for previous lumbar spine surgery

S. Kim, SJ. Lee, JH. Kim, YS. Shin

Anesthesiology and Pain medicine, Yonsei University Health Care System, Seoul, Republic of Korea

Background and Goal of Study: Spinal anesthesia is no more contraindicated in patients having undergone previous lumbar spinal surgery (1); although there are still problems that make spinal anesthesia technically more difficult, with the possibility of worsening the patient's back pain (2). Herein, spinal anesthesia in patients with/without previous lumbar surgery was investigated and compared with regard to the success rates and possible complications.

Materials and Methods: Thirty patients with previous lumbar surgery (Group I), and thirty patients without surgery were studied (Group II) prospectively. Spinal anesthesia was performed, using a $27 \mathrm{G}$ spinal needle with $0.5 \%$ isobaric tetracaine $10 \mathrm{mg}$, at the left lateral decubitus position. The attempt times of spinal anesthesia and its onset were marked, and the maximal sensory block level (MSBL), regression time of anesthesia, and its success rate and complications observed.

Results and Discussions: The success rates of spinal anesthesia were $92.9 \%$ and $100 \%$ in group I and II, respectively. The MSBLs were T4 (T3-T12) and T5 (T3-T10) in group I and II, respectively, in the median range. The regression times from MSBL to 2-segment below were $125.04 \pm 43.04$ min (minutes) and $110.28 \pm 33.23 \mathrm{~min}$ in group I and II, respectively.

Conclusion(s):

1. Success rate in patients with previous lumbar spine surgery experience was $92.9 \%$. The patients experienced no significant neurological complications.

2. There was no statistically significant difference in MSBLs between two groups.

References:

1 Berkowitz S, Gold MI. Anesth Analg 1980; 18: 768-774.

2 Sun KO. Eur J Anaesthesiol 1994; 11: 321-323.

\section{AP7-1}

Efficacy of $15 \mathrm{~mm}$ needle length for peribulbar anaesthesia in cataract surgery

W.R. Soliman, M. Schreiber, N. Ahmed

Anesthesia, King Khaled Eye Specialist Hospital, Riyadh, Saudi Arabia

Background and Goal of Study: Needle length is an important for the safe conduct of peribulbar blocks. Shorter needles may reduce damage to vital structures behind the globe. At present, administration of extraconal injection with $25 \mathrm{~mm}$ needles is recommended. Peribulbar block is usually performed using two injections inferior and superior to the globe. The aim of this work is to demonstrate the efficacy of $15 \mathrm{~mm}$ needle for peribulbar anesthesia. Materials and Methods: This is a descriptive study, involves a 150 patients. The technique of peribulbar block involves an infero-temporal and a supero-medial injection using $15 \mathrm{~mm}$ length, 25 gauge needle. The inferior injection was performed through the lower eyelid in the infero-temporal quadrant. A volume of $8-10 \mathrm{ml}$ of local anesthetic solution was injected. A modified supero-medial injection site situated infero-lateral to the superior orbital notch was used. The needle was directed over the globe. Three to five $\mathrm{ml}$ of local anesthesia was injected. After the second injection a pressure reducing device was applied. Ocular movement was assessed $10 \mathrm{~min}$ after the block using a Simple Akinesia Score. A score of one or zero was considered satisfactory to commence surgery.

Results:

\begin{tabular}{lc}
\hline Observation & Blockade using $15 \mathrm{~mm}$ needle \\
\hline Initial Volume injected $(\mathrm{ml})$ & $9.5 \pm 0.5$ \\
Infero-lateral & $4.9 \pm 0.8$ \\
Supero-medial & $14.1 \pm 1.1$ \\
Total & $15.9 \pm 3.8$ \\
Total volume injected $(\mathrm{ml})$ & $132(88 \%)$ \\
Acceptable akinesia after double injection (Score 1 or 0) & $18(12 \%)$ \\
No. of patients requiring supplementary injection & \\
\hline
\end{tabular}

Data expressed as a mean value and standard deviation or number and percentages.

Conclusion: Using $15 \mathrm{~mm}$ length needle for double injection peribulbar block has showed satisfactory results. This technique is effective for patients undergoing phacoemulsification procedure.

\section{AP7-2}

Epidural analgesia decreases pulmonary complications after abdominal and thoracic surgery a meta-analysis

D.M. Pöpping, N. Elia, E. Marret, C. Remy, M.R. Tramèr

Anaesthesiology and Intensive Care Medicine, University Hospital of Münster, Münster, Germany

Background and Goal: Whether epidural analgesia offers advantages over systemic analgesia regarding the occurrence of pulmonary complications after abdominal or thoracic surgery remains controversial.

Methods: We searched for all randomised controlled trials comparing epidural with systemic analgesia and reporting on pulmonary complications, lung function or gas exchange parameters. Inclusion criteria were epidural analgesia lasting more than 24 hours postoperatively, adult patients ( $\geqslant 18$ years) undergoing abdominal or thoracic surgery, without language restriction.

Results: 58 trials (5,904 patients), published 1971-2006, met all inclusion criteria. Epidural analgesia protected better against pneumonia (odds ratio $0.54 ; 95 \%$ confidence interval $0.43-0.68$ ), independent of site of surgery and catheter insertion, or duration of analgesia. The effect was significantly lower in trials that used patient controlled analgesia in controls (odds ratio 0.64; 0.49-0.83) compared with trials that did not (odds ratio $0.30 ; 0.18-0.49$ ), and in larger studies (odds ratio 0.62; 0.47-0.81) compared with smaller studies (odds ratio $0.37 ; 0.23-0.58$ ). From 1971-2006, the incidence of pneumonia in patients receiving epidural analgesia remained stable (about $8 \%$ ), but significantly decreased from $34 \%$ to $12 \%$ in those receiving systemic analgesia $(P<0.001)$; consequently, the relative benefit of epidural analgesia decreased also. Epidural analgesia reduced the need for prolonged ventilation or re-intubation, and improved lung function and blood oxygenation. The risk of hypotension, urinary retention, and pruritus was increased. Technical failures occurred in about $7 \%$.

Conclusion: Although the impact of epidural analgesia on pneumonia has lessened due to a decrease in the baseline risk over the last 35 years, it should be regarded as the gold-standard analgesia technique in patients undergoing abdominal or thoracic surgery.

\section{AP7-3}

\section{Anaesthesia for hip fracture surgery, a comparison between GA and spinal anaesthesia}

\section{A. Gulati, M. Parker, R. Griffiths}

Anaesthesia, Peterborough District Hospital, Peterborough, United Kingdom

Background and Goal of Study: Continuing controversy exists with regards to the preferred method of anaesthesia for emergency hip surgery. The Cochrane review ${ }^{1}$ of all randomised trials on hip fracture patients comparing general versus spinal anaesthesia summarised the results for 2,500 patients. Whilst there was a tendency to a slightly lower mortality at 30 days after surgery for those allocated to spinal anaesthesia, there were no long term differences in mortality and no notable differences in morbidity.

A large randomised controlled study may now be difficult to conduct as evidence suggests that up to $40 \%$ of the mortality from these patients is unavoidable ${ }^{2}$.

Materials and Methods: We audited the results for 4,723 consecutive patients admitted to one institution between 13/01/1989 to 19/04/05 to see if any outcome measures differed between the different forms of anaesthesia. Results and Discussions: 2548 patients had General Anaesthesia and 1541 had spinal anaesthesia. The 30 days mortality and 1 year mortality for the general and spinal anaesthesia groups were $6.2 \% / 8.4 \%$ and $27.5 \% / 29.6 \%$ respectively. Regarding post operative complications(GA/Spinal), the incidence of pneumonia was $3.8 \% / 5.0 \%$, myocardial infarction $0.3 \% / 0.3 \%$, congestive cardiac failure $1.8 \% / 2.3 \%$, pulmonary embolism $1.0 \% / 0.6 \%$, DVT $1.6 \% / 1.6 \%$ and confusion $5.4 \% / 4.0 \%$.

Multivariate analysis by stepwise logistic regression analysis was performed using SPSS 14.0. Appropriate higher interaction terms were included in the analysis if the correlation between the variables was $>0.40$. A p value $<0.05$ was considered statistically significant for the regression analysis. Variables that affected the mortality included age, gender, ASA, hemoglobin, mobility score and mental test score.

Conclusion: Mortality was not significantly affected by the type of anesthesia that the patients received after correcting for age, gender, ASA, mobility score and mental test score.

References:

1 Parker MJ, Handoll HHG, Griffiths R

2 Foss \& Kehlet British Journal of Anaesthesia 94 (1): 24-29; 200 


\section{AP7-7}

Local anaesthesia distribution in retrobulbar space following a Sub-tenons injection

M. Kendrisic, N. Tomanovic

Anaesthesia and Intensive care, Health Centre Sremska Mitrovica, Sremska Mitrovica, Serbia

Background and Goal of Study: Sub-tenon Block is nowadays commonly used in ophthalmic surgery because of its safety and efficacy. The aim of this study is to investigate the distribution of the anaesthetic in the retrobulbar space following an injection into the Sub-tenon space.

Materials and Methods: After approval of the local ethics committee, 19 pig cadaver heads were used (38 eyeballs). An incision was made at the infero-nasal quadrant of the eyeball, $5 \mathrm{~mm}$ from the corneal limb. $4.5 \mathrm{ml}$ of a mixture of $2 \%$ Lignocaine, $0,5 \%$ Bupivacaine, $75 \mathrm{mg} / \mathrm{ml}$ hyaluronidase and $0,5 \mathrm{ml}$ of India Ink was administered. The orbit content was enucleated and then kept in formaline for a period of 24 hours, after which, a cross-section was made $2.6 \mathrm{~mm}$ posterior to the hind surface of the globe. From here, samples were taken and stained with a haematoxylin-eosin stain. After being analysed using the standard histopathological procedure, they were also analysed by computer using the Adobe Photoshop program. The retrobulbar space is divided into 4 quadrants by 2 perpendicular lines, which cross in the centre of the optic nerve. The presence of ink in connective and muscle tissues and in the neural sheath of the optic nerve was observed in each of the 4 quadrants: inferonasal (IN), inferolateral (IL), supranasal (SN) and supralateral (SL).

Results:

\begin{tabular}{lrrrrl}
\hline & $\mathrm{IN}$ & $\mathrm{IL}$ & $\mathrm{SN}$ & $\mathrm{SL}$ & $\mathrm{p}$ value \\
\hline Connective tissue & 37 & 16 & 37 & 26 & $\mathrm{p}<0,01$ \\
Muscle tissue & 33 & 5 & 31 & 10 & $\mathrm{p}<0,01$ \\
Optic nerve sheath & 2 & 2 & 3 & 1 & $\mathrm{NS}$ \\
\hline
\end{tabular}

Conclusions: Following a Sub-tenon block, local anaesthetic was present in the retrobulbar space in a high percentage of cases $(97.3 \%$ or $37 / 38)$. The highest concentration was found in the IN segment (place of original injection) but significant amounts were also found in the muscle and connective tissue of the SN and SL regions. Statistically significantly smaller amounts were seen in the IL segment, which would be expected to lead to increased preservation of lateral rectus muscle function. The presence of the anaesthetic in the optic nerve sheath was insignificant but it is important to mention that higher concentrations could be potentially dangerous, due to the possibility of diffusion of the anaesthetic into the subarachnoid space.

\section{AP7-8}

\section{Infraorbital nerve block for cleft lip surgery}

L. Bouattour, M. Smaoui, S. Belhaj, K. Khemakhem, H. Chikhrouhou

Anesthesiology, University Habib Bourguiba Hospital, Sfax, Tunisia

Background and Goal of Study: Cleft lip repair is a common operation in infants and requires that the child is pain-free during the postoperative period. The efficacy of infraorbital nerve block in reducing opioids consumption and postoperative pain was evaluated in children undergoing primary cleft lift repair under general anaesthesia $(1,2)$.

Materials and Methods: After ethical committee approval and informed parental consent, we conducted a prospective randomized double blind study including fourteen ASA I-II infants, aged from 6 to 12 months, and scheduled for elective primary cleft lip repair under general anesthesia. Patients were randomly allocated to either the block group $(n=7)$ or the control group $(n=7)$. General anesthesia was standardized using halothane, alfentanil and rocuronium. The patients received infraorbital nerve block with $0.5 \mathrm{ml}$ of either $0.5 \%$ bupivacaine (block group) or normal saline solution (control group) administered into the soft tissue in front of the infraorbital foramen. Alfentanil was injected if systolic blood pressure or heart rate increases more than $20 \%$ of the base values. Consumption of alfentanil and morphine was evaluated in both groups. Pain intensity was also evaluated with the Objective Pain Scale (OPS) every 15 min after the end of anesthesia. Statistical analysis have used Fisher and Student test. $p<0.05$ was significant. Results and Discussions: The consumption of alfentanil was $201.4 \pm 63.6 \mu \mathrm{g}$ in the block group and $357.8 \pm 166.7 \mu \mathrm{g}$ ) in the control group during surgery $(p<0.05)$. Postoperative pain intensity was lower in the block group than in the control group, this difference was significant at 15, 30, 60 and 105 minutes. The consumption of morphine was $42.8 \pm 113.3 \mu \mathrm{g}$ in the block group and $557.1 \pm 269.9 \mu \mathrm{g}$ in the control group $(p<0.01)$.

Conclusions: General anesthesia combined with infraorbital nerve block is effective in reducing the consumption of opioids and postoperative pain intensity in children undergoing primary cleft lip repair.
References:

1 Parbhu KP. Scand J Plast Reconstr Surg Hand Surg 1999; 33: 83-7.

2 Epine N. Cleft Palate Craniofac J 2006; 43: 138-41.

\section{AP7-9}

Influences of remifentanil on hyperglycemic and endocrine response to surgery. A comparison with epidural block

A. Papadima, V. Tsirtsiridou, E. Lagoudianakis, E. Samanta, Ch. Loizou Anesthesiology, Hippocration, Athens, Greece

Background and Goal of Study: Neuraxial block with epidural local anaesthetic is capable of obtunding the metabolic and endocrine response induced by surgery. ${ }^{1}$ The purpose of this study was to investigate the effect of sevoflurane anaesthesia combined with remifentanil or epidural blockade on plasma concentration of glucose and cortisol during colorectal surgery. Materials and Methods: Thirty eight patients undergoing elective colorectal resection for non metastatic adenocarcinoma were admitted to the study. Patients were randomly assigned to receive sevoflurane anaesthesia combined with epidural blockade (group A) or with intravenous remifentanil (group B). Anaesthesia in both groups was maintained with sevoflurane at end tidal concentration between 1.2 and $1.5 \%$. The epidural block was maintained with ropivacaine $0.75 \%$ to achieve a bilateral sensory block from thoracic dermatome level six (T6) to sacral level five (S5). Patients in the group $B$ received remifentanil with infusion rate of 0.15 to $0.2 \mathrm{mcg} / \mathrm{kg} / \mathrm{min}$. Blood pressure and heart rate were maintained within $20 \%$ of the preoperative measurements. During surgery normothermia and normocapnia were maintained. Plasma concentration of glucose, triglycerides, lactate and cortisol were determined preoperatively, at the end of surgery and $60 \mathrm{~min}$ postoperatively. Furthermore, blood glucose and lactate were measured every 30 min during surgery. Differences between the groups were analysed using ANOVA, and $x^{2}$. Results and Discussions: Plasma glucose concentration increased during and after surgery in both groups, without showing any differences between the two group [group A: 136 (sd) $19 \mathrm{mmol} / \mathrm{dL}$ vs. group B: 142 (sd) $21 \mathrm{mmol} / \mathrm{dL}$, $p>0.05]$. Plasma concentration of lactate and triglycerides remained unaltered. Plasma concentration of cortisol increased in all patients after surgery: group A: 29 (sd) $7 \mathrm{mcg} / \mathrm{dL}$ vs. group B: 34 (sd) $14 \mathrm{mcg} / \mathrm{dL}, \mathrm{p}<0.05$.

Conclusion: Combined anaesthesia with epidural blockade significantly reduces blood cortisol compared to remifentanil in combination with sevoflurane but there was no difference on postoperative blood glucose concentration. Reference:

1 Latterman R et al. Anesthesiology 2002; 97: 374-81.

\section{AP8-1}

\section{Influence of heart adrenergic blockade obtained by thoracic epidural anaesthesia on QT, QTc intervals and their dispersions}

R. Owczuk, M.A. Wujtewicz, M. Steffek, W. Szymanowicz, T. Marjanski

Department of Anaesthesiology and Intensive Therapy, Medical University of Gdansk, Gdansk, Poland

Background and Goal of Study: QT interval prolongation is connected with the risk of development of grave cardiac arrhythmias - torsade de pointes ventricular tachycardia and ventricular fibrillation. Dispersion of QT and corrected QT (QTc) interval may promote re-entry phenomenon occurrence. It is known, that sympathetic tone may influence on QT and QTc duration as well as on their dispersion, but it has never been studied, how reversible adrenergic blockade provided by thoracic epidural anaesthesia influences on ventricular repolarization. We wanted to verify hypothesis, that thoracic epidural anaesthesia (TEA) comprising adrenergic fibres which innervate heart (T1-T4) produces decrease in duration of parameters of ventricular repolarization.

Materials and Methods: Twenty male, non-premedicated patients $(54.7 \pm 4.7$ years) scheduled for lung surgery were introduced into the study. 12-lead continues ECG registration ( $\mathrm{H} 12+$, Mortara Instrument Inc, Milwaukee, USA; sampling frequency of 10000 samples per second per channel) started before anaesthesia. Epidural catheter $(E C)$ was inserted into epidural space at level T6-T7, T7-T8 or T8-T9. After negative test with $3 \mathrm{ml}$ of lidocaine, isobaric bupivacaine was administered via EC. Level of anaesthesia was checked with cold sensation. Values measured before anesthesia and after obtaining T1 level of blockade were taken to the analysis. QT interval was corrected using Bazett's and Fridericia's formula. Results are presented as mean $\pm \mathrm{SD}$. Comparisons were performed using Student's t-test for dependent data.

Results and Discussions: Obtained results are presented in table below. All values are presented in milliseconds. 


\begin{tabular}{lccc}
\hline parameter & value before TEA & value after T1-4 block & P value \\
\hline RR interval & $768 \pm 110$ & $851 \pm 161$ & $\mathbf{0 . 0 0 1 6}$ \\
QT interval & $330 \pm 37$ & $331 \pm 38$ & 0.76 \\
QT disp. & $33.5 \pm 13$ & $26.5 \pm 13$ & 0.13 \\
QTcb interval & $380 \pm 41$ & $364 \pm 38$ & $\mathbf{0 . 0 3}$ \\
QTcb disp. & $39 \pm 16$ & $29 \pm 14$ & 0.08 \\
QTcf interval & $361 \pm 37$ & $350 \pm 32$ & $\mathbf{0 . 0 5}$ \\
QTcf disp. & $36.7 \pm 15$ & $27.9 \pm 14$ & 0.08 \\
\hline
\end{tabular}

Conclusion: Reversible heart adrenergic blockade obtained by TEA results in shortening of corrected QT interval.

\section{AP8-2}

\section{Awake off pump coronary artery bypass surgery}

V. Petrovski, T. Anguseva, V. Belostotskii, N. Hristov, Z. Mitrev

Anesthesia, Special hospital for Cardiosurgery "Filip Vtori", Skopje, The former Yugoslav Republic of Macedonia

Background and Goal of Study: To describe our initial experience in awake off pump coronary artery bypass surgery (ACAB).

Materials and Methods: Ten patients were selected for ACAB, based on their preoperative status and assessment for increased risk for complications or contraindication for general anesthesia and endotracheal intubation. High thoracic epidural catheters were inserted in all patients prior to surgery and high thoracic epidural anesthesia (HTEA) was administered during surgery, and in the immediate postoperative period.

Results and Discussions: Mean preoperative NYHA classification was $2.7 \pm 0.5$. All patients underwent $A C A B$ with 10-point visual analog scale pain index of mean $2.1 \pm 0.8$. Median sternotomy was used in all patients with average surgery duration of $71 \pm 22$ minutes. All patients received left internal mammary artery (LIMA) to left anterior descending artery (LAD), one patient received additional left radial artery to left marginal branch of the left circumflex artery. Left pleural spaces were accidentally entered in two patients during LIMA dissection, which required placement of pleural drainage. There were no respiratory complications or hemodinamyc instability during surgery and in the postoperative period. There was no mortality. Using only HTEA, all patients were mobilized in the first 4 hours after surgery, with 10-point visual analog scale pain index of $1.3 \pm 0.5$. Average in hospital stay was $4.2 \pm 1.1$. Mean patient follow up was $34 \pm 6$ months.

Conclusion(s): Awake off pump coronary artery bypass surgery can be safely done using HTEA, in selected group of patients in which there is an increased risk of complications from general anesthesia. ACAB with HTEA has shown to be safe for patients during surgery and postoperative period, with minimal pain and no complications, with early mobilization and short hospital stay.

Reference:

Karagoz HY, Sonmez B, Bakkaloglu B et al. Coronary artery bypass grafting in the conscious patient without endotracheal general anesthesia. Ann Thorac Surg. 2000; 70: 91-96.

\section{AP8-3}

\section{Epidural anesthesia in cardiac surgery}

V. Petrovski, E. Stoicovski, T. Manailova, N. Hristov, Z. Mitrev

Anesthesia, Special hospital for Cardiosurgery "Filip Vtori", Skopje, The former Yugoslav Republic of Macedonia

Background and Goal of Study: The purpose of this study was to present our initial experience with the use of combined high epidural thoracic anesthesia (HTEA) with general anesthesia (GA) in unselected patients for openheart surgery.

Materials and Methods: From November 2005 to January 2006, 75 patients were subjected to open heart surgery using HTEA and GA. Unstable angina was present in 42 (56\%). Forty eight (64\%) were classified as New York Heart Association class III or IV. Preoperative ejection fraction was $39 \pm 13$. Median sternotomy was performed in 70 (93\%) patients, right antero-lateral thoracotomy in $4(5 \%)$ and median laparotomy in 1 patient. Aorto coronary bypass (ACB) was done in $45(60 \%)$ of the patients with $5(7 \%)$ off pump cases, ventriculoplasty and valve reconstructive surgery in $8(11 \%)$ of the patients, valve replacement was done in $12(16 \%)$ of the patients, mitral valve reconstruction in $6(8 \%)$ of the patients. Two patients required intra aortic balloon pump support.

Results and Discussions: All patients remained stable throughout the procedure, with 29 (39\%) extubated in the operating theater, $15(20 \%)$ extubated within 5 hours following the procedure, 17 (23\%) within 10 hours and $14(18 \%)$ after 10 hours. Average first mobilization time was $31 \pm 17$ hours. Average intensive care unit stay (ICU) was $40 \pm 31$ hours and hospitalization time of $8.6 \pm 4.6$ days. Average postoperative visual analog scale for pain was $3.5 \pm 0.9$.
Conclusion(s): HTEA with GA in unselected patients has shown to be safe during surgery and postoperative period, allowing fast extubation with minimal pain, no complications, with early mobilization and short ICU and hospital stay.

Reference:

Fawcett WJ, Edwards RE, Quinn AC, MacDonald IA, Hall GM. Thoracic epidural analgesia started after cardiopulmonary bypass. Adrenergic, cardiovascular and respiratory sequelae. Anaesthesia 1997; 52(4):294-299.

\section{AP8-5}

Pharmacodynamic safety of Ropivacaine during continuous epidural infusion

C. Baldi, M. Parise, M. Cusato, M. Allegri, A. Braschi

Anaesthesiology, Fondazione IRCCS Policlinico San Matteo, Pavia, Italy

Background and Goal of Study: In many preclinical studies Ropivacaine shows less central nervous system and cardiac toxicity than other local anaesthetics. The objective of this study is to value the systemic absorption of Ropivacaine in continuous epidural infusion for post-operative pain control and determine how much our arterial plasma concentrations are different from which are considered toxic for the literature $(4,3 \mathrm{mg} / \mathrm{lt})$.

Materials and Methods: In this study we analysed 12 patients ASA I-II with epidural catheter for pain control in post-operative period of major abdominal and urological surgery.

The pharmacological protocol was as follow: First bolus 45 minutes before ending of surgery with $10 \mathrm{mg}$ of Ropivacaine $0,2 \%+10 \mathrm{mcg}$ of Sufentanyl; second bolus after $15-20$ minutes with $10 \mathrm{mg}$ of Ropivacaine $0,2 \%$. At the end of surgery we start an infusion $5 \mathrm{ml} / \mathrm{h}$ with Ropivacaine $0,2 \% 500 \mathrm{mg} / 250 \mathrm{ml}$ and Sufentanyl $200 \mathrm{mcg}$ with an electronic device. During post-operative period we obtained arterial samples done before first local anaesthetic administration and at time $+3,+6,+12,+24,+48,+54,+60$, and we studied the systemic absorption of local anaesthetic with HPLC-UV technique.

All patients were monitored after surgery for eventual side effects. Result and Discussion:

\begin{tabular}{lccc}
\hline & $\begin{array}{l}\text { Median arterial } \\
\text { plasma level } \\
\text { of Ropivacaine }\end{array}$ & $\begin{array}{l}\text { \% of toxic level } \\
(4,3 \mathrm{mg} / \mathrm{lt})\end{array}$ & Side effects \\
\hline Time of sample & 0.29000 & $6,74 \%$ & 0 \\
$\mathbf{A L}+\mathbf{3}$ & 0.44500 & $10,35 \%$ & 0 \\
$\mathbf{A L}+\mathbf{6}$ & 0.73400 & $17,07 \%$ & 0 \\
$\mathbf{A L}+\mathbf{1 2}$ & 0.93000 & $21,63 \%$ & 0 \\
$\mathbf{A L}+\mathbf{2 4}$ & 1.70500 & $39,65 \%$ & 0 \\
$\mathbf{A L}+\mathbf{4 8}$ & 1.17000 & $27,21 \%$ & 0 \\
$\mathbf{A L}+\mathbf{5 4}$ & 0.53000 & $12,32 \%$ & 0 \\
$\mathbf{A L}+\mathbf{6 0}$ & &
\end{tabular}

From preliminary results we can notice that arterial plasma levels obtained in our patients at different time of evaluation are really inferior to the neurocardiotoxical one. Even at plasma peak $(+48)$ the levels observed are of only 39,65 of toxic one.

Conclusions: The preliminary data obtained show that plasma concentration of Ropivacaine in continuous epidural infusion are really inferior to toxic level for literature and it's result in total absence of side effect in our study. Therefore, we can consider that the continuous epidural infusion of Ropivacaine at concentration used is clinically and pharmacologically sure and safety. Reference:

K. Knudsen, M. Beckman Suurkula, S. Blomberg, J. Sjovall, N. Edvardsson, British Journal of Anaesthesia 1997; 78: 507-514.

\section{AP8-6}

\section{Effects of levosimendan on oxygen transport indices in patients with low output heart failure under lumbar epidural} anaesthesia

E. Samanta, A. Krana, C. Loizou, S. Katsaragakis, L. Georgiou Anaesthesiology, G.H.A. Hippocration, Athens, Greece

Background and Goal of Study: The goal of our study was to assess the effects of preoperative infusion of LS (levosimendan) on perioperative oxygen transport in patients with LVEF $<22 \%$ undergoing elective minor abdominal surgery under lumbar epidural anaesthesia.

Materials and Methods: Five male patients, ASA III (mean age 76, range 64-85 years) with low output heart failure (mean LVEF 20, range 17-22\%) were studied. Preoperatively: Before LS administration all patients had arterial and pulmonary artery catheters. LS was given as a bolus $(6-24 \mathrm{mcg} / \mathrm{kg})$ followed by continuous infusion $(0,1 \mathrm{mcg} / \mathrm{kg} / \mathrm{min})$ lasted 24 hours.

Day of surgery: All patients received lumbar epidural anaesthesia with lidocaine $2 \%$ (loading dose $14 \pm 3 \mathrm{ml}$ ) for minor low abdominal surgery 
lasted $60 \pm 10 \mathrm{~min}$. Intraoperatively patients were spontaneously ventilated with $\mathrm{FiO} 2: 50 \%$ (in air) via Venturi mask. Fluids were given PRN to maintain sufficient MAP and PCWP.

Baseline data were collected before LS administration. The second data were obtained in 24 hours, the third after lumbar epidural anaesthesia and the fourth at the end of surgery.

Results and Discussions: After LS infusion CO significantly increased (*one way ANOVA on ranks BL vs LS). After epidural anaesthesia DO2 and VO2 increased, the first mare significantly (**one way ANOVA on ranks LS vs EA). Results are given as mean \pm standard deviation, 95\% confidence interval.

(BL-baseline, LS-after levosimendan administration, EA-after epidural anaesthesia, ES-end of surgery.)

\begin{tabular}{lllll}
\hline & $\mathrm{BL}$ & LS & EA & ES \\
\hline $\mathrm{CO}(\mathrm{lt} / \mathrm{min})$ & $3,7 \pm 0,5$ & $6,5 \pm 1^{\star}$ & $5,9 \pm 0,2$ & $5,9 \pm 2,2$ \\
$\mathrm{SvO} 2(\%)$ & $65 \pm 7$ & $72 \pm 4$ & $75 \pm 7$ & $69 \pm 8$ \\
$\mathrm{DO} 2(\mathrm{ml} \mathrm{O} 2 / \mathrm{min})$ & $786 \pm 126$ & $1095 \pm 74^{\star \star}$ & $889 \pm 127$ & $833 \pm 248$ \\
$\mathrm{VO} 2(\mathrm{ml} \mathrm{O} 2 / \mathrm{min})$ & $221 \pm 33$ & $246 \pm 57$ & $184 \pm 73$ & $233 \pm 24$ \\
\hline
\end{tabular}

$\left({ }^{\star} p<0.01,{ }^{\star \star} p<0.04\right)$.

Conclusion(s): The beneficial effects of preoperative administration of LS on oxygen transport lasts during epidural anaesthesia in patients undergoing minor abdominal surgery.

\section{AP8-7 \\ High resolution ultrasound guided perivascular and cervical block for carotid artery surgery \\ T. Roessel, D. Wiessner, M. Scheit, T. Koch, R.J. Litz \\ Anaesthesiology, University of Technology, Dresden, Germany}

Background and Goal of Study: Cervical nerve blocks performed for carotid artery surgery (TEA) are insufficient for surgery in more than $50 \%$ of patients and require intraoperative supplementation with local anesthetics (LA). Underlying reasons may be the wide range of variation in cervical anatomy and the individual pattern of local anesthetic spread. We report our experiences with an ultrasound (US) guided combined perivascular and superficial cervical block for TEA.

Materials and Methods: After IRB approval and written informed consent 30 consecutive unpremedicated patients (ASA II-III, $68 \pm 4$ years, BMI $32 \pm 4)$ received RA for TEA. Patients were placed supine with the head slightly turned to the opposite side. Using a high resolution US device with a 12.5 or $15 \mathrm{MHz}$ linear transducers (HD11XE, Philips Medizin Systeme GmbH, Hamburg, Germany) the bifurcation of the carotid artery was identified. Then, the needle was advanced from the lateral border of the sternocleidomastoid muscle under visualisation close above the bifurcation. After negative aspiration test $10-15 \mathrm{ml}$ of ropivacaine $0.5 \%$ was applicated perivascularly. Then, the needle was withdrawn and $10 \mathrm{ml}$ of ropivacaine $0.5 \%$ were applicated below the lateral border of the sternocleidomastoid muscle. The incidence of intraoperative LA supplementation as well as pain scores according to a numeric rating scale (1-10) and complications were recorded.

Results and Discussions: Target structures could be identified in all patients. Likewise, observation of needle advancement as well as local anesthetic spread was always possible, thus blocks could be performed successfully in all patients without intraoperative supplementation or conversion to general anesthesia (NAS 1[0-3]). A high variability was found regarding the distance between the carotid bifurcation and the spine $(1.6 \pm 0.5 \mathrm{~cm})$ as well as the distance of the bifurcation to the skin $(2 \pm 0.6 \mathrm{~cm})$. Aspiration test during the procedure was negative in all blocks and no block related complications occurred.

Conclusion(s): The combination of a perivascular and cervical block is a highly effective technique for TEA. The use of high resolution US for block performance provides a high margin of safety by visualization of needle advancement, observation of LA spread, thus avoidance of accidental intravascular LA application.

\section{AP8-8}

The role of ropivacaine and fentanyl epidural analgesia on reducing perioperative myocardial ischaemia in hip fracture C. Berteanu, D. Cernea, D. lonescu, C. Roiu, M. Berteanu Department of Anesthesiology and Intensive Care, Emergency University Hospital, Bucharest, Romania

Background and Goal of Study: Perioperative myocardial ischaemia is an important risk factor for cardiac morbidity and mortality after noncardiac surgery. The aim of the study was to evaluate the influence of combined fentanyl/ropivacaine epidural analgesia on reducing the incidence and severity of cardiac ischaemia in patients undergoing surgical treatment for traumatic hip fracture.

Materials and Methods: After Ethics Committee approval and written consent,79 ASA II-IV patients were randomly assigned to conventional analgesic regimen(tramadol,CON group) or continuous epidural infusion of ropivacaine/fentanyl(EPI group). These regimens were started preoperatively and continued three days postoperatively, after spinal anesthesia. ECG was continuously recorded. STsegment depression of $>0.1 \mathrm{mV}$ or elevation of $>0.2 \mathrm{mV}$, lasting $>1 \mathrm{~min}$ were considered as ischaemic episodes. Nocturnal arterial oxygen saturation(SaO2) was recorded perioperatively and subjective pain was assessed using visual analogue scale(VAS).

Results and Discussions: 13 patients(43\%) in the CON and 12 patients in the EPI group had ischaemic episodes. However, significantly more patients in the CON group had ischaemia during surgery( 8 vs 0 in the EPI group, $p=0.005$ ). The median (quartal deviation) total ischaemic burden(i.e. integral of ST-change vs time) in patients with ischaemic episodes was ten times larger in the CON group $(340 \mathrm{~mm} / \mathrm{min})(p=0.002)$. There were no significant differences between the groups in the average heart rates(HR) at the start of ischaemic episodes or in the maximal HR during the attacks. Average nocturnal SaO2 was similar in the two groups and there were no differences in subjective pain but postoperative and average perioperative VAS scores were almost $40 \%$ lower in the EPI group $(p=0.006)$. Perioperative myocardial infarctions were not detected.

Conclusions: Continuous epidural ropivacaine/fentanyl analgesic regimen, started preoperatively, reduces the amount of myocardial ischaemia in elderly patients with hip fracture.

\section{AP8-9}

Thoracic epidural anesthesia improves intestinal mucosal capillary perfusion in acute oedematous pancreatitis in the rat H. Freise, A.W. Sielenkaemper, H. Van Aken, M.M. Lerch, L.G. Fischer Anesthesiology and Intensive care medicine, University Hospital of Muenster, Muenster, Germany

Background and Goal of Study: Thoracic epidural anaesthesia (TEA) improved mucosal perfusion and survival in severe AP (1). Mild acute pancreatitis (AP) is also associated with impaired intestinal perfusion and motiltiy. Therefore, the effects of TEA on mucosal circulation in mild AP were investigated.

Materials and Methods: 21 male rats were equipped with central veanous and arterial lines and a thoracic epidural catheter. Rats were randomly assigned to Sham ( $2 \mathrm{ml} / \mathrm{h} \mathrm{NaCl}, 9 \%$ i.v., $15 \mu \mathrm{l} / \mathrm{h} \mathrm{NaCl0,9 \%}$ epidural) untreated AP $(10 \mu \mathrm{g} / \mathrm{kgKG} / \mathrm{h}$ Caerulin i.v., $15 \mu \mathrm{l} / \mathrm{h} \mathrm{NaCl} 0,9 \%$ epidural) and AP+TEA $(10 \mu \mathrm{g} / \mathrm{kgKG} / \mathrm{h}$ Caerulin i.v., $15 \mu \mathrm{l} / \mathrm{h}$ Bupivacain $0,5 \%$ epidural). After $4 \mathrm{~h}, 20 \mathrm{mg}$ FITC-albumin was injected and ileal mucosal perfusion was evaluated by intravital microscopy. The intercapillary area (ICA), which is inversely proportional to capillary density, of all perfused capillaries $\left(\right.$ ICA $\left._{\text {total }}\right)$ and of continuously perfused capillaries only $\left(\mathrm{ICA}_{\text {cont }}\right)$ and mucosal terminal arteriolar blood flow (ABF) was evaluated. Pancreatic water content, trypsinand MPO-content were recorded.

Results and Discussions: While $\mathrm{ICA}_{\text {total }}$ was not increased in untreated $\mathrm{AP}$, a marked reduction in continuous capillary perfusion reflected by an increase in ICA $_{\text {cont }}$ from $842 \pm 67 \mu \mathrm{m}^{2}$ to $1212 \pm 152 \mu \mathrm{m}^{2}$ ( $p<0.05$ vs. Sham) was recorded. In AP+TEA, ICA total dropped to $824 \pm 81 \mu \mathrm{m}^{2}$ ( $\mathrm{p}<0.05 \mathrm{vs}$. AP). Mucosal ABF increased from $2.6 \pm 0.3 \mathrm{nl} / \mathrm{s}$ to $3.6 \pm 0.3 \mathrm{nl} / \mathrm{s}$ in untreated AP $(p<0.05$ vs. Sham). This increase was reduced to $2.4 \pm 0.3 \mathrm{nl} / \mathrm{s}$ in untreated AP ( $p<0.05$ vs. untreated AP). Increased ABF associated with decreased capillary perfusion indicates increased mucosal shunt. Untreated AP increased pancreatic water content from $69 \pm 3 \%$ to $91 \pm 2 \%(p<0.05$ vs. Sham), which was not affected in AP + TEA. Neither AP-induced increase in MPO nor the trypsin-content was altered in AP+TEA.

Conclusion(s): TEA reduces AP-induced alterations of mucosal microcirculation in mild AP. The extrapancreatic therapeutic effects are not related to reduced pancreatic injury and might be due to regional sympathetic block (2). References:

1 Freise H, Lauer S, Anthonsen S et al. Anesthesiology 2006; 105: 354-359.

2 Freise H, Anthonsen S, Fischer LG et al. Anest. Analg. 2005; 100: 255-262.

\section{AP9-1}

A national survey: peri-operative anaesthetic management of patients with femoral neck fractures in the UK

M. Sandby-Thomas, G. Sullivan, J. Hall

Anaesthetics, Cardiff University, Cardiff, United Kingdom

Background and Goal of Study: Surgical correction of a fractured neck of femur is an extremely common orthopaedic procedure. The incidence of hip fractures worldwide is predicted 4.5 million by 2050 with some estimates 
putting the figure as high as 21.3 million (1). The standardised mortality ratio is much higher in these patients than in the general population of comparable age (2). There are various options for the anaesthetic management of these but no one anaesthetic technique has been shown to be clearly superior to any other (3). Our survey gives a national snapshot of current anaesthetic practice in the UK which will enable us to focus and direct further research into this important area.

Materials and Methods: We randomly selected $20 \%$ of acute hospitals in the UK and sent postal tick-box questionnaires to the trauma anaesthetists working there with stamp addressed envelopes for replies. The questions pertained to peri-operative aspects of anaesthetic care.

Results and Discussions: We received 155 replies from 218 questionnaires sent $(71.1 \%$ response rate). Regional anaesthesia (RA) was preferred by $75.8 \%$ of respondents, $14.4 \%$ combined general anaesthesia (GA) with a regional technique and $9.8 \%$ used GA alone. A spinal was the preferred RA in $95.5 \%$ of cases. This was generally performed bad side down (45.7\%) using ketamine $(37.3 \%)$ and/or midazolam (41.2\%) to aid positioning. When GA was employed, either alone or in combination, respondents used a laryngeal mask airway (71.3\%) with the patient breathing spontaneously $(56.2 \%)$ on volatile agents (84.3\%). If GA and RA were used together, a 3 -in-1 block was the most popular adjunct (47.1\%). Paracetamol and morphine were the most common post-operative analgesic regimes with continuous epidural or nerve block infusions used rarely.

Conclusion(s): Spinal Anaesthesia is the most commonly employed anaesthetic technique for hip fracture in the UK despite clear evidence of its benefits. References:

1 Gullberg B, Johnell O, Kanis JA. Osteoporosis int. 1997; 7(5): 407-13

2 Michael J Goldacre, Stephen E Roberts, David Yeates. BMJ 2002; 325: 868-869

3 Urwin SC, Parker MJ, Griffiths R. Br J Anaesth 2000; 84: 450-455

\section{AP9-2}

Ultrasound guidance reduces the minimum effective volume of local anaesthetic required to block the femoral as compared with nerve stimulation

A. Casati, G. Danelli, M. Baciarello, S. Leone, M. Rossi

Anesthesiology and Pain Therapy, University of Parma, Parma, Italy

Background and Goal of Study: This prospective, randomized, up-and-down study tested the hypothesis that using ultrasound guidance may reduce the minimum effective anesthetic volume $\left(\mathrm{MEAV}_{50}\right)$ required to block the femoral nerve as compared with nerve stimulation guidance.

Materials and Methods: After standard premedication and sciatic nerve block were given, 60 patients undergoing knee arthroscopy were randomly allocated to receive a femoral nerve block with $0.5 \%$ ropivacaine using either nerve stimulation (group NS, $\mathrm{n}=30$ ) or ultrasound (group US, $\mathrm{n}=30$ ) guidance. The volume of the injected solution was varied for consecutive patients based on an up-and-down staircase method according to the response of the previous patient. The initial volume was $12 \mathrm{~mL}$. A blind observer evaluated the occurrence of complete loss of pinprick sensation in the femoral nerve distribution, with concomitant block of the quadriceps muscle: positive or negative responses within $20 \mathrm{~min}$ after the injection determined a $3 \mathrm{ml}$ decrease or increase for the next patient, respectively.

Results and Discussions: The MEAV ${ }_{50}$ for femoral nerve block was $15 \pm 4 \mathrm{~mL}\left(\mathrm{Cl}_{95}: 7-23 \mathrm{~mL}\right)$ in group US and $26 \pm 4 \mathrm{~mL}\left(\mathrm{Cl}_{95}: 19-33 \mathrm{~mL}\right)$ in group NS $(P=0.002)$. The $\mathrm{ED}_{95}$ calculated with probit transformation and logistic regression analysis was $22 \mathrm{~mL}\left(\mathrm{Cl}_{95}: 13-36 \mathrm{~mL}\right)$ in group US, and $41 \mathrm{~mL}\left(\mathrm{Cl}_{95}\right.$ : 24-66 mL) in group NS.

Conclusion: Ultrasound guidance provided a $42 \%$ reduction of the minimum effective anesthetic volume required to block the femoral nerve as compared with the nerve stimulation guidance.

\section{AP9-3}

\section{Combined lower extremity regional anaesthesia: a 10 year review of 6000 nerve blocks}

L. Driesz, G. Bencsik, A. Tölgyes, S. C. Dashti

Department of Anesthesiology and Intensive Care Unit, Geza Hetényi County Hospital, Szolnok, Hungary

Background and Goal of Study: Recently, more and more studies are dealing with lower extremity regional blocks as alternatives to general and spinal anesthesia (1). In our retrospective study - that covers 10 years of practice we summarize the experience gained from the above mentioned regional blocks.

Materials and Methods: We performed 6000 combined lower extremity regional anesthesia from September 1995 to December 2006. In the first 4 years we used mainly the " 3 in 1" Winnie's method, and in the last 6 years we gradually switched to the Psoas compartment block. Sciatic nerve block was performed using the Labat approach. Performing blocks we used the nerve stimulators Stimuplex S, HNS (B.Braun), and Pajunk Multistim LA, and Stimuplex A 100, A 150 needles. The administered local anesthetic doses were calculated for weight groups and recorded on a chart for everyday use. We didn't exceed the dose of $4 \mathrm{mg} / \mathrm{kg}$ bodyweight for lidocain $1 \%$, and $3 \mathrm{mg} / \mathrm{kg}$ bodyweight for bupivacain $0.5 \%$ or ropivacain $0.75 \%-1 \%$.

Results and Discussion: 16 of 21 full-time anesthesiologists routinely perform the above mentioned combined nerve-block in our hospital. There was no significant difference regarding the total anesthetic procedures performed in 1996 and 2005 (11246 vs. 11638). The proportion of regional anesthesia was constant in 1996 and 2005 (28.11\% vs. $28.6 \%$ respectively). The share of lower limb regional blocks to total anesthetic procedures increased from $2.06 \%$ to $5.67 \%$, and within the field of regional anesthesia increased from $7.34 \%$ to $19.67 \%$. On the basis of the data of the last 5 years, the rate of complications has been between $0.6-2.5 \%$ (2).

Conclusions: Lower extremity regional anesthesia developed rapidly during the last 10 years in our hospital. Combined Psoas compartment and sciatic nerve block is easy to learn, and perform with appropriate training. Patient's trust, comfort and especially postoperative analgesia is very good.

References:

1 F. Heid et al. European Journal of Anaesthesiology 2006; 23: 346-350.

2 Y. Auroy et al. Anesthesiology 2002; 97: 1274-1280.

\section{AP9-4}

\section{New indication for obturator nerve blockade}

B. Krivic, N. Ladjevic, J. Filimonovic, I. Likic Ladjevic, N. Popovic

Urology Department, Institute of Anesthesia, Belgrade, Serbia

Background and Goal of study: N. obturator is a branch of lumbar plexus and he is in close contact with lateral wall and bladder neck and external side of prostatic urethra. Unwonted high frequency electro stimulation of $n$. obturator provokes very often a leg movement and can be dangerous during transurethral surgery. The goal of study was: can we provide better and safer condition for surgeon work without additional complications and can we establish new indication for $\mathrm{n}$. obturator blockade?

Materials and Methods: 60 patients ( 38 men and 15 women) with bladder tumor of lateral wall were divided in two groups: First group (20 men and 10 women) have received only spinal anesthesia ( $3 \mathrm{ml}$ of Bupivacain $0.5 \%$, needle $G$ 25, $L_{1}-L_{2}$ space) and Second group (19 men and 11 women) have received spinal anesthesia and $\mathrm{n}$. obturator blockade $(20 \mathrm{ml}$ of Bupivacain $0.25 \%-$ Labatsu technique).

Results and Discussion: Between groups there were no significant difference according average years, ASA status, sex, blood arterial pressure, heart and respiratory rates changes. We have noticed 8 times more $n$. obturator stimulation and consequent leg movements in first group (26.67\%) than in group two $(3.33 \%)$ and this difference is statistically significant $(p<0.05)$. Average electro stimulation for nerve identification was $2.35 \mathrm{Ma}$. We did not notice any complication.

Conclusion: This is very cheep, short and simply, technique which provide better and safer condition for surgeon work during transurethral resection and we like to emphasize and add this indications as a new and beneficial. References:

Auroy $Y$ et al: Serious complications related to regional anesthesia: Results of a prospective survey in France. Anesthesiology 1997; 87: 479-86.

Hadzic $\mathrm{A}$, Vloka J: The use of peripheral nerve blocks in anesthesia practice. A natinal survey. Reg Anesth Pain Med 1998; 23: 241-246.

\section{AP9-5}

\section{A prospective, randomized double-blinded comparison between ropivacaine and lidocaine for outpatient knee arthroscopy}

G. Danelli, M. Zasa, S. Adamanti, M. Gaudio, M. Gatti

Department of Anesthesiology, University of Parma, Parma, Italy

Background and Goal of study: Intrathecal lidocaine is a commonly used drug for outpatient orthopaedic procedures, but its use is associated with a high incidence of transient neurologic symptoms (TNS). The aim of this prospective, randomized, double blind study was to compare the evolution of spinal block obtained with either lidocaine or ropivacaine for outpatient knee arthroscopy.

Materials and Methods: With IRB approval and patients written consent, 30 ASA physical status I-III outpatients undergoing outpatient knee arthroscopy were randomly allocated to receive spinal anaesthesia with either $50 \mathrm{mg}$ lidocaine $1 \%(n=15)$ or $10 \mathrm{mg}$ ropivacaine $0.5 \%(n=15)$. A blinded observer recorded the evolution of the spinal block until achievement 
of home discharge criteria. Sensory block was assessed using loss of pinprick sensation. Motor block was assessed using a modified Bromage score. Times from spinal injection to recovery of sensory and motor functions, unassisted ambulation and micturition were recorded. A phone-call follow up was also performed 24 hours and 7 days after surgery, in order to evaluate the incidence of TNS.

Results and Discussion: Median (1st-3rd quartile) onset time was 15 (10-21) min with lidocaine and $24(11-37) \mathrm{min}$ with ropivacaine $(P=0.11)$. Recovery of motor function required 113 (95-131) $\mathrm{min}$ with lidocaine and 135 (87-183) with ropivacaine $(P=0.21)$. Lidocaine resulted in faster resolution of sensory block [148 (130-167) min vs $188(146-231)(P=0.02)]$, unassisted ambulation [176 (144-208) $\mathrm{min}$ vs $240(179-302) \mathrm{min}(\mathrm{P}=0.014)]$, and voiding [208 (163-254) min vs $293(242-343) \min (P=0.001)]$. Six lidocaine patients reported TNS (40\%) versus no ropivacaine patients $(P=0.005)$.

Conclusion(s): $10 \mathrm{mg}$ ropivacaine $0.5 \%$ represents an efficient alternative to $50 \mathrm{mg}$ lidocaine $1 \%$ for outpatient spinal anaesthesia and is associated with less TNS.

\section{AP9-6}

A new approach to blockade of the posterior tibial nerve

A. Vescovo, R. Castro, X. Dominguez, S. Pérez, H. Turene

Anesthesiology, Italian Hospital, Buenos Aires, Argentina

Background and Goal: An ankle block is a form of regional analgesic technique. The blockade of the tibial posterior nerve has been described in many text books. Most of the approaches to the nerve are in the ankle joint, but it depends on the posterior tibial artery as a landmark, which in some patients it is not palpable.

The aim was to describe a new approach to the tibial posterior nerve distal to the internal malleolus using a nerve stimulator as a unique technique. Materials and Methods: Ten patients (ASA I-III) scheduled for elective foot surgery were included. With the patient in dorsal position the foot was externally rotated. We traced two lines, one from the medium point of the internal malleolus to the inferior angle of calcaneum, and other from the tuberosity of the scaphoid to the distal insertion of the tendo Achillis.

The puncture was done in the intersection of both lines with a needle of $25 \mathrm{~mm}$ conected to a nerve stimulator. The needle was inserted in direction to the external malleolus until the plantar flexion response was obtained $(0.5 \mathrm{~mA}, 1 \mathrm{~Hz})$. We injected $5 \mathrm{ml}$ de bupivacaíne $0,5 \%$ and $5 \mathrm{ml}$ lignocaíne $2 \%$ without adrenaline after a negative aspiration test.

Results: The new approach was completely successful in our patients. They did not require local analgesic supplementation or intravenous analgesic. The maneuver was completed in 2 minutes with acceptable tolerance for the patient.

Conclusion: We described a approach to the distal posterior tibial nerve with nerve stimulator. The anatomic parameters were easily recognised independently of the posterior tibial artery landmark. This approach is useful as a unique technique or as supplement when proximal techniques failed.

References:

Wassef MR. Anaesthesia.19910ct; 46(10): 841-4.

Larrabure P and cols.CanJAnaesth.2005Mar; 52(3): 276-80.

\section{AP9-7}

\section{Placement of catheters in the popliteal fossa through the medial approach: a preliminary study \\ L. Di Marco, E. Guntz, E. Dereeper, M. Sosnowski \\ Anesthesiology, Hospital Saint Pierre, Brussels, Belgium}

Background and Goal of Study: Medial approach of the sciatic nerve in the popliteal fossa has been described for single injection (1) and continuous block in patients unable to be placed prone (2). We studied catheter placement through the medial approach and compared this technique to the posterior approach (3).

Materials and Methods: 12 patients scheduled for hallux valgus surgery were randomly allocated in 2 groups. In the medial approach group, a $100 \mathrm{~mm}$ Tuohy needle was inserted through the Jobert fossa of 6 patients in supine position (1). When a plantar flexion was obtained with a stimulation of $0.5 \mathrm{~mA}, 2 \mathrm{~Hz}, 0.1 \mathrm{~ms}$, the catheter was inserted and a bolus of $30 \mathrm{~mL}$ of L-bupivacaïne $0.5 \%$ was administered. Surgery was performed under general anaesthesia (propofol, remifentanil), a continuous infusion of $5 \mathrm{~mL}$ per hour of L-bupivacaïne $0.125 \%$ with optional $5 \mathrm{~mL}$ boli every 40 minutes was initiated at the end of surgery. In the posterior approach group, the same protocol was performed using Singelyn's landmarks (3). Time to perform the catheter placement, post-operative pain, additional analgesic requirements, volume of infused L-bupivacaïne and patient acceptance were recorded. Statistical comparisons (mean \pm s.e.m.) were performed using unpaired Student $t$-test $(\mathrm{P}>0.05$ not significant).
Results and Discussion: Groups are similar in terms of age, weight, height and sex $(P>0.05)$. There are no statistical differences concerning the time to perform catheter placement ( $10 \mathrm{~min} \pm 0.74$ vs $12 \mathrm{~min} \pm 0.89$ ), post-operative pain in the first 12 hours (VAS $=0$ ), additional analgesic requirements (no additional analgesics) and the volume of infused L-bupivacaïne (162 \pm 23 vs $167 \pm 25)$ between the 2 groups $(P>0.05)$. No vascular puncture occurred in both groups. In the posterior approach, 2 patients reported discomfort related to the presence of the catheter at the posterior part of the thigh.

Conclusion: Our results suggest that continuous sciatic nerve block through the medial approach is an efficient technique that offers the patient the comfort to remain supine during the procedure and allows an easy monitoring of the catheter.

References:

Guntz E. Can J Anaesth 2004; 51: 817-20.

Eurin M. Can J Anaesth 2006; 53: 1165-6.

Singelyn F. Anesth Analg 1997; 84: 383-6.

\section{AP9-8}

Intravenous regional anesthesia for knee arthroscopy

Y. Pala, M. Gamli, M. Arslan, M. Canturk, M. Pekel

II. Anesthesiology and Reanimation, Ankara Numune Education and Research Hospital, Ankara, Turkey

Background and Goal of Study: Intravenous regional anesthesia (IVRA) has been used successfully for foot and ankle surgery (1), there was only one case report in the literature with IVRA for knee arthroscopy (2). We aimed to find out whether IVRA would be effective for knee arthroscopy and the effects of adding ketamine to local anesthetics on block properties and tourniquet pain Materials and Methods: Forty patients undergoing knee arthroscopy were included. IVRA was perfomed with the modified inter-cuff technique described by Al-Metwalli. Patients were randomized to receive $80 \mathrm{~mL}$ solutions containing either $0.5 \%$ prilocaine (Group I) or $0.15 \mathrm{mg} / \mathrm{kg}$ ketamine plus $0.5 \%$ prilocaine (Group II). 15 minutes after drug injection distal cuff of the thigh tourniquet was inflated, proximal cuff and calf tourniquet were released then surgery was started. IVRA block characteristics, tourniquet and surgical pain, perioperative fentanyl consumption, first analgesic request time, hemodynamic parameters were assessed. Statistical analyses were performed with Mann-Whitney $U$ and Fisher's Chi-squared tests. $\mathrm{P}<0.05$ was considered significant.

Results and Discussions: Demographic data, operation times, fentanyl consumptions were similar. 5 patients in group I and 6 patients in group II received general anesthesia because of inadequate block. Body mass indexes $(\mathrm{BMI})$ of these 11 patients were higher than the others $(29.3-25.1$, $p=0.035)$. Sensory block onset time was shorter and first analgesic request time was longer in group II.

Conclusion: We think that, IVRA may be considered as an alternative regional anesthesia technique for knee arthroscopy provided that volume and dosage of the drug is adjusted according to BMI. In addition, adding ketamine to local anesthetic solution may have partial beneficial effects on block quality by decreasing sensory block onset time and increasing first analgesic request time.

References:

1 Kim DD: Orthopedics. 1993; 16: 1109-113

2 Al-Metwalli R: CJA 2002;49: 687-9

\section{AP9-9}

\section{Effectiveness of ropivacaine and L-bupivacaine for continuous postoperative analgesia with " 3 in 1 nerve block" after total knee arthroplasty}

\section{Quattrone, C. Galletti, G. Filoni, G. Mazzeo, T. Mandolfino}

Neurosciences, Psychiatric and Anesthesiological Sciences, Ausl 5 Presidio Ospedaliero San Vincenzo - Taormina and Policlinico Universitario G. Martino, Messina, Italy

Background and Goals: Severe postoperative pain is associated with total knee arthroplasty (TKA). Adequate pain management is necessary to achieve early mobilization and physical therapy. The use of peripheral nerve blocks is widely recommended after orthopaedic surgery. Our purpose was to compare the effectiveness of ropivacaine and L-bupivacaine, used for continuous " 3 in 1 nerve block" as a form of postoperative analgesia.

Materials and Methods: For this retrospective study we considered 80 patients (pts), ASA II-IV, mean age $53.6 \pm 10.8 \mathrm{yrs}$, undergoing elective unilateral TKA under subarachnoid block from January 2003 to June 2006. Postoperative analgesia was performed with L-bupivacaine $0.125 \%$ in 40 pts (group L) or ropivacaine $0.2 \%$ in the other 40 pts (group R) by a perineural catheter placed to obtain a continuous 3-in-1 femoral nerve block for 72 hours after surgery, using a portable infusion pump settled at $6 \mathrm{ml} / \mathrm{h}$. 
Pain relief was assessed by using a visual analog scale.

Results: At the infusion dose of $6 \mathrm{ml} / \mathrm{h}$ the risk for systemic central nervous toxicity is very low and we observed a good quality of analgesia and functional outcome in both groups, without any significant differences about sensorimotor block.

Conclusions: According with the current literature, with this study we demonstrate that peripheral nerve block techniques are the optimal analgesic method after TKA and that the same volume of ropivacaine and L-bupivacaine, used at different concentration leads to similar postoperative pain relief, producing a good patient satisfaction and early physical therapy.

\section{References:}

Ekatodramis G, Borgeat A, Huledal G et al. Anesthesiology 2003; 98: 143-50.

Horlocker TT, Kopp SL, Pagnano MW et al. J Am Acad Orthop Surg. 2006; 14: 126-35.

\section{AP9-10}

\section{A study of complications of continuous femoral block in total} knee replacement

E. Antonopoulou, K. Papaioannou, K. Tataraki, D. Papadopoulos, V. Karamoulas

Anaesthesiology, General Hospital of Xanthi, Xanthi, Greece

Background and Goal of Study: To study the incidence and the severity of side-effects of continuous femoral block as part of a polymodal analgesia in total knee replacement.

Materials and Methods: We studied 150 women, aged 65-89 yrs, ASA I-III, scheduled for TKR. All patients had spinal anesthesia for the operation. In the PACU after sensory and motor blockade had warn off a femoral block was performed with a nerve stimulator using an aseptic technique. A bolus dose of $20 \mathrm{ml}$ of levobupivacaine $5 \mathrm{mg} / \mathrm{ml}$ was given in increments of $5 \mathrm{mls}$ following a negative aspiration and a continuous infusion of $4-5 \mathrm{ml} / \mathrm{h}$ levobupivacaine $0,125 \%$ was set. The infusion lasted 48 hours. Vital signs were recorded every 4 hours and the patients were examined for signs of local pain, motor and sensory blockade, local haematoma infection, inadvertent arterial puncture, cardiovascular or neurologic toxicity.

Results and Discussions: Despite the fact that continuous femoral block seems to be a very satisfactory technique we observed the following complications.

Failure to perform the block in 1 patient. There was accidental removal of the catheter during its fixation in 3 patients and in other 3 patients in the ward.

The procedure was stopped in 1 patient after the appearance of blood during aspiration of he last $5 \mathrm{mls}$ of the bolus injection with no other complications.

In one patient there was a severe complication. After removing of the catheter there was neuropraxia of the femoral nerve which lasted six weeks. No other complications were observed.

Conclusion(s): The most severe complication seems to be neuroapraxia There was no pain during the injection, the block succeeded after 3 attempts although in retrospect there was excessive resistance during the injection. The incidence happened during the learning curve of the procedure.

Accidental removal of the catheter was the commonest problem and we changed the mode of fixation. Overall continuous femoral block seems to be a safe and effective technique in providing analgesia in TKR.

\section{AP10-1}

\section{Plasma bupivacaine levels during continuous iliac crest wound infusion}

V. Espinal, J. Hayes, B. Bissonnette

Anesthesia, The Hospital for Sick Children, Toronto, Canada

Background and Goal of Study: Infusion of local anesthetic can be used for postoperative pain control; however, absorption into systemic circulation may result in serious toxicity. Plasma levels of bupivacaine infused through an iliac crest graft wound catheter for pain management have not been reported. The purpose of this study is to measure plasma bupivacaine levels during an iliac crest infusion over 48 hours.

Materials and Methods: 36 patients requiring an iliac crest bone graft (ICBG) for alveolar cleft repair or Lefort I osteotomy will be studied. Patients will receive a bolus of $0.1 \mathrm{ml} / \mathrm{kg}$ of plain bupivacaine $0.25 \%$ followed by an infusion at $0.1 \mathrm{ml} / \mathrm{kg} / \mathrm{hour}$ for 48 hours. Bupivacaine assays will be performed prior to and $0.5,1,6,12$ and 48 hours after the initial bolus. Bupivacaine levels will be measured with an assay developed on the 4000 QTrap Tandem Mass Spectrometer (Applied Biosystems/MDS Sciex).

Results and Discussions: Plasma bupivacaine levels were undetectable until 6 hours after the bolus and start of infusion in the two patients studied to date. After 48 hours, levels were lower than the toxic threshold of $4 \mathrm{mg} / \mathrm{L}$ (1).

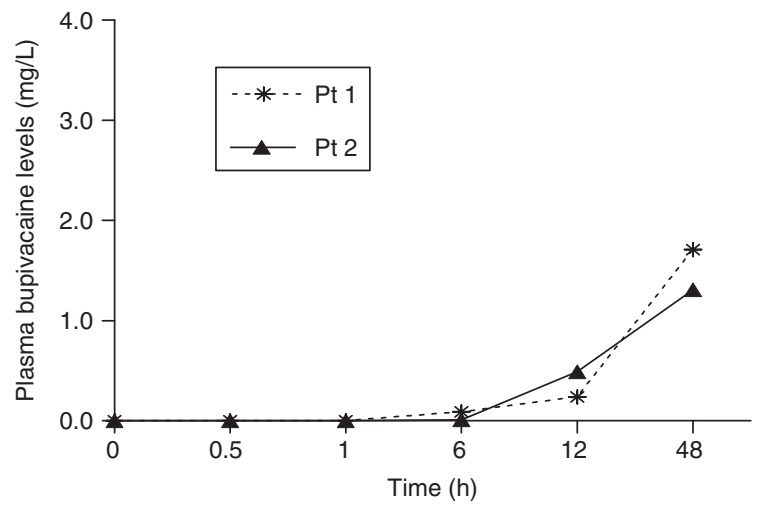

Conclusion: In the patients studied to date, a bolus followed by an infusion of bupivacaine $0.25 \%$ plain through an iliac crest graft wound catheter for 48 hours resulted in plasma concentrations below toxic levels.

Reference:

1 Gunter JB. Pediatr Drugs 2002; 4: 649-672.

\section{AP10-2}

Intraoperative low dose of ketamine improves the transition from epidural to systemic analgesia after major abdominal surgery

P. Janin, S. Lauwick, A. Kaba, M. Lamy, J. Joris

Anesthesia and Intensive Care Medicine, CHU Liège, Liège, Belgium

Background and Goal of Study: The interruption of epidural analgesia after surgery coincides frequently with the appearance of moderate to severe pain. We investigated whether intraoperative ketamine (KET) smoothened the transition from epidural to systemic analgesia after major abdominal surgery. Materials and Methods: With IRB approval and informed consent, 40 patients scheduled for major abdominal surgery were included in this randomized double blind placebo-controlled study. General anesthesia (sevoflurane in $\mathrm{O}_{2}$ :air) combined with thoracic (T9-T10) epidural anesthesia was used in all patients. After the induction of anesthesia patients were randomly allocated in two groups ( $\mathrm{n}=20$ in each group): $0.5 \mathrm{mg} / \mathrm{kg}$ then $0.25 \mathrm{mg} / \mathrm{kg} / \mathrm{h} \mathrm{KET}$ or the same volume of saline (SAL). Postoperative analgesia was provided with a standardized patient-controlled epidural analgesia (PCEA) with ropivacaine $0.2 \%$ for three days postoperatively. Then PCEA was stopped and replaced by piritramide (a synthetic opioid) PCA. The following parameters were measured during the four first days postoperatively: pain scores (100 mm VAS) at rest, during mobilisation, and when coughing, consumption of ropivacaine solution and of piritramide. Data (mean \pm SD) were analyzed by ANOVA; $P<0.05=$ statistically significant.

Results and Discussions: Patient data and pain scores at rest were similar in the two groups. Pain scores when coughing $(P<0.01)$ and ropivacaine consumption ( $398 \pm 66$ vs $466 \pm 115 \mathrm{~mL}$; $\mathrm{P}<0.001)$ were significantly reduced in the KET group during PCEA. After PCEA interruption pain scores during activity $(P=0.001)$ and piritramide consumption during the first $24 \mathrm{~h}$ were signicantly lower in the KET group $(P=0.03$; table).

\begin{tabular}{llllll}
\hline Time & $+1 \mathrm{~h}$ & $+2 \mathrm{~h}$ & $+4 \mathrm{~h}$ & $+8 \mathrm{~h}$ & $+24 \mathrm{~h}$ \\
\hline SAL & $2 \pm 2$ & $5 \pm 3$ & $9 \pm 5$ & $16 \pm 10$ & $37 \pm 25$ \\
KET & $1 \pm 1$ & $4 \pm 4$ & $7 \pm 6$ & $12 \pm 10$ & $23 \pm 17$ \\
\hline
\end{tabular}

Conclusion(s): Intraoperative small dose of ketamine significantly improves postoperative analgesia provided by ropivacaine thoracic PCEA and the transition from epidural to systemic analgesia.

\section{AP10-3}

\section{Calcium chloride prolongs the effects of lidocaine in rat sciatic nerve}

Y.C. Hung, C.C. Chen, G.K. Wang, P. Gerner

Department of Anesthesiology, Mackay Memorial Hospital, Taipei, Taiwan

Background and Goal of Study: The effect of the divalent calcium ion coadministered with local anesthetics is unknown. We hypothesized that calcium chloride $\left(\mathrm{CaCl}_{2}\right)$ could be used as an adjuvant to prolong and intensify the block by lidocaine. We therefore investigated its effect on block and assessed potential histopathological damage in rat sciatic nerves, and the mechanism of the lidocaine-calcium interaction in cultured $\mathrm{GH}_{3}$ cells. 
Materials and Methods: Rats weighed 250-300 grams were anesthetized, and the sciatic nerves were exposed by lateral incision. $0.2 \mathrm{~mL}$ of the test dose was injected with $1 \%$ lidocaine which was used alone or coadministered with $0 \%$ (no $\mathrm{CaCl}_{2}$ added), $0.625 \%, 1.25 \%, 2.5 \%$, or $5 \% \mathrm{CaCl}_{2}$, respectively ( $n=8-10$ per group). The behavioral test of proprioception, motor function, and nocifensive reaction were used to assess the complete blockade time and complete recovery time. The data were analyzed by ANOVA using the F-test. For pathological evaluation, the nerves were taken out 7 days after receiving the test dose ( $n=6 /$ group). To elucidate the lidocaine-calcium interaction, voltage-dependent inactivation curves were determined in cultured rat $\mathrm{GH}_{3}$ cells expressing neuronal sodium channels.

Results and Discussions: The addition of $\mathrm{CaCl}_{2}$ prolonged the duration of blockade by lidocaine that was significantly longer for $5 \%$ than $0 \% \mathrm{CaCl}_{2}$. Histologically, rat sciatic nerves treated with $1 \%$ lidocaine coadministered with $5 \% \mathrm{CaCl}_{2}$ showed activated Schwann cells, some endoneurial edema and the epineurium thickened. However, the voltage-dependence of the steadystate $\mathrm{Na}^{+}$channel inactivation in the presence of $300 \mu \mathrm{M}$ lidocaine was shifted rightward by $10 \mathrm{mM} \mathrm{CaCl}_{2}$, indicating that the $\mathrm{CaCl}_{2}$ reduces the potency of lidocaine toward the inactivated $\mathrm{Na}^{+}$channel.

Conclusion(s): $\mathrm{CaCl}_{2}$ coadministered with lidocaine prolongs the duration of sciatic nerve blockade in rats with major histopathological changes. It appears not to be useful as an adjuvant for peripheral nerve blockade because of neurotoxicity. The mechanism is independent of the action of $\mathrm{CaCl}_{2}$ on the $\mathrm{Na}^{+}$channel.

\section{AP10-4}

Involvement of glutamate in bupivacaine-induced myotoxicity in rats

C. Cherng, C. Wong

Anesthesiology, Tri-Service General Hospital, Taipei, Taiwan

Background and Goal: Local anesthetics may cause reversible skeletal muscle injury and bupivacaine produce the most degree of injury (1). Glutamate is widely existed in the skeletal muscle and has its metabolic function (2). Using microdialysis, this study was to examine whether bupivacaine-induced myotoxic effect involves intramuscular glutamate release.

Materials and Methods: Twenty four male Wistar rats were divided into 4 groups (control, $0.25 \%, 0.5 \%$, and $1 \%$ bupivacaine), 6 rats in each. A microdialysis tube was implanted into the right tibialis anterior (RTA) muscle of each rat. After two hours of equilibrium of the microdialysis system, $0.2 \mathrm{~mL}$ of normal saline or $0.25 \%, 0.5 \%$, and $1 \%$ bupivacaine was injected into the RTA muscle where the microdialysis tube implanted, then the samples were collected every 30 min for 4 hours. Glutamate was measured by HPLC. Three days later, the RTA muscles were dissected at the injection sites and were examined for the severity of muscle damage.(score 0 to 3 : no damage to severe myonecrosis). Results: Bupivacaine produced a significant increase of glutamate release in the muscle and the increase was in a dose-dependent manner. The histological findings also showed a dose-dependently injury of muscular fibers caused by bupivacaine. (score, N/S:0, 0.25\% bupivacaine:1.2 $\pm 0.6,0.5 \%$ bupivacaine: $1.8 \pm 0.7,1 \%$ bupivacaine: $2.4 \pm 0.8, \mathrm{P}<0.05$ ).

Conclusion: The bupivacaine-induced myotoxicity could be mediated with glutamate release in the muscle.

References:

1 Zink W, Graf BM. Reg Anesth Pain Med 2004; 29: 333-340.

2 Rutten EP, Engelen MP, Schols AM, et al. Curr Opin Clin Nutr Metab Care 2005; 8: 41-51.

\section{AP10-5}

Effect of melatonin administered by intrathecal injection - an experimental study in the rat

M. Chanimov, Z. Chaitov, S. Stoleru, Z. Fanus, M. Bahar

Department of Anesthesiology, Assaf Harofeh Medical Center, Zerifin, Israel

Background and Goal of Study: Recently, a species-dependent distribution of melatonin-binding sites was found in the dorsal and ventral horn of the spinal cord $(1,2)$. We sought to examine the hypothesis that intrathecally injected melatonin might produce reversible spinal anesthesia comparable to that produced by local anesthesia.

Materials and Methods: Seventy two male Wistar strain rats were divided into 6 groups of 12 animals each. A well-known spinal cannulation technique was used (3). Group 1 received $0.02 \mathrm{~mL}$ intrathecal melatonin $0.1 \%$; Group 2 received $0.01 \mathrm{~mL}$ melatonin $0.1 \%$ plus $0.01 \mathrm{~mL}$ fentanyl $0.005 \%$; Group 3 received $0.02 \mathrm{~mL}$ melatonin $0.1 \%$ intravenously; Group 4 received $0.02 \mathrm{~mL}$ lidocaine $2 \%$ intrathecally; Group 5 received $0.02 \mathrm{~mL}$ fentanyl $0.005 \%$ intrathecally and Group 6, a control group, received no intrathecal or intravenous test substances. The hemodynamic parameters were recorded throughout the experiment by means of direct arteriovenous cannulation and via a Gould
PE50 transducer. The presence of motor and sensory block of the abdominal wall was determined by the animals' response to lower laparotomy. Results and Discussions: We have demonstrated in a rat model that the intrathecal injection of melatonin plus fentanyl can achieve anesthesia sufficient for a laparotomy, comparable to that produced by intrathecal lidocaine. The lack of sensory response over the lower limbs and trunk lasted for $20 \pm 3.6$ min. The respiratory and hemodynamic functions were minimally changed. Conclusions: Intrathecal melatonin enhanced the antinoceptive effect of fentanyl, indicating that melatonin acts as a neuromodulator in the spinal cord producing spinally-mediated antinoception.

References:

1 Sohn PK, Lansmann T, Berger E, et al. J Pineal Res 2003; 35: 24-31.

2 Genovese T, Mazon E, Muia C, et al. J Pineal Res 2005; 38: 198-208.

3 Bahar M, Rosen M, Vickers MD. Br J Anaesth 1983; 55: 541-547.

\section{AP10-6}

\section{The influence of General Anaesthesia on plasma pharmacokinetics of ropivacaine}

M. Ratajczak, J.P. Estebe, F. Chevanne, M. Laurentie, G. Dollo

Laboratoire de Pharmacie Galénique, Biopharmacie et Pharmacie Clinique, Université de Rennes 1, Rennes, France

Background and Goals: The effect of general anaesthesia (GA) on the pharmacokinetics of local anaesthetics administered epidurally remains unclear. We evaluated the influence of isoflurane on plasma pharmacokinetics of epidural ropivacaine with $(R+E)$ and without $(R)$ epinephrine in a sheep model. Material and Methods: The epidural catheter was inserted and secured (2 weeks) before experiment. Animals received epidural $\mathrm{R}(50 \mathrm{mg} / 15 \mathrm{ml})+\mathrm{E}$ $(5 \mu \mathrm{g} / \mathrm{ml})$ and $\mathrm{R}$ under $\mathrm{GA}$ (intubation, ventilation with isoflurane: $1-2 \%$ in oxygen/air $50 \% / 50 \%$ ) and epidural $R+E$ and $R$ without GA. Then an intravenous administration of ropivacaine was performed to study the systemic absorption of ropivacaine by deconvolution method. For each animal, the five administrations were performed with one week intervals. A non-compartmental analysis was performed for individual concentration data sets $(n=3)$.

ANOVA test was used to compare different administrations.

Results: Data (Mean $\pm \mathrm{SD}$ ) are presented in the table.

\begin{tabular}{|c|c|c|c|c|}
\hline Mean \pm SD & $\mathrm{R} / \mathrm{GA}+$ & R/GA - & $\mathrm{R}+\mathrm{E} / \mathrm{GA}+$ & $\mathrm{R}+\mathrm{E} / \mathrm{GA}-$ \\
\hline $\begin{array}{l}\text { AUC last } \\
\text { (min. ng/ml) }\end{array}$ & $29861 \pm 11923$ & $20461 \pm 5514$ & $30055 \pm 21414$ & $26119 \pm 10578$ \\
\hline Clast (ng/ml) & $109 \pm 42$ & $89 \pm 15$ & $110 \pm 62$ & $125 \pm 53$ \\
\hline Cmax (ng/ml) & $309 \pm 196$ & $182 \pm 40$ & $282 \pm 216$ & $200 \pm 51$ \\
\hline $\mathrm{T} 1 / 2 \beta$ (min) & $151 \pm 61$ & $194 \pm 50$ & $204 \pm 249$ & $312 \pm 16$ \\
\hline $\operatorname{Tmax}(\min )$ & $18 \pm 11$ & $12 \pm 4$ & $25 \pm 14$ & $18 \pm 11$ \\
\hline $\mathrm{Cl} / \mathrm{F}(\mathrm{I} / \mathrm{min})$ & $0.9 \pm 0.4$ & $1.0 \pm 0.1$ & $0.9 \pm 0.7$ & $0.6 \pm 0.2$ \\
\hline
\end{tabular}

Mean systemic absorption time plots of ropivacaine are presented in the figure.

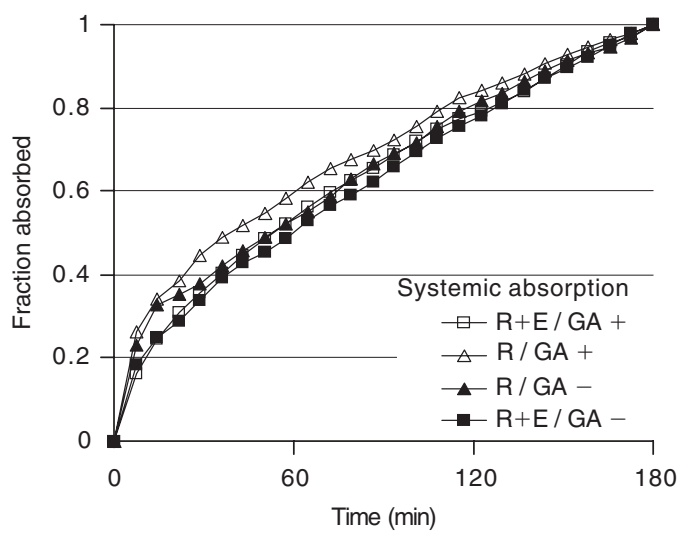

Conclusions: No significant influence of GA on plasma pharmacokinetics of epidurally administrated ropivacaine either with or without epinephrine was evidenced. The systemic absorption of epidural ropivacaine was not modified neither by epinephrine nor by GA.

\section{AP10-7}

Neurotoxic change neurons after intrathecal introduction local anesthetics in rats

K. Repin, N. Davidova

Anesthesiology, The Ural Medical Academy, Ekaterinburg, Russian Federation 
Background and Goal of Study: Neurological picture after using lidocaine intrathecally made a concern about the toxicity of local anaesthetics. Recent researches demonstrate that lidocaine is more connected with neurotoxicity than other local anaesthetics.

Method: In our research we tried to compare the neurotoxical effect of different local anaesthetics in rats. As a criteria of neurotoxicity the structural changes of spinal cord motoneurones in light and electronic microscopy have been chosen. After the permission of our ethical committee 21 male rats were included in this research. They were divided in three groups of 7 . $15 \mathrm{mg} 2 \%, 5 \%, 10 \%$ of lidocaine, $0,5 \mathrm{ml}$ of normal solution, $3 \mathrm{mg} 0,5 \%$ and $0,75 \%$ of bupivacaine, $4,5 \mathrm{mg} 0,5 \%$ ropivacaine were injected intrathecally. The lumbar spinal cord with both anterior and posterior roots and dorsal ganglion were excised for light and electron microscopic examination on the following day.

Results: The histological abnormalities were observed histological in the posterior roots and column of spinal cord in rats that received $10 \%$ lidocaine. These lesions were characterized by destruction motoneurons. In group with $5 \%$ lidocaine hyperchromatolis singular neurons with destruction mitochondria and endoplasmatic net were founded. Other groups did not illustrate any histological impairment neurons. Insignificant changes of myelonic neurofibers were founded excluding normal solution group.

Conclusion: The parts of spinal cord after intrathecal $10 \%$ and $5 \%$ lidocaine were presented as intracellular destructive changes of motoneurones. Our results suggest that $5 \%$ lidocaine administered intrathecally may cause damage to neurons in rats. Other intrathecal anaesthetics including $2 \%$ lidocaine did not show any neurotoxic effects in rats.

\section{AP10-8}

Mitigation of lidocaine-induced neurotoxicity - a comparison of compartmented and dissociated sensory neuron cultures

I. Haller, H.P. Colvin, P. Gerner, L. Klimaschewski, P. Lirk

Department of Anesthesiology \& Critical Care Medicine, Medical University of Innsbruck, Innsbruck, Austria

Background and Goal of Study: All currently used local anesthetics are, to some extent, neurotoxic. So far, toxicity studies were mainly carried out in dissociated neuron cultures, immersing both axon and soma in local anesthetic. This approach, however, is not fully satisfying, since local anesthetic is applied to the axon alone for peripheral nerve block in the in vivo situation. Therefore, we investigated lidocaine neurotoxicity both in compartmental and dissociated sensory neuron cultures, focussing on possible ways of neuroprotection.

Materials and Methods: Lidocaine $(40 \mathrm{mM} / \sim 1 \%)$ was added to the central and/or the peripheral compartment of three-chambered dishes as well as to dissociated cultures. Additionally, we co-applied several inhibitors, such as inhibitor of caspase or Rho, to determine their effect both locally at the axon, and the neuron's soma. Neuronal survival, axonal length and morphology were used as outcome measures.

Results and Discussion: In dissociated cultures, caspase-inhibition had a significant neuroprotective effect. Contrary, in compartmented cultures controls showed an increase of maximal axonal distance from $100 \%$ at day 7 to $133 \pm 5 \%$ at day 8 . Co-incubation of compartmented cultures with $40 \mathrm{mM}$ lidocaine in the peripheral compartment and the inselective caspase-inhibitor z-vad-fmk at $10 \mu \mathrm{M}$ in the peripheral compartment $(n=3)$ showed that peripheral inhibition of caspase activation had no significant effect on the axonotoxicity induced by lidocaine. The maximal axonal distance of these cultures was $77 \pm 8 \%$ at day 8 , and the cultures treated with $40 \mathrm{mM}$ lidocaine alone in the peripheral chamber had a maximal axonal distance $60 \pm 9 \%$. Moreover, simultaneous incubation of cultures with $40 \mathrm{mM}$ lidocaine in the peripheral and $10 \mu \mathrm{M} z$-vad-fmk in the central compartment showed a slight, but not significant protective effect on axon outgrowth (maximal axonal distance $72 \pm 9 \%$ ).

Conclusion: We conclude that processes localized to the axon, such as selective axonal degeneration, which do not involve the neuronal soma or caspase activation, may mediate lidocaine-induced axonal neurotoxicity. Cell death after local anesthetic application is caused by different processes depending upon whether the entire cell, or only the axon, is incubated. The compartmental culture model is potentially useful to determine in detail mechanisms involved in local anesthetic-induced neurotoxicity in vitro.

\section{AP10-9}

\section{Local Anesthetic effects on human neutrophil morphology and activation}

A. Ploppa, M.D. Smith, R.T. Kiefer, K.E. Unertl, M.E. Durieux

Department of Anesthesia and Intensive Care Medicine, University Hospital Tuebingen, Germany, Tuebingen, Germany
Background and Goal of the Study: Local Anesthetics (LA) inhibit neutrophil chemotaxis and accumulation in inflamed tissues, but the underlying mechanisms are not well understood. Neurons have been shown to change morphology after LA exposure, and we hypothesized that changes in cell morphology might also occur in neutrophils. If so, these might affect their motility and be relevant to the observed decrease in chemotaxis and accumulation of leukocytes in inflamed tissues. We therefore investigated the effects of LA on changes in leukocyte morphology and activation in a whole blood model.

Materials and Methods: After IRB approval and informed consent was obtained, whole blood samples were obtained from healthy volunteers and incubated for 1 or 3 hours with different concentrations (10-7 to 10-4 M) of either lidocaine, ropivacaine, QX314, or saline as control. Blood was primed with platelet activating factor (10-5 M) and activated with fMLP (10-5 M) for 15 min each. Thereafter, cells were staining with a monoclonal antibody against CD11b. After lysis of erythrocytes, samples were analyzed by flow cytometry. Changes in cell morphology were quantified as changes in Side Scatter values. Results and Discussion: QX314, an uncharged LA that is not able to penetrate the cell wall, did not alter cell morphology and CD11b-expression. Ropivacaine, also did not affect CD11b-expression, however a significant change in cell morphology was observed at the highest concentration $(p<0.05)$. Lidocaine induced the most pronounced effects on cell morphology. In addition, lidocaine $(10-4 \mathrm{M}, \mathrm{p}<0.05)$ significantly inhibited priming and activation of CD11b-expression after 1 and $3 \mathrm{~h}$. Changes in cell morphology were observed even at the lower concentration of lidocaine (10-4 and 10-5 M, p 0.05).

Conclusions: Our results suggest that CD11b-expression can be primed and that priming of CD11b-expression can be significantly attenuated by LA. Second, leukocyte morphology is more sensitive to LA than neutrophil activation. The inhibition of CD11b-expression and changes in cell morphology might contribute to the decreased accumulation in inflamed tissues under LA exposure.

Reference:

Kasaba T. Onizuka S. Takasaki M. AnesthAnalg 2003; 97(1): 85-90.

\section{AP10-10}

\section{Muscle regeneration following local anesthetic myotoxic insult. Morphometric evaluation}

E. Amaniti, D. Kapoukranidou, I. Kalpidis, D. Vasilakos, M. Albani Laboratory of Experimental Physiology, Anesthesiology Department, Aristotle University of Thessaloniki, Thessaloniki, Greece

Background and Goal of Study: Local anesthetic myotoxicity is a frequent complication following muscle infiltration. Although considered reversible, cases of permanent damage have been reported. Aim of the present study was the microscopic evaluation of muscle regeneration after bupivacaine muscle infiltration in rats.

Materials and Methods: Wistar rats underwent muscle injection with $100 \mu \mathrm{l}$ of bupivacaine $0.5 \%$ (B05 group). The left tibialis anterior muscle of each rat was injected while the controlateral muscle was used as control (Control group). One month later, rats were sacrificed and the muscles were dissected, frozen and cut into $10 \mu$ thick sections. Afterwards, they were stained using acid ATPase and examined using light microscope with a computer imaging system for morphometric analysis. Samples were evaluated regarding type of muscle fibers, mean cross-section fiber area and fiber perimeter. Statistical analysis was conducted using t-test. Level of significance was set at $\mathrm{p}<0.05$.

Results and Discussions: Groups were comparable regarding muscle fiber types percentages $(p>0.05)$. However, regenerated muscle fibers, except for type I fibers, had smaller cross-sectional fiber area and perimeter $(p<0.05)$.

\begin{tabular}{|c|c|c|c|}
\hline & \multicolumn{3}{|c|}{ Fiber area: Mean \pm S.D. $\left(\mu \mathrm{m}^{2}\right)$} \\
\hline & Type I & Type Ila & Type Illb \\
\hline B05 & $675.25 \pm 163.14$ & $860.95 \pm 192.49$ & $983.98 \pm 307.27$ \\
\hline \multirow[t]{3}{*}{ Control } & $575.58 \pm 158.89$ & $814.67 \pm 210.42$ & $1317.66 \pm 451.10$ \\
\hline & \multicolumn{2}{|c|}{ Fiber perimeter: Mean \pm S.D. $(\mu \mathrm{m})$} & \\
\hline & Type I & Type Ila & Type Ilb \\
\hline B05 & $103.48 \pm 12.60$ & $118.19 \pm 14.04$ & $127.11 \pm 20.96$ \\
\hline Control & $96.56 \pm 13.97$ & $116.00 \pm 15.95$ & $149.95 \pm 27.90$ \\
\hline
\end{tabular}

Conclusion(s): Although seemed to fully recover after a myotoxic insult, regenerated fibers of types $\mathrm{Ila}$ and Ilb are characterized by smaller crosssectional area and diameter, corresponding to smaller size. The latter could possibly lead to impaired function.

Reference:

Zink W. Anesth Analg 2003; 97: 1173-1179. 


\section{AP10-11}

\section{Reversal of central nervous system and cardiac toxicity following local anesthetic intoxication by lipid emulsion application}

R.J. Litz, T. Roessel, A.R. Heller, S. Stehr, T. Koch

Anaesthesiology, University of Technology, Dresden, Germany

Background and Goal of Study: Lipid emulsion infusion has been proposed as the last treatment option for local anesthetic (LA)-induced systemic toxicity after failure of established resuscitation measures. The successful use of lipid emulsion following bupivacaine-induced cardiac arrest has been demonstrated in animal models. Recently, two case reports were published reporting on successful resuscitation of patients after ropivacaine- and bupivacainerelated cardiac arrest, respectively. We report of a case of successful treatment of systemic local anesthetic intoxication following brachial plexus block.

Materials and Methods: A 91 year old male $(57 \mathrm{~kg}, 156 \mathrm{~cm}$, ASA III) received vertical infraclavicular brachial plexus block for olecranon surgery. Fifteen min after application of $30 \mathrm{ml}$ of mepivacaine $1 \%$ the block was supplemented by administration of $10 \mathrm{ml}$ of prilocaine $1 \%$. Within the next five min the patient complained of nausea and dizziness and became unresponsive to verbal commands. Heart rate increased from 76 to $92 \mathrm{bpm}$ and supraventricular extrasystoles with intermittend bigeminus appeared. Blood pressure increased from $160 / 70$ to $190 / 90 \mathrm{mmHg}$.

Results and Discussions: Local anesthetic toxicity was suspected and a bolus injection of $1 \mathrm{~mL} / \mathrm{kg}$ Intralipid $20 \%{ }^{\circledR}$ was intravenously applicated and repeated after three min (total of $100 \mathrm{~mL}$ ). A continuous Intralipid ${ }^{\circledR}$ infusion was started at a rate of $14 \mathrm{~mL} / \mathrm{min}\left(0.25 \mathrm{~mL} / \mathrm{kg}^{*} \mathrm{~min}\right)$. The patient regained consciousness within 5 min following the first lipid bolus injection. Because extrasystoles were still apparent lipid infusion was carried on up to a total dose of $200 \mathrm{~mL}$ until extrasystoles disappeared. Plasma concentrations of mepivacaine/ prilocaine at the start of lipid infusion and 20 and 40 min later were 4.08/0.92, 2.30/0.35 and 1.73/0.24 $\mu \mathrm{g} / \mathrm{ml}$.

Conclusion(s): This case report demonstrates the successful use of lipid emulsion infusion with the isochronal decrease of plasma concentrations of mepivacaine and prilocaine in a patient at the onset of CNS and cardiac toxicity. Our findings may support the theory of a "lipid sink" effect as a causative mechanism of action in the treatment of LA toxicity.

\section{Pharmacology}

\section{AP1-1}

\section{Investigation of the antibacterial effect of ketamine}

Ü. Buyukkocak, J.S. Gocmen, O. Caglayan

Anaesthesiology and Reanimation, Kirikkale University School of Medicine, Kirikkale, Turkey

Background: Anaesthetic agents have activity on not only excitable cells affecting cellular membranes but all cells as well. Antibacterial activity of local anaesthetics especially lidocaine have been shown previously (1). In this study, the antibacterial effect of ketamine a general anaesthetic agent was investigated.

Material and Methods: The antibacterial effect of ketamine was studied using six different strains of bacteria (S. aureus, S. epidermidis, E. faecalis, S. pyogenes, $P$. aeruginosa and $E$. coli) with disc diffusion method. Discs containing $500,250,125$ and $62.5 \mu \mathrm{g}$ of ketamine were prepared. Ketamine discs were placed in the plates into which bacteria were inoculated. Ciprofloxacin discs (CIP, oxoid) were used as controls for checking of the methodology. Results of different drug concentrations were compared within the ketamine group and with the results obtained from the CIP discs.

Results: CIP affected all bacteria as expected. Ketamine with the doses of $250 \mu \mathrm{g}$ and $500 \mu \mathrm{g}$ which was more prominent inhibited all bacteria resembling CIP. Ketamine with the doses of $125 \mu \mathrm{g}$ had also activity on all bacteria with the exception of $E$. coli. No inhibition was evident in discs containing $62.5 \mu \mathrm{g}$ of ketamine.

Conclusions: Ketamine exhibited antibacterial activity with higher doses which were very close to the doses used to induce anaesthesia in experimental animal studies. When the dosages used in this study are considered, rather than studies concerning humans, the results will be meaningful in animal studies in which ketamine is used for anaesthesia (2). Therefore antibacterial activity of ketamine should be kept in mind in experimental animal studies. References:

1 Parr AM, Zoutman DE, Davidson JS. Ann Plast Surg 1999; 43: 239-245.

2 Fujimoto T, Nishiyama T, Hanaoka K. Anesth Analg 2005; 101: 1054-1059.

\section{AP1-2}

High performance liquid chromatography (HPLC) determination of a ketamine and remifentanil mixture

M.A. Fuentes-Pradera, F. García-Torrado, D. Villegas-Duque, F. Soler-Cantos, J.L. López-Romero

Anesthesiology, University Hospitals Virgen Del Rocio, Seville, Spain

Background and Goals: The administration of small doses of ketamine during a remifentanil based anaesthesia in different surgical processes, seems to diminish the need for postoperative opiate. Our target is to check the noninteraction between the two compounds, if they are simultaneously and continuously supplied with an only infusion pump.

Materials and Methods: An HPLC system Waters 2695 fitted with a Photodiode Array Detector (PDA) and an Empower Software, was used. The chromatography of both compounds (remifentanil hydrochloride and ketamine hydrochloride) was carried out in reversed phase with a Nova-Pak column. The mobile phase was prepared with acetonitrile $(180 \mathrm{~mL})$, methanol $(120 \mathrm{~mL})$, phosphate buffer $50 \mathrm{mM}(48 \mathrm{~mL})$, and water $(352 \mathrm{~mL}, \mathrm{pH} 3)$. To prepare the problem solutions, remifentanil hydrochloride $(1.5 \mathrm{mg})$ and ketamine hydrochloride $(1.0 \mathrm{mg})$ were respectively dissolved into mobile phase $(10 \mathrm{~mL})$. Samples of $10 \mu \mathrm{L}$ of each solution were introduced into the HPLC system, and the flow rate of the mobile phase was kept, in both cases, at $1 \mathrm{mLmin}^{-1}$. Under these conditions the chromatograms 1 (remifentanil hydrochloride) and 2 (ketamine hydrochloride) were obtained. The two problem solutions were mixed at room temperature and the chromatographies were repeated under the same conditions after 3 (chromatogram 3) and 24 (chromatogram 4) hours. Results: Chromatogram number 1; remifentanil hydrochloride retention time $2.5 \mathrm{~min}$. Chromatogram number 2: ketamine hydrochloride retention time $1.7 \mathrm{~min}$. The chromatograms 3 and 4 show no changes in the retention times of either compound and no formation of any new product.

Conclusions: No reaction has been observed between the two studied compounds, under the described conditions. Consequently remifentanil hydrochloride and ketamine hydrochloride can be simultaneously administered, in a safe way, using a single infusion pump. This fact facilitates the intraoperatory management.

\section{AP1-3}

\section{Determination of the blood-gas partition coefficients for} isoflurane, sevoflurane and desflurane

T. Esper, M. Wehner, H. Rueffert, D. Geier, F. Koenig

Anaesthesiology and Intensive Care Medicine, University Hospital Leipzig, Leipzig, Germany

Background and Goal of Study: The blood-gas partition coefficient is a crucial parameter of pharmacokinetics of volatile anaesthetics. The parameter is given in textbooks (e.g. 1) as a singular value and has been confirmed in the recent year (2). The goal of the study was the determination of the range of the blood-gas partition coefficients in a clinical relevant population.

Materials and Methods: After informed consent $30 \mathrm{ml}$ venous blood was drawn from individuals scheduled for elective surgical procedures. A probe of $10 \mathrm{ml}$ was incubated with isoflurane, sevoflurane and desflurane in $20 \mathrm{ml}$ vial under stirring at $37^{\circ} \mathrm{C}$. For calculation of the blood-gas partition coefficient the concentration of the volatile anaesthetic in the gas phase was determined by headspace gas chromatography on an Agilent HP 6890 set with a GasPro column (Agilent). Each measurement was done threefold. The ethics committee of the Leipzig Medical Faculty approved the protocol.

Results: Results of the blood-gas partition coefficient are shown in table in comparison with the values given in a popular German Anaesthesia textbook.

\begin{tabular}{llllll}
\hline & $\mathrm{n}$ & median & Min-Max & $25^{\text {th }} / 75^{\text {th }}$ percentile & textbook value(1) \\
\hline isoflurane & 37 & 1,44 & $1,14-1,69$ & $1,39-1,52$ & 1,46 \\
sevoflurane & 34 & 0,76 & $0,61-0,89$ & $0,72-0,79$ & 0,69 \\
desflurane & 28 & 0,57 & $0,48-0,68$ & $0,55-0,59$ & 0,42 \\
sum & 99 & & & &
\end{tabular}

Conclusion: For sevoflurane and for desflurane we determined higher bloodgas partition coefficients than reported $(1,3)$. The observed variation of the coefficient may contribute in part to individual recovering from anaesthesia. References:

1 Larsen R. Anästhesie, Urban \& Fischer, 2002, 24.

2 Eger II E1, Saidman LJ. Anesth Analg 2005, 100, 1022.

3 Eger II E1. Suprane ${ }^{\circledR}$ product monograph, Pharmacia, 1994: 19. 


\section{AP1-4}

Reduced propofol sensitivity in $\mathbf{S 2 6 7}$ mutant glycine receptors

J. Ahrens, M. Leuwer, J. Lambert, G. Haeseler

Anaesthesiology, Hannover Medical School, Hannover, Germany

Background and Goal of Study: The aromatic alcohol propofol (2,6 diisopropylphenol) positively modulates GABAA and glycine receptors (1). While molecular determinants for binding of propofol to GABAA receptors have been extensively studied (2), little is known about the binding site of propofol on glycine receptors.

Material and Methods: We investigated the effects of propofol on chloride inward currents via two specific mutations within the $\alpha_{1}$ subunit of the rat glycine receptor ( $\alpha_{1}$ S267I and $\alpha_{1}$ S267M) with standard whole-cell experiments. Glycine receptor $\alpha_{1}$ subunits (wild type or mutants) were heterologously expressed in human embryonic kidney cells.

Results: Both mutants (a) decreased sensitivity to the natural agonist glycine (glycine EC50 12.8 $\pm 2.3 \mu \mathrm{M}$ in wild type (WT), 81.5 $\pm 21.2 \mu \mathrm{M}$ in $\alpha_{1}$ S267। and $122.8 \pm 18.5 \mu \mathrm{M}$ in $\left.\alpha_{1} \mathrm{~S} 267 \mathrm{M}\right)$; (b) decreased sensitivity to propofol with respect to co- activation of an EC20 glycine response (propofol EC50 $4.8 \pm 1.2 \mu \mathrm{M}$ in WT, $36.3 \pm 17.5 \mu \mathrm{M}$ at the $\alpha_{1}$ S267I and $9.5 \pm 3.2 \mu \mathrm{M}$ at the $\alpha_{1}$ $\mathrm{S} 267 \mathrm{M}$ receptor); and (c) abolished direct receptor activation by propofol. Conclusions: These results suggest that the serine residue at the position 267 in the transmembrane domain 2 of the $\alpha_{1}$ glycine receptor is crucial for receptor activation by the natural agonist glycine and by the anaesthetic propofol in the absence of glycine. The fact that the exchange of serine for the bulkier isoleucine had a stronger impact on the co-activating actions of propofol than the exchange for the polar methionine suggests that larger hydrophobic side chains inhibit access to the co-activating binding site.

References:

1 Krasowski MD, Harrison NL Cell Mol Life Sci 1999; 55: 1278-1303.

2 Mihic SJ, Ye Q, Wick MJ et al. Nature 1997; 389: 385-389.

\section{AP1-5}

\section{Interaction of the Ubiquitin Carboxyl-terminal Hydrolase-L1} with the alpha2-adrenergic receptor

C. Schaper, B. Weber, J. Scholz, B. Bein

University Hospital Schleswig-Holstein, Campus Kiel, Anaesthesiology and Intensive Care Medicine, Kiel, Germany

Background and Goal of Study: In contrast to most currently used anaesthetics, $\alpha_{2}$-adrenergic receptor (AR) agonists exert their potent clinical effects via a single type of receptors. In addition, neuroprotective effects of $\alpha_{2}$-AR agonists are mediated via the $\alpha_{2 A}$-AR subtype (1), but the molecular mechanisms underlying these actions are still not elucidated. In this study, we investigated functional effects of the $\alpha_{2}$-AR interaction with the Ubiquitin Carboxyl-terminal Hydrolase-L1 gene (UCHL1), a protein that is involved in Parkinson disease. Materials and Methods: The third intracellular loop of the $\alpha_{2 A}$-AR was used in a two-hybrid system to screen a human brain cDNA for protein-protein interactions. DNA sequences were obtained from positive clones and protein-protein interactions were confirmed by GST pull down assays and co-immunoprecipitation.

Results and Discussions: Two identical clones that were $100 \%$ homologous to the gene encoding UCHL1 were identified. GST pull down assays demonstrated that UCHL1 binds preferentially to the $\alpha_{2 A}-A R$ subtype and only with much less affinity to $\alpha_{2 B}-A R$ and $\alpha_{2 C}-A R$. Co-immunoprecipitation and immunofluorescence results confirmed the specificity of this interaction in vivo.

Conclusion(s): UCHL1 is a key enzyme in the protein degradation pathway. Therefore, the interaction between $\mathrm{UCHL} 1$ and $\alpha_{2 \mathrm{~A}}$-AR may play an important role in trafficking of the receptor and its response to agonist stimulation. Furthermore, mutations in the UCHL1 gene have been associated with Parkinson and other conformational diseases (2). UCHL1 binds preferentially to $\alpha_{2 A}-A R$, the subtype mediating the neuroprotective actions of $\alpha_{2}-A R$ agonists. For this reason, one may speculate that this interaction presents a potential molecular mechanism for the neuroprotective effects of $\alpha_{2}$-AR agonists. References:

1 Ma et al.: Eur J Pharmacol 2004; 502: 87-97.

2 Facheris et al.: Neurosci Lett. 2005; 381:131-134.

\section{AP1-6}

An investigation of possible neurotoxic effects of intrathecal melatonin combined with fentanyl in a rat model

Z. Fanous, S. Stoleru, Z. Haitov, M. Chanimov, M. Bahar

Department of Anesthesiology, Assaf Harofeh Medical Center, Zerifin, Israel Background and Goal of Study: We have demonstrated in a rat model that intrathecal injection of melatonin plus fentanyl intrathecally can achieve anesthesia sufficient for a laparotomy. We sought to establish whether this would produce neurologic damage or neurotoxic injury.

Materials and Methods: Thirty Wistar strain rats with nylon catheter chronically implanted in the lumbar subarachnoid space were divided into 5 groups $(1,2)$. Group 1 received $0.02 \mathrm{~mL}$ intrathecal melatonin $0.1 \%$; Group 2 received $0.01 \mathrm{~mL}$ melatonin $0.1 \%$ plus $0.01 \mathrm{~mL}$ fentanyl $0.005 \%$ intrathecally; Group 3 received $0.02 \mathrm{~mL}$ fentanyl $0.005 \%$ intrathecally; Group 4 received $0.02 \mathrm{~mL}$ lidocaine 2\% intrathecally; Group 5 received $0.02 \mathrm{~mL}$ phenol in water $5 \%$ intrathecally. The neurotoxic effect of melatonin alone or combined with fentanyl injected intrathecally repeatedly on 15 occasions over a period of 1 month was studied. Histopathological examination of the excised spinal cord and paraspinal tissues was carried out.

Results and Discussions: There were no significant differences in histological changes in the neural tissues of the first 4 groups. Group 5 demonstrated the typical neurolytic lesions of phenol when injected intentionally into the subarachnoid space.

Conclusions: No clinical effect or histopathological changes were noted in this rat model when melatonin and fentanyl were injected intrathecally.

References:

1 Bahar M, Rosen M, Vickers MD. Br J Anaesth 1985; 55: 541-547.

2 Yaksh TL, Rudy TA. J Pharmacol Exp Ther 1977; 202: 411-427.

\section{AP1-8}

Neurotoxic effects of intrathecally $\mathrm{MgSO}_{4}$

L. Ozdogan, H. Sastim, T. Ayerden, A. Postaci, B. Dikmen

Anaesthesiology and Reanimation, Numune Education and Research Hospital, Ankara, Turkey

Background and Goal of the Study: $\mathrm{MgSO}_{4}$ is a non competitive antagonist of $\mathrm{N}$ - methyl-D-aspartate receptors, has been investigated for intrathecal anesthetic, analgesic and neuroprotective effects in animal and human studies $(1,2)$. There is inadequate data concerning the intrathecal neurotoxicity of the drug. In this study we aimed to investigate the neurotoxic effects of intrathecally $\mathrm{MgSO}_{4}$

Material and Methods: After ethics committee approval 24 male Spraque Dawley rats weighing $250-300 \mathrm{~g}$ were randomly divided into 3 groups. Following intraperitoneal anaesthesia with ketamine $\mathrm{HCl}$ and $\mathrm{Xylasin} \mathrm{HCl}$ rats in group II received $0.02 \mathrm{ml} 15 \% \mathrm{MgSO}_{4}$ on day 7 as a sole injection; in group I received $0.02 \mathrm{ml} 0.9 \%$ saline and group III received $0.02 \mathrm{ml} 15 \% \mathrm{MgSO}_{4}$ once a day for 7 days intrathecally at the lumbar region. Rats observed for clinical neurotoxicity throughout the study period were sacrificed at day 8 and medulla spinalis sections were assessed by electron microscopy. Data were analyzed by Kruskal-Wallis test.

Results: While no ultrastructural changes were observed in group I (control); there was mitochondrial degeneration in group II (single dose) and significant neurodegeneration in group III (repetitive dose). While histopathological assessment score was significantly higher in groups II and III compared with group I ( $p=0.002$ and $p=0.002$, respectively), it was significantly higher in group III compared with group II $(p=0.008)$.

Conclusion: In our study we observed by electron microscopy that $\mathrm{MgSO}_{4}$, especially after repetitive administrations, causes significant neurodegeneration. Therefore we came to the conclusion that intrathecal $\mathrm{MgSO}_{4}$ can cause neurotoxicity and undesirable effects on clinical practice in humans.

References:

1 Karasawa S. Anaesthesia, 1998; 53: 878-886.

2 Chanimov M. Anaesthesia, 1997; 52: 223-228.

\section{AP1-9}

Chelation of intra neuronal free calcium opposes anaesthetic actions in aged animals

H. El Beheiry, SS. Jahromi, J. El Hayek

Anesthesia \& Pain Management, Toronto Western Hospital, Toronto, Canada

Background and Goal of Study: Our previous reports indicated that ageing potentiation of anaesthetic actions can be opposed by decreasing intra neuronal $\mathrm{Ca}^{2+}$ concentration $\left(\left[\mathrm{Ca}^{2+}\right]_{\mathrm{i}}\right)$ using manoeuvres to block voltagedependent $\mathrm{Ca}^{2+}$ influx ${ }^{1}$. In keeping with this concept, we hypothesize that decreasing $\left[\mathrm{Ca}^{2+}\right]_{\mathrm{i}}$ using intra neuronal $\mathrm{Ca}^{2+}$ chelators can reverse ageinginduced enhancement of anaesthetic actions.

Materials and Methods: First, we determined the effects of intraneuronal $\mathrm{Ca}^{2+}$ chelator, BAPTA-AM $(50 \mu \mathrm{M})$ on anesthetic actions in hippocampal slices taken from young and aged Fischer 344 rats. Anesthetic actions were measured by analyzing the changes in field excitatory postsynaptic potentials (fEPSPs) evoked in hippocampus slices. Slices were incubated either in BAPTA-AM or its vehicle DMSO (0.12\%). Second, we determined the effects of 
intraperitoneal administration of BAPTA-AM and its vehicle DMSO on MAC values for isoflurane as well as isoflurane-induced electroencephalographic (EEG) burst suppression. We also determined latency to righting reflex and eye opening after isoflurane anesthesia. Mann-Whitney test was used for statistical significance.

Results and Discussions: Aged slices incubated and perfused with BAPTAAM showed that isoflurane actions were partially reversed compared to those perfused with DMSO. On the contrary, isoflurane actions were enhanced in young slices perfused with BAPTA-AM.

Intraperitoneal injections of BAPTA-AM enhanced MAC for isoflurane by $28.78 \pm 4.6$ (mean $\pm S D$ ) $\%$ and increased the number of bursts during isoflurane-induced EEG burst suppression in old animals. Such effects of BAPTA-AM were not observed in young animals. The latency to eye opening and righting reflex after administration of isoflurane 2 MAC for 1 hour was not affected by BAPTA-AM injections in old and young animals.

Conclusion(s): The intraneuronal $\mathrm{Ca}^{2+}$ chelator, BAPTA-AM improves synaptic efficiency and plasticity in aged neurons ${ }^{2}$. Such effect might have attenuated the aging-induced decrement in the MAC values for isoflurane. References:

1 Ouanounou A, Carlen PL, El Beheiry H. Br. J. Pharmacol. 1998; 123 :1075-1082.

2 Tonkikh A, Janus C, El Beheiry H, et al. Exp. Neurol. 2006; 197: 291-300.

\section{AP1-10}

Local anesthetic effects on intracellular release of reactive oxygen species in human neutrophils

A. Ploppa, MD. Smith, RT. Kiefer, KE. Unertl, ME. Durieux

Department of Anesthesia and Intensive Care Medicine, University Hospital Tuebingen, Tuebingen, Germany

Background and Goal of the Study: Anti-inflammatory effects of Local Anesthetics (LA) are well documented. LA inhibit extracellular oxygen release in isolated neutrophils; the underlying mechanism appears to be an inhibition of leukocyte priming. Since the intracellular generation of oxygen species is a central part of the host defense, its inhibition by LA could potentially be detrimental. We therefore investigated the effects of LA on intracellular oxygen release. To avoid the cellular activation associated with neutrophil isolation, we used a whole blood model.

Materials and Methods: After IRB approval and informed consent was obtained, whole blood samples were collected from healthy volunteers and incubated for 1 or 3 hours with different concentrations $\left(10^{-7}\right.$ to $\left.10^{-4} \mathrm{M}\right)$ of either lidocaine, ropivacaine, QX314 (an uncharged LA unable to penetrate the cell wall), or saline as control. Dihydroethidium was added to quantify oxidative burst. Samples were primed with platelet activating factor $\left(10^{-5} \mathrm{M}\right)$ and activated with fMLP $\left(10^{-5} \mathrm{M}\right)$ for 15 min each. After lysis of erythrocytes, samples were analyzed by flow cytometry.

Results and Discussion: Ropivacaine and QX314 did not affect leukocyte oxidative burst. Lidocaine did not affect oxidative burst at 1 hour incubation, but after 3 hours incubation, $10^{-4} \mathrm{M}$ lidocaine significantly inhibited the generation of intracellular reactive oxygen species. No effects of LA on leukocyte priming could be observed.

Conclusions: Intracellular generation of reactive oxygen species remains largely unaffected by LA in clinical concentrations. This supports the hypothesis that the anti-inflammatory effects of LA do not interfere with the host defense.

References:

1 Hollmann MW, Gross A, Jelacin N, Durieux ME: Local Anesthetic Effects on Priming and Activation of Human Neutrophils, Anesthesiology 2001; 5: 113-22.

2 Fischer LG, Bremer M, Coleman EJ, Conrad B, Krumm B, Gross A, Hollmann MW, Mandell G, Durieux ME: Local anesthetics attenuate lysophosphatidic acid-induced priming in human neutrophils. Anesth Analg 2001; 92: 1041-7.

\section{AP2-1}

\section{Reversal of pancuronium-induced block by the selective} relaxant binding agent sugammadex

M. Decoopman, G. Cammu, K. Suy, M. Heeringa, I. Demeyer Anaesthesiology, OLV Clinic, Aalst, Belgium

Background and Goal of Study: Sugammadex is the first selective relaxant binding agent that has been designed to antagonize rocuronium ${ }^{1}$. This exploratory study evaluated the effects of sugammadex on reversal of shallow neuromuscular block by pancuronium, in surgical patients.

Materials and Methods: After EC approval and written IC, 20 patients (ASA 1-2;20-81 yrs) received $0.1 \mathrm{mg} / \mathrm{kg}$ pancuronium. Neuromuscular block was monitored by acceleromyography (TOF-Watch ${ }^{\circledR} \mathrm{SX}$ ). Seventeen of these patients (per-protocol population) randomly received a dose of sugammadex $(1.0 ; 2.0 ; 4.0 ; 6.0 ; 8.0 \mathrm{mg} / \mathrm{kg})$ at reappearance of T2. The primary end-point was the time from administration of sugammadex until recovery of the T4/T1 ratio to 0.9 . Safety of sugammadex was evaluated by analyzing adverse events and vital signs.

Results and Discussions: The table shows the time intervals of the T4/T1 ratio to 0.9 after pancuronium-induced shallow neuromuscular block following different doses of sugammadex. For two subjects, serious adverse events were reported. They were not considered to be related to sugammadex.

\begin{tabular}{ll}
\hline & $\begin{array}{l}\text { Time Interval [mean (SD)] of T4/T1 ratio } \\
\text { to } 0.9(\text { min:sec })\end{array}$ \\
\hline $1.0(n=2)$ & $44: 02(53: 32)$ \\
$2.0(n=3)$ & $5: 17(0: 48)$ \\
$4.0(n=4)$ & $2: 46(1: 32)$ \\
$6.0(n=4)$ & $1: 24(0: 20)$ \\
$8.0(n=4)$ & $1: 22(0: 17)$ \\
\hline
\end{tabular}

Sugammadex has provided reliable and predictable reversal of neuromuscular block induced by rocuronium or vecuronium ${ }^{2}$. This study indicates that sugammadex decreases recovery time when administered at shallow pancuronium block, but no significant dose-response relation could be shown. Sugammadex was well tolerated.

Conclusion: Sugammadex decreases mean recovery times at shallow pancuronium-induced block. No significant dose-response relation could be shown. Sugammadex was well tolerated and had a good safety profile.

References:

1 Bom A, et al. Angew Chem Int Ed 2002;41:276-280.

2 Suy K, et al. Anesthesiology, in press.

\section{AP2-2}

\section{Neuromuscular pharmacodynamics of mivacurium in adult} patients with major burns

A. Han, JAJ. Martyn

Department of Anesthesia, University of lowa Hospitals and Clinics, lowa City, USA

Background and Goal of Study: Burned patients are resistant to nondepolarizing muscle relaxants (NDMRs). Mivacurium, however, has not been shown to be resistant in burned children. Neuromuscular (NM) response to mivacurium in burned adults is unknown. This study examined the pharmacodynamics of mivacurium bolus in relation to size of burn, time after burn, plasma pseudocholinesterase (PCHE) activity and dibucaine number, in adult major burns.

Materials and Methods: Adults (M/F $=22 / 8)$, aged $44.0 \pm 10$ years, with total burn area (TBA) $35.0 \pm 13 \%$, were studied at $40 \pm 29$ postburn days. Age and sex matched 30 nonburns served as controls. Anesthesia consisted of propofol and fentanyl infusion with $\mathrm{N}_{2} \mathrm{O}$ and $\mathrm{O}_{2}$. Mivacurium $0.2 \mathrm{mg} / \mathrm{kg}$ bolus was given. T1 of TOF Watch ${ }^{\circledR}$ monitored NM block. Onset time was the interval from beginning of drug administration to maximal twitch suppression. Intubation at 1 minute was attempted to simulate rapid sequence induction with recording of either failure or success. Spontaneous recovery profiles from paralysis were measured. Pearson correlations assessed relationship of recovery profiles to PCHE activity, dibucaine number, TBA and elapsed time after the injury.

Results and Discussions: Demographics of patients were similar in both groups. Onset time and all recovery profiles were prolonged in the burned patients. Intubation was successful with difficulty in approximately $70 \%$ of both groups. PCHE activity was significantly decreased in burns. Dibucaine number was similar in both groups. Recovery profiles showed high negative correlation to PCHE activity and positive correlation to TBA and time after the burn injury.

Conclusion(s): Unlike children and adolescents who have onset of NM paralysis similar to unburned, mivacurium onset time was prolonged in adult burns. Prolonged onset time suggests resistance to the NM effects. Conversely, prolonged recovery profiles suggest increased sensitivity. These contradictory findings can be explained by AChR proliferation and decreased level of plasma PCHE activity, which seem to depend on time after injury. In view of the prolonged onset time of almost two minutes for maximal paralysis, mivacurium does not appear to be a good drug for rapid onset of paralysis in burned adults.

\section{AP2-3}

\section{Incidence of residual block in recovery room (RR) after} neuromuscular blockade: a prospective study

P.C. Bergonzi, F. Ruggieri, A.F. Garozzo, C. Martani, R. Valeri

Department of Anesthesia, San Raffaele Scientific Institute, Milano, Italy 
Background and Goal of study: The aim of this observational, prospective study is to assess, by accelerometry, the incidence of residual block in recovery room (RR) after the use of intermediate-action neuromuscular block-

Materials and Methods: 252 adults, undergoing general anesthesia and neuromuscular blockade with atracurium or cisatracurium, were enrolled. At the patient's admission in RR (RR time). The Train of four ratio (TOF) was determined by stimulating the ulnar nerve at the wrist with surface electrodes. Each patient was asked to perform the head lift test (HLT) and was classified by a modified Aldrete score (1). TOF ratio was then calculated as the arithmetic mean of the results of four tests. The observation was terminated if TOF ratio was 0.9 . Otherwise the same procedure was repeated after $5 \mathrm{~min}(\mathrm{RR}+5)$ and eventually after $10 \mathrm{~min}(\mathrm{RR}+10)$. Primary endpoint was to define the incidence of TOF ratio $<0.9,<0.8,<0.7$ at RR time, $\mathrm{RR}+5$ and $\mathrm{RR}+10$. Secondary aim was to find any relationship between cholinesterase or time delay from last NMBA dose to admission in recovery room.

Results and Discussions: TOF ratio $<0.9,0.8,0.7$ at RR time was found respectively in $133(57 \%), 84(36 \%), 53(23 \%)$ patients. More than $20 \%$ of patients admitted in RR had a TOF ratio suitable for a safe extubation of the trachea (2). Furthermore, at RR $+10,20 \%$ of patients had a TOF ratio $<0.9$, which indicates an incomplete recovery (3). In our cases a successfully performed HLT is significantly related to an Aldrete score $>0.8$ but not to a TOF ratio $>0.7$.

Conclusions: The rates of residual block after atracurium and cisatracurium are not statistically different. Multivariate analysis shows that the short time delay from last NMBA injection to RR admission and abdominal surgery are the only factors related to TOF ratios in RR.

References:

1 Murphy et al. Anesth Analg 2004; 98: 193-200.

2 Ali HH et al. BJA 1971; 43: 478-485.

3 Eikermann M et al.: Anesthesiology 2006; 102: 937-942. ing agents (NMBA). TOF ratio and factors as atracurium versus cisatracurium, use of anthi-

\section{AP2-5}

Sugammadex achieves fast recovery from shallow neuromuscular blockade induced by rocuronium or vecuronium: dose-response studies

F. Pühringer, M. Blaszyk, G. Cammu, H. Sparr, M. Heeringa

Anaesthesia and Intensive Care Medicine, Klinikum am Steinenberg, Reutlingen, Germany

Background and Goal of Study: Sugammadex is the first selective relaxant binding agent (SRBA) designed to reverse the effects of rocuronium-induced neuromuscular blockade. This study assessed the effects of sugammadex on reversal of rocuronium- and vecuronium-induced shallow neuromuscular blockade.

Materials and Methods: One-hundred surgical patients (age 20 $-<65$ years, ASA class $1-3)$ were randomized to rocuronium $(0.9 \mathrm{mg} / \mathrm{kg}, \mathrm{n}=50)$ or vecuronium $(0.1 \mathrm{mg} / \mathrm{kg}, \mathrm{n}=50)$. Anaesthesia was induced using propofol and fentanyl and maintained with sevoflurane. Neuromuscular activity was monitored by acceleromyography (TOF-Watch ${ }^{\circledR} \mathrm{SX}$ ). Patients were randomised to sugammadex $(0.5,1.0,2.0$ or $4.0 \mathrm{mg} / \mathrm{kg})$ or placebo at reappearance of $T_{2}$. The primary end-point was the time from administration of sugammadex to recovery of the $T_{4} / T_{1}$ ratio to 0.9 . An exponential model (and weighted non-linear regression) was used to explore the dose-response relation. Possible recurarization or residual curarization was assessed. Safety was evaluated by adverse events, vital signs and laboratory parameters.

Results and Discussion: Significant dose-response relations were found between sugammadex dose and time to T4/T1 ratio to 0.9 (Table).

\begin{tabular}{lllll}
\hline Sugammadex & \multicolumn{4}{l}{ Mean time $(\mathrm{SD})$ to $\mathrm{T}_{4} / \mathrm{T}_{1}$ ratio of $0.9(\mathrm{~min})$} \\
\cline { 2 - 5 } Dose $(\mathrm{mg} / \mathrm{kg})$ & Rocuronium & \multicolumn{3}{l}{ Vecuronium } \\
\hline placebo & $(\mathrm{n}=7)$ & $96.3(33.1)$ & $(\mathrm{n}=8)$ & $79.0(26.0)$ \\
0.5 & $(\mathrm{n}=8)$ & $16.3(20.6)$ & $(\mathrm{n}=9)$ & $35.5(42.1)$ \\
1.0 & $(\mathrm{n}=8)$ & $4.6(6.0)$ & $(\mathrm{n}=10)$ & $5.1(2.4)$ \\
2.0 & $(\mathrm{n}=9)$ & $1.4(0.5)$ & $(\mathrm{n}=7)$ & $3.4(1.9)$ \\
4.0 & $(\mathrm{n}=8)$ & $1.5(0.4)$ & $(\mathrm{n}=9)$ & $3.0(2.2)$ \\
\hline
\end{tabular}

A decrease from a $T_{4} / T_{1}$ ratio of $\geqslant 0.9$ to $<0.8$ (indication for recurarisation) was reported for 7 subjects, predominantly in the $0.5 \mathrm{mg} / \mathrm{kg}$ group. SAEs were reported for three subjects, but none was considered related to sugammadex.

Conclusions: Sugammadex decreased recovery time from rocuronium- or vecuronium-induced neuromuscular blockade in a dose dependent manner. Sugammadex was safe and well tolerated.

\section{Lossen, A. Pape, H. Kertscho, B. Zwissler, O. Habler}

Clinic of Anaestesiology, Intens. Care Med. and Pain Management, J.W. Goethe-University Hospital, Frankfurt/Main, Germany

Background and Goal of Study: The utilisation of the patient's individual anemia tolerance represents a cornerstone in every allogeneic blood conservation strategy. Whether muscular relaxation has an effect on anemia tolerance by lowering skeletal muscular $\mathrm{O}_{2}$-consumption and total body $\mathrm{O}_{2}$-demand ${ }^{1}$ has not been investigated so far. The goal of the present study was to investigate the influence of rocuronium bromide on the tolerance of acute normovolemic anemia.

Materials and Methods: 16 anesthetised and mechanically ventilated juvenile domestic pigs were randomised to receive either $0.378 \mathrm{~mL} / \mathrm{kg}$ of a $1 \%$ rocuronium bromide preparation $\left(\right.$ Esmeron $^{\mathrm{TM}}$ ) followed by continuous infusion of $0.1 \mathrm{~mL} / \mathrm{kg} / \mathrm{min}$ (ROC-group, $\mathrm{n}=8$ ) or the same volume of normal saline (N.S.-group, $n=8$ ). Acute normovolemic anemia was induced by exchange of whole blood for a $6 \%$ HES-solution (130/0.4) until a sudden decrease of total body $\mathrm{O}_{2}$-consumption $\left(\mathrm{VO}_{2}\right)$ indicated a critical limitation of $\mathrm{O}_{2}$-delivery to the tissues $\left(\mathrm{DO}_{2}\right)^{2}$. The $\mathrm{Hb}$-concentration quantified at this time point $\left(\mathrm{Hb}_{\text {crit }}\right)$ was defined as the primary endpoint of the protocol. Secondary endpoints were parameters of central hemodynamics, $\mathrm{O}_{2}$-transport and tissue oxygenation.

Results: $\mathrm{Hb}_{\text {crit }}$ was significantly lower in the ROC-group $(3.4 \pm 0.8 \mathrm{~g} / \mathrm{dL}$ vs. $2.5 \pm 0.5 \mathrm{~g} / \mathrm{dL}$ ). The implementation of neuromuscular block did not exert any alterations on secondary endpoints. At $\mathrm{Hb} 3.4 \mathrm{~g} / \mathrm{dL}$ (i.e., $\mathrm{Hb}_{\text {crit }}$ of the N.S.-group), no significant differences between the groups were detected. At $\mathrm{Hb} 2.5 \mathrm{~g} / \mathrm{dL}$ (i.e. $\mathrm{Hb}_{\text {crit }}$ of the $\mathrm{ROC}$-group), $\mathrm{CaO}_{2}$ and MAP were significantly lower than in the N.S.-group.

Conclusion(s): Deep neuromuscular blockade with rocuronium bromide increases the tolerance of acute normovolemic anemia.

Disclosure: The study was sponsored by a research grant from Organon Inc., Oberschleißheim, Germany.

References:

1 Richardson RS, Adv.Physiol Educ 2003; 27: 183-191.

2 Meier J, Woelkhammer S, Habler O, Comp. Biol. Med. 2003; 33: 395-405.

\section{AP2-6}

\section{Propranolol and isoprenaline do not modify the sugammadex-induced fast recovery from rocuronium- induced neuromuscular blockade}

A. Bom, F. Hope

Department of Pharmacology, Organon Laboratories Ltd, Newhouse, United Kingdom

Background and Goal of Study: Sugammadex is the first selective relaxant binding agent (SRBA) for the reversal of neuromuscular blockade induced by steroidal neuromuscular blocking agents. Beta-adrenoceptor agonists can accelerate the onset time of rocuronium, whereas beta-adrenoceptor antagonists can slow it down ${ }^{1,2}$. The aim of this study was to determine whether pretreatment with isoprenaline or propranolol alters the recovery time from rocuronium-induced neuromuscular blockade after administration of sugammadex in rats.

Materials and Methods: Male Sprague-Dawley rats were anaesthetized with pentobarbitone sodium and artificially ventilated. Single twitch contractions of $\mathrm{M}$. gastrocnemius were induced by stimulation of the sciatic nerve. Animals were pretreated with saline $(n=6)$, propranolol $(1 \mathrm{mg} / \mathrm{kg}$; i.v. bolus; $\mathrm{n}=6)$ or isoprenaline $(0.15 \mu \mathrm{g} / \mathrm{kg} / \mathrm{min}$; i.v. infusion; $\mathrm{n}=6)$. Complete blockade was induced by i.v. bolus administration of a $3 \mathrm{XED}_{90}$ dose of rocuronium $(3.9 \mu \mathrm{mol} / \mathrm{kg})$. One minute after obtaining complete blockade, sugammadex was administered as a single i.v. bolus dose $(1 \mu \mathrm{mol} / \mathrm{kg})$.

All data are expressed as Mean \pm SEM. A one-way ANOVA ( $p 0.05$ level) was used to determine differences between groups.

Results and Discussions: No statistically significant differences in recovery index and time to $25,50,75$ and $90 \%$ recovery were observed between the three treatment groups. 


\begin{tabular}{llll}
\hline $\begin{array}{l}\text { Recovery after } \\
\text { sugammadex }\end{array}$ & saline & propranolol & isoprenaline \\
\hline $25 \%(\min )$ & $1.5 \pm 0.2$ & $1.6 \pm 0.4$ & $2.1 \pm 0.5$ \\
$50 \%(\min )$ & $2.6 \pm 0.3$ & $2.4 \pm 0.6$ & $2.8 \pm 0.6$ \\
$75 \%(\min )$ & $4.1 \pm 0.6$ & $3.6 \pm 0.8$ & $3.8 \pm 0.8$ \\
$90 \%(\min )$ & $5.2 \pm 0.8$ & $6.9 \pm 1.9$ & $5.8 \pm 1.1$ \\
Recovery index & $2.6 \pm 0.5$ & $1.9 \pm 0.4$ & $1.8 \pm 0.3$ \\
\hline
\end{tabular}

Conclusion: Changes in haemodynamics induced by propranolol or isoprenaline do not modify the sugammadex-induced rapid recovery from rocuronium-induced neuromuscular blockade.

References:

1 Szmuk et al., Anaesth Analg 2000; 90(5): 1217.

2 Eszri et al., Acta Scand Anaesth 2003; 47(9): 1067

\section{AP2-7}

Pharmacokinetics of the selective relaxant binding agent sugammadex, administered for reversal of shallow neuromuscular blockade induced by rocuronium or vecuronium

M. Gordon, C. Rex, J. Ingimarsson, B. Klarin, J. Smeets

Anaesthesiology, Intensive Therapy and Treatment of Pain, Poznan University of Medical Sciences, Poznañ, Poland

Background and Goal of Study: Sugammadex is a modified $\gamma$-cyclodextrin and the first selective relaxant binding agent (SRBA), designed to reverse the effects of rocuronium-induced neuromuscular blockade. An objective of this study was to explore the pharmacokinetics of rocuronium, vecuronium and sugammadex in ASA class 1-3 patients who received sugammadex for reversal of shallow neuromuscular blockade induced by rocuronium or vecuronium.

Materials and Methods: This randomised, multicentre, parallel-group trial included 100 surgical patients (age $\geqslant 20$ years and $<65$ years, ASA class $1-3$ ) who had given written informed consent. Anaesthesia was induced with propofol and maintained using sevoflurane. Patients received rocuronium $(0.9 \mathrm{mg} / \mathrm{kg})$ or vecuronium $(0.1 \mathrm{mg} / \mathrm{kg})$ during induction of anaesthesia. Additional doses of the neuromuscular blocking agent (NMBA) were administered if necessary. Neuromuscular activity was monitored by acceleromyography (TOFWatch $^{\circledR}$ SX). At reappearance of T2 after the last dose of the NMBA, either sugammadex $(0.5,1.0,2.0$ or $4.0 \mathrm{mg} / \mathrm{kg})$ or placebo was administered in randomised order. Rocuronium, vecuronium and sugammadex concentrations in plasma were determined using validated liquid chromatography assay methods with mass-spectrometric detection. These essays do not discriminate between complexed and non-complexed sugammadex and rocuronium. Pharmacokinetic analysis was performed with data from 98 of the 100 included subjects (49 subjects for both NMBA groups).

Results and Discussions: Median rocuronium plasma concentrations, and to a lesser extent vecuronium plasma concentrations, showed a temporary increase after sugammadex administration, which was not seen in the placebo group. Plasma concentrations of sugammadex increased with increasing dose of sugammadex over the dose range $0.5-4.0 \mathrm{mg} / \mathrm{kg}$, both in the rocuronium and the vecuronium group.

Conclusion(s): Plasma concentrations of rocuronium showed an increase after administration of sugammadex. Plasma concentrations of sugammadex were approximately dose-proportional over the dose range of 0.5 to $4.0 \mathrm{mg} / \mathrm{kg}$, independent of the NMBA used.

\section{AP2-8}

The effects of ephedrine on intubating conditions following low dose priming with cisatracurium

Y. Leykin, M. Dalsasso, T. Setti, T. Pellis

Anesthesia and Intensive Care, S.Maria degli Angeli Hospital, Pordenone, Italy Background and Goals: Both ephedrine $(E)$ in small dose and the "priming principle" decrease the onset time of cisatracurium (1,2). E improves clinical intubating conditions following priming $(P)$ with rocuronium when compared with $P$ or $E$ alone (3). We investigated the effects of $E$ plus $P$ with cisatracurium on clinical intubating conditions.

Material and Methods: 124 ASA I-II, aged 18 to 65 years, undergoing elective surgery were randomly allocated to 4 groups $(n=31)$. All patients were induced with propofol $2 \mathrm{mg} / \mathrm{kg}$ and sulfentanil $0.15 \mu \mathrm{g} / \mathrm{kg}$. In group A a P dose of $0.005 \mathrm{mg} / \mathrm{kg}$ (10\% ED95) was followed 3 minutes later by an intubating dose of $0.145 \mathrm{mg} / \mathrm{kg}$ of cisatracurium. E, $70 \mu \mathrm{g} / \mathrm{kg}$, was injected together with the induction agents over $30 \mathrm{~s}$ just before the intubating dose. After 60 seconds intubation was attempted. In group B the same sequence was repeated except for sham $E$ administration. Group $C$ received sham $P$ and an intubation dose was of $0.15 \mathrm{mg} / \mathrm{kg}$., while group $D$ sham $P$ and sham $E$. Intubating conditions were graded as excellent, good, poor, and bad. Neuromuscular transmission was monitored by acceleromyography of the adductor pollicis muscle.

Results: There were no demographic and baseline differences among groups. All patient in group A were intubated $60 \mathrm{~s}$ after the intubating dose, compared to $74 \%$ of group $B, 77 \%$ of $C$, and $64 \%$ of $D$. In group $A$ the proportion of good to excellent intubating conditions was significantly higher compared to the other group (group A 100\%, B 52\%, C 52\%, and D 48\%; $p<0.01$ ). No significant difference between groups was observed with regard to twitch suppression at 60 seconds and onset time, as well as haemodynamic parameters (heart rate and mean blood pressure).

Conclusions: Low dose ephedrine combined with low dose priming significantly improved clinical intubating conditions $60 \mathrm{~s}$ after intubating dose of cisatracurium.

References:

1 Albert F et al. Acta Anaesth.Belg 2000; 51: 167.

2 Puhringer FK et al. Anaesthetist 2000; 49: 102.

3 Leykin Y et al. Acta Anaesthesiol Scad 2005; 49: 792.

\section{AP2-9}

\section{Modification of posttetanic count (PTC) for monitoring deep} neuromuscular block

C. Kjaer, LT. Skovgaard, Y. Gurkan, J. Viby-Mogensen

Department of Anaesthesia, HOC 4231, Copenhagen University Hospital, Rigshospitalet, Copenhagen, Denmark

Background and Goal of Study: Posttetanic count (PTC) is the gold standard for quantifying deep neuromuscular block. The purpose of this study was to evaluate whether the duration of $1 \mathrm{~Hz}$ stimulation as used in the original PTC method could be decreased without influencing recovery of block and the relation between PTC and time to first reaction to Train-of-four (TOF) (1). Materials and Methods: Fifteen adult patients were studied. Anesthesia was induced and maintained with propofol and remifentanil. Acceleromyography was used for evaluation of neuromuscular block, following rocuronium $0.6 \mathrm{mg} / \mathrm{kg}$ intravenously. At one hand a TOF Watch SX nerve stimulator recorded the PTC response using the original PTC method. At the contralateral hand a modified PTC sequence was applied. The $1 \mathrm{~Hz}$ single stimulation was decreased from $60+60$ stimulations to $10+30$ stimulations, before and after the tetanic stimulation, respectively.

Results and Discussions: For the primary endpoint, time to first response in TOF, the mean bias between the two stimulation sequences was $0.72 \mathrm{~min}$ (c.i. $-0.43-1.87 \mathrm{~min}), \mathrm{p}=0.20$.

The relationship between PTC and time to first reaction to TOF was similar with the two methods, $\mathrm{p}=0.89$.

Conclusion(s): The duration of $1 \mathrm{~Hz}$ stimulation and thus the total duration of PTC stimulation could be decreased significantly (from 127 to only 47 seconds), without influencing recovery and the relationship between PTC and time to first TOF response. The modified PTC method thus allows the clinician to obtain a result from a PTC stimulation in less than half the time of the original method.

Reference:

1 Viby-Mogensen J et al. Anesthesiology 1981; 55: 458-61.

\section{AP2-10}

\section{Optimal dosage of cisatracurium in obese patients}

Y. Deshko, V. Stamov, V. Mizikov

Department of Anaesthesiology, Russian Research Center of Surgery, Moscow, Russian Federation

Background and Goal of the Study: Whether the ideal body weight (IBW) or the real body weight (RBW) to be used as a basis to calculate the reduced dosage of relaxants for obese patients - this was always under discussion. The aim of this study is to estimate the optimal dosage of cisatracurium (CisA) based on corrected body weight (CBW).

Patients and Methods: 57 patients (age 27-70, ASA I-III) to undergo elective abdominal surgery under balanced anesthesia or TIVA were divided into four random groups after RRCS's Commission on ethics approval. All patients received $0.15 \mathrm{mg} / \mathrm{kg}$ CisA on the basis of RBW (group I, $\mathrm{n}=14$ ), CBW (group II, $\mathrm{n}=12$ ), IBW (group III, $\mathrm{n}=12$ ) or RBW (control group, $\mathrm{n}=19$ ). $\mathrm{BMI}$ in groups I-III was 30.1-51.1 and $\leqslant 25$ in the controlled group. CBW was calculated as IBW $+0.4 \times(\text { RBW-IBW })^{1}$. Monitoring of NMB: TOF-Watch ${ }^{\circledR} \mathrm{SX}$. The onset time, the durations $25 \%$, spontaneous or induced recovery (RI and RT), and occurrence of antagonists injections were registered. 
Results: Induction dose of CisA based on CBW calculus allowed perform of tracheal intubation as long as if it was based on RBW figures and shorter period of action was achieved at the same time (see Table). Induction dose of CisA based on IBW extended onset time and obviously reduced its duration $25 \%$. Body weight value had no any significant impact on the recovery rate.

\begin{tabular}{lllll}
\hline & group I & group II & group III & Control \\
\hline The onset time & $211 \pm 28$ & $242 \pm 48$ & $305 \pm 20^{*}$ & $233 \pm 41$ \\
Duration 25\% & $57.4 \pm 5.4^{\star}$ & $48.3 \pm 8.2$ & $37.4 \pm 7.8^{\star}$ & $47.2 \pm 9.5$ \\
RI & $16.7 \pm 6.9$ & $19.1 \pm 9.0$ & $17.9 \pm 4.5$ & $16.7 \pm 7.2$ \\
RT & $23.7 \pm 8.4$ & $25.2 \pm 9.6$ & $23.9 \pm 6.6$ & $24.5 \pm 6.2$ \\
Reversal agent & $21 \%^{*}$ & $8 \%$ & $8 \%$ & $10 \%$ \\
\hline
\end{tabular}

Statistics: ANOVA with ${ }^{*} p<0.05$, data are mean \pm SD

Conclusions: When CBW was used as the CisA dosage calculation basis for obese patients more controlled and secure relaxation was achieved. We suggest that feminine or masculine constitutional patterns, muscular mass of the obese patients may have been of great importance.

Reference:

1 Servin F et al. Anesthesiology 1993; 78: 657-665.

\section{AP3-1}

Propofol and remifentanil vs. isoflurane and nitrous oxide: which can provide better surgical field in FESS?

X. Li, X. Song, T. Li, B. Zhou, B. Zhang

\section{Anesthesiology, Beijing Tongren Hospital, Beijing, China}

Background and Goal of Study: Both total intravenous anesthesia (TIVA) and inhaled anesthesia are widely used in functional endoscopic sinus surgery (FESS). However, they can provide different surgical field quality even under the same depth of anesthesia. This study is to compare the nasal mucus blood flow under different anesthesia methods.

Materials and Methods: The study is randomized, controlled, double-blinded and prospective. 40 patients (ASA I-II, aged from 18-45, male 26, female 14) underwent elect FESS were allocated into TIVA (propofol and remifentanil) group and inhaled (isoflurane and nitrous oxide) group, $n=20$ each. Anesthesia depth was monitored with bispectral analysis and controlled between the BIS value between 40 and 50 in both groups during the operation. Nasal mucosal blood flow was measured with laser Doppler flowmetry. Surgical field was assessed by the same pointed surgeon according to the Fromme score ${ }^{(1)}$.

Results and Discussions: Perfusion Unit (PU) and flow velocity ( $)$ value of TIVA group were significantly lower than those of inhaled group (343.33 \pm 62.23 vs. $448.60 \pm 66.68$ and $96.46 \pm 13.87$ vs. $122.07 \pm 21.41), p=0.00015$ and $\mathrm{p}=0.00021$ respectively.

Visual field score in TIVA group $(2.07 \pm 0.59)$ was significantly lower than those of inhaled group $(2.8 \pm 0.56), \mathrm{p}=0.0016$.

Conclusion(s): Under the same depth of anesthesia, propofol and remifentanil can provide better surgical field than isoflurane by decreasing the local blood flow of the nasal mucosa.

Reference:

1 Fromme GA, MacKenzie RA, Gould AB Jr, et al. Anesth Analg 1986; 65: 683-6.

\section{AP3-2}

Effect of nitrous oxide on the desflurane requirement for blunting sympathetic responses after surgical incision combined with two target-controlled concentrations of remifentanil (1 and $3 \mathbf{n g} / \mathrm{ml}$ )

E. Dedola, G. Bruno, A. Albertin, S. Turi, A. Gandolfi

Department of Anesthesia, San Raffaele Scientific Institute, Milano, Italy

Background and Goal of Study: The aim of this prospective, randomized, double blind study was to determine the effects of adding nitrous oxide on the desflurane requirement for blunting sympathetic responses after surgical incision $\left(\mathrm{MAC}_{\mathrm{BAR}}\right)$ combined with two different target-controlled concentrations of remifentanil (1 and $3 \mathrm{ng} / \mathrm{ml})$.

Materials and Methods: 100 patients, aged 20-50 years, ASA physical status I, undergoing general anaesthesia for elective abdominal surgery were enrolled and randomly allocated to receive desflurane anesthesia alone (Group A, $n=52$ ), or with the addition of $60 \%$ nitrous oxide (Group $N, n=46$ ). Patients of both groups were further assigned to receive a target controlled effect-site concentration of $1 \mathrm{ng} / \mathrm{ml}$ (Group A1, $\mathrm{n}=26$; Group N1, $n=26$ ) or $3 \mathrm{ng} / \mathrm{ml}$ remifentanil (Group A3, $\mathrm{n}=26$, Group N3, $n=22$ ). Sympathetic responses to surgical incision were determined after a $20 \mathrm{~min}$ period of stable end-tidal desflurane and target-controlled remifentanil concentrations. Predetermined end-tidal desflurane concentrations and the $\mathrm{MAC}_{\mathrm{BAR}}$ for each group were determined using an up-and-down sequential allocation technique.
Results and Discussions: The MAC $\mathrm{MAR}_{\mathrm{BA}}$ of desflurane was 5.2\% (95\% confidence interval, $\left.\mathrm{Cl}_{95}: 4.9-5.5 \%\right)$ in Group $\mathrm{A} 1$ and $2.7 \%\left(\mathrm{Cl}_{95}: 2.6-2.8 \%\right)$ in Group N1 $(P<0.001)$, while in Groups A3 and N3 the MAC BAR $_{\text {ar }}$ of desflurane was $2.2 \%\left(\mathrm{Cl}_{95}: 2-2.4 \%\right)$ and $2 \%\left(\mathrm{Cl}_{95}: 1.9-2.2 \%\right)$, respectively $(P<0.01)$. When considering a minimum anesthetic concentration (MAC) value in this age population and the contribution of $60 \%$ nitrous oxide ( $0.55 \mathrm{MAC})$, the combined MAC $\mathrm{BAR}_{\mathrm{R}}$ values, expressed as multiples of the MAC, were 1.9 MAC, $1 \mathrm{MAC}$ in $\mathrm{A} 1$ and $\mathrm{N} 1$ groups and $0.8 \mathrm{MAC}$ and $0.74 \mathrm{MAC}$ in $\mathrm{A} 3$ and N3 groups. Conclusions: Adding $60 \%$ nitrous oxide reduces the $\mathrm{MAC}_{\mathrm{BAR}}$ of desflurane by $52 \%$ when using a remifentanil concentration of $1 \mathrm{ng} / \mathrm{ml}$ and $10 \%$ when using a remifentanil concentration of $3 \mathrm{ng} / \mathrm{ml}$.

\section{AP3-3}

\section{Evidence for a daily rest-activity rhythm disruption the days} following general (propofol) anaesthesia in patients.

L. Pain, G. Dispersyn, O. Coste, J. LLeu, E. Challet

Faculty of Medicine, INSERM U666;CHRU Strasbourg, Strasbourg, France

Background and Goal of Study: Because of evidenced chronobiotic properties of propofol (effect on biological clock) in animal (1), we examined the daily rest-activity rhythm before and after anesthesia, in patients.

Materials and Methods. Patients (age 40-60 years, ASA 1-2), with no prior sleep disorders, shift-work, cancer, benzodiazepines or beta blockers treatment, scheduled for systematic ambulatory digestive endoscopy (under intravenous propofol $1.7-2.0 \mathrm{mg} / \mathrm{kg}$ ) were included. After informed consent, participants were asked to wear an actigraph (Cambridge Neurotechnology LtD, UK) the week before and the week of procedure. We assessed the restactivity rhythms on $1 /$ the interdaily stability (IS), a measure of the strength of coupling the rest-activity rhythm to Zeitgebers (environmental time cues); high values representing a stable rhythm, 2 / the intradaily variability (IV) serves as a measure of fragmentation of the rhythm, a normal activity showing a low IV (2). Based on the fact that group differences for these variables have been previously reported using only 3 days of actigraphy, we choose to compare the same four consecutive days (6 P.M.:6P.M.) before and after anaesthesia. Results and Discussions: Data obtained from the first set of 10 patients evidenced a main effect of anaesthesia $(F(1,9)=9.036 ; p=0.015)$ on the rest-activity rhythm variables: $1 /$ a significant decrease of IS $(F(1,9)=5.68$; $\mathrm{p}=0.04$ ) indicative of a lower coupling of the rest-activity rhythm to Zeitgebers and $2 /$ a significant increase of $\operatorname{IV}(F(1,9)=5.78, p=0.04)$, indicative of more fragmentation of the daily rhythm the days following anaesthesia.

\begin{tabular}{lll}
\hline & IS mean (standard deviation) & IV mean (standard deviation) \\
\hline pre & $0.79(0.10)$ & $0.53(0.17)$ \\
post & $0.67(0.14)$ & $0.69(0.18)$ \\
\hline
\end{tabular}

Conclusion(s): Propofol anesthesia was associated with a disruption of the daily rest-activity rhythm in ambulatory patients; such a disruption might participate to postoperative fatigue and sleep disorders.

References:

1 Challet et al. Neuropsychopharmacology 2006.

2 Van Someren et al. J sleep Res 2006, 415-423.

\section{AP3-4}

\section{Remifentanil can prevent the withdrawal movements due to} the intravenous injection of rocuronium

B. Choi, K. Lee, S. Shin

Department of Anesthesiology and Pain Medicine, Yonsei University Medical College, Seoul, Republic of Korea

Background and Goal of Study: We investigated the effect of remifentanil pretreatment on the withdrawal movements due to the intravenous (IV) injection of rocuronium during anesthetic induction $(1,2)$.

Materials and Methods: Ninety adult females undergoing thyroidectomy were randomly allocated to three groups. Each patient received one of three solutions of $4 \mathrm{~mL}$ : saline (Group I, $\mathrm{n}=30$, control), remifentanil $0.5 \mu \mathrm{g} / \mathrm{kg}$ in saline (Group II, $n=30$ ) or remifentanil $1 \mu \mathrm{g} / \mathrm{kg}$ in saline (Group III, $\mathrm{n}=30$ ), through a 20G IV cannula inserted into the forearm cephalic vein over $30 \mathrm{sec}-$ onds. Thirty seconds after remifentanil injection, anesthesia was induced with $I . V$ thiopental $5 \mathrm{mg} / \mathrm{kg}$. Twenty seconds after thiopental injection, I.V rocuronium $0.6 \mathrm{mg} / \mathrm{kg}$ was administered at the injection rate of $0.5 \mathrm{ml} / \mathrm{sec}$ and patients' withdrawal movements were assessed. Mean arterial pressures and heart rates before and during the anesthetic induction were measured to clarify the effect of remifentanil on the cardiovascular response following by laryngoscopy and endotracheal intubation.

Results and Discussions: The incidence of withdrawal movements was significantly reduced in both of the remifentanil groups (3 and $0 \%$ in Group II and III, respectively) than in the control group (70\%). Remifentanil in both 
of the remifentanil groups attenuated the increase in heart rate and MAP immediately after and 1 minute after endotracheal intubation.

Conclusion(s): The pretreatment with remifentanil in both 0.5 and $1.0 \mu \mathrm{g} / \mathrm{kg}$ of bolus doses dramatically prevented the withdrawal movements caused by the rocuronium injection, and effectively blunted the cardiovascular activation following by laryngoscopy and endotracheal intubation.

\section{References:}

1 Chiarella AB, Jolly DT, Huston CM, et al. Br J Anaesth 2003:90:377-379.

2 Memis D, Turan A, Karamanlioglu B, et al. Anesth analg 2002;94:1517-1520.

\section{AP3-5}

\section{The effects of two different doses of dexmedetomidine} on extubation

C. Turan, G. Turan, A. Ozgultekin, E. Dýncer, G. Yuksel

\section{Anesthesiology and Intensive Care Unit, Istanbul Haydarpapa Numune} Teaching and Research Hospital, Istanbul, Turkey

Background and Goal of study: We studied the effects of dexmedetomidine with two different doses in extubation period, regarding hemodynamics and recovery characteristics in patients operated for intracranial lesions.

Material and Methods: Following the approval of hospital ethics committee, 45 patients aged 18-75, ASA I-III, who were undergoing intracranial surgery were randomly selected and divided into three groups. $50 \% \mathrm{O}_{2}-\mathrm{N}_{2} \mathrm{O}$ and $1 \%$ isoflurane used for the maintanance. In Group I; isoflurane reduced to $0.5 \%$ 15 minutes before the end of surgery and dexmedetomidine infusion installed at the dose of $1 \mu \mathrm{g} \mathrm{kg}^{-1}$ for $10 \mathrm{~min}$ then $0.5 \mu \mathrm{g} \mathrm{kg}^{-1} \mathrm{hr}^{-1}$ until extubation. In Group II; isoflurane reduced to $0.5 \% 5$ minutes before the end of the surgery and dexmedetomidine used $0.5 \mu \mathrm{g} \mathrm{kg}^{-1}$ was given in $1 \mathrm{~min}$. just before extubation. In Group III; $20 \mathrm{ml} \mathrm{hr}^{-1} 0.9 \% \mathrm{NaCl}$ infusion was started 15 minutes before the end of the surgery. Mean arterial pressure (MAP), heart rate (HR) recorded at $1 \mathrm{~min}$ before and $1,3,5,10,15,20,30^{\text {th }} \mathrm{min}$. after extubation. Isoflurane was stopped at the last stitch. Time to extubation and verbal responses were recorded. Oneway ANOVA, Tukey HDS, paired t test and $x^{2}$ square test in the SPSS were used for statistical analysis.

Results and Discussion: MAP and HR were found significantly higher in gr III and II compared gr I. There were no significant differences in extubation times between groups. Verbal response time was significantly longer in gr I than gr II and III. ( $\mathrm{p}<0.01)$.

Conclusion: When Dexmedetomidine used $0.5 \mu \mathrm{g} \mathrm{kg}^{-1}$ (in $1 \mathrm{~min}$ ) before the extubation it is a suitable agent for optimal hemodynamic state and good recovery condition for intracranial operations.

References:

1 Arain SR, Ebert TJ. Anesth Analg. 2002; 95: 461-466.

2 Wijeysundera DN, Naik JS, Beattie WS. Am J Med. 2003; 114: 742-52.

3 Jaakola ML, Ali-Melkkila T, Kanto J, et al.. Br J Anaesth. 1992; 68: 570-575.

\section{AP3-6}

\section{Clonidine modifies the BIS response to trachel intubation}

I. Soumpasis, E. Antypa, Th. Georgiadou, A. Mademli, F. Kanakoudis Anaesthesia, G. Gennimatas General Hospital of Thessaloniki, Thessaloniki, Greece

Background and Goal of Study: Clonidine has been used as an adjunct to anaesthetics, because of its beneficial effects during and after anaesthe$\mathrm{sia}^{1}$, including sparing effect on propofol infusion guided with Bispectral Index (BIS) values ${ }^{2}$. The aim of the study was to evaluate the effect of clonidine pre-treatment on BIS during induction of anaesthesia and tracheal intubation.

Materials and Methods: Forty patients, ASA I-II, aged $49 \pm 17$ years, scheduled for elective surgery under general anaesthesia, were randomly allocated in group CLO and PLA. Pre-treatment regime (clonidine $2 \mathrm{mcg}^{\star} \mathrm{Kg}^{-1}$ in $5 \mathrm{ml}$ or same volume of normal saline respectively) was administered $3 \mathrm{~min}-$ utes before induction of anaesthesia, which was performed with propofol $\left(2 \mathrm{mg}^{\star} \mathrm{Kg}^{-1}\right)$, rocuronium $\left(0.9 \mathrm{mg}^{\star} \mathrm{Kg}^{-1}\right)$ and remifentanil $\left(0.5 \mathrm{mcg}^{\star} \mathrm{Kg}^{-1}\right.$ bolus, $0.05 \mathrm{mcg}^{*} \mathrm{~kg}^{-1 *} \mathrm{~min}^{-1}$ infusion). BIS values, along with heart rate, systolic and diastolic blood pressure, were recorded in 6 phases: before and after administration of clonidine or saline, after propofol, muscle relaxant and opioid administration and 1 and 3 minutes after tracheal intubation. All data were analyzed with 2-way ANOVA, and $p<0.05$ was considered as level of significance.

Results and Discussions: Demographic data were similar in both groups. Changes on BIS are shown in figure 1 and Systolic Arterial Pressure/Heart Rate in figure 2. There was an increase at BIS at both groups after intubation, but this seems to be significantly smaller at the CLO group $(p<0.001)$. There was also similar difference in SAP $(p<0.05)$ and in HR $(p<0.05)$.

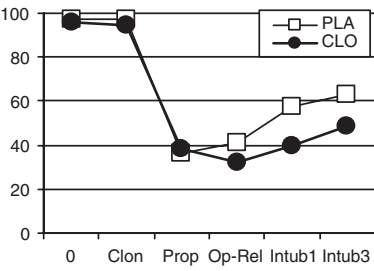

Fig. 1. (BIS)

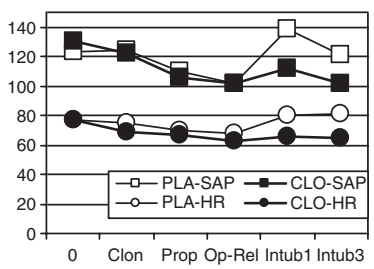

Fig. 2. (SAP-HR)

Conclusion(s): Pretreatment with clonidine, does not influence the BIS reduction after propofol administration but provides a lower level of BIS values after intubation.

References:

1 Hall JE: Br J Anaesth 2001; 86: 5-11.

2 Fehr SB, et al: Br J Anaesth 2001; 86: 627-32.

\section{AP3-7}

\section{Endocrine stress response modification by clonidine during laparoscopic cholecystectomy}

I. Soumpasis, M. Georgiou, P. Ziaga, Th. Georgiadou, F. Kanakoudis

Anaesthesia, G. Gennimatas General Hospital of Thessaloniki, Thessaloniki, Greece

Background and Goal of Study: One of anaesthesia aims is to suppress the stress response to surgery. This study tries to reveal and evaluate possible suppressive effect of clonidine on stress response because of pneumoperitoneum during laparoscopic procedures.

Materials and Methods: Twenty patients ASA I-II, aged $58 \pm 15$ years, scheduled for laparoscopic cholecystectomy were randomly allocated to receive intravenously either clonidine $2 \mu \mathrm{g} / \mathrm{Kg}$ in $5 \mathrm{ml}$ volume (Group CLO, $n=10$ ), or equal volume of normal saline as placebo (Group PLA, $n=10$ ), $2 \mathrm{~min}$ prior to anesthesia induction. In both groups general anesthesia was induced with propofol ( $1.5 \mathrm{mcg} / \mathrm{Kg}$ bolus followed by $0,1 \mathrm{mg} / \mathrm{Kg} / \mathrm{min}$ infusion) and remifentanil $(0.5 \mathrm{mcg} / \mathrm{Kg}$ bolus followed by $0.1 \mathrm{mcg} / \mathrm{kg} / \mathrm{min}$ infusion). Muscle relaxation was achieved with rocuronium and tracheal intubation was performed. Anaesthesia was maintained with infusions of propofol and remifentanil, as well as repeated bolus doses of rocuronium. Cortisol and blood glucose levels were estimated at 4 time points: prior to induction, $5 \mathrm{~min}$ after intubation, $5 \mathrm{~min}$ after pneumoperitoneum establishment and $5 \mathrm{~min}$ after the end of pneumoperitoneum. Blood pressure and Heart Rate were also recorded at the same time points. Two-way ANOVA followed by Bonferroni posthoc test were used to evaluate differences between groups.

Results and Discussions: Demographic data were similar between groups. Group CLO demonstrated lower cortisol levels $(p<0.01)$ with difference to be obvious after pneumo-peritoneum ( $<0.05)$, as well as after the end of pneumo-peritoneum $(p<0.05)$. There was no difference between groups at all time points in blood glucose concentration.

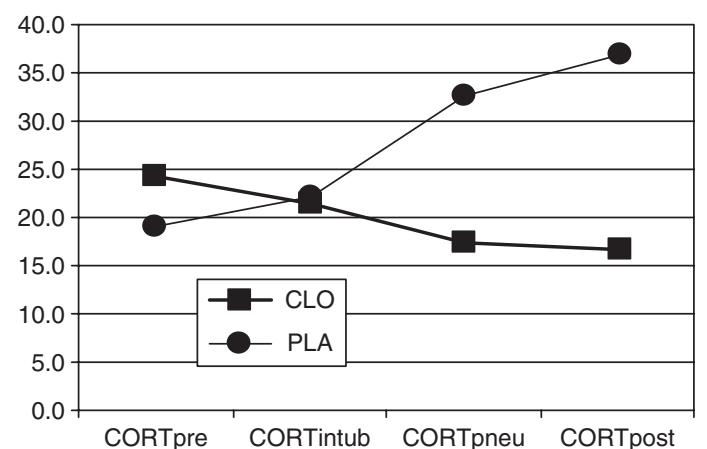


Conclusion(s): The preoperative administration of clonidine suppresses the endocrine stress response during laparoscopic cholecystectomy.

\section{AP3-9}

The effects of different doses of remifentanil pretreatment on etomidate injection pain and myoclonus

N.M. Mutlu, N. Ediz, E. Karabulut, N. Gogus

Anesthesiology and Reanimastion, Ankara Numune Trainng and Resarch Hospital, Ankara, Turkey

Background and Goal of Study: Etomidate is a hypnotic agent causing minimal histamine release and very stable hemodynamic profile. However, pain on injection and myoclonus are the most common side effects of this drug. The aim of this study is to compare the effect of different doses of remifentanil on preventing the injection pain and myoclonus after anesthesia induction with etomidate.

Materials and Methods: 120 adult patients, ASA I-II, scheduled for minor surgery procedures under general anesthesia, were randomly allocated into four groups. Patients received $1 \mu \mathrm{g} \mathrm{kg}^{-1}$ remifentanil (group $\mathrm{REM}_{1}$ ), $0.75 \mu \mathrm{g}$ $\mathrm{kg}^{-1}$ remifentanil (group $\mathrm{REM}_{2}$ ), $0.5 \mu \mathrm{g} \mathrm{kg}^{-1}$ remifentanil (group $\mathrm{REM}_{3}$ ) and isotonic saline (group $\mathrm{S}$ ) prior to administration of etomidate $0.3 \mathrm{mg} \mathrm{kg}^{-1}$. A study-blinded anesthesiologist asked patients to evaluate their pain during etomidate injection according to a 4 point scale $(0=$ no, $1=$ mild, $2=$ moderate, $3=$ severe $)$ and myoclonus was recorded with a 2 point scale $(0=$ no, $1=$ present). Data were analysed using Chi-square test, with $p<0.05$ considered statistically significant.

Results and Discussions: Data are shown in the table

\begin{tabular}{llrrrrr}
\hline & & REM $_{1}$ & REM $_{2}$ & REM $_{3}$ & \multicolumn{1}{l}{$\mathrm{S}$} & $\mathrm{p}$ \\
\cline { 3 - 7 } Myoclonus $(\mathrm{n})$ & & 0 & 0 & 5 & 4 & $0.031^{\star}$ \\
\hline injection pain $(\mathrm{n})$ & no & 40 & 36 & 27 & 21 & $0.000^{\star}$ \\
& Mild & 0 & 0 & 1 & 11 & \\
& Moderate & 0 & 4 & 10 & 6 & \\
& Severe & 0 & 0 & 2 & 2 & \\
\hline
\end{tabular}

${ }^{*} p<0.05$

When compared with the REM 3 and $S$ groups, $R E M_{1}$ and $R E M_{2}$ showed less frequency and severity of pain $(p<0.05)$. Injection pain was not observed in group $R E M_{1}$ and there was no statistically difference between groups $R_{E M}$ and $\mathrm{REM}_{2}$ with regard to injection pain.

Conclusion(s): $1 \mu \mathrm{g} \mathrm{kg}^{-1}$ or $0.75 \mu \mathrm{g} \mathrm{kg}^{-1}$ doses of remifentanil would be a good alternative to prevent myoclonus and pain injection due to etomidate. Reference:

1 Kelsaka E., Karakaya D., et al. Journal of Clinical Anesthesia 2006; 18: 83-86.

\section{AP3-10}

Lower isoflurane concentrations may have a stronger effect than higher concentrations in spatial learning in mice

A.M. Valentim, H.C. Alves, I.A.S. Olsson, L.M. Antunes

Laboratory Animal Science Group, Universidade de Trás-os-Montes e Alto Douro; Instituto de Biologia Molecular e Celular, Porto, Portugal

Background and Goal of Study: Anaesthesia is a reversible process, but it could have long lasting effects because it affects the brain at several levels. Studies made in humans and rodents indicate that surgery and/or general anaesthesia can impair the cognitive functions, especially in older subjects. The objective of this study is to evaluate the effects of different levels of depth of anaesthesia with isoflurane in the spatial learning/memory of adult mice, without the influence of all the variables inherent to clinical studies.

Materials and Methods: Twenty-six males mice divided in two groups of nine and one group of eight animals were used in t-maze test 28 hours and 1 week after two different anaesthetic procedures. The protocol consisted in two different isoflurane concentrations: $0.8 \%$ and $1.8 \%$. The control group was not exposed to anaesthesia. The number of trials to achieve the criterion established to complete the t-maze task was recorded.

Results and Discussions: Twenty-eight hours after anaesthesia the performance from the group anaesthetized with $0.8 \%$ isoflurane was significantly worst compared with the control group. No significantly differences were observed in the $1.8 \%$ isoflurane group. One week after anaesthesia no differences were detected between groups.

Conclusion(s): We conclude that lower isoflurane concentrations may have a stronger effect than higher concentrations in spatial learning in mice, and it has no long term effect on t-maze memory performance.

\section{AP4-1}

Ultra-low dose of naloxone preserves morphines antinociceptive effect in PTX-treated rats: the role of glutamate transporter and glutamate metabolic enzyme R. Tsai, Y.H. Tai, C.P. Yang, C.H. Shen, C.S. Wong Graduate Institute of Medical Science, National Defense Medical Center, Taipei, Taiwan

Background and Goal Study: Morphine is less effective for treating neuropathic pains. Opioid antagonist naloxone, at ultra-low nanogram doses, enhances the analgesic effect of opioid agonists. Pertussis toxin (PTX) not only decreases the antinociceptive effect of opioid agonists, but also produces a thermal hyperalgesia. Studies have shown that glutamate transporters (GTs) play a critical role in the prevention of glutamate neurotoxicity under both physiological and pathological conditions. However, there has no study examined GTs expression by the PTX-treatment. Ultra-low dose of naloxone enhances morphine's antinociceptive effect and reduces morphine's side effects as well, the detail mechanisms, such as the involvement of GTs expression are examined.

Materials and Methods: Rats implanted two intrathecal (i.t.) catheter, with or without a microdialysis probe were used. PTX $(1 \mu \mathrm{g})$ or saline was injected via the i.t. catheter to induce hyperalgesia. The antinociceptive effect of i.t. injection of saline, naloxone $(15 \mu \mathrm{g}$, or $15 \mathrm{ng})$, and morphine $(10 \mu \mathrm{g})$ were tested on day 4 after PTX or saline injection.

Results and Discussion: After PTX i.t. injection, a significant thermal hyperalgesia was observed with an increase in excitatory amino acids (EAAs) concentration in the spinal CSF dialysates. Naloxone, at $15 \mu \mathrm{g}$, had no effect on morphine's antinociceptive effect but, at ultra-low dose, it enhanced the analgesic effect of morphine. Moreover, ultra-low dose of naloxone treatment suppressed the morphine-induced EAAs release and prevented the downregulation of GTs in PTX-treated rats.

Conclusion: We suggest that the mechanism, at least in part, of ultra-low dose of naloxone on presenting morphine's antinociceptive effect is via affecting the EAAs uptake and metabolism.

References:

1 Finn AK, Whistler JL. Neuron 2001; 32(5): 829-839.

2 Ossipov MH, Lai J, King T, et al., J Neurobiol 2004; 61(1): 126-148.

3 Gilron I, Bailey JM, Tu D, et al., N Engl J Med 2005; 352(13): 1324-1334.

\section{AP4-2}

Potentiation of $5-\mathrm{HT}_{3 \mathrm{AB}}$ receptors by small n-alcohols is caused by shifting the open-close equilibrium to the open state

D. Ruesch, A. Schuster, J. Spodeck, H. Wulf, D. Raines

Department of Anaesthesia and Critical Care, University Hospital GiessenMarburg GmbH, Marburg, Germany

Background and Goal of Study: 5-hydroxytryptamine type $3\left(5-\mathrm{HT}_{3}\right)$ receptors belong to the alcohol- and anaesthetic-sensitive family of Cys-loop ligandgated ion channels ${ }^{1}$. Potentiation of currents evoked by low concentrations of agonist and leftward shift of the agonist concentration response curve by small $\mathrm{n}$-alcohols in $5-\mathrm{HT}_{3}$ receptors is subunit dependent ${ }^{2}$. Receptor activation can be described using a linear model that consists of two steps: 1) the agonist binding step, and 2) the channel opening step. Partial agonists can be used as a tool to distinguish whether a modulator increases a receptor's sensitivity to agonist by enhancing either agonist binding affinity or channel gating efficacy ${ }^{3}$. The mechanisms of how n-alcohols potentiate $5-\mathrm{HT}_{3 A B}$ receptors are unknown.

Materials and Methods: Currents from Xenopus oocytes expressing recombinant human $5-\mathrm{HT}_{3 A B}$ receptors were recorded using the twoelectrode voltage-clamp technique. The effects of several small $n$-alcohols (C2-C5) at $0.5,1,2$, and 4 times their anaesthetizing concentration on peak currents elicited by receptor-saturating concentrations $(3 \mathrm{mM})$ of the partial agonist dopamine (DA) were studied. A Student's t-test was used to compare DA-evoked peak currents in the absence and presence of alcohols. $\mathrm{P}<0.05$ was considered statistically significant.

Results and Discussions: All alcohols potentiated $(p<0.01)$ DA-evoked peak currents at the four concentrations studied ( $n=5-10$ cells per concentration in every alcohol). Enhancement of peak currents was alcohol concentration dependant in every alcohol. Potentiation of DA-evoked peak currents by equianaesthetic concentrations of $n$-alcohols differed among the studied $\mathrm{n}$-alcohols with enhancement being strongest in the following order: propanol $>$ butanol $>$ pentanol $>$ ethanol.

Conclusion(s): Enhancement of the 5- $\mathrm{HT}_{3 A B}$ receptor function by small $\mathrm{n}$-alcohols is caused by facilitating receptor gating without altering agonist 
binding affinity. Moreover, the magnitude of potentiation of agonist evoked currents depends on the size of the n-alcohol.

References:

1 Jenkins et al. (1996) Br J Pharmacol 117(7): 1507-15.

2 Stevens et al. (2005) J Pharmacol Exp Ther 314: 1696-1703.

3 Rüsch et al. (2004) J Biol Chem 279(20): 20982-92.

\section{AP4-3}

Antinociceptive activity of vigabatrin in chronic neuropathic pain in the rat

M. Czuczwar, J. Kis, P. Plaza, K. Przesmycki

II Department of Anaesthesiology and Intensive Care, F. Skubiszewski Medical University in Lublin, Poland, Lublin, Poland

Background and Goal of Study: The aim of study was to assess antiallodynic and anti-hyperalgesic activity of vigabatrin (VGB), and its effect on the duration of mechanical allodynia and thermal hyperlgesia in Seltzer model of chronic neuropathic pain in the rat.

Materials and Methods: Experiments were performed on male Wistar rats, initially weighing 250-300 g. The experimental groups consisted of 14 animals at the beginning of experiment. VGB were administered intraperitoneally once a day at doses of 50,100 , and $200 \mathrm{mg} / \mathrm{kg}$, starting from the second day after the operation, one hour prior to behavioral tests. Mechanical allodynia and thermal-hyperalgesia, were assessed every second day after surgery, with the use of von Frey filaments and hot plate test, respectively.

Results and Discussion: Nerve injury resulted in exhibited foot withdrawal responses to von Frey hair and decreased latency to noxious thermal stimuli 2-16 days after surgery. The anti-allodynic activity of VGB was significant at $200 \mathrm{mg} / \mathrm{kg}$, however, no effect on the duration of mechanical allodynia was observed. VGB at doses of 100 and $200 \mathrm{mg} / \mathrm{kg}$ significantly elevated the nociceptive thresholds in hot plate test. In no case, there was a complete reversal of thermal hyperalgesia in the VGB-treated group.

Conclusion: VGB proved to be more efficient in relieving thermal hyperalgesia than mechanical allodynia in the Seltzer model of chronic neuropathic pain.

\section{AP4-4}

\section{Protective effect of nefopam on seizure activity in mice}

M. Czuczwar, K. Czuczwar, J. Kis, A. Kolacz, K. Przesmycki

II Department of Anaesthesiology and Intensive Care, F. Skubiszewski Medical University in Lublin, Poland, Lublin, Poland

Background and Goal of Study: Nefopam is a centrally acting non-opioid analgesic with not completely understood mechanism of action. Adverse effects associated with therapeutic use and overdose of nefopam are mainly associated with central nervous system and include hallucinations, cerebral oedema and convulsions. To the best of our knowledge, no research was conducted on influence of nefopam on seizure activity nor possible interactions between nefopam and antiepileptic drugs. Therefore, the aim of this study was to assess the effect of nefopam administration on electrical threshold and its influence on protective activity of antiepileptic drugs in the maximal electroshock test in mice.

Materials and Methods: Experiments were performed on male Swiss mice, weighing $20-25 \mathrm{~g}$. The following antiepileptics were used: valproate, carbamazepine, phenobarbital, and phenytoine. The convulsive threshold was evaluated as CS50, which is the current strength (in $\mathrm{mA}$ ), necessary to produce tonic hindlimb extension in $50 \%$ of the animals tested. In order to estimate the anticonvulsant ED50 values (50\% effective anticonvulsant dose) of studied antiepileptics (given alone or in combination with nefopam) mice were pretreated with different doses of the drugs and then challenged with maximal electroshock (25 mA)

Results and Discussion: Nefopam significantly elevated the electric seizure threshold at the dose of $5 \mathrm{mg} / \mathrm{kg}$, whilst the dose of $1 \mathrm{mg} / \mathrm{kg}$ had no effect on seizure activity. The protective activity of studied antiepileptics were significantly enhanced by co-administration of nefopam at the dose of $5 \mathrm{mg} / \mathrm{kg}$. Nefopam at the dose of $1 \mathrm{mg} / \mathrm{kg}$ had no effect on the protective activity of studied drugs.

Conclusion: Nefopam exerts anticonvulsive effect when given alone and potently enhances the protective activity of valproate, carbamazepine, phenobarbital, and phenytoine in the experimental seizure models in mice.

\section{AP4-6}

Do parecoxib sodium and ketoprofen modulate the immune response and therapeutic outcome after abdominal hysterectomy

B. Tablov, V. Tablov, G. Stavreva, J. Popov, E. Konova

Anesthesiology and Intensive Care, Medical University - Pleven, Pleven, Bulgaria
Background and Goal: Surgery is associated with immune alterations, which are the combined result of tissue damage, anesthesia, postoperative pain and psychological stress (1). There are insufficient empirical data on the effects of postoperative pain management on immune function. Previous studies concluded that some NSAID (ketorolac, diclofenac) have immunomodulatory effect, affecting the therapeutic outcome (2); there is a lack of experience about the influence of COX-2 selective inhibitors on postoperative immune response. The aim of our study was to determine how the use of selective COX-2 inhibitor parecoxib sodium for postoperative analgesia after abdominal hysterectomy affected the immune response and to compare such modulation with the effect of ketoprofen.

Materials and Methods: Forty patients who underwent abdominal hysterectomy were randomly divided into two groups: one in which the postoperative pain was managed with opioid and IV parecoxib sodium, and in the other group - with opioid and IV ketoprofen. Blood samples were collected at three points: before surgery, 24 and $72 \mathrm{~h}$ postoperatively. Plasma was separated and frozen; the concentrations of cytokines were evaluated via enzyme-linked immunosorbent assays. Temperatures, some populations of T-lymphocytes (CD4, CD8, total CD3 and activated CD3) were measured. Postoperative pain was assessed by VAS; sedation - by Ramsay sedation score.

Results and Discussions: IL-6 concentrations increased, reaching peak levels at $24 \mathrm{~h}$ postoperatively and decreased to the initial levels at $72 \mathrm{~h}$ in both groups. At $24 \mathrm{~h}$ and $72 \mathrm{~h}$, the IL-10 concentration was not significantly higher than before surgery in both groups. The pain intensity, sedation and side effects were similar in two groups of women.

Conclusion: Selective COX-2 inhibitor parecoxib sodium and traditional NSAID ketoprofen as components of postoperative analgesic strategy have demonstrated similar modulation of cytokine response. Parecoxib sodium may have beneficial effect on therapeutic outcome after abdominal hysterectomy. References:

1 Beilin B, Shavit Y, Trabekin E, et al. Anesth Analg 2003; 97: 822-827.

2 Kim MH, Hahm TS. Clin J Pain 2001; 17: 72-77.

\section{AP4-7}

\section{Rekombinant NMDA receptor signaling is influenced by intracellular injection of DAMGO}

C.A. Gronwald, Helen. Terbille, Robert M. Radke, Vladimir. Vegh, Klaus. Hahnenkamp

Department of Anaesthesiology and Intensive Care, University Hospital Muenster, Muenster, Germany

Background and Goal of Study: DAMGO is a synthetic opioid peptide widely used as a selective $\mu$ opioid receptor agonist in pain research. Previous research on DAMGO effects on NMDA receptor signaling yielded heterogenous results, describing inhibiting as well as potentiating effects (1). The goal of this study was to investigate these opposite effects and to generate dose-response curves as a first step towards clarification of the involved mechanismens.

Materials and Methods: After approval of the Animal and Use Committee human NR1A/NR2B (1:5 weight ratio) NMDAR subunits were recombinantly expressed in Xenopus laevis oocytes in the absence of opioid receptors. Cells were incubated in DAMGO $\left(10^{-5} \mathrm{M}\right)$ or injected with DAMGO $10^{-4} \mathrm{M}$ (ic concentration $\sim 10^{-5} \mathrm{M}$ ). After 10 minutes inward currents induced by glutamate $\left(10^{-9}-10^{-1} \mathrm{M}\right)$ in the presence of the co-agonist glycin $\left(10^{-5} \mathrm{M}\right)$ were measured by 2 -electrode voltage clamp and compared to control measurements of the same cells.

Results and Discussions: Our results show a non-competitive potentiation of glu/gly induced NMDAR currents after injection of DAMGO while extracellular incubation for 10 minutes lead to an inhibition resembling a competitive curve. (curve not shown).

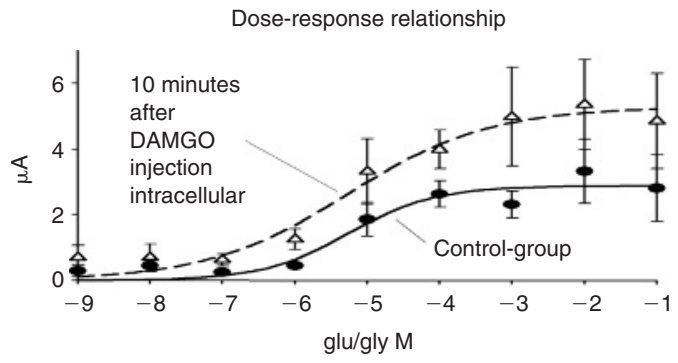

Conclusion(s): The dose-response relationship suggests a non-competitive mechanism of intracellular potentiation. We hypothesize that PKC might be involved in this step (2). The apparent opioid receptor independence of this effect should be considered on DAMGO and NMDAR interaction in future.

References:

1 Mao J, Brain Res Rev. 1999 Nov; 30(3): 289-304.

2 Grosshans, DR, Browning MD, J.Neurochem. 2001 Feb; 76(3): 737-44. 


\section{AP4-8}

Methylnaltrexone Potentiates Inhibition of VEGF-Induced Angiogenesis by 5-Fluorouracil (5-FU) and Bevacizumab

J. Moss, P.A. Singleton, J.G.N. Garcia

Anesthesia and Critical Care, University of Chicago, Chicago, USA

Background and Goal of Study: It has been recently shown that opioids cause endothelial cell (EC) proliferation and migration (EC-PM), two key components in tumor-associated angiogenesis. Further, methylnaltrexone (MNTX), a peripheral mu opioid antagonist being developed for opioid-induced constipation in advanced illness and in phase 3 trials for postoperative ileus, has been shown to inhibit opioid-induced EC-PM by VEGF receptor transactivation. ${ }^{1}$ In that study, MNTX had effects beyond the receptor level, suggesting a possible potentiation of chemotherapeutic agents. Because many cancer patients receive combinations of drug treatments which include 5-fluorouracil (5-FU) and bevacizumab (B), we examined whether MNTX had a synergistic effect with these chemotherapeutic agents on angiogenic events.

Materials and Methods: Human pulmonary microvascular EC proliferation assay was performed as previously described. HPMVEC $\left(\sim 1 \times 10^{4}\right.$ cells/well) were plated in serum-free media containing various concentrations of 5-FU, MNTX or both ( $1 \mathrm{nM}$ to $100 \mu \mathrm{M})$ to the upper chamber and $100 \mathrm{nM}$ VEGF was added to the lower chamber. Cells were allowed to migrate for $18 \mathrm{hrs}$. Results and Discussions: MNTX inhibited EC proliferation with an IC50 of $\sim 100 \mathrm{nM}$. Adding $100 \mathrm{nM}$ MNTX to EC shifted the IC50 of 5-FU from $\sim 5 \mu \mathrm{M}$ to $\sim 7 \mathrm{nM}$ while adding $10 \mathrm{nM}$ MNTX shifted the IC50 of 5 -FU from $\sim 5 \mu \mathrm{M}$ to $\sim 40 \mathrm{nM}$. Further, adding $50 \mathrm{ng} / \mathrm{ml}$ MNTX shifted the IC50 of B on inhibition of EC migration from $\sim 25 \mathrm{ng} / \mathrm{ml}$ to $\sim 6 \mathrm{ng} / \mathrm{ml}$ while adding $10 \mathrm{ng} / \mathrm{ml}$ MNTX shifted the IC50 of B from $\sim 25 \mathrm{ng} / \mathrm{ml}$ to $\sim 9 \mathrm{ng} / \mathrm{ml}$. Unlike MNTX, naltrexone did not exhibit synergy of 5-FU or B.

Conclusion(s): Taken together, these results indicate that in addition to blocking opioid-induced angiogenesis, MNTX is synergistic with 5-FU and B on EC-PM. Agents that reduce the therapeutic concentration of these drugs can have clinical utility by reducing cost and toxicity.

Reference:

1 Singleton PA, Lingen MW, Fekete MJ, et al. Microvasc Res 2006; 72: 3-11.

\section{AP4-9}

\section{Cannabinoid 1 receptor antagonist reduces THC/propofol interaction}

P. Brand, P. Meybohm, J. Scholz, C. Werner, B. Bein

Department of Anaesthesiology and Intensive Care Medicine, University Hospital of Schleswig-Holstein, Campus Kiel, Kiel, Germany

Background and Goal of Study: $\Delta 9$-Tetrahydrocannabinol (THC) is an exogen cannabinoid that acts as an agonist at both primary cannabinoid receptors (CB 1 and CB 2). It has been reported that THC reduces propofol induced sedation. The mechanism of this interaction however, remains unclear. Thus, we studied the interaction of propofol and THC in presence of a specific cannabinoid 1 receptor antagonist, AM 251 in a mouse model.

Materials and Methods: Twenty SV 129 male mice received an intraperitoneal injection of $75 \mathrm{mg} / \mathrm{kg}$ propofol, $50 \mathrm{mg} / \mathrm{kg}$ THC and $20 \mathrm{mg} / \mathrm{kg}$ AM 251 with permission and according to the state laws of animal safety. Propofol injection was defined as time point 0 . THC was injected at -15 min and AM 251 at $-25 \mathrm{~min}$. Sedation was monitored with a rotating rod with $16 \mathrm{rpm}$. Mouse resting on rotating rod for $60 \mathrm{~s}$ was defined as no sedation.

Results and Discussion: Neither $50 \mathrm{mg} / \mathrm{kg}$ THC nor $20 \mathrm{mg} / \mathrm{kg}$ AM 251 alone exerted an effect on sedation. $75 \mathrm{mg} / \mathrm{kg}$ propofol induced sedation $1 \mathrm{~min}$ after injection and reached a maximum after $2.5 \mathrm{~min}$ with $17.1 \mathrm{~s}$ on the rotating rod. Thereafter depth of sedation decreased constantly and was no longer present after $20 \mathrm{~min}$.

The sedative effect of propofol was completely abolished in the presence of THC. Mice remained on the rotating rod for $60 \mathrm{~s}$ throughout the experiment. After injection of $20 \mathrm{mg} / \mathrm{kg}$ of the THC antagonist AM 251 in presence of THC, propofol sedation was reestablished from $1 \mathrm{~min}$ until $12.5 \mathrm{~min}$ post injection of propofol. Maximum sedation was reached after 2.5 until $7.5 \mathrm{~min}$ post injection with rotating rod times of $46.6 \mathrm{~s}$ and $45.4 \mathrm{~s}$, respectively. Thereafter sedation decreased and was abolished after $15 \mathrm{~min}$.

Conclusion: THC reduces propofol sedation as reported before. Administration of a CB 1 antagonist reestablished propofol sedation in presence of THC. The results of this study indicate that the interaction of THC and propofol is at least in part due to effects on the CB 1 receptor.

\section{AP4-10}

Effect of ketamine on IL-6 plasmatic levels in patients undergoing hepatectomies with Pringle's maneuver

F. Bonofiglio, S. Pérez, F. Spak, S. Salgado, J. Andrade

Anesthesiology, Italian Hospital, Buenos Aires, Argentina
Background and Goal: It has been studied the role of ketamine in diminishes the synthesis of IL- 6 and therefore, the plasmatic level. The aim was to evaluate if ketamine reduce IL-6 synthesis and plasmatic level in patients undergoing hepatectomy with Pringle's maneuver.

Materials and Methods: We included 31 patients after the informed consent. They were randomly assigned to: ketamine group (KG) or placebo group (PG). The study was prospective and double blinded. IL-6 plasmatic level was evaluated at different times: basal, 4,12 and 24 hours and at 3 and 5 days after the surgery. We applied the intention to treat principle and Mann Whitney test to evaluate significant differences.

Results: The blood was centrifuged after the extraction and it was freezed until performing the ELISA determinations. We obtained the following results $(X \pm S D):$

\begin{tabular}{lcc}
\hline Time & Ketamine $(\mathrm{pg} / \mathrm{ml})$ & Placebo $(\mathrm{pg} / \mathrm{ml})$ \\
\hline Basal & $6.54 \pm 6.5$ & $5.28 \pm 5.1$ \\
4 hs. & $162.07 \pm 149$ & $164.79 \pm 150.6$ \\
12 hs. & $132.43 \pm 137.2$ & $175.44 \pm 156.6$ \\
24 hs. & $108.32 \pm 85.2$ & $122.76 \pm 107.1$ \\
3 days & $79.38 \pm 59.7$ & $97.83 \pm 101.1$ \\
5 days & $43.31 \pm 32.7$ & $51.49 \pm 68.1$ \\
\hline
\end{tabular}

Although the data of the ketamine group is less than the placebo, there is not significant difference.

Conclusion: In our study ketamine does not appear to reduce the plasmatic level of IL- 6 in patients undergoing hepatectomy with Pringle's maneuver. References:

1 Chouker A. British Journal of Anaesthesia 93 (2): 204-11 (2004).

2 Faybik P. Transplantation Proceedings, 35, 3019-3021 (2003).

\section{AP5-1}

Maturational pharmacokinetics of single intravenous bolus administration of propofol during childhood

K. Allegaert, J. de Hoon, G. Naulaers, R. Verbesselt, I. Murat

Neonatal Intensive Care Unit, University Hospital Gasthuisberg, Leuven, Belgium

Background and Goal of Study: to describe maturational propofol pharmacokinetics following single intravenous bolus administration during childhood.

Materials and Methods: recently reported observations following intravenous bolus administration of propofol in neonates $(n=9)$ were combined with pharmacokinetic estimates in toddlers $(n=12)$ and young children $(n=10)(r e f 1,2,3)$. Data were reported by median and range. Mann-Whitney $U$ test or linear correlation was used to analyse pharmacokinetic findings. Results and Discussions: Concentration-time profiles obtained were interpreted by two-stage analysis, three compartment open model in 31 patients with a median weight of 11.2 (range $0.91-24$ ) $\mathrm{kg}$ and median postmenstrual age (PMA) of 108 (range 27-405) weeks. Median clearance (Cl) was 36.8 (range $3.7-78.1$ ) $\mathrm{ml} / \mathrm{kg} / \mathrm{min}$ or 67.4 (range $2.04-135.8$ ) $\mathrm{ml} / \mathrm{kg}^{0.75} / \mathrm{min}$. Median apparent volume of distribution at steady state $\left(\mathrm{V}_{\mathrm{ss}}\right)$ was $7.6(1.33-15.6) \mathrm{L} / \mathrm{kg}$ and median final serum elimination half life $\left(t_{1 / 2 \text { gammal }}\right)$ was 377 (range 27-1134) minutes. Median clearance was significantly lower in neonates compared to toddlers and older children $(p<0.01)$ and these differences remained significant after allometric scaling $\left(\mathrm{ml} / \mathrm{kg}^{0.75} / \mathrm{min}\right)$. A significant correlation between $\mathrm{V}_{\mathrm{ss}}$ and PMA $(r=0.61,95 \% \mathrm{Cl} 0.32-0.8, \mathrm{p}<0.004)$ was observed.

Conclusion(s): Propofol disposition is significantly different in neonates compared to toddlers and young children, reflecting both ontogeny and differences in body composition. Based on the reduced clearance of propofol, accumulation and longer recovery time are more likely to occur in neonates.

References:

1 Allegaert K. Br J Anaesth (submitted).

2 Murat I. Anesthesiology 1996; 84: 526-532.

3 Saint-Maurice C. Br J Anaesth 1989; 63: 667-670.

\section{AP5-2}

Individual variances of body composition in patients with same sex, height and weight can affect the concentrations of plasma propofol using TCI

Y.G. Choi, S.H. Lim, C.M. Shin, Y.J. Kim, S.H. Cheong

Department of Anesthesiology, Paik Hospital Inje University, Busan, Republic of Korea

Background: Height and weight are two important variables to determine infusion rate of propofol according to the estimated lean body mass (LBMe) using target controlled infusion (TCl). But there are individual differences in body composition of patients with same height and weight, and those variances 
can make errors to estimate central volume of distribution and clearance of propofol. The concentrations of plasma propofol would be affected by the differences between LBMe and actual lean body mass (LBMa).

Materials and Methods: 65 Korean women(age $42 \pm 8$ yrs) scheduled for low abdominal surgery received a combined $\mathrm{TCl}$ of propofol and remifentanil. Target concentration of plasma propofol was $3 \mu \mathrm{g} / \mathrm{ml}$. Propofol plasma concentration, hemodynamic variables and bispectral index score (BIS) were determined at 60 minutes after induction. We calculated LBMe in according to Schnider model formula, checked LBMa with body composition analyzer (Inbody 3.0, Biospace, Korea) and subtracted LBMa from LBMe (delta lean body mass; LBMd). Correlation analyses were taken between LBMd and each variable.

Results: LBMd showed positive correlation with plasma concentrations of propofol (Figure). There were no significant correlations between LBMd and blood pressure, heart rate, BIS, respectively. Mean plasma concentration of propofol $(4.1 \pm 1.08 \mu \mathrm{g} / \mathrm{ml})$ was higher than target concentration $(3 \mu \mathrm{g} / \mathrm{ml})$.

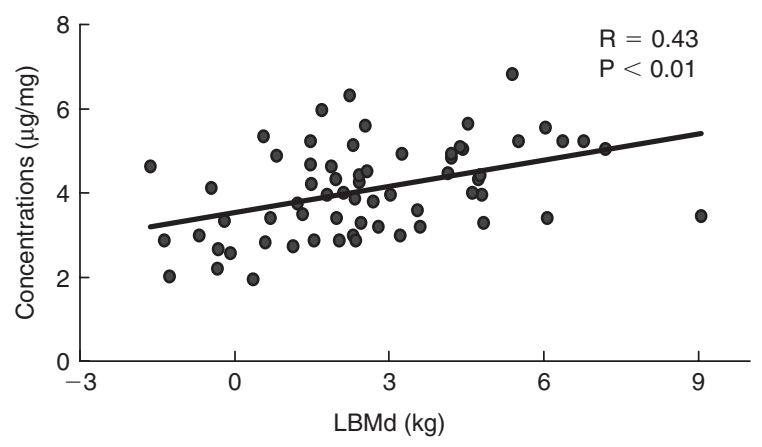

Conclusions: Individual variances of body composition in patients with same sex, height and weight can affect the concentrations of plasma propofol using TCl. In addition, Schnider parameter set underestimates plasma concentration of propofol in Korean women.

\section{AP5-3}

Pharmacokinetics of Rocuronium in patients with Duchenne muscular dystrophy

T. Munster, H. Ihmsen, V. Viethen, H. Schmitt

Department of Anaesthesiology, University Hospital Erlangen, Erlangen, Germany

Background and Goal of Study: In patients with Duchenne muscular dystrophy (DMD) time course of rocuronium(ROC)-induced neuromuscular block (NMB) has been found to be different from healthy subjects. ${ }^{1,2}$ The aim of this study was to clarify whether pharmacokinetics or pharmacodynamics are responsible for this different time course.

Materials and Methods: After approval of the local Ethics Committee and signed consent 6 boys with DMD (age: $15 \pm 2$ yrs, weight: $59 \pm 10 \mathrm{~kg}$ ) were studied. Following ROC $0.3 \mathrm{mg} / \mathrm{kg}$ NMB was measured according to a standard protocol at the adductor pollicis muscle using acceleromyography. Blood samples were drawn from an arterial line 2, 4, 7, 10, 15, 20, 30, 60, 90, 120 and 240 min after administration of ROC and analyzed by HPLC.

Results and Discussions: The measured concentrations of ROC could be described by a two-compartment model (table: mean \pm SD and range, respectively).

\begin{tabular}{lll}
\hline & DMD & $\begin{array}{l}\text { Healthy subjects } \\
\text { Literature }^{3}\end{array}$ \\
\hline $\mathrm{Vc}(\mathrm{ml} / \mathrm{kg})$ & $69 \pm 20$ & $39-77$ \\
$\mathrm{Vss}(\mathrm{ml} / \mathrm{kg})$ & $281 \pm 82$ & $207-292$ \\
$\mathrm{Cl}(\mathrm{ml} / \mathrm{min} / \mathrm{kg})$ & $3.7 \pm 2.3$ & $2.9-5.4$ \\
$\mathrm{~T} 1 / 2 \alpha(\mathrm{min})$ & $3.6 \pm 1.6$ & $1.2-2.2$ \\
$\mathrm{~T} 1 / 2 \beta(\mathrm{min})$ & $84 \pm 53$ & $11.1-17.2$ \\
$\mathrm{~T} 1 / 2 \gamma(\mathrm{min})$ & & $61-94$ \\
\hline
\end{tabular}

Conclusion: The pharmacokinetics of ROC in DMD patients were in agreement with those described for healthy subjects. Therefore, in DMD patients the altered time course of NMB is most likely caused by different pharmacodynamics.

References:

1 Wick et al. Anesthesiology 2005; 102: 915-9.

2 Muenster et al. Peadiatr Anaesth 2006; 16: 840-5.

3 Vermeyen et al. Br J Anaesth 2003; 90: 183-8.

\section{AP5-4}

EEG Entropy Monitoring: pharmacokinetic and dynamic modelling

A. Castro, N. Bressan, L. Antunes, C.S. Nunes

Matematica Aplicada, Faculdade de Ciencias da Universidade do Porto, Porto, Portugal

Background and Goal of Study: The entropy module is an EEG based monitor for humans, that uses the Shannon entropy (Ent) [1] as an indicator of depth of anaesthesia (DOA). In this study Ent was applied to EEG collected in rats anesthetized to evaluate how it responds to variations in the propofol level, and model the drug effect on DOA.

Materials and Methods: Four male Wistar rats weighing $477 \pm 11 \mathrm{~g}$ (mean \pm SD) were used. The lateral tail vein was cannulated and anaesthesia maintained with a propofol infusion $\left(62.5 \mathrm{mg} \cdot \mathrm{kg}^{-1} \cdot \mathrm{h}^{-1}\right)$ for electrode implantation and endotracheal intubation. Raw EEG was recorded during different propofol infusions for each rat. The Ent algorithm was applied to the EEG records to obtain the entropy index [0-100]. Two and three-compartmental models merged with a Hill equation were used to translate the relation between the propofol infusions and Ent signal. Least squares was used to identify the model parameters and the relative mean square error was used to compare the performance of the models. [2].

Results and Discussions: Models adjusted well to Ent, but the 3-compartmental (Fig.1) model performed better in all rats. The 3-compartmental model for each rat was applied to other rat data, testing its prediction abilities. For each rat the best performance was obtained with its own adjusted model. The 3-compartmental model of rat 2 adjusted well to all other rats.
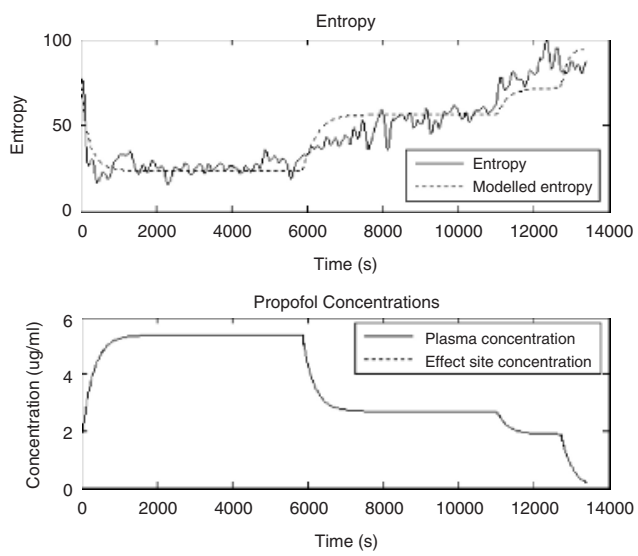

Fig.1 Real and modelled Ent values and modelled propofol concentrations for rat 4 (3-compartmental model)

Conclusion(s): Ent is a good indicator of depth of anaesthesia in rats, responding adequately to changes in the hypnotic infusion rates. Models identified for one set of data can be used in data from other animals with reasonable performance.

Reference:

1 IEEE Trans on Inf Theory 1992, 38: 713-718.

2 J Neurosurg Anesthesiol 2006, 18: 333-334.

\section{AP5-5}

Population pharmacokinetics and pharmacodynamics of short term etomidate infusion in healthy volunteers

T.H. Han, M. Todd

Department of Anesthesia, University of lowa Carver College of Medicine, lowa City, USA

Background and Goal of Study: Etomidate is used as a fast-acting hypnotic to induce anesthesia in patients with a poor cardiovascular reserve. Bispectral index (BIS) has been suggested to be a measure of the depth of anesthesia and correlates well with the level of consciousness. This study examined the population pharmacokinetics (PK) and pharmacodynamics (PD) of etomidate during and after a brief infusion, aiming at predetermined clinical endpoints.

Materials and Methods: Eighteen middle aged adults, with ASA physical status I or II, scheduled for elective surgery, were included. Etomidate $2 \mathrm{mg} / \mathrm{ml}$ was administered at $25 \mathrm{ml} / \mathrm{min}$ until loss of consciousness (LOC). The patients 
were allowed to recover spontaneously until they regained consciousness, as determined by response to verbal command. BIS was recorded every minute and arterial blood samples were collected simultaneously. The plasma concentrations were measured with high performance liquid chromatograhy (HPLC). Nonlinear mixed effect modeling (NONMEM) was used for population PK and sigmoid Emax for PD analysis.

Results and Discussions: The induction dose for LOC was $0.38 \mathrm{mg} / \mathrm{kg}$. The time from beginning of drug infusion to $\mathrm{LOC}$ took approximately $3.5 \mathrm{~min}$ utes. The whole study took approximately 8.5 minutes from the start of drug infusion to the recovery of consciousness. BIS and etomidate plasma concentrations were $50 \pm 17$ and $1519.4 \pm 458.8 \mathrm{ng} / \mathrm{ml}$ at LOC, and $75 \pm 16$ and $239.6 \pm 104.5 \mathrm{ng} / \mathrm{ml}$ at recovery, respectively. PK parameters were $\mathrm{t}_{1 / 2 \alpha}=1.1 \mathrm{~min}, \quad \mathrm{t}_{1 / 2 \beta}=1.9 \mathrm{~min}, \quad \mathrm{t}_{1 / 2 \gamma}=106.5 \mathrm{~min}, \quad \mathrm{k}_{21}=0.36 \mathrm{~L} / \mathrm{min}$, $\mathrm{k}_{31}=0.009 \mathrm{~L} / \mathrm{min}, \mathrm{V}_{1}=6.43 \mathrm{~L}, \mathrm{~V}_{\text {area }}=426 \mathrm{~L}, \mathrm{Cl}=2.77 \mathrm{~L} / \mathrm{min}$. PDs' were $k_{e o}=0.40 \mathrm{~L} / \mathrm{min}, \mathrm{EC}_{50}=1.0 \mathrm{ug} / \mathrm{mL}, \mathrm{E}_{0}=94, \mathrm{E}_{\max }=94$, and $\gamma=1.2$. The performance error for etomidate concentration was $0.14 \pm 0.99$ (typical prediction) and $-0.03 \pm 0.40$ (individual prediction) and for BIS scores, $-0.09 \pm 1.00$ and $-0.001 \pm 0.13$, respectively. Mild myoclonus $(n=12)$ had no significant impact on BIS determined by observation.

Conclusions: When compared with other reports, our PK parameters demonstrated a shorter $t_{1 / 2}$, a larger $V d$, and an increased $C /$ with significant interindividual differences. The PD showed a large interindividual variability. These discrepancies might be due to relatively short sampling time. Further study will be warranted to improve the final model performance on clinical application.

\section{AP5-6}

Pain on injection does not affect the effect site concentration of propofol at loss of consciousness

S. Hagihira, H. Yamanaka, O. Nagata, M. Ozaki, T. Mashimo

Anesthesiology, Osaka University Graduate School of Medicine, Suita City, Japan

Background and Goal of Study: We previously reported ${ }^{1)}$ that fentanyl deceased the effect site concentration $(\mathrm{Ce}$ ) of propofol at loss of consciousness (LOC). When Ce of fentanyl was kept at 0,1 and $2 \mathrm{ng} / \mathrm{ml}, \mathrm{Cp} 50$ of $\mathrm{Ce}$ of propofol at LOC was $1.81 \mu \mathrm{g} / \mathrm{ml}, 1.51 \mu \mathrm{g} / \mathrm{ml}$ and $1.04 \mu \mathrm{g} / \mathrm{ml}$, respectively (each $\mathrm{n}=20$ ). Then we made a hypothesis that this effect of fentanyl was due to the inhibition of the pain on injection of propofol during induction of anesthesia. Here we investigated the effect of administration of lidocaine just before induction on $\mathrm{Ce}$ of propofol at $\mathrm{LOC}$ and compared our previous data. Materials and Methods: After IRB approval and obtained informed consent from the participants, we enrolled 20 patients (either gender; 21-76 yr; American Society of Anesthesiologists physical status I or II) who underwent elective surgery in the current study. Premedication was not applied. Just before we started the infusion of propofol, we administered $0.5 \mathrm{mg} / \mathrm{kg}$ of $2 \%$ lidocaine. Then using TCI (target controlled infusion) system, we controlled the increase in propofol Ce to $0.2-0.4 \mu \mathrm{g} / \mathrm{ml}$ per minute. At LOC, defined by lack of response when the patient was addressed by name and loss of eyelash reflex, we recorded Ce defined here as $\mathrm{P}_{-}$sleep. Cp50 was calculated from the non-linear regression to sigmoid curve. We also asked the patients of the severity of the pain on injection and recorded it.

Results and Discussions: There were no significant differences between the current group and our previous groups. In the current study, Cp50 of P_sleep was $1.82 \mu \mathrm{g} / \mathrm{ml}$, and the regression curve was almost identical to our previous data obtained from the patients without pretreatment with lidocaine. The number of patients who felt the pain on injection was 4 (mild), 2 (moderate) and 0 (severe), respectively. Although pretreatment of lidocaine markedly reduced the pain on injection, it had no effect on P_sleep of propofol.

Conclusion(s): The effect of fentanyl on Ce of propofol at LOC did not due to the inhibition of the pain on injection. It might be the synergic effect of fentanyl and propofol on the central nervous system.

Reference:

1 http://www.eurosiva.org/Archive/Vienna2005/Posters/EuroSIVA2005_abst.htm

\section{AP5-7}

\section{Determination of the optimum ke0 value for use with the Marsh PK model for propofol}

A.J. Thomson, A.F. Nimmo, J.B. Glen

Department of Anaesthesia, Critical Care and Pain Medicine, Royal Infirmary of Edinburgh, Edinburgh, United Kingdom

Background and Goal of Study: Effect-site target-controlled infusion (TCl) of propofol requires the use of a blood /brain equilibration rate constant $\left(\mathrm{k}_{\mathrm{e} 0}\right)$. A wide range of $k_{e 0}$ values has been reported for the Marsh PK model $(0.2-1.2 / \mathrm{min})^{1,2}$. This study aimed to determine the optimum $\mathrm{k}_{\mathrm{e} 0}$.
Materials and Methods: Six groups of ASA I and II patients were studied in sequence. Patients were sedated with propofol using a customised Graseby 3500 pump for effect-site $\mathrm{TCl}$ using the Marsh model. For each group, the pump was programmed with a different $\mathrm{k}_{\mathrm{e} 0}$ value $(1.2,0.8,0.7,0.6,0.5$ or 0.2 /min). The initial target concentration $\left(\mathrm{C}_{\mathrm{eT}}\right)$ was $0.6 \mathrm{mcg} / \mathrm{ml}$. This was increased in $0.2 \mathrm{mcg} / \mathrm{ml}$ increments each time the calculated concentration $\left(\mathrm{C}_{\text {eCALC }}\right)$ had reached the target until a sedation score (OAA/S $)^{3}$ of 3 was reached. The $\mathrm{C}_{\mathrm{eT}}$ was then held constant for $15 \mathrm{~min}$. If the $\mathrm{k}_{\mathrm{e} 0}$ was appropriate, the level of sedation should then have remained stable. An incorrect $k_{e 0}$ would lead to deepening or lightening sedation. Degree of sedation was assessed by measuring visual reaction time (VRT). The VRT after $15 \mathrm{~min}$ was used to classify the level of sedation as stable, deepening ( $>23 \%$ increase in VRT), or lightening ( $>25 \%$ decrease). These criteria were based on the changes in VRT which occurred when $\mathrm{C}_{\text {eCALC }}$ was changed by $0.2 \mathrm{mcg} / \mathrm{ml}$ in a pilot study. The proportion of patients with deepening levels of sedation was noted for each group with 'steady' contributing 0.5 to a group score. Probit analysis was used to calculate the optimum $\mathrm{k}_{\mathrm{e} 0}$.

Results and Discussion: 64 patients (30M, 34F) aged $41 \pm 11$ (21-65) (mean; $\mathrm{SD}$; range) were studied. Inter-individual variation in $\mathrm{k}_{\mathrm{e} 0}$ was marked In general, sedation deepened in more patients in the higher $\mathrm{k}_{\mathrm{e} 0}$ groups than in the lower $\mathrm{k}_{\mathrm{e} 0}$ groups. We calculated a median $\mathrm{k}_{\mathrm{e} 0}$ of $0.59 / \mathrm{min}(95 \% \mathrm{Cl} 0.36-0.76)$, $\mathrm{p}=0.039$.

\begin{tabular}{lllllll}
\hline$k_{\mathrm{e} 0}$ & 1.2 & 0.8 & 0.7 & 0.6 & 0.5 & 0.2 \\
\hline $\begin{array}{l}\mathrm{N}^{\circ} \text { with } \\
\text { deeper sedation/ }\end{array}$ & $7.5 / 8$ & $7.5 / 12$ & $7.5 / 12$ & $4.5 / 12$ & $6.5 / 12$ & $1.5 / 8$ \\
\hline \begin{tabular}{l} 
group size \\
\hline
\end{tabular}
\end{tabular}

Conclusion: Propofol infusion pumps incorporating the Marsh model should use a $\mathrm{k}_{\mathrm{e} 0}$ of approximately 0.6 /min to allow most accurate effect-site TCI. References:

1 White $\mathrm{M}$ et al. Br J Anaesth 1999; 82: 333-9.

2 Struys M et al. Anesthesiology 2000; 92: 399-406.

3 Chernick D et al. J Clin Psychopharmacol 1990; 10: 244-51.

\section{AP5-8}

\section{Ketamine Pharmacokinetics in Healthy Volunteers: Model Evaluation}

A. Rigby-Jones, J.R. Sneyd, A.R. Absalom

Anaesthesia Research Group, Peninsula Medical School, Plymouth, United Kingdom

Background and Goal of Study: During 3 neuroimaging and behavioural studies the Domino model ${ }^{1}$ performed poorly when controlling a low-dose target controlled infusion $(\mathrm{TCl})$ of ketamine ${ }^{2}$. We used the data acquired during these 3 studies to develop a new pharmacokinetic model for ketamine ${ }^{3}$. The purpose of the current study was to retrospectively evaluate the performance of this model in healthy volunteers who were administered ketamine (by TCl controlled by the Domino model) during a 4th study.

Materials and Methods: The 4th study comprised 49 observations from 11 individuals. Simulation was used to generate model predicted ketamine plasma concentrations for each individual using both our new model ${ }^{3}$ and the Domino model ${ }^{1}$. The median performance error (MDPE) and the median absolute performance error (MDAPE) were calculated ${ }^{4}$. Finally, a revised population PK model was developed using the data from all 4 studies (227 observations, 47 individuals).

Results and Discussions: Using our original model ${ }^{1}$, the MDPE for the 4 th study was $20.5 \%$ and the MDAPE was $23.1 \%$. Predictions based on the Domino model had an MDPE of $-9.4 \%$ and a MDAPE of $31.6 \%$. The PK analysis of the data from all 4 studies resulted in typical parameter values (Table) that were similar to our previous model ${ }^{3}$. However, unlike our previous model, weight was not a significant model covariate.

\begin{tabular}{lll}
\hline Parameter & Typical value & $95 \% \mathrm{Cl}$ \\
\hline Clearance $(\mathrm{CL}), \mathrm{L} / \mathrm{min}$ & 1.27 & $0.978-1.560$ \\
Distributional CL, L/min & 7.16 & $4.51-9.81$ \\
Central volume, L & 63.4 & $51.0-75.8$ \\
Peripheral volume, L & 248 & $201-295$ \\
Random error (ug/mL) & 0.0158 & $0.012-0.019$ \\
\hline
\end{tabular}

Conclusion(s) An MDPE (bias) of $20.5 \%$ indicates that the concentration predicted by our original model tended to be less than the measured concentration. Prospective clinical studies are required to further refine this model. References:

1 Domino EF et al. Clin Pharmacol Ther 1984;36:645-53

2 http://sivauk.org/PreviousMeetings/Birmingham/Lee.htm

3 http://sivauk.org/PreviousMeetings/Edinburgh/Rigby-Jones.htm

4 Varvel JR et al. J Pharmacokinetic Biopharm 1992; 20(1):63-94 


\section{AP5-9}

Loss of consciousness with intravenous induction of general anesthesia with remifentanil and propofol and its relationship with C-reactive protein

F. Lobo, A. Beiras, P. Amorim

Anesthesiology, Hospital Geral de Santo António, Porto, Portugal

Background and Goal of Study: Some published data suggested that inflammation and C-Reactive Protein (CRP) plasmatic concentration might be related with cognitive impairment after cardiac surgery ${ }^{1}$. We hypothesized that anesthetic needs for loss of consciousness (LOC) are influenced by a pre-operative inflammatory state.

Materials and Methods: We studied 78 patients, 36 female, age between 21 and 81 years old, ASA I- IV, Glasgow Coma Score 14-15, submitted to brain and non-brain surgery. Anesthetic management for induction of general anesthesia was the same: using Rugloop $\| \circledast$ software, anesthesia started with $\mathrm{TCl}$ of remifentanil with an effect-site target concentration of $2.5 \mathrm{ng} / \mathrm{ml}$ and a constant infusion of $1 \%$ propofol at $200 \mathrm{ml} / \mathrm{hr}$ until LOC; for each patient, predicted effect-site concentration of propofol at LOC was registered. Pre-operative plasmatic concentration of CRP was determined, but until LOC anesthesiologists in charge was blind for that value.

Quadratic regression was done to correlate pre-operative plasmatic concentration of CRP and predicted effect-site concentration of propofol at LOC; $p<0.05$ was considered significant.

Results: Pre-operative plasmatic concentration of CRP and predicted effectsite concentration of propofol at LOC showed an inverse correlation $(r=0,79, p<0.01)$ (Figure 1). Similar correlations were found when patients were divided into brain surgery group $(r=0,64, p<0.01)$ and non-brain surgery group $(r=0,86, p<0.01)$.

Relationship between baseline blood levels of Creactive protein and Effect-Site concentration of propofol at LOC

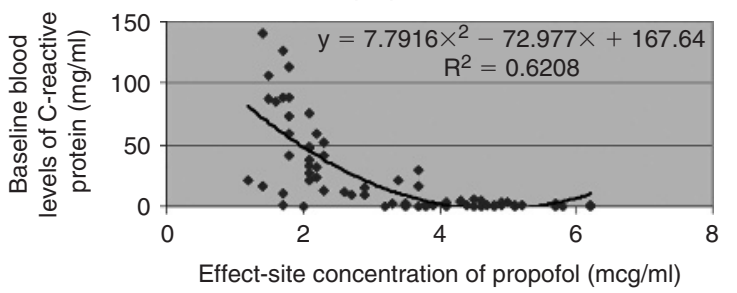

Fig. 1

Conclusion(s): Assuming that brain is an important target of inflammation², general anesthesia mechanisms that cause loss of consciousness might be changed if an inflammatory state co-exists. To our knowledge, our data are the first to show that inflammation decreases propofol needs for LOC.

References:

1 Anesth Analg 2006; 102: 1602-8.

2 Trends Neurosci. 2006; 29: 518-27.

\section{AP5-10}

The use of remifentanil changes the propofol requirements for loss of consciousness and correlation with patient baseline heart rate

C.S. Nunes, D.A. Ferreira, F. Lobo, P. Amorim, L. Antunes

Departamento de Matematica Aplicada, Faculdade de Ciencias da Universidade do Porto, Porto, Portugal

Background and Goal of Study: We observed that baseline heart rate (HR) had a high correlation with the propofol (Prop) effect-site concentration (Ce) required for loss of consciousness (LOC) [1], when using Prop alone. In this study, we investigate if this correlation holds when remifentanil (Remi) is also used in induction.

Materials and Methods: Data from 66 neurosurgeries collected until LOC (RugLoop II $\circledast$ every 5 s from Datex AS5, Aspect XP). TCI: Schnider[2] for Prop; Minto[3] for Remi. 2 groups of 33 patients (ASA 1-3). Group1 (G1): induction with Prop at $200 \mathrm{ml} / \mathrm{h}$ until LOC. Group2 (G2): induction with Remi TCl target $\mathrm{Ce}=2.5 \mathrm{ng} / \mathrm{ml}$; when Remi target was reached, Prop started at $200 \mathrm{ml} / \mathrm{h}$ until LOC. Patients with interfering pathologies were excluded. Statistics used correlation analysis and t-test (data:mean $\pm \mathrm{SD}$ ).

Results and Discussions: G1: $50 \pm 14$ years, $49 \pm 10 \mathrm{~kg}$ (LBM), 20 female. G2: $52 \pm 18$ years, $51 \pm 8 \mathrm{~kg}$ (LBM), 15 female. No statistical difference for age and LBM.
Table 1 Average values for the 2 groups. (SAP- systolic arterial pressure) ( $2 \mathrm{P}<0.01-\mathrm{t}-$ test, ${ }^{\star} \mathrm{P}<0.05$-paired t-test; ${ }^{* \star} \mathrm{P}<0.01$-paired t-test)

\begin{tabular}{llllll}
\hline & Group 1 & & \multicolumn{3}{c}{ Group 2 } \\
\cline { 2 - 6 } & Awake & At LOC & Awake & Start of Prop & At LOC \\
\hline $\begin{array}{l}\text { Prop Ce } \\
\mu \mathrm{g} / \mathrm{ml}\end{array}$ & 0 & $5 \pm 0.97$ & 0 & 0 & $3.5 \pm 1.18 \boldsymbol{}$ \\
Remi Ce & 0 & 0 & 0 & $2.5 \pm 0.01$ & $2.51 \pm 0.01$ \\
$\mathrm{ng} / \mathrm{ml}$ & & & & & \\
SAP mmHg & $140 \pm 17$ & $125 \pm 20^{\star \star}$ & $138 \pm 11$ & $141 \pm 16$ & $128 \pm 16$ \\
HR bpm & $72 \pm 12$ & $70 \pm 12$ & $73 \pm 13$ & $69 \pm 15^{\star \star}$ & $65 \pm 13^{\star \star}$ \\
BIS & $97.4 \pm 0.59$ & $53.5 \pm 16^{\star \star}$ & $96.2 \pm 2.7$ & $92.7 \pm 5.1^{\star \star}$ & $72.9 \pm 12^{\star \star}$ \\
\hline
\end{tabular}

Start of Prop until LOC: $3.4 \pm 0.8 \min (\mathrm{G} 1) ; 2.5 \pm 0.8 \mathrm{~min}(\mathrm{G} 2)$. In G1 Prop Ce at LOC correlated to awake HR $(R=0.61, P=0.0002)$. In $G 2$ Prop Ce at LOC correlated with awake SAP $(R=0.51, P=0.002)$, SAP before Prop $(R=0.37, P=0.03)$, and BIS before Prop $(R=0.46, P=0.007)$.

Conclusion(s): The use of Remi showed relation between both SAP and $\mathrm{BIS}$ and Prop Ce. It also changed the Prop requirements and relation with $\mathrm{HR}$. Reference:

1 Anesthesiology 2006; 105: A620.

2 Anesthesiology 1998; 88: 1170-82.

3 Anesthesiology 1997; 86: 24-33.

\section{AP6-1}

Propofol-induced changes of myoplasmic calcium concentration in cultured human skeletal muscles from RYR1 mutation carriers

T. Migita, K. Mukaida, M. Kawamoto, M. Kobayashi, O. Yuge

Anesthesiology and Critical Care, Hiroshima University Hospital, Hiroshima, Japan

Background and Goal of Study: Malignant hyperthermia $(\mathrm{MH})$ is a pharmacokinetic disorder during general anesthesia. We investigated whether propofol triggers $\mathrm{MH}$ by changing calcium homeostasis using human cultured myotubes isolated from the patients harboring the native RYR1, R2508C and L4838V mutations linked to $\mathrm{MH}(1)$.

Materials and Methods: Muscle specimens were obtained from the patients for diagnosing $\mathrm{MH}$ disposition. By using calcium imaging with the calcium sensitive probe Fura 2, calcium homeostasis in response to propofol was measured. The propofol concentrations tested were 1, 3, 10, 30, 100, 300, 1000,3000 , and $5000 \mu \mathrm{M}$.

Results and Discussions: The intracellular calcium concentration did not increase when propofol concentrations were within 1 to $30 \mu \mathrm{M}$, while it increased when propofol exceeded over $100 \mu \mathrm{M}$. The half-maximal activation concentrations $\left(\mathrm{EC}_{50}\right)$ were $181.1 \mu \mathrm{M}$ and $420.5 \mu \mathrm{M}$ in R2508C and L4838V mutations, respectively. Because serum-protein combination rate are between 97 and $98 \%$, free concentrations are assumed to be approximately $1 \mu \mathrm{M}$.

Conclusion(s) The rise in calcium concentration in response to propofol dosage was limited with doses up to 100 -fold greater than those used in clinical settings. We concluded that propofol is safe in MHS patients for clinical use. Reference:

1 Ibarra M.C.A., Wu S., Murayama K., et al. Anesthesiology 2006; 104: 1146-1154.

\section{AP6-2}

Comparative study of granisetron alone and in combination with droperidol and dexamethasone for prevention of postoperative nausea and vomiting in laparoscopic bariatric surgery

A. Moussa, P. Oregan

Anesthesiology, King Faisal Specialist Hospital and Research Center, Riyadh, Saudi Arabia

Background and Goal of Study: Laparoscopic bariatric surgeries are associated with an appreciably high incidence of postoperative nausea and vomiting (PONV). This study was designed to compare the effectiveness of granisetron alone and in combination with droperidol or dexamethasone for the prevention of post operative nausea and vomiting in patients undergoing laparoscopic bariatric surgeries.

Materials and Methods: After obtaining approval from the Hospital Ethics Committee and patients' informed consent, a randomized, double-blind, placebo-controlled trial was designed. 120 patients, aged between 18-44 years, ASA class II and III were randomly assigned into 4 equal groups to receive either granisetron $1 \mathrm{mg}$, granisetron $1 \mathrm{mg}$ plus droperidol $1.25 \mathrm{mg}$, granisetron $1 \mathrm{mg}$ plus dexamethasone $8 \mathrm{mg}$ or Placebo (saline), intravenously immediately before induction of anesthesia. Perioperative anesthetic care was standardized in all patients. Patients were then observed for 24 hours after administration of the study drugs. 
Results: Demographic and clinical characteristics of the study patients were statistically similar between the four studied groups. The incidence of PONV was $30 \%$ with granisetron alone, $30 \%$ with granisetron plus droperidol, $20 \%$, with granisetron plus dexamethanone, and $67 \%$ with placebo. ( $P$ value $<0.05)$. The incidence of adverse events was statistically similar among the 4 groups.

Conclusion: Graniserton is effective and safe drug for reducing the incidence of PONV in patients undergoing bariatric surgeries, and becomes highly effective when combined with dexamethasone.

\section{AP6-3}

A comparative study of haloperidol or dexamethasone plus ondansetron as prophylactic antiemetic therapy in patients at high risk of postoperative nausea and vomiting

Y. Lee, P.K. Huang, Y.L. Yang, H.Y. Lai, J.J. Wang

Anesthesiology, Buddist Tzu Chi Medical Center, Hualien, Taiwan

Background and Goal of Study: The combination of antiemetic drugs could be a solution to prevent severe postoperative nausea and vomiting (PONV) (1). Haloperidol has been used in the treatment of chemotherapy-, radiotherapy-, and opioid-related nausea and vomiting. We compared the prophylactic antiemetic efficacy of combining ondansetron with haloperidol or dexamethasone for patients at high risk of PONV.

Materials and Methods: A total of 88 non-smoking female patients with a history of PONV or motion sickness were included in this prospective, randomized, double-blinded study. Group $\mathrm{H}+\mathrm{O}$ received haloperidol $2 \mathrm{mg}$ and Group D + O received dexamethasone $8 \mathrm{mg}$ during induction of anaesthesia. All patients received ondansetron $4 \mathrm{mg}$ before the end of anaesthesia. The reported incidences of PONV ( $0-24 \mathrm{~h}$ ) were compared to the predicted incidences in these patient groups if no prophylactic antiemetic was given. The predicted incidence of PONV was obtained by calculation according to the Apfel' risk score. Sample size calculation was performed by using a statistical power analysis $(\mathrm{N}=45)$. Data were analyzed using Fisher's exact test and Chi square test.

Results and Discussions: Population characteristics were similar between groups. Efficacy results are shown in the table.

\begin{tabular}{lcll}
\hline Incidence of PONV & $\mathrm{H}+\mathrm{O}$ & $\mathrm{D}+\mathrm{O}$ & $\mathrm{P}$ \\
\hline Actual incidence & $9(20 \%)$ & $14(33 \%)$ & 0.180 \\
Predicted incidence & $34(76 \%)$ & $32(74 \%)$ & 0.262 \\
$\mathrm{P}$ & $<0.001$ & $<0.001$ & \\
\hline
\end{tabular}

Conclusion(s): Haloperidol $2 \mathrm{mg}$ combined with ondansetron $4 \mathrm{mg}$ is effective in preventing PONV and its efficacy is comparable to dexamethasone $8 \mathrm{mg}$ plus ondansetron $4 \mathrm{mg}$.

References:

1 Hefferman AM, Rowbotham DJ. Br J Anaesth 2000; 85: 675-7

2 Rüsch D, Eberhart L, Biedler A, et al. Can J Anesth 2005; 52: 478-84.

\section{AP6-4}

\section{A prospective randomised trial to determine if inhalational} anaesthetics have any effects on hearing function

Ü. Buyukkocak, R. Kilic, O. Arikan, O. Sert, F. Datli

Anaesthesiology and Reanimation, Kirikkale University School of Medicine, Kirikkale, Turkey

Background and Goals: Although uncommon, general anaesthesia is known to impair the hearing status in humans $(1,2)$. The purpose of this study was to investigate and compare the effects of inhalation anaesthetics (sevoflurane and isoflurane) on hearing function by using objective audiometric tests.

Patients and Methods: A prospective series of 53 adult patients (ASA I-II) scheduled for sinonasal surgery under intratracheal general anaesthesia were enrolled in the study. An exclusion criterion was history of hearing problems. Patients were premedicated intramuscularly with diazepam. Propofol $(2 \mathrm{mg} / \mathrm{kg})$ was given intravenously (iv.) for induction. After endotracheal intubation with vecuronium iv $(1 \mathrm{mg} / \mathrm{kg})$, sevoflurane $2 \%$ in group $1(\mathrm{n}=27)$ and isoflurane $1.2 \%$ in group $2(n=26)$ were used to maintain general anaesthesia. All patients received nitrous oxide during maintenance. Hearing function of the patients was measured before anaesthesia and 24 hours after surgery by means of pure tone audiometry, high frequency pure tone audiometry and transient evoked otoacustic emissions (TEOAE) by the same clinician. The data of hearing levels obtained from the left and right ears for each frequency were used for statistical analysis.

Results: There was no statistically significant difference in demographic data, haemodynamic and respiratory parameters between the groups. Although we observed a decrease at low frequencies $(250,500 \mathrm{~Hz})$ in patients receiving isoflurane, when they were assessed before and after surgery, this decrease was not found to be statistically significant $(p>0.05)$. In addition, no statistically significant differences in hearing levels were detected between the groups (Mann Whitney - U test, $p>0.05$ ).

Conclusions: It was audiometrically demonstrated that general anaesthesia did not affect the hearing function in any of the patients undergoing sinonasal surgery. These findings encourage the use of sevoflurane and isoflurane as a safe agent without any ototoxic effects in general anaesthesia related to otorhinolaryngologic surgery.

References:

1 Schaffartzik W. Anesth Analg 2000; 91: 1466-1472.

2 Ferber-Viart C. Hear Res 1998; 121: 53-61.

\section{AP6-5}

\section{A low-level occupational exposure to sevoflurane is associated with genotoxicity in the sister chromatid exchange but not in the micronucleus assay}

G. Wiesner, F. Schiewe-Langgartner, R. Lindner, M. Gruber

Department of Anaesthesiology, German Heart Centre, Munich, Germany

Background and Goal: The genotoxicity of the occupational exposure to inhaled, especially volatile anaesthetics, is still debated. This is the first study assessing genotoxic effects measured by sister chromatid exchanges (SCE) and micronuclei $(\mathrm{MN})$ in anaesthetists exposed to low levels of sevoflurane without nitrous oxide.

Materials and Methods: Fourteen anaesthetists wore a diffusion sampler to measure trace concentrations of sevoflurane for one working week. At the end of the working week blood samples were obtained from the anaesthetists and 14 internists (non-exposed controls) to determine SCE and MN in lymphocyte cultures. Confounding factors such as age, sex and smoking habits were assessed by questionnaires. Normally distributed data (age and SCE) were compared by t-tests, MN (not normally distributed) by Mann-Whitney U-test and ordinal data (sex, smoking habits) by Chi square analysis.

Results: See Table. Data is presented as median [range] and mean \pm SD. ${ }^{*} \mathrm{P}<0.05$ vs Internists

\begin{tabular}{lll}
\hline & Anaesthetists & Internists \\
\hline Sevoflurane (ppm) & $0.2[0.1-2.2]$ & $\mathrm{n} / \mathrm{a}$ \\
Age (years) & $32 \pm 5$ & $32 \pm 4$ \\
Sex (male/female) & $8 / 6$ & $9 / 5$ \\
Smoker (yes/no) & $4 / 10$ & $2 / 12$ \\
SCE/cell & $6.6 \pm 0.9^{*}$ & $5.1 \pm 0.8$ \\
MN/1000 cells & $9.5[2-15.5]$ & $8.5[3-25.5]$ \\
\hline
\end{tabular}

Conclusions: A low-level exposure to sevoflurane (NIOSH threshold value: $2 \mathrm{ppm}$ ) was associated with an increased formation of SCE but not of MN. These results are comparable to former studies with a low-level exposure to isoflurane and nitrous oxide $(1,2)$. However, discussing the impact of genotoxic effects it has to be taken into account that SCE are of the limited value for health risk assessment (3).

References:

1 Hoerauf KH, Wiesner G, Schroegendorfer KF, et al. Br J Anaesth 1999; 82: 764-766.

2 Wiesner G, Hoerauf K, Schroegendorfer K, et al. Anesth Analg 2001; 92: 118-122.

3 Tucker JD, Preston RJ. Mutat Res 1996; 365: 147-159.

\section{AP6-6}

Effects of ondansetron and acupuncture on postoperative nausea and vomiting

A. Cardone, P. Aceto, E. Congedo, E. Chinè, G. De Cosmo

Anaesthesiology and Intensive Care, Catholic Univesity of Sacred Heart, Rome, Italy

Background and Goal of Study: The authors investigated the efficacy of ondansetron and acupuncture in preventing postoperative nausea and vomiting (PONV) in this randomized and observer blind study.

Materials and Methods: After obtaining informed consent, 80 female patients, aged 18-70 years, ASA I-II, undergoing laparoscopic colecistectomy, were enrolled. General anaesthesia was standardized. Patients were randomly assigned to four groups: placebo (A group); $4 \mathrm{mg}$ of i.v. ondansetron (B group); $4 \mathrm{mg}$ of i.v. ondansetron plus acupuncture at the point P6 (C group); acupuncture at the point P6 (D group). All PONV prevention treatments were administered before surgical incision. Evaluation of PONV was performed with the VRS (verbal rating scale) ranging from $0=$ no nausea and $3=$ the worst immaginable nausea. Every hour for the first 4 hours and every four hours up to 24 hour. Logistic regression analysis was used to evaluate individual variables influence in determining PONV incidence. The independent variables considered were: treatment for PONV preventing (group) for univariate analysis; smoking, PONV history, anaesthesia duration for multivariate analysis. 
Results: Univariate analysis: probability to suffer from nausea was 7.5-12 times greater for patients of $A$ group than those of $B$ group and 16.4-20 times larger than those of $C$ group (respectively at first-second hour) $(p<0.01)$. Multivariate analysis: probability to find nausea was 17.8-25.6 times larger in A group than $\mathrm{C}$ group (at first-second hour) and 18.5 times greater than $B$ group at the second hour $(p<0.01)$. Vomiting did not show significantly results. Conclusions: Acupuncture plus ondansetron was the best treatment to prevent nausea. Ondansetron alone prevented nausea while acupuncture alone was not efficacy as antiemetic procedure.

Reference:

1 K. Streitberger, M. Diefenbacher, A. Bauer et al Anaesthesia 2004; 59: 142-149.

\section{AP6-7}

\section{Propofol anesthesia modifies corticosterone secretion} differently according to the circadian time

G. Dispersyn, L. Pain, Y. Touitou

Inserm U 713 Laboratoire de Biochimie Médicale et Biologie Moléculaire, Faculté de Médecine Pitié-Salpêtrière, Paris, France

Background and Goal of Study: Because of potential chronobiotic properties of propofol (effect on biological clock) (1), we examined the effects in rats of a short duration propofol anaesthesia on corticosterone, the major glucocorticoid.

Materials and Methods: Forty male Wistar rats were synchronized under a LD 12:12 cycle ( $12 \mathrm{~h}$ light, $12 \mathrm{~h}$ dark) during 4 weeks. One group was injected in the middle of the rest phase (CT6), another one was injected 2 hours before the beginning of the activity phase (CT10) and the third group was injected in the middle of the activity phase (CT16). On day 29, rats received either propofol injection $(120 \mathrm{mg} / \mathrm{kg})$ or intralipids and they were sacrificed by decapitation one hour after drug administration. Trunk blood was collected and centrifugated; serum was then separated and kept at $-30^{\circ} \mathrm{C}$ until corticosterone assayed by using 125I RIA kits (MP Biomedicals, NY, USA).

Results and Discussions: Propofol induced a significant increase in corticosterone secretion and disturbed its circadian rhythm. Propofol resulted in an increase of $108 \%$ (Student test, $p<0.05), 61 \%(p<0.03)$ and $157 \%$ $(p<0.006)$ of corticosterone in CT6, CT10 and CT16 groups respectively (see figure).

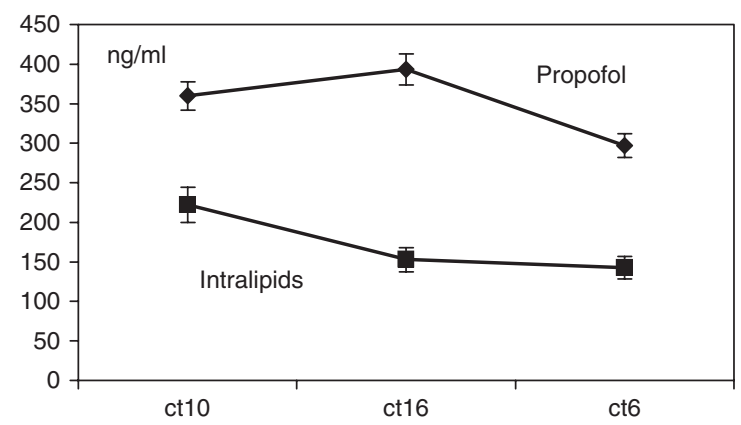

Conclusion(s): Propofol anaesthesia seems induce a desynchronization of corticosterone circadian rhythm by abolishing its morning peak and also increasing the total amount of corticosterone secretion, in rats.

Reference:

1 Challet et al. Neuropsychopharmacology 2006.

\section{AP6-8}

Multimodal analgesia after laparoscopic cholecystectomy

I. Grecu, A.E. Nicolau, V. Merlan, M. Nicolau, I. Grintescu

Anesthesiology and ICU, Clinical Emergency Hospital of Bucharest, Bucharest, Romania

Background and Goal of Study: Multimodal analgesia is an important prerequisite for accelerated recovery after elective surgery. Our study goal was to investigate the effects of locally injected $0.25 \%$ ropivacaine $(R)$ on postoperative pain after elective laparoscopic cholecystectomy.

Materials and Methods: After Hospital Ethics Committee approval, 60 adult patients scheduled for laparoscopic cholecystectomy performed by the same surgeon were enrolled in a prospective, randomized, double-blind, placebocontrolled study over a period of 6 months (Jan-June 2006).
All patients received the same general anesthesia protocol and $1 \mathrm{~g}$ iv paracetamol was infused after induction of anesthesia for postoperative analgesia, repeated each $6 \mathrm{hs}$ postoperatively, up to $4 \mathrm{~g} / 24 \mathrm{hs}$. Tramadol $50 \mathrm{mg}$ iv bolus was used as rescue analgesic. After anesthesia induction, patients were randomized in 4 groups ( $\mathrm{n}=15 \mathrm{pts}$, each): group $\mathrm{A}(20 \mathrm{ml}$ preincisional saline infiltration at the abdominal wall and $20 \mathrm{ml} \mathrm{R} 0.25 \%$ intraperitoneal instillation), group $B(20 \mathrm{ml} \mathrm{R} 0.25 \%$ preincisional infiltration and $20 \mathrm{ml}$ saline intraperitoneal instillation), group $C(A+B)$ and group $P$ (placebo, both sites saline infiltration). Primary endpoints were VAS at movement at 0, 2, 6, 12 and 24 postoperative hs $(0-10 \mathrm{~cm})$, rescue analgesic requirements, PONV and length of hospital stay. Statistics: chi-square, one-way ANOVA (SPSS 13.0).

Results and Discussions: We found no differences in demographics, length of surgery and of hospital stay (total: $3.38 \pm 0.22$ days). VAS was significantly lower at all intervals in group $C$ versus $P$ and at 0,6 and 12 hs in group $C$ versus both groups $A$ and $B(p<0.05)$. Tramadol boluses required were significantly fewer in group $C$ vs $P(0.73 \pm 1.10$ vs $1.93 \pm 1.03$, $p=0.017$ ) and also the incidence of PONV (1 pt in group $C$ vs 7 pts in group $P, p=0.01)$.

Conclusion(s): Ropivacaine $0.25 \%$ shows significant favorable effects on postoperative pain after laparoscopic cholecystectyomy only when using combined preincisional local infiltration and intraperitoneal instillation.

Reference:

1 Louizos A.A et al. Surg Endosc 2005; 19: 1503-6.

\section{AP6-9}

\section{Safety of metoclopramide in PONV prophylaxis using doses higher than $10 \mathrm{mg}$ intraoperatively}

J. Wallenborn, G. Gelbrich, M. Rudolph, D. Olthoff

Department of Anaesthesiology and Intensive Care, University of Leipzig, Leipzig, Germany

Background and Goal of Study: Doses of 25 and $50 \mathrm{mg}$ metoclopramide (MCP) in combination with $8 \mathrm{mg}$ dexamethasone (DEX) are effective in prophylaxis of postoperative nausea and/or vomiting (PONV; [1]). Safety and a fair trade-off of avoidance of PONV against adverse effects needs to be highlighted [2].

Materials and Methods: We reanalyzed the data of 3140 patients participating in a multi-centre study on PONV [1] and receiving randomized $0,10,25$ or $50 \mathrm{mg}$ MCP with special consideration of MCP-related immediate and delayed adverse events $(\mathrm{AE})$. The case report forms contained fields of 18 expected $\mathrm{AE}$ and one free text item each for intraoperative and postoperative suspected AE. We performed a trend test using Kendall's Tau (for event rate increasing with dose) and Fisher's exact test (for increased event rate of any dose). Stepwise multiple regression analysis was used to identify predictors of AE. Results and Discussions: Only hypotension and tachycardia following study drug administration were clearly related to MCP and increasing dose. The incidence (95\%-Confidence Interval) of either of these events were $8.8 \%$ (6.8-10.8), 11.2\% (9.0-13.4), 12.9\% (10.5-15.3), and 17.9\% (15.2-20.6) for $0,10,25$, and $50 \mathrm{mg}$ MCP. These AE were more frequent in patients older than 50 years, but obesity seemed to be protective. MCP was also associated with movement disorders (MCP-MD): number needed to harm $(\mathrm{NNH})=147$ for any dose $(78-\infty)$ and $112(60-1111)$ for 25 or $50 \mathrm{mg}$, impaired taste and smell: $\mathrm{NNH}=167$ (93-5000), and hot flushes: $\mathrm{NNH}=182$ (106-345). Duration of MCP-MD was predominantly $<1$ hour with subjective impairment of 4.7 (range 2-7). MCP-MD was associated with age $<30$ years. Gender, opioids, type of volatile anesthetic, and diabetes have no influence of MCP-MD. 3 definite and 3 suspected movement disorders occurred in the control group receiving only $8 \mathrm{mg}$ DEX.

Conclusion(s): Because of short-lasting and mostly self-limiting immediate AE and only occasionally occurring MCP-MD a single dose of 25 or $50 \mathrm{mg}$ MCP given intraoperatively can be recommended even under aspects of safety. These results cannot be transferred to pediatric anesthesia or awake patients.

References:

1 Wallenborn J, Gelbrich G, Bulst D et al. BMJ 2006; 333: 324-327.

2 Carlisle JB, Stevenson CA. Cochrane 2006; CD004125.

\section{AP7-1}

The efficacy of sugammadex in subjects with impaired renal function

L. Staals, MMJ. Snoeck, E. Flockton, M. Heeringa, JJ. Driessen

Anesthesiology, Radboud University Medical Center, Nijmegen, The Netherlands

Background and Goal of Study: Sugammadex is a modified gammacyclodextrin that has been designed to encapsulate the neuromuscular 
blocking agent rocuronium bromide, thereby reversing neuromuscular blockade. ${ }^{1}$ As the complex of sugammadex and rocuronium is cleared by the kidneys ${ }^{2}$, we studied the efficacy and safety of sugammadex in reversing the effect of rocuronium in patients with renal failure.

Materials and Methods: Following Ethics Committee approval, 16 female and 14 male patients (29-81 years) consented to participate in a multicentre, parallel-group, comparative trial. Fifteen ASA II-III patients had impaired renal function (creatinine clearance $\left[\mathrm{CR}_{\mathrm{Cl}}\right]<30 \mathrm{ml} \mathrm{min}^{-1}$ ) and $15 \mathrm{ASA}$ I-II patients had normal renal function $\left(\mathrm{CR}_{\mathrm{Cl}}>80 \mathrm{ml} \mathrm{min}^{-1}\right)$. Patients were anaesthetized with propofol and opiates. Rocuronium $0.6 \mathrm{mg} \mathrm{kg}^{-1}$ was given prior to intubation. Neuromuscular monitoring was performed using train-offour (TOF) nerve stimulation and acceleromyography (TOF-Watch ${ }^{\circledR} \mathrm{SX}$ ). A single dose of sugammadex $2.0 \mathrm{mg} \mathrm{kg}^{-1}$ was given at reappearance of $T_{2}$. The primary efficacy variable was time from administration of sugammadex to recovery of $T_{4} / T_{1}$ to 0.9 . Clinical signs of recurarization were monitored for 48 hours postoperatively. Safety was assessed 2-4 weeks postoperatively. Results and Discussions: Mean (SD) $\mathrm{CR}_{\mathrm{Cl}}$ was 12 (5) $\mathrm{ml} \mathrm{min}^{-1}$ in renallyimpaired patients and $103(24) \mathrm{ml} \mathrm{min}^{-1}$ in controls. The mean (SD) time from start of administration of sugammadex $2.0 \mathrm{mg} \mathrm{kg}^{-1}$ to recovery of $T_{4} / T_{1}$ to 0.9 was $2.0(0.7) \mathrm{min}$ in renally-impaired patients and $1.7(0.6) \mathrm{min}$ in controls. No signs of recurarization were observed in patients with renal impairment. No sugammadex-related serious adverse events were reported.

Conclusion(s): Sugammadex $2.0 \mathrm{mg} \mathrm{kg}^{-1}$ is well tolerated, and causes rapid and complete recovery from rocuronium-induced blockade in patients with normal or impaired renal function. Further studies of sugammadex in renal failure patients are indicated.

References:

1 Giisenbergh F, et al. Anesthesiology 2005; 103: 695-703.

2 Sorgenfrei I, et al. Anesthesiology 2006; 104: 667-674.

\section{AP7-2}

Sugammadex after rocuronium provides faster recovery from neuromuscular blockade than neostigmine after cisatracurium

E. Flockton, E. Scanni, C. Gomar, M. Shields, L. Aguilera

University Department of Anaesthesia, Royal Liverpool University Hospital, Liverpool, United Kingdom

Background and Goal of Study: The use of anticholinesterase inhibitors such as neostigmine for reversal of neuromuscular blockade accelerates recovery but can be relatively slow and be associated with muscarinic sideeffects. Sugammadex, a modified $\gamma$-cyclodextrin, rapidly reverses the effects of the aminosteroidal neuromuscular blocking agent rocuronium by encapsulation (1). The aim of this study was to assess reversal with sugammadex after rocuronium compared with neostigmine-glycopyrrolate after cisatracurium. Materials and Methods: Eighty-four ASA 1-3 adult patients were anaesthetized with propofol and opioids and randomised to receive rocuronium $0.6 \mathrm{mg} \mathrm{kg}^{-1}$ or cisatracurium $0.15 \mathrm{mg} \mathrm{kg}^{-1}$ with a maximum of two maintenance doses if required. Neuromuscular blockade was monitored using a TOF-Watch ${ }^{\circledR}$ SX. Sugammadex $2.0 \mathrm{mg} \mathrm{kg}^{-1}$ or neostigmine $50 \mu \mathrm{g} \mathrm{kg}^{-1}$ (with glycopyrrolate $10 \mu \mathrm{g} \mathrm{kg}^{-1}$ ) was administered to the rocuronium and cisatracurium groups respectively at reappearance of $T_{2}$ after the last dose of NMBA. Times to onset of blockade after the NMBA and for recovery of $T_{4} / T_{1}$ to 0.9 after reversal were recorded. All subjects were monitored for adverse events and any clinical evidence of recurarization.

Results and Discussions: Mean time (sec [SD]) to onset of neuromuscular blockade was significantly faster with rocuronium (91 [33] vs 171 [46]; $p<0.0001)$. The time to $T_{4} / T_{1}$ ratio of 0.9 in the intent-to-treat population was significantly shorter in the rocuronium/sugammadex patients (Table). There were no serious adverse events or any evidence of recurarization in either group.

Median (range) times to $T_{4} / T_{1}$ ratio of $0.9(\mathrm{~min})$

\begin{tabular}{ll}
\hline Rocuronium/sugammadex $(n=34)$ & Cisatracurium/neostigmine $(n=39)$ \\
\hline $1.9(0.7-6.4)^{\star}$ & $7.2(4.2-28.2)$ \\
\hline${ }^{*} \mathrm{P}<0.0001$ &
\end{tabular}

Conclusion(s): Rocuronium is a faster acting NMBA than cisatracurium. Its reversal with sugammadex is 4 times faster than cisatracurium with neostigmine.

Reference:

1 Sorgenfrei et al. Reversal of rocuronium-induced neuromuscular block by the selective relaxant binding agent sugammadex: a dose-finding and safety study. Anesthesiology 2006; 104: 667-74.

\section{AP7-3}

Sugammadex achieves fast recovery from profound neuromuscular blockade induced by rocuronium or vecuronium: a dose-response study

P. Duvaldestin, K. Kuizenga, CC. Kjaer, V. Saldien, B. Debaene Anesthesia and ICU, Hopital Henri Mondor, Assistance Publique Hopitaux de Paris, Creteil, France

Background and Goal of Study: Sugammadex is the first selective relaxant binding agent to prevent steroidal neuromuscular blocking agents from acting at their receptors. This study assessed the effect of different doses of sugammadex given after profound neuromuscular blockade (NMB) induced by rocuronium or vecuronium.

Materials and Methods: After written informed consent, 102 patients (age 21-64 yr, ASA class 1-3) undergoing general anesthesia with sevoflurane for maintenance were randomised to receive either rocuronium $(0.9 \mathrm{mg} / \mathrm{kg}$ bolus and repeated doses of $0.1-0.2 \mathrm{mg} / \mathrm{kg})$ or vecuronium $(0.1 \mathrm{mg} / \mathrm{kg}$ bolus and repeated doses of $0.02-0.03 \mathrm{mg} / \mathrm{kg}$ ). NMB was monitored by accelerometry (TOF-Watch ${ }^{\circledR} \mathrm{SX}$ ). Sugammadex $(0.5$ to $8.0 \mathrm{mg} / \mathrm{kg})$ was administered after the last dose of muscle relaxant, at 1-2 PTCs. The time from sugammadex administration to recovery of the $\mathrm{T} 4 / \mathrm{T} 1$ ratio to 0.9 was recorded. Dose response was analysed using weighted nonlinear regression. All patients were monitored for residual curarization, recurarization, and adverse events. Results and Discussion: A statistically significant dose-response relationship was found between the dose of sugammadex and recovery time (table, per protocol population).

\begin{tabular}{lll}
\hline Sugammadex Dose $(\mathrm{mg} / \mathrm{kg})$ & \multicolumn{2}{l}{ Mean $(\mathrm{SD})$ time to T4/T1 ratio 0.9 $(\mathrm{min})$} \\
\cline { 2 - 3 } & rocuronium & vecuronium \\
\hline 0.5 & $(\mathrm{n}=8) 79.8(33.0)$ & $(\mathrm{n}=7) 68.4(31.9)$ \\
1.0 & $(\mathrm{n}=9) 28.0(43.7)$ & $(\mathrm{n}=9) 25.1(24.9)$ \\
2.0 & $(\mathrm{n}=10) 3.2(1.5)$ & $(\mathrm{n}=11) 9.1(20.6)$ \\
4.0 & $(\mathrm{n}=10) 1.6(0.7)$ & $(\mathrm{n}=8) 3.3(3.5)$ \\
8.0 & $(\mathrm{n}=10) 1.1(0.3)$ & $(\mathrm{n}=101.7(0.7)$ \\
\hline
\end{tabular}

A subsequent decrease of $\mathrm{T} 4 / \mathrm{T} 1$ from $\geqslant 0.9$ to $<0.8$ (indication for recurarisation) was observed in four patients after the low dosages of sugammadex 0.5 and $1 \mathrm{mg} / \mathrm{kg}$. Serious adverse events were reported for four patients, but none was considered related to sugammadex.

Conclusion(s): Sugammadex safely decreased recovery time from profound NMB by rocuronium or vecuronium in a dose-dependent manner.

\section{AP7-4}

\section{The effect of preoperative consumption of potatoes on succinylcholine-induced paralysis and recovery from anesthesia}

A. Bestas, H. Goksu, O.L. Erhan

Department of Anesthesiology and Reanimation, Firat University School of Medicine, ELAZIG, Turkey

Background and Goal of Study: Potatoes contain solanaceous glycoalkaloids (SGAs), which inhibit both butyrylcholinesterase (BuChE) and acetylcholinesterase (AChE). Potatoes also contain a series of benzodiazepines. The aim of our study was to investigate the effect of preoperative consumption of potatoes on succinylcholine-induced paralysis and recovery from anesthesia. Materials and Methods: After the approval of ethic commitee of Faculty, forty-four ASA I-II, adult patients, scheduled for elective surgery, were included in a randomized, blind and controlled study. Patients were randomly divided into two groups. Patients in Group $P(n=21)$ were eaten a standard portion of potatoes in the last meal prior to fasting while patients in Group C were eaten with food not containing SGAs. Patients were premedicated with midazolam $\left(0.05 \mathrm{mg} \mathrm{kg}^{-1}\right.$, im). Anesthesia was induced with thiopental $\left(5 \mathrm{mg} \mathrm{kg}^{-1}\right)$ and fentanyl $\left(2 \mu \mathrm{g} \mathrm{kg}^{-1}\right)$, and maintained with $2.5 \%$ sevofluran in $50 \% \mathrm{O}_{2}$ /air and fentanyl as needed. Succinylcholine $1 \mathrm{mg} \mathrm{kg}^{-1}$ was administered to facilitate endotracheal intubation. Duration of succinylcholine blockade and recovery time from anesthesia were measured. Serum BuChE levels were also measured at before and four time points within $24 \mathrm{~h}$ postconsumption start.

Results and Discussions: Duration of succinylcholine-induced paralysis and recovery time from anesthesia was significantly longer in Group $P$ than in Group C $(p<0.05)$. In Group P, Serum BuChE levels decreased at $6 \mathrm{~h}$ from consumption start. In addition, in both groups, BuChE levels markedly decreased immediately after succinylcholine blockade, thereafter increased, but did not return to baseline within $24 \mathrm{~h}$ from consumption start. None of these differences observed in BuChE levels was statistically significant. 
Conclusion(s): This study suggests that potatoes eaten before surgery can slow down the metabolism of succinylcholine and delay recovery from anesthesia.

\section{AP7-5}

Reversal of rocuronium-induced neuromuscular blockade with sugammadex in paediatric and adult patients

B. Plaud, O. Meretoja, B. Pohl, R.K. Mirakhur, J. Raft

Anaesthesia department, CHU Cote de Nacre, Caen, France

Background and Goal of Study: Sugammadex is a modified $\gamma$-cyclodextrin that reverses rocuronium-induced neuromuscular blockade (NMB) in adults in a dose-dependent manner (1). This study explored the effects of sugammadex on reversal of rocuronium-induced NMB in paediatric and adult patients.

Materials and Methods: Eight infants (INF, 28 days-23 months), 24 children ( $\mathrm{CHI}, 2-11$ years), 31 adolescents (ADO, 12-17 years) and 28 adults (ADU, 18-65 years) (ASA class I/II) were anaesthetised with propofol and opioids or caudal analgesia (INF) and received rocuronium $0.6 \mathrm{mg} / \mathrm{kg}$. NMB was monitored using TOF-Watch ${ }^{\circledR}$ SX. Patients were randomised to sugammadex $0.5,1.0,2.0$ or $4.0 \mathrm{mg} / \mathrm{kg}$ or placebo at reappearance of $\mathrm{T}_{2}$. Time from sugammadex/placebo to recovery of $T_{4} / T_{1}$ ratio to 0.9 was recorded. Safety was assessed by recording vital signs, ECGs, laboratory data and adverse events.

Results and Discussions: The recovery time decreased in a dose-related manner in all age groups (per-protocol population) (not formally shown for infants due to the low numbers), see Table. Sugammadex was well tolerated with no recurrence of NMB. Serious adverse events in 1 infant and 1 child were not considered related to sugammadex.

\begin{tabular}{llllll}
\hline \multicolumn{5}{c}{ Median (range) time to $\mathrm{T}_{4} / \mathrm{T}_{1}$ ratio of $0.9(\mathrm{~min})$} \\
\hline \multicolumn{5}{c}{ Sugammadex $(\mathrm{mg} / \mathrm{kg})$} \\
\cline { 3 - 6 } & Placebo & 0.5 & 1.0 & 2.0 & 4.0 \\
\hline INF & 21.0 & 3.7 & 2.4 & 0.6 & 0.7 \\
& $(13.0-29.0)$ & $(3.3-4.2)$ & $(1.9-2.9)$ & & \\
& $(\mathrm{n}=2)$ & $(\mathrm{n}=2)$ & $(\mathrm{n}=2)$ & $(\mathrm{n}=1)$ & $(\mathrm{n}=1)$ \\
$\mathrm{CHI}$ & 19.0 & 3.7 & 2.7 & 1.2 & 0.6 \\
& $(8.4-31.8)$ & $(2.4-10.9)$ & $(1.9-9.6)$ & $(0.9-1.6)$ & $0.6-4.4)$ \\
& $(\mathrm{n}=4)$ & $(\mathrm{n}=5)$ & $(\mathrm{n}=5)$ & $(\mathrm{n}=4))$ & $(\mathrm{n}=4)$ \\
& 23.4 & 4.6 & 1.7 & 1.1 & 1.1 \\
& $(6.8-41.7)$ & $(1.9-43.5)$ & $(1.5-2.5)$ & $(0.7-5.2)$ & $(0.7-1.4)$ \\
& $(\mathrm{n}=5)$ & $(\mathrm{n}=5)$ & $(\mathrm{n}=6$ & $(\mathrm{n}=6$ & $(\mathrm{n}=6)$ \\
& 28.5 & 4.2 & 1.7 & 1.4 & 1.2 \\
& $(19.6-44.0)$ & $(2.3-4.8)$ & $(1.2-2.0)$ & $(1.0-2.0)$ & $(0.9-1.6)$ \\
& $(\mathrm{n}=2)$ & $(\mathrm{n}=5)$ & $(\mathrm{n}=5)$ & $(\mathrm{n}=5)$ & $(\mathrm{n}=5)$ \\
\hline & & & & &
\end{tabular}

Conclusion(s): Sugammadex was effective and safe for reversal of a shallow rocuronium-induced NMB in paediatric and adult patients.

Reference:

1 Sorgenfrei et al. Anesthesiology 2006; 104: 667-674.

\section{AP7-6}

Histamine-induced hemodynamic changes after vecuronium, rocuronium, and cisatracurium during cardiac surgery

D. Vershuta, I. Kozlov, A. Ermolenko, V. Ilnitsky

Anaesthesiology and Intencive Care, Research Institutetransplantology and Artificial Organs, Moscow, Russian Federation

Background and Goal of Study: Choice of muscle relaxants for anaesthesia is determined by their clinical profile and side effects. The aim of our study was to evaluate the effects of different neuromuscular blocking agents (NMBA) on histamine plasma concentration, mean arterial blood pressure (MABP) and heart rate (HR) in cardiosurgical patients.

Materials and Methods: After obtaining written informed consent and approval from the Ethics Committee, we studied 35 adult patients ASA III-IV underwented elective coronary artery bypass grafting or valvular surgery. Anaesthesia was induced by midazolam and fentanyl in standard doses. Patients received vecuronium $(n=13) 0.15 \mathrm{mg} / \mathrm{kg}$, or rocuronium $(n=10)$ $0.6 \mathrm{mg} / \mathrm{kg}$, or cisatracurium $(n=12) 0.15 \mathrm{mg} / \mathrm{kg}$. ECG, HR, and invasive MABP were monitored continuously wits UCW monitoring system (Space Labs). Venous blood samples were obtained in $1 \mathrm{~min}$ after induction of anaesthesia (baseline) and in 1 and $3 \mathrm{~min}$ after relaxant administration. The plasma samples were analyzed by radioenzymatic assay (Histamine ELISA). Data were analyzed using Student's t-test. Statistical significance was assumed for $\mathrm{p}<0.05$.
Results: Vecuronium, rocuronium, and cisatracurium administration did not induce any changes in either plasma histamine (table) haemodynamic parameters (mean \pm SEM).

\begin{tabular}{lllll}
\hline & Baseline & $+1 \mathrm{~min}$ & $+3 \mathrm{~min}$ & $\mathrm{p}$ \\
\hline $\begin{array}{l}\text { Histamine } \mathrm{ng} / \mathrm{ml} \\
\text { Vecuronium }\end{array}$ & $0.4 \pm 0.06$ & $0.4 \pm 0.07$ & $0.4 \pm 0.07$ & $\mathrm{~ns}$ \\
$\begin{array}{l}\text { Rocuronium } \\
\text { Cisatracurium }\end{array}$ & $0.4 \pm 0.08$ & $0.4 \pm 0.09$ & $0.3 \pm 0.09$ & $\mathrm{~ns}$ \\
HR (beat min $^{-1}$ ) & $0.3 \pm 0.05$ & $0.3 \pm 0.05$ & $0.4 \pm 0.05$ & $\mathrm{~ns}$ \\
$\begin{array}{l}\text { Vecuronium } \\
\text { Rocuronium }\end{array}$ & $75 \pm 3.5$ & $71 \pm 3.8$ & $74 \pm 4.6$ & $\mathrm{~ns}$ \\
Cisatracurium & $73 \pm 3.3$ & $74 \pm 3.1$ & $74 \pm 3$ & $\mathrm{~ns}$ \\
MABP (mmHg) & $76 \pm 3.4$ & $71 \pm 3.1$ & $73 \pm 2.9$ & $\mathrm{~ns}$ \\
Vecuronium & $74 \pm 4.1$ & $72 \pm 4.1$ & $73 \pm 3.4$ & $\mathrm{~ns}$ \\
$\begin{array}{l}\text { Rocuronium } \\
\text { Cisatracurium }\end{array}$ & $79 \pm 3$ & $74 \pm 2.4$ & $76 \pm 2.6$ & $\mathrm{~ns}$ \\
\hline & $74 \pm 3.2$ & $74 \pm 4.3$ & $76 \pm 4.7$ & $\mathrm{~ns}$ \\
\hline
\end{tabular}

Conclusions: Vecuronium, rocuronium and cisatracurium proved to be safe for use during induction of anaesthesia in cardiosurgical patients ASA III-IV.

\section{AP7-7}

\section{The neuromuscular effects of rocuronium in elderly and young adults with or without renal failure}

S. Kocabas, D. Yedicocuklu, F.Z. Askar

Anaesthesiology and Reanimation Department, Ege University Faculty of Medicine, Bornova, Izmir, Turkey

Background and Goal of Study: This study aims to investigate the neuromuscular effects of $0.6 \mathrm{mg} . \mathrm{kg}^{-1}$ rocuronium under propofol anaesthesia in elderly and young adults either with renal failure or normal renal function.

Materials and Methods: After obtaining ethics committee approval and informed consent, 40 patients with renal failure undergoing arteriovenous shunt surgery and 40 patients with normal renal function undergoing peripheral venous surgery were included in the study. Patients were grouped as follows: Younger adult (18-50 yr) with renal failure $(n=20)$, elderly ( $>65 \mathrm{yr}$ ) with renal failure $(n=20)$, younger adult with normal renal function $(n=20)$ and elderly with normal renal function $(n=20)$. Anaesthesia was induced with propofol $1-2 \mathrm{mg} \cdot \mathrm{kg}^{-1}$ and fentanyl $2 \mu \mathrm{g} \cdot \mathrm{kg}^{-1}$ and maintained with propofol 6-12 $\mathrm{mg} \cdot \mathrm{kg}^{-1} \cdot \mathrm{h}^{-1}, \mathrm{~N}_{2} \mathrm{O}$ in oxygen and supplemental fentanyl. The ulnar nerve was stimulated supramaximally at the wrist via cutaneous electrodes using train-of-four nerve stimulation. Rocuronium $0.6 \mathrm{mg} \cdot \mathrm{kg}^{-1}$ was administered and intubation was performed at the onset of maximal neuromuscular block. The onset time, the time to recovery of the first twitch $\left(\mathrm{T}_{1}\right)$ to $25 \%, 50 \%, 75 \%$ and $90 \%$ and recovery index were recorded. T-test and Fisher's Exact test were used for statistical analysis. $\mathrm{P}<0.05$ was significant. Results and Discussions: The time to recovery of $\mathrm{T}_{1}$ to $25 \%, 50 \%, 75 \%$ and $90 \%$ and recovery index were found to be prolonged in both the young and elderly patients with renal failure when compared to those with normal renal function $\left(T_{1} 25 \%\right.$ : $58.4 \pm 20.2 \mathrm{~min}$ and $80.1 \pm 7 \mathrm{~min}$ vs $32.8 \pm 5.6 \mathrm{~min}$ and $46.3 \pm 9.0 \mathrm{~min}$, respectively) $(p<0.05)$. The recovery parameters were found to be prolonged in the elderly when compared with young adults in both the renal failure and the non-renal failure groups $(p<0.05)$.

Conclusion(s): The neuromuscular effects of $0.6 \mathrm{mg} . \mathrm{kg}^{-1}$ rocuronium under propofol anaesthesia were markedly prolonged in elderly patients when compared with young adults and in the presence of renal failure.

Reference:

1 Robertson EN, Driessen JJ, Booij LHDJ. Pharmacokinetics and pharmacodynamics of rocuronium in patients with and without renal failure. Eur J Anaesth 2005; 22: 4-10.

\section{AP7-8}

Reversal of vecuronium-induced shallow neuromuscular blockade is significantly faster with sugammadex compared with neostigmine

J.A. Alvarez-Gómez, M. Wattwill, B. Vanacker, Jl. Lora-Tamayo, KS. Khünl-Brady

Anestesia-Reanimación y T. Dolor, Hospital Santa Maria Rosell, Cartagena, Spain

Background and Goal of Study: Sugammadex, a modified $\gamma$-cyclodextrin, is the first selective relaxant binding agent (SRBA) and was originally designed to reverse the effects of the steroidal neuromuscular blocking agent rocuronium (1). In this phase 3 a study, the efficacy of sugammadex was compared with that of neostigmine for the reversal of shallow neuromuscular blockade (i.e., at reappearance of $T_{2}$ ) induced by single or multiple bolus doses of vecuronium during sevoflurane maintenance anaesthesia for scheduled surgery. 
Materials and Methods: A total of 100 subjects (age $\geqslant 18$ years, ASA class 1 to 3 ) who had given written informed consent were included in this randomised, multicentre, parallel-group trial. Neuromuscular blockade was monitored continuously using acceleromyography (TOF-Watch ${ }^{\circledR}$ SX, Organon Ltd, Dublin, Ireland). At reappearance of $\mathrm{T}_{2}$ after the last dose of vecuronium, either sugammadex $2.0 \mathrm{mg} \mathrm{kg}^{-1}$ or neostigmine $50 \mathrm{\mu g} \mathrm{kg}^{-1}$ plus glycopyrrolate $10 \mu \mathrm{g} \mathrm{kg}^{-1}$ were administered intravenously in randomised order. The primary efficacy variable was time from start of administration of sugammadex or neostigmine to recovery of the $T_{4} / T_{1}$ ratio to 0.9 . Clinical signs of residual or re-curarisation were also assessed. Statistical analysis was performed using an ANOVA model on logarithmic transformed recovery times. Safety was evaluated by (serious) adverse events, laboratory variables, and vital signs. Results and Discussion: Median (range) time to recovery in the intent-totreat population was statistically significantly shorter with sugammadex $(n=48)$ versus neostigmine $(n=45): 2.1(1.2-64.2)$ min versus 18.9 (2.9-76.2) $\min (p<0.0001)$. There were no clinical events due to residual or re-curarisation. No serious adverse events were reported.

Conclusion(s): After neuromuscular blockade with vecuronium, sugammadex achieved significantly faster recovery of the $T_{4} / T_{1}$ ratio to 0.9 compared with neostigmine when administered at reappearance of $\mathrm{T}_{2}$.

Reference:

1 Bom A, et al. Angew Chem Int Ed Engl 2002; 41: 266-70.

\section{AP7-9}

Neostigmine use: audit of the clinical decision compared to acceleromyography

R. Videira, F. Oda, C. Biazotto, J. Vieira, M. Carmona

Anesthesiology, Hospital das Clinicas - University of Sao Paulo, Sao Paulo, Brazil

Background and Goal of Study: Residual neuromuscular blockade (NMB) defined as train-of-four(TOF) $<0,9$ has an incidence higher than $80 \%$ right before tracheal extubation. ${ }^{1}$ The objective of this study was to evaluate the anesthesiologist's clinical decision of using neostigmine, as a diagnostic test, compared to acceleromyography, in a university hospital of a developing country.

Materials and Methods: Adult patients under general anesthesia and endotracheal intubation with nondepolarizing NMB were studied. At the end of the surgery, the anesthesiologist was asked if neostigmine would be administered. The ulnar nerve was stimulated while a thumb transducer $\left(\right.$ TOF-Watch ${ }^{\circledR}$ ) quantified NMB. If TOF $\leqslant 0,9$ the anesthesiologist was informed. Kappa and McNemar tests were used and $\mathrm{P}<0,05$ was considered significant.

Results and Discussions: A total of 150 patients were studied, but 78 were excluded due to peripheral hypothermia $\left(T<32^{\circ} \mathrm{C}\right), 6$ due to transducer instability and 2 because neostigmine had already been used. The 64 remaining patients had a mean \pm sd age of $49 \pm 18$ years, $68 \pm 15 \mathrm{~kg}, 166 \pm 9 \mathrm{~cm}$, and were grouped as:

\begin{tabular}{lll}
\hline Will neostigmine be used? & Yes & No \\
TOF $\leqslant 90 \%$ & 7 & 32 \\
TOF $>90 \%$ & 3 & 2 \\
\hline
\end{tabular}

The control event rate (CER) was $77 \%(49 / 64)$ and the experimental event rate (EER) was $50 \%$ (32/64), with absolute risk reduction (ARR) of $27 \%$ and relative risk reduction (RRR) of $35 \%$. The positive predictive value (PPV) of the clinical evaluation was $85 \%$, while the negative predictive value was $27 \%$. (McNemar $p=0,000$ while kappa $p=0,088$ )

Conclusion(s): The qualitative clinical decision is inappropriate to diagnose residual NMB. In our setting, hypothermia was a limiting factor for the routine use of acceleromyography. ${ }^{2}$

References:

1 Beaussier M. Ann Fr Anesth Reanim 2005: 1266-1274.

2 Black N. BMJ 1996; 312: 1215-1218.

\section{AP7-10}

Sugammadex $(2.0 \mathrm{mg} / \mathrm{kg})$ significantly faster reverses shallow rocuronium-induced neuromuscular blockade compared with neostigmine $(50 \mu \mathrm{g} / \mathbf{k g})$

M. Blobner, L. Eriksson, J. Scholz, H. Hillebrand, L. Pompei

Klinik für Anaesthesiologie, Technische Universität München, Munich, Germany Background and Goal of Study: Sugammadex, a modified $\gamma$-cyclodextrin, is the first selective relaxant binding agent (SRBA) and was designed to reverse the effects of the steroidal neuromuscular blocking agent rocuronium (1). This phase 3 a study compared the efficacy of sugammadex versus neostigmine for the reversal of shallow neuromuscular blockade (i.e., at reappearance of $T_{2}$ ) induced by single or multiple bolus doses of rocuronium during sevoflurane maintenance anaesthesia for elective surgery.

Materials and Methods: This randomised, multicentre, parallel-group trial included 98 subjects (age $\geqslant 18$ years, ASA class 1 to 3 ). Neuromuscular activity was monitored continuously using acceleromyography (TOFWatch ${ }^{\circledR}$ SX, Organon Ltd, Dublin, Ireland). At reappearance of $T_{2}$ after the last dose of rocuronium, either sugammadex $2.0 \mathrm{mg} \mathrm{kg}^{-1}$ or neostigmine $50 \mu \mathrm{g} \mathrm{kg}^{-1}$ plus glycopyrrolate $10 \mu \mathrm{g} \mathrm{kg}^{-1}$ were administered intravenously in randomised order. The primary efficacy variable was the time from start of administration of sugammadex or neostigmine to recovery of the $T_{4} / T_{1}$ ratio to 0.9. Clinical signs of residual or re-curarisation were also assessed. Statistical analysis was performed using an ANOVA model on logarithmic transformed recovery times. Safety was evaluated by assessing (serious) adverse events, laboratory variables and vital signs.

Results and Discussions: In the intent-to-treat population ( $n=48$ in each group), there was a markedly shorter recovery pattern after sugammadex compared to neostigmine, the time to TOF (median and range) being 1.4 $(0.9-5.4)$ versus $17.6(3.7-106.9) \mathrm{min}(\mathrm{p}<0.0001)$. No clinical events due to residual or re-curarization occurred. Serious adverse effects were reported for 2 subjects in the sugammadex group and 3 in the neostigmine group but none were considered related to the study drug.

Conclusion(s): Sugammadex achieved significantly faster recovery of $\mathrm{T}_{4} / \mathrm{T}_{1}$ ratio to 0.9 compared with neostigmine after neuromuscular block with rocuronium. In addition outliers and variability of recovery times were significantly lower following reversal with sugammadex.

Reference:

1 Bom A, et al. Angew Chem Int Ed Engl 2002; 41: 266-70.

\section{AP8-1}

The anti-oxidative effect of isosteviol on angiotensin-IIinduced reactive oxygen species generation in hypertensive injury of aortic smooth muscle cells

K.L. Wong, K.C. Wu, E.C. So, R.S.C. Wu, T.H. Cheng

Anesthesia \& Institute of Medical Sciences, China Medical University \& Hospital, Taichung, Taiwan

Background and Goal of Study: Isosteviol is an active derivative of stevioside, a constituent of Stevia rebaudiana, and also possessing an antihypertensive effect. This study is to search the anti-proliferative mechanisms of isosteviol related to the Involvement of reactive oxygen species (ROS) in signal-regulation on endothelin-1 in modulating hypertensive vascular injury. Materials and Methods: Cultured rat aortic smooth muscle cells (SMCs) were stimulated with Ang II, [3H]thymidine incorporation and the ET-1 gene expression was examined. Isosteviol and antioxidants pretreatment on Ang-II induced extracellular signal-regulated kinase (ERK) phosphorylation were performed to elucidate the redox-sensitive pathway in proliferation and ET-1 gene expression.

Results and Discussions: Cultured rat aortic SMCs were preincubated with isosteviol then stimulated with angiotensin II, after which $[3 \mathrm{H}]$ thymidine incorporation and endothelin-1 secretion were examined. Isosteviol (1-100 $\mu \mathrm{M})$ inhibits angiotensin-II -induced DNA synthesis and endothelin-1 secretion. Measurements of 2'7'-dichlorofluorescin diacetate, a redox sensitive fluorescent dye, showed an isosteviol- mediated inhibition of intracellular ROS generated by the effects of angiotensin II. The inductive properties of angiotensin II on ERK phosphorylation were found reversed with isosteviol and antioxidants such as $\mathrm{N}$-acetyl-cysteine.

Conclusion(s): Our data suggest that isosteviol inhibit the ROS which are involved in Ang II-induced proliferation and the redox-sensitive ERK pathway plays a role in ET-1 gene expression in rat aortic SMCs that contribute to the hyperproliferation of vascular smooth muscle cells and vascular plaque formation. Thus, this study provides important insight that may contribute to the effects of isosteviol on the cardiovascular system

References:

1 Cheng TH J AM Cell Cardiol 2003; 42: 1845-1854.

2 Wong KL Life Sci 2004; 74: 2379-2387.

\section{AP8-2}

Effects of 5-hydroxytryptamine on rocuronium-induced neuromuscular blockade in the rat phrenic nervehemidiaphragm preparation

W.C. Choi, Y.K. Lee, H.S. Yang

Department of Anesthesiology and Pain Medicine, Asan Medical Center, College of Medicine, University of Ulsan, Songpa-gu, Republic of Korea

Background and Goal of Study: The 5-Hydroxy-tryptamine 3 receptor is a member of a superfamily of the ligand-gated ion channels. It has structural 
similarities and common evolutionary origin to the nicotinic acetylcholine receptor. 5-Hydroxytryptamine (5-HT) and muscle relaxants may have cross reaction. This study was investigated the effects of $5-\mathrm{HT}$ on rocuroniuminduced neuromuscular blockade.

Materials and Methods: Institutional approvement was obtained. Fifty male Sprague Dawley rats (150-200 g) were divided into 5 groups (control, 0.1, 1, $10,20 \mu / \mathrm{ml}$ of $5-\mathrm{HT}$ ). The animals were injected with phenobarbital $40 \mathrm{mg} / \mathrm{kg}$ in peritoneal cavity. The hemidiaphragm with the phrenic nerve was dissected and mounted within 5 minutes in a bath containing $100 \mathrm{ml} \mathrm{Kreb's} \mathrm{solution} \mathrm{at}$ room temperature. The phrenic nerve was stimulated at supramaximal intensity by a Grass ${ }^{\circledR}$ S88 stimulator through an SIU5 isolation unit. A twitch height was measured by a precalibrated Grass FT88 force displacement transducer and recorded with a Grass 79 polygraph. In the cumulative doseresponse study, $100 \mu \mathrm{g}$ of rocuronium and each doses of 5-HT were administered simultaneously, and incremental $50 \mu \mathrm{g}$ doses of rocuronium were added to obtain more than $95 \%$ twitch inhibition. $\mathrm{ED}_{5}, \mathrm{ED}_{50}, \mathrm{ED}_{90}, \mathrm{ED}_{95}$ of rocuronium in each group were calculated using a logistic model.

Results and Discussions: ED50 of rocuronium was significantly reduced in the $20 \mu / \mathrm{ml} 5$-HT group $(\mathrm{p}<0.05)$.

Conclusion(s): $5-\mathrm{HT} 20 \mu / \mathrm{ml}$ enhanced the neuro-muscular blockade of rocuronium.

References:

1 Cross KM. Br J Pharmacol 1995; 114: 1636-40.

2 Colomo F. Eur J Pharmacol 1968; 3: 272-4.

\section{AP8-3}

Verapamil-fentanyl interaction on rat neuromuscular junction

E. Amaniti, D. Vasilakos, K. Karakoulas, N. Gorgias, E. Matsaridou

Anaesthesiology, AHEPA University Hospital, St Demetrios Gen. Hospital, G. Gennimatas Gen. Hospital, Thessaloniki, Greece

Background and Goal of Study: Verapamil is a calcium channel blocker Previous studies have shown that verapamil may inhibit neuromuscular transmission. On the other hand, opioids have also been found to exert complex action on neuromuscular junction. Aim of the study was the in vitro evaluation of possible synergism between verapamil and fentanyl on diaphragm contraction. Furthermore, the action of neostigmine, naloxone, $\mathrm{CaCl}_{2}$ and isoproterenol was studied regarding their potential interaction with verapamil-fentanyl. Materials and Methods: 24 Wistar rats were sacrificed; their phrenic nerves and the diaphragm were dissected and suspended in Krebs' solution. The nerves were stimulated with 4 Volts, $4 \mathrm{~Hz}$, square wave pulses and the isotonic outputs were recorded. Verapamil and fentanyl were added in the bath at concentrations of $5.09 \times 10^{-5} \mathrm{M}$ and $1.4 \times 10^{-6} \mathrm{M}$ respectively and the muscles isotonic outputs were recorded again. Afterwards, preparations were assigned into four groups to be subjected to naloxone $0.5 \times 10^{-6} \mathrm{M}, \mathrm{CaCl}_{2} 3.40 \times 10^{-3} \mathrm{M}$, neostigmine $1.1 \times 10^{-6} \mathrm{M}$ and isoproterenol $1.0 \times 10^{-6} \mathrm{M}$ respectively. Isotonic muscle outputs were also recorded. Student t-test was conducted for the statistical analysis. Level of significance was set at $p<0.05$.

Results and Discussions: The mean reduction of twitch height observed after verapamil addition was $38.59 \pm 15.83 \%(p<0.005)$. Fentanyl addition lead to a $44.80 \pm 2.30 \%$ reduction $(p<0.005)$. Neither naloxone, nor neostigmine and $\mathrm{CaCl}_{2}$ produced any change in the twitch height, whereas isoproterenol produced an increase on the twitch height of $51.00 \pm 3.12 \%(p<0.005)$.

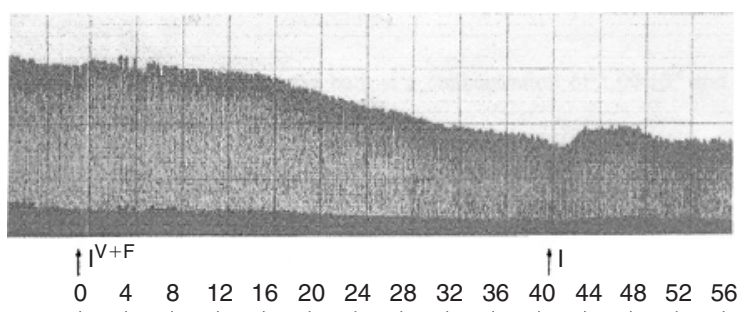

Fig 1. V+F: Verapamil fentanyl action. I: Isoproterenol action.

Conclusion(s): Verapamil and fentanyl act synergistically on the neuromuscular junction, action that is partially reversed by isoproterenol.

Reference:

1 Sim M.K, Chua M.E. Clin Exp Pharm Physiol 1986; 13: 159-162.

\section{AP8-4}

Amitriptyline attenuates smooth muscle contraction of rat trachea through the Rho-kinase pathway

O. Shibata, K. Nishioka, M. Yamaguchi, T. Makita, K. Sumikawa

Anesthesiology, Nagasaki University School of Medicine, Nagasaki, Japan
Background and Goal of Study: Amitriptyline, a tricyclic antidepressant, abolishes smooth muscle contraction induced by acetylcholine (Ach) ${ }^{(1)}$, bradykinin, histamine or norepinephrine. On the other hand, landiolol, a beta ${ }_{1}$ adrenoceptor antagonist, causes airway smooth muscle contraction through the activation of Rho-kinase pathway ${ }^{(2)}$.

It is not clear whether amitriptyline would induce relaxation of airway smooth muscle through Rho-kinase pathway. We examined the effects of amitriptyline on the airway smooth muscle contraction induced by landiolol.

Materials and Methods: This study was conducted following guidelines approved by our Institutional Animal Care Committee. Sixteen male Wistar rats weighing 250-350 g were used for the experiments. Their tracheas were cut into 3-mm-wide ring segments. The tracheal ring was suspended between two stainless steel hooks and placed in organ chamber containing Krebs-Henseleit solution. The resting tension was adjusted periodically to $1.0 \mathrm{~g}$ during equilibration period. Landiolol, $700 \mu \mathrm{M}$, or Ach, $10 \mu \mathrm{M}$, was added, and 30 minutes later, tension was measured by stepwise cumulative additions of amitriptyline. Data are expressed as mean $\pm \mathrm{SD}$, and statistical significance $(P<0.05)$ was determined using ANOVA.

Results and Discussions: Landiolol- and Ach-induced contraction was completely inhibited by amitriptyline. The $\mathrm{ID}_{50}$ values for amitriptyline on landiololand Ach-induced tracheal ring contraction were $64 \pm 26 \mu \mathrm{M}$ and $2.6 \pm 1.3 \mu \mathrm{M}$, respectively.

Conclusion(s): Amitriptyline, in part, attenuates airway smooth muscle contraction through the Rho-kinase pathway.

\section{References:}

1 Huang Y. Pharmacology 1997; 54: 312-318.

2 Shibata O. Anesthesiology 2006; 105: A726.

\section{AP8-5}

Direct increase of the intracellular calcium concentration in porcine vascular smooth muscle cells by protamine

M. Wehner, H. Rueffert, D. Geier, K. Krist, F. Koenig

Anaesthesiology and Intensive Care Medicine, University Hospital Leipzig, Leipzig, Germany

Background and Goal of Study: Protamine is a polycationic protein used in clinical practice for antagonizing the anticoagulatory effect of heparin. Acute pulmonary arterial hypertension by vasoconstriction is one side effect of protamine $(1,2)$. Allergic reactions, liberation of thromboxane or serotonin were assumed to contribute to this side effect, but the exact mechanism could not revealed (1,2). We investigated if protamine is able to increase the calcium concentration in vascular smooth muscle cells as a mechanisms of vasoconstriction.

Materials and Methods: Vascular smooth muscle cells were obtained from immediately slaughtered pigs from two different German breeds. The smooth muscle cells were cultured in smooth muscle medium (PromoCell, Germany). Intracellular calcium concentration in single myocytes was measured with FURA 2 (Invitrogen) at an imaging workstation (Till Photonics, Germany). Results and Discussions: Protamine increased the intracellular calcium concentration in myocytes in a dose-dependent manner. The half-maximal effect concentration $\mathrm{EC}_{50}$ was $46.6 \mathrm{mg} / \mathrm{ml}$. In a calcium-free extra cellular solution the calcium transient was not detected. The intracellular stores did not contribute significantly to the protamine induced calcium transient.

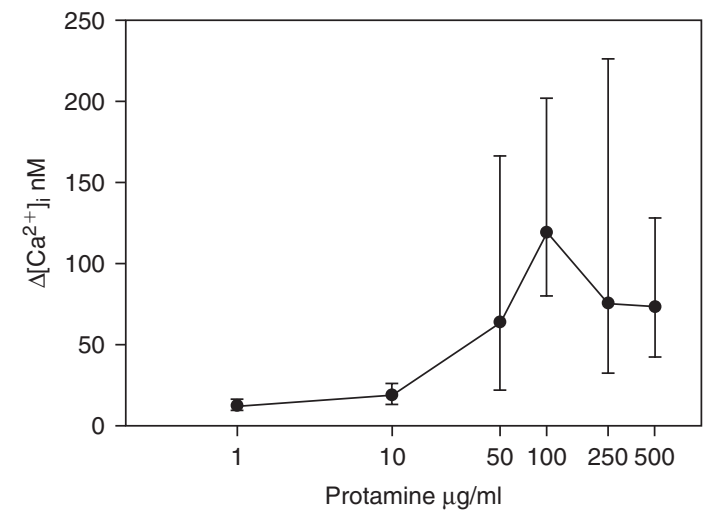

Fig 1. Dose-effect curve of protamine (median, 25th/75th percentile, $\Delta\left[\mathrm{Ca}^{2+}\right]^{\mathrm{i}}$ increase of intracellular calcium).

Conclusion: The protamine evoked intracellular calcium transient in porcine vascular myocytes may induce a vasoconstriction in a direct manner. This 
mechanism can contribute to the known side effect acute pulmonary arterial hypertension of protamine.

References:

1 Comunale M.E., Malow A., Robertson L.K., et al. J Cardiovasc Anesth 2003, 17, 309-313.

2 Habazettl H., Conzen P.F., Vollmar P., et al. Anesth Analg 1990, 71, 637-644.

\section{AP8-6}

The relationship between $\mathrm{Ca}$-induced Ca release function in skinned fibers and calcium homeostasis in human myotubes

K. Mukaida, T. Migita, M. Kobayashi, M. Kawamoto, O. Yuge

Anesthesiology, Hiroshima Prefectural Rehabilitation Center, Higashi-hiroshima, Japan

Background and Goal of Study: Malignant hyperthermia is an inherited disorder of calcium homeostasis in the skeletal muscle caused by enhanced rate of Ca-induced Ca release (CICR) via ryanodine receptor 1 (RYR1). We assessed the relationship between CICR rate and sensitivity of human myotubes to caffeine, halothane and 4-chloro-m-cresol (4-CmC).

Materials and Methods: We measured CICR rate in chemically skinned fibers using Endo's method and divided 18 individuals into 'Enhanced' and 'Normal' groups according to their CICR rate. The intracellular calcium concentration of myotubes was determined with fluorescent calcium imaging system using fura-2. To stimulate myotubes, caffeine, halothane and $4-\mathrm{CmC}$ were used as RYR1 agonists. Half-maximal activation concentrations $\left(\mathrm{EC}_{50}\right)$ were determined in myotubes.

Results and Discussions: 'Enhanced' and 'Normal' group contained 9 individuals each. EC50 for caffeine, halothane and 4-CmC were significantly $(p<0.001$ ) lowered showing keen sensitivity to RYR1 (Table 1). The highest correlation coefficient $(|r|=0.757)$ was observed between CICR rate (pCa5.0) and $\mathrm{EC}_{50}$ for $4-\mathrm{CmC}$.

Table 1. A comparative data in the two groups

\begin{tabular}{lcc}
\hline Groups & Enhanced & \multicolumn{1}{c}{ Normal } \\
\hline Resting Ca Conc. $(\mathrm{nM})$ & $79.5 \pm 17.8$ & $51.8 \pm 9.6$ \\
EC $_{50}$ for Caffeine $(\mathrm{mM})$ & $2.72 \pm 0.88$ & $5.78 \pm 0.75$ \\
$\mathrm{EC}_{50}$ for Halothane $(\mathrm{mM})$ & $1.89 \pm 0.28$ & $3.72 \pm 0.76$ \\
EC $_{50}$ for 4-CmC $(\mu \mathrm{M})$ & $141.2 \pm 46.5$ & $349.2 \pm 76.1$ \\
\hline
\end{tabular}

Conclusion(s): There was significant relationship between CICR rate in skinned fibers and sensitivity to RYR1 agonists in myotubes.

References:

1 Endo M, Yagi S, Ishizuka T, et al. Biomed Res 1983; 4: 83-92.

2 Wehner M, Rueffer H, Koenig F, et al. Neuromuscul Disord. 2004; 14429-14437.

\section{AP8-7}

\section{Levosimendan in septic myocardial depression:} hemodynamics and neurohormonal effects

F. Guarracino, C. Cariello, L. Giannecchini, R. Baldassarri, A. Danella Cardiothorax Department, Cardiothoracic Anesthesia and ICU, Pisa, Italy

Background and Goal of the Study: Septic shock is characterized by profound cardiovascular alterations including myocardial depression. Levosimendan has recently showed to improve cardiac function in septic shock..$^{(1,2)}$

The aim of this retrospective study is to evaluate hemodynamic and neurohormonal effects of levosimendan in cardiac patients with sepsis-induced cardiac dysfunction.

Materials and Methods: Fifteen patients with myocardial depression related to septic shock were enrolled. All patients had SIRS criterias, cultures isolation of 1 or more pathogens, positive PCT, SBP $<90 \mathrm{mmHG}$ unresponsive to load challenge.

We defined myocardial depression as reduced $\mathrm{SvO}_{2}$ in presence of increased BNP secretion and Trop I release, and systolic and/or diastolic dysfunction by TEE evaluation of EF and mitral annulus TDI velocities. All pts. received Levosimendan infusion for 24 hours at $0.1 \mathrm{mcg} \mathrm{kg}^{-1} \mathrm{~min}^{-1}$ combined with norepinephrine.

Results and Discussion: Data were obtained by evaluating the average of percentual variation between T0 (starting infusion) and T1 (24h after infusion) T2 (48h), T3 (72 h), T4 (96h), T5 (120h) and T6 (144h). Levosimendan significantly increased $\mathrm{SvO}_{2}$ and EF, and decreased Trop I and BNP. Levosimendan improved diastolic function by increasing E' velocity at TDI at $48 \mathrm{~h}$. All data were analysed by Fisher F test.

\begin{tabular}{llllllll}
\hline & $\mathrm{T} 0$ & $\mathrm{~T} 1$ & $\mathrm{~T} 2$ & $\mathrm{~T} 3$ & $\mathrm{~T} 4$ & $\mathrm{~T} 5$ & $\mathrm{~T} 6$ \\
\hline $\mathrm{SvO}_{2} \%$ & $0 \%$ & $4 \%$ & $10 \%$ & $17 \%$ & $22 \%$ & $19 \%$ & $22 \%$ \\
Trop I & $0 \%$ & $-65 \%$ & $-86 \%$ & $-82 \%$ & $-78 \%$ & $-62 \%$ & $-61 \%$ \\
ng ml & & & & & & & \\
$\mathrm{BNP}$ & $0 \%$ & $-55 \%$ & $-41 \%$ & $-56 \%$ & $-50 \%$ & $-42 \%$ & $-44 \%$ \\
E' cm & $<8$ & $>8$ & $>8$ & $>8$ & $>8$ & $>8$ & $>8$ \\
sec & & & & & & & \\
EF $\%$ & $<30 \%$ & $>40 \%$ & $>40 \%$ & $>40 \%$ & $>40 \%$ & $>40 \%$ & $>40 \%$ \\
\hline
\end{tabular}

Conclusions: Levosimendan seems to improve systemic hemodynamics and neurohormonal cardiac function in patients with septic cardiac dysfunction.

References:

1 Noto A, Giacomini M, Palandi A, et al: Levosimendan in septic cardiac failure. Intensive Care Med 2005; 31: 164-165.

2 Morelli A, De Castro S, Teboul JL, et al. Effect of levosimendan on systemic and regional hemodynamics in septic myocardial depression. Intensive Care Med 2005; 31: $638-644$.

\section{AP8-8}

\section{The neurofilament cytoskeleton is essential to the hypnotic sensitivity}

D. Pietrin, F. Letournel, J.C. Granry, J. Eyer, A. Barthelaix

1-Department of Anaesthesia and Critical Care 2-2-Neurobiology and Transgenesis Laboratory UPRES 314, Hospital University Angers, Angers, France

Background and Goal of Study: Molecules and mechanisms related to differences of hypnotic sensibility remain largely unclear (1). To explore whether the neurofilament cytoskeleton could be involved in anesthesia susceptibility, we used a transgenic model (NFHLacZ) in which the expression of a fusion protein between B-galactosidase and the high molecular weight neurofilament subunit (NFH) induces neurofilament aggregation in cell bodies and their axonal deficiency (2). Transgenic and normal mice from the same age and the same genetic background were tested for their sensitivity to three different hypnotic drugs.

Materials and Methods: Protocols were approved by regional ethic committee for animal experimentation. Behavioral test and LORR were performed before and during anaesthesia on adult (10-weeks-old) transgenic and normal mice using video monitoring. Anesthesia was induced by intra-peritoneal injection of ketamine $(200 \mathrm{mg} / \mathrm{kg} ; \mathrm{n}=16)$, thiopental $(100 \mathrm{mg} / \mathrm{kg} ; \mathrm{n}=10)$, or midazolam $(20 \mathrm{mg} / \mathrm{kg} ; \mathrm{n}=10)$ in a blind fashion concerning the genotype of mice. Each movie was analyzed by a second experimenter blind concerning the anesthetics used and the genotype. Endpoint of study was duration of LORR. Statistical analysis was performed by unpaired t test with SAS software (SAS®, USA). A P value less than 0.05 was considered as significant. Results and Discussion: Data were expressed as mean \pm SEM. Behavioral test showed no difference between transgenic and control mice before anesthesia $(p=0.9)$. While duration of LORR was equal between transgenic and normal mice using midazolam ( $6 \pm 3$ min vs $5 \pm 2 \mathrm{~min} ; p=0.8$ ), the duration of LORR was shorter for transgenic mice with ketamine ( $14 \pm 3$ min vs $26 \pm 3 \mathrm{~min}$; $p=0.02)$, but longer with thiopental ( $98 \pm 12$ min vs $39 \pm 13 \mathrm{~min} ; \mathrm{p}=0.01$ ). Conclusion: This study clearly shows that the absence of axonal neurofilaments affects differently the anesthesia due to ketamine and thiopental injections. This indicates a fundamental role of neurofilaments, and/or associated molecules, to the hypnotic drugs susceptibility. The possible perturbation of distribution, accessibility, and function of NMDA or GABA ${ }_{-}$receptors is presently investigated in these animals.

References:

1 Sato Y et al Acta Anaesthesiol Scand 2006

2 Eyer $\mathrm{J}$ et al Nature 1998

\section{AP8-9}

Impact of cyclosporine upon emotional behavior in mice

Y. Sato, T. Onaka, E. Kobayashi, N. Seo

Department of Anesthesiology, Jichi Medical School, Shimotsuke, Japan

Background and Goal of Study: The calcineurin inhibitor, cyclosporine, is widely used for preventing allograft rejection in organ transplantation. Several studies report that cyclosporine induce neuropsychological side effects (1) and prolong sleep times after administration of barbiturate (2). However, its mechanisms of action remain to be elucidated.

Materials and Methods: Systemically administered cyclosporine is prevented from entering into the brain by the action of P-glycoprotein. In the present study, we developed behavioral models in wild-type and mdr1 (multidurg registrant 1) knockout mice that lack P-glycoprotein (3) to examine central effects of cyclosporine upon anxiety. After intraperioneal administration of cyclosporine, open field test and elevated-plus maze test were performed. 
Results: Administration of cyclosporine decreased motor activity in both wildtype and knockout mice. In wild-type mice, cyclosporine did not significantly change percent entries into open arms and time spent on open arm of the elevated plus maze, and in the center of the open field. On the other hand in mdr1a knockout mice, cyclosporine decreased the percentage of stay time in the open arm of the elevated plus maze, and in the center of the open field. Conclusion: We found that centrally located cyclosporine increase anxietyrelated behavior. The present finding might be pathologically relevant in patients who take cyclosporine as immunosuppressant therapy.

1 Hughes RL. N Engl J Med 1990; 323: 420-421.

2 Cirella VN. Anesth Analg 1987; 66: 703-706.

3 Schinkel AH. J Clin Invest 1996; 97: 2517-2524.

\section{AP8-10}

\section{Chronic therapy by statin before vascular surgery: are} patients really treated?

S. Perbet, A. Soummer, Y. Le Manach, P. Coriat, G. Godet

Department of anesthesiology and critical care, Centre Hospitalier Universitaire Pitié-Salpétrière, PARIS, France

Background and Goal of Study: Statins are a common chronic therapy of dyslipemic patients (primary prevention) and of cardiovascular risk patients (secondary prevention). The goals of this prospective study on patients undergoing noncardiac vascular surgery were 1) to evaluate the real incidence of chronic therapy by statin (CTS) 2) to see if objectives in low-density lipoprotein(LDL) cholesterol lowering were reached.

Materials and Methods: All cardiovascular risk factors (RF) [coronaropathy, diabetes mellitus, tabagism, age, gender, peripheral atherosclerosis, cerebrovascular disease] and the rates of HDL- and LDL-cholesterol ( $\mathrm{g} / \mathrm{L})$ of 213 patients undergoing vascular surgery were recorded. Inflammatory, congenita and traumatic pathologies were excluded. The existence of CTS and his efficacity on LDL rate were evaluated in different risk groups of patients.

Results and Discussions: All patients of our study had at least the indication of secondary prevention by CTS.

\begin{tabular}{llcclcr}
\hline Groups & $\begin{array}{l}\text { LDL } \\
\text { objective } \\
(\mathrm{g} / \mathrm{L})\end{array}$ & $\begin{array}{l}\text { Patients } \\
\text { with CTS } \\
\mathrm{N}(\%)\end{array}$ & $\begin{array}{l}\text { Patients } \\
\text { without } \\
\text { CTS N (\%) }\end{array}$ & $\begin{array}{l}\text { LDL } \\
\text { reached if } \\
\text { CTS N (\%) }\end{array}$ & $\begin{array}{l}\text { LDL } \\
\text { reached If no } \\
\text { CTS N (\%) }\end{array}$ & Total N (\%) \\
\hline 0 or 1 RF & $<2.2$ & $0(0)$ & $2(100)$ & $0(0)$ & $2(100)$ & $2(0.9)$ \\
2 RF & $<1.6$ & $2(15)$ & $11(85)$ & $2(100)$ & $11(100)$ & $13(6.1)$ \\
$>2$ RF & $<1.3$ & $3(30)$ & $7(70)$ & $3(100)$ & $5(71)$ & $10(4.7)$ \\
High risk & $<1$ & $113(60)$ & $75(40)$ & $68(60)$ & $49(65)$ & $188(88)$ \\
Total & & $118(55)$ & $95(45)$ & $73(62)$ & $67(71)$ & 213 \\
\hline
\end{tabular}

Conclusion: Patients with cardiovascular high risk undergoing vascular surgery don't received all a CTS despite recommendations. Objectives in LDL rates are not reached in preoperative time in one third of the most severe patients. Preoperative introduction of statin therapy and control of his efficacity could be discussed in these patients.

\section{AP8-11}

\section{Genotyping patients with prolonged duration of action of succinylcholine by dHPLC}

E. Schobinger, S. Levano, A. Urwyler, T. Girard

Anaesthesia and Research, University hospital Basel, Basel, Switzerland

Background and Goal of Study: The short duration of action of succinylcholine (SCh) is crucial if intubation and ventilation are impossible and is due to a rapid hydrolysis by the enzyme butyrylcholinesterase (BCHE) (1).

A prolonged duration of action can be due to acquired or inherited reductions in BCHE activity (2)

The aim of this study was to determine the incidence of genetic BCHE variants in patients with prolonged duration of action of SCh.

Materials and Methods: Duration of action of SCh $1 \mathrm{mg} / \mathrm{kg}$ until recovery of neuromuscular function was recorded.

Blood samples were taken from patients with a prolonged duration of neuromuscular block.

Genomic DNA was isolated and amplified by PCR (7 PCR-reactions, covering all the coding regions of the gene) in patients with a duration of action of 18 minutes or beyond. PCR products were analyzed by dHPLC.

Aberrant dHPLC profiles were controlled by automated sequencing in both directions.

Results and Discussions: Out of 1490 patients 24 (2.3\%) showed a neuromuscular block $\geqslant 18 \mathrm{~min}$. 12 patients had a wild-type genotype, while the other 12 showed different combinations of the following variants: 5 heterozygous for the A-variant, 9 carrying the K-variant ( 7 heterozygous and 2 homozygous) and one with the F-variant G417V. In addition we detected a novel variation W239S.

Conclusion(s): Variations of the BCHE gene were present in $50 \%$ of patients with prolonged muscle relaxation. The dHPLC technique seems to be an adequate and also rapid method for detection of known and novel variations.

References

1) Levano, S., et al. Anesthesiology 2005; 102: 531-5.

2) Davis, L. Anaesthesia 1997; 52: 244-60.

\section{Paediatric Anaesthesia and Intensive Care}

\section{AP1-1}

\section{EEG spectral entropy during anaesthesia in children:} halothane versus sevoflurane

S. Leclercq, C. Hanart, A. De Villé, S. De Hert, P. Van der Linden Anaesthesiology, CHU Brugmann-HUDERF, Brussels, Belgium

Background and Goal of Study: The spectral entropy of the EEG (state entropy: SE) and frontal electromyogram (response entropy: RE) have been promoted as monitors of anaesthetic depth, but their characteristics in children remain poorly defined. This prospective randomized double-blind study compared SE and RE values during halothane and sevoflurane anaesthesia administered at equipotent dose in children.

Materials and Methods: Following institutional Ethics Committee approval and parental written informed consent, 40 ASA I/II children aged 6 months -5 years undergoing general anaesthesia for lower abdominal surgery were studied. Children were randomized to undergo halothane (group $\mathrm{H}: \mathrm{N}=20$ ) or sevoflurane (group $\mathrm{S}: \mathrm{N}=20$ ) All received caudal analgesia (bupivacaine $2 \mathrm{mg} / \mathrm{kg}$ ) after anaesthetic induction. Entropy values were recorded by a blinded anaesthesiologist before induction and at 1 and 2 MAC steady state end tidal concentrations, and at awakening. Data were compared across the two groups with a two-way analysis of variance followed by a post hoc Tuckey test. A $p<0.05$ was considered significant. Data: mean \pm SD.

Results and Discussions: Demographic and surgical characteristics did not differ between groups.

\begin{tabular}{llll}
\hline & Entropy & $\begin{array}{l}\text { Group H } \\
(\mathrm{N}=20)\end{array}$ & $\begin{array}{l}\text { Group S } \\
(\mathrm{N}=20)\end{array}$ \\
\hline Before induction & SE & $84 \pm 8$ & $87 \pm 4$ \\
1 MAC end-tidal & RE & $93 \pm 8$ & $95 \pm 3$ \\
2 MAC end-tidal & SE & $63 \pm 9$ & $38 \pm 13^{*}$ \\
Awakening & RE & $71 \pm 11$ & $48 \pm 15^{*}$ \\
& SE & $50 \pm 16$ & $31 \pm 22^{*}$ \\
& RE & $54 \pm 17$ & $40 \pm 24$ * \\
\hline
\end{tabular}

Conclusion(s): Entropy values are higher during halothane vs. sevoflurane anaesthesia in children. This may be due to different pattern of EEG depression with halothane and sevoflurane. Similar results have been reported for bispectral index values ${ }^{1}$.

Reference:

1 Edwards JJ et al. Acta Anaesthesiol Scand 2005; 49: 1084-7.

\section{AP1-2}

EEG spectral entropy during anaesthesia in children: effects of nitrous oxide

C. Hanart, S. Leclercq, M. Gerin, S. De Hert, P. Van der Linden Anaesthesiology, CHU Brugmann-HUDERF, Brussels, Belgium 
Background and Goal of Study: The spectral entropy of the EEG (state entropy: SE) and frontal electromyogram (response entropy: RE) have been promoted as monitors of anaesthetic depth, but their characteristics in children remain poorly defined. This prospective randomized double-blind study assessed the effects of $50 \%$ N20 on SE and RE values during halothane and sevoflurane anaesthesia in children.

Materials and Methods: Following institutional Ethics Committee approval and parental written informed consent, 40 ASA I and II children aged 6 months -5 years undergoing general anaesthesia for lower abdominal surgery were studied. Children were randomized to undergo halothane (group $\mathrm{H}: \mathrm{N}=20$ ) or sevoflurane (group $\mathrm{S}: \mathrm{N}=20$ ) All received caudal analgesia (bupivacaine $2 \mathrm{mg} / \mathrm{kg}$ ) after anaesthetic induction. Entropy values were recorded by a blinded anaesthesiologist at $1 \mathrm{MAC}$ steady state end tidal concentrations, in $50 \% \mathrm{O}_{2} /$ air and $50 \% \mathrm{O}_{2} / \mathrm{N}_{2} 0$ conditions. Data were compared with a paired Student $t$ test. A $p<0.05$ was considered significant. Data are presented as mean \pm SD.

Results and Discussions: Demographic and surgical characteristics were not different among groups.

\begin{tabular}{llll}
\hline & Entropy & $\begin{array}{l}\text { Group H } \\
(\mathrm{N}=20)\end{array}$ & $\begin{array}{l}\text { Group S } \\
(\mathrm{N}=20)\end{array}$ \\
\hline $\mathrm{O}_{2} /$ air & $\mathrm{SE}$ & $55 \pm 11$ & $35 \pm 19$ \\
& $\mathrm{RE}$ & $62 \pm 13$ & $44 \pm 21$ \\
$\mathrm{O}_{2} / \mathrm{N}_{2} \mathrm{O}$ & $\mathrm{SE}$ & $52 \pm 11$ & $29 \pm 13$ \\
& $\mathrm{RE}$ & $57 \pm 12$ & $38 \pm 15$ \\
\hline
\end{tabular}

Conclusion(s): Administration of $\mathrm{N}_{2} \mathrm{O}$ did not influence $\mathrm{SE}$ and $\mathrm{RE}$ values during halothane or sevoflurane anaesthesia in children.

\section{AP1-3}

\section{EEG spectral entropy during sevoflurane anaesthesia in} children: influence of age

S. Leclercq, F. De Groote, A. De Villé, S. De Hert, P. Van der Linden Anaesthesiology, CHU Brugmann-HUDERF, Brussels, Belgium

Background and Goal of Study: The spectral entropy of the EEG (state entropy: SE) and frontal electromyogram (response entropy: RE) have been promoted as monitors of anaesthetic depth, but their characteristics in children remain poorly defined. This prospective randomized double-blind study evaluated the effects of age on SE and RE values during sevoflurane anaesthesia. Materials and Methods: Following institutional Ethics Committee approval and parental written informed consent, 20 ASA I and II children aged 6 months- 5 years undergoing general anaesthesia for lower abdominal surgery were studied. Children were divided into 2 age groups: 6 months2 years old (infants; $N=10$ ) and $2-5$ years old (children; $N=10)^{1}$. All children received caudal analgesia (bupivacaine $2 \mathrm{mg} / \mathrm{kg}$ ) after anaesthetic induction. Entropy values were recorded by a blinded anaesthesiologist before induction, at $1 \mathrm{MAC}$ and 2 MAC steady state end tidal sevoflurane concentrations, and at awakening. Data were compared across the two groups with a two-way analysis of variance followed by a posthoc Tuckey test. A $p<0.05$ was considered significant. Data are presented as mean \pm SD.

Results and Discussions: Demographic and surgical characteristics were not different among groups.

\begin{tabular}{llll}
\hline & Entropy & $\begin{array}{l}\text { Infants } \\
(\mathrm{N}=10)\end{array}$ & $\begin{array}{l}\text { Children } \\
(\mathrm{N}=10)\end{array}$ \\
\hline Before induction & $\mathrm{SE}$ & $85 \pm 5$ & $88 \pm 2$ \\
& $\mathrm{RE}$ & $94 \pm 4$ & $95 \pm 3$ \\
$1 \mathrm{MAC}$ end-tidal & $\mathrm{SE}$ & $42 \pm 16$ & $34 \pm 9$ \\
& $\mathrm{RE}$ & $53 \pm 16$ & $43 \pm 12$ \\
$2 \mathrm{MAC}$ end-tidal & $\mathrm{SE}$ & $32 \pm 21$ & $30 \pm 26$ \\
& $\mathrm{RE}$ & $42 \pm 25$ & $38 \pm 25$ \\
Awakening & $\mathrm{SE}$ & $83 \pm 7$ & $77 \pm 21$ \\
& $\mathrm{RE}$ & $93 \pm 10$ & $89 \pm 15$ \\
\hline
\end{tabular}

Conclusion(s): For children aged over 6 months, entropy values did not seem to be influenced by age during sevoflurane anaesthesia.

References

1. Edwards JJ et al. Acta Anaesthesiol Scand 2005; 49: 1084-7.

\section{AP1-4}

A comparative study between cerebral state index (CSI) and bispectral index (BIS), in children anesthetised with sevoflurane F. Piana, A. Rigouzzo, S. Lestrat, M. Sammartino, I. Constant Anesthesiology, policlinico Universitario A. Gemelli, Roma, Italy
Background and Goal of Study: The CS Monitor (CSM) has been recently introduced for monitoring depth of anaesthesia. Data on CSM are lacking in children. This study compare the CSI with the BIS in children anesthetised with sevoflurane.

Materials and Methods: After CPP approval, 17 patients, $7.5 \pm 3 \mathrm{yr}$ (mean $\pm \mathrm{sd}$ ) scheduled for tonsillectomy were included. Anesthesia was induced with $6 \%$ sevoflurane in $\mathrm{O}_{2}-\mathrm{N}_{2} \mathrm{O}(50-50)$, tracheal intubation was performed at central pupils, no opioid or muscle relaxant was used. Then, five 10 min-periods with a stable expired concentration of sevoflurane (Etsevo: $1,2,3,4$ and $5 \%$ ) were obtained in each children. The surgery started after completion of the study. In addition to the standard monitoring, the BIS (Aspect medical Systems, Newton, MA) and CSI (Danmeter, Odense, Denmark) were continuously recorded in all children. Mean values of BIS and CSM were calculated on the last minute of each stable $10 \mathrm{~min}$ period, and compared for each Etsevo using paired $t$ test. In addition the relationship between both monitor indices and Etsevo was investigated using the most appropriate model of regression (Statview, Abacus)

Results and Discussions: Mean values of BIS and CSM decreased when ETsevo increased up to $3 \%$, and then going up again, especially for the BIS (figure) A second order regression described the relationship between BIS and CSI, and Etsevo, $r^{2}=0.85$ and $r^{2}=0.87$, respectively.

Conclusion(s): The CSI might be an interesting alternative to the BIS during the sevoflurane anesthesia in children. Further studies are required to understand BIS and CSM changes at high concentrations of sevoflurane

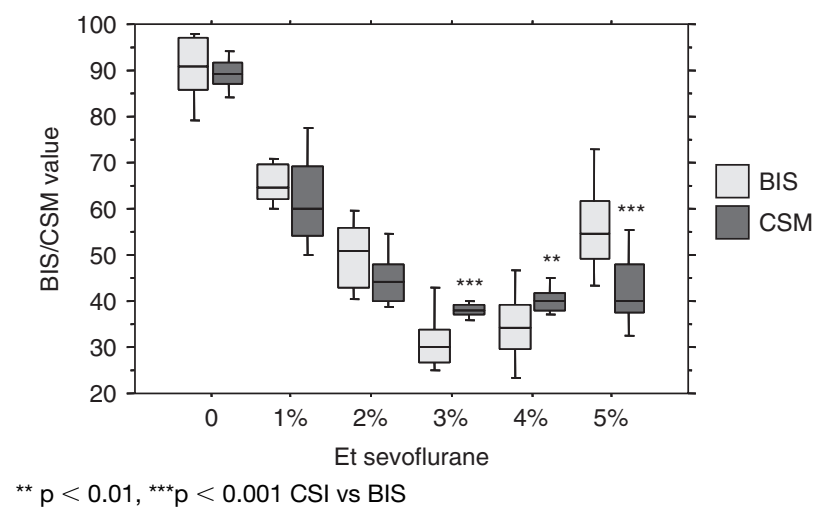

\section{AP1-5}

Reliability of the $\mathrm{VO}_{2}$ measurement using the deltatrec II module for cardiac output calculation in intubated children undergoing cardiac catheterisation

A. Schmitz, O. Kretschmar, W. Knirsch, U. Bauersfeld, M. Weiss Department of Anaesthesia, University Childrens Hospital Zurich, Zurich, Switzerland

Background and Goal of Study: To compare cardiac output values calculated with measured $\mathrm{VO}_{2}$ with cardiac output values calculated with formula-based $\mathrm{VO}_{2}$ in intubated children in a cardiac catheter laboratory setting.

Materials and Methods: With ethical review board approval and written parental consent, $\mathrm{VO}_{2}$ was measured using the Datex-Ohmeda Compact Airway Module $\mathrm{AS} / \mathrm{3}^{\mathrm{TM}}$ (Datex-Ohmeda, Helsinki) in children undergoing interventional cardiac catheterisation under general anaesthesia. Cuffed tubes were used for tracheal intubation. Measurements were performed in patients without cardiac shunts. Arterial and mixed venous blood was sampled for oxygen saturation measurement in a bedside oximeter. Formula-based $\mathrm{VO}_{2}$ values were chosen according to LaFarge (1). Cardiac output was calculated using the Fick's principle. Data are compared using linear regression and Bland-Altman analysis.

Results: 31 patients aged from 1.0-16.7 years (median 4.9 years) were studied. Comparison of cardiac output values calculated with measured $\mathrm{VO}_{2}$ and formula-based $\mathrm{VO}_{2}$ revealed significant correlation $(r=0.93$; $p<0.0001)$. Bias and precision were -0.08 and $1.59 \mathrm{~L} / \mathrm{min}(95 \%$ limits of agreement: -1.67 to $1.51 \mathrm{~L} / \mathrm{min}$ ).

Conclusion: Cardiac output values calculated with measured $\mathrm{VO}_{2}$ using the Deltatrec $\| \mathrm{VO}_{2}$ module did reliably correspond to formula-based $\mathrm{VO}_{2}$ cardiac output calculations.

Reference:

1 La Farge CG, Miettinen OS. Cardiovascular Research 1970; 4: 23-30. 


\section{AP1-6}

\section{Electrical velocimetry compared with ficks principle for cardiac output measurement in children undergoing cardiac catheterisation}

A. Schmitz, O. Kretschmar, W. Knirsch, U. Bauersfeld, M. Weiss Department of Anaesthesia, University Childrens Hospital Zurich, Zurich, Switzerland

Background and Goal of Study: To compare cardiac output values measured by electrical velocimetry (EV-CO) (Aesculon, Osypka Medical GmbH, Berlin, Germany) $(1,2)$ with cardiac output values calculated from measured $\mathrm{VO}_{2}$ using the Fick's Principle $\left(\mathrm{VO}_{2}-\mathrm{CO}\right)$ in children.

Materials and Methods: With ethical review board approval and written parental consent, EV-CO using the Aesculon Monitor and $\mathrm{VO}_{2}-\mathrm{CO}$ using the Fick's Principle were simultaneously recorded in children without intra-cardiac shunts undergoing cardiac catheterisation. Measurements were performed under general anaesthesia. Cuffed tubes were used for tracheal intubation. Arterial and mixed venous blood was sampled for oxygen saturation assessment in a bedside oximeter. Linear regression analysis and BlandAltman analysis were used to compare EV-CO values with $\mathrm{VO}_{2}-\mathrm{CO}$ values. Results: 31 patients aged from 1.0-16.7 years (median 4.9 years) were studied. Only a moderate correlation between EV-CO and $\mathrm{VO}_{2}-\mathrm{CO}(\mathrm{r}=0.64$ $\mathrm{p}<0.0001)$ was found. Bias and precision were 2.05 and $3.67 \mathrm{~L} / \mathrm{min}(95 \%$ limits of agreement: -1.62 to $5.72 \mathrm{~L} / \mathrm{min}$ ).

Conclusion: Based on our preliminary experience, cardiac output values measured by electrical velocimetry using the Aesculon Monitor did not reliably correlate to cardiac output values calculated with Fick's Principle in children. Because of its user-friendly application and the simple underlying principle the Aesculon device may be used as a trend monitor for cardiac output. To support this, further investigations, using continuous measurement of EV-CO in patients with changing hemodynamics will be needed.

References:

1 Bernstein DP, et al. Crit Care Med 1986; 14: 904-9.

2 Osypka M, Bernstein D. AACN 1999; 10: 385-99.

\section{AP1-7}

Evaluation of the simultaneous performance of two monitors of depth of anaesthesia in children

H. Muñoz, P. León, L. Cortínez

Anestesiología, Pontificia Universidad Católica de Chile, Santiago, Chile

Background and Goal of Study: The $\mathrm{k}_{\mathrm{e} 0}$ of propofol in children has been determined with the use of the tpeak method measured with the Alaris AEP monitor (v1.4).(1) This parameter, however, might be different when the effect of propofol is measured with different monitors. We simultaneously measured the performance of two monitors of depth of anaesthesia after a bolus dose of propofol in children.

Materials and Methods: Children aged 3 to $11 \mathrm{yr}$, ASA I scheduled to undergo surgery under general anaesthesia were prospectively studied. In addition to routine monitoring, all patients were monitored with the $A 2000$ BIS $®$ monitor and the Alaris AEP monitor (v1.6). A bolus dose of propofol $3 \mathrm{mg} / \mathrm{kg}$ was injected in 3 seconds (s), the study finished when patients had partially recovered from propofol defined as the presence of movements of extremities. Tpeak was considered the time from the start of propofol injection until the minimum value of BIS and AAI. BIS and AAI values at baseline, tpeak and at the end of the study were compared. Values are mean \pm SD.

Results and Discussions: Twenty children of $7.3 \pm 2.4 \mathrm{yr}$ and $28 \pm 8 \mathrm{~kg}$ were studied. In all patients, the BIS values were obtained during all the study. Because of poor signal quality, the AAI was obtained in only 12 children and the tpeak could not be determined in 2. All patients lost consciousness very fast after propofol and had signs of recovery before the end of study. Basal values were $94 \pm 4$ for BIS and $69 \pm 22$ for AAI. The minimum values were $\mathrm{BIS}=15 \pm 7$ and $\mathrm{AAI}=21 \pm 13$. The tpeak with $\mathrm{BIS}$ was $65.7 \pm 4.5 \mathrm{~s}$ and with AAI $202 \pm 74 \mathrm{~s}$. At the end of study BIS was $65 \pm 7$ and AAl was $22 \pm 5$. The differences in the measurements obtained with both monitors were statistically significant with the exception of the minimum values.

Conclusion(s): Under the dynamic conditions of this study in children, the performance of these two monitors is clearly different. While the BIS seems to display values more consistent with the clinical condition of the patient, more studies are needed to determine which monitor better reflects the state of consciousness in this population.

Reference:

1 Muñoz HR, Cortínez LI, Ibacache RE, Altermatt FR. Anesthesiology 2004; 101: 1269-74.

\section{AP2-1}

Recommended inflation volumes result in lower cuff pressures in paediatric patients with Ambu AuraOnce when compared to LMA-Classic

H.V. Genzwuerker, J. Sinkowitsch, J. Hinkelbein, O. Jandewerth, E. Ch. Hohl Clinic of Anaesthesiology and Intensive Care Medicine, University Hospital Mannheim, Mannheim, Germany

Background and Goal of Study: Two laryngeal masks, LMA-Classic (LMA, LMA Company) and the single-use Ambu AuraOnce (Aura, Ambu) are compared for ventilation in paediatric patients. Besides ease of insertion, the cuff pressures resulting when the recommended inflation volumes are applied are assessed. Materials and Methods: After approval of the local ethics committee and written consent of the guardians, $100 \mathrm{ASA}$ I/II patients, 2 to 8 years, scheduled for elective ambulatory interventions were randomized to be ventilated with LMA or Aura. Following standardized induction of general anaesthesia, the completely deflated airway devices were placed according to manufacturer's instructions. Cuffs were inflated with $10 \mathrm{ml}$ for size $2,14 \mathrm{ml}$ for size 2.5 and $20 \mathrm{ml}$ for size 3. Number of attempts (maximum 2), time until first tidal volume, initial cuff pressure and intraoperative tidal volumes (goal: petCO $\mathrm{C}_{2}$ of $35 \mathrm{mmHg}$ ) were recorded. Cuff pressures were adjusted to $60 \mathrm{cmH}_{2} \mathrm{O}$ for measurement of airway leak pressure. Devices were inspected for traces of blood after removal. Patients were questioned for postoperative complaints.

Results and Discussions: 50 patients were ventilated with either device. Average age was 5.1 (2.2-8.0) for LMA and 5.1 (2.5-7.9) years for Aura. 44 patients in the LMA group and 48 patients in the Aura group were male. Weight, height, baseline heart rate, blood pressure and oxygen saturation were comparable for both groups. Size 2 was used in LMA/Aura in 7/4, size 2.5 in 41/45 and size 3 in 2/1 patients. Insertion was successful in all patients (first attempt LMA 45, Aura 47). Time until first tidal volume for LMA and Aura was $8.7 \pm 2.3$ and $8.0 \pm 2.3$ seconds. Initial cuff pressures were $104.2 \pm 19.0 \mathrm{cmH}_{2} \mathrm{O}$ for LMA and $74.4 \pm 24.3 \mathrm{cmH}_{2} \mathrm{O}$ for Aura $(\mathrm{p}<0.001)$. Tidal volumes were 8.7 and $9.1 \mathrm{ml} \mathrm{kg}^{-1}$ for LMA and Aura, airway leak pressures were $32.7 \pm 9.4$ and $34.1 \pm 8.4 \mathrm{cmH}_{2} \mathrm{O}$. Intraoperative dislocation occurred in $1 \mathrm{LMA}$ patient. No traces of blood were found after removal of the devices. No postoperative complaints were stated.

Conclusion(s): In paediatric patients, LMA-Classic and Ambu AuraOnce are found to be comparable in all respects except cuff pressures resulting from recommended inflation volumes. Excess of maximum recommended cuff pressure is considerably higher with LMA-Classic.

\section{AP2-2}

\section{Effectiveness of a new paediatric sedation device}

S.C. Brown, S. Schneeweiss, D. Chastain, G. Hart, P.A. McGrath

\section{Department of Anesthesia, Hospital for Sick Children, Toronto, Canada}

Background and Goal of Study: Despite significant progress in pain management for children requiring emergency treatment, procedural pain control remains problematic for young children, especially for anxiety-causing procedures for which children should not be deeply sedated. A new pediatric Sedation Device (SD) was designed to address this problem by delivering $50 \%$ nitrous oxide in oxygen through a simple nose piece, combined with an interactive video component so that children can use distraction with drug delivery. We conducted a Randomized Clinical Trial (RCT) to evaluate the effectiveness of this device in comparison to Standard Care (SC).

Materials and Methods: Children ages 3-9 years old who required invasive procedures typically associated with high levels of anxiety and lower levels of pain such as sutures, IV's, and lumbar punctures were eligible for randomization. 36 children were randomized to receive either $\mathrm{SC}$ - physician decision re: comforting, topical or local anesthetic, nitrous oxide or the SD. Primary outcomes include children's overt distress (Observational Scale of Behavioral Distress), and sedation level (Bromage scale). Secondary outcomes were pain, level of child's co-operation and satisfaction with the sedation approach. Results and Discussions: Children randomized to the device had significantly less distress $(M=1.8, S D=3.2)$ than children receiving $S C(M=9.3$, $\mathrm{SD}=5.6$, ANOVA $\mathrm{p}<0.001)$. Children who used the device reported significantly less pain $(M=1.6, S D=0.8)$ than those receiving $S C(M=2.4$, $\mathrm{SD}=0.9,(\mathrm{t}(19)=2.07, \mathrm{p}=0.05$.) Physicians $(\mathrm{t}(15)=4.04, \mathrm{p}<0.001)$, nurse $(t(29)=5.03, p<0.0001)$, and parents $(t(34)=6.69, p<0.0001)$ rated children in the device group as significantly more cooperative during invasive procedures than children receiving SC. Children who used the device had no adverse effects from the sedation.

Conclusion(s): This study shows that emergency care regimens including administration of continuous flow nitrous oxide using a novel sedation device significantly reduce distress and pain in young children, while increasing their cooperation during emergency procedures. 


\section{AP2-3}

Cerebral perfusion in newborn infants treated with non-invasive ventilation (NIV)

D. Dmytriiev, I. Kuchyn

Anaesthesiology, Vinnitsa National Medical University, Vinnitsa, Ukraine

Background: The effects of non-invasive ventilation (NIV) on hemodynamic parameters have been shown in clinical studies. In a further study we could demonstrate, that after change of a conventional positive pressure ventilation (CMV) to non-invasive ventilation (NIV) flow velocities in cerebral arteries decreased. In the following we added to the dopplerflow method the continuous examination of cerebral oxygenation with near infrared spectroscopy (NIRS).

Methods: 19 measurements were prospectively conducted in 12 neonates. The infants were mechanically ventilated with (NIV) and were in a stable condition. Before change from NIV to CMV doppler signals of the anterior cerebral artery were measured. We repeated this at the end of the study in each patient. NIRS-optodes were placed on the front and the os parietale of each infant. After stabilization of the system we changed from NIV to CMV without disconnection of the patient from the machine. $\mathrm{PCO}_{2}$ was registered continuously via a transcutaneous probe, as well as oxygen saturation via pulse oxymetry. Statistical analysis was performed with Wilcoxon test.

Results: There were no significant changes of doppler - signals during the study (median vs. $20 \mathrm{~cm} / \mathrm{s} \pm 6$ ) during NIV, $28 \mathrm{~cm} / \mathrm{s}$ ( \pm ) during CMV). The parameter of NIRS, oxygenated hemoglobin $\mathrm{HbO}[-1.3 \mathrm{U} \pm 20.1)]$ at $15 \mathrm{~min}-$ utes after change), reduced hemoglobin $\mathrm{HbR}[-1.1 \mathrm{U} \pm 4.2)]$ and total hemoglobin $\mathrm{HbT}[-2.68 \mathrm{U} \pm 19.7)]$ remained stable during the change from NIV to $\mathrm{CMV}$, too. In two infants there was a decrease and in one an increase of $\mathrm{HbO}$ 15 minutes after change, which correlated with decrease or increase of $\mathrm{pCO}_{2}$. Conclusion: In a combined measurement of dopplerflow and NIRS we found no special effect of NIV on cerebral hemodynamics comparing with CMV. Changes of cerebral oxygenation in NIRS correlated with $\mathrm{pCO}_{2}$.

Reference:

1 H. Zang, V. Ranieri, A. Slutsky/Cellular effects of ventilator induced lung injury// Current Opinion in Critical Care. 2000. p. 71-74.

\section{AP2-4}

\section{Correlation between radiological an clinical parameters with} AMBU laryngeal mask in paediatric patients

E. Monclus, A. Garcés, D. Artés, S. Serrano, M. Mabrock Anaesthesia, Hospital Sant Joan de Deu, Esplugues del Llobregat, Spain Background and Goal of Study: The object of the study is to carry out a radiological confirmation of the correct placement of the laryngeal masks in paediatric patients, correlating clinical parameters as the seal pressure and introduction easiness, with radiological studies (NMR).

Materials and Methods: They were included in the study 114 patients ASA I and II of ages from 4 months to 10 years programmed for cranial NMR. Under general anaesthesia with sevoforane, an AMBU laryngeal mask was placed for ventilatory control. The size chosen was according to the patient weight. No muscle relaxant was used. Seal pressure and introduction attempts were registered. Radiological images obtained in NMR were valued, calculating distances from proximal and distal cuff to trachea. Angles of the laryngeal mask, four first cervical vertebrae, and hypophysis were also calculated.

Results and Discussions: The data were classified based on the used mask.

\begin{tabular}{lllll}
\hline & $\begin{array}{l}\text { Group 1 } \\
(\mathrm{ML} \mathrm{1})\end{array}$ & $\begin{array}{l}\text { Group 2 } \\
\left(\mathrm{ML} \mathrm{1} 1 \frac{2}{2} \mathrm{z}\right)\end{array}$ & $\begin{array}{l}\text { Group 3 } \\
(\mathrm{ML} \mathrm{2})\end{array}$ & $\begin{array}{l}\text { Group 4 } \\
\left(\mathrm{ML} \mathrm{2} \frac{1}{2}\right)\end{array}$ \\
\hline Weight & $4.5 \pm 1.88$ & $7.65 \pm 1.73$ & $13.64 \pm 2.55$ & $20.88 \pm 4.05$ \\
Aye $(\mathrm{m})$ & $3 \pm 2$ & $9 \pm 8$ & $36 \pm 15$ & $63 \pm 22$ \\
Rad Disp & $60 \%$ & $30.7 \%$ & $14.03 \%$ & $3.22 \%$ \\
Seal P & $21 \pm 1$ & $21.5 \pm 4.3$ & $22.1 \pm 4.89$ & $23.7 \pm 3.04$ \\
\hline
\end{tabular}

The radiological displacement index (table1: rad dis) was calculated over the distances and angles studied and increases when diminishing the size of the used mask. First attempt introduction was higher than $90 \%$ and seal pressures achieved with AMBU laryngeal mask were similar to those obtained with other devices and no statistical differences were found between displaced laryngeal masks and normal fitted ones.

Conclusion(s): The anomalous placement of the mask, in paediatric patients, seen in NMR, have none correlation with clinical parameters, as modification of the seal pressure, or difficult introduction.

\section{Reference:}

Gaku Inagawa, Koji Okuda, Takaaki Miwa, Koichi Hiroki. Higher airway seal do not imply adequate positioning of laryngeal mask airways in paediatric patients. Paediatr Anaesth 2002, 12: 322-26.

\section{AP2-5}

Reinforced laryngeal mask airway and tonsillectomy. A retrospective study

D. Molies, A. Artigas, S. López, J. Torrallardona, J. Marco

Anesthesiology, Hospital Parc Taulí, Sabadell, Spain

Background and Goal of study: Endotracheal intubation with an uncuffed tracheal tube is routinely used to maintain the airway during tonsillectomy in children and is associated with complications. These problems can be avoided by using the reinforced laryngeal mask (rLMA).

Material and Methods: A retrospective, descriptive study was performed on 91 children undergoing tonsillectomy in whom the use of the rLMA was indicated, in our hospital between January 2005 and December 2006. Children were premedicated with intranasal midazolam $0.2 \mathrm{mg} \mathrm{kg}^{-1}$ and EMLA cream was applied to the dorsum of both hands. Anaesthesia was induced in all patients with propofol 3-6 $\mathrm{mg} \mathrm{kg}^{-1}$, preceded by fentanyl $1.5-2 \mu \mathrm{g} \mathrm{kg}^{-1}$ and midazolam. The rLMA was inserted without muscle relaxant. Anaesthesia was maintained by spontaneous breathing with $50 \%$ oxygen in air and 1.5-2\% sevoflurane. LMA were removed with de cuff inflated.

Results: Data are shown in the table:

\begin{tabular}{llllll}
\hline & Number of patients & Age (years) & ASA 1 & ASA 2 & ASA 3 \\
\hline Males & 54 & 5.7 & 11 & 41 & 2 \\
Females & 37 & 6 & 10 & 27 & 0 \\
\hline \multicolumn{5}{c}{ Number of patients } \\
\cline { 2 - 5 } & Size of LMA used idem to & Size of LMA used inferior to \\
& recommended & recommended & \\
\hline $10-20 \mathrm{~kg}$ & 38 & 18 & \\
$21-30 \mathrm{~kg}$ & 21 & 5 & \\
$31-50 \mathrm{~kg}$ & 4 & 5 & \\
$51-70 \mathrm{~kg}$ & 0 & & & \\
\hline
\end{tabular}

A smaller sized rLMA was placed in 28 children.

In three occasions, the technique was judged unsatisfactory and the trachea was intubated. In one occasion the surgeon cut the pilot tube of the rLMA. There were no episodes of laryngospasm neither haemorrhage or aspiration. Conclusions: Our study suggests that there are advantages using rLMA during tonsillectomy. It was good surgical acces without increasing the risk of aspiration. Neuromuscular blocking agents were not required. It also provides an improved recovery profile and perhaps most important a reduction in the incidence of laryngospam.

Reference:

1 Williams W. Br J Anaest 1993 Jan; 70(1): 30-33.

\section{AP2-6}

The laryngeal mask airway proseal for presure controlled ventilation in children

B. Mamaya, J. Stepanovs

Anaesthesiology and Reanimatology, Riga Stradins University, Riga, Latvia

Background and Goal of Study: The ProSeal (PLMA) is new laryngeal mask device with modified cuff to improve the seal and drain tube to provide access to the gastrointestinal tract. We assessed the use of PLMA for children.

Materials and Methods: Following acceptance by the Institutional Ethics Committee written consent from parents was obtained. 55 children (weight $25-50 \mathrm{~kg}$ ) were managed with the size 3 and 15 children (weight $10-25 \mathrm{~kg}$ ) with the size 2, ASA I or II and were undergoing elective plastic and reconstructive surgery in the supine position. Induction was with $\mathrm{i} / \mathrm{v}$ Propofol and Fentanyl until an adequate level of anaesthesia was achieved for PLMA placement. Intracuff pressure was set at $60 \mathrm{mmH}_{2} \mathrm{O}$ using Mallinckrodt manometer. Maintenance was of Sevoflurane and Fentanyl $3 \mu \mathrm{g} / \mathrm{kg}$ and pressure controlled ventilation at $15 \mathrm{~cm} \mathrm{H} \mathrm{H}_{2} \mathrm{O}$ without muscle relaxants, with any oropharyngeal leaks or gastric insufflation. Routine monitoring applied including NIBP, HR, ECG, $\mathrm{SpO}_{2}$, ETCO 2 , PIP, PP, PEEP, $\mathrm{R}_{\mathrm{aw}}$ and $\mathrm{C}_{\mathrm{R}}$.

Results and Discussions: First attempt success rates of achieving an effective airway were $90 \%$ but after 3 attempts $100 \%$. The time to achieve effective airway was $32 \pm 10 \mathrm{sec}$. A failed attempt wasn't observed. Displacement of PLMA intraoperatively -4 and alternative airway device was used. Drain tube air leaks -4 cases. Tidal volume and gas exchange was adequate in all patients. During PCV by PIP $=15 \mathrm{~cm} \mathrm{H}_{2} \mathrm{O}$; $V_{\mathrm{T}}$ was $7 \pm 2 \mathrm{ml} / \mathrm{kg} ; \mathrm{R}_{\mathrm{aw}}$ was $14 \pm 2 \mathrm{~cm} \mathrm{H} \mathrm{H}_{2} \mathrm{O} / \mathrm{l} / \mathrm{sec} ; \mathrm{C}_{\mathrm{R}}$ within $19 \pm 5 \mathrm{ml} / \mathrm{cm} \mathrm{H} \mathrm{H}_{2} \mathrm{O}$. Such intraoperative complications as: aspiration/regurgitation, hypoxia $\left(\mathrm{SpO}_{2}<92 \%\right)$, bronchospasm were not observed. Airway obstruction -1 , gastric insufflation -1 , coughing during removal -2 , blood staining after removal -3 . Success rate for gastric tube insertion was $80 \%$, more difficult at the first attempt. Incidence 
of postoperative complications was: sore throat mild and moderate -3 , dysphonia - 1, jaw and neck sore - 0 . Anaesthesia time was 20-245 min. Conclusion: The laryngeal mask airway ProSeal is an effective airway device for pressure controlled ventilation in nonparalyzed anaesthetised children providing adequate gas exchange.

\section{AP2-7}

\section{Cost effectiveness of cuffed versus uncuffed tracheal tubes in children}

S. Eschertzhuber, B. Salgo, W. Roth, A. Gerber, M. Weiss

Anaesthesia and Intensive Care, Medical University Innsbruck, Innsbruck, Austria

Background and Goal: The increased costs of cuffed tracheal paediatric tubes are often cited reasons to avoid them in clinical practice (1). The aim of this study was to compare cost effectiveness of cuffed versus uncuffed tracheal tubes (TT) in paediatric surgical patients.

Materials and Methods: After institutional ethics committee approval and written parental consent the tracheas of patients from birth to 5 years undergoing elective surgery were intubated with either uncuffed (Sheridan) or cuffed TT (Microcuff PET) in randomised order. Number of TT used per patient, time required for intubation until a $\Pi T$ with appropriate fit and seal was placed, lowest possible fresh gas flow (minimal allowed FGF: $0.5 \mathrm{Lt} / \mathrm{min}$ ) and Sevorane ${ }^{\circledR}$ concentration used were noted. Consumption and costs for Sevorane ${ }^{\circledR}$ were calculated (2). Data were compared using unpaired, two-sided T-test $(\mathrm{P}<0.05)$ and are presented as mean $\pm \mathrm{SD}$.

Results: 58 children aged $1.94 \pm 1.52$ years were enrolled. No significant differences in age $(p=0.28)$, weight $(p=0.53)$ or Sevorane ${ }^{\circledR}$ concentrations used $(p=0.84)$ were found between the two groups.

\begin{tabular}{llll}
\hline & Unit & Uncuffed TT & Cuffed TT \\
\hline Patients & $\mathrm{n}$ & 27 & 31 \\
Intubation time & $\mathrm{sec}$ & $114.0 \pm 103.7$ & $39.5 \pm 35.1^{\#}$ \\
Number TT used & $\mathrm{n} /$ patient & $1.6 \pm 0.6$ & $1.0 \pm 0.0^{\#}$ \\
Costs for TT & $€ /$ patient & $2.9 \pm 1.4$ & $9.5 \pm 0.0^{\#}$ \\
Fresh gas flow & $\mathrm{Lt} / \mathrm{min}$ & $2.41 \pm 0.85$ & $0.88 \pm 0.34^{\#}$ \\
Assessment period & Min & $59.7 \pm 23.4$ & $67.5 \pm 36.8^{\text {ns }}$ \\
Sevo $^{\circledR}$ liquid/min & $\mathrm{ml} / \mathrm{min}$ & $0.32 \pm 0.14$ & $0.11 \pm 0.04^{\#}$ \\
Sevo $^{\circledR}$ costs/min & $€ /$ min & $0.23 \pm 0.10$ & $0.08 \pm 0.03^{\#}$ \\
Total Sevo ${ }^{\circledR}$ costs & $€ /$ patient & $14.6 \pm 11.7$ & $5.1 \pm 2.91^{+}$ \\
Effective costs & $€ /$ patient & $17.2 \pm 11.9$ & $14.6+2.9^{\text {ns }}$ \\
\hline
\end{tabular}

P: cuffed vs uncuffed $T:{ }^{+}<0.001,{ }^{\#}<0.0001,{ }^{\text {ns }}>0.05$

Conclusions: In this study the increased cost of the cuffed tracheal tube was fully compensated by a lower cost of Sevorane ${ }^{\circledR}$ consumption. In addition, there are further savings due to reduced consumption of oxygen, air and nitrous oxide as well as reduced indirect costs due to primary success of intubation, reliably sealed airway and less environmental pollution by the use of cuffed tracheal tubes.

References:

1 James I. Paediatr Anaesth 2001; 11: 259-63.

2 Rapeport D. Anaesth Intensive Care 2005; 33: 144-45.

\section{AP3-1}

The effect of varying continuous propofol infusion on plasma cGMP concentrations in anesthetized children

T. Engelhardt, AJ. McCheyne, C. Karsli, I. Luginbuehl, B. Bissonnette Department of Anesthesia, Hospital for Sick Children, Toronto, Canada

Background and Goal of Study: The glutamate-nitric oxide-cyclic GMP pathway is potentially an effective target for general anesthesia agents. Plasma cyclic GMP (cGMP) concentrations are reduced following an increase in predicted plasma propofol concentrations during sedation in healthy adult volunteers. This study hypothesized that an increase in measured plasma propofol concentration leads to a reduction in plasma cGMP in anesthetized children

Materials and Methods: With Research Ethics Board approval and written parental consent, a total of eighteen healthy children aged $46.8( \pm 19.6)$ months, requiring general anesthesia for lower body surgical procedures were enrolled. Following inhalational induction, tracheal intubation and initiation of intermittent positive pressure ventilation, caudal epidural analgesia was performed. Anesthesia was maintained using a continuous propofol infusion adapted from a previously published regimen to achieve predicted propofol plasma concentration of 6,3 and $1.5 \mu \mathrm{g} / \mathrm{ml}$ after 30,50 and $70 \mathrm{~min}$, respectively. Samples for propofol and cGMP plasma concentrations were collected and analyzed using high-performance liquid chromatography and an enzyme immunoassay system.
Results and Discussions: The plasma cGMP concentrations varied significantly (median [range]) 19.2 [11.8-23.5], 21.3 [14.6-30.8] and 24.9 [15.7-37.8] $\mathrm{nmol} / \mathrm{L}$ between each predicted plasma propofol concentrations, $P<0.0001$. The correlation coefficient ( $r$ ) was -0.62 .

Conclusion: This study demonstrates that an increase in plasma propofol concentration leads to a decrease in plasma cGMP in healthy children and may potentially serve as a biochemical marker for depth of propofol anesthesia in children.

\section{AP3-3}

Evaluation of continuous NIRS cerebral oxygenation reading for global oxygenation monitoring during major paediatric surgery

A. Schmitz, K. Stutz, O. Kretschmar, W. Knirsch, M. Weiss

Anaesthesia, University Childrens Hospital Zurich, Zurich, Switzerland

Background and Goal: To evaluate continuous near-infrared spectroscopy (NIRS) cerebral oxygenation reading using the INVOS 5100 oximeter for noninvasive estimation of central venous oxygen saturation $\left(\mathrm{SvO}_{2}\right)$ in children undergoing major orthopaedic surgery (1-3).

Materials and Methods: With approval of the Local Ethical Committee and written parental and/or patient consent, patients aged from birth to 16 years undergoing major orthopaedic surgery were enclosed into the study.

The $\mathrm{rSO}_{2}$ measurements were performed at the right forehead using the INVOS self-adhesive NIRS probes. Central venous blood samples were drawn every 30 minutes and analysed for $\mathrm{SvO}_{2}$ by a blood gas analyser (ABL 700, Radiometer, Copenhagen, Denmark). Simultaneously, the $\mathrm{rSO}_{2}$ values were recorded. Values of $\mathrm{rSO}_{2}$ and $\mathrm{SvO}_{2}$ were compared inter- and intraindividual using linear regression analysis. Sensitivity and specifity of $\mathrm{rSO}_{2}$ between two measurements to indicate a fall in $\mathrm{SvO}_{2}$ were calculated.

Results: 18 patients aged from 0.1 to $15.5 \mathrm{yr}$ (median $10.1 \mathrm{yr}$ ) and weighing 4.8 to $57.4 \mathrm{~kg}$ (median $22.0 \mathrm{~kg}$ ) were studied. Values for $\mathrm{rSO}_{2}$ ranged from 59.0 to $92.0 \%$ (median $74.0 \%$ ) for $\mathrm{Hb}$ from 5.1 to $14.6 \mathrm{~g} / \mathrm{dl}$ (median $9.5 \mathrm{~g} / \mathrm{dl}$ ) and for $\mathrm{SvO}_{2}$ from 69.3 to $89.0 \%$ (median $80.0 \%$ ). Among all subjects only weak correlation was found between $\mathrm{rSO}_{2}$ and $\mathrm{SvO}_{2}\left(\mathrm{R}^{2}=0.073\right)$. Intra-individual measured $\mathrm{rSO}_{2}$ values demonstrated various correlations to $\mathrm{SvO}_{2}$ values $\left(R^{2}:<0.001\right.$ to 0.987 ; median 0.424$)$. Sensitivity and specifity to indicate a fall of $\mathrm{SvO}_{2}$ by $\mathrm{rSO}_{2}$ was $44.3 \%$ and $57.1 \%$, respectively.

Conclusion: Based on our preliminary findings continuous near-infrared spectroscopy (NIRS) cerebral oxygenation reading using the INVOS 5100 oximeter does not allow to monitor the course of global oxygenation in children during major surgery.

References:

1 Weiss M. Paediatric Anaesth 2004: 14: 989-95.

2 Nagdyman N. Intensive Care Med 2004; 30: 468-71.

3 Weiss M. Paediatric Anaesth 2003; 13: 184-91.

\section{AP3-4}

Efficacy and safety of sevoflurane and propofol anesthesia for MRI in pediatric patients

I. Ozdemir KOI, H. Egilmez*, K. Kaygusuz, S. Gursoy, C. Mimaroglu Department of Anesthesiology and *Radiology, Cumhuriyet University School of Medicine, Sivas, Turkey

Background and Goal of Study: Magnetic resonance imaging (MRI) for children often require deep sedation or anesthesia because the patients must remain completely immobile for a relatively long period to obtain high quality images. The laryngeal mask (LMA) has proved to be a safe and effective technique for airway management in both neonatal and pediatric patients. The aim of this randomized controlled study was to compare effects of propofol anesthesia or sevoflurane anesthesia with the LMA for children undergoing MRI.

Materials and Methods: After the approval of ethical committee and the patient, seventy-nine patients of ASA physical status I and III, with age ranging 6 mounts- 10 years, who were scheduled for cranial MRI, were enrolled in this study consecutively. Group propofol (group $P, \mathrm{n}=20$ ) were given $4 \mathrm{mg} \cdot \mathrm{kg}^{-1}$ propofol i.v. in 30s after insertion i.v. catheter and followed by $150 \mu \mathrm{g} \cdot \mathrm{kg}^{-1}$. $\min ^{-1}$ infusion. Group sevoflurane (group S) was induced with $8 \%$ sevofluorane by facemask and maintained with an air/oxygen mixture and followed by 1-2 sevofluorane. Then i.v. catheter was insertioned. The LMA was inserted with the traditional technique described by Brain (17), After insertion the lungs were manually expanded and the endtidal $\mathrm{CO}_{2}$ curve was evaluated. Heart rate, blood pressure, respiratory rate, endtidal $\mathrm{CO}_{2}$ and oxygen saturation were recorded at the start and finish of anesthesia. Imaging quality were assessed (1: very poor, 5: excellent). In addition, LMA insertion, removal, and recovery times were noted. 
Results and Discussions: There were no difference between groups about demografic data, duration of scan and hemodynamic parameters. There was no significant difference in imaging quality between study groups. No significant differences were found in the incidence of PONV. Induction times and recovery times were significantly shorter in the sevoflurane group than in the propofol group $(p<0.05)$.

Conclusion: Propofol or sevoflurane with larengeal mask provides satisfactory anesthesia during MRI in children. Sevoflurane provides more rapid induction and recovery than propofol.

\section{AP3-5}

A pharmacokinetic study of i.v. tramadol continuous infusion in children

A. Rochette, J. Ouaki, O. Raux, Ch. Dadure, X. Capdevila

Hôpital Lapeyronie, Department of Anesthesia \& critical care, CHU de Montpellier, Montpellier, France

Background and Goal: Clinical studies encourage the use of i.v. tramadol (T) in children for post operative pain relief (1), but pharmacokinetic data remain rare. Stereo-selective plasma concentration and elimination profile of $\mathrm{T}$ and its main metabolite, O-desmethyl-T (ODT), i.e (+) \& (-) enantiomers, were determined after 24-h T infusion.

Methods: 24 children, $3.76+/-1.9 \mathrm{yrs}, 15.8+/-5.5 \mathrm{~kg}, 0.66+/-0.16 \mathrm{~m}^{2}$, underwent gastro-oesophageal reflux laparoscopic surgery under standard anesthetic conditions. They received T, $2 \mathrm{mg} / \mathrm{kg}$ in $10 \mathrm{~min}$, followed by $8 \mathrm{mg} / \mathrm{kg}$ as a 24-h continuous infusion and $1 \mathrm{mg} / \mathrm{kg}$ bolus if CHIPPS was $>3$ ( $\max 5$ times). $1.5 \mathrm{ml}$ plasma sample was taken at $0.3,12 \& 24 \mathrm{~h}$ during and 0.25 , $0.5,1,2,4,6$ \& $12 \mathrm{~h}$ after infusion for HPLC assay using tandem mass spectrometry detection. Pk-fit software was used for pharmacokinetic analysis (2). Results: 18 patients received bolus $T, 17$ within $1.5 \mathrm{~h}$ of initial dose, 6 had 2 to 4 boluses. Infusion resulted in a steady state for $\mathrm{T}+, \mathrm{T}-$, ODT+, ODT - plasma concentrations. Pharmacokinetic data are reported in the table.

\begin{tabular}{|c|c|c|c|c|}
\hline & $\begin{array}{l}\text { Plasma conc } \\
\mathrm{ng} / \mathrm{ml}\left(\mathrm{H}_{2} 4\right)\end{array}$ & $\begin{array}{l}\text { VDss } \\
I\end{array}$ & $\begin{array}{l}\text { Clearance } \\
I / h\end{array}$ & $\begin{array}{l}\mathrm{t}_{1 / 2} \\
h\end{array}$ \\
\hline \multicolumn{5}{|l|}{$\overline{(+) \text { tramadol }}$} \\
\hline mean(SD) & $166.9(77.1)$ & 51 (32.8) & $13.3(5.9)$ & $2.6(.79)$ \\
\hline range & $73.4-390$ & $7.7-159$ & $5.24-27.1$ & $1.0-4.1$ \\
\hline $\begin{array}{l}(-) \text { tramadol } \\
\text { mean(SD) }\end{array}$ & 169.9 (69.3) & $53(30.1)$ & $14.5(6.6)$ & $2.5(.7)$ \\
\hline $\begin{array}{l}\text { range } \\
(+) \text { ODT }\end{array}$ & $62.7-350$ & $7.9-126$ & $5.69-28.2$ & $.96-3.8$ \\
\hline mean(SD) & 26.2 (13) & 852(822) & $121(103)$ & $4.7(1.4)$ \\
\hline $\begin{array}{l}\text { range } \\
(-) \text { ODT }\end{array}$ & $4.51-54.1$ & $248-3563$ & $49.5-499$ & $2.8-7.7$ \\
\hline mean(SD) & $32.8(15.2)$ & $539(417)$ & 86.7 (52) & $4.14(1)$ \\
\hline range & $8.11-86.9$ & $180-1984$ & $23.4-278$ & $2.31-5.8$ \\
\hline
\end{tabular}

( - ) ODT clearance correlated to body surface $(p=0.035)$.

Conclusions: 1 - no accumulation of T or ODT occurred. 2 - no kinetic difference appeared between enantiomers of both T and ODT. 3 - data were comparable to adults. $4-t_{1 / 2}$ (ODT) is about twice $t_{1 / 2}(T) .5$ - clinically relevant kinetic-demographic correlations were not found.

References:

1 Bozkurt P. Pediatr anesth 2005. 15: 1041-7.

2 Farenc $\mathrm{C}$ et al. Comput biomed res 2000. 33; 315-30.

\section{AP3-6}

The role of octreotide in the treatment of pancreatic pseudocyst in pediatric patients

I. Budic, A. Slavkovic, D. Simic, V. Djordjevic, D. Novakovic

Anesthesiaology Department, Clinic for Pediatric Surgery and Orthopedics, Clinical Centre Nis, Serbia, Nis, Serbia

Background and Goal of Study: Pancreatic pseudocysts in children are rare and known as complications of acute pancreatitis and pancreatic trauma. Lately, the use of somatostatin and its long-acting analogue octreotide have proved useful in the treatment of pancreatc pseudocysts in children.

Materials and Methods: In a recent multicenter randomized controlled study we investidgated the effects of short and long-term treatment of pancreatic pseudocysts with octreotide.

Complete records were available for 9 patients. Data pertaining to their admission, plus long-term radiologic and clinical outcome were analyzed. All patients were treated with octreotide acetate (2-3 $\mu \mathrm{g} / \mathrm{kg} \mathrm{SQ} \mathrm{QD).}$

Results and Discussions: Five children were treated conservatively with bowel rest and hyperalimentation. Two patients required percutaneous drainage, one patient needed surgical intervention. One patient had abdominal pain and surgical re-intervention not related to the pancreatic injury. The median length of hospitalization was 22 days. In three children significant decrease of the pseudocyst size was noticable 7 days after the conservative treatment with octreotide acetate, with almost complete resolution of the pseudocysts occurred within 6 weeks.

Conclusion(s): Octreotide is a safe and potentially effective adjunct in the treatment of pediatric pancreatic pseudocyst, by which means surgical re-intervention could be avoided.

References:

1) Uhl W, Anghelacopoulos SE, Friess H, Buchler MW. Digestion 1999; Suppl 2:23-31.

2) Cheruvu CV, Clarke MG, Prentice M, Eyre-Brook IA. Ann R Coll Surg Engl 2003; 85(5):313-6.

\section{AP3-7}

\section{Paediatric propofol pharmacokinetics: a multicentre study}

A. Rigby-Jones, M.J. Priston, A.R. Wolf, J.R. Sneyd

Anaesthesia Research Group, Peninsula Medical School, Plymouth, United Kingdom

Background and Goal of Study: Schuttler and Imhsen's propofol pharmacokinetic (PK) model ${ }^{1}$ based on pooled adult and paediatric data lacked information for the smallest patients. We conducted a pooled population analysis of available neonatal and paediatric propofol PK data. The variable clinical circumstances of the individual studies allowed us to explore health status as a covariate.

Materials and Methods: We combined propofol blood/plasma concentration vs. time data, dosing information and demographic data from 8 paediatric studies conducted by 6 research groups, with our data ${ }^{2,3}$. The pooled data set comprised 197 individuals ( 2315 observations), aged 0.02 to 12.25 years ( 2.75 to $60.5 \mathrm{~kg}$, median $15 \mathrm{~kg}$ ). In this preliminary PK analysis using NONMEM, the basic model structure was established before all model parameters were allometrically scaled to body weight. The influence of health status on paediatric propofol PK was explored.

Results and Discussions: In this 3-compartment preliminary model, postcardiac surgery patients have significantly reduced metabolic clearance rates (31 to $45 \%$ less when compared to healthy children or non-cardiac PICU patients). The volume of the deep peripheral compartment in critically ill and post-cardiac surgery children is $319 \%$ and $205 \%$ larger, respectively, than in healthy children, see Table 1.

Table 1. PK values for a child weighing $15 \mathrm{~kg}$

\begin{tabular}{llll}
\hline Parameter & & Typical Value & $95 \% \mathrm{Cl}$ \\
\hline $\mathrm{CL}(\mathrm{L} / \mathrm{min})$ & Healthy & 0.614 & $0.563-0.665$ \\
& PICU & 0.767 & $0.628-0.906$ \\
& PICU cardiac & 0.421 & $0.366-0.476$ \\
Q2 (L/min) & & 0.839 & $0.703-0.975$ \\
Q3 (L/min) & & 0.252 & $0.221-0.283$ \\
V1 (L) & & 7.76 & $6.33-9.19$ \\
V2 (L) & & 14.4 & $12.8-16.0$ \\
V3 (L) & Healthy & 83.9 & $61.4-106$ \\
& PICU & 268 & $183-353$ \\
& PICU cardiac & 172 & $117-227$ \\
\hline
\end{tabular}

Conclusion(s):

Health status may materially influence paediatric propofol PK.

References:

1 Schuttler J, Ihmsen H Anesthesiology 2000; 92: 727-38.

2 Rigby-Jones AE et al Anesthesiology 2002; 97: 1393-400.

3 Murray DM et al. Paediatr Anaesth 2004; 14: 143-51.

\section{AP4-1}

Behavioral reinforcement and play in paediatric outpatients: effects on anxiety, parental separation and induction

A. Dutta, S.K. Malhotra, J. Sood, D. Kumar

Anaesthesiology, Pain and Perioperative Medicine, Sir Ganga Ram Hospital, New Delhi, India

Background and Goal of study: Coping skills, modeling and play are effective behavioral modalities in decreasing preoperative anxiety amongst pediatric inpatients. In outpatient setting, these may be inadequate and difficult to apply. This investigation determines the effectiveness of behavioral reinforcement with play versus play alone, in respect to anxiety, parental separation and induction in pediatric outpatients.

Material and Methods: Thirty two children aged 3-7 years, of either sex were randomly divided into two groups: play with behavioral reinforcement ( $n=16$, group I) and play alone ( $n=16$, group II). Standardised measures for anxiety assessment were applied in the outpatient clinic and pre-anaesthesia 
room. The time for parental separation and induction were observed. A bold $2 \mathrm{~cm}$ line, colored green, drawn on the dorsum of hand during outpatient clinic visit, acted as reinforcer in group I.

Results: Both the groups were comparable for age, sex, body weight, parental literacy, socio-economic status, proposed procedure, surgical history and preoperative anxiety. Anxiety levels in pre-anesthesia room decreased in both the groups, though significant only in group I $(p<0.001)$, when compared to the baseline. The time, in seconds, for parental separation and induction were significantly higher in group II $(111.75 \pm 50.22$ and $187.5 \pm 25.92)$ as compared to group I $(63.81 \pm 35.49$ and $140.31 \pm 40.64)(p<0.01)$. Children in group I were also more cooperative during induction.

Conclusion(s): Behavioral reinforcement when added to play results in lesser anxiety, faster parental separation and induction time as compared to play alone in pediatric outpatients.

References:

1 Saile H, Burgmeier R, Schmidt LR. Psychol Health 1997; 2: 107-32.

2 Kain ZN, Wang SM, Mayes LC, et al. Anesth Analg 1999; 88: 1042-47.

\section{AP4-2}

Perioperative glycemia in paediatric ambulatory surgery

F. Munar, S. Manrique, N. Montferrer, M. De Miguel, E. Andreu

Anaesthesiology, Hospital Vall dHebron, Barcelona, Spain

Background and Goal of Study: Intravenous glucose administration during paediatric surgery remains controversial (1). We studied changes in capillary blood glucose concentrations by administering different intravenous solutions in pediatric ambulatory surgery under general anaesthesia and peripheral nerve block.

Materials and Methods: After institutional approval and parental informed consent, a prospective study was carried out on 46 patients aged 2-16 years, ASA I, II or stable III, undergoing circumcision or herniorrhaphy under general anaesthesia and peripheral nerve block. The patients were randomly placed in group $A(n=25): 1 / 5$ glucosaline $(4.7 \%$ glucose) or in group $B$ $(n=21)$ : glucosaline (3.3\% glucose). We started immediately after induction at $4 \mathrm{ml} \cdot \mathrm{kg}^{-1} \cdot \mathrm{h}^{-1}$ for the first ten $\mathrm{kg}, 2 \mathrm{ml} \cdot \mathrm{kg}^{-1} \cdot \mathrm{h}^{-1}$ for the second ten and $1 \mathrm{ml} \cdot \mathrm{kg}^{-1} \cdot \mathrm{h}^{-1}$ for upwards of twenty $\mathrm{kg}$ until thirty minutes post-extubation. Capillary blood glucose was determined before induction $\left(T_{0}\right)$ and three minutes afterward $\left(T_{3}\right)$, fifteen minutes post-incision $\left(T_{15}\right)$ and thirty minutes post-extubation $\left(\mathrm{T}_{30}\right)$. Urinary ketones were recorded. Data were analyzed by Mann-Whitney rank sum test, Friedman's statistic and Chi square, and were expressed as median, interquartile range and range. $\mathrm{P}<0.05$ was considered significant.

Results and Discussions: There were no characteristic differences, or any significant variations in glycemia between the groups. There was a significant change in the time course of glycemia in group $B(p=0.004)$ at $T_{0}-T_{3}$ $(p=0.007)$ and at $T_{0}-T_{15}(p=0.049)$ but not in group $A(p=0.066)$. The urinary ketones $(n=6)$ and dizziness, nausea or vomiting $(n=10)$ did not depend on the fluid administered.

Conclusion(s): In paediatric patients undergoing circumcision or herniorrhaphy under general anaesthesia and peripheral nerve block, significan changes in capillary glycemia do not exist between groups that received either $1 / 5$ glucosaline or glucosaline. The occurrence of dizziness, nausea or vomiting and the presence of urinary ketones do not depend on the solution administered.

Reference:

1 O. Paut and F. Lacroix. Recent developments in the perioperative fluid management for the paediatric patient. Curr Opin Anaesthesiol 2006; 19 (3): 268-277.

\section{AP4-3}

Intravenous lidocaine prior to extubation reduces postextubation cough and agitation in children undergoing tonsillectomy and adenoidectomy

J. Lee, K. Lee

Department Anesthesiology and Pain Medicine, Yonsei University Medical College, Seoul, Republic of Korea

Background and Goals: Sevoflurane is widely used to ambulatory pediatric anesthesia. But, sevoflurane is associated with a high incidence of emergence agitation in children (1). In this study, we examined the effect of single intravenous lidocaine prior to extubation on postoperative cough and agitation in children undergoing tonsillectomy and adenoidectomy.

Materials and Methods: All patients received a standardized anesthetic regimen with 2-3\% sevoflurane in 50\% $\mathrm{O}_{2} / \mathrm{N}_{2} \mathrm{O}$ after anesthetic induction with intravenous glycopyrrolate $0.004 \mathrm{mg} / \mathrm{kg}$, thiopental $5 \mathrm{mg} / \mathrm{kg}$ and vecuronium
$0.1 \mathrm{mg} / \mathrm{kg}$. In a double-blinded trial, 120 children (age 3-9 years) were randomly assigned to receive normal saline $0.1 \mathrm{~mL} / \mathrm{kg}$ (control, $\mathrm{n}=40$ ), $1 \%$ lidocaine $1 \mathrm{mg} / \mathrm{kg}(\mathrm{L} 1, \mathrm{n}=40)$ or $2 \%$ lidocaine $2 \mathrm{mg} / \mathrm{kg}(\mathrm{L} 2, \mathrm{n}=40)$ at 1 minute after beginning of spontaneous respiration. After extubation, the sedation score and the incidence of cough and agitation were recorded.

Results and Discussions: The incidence of severe cough and agitation in the L1 and L2 groups were significantly less compared with the control group $(P<0.05)$. The sedation score in the $L 2$ group was higher than the $L 1$ and control groups at $5 \mathrm{~min}$ after extubation. And at $10 \mathrm{~min}$ after extubation, the sedation score in the L1 and L2 groups was higher than the control group.

Conclusions: We conclude that intravenous lidocaine prior to extubation reduces cough and agitation after sevoflurane anesthesia in children undergoing tonsillectomy and adenoidectomy.

Reference:

1) Aono J, Ueda W, Mamiya K, et al. Anesthesiology 1997; 87: 1298-1300.

\section{AP4-4}

The effect of subtenons lidocaine injection on emergence agitation after general anesthesia in pediatric strabismus surgery

I.S. Seo, C.L. Seong, D.L. Jee, D.H. Lee

Deptartment of Anesthesiology, Yeungnam University Hospital, Daegu, Republic of Korea

Background and Goal of Study: Inhalation anesthesia with sevoflurane is widely used for pediatric patients. However, emergence agitation after sevoflurane anesthesia has been reported. This study examined whether or not a lidocaine injection into subtenons space affects emergence agitation after pediatric strabismus surgery with sevoflurane or balanced anesthesia.

Materials and Methods: We studied 160 children receiving muscle surgery for strabismus randomized to one of four groups (Group SS: sevoflurane and saline injection, Group SL: sevoflurane and lidocaine injection, Group BS: remifentanil \& propofol and saline injection, Group BL: remifentanil \& propofol and lidocaine injection). Anesthesia was induced with $4-6 \mathrm{mg} / \mathrm{kg}$ thiopental sodium and $0.8 \mathrm{mg} / \mathrm{kg}$ rocuronium. Before the end of surgery, a surgeon injected the subtenons space with $2 \%$ lidocaine or normal saline $1 \mathrm{ml}$. The degree of emergence agitation was assessed at the postanesthetic care unit using a five point scoring scale. (score 1: asleep, score 2: awake \& calm, score 3: irritable or consolable crying, score 4: inconsolable crying, 5: severe restlessness) We defined a score of 4 or 5 as emergence agitation.

Results and Discussions: There were no differences in age, weight, and duration of anesthesia. The incidence of emergence agitation was significantly decreased in patients who received subtenons lidocaine injection compared with saline injection.(P.0.05)

\begin{tabular}{lllll}
\hline & GP SS & GP SL & GP BS & GP BL \\
\hline Anesthesia time (minutes) & 60.8 & 61.6 & 61.4 & 63.6 \\
EA incidence & $14 / 40$ & $7 / 40^{*}$ & $11 / 40$ & $3 / 40+$ \\
\hline
\end{tabular}

${ }^{*} \mathrm{P}<0.05$ vs GP SS; $+\mathrm{P}<0.05$ vs GP BS

Conclusion(s): A lidocaine injection into the subtenons space reduces emergence agitation after general anesthesia in pediatric strabismus surgery.

References:

1 Cole JW. Paediatr Anaesth. 2002; 12: 442-7.

2 Voepel-Lewis T. Anesth Analg. 2003; 96: 1625-30.

\section{AP4-5}

Severe infections in postoperative period after pediatric cardiac operations - value of biochemical monitoring

E. Urbanska, A. Grzybowski, P. Knapik, J. Skalski, M. Zembala Department of Cardioanesthesia, Silesian Center of Heart Diseases, Zabrze, Poland

Background and Goal of Study: Children are more prone to develop severe infection during postoperative period after cardiac operations. Systemic inflammatory response caused by cardiopulmonary bypass can impede early recognition of severe infection. The aim of the study was to established importance of biochemical parameters monitoring during children's cardiosurgical postoperative period as a help to reveal infectious process.

Materials and Methods: 63 consecutive children after cardiac operation with ICU stay no less than 3 days. Children were divided into two groups: A - control group without any complications during postoperative period (25 pt) and $\mathrm{B}$ - children who developed sepsis or severe infection in postoperative period 
(38 pt). Each child had monitored several biochemical parameters daily (CPK, LDH, AIAT, AspAT, urea, creatinine, bilirubine). Definition of severe infection (sepsis, severe sepsis, septic shock) was according to [1]. Changes in time of biochemical values from children without infection and with severe infection were observed during postoperative period. For statistical analysis we have used Friedman ANOVA test.

Results and Discussions: Significant difference was found between groups in children's age (64 ms vs. $12 \mathrm{~ms} p<0,001$ ) but there were no statistical differences between times of cardiopulmonary bypass (102 min vs. $92 \mathrm{~min}$ $p>0,05)$. In control group biochemical values did not rise significantly during first four days after operation. Children in studied group developed severe infection on 2 nd day after operation on average (1st to 6 th day). In this group we have observed significant rise of some biochemical markers (AspAT, AIAT, LDH, urea, creatinine), beginning just after operation. This rise preceded diagnostic evaluation of the infection.

Conclusion(s): Elevation of biochemical markers during postoperative period after cardiac operations in children could be used as sign of impending infection.

Reference:

1 Crit. Care Med. 1992; 20: 864-875.

\section{AP4-6}

The effect of anesthetic agents on emergence delirium in pediatric strabismus surgery

S.R. Choi, S.C. Lee, C.J. Chung, S.I. Lee, Y.J. Chin

Anesthesiology and Pain Medicine, Dong-A University Hospital, Busan,

Republic of Korea

Background and Goal of Study: This study was designed to compare the effects of anesthetic agents used recently on emergence delirium (ED) in pediatric strabismus surgery.

Materials and Methods: Two-hundred thirty two children, aged 2-10 years, undergoing strabismus surgery, were randomly assigned to one of eight groups; ketamine-desflurane, ketamine-sevoflurane, ketamine-propofol, ketamineremifentanil, midazolam-desflurane, midazolam-sevoflurane, midazolanpropofol, and medazolam-remifentanil. Anesthesia was induced with ketamine $1.0 \mathrm{mg} / \mathrm{kg}$ or midazolam $0.15 \mathrm{mg} / \mathrm{kg}$. Laryngeal mask airway was placed with rocuronium $0.5 \mathrm{mg} / \mathrm{kg}$. Anesthesia was maintained with desflurane 5-6 vol\%, sevoflurane $2-3$ vol\%, propofol $6-9 \mathrm{mg} / \mathrm{kg} / \mathrm{h}$, or remifentanil $0.5 \mu / \mathrm{kg} / \mathrm{min}$ under $\mathrm{N}_{2} \mathrm{O} 66 \%$ in $\mathrm{O}_{2}$. The status of $\mathrm{ED}$ was evaluated by a blinded observer until discharge from postanesthetic care unit. Data were analyzed using t-test, ANOVA, and $\chi^{2}$-test.

Results and Discussions: There was no difference in age, sex, weight, height, anesthetic time, and recovery time among the eight groups. ED occurred in 54 children $(23.3 \%)$. Compared with the ketamine group, the midazolam group showed less incidence of ED. The propofol and the remifentanil groups showed less incidence of ED compared with the desflurane and the sevoflurane groups. ED group was younger and more temperamental compared with nonED group.

Conclusions: Propofol or remifentanil provided less incidence of ED compared with desflurane or sevoflurane anesthesia in pediatric strabismus surgery.

Table 1. Factors Related Emergence Delirium (ED)

\begin{tabular}{llll}
\hline & NonED $(\mathrm{n}=178)$ & ED $(\mathrm{n}=54)$ & P value \\
\hline Age $(\mathrm{yr})$ & $5.5 \pm 1.9$ & $4.0 \pm 1.9$ & 0.00 \\
Sex $(\mathrm{M} / \mathrm{F}, \mathrm{n})$ & $100 / 78$ & $27 / 27$ & 0.42 \\
Emotional status (1/2/3, $\mathrm{n})$ & $128 / 32 / 18$ & $19 / 27 / 8$ & 0.00 \\
Anesthetic time (min) & $26.6 \pm 4.1$ & $27.3 \pm 4.6$ & 0.31 \\
Recovery time (min) & $9.6 \pm 3.9$ & $9.8 \pm 3.1$ & 0.83 \\
Ketamine/midazolam (n) & $83 / 95$ & $34 / 20$ & 0.035 \\
Des/Sevo/Propofol/remifentanil (n) & $35 / 41 / 50 / 52$ & $23 / 19 / 7 / 5$ & 0.00 \\
Inhalational/intravenous ( $\mathrm{n})$ & $76 / 102$ & $42 / 12$ & 0.00 \\
\hline
\end{tabular}

Data are mean \pm SD or number.

\section{AP4-7}

Evaluation and validation of the FLACC preverbal patient pain scale in comparison with the VAS pain scale for pediatric patients in the PACU - A preliminary study

G. Minas, E. Goutziomitrou, A. Douvantzi, A. Foulidou, P. Petropoulou Anesthesiology Department, Papageorgiou General Hospital, Thessaloniki, Greece

Background and goal: FLACC Pain Assessment Tool is an observational scale for quantifying pain behaviors. Facial expressions, Leg movement,
Activity, Cry and Consolability are each scored 0-2, for a total FLACC score of $0-10$.

The goal of this study is to evaluate the correlation of the FLACC pain assessment scale with the Visual Analogue Scale VAS 0-10, in assessing pain for pediatric patients in the Post Anesthesia Care Unit (PACU)2.

Materials and Methods: 16 male pediatric patients aged from 1-7 years, who underwent minor surgical operations of less than 60 minutes, were studied.

They were all given the same anesthetic scheme and intraoperative analgesia with i.v fentanyl $(4 \mu \mathrm{g} / \mathrm{kg})$, per rectum paracetamol $(30 \mathrm{mg} / \mathrm{kg})$ and i.v clonidine $(2 \mu \mathrm{g} / \mathrm{kg})$.

All children were evaluated for pain using the two scales, by two different observators - in order to achieve interrater reliability - at 5, 10, 20, 30, 40 and 50 minutes from their arrival to the P.A.C.U. Additional analgesia $0,10 \mathrm{mg} / \mathrm{kg}$ iv morphine was given if the score in either scale exceeded 5 (moderate to severe pain). Data was analysed by SPSS 12.0 program using analysis of bivariate correlation (PEARSON correlation).

Results and Discussion: Data results are shown in the tables:

\begin{tabular}{llll}
\hline & & FLACC 10 & VAS 10 \\
\hline FLACC 10 & Pearson Correlation & 1 &, 689 \\
& Sig. (2-tailed) &. &, 003 \\
\hline & & FLACC 40 & VAS 40 \\
\hline FLACC 40 & Pearson Correlation & 1 &, 858 \\
& Sig. (2-tailed) &. &, 000 \\
\hline & & & \\
\hline FLACC 50 50 & FLACC 50 & VAS 50 \\
& & 1 &, 752 \\
& Pearson Correlation &. &, 005 \\
\hline
\end{tabular}

Positive correlation between the FLACC and VAS scales was found at all time intervals and was stronger and correlated significantly $(p<0,01)$ at 10 , 40 and 50 minutes (Pearson $r_{10}=0,689, r_{40}=0,858, r_{50}=0,752$ ).

Conclusion: FLACC pain assessment scale can be an appropriate and valid alternative tool for assessing pain especially in preverbal children in the PACU, when other pain scales are not effective.

References:

1 Merkel SI, Voepel-Lewis T, Shayevitz JR, Malviya S. The FLACC: A behavioral scale for scoring postoperative pain in young children. Pediatr Nurs 1997; 23(3): 293-297.

2 Manworren RCB, Hynan LS. Clinical validation of FLACC: Preverbal Patient Pain Scale. Pediatr Nurs 2003; 29(2): 140-146.

\section{AP5-1}

Perioperative assessment of coagulation in paediatric neurosurgical patients using thromboelastography M. Yosry, H. Khedr, N. El Kady, S. El Mekawi

Anesthesia* and Neurosurgery ${ }^{+}$, Faculty of medicine Cairo University. -Egypt, Cairo, Egypt

Background and Goal of study: Primary brain tumors may be associated with coagulation disorders which can pose intraoperative and postoperative management difficulties. Thromboelastography (TEG) is a useful technique for evaluating coagulability.

Materials and Methods: In this study we evaluated the perioperative coagulation profile using both standard laboratory work and TEG in pediatric patients undergoing craniotomy for primary brain tumors. 40 Pediatric patients were enrolled in the study. All patients received standard anesthesia. Blood was analyzed for both standard laboratory work and TEG at three points for each patient: preoperatively, intraoperatively, and postoperatively. Post operatively patients were divided into two groups according to occurrence or not of postoperative haematomas.

GNH (non haematoma group) and GH (haematoma group). The standard blood work and TEG values for both groups were compared.

Results and Discussion: Perioperative standard blood work was within normal limits for all patients with no significant difference between both groups. In GNH TEG values were indicative of a hypercoagulable state which started intraoperative and continued into the 1st postoperative day. In GH TEG values were indicative of a hypocoagulable state which was evident in the preoperative TEG values and continued into the intraoperative as well as post operative period.

Conclusion: TEG may be useful in the perioperative assessment and monitoring of coagulation in pediatric neurosurgical patients and helps in identifying patients at increased risk of bleeding or thromboembolic events. 


\section{AP5-2}

Perioperative fluid prescribing in paediatrics

D. Pappin, P. Dix

Anaesthetic, Royal Devon and Exeter Hospital, Exeter, United Kingdom

Background and Goal of Study: The use of hypotonic intravenous fluids has been associated with more than 50 international case reports of death or neurological injury in children. In 2003 the Royal College of Anaesthetists warned of the risk of iatrogenic hyponatraemia and water overload developing after the use of sodium chloride $0.18 \%$ with glucose $4 \%$ in children. A followup survey found less than half of consultant anaesthetists had been aware of this warning. ${ }^{1}$ Our audit looked at whether anaesthetists' fluid prescriptions intraoperatively and postoperatively reflected these concerns in our department.

Materials and Methods: The audit examined the fluid administration in paediatric operations over a 4 month period. Data collected included the age and weight of the child, the volume, rate and type of fluid prescribed intraoperatively and postoperatively, whether there was any estimate of blood or fluid loss, and whether capillary blood sugar was recorded. We also recorded the current locations of sodium chloride $0.18 \%$ with glucose $4 \%$.

Results and Discussions: Over the 4 month period, 35 patients received intraoperative fluids: none had a capillary blood sugar recorded and only 1 had an estimate of blood or fluid loss. 31 were given isotonic solutions, at volumes between 3-34 ml/kg. 4 were given hypotonic solutions (2 at 10-20 ml/kg, 2 at greater volumes).

Only 17 patients were prescribed postoperative fluids: 13 were given isotonic solutions, of which only 4 were prescribed according to the Holliday and Segar formula. ${ }^{2}$ The majority were prescribed at greater rates. 4 were given hypotonic fluids, 1 at the predicted rate, 2 at a greater rate and 1 at almost half the calculated rate. ${ }^{2}$ Sodium chloride $0.18 \%$ with glucose $4 \%$ solution was freely available in all clinical areas.

Conclusion(s): There is no restriction in the availability of sodium chloride $0.18 \%$ with glucose $4 \%$ solution. The majority of paediatric patients are given isotonic fluid intraoperatively. However, despite the concerns about hyponatraemia, post-operatively the majority are being prescribed either hypotonic or excessive rates of fluid.

References:

1 Way C et al. BJA 2006; 97 (3): 371-379.

2 Holliday MA, Segar WE. Pediatrics 1957; 19: 823-832.

\section{AP5-3}

\section{Anesthesia-related cardiac arrest in children: the thai anesthesia incidents study (THAI Study)}

N. Bunchungmongkol, Y. Punjasawadwong, S. Chumpathong, W. Somboonviboon, S. Suraseranivongse

Anesthesiology, Faculty of Medicine, Chiang Mai University, Chiang Mai, Thailand

Background and Goal of Study: A higher incidence of perioperative cardiac arrest and mortality has been recognized in children than in adults (1-2). This survey evaluated the incidence, causes, outcome and corrective strategies associated with anesthesia-related cardiac arrest in children from The Thai Anesthesia Incidents Study (THAI Study), the first national study of anesthesia outcomes in Thailand.

Materials and Methods: A multi-centered prospective study was conducted among 20 hospitals across Thailand over a year from 2003 to 2004. Data of cardiac arrests in children aged 15 years and younger were collected during anesthesia, in the recovery room and 24 hours postoperative period, and reviewed independently by at least 2 reviewers.

Results and Discussions: From the database of 25,098 cases, cardiac arrest related to anesthesia had an incidence of 5.1 (95\% Cl: 2.7-8.8) per 10,000 anesthetics and a mortality rate of $46 \%$. Medication-related $(31 \%)$ and respiratory-related $(31 \%)$ causes of cardiac arrest were most common. Most of anesthesia-related arrests occurred in operating room (61\%) during induction or maintenance of anesthesia (84\%). Infants younger than 1 year of age accounted for $61 \%$ of all anesthesia-related arrests. Incidences of anesthesia - related arrest were significant higher in infants than older children and in children with ASA physical status $3-5$ than those with ASA physical status $1-2(p<0.05)$. Improving supervision, additional training in airway management, practice guideline, efficient blood blank, equipment maintenance and quality assurance activity are suggested corrective strategies to improve quality of care in pediatric anesthesia.

Conclusions: Anesthesia-related cardiac arrest occurred most often in children younger than 1 year of age and in patients with severe underlying disease. The identification of airway management and medication-related problems as the most frequent cause of anesthesia-related cardiac arrest has important implications for preventive strategies.

References:

1 Cohen M. Anesth Analg 1990; 70: 160-167.

2 Morray JP. Anesthesiology 2000; 93: 6-14.

\section{AP5-4}

Replacement of preoperative NPO deficit with ringers lactate maintains the blood glucose concentration within physiologic range in children undergoing strabismus surgery

K. Lee, S. Choi

Department of Anesthesiology and Pain Medicine, Yonsei University Medical Center, Seoul, Republic of Korea

Background and Goal of Study: We investigated the effect that the replacement of preoperative NPO deficit with Ringer's Lactate (RL) would have on blood glucose concentration in children undergoing a brief surgery.

Materials and Methods: Sixty children scheduled for strabismus surgery, from 3 to 9 years of age, were enrolled and randomly assigned to three groups of 20 patients according to the types of fluid therapy employed.

\begin{tabular}{lll}
\hline & Replacement & Maintenance \\
\hline Group 1 & $\mathrm{RL}$ & $\mathrm{RL}$ \\
Group 2 & $\mathrm{RL}$ & $\mathrm{D}_{5} 1 / 4 \mathrm{NS}$ \\
Group 3 & $\mathrm{RL}$ & $1 / 2$ of $\mathrm{D}_{5} 1 / 4 \mathrm{NS}+1 / 2$ of RL \\
\hline
\end{tabular}

$\mathrm{D}_{5} 1 / 4 \mathrm{NS}: 5 \%$ dextrose in $1 / 4$ strength normal saline

RL for replacement of preoperative NPO deficit was administered with the rate of $1 / 2$ of the estimated amount for the first $1 \mathrm{hr}, 1 / 4$ for the each $2 \mathrm{nd}$ and $3 \mathrm{rd}$ hour (1). The blood samples to measure blood glucose were taken from a saphenous vein during anesthetic induction and at 30 and 60 min after anesthetic induction. Hypoglycemia was defined as a blood glucose concentration of less than $60 \mathrm{mg} / \mathrm{dL}$ and hyperglycemia, greater than $200 \mathrm{mg} / \mathrm{dL}$.

Results and Discussions: There were no significant differences in blood glucose levels at anesthetic induction among the three groups, and the mean blood glucose concentrations remained unchanged throughout the study period in all groups. No patients were found to be hypoglycemic or hyperglycemic throughout the study period.

Conclusion: We conclude that the replacement of NPO deficits with RL maintained the blood glucose concentration within physiological range throughout the operation, regardless of the types of maintenance fluid. So, We suggest that RL was suitable for replacement of NPO deficits in children undergoing a brief surgery.

Reference:

1 Berleur MP, Dahan A, Murat I, et al. J Clin Pharm Ther 2003; 28: 31-40.

\section{AP5-7}

Risk and predictors of blood transfusion in patients undergoing paediatric open heart surgery

A. Székely, Zs. Cserép, E. Sápi, T. Breuer, A. Szatmári

Department of Paediatric Anaesthesia and Intensive Care, Gottsegen György National Institute of Cardiology, Budapest, Hungary

Background and Goal of Study: Transfusion was found to increase morbidity and mortality after adult cardiac surgery. Only few data are available about the risk of transfusion in pediatric population undergone operation with cardiopulmonary bypass (CPB).

Materials and Methods: The prospectively and consecutively collected database of our pediatric intensive care department (September 2001 December 2003) was analyzed in regard of possible relationship between blood transfusion $(\mathrm{ml} / \mathrm{kg})$ and adverse outcome. The main predictors of blood transfusion were also investigated. Spearman correlation, linear and logistic regression were applied, as appropriate for statistical analysis.

Results and Discussions: The median need for blood transfusion during the operation and the first postoperative 24 hours in the patients $(n=657)$ was $33.2 \mathrm{ml} / \mathrm{kg}$ (interquartile range $10-75 \mathrm{ml} / \mathrm{kg}$ ). Every given $10 \mathrm{ml} / \mathrm{kg}$ of blood increased the risk for peritoneal dialysis (risk ratio $\{R R\}, 1.55 ; 95 \%$ confidence interval $\{\mathrm{Cl}\}, 1.34-1.78)$ the risk for nonvascular pulmonary failure (RR, 1.31; $95 \% \mathrm{Cl}, 1.21-1.42)$ and the risk for serious infection $(\mathrm{RR}, 1.18 ; 95 \% \mathrm{Cl}$, 1.13-1.24). Amount of blood transfusion was correlated with the postCPB arterial oxygen tension $(r=0.29 ; p<0.0001)$ and with the 24 th hour creatinine clearance $(r=0.75 ; p<0.0001)$. Linear regression showed that age 
( $p<0.001)$, weight $(p=0.001)$, operative complexity $(p=0.008)$ and duration of CPB $(p<0.001)$ were independent factors of the amount of transfusion (model R square $=0.57$ ).

Conclusion(s): Our findings indicate that blood transfusion increased the incidence of adverse postoperative outcomes, as renal, pulmonary events and infections after pediatric cardiac surgery. The most vulnerable group was infants having undergone complex procedures and long lasting CPB.

\section{AP6-1}

\section{A comparison of clonidine $1 \mathrm{mcg} / \mathrm{kg}$ vs clonidine $2 \mathrm{mcg} / \mathrm{kg}$ for caudal block with levobupivacaine $0.25 \%$ in paediatric patients}

\author{
D. Galante, M. Dambrosio, G. Pellico, A. Federico
}

University Department of Aneshtesia and Intensive Care, University Hospital Ospedali Riuniti of Foggia, Foggia, Italy

Background and Goal of Study: A caudal block is commonly performed for pediatric postoperative analgesia. Adjuvants are safe and effective in pain control but are associated with troublesome side effects. We hypothesized that patients receiving caudal levobupivacvaine $0.25 \%$ plus clonidine $1 \mathrm{mcg} / \mathrm{kg}$ versus levobupivacvaine $0.25 \%$ plus clonidine $2 \mathrm{mcg} / \mathrm{kg}$ would experience fewer side effects and the same analgesic and rehabilitation profile.

Materials and Methods: After IRB approval and informed consent, 24 patients 6 months to 4 years of age undergoing genitourinary surgery were studied in a prospective, randomized, double-masked manner. The levobupivacaine/clonidine 1 group (LC1 = 12 patients) received $1 \mathrm{mcg} / \mathrm{kg}$ of clonidine and $2 \mathrm{mg} / \mathrm{kg}$ of levobupivacaine $0.25 \%$ while the levobupivacaine/ clonidine 2 group (LC2 = 12 patients) received $2 \mathrm{mcg} / \mathrm{kg}$ of clonidine and $2 \mathrm{mg} / \mathrm{kg}$ of levobupivacaine $0.25 \%$. All caudal blocks were performed with "no turn technique" prior to surgical incision. All subjects received a sevoflurane/oxygen/air anesthetic by mask or laryngeal mask airway (LMA). No other hypnotics, analgesics or antiemetics were administered intraoperatively. CHEOPS (1-7 years) and NIPS ( $<1$ years) scales for were used for postoperative pain measurement at the end of the surgical procedures and then every 4 hours for the first 24 hours.

Results and Discussions: There were no differences in demographic or surgical characteristics. Subjects in the LC1 group experienced less postoperative nausea and vomiting (PONV) and no excessive sedation than LC2 group. Subjects in the LC2 group experienced more sustained initial postoperative analgesia and sedation than the LC1 group. However, no difference was observed in pain scores or rescue analgesic use during the initial 24 hours, or in the time to first oral liquid/solid intake or discharge home. No postoperative respiratory depression, hypotension or bradycardia was identified.

Conclusion(s): While $2 \mathrm{mcg} / \mathrm{kg}$ clonidine may produce more sustained initial analgesia and sedation, $1 \mathrm{mcg} / \mathrm{kg}$ clonidine appears to provide comparable analgesia with fewer side effects.

\section{AP6-2}

\section{Preoperative piroxicam for postoperative analgesia in} children undergoing orthopaedic surgery

O. Kaabachi, B. Ghrab, W. Koubaa, R. Ouezzini, O. Chettaoui

Anesthesiology and Intensive Care, Institut Kassab dOrthopédie, Tunis, Tunisia

Background and Goal of Study: The use of non-steroidal anti-inflammatory drugs for pre-emptive analgesia in children is controversial. Piroxicam is a non-steroidal anti-inflammatory drug with a long plasma half-life of about 22-40 hours in children [1]. A pharmacokinetic study concluded that $1 \mathrm{mg} / \mathrm{kg}$ of piroxicam achieved adequate plasma levels and was safe for short-term administration in children [2]. We hypothesized that preoperative administration of $1 \mathrm{mg} / \mathrm{kg}$ fast-dissolving piroxicam would reduce postoperative pain scores and analgesic requirements in children undergoing orthopaedic surgery.

Materials and Methods: Following ethical committee approval and informed parental consent, we conducted a prospective randomized double blind study including 53 children 2 to 12 years old randomly allocated to two groups: Group T(25 patients): received $5 \mathrm{ml}$ of water 1 hour before surgery and $2 \mathrm{mg} / \mathrm{kg}$ of IV tramadol $10 \mathrm{~min}$ before the end of surgery. Group P (28 patients): received $1 \mathrm{mg} / \mathrm{kg}$ of piroxicam (dissolved in $5 \mathrm{ml}$ of water) 1 hour prior to the surgery and $5 \mathrm{ml} \mathrm{IV}$ saline $10 \mathrm{~min}$ before the end of surgery. Children with CHEOPS modified score $\geqslant 4$ were treated postoperatively with IV paracetamol $(15 \mathrm{mg} / \mathrm{kg})$. The time to the first analgesic supplementation, the analgesic use and the frequency of side effects were recorded. Anova and Fisher exact tests were used. $P<0.05$ were significant.

Results and Discussions: Demographic data and mean surgery time were similar in both groups. Significantly lower rate of rescue analgesia was found in group P; 6 children in group T (24\%) and 18 children in group P $(64 \%)$ did not required rescue analgesic during the 48 hours postoperative observation period $(p<0.01)$. Time for the first analgesic requirement was longer in the group P $(489.0 \pm 477.5$ min versus $356.3 \pm 276.6 \mathrm{~min})$, but difference was not significant $(p=0.34)$. Eight patients in group $T(32 \%)$ and 2 patients in group $P(7 \%)$ suffered from nausea and vomiting $(p=0.02)$.

Conclusion(s): Our findings suggest that preoperative administration of $1 \mathrm{mg} / \mathrm{kg}$ fast-dissolving piroxicam improves postoperative analgesia after orthopedic surgery in children compared with $2 \mathrm{mg} / \mathrm{kg}$ of tramadol. References:

1 Makela AL. Eur J of Clin Pharmacol 1991; 41: 79-81.

2 Dix P. Anaesthesia, 2004, 59: 984-987.

\section{AP6-3}

Ultrasound-guided paediatric epidural anaesthesia in the Nuss procedure for pectus excavatum

S. Sugino, N. Kanaya, M. Yamauchi, N. Tsukigase, A Namiki Department of Anesthesiology, Sapporo Medical University School of Medicine, Sapporo, Japan

Background and Goals: Epidural analgesia is often used for postoperative pain relief after the Nuss procedure in pediatric patients. However, epidural puncture after induction of general anesthesia may cause various complications, such as nerve injury, and this risk is a concern for anesthesiologists. It has recently been reported that ultrasound guidance has some advantages for neuraxial blockade compared with the classical blind technique (Grau et al. 2002). The aim of the present study was to determine the usefulness of ultrasound-guided epidural puncture for the Nuss procedure.

Materials and Methods: 15 children were scheduled to undergo surgery using the Nuss procedure were randomly assigned to an ultrasound group $(n=7)$ or a control group $(n=8)$. In the ultrasound group, after general anesthesia had been induced, ultrasound examination was performed to identify the epidural space for puncture (at Th4-9 level). Neuraxial structures, including the ligamenta flava and facet joints, were visualized in proper transversal view. Depth from the skin to the dura matter was measured. Epidural puncture was performed by the loss of resistance technique with reference to the ultrasound-guided puncture point, angle, and depth. In the control group, the puncture point was decided by palpation, and epidural puncture was performed. Number of epidural punctures required and time to reach the epidural space were recorded. The difficulty in epidural puncture was scored as a five-point scale follows: $1=$ very easy, 2 = easy, $3=$ equal to blind puncture, 4 = difficult, 5 = very difficult.

Results: Data (mean $\pm \mathrm{SD}$ ) are shown in the table:

\begin{tabular}{lcc}
\hline & Ultrasound $(\mathrm{n}=7)$ & Control $(\mathrm{n}=8)$ \\
\hline No. of Punctures & $1.4 \pm 0.9$ & $2.2 \pm 1.6$ \\
Time $(\mathrm{sec})$ & $60.1 \pm 58.0^{\star}$ & $146.9 \pm 51.0$ \\
Puncture Scale & $1.4 \pm 0.3^{*}$ & $3.1 \pm 1.5$ \\
\hline
\end{tabular}

*: $P<0.05$ vs. Control.

Conclusions: The results of the present study showed that ultrasound imaging for pediatric epidural anesthesia in the Nuss procedure has the advantages of direct visualization of neuraxial structures and reduced performance time. Ultrasound guidance may overcome the difficulty in pediatric epidural anesthesia and reduce the anxiety of anesthesiologists.

\section{AP6-4}

Pain control after iliac crest bone graft surgery in children: intravenous ketorolac vs. continuous bupivacaine infusion V. Espinal, J. Hayes, B. Bissonnette Anesthesia, The Hospital for Sick Children, Toronto, Canada

Background and Goal of Study: Iliac Crest Bone Graft surgery (ICBG) is painful. Postoperative pain management includes the infusion of local anesthetics into the wound via an indwelling catheter $(1,2)$ and/or non steroidal anti-inflammatory drugs (NSAIDs). The purpose of this study is to compare the efficacy of intravenous ketorolac to local bupivacaine infusion for pain control after ICBG.

Materials and Methods: To date, four patients older than 10 years requiring ICBG for alveolar cleft repair or Lefort I osteotomy have been studied. Patients were randomly assigned in a blinded fashion to receive either: IV 
ketorolac $0.5 \mathrm{mg} / \mathrm{kg} \mathrm{q} 8 \mathrm{~h}$ (Group K); IV ketorolac and local infusion of plain bupivacaine $0.25 \%$ at $0.1 \mathrm{ml} / \mathrm{kg} /$ hour for 48 hours (Group K + B); or bupivacaine infusion alone (Group B). All patients received PCA morphine and oral acetaminophen.

Results and Discussions:

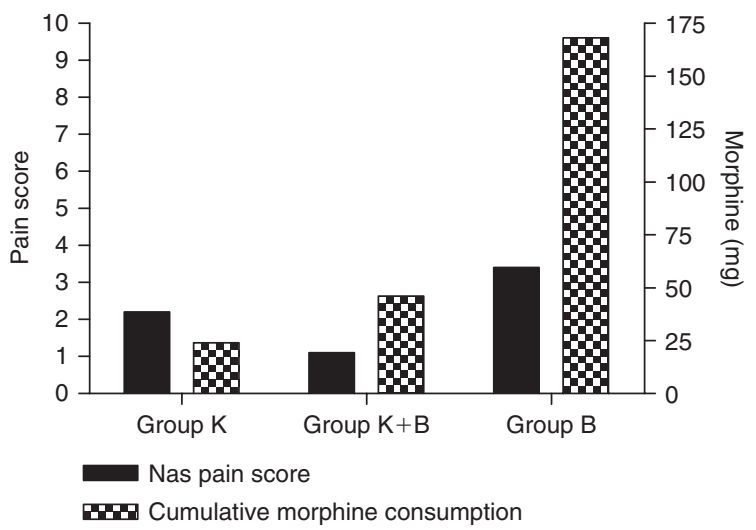

Conclusion: The patient who received bupivacaine infusion alone had the highest average pain score and cumulative morphine consumption over 48 hours when compared to the patients who received ketorolac $+/$ - bupivacaine infusion.

References:

1 Blumenthal S et al. Anesthesiology 2005; 102: 392-7.

2 Puri R et al. Am J Orthop 2000; 29: 443-6.

\section{AP6-5}

The effect of pre-incisional infiltration of tonsils with dexamethasone on post-tonsillectomy pain in children

K. Montazeri, A. Okhovat, L. Ashrafi

Aneasthesiology, Alzahra hospital, Isfahan, Iran

Background and Goals: Previous studies on dexamethasone's antiemetic and analgesic potential in children undergoing tonsillectomy have produced conflicting results(1). The aim of this study was to evaluate the effects of peritonsillar infiltration of dexamethasone on the incidence and severity of postoperative vomiting and pain in children undergoing electrocautery tonsillectomy under standardized general anesthesia(2).

Material and Methods: In a double-blinded study, 62 patients were randomly allocated to infiltrate dexamethasone $(0.5 \mathrm{mg} / \mathrm{kg}$, maximum dose, $12 \mathrm{mg})$ or an equivalent volume of saline at the peritonsillar region. All infiltrations were performed following the induction of general anesthesia and 5 minutes prior to the onset of surgery. Anesthetic agents, end-tidal carbon dioxide levels, and the administration of intravenous fluids were carefully regulated. Surgery was performed by one attending otolaryngologists using the same dissection and snare technique. Hemostasis was managed by suction-electrocautery and packs. The incidence of vomiting, need for rescue antiemetics, and analgesic consumption were compared in both groups. Pain scores used included Children's Hospital Eastern Ontario Pain Scale, "faces", and a 0-10 visual analogue pain scale.

Results: Demographics of dexamethasone and placebo groups were similar. No statistically significant difference was found between the dexamethasone and placebo groups in pain score, nausea, vomiting, or analgesic requirement postoperatively.

Conclusions: This study has shown that preincisional infiltration of the tonsils with dexamethasone play a limited role in the recovery phase from tonsillectomy, but further prospective, randomized studies are needed to support it. Assessment of pain, nausea and vomiting in a prospective study with larger groups of patients may reflect different results.

References:

1 Goldsher M, Podoshin L. et al. Ann Otol Rhinol Laryngol1996 Nov; 105(11): 868-70. 2 Naja MZ, El-Rajab M: et al. Int J Pediatr Otorhinolaryngol 2005 Jan; 69(1): 35-41.

\section{AP6-6}

\section{Single-shot Paravertebral blockade for postoperative} analgesia after urologic surgery in children

E. Berta, J. Spanhel, T. Gabrhelik, O. Smakal, V. Smolka

Clinic of Anaesthesia and Intensive Care, University Hospital Olomouc, Olomouc, Czech Republic
Background and Goal of Study: Paravertebral blockade (PVB) has been employed for analgesia in children since 1992 (1). However, efficacy of the "single-shot" technique has not yet been established.

Materials and Methods: Duration of analgesia following "single-shot" PVB was prospectively studied in 28 children aged $11.8 \pm 7.06$ (mean \pm SD) months scheduled for major urologic surgery. Bolus $0.5 \mathrm{ml} . \mathrm{kg}^{-1}$ of levobupivaciane $2.5 \mathrm{mg} \cdot \mathrm{ml}^{-1}$ with epinephrine $5 \mathrm{mcg} \cdot \mathrm{ml}^{-1}$ was administered after paravertebral space had been identified by loss of resistance to saline with 20G Tuohy needle at the end of surgery. Postoperative analgesia defined as interval between PVB and first administration of an opioid was evaluated by behavioral FLACC score (2) and Nurse score $(0=$ no pain, $1=$ mild pain, $2=$ moderate pain, 3 = severe pain) in observer-blinded manner for 12 hours. In case that an opioid had to be administered within first 120 min., the blockade was considered as failed. Incidence of complications, PONV and motor block were recorded. Both anaesthesia and postoperative care were standardized. Results and Discussions: 10 children (35.7\%) required no additional analgesia during the study period. In 16 children (57.1\%) the duration of analgesia was $361.3 \pm 132.1 \mathrm{~min}$. In one child $(3.6 \%)$ the blockade failed. Vascular puncture was observed in 3 children (10.7\%), one of them received no PVB. PONV occurred in 6 children (21.4\%), mostly after administration of an opioid. Motor block appeared in 3 children (10.7\%) which can be explained by epidural spread of local anaesthetic. All complications were considered minor and did not influence patients' recovery.

Conclusion(s): "Single-shot" PVB provided long-lasting analgesia with a favourable safety profile in children undergoing urologic surgery.

References:

1 Lönnqvist PA. Anaesthesia, 1992; 47: 607-609.

2 Manworren RC. Pediatr Nurs, 2003; 29(2): 140-146.

\section{AP6-7}

General and caudal anesthesia in children during appendectomies

A. Hasani, Sh. Azizi, N. Baftiu

Anaesthesiology, University Clinical Center of Kosova, Prishtina, UNMIK Kosovo

Background and Goal of study: Single-shot caudal epidural blockade is one of the most widespread techniques to provide intra and postoperative analgesia in paediatric patients $(1,2)$. The aim of this study is to evaluate the duration of postoperative analgesia after caudal blocks in children with different concentrations of bupivacaine during emergent appendectomies. Materials and Methods: After local ethical committee approval and written parental consent, 84 children (ASA I-II, 1-9 years old), undergoing appendectomies were enrolled in study. Induction of general anaesthesia is performed using oxygen, $\mathrm{N}_{2} \mathrm{O}$, and halothane, without using opioids in both groups. The airway was secured by tracheal intubations. After identifying the sacral hiatus, the caudal space is entered using a short (1-inch), 20-gauge short-bevel needle that has been attached to a labelled syringe containing the solution of $0.125 \%$ bupivacaine in volume $1 \mathrm{ml} / \mathrm{kg}$ (group I, $\mathrm{n}=47$ ) and $0.20 \%$ bupivacaine, $1 \mathrm{ml} / \mathrm{kg}$ (group II, $\mathrm{n}=37$ ). The needle was placed in the midline and inserted at a 45-60 degree angle to the coronal plane, perpendicular to all other planes and maintaining a rostral direction and prepared solution was injected. Continual monitoring of vital signs, observational paediatric pain score (OPS), modified Bromage scale and postoperative sedation were assessed.

Results and Discussions: Patients characteristics were similar, as well as surgical time. Analgesics were needed after $662 \pm 395 \mathrm{~min}$ in the first group (lower concentration) and $887 \pm 607 \mathrm{~min}$ (higher concentration) in the second group $(p<0.05)$. Motor block was less in the first group $(p<0.05)$. Emergency agitation was present only in two cases, in both groups. Urine incontinence was present in 17 (45.9\%) children in second group and only in 3 cases $(6.4 \%)$ in first group.

Conclusion: In children undergoing appendectomies caudal block with $0.125 \%$ bupivacaine in volume $1 \mathrm{ml} / \mathrm{kg}$ produced shorter analgesic effect, but without motor block, muscle weakness and the other side effects.

References:

1 Ivani G., et al. Anesth Analg 2003; 97: 368-371.

2 Verghese ST, Anesth Analg 2002; 95: 1219-24.

\section{AP6-8}

Tramadol vs nalbuphine: analgesic efficacy and side effects. A prospective, randomized, double-blinded study in children. J. Ouaki, A. Rochette, O. Raux, Ch. Dadure, X. Capdevila Department of Anesthesia and Critical Care, Chu Montpellier, Hopital Lapeyronie, Montpellier, France 
Background and Goal of Study: Tramadol (T), a central analgesic with low affinity for opioïd $\mu$ receptors, has been recently suggested for moderate-tosevere post operative pain relief in children (1). However, nalbuphine (N) remains a gold standard. We compared in a prospective, randomized, doubleblinded trial analgesic efficacy and side effects of intravenous continuous infusion of equipotent doses of T and $\mathrm{N}$ in children, 1 to 10 years old, ASA 1 to 3 , undergoing gastro-oesophageal reflux laparoscopic surgery under standard anesthetic procedure.

Methods: 47 children were randomly allocated to receive $\mathrm{T}(2 \mathrm{mg} / \mathrm{kg}$ in $10 \mathrm{~min}$, followed by infusion of $8 \mathrm{mg} / \mathrm{kg} / 24 \mathrm{~h}$ and bolus $1 \mathrm{mg} / \mathrm{kg}$ if pain score $>3$ ) or $\mathrm{N}(0.2 \mathrm{mg} / \mathrm{kg}$, infusion $0.8 \mathrm{mg} / \mathrm{kg} / 24 \mathrm{~h}$, bolus $0.1 \mathrm{mg} / \mathrm{kg})$. All were given paracetamol $60 \mathrm{mg} / \mathrm{kg}$ and niflumic acid $40 \mathrm{mg} / \mathrm{kg}$ per day. Pain score (CHIPPS), sedation, bolus requirements, Respiratory- and heart rate (RR \& HR), $\mathrm{SpO}_{2}$, MAP, nausea and vomiting were recorded upon arrival in recovery room and at $\mathrm{H} 2, \mathrm{H} 4, \mathrm{H} 6, \mathrm{H} 8, \mathrm{H} 12, \mathrm{H} 16, \mathrm{H} 2 \mathrm{O}, \mathrm{H} 24$.

Results: Groups were not statistically different: $3.6(1.7-5.2)$ years old, 14.0 (11-17) kg, ASA 2 (1-2) [median (25-75) interquartile]. CHIPPS, hemodynamic \& respiratory data, PONV, number of analgesic bolus were comparable in both groups during the whole study. $\mathrm{SpO}_{2}$ was lower in recovery room and $\mathrm{RR}$ was lower at $\mathrm{H} 2$ in group $\mathrm{N}$ while not significantly $(p=0.06$ and 0.09 , respectively). T caused less sedation at $\mathrm{H} 2(p=0.01)$. A second bolus was required earlier in group T $(p=0.02)$. Hemodynamic data were similar to preoperative records in both groups.

Conclusion: T appears at least as efficient as $\mathrm{N}$ to treat moderate to severe postoperative pain in children. $T$ causes less early sedation, and does not increase PONV.

Reference:

1 Bozkurt P. Pediatr Anesth 2005. 15: 1041-7.

\section{AP6-9}

Penile block for postoperative analgesia of hypospadias repair in children

I. Budic, A. Slavkovic, D. Simic, V. Djordjevic, D. Novakovic

Anesthesiology Department, Clinic for Pediatric Surgery and Orthopedics, Clinical Centre Nis, Nis, Serbia
Background and Goal of Study: Penile block is the regional anesthetic technique recommended for providing long-lasting pain relief after hypospadias repair. In the recent time, several studies have reported that two penile nerve block, one at prior to incision and one at the conclusion of surgery provide satisfactory intra and even more postoperative analgesia. The aim of our study was to evaluate the penile block with respect to penile block timing and the puncture technique.

Materials and Methods: The study population consisted of 40 ASA 1 and 2 boys aged 1 to 14 years undergoing hypospadias repair. The participants were allocated randomly into two groups of 20 children each. Group 1 received penile block at the beginning of hypospadias repair and at the completion of surgery, group 2 received the block at the completion of surgery only. Intraoperative analgesia in group 2 was achieved by alfentanyl. Each group was subdivided into two groups according to the punction technique (one or two punction sites). In all participants, inhalation anesthesia was administered with oxygen : nitrous oxide $(1: 2)$ and sevoflurane. The dorsal nerves of the penis were blocked with $0.5 \%$ marcaine in a dose of $0.1 \mathrm{ml} / \mathrm{kg}$ per side (two punction sites) or $0.2 \mathrm{ml} / \mathrm{kg}$ (one punction site). To measure postoperative pain, we used the Faces Pain Scale and as a second measurement tool we used the CHEOPS behavioral scale.

Results: There was no difference in paracetamol doses reqiured from 15 minutes to 3 hours in two groups when the two punction sites technique was used. Data collection showed that when one punction technique was used the need for analgesia was significantly higher in both subgroups, especially in group 2 subgroup. The reason for this finding is probably the fact that subpubic space can occasionaly be devided into two noncommunicating compartments. By 12 hours after surgery the number of paracetamol doses required for pain control were significantly lower in group 1.

Conclusion(s): Two penile blocks performed at the beginning and conclusion of hypospadia repair provide better postoperative pain control and earlier mobilization than one penile block done by one punction site after surgery.

Reference:

1 Johr M. Anaesthesiol Reanim 2003; 28(3): 69-73

\section{Obstetric Anaesthesia}

\section{AP1-1}

\section{Botox and obstetric anesthesia: is there cause for concern?} K. Kuczkowski

Ansthesiology and Reproducitve Medicine, University of California, San Diego, San Diego, USA

Introduction: Botulinum toxin is a neurotoxin produced by the bacterium Clostridium botulinum. It produces flaccid muscle paralysis by blocking the release of acetylcholine at the neuromuscular junction. Botulinum toxin type A (BTA) (Botox; Allergan Inc., Irvine, CA) is commonly used for the treatment of hyperfunctional facial lines resulting from repeated contractions of certain muscles (e.g., orbicularis oculi) (1). In anesthesia peripheral ulnar nerve stimulation of the adductor pollicis muscle and facial nerve stimulation of the orbicularis oculi muscle are most commonly monitored to access the degree of neuromuscular blockade. It is therefore reasonable to speculate that cosmetic BTA injection-induced flaccid muscle paralysis may interfere with neuromuscular blockade monitoring under anesthesia.

Report of Case: Indeed, I encountered a 46-year-old parturient who required Cesarean section under general anesthesia for HELLP syndrome. Following routine rapid sequence induction of general anesthesia with standard dosages of etomidate and succinylcholine $(140 \mathrm{mg})$ anesthesia was maintained with fentanyl and sevoflurane in oxygen. Ten minutes later an attempt to determine the recovery from the neuromuscular blockade [facial nerve stimulation (trainof-tour pattern) to detect contraction of the orbicularis oculi muscle] was made by the anesthesiologist; however, no twitches were noted. The train-of-four test was repeated 15 minutes later still yielding no twitch response. Ulnar nerve stimulation at that time confirmed full recovery from the neuromuscular blockade. Following emergence from anesthesia it was discovered that the patient had undergone multiple bilateral cosmetic facial BTA injections in early pregnancy. To the best of my knowledge this complication of BTA injections in pregnancy has not been previously reported.

Discussion: Facial enhancement by the use of BTA has revolutionized the treatment of "the aging face", and it remains the most popular aesthetic procedure performed in the United States. In the year 2005 over 3.8 million of BTA procedures were preformed in the United States (1). The duration of BTA effect when used for the treatment of facial lines is generally 6-11 months.
As the incidence of pregnancy in older women is increasing, preoperative questioning regarding BTA injections in this group should be considered. Reference:

1 Arch Facial Plast Surg 2006; 8: 426-431.

\section{AP1-2}

Ten years of experience with post dural puncture headache and accidental dural perforation in a tertiary obstetric anaesthesia department

M. Van de Velde, R. Schepers, N. Berends, R. Devlieger, E. Vandermeersch

Anesthesiology, UZLeuven - KULeuven, Leuven, Belgium

Background and Goal: Accidental dural perforation (DT) and post dural puncture headache (PDPH) are two common complications of obstetric regional anaesthesia $(1,2)$. PDPH is incapacitating, interferes with maternal-infant interaction and is a cause of increased staff workload and prolonged hospitalisation. We report on all cases of DT and PDPH in a tertiary care obstetric anaesthesia unit, using predominantly combined spinal epidural anaesthesia (CSE), over a 10 year period.

Material and Methods: Following institutional approval, the obstetric anaesthesia database in a tertiary care teaching hospital was searched to identify patients that experienced a DT or PDPH between Jan 1st 1997 and Oct 31st 2006. Since 1997 all patients that received obstetric anaesthesia are visited or contacted on the second postpartum day and data is prospectively gathered on all patients. The anaesthetic and obstetric charts of all patients with the diagnosis of DT and PDPH were retrospectively evaluated. Data were analysed using Chi-square analysis.

Results and Discussion: During the study period, 17610 patients received regional anaesthesia. We excluded those lost to follow-up and thus 17198 patients remained. An accidental DT occurred in 55 patients (DT-rate of $0.32 \%$ ). Of these $31(56 \%)$ developed PDPH and $26(47 \%)$ required a blood patch (BP) and 4 (7\%) needed a repeat BP. Inserting an epidural catheter intrathecally and leaving it there for at least 24 hours does not reduce the incidence of PDPH or BP. A further 34 patients developed PDPH without a clear DT. Overall 
65 patients in our series developed PDPH (PDPH-rate 0.38\%). The need for BP occurred in $82 \%$ of patients and in $15 \%$ a repeat $B P$ was required. The interval between regional block and onset of PDPH was $32 \pm 20$ hours. Caffeine was not successful in treating PDPH and in 1 patient caused seizures.

Conclusion: In our teaching unit, using predominantly CSE, PDPH and DT occur with a similar incidence as reported in the literature $(1,2,3)$. Contrary to some evidence, intrathecal catheters do not protect against PDPH or BP in our series. The incidence of $\mathrm{BP}$ and repeat $\mathrm{BP}$ in the present study confirms previous data.

References:

1 Lybecker $\mathrm{H}$ et al. Acta Anaesthesiol Scand 1995; 39: 605-612.

2 Paech M et al. Int J Obstet Anesth 2001; 10: 162-167.

3 Gleeson et al. IJOA 1998; 7: 242-246.

\section{AP1-3}

\section{Obstetric and neonatal outcome of twin pregnancies in a} tertiary care obstetric unit in relationship to the anaesthetic technique used during labour and delivery

M. Van de Velde, H. Cooreman, J. Decoster, M. Hanssens, E. Vandermeersch Anesthesiology, UZLeuven - KULeuven, Leuven, Belgium

Background and Goal: Twin pregnancies are associated with more obstetric risks, such as Caesarean section (CS) and neonatal risks. The liberal use of epidural analgesia may reduce the need for CS in twins considered good candidates for vaginal delivery (1). The purpose of this trial was to determine the relationship between obstetric and neonatal outcome and anaesthetic technique in twins.

Material and Methods: Following institutional approval, the obstetric database of a tertiary care hospital was searched to identify all patients that delivered a twin pregnancy of at least 24 weeks gestation between Jan 1 st 1997 and Aug 31st 2004. A retrospective chart analysis was performed and obstetric anaesthetic and neonatal outcome data were recorded. Data were analysed using the Fisher's exact test and Chi-square analysis.

Results and Discussion: A total of 553 twins were identified. Sixty-one patients were excluded from analysis due to incomplete datasets. Final analysis consisted of 492 patients. Planned CS was performed in 229 parturients. From 263 patients undergoing planned vaginal delivery (VD), 70 required an unplanned CS. Ultimately, 299 mothers underwent CS and 193 VD. Neuraxia analgesia was used in 179 patients undergoing planned VD. The incidence of neuraxial labour analgesia was much higher in patients that ultimately delivered vaginally then in those that needed an unplanned CS ( $77 \%$ vs $50 \%$, $\mathrm{p}<0.01$ ). Regional anaesthesia was used in $253 \mathrm{CS}$, whilst general anesthesia (GA) in 46 patients (15\%). The risk for GA was increased in patients that underwent unplanned CS as compared to patients that underwent planned CS $(33 \%$ vs $10 \%, p<0.05)$. The risk increased if neuraxial analgesia was not used during labour in planned VD patients as compared to patients that did use neuraxial analgesia ( $51 \%$ vs $15 \%, p<0.05)$. Regional anaesthesia during CS resulted in better neonatal outcome when compared to GA

Conclusion: Despite the retrospective design of our study, several conclusions can be drawn. Firstly, many vaginal deliveries ended in an unplanned CS. Secondly, established labour analgesia seems to protect against unplanned CS and GA during CS. Finally, neonatal outcome was better in planned CS deliveries and in CS under regional anaesthesia.

Reference:

1 Williams KP, Galerneau F. Intrapartum influences on cesarean delivery in multiple gestation. Act Obstet Gynecol Scand. 2003 Mar; 82(3): 241-5.

\section{AP1-4}

Multidimensional evaluation of pain during early and late labor: a comparison of nulliparous and multiparous women

S. Stirparo, M. Camorcia, C. Berritta, C. Cifarelli, G. Capogna

Anesthesiology, Città di Roma, Roma, Italy

Background and Goal of Study: This study evaluates and compares the characteristics of labor pain during early and late labor in nulliparous $(\mathrm{N})$ and multiparous (M) women using the McGill Pain Questionnaire (MPQ) (1).

Materials and Methods: After informed consent we interviewed 83 parturients divided in 4 groups according to parity and stage of labor. According to the standard MPQ the words describing qualities of pain were categorized into sensory, affective, evaluative and miscellaneous subclasses. Pain Rating Index (PRI), was calculated and scored by dividing the sum of the obtained ranks within each dimension by the total possible score for a particular dimension (2). Intensity of pain was evaluated by both VAPS (0-100) and Present Pain Intensity (PPI) (0-5). Statistical analisys was performed using Student's test and linear regression.
Results and Discussions: Both $\mathrm{N}$ and $\mathrm{M}$ had mean intensity of pain which increased with greater cervical dilatation, $\mathrm{N}$ having more pain in early labor and $\mathrm{M}$ in late labor $(\mathrm{P}<0.05)$. In $\mathrm{M}$ a significant correlation between the values of VAPS and PPI was noted $(\mathrm{P}<0.01)$. Concerning parity, in $\mathrm{N}$ sensory and evaluative $P R I s$ were higher during early labor while affective PRI was higher in late labor $(P<0.05)$. In $M$ all the subclasses had higher $P R l s$ during early labor $(\mathrm{P}<0.05)$. Concerning the stage of labor, during early labor $\mathrm{N}$ reported higher evaluative and lower sensory and affective PRIs than $M(P<0.05)$. During late labor $N$ reported lower sensory and higher affective PRIs than $M$ $(P<0.05)$. In all groups pain was described in more than $50 \%$ of cases as: jumping, pricking, lacerating, tugging, stinging, tiring and unbearable.

Conclusion: Intensity of pain increases with cervical dilatation. $\mathrm{N}$ reported more pain in early labor and $M$ in late labor. Perceptual, motivational and cognitive aspects of pain may vary depending on parity and stage of labor.

References:

1 Melzack R. Pain 1975; 1: 275-99.

2 Kremer EF. Pain 1981; 11:93-100.

\section{AP1-5}

\section{Preoxygenation in pregnancy: a computational modelling investigation}

S.H. McClelland, J.G. Hardman

University Department of Anaesthesia, Queen's Medical Centre, Nottingham, United Kingdom

Background and Goal of Study: Hypoxaemia during anaesthetic induction in obstetrics is hazardous for mother and baby. Ethical and practical issues constrain clinical studies. Following configuration of a computational model to replicate pregnant physiology, we investigated the optimal technique for preoxygenation.

Materials and Methods: Using the Nottingham Physiology Simulator (1), we modelled three pregnant and three non-pregnant women, representing normal population variation according to published physiological values. They underwent preoxygenation by tidal and vital capacity (VC) breathing of $100 \%$ oxygen for 10 minutes.

Results and Discussion: Results for the six subjects during the first $3 \mathrm{~min}$ of non-VC preoxygenation are shown in the figure. The median time to achieve $95 \%$ of the maximum end-tidal $\mathrm{PO}_{2}\left(\mathrm{PEO}_{2}\right)$ was $1 \mathrm{~min} 40 \mathrm{sec}$ in pregnant compared to $3 \mathrm{~min} 6 \mathrm{sec}$ in non-pregnant women. Vital capacity preoxygenation required 11 breaths in $1 \mathrm{~min} 10 \mathrm{sec}$ to achieve $95 \%$ of the maximum $\mathrm{PE}^{\prime} \mathrm{O}_{2}$ for both pregnant and non-pregnant women.

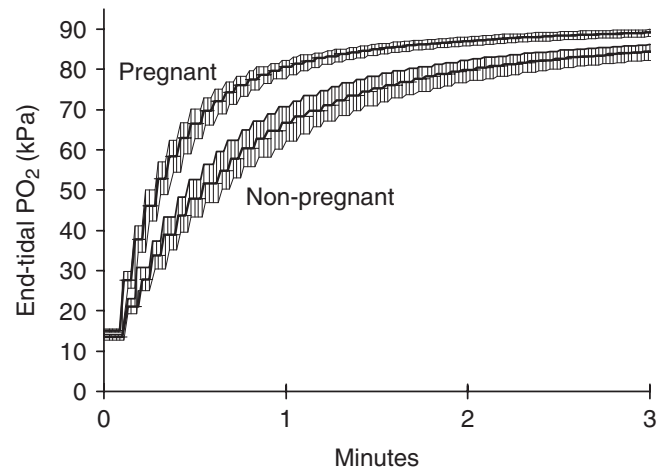

Conclusions: Preoxygenation is more rapid in pregnancy. During tidal breathing, the $\mathrm{PE}^{\prime} \mathrm{O}_{2}$ plateau was reached approximately twice as quickly. Vital capacity breathing accelerates preoxygenation to produce a similar rate in pregnant and non-pregnant women. The traditionally-advised four vital capacity breaths do not achieve optimal preoxygenation.

Reference:

1 Hardman JG, Wills JS, Aitkenhead AR. Anesth Analg 2000; 90: 614-618.

\section{AP1-6}

\section{Factors affecting women's ambulation during labor}

C. Berritta, M. Camorcia, G. Capogna, C. Cifarelli

Anesthesiology, Città di Roma, Roma, Italy

Background and Goal of Study: It is commonly believed that upright position may be beneficial for labor although it has not been demonstrated (1). This study was designed to investigate the factors affecting the parturient's ambulation during labor and its incidence and duration. 
Materials and Methods: This study was conducted on 124 parturients in active labor, 65 nulliparous $(\mathrm{N})$ and 59 multiparous $(\mathrm{M})$ women, who gave their informed consent to answer a questionnaire during their labor. The logistic linear and the regression logistic models were used to evaluate the factors affecting the ambulation.

Results: Epidural analgesia was received by $90 \%$ of $\mathrm{N}$ and by $60 \%$ of $\mathrm{M}$. During I stage the majority of parturients walked ( $99 \% \mathrm{~N}$ and $60 \% \mathrm{M})$. Each mean single continual period of time women ambulated during labor was 32 min (range 15-50) in N and 12 min (range 2-34) in M. The mean total duration of walking throughout the entire labor in $\mathrm{N}$ was $88 \mathrm{~min}$ (range 0-122) and

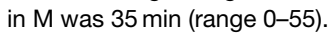

Factors affecting the choice in favour of parturient's ambulation were: midwife's suggestion $(P<.0001)$, absence of pain due to epidural analgesia $(P<.005)$, participation in active management labor classes $(P<.05)$, on call gynecologist's opinion $(\mathrm{P}<.0001)$. Factors discouraging or preventing parturient's ambulation were: longer labors $(P<.0001)$, dystocic labors $(P<.001)$, the frequency of obstetrical assessment and the need for FHR monitoring $(P<.0001)$, longer duration of prodromic phase of labor $(P<.0001)$. Formal education and socioeconomic status were not significant factors for all the groups.

Conclusion: Many factors other than epidural analgesia may affect the parturient's decision to walk during labor and its duration.

Reference:

1 Bloom SL et Al. NEJM 1998; 339: 76-9.

\section{AP1-7}

\section{Efficacy of a single dose of epidural neostigmine for postpartum pain}

A. Richard, F. Roelants, P. Lavandhomme

Anesthesiology Department, Cliniques Universitaires St-Luc Université Catholique de Louvain, Brussels, Belgium

Background and Goal of Study: Severe perineal pain is a common problem after vaginal delivery. Single dose epidural morphine decreases postpartum use of oral pain medication (1). Epidural neostigmine produces analgesia devoid of side effects during labor (2). The study evaluates the benefit of a single dose epidural neostigmine on postpartum pain and analgesics requirements.

Material and Methods: After informed consent, 26 parturients, with epidural analgesia for labor (ropivacaine combined with sufentanil), were randomly allocated to receive after delivery $5 \mathrm{~mL}$ saline (group $\mathrm{S} ; \mathrm{n}=11$ ) or neostigmine $500 \mu \mathrm{g}$ in $5 \mathrm{~mL}$ saline (group $\mathrm{N} ; \mathrm{n}=15$ ). All the parturients were allowed to receive diclofenac $(\max 150 \mathrm{mg} / \mathrm{d})$, with oral paracetamol $(\mathrm{max} 3 \mathrm{~g} / \mathrm{d}$ ) at their discretion as rescue analgesia. Postpartum pain scores (VAS:0-10) and analgesics consumption were recorded for 72 hours. Statistical analysis used unpaired T-test, $P<0.05$ was considered significant.

Results and Discussion: Demographic data (including duration of first and second stage of labor, neonatal weight, episiotomy, perineal lacerations and instrumentation rate) did not differ between the groups. Postpartum VAS were similar between the groups. Expressed as mean value $(95 \% \mathrm{Cl})$, total diclofenac consumption was $300 \mathrm{mg}(176-723)$ in $\mathrm{S}$ group and $283 \mathrm{mg}$ (173-393) in N group; total paracetamol consumption was $4454 \mathrm{mg}$ (1227-7681) in S group and $2366 \mathrm{mg}$ (834-3899) in N group. Paracetamol consumption (mean $\pm \mathrm{SD}$ ) was significantly reduced in $\mathrm{N}$ group, at $48 \mathrm{~h}$ $(536 \pm 842 \mathrm{mg}$ vs $1944 \pm 1703 \mathrm{mg} ; \mathrm{p}=0.014)$ and at $72 \mathrm{~h}(500 \pm 975 \mathrm{mg}$ vs $1667 \pm 1650 \mathrm{mg} ; \mathrm{p}=0.045)$.

Conclusions: Single dose epidural neostigmine $500 \mu \mathrm{g}$ administered after delivery allowed a reduction of postpartum paracetamol consumption but did not affect pain scores as previously observed with epidural morphine (1). In contrast with epidural morphine, epidural neostigmine administration was devoid of side effects.

References:

1 Goodman S, Drachenberg A, Johnson S et al. Reg Anesth Pain Med. 2005; 30: 134-9. 2 Roelants F, Lavand'homme P Anesthesiology 2004; 101: 439-44.

\section{AP1-9}

\section{Closing the gap between decision an delivery - 18 months of rescue service for obstetrical emergencies}

\section{R.J. Litz, R. Rehnig, G. Kamin, S. Stehr, T. Koch}

Anaesthesiology, University of Technology, Dresden, Germany

Background and Goal of Study: After implementation of a reporting system for critical incidents at our institution an optimized alerting system for obstetrical emergencies (cesarean delivery, ECD) was introduced in 1/2005. Midwives contact the switchboard via a special number and a team consisting of a staff anesthesiologist, anesthesia nurse, gynecologist and operating room (OR) nurse are contacted by special pagers and dispatch immediately to the OR without returning the call. We report our experience with the ECD alerting system in our institution.

Materials and Methods: Data from the on-line anesthesia documentation system as well as the midwifes' records were analyzed regarding time course and outcomes of ECD since the introduction of the alerting system (group 1). For comparison the records of the past 2 years-period (2003-2004) were used (group 2, $\mathrm{n}=57$ ). For maternal and fetal outcomes the charts from the ward were analyzed.

Results and Discussions: Within a 18 month period from 1/2005 to 6/2006 this system was used 24 times, representing an incidence of approximately $1 \%$ of all life births and $3 \%$ of all cesarean section $(n=702)$. The most common causes were eclampsia, maternal bleeding or fetal asphyxia and one case of maternal and neonatal cardiac arrest following amniotic fluid embolism (AFE). Time from decision to incision was shorter in group 1(9.8 $\pm 3.5 \mathrm{~min}$ vs. $17.5 \pm 10.7 \mathrm{~min} ; \mathrm{p}<0.001)$ as was time from decision to delivery $(12.7 \pm 3.9 \mathrm{~min}$ vs. $21 \pm 12 \mathrm{~min} ; \mathrm{p}<0.001)$. Maternal outcome did not differ between groups. All mothers were discharged home after 8 (7-11.7) vs. 9 (8-13) days, $p=0.3$ (median (interquartile) range). Neonates had good outcome in both groups. In group 1 two premature infants (weight $<500 \mathrm{~g}$ ) with asphyxia did not survive. Apgar scores (median [range]) at 1, 5 and $10 \mathrm{~min}$ did not significantly differ between groups (7 [0-9] vs. 6 [0-9]; 8 [0-10] vs. 8 [3-10]; 9 [0-10] vs. 9 [5-10] $\mathrm{p}=0.3)$. Likewise umbilical $\mathrm{pH}$-values did not show statistical differences $(7.16 \pm 0.6$ vs. $7.15 \pm 0.2)$.

Conclusion(s): The introduction of an ECD service led to a significant reduction in time between decision and delivery. Despite comparable results concerning outcome we are convinced that in the individual (AFE) patient(s), the short decision to delivery time may be crucial for superior outcome of both mother and child.

\section{AP1-10}

\section{Maternal morbidity after pregnancy suffering from HELLP syndrome}

P. Hupuczi, B. Rigó, Z. Papp

I. Department of Obstetrics and Gynecology, Semmelweis University, Budapest, Hungary

Background and Goal of Study: HELLP syndrome is a severe, lifethreatening form of preeclampsia, described by Weinstein in 1982 (1). Its importance lies in the fact that maternal and fetal morbidity, as well as mortality, are significantly elevated (2).

Materials and Methods: Between January 1st, 1995 and December 31th, 2004, 107 patients were treated for HELLP syndrome at the 1st Department of Obstetrics and Gynecology of Semmelweis University. We retrospectively analyzed the incidence of complications and necessary interventions due to these complications.

Results and Discussions: Our patients on average spent 3 days in our intensive care unit. Pulmonary oedema was the most common cardiopulmonary complication (11\%), and it occurred mostly in group I. (21\%). Infection occurred in all of the tree groups in a notable proportion. The complications of HELLP syndrome are shown in the table.

\begin{tabular}{llllll}
\hline & $\begin{array}{l}\text { Mississippi I. } \\
\mathrm{n}=38\end{array}$ & $\begin{array}{l}\text { Mississippi II. } \\
\mathrm{n}=52\end{array}$ & $\begin{array}{l}\text { Mississippi III. } \\
\mathrm{n}=17\end{array}$ & $\mathrm{p}$ & Total \\
\hline $\begin{array}{l}\text { Pulmonary } \\
\text { oedema }\end{array}$ & $8(21 \%)$ & $3(6 \%)$ & $1(6 \%)$ & ${ }^{*} \mathrm{p}=0,029$ & $12(11 \%)$ \\
$\begin{array}{l}\text { Thrombo- } \\
\text { emboly }\end{array}$ & $4(10,5 \%)$ & 0 & 0 & ${ }^{*} \mathrm{p}=0,028$ & $4(4 \%)$ \\
$\begin{array}{l}\text { Eclampsy } \\
\text { Oligury }\end{array}$ & $4(11 \%)$ & $4(8 \%)$ & 0 & $\mathrm{NS}$ & $8(7 \%)$ \\
$\begin{array}{l}\text { Infection } \\
\text { DIC }\end{array}$ & $20(52,6 \%)$ & $5(10 \%)$ & $2(12 \%)$ & $\mathrm{NS}$ & $12(11 \%)$ \\
$\begin{array}{l}\text { Intensive } \\
\text { care (day) }\end{array}$ & $6(16 \%)$ & $1(2 \%)$ & 0 & $\mathrm{NS}$ & $43(40 \%)$ \\
\hline
\end{tabular}

$62 \%$ of our patients received transfusion during treatment. In five cases, hysterectomy was performed. Intrauterine examination and abrasion was necessary in $5 \%$ of all cases.

Conclusion(s): Our study shows that the rate of cardiopulmonary and haematology complications are significantly higher in Mississippi I. group than in other cases. The increased need for transfusion of erythrocyte concentrate and FFP in the postpartum time of Mississippi I. group patients must be considered.

References:

1 Weinstein L. Am J Obstet Gynecol 1982; 142: 159-167.

2 Sibai BM at all. Am J Obstet Gynecol 1993; 169: 1000-1006. 


\section{AP2-1}

Epidural tramadol for controlling pain after caesarean section T. Sanjoaquín, A. Hernández, C. Teixeira, R. López

\section{Anestesiología y Reanimación, Hospital MAZ., Zaragoza, Spain}

Objectives and Goal of the Study: Epidural tramadol has been used for controlling pain, establishing $100 \mathrm{mg}$ as optimal dose. The aim of the study is comparing the analgesic quality obtained with $100 \mathrm{mg}$ of epidural tramadol and with IV non-steroidal anti-inflammatory drugs (NSAD) after cesarean section. Material and Method: 120 patients who underwent cesarean section with epidural anesthesia were randomly allocated into two groups: $T(n=60)$ received $100 \mathrm{mg}$ epidural tramadol and $S(n=60)$ epidural saline when arriving at post-anesthetic care unit (PACU). Supplementary analgesia (IV NSAD) was prescribed for both groups, registering the moment it was supplied. Pain scores (PS) $(0=$ no pain, $10=$ maximum pain) and medium blood pressure (MBP) were recorded at 1, 6 and 12 hours of arriving at PACU, at rest and with movement, as well as side effects.

Results: Both groups were compaired in population variables. Surgical incision and epidural anesthetic used were the same. When arriving at PACU PS and MBP were similar in both groups. At 1, 6 and 12 hours statistically relevant differences were found in PS at rest and in movement $(p<0.01)$.

Nausea appeared in $15 \%$ patients of $\mathrm{T}$, without requiring treatment. One hundred per cent patients of S demanded analgesia from the third hour of receiving epidural saline. Forty five per cent patients of $\mathrm{T}$ did not require supplementary analgesia. Fifty five patients of $T$ demanded analgesia from the 10th hour of receiving epidural tramadol.

Conclusions: $100 \mathrm{mg}$ of epidural tramadol provide an adequate analgesia for controlling pain the first hours after cesarean section, without severe side effects, achieving a better analgesia quality than using only IV NSAD.

\section{AP2-2}

Relation between the direction of Whitacre needle hole and Mean Local Anaesthetic Dose of subarachnoid Ropivacaine $\mathbf{0 , 7 5 \%}$ in Caesarean section

D. Valsamidis, S. Leontopoulou, P. Klimi, O. Petropoulou, A. Karathanos Anaesthesiology, General Hospital Alexandra, Athens, Greece

Background and Goal of Study: The relation between the direction of spinal needle hole to the Mean Local Anaesthetic Dose [MLAD] in Caesarean Section has not yet been established. We measured the MLAD of subarachnoid Ropivacaine $0,75 \%$ plus $15 \mu \mathrm{g}$ fentanyl when given through a $26 \mathrm{G}$ Whitacre needle, with cephalad or caudad direction.

Materials and Methods: Lumbar [L2-3] puncture was performed in lateral decubitus position in 56 health parturients scheduled for elective CS. The epidural and subarachnoid space was identified with Combined Spinal Epidural technique [needle through needle]. In the first 28 parturients [group $\mathrm{Ca}$ the Whitacre needle hole was directed caudad and in the rest cephalad [Group Ce]. The dose of Rc $0,75 \%$ was administered according to the Dixon and Massey up-and-down method[1]. The dose for the first parturient of each group was 2,05 and $1,00 \mathrm{ml}$ [group $\mathrm{Ca}$ and $\mathrm{Ce}$ respectively]. In both groups fentanyl $15 \mu \mathrm{g}$ was added to the solution. An ineffective dose, defined as a verbal analog pain score of greater than $2 / 10$, no spread of dermatomal level of block up to T4 and duration of anaesthesia of less than $60 \mathrm{~min}$ after injection, directed an increase of $0.05 \mathrm{ml}$ to the next parturient and vice versa. Two tailed t-test was performed for statistical analysis and $P<0,01$ was considered significant.

Results: The results are shown in the above figures. $\mathrm{Cl}=$ confidence interval $95 \%$

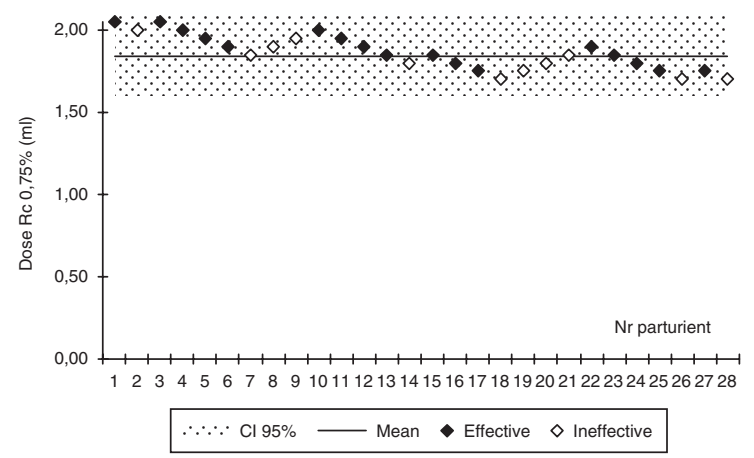

Group Ca [caudad]

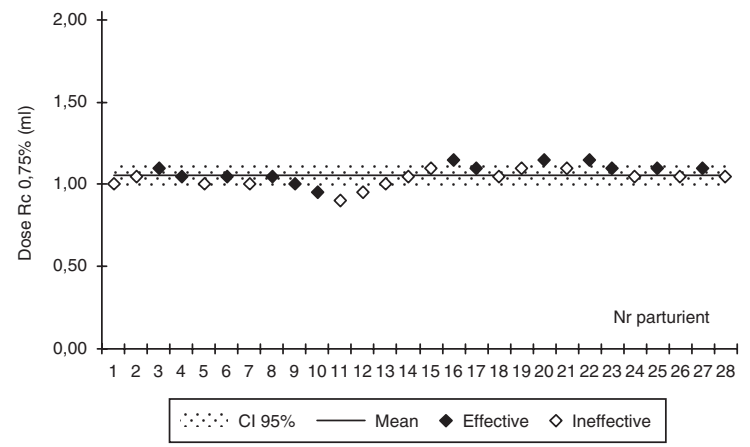

Group Ce [cephalad]

In Group $\mathrm{Ca}$ the mean dose[SD] was $1,84[0,35] \mathrm{ml}$ and in Group Ce $1,04[0,14] \mathrm{ml}$ with $\mathrm{P}<0,001$.

Conclusion: When subarachnoid anaesthesia is used for CS, different doses of local anaesthetic should be chosen according the hole direction of the Whitacre needle and when different doses are compared, the hole direction must be taken into account.

Reference:

1 Dixon WJ, Massey FJ. In: Introduction to statistical analysis. 1983:426-41.

\section{AP2-3}

A randomised comparison of $0.5 \%$ levobupivacaine with Lidocaine/Epinephrine/Fentanyl mixture for epidural top-up for emergency caesarean section after "low dose" epidural analgesia for labour

P. Balaji, P. Dhillon, I.F. Russell

Anaesthetics, Hull Royal Infirmary, Hull, United Kingdom

Background and Goal of Study: Levobupivacaine, an enantiomer of bupivacaine is now commonly used for epidural top-up for emergency caesarean section for women who have been receiving "low dose" labour analgesia. We decided to compare our standard epidural top-up solution [Lidocaine/ Fentanyl/Epinephrine (LEF)] with $0.5 \%$ levobupivacaine in those patients. Materials and Methods: In a prospective, single blind study, we compared $0.5 \%$ levobupivacaine (50 patients) with LEF mixture (50 patients) for epidural top-up in 100 patients. We compared the time taken for top-up drug preparation, time to inject via epidural catheter, time duration to achieve T6 for touch (anaesthesia level), supplementation rates and complication. Results: One patient had spinal anaesthesia as the block was not sufficient even after 30 minutes in levobupivacaine group. Data (Mean \pm SD) are in the table:

\begin{tabular}{llll}
\hline & $\begin{array}{l}\text { Levobupi } \\
(\mathrm{n}=49)\end{array}$ & $\begin{array}{l}\text { LEF } \\
(\mathrm{n}=50)\end{array}$ & $\begin{array}{l}\text { Statistical } \\
\text { Significance }\end{array}$ \\
\hline $\begin{array}{l}\text { Time - start of drug } \\
\text { preparation to } \\
\text { inject (min) }\end{array}$ & $3.66 \pm 1.31$ & $6.9 \pm 2.42$ & $\mathrm{P}<0.0001$ \\
$\begin{array}{l}\text { Time to achieve } \\
\begin{array}{l}\text { T6 block from } \\
\text { injection (min) }\end{array}\end{array}$ & $15.2 \pm 6.21$ & $10.7 \pm 5.08$ & $\mathrm{P}<0.0001$ \\
$\begin{array}{l}\text { Time from } \\
\text { preparation to }\end{array}$ & $18.8 \pm 6.4$ & $17.6 \pm 5.72$ & $\mathrm{NS}$ \\
$\begin{array}{l}\text { T6 (min) } \\
\text { Supplementation }\end{array}$ & $14 / 50$ & & \\
\hline
\end{tabular}

Conclusion(s): 1) Speed of onset was faster and 2) intraoperative supplementation with opioids or entonox was significantly less with LEF group. In order to get clinical benefit time-wise, we would need to have LEF mixture ready and drawn up.

References:

1 Levy DM. Anaesthesia 2006; 61(8): 786-91.

2 Bader AM. Anesthesiology 1999; 90(6): 1596-1601.

\section{AP2-4}

\section{Audit of side effects of neuraxial opioids used for analgesia} after Caesarean section

P. Lee, R. Fanning, M. Carey

Perioperative Medicine, Coombe Womens Hospital, Dublin, Ireland 
Background and Goals: It is common practice to add spinal and epidural opioids to local anaesthetic solutions for post caesarean section (CS) analgesia. However, there is an increased incidence of side effects with increasing doses of neuraxial opioids, with no difference in quality of analgesia ${ }^{1}$.This audit was undertaken to determine the incidence of opioid induced adverse effects after CS as part of an ongoing quality assurance measure within our hospital, where intrathecal doses higher than $0.1 \mathrm{mg}$ are still used.

Material and Methods: A prospective audit was undertaken over a four month period in 2006. The setting was an obstetric unit with 8,000 deliveries a year, $23.6 \%$ of which are by CS. 174 parturients were included. Method involved follow-up by anaesthetists and self assessment of adverse effects by the parturients.

Results: Caesarean sections were performed under spinal (83.9\%) and epidural (16.1\%) anaesthesia. The intra-operative epidural dose of morphine was $2 \mathrm{mgs}$ and the intrathecal dose of morphine ranged from 100-200 mcgs. The incidence of recorded side effects at 24 hours is given in the table. At 24 hours median (range) dynamic visual analogue pain scores were similar for each group, and were $3(0-9)$ in the intrathecal group and $4(1-8)$ in the epidural group.

\begin{tabular}{llll}
\hline & Pruritus & Nausea & Sedation \\
\hline Intrathecal $\mathrm{n}(\%)$ & $128 / 146(87.7)$ & $69 / 146(47.3)$ & $45 / 146(30.8)$ \\
Epidural $\mathrm{n}(\%)$ & $20 / 28(71.4)$ & $13 / 28(46.4)$ & $5 / 28(17.9)$ \\
\hline
\end{tabular}

Conclusions: With the exception of the incidence of pruritus in patients treated with epidural morphine, the incidence of pruritis in the intrathecal group and nausea and vomiting in the epidural and intrathecal groups are higher than the ranges reported in literature. Mild sedation was noted in $18-31 \%$ of our patients. This audit has shown the need to reduce the dose of intrathecal morphine and to consider the use of prophylactic anti-emetics and anti-pruritics.

Reference:

1 Dahl JB, Jeppesen JS, Jorgensen H et al. Anesthesiology 1999;91:1919-1927

\section{AP2-5}

The effect of intrathecal S (+) ketamine addition to spinal anaesthesia with ropivacaine or bupivacaine in parturients undergoing caesarean section

S. Gunastý, H. Unlugenc, I. Urunsak, M. Ozalevli, T. Guler

Anaesthesiology, Cukurova University, Adana, Turkey

Background and Goal of Study: In this prospective, randomized, doubleblind, controlled study, we investigated the sensory, motor, analgesic block characteristics and side effects of S $(+)$ ketamine given intrathecally (IT)in addition to $0.75 \%$ plain ropivacaine $(15 \mathrm{mg})$ for spinal analgesia and compared with $\mathrm{S}(+)$ ketamine $+0.5 \%$ plain bupivacaine $(10 \mathrm{mg})$ combination given IT in parturients undergoing caesarean section (CS).

Materials and Methods: Following faculty ethic committee approval and written parturient consent, 120 ASA I or II adult parturients undergoing CS were randomly allocated to one of the four groups. Group I $(n=30)$ received $10 \mathrm{mg}$ of $0.5 \%(2 \mathrm{ml})$ plain bupivacaine +0.9 saline $(1 \mathrm{ml})$ in group B, Group II $(\mathrm{n}=30)$ received $10 \mathrm{mg}$ of $0.5 \%(2 \mathrm{ml})$ plain bupivacaine $+0.05 \mathrm{mg} \mathrm{kg}^{-1} \mathrm{~S}$ $(+)$ ketamine $(1 \mathrm{ml})$ in group BK, Group III $(\mathrm{n}=30)$ received $15 \mathrm{mg}$ of $0.75 \%$ $(2 \mathrm{ml})$ plain ropivacaine +0.9 saline $(1 \mathrm{ml})$ in group $R$, Group II $(n=30)$ received $15 \mathrm{mg}$ of $0.75 \%(2 \mathrm{ml})$ plain ropivacaine $+0.05 \mathrm{mg} \mathrm{kg}^{-1} \mathrm{~S}(+)$ ketamine (1 ml) in group RK, intrathecally. We recorded onset and duration of sensory and motor block, the level of maximal sensory height, duration and quality of spinal analgesia, pain and sedation scores at 5, 10, 15, 20, 25 and 30 min after the injection, and subsequently every $15 \mathrm{~min}$ for 120 minutes.

Results and Discussions: The onset time of sensory and motor block was significantly shorter in BK and RK groups than in $B$ and $R$ groups $(p<0.001)$. The duration of sensory and motor block was significantly longer in $B$ and $R$ groups than in RK group $(p<0.007)(p<0.001)$; it was similar in RK and BK groups. The level of maximal sensory height, were significantly higher in parturients receiving $S(+)$ ketamine $(p<0.003)$. Duration of spinal analgesia was similar between groups. Mean sedation scores were found significantly higher in BK and RK groups at 10, 15, 20, 25, 30 and 45 minutes than in groups $B$ and $R(p<0.001)$.

Conclusion(s): In patients undergoing CS with spinal anaesthesia, the addition of $S(+)$ ketamine $\left(0.05 \mathrm{mg} \mathrm{kg}^{-1}\right)$ intrathecally to $15 \mathrm{mg}$ of spinal plain ropivacaine $(0.75 \%)$ led to rapid onset of both sensory and motor blockade and enhanced the segmental spread of spinal block without prolonging the duration of spinal analgesia, but provided sedation in the dose used in this study $\left(0.05 \mathrm{mg} \mathrm{kg}^{-1}\right)$.

\section{AP2-6}

Randomized double-blinded comparison of phenylephrine versus ephedrine for treating hypotension during spinal anaesthesia for emergency Caesarean section

W. Ngan Kee, K. Khaw, K. Ng, K. Chui, T. Lau Department of Anaesthesia and Intensive Care, The Chinese University of Hong Kong, Hong Kong, China

Background and Goal of Study: Recent studies suggest phenylephrine (Phe) may have advantages over ephedrine (Eph) for maintaining blood pressure (BP) during spinal anaesthesia (SA) for Caesarean section (CS). ${ }^{1}$ However, most data are from elective CS. Few data are available for emergency CS where potential for fetal compromise may influence the relative risks and benefits of vasopressors. Therefore, we designed this double-blinded study to compare Phe and Eph in patients having emergency CS.

Materials and Methods: After ethics approval, we obtained consent from 869 patients admitted to the labour ward. Of these, 204 progressed to emergency CS under SA and were randomized to receive a bolus of either Eph $10 \mathrm{mg}$ or Phe $100 \mu \mathrm{g}$ each time systolic BP, measured Q1min, decreased to $<100 \mathrm{mmHg}$. The study terminated at delivery. We compared maternal haemodynamics and neonatal outcome including umbilical arterial (UA) and venous (UV) blood gases and lactate concentration.

Results and Discussions: 148 patients required a vasopressor. For the Phe group $(n=74)$ vs the Eph group $(n=74)$, there was less nausea/vomiting (4 vs $30 \%, P=0.0001$ ) despite similar minimum recorded BP; lower UA lactate (median 2.3 vs $2.7 \mathrm{mmol} / \mathrm{l}, P<0.0001)$ and UV lactate $(2.2 \mathrm{vs} 2.6 \mathrm{mmol} / \mathrm{l}$, $P<0.0001)$ but lower UA PO $2(2.2$ vs $2.5 \mathrm{kPa}, P=0.027)$ and UV $\mathrm{PO}_{2}$ (3.9 vs $4.1 \mathrm{kPa}, P=0.012$ ) although $U A$ and $U V$ oxygen content were similar. There were no differences in UA pH (7.29 vs 7.28), UA base excess $(-2.6$ vs $-3.2 \mathrm{mmol} / \mathrm{l})$ or other biochemical outcomes. Apgar scores were similar. Conclusion(s): The results of this study support the safety and efficacy of Phe in emergency CS.

Reference:

1 Lee A et al. Anesth Analg 2002; 94: 920-6.

\section{AP2-7}

\section{Epidural volume extension with low dose spinal anesthesia} for cesarean section

E. Guasch, E. Alsina, A. Domínguez, J. Díaz, F. Gilsanz

Anesthesia Reanimación, Hospital Universitario Maternal La Paz, Madrid, Spain

Background and Goal of Study: Low dose spinal anesthesia is not widely used for caesarean section (CS). (1) Epidural volume extension (EVE), can contribute to the intrathecal drug spread,(1)(2). Our primary outcome was the incidence of maternal hypotension with three different doses of spinal bupivacaine or levo-bupivacaine and EVE. Motor and sensitive blockade, epidural rescue analgesia (ERA) and neonatal effects were also studied.

Methods: After approval of the local ethics committee, an open prospective study was performed in women scheduled for CS assigned to one of 3 groups depending on the intrathecal hyperbaric local anesthetic dose: B5: Bupivacaine $5 \mathrm{mg} \mathrm{0,25 \%} \mathrm{(} n=51)$, L5: L-bupivacaine $5 \mathrm{mg} \mathrm{0,25 \%} \mathrm{(}=50)$ and L6: L-bupivacaine $6 \mathrm{mg} \mathrm{0,3 \%}(\mathrm{n}=50)$. Each with $25 \mu \mathrm{g}$ Fentanyl mixed with hyperbaric $2 \mathrm{ml}$ intrathecal solution. $10 \mathrm{ml}$ of saline EVE was performed to improve local anesthesia spread within the first 5 minutes after the intrathecal injection. Hypotension was defined as a decrease in systolic blood pressure of $>20 \%$ below baseline. Hypotension incidence, dose of ephedrine, sensory (time to reach T4) and motor blockade (modified Bromage scale), ERA and neonatal outcome, were recorded. Data were analyzed using $\chi^{2}$, and one way ANOVA. Statistical signification was assumed when $p<0,05$.

Results: Demographic data, cause of CS, duration of surgery and anesthesia, incision to delivery interval and Apgar scores were comparable.

\begin{tabular}{llllc}
\hline & $\begin{array}{l}\text { Hypotension } \\
(\mathrm{n} \text { and \%) }\end{array}$ & ERA $(\mathrm{n})$ & $\begin{array}{l}\text { Bromage end } \\
\text { surgery block }=0 \\
(\mathrm{n} \text { and \%) }\end{array}$ & $\begin{array}{l}\text { Time } \\
\text { to T4(min.) }\end{array}$ \\
\hline B5 & $27(52,9 \%)$ & 12 & $19 \bullet \mathrm{p}(37.3 \%)$ & $9.89 \pm 3.635$ \\
L5 & $13^{*}(26 \%)$ & $23^{* *}$ & $29(58 \%)$ & $10.47 \pm 2.896$ \\
L6 & $28(56 \%)$ & 14 & $35(70 \%)$ & $7.83 \dagger \pm 3.226$ \\
\hline
\end{tabular}

${ }^{*} p=0,04\left(\chi^{2}\right),{ }^{* \star} p=0,039\left(\chi^{2}\right), \bullet p=0,004\left(\chi^{2}\right), \uparrow p=0,001$ ANOVA

Hypotension was correlated to lower umbilical cord gases $(p=0,001)$ (ANOVA), without differences among groups. Maternal ephedrine doses over $20 \mathrm{mg}$. were associated to lower umbilical cord gases $(p=0,031)$ ANOVA. 
Conclusions: The incidence of hypotension is less in L5 group although the need of ERA is higher. Hypotension is strongly correlated to lower umbilical cord blood gases. EVE is not adequate for CS with these low doses.

References:

1 Rawal N Best Pract Res Clin Anestesiol 2003; 17: 347-64.

2 Beale N et al. Br J Anaesth 2005; 95: 500-3.

\section{AP2-8}

\section{Low-dose S-ketamine for postoperative pain in cesarean} section with spinal anesthesia

\section{E. Suppa, R. Pinto, B.A. Zanfini, A. Valente, G. Draisci}

Anesthesia and Intensive Care, Catholic University of the Sacred Heart, Rome, Italy

Background and Goal of the study: Numerous studies showed the antihyperalgesic effects of small-dose ketamine on postoperative pain in patients under general anaesthesia. We investigated the analgesic efficacy and safety of low-dose S-ketamine infusion in patients undergoing cesarean section with spinal anesthesia.

Material and Methods: Twenty-eight ASA I-II women, 18-40 years old, with uncomplicated pregnancies at term, scheduled for elective repeated cesarean section, were randomized to receive S-ketamine or placebo. In both groups subarachnoid anesthesia was performed with $0.5 \%$ hyperbaric bupivacaine $(8-10 \mathrm{mg}$ ) plus sufentanyl $5 \mu \mathrm{g}$. Study group received $0.5 \mathrm{mg} / \mathrm{kg}$ i.m. S-ketamine bolus $10 \mathrm{~min}$ after birth, followed by $2 \mu \mathrm{g} / \mathrm{kg} / \mathrm{min}$ continuous i.v. infusion for $12 \mathrm{~h}$. Control group received normal saline in the same way. At the end of surgery a $24 \mathrm{~h}$ PCA i.v. morphine analgesia was started in all patients. Time to first request of analgesia, total morphine consumption, VAS, Ramsay Sedation Scores (RSS) and side effects were collected at 1, 4, 8, 12, $24 \mathrm{~h}$. RM- and M-ANOVA and Student's T-tests were used for statistical analysis.

Results and Discussion: Patients characteristics (mean \pm SD) were: age $33.9 \mathrm{yrs} \pm 4.3$, weight $74.5 \mathrm{~kg} \pm 10.2$, height $157 \mathrm{~cm} \pm 4.6$.

Mean time to first morphine request was $382 \mathrm{~min} \pm 275$ for the S-ketamine group, versus $220 \mathrm{~min} \pm 102$ for the control group $(P=0.022)$. Cumulative morphine consumption in S-ketamine and control group was respectively $9.86 \mathrm{mg} \pm 6.18$ and $15 \mathrm{mg} \pm 5.43$ at $12 \mathrm{~h}(\mathrm{P}=0.014)$ and $21.71 \mathrm{mg} \pm 10.2$ and $32.72 \mathrm{mg} \pm 6.53$ at $24 \mathrm{~h}(\mathrm{P}=0.026)$. Side effects observed in S-ketamine group only were: dizziness and drowsiness in $100 \%$, diplopia in 43\% $(P=0.004)$ and nystagmus in $36 \%(P=0.012)$ of patients. All side effects were mild and transient. There were no significant differences in VAS and RSS.

Conclusions: Low-dose S-ketamine, administered by i.m. bolus and continuous i.v. infusion, reduced morphine consumption and prolonged postoperative analgesia after cesarean section with spinal anesthesia. Only minor side effects were detected. Low-dose S-ketamine has shown to be beneficial in awake surgical patients with neuraxial block.

\section{AP2-9}

Low-dose combined spinal-epidural anaesthesia vs. conventional epidural anaesthesia for caesarean section in preeclampsia patients

M. Lerintiu, O. Karoly, A. Ilie, F. Stamatian

Department of Anesthesia and Intensive Care, University Emergency Hospital, Cluj-Napoca, Romania

Background and Goal of Study: Epidural anesthesia is the anesthetic technique of choice for cesarean section in pregnant women with preeclampsia. Low-dose combined spinal-epidural anaesthesia ensures spinal block with minimum hemodynamic impact. The question is whether or not this is an alternative to conventional epidural anesthesia for caesarean section in preeclampsia patients.

Materials and Methods: This prospective, randomized study included 30 pregnant women with preeclampsia who underwent cesarean section between 2005 and 2006. The patients were divided into two groups. The CSE group $(n=15)$ received low-dose combined spinal-epidural anesthesia with $7.5 \mathrm{mg}$ bupivacaine $0.5 \%$ and $25 \mu \mathrm{g}$ fentanyl. Epidural volume extension was performed in case of insufficient spinal block. The EA group $(n=15)$ underwent conventional epidural anesthesia with $20 \mathrm{ml}$ lidocaine $2 \%, 1 / 200000$ epinephrine and $100 \mu \mathrm{g}$ fentanyl. All patients received $3 \mathrm{mg}$ of epidural morphine for postoperative anesthesia. Low blood pressure was corrected with ephedrine according to the same protocol. The data were analyzed using the 3.3.2 Epiinfo program. The statistical significance level was $p<0.05$ Results and Discussions: The demographic data were similar in the two groups.

\begin{tabular}{llll}
\hline & CSE gr. & EA gr. & $\mathrm{P}$ \\
\hline $\begin{array}{l}\text { Successful anesthesia } \\
\text { induction at } 10 \text { minutes }\end{array}$ & $100 \%$ & $100 \%$ & \\
$\begin{array}{l}\text { Initial SAP (mean- } \mathrm{mmHq} \text { ) } \\
\text { Lowest SAP(mean- mmHq) }\end{array}$ & 153.33 & 158.66 & $\mathrm{P}=0.41$ \\
$\begin{array}{l}\text { SAP at } 10 \text { minutes } \\
\text { (mean-mmHq) }\end{array}$ & 121.66 & 128.33 & $\mathrm{P}=0.39$ \\
$\begin{array}{l}\text { Ephedrine requirement (mg) } \\
\text { Apgar score at 5 minutes }\end{array}$ & 135.33 & 132 & $\mathrm{P}=0.55$ \\
$>8$ (no.) & 16.66 & 18.66 & $\mathrm{P}=0.82$ \\
\hline
\end{tabular}

SAP- systolic arterial pressure

Conclusion(s): Low-dose combined spinal-epidural anesthesia administered in preeclampsia patients ensures a spinal block that is similar in terms of quality and hemodynamic involvement with that provided by conventional epidural anesthesia. The use of anesthetics is also decreased.

\section{AP2-10}

Postoperative analgesic effects of epidural neostigmine after non-elective cesarean section

E. Hardy, Fabienne. Roelants, Patricia. Lavandhomme

Anesthesiology, Cliniques Universitaires St Luc, Brussels, Belgium

Background: Epidural neostigmine $(\mathrm{N})$, a cholinesterase inhibitor, produces antinociceptive effects. At dose of $4 \mu \mathrm{g} / \mathrm{kg}$, epidural $\mathrm{N}$ provides modeste analgesia after elective cesarean section (CS) (1). Because pain may activate spinal noradrenergic and hence cholinergic pathways (2), the present study evaluates the postoperative effects of epidural $\mathrm{N}$ administered in laboring women undergoing semi-urgent CS under epidural anesthesia.

Materials and Methods: After informed consent, healthy parturients in labor with indwelling epidural catheter (ropivacaine/sufentanil analgesia) who needed semi-urgent CS (ropivacaine $0.75 \%$ used for epidural anesthesia) were randomly assigned to receive after cord clamping either saline $5 \mathrm{~mL}$ $(n=13)$ or $N 500 \mu \mathrm{g}$ in $5 \mathrm{~mL}$ saline $(n=12)$. Postoperative pain scores at rest and movement (VAS:0-10), time to first analgesic request and PCA morphine needs were recorded at 4,6,12,24 and $48 \mathrm{~h}$ after injection. Hemodynamic parameters and side effects (sedation, nausea) were also noticed. Statistical analysis used one way ANOVA, $\mathrm{P}<0.05$ was significant.

Results: Demographic data did not differ (age, pregnancy term, history of previous CS). Labor duration (400 $\pm 200 \mathrm{~min})$, cervical dilatation $(5 \pm 1.7 \mathrm{~cm})$ and epidural doses of ropivacaine $(80 \pm 45 \mathrm{mg})$ and sufentanil $(20 \mu \mathrm{g})$ received at the time of CS were similar in both groups. Evaluation of labor analgesia demonstrated similar average VAS in saline $(1 \pm 0.7)$ and $\mathrm{N}$ group $(2 \pm 1.5)$. VAS at rest at $4 \mathrm{~h}(1 \pm 0.6$ vs $5 \pm 1)$ and at $6 \mathrm{~h}$ with movement $(3 \pm 2$ vs $6 \pm 1)$ were significantly decreased in $\mathrm{N}$ group. Respectively in saline and $\mathrm{N}$ group, time for first analgesic request was $174 \pm 103 \mathrm{~min}$ and $157 \pm 123 \mathrm{~min}$, and PCA morphine use was similar with $48 \mathrm{~h}$ total dose of $32 \pm 17 \mathrm{mg}$ and $38 \pm 19 \mathrm{mg}$. No particular side effects occurred related to $\mathrm{N}$ use.

Discussion and Conclusion: As previously reported with elective CS (1), epidural $\mathrm{N}$ only provides short-lasting postoperative analgesia in laboring women undergoing CS. The results are in agreement with the lack of evidence for spinal cholinergic system activation during labor (3).

References:

1 Kaya et al. Anesthesiology 2004; 100: 381-5

2 Eisenach et al. Pain 1990; 43: 149-54

3 Eisenach et al. Anesth Analg 1996; 82: 621-6

\section{AP2-11}

Spinal anesthesia for cesarean delivery: effects of low dose isobaric bupivacaine on maternal hemodynamics

Z. Triki, S. Kallel, F. Amouri, H. Chikrouhou, A. Karoui

Anesthesiologie, CHU hABIB Bourguiba, Sfax, Tunisia

Background: Hypotension remains an important side effect of spinal anesthesia for caesarean delivery. There is. We designed the present randomized trial to test the hypothesis that reducing the spinal dose of local anesthetics results in equally effective anesthesia, less maternal hypotension and reduced incidence of nausea and vomiting (1)

Material and Methods: After ethical committee approval and written informed consent, 60 term pregnant patients were randomly assigned to two study groups. In the HIGH group spinal anesthesia was performed using $10 \mathrm{mg}$ isobaric bupivacaine combined with $10 \mu \mathrm{g}$ fentanyl and $100 \mu \mathrm{g}$ morphine. In the LOW-group spinal anesthesia was performed using $8 \mathrm{mg}$ isobaric bupivacaine combined with same doses of morphiniques. Spinal anesthesia was achieved in the L3/L4 interspace. In both groups, $6 \mathrm{mg}$ of ephedrine in bolus are administrated if systolic arterial blood pressure decreased more than $90 \mathrm{mmHg}$ or $25 \%$ than the reference value. 
Demographic data, obstetrical data, maternal hemodynamics, incidence of nausea and vomiting and neonatal outcome were recorded. Comparisons between groups were evaluated with the chi-square test and Student $t$ test, $\mathrm{p}<0,05$ was considered significant.

Results: Anaesthetic and hemodynamic data in the table:

\begin{tabular}{lll}
\hline & $\begin{array}{l}\text { HIGH group } \\
(\mathrm{n}=30)\end{array}$ & $\begin{array}{l}\text { LOW-group } \\
(\mathrm{n}=30)\end{array}$ \\
\hline Incidence hypotension (\%) & 93,3 & $60^{\star}$ \\
Duration hypotension (min) & $6,36 \pm 4,14$ & $2,03 \pm 2,34^{\star}$ \\
Ephedrine (mg) & $40,03 \pm 25,9$ & $11,4 \pm 12,83^{\star}$ \\
Nausea (\%) & 66,7 & $16,7^{\star}$ \\
Vomiting (\%) & 23,3 & $3,3^{\star}$ \\
Block $>$ T4 (\%) & 83,3 & $53,4^{\star}$ \\
Adequate anaesthesia ( $\mathrm{n})$ & 30 & 30 \\
Apgar $<7(\mathrm{n})$ & 0 & 0 \\
\hline
\end{tabular}

Data are presented as a mean $\pm S D$

Block > T4: block higher than dermatoma level T4

${ }^{*} \mathrm{p}<0,05$ versus High-group
Conclusion: small-dose spinal anesthesia ( $8 \mathrm{mg}$ isobaric bupivacaine + fentanyl) better preserves maternal hemodynamic stability with equally effective anesthesia and reduce the incidence of nausea and vomiting but does not improve neonatal outcome.

Reference:

1 Ben-David B. Reg Anesth Pain Med 2000; 25: 235-9.

\section{Intensive Care Medicine}

\section{AP1-1}

The effect of time on the estimation of lung recruitability

D. Chiumello, F. Tallarini, M. Chierichetti, F. Polli, S. Azzari

Institute of anesthesia and critical care, Policlinico Hospital, milano, Italy

Background and Goal of Study: The Acute Respiratory Distress Syndrome (ARDS) is a clinical syndrome characterized by inflammatory pulmonary edema, severe hypoxemia, small lungs and a diffuse endothelial - epithelial lung injury. Although PEEP may improve oxygenation and reduce barotrauma may also cause lung injury. Thus the effect of PEEP depends on the lung recruitability. We recently found that the lung recruitability can be estimated with a certain degree of accuracy based on physiological respiratory variables (1).

Aim of this study was to evaluate the effect of time on the prediction of lung recruitability.

Materials and Methods: Intubated, sedated and paralysed ALI/ARDS patients were studied during controlled mechanical ventilation at constant minute ventilation at two level of PEEP $\left(5\right.$ and $\left.15 \mathrm{cmH}_{2} \mathrm{O}\right)$. Blood gas analysis and dead space measurements were performed at 5, 15, 30, 60 minutes. Patients were defined as responder (i.e., a patient who presented a high lung recruitability) when the $\mathrm{PaO}_{2} / \mathrm{FiO}_{2}$ ratio was less than $150 \mathrm{mmHg}$ at PEEP of $5 \mathrm{cmH}_{2} \mathrm{O}$, the alveolar dead space decreased and respiratory compliance increased when PEEP was changed from 5 to $15 \mathrm{cmH}_{2} \mathrm{O}$.

Results and Discussions: Fourteen patients (10 males) with a mean weight of $68 \pm 10 \mathrm{Kg}_{\mathrm{IBW}}$, age of $60 \pm 12 \mathrm{yrs}, \mathrm{PaO}_{2}$ of $89.1 \pm 20.2 \mathrm{mmHg}, \mathrm{PaCO}_{2}$ of $41.6 \pm 7.3 \mathrm{mmHg}$ with a PEEP level of $10 \mathrm{cmH}_{2} \mathrm{O}$ were enrolled. The distribution of responders and non responders was the same at 5,15 and 30 minutes. Conclusion(s): These data show that the computation of these respiratory variables allows the clinician, to estimate the lung recruitability, just after only five minutes.

Reference:

1 Gattinoni L et al. N Engl J Med 2005.

\section{AP1-2}

The accuracy of dead space measurements in critically ill patients

D. Chiumello, F. Pagan, M. Chierichetti, F. Tallarini, M. Cressoni

Institute of Anesthesia and Critical Care, Policlinico Hospital, Milano, Italy

Background and Goal of Study: The dead space is defined as the fraction of tidal volume that does not participate to the gas exchange (i.e., the anatomical and alveolar dead space). Being a fundamental index in patients with acute lung injury it is essential to have a reasonable measurement at the bedside.

Aim of this study was to evaluate the accuracy of the dead space measurements in critically ill patients.

Materials and Methods: Intubated, sedated patients with acute lung injury were enrolled. A blood gas analysis was taken at the same time to the measurement of expired $\mathrm{CO} 2$ by a CO2SMO monitor (CO2SMO Monitor Novametrix Medical Instrumentation USA).

Results and Discussions: 60 measurements were obtained from 20 patients (mean age $61 \pm 15 \mathrm{yrs}$, BMI $27.5 \pm 7.3 \mathrm{Kg} / \mathrm{m}^{2}$, PEEP $9 \pm 3 \mathrm{cmH}_{2} \mathrm{O}$ and tidal volume of $608 \pm 146 \mathrm{ml}$ ) during mechanical ventilation. The variability was computed as the coefficient of variability $\left(C_{v}\right.$, i.e., standard deviation divided per mean).

Regarding the physiological dead space the $C_{V}$ was 0,02 , for the alveolar dead space was 0,14 and for the anatomic dead space was 0,02 .
Conclusion(s): Using the same functional respiratory monitor we did not observe any significant variability in the measures of dead space.

\section{AP1-3}

\section{Ventilatory management of patients with Pulmonary} Contusion: influence position on gas exchange

I. Kuchyn, F. Glumcher, D. Dmytriev

Anesthesiology and Intensive Care, National Medical University, Kyiv, Ukraine Background and Goals: Pulmonary Contusion (PC) is the main cause of respiratory failure after blunt thoracic trauma (1). Prone position (PP) was proposed to improve the arterial oxygenation in patients with various forms of acute respiratory failure but there are very little data of use this method in patient with Pulmonary Contusion. The purpose of our research was study changes in arterial blood gas during the mechanical ventilation in PP. Material and Methods: We studied 24 patients with sever PC (Chest X-ray and CT-scan local infiltration, $\mathrm{AIS}_{\text {thorax }} \geqslant 3$, admission $\mathrm{PaO}_{2} / \mathrm{FiO}_{2} \leqslant 100 \mathrm{mmHg}$ ). Repeated arterial gas blood tests were made in supine position and after 30 minutes ventilation in PP on 1, 3, 5 and 7 day of observation. Changes in intrapulmonary shunting $\left(\mathrm{Q}_{\mathrm{s}} / \mathrm{Q}_{\mathrm{t}}\right)$ and $\mathrm{PaO}_{2} / \mathrm{FiO}_{2}$ were analyzed. Statistical analysis of difference of the data arterial blood gas (before and after the change of position) was performed by Wilcoxon Matched Pairs Test, $p$-value less than 0.05 was considered to indicate statistical significance. Results: Data (Median [25-75 percentages]) are shown in the table:

\begin{tabular}{lllll}
\hline Day \# & \multicolumn{2}{c}{$\mathrm{PaO}_{2} / \mathrm{FiO}_{2}, \mathrm{mmHg}$} & \multicolumn{2}{c}{$\mathrm{Qs} / \mathrm{Qt}, \%$} \\
\cline { 2 - 5 } & Supine & Prone & Supine & Prone \\
\hline $\mathbf{1}$ & 105.7 & 150.4 & 21.5 & 15.7 \\
& {$[83.9-125.8]$} & {$[129.7-171.3]$} & {$[17.2-24.2]$} & {$[14.2-17.3]$} \\
& & $\mathrm{p}<0.001$ & & $\mathrm{p}<0.001$ \\
$\mathbf{3}$ & 140.7 & 235.7 & 11.1 & 18.0 \\
& {$[136.8-147.8]$} & {$[224.3-252.7]$} & {$[9.4-14.0]$} & {$[14.4-19.4]$} \\
& & $\mathrm{p}<0.001$ & & $\mathrm{p}<0.001$ \\
$\mathbf{5}$ & 157.5 & 325.1 & $8.3[7.7-9.6]$ & 13.6 \\
& {$[154.5-196.4]$} & {$[253.7-344.3]$} & & {$[12.2-14.9]$} \\
& & $\mathrm{p}<0.001$ & & $\mathrm{p}<0.001$ \\
$\mathbf{7}$ & 211.0 & 330.7 & $6.9[6.2-8.3]$ & 10.8 \\
& {$[207.6-214.2]$} & {$[280.9-344.3]$} & & {$[9.6-12.2]$} \\
& & $\mathrm{p}=0.002$ & $\mathrm{p}=0.046$ \\
\hline
\end{tabular}

We observed a significant improvement of arterial oxygenation and decreased the value of intrapulmonary shunt during all days of using prone position. Influence ventilation in sit-in position on gas exchange was like the effect of the prone position.

Conclusions: PP improves oxygenation with decrease of intrapulmonary shunt and increase $\mathrm{PaO}_{2} / \mathrm{FiO}_{2}$ ratio in patients with $\mathrm{PC}$.

References:

1 Pinella J.J Trauma 1982; 22: 221-226.

2 Herman C., et al. Anaesthesist 1994; 43: 454-462.

\section{AP1-4}

Prevention of nosocomial pneumonia in patients with Pulmonary Contusion: role of early fiberoptic bronchoscopy I. Kuchyn, F. Glumcher, D. Dmitriiev

Anesthesiology and Intensive Care, National Medical University, Kyiv, Ukraine 
Background and Goals: Impairment of patency of airways by blood and sputum in patients with Pulmonary Contusion (PC) reduces regional lung ventilation with increase of lung dyslectasis and intrapulmonary shunt. It creates conditions for infection. The most effective strategy of maintenance of patency of airways and prevention of atelectasis is the Fiberoptic bronchoscopy $(\mathrm{FOBr})(1)$. The purpose of our research was estimation the role of early FOBr in prevention of nosocomial pneumonia (NP) in patient with PC. Material and Methods: 158 patients with PC were observed. We used early $\mathrm{FOBr}$ (in first 3 days) in 82 patients. In 32 patients $\mathrm{FOBr}$ was done in deferred term (more than 3 days). The analysis of incidence of NP was done. Statistical analysis included Chi-square test, Kaplan\&Meier method and Cox's F-Test, $\mathrm{p}$-value less than 0.05 was considered to indicate statistical significance. Results: Total rate of NP was $14.6 \%$ (23 pts): $9.6 \%$ (8pts) in early FOBr group and $46.9 \%$ (15 pts) in deferred FOBr group ( $p<0.001$ by Chi-square test). In order to exclude the influence of differences in terms of hospitalization we used Kaplan\&Meier method and Cox's F-Test to estimate the differences in NP incidence. Results of Kaplan\&Meier method are shown in the picture.

Cumulative proportion surviving (Kaplan-Meier) - Complete a Censored

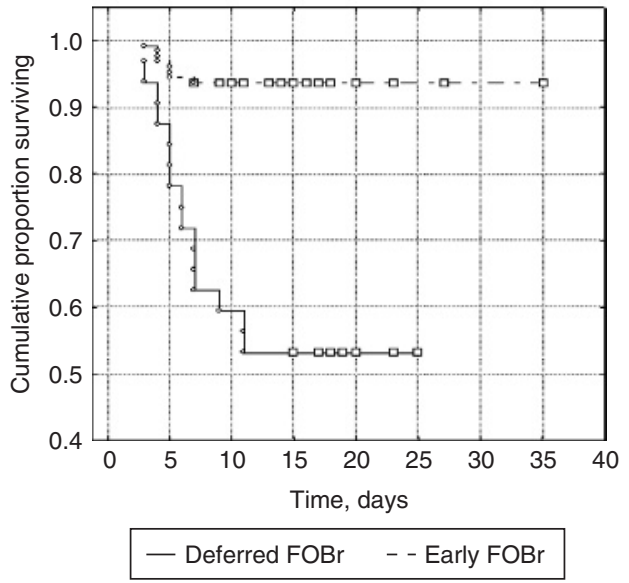

The result of Cox's F-Test confirmed differences in incidence rate of NP in patients with $\mathrm{PC}: \mathrm{F}=8.98, \mathrm{p}=0.00002$.

Conclusion(s): Deferred in comparison with Early Fiberoptic bronchoscopy may be the risk factor of NP in patients with PC.

Reference:

1 Clinical practice guideline Respir Care1993; 38: 1173-1178.

\section{AP1-5}

Propofol attenuates pulmonary edema induced by collapse and reventilation of lung in rabbits

S. Kwak, H. Bae, S. Kim, S. Chung

Anesthesiology and Pain Medicine, Chonnam National University Medical School, Gwangju, Republic of Korea

Background and Goal of Study: To study the effects of propofol on the pulmonary edema caused by collapse-reventilation injuries of the lungs in rabbits.

Materials and Methods: There were five groups of animals: (1) the Sham group - normal ventilation $(n=14)$; (2) the collapse group - the right lung was collapsed $(n=14) ;(3)$ the collapse-reventilation group - the right lung was collapsed for 3 hours then reventilated for 3 hours $(n=14)$; (4) the propofol group - collapse-reventilation for 3 hours with administration of propofol in 5 or $20 \mathrm{mg} / \mathrm{kg} / \mathrm{hr}$ dosing schedules $(n=28)$. Mean arterial pressure, heart rate, arterial blood oxygen tension, leukocyte and platelet counts in the peripheral blood were recorded hourly for 6 hours starting at the 0 hour of experimentation.

The wet to dry (W/D) weight ratio of the lung and lung injury score were calculated to assess tissue edema. In addition, the bronchoalveolar lavage fluid (BALF) from both lungs was analyzed for leukocytes, albumin, malondialdehyde (MDA) and interleukin-8 (IL-8) 6 hour after the start of experiment in both lungs.

Results and Discussions: There was a global elevation of all parameters in the collapse-reventilation group, namely the W/D weight ratio of the lungs, the lung injury scores, the counts and percentages of leukocytes, and concentrations of albumin, MDA, and IL-8 in BALF. These changes were greater in the fluids from the collapse-reventilated right lung than those from the left lung. Significantly, there was a global improvement in these parameters with the administration of propofol. The only exception to this response was the leukocyte counts which remained elevated.

Conclusion(s): The findings of our study indicated that collapse-reventilation of the lungs leads to pulmonary edema in rabbits. In addition, we showed that propofol has a protective effect on the pulmonary edema caused by collapse-reventilation injuries in rabbits.

\section{AP1-6}

Chaotic variation of tidal volumes adds further benefit to the open lung approach in experimental acute lung injury

P.M. Spieth, C. Meissner, M. Kasper, T. Koch, M. Gama de Abreu Clinic for Anaesthesiology and Intensive Care Therapy, University Hospital Dresden, Dresden, Germany

Background and Goal of Study: Biological systems seems to benefit from chaotic variability. We tested if chaotic variation of tidal volumes (noisy ventilation) adds further benefit to a lung protective ventilatory strategy aimed at keeping the lungs open after a recruitment maneuver (open lung approach) in experimental acute lung injury.

Materials and Methods: Acute lung injury was induced in 12 pigs by repetitive saline lung lavage. Following injury, animals were randomly assigned to a standard lung protective therapy with $(n=6)$ or without $(n=6)$ administration of chaotic variable tidal volumes (mean $V_{T}=6 \mathrm{ml} / \mathrm{kg}$ ). Gas exchange, lung mechanics and hemodynamics were assessed. After $6 \mathrm{~h}$, animals were killed and lung damage was evaluated histologically. Gas exchange and lung mechanics were tested with univariate analysis of variance, histological results were tested by means of the Mann-Whitney-U-Test.

Results and Discussions: Groups were comparable at baseline and after injury. Variables of gas exchange $\left(\mathrm{PaO}_{2}, \mathrm{PaCO}_{2}, \mathrm{SvO}_{2}\right)$, hemodynamics as well as peak and mean airway pressures did not differ significantly between groups. Mean lung elastance and alveolar stress index (\%E2) were significantly reduced in the noisy ventilated animals $(p<0.05)$. Histological analysis of diffuse alveolar damage showed a trend toward better lung protection with noisy ventilation ( $p=0.08)$, and less inflammatory infiltration was observed in noisy ventilated lungs $(p<0.05)$.

Conclusion(s): Chaotic variation of tidal volume adds further benefit to protective ventilation according to the open lung approach.

\section{AP1-7}

Levosimendan pretreatment improves systemic hemodynamics and metabolism during acute hypoxia in dogs

L.A. Schwarte, I. Schwartges, T.W.L. Scheeren, A. Fournell, O. Picker Anesthesiology, University Hospital Duesseldorf, Duesseldorf, Germany

Background \& Goal of Study: Levosimendan (LEVO) improves regional tissue oxygenation under physiological, normoxic conditions, as we demonstrated recently [1]. It is unclear, however, if LEVO exerts beneficial effects also under pathological, hypoxic conditions, particularly in respect to systemic hemodynamics and metabolism. Thus we studied the effects of LEVO pretreatment during acute hypoxia on systemic hemodynamics and metabolism.

Materials \& Methods: Chronically instrumented dogs (bodyweight $\sim 30 \mathrm{~kg}$, $\mathrm{n}=10$ experiments) were anesthetized (sevoflurane, 1.5 MAC) and mechanically ventilated $\left(\mathrm{FiO}_{2}=0.3\right.$; etCO $\left.=35 \mathrm{mmHg}\right)$. The animals were randomized to undergo hypoxia $\left(\mathrm{FiO}_{2}=0.1\right.$ for $15 \mathrm{~min}$ ) with or without LEVO pretreatment [1]. To assess systemic hemodynamics we measured cardiac output (CO), stroke volume (SV) and the inotropy marker dP/dTmax. To assess systemic metabolism, we measured $\mathrm{O}_{2}$-consumption $\left(\mathrm{VO}_{2}\right.$, Deltatracll) and arterial lactate levels. Data are mean \pm sem.

Results \& Discussions: Hypoxia $\left(\mathrm{FiO}_{2}=0.1\right)$ reduced arterial $\mathrm{PO}_{2}$ from $\sim 130 \mathrm{mmHg}$ to $\sim 30 \mathrm{mmHg}$ in both groups, i.e., hypoxia with and without LEVO pretreatment. LEVO pretreatment significantly improved systemic hemodynamics during hypoxia, i.e., CO (104 $\pm 4 \mathrm{vs} 83 \pm 5 \mathrm{ml} / \mathrm{kg} / \mathrm{min}), \mathrm{SV}(25.2 \pm 1.7$ vs $19.8 \pm 1.2 \mathrm{ml}$ ) and $\mathrm{dP} / \mathrm{dTmax}(553 \pm 41$ vs $377 \pm 36 \mathrm{mmHg} / \mathrm{sec})$. These hemodynamic improvements were not fueled by increased $\mathrm{O}_{2}$-consumption, i.e., $\mathrm{VO}_{2}$ did not differ between groups ( $3.1 \pm 0.2 \mathrm{vs} 3.0 \pm 0.2 \mathrm{ml} / \mathrm{kg} / \mathrm{min}$ ). Interestingly, LEVO pretreatment prevented an increase in arterial lactate during hypoxia ( $1.8 \pm 0.1$ to $1.9 \pm 0.1 \mathrm{mmol} / \mathrm{L})$, whereas lactate significantly increased in the group without LEVO $(1.7 \pm 0.1$ to $2.1 \pm 0.1 \mathrm{mmol} / \mathrm{L})$.

Conclusion(s): LEVO pretreatment markedly improved systemic hemodynamics during acute hypoxia, without increasing oxygen consumption. Furthermore, LEVO pre-treatment prevented the increase in arterial lactate levels during acute hypoxia, indicating an optimized $\mathrm{O}_{2}$-distribution and/or utilization by LEVO. Thus, if our data apply to the clinical setting, LEVO pretreatment is a promising option to improve systemic hemodynamics without 
increasing $\mathrm{VO}_{2}$ and furthermore to optimize lactate balance in patients at risk of hypoxia.

Reference:

1 Schwarte LA et al. Crit Care Med. 2005; 33: 135-142.

\section{AP1-9}

Comparative effect of acute hypoxia on contractile and energetic properties of diabetic diaphragm

P. Michelet, N. Salvy, B. Riou, G. Orliaguet, O. Langeron

Département danesthésie Réanimation, Hôpital de la Pitié Salpetrière, Paris, France

Background and Goal of Study: Hypoxia adversely affects respiratory muscle function. In the rat diaphragm, diabetes results in an increase in low (Type I) myosin heavy chain characterized by an aerobic metabolism. Therefore, a different effect of acute hypoxia between diabetic and control rat diaphragm could be hypothesized.

Materials and Methods: In vitro contractile and energetic properties were measured with ventral diaphragm strips obtained from 2 months streptozotocin-induced diabetic ( $4 \mathrm{mo}$ old, $\mathrm{n}=12$ ) and control ( $4 \mathrm{mo}$ old, $\mathrm{n}=12)$ male Wistar rats submitted to acute hypoxia $\left(\mathrm{Po}_{2} \sim 6.5 \mathrm{kPa}\right)$. Data are mean $\pm \mathrm{SD}$ and comparison between groups was performed using ANOVA. Results and Discussions: Compared to control animals, diabetic rats were characterized by an increase in active force $\left(100 \pm 6\right.$ vs $\left.79 \pm 10 \mathrm{mN} \cdot \mathrm{mm}^{-2}\right)$ associated with an increase in the total number of cross-bridges per sectional area $\left(11.1 \pm 1.2\right.$ vs $\left.8.5 \pm 1.310^{9} \cdot \mathrm{mm}^{-2}\right)$. Moreover a decrease in maximal unloaded shortening velocity was observed between diabetic and control rats $\left(6.3 \pm 0.9\right.$ vs $\left.7.9 \pm 1.0 \mathrm{Lmax} \cdot \mathrm{s}^{-1}\right)$. Under hypoxia, diabetic rats were characterized by a slower decrease in active force and a faster recovery in re-oxygenation conditions (Figure).

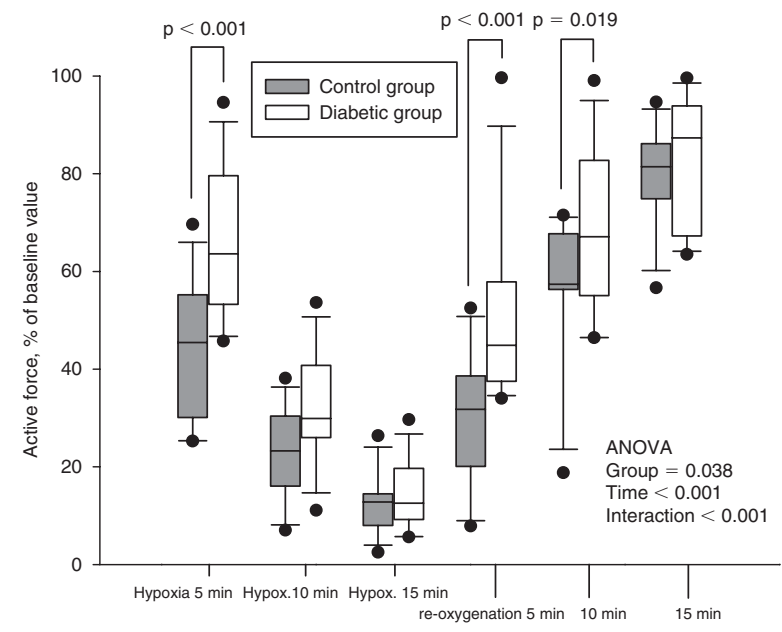

Conclusion(s): These results suggest that diabetic induced modifications in diaphragm contractility are associated with a better resistance to acute hypoxia.

\section{AP2-1}

\section{High dependency care unit after major cancer surgery:} medical and economic impact

\section{F. Lefebvre, JL. Bourgain, G. De Pouvourville}

Service dAnesthesie, Institut Gustave-Roussy, Villejuif, France

Background and Goal of Study: The intensity of care is part of a better postsurgery rehabilitation $(1,2)$. The aim of this study was to evaluate the impact of the introduction of a high dependency care unit (HDU) for postoperative care on patient outcome and cost.

Materials and Methods: 1506 patients who have undergone major cancer surgery were studied. 753 were hospitalised in the HDU during 2004 and 2005 (After Group). They were compared to a control group (753 patients hospitalised during 2002 and 2003 before the opening of HDU: Before Group) matched on sex, age, type of surgery (LAS = Lower Abdominal Surgery, UAS = Upper Abdominal Surgery, GS = Gynaecological Surgery). Length of hospital stay was defined as number of days between entrance and discharge from the hospital. Data from the analytic accounts of our hospital for the year 2004 were used to estimate the cost.
Results and Discussions: are shown in the table (data are expressed as mean $\pm S D$ ).

\begin{tabular}{llll}
\hline & Before group & Aftergroup & \\
\cline { 2 - 4 } & $\mathrm{n}=753$ & $\mathrm{n}=753$ & $p$ value \\
\hline Length of hospital stay, days & $15.8 \pm 12.2$ & $14.2 \pm 9.8$ & $p<0.0001$ \\
Patients admitted in ICU & $96(12.7 \%)$ & $41(5.4 \%)$ & $p<0.000001$ \\
Outcome at discharge & & & NS \\
Home & $724(96.1 \%)$ & $735(97.6 \%)$ & \\
Another hospital & $17(2.3 \%)$ & $11(1.5 \%)$ & \\
Died & $12(1.6 \%)$ & $7(0.9 \%)$ & \\
\hline
\end{tabular}

For the patients admitted in HDU, total cost of the hospital stay was reduced by $15.68 \%$.

Conclusion(s): The creation of a HDU was effective to improve the cost of care by decreasing the length of stay and the rate of ICU hospitalisation, without change in patient outcome.

References:

1 Kehlet H. et al. Br J Surg 2002; 89(4): 446-53.

2 Collins KS. et al. Lancet 2002; 359(9314): 1276-82.

\section{AP2-3}

\section{How frequent and important are iv medication errors} in an ICU?

L. Vimláti, Zita. Czikajló

Anaesthesia and Intensive Care, University of Szeged, Medical Faculty, Szeged, Hungary

Background and Goal of Study: Medication safety in ICU practice is of utmost importance (1), but hard data are missing on the prevalence of errors during the infusion process (2). Also there are no proven solutions on guarding and preventing this type of serious errors.

Aim of study: to present data on frequency and nature of iv drug administration errors during daily ICU practice, and study the potential benefit of a guarding system.

Materials and Methods: A 6-bedded ICU unit was equipped with a new infusion pump set which records automatically all infusion administrations. Via its pre-adjustable drug library it also controls infusion pump settings: it warns by overriding the "soft limits", and intercepts drug administration automatically if "hard limits" are intended to exceed.

All infusion administrations of a study period from 31st Jan to 13th of Sep 2006 were recorded. Each automatic data recordings were downloaded and the events which occurred were analysed in detail according to type, frequency, timing, etc.

Results and Discussions: Total № of infusions during study period: 6957. There were 688 alerts generated over this period $(9,88 \%$ !), including 64 hard limit alerts $(\mathbf{0 , 9 1} \%)$ : nearly $10 \%$ of all events were for infusions attempted above hard max. limits, insulin and cordarone had $>68 \%$ of them. Analysis of events by day time, by week days and by months of study timeframe revealed typical risky periods, demanding modifications of working conditions. On the other hand our system prevented successfully all of 64 severe medication errors, including e.g.: 7 overdosing of cordarone 10-50 times the max. dose; 3 of vancomycin incl. one of 10 times the max. dose; 1 of morphine 5 times the max. dose; and 5 of dobutamine, one of 5 times the max. dose.

Conclusion: According to our data prevalence of IV medication errors is substantially high at an ICU. As without an automated documentation system far most of errors remain undiscovered and therefore unavoidable, our data probably support the use of intelligent monitoring and warning devices as mandatory for prevention of potential harm of patients(3).

References:

1 Tissot E: Intens. Care Med 1999; 2S: 353-359.

2 Bates DW et al. JAMA 1995; 274: 29-34.

3 Berwick and Leape, BMJ 1999; 319: 136-7.

\section{AP2-4}

\section{Weaning from mechanical ventilation: protocol vs physician} decision

A. Ogica, G. Droc, D. Tomescu, H. Popescu, D. Tulbure

Anesthesiology and Intensive Care, Fundeni Clinical Institute, Bucharest, Romania

Background and Goal of Study: Due to the increased risk of complications associated with prolonged mechanical ventilation (MV), early liberation from the ventilator is important and protocol directed weaning seems to be the answer (1). We decided to evaluate a ventilator weaning protocol in our intensive care unit (ICU) in terms of length of MV, ICU stay and reintubation rate. Materials and Methods: After Ethical Committee approval, 103 patients starting MV (EVITA IV Dräger) were included over a 6 months period, being 
randomly distributed in two groups: group A - undergoing classical ventilator disconnection and group B - assigned to the weaning protocol. Patients already on ventilatory support as well as pediatric subpopulation ( $<18$ years) were excluded. Severity of illness was assessed using the APACHE II and SOFA scores. Demographic data, duration of MV and superficial breathing index were analyzed. Secondary analysis focused on reintubation rate and possible correlation with mortality. Means and standard deviations were used. Comparison between groups was made with the t-test $(p<0.05)$.

Results and Discussions: The 103 patients represented $78 \%$ of the total number of hospitalized patients in the ICU during the study period. Group A had 52 patients, 37 survivors and group B had 51 patients, 33 survivors. By applying the weaning algorithm, the average duration of the MV decreased with 1.24 days.

\begin{tabular}{lll}
\hline & Group A & Group B \\
\hline Mean days of MV & 6.702 & 5.468 \\
Standard error & 1.130 & 0.8460 \\
Standard deviation & 6.891 & 4.785 \\
\hline
\end{tabular}

The patients having survived were ventilated $5.83 \pm 5.15$ days, while the length of ventilation in non-survivors was $12.79 \pm 13.71$ days $(p=0.002)$. Regarding reintubation analysis, group A had a $24.32 \%$ ventilator reconnection, compared to $15.15 \%$ in the group B. No influence on mortality rate was noticed.

Conclusion(s): Using a weaning protocol led to a decrease in the number of $\mathrm{MV}$ days and to a lower reintubation rate.

Reference:

1 Ely WE. Weaning from mechanical ventilation (part 1): evidence supports the use of protocols. In: J.L.Vincent, Yearbook of Intensive Care and Emergency Medicine Springer; 2001: 481-95.

\section{AP2-5}

\section{Cost reduction in paediatric parenteral nutrition}

T. Gamsjager, E. Schaden, C. Sitzwohl, C. Weinstabl

Anaesthesiology and Intensive Care, Medical University of Vienna, Vienna, Austria

Background and Goal of Study: In paediatric intensive care, two approaches to parenteral nutrition are available: individualized admixtures or commercial standard solutions. Even though individualized admixtures can be tailored to sometimes highly intricated requirements, standard solutions are able to meet the demands (1) of the majority of paediatric patients. To address the growing importance of costs in intensive care, we investigated whether relevant differences between individualized admixtures and standard solutions in terms of costs can be found.

Materials and Methods: Actual costs of individualized admixtures (prepared following national guidelines) in 20 postoperative paediatric patients with uncomplicated requirements were compared with retrospectively determinded theoretical costs of standard solutions. As standard solutions do not contain vitamins and trace elements, these had to be added in the calculation.

Daily costs (in 2006 Euro values) per patient were calculated as means \pm standard deviation. Student's t-test $(p<0.05)$ was used to evaluate any differences between the two groups (actual costs vs. theoretical costs). Results and Discussions: Mean actual costs of individualized admixtures of $€ 75.92( \pm 12.50)$ per patient and day were significantly higher $(p<0.05)$ than the theoretical costs of standard solutions, which amounted to $€ 58.55$ $( \pm 8.85)$. In the setting of an intensive care unit with 400 patient days per year of paediatric parenteral nutrition, this difference would add up to a cost reduction of approximately $€ 7,000$ per year.

Conclusions: The use of standard solutions instead of individualized admixtures is able to markedly decrease the costs of paediatric parenteral nutrition. Reference:

1 Koletzko B. J Pediatr Gastroenterol Nutr 2005; 41 Suppl 2: S1-S87.

\section{AP2-6}

\section{Impact of one-year Surviving Sepsis Campaign (SSC)} guideline on implementation of 'Sepsis Care Bundles' on outside Critical Care Units

L. Linhartova, F. Gao, J. Pooni, D. Daniels, C. Jones

Anaesthesia and Critical Care, Birmingham Heartlands Hospital, Birmingham, United Kingdom

Background and Goal of Study: 'Surviving Sepsis Campaign (SSC) guideline for management of severe sepsis and septic shock' was launched in 2004, which has introduced the 'sepsis care bundle' into clinical practice. Our group conducted a prospective observational study in 2005 to evaluate compliance of 6-hour and 24-hour sepsis care bundles and their impact on hospital mortality. Over the year, a great deal of work has been carried out to promote for implementation of Sepsis Care Bundles worldwide. We have therefore conducted another prospective observational study aiming to assess impact of one-year Surviving Sepsis Campaign (SSC) guideline on implementation of 'Sepsis Care Bundles' on outside Critical Care Units.

Material and Methods: Total of 102 patients was recruited from medical, surgical wards or emergency departments of four acute NHS Trust Teaching hospitals in England, using proximate look-back data extraction. The results of 2006 with 2005 using unpaired t-test or Fisher's Exact test.

Results and Discussion: Patients' demographic data, early warning scores (MEWS), rate of ICU admissions and type of infections are identical between two studies. The Table shows improved compliance with 6-hour sepsis care bundle. Hospital mortality remains unchanged.

\begin{tabular}{|c|c|c|}
\hline & 2005 study & 2006 study \\
\hline Numbers & 101 & 102 \\
\hline $\begin{array}{l}\text { Compliance of } \\
6 \text {-hour bundle }\end{array}$ & $52 \%$ & $64 \%$ \\
\hline Lactate & $52 / 101(51 \%)$ & $70 / 102$ (69\%) \\
\hline Blood cultures & 74/101 (74\%) & 56/102 (54\%) \\
\hline Antibiotics given & 74/101 (74\%) & 96/102 (94\%) \\
\hline Fluid resusc. & 84/101 (84\%) & 86/102 (84\%) \\
\hline CVP $>8$ & $2 / 101(2 \%)$ & $91 / 102(89 \%)^{\star \star}$ \\
\hline $\mathrm{SvO}_{2}>70 \%$ & $1 / 101(1 \%)$ & $10 / 102(9.8 \%)^{*}$ \\
\hline Outreach & $33 / 101(33 \%)$ & $61 / 102(60 \%)^{\star}$ \\
\hline Hosp. mortality & $37 \%$ & $40 \%$ \\
\hline
\end{tabular}

${ }^{\star} \mathrm{P}<0.05,{ }^{\star \star} \mathrm{P}<0.01$

Conclusion: Surviving Sepsis Campaign (SSC) guideline appears to improve implementation of 'Sepsis Care Bundles' on outside Critical Care Units.

\section{AP2-7}

\section{A method for contact-less quantitation of movements of} intensive care patients

S. Friedl, H. Schwilden, G. Braun, T. Wittenberg

Department for Anaesthesiology, Friedrich-Alexander-University ErlangenNuernberg, Erlangen, Germany

Background and Goal of Study: The factor self-movements of patients during the intensive care therapy is widely unattended. Complete bed rest has been found as contraindicated in many clinical cases [1]. For further evaluation of the impact of movements to the outcome of the therapy, a quantitative measurement of the movements of patients is needed. In this work, a contactless method for measuring those movements is presented.

Materials and Methods: Using a digital video camera, movements of patients are acquired and recorded. An adopted image-differencing technique [2] is applied to determine the amount of motion during the day as the sum of absolute intensity differences. For this work, 5 patients were observed from $6 a m$ to $6 p m$ for 4 days each.

Results and Discussions: The cumulative amount of movements during the day and a comparison of the daily variation is shown in the figure below. It can be seen how the amount of movements of the patient changes during and in between the days. Subjective comparisons to the simultaneously recorded SAS (sedation agitation scale) values have been done and demonstrated similar variances.

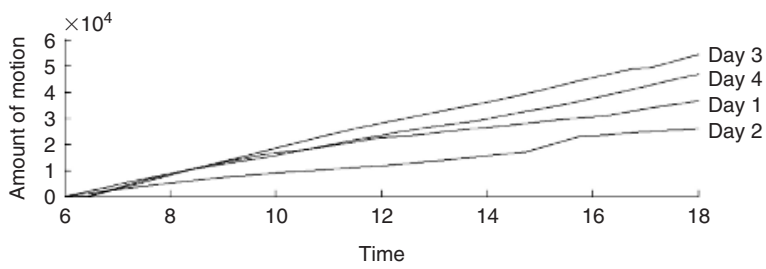

Conclusion: Compared to various clinical scales like RSS, SAS, VICS... [3], the presented method is a first automated approach to study the impact of movement for intensive care therapy with the final goal to study its impact on outcome.

References:

1 Allen C, et al.: Bed rest: a potentially harmful treatment needing more careful evaluation. Lancet 345 (1999) 1229-33

2 Bobick, $\mathrm{A}$, et al.: The recognition of human movement using temporal templates. IEEE PAMI 23 (2001) 257-67

3 Sessler CN: Sedation Scales in the ICU, Chest 126 (2004) 1727-30 


\section{AP2-8}

Effects of staff training regarding care of mechanically ventilated patients on duration of mechanical ventilation

F. Bloos, A. Harz, S. Mueller, K. Reinhart, G. Marx

Department of Anesthesiology and Intensive Care Medicine, University Hospital Jena, Jena, Germany

Background and Goal of Study: Reduction of time on the ventilator is a key concept to avoid complications in critically ill patients.

Recommendations include semirecumbent positioning and low tidal volume ventilation (tidal volumes $<6 \mathrm{ml} / \mathrm{kg}$ ) [1]. Care of patients on mechanical ventilation also consists of prophylaxis for stress ulcer [2] and deep vein thrombosis [3]. The goal of this study was to investigate whether staff training regarding a bundle of predefined treatments would decrease days on mechanical ventilation.

Materials and Methods: All Patients of a 50 bed ICU with mechanical ventilation for $>24 \mathrm{hrs}$ were included. From 06/2005 to 09/2005 (Audit I), patients were examined daily for semirecumbent position $\left(>30^{\circ}\right)$, low tidal volume ventilation, deep vein thrombosis prophylaxis, and stress ulcer prophylaxis by an independent task force. After completion of the audit, all nurses and physicians were trained about importance and methods of the monitored treatments. A second audit was then performed from 03/2006 to 06/2006 (Audit II).

Results and Discussions: 133 patients (1389 ventilator days) were included in Audit I and 141 patients (1002 ventilator days) in Audit II.

\begin{tabular}{llll}
\hline & Audit I & Audit II & $\mathrm{p}$ \\
\hline APACHE II & $24(10)$ & $25(11)$ & 0.387 \\
$\begin{array}{l}\text { Semirecumbent position } \\
\text { Tidal volume }(\mathrm{ml} / \mathrm{kg})\end{array}$ & $24.9 \%$ & $49.6 \%$ & $<0.001$ \\
$\begin{array}{l}\text { Deep vein thrombosis } \\
\text { prophylaxis }\end{array}$ & $6.3(2.2)$ & $6.4(2.3)$ & 0.154 \\
Ulcer prophylaxis & $89.5 \%$ & $91.9 \%$ & 0.048 \\
Tracheotomy rate & & & \\
Days on ventilator & $94.5 \%$ & $94.9 \%$ & 0.712 \\
\hline
\end{tabular}

There was no effect on frequency of pneumonia, ICU length of stay, or survival. Conclusions: Semirecumbent positioning could be successfully improved by staff training. Enhanced implementation was associated with reduction in days on ventilator.

References:

1 Dellinger RP. Intensive Care Med 2004; 30: 536.

2 Cash BD. Crit Care Med 2002; 30: S373.

3 Samama MM. NEJM 1999; 341: 793.

\section{AP2-9}

\section{Influence of remifentanil on duration of mechanical ventilation, ICU lenght of stay and ICU costs}

M. Al, L. Hakkaart, SS. Tan, P. Mulder, J. Bakker

Institute for Medical Technology Assessment, Erasmus MC, Rotterdam, The Netherlands

Background and Goal of Study: To estimate the impact of remifentanil-based sedation (RS) vs. conventional sedation (CS) on the direct medical costs, the length of stay (LOS) and the duration of mechanical ventilation (MV) in ICU patients requiring $\mathrm{MV}$ for 2-3 days.

Materials and Methods: A Markov model was employed to describe the patient flow on the ICU. Three states were defined: MV before start of weaning, MV after start of weaning, and post-MV before ICU discharge. Data to derive the hourly transition probabilities was obtained from UltiSAFE, a recent Dutch open label, centre-randomized, centre-crossover trial including patients with an expected MV-time of 2-3 days. Study medication was either CS (morphine or fentanyl combined with propofol, midazolam or lorazepam according to Dutch guidelines) or RS (remifentanil, combined with propofol when required). ICU LOS, time of start weaning and of extubation, plus all study medication were recorded. Study drug costs were derived from the trial, whereas all other ICU costs (with and without MV) were estimated in a Dutch micro-costing study, All costs were measured from the hospital perspective (price level of 2006). According to the study target population we only included those patients in the analysis who started weaning within 72 hours of start of treatment. Patients were followed for 28 days.

Results and Discussions: The average total 28 -day costs were $€ 11,932$ with CS versus $€ 10,356$ with RS, meaning a difference in costs of $€ 1576(95 \%$ $\mathrm{Cl} 42-3110$ ). The average length of stay on the ICU was 7.7 days in the CS group versus 6.6 days in the RS group (difference 1.1, 95\% $\mathrm{Cl} 0.6-1.6$ ), while the average MV time was 3.8 days for CS versus 2.8 days for RS.
Conclusion: Compared to CS, RS significantly decreases the ICU LOS, the overall costs and the duration of MV. As a decreased duration of MV reduces the risk of ventilator-associated morbidity, RS seems to be the preferred regimen.

\section{AP3-2}

Association of TNF - 308A/G polymorphism with TNF alpha level, illness severity and outcome in septic patient

O. Sabelnikovs, I. Vanags, L. Nikitina-Zake, I. Jaunalksne, P. Oss Department of the Anaesthesiology and Reanimatology, Riga Stradins University, Riga, Latvia

Background and Goal of Study: Several studies have found association of prevalence of the TNF - 308A allele and susceptibility to and outcome from severe sepsis and septic shock. The aim of this study was to examine association of the TNFA - 308A/G polymorphism with TNF $\alpha$ level, illness severity and clinical outcome among septic patients in our clinic.

Materials and Methods: 15 critically ill patients with confirmed severe sepsis or septic shock were included. TNF $\alpha$ and severity of the patients' condition (SOFA score) was measured at the day of admission to the ICU. Patients were followed up throughout their stay in the ICU for clinical outcome. TNFA - 308A/G polymorphism was detected by direct sequencing using ABI prism sequencer.

Results and Discussions: Table 1 Patient characteristic according to TNF $-308 \mathrm{~A}$ allele carriage

\begin{tabular}{llll}
\hline & $\begin{array}{l}\text { TNFA }-308 \mathrm{G} / \mathrm{G} \\
(\mathrm{n}=10)\end{array}$ & $\begin{array}{l}\text { TNFA }-308 \mathrm{~A} / \mathrm{G} \\
(\mathrm{n}=5)\end{array}$ & $\mathrm{P}$ \\
\hline SOFA $( \pm \mathrm{SD})$ & $8.2( \pm 4.6)$ & $10.2( \pm 4.9)$ & 0.39 \\
TNF $\alpha(\mathrm{pg} / \mathrm{L})( \pm \mathrm{SD})$ & $129.8( \pm 271.6)$ & $199.8( \pm 335.9)$ & 0.27 \\
Shock $(\%)$ & 50 & 60 & 1.0 \\
Mortality $(\%)$ & 50 & 80 & 0.58 \\
\hline
\end{tabular}

Conclusion(s): The present study was failed to demonstrate association of TNFA - 308A/G polymorphism with TNF $\alpha$ level at the day of admission to ICU, severity of illness (SOFA score) and mortality in septic patients.

More investigations are needed to determine the role of TNFA - 308A/G polymorphism analysis in the ICU.

\section{AP3-3}

Microcirculatory changes in patients with septic shock treated with vasopressin

S. Klinzing, C. Reinhard, T.P. Simon, K. Reinhart, G. Marx

Anaesthesiology and Intensive Care, Friedrich Schiller University, Jena, Germany

Introduction: Microcirculatory dysfunction leads to inadequate tissue oxygenation and multi organ failure during sepsis or septic shock.

We tested the effects of arginine vasopressin (AVP) on tissue oxygenation $\left(\mathrm{StO}_{2}\right)$, micro vascular reactivity and oral mucosa microcirculation in patients with septic shock.

Methods: In 20 critically ill patients (12M/8F, age 63; 35-78 years) with severe sepsis or septic shock tissue microcirculation was determined before treatment with AVP $(2 \mathrm{IU} / \mathrm{h})$, after $2 \mathrm{~h}$ of treatment and $2 \mathrm{~h}$ after AVP was stopped. Thenar muscle $\mathrm{StO}_{2}$ was measured by near-infrared spectroscopy (InSpectra ${ }^{\mathrm{TM}}$ ), using the artery occlusion method (3 minutes of brachial artery occlusion). Microvascular reactivity alterations was evaluated by the calculation of the slope of the decrease in $\mathrm{StO}_{2}$ during the first 14 seconds after the occlusion of the brachial artery and the calculation of the slope of the increase in $\mathrm{StO}_{2}$ during the first 14 seconds following the ischemic period. Oral mucosal tissue oxygen saturation, microcirculatory blood flow and blood flow velocity were measured in depth of 1 and $4 \mathrm{~mm}$ with a laser Doppler flowmetry and remission spectroscopy system $\left(\mathrm{O}_{2} \mathrm{C}^{\mathrm{TM}}\right)$. Time points: Before, during and $2 \mathrm{~h}$ after the treatment with AVP. Statistics were performed with Wilcoxon test. Results: Vasopressin infusion led to a significant decrease of oral mucosal oxygen saturation, blood flow and flow velocity. There were no changes in thenar tissue perfusion.

\begin{tabular}{llllll}
\hline & Base level & 2h AVP & 2h after AVP & $\begin{array}{l}\mathrm{p} \text {-value } \\
\text { step 1 }\end{array}$ & $\begin{array}{l}\mathrm{p} \text {-value } \\
\text { step 2 }\end{array}$ \\
\hline $\mathrm{SO}_{2}$ 1 mm [\%] & $79 ; 40-99$ & $72.5 ; 59-88$ & $83 ; 45-93$ & $\mathrm{P}<0.05$ & $\mathrm{P}<0.05$ \\
$\mathrm{SO}_{2} 4 \mathrm{~mm}[\%]$ & $79 ; 48-97$ & $68 ; 50-93$ & $81 ; 24-99$ & $\mathrm{P}<0.05$ & $\mathrm{P}<0.01$ \\
$\mathrm{Flow} 1 \mathrm{~mm}$ & $56 ; 11-390$ & $33: 10-212$ & $39 ; 10-249$ & $\mathrm{P}<0.01$ & $\mathrm{P}<0.01$ \\
Flow 4 mm & $332.5 ;$ & $280 ; 119-511$ & $331 ; 150-581$ & $\mathrm{P}<0.05$ & $\mathrm{P}<0.05$ \\
& $149-517$ & & & & \\
Velocity 1 mm & $22.5 ; 12-45$ & $17.5 ; 11-33$ & $20 ; 11-33$ & $\mathrm{P}<0.01$ & $\mathrm{P}<0.05$ \\
Velocity 4 mm & $48 ; 23-74$ & $43 ; 21-105$ & $45 ; 23-69$ & $\mathrm{P}>0.05$ & $\mathrm{P}>0.05$ \\
\hline
\end{tabular}


Conclusion: Vasopressin infusion causes deterioration of oral mucosal blood flow and not of peripheral perfusion independent of mean arterial pressure.

\section{AP3-4}

\section{Removal of inflammatory cytokines with high volume} hemofiltration

M. Lonardo, O. Piazza, E. Zamparelli, G. Servillo, R. Tufano

Anesthesia and Intensive Care, Università Degli Studi di Napoli Federico II, Napoli, Italy

Background and Goal of the Study: The removal of inflammatory mediators in septic patients treated by high volume hemofiltration might depend by the adsorption of the membrane (1) and by the filtration rate (2).

The aim of this study was to evaluate the efficiency of high volume continuous veno-venous hemofiltration (CVVH), with a $12 \mathrm{Kd}$ cut-off filter, in removing pro-inflammatory and anti-inflammatory cytokines from the blood compartment of severe sepsis patients.

Materials and Methods: We enrolled 31 severe sepsis patients. 6 out of 31 developed ARF and required CVVH (filtration rate of $40 \mathrm{ml} / \mathrm{kg} / \mathrm{h}$ ). Cytokines serum levels were measured at the beginning and after 48 hours of the CVVH treatment. Pre-filter, post-filter venous blood and filtrate samples were collected.

Cytokines (IL-2, IL-18, IL-10 and TNF- $\alpha$ ) serum levels were quantified by ELISA.

Results: In septic patients not treated by $\mathrm{CVVH}$, at day 1 median cytokines levels $(\mathrm{pg} / \mathrm{mL})$ were: IL-18 $(1028,9), \mathrm{IL}-2$ (18,86), TNF- $\alpha(17,92)$, IL-10 $(185,7)$ Cytokine concentrations $(\mathrm{pg} / \mathrm{mL})$ in patients treated by $\mathrm{CVVH}$ are expressed, as median, in the table below:

\begin{tabular}{llllll}
\hline & Before CVVH & After CVVH & Pre-filter & Post-filter & Filtrate \\
\hline IL-18 & 902 & 1016 & 1490 & 1276 & $*$ \\
IL-2 & 15,6 & $*$ & $*$ & $*$ & $*$ \\
TNF- $\alpha$ & 15,5 & 14 & 15,2 & 14 & $*$ \\
IL-10 & 268 & $*$ & 189 & 130 & 28 \\
\hline
\end{tabular}

*not measurable

Conclusions: After 48 hours of high volume hemofiltration cytokines serum levels are not significantly decreased.

In our opinion adsorption is the main mechanism by which extracorporeal elimination techniques can remove mediators from the blood compartment: the conventional hemofilters are able of a low removal of mediators, because of the low cut-off point of the membrane.

References:

1 Morgera S. Crit Care Med 2006; 34: 2099-2104.

2 Ronco C. Artif Organs 2003; 27: 92-801.

\section{AP3-5}

\section{Upregulation of TLR2 and LPS receptor TLR4 in septicemic} patients

T. Munster, S. Kunkele, A. Pahl

Department of Anaesthesiology, University Hospital Erlangen, Erlangen, Germany

Background and Goal of Study: Sepsis is a systemic response to a generalized infection leading to multiple organ dysfunction. Hyporesponsiveness towards lipopolysaccharide (LPS) has been claimed to contribute to disease progression. Recent data indicate that members of the transmembrane toll-like receptor (TLR) family, called TLR2 and TLR4, are involved in LPS signalling. These receptors are necessary for LPS-signalling and endotoxin tolerance in mouse peritoneal macrophages correlates with down-regulation of surface TLR4 expression. Therefore we investigated the expression of proinflammatory cytokines and TLRs during sepsis.

Materials and Methods: After approval of the local Ethics Committee and signed consent we studied septic patients and healthy subjects. Septic patients were enrolled within 24 hours after meeting criteria for diagnosis (1). Blood samples were collected on days 1, 3, 7, 10 and 14. PBMCs from blood samples of sepsis patients were prepared. RNA was prepared from either LPS stimulated or unstimulated monocytes. The mRNA levels of IL1 $\beta$, IL-6 and TNF and of TLR2 and TLR4 were determined by quantitative real-time RT-PCR. Results and Discussion: We measured IL- 6 and TNF $\alpha$ mRNA level in PBMCs of septic patients compared to healthy controls. TNF $\alpha$ mRNA level were significantly elevated in septic patients. Transcript levels of both TLRs were elevated significantly in septic patients compared to healthy donors We could not detect LPS tolerance ex vivo in our study population. TLR2 and TLR4 mRNA level from PBMCs of healthy donors treated in vitro with LPS did not reach mRNA levels of untreated PBMCs from septic patients. Therefore an unknown mechanism must account for elevated TLR2 and TLR4 levels in septic patients. Correlations with clinical parameters demonstrate the possible use of TLR2 and TLR4 as new diagnostic markers for the outcome of sepsis.

Conclusion: The upregulation of TLR2 and TLR4 in human patients may contribute to the pathophysiology of sepsis and may result in a self perpetuating cycle leading to septic shock. Interference of this vicious cycle may lead to new therapeutic options for the treatment of sepsis.

Reference:

1 ACCP/SCCM Consensus Conference. Crit Care Med 1992; 20: 864-74.

\section{AP3-6}

Protein $\mathbf{C}$ correlation with SOFA score and etiology of sepsis G. Opramolla, E. De Robertis, O. Piazza, G. Servillo, R. Tufano

Department of Anaesthesia and Intensive Care, University Federico II, Napoli, Italy

Background and Goal of study: Protein C plays a central role in hemostasis and in host response to infection. Protein $C$ deficiency is characteristic of sepsis and has been shown to predict morbidity and mortality. Aim of the study was to check if Protein $\mathrm{C}$ deficit correlate with the pathogens cause of sepsis (Gram + vs Gram-).

Materials and Methods: All patients (pts) consecutively admitted in our ICU were included prospectively at the first appearance of SIRS $\left(T_{0}\right)$. Exclusion criteria: hepatic failure, pre-existent hemostasis alteration, plasma administration. Pts were divided into 2 groups depending on bacteriologic cultures: Group A $(\mathrm{Gram}+)$ and Group B (Gram-). Pts with mixed infections were excluded. Protein $C$ plasmatic levels (daily from $T_{0}$, using a cromogenic method) and clinical evaluation scores (SAPS II, at $\mathrm{T}_{0}$, and SOFA, daily from $\mathrm{T}_{0}$ ) were measured. T-Student test for not paired data was used.

Results: 10 pts were studied. Group A: 5 pts (M3/F2; age 53,4 \pm 22 y.; SAPSII: $48 \pm 4$ ). Group B: 5 pts (M4/F1; age 69,4 \pm 14 y.; SAPSII: 48,8 \pm 13 ). Protein C (\% activity) was lower in Group B (statistically significance from T2: $\left.{ }^{*} p<0.05\right)$. Mean SOFA score was higher in Group A (ns).

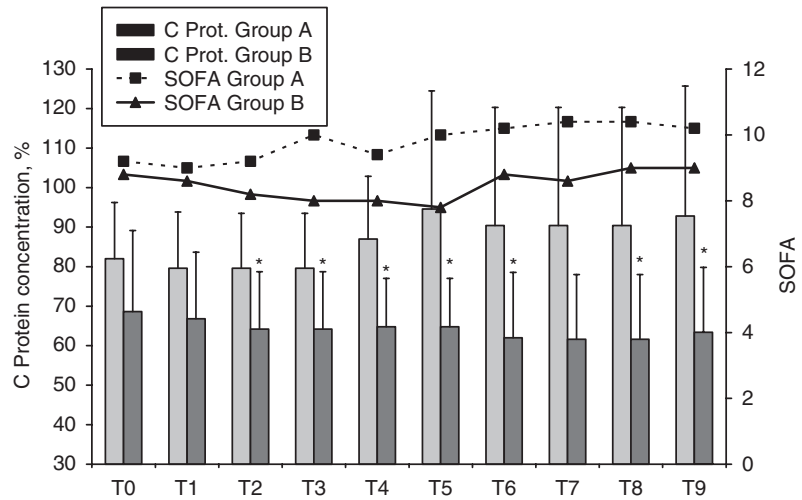

Mortality in Group A was 80\%; in Group B 40\%.

Conclusions: Although the limited number of pts, our data show that Gram - infections seem to be linked with lower Protein C concentration. No correlation with severity and/or outcome was observed. Correlation between protein $\mathrm{C}$ level and type of infection may have interesting clinical applications. More focused studies are needed.

\section{AP3-7}

Central venous catheter colonization and catheter-related bloodstream infection in intensive care unit: comparing standard with silver integrated catheter

N. Hagau, D. Studnicska, M. Flonta, A. Slavcovici, S. Cocu

Anaesthesia and Intensive Care, County Emergency Hospital Cluj, University of Medicine and Pharmacy Iuliu Hatieganu, Cluj-Napoca, Romania

Background and Goal of Study: We compared the rates of positive quantitative culture (PQC) and catheter-related bloodstream infection of standard polyurethane central venous catheters (S-CVC) with rates for silver integrated catheters (SI-CVC) (1). 
Materials and Methods: A prospective, descriptive study. Data was collected over 12 months from 20 surgical-medical ICU beds. Double and triple lumen standard Vygon catheters and silver integrated MultiCath expert Vygon catheters were used. After their removal the tips of the catheters were cultured by the roll-plate method and the quantitative culture technique. Peripheral blood cultures were obtained at the time of catheter removal. Continuous demographic and clinical data were analyzed. The CVC colonization was expressed in terms of cumulative incidence and incidence density. The Kaplan-Meier test was used to compare the risk of PQC over time between s-CVC and SI-CVC.

Results and Discussions: A total of 175 CVC, 75 SI-CVC, 714 catheters days and $100 \mathrm{~s}-\mathrm{CVC}, 846$ catheter days, in 147 patients, were studied. There was no significant difference in CVC indwelling times between s-CVC and SI-CVC $(p=0.19)$. The mean duration of $s-C V C$ was 8.46 days and of $\mathrm{SI-CVC}$ was 9.52 days. There was no significant difference in the colonization rate between S-CVC and SI-CVC. The cumulative incidence of catheter infection was $2.67 \%$ for SI-CVC and $8 \%$ for s-CVC $(p=0.24)$. The incidence density of catheter infection was 2.8 for SI-CVC and 9.5 for s-CVC $(p=0.19)$. The cumulative incidence of catheter colonization was $24 \%$ for SI-CVC and $25 \%$ for s-CVC $(p=0.88)$ and the incidence density was 25 for $\mathrm{SI}-\mathrm{CVC}$ and 29 for s-CVC $(\mathrm{p}=0.60)$. Using the Kaplan Meier test there was a significant difference in the incidence of infection and colonization over time between SI-CVC and S-CVC $(p=0.04)$.

Conclusion(s): SI-CVC could not prevent catheter colonization in critical care patients, but it is a significant difference in the incidence of colonization over time between s-CVC and SI-CVC.

Reference:

1 Martin W. Anesth Analg 2005; 101: 1778-1784.

\section{AP3-8}

Meropenem administered in 3-hour infusion significantly shortens ICU stay of patients with severe intraabdominal infection \&\#8211; initial report.

A. Lukaszewska, P. Sowinski, E. Hagmajer, M. Symonides

Department of Anaesthesia and Intensive Care Unit, The Maria SklodowskaCurie Memorial Cancer Center - Institute of Oncology, Warsaw, Poland

Background and Goal of Study: Due to the increase of antibiotic resistance the direct application of the rules of pharmocodynamics (PD) calls for extra attention and may improve results. The aim of the study was to assess the impact of the change in meropenem administration on the rate of the disappearance of symptoms of severe abdominal infection.

Material and Methods: 42 pts with recognized cancer (age: $48-76$ yrs) were admitted post-operatively to the ICU due to severe intra-abdominal infection. The patients all received meropenem $3 \times 1 \mathrm{~g}$ but were randomized into two groups regarding the mode of administration; group I received meropenem in 20 minute infusions, group 2 - in 3-hour infusions (time set acc. to literature data - calculations of $\mathrm{T}>\mathrm{MIC}$ ). Additionally all pts. received fluconazole $400 \mathrm{mg}$ per die. No other differences were noted between the groups as to postoperative treatment. During the ICU stay clinical evaluation (APACHE II $2 \times$ daily) and procalcitonine level (PCT $-1 \times$ daily) were performed at pre-set time points and all complications were noted. ICU discharge criteria included a PCT level of less thsan $2 \mathrm{ng} / \mathrm{ml}$, normal WBC and no fever. The efficacy of eradication was assessed microbiologically (complete culture sets $2 \times$ weekly). Patient observation terminated on discharge from ICU.

Results: Mean post-op. ICU stay of pts. treated for severe intraabdominal infections was, on average, 7 days in Group I and 5 days in Group II, in which the mode of antibiotic administration followed the PD-dicateted appraoch. Conclusion: Although this prospective study is still being conducted the intial observations suggest a very significant positive impact of meropenem administered in a prolonged infusion on the duration of ICU stay in case of pts. with severe abdominal infections. We believe that the clinical implication of this finding call for such an early report.

\section{AP4-1}

\section{Melatonin preconditioning improves liver function after hemorrhagic shock}

A. Mathes, S. Pradarutti, A. Bentley, I. Bauer, H. Rensing

Department of Anesthesiology and Critical Care Medicine, Saarland University Hospital, Homburg, Germany

Background and Goal of Study: Exogenous administration of pineal hormone melatonin has been demonstrated to attenuate organ damage in septic shock and ischemia-reperfusion models. Here, we investigated whether melatonin pretreatment before hemorrhagic shock improves liver function, hepatic perfusion index and hepatocellular redox state.
Materials and Methods: Sprague-Dawley rats underwent hemorrhagic shock (MAP $\pm 35 \mathrm{~mm} \mathrm{Hg}$ ) for $90 \mathrm{~min}$. Animals were resuscitated with shed blood and Ringer's. Pretreatment was performed with melatonin $10 \mathrm{mg} / \mathrm{kg}$, melatonin plus melatonin receptor antagonist luzindole $2.5 \mathrm{mg} / \mathrm{kg}$, or vehicle (each $n=10)$. Sham-operated controls with and without melatonin were included (each $n=8$ ). After 2 hours of resuscitation, animals underwent either measurement of Plasma Disappearance Rate of indocyanine green $\left(\mathrm{PDR}_{\mathrm{ICG}}\right)$ as a sensitive marker of liver function, or intravital microscopy for assessment of hepatocellular redox state (measured by $\mathrm{NAD}(\mathrm{P}) \mathrm{H}$ autofluorescence) and hepatic perfusion index.

Results and Discussion: Melatonin preconditioning before hemorrhage improved $\mathrm{PDR}_{\mathrm{ICG}}$ (melatonin/shock 15.02\%/min $\pm 1.2 \mathrm{SD}$ vs. vehicle/shock $6.18 \% / \mathrm{min} \pm 1.9 \mathrm{SD} ; \mathrm{p}=0.003$ ), hepatic perfusion index (melatonin/shock $516.6 \mathrm{pl} / \mathrm{sec} / \mathrm{mm} \pm 103.9 \mathrm{SD}$ vs. vehicle/shock $361.0 \mathrm{pl} / \mathrm{sec} / \mathrm{mm} \pm 25.5 \mathrm{SD}$; $\mathrm{p}=0.002$ ) and redox state (melatonin/shock $110.27 \mathrm{aU} \pm 2.36 \mathrm{SD}$ vs. vehicle/shock $129.37 \mathrm{aU} \pm 3.94 \mathrm{SD} ; \mathrm{p}=0.006$ ), compared to vehicle controls. Pretreatment with luzindole completely abolished this protective effect with respect to $P_{D R_{I C G}}(7.31 \pm 1.4)$. Improvements regarding hepatic perfusion index and redox state were not affected by luzindole administration.

Conclusion(s): These data suggest that the beneficial effects of melatonin are not only due to oxygen radical scavenging properties. With respect to liver function, melatonin preconditioning exhibits protective mechanisms that are dependent on melatonin receptor activation.

\section{AP4-2}

Maintaining normoglycemia and not glycemia-independent actions of insulin preserves myocardial performance by protecting mitochondrial function

B. Ellger, I. Vanhorebeek, Y. Debaveye, R. De Voss, G. Van den Berghe Department of Intensive Care Medicine, Catholic University of Leuven, Leuven, Belgium

Background and Goal of Study: Intensive insulin therapy (IIT) reduces mortality and morbidity of critically ill patients. Several investigations suggest that insulin might ameliorate myocardial contractility independently of its actions on blood glucose.

Materials and Methods: In a rabbit model of prolonged critical illness using a 4 -arm design ( $n=8$ per group), blood glucose (normal NG-high HG) and plasma insulin levels (normal NI-high $\mathrm{HI}$ ) were independently manipulated over 7 days, to elucidate relative impact of maintaining normoglycemia and glycemia-independent actions of insulin on left ventricular contractility in an open chest preparation, cellular ultra-structure by electron microscopy, the activities of the respiratory chain complexes in biopsies from the left ventricle, and plasma levels of serum heart-fatty-acid-binding-protein.

Results and Discussions: Contractility increased in $\mathrm{HI} / \mathrm{NG}$ animals and deteriorated in $\mathrm{HI} / \mathrm{HG}$ animals compared to other groups and healthy controls. Cardiac output and surrogate parameters of preload and afterload did not differ among groups. Electron microscopy revealed severely damaged mitochondria in cardiac myocytes in particular in $\mathrm{HI} / \mathrm{HG}$ rabbits. Concomitantly, the activities of complex I, III and V were compromised in the left ventricle biopsies of both hyperglycemic groups, in particular in the $\mathrm{HI} / \mathrm{HG}$ group. Both normoglycemic groups revealed no changes in ultra-structure and complex activity compared to healthy controls. Compromised mitochondrial enzyme activities correlated with cardiac damage assessed by plasma levels of heartfatty-acid-binding-protein, suggesting that mitochondrial protection mediated part of the prevention of organ failure.

Conclusions: In our animal model of prolonged critical illness, insulin ameliorated myocardial contractility but only when normoglycemia was maintained concomitantly. Maintaining normoglycemia and not glycemia-independent actions of insulin appear crucial for preserving mitochondrial function in the myocardium.

\section{AP4-3}

Normoglycemia and not glycemia-independent actions of insulin maintain physiologic NOS-activity by preserving physiological regulation of ADMA-levels

B. Ellger, M. Richir, L. Langouche, P. Van Leeuwen, G. Van den Berghe Department of Intensive Care Medicine, Catholic University of Leuven, Leuven, Belgium

Background and Goal of Study: In critical illness, both hyperglycemia and elevated levels of asymmetric dimethylarginine (ADMA), an endogenous inhibitor of NO-synthase (NOS) that is released during catabolism, are associated with organ dysfunction and increased mortality. Controlling glycemia by intensive insulin therapy (IIT) reduces morbidity and mortality, lowers 
ADMA plasma levels, and thus presumably preserves local NO bio-availability. The effects on ADMA-levels can be mediated by affecting proteolysis and/or the activity of dimethyl-arginine-dimethyl-aminohydrolase (DDAH), the enzyme breaking down ADMA. We thus hypothesized that controlling glycemia or glycemia-independent actions of insulin preserve regional NOS-activity by modulating ADMA levels in tissues and plasma.

Materials and Methods: In a rabbit model of prolonged critical illness we studied the impact of maintaining normoglycemia/normoinsulinemia, normoglycemia/hyperinsulinemia, hyperglycemia/normoinsulinemia and hyperglycemia/hyperinsulinemia over 7 days on NOS-activity (by radiolabelled enzymatic assay), NOS transcription (by real time PCR) and ADMA levels (by High-Performance Liquid Chromatography (HPLC)) in muscle, kidney and liver biopsies and plasma, plasma urea/creatinine ratio (U/C) as a parameter for proteolysis, and the DDAH-activity (by HPLC) in the biopsies. Results and Discussions: In both hyperglycemic groups NOS-activity was reduced despite up-regulated NOS-transcription, independently from insulin levels. In both normoglycemic groups NOS-activity and -transcription remained unchanged compared to healthy controls. ADMA-levels in plasma and tissues were increased in both hyperglycemic groups and not affected in both normoglycemic groups. ADMA-levels correlated with NOS-activity in the biopsies. U/C was low in hyperglycemic groups, indicating minor proteolysis. DDAH-activity was preserved in normoglycemic groups and deteriorated in both hyperglycemic groups. DDAH-activity correlated with ADMA-levels. Conclusion: Maintained normoglycemia and not glycemia-independent actions of insulin preserved physiologic tissue NOS-activity not by modulating NOS transcription but by preventing increased levels of ADMA through preserving physiological DDAH-activity.

\section{AP4-5}

Recovery of temperature homeostasis and metabolism after long abdominal surgery under combined anesthesia

I.B. Zabolotskikh, T.S. Musayeva, S.V. Grigoriev

Chair of Anesthesiology, Intensive Care and Transfusiology, Kuban State Medical University, Krasnodar, Russian Federation

Background and Goal of Study: The seasonable diagnosis and adequate correction of homeostasis disorders influence significantly on surgical operations outcomes. It is important to assess the postoperative recovery, too. The aim of the study is to evaluate recovery of temperature homeostasis and metabolism.

Materials and Methods: Were study 66 patients, underwent long abdominal surgery under combined anesthesia. The mean age was $62,1(34,0-69,0)$ yrs. A core and peripheral temperature, central hemodynamics, tissue delivery $\left(\mathrm{DO}_{2}\right)$, consumption $\left(\mathrm{VO}_{2}\right)$ and extraction of oxygen $\left(\mathrm{ERO}_{2}\right)$, glucose profile and nitrogen balance were assessed in all patients up to 24 hours after surgery end. Depending on the oxygen extraction ratio $\left(\mathrm{ERO}_{2}\right)$ at the admission into $\mathrm{ICU}$, all patients were divided in three groups: group $1(\mathrm{n}=12)-\mathrm{ERO}_{2}$ $10-21 \%$; group $2(\mathrm{n}=26)-\mathrm{ERO}_{2} 24-30 \%$, and group $3(\mathrm{n}=27)-\mathrm{ERO}_{2}$ $35-40 \%$. Statistical analysis include Kruskall-Wallis test.

Results and Discussions: Heterogenity of metabolism recovery was revealed between the groups. After admission in ICU there were differences in core temperature between groups 1,2 and $3\left(35,1(34,9-35,6)^{\circ} \mathrm{C}, 35,2(35,0-35,6)^{\circ} \mathrm{C}\right.$ and $34,3(33,7-34,9)^{\circ} \mathrm{C}$, respectively), which determine delayed recovery in group 3 by $4-7$ hour, compared with 1 and 2 group, in which recovery occurred in 1-3 hour. Also was found differences in $\mathrm{VO}_{2}$ between groups $(0,9$ $\left.(0,75-1,2) \mathrm{ml} / \mathrm{kg}^{*} \mathrm{~min}\right), 2,58(2,3-4,5) \mathrm{ml} / \mathrm{kg}^{*} \mathrm{~min}$ and $2,5(2,8-3,7) \mathrm{ml} / \mathrm{kg}^{*} \mathrm{~min}$, respectively), $\mathrm{DO}_{2}$ in all groups was decreased. In oxygen status there were following differences $\mathrm{SvO}_{2}$ between the groups: 1 - $(85,9(81,4-87,7) \%)$, 2 - $(70,1(69,5-74,4) \%), 3-(60,8(56,7-62,8) \%)$. The glycemia level was not differ between the groups. All the patients had negative nitrogen balance after admission in ICU. After the 12th postoperative hour the nitrogen balance in group 3 was significant less $(-5,6[-8,0-(-3,2)] \mathrm{g} /$ day) than in groups 1 and $2(-2,4[-2,9-(-1,9)] \mathrm{g} /$ day $),(-2,5[-4,3-(-0,8)] \mathrm{g} /$ day $)$.

Conclusions: The state of the patients, who had normal values $\mathrm{ERO}_{2}$ after the long abdominal surgery under combined anesthesia may be characterized as compensated. The decreased $\mathrm{ERO}_{2}$ allow to consider this patients as high risk of delayed metabolism recovery. Patients with increased $\mathrm{ERO}_{2}$ requires correction of hemodynamic branch of oxygen transport system.

\section{AP4-6}

Infusion of hydroxyethl starch maintained the blood flow in the villi in the severe hemorragic shock in rats

S. Suzuki, T. Hitosugi, T. Shimizu, T. Saito, Y. Oi

Anesthesiology, Nihon University Graduate School of Dentistry, Chiyoda-ku, Japan
Background and Goal of Study: Severe hemorrhagic shock could induce intestinal ischemia which impairs barrier function, leads to multiple organ failure(1). We compared the several kinds of fluids to find the optimal initial fluid to minimize the intestinal ischemia in the severe hemorrhagic shock in rats. Materials and Methods: We studied 25 male Sprague Dawley rats. Hemorrhage was started with bleeding through a line until mean arterial pressure (MAP) at $40 \mathrm{mmHg}$. At $30 \mathrm{~min}$ point, the rats were received following initial fluid of several volume substitutes for $15 \mathrm{~min}$. Control group: no initial fluid, Blood transfusion group (Blood): $50 \%$ volume of bleeding. $6 \%$ hydroxyethyl starch (HES) (17.5-22 kDa) group: 100\% volume of bleeding, Aceted Ringer's solution (AR) group: $300 \%$ volume of bleeding, $7.5 \%$ hypertonic saline (HTS) group: $18 \%$ volume of bleeding. Rats were received maintainance infusion of $A R$ at the rate of $5 \mathrm{ml} / \mathrm{kg} /$ hour from $45 \mathrm{~min}$ point. At $90 \mathrm{~min}$ point, rats were resuscitated with blood, which is equal to $50 \%$ volume of bleeding in HES, AR and HTS groups. Statistical analysis: ANOVA followed by Scheffe test $(P<0.05)$.

Results and Discussions: All rats without any regimen of fluid died within $90 \mathrm{~min}$. MAP was kept $89 \pm 16$ (mean $\pm \mathrm{SD}$ ) $\mathrm{mmHg}$ after initial fluid with blood, HES and $A R$ at 90 min point.

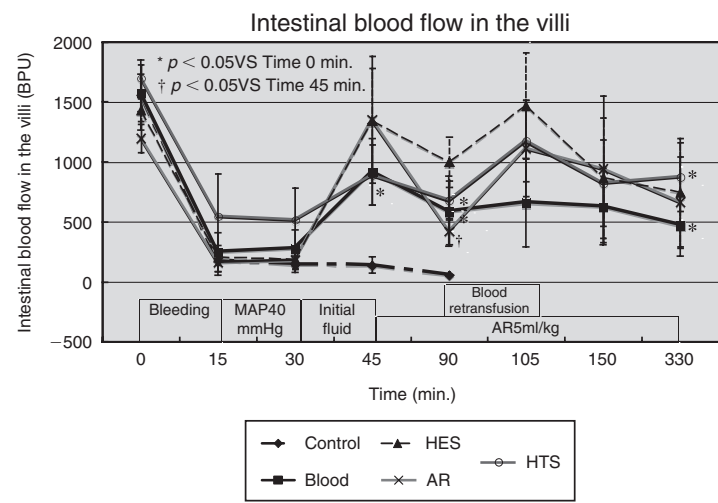

Conclusion: HES is the optimal initial fluid to recovered and maintained blood flow in the villi in the severe hemorrhagic shock in rats. Reference:

1 L. Landow. L.W. Andersen. Acta. Anaesth. Scand. 1994; 38: 626-639

\section{AP4-7}

Serum sodium concentration into model for end-stage liver disease and liver transplantation outcome

H. Serrano, S. González, C. Cortés, C. Bosch, A. Mora

Anesthesiology, Hospital Vall d'Hebron, barcelona, Spain

Background and aim: Actually have been demonstrate the efficay to incorporate serum sodium $(\mathrm{Na})$ into model for end-stage liver disease (MELD). This new score ("MELD-Na" = MELD + $1.59(135-\mathrm{Na})$ seems to provide more accurate survival prediction in patients with liver disease than MELD alone. So, "MELD-Na" scores $>20$ were associated with higher risk of death(1). The aim of this study was to determine the effects of MELD-Na on posttransplantation outcome in patients with cirrhosis.

Methods: In this retrospective study we enrolled patients with end-stage liver disease undergoing liver transplantation during a 2-year period. This patients were separated in two groups according to the MELD-Na score $>$ or $<20$. We compared the postoperative complications between groups during the first postoperative month, and the overall survival of both groups.

Results: Complete date were available in 148 patients in whom the median "MEL-Na" score was $14.84 \pm 12.71$. "MELD-Na" $>20$ was present in 47 patients (31.8\%) of whom $36.6 \%$ developed renal failure and $28.6 \%$ liver failure; there were no significant differences between groups for these complications ( $p=0.148$ and $p=0.368$, respectively). Also, the overall survival was similar between groups (Kaplan-Meier, $p=0.777$ ).

Conclusions: In this study, the patients who were going to receive liver transplantation, MELD-Na score $>20$ wasn't associated with a higher rate of renal and liver failures during the first month after transplantation. Also, we can't demonstrate that the incorporation of $\mathrm{Na}$ into MELD provides an accurate survival prediction in these postoperative patients.

Reference:

1 Scott W. Gastroenterology 2006; 130: 1652-60. 


\section{AP5-1}

\section{Regulation of renal sodium transporters during severe inflammation}

C. Schmidt, K. Hoecherl, A. Kurtz, M. Bucher

Anesthesiology, University of Regensburg, Regensburg, Germany

Background and Goal of Study: The pathogenesis of endotoxemic tubular dysfunction with failure in urine concentration and increased fractiona sodium excretion is poorly understood (1). Since expression of NHE3, ROMK, NKCC2, ENaC and $\mathrm{Na}^{+} / \mathrm{K}^{+}$-ATPase is essential for tubular sodium reabsorption (2), we investigated the regulation of these transporters during severe inflammation.

Materials and Methods: By agreement of the animal protection committee C57BL/6J mice were injected with lipopolysaccharides (LPS) or proinflammatory cytokines. Hemodynamic, renal parameters and the expression of renal sodium transporters were investigated. To clarify the role of cytokines and renal ischemia in the regulation of renal sodium transporters, experiments with cytokine knock-out mice, glucocorticoid-treated mice, and mice with renal artery clipping serving as model for renal ischemia were performed. The influence of cytokines on the expression of renal sodium transporters in cultured cortical collecting duct (CCD) cells was investigated.

Results and Discussions: LPS reduced blood pressure and GFR, increased fractional sodium excretion and strongly decreased the expression of NHE3, ROMK, NKCC2, ENaC and $\mathrm{Na}^{+} / \mathrm{K}^{+}$-ATPase. Injection of TNF- $\alpha$, IL-1 $\beta$ or IFN- $\gamma$ decreased renal function and expression of renal sodium transporters. LPS-induced downregulation of sodium transporters was not affected in knockout mice with deficiencies for TNF- $\alpha$, IL-receptor1 or IFN- $\gamma$. Glucocorticoid pretreatment, which inhibited LPS-induced increase of tissue TNF- $\alpha, \mathrm{IL}-1 \beta$ or IFN- $\gamma$ concentration, attenuated LPS-induced renal dysfunction and downregulation of tubular sodium transporters. Renal ischemia did not influence sodium transporter expression. In vitro, proinflammatory cytokines decreased expression of ROMK, ENaC and $\mathrm{Na}^{+} / \mathrm{K}^{+}$ATPase in CCD cells

Conclusion(s): Our findings demonstrate downregulation of renal sodium transporters which likely accounts for tubular dysfunction during sepsis and suggest that this regulation is mediated by proinflammatory cytokines.

References:

1 Hotchkiss RS, Karl IE: NEJM. 2003; 348: 138-50.

2 Lang F, Capasso G, Schwab M, et al. Clin Exp Nephrol. 2005; 9: 91-9.

\section{AP5-2}

Prophylactic adrenomedullin infusion prevents pulmonary hypertension and lactic acidosis in ovine endotoxemia

C. Ertmer, M. Lange, H. Van Aken, K. Bröking, M. Westphal

Department of Anaesthesiology and Intensive Care Medicine, University of Muenster, Muenster, Germany

Background and Goal of Study: Sepsis-associated arterial hypotension is often complicated by an impaired oxygen supply/demand relationship, as reflected by increased arterial lactate concentrations.(1)

We hypothesized that prophylactic infusion of the endogenous vasodilatory peptide hormone adrenomedullin (AM) may improve tissue oxygenation and thereby limit arterial lactate production in ovine endotoxemia.

Materials and Methods: Fourteen adult female sheep were instrumented for chronic hemodynamic monitoring. Thereafter, the sheep were randomized to receive either $\mathrm{AM}$ at incremental doses $\left(10,50,100 \mathrm{ng} \cdot \mathrm{kg}^{-1} \cdot \mathrm{min}^{-1}\right.$; each for 30 minutes; doses were chosen for dose/response studies) or the vehicle (normal saline). Twenty-four hours later, endotoxin (Salmonella typhosa endotoxin, $10 \mathrm{ng} \cdot \mathrm{kg}^{-1} \cdot \mathrm{min}^{-1}$ ) was infused for 24 hours in all sheep.

Results and Discussions: Endotoxin infusion increased heart rate, cardiac index and decreased mean arterial pressure and systemic vascular resistance index to the same extent in AM pre-treated and control animals. However, AM pre-treatment prevented the endotoxin-associated increase in mean pulmonary arterial pressure (18 \pm 1 vs. $25 \pm 1 \mathrm{mmHg}, \mathrm{p}<0.01 \mathrm{AM}$ vs. control), pulmonary vascular resistance index ( $86 \pm 7 \mathrm{vs} .132 \pm 18 \mathrm{dyne} \cdot \mathrm{s} \cdot \mathrm{cm}^{-5} \cdot \mathrm{m}^{2}$; $\mathrm{p}<0.05 \mathrm{AM}$ vs. control) and arterial lactate concentrations $(0.6 \pm 0.1$ vs. $1.4 \pm 0.3 \mathrm{mg} \cdot \mathrm{dL}^{-1} ; \mathrm{p}<0.01 \mathrm{AM}$ vs. control).

Conclusion(s): These data demonstrate that prophylactic infusion of AM prevents sepsis-associated pulmonary hypertension and lactic acidosis in ovine endotoxemia.

Reference:

1 Levy B, Sadoune LO, Gelot AM, et al. Crit. Care Med. 2000; 28: 114-119.

\section{AP5-3}

Benefical effects of low-dose dexamethasone on cardiovascular and kidney function in septic shock in the rat are not related to iNOS inhibition

T. Johannes, E.G. Mik, H.-J. Dieterich, K.E. Unertl, C. Ince Anesthesiology and Critical Care, University Hospital Tuebingen, Tuebingen, Germany

Background and Goals: The pathogenesis of acute renal failure (ARF) in sepsis is multi-factorial and only partially understood. A controversially discussed molecule to play a role in ARF is nitric oxide (NO), which is known to be suppressed by dexamethasone (1). The hypothesis of this study was that inhibition of iNOS by low-dose dexamethasone would improve an impaired cardiovascular and kidney function in sepsis.

Materials and Methods: 12 male Wistar rats received a 30-min iv-infusion of lipopolysaccharide (LPS). $2 \mathrm{~h}$ later, in all rats fluid resuscitation was started. Additionally to the fluid (Voluven ${ }^{\circledR}$ ) 6 animals received dexamethasone (DEX; $0.1 \mathrm{mg} / \mathrm{kg}$ ). Systemic hemodynamic parameters were continuously recorded. Furthermore plasma NOx levels (Sievers NO-analyzer), renal iNOS RNA expression (in situ hybridization) and creatinine clearance $\left(\mathrm{Cl}_{\text {crea }}\right)$ were determined. Statistics were performed using two-way ANOVA for repeated measurements. Results: Data are presented as mean \pm SD.

\begin{tabular}{|c|c|c|c|}
\hline & $\begin{array}{l}\text { Baseline } \\
\text { (0 min) }\end{array}$ & $\begin{array}{l}\text { Endotoxemia } \\
\text { (120 min) }\end{array}$ & $\begin{array}{l}\text { Resuscitation } \\
(300 \text { min) }\end{array}$ \\
\hline \multicolumn{4}{|c|}{ MAP (mmHg) } \\
\hline Voluven ${ }^{\circledR}$ & $105 \pm 4$ & $67 \pm 12^{*} \dagger$ & $59 \pm 12^{*} \dagger$ \\
\hline DEX & $110 \pm 10$ & $61 \pm 8^{*} \dagger$ & $80 \pm 7^{*} † \ddagger$ \\
\hline \multicolumn{4}{|c|}{$\mathbf{R B F}\left(\mathrm{mL}{ }^{*} \mathrm{~min}^{-1}\right)$} \\
\hline Voluven ${ }^{\circledR}$ & $6.3 \pm 1.4$ & $1.9 \pm 0.9^{*} \dagger$ & $2.4 \pm 1.3^{\star} \dagger$ \\
\hline DEX & $6.1 \pm 0.9$ & $2.5 \pm 1.2^{*} \dagger$ & $4.7 \pm 1.3^{*} \ddagger$ \\
\hline \multicolumn{4}{|c|}{$\mathrm{Cl}_{\text {crea }}\left(\left.\mu\right|^{*} \min ^{-1 *} \mathrm{~g}^{-1}\right)$} \\
\hline Voluven ${ }^{\circledR}$ & $751 \pm 234$ & 1 & $231 \pm 59^{\star} \dagger$ \\
\hline DEX & $656 \pm 150$ & 1 & $753 \pm 254^{\star} \ddagger$ \\
\hline \multicolumn{4}{|c|}{$\operatorname{NOx}\left(\mu \mathrm{M}^{\star} \mathrm{L}^{-1}\right)$} \\
\hline Voluven ${ }^{\circledR}$ & $16 \pm 9$ & $26 \pm 11$ & $217 \pm 54{ }^{*} \dagger$ \\
\hline DEX & $17 \pm 3$ & $31 \pm 6$ & $227 \pm 36^{\star} \dagger$ \\
\hline
\end{tabular}

${ }^{\star} P<0.05$ vs baseline. $\dagger P<0.05$ vs control. $\ddagger P<0.05$ vs Voluven ${ }^{\circledR} .{ }^{1}=$ anuria.

DEX did not reduce renal iNOS RNA expression.

Conclusion: The restoration of systemic hemodynamics and kidney function in dexamethasone-treated animals was not related to iNOS inhibition. Reference:

1 Wang $\mathrm{H}$. et al. Effect of dexamethasone on nitric oxide synthase and Caspase-3 gene expressions in endotoxemia in neonate rat brain. Biomed Environ Sci 2005; 18(3):181-6.

\section{AP5-4}

\section{Nicotine protects from renal ischemia/reperfusion injury}

C. Sadis, G. Teske, M. Goldman, S. Florquin, L. Barvais

Anesthesiology, Erasme Hospital, Brussels, Belgium

Background and Goal: Kidney ischemia/reperfusion (l/R) injury is responsible for delayed graft function and worsens long-term allograft survival. Recently, nicotine has been shown to protect mice from the toxemia-induced shock through the modulation of innate immune response. As sepsis and renal I/R share similar inflammatory patterns, we tested the potential protective effect of nicotine in renal I/R.

Material and Methods: I/R was induced by a bilateral 35 minutes clamping of renal arteries in mice. Sham animals have been operated without clamping ( $n=5$ /group). Nicotine was administrated 30 minutes before surgery. Control and nicotine pretreated animals underwent a bilateral renal artery clamping and were sacrificed 24 and 72 hours after reperfusion ( $n=12$ to 15/group). Renal dysfunction was assessed by plasmatic creatinine levels. Tubular damage was scored from 0 to 5 according to the presence of 4 criteria: loss of brush border, tubular casts, necrosis and tubule dilatation. TNF- $\alpha$ levels were measured using ELISA and neutrophil infiltration was assessed by Ly$6 \mathrm{G}$ immunostaining.

Results: Nicotine pretreatment significantly improved renal function as shown by lower creatinine plasmatic levels in comparison with controls $(71.0 \pm 11.7$ vs $41.1 \pm 7.7 \mu \mathrm{mol} / \mathrm{l}$ and $54.3 \pm 14.9$ vs $28.1 \pm 7.4 \mu \mathrm{mol} / \mathrm{l} ; \mathrm{p}<0.05$, mean \pm SEM, day 1 and day 3 after IRI, respectively). Similarly, tubular damage score was lower in nicotine pretreated groups compared with controls $(3.6 \pm 0.2$ vs $2.4 \pm 0.5$ and $4.0 \pm 0.1$ vs $1.9 \pm 0.6 ; p<0.05$, day 1 and day 3 after IRI, respectively). The anti-inflammatory effect of nicotine was attested by a downregulation of renal TNF- $\alpha(25.2 \pm 1.9$ and $9.2 \pm 0.4 \mathrm{pg} / \mathrm{mg}$ protein, untreated IRI vs treated IRI, $\mathrm{p}<0.01$ ) and by a suppression of neutrophil infiltration $(247.7 \pm 14.6$ and $96.2 \pm 41.7$ cells/10 fields, untreated IRI vs treated IRI, $\mathrm{p}<0.05)$. 
Conclusion: Nicotine protects kidney from renal I/R through the dampening of innate immune response.

\section{AP5-5}

\section{Flecanide acetate reduces mortality to endotoxin induced} acute lung injury in rats

H. Bae, S. Kwak, S. Kim, S. Chung

Anesthesiology and Pain Medicine, Chonnam National University Hospital, Gwangju, Republic of Korea

Background and Goal of Study: To clarify the effects of flecanide acetate, an antiarrythmic drug, on mortality and interleukin-8 (IL-8) response to endotoxin induced acute lung injury in rats.

Materials and Methods: Animals were randomly assigned to one of five groups: rats receiving subcutaneous (SC) infusion of saline and intraperitoneal (IP) injection saline (S-S group, $n=14$ ), those receiving SC infusion of saline and IP injection of Escherichia coliendotoxin ( $20 \mathrm{mg} / \mathrm{kg})(\mathrm{S}-\mathrm{E}$ group, $\mathrm{n}=28$ ), those receving SC infusion of flecanide acetate $(0.2 \mathrm{mg} / \mathrm{kg} / \mathrm{hr})$ and IP injection of saline (F-S group, $n=14$ ), those receiving SC infusion of flecanide acetate $(0.1 \mathrm{mg} / \mathrm{kg} / \mathrm{hr})$ and IP injection of endotoxin ( $\mathrm{F} \mathrm{0.1-E}$ group, $\mathrm{n}=14)$, those receiving SC infusion of flecanide acetate $(0.2 \mathrm{mg} / \mathrm{kg} / \mathrm{hr})$ and IP injection of endotoxin ( $F$ 0.2-E group, $n=17$ ) SC influsion of saline or flecanide acetate using by mini-osmotic pump was started 3 hours before IP injection of saline or endotoxin and continued until 24 hours after IP injection of saline or endotoxin when all rats were killed. The wet weight/dry weight (W/D) ratio of lung, lung injury score and number of WBC, \% polymorphonuclear cells and concentration of IL-8 in bronchoalveolar lavage fluid (BALF) and mortality rate were calculated 24 hours after IP injection of saline or endotoxin.

Results and Discussions: The mortality rates for $F$ 0.1-E groups $(0 \%)$ and F 0.2-E groups (17.7\%) were significantly lower than that for the S-E group (43\%). The increases in W/D ratio, lung injury score and the number of WBC, $\%$ polymorphonuclear cells and concentration of IL-8 in BALF were attenuated for the F-E group than the S-E group.

Conclusion(s): Flecanide acetate dramatically reduced the mortality rate and attenuated IL-8 and inflammatory response to the endotoxin induced acute lung in rats. These findings suggest that flecanide acetate has a therapeutic effect to the acute lung injury to sepsis.

\section{AP5-6}

Inhibitor of apoptosis proteins cIAP1, cIAP2 and XIAP regulate apoptosis in sepsis

S. Weber, J.C. Schewe, L.E. Lehmann, A. Hoeft, F. Stueber Anesthesiology and Intensive Care Medicine, University of Bonn Medical Center, Bonn, Germany

Background and Goal of Study: During sepsis lymphocytes undergo accelerated apoptosis, which contributes to immunosuppression. Inhibitor of Apoptosis Proteins (IAP) are endogenous inhibitors of caspase activity (1) but their role in sepsisis unknown. The aim of the study was to investigate the expression of IAP's and their regulator smac/diablo in patients with severe sepsis.

Materials and Methods: With approval by the local ethics committee 16 patients with severe sepsis were included and blood was obtained as soon as the criteria of severe sepsis were fulfilled. 10 patients with mild systemic inflammatory response syndrome (SIRS) and 11 healthy volunteers served as two separate controls. Caspase activity and antigen expression in lymphocytes were measured by flowcytometry, and mRNA expression in whole blood was quantified by light-cycler rtPCR. ANOVA-analysis was performed to test for statistical significance.

Results and Discussions: In severe sepsis but not in SIRS phosphatidyl serine externalisation increased and the central executioner caspase 3 was found to be active in an expanded subpopulation of T- and B-cells. In healthy controls, mRNA's for cIAP1, clAP2 and XIAP were expressed at relatively low levels. While all three may inhibit caspase 3, xIAP is also known to inhibit caspase 9 . In patients suffering from severe sepsis, the transcription of cIAP1, cIAP2 and XIAP was increased significantly $(p<0.001) 2.4$-fold, 4.8-fold and 9.5-fold. Surprisingly, in SIRS the respective transcripts were elevated even more $(8.5,8.8$ and 20.5 -fold, $p<0.001)$. The expression of the IAP-inhibitor smac/diablo remained unchanged.

Conclusions: We hypothesize that elevated levels of IAP's in SIRS aide to abrogate caspase- 3 activation. In sepsis, the compensatory increase in IAP transcripts may not be strong enough to efficiently block caspase-3 activation, thereby rendering lymphocytes susceptible to cell death.

Reference:

1 Huang Y, Park YC, Rich RL, Segal D, Myszka DG, and Wu H. Cell. 2001; 104: 781-79

\section{AP5-7}

Impact of food-restriction on the outcome after abdominal sepsis in the rat

A. Torossian, S. Rühlmann, C. Arndt, H. Wulf, A. Bauhofer

Anesthesia and Critical Care, University Hospital Marburg, Marburg, Germany Background and Goal of Study: We sought to evaluate the differences in outcome 120 hours after induction of abdominal sepsis between well-nourished (WN) and food-restricted (FR) rats.

Materials and Methods: After approval, in Trial $130 \mathrm{FR}$ and $30 \mathrm{WN}$ rats of the same age received antibiotic prophylaxis and underwent peritoneal infection on day 20. In Trial 220 FR rats were weight matched to $20 \mathrm{WN}$, but younger rats. All had i.v. antibiotic prophylaxis with cefuroxime $3 \mathrm{mg} / \mathrm{metronidazole}$ $10 \mathrm{mg} / \mathrm{kg} 1 \mathrm{~h}$ before surgery and peritoneal stool infection. In 10 rats of Trial 2, plasma cytokines TNF- $\alpha$, IL- 6 and MIP-2 were measured (ELISA) $1 \mathrm{~h}$ after infection. Primary endpoint was the 120 hour survival rate analysed using Chi-square test. Cyto-kine data were analysed with the Kruskal-Wallis test and post hoc Bonferroni correction. Means + SEM are shown.

Results and Discussions

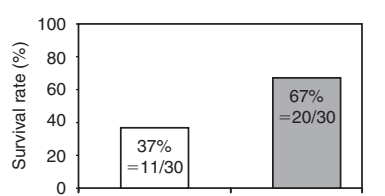

WN

Trial 1

Cytokine levels in Trial 2:

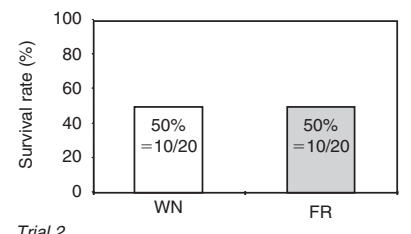

Trial 2

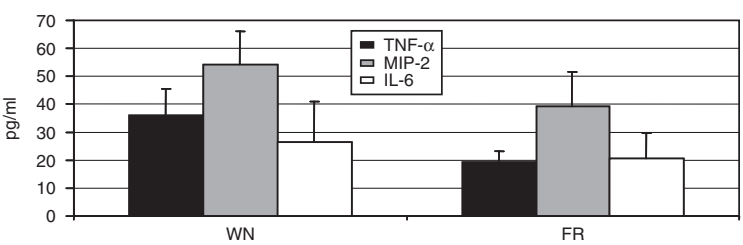

FR rats were more resistant to infection than $\mathrm{WN}$ rats of same age (others found that FR mice had less neoplasms, auto-immune disease and lived longer compared with WN controls, 1). However this advantage disappeared when we compared weight-matched rats in Trial 2, but pro-inflammatory cytokines were reduced in FR rats (as in 2).

Conclusion(s): Food restriction per se appears to be no independent risk factor for postoperative sepsis. Immuno-modulatory effects should be further evaluated.

Reference:

1 Science 1982; 215: 1415-8.

2 Neuroimmunomodulation 2000; 7: 92-8.

\section{AP5-8}

\section{Caffeic acid phenethyl ester reduces mortality and sepsis} induced injury in rats

H. Fidan, O. Sahin, Y. Yavuz, A. Kilbas, Z. Cetinkaya

Department of Anesthesiology, Afyon Kocatepe University, Afyon, Turkey

Background and Goal of study: Sepsis and ensuing multi organ failure continue to be the major causes of morbidity and mortality in the intensive care units. Nuclear factor-Kappa beta (NFKB) activation is supposed to be one of the targets in the treatment of sepsis and ensuing mortality. We studied the effectiveness of caffeic phenethyl ester (CAPE), a known NFKB inhibitor, in cecal ligation and puncture (CLP) induced sepsis and lung injury.

Materials and Methods: 80 rats are randomized to 5 groups that included 8 rats for the mortality study and the rest 8 rats for histopathological and biochemical study. All rats were operated to induce sepsis with cecal ligation and puncture (CLP) except control and CAPE groups that were operated just with laparotomy. CAPE $(50 \mu \mathrm{g} / \mathrm{kg})$ was administered to rats intramuscularly at the time of operation in CAPE and CAPE + Sepsis(0) groups. CAPE was administered to rats in CAPE + Sepsis (12)group 12 hours after CLP. Rats were observed for mortality. 8 rats from each group were sacrificed 24 hours after CLP; blood was taken for interleukin 1, IL-6, IL-10 and TNF- $\alpha$ study and right lung was taken out for histopathological, and left lung was taken out for oxidative stress parameters. Apoptosis was examined with Tunnel staining. Induced nitric oxide synthase(iNOS) and heat schock protein (HSP70) were examined with immunohistochemistry. Malonlydialdehyde (MDA), catalase (CAT), superoxide dismutase (SOD) and glutathione peroxidase (GSH-Px) were studied for oxidative stress evaluation. 
Results and Discussion: Mortality was significantly decreased in CAPE + sepsis(0) (3deaths/8rats) and CAPE + sepsis(12) (3deaths/8rats) groups compared to the sepsis group (8deaths/8rats). IL-1, IL-6, and IL-10 increased except TNF- $\alpha$ levels in sepsis group compared to control group. All cytokine levels were similar to control levels only in CAPE + sepsis(12) group. Apoptosis, iNOS and HSP70 evaluation were significantly changed between all groups in following order; control $<$ CAPE $<$ CAPE + sepsis(12) < CAPE + sepsis(0) < sepsis. SOD and GSH-Px levels were not different among groups. MDA, CAT were increased sepsis.

Conclusion: CAPE reduced mortality in sepsis and histopathological changes best when it was administered after sepsis formation.

\section{AP6-1}

\section{Microbiology Ward rounds within Critical Care}

L. Wilson, G. Dempsey

Critical Care, University Hospital Aintree, Liverpool, United Kingdom

Background: Direct microbiological input to critical care is essential for the management of the septic patient. Early broad spectrum antimicrobial therapy with appropriate diagnostic studies to ascertain causative organisms is well established, there should be reassessment with the aim of using narrow spectrum antibiotics to prevent the development of antimicrobial resistance, reduce toxicity and reduce costs(1). In systematic analysis of ward rounds in ICUs the information most commonly missing from a patient's file concerned microbiology findings(2).

Methods: We performed a telephone survey of all NHS critical care units in the North West of England, $(n=31)$. Each unit was telephoned and the duty consultant was asked a series of questions relating to the type of microbiology input to their critical care unit.

Results: We achieved a 100\% response rate.

The study looked at 11 teaching Hospitals \& 21 DGHs. representing $12 \%$ of UK ICUs.

$26(83 \%)$ critical care units had live computerised access to microbiology data.

$21(68 \%)$ units had an antibiotic policy in place.

$19(61 \%)$ units had a formal microbiogy ward round.

Frequency ranging from once per week, ( 1 unit) to 7 days per week, ( 4 units), most units with a microbiology ward round had this service monday-friday, (12 units).

When asked to rate the value of this ward round the mean score was 8.6 out of a possible 10. (Range 10-5, mode 9).

In those units without a microbiology ward round the desirability of such a service was scored on average at 8.5 out of 10. (Range 10-3, mode 9).

Conclusion: Direct microbiological advice at the bedside is highly valued by ICU consultants. Antibiotic prescribing is generally well controlled with $2 / 3$ rds of units having an agreed antibiotic policy in place. Work will continue to determine whether these results reflect the national picture in the UK.

\section{References:}

1 AF Widmer, Intensive Care Medicine, June 2005 pS7-11.

2 W Friesdorf J Clin Monit May 1994, 10(3): 201-9.

\section{AP6-2}

\section{Microbiology of intra-peritoneal comminatory peritonitis}

M. Daghfous, A. Cherif, Y. Saidi, M. Bamadhaj, A. Daoud

Anesthésie- réanimation, Hôpital Charles Nicolle, Tunis, Tunisia

The delay of surgery and efficient antibiotherapy may increase the severity of the peritonitis. That's why it's important for the physicians to check periodically the ecology of bacteria in the peritonitis to adapt the antibiotic strategy.

The aim of our study was to evaluate the bacteria ecology of the comminatory peritonitis treated in the abdominal surgery unit.

Materials and Methods: Study design: prospective. Inclusion criteria: all patients admitted in the visceral surgery unit with the diagnosis of peritonitis along three months. All patients included received firstly an association of three antibiotics including cefotaxim, gentamicin and imidazole before undergoing surgery. At the beginning of the intervention, the surgeon took a sample from peritoneal liquid witch was immediately underwent to bacteriological analysis.

Results: We included in this study 30 consecutive patients (23 males and 7 females). The diagnosis of peritonitis was confirmed by the surgery for all patients. The origin of the peritonitis was perforate duodenal ulcer $(20 \%)$, appendicitis $(23 \%)$, biliar peritonitis $(37 \%)$ and intestinal perforation for the other cases. The bacteriological analysis was positive for 16 patients $(53.3 \%)$ and negative for the others. The bacteriological analysis isolated 6 types of bacteria (4 streptococcus, 3 staphylococcus, 2 enterococcus, $4 \mathrm{E}$ coli, 2 klebsiella and 2 pseudomonas) and in one case, we identified a candida albicans. In three patients, the isolated bacteria were resistant to primary antibiotics and 2 patients died in the few postoperative days. We noted too, a high level of resistance to amoxicillin-clavulanic acid (31\%).

Conclusion: These results invite us to change our antibiotic strategy for comminatory peritonitis because of the high level of resistance of the bacteriological ecology mainly in severe peritonitis with organ failure.

\section{AP6-3}

\section{Effect of Enteral Nutrition in VAP and Immunologic Status}

Q. Morina, Z. Bukoshi, F. Sada, B. Bruqi, A. Morina

Anesthesia and ICU, University Clinical Center Prishtina, Prishtina, Serbia

Background and Goal of Study: Even the improvement of diagnosis, therapy and prevention measures the hospital acquired pneumonias cause high morbidity and mortality in the ICU. The aim of this study is the effect of enteral nutrition in VAP and immunologic status.

Materials and Methods: This prospective study is made in the ICU of the UCC in Prishtina. 43 mechanically ventilated patients are divided in two groups, gr. $A(n=21)$ did received enteral nutrition and gr. $B(n=22)$ received parenteral nutrition. At all of the patients included in the study is been evaluated the prognosis by APACHE II system in the first 24 hours from the admittance. The aim of the nutrition in both groups was $0.2 \mathrm{~g} \mathrm{~N} / \mathrm{kg}$ bw/day and $30-40 \mathrm{kcal} / \mathrm{kg} / \mathrm{day}$ of the non proteinic calories. The levels of the $\mathrm{lg}$ A and lymphocytes were followed up. Samples for microbiological studies have being taken from oropharyngs, aspirate of the NGS and trachea, in order to identify the path of colonization and later on the respiratory infections in two groups of patients.

Results and Discussions: Pneumonia had only 9 (42.8\%) of the enterally feeded patients compared with $18(81.1 \%)$ with parenteral nutrition, which is statistically significant. In gr. A 6 patients died, while 10 of them died from the gr. B. From the beginning levels of $\lg A$ from $1.84 \mathrm{~g} / \mathrm{L}$ on the $\mathrm{gr} A$ were raised on $2.10 \mathrm{~g} / \mathrm{L}$, while on the group $B$ the beginning levels of $\lg A$ from $1.85 \mathrm{~g} / \mathrm{L}$ were raised on $1.92 \mathrm{~g} / \mathrm{L}$.

Lower respiratory tract colonization in the gr. A was $33 \%$ and in the gr. B $50.1 \%$, with more pronounced from oropharynx flora than gastrointestinal flora.

Conclusion(s): Because enteral feeding is physiologic, less invasive, safer, has a good outcome in immunoglobulin secretion and for us it's important that is cheaper, lowers the incidence of septic complications and hospital acquired pneumonias.

\section{AP6-4}

\section{TPN as a vector of infection in ICU}

M. Staber, M. Whitelaw, F. J. deVilliers

Anaesthesia, Inverclyde Royal Hospital, Greenock, United Kingdom

Background and Goal of study: In our hospital ICU, TPN (total parenteral nutrition) infusions are administered over 24 hours at room temperature. We postulated that TPN it is a potential growth medium for pathogens and that contamination of infusion systems could lead to bloodstream infections. This study was undertaken to investigate the ability of common pathogens to establish and grow in TPN solution at room temperature.

Materials and Methods: NuTRIflex $®$ is the currently used TPN in our institution. It contains $20 \mathrm{~g} / \mathrm{l}$ soya oil, $20 \mathrm{~g} / \mathrm{l}$ medium chain triglycerides, $38 \mathrm{~g} / \mathrm{l}$ amino acids, and $120 \mathrm{~g} / \mathrm{l}$ glucose. Inoculated NuTRIflex ${ }^{\circledR}$ was incubated at constant room temperature $\left(21 \pm 1^{\circ} \mathrm{C}\right)$ and at $35^{\circ} \mathrm{C}$. Four common local pathogens (MRSA, $S$ maltophilia, $P$ mirabilis and $C$ albicans) were used at inoculum weights of $10^{4} \mathrm{cfu} / \mathrm{ml}$ throughout. Periodic subcultures were made onto blood (Oxoid), sabouraud (Oxoid) and cled (Oxoid) agars, Antimicrobial activity was determined using $10 \mathrm{ml}$ NuTRIflex® onto a blood agar seeded with $S$ aureus (ATCC 25923). Growth curves were performed with all four pathogens in undiluted NuTRIflex®. Subsequently, administration setups were contaminated at potential contamination sites (mechanical junctions) with equal inocula of the four pathogens. Systems were maintained at room temperature, sampled at $0,1,2,4,8$ and 24 hours onto appropriate media as above.

Results and Discussions: At room temperature $\left(21 \pm 1^{\circ} \mathrm{C}\right) \mathrm{C}$ albicans grew poorly, whilst MRSA, $S$ maltophilia, and $P$ mirabilis merely survived, even at inoculum weights 100 times greater than found on the hands of Health Care Workers in our hospital. At $35^{\circ} \mathrm{C}$ none of the pathogens grew exponentially in NuTRIflex® and no antibacterial activity was detected. No upstream migration occurred at room temperature over a 24-hour period.

Conclusions: $C$ albicans, MRSA, $S$ maltophilia, and $P$ mirabilis survived, but failed to grow exponentially and failed to migrate upstream in NuTRIflex $®$ infusion systems. At room temperature during the first 24 hours of administration, NuTRIflex $\AA$ can act as a vector but does not multiply the risk of bloodstream infection. 


\section{AP6-6}

The use of the laryngeal mask airway during percutaneous dilational tracheostomy: an alternative to the endotracheal tube

G. Thomaidis, K. Konstadinidis, V. Wiltzinski, M. Giaourakis, V. Bekos Intensive Care Unit, Naval \& Veterans Hospital of Athens, Athens, Greece Background and Goal of Study: Percutaneous Dilational Tracheostomy (PDT) facilitates the bedside insertion of a tracheostomy tube in the Intensive Care Unit. Accidental puncture of endotracheal tube (ETT) cuff and unintended tracheal extubation are the potential complications during (PDT) our purpose was to evaluate the safety and efficiency of the use of the laryngeal mask airway (LMA) during percutaneous dilatational tracheostomy under bronchoscopic guidance comparing with the ventilation via (ETT).

Materials and Methods: The bedside PDT was performed in 30 critically ill patients-15 in each group: LMA group and ETT group- that fulfilled the criteria for this study: $\mathrm{PaO}_{2}>100 \mathrm{mmHg}, \mathrm{PaCO}_{2}<45 \mathrm{mmHg}$ under intermittent positive pressure ventilation (IPPV) with a mean ventilation pressure of $<25 \mathrm{mmHg}$.

The positioning of the LMA was done at least 30 min before the tracheostomy was performed.

Blood samples for arterial blood gas analyses were taken $5 \mathrm{~min}$ before the procedure (first value) and just after $(1 \mathrm{~min})$ the insertion of tracheostomy tube (second value). Mean arterial pressure (MAP), heart frequency (HF) and peripheral oxygen saturation $\left(\mathrm{SpO}_{2}\right)$, endexpiratory $\mathrm{CO}_{2}$ and minute ventilation volume (MVV) were registered every 60 seconds.

Results and Discussions: There was no significant difference in MAP, HF $\mathrm{SpO}_{2}, \mathrm{pH}, \mathrm{PaO}_{2}$, or $\mathrm{PaCO}_{2}$ between groups before the procedure. The operating time was significantly shorter in LMA group $(5.5 \pm 0.8 \mathrm{~min}$ versus $7.9 \pm 1.4 \mathrm{~min}, \mathrm{P}<0.05$.). Hypercarbia was noted in $33.3 \%$ in the LMA group and $26.7 \%$ in the ETT group.

Conclusion(s): The LMA provides a safe and effective alternative to an endotracheal tube for airway management during guidewire dilatating forceps tracheostomies in selected patients and prevents the difficulties associated with the use of ET such as cuff puncture, tube transection by the needle, and accidental extubation.

\section{AP6-7}

Comparison of sensitivity and specificity of the protected specimen brush (PSB) technique and the tracheo-bronchial aspirate (TBAS) in the diagnostics of ventilator associated pneumonia (VAP)

B. Tamowicz, K. Wronka, J. Tyczka, A. Mikstacki

Department of Anaesthesiology and Intensive Therapy, Karol Marcinkowsk University of Medical Sciences, Poznan, Poland

Background and Goal of Study: Invasive bronchoscopic techniques improve early prognosis in critically ill patients with suspected VAP by reducing antibiotic therapy and its costs and suppressing the growth of resistant microorganisms (1). The aim of the study was to compare sensitivity and specificity of the PSB technique with the TBAS method in the diagnostics of VAP.

Materials and Methods: The prospective study included 50 patients with the predicted ventilation period of at least 6 days. VAP was confirmed using clinical, radiological and microbiological criteria. Material from the lower respiratory tract was obtained using the TBAS technique and PSB on the $1^{\text {st }}, 4^{\text {th }}$ and $6^{\text {th }}$ day of therapy (a total of 150 samples). Significance of differences in specificity and sensitivity of used methods was assessed by test of structural indicators.

Results and Discussions: VAP was confirmed in 16 patients. The following microorganisms were isolated from the material obtained using the PSB technique: MSSA, S. agalactiae, P. aeruginosa, P. mirabilis, A. baumanii, E. aerogenes. The following were isolated using the TBAS technique: MSSA, MRSE, S. agalactiae, P. aeruginosa, Enterobacter spp., P. mirabilis, A. baumanii, K. pneumoniae, E. coli, E. faecalis. The sensitivity of the PSB technique was $90 \%$ and its specificity - 95\%, while the sensitivity of the TBAS technique was $85 \%$ and its specificity - $65 \%$. The difference between specificity of both techniques was statistically significant $(p<0,01)$. Analogical difference between sensitivity was not statistically significant.

Conclusion(s): High sensitivity and specificity of the PSB technique in comparison with the TBAS method confirm the suitability of invasive techniques in the diagnostics of VAP.

Reference:

1 Torres A, Ewig S: Diagnosing ventilator-associated pneumonia. N Engl J Med, 2004; 350: 433-435.

\section{AP6-8}

Central venous catheter infections in cancer patients - the ICU and the Wards or is management an issue?

R. Zielinski, M. Symonides, M. Misiak, A. Lukaszewska, P. Sowinski

Anaesthesiology and Intensive Care, Institute of Oncology, Warsaw, Warsaw, Poland

Background: Central venous catheters (CVC) are a common site of infection, with inadequate management listed as one of the most common causes. Cancer pts. are believed to be at an increased risk of infection due to immunosuppression.

Goal of the study: To assess the risk of CVC infections in cancer pts and to evaluate whether upgraded management (by anaesthesiologists \& ICU nurses) reduces the risk of CVC infection.

Material and method: We performed a prospective analysis of all catheters introduced and removed over a period of 12 months in a 750-bed oncological hospital. Cultures were obtained from all removed catheters.

Results: Overall number of inserted catheters - 253; overall CVC infection rate $-5.92 \%$ ( 15 cases). The CVC patients were divided into 2 groups: group I-204 pts. after elective CVC introduction in the operating theatre (189 jugular, 14 subclavian, 1 femoral) for chemotherapy and/or parenteral nutririon and group II - 49 pts. - non-elective introduction in the operating theatre due to intraoperative complications (28 pts. -24 jugular, 4 subclavian) and in the ICU due to emergency admissions - (21 pts - 19 jugular, 2 subclavian). All group II pts. were hospitalised in the ICU (duration 6-513 days). In Group I the CVC infection rate was $1.47 \%$ ( 3 cases) and in Group II - 24\% (12 cases), of which 5 cases $(10 \%)$ occurred in the ICU and $7(14 \%)$ cases in the wards after discharge from ICU. Summarising: in the ICU there were 5 infections per 1630 CVC-patient-days (all emergency infections); in the wards there were 10 infections per a total of 1630 CVC-patient-days ( 3 elective insertions and 7 emergency insertions).

Conclusions: (I) Non-elective, emergency-related CVC introduction in cancer pts. is associated with a high infection rate. (II) Correct CVC maintenance as recommended and performed in the ICU facilitates an acceptable infection rate.

\section{AP7-1}

Intra-peritoneal microdialysis for detection of post-operative mesenteric ischaemia

J. Mccormack, P. Andrews, T. Aziz, G. Wilson

Anaesthesia, Critical Care and Pain Medicine, University of Edinburgh, Edinburgh, United Kingdom

Background and Goal of Study: Intra-peritoneal microdialysis (IPMD) identifies elevations of peritoneal fluid lactate/pyruvate ratio, a maker of mesenteric ischaemia (1). This observational study assessed the utility of IPMD following colorectal surgery.

Materials and Methods: 11 patients having elective left sided colorectal resection with primary anastomosis in a university teaching hospital were recruited to have IPMD monitoring for up to 48 hours post-operatively. Concurrent physiological and biochemical parameters were recorded.

Results and Discussions: Median (range) age was 60 (29-76) years and median (range) length of hospital stay was 11 (6-32) days. IPMD sample collection demonstrated a mean $(95 \% \mathrm{Cl})$ intraperitoneal lactate/pyruvate ratio of 24 (17-30), normal range being $19 \pm 4$. Two patients developed early postoperative anastamotic complications, displaying elevations in lactate/pyruvate ratio of $240 \%$ and $177 \%$ over baseline, these episodes associated with concurrent periods of systemic hypotension. A rise of greater than $60 \%$ in lactate/ pyruvate ratio has been shown to be suggestive of mesenteric ischaemia (1). No other patients demonstrated elevations of lactate/pyruvate ratio to this extent. Primary pathology, volume of fluid administration, use of vasopressor therapy and use of epidural analgesia was not shown to significantly affect IPMD results.

Conclusion(s): Post-operative intra-peritoneal microdialysis analysis performed in the critical care unit highlighted that in two patients, one with clinical suggestion of mesenteric ischaemia and one with a confirmed anastamotic leak, an early elevation of lactate/pyruvate ratio in the post-operative period was recorded. Further evaluation of IPMD as an adjunct to guiding administration of fluid or vasoactive therapies in the critical care unit following colorectal resection is warranted.

Reference:

1 Sommer T, Larsen JF. Validation of intramural intestinal microdialysis as a detector of intestinal ischaemia. Scand.J.Gastroenterol. 2004; 39: 493-9. 


\section{AP7-2}

Is intrathoracic blood volume index an ideal target parameter of resuscitation after burn injury?

V. Foldi, C. Csontos, T. Fischer, L. Bogar

Department of Anaesthesia and Intensive Care, Faculty of Medicine, University of Pécs, Hungary, Pecs, Hungary

Introduction: A number of target parameters are used for fluid replacement in severely burned patients. The aim of our prospective randomised study was to examine the effect of two types of fluid resuscitation regimes on the central venous oxygen saturation of the haemoglobin $\left(\mathrm{ScvO}_{2}\right)$ and multiple organ dysfunction score (MODS).

Materials and Methods: Twenty four consecutive patients were involved in the study. Inclusion criteria were the presence of burn injury affecting more that $15 \%$ of body surface area. Exclusion criteria were chronic left heart or renal insufficiency, age under 18 years, and acute renal failure developed within three days after injury. In Group I $(n=12)$ the fluid resuscitation was guided by the hourly urine output, in Group II $(n=12)$ by the intrathoracic blood volume index (ITBVI). Results: Mean $\mathrm{ScvO}_{2}$ was significantly lower in Group I than in Group II $(p<0.05)$ in the first 24 hours. MODS was significantly higher in Group I than in Group II calculated at 48 hours $(p<0.05)$ and 72 hours after injury $(p<0.05)$. The two main outcome parameters i.e. MODS calculated at 48 and 72 hours after injury were in a significant negative linear correlation with $\mathrm{ScvO}_{2}$ measured on day $1(r=-0.684, p<0.01 ; r=-0.677, p<0.01$, respectively). Significant linear correlation was found between ITBVI and $\mathrm{ScvO}_{2}(r=0.855$, $p<0.001)$, ITBVI and cardiac index $(r=0.491, p<0.001)$, ITBVI and left ventricular contractility index $(r=0.523, p<0.001)$. Extravascular lung water index did not show significant differences between Group I and II on the first and second days after admission. No sign of fluid overload were detected neither in Group I nor in Group II. Hourly urine output, central venous pressure and central venous pressure minus positive end expiratory pressure did not show any correlation neither with $\mathrm{ScvO}_{2}$ nor with other hemodynamic parameters.

Conclusion: Our data suggest that ITBVI may be a better target parameter than hourly urine output in fluid resuscitation of severely burned patient in the first two days.

Reference:

Holm C, Mayr M, Tegeler J, et al. A clinical randomised study on the effects of invasive monitoring on burn shock resuscitation. Burns 2004; 30: 798-807.

\section{AP7-3}

Picco PLUS monitoring hemodynamic parameters in critically ill patients

S. Pirri, A. Mazzola, A. Barcia, S. Salemi

Anesthesiology and Intensive Care, POliclinico Universitario, Palermo, Italy

Background and Goal of Study: To assess Picco PLUS monitoring hemodynamic parameters in critically ill patients.

Materials and Methods: 10 patients with hemodynamic instability $(7 \mathrm{M}, 3 \mathrm{~F}$, age 52-79 years) admitted to ICU for more than 48 hours, were enrolled and divided into 3 groups. Gr1: cardiac pathology (5); Gr2: septic with a positive haematic, BAL or urinary colture; Gr3: post-surgicals with history of intraoperative haemorrhage (2). All the patients have been subjected to a careful clinical surveillance, a baseline and a Picco PLUS monitoring hemodynamic.

Results and Discussions: Gr1: impressive hypotension with cardiac index (CI)(I/min $/ \mathrm{m}^{2}$.) between 1.86 and 2.94, CFI (l/min) under 4.5, GEDVI $\left(\mathrm{ml} / \mathrm{m}_{2}.\right)>$ 1000, ITBVI $\left(\mathrm{ml} / \mathrm{m}_{2}.\right)>1200$, EVLWI between 8 and 18. Echocardiography M-B mode and trans-thoracic Doppler evidenced indices of depressed systolic function (EF between 22 and 25\%). Such parameters have confirmed a deficit of cardiac pump. In particular on the base of the $\mathrm{Cl}$ and CFI values we have been able to establish a treatment with inotropic and vasoactive drugs, to titrate their administration, and by the GEDVI and its relationship with EVLW to manage the fluid budget accurately. Values of EVLWI $(\mathrm{ml} / \mathrm{kg})<10$ have been considered normal, while values $>10$ came deals with diuretic on indication of the other hemodynamic and volumetric parameters: some patients have been treated with dobutamine, others with levosimendan. Gr2: hemodynamic instability with $\mathrm{Cl}$ between 2.04 and 4.02, CFI between 4 and 7, SVRI $<1000$. Such parameters have confirmed the diagnosis of massive peripheral vasodilatation (septic origin), and in relation to the values suggested the necessity to associate vasopressor and inotropic drugs. Gr3: hypotension with values of $\mathrm{Cl}<3$, GEDVI $<500$, ITBVI $<600$, SVRI $>1500$ : presence of detached hypovolaemia from intraoperative haematic losses. The evidence of a reduction of preload let us concurred an accurate management of the fluid budget.

Conclusion(s): Our data suggest that Picco PLUS monitoring hemodynamic parameters is an accurate less invasive monitoring technique.

Reference:

Intensive Crit Care Nurs 2003, 19: 301-307.

\section{AP7-6}

Compliance with NICE guidelines on the use of ultrasound locating machines in central venous line placement.

J. Kurian, A. Rashid, C. Oti, G. Kumar, M. MacGregor

Anaesthetics and Critical Care, East Surrey Hospital NHS trust, Redhill, United Kingdom

Background and Goal of Study: In August 2002 NICE (National Institute for Health and Clinical Excellence) issued guidance, on the use of ultrasound locating devices for placing central venous catheters (CVC) (1). The goal was to observe compliance with NICE guidelines.

Materials and Methods: Data base analysis of $50 \mathrm{CVC}$ insertion procedures done on adult patients during a one month period from 21/12/05 to 20/01/06 to observe the compliance with NICE guidelines.

Results and Discussions: Of the 50 procedures on which data was collected, only 20 were documented to be done under ultrasound guidance. 9/13 (69.23\%) elective procedures and $7 / 17$ (41.2\%) of emergency procedures done in theatres were done under ultrasound guidance. In the ITU only 4/17 (23.57\%) procedures were documented to have been done under ultrasound guidance.

Table 1 Ultrasound guided CVC insertions

\begin{tabular}{lll}
\hline & No of patients & Documented use of Ultrasound \\
\hline Total no of Patients & 50 & 20 \\
Elective & 13 & 9 \\
Emergency & 17 & 7 \\
ITU/SSDU/A\&E & $17+2+1$ & $4+0+0$ \\
Theatre & 28 & 14 \\
Recovery & 2 & 2 \\
A\&E & 1 & 0 \\
Surgical step down unit & 2 & 0 \\
ITU & 17 & 4 \\
More than one attempt. & 6 & 3 \\
\hline
\end{tabular}

Conclusion(s): In spite of adequate provision of equipment and consumables there is poor compliance with NICE guidance by the anaesthetists in on using ultrasound guidance for CVC insertion.

Reference:

1 National Institute for Clinical Excellence. Guidance on the use of ultrasound locating devices for placing central venous catheters. London: NICE, 2002. [NICE Technology Appraisal No 49.]

\section{AP7-7}

Intra-abdominal preasure as risk factor for hemodynamic, respiratory and renal complications after emergency major abdominal surgery $\mathbf{4 8}$ hours postopertive

I. Filipovic-Grcic, D. Tonkovic, V. Majeric Kogler

Anesthesiology and Intensive Care, Clinical Hospital Center Zagreb,

Zagreb, Croatia

Background and Goal of Study: Intraabdominal hypertension and compartment syndrome are considered as a risk factors for poor postoperative outcome after abdominal surgery. Clinical evaluation of intraabdominal hypertension can easily be done by the measurement of intraabdominal pressure (IAP) via urinary bladder. The aim of this study was to evaluate IAP as a risk factor for hemodynamic, respiratory and renal complications after emergency major abdominal surgery $48 \mathrm{hr}$ postoperative.

Materials and Methods: In this prospective study, to date, are reported 30 patients.

IAP was measured after $24 \mathrm{hr}$ and $48 \mathrm{hr}$ postoperative. Intra-abdominal hypertension were graded as mild $(10-14 \mathrm{mmHg})$ and severe $(15-25 \mathrm{mmHg})$. We considered hemodynamic, respiratory and renal complications as follows: MAP $<60 \mathrm{~mm} \mathrm{Hg}$, need for mechanical ventilation and urinary output $<$ $1 \mathrm{ml} / \mathrm{kg} / \mathrm{h}$. Data are reported as mean values $+/-$ standard deviation. For comparison of IAP and organ functions we used Student's t-test and MannWhitney U-test.

Results and Discussions: Average age of patients was $66.35+/-16.33$, range 19-84. All patients had increased values of IAP, 18 patients mild and 12 patients severe one. Hemodynamic complications were found in 4 patients, respiratory in 12 patients and renal in 13 patients. Values of IAP were not statistically significant associated with hemodynamic, respiratory and renal complications $(p>0.5)$.

Conclusion(s): Increased IAP was not found to be a risk factor for hemodynamic, respiratory and renal complications after emergency major abdominal surgery 48 hours postoperative.

References:

Cullen DJ, Coyle JP, Teplick R, Long MC. Crit Care Med 1989; 17: 118-21.

Malbrain ML. Curr Opin Crit Care 2004; 10: 132-45.

Malbrain ML, Chiumbello D, Pelosi P, Wilmer A, Brienza N, Malcangi V et al. Intensive Care Med 2004; 30: 822-9. 


\section{AP7-8}

Changes in serum lactate, mix-venous oxygen saturation and indocyanin green plasma disappearance rate in postoperative cardio surgical patients

O. Thuemer, C. Schelenz, S. Sakka

Anaesthesiology and Intensive Care, Friedrich-Schiller-University Jena, Jena, Germany

Background and Goals: Parameters of global and regional, in particular hepato-splanchnic, blood flow may be used for guiding therapy in critically ill patients. However, regional conditions may not be adequately reflected by global parameters. In this study, we analysed changes in cardiac index (Cl), mixed-venous oxygen saturation $\left(\mathrm{SvO}_{2}\right)$, serum lactate, gastric mucosal $\mathrm{PCO}_{2}\left(\mathrm{P}_{\mathrm{R}} \mathrm{CO}_{2}\right)$ and indocyanine green plasma disappearance rate (ICGPDR) in patients after cardiac surgery.

Methods: With ethics approval and written consent we studied 52 patients (39 male, 13 female, age $65 \pm 10$ years). All patients underwent pulmonary artery catheterisation for clinical indication. Serum lactate and $\mathrm{SvO}_{2}$ were determined by a blood gas analyser. ICG-PDR was measured by a transcutaneous system and $\mathrm{P}_{\mathrm{R}} \mathrm{CO}_{2}$ by air tonometry. All parameters were determined immediately postoperatively on the ICU and 2 hours later. Linear regression was used for statistical analysis. A $p<0.05$ was considered statistically significant.

Results: Cardiac index $\left(2.8 \pm 0.8\right.$ vs. $\left.3.2 \pm 0.7 \mathrm{l} / \mathrm{min} / \mathrm{m}^{2}\right)$, serum lactate $(1.9 \pm 1.2$ vs. $2.4 \pm 1.6 \mathrm{mmol} / 1)$ and $\mathrm{P}_{\mathrm{R}} \mathrm{CO}_{2}(5.2 \pm 1.0$ vs. $5.6 \pm 1.1 \mathrm{kPa}) \mathrm{sig}$ nificantly increased during the study period while $\mathrm{SvO}_{2}(66 \pm 7$ vs. $68 \pm 6$ $\%)$ and ICG-PDR ( $21.4 \pm 6.9$ vs. $21.8 \pm 7.4 \% / \mathrm{min})$ remained unchanged Furthermore, central venous pressure $(8 \pm 3$ vs. $8 \pm 4)$ and haemoglobin content $(6.2 \pm 0.9$ vs. $6.0 \pm 0.8 \mathrm{mmol} / \mathrm{l})$ did not change significantly. However body temperature $\left(36.0 \pm 0.8\right.$ vs. $\left.36.6 \pm 0.7^{\circ} \mathrm{C}\right)$ and dosages for norepinephrine (mean 0.04 vs. $0.02 \mu \mathrm{g} / \mathrm{kg} / \mathrm{min}$ ) and epinephrine (mean 0.02 vs $0.03 \mu \mathrm{g} / \mathrm{kg} / \mathrm{min}$ ) changed significantly. The changes in $\mathrm{SvO}_{2}$ and $\mathrm{Cl}$ correlated moderately $(r=0.43, p<0.001)$. However, no correlation was found between changes in $\mathrm{Cl}$ and ICG-PDR $(r=0.07, \mathrm{p}=0.62)$ and $\mathrm{Cl}$ and lactate $(r=0.37, p=0.91)$. The correlation between changes in $\mathrm{Cl}$ and $\mathrm{P}_{\mathrm{R}} \mathrm{CO}_{2}$ was $r=0.37(p=0.006)$. There was no relationship between changes in variables of global oxygen transport and regional blood flow: $r=0.06(p=0.65)$ for $\Delta \mathrm{ICG}-\mathrm{PDR} / \Delta \mathrm{SvO}_{2}$, and $\mathrm{r}=0.009(\mathrm{p}=0.95)$ for $\Delta \mathrm{ICG}-\mathrm{PDR} / \mathrm{\Delta lactate}$.

Conclusion: Changes in serum lactate, $\mathrm{SvO}_{2}$, ICG-PDR and $\mathrm{P}_{\mathrm{R}} \mathrm{CO}_{2}$ do not correlate with changes in cardiac index and also changes within regional parameters are not reflected by each other. Thus it is not possible to draw definite conclusions from one to the other parameters.

\section{AP7-9}

Stroke volume variations obtained with Vigileo ${ }^{\circledR}$ monitor predicts fluid responsiveness in critically ill ventilated patients

M. Biais, K. Nouette-Gaulain, V. Cottenceau, P. Revel, F. Sztark

Réanimation chirurgicale, Pellegrin, Bordeaux, France

Background and Goal of Study: Optimal monitoring of cardiac preloaded is paramount for precise hemodynamic management. Dynamic indices such as stroke volume variation (SWV) usually predict adequately the individual response to fluid challenge FC. The aim of this study was to assess whether SW obtained with the new Vigileo $₫$ monitor can predict fluid responsiveness.

Materials and Methods: Inclusion criteria were mechanically ventilated patients undergoing liver transplantation and clinical requirement for a rapid fluid challenge $(20 \mathrm{ml} x$ body mass index (BMI) of $4 \%$ human albumin over $20 \mathrm{~min}$ ). Exclusion criteria were patients younger than 18 years, arrhythmias, BMl upper than $40 \mathrm{~kg} / \mathrm{m}^{2}$ or less than $15 \mathrm{~kg} / \mathrm{m}^{2}$, left ventricular systolic dysfunction, valvular heart disease, intracardiac shunt and spontaneous breathing activity. Patients were monitored with pulmonary arterial catheter (PAC) and with Vigileo ${ }^{\circledR}$ monitor. Measurements of CO, CVP, PAOP with PAC, of $\triangle$ VPeak with transthoracic echocardiography, of MAP and DeltaPP, and of SV with Vigileo ${ }^{\circledR}$ were made before and after FC. Patients were separated into responders $(\mathrm{R})$ and non-responders $(\mathrm{NR})$ according to the change in CO: $\geqslant 15 \%$ or $<15 \%$ after FC. Statistical analysis was made using Wilcoxon test and Spearman rank test. $\mathrm{p}<0.05$ was considered significant.

Results and Discussions: Sixteen patients $(13$ males $/ 3$ females; age $50 \pm 8$ years) were enrolled. 9 were NR and 7 were R. SVV before FC were significantly higher in responder patients $(p<0.05)$. Change in cardiac output after FC was significantly correlated with SVV before FC $\left(r^{2}=0.88\right.$ $\mathrm{p}<0.001)$. A SVV value up to $9 \%$ predicted responsiveness in FC with $100 \%$ sensitivity and $89 \%$ specificity.
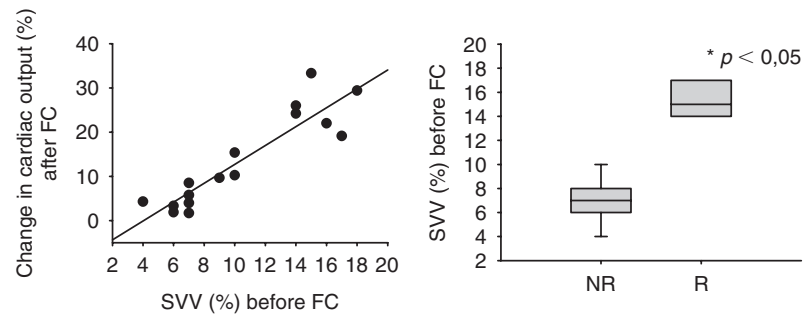

Conclusion(s): SVV obtained with Vigileo ${ }^{\circledR}$ monitor is an accurate method for predicting hemodynamic effects of volume expansion in mechanically ventilated patients.

\section{AP7-10}

"Liver dysfunction in critical Care Unit: relationship between PDR (indocyanine green plasma disappearance rate), with LIMON $^{\odot}$ monitor (Pulsion ${ }^{\odot}$ ), bilirubin and mortality"

L. Blasco, G. Aguilar, M. Ballester, J. Llorens

Anesthesiology and Critical Care, Hospital Clinico Valencia, Valencia, Spain Background and Goal of Study: In Critical Care Units (CCU), liver function (LF) is evaluated with bilirubin which can be easily modified and it wastes time in laboratory. Indocyanine Green (IG) Plasma Disappearance Rate (PDR) measured with LIMON $^{\odot}\left(\mathrm{PULSION}^{\odot}\right)$ by pulse densitometry is a newly non-invasive technique to assess LF at patient's bedside in few minutes (1). This prospective study was aimed to evaluate whether this method could be comparable to bilirubin as a marker of liver dysfunction in CCU.

Materials and Methods: 15 patients (8M, 7F) were included in 4 groups according to their initial diagnosis: 6 Cardiac Surgery (age 42-81); 1 Severe Brain Trauma (age 82); 6 Septic Shock (age 51-79); 2 Liver/Bile/Pancreatic Surgery (age 65-69). We measured PDR $(0.5 \mathrm{mg} / \mathrm{kg}$ of IG in a central catheter twice a day) and bilirubin levels once a day. Survivor: patient who was discharged from the unit alive. APACHE II was performed in SFAR web. Taking into account the worst results, Pearson coefficient of correlation was used for statistical analysis.

Results and Discussions: Preliminary data are given as Mean \pm SD. PDR and bilirubin are correlated $\left(r_{1}\right)$. Lower values of PDR were founded in non survivors and liver surgery, followed by sepsis. PDR and severity is not enough $\left(r_{2}\right)$. At least $50 \%$ of patients had liver dysfunction (PDR $<18 \% / \mathrm{min}$ ).

\begin{tabular}{lccc}
\hline & PDR $(\% / \mathrm{min})$ & $\mathrm{BL}(\mathrm{mg} / \mathrm{dl})$ & APACHE II \\
\hline Cardiac. & $17,1 \pm 4,8$ & $1,1 \pm 0,63$ & $15,6 \pm 5,1$ \\
Liver & $8,4 \pm 5,8$ & $10,8 \pm 13$ & $12,5 \pm 9,2$ \\
Sepsis & $15,6 \pm 5,7$ & $1,1 \pm 1,1$ & $19,17 \pm 3,2$ \\
BT & $\mathbf{1 6 , 3}$ & $\mathbf{0 , 5}$ & $\mathbf{2 5}$ \\
Survivor & $16,3 \pm 4,9$ & $1,1 \pm 0,57$ & $16,8 \pm 5,6$ \\
No Surv & $8,9 \pm 6,6$ & $11,8 \pm 11,6$ & $20,5 \pm 2,1$ \\
Total & $15,3 \pm 5,5$ & $2,5 \pm 4,9$ & $17,3 \pm 5,4$ \\
\hline
\end{tabular}

(PDR vs BL) $r_{1}=-0.581$ (significant for $n=15$ with $p<0,05$ )

(PDR vs APACHE II) $r_{2}=0,21$ (non-significant with $p<0,05$ )

Conclusions: In critically ill patients, increased bilirubin levels are wellcorrelated with a poor percentage of PDR, but not with severity. PDR could be used as a fast marker of global LF in scores. More patients must be studied. Reference:

1 Sakka SG. Chest 2002; 122:1715-1720.

\section{AP8-1}

\section{HDU bed requirement after major spine surgery}

S. Sebastian, A. Kozhikunnath, M. Peters

Anaesthetics, Manor Hospital, Walsall, United Kingdom

Background and Goal of Study: Post spinal surgery intensive care requirement in a general hospital is different from that of a spine unit $(1,2)$. Aim of this study was to find out the HDU admission rate after elective major spine surgery and to determine any surgical factors contributing to the need for HDU admission in a UK District General Hospital.

Materials and Methods: Retrospective chart review of elective spine surgery patients booked for HDU bed, during an 18 month period in 2005-06.

Results and Discussions: Of the 32 cases, only $13(41 \%)$ went to HDU or ICU and only $7(22 \%)$ of them needed any specific intervention there. HDU admission rate was $13 \%$ for ASA grades 1 and 2 , but $65 \%$ for grade 3 patients. The 
type of surgery did not show any specific correlation with HDU requirement. None of the patients who went back to the ward needed HDU care later. Conclusion(s): The preoperative general condition of the patient is more important than the type of surgery determining the need for HDU care after major spine surgery and most of the patients could be managed in an appropriate surgical extended care unit.

References:

1 Helm RH, Newman RJ. Injury. 1992;23(8):515-7

2 Butler J, McMahon C, Marsh B, et al. Critical Care 2006, 10(Suppl 1):P37

\section{AP8-2}

New-onset atrial fibrillation in the Intensive Care Unit: a survey of current practice in the United Kingdom

J. Kinnear, D. Higgins, A. Stone, M. Armstrong

Anaesthetics, Southend Hospital NHS Trust, Westcliff-on-sea, United Kingdom

Background and Goal of Study: New-onset atrial fibrillation (AF) is a common problem in the ICU environment, with no universally accepted guidelines on its management. This study sets out to elucidate current practice in its management in the UK.

Materials and Methods: The lead clinicians of 126 ICUs in the UK responded to a questionnaire regarding their management of new-onset AF. It addressed: type of hospital and unit, whether there was a formal treatment protocol in place, satisfaction with the current management plan, the therapeutic target, and the immediate management strategy for the unstable and stable patient. Participants were then asked to rank their treatment preference from a list of drugs that have been described in the literature for the management of AF. Results and Discussions: Of the 126 respondents, 96 described their hospitals as District General Hospitals, with the remaining 30 as teaching institutions. Most units (122) were mixed medical-surgical. When asked about which would be regarded as a satisfactory outcome following treatment, reversion to sinus rhythm was chosen by 112 , with rate control of $90-100 /$ minute (56) as a second best option. With regard to the immediate treatment of acuteonset $\mathrm{AF}$, there was a preference for electrical cardioversion for the unstable patient (92), and chemical cardioversion for the stable (83). Where drugs were thought appropriate, there was a clear preference for either amiodarone (73) or magnesium (51) as the first choices [ranked results shown in the table].

\begin{tabular}{lrrrr}
\hline Ranked choice of drug & 1st & 2nd & 3rd & 4th \\
\hline Amiodarone & 73 & 38 & 8 & 3 \\
Magnesium & 51 & 28 & 20 & 4 \\
Digoxin & 6 & 37 & 44 & 19 \\
B-Blockers & 3 & 14 & 19 & 32 \\
Calcium channel blocker & & 3 & 6 & 6 \\
Flecainide & & 1 & 2 & 4 \\
sotalol & 1 & 6 & 7 \\
\hline
\end{tabular}

Conclusion(s): Our survey has confirmed that a uniform approach to the management of new-onset AF is lacking, and that it may be time to arrive at a consensus for the treatment of this common problem.

\section{AP8-3}

Applicability of the Acute Physiology and Chronic Health Evaluation II (APACHE II) and simplified Acute Physiology Score II (SAPS II) in predicting hospital mortality for neurosurgical Intensive Care Unit (ICU) patients

D. Kim, J. Shim, J. Suh, H. Yi

Anesthesiology, Neurosurgery, Hanyang University Medical Center, Seoul, Republic of Korea

Background and Goal of Study: To study the applicability of APACHE II and SAPS II in whole patients and neurosurgical patients who were admitted to the surgical ICU (1).

Materials and Methods: Retrospective investigation was conducted to 672 patients who were admitted to the neurosurgical ICU. Data were collected during the first hours of the admission and were calculated to obtain predicted mortality. First, in a whole ICU patients predicted mortality was compared each other and, univariate and multivariate analyses were conducted on patients with SAH and TBI.

Results and Discussions: Observed mortality was $24.8 \%$ whereas predicted mortalities by the APACHE II and SAPS II were $37.7 \%$ and $38.4 \%$. Calibration curve was close to the line of perfect prediction. SAPS II was not statistically significant in Lemeshow-Hosmor test, but slightly favor in AUC. In 2 subsets of patients, univariate and multivariate analyses showed that the strongest independent predictor for mortality was SAPS II.

Conclusion(s): In this study, both systems can be used to predict mortality and measure performance in the neurosurgical ICU some extent, but not in the individual basis. The main reason for such discrepancy is the fact that the outcome of NICU patients would not simply follow the initially given physiologic parameters. A certain factor (natural healing or following resuscitation) are deemed responsible for improvement after initial ictus(2).

References:

1 Knaus WA, et al. Crit Care Med; 1985; 13: 818-829.

2 Yi HY, et al. J Neurosurgery 2006; 59: 838-845.

\section{AP8-4}

Quality of life 6 months after surgical intensive care

F. Abelha, P. Maia, C. Santos, M. Castro

Anaesthesiology and Intensive Care, Hospital de São João, Porto, Portugal

Background and Goal of Study: Apart from mortality, Health Related Quality of Life (HRQOL) has increasingly been claimed to be an important outcome variable. The aim of this study was to assess HRQOL and activities of daily living (ADL) and identify its determinants after discharge from a surgical intensive care unit (ICU).

Material and Methods: All 375 post-operative adult patients, admitted to a surgical ICU between October 2004 and July 2005, were eligible for the study. From 333 hospital survivors, 226 completed the questionnaires. The following variables were recorded on admission: age, gender, ASA physical status (ASA), type and magnitude of surgical procedure; ICU and hospital length of stay (LOS), mortality and Simplified Acute Physiology Score II (SAPSII). Six months after discharge, a Short Form-36 questionnaire (SF-36) and a questionnaire to assess the dependency on ADL was sent to all survivors. Means, medians, standard deviations, and ranges were calculated for SF-36. Comparisons between patients groups were performed with nonparametric tests. A logistic regression analysis was performed to identify covariate effects of each variable on dependency for ADL tasks and for the change in health question of SF-36.

Results and Discussion: From all the patients who survived (333), 226 completed the questionnaires. Fifty nine percent of patients reported that their general level of health was better six months after discharge from hospital. Patients with greater co-morbidities (ASA III/IV), had lower SF-36 scores in all domains and were more often dependent in instrumental and personal ADL tasks. Logistic regression showed that SAPS II was associated with health changes (OR 1.06, 95\% Cl 1.01-1.11, $\mathrm{p}=0,016)$. Six months after discharge from ICU, $60 \%$ and $34 \%$ of patients were dependent in at least one activity in instrumental ADL, and in personal ADL, respectively. ASA (OR de 3.00, $95 \% \mathrm{Cl} 1.31-6.87, \mathrm{p}=0.009$ ) and age (OR de $2.36,95 \% \mathrm{Cl}$ de 1.04 a 5.34 , $\mathrm{p}=0.04)$ were associated with dependency in ADLI and for ADLP only ASA (OR de $4.58,95 \% \mathrm{Cl}$ de $1.68-12.46, \mathrm{p}=0.003$ ) was associated with more dependency.

Conclusions: ICU variables and patients' background were significant determinants of HRQOL. ASA physical status, age, type of surgery and LOS could be seen as determinants of HRQOL.

\section{AP8-5}

\section{National Survey of Standards of Inter-hospital Transfers followed by Critical Care Networks in England}

R. Ramaiah, Z. Jummani, W. Lum Hee

\section{Anaesthesia, Bradford Royal Infirmary, Bradford, United Kingdom}

Background and Goal of study: The Intensive Care Society (ICS) of England has published guidelines for the transport of critically ill patients outside of the normal critical care environment (1). It recommends that all equipment and transport documentation across a critical care network be standardized. Our objective was to find out if the ICS recommendations were followed by the Critical Care Networks in England.

Materials and Methods: A survey of the 29 Critical Care Networks within England was made to establish whether the transfer equipment used by the hospitals within their networks were standardised and conformed to ICS guidelines. We asked whether the networks were using local or Intensive Care Society checklists for transferring critically ill patients. We also checked whether the networks had any guidelines for the provision of emergency drugs for use during the inter-hospital transfer of critically ill patients.

Results and Discussion: 16 out of 29 networks responded to our questionnaire. Only 6 out of the 16 networks had their transfer equipment standardised as recommended by the ICS. 10 of the 29 networks had recommendations for the checking of the transfer equipment but these varied. All 16 networks had local checklists which conformed to the pre-transfer checklists recommended by the ICS, and which they claimed were used by all hospitals within their networks for transferring patients between hospitals. However, only 6 of them had audited the use of these checklists. None of the 16 networks had any 
guidelines for the provision of emergency drugs for the inter-hospital transfer of patients within the network.

Conclusion: More than one third of the Critical Care Networks in England did not meet the ICS recommendation of having their transfer equipment and documents standardised. Many of the Critical Care Networks do not have any guidelines in place for checking the transfer equipment. All Critical Care Networks should adhere to Intensive Care Society recommendations and work towards standardising their transfer equipment and documentation.

Reference:

1 Intensive Care Society. Guidelines for the transport of the critically ill adult. London: Intensive Care Society, 2002.

\section{AP8-6}

Patients aged 65 years and more treated in university Intensive Therapy Unit (ITU) in 2001-2005

M.A. Wujtewicz, A. Suszyñska-Mosiewicz, W. Sawicka, A. DylczykSommer, R. Owczuk

Anaesthesiology and Intensive Therapy, Medical University of Gdañsk, Gdañsk, Poland

Background and Goal of Study: Progress in medicine results in increasing expectation of life duration and quality. More and more older patients undergo surgery and ITU treatments. In this study authors analyzed reasons for admission to the ITU and hospital mortality in a group of patients aged 65 and more and compared results to the same parameters in a group of patients below 65 years old

Materials and Methods: There were 1770 admissions to the mixed university, adult ICU from 01.01.2001 to 31.12.2005. 92 patients were readmitted, four of them more than twice. Population of 1674 patients was divided into two groups; I-patients $\geqslant 65$ years old $(n=726)$, II-patients $<65$ years old $(n=948)$. Statistical analysis was performed using chi-square test for categorical data and $U$ Mann-Whitney test for continuous data (none of the data had normal distribution as checked with $\mathrm{W}$ Shapiro and Wilk test). $\mathrm{p}<0.05$ was considered significant.

Results: Data are presented in the table as median (range) - age, apache II, length of ITU stay and as percents - the rest of the parameters.

\begin{tabular}{|c|c|c|c|c|c|c|}
\hline & Age in years & Apache II & $\begin{array}{l}\text { Male } \\
\text { patients }\end{array}$ & $\begin{array}{l}\text { Death in } \\
\text { ITU }\end{array}$ & $\begin{array}{l}\text { Death } \\
\text { after ITU } \\
\text { discharge }\end{array}$ & $\begin{array}{l}\text { stay in ITU } \\
\text { in days } \\
\text { days }\end{array}$ \\
\hline 1 & 73 (65-92) & $16(2-102)$ & 68 & 25.1 & 20.3 & $\begin{array}{l}1.9 \\
(0.014-149)\end{array}$ \\
\hline ॥ & $51(14-65)$ & $15(0-103)$ & 67.4 & 26.1 & 12.1 & $\begin{array}{l}3.2 \\
(0.01-231)\end{array}$ \\
\hline \multirow[t]{2}{*}{$\mathrm{p}$} & $\begin{array}{l}\mathrm{p}< \\
0.000001\end{array}$ & $\mathrm{p}<0.001$ & N.S. & N.S. & $p=0.0002$ & $\begin{array}{l}p< \\
0.00005\end{array}$ \\
\hline & readmission & $\begin{array}{l}\text { head } \\
\text { trauma }\end{array}$ & after CPR & $\begin{array}{l}\text { resp. } \\
\text { insuff }\end{array}$ & $\begin{array}{l}\text { urg./ } \\
\text { planned } \\
\text { surg. }\end{array}$ & $\begin{array}{l}\text { multiple } \\
\text { trauma }\end{array}$ \\
\hline I & 7.16 & 5.2 & 16.1 & 54.1 & $30.85 / 34.02$ & 2.2 \\
\hline II & 5.6 & 23.8 & 12.65 & 59.5 & $33.8 / 24.3$ & 9.7 \\
\hline $\mathrm{p}$ & $p=0.009$ & $p<0.0001$ & $p=0.04$ & $p=0.03$ & $p=0.006$ & $\begin{array}{l}p< \\
0.0001\end{array}$ \\
\hline
\end{tabular}

Conclusion(s): There was no difference in ITU mortality between analyzed groups of patients. Older patients were treated in ITU for a shorter period of time. Mortality after ITU discharge was higher in group I. Readmission was more frequent in group I.

\section{AP8-7}

\section{Quality of life after surviving sepsis}

E. Agustin, M. Rodriguez, S. Pico, E. Lopez-Zayas, J. Rico-Feijoo Anaesthesiology and Postoperative Critical Care, Rio Hortega University Hospital, Valladolid, Spain

Background and Goal of Study: The aim of our study is to assess the quality of life of patients that survived from severe sepsis and septic shock in our Postsurgical Critical Care Unit by analyzing several medical factors $(1,2)$. Materials and Methods: We studied 78 surviving patients from our Surgical Critical Care Unit between January 1999 and July 2006 by means of the Short Form 36 questionnaire (SF-36) (3). Data from 5 of the 8 subscales (physical functioning, emotional role, physical role, general health perception, social functioning and transition to the state of health) were surveyed by phone. Age, APACHE II score, medical-surgical records, length of stay in our unit and presence of cancer pathology were registered. Statistical analysis was carried out with the SPSS v.10.0 package using $\mathrm{p}<0.05$.
Results and Discussions: The mean age was $67.91 \pm 14.59$ years. The mean stay in our Unit was $9.59 \pm 8.36$ days and the mean score of APACHE II was $17.94 \pm 6.60$. The mean number of comorbidities was $2.13 \pm 1.35$. $39.5 \%$ presented some kind of malignance. Statistical significance was found between APACHE II scores and general health and social functioning scores (Fig. 1) and between social functioning scores and the number of comorbidities.

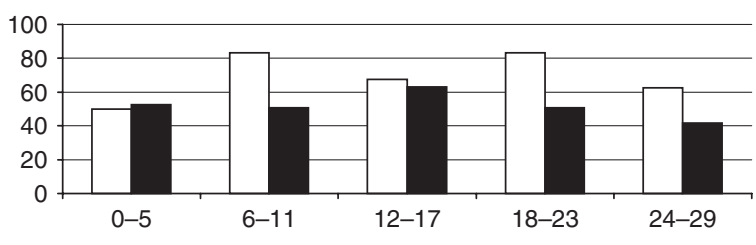

$\square$ Social functioning scores General health scores

Figure 1. Relation by APACHE II.

Conclusion(s): Patient that survived septic shock and severe sepsis had good scores regarding physical and social functioning. The physical and emotional roles and general health perception obtained lower scores. Severity and previous state of health correlates well with social adaptation after discharge from the ward.

References:

1 Heyland D, Hopman W, Coo H, et al. Crit Care Med. 2000; 28: 3599-3605.

2 Orlando R. Crit Care Med. 2000 Nov; 28: 3755-6.

3 Alonso J, Prieto L, Anto JM. Med Clin (Barc). 1995; 104: 771-6.

\section{AP8-8}

Postoperative outcomes in patients with Pseudomyxoma Peritonei undergoing cytoreductive surgery and hyperthermic intraoperative chemotherapy - a UK perspective

P. Kochhar, S. Sagadei, S. Beards, D. Tansey, S.L. Maguire

Anaesthesia, South Manchester University Hospital, Manchester, United Kingdom

Background and Goal of Study: Definitive treatment for Pseudomyxoma Peritonei (PMP) involves extensive cytoreductive surgery with heated intraoperative intra-peritoneal chemotherapy (HIPEC) and only occurs in two centres in the UK. Anaesthesic issues include long operative times, extensive fluid shifts, blood loss, temperature swings and disturbances in coagulation and biochemical parameters. We wanted to establish the pattern of postoperative complications.

Materials and Methods: We prospectively analysed 28 patients who had undergone elective cytoreductive surgery and HIPEC at our institution detailing critical care use and adverse outcomes.

Results and Discussion: Patients were predominantly female (71.4\%) with a mean age of 54 years (range 16-71). Mean surgical time was 445 mins. Mean fluid use was $7687 \mathrm{mls}$ crystalloid and $1358 \mathrm{mls}$ of colloid. The average stay in a critical care facility was 5.9 days (2-10d) with a mean stay of 3.0 days in ITU (2-7d) and 3.7 days in HDU (1-8d). 19 patients were extubated in theatre but 9 taken to ITU and ventilated for a mean of $5.43 \mathrm{hrs}(1-18 \mathrm{~h}$ ). Reasons for ventilation: facial/laryngeal oedema (4); unstable cardiovascular status (3); massive blood transfusion (1); metabolic derangements (1). Organ failure: no organ failure (25); liver failure (1); respiratory failure (1); LVF (1). Other complications: dysrhythmias (3); epithelial damage due to cooling packs (1); pneumothorax (1); confusion (1). Intra-operative serum lactate was high $(>2 \mathrm{mmol} / \mathrm{L})$ in 9 patients and very high $(>3 \mathrm{mmol} / \mathrm{L})$ in 5 . These correlated with patients having upper abdominal disease and stripping of peritoneum around the liver. Patients with a lactate greater than 3 had a higher rate of complications in the immediate post-operative period. 7 patients developed an abnormality in LFTs (raised AST, LDH and gGT).

Conclusion: The majority have an uneventful recovery. The surgical process of stripping quantities of peritoneum, organ resection and liver trauma, allied to the process of HIPEC may leave some patients at a higher risk of liver damage, and this may be detected by lactate rises peri-operatively.

\section{AP8-9}

The impact of risk factors for the development of pneumonia in burned patients

M.I. Rochera Villach, A. Lacasta Fornells, I. Coll Rosell, O. Martínez Silva, J. Roige Sole

Anaesthesiology, Hospital Universitari Vall d'Hebron, Barcelona, Spain 
Background and Goal of Study: Clinical experience has shown that concomitant diseases and risk factors have a significant influence on the burned patient's complications. Pneumonia is one of the most frequent complications of the critically ill patients (1). The aim of this study was to identify the risk factors and their significance for the development of pneumonia in burned patients.

Materials and Methods: Retrospective, observational study in burned patients. Patients were eligible for inclusion if they have had an acute burn injury and had been admitted to a Burn Unit of a $3^{\text {rd }}$ Level Universitary Hospital between Jan. 1997 and Sep. 2006. Patients with symptoms of respiratory tract infection at the moment of admittance were excluded. The following variables were recorded: sex, age, smoking habit, COPD antecedents, TBSA burn, presence of inhalation injury, surgery, enteral nutrition (EN), the number of transfusions. A logistic regression model was used to identify the risk factors and their significance for the diagnostic of pneumonia.

Results and Discussion: Among the 4.268 patients, 3.894 were included in the study. Results are shown in the table:

\begin{tabular}{lrrrr}
\hline & & & \multicolumn{2}{c}{$95 \%$ confidence int. } \\
\cline { 5 - 5 } & $\mathrm{p}$ & Odds ratio & Lower & Upper \\
\hline COPD & 0,046 & 2,273 & 1,014 & 5,099 \\
Enteral Nutr. & $<0,0001$ & 3,595 & 2,171 & 5,953 \\
Surgery & 0,001 & 2,842 & 1,522 & 5,308 \\
Smoking & 0,379 & 0,806 & 0,499 & 1,303 \\
Inhalation injury & $<0,0001$ & 5,181 & 3,207 & 8,369 \\
Age & 0,001 & 1,021 & 1,008 & 1,033 \\
TBSA & & & & \\
$<10 \%$ & 0,001 & 2,712 & 1,493 & 4,924 \\
$11-20 \%$ & 0,014 & 2,625 & 1,217 & 5,664 \\
$21-30 \%$ & $<0,0001$ & 5,796 & 2,562 & 13,113 \\
$31-40 \%$ & 0,005 & 4,577 & 1,602 & 13,072 \\
$41-50 \%$ & $<0,0001$ & 13,293 & 4,488 & 39,368 \\
$51-60 \%$ & $<0,0001$ & 15,652 & 3,681 & 66,557 \\
$61-70 \%$ & $<0,0001$ & 36,690 & 11,087 & 121,417 \\
$71-80 \%$ & $<0,0001$ & 12,639 & 3,431 & 46,557 \\
$>80 \%$ & 0,058 & 5,369 & 0,941 & 30,624 \\
Sex (woman) & 0,293 & 0,774 & 0,480 & 1,248 \\
Total nr. transfusions & 0,018 & 1,027 & 1,005 & 1,051 \\
\hline & & & &
\end{tabular}

TBSA as an indicator of burn severity is a significant risk factor increasing with the $\%$ of burned area, except for the gravest patient, probably because they die soon.

Conclusion(s): Among all risk factors, EN is the only one that can be controlled during the evolution. Special care should be put on EN administration in order to minimize hazards. Other factors, although relevant, are not modifiable. Reference:

1 J Burn Care Res 2006; 27(2):152-60.

\section{AP9-1}

\section{Is ketamine the right sedative for intensive care unit} patients?

\section{E. Elamin, D. Drew}

Anesthesia, University of South Florida, Tampa, USA

Background and Objectives: Little is known about the potential hemodynamic benefits of continuous ketamine sedation and analgesia in adult ICU patients.

Methods: In a pilot multi-center, prospective, double-blind, randomized controlled trial ${ }^{*}$ we screened 66 adult ICU patients who required sedation and analgesia. Patients meeting entry criteria were randomized and received continuous infusion of ketamine or fentanyl for $\geqslant 24$ hours to achieve a Ramsay Sedation Scale of " 4.

Measurements and Main Results: In addition to demographic data, we recorded mean blood pressure, (MBP), heart rate, requirements of any intravenous (IV) vasopressor or any additional sedatives.

Sixty $\%$ of patients $(3 / 5)$ received ketamine for 24 hours followed by midazolam while $40 \%(2 / 5)$ received fentanyl infusion. MAP was statistically significant higher in the study compare to the control group $(p<.05)$, figure 1 . In addition, patients who received ketamine did not required any additional vasopressors \& were less likely to be diagnosed with shock state, comparing to the fentanyl group $(p<.05)$.

Conclusions: This preliminary report illustrated the possible value of ketamine for continuous ICU sedation and analgesia. The results of our ongoing study will provide more information about the impact of ketamine in early weaning from mechanical ventilation and hence decrease in ICU length of stay.

*Supported by a grant from the American Lung Association.

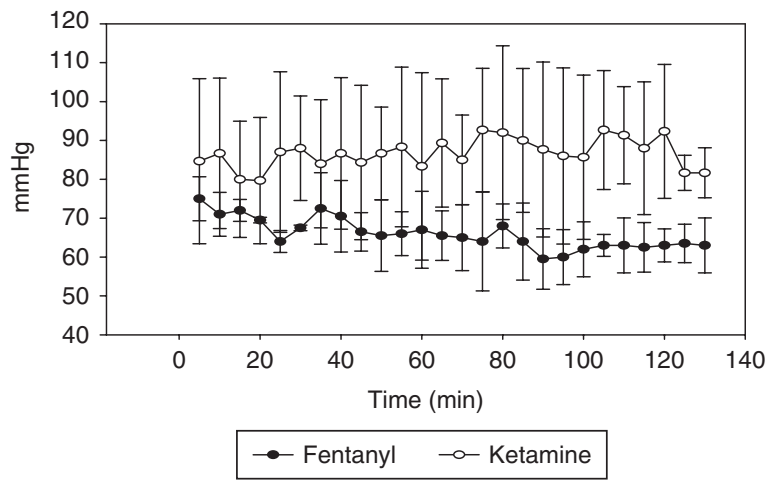

12AP9-2

Treatment of peptic ulcer bleeding in ICU settings: an algorithmic approach

S. Spiridonov, V. Ivanova, E. Nedashkovskiy

Chair of Anaesthesiology and Intensive Care, Northern State Medical University, Arkhangelsk, Russian Federation

Background and Goal of Study: This study assessed outcomes of acute peptic ulcer bleeding (PUB) in patients treated in the intensive care unit (ICU) of a city emergency hospital before and after implementation of an internal diagnostic and treatment algorithm based on the current published literature (1).

Materials and Methods: We studied 104 patients, who presented with bleeding gastric and duodenal ulcers between October 2003 and October 2005 [56 retrospective pts (control group, age $49 \pm 16 \mathrm{yrs}$ ); 48 prospective pts (study group, age $51 \pm 15 \mathrm{yrs}$ ), admitted after the algorithm implementation in October 2004]. The algorithm rested on Rockall risk scoring and use of parenteral proton pump inhibitors (omeprazole) as anti-secretion agents. Pts with Clinical Rockall Scores $\geqslant 3$ were assigned for ICU admission and urgent endoscopy. Total Rockall Scores (TRS) were calculated, and lower risk $(T R S \leqslant 4)$ or high-risk (TRS $\geqslant 5$ ) pts were recognised and treated differentially. The control group was stratified in the same manner retrospectively; parenteral PPI were not applied. Both groups were similar on age, gender, co-morbidities, shock presence on admission and mean TRS's.

Results and Discussions: Mortality and rebleeding (RB) were lower in the study group. RB difference reached statistical significance owing to improvement in high-risk pts' outcomes. Data [cases and controls (totals); Odds Ratios $(95 \% \mathrm{Cl}) ; \mathrm{p}(\chi 2)]$ are shown in the table:

\begin{tabular}{lrcll}
\hline & Cases & Controls & OR & $\mathrm{p}$ \\
\hline Mortality & $2(48)$ & $7(56)$ & $0,35(0,08-1,57)$ & 0,25 \\
RB, Total & $15(48)$ & $31(56)$ & $0,37(0,17-0,83)$ & 0,02 \\
RB, TRS $\geqslant 5$ & $9(27)$ & $21(29)$ & $0,20(0,07-0,62)$ & 0,007 \\
\hline
\end{tabular}

Mean ICU stay was significantly lower in the study group $(69,9 \pm 11,8$ vs. $86,0 \pm 13,9$ hours, $p<0,05)$ due to rare rebleeding and readmissions. Mean hospital stay $(17 \pm 2,5$ vs. $18 \pm 2,8$ days, $p>0,05)$ and surgery rate (16 vs. $14 \%$; OR $=1,49(0,64-3,49) ; p>0,05)$ were similar in both groups. Conclusions: In ICU management of acute PUB: (1) a structured algorithmic approach improves outcomes; (2) Rockall scoring system and parenteral PPI use is preferable for rebleeding prevention in high-risk patients. Reference:

1 Barkun AN, et al. Ann Intern Med 2003; 139: 843-57.

\section{AP9-3}

What can predict atrial fibrillation after coronary artery bypass grafting (our experience)?

S. Straus, M. Kacila, M. Kulic, A. Cimic, E. Omerbasic

Heart Center Sarajevo, KCUS, Sarajevo, Bosnia and Herzegovina

Background and Goal of Study: Postoperative atrial fibrillation (AF) occurs in up to $50 \%$ of coronary artery revascularization and presents the most common postoperative arrhythmic complication. Its ethiology in open-heart surgery is incompletely understood, so prevention remains suboptimal. The goal of our study was to find correlation of AF with pre- and intraoperative factors.

Materials and Methods: Two comparable groups who underwent coronary artery bypass grafting (CABG) in period January 1st to November 15th 2006 
were stidied. Group I consisted of 37 patients who developed AF postoperatively and group II 37 patients who did not develope AF postoperatively. We analized preoperative and intraoperative factors in both groups relevant for AF appearance as follows below. Parson chi-squared test was used.

Results and Discussions: Results of correlation of preoperative factors and AF development shown in Table1.

\begin{tabular}{llll}
\hline Preoperative factors & Group I & Group II & $\mathrm{P}$ \\
\hline Low ejection fraction $(\mathrm{EF}<40 \%)$ & 12 & 5 & 0,05 \\
$\mathrm{BMI}>30$ & 13 & 5 & 0,03 \\
\hline
\end{tabular}

Results of correlation of intraoperative factors and AF development shown in Table 2.

\begin{tabular}{lllc}
\hline Intraoperative factors & Group I & Group II & $\mathrm{P}$ \\
\hline Long extracorporeal circulation & 12 & 5 & 0,05 \\
time $(>100$ min & & & min) \\
Increased inotropic support & 13 & 5 & 0,03 \\
\hline
\end{tabular}

Conclusion(s): Results suggest that the incidence of AF in patients undergoing CABG can be predicted by some pre- and intraoperation factors - in our case low EF, BMI over 30, long extracorporeal circulation time ( $>100 \mathrm{~min}$ ) and increased inotropic support caused postoperative AF appearance, so in future we can plan prophylactic measures for this factors.

Reference:

1 Aner $\mathrm{J}$, Weber $\mathrm{T}$, Bernet $\mathrm{R}$ et al. Risk factors of postoperative atrial fibrillation after cardiac surgery. J Card Surg 2005; 20(5): 425-31.

\section{AP9-4}

High thoracic epidural anesthesia versus general anesthesia in surgery for ischemic dilatative cardiomyopathy

V. Petrovski, E. Stoicovski, D. Slavevski, N. Hristov, Z. Mitrev Anesthesia, Special hospital for Cardiosurgery "Filip Vtori", Skopje, The former Yugoslav Republic of Macedonia

Background and Goal of Study: The purpose of this study was to compare the results high epidural thoracic anesthesia (HTEA) versus general anesthesia (GA) in treatment of patients with ischemic dilatative cardiomyopathy (IDC).

Materials and Methods: From May 2006 to November 2006, 40 patients were subjected to open-heart surgery for IDC. Unstable angina was present in 20 (50\%). Thirty-three (82\%) were classified as New York Heart Association class III or IV. Previous myocardial was diagnosed in 24 (60\%). Preoperative ejection fraction was $27 \pm 3$. Group 1 consisted of 20 patients subjected to HTEA, and group 2 the other 20 subjected to GA. Median sternotomy was performed in all. Aorto coronary bypass (ACB) was done in all patients, with 1 off pump case and $5(12 \%)$ with assisted circulation, ventriculoplasty and valve reconstructive surgery in $8(20 \%)$, valve replacement in $4(10 \%)$ of the patients, valve reconstruction in $2(5 \%)$. Three patients required intra aortic balloon pump support.

Results and Discussions: All patients remained stable throughout the procedure, with $3(7 \%)$ patients from group 1 extubated in the operating theater versus 0 for group 2, $4(10 \%)$ extubated within 5 hours following the procedure in group 1 versus 1 (2\%) for group 2, 7 (17\%) within 10 hours in group 1 versus $6(15 \%)$ in group 2 . Average Sufentanil usage was $25 \mu \mathrm{g}$ per patients in group 1 versus $250 \mu \mathrm{g}$ in group 2 . Average first mobilization time was $33 \pm 16$ hours for group 1 versus $75 \pm 57$ for group 2 . Average intensive care unit stay (ICU) was $36 \pm 17$ hours for group 1 and hospital stay of $6.1 \pm 1.4$ days versus $71 \pm 50$ and $6.6 \pm 1.7$ for group 2 . Average postoperative visual analog scale for pain was $2.7 \pm 0.3$ for group 1 versus $3.2 \pm 0.5$ in group 2 . Conclusion(s): HTEA in patients with IDC has shown to be safe during surgery and postoperative period, allowing fast extubation with minimal pain, with early mobilization and shorter ICU and hospital stay compared to GA. Reference:

Kirno K, Friberg P, Grzegorczyk A, Milocco I, Ricksten SE, Lundin S. Thoracic epidural anesthesia during coronary artery bypass surgery: effects on cardiac sympathetic activity, myocardial blood flow and metabolism, and central hemodynamics. Anesth Analg 1994; 79(6): 1075-1081.

\section{AP9-5}

Time-course of recovery from botulinum toxin-induced neuromuscular disorder

C. Frick, M. Blobner, J.A.J. Martyn

Anesthesia \& Critical Care, Massachusetts General Hospital \& Klinkum rechts der Isar, TU München, Boston, USA
Background and Goals: Infection with $C$. botulinum causes paralysis of the affected muscles, leading to respiratory failure. Recovery typically takes place over weeks to months. Therefore, we studied the effects of botulinum toxin (BTX) on time-course and extent of neuromuscular block, and muscle fatigability.

Methods and Methods: Rats $(n=36)$ were injected with 2.5U BTX into the tibialis muscle. The contralateral side received no injection and served to study distant effects. Control animals $(n=34)$ received an equivalent volume of saline. At $0,4,16$ and 128 days after injection, neuromuscular transmission was tested by mechanomyography.

Results: On day 0 after injection, tibialis muscle tensions and muscle mass did not differ between groups and sides. On day 4, there was complete paralysis of the BTX-injected side, while its contralateral side showed a decrease in muscle contraction. On day 16 and 128, evoked and tetanic tensions, including muscle mass were decreased on the toxin-injected side relative to the contralateral, side and to saline injected controls. At day 16, tetanic fade was increased on the toxin-injected side. Normalized to muscle mass, specific evoked and tetanic tensions (tensions/muscle mass) were reduced on the contralateral side at day 4 and the toxin-injected side at day 16. At 128 days, there were no differences in specific tensions between groups.

Conclusion: Infection with BTX follows a time-course from complete paralysis to severely depressed muscle dysfunction. Muscle contraction partially recovers by 128 days. At this time, loss of tension is almost exclusively related to muscle atrophy, as the specific tension did not change. The presence of fade at day 16 and its absence at 128 days suggest that pre-junctional related muscle dysfunction disappears by 128 days.

\section{AP9-6}

\section{Anesthesia for combined liver/kidney transplantation in primary hyperoxalurie}

O. Collange, D. Vallaud, M.C. Gillon, J. Mantz, K. Hasehnoune

Anesthesiology and Critical Care, Strasbourg University Hospital, Strasbourg, France

Background and Goal of Study: Primary hyperoxaluria type-1 $(\mathrm{PH}-1)$ is an autosomal recessive inborn error of glyoxylate hepatic metabolism in which excessive production of oxalate results in nephrocalcinosis followed by endstage renal failure. Combined liver-kidney transplantation (LKTx) corrects the metabolic defect and prolongs kidney graft survival. $\mathrm{PH}-1$ transplanted patients require particular care to avoid oxalate precipitation and acute renal dysfunction during the immediate postoperative period. There is no literature on anaesthesia management during LKTx for $\mathrm{PH}-1$ patients. The goals of our study were to compare anaesthesia and postoperative data in two Tx centers and to propose specific rules to conduct anaesthesia during LKTX for $\mathrm{PH}-1$ patients.

Materials and Methods: We retrospectively included all $\mathrm{PH}-1$ patients with combined liver/kidney transplantation in two large French transplantation centers of (C1 and C2). Anesthesia management (anesthetic and antibioprophylactic drugs, hemodynamic monitoring etc.) was recorded. Postoperative data were hospital length of stay and plasma creatinine levels at three months. Results and Discussions: Between 1991 and 2006, 17 adult patients were LK transplanted ( 6 in $\mathrm{C} 1$ and 11 in C2). There was no difference in age population ( $41 \pm 11$ vs $40 \pm 15$ years, $p=0.9$ ) between the two centers. Differences in management were mainly found in hemodynamic monitoring (0/6 vs 11/11 right catheter), prophylactic antibiotics (6/6 vs $1 / 11$ associations of two nephrotoxic antibiotics) and the use of aprotinin (0/6 vs 11/11). Patients from C1 had a longer non-ICU $(33 \pm 23$ vs $19 \pm 18$ days, $\mathrm{p}=0.049)$ and total hospital length of stay $(57 \pm 30$ vs $32 \pm 12$ days, $p=0.032)$. At 3 months, plasma creatinine was significantly lower $(170 \pm 48$ vs $117 \pm 39 \mu \mathrm{mol} / \mathrm{l}, \mathrm{p}=0.048)$ for the patients transplanted in $\mathrm{C} 2$. Aprotinin does not appear to alter renal function. Conclusions: The role of anesthesia management on the observed differences remains unclear. However, our results suggest that hemodynamic monitoring and avoidance of nephrotoxic drugs during LKtx are important for $\mathrm{PH}-1$ patients.

\section{AP9-7}

\section{Is ventilator therapy mandatory for anaesthesia management in staged abdominal repair: intubation- extubation and re-intubation}

Ü. Buyukkocak, C. Daphan, K. Aydinuraz, F. Agalar

Anaesthesiology and Reanimation, Kirikkale University School of Medicine, Kirikkale, Turkey

Background and Goals: Ventilator therapy is usually considered a "must" for ICU patients with a high APACHE score who require Staged Abdominal Repair 
(S.T.A.R) (1). Yet such patients can be managed with limited duration of ventilator therapy namely by sequences of intubation-extubation and re-intubation which might be helpful for weaning.

Materials and Methods: Ten consecutive patients who underwent S.T.A.R for moderate to severe secondary peritonitis and mesenteric vascular occlusion were evaluated retrospectively for anaesthesia management.

Results: The mean age of the patients was 54.2 years (range 23-78 years), and 7 of them were male. All APACHE scores were over 10. Eight of the patients had postoperative peritonitis and 2 had mesenteric vascular occlusion. All patients underwent STAR every 24 hours and abdomen was covered temporarily with "Bogota Bag" until definitive closure was done. Mean number of operation was 5 (range 2-11). Nitrous oxide was not used in any of the patients. Endotracheal intubation was performed only for index operation and definitive abdominal closure. Other than these operations, intravenous general anaesthesia or general anaesthesia with mask was preferred for STAR which usually lasted for approximately 20 minutes. When respiratory parameters were acceptable after index operation, the patients were extubated until definitive abdominal closure. Five patients who could not be extubated underwent ventilator therapy during their ICU stay and two of them had tracheotomy. Mean ICU stay was 12.2 (3-23) days. Overall mortality rate was $50 \%$. The reason for death was respiratory failure in three patients whereas urinary sepsis and AMI were encountered in the other two. Remaining patients recovered uneventfully.

Conclusions: Patients with an APACHE score $>10$ undergoing STAR can be managed without ventilator therapy but with sequences of intubationextubation and re-intubation when respiratory parameters are acceptable. Patients who required ventilator support had a higher APACHE score and mortality rate.

Reference:

1 Agalar F, Eroglu E, Bulbul M, Agalar C, Tarhan OR, Sari M. World J Surg 2005; 29(2): 240-244.

\section{AP9-8}

\section{Severe acute pancreatitis - is there a place for HBO treatment?}

I. Lisagors, A. Sondore, P. Oss, A. Shkesters, I. Jaunalksne

Anaesthesiology and Reanimatology, Riga Stradins University, Riga, Latvia
Background and Goal of Study: Despite recent advances in the management of SAP it remains a life-threatening disease. Recently the potential benefit of hyperbaric oxygen therapy (HBOT) in the treatment of SAP has been demonstrated (1). Our goal was to investigate the effects of HBOT as an adjunct to the standard treatment on the severity of disease, oxidative stress (OS) and inflammatory reactions.

Materials and Methods: A prospective case-controlled study was performed on 8 patients ( $p$ ) with SAP. $4 \mathrm{p}$ were included in HBOT group (gr). Another 4 -in the control gr ( $\mathrm{C} \mathrm{gr}$ ). HBOT course (c) comprising of 6 sessions (s), once a day (d), under 1,7 ATA in the monoplace chamber has been initiated within $24 \mathrm{~h}$ after admission. Severity of SAP (SOFA score), oxidative stress (MDA, luminol-enhanced chemiluminescence (CL), vit $E$, selenium (SE), as well as pro- and anti-inflammatory reaction (serum amyloid-SAA, IL-6, TNF a, sTNFR2) were assessed.

Results and Discussions: The demographics and severity of SAP were similar in two gr. The SOFA score improved faster in HBO gr. MDA decreased on average for $41.69 \%$ in HBOT gr, for $66.46 \%$ in $C$ gr. Reduction of CL was more intensive in HBOT gr on av for $15.78 \%$ v $12.23 \%$ in $\mathrm{C}$ gr. Level of vit $\mathrm{E}$ was normal in $7 \mathrm{p}$, but in $1 \mathrm{p}$ increased for $32.64 \%$ after HBOT c. An early low plasma Se concentration, established in 8 p (100\%), wasn't affected by HBOT c. IL-6 was increased in all patients with SAP. HBOT induced no significant elevation of IL- 6 during first 3 s for $13.9 \%$ with consecutive diminishing after 6 th s. sTNFR2 and SAA were elevated in all $8 \mathrm{p}(100 \%)$, though TNF-a only in $2 \mathrm{p}(50 \%)$ in each gr.

\section{Conclusion(s):}

1. Addition of HBOT to the therapy of SAP diminished the severity and formation of lipid peroxides and hydroperoxides, possibly due to improvement of tissue oxygenation and metabolism

2. HBOT did not affect deficiency of selenium

3. We noted putative immuno-modulating effect of HBOT because of IL-6 temporary elevation and decrease

4. It seems that HBOT induced some favorable effects as an adjunct method of IC in patients with SAP.

Reference:

1 Cuthbertson C, Christophi C. ANZ J. Surg. 2006; 76: 625-630.

\section{Resuscitation and Emergency Medicine}

\section{AP1-1}

The trauma room - a "one stop shop" for diagnosis and therapy?

M. Struck, R. Stuttmann, P. Hilbert

Department of Anaesthesiology, Trauma Center Bergmannstrost, Halle, Germany

Background: Complete diagnostic tests and surgical care during the first golden hour [1] is of the utmost importance to increase survival in multiple trauma patients. Multi-slice CT (MSCT) allows a complete diagnostic assessment of injured patients in 90-240 sec [2], but is normally carried out only at the conclusion of basic diagnostics.

Materials and Methods: We modified the work flow in the emergency room (ER) at our trauma center and investigated the effects of a clinical algorithm using MSCT (native head CT + contrast medium-supported wholebody CT) scanning ahead of other measures like conventional radiography and ultrasound in seriously injured patients.

Results: In a two years period ( 2004 / 2005) $n=256$ patients ( 198 male / 58 female) were enrolled according to the aforementioned procedure. Mean ISS was $26.76, n=25(9.7 \%)$ patients died. After patients arrival MSCT was started $8 \mathrm{~min}$ (SD 5.47) and completed at an average of $13 \mathrm{~min}$ (SD $+/-6.74)$. Mean duration of complete CT diagnostics was $4 \mathrm{~min}(+/-\mathrm{SD} 5.71)$. Length of stay in the trauma room was significantly reduced from $87 \mathrm{~min}$ (own data from $2000 / 2001$, before integration of MSCT into the ER) to $38 \mathrm{~min}$ (SD + / - 19.3).

Conclusion: Seriously injured patients treatment should be initiated as soon as possible and saving time for clinical diagnostics is vital. Rapid CT scanning is therefore a sensible and practicable tool. It reduces the length of stay in the ER markedly and should significantly improve outcome. Life-threatening injuries can be identified within a matter of minutes and appropriate interventional therapy be initiated. Prerequisite for installing the concept introduced is the integration of a MSCT into the trauma room, transforming it into a "one stop shop" for diagnosis and therapy.
References:

1 Cowley RA. Clin Med 1976; 83:16-22.

2 Hessmann M. Eur J Trauma 2006; 31: 231-238.

\section{AP1-3}

\section{Daytime Efficiency of Emergency (CEPOD) theatre}

S. Gupta, R. Davies, J. Srinivas, O. Adekanye

Department of Anaesthetics, University Hospital of Wales, Cardiff, United Kingdom

Background and Goal: The provision of a $24 \mathrm{~h}$ emergency theatre (CEPOD theatre) is common practice in most United Kingdom (UK) acute hospitals. An efficient use of the CEPOD theatre during daytime sessions (when enough staff and support of appropriate seniority are available) should reduce the need for risky operations in high risk patients after midnight [1, 2]. The aim of this study is to evaluate the efficiency of the daytime CEPOD theatre, identify reasons for inefficiency and recommend possible remedies to effect a change.

Methods: After Trust Audit Department approval, data was collected from the University Hospital of Wales database. Using a pro-forma, the number of cases booked/day, done/day, cancelled/day, the theatre utilization time between 8.00 and $18.00 \mathrm{~h}$, and the average delay in start of the Emergency Theatre (CEPOD theatre) during the month of March 2005 and July 2006 were prospectively extracted and compared.

Results and Discussions:

\begin{tabular}{lll}
\hline & $03 / 2005$ & $07 / 2006$ \\
\hline Average cases booked/day & 11 & 15 \\
Average cases done/day & 3 & 4.1 \\
Average cases postponed/day & 4 & 4 \\
Theatre utilization time (h) & 4.6 & 4 \\
Average delay in start time (h) & 2.5 & 1.5 \\
\hline
\end{tabular}


The level of urgency of booked cases for emergency operation in our hospital is determined by a categorical scale chosen by the surgeon booking the case. $70 \%$ of patients booked as category 1 (cases to be done within $1 \mathrm{~h}$ ) were done on time, $66 \%$ of $2 \mathrm{a}$ (within $4 \mathrm{~h}$ ) were done on time, $70 \%$ of $2 \mathrm{~b}$ (within $12 \mathrm{~h}$ ) and $50 \%$ of category $3(24 \mathrm{~h}$ ) were done within the stipulated time. Conclusion(s): There is a $25 \%$ increase in number of cases done per day as well as an overall improvement of theatre efficiency. This can be attributed to increased awareness (brought about by the 2005 audit) of cancellation of booked cases and under-utilization of CEPOD theatre and the introduction of a dedicated CEPOD consultant anaesthetist.

References

1 Magee TR et al. Ann R Coll Surg Engl. 95; 77: 121-4.

2 Barlow AP et al. Ann R Coll Surg Engl. 93; 75: 441-4.

\section{AP1-5}

\section{Comparison of different strategies for ventilation in a} simulation of an entrapped car accident victim

H.V. Genzwuerker, S. Krahl, F. Finteis, J. Hinkelbein

Clinic of Anaesthesiology, University Hospital Mannheim, Mannheim, Germany

Background and Goal of Study: Airway management, considered of highest priority in trauma victims, may be impaired when patients are entrapped in vehicles. Different supraglottic airway devices are compared with facemask ventilation and tracheal intubation for ventilation of a manikin placed in a car. Materials and Methods: A manikin (Ambu Megacode Trainer) with an extrication collar was fixed in the driver seat of a Volkswagen Golf IV. Ventilation was attempted by 21 paramedics and 17 emergency physicians through the window of the driver's door and from the backseat with facemask, tracheal intubation, LMA-Fastrach (LMA), Combitube (CT), Easytube (EZT), and Laryngeal Tube Suction (LTS II). Number of attempts (maximum 3) until successful insertion (tidal volume $>400 \mathrm{ml}$ achieved by the participant), time (picking up the device until first tidal volume), and signs of gastric inflation were recorded.

Results and Discussions: 8 (21.1\%) participants succeeded with facemask ventilation from the side (1st/2nd/3rd attempt: 3/4/1), 21 participants $(55.3 \%)$ from the backseat (5/5/11). Gastric inflation from side/backseat was recorded in $97.4 / 100 \%$. From the side, 29 participants achieved tracheal intubation (76.3\%; 14/9/6) with a duration of $46.6(20-74)$ sec for the successful attempt. From the backseat, 35 participants succeeded with intubation $(92.1 \% ; 24 / 6 / 5)$, duration 40.8 (23-93) sec. Gastric inflation by failed intubation attempts from side/backseat occured in $55.3 / 39.5 \%$. From the side position, the following results were found for the supraglottic airway devices: LMA $1 \mathrm{st} / 2 \mathrm{nd} / 3 \mathrm{rd}$ attempt 97.4/2.6/0\%, 19.4 (11-45) sec, no gastric inflation; CT 92.1/5.3/2.6\%, 35.7 (17-100) sec, no gastric inflation; EZT 92.1/5.3/2.6\%, 30.9 (18-61) sec, gastric inflation 2.6\%; LTS II 94.7/5.3/0\%, 18.8 (11-34) sec, gastric inflation $2.6 \%$. From the backseat, results were as follows: LMA 97.4/2.6/0\%, 20.1 (9-39) sec, no gastric inflation; CT 100/0/0\%, 29.6 (15-57) sec, no gastric inflation; EZT 100/0/0\%, 28.2 (13-52) sec, no gastric inflation; LTS II 94.7/5.3/0\%, 20.1 (12-38) sec, no gastric inflation.

Conclusion(s): Supraglottic airway devices offer ventilation more rapidly and with higher reliability than facemask ventilation and tracheal intubation in this simulation of a patient entrapped in a car. With LMA and LTS II, ventilation is faster than with CT and EZT.

\section{AP1-6}

M-GAP, a new score to establish the vital prognosis of multiple trauma patients

D. Sartorius, M. Thicoïpé, E. Wiel, E. Rancurel, B. Riou

Anesthesiology and Intensive care, Hôpital Universitaire De Genève, Geneva, Switzerland

Background and goals: In order to improve the survival of multiple trauma patients, they have to be sent to level 1 trauma centres where rapid intervention and prompt definitive care can be purveyed (1). It is therefore of particular importance for them to be evaluated quickly and accurately. The purpose of this study was to develop a triage tool predictive of mortality for multiple trauma patients that would be easy to use and that would encompass objective clinical and contextual elements available in the prehospital setting. Material and methods: 1360 multiple trauma patients were included from january 2003 to march 2004 by 22 french centres. We performed a multivariate analysis taking into account only elements available in the prehospital setting to identifie factors predictive of mortality. The score was built with the coefficients obtained. We used ROC curves to compare the new score (MGAP) with the Revised Trauma Score (RTS) (2).
Results: Global mortality was of $18,4 \%$.

Data are shown in the table.

\begin{tabular}{llc}
\hline & OR & CI 95\% \\
\hline Penetrating Mechanism & 3,7 & $2,1-6,6$ \\
GCS $<12$ & 18 & $12,0-27,2$ \\
Age $>40$ & 1,9 & $1,4-2,7$ \\
Blood Pressure $<120 \mathrm{mmHg}$ & 2,8 & $2,0-4,0$ \\
\hline
\end{tabular}

The surface under the ROC curve of the RTS and the new score (M-GAP) were $0,78 \pm 0,025$ and of $0,89 \pm 0,014(p<0,05)$.

Conclusions: The new score M-GAP is calculated from 4 objective factors all available in the prehospital setting . It is much more easier to use than the other existing triage scores and is as predictive in terms of mortality as the Revised Trauma Score (RTS).

References:

1 MacKenzie EJ, Rivara FP, Jurkovich GJ et al. NEJM 2006; 366-378.

2 Champion HR, Sacco WJ, Copes WS et al. J Trauma 29: 623-629.

\section{AP2-1}

Carotid-pulse-check performance in soldiers: Effects of CPR-training and effects of physical or combined physical/psychological stress

L.A. Schwarte, P. Schober

Anesthesiology, University Hospital Duesseldorf, Duesseldorf, Germany

Background and Goal of Study: Recommendation of the carotid-pulsecheck is currently restricted by international resuscitation guidelines to health professionals, mainly caused by poor performance of non-health professionals [1]. Whether soldiers undergoing CPR-training should apply carotidpulse-check remains controversial, but decision may be affected by soldiers carotid-pulse-check trainability and-performance. Thus we tested both the impact of CPR-training and the impact of stress (physical and psychological) on the carotid-pulse-check performance of soldiers.

Materials and Methods: Soldiers $(n=86)$ underwent standardized theoretical CPR-instructions, including demonstration of the carotid-pulse-check technique. Thereafter, the soldiers had to perform carotid-pulse-check on a supine normotensive, normo-frequent person under 5 conditions (A-E): Before (A) and after (B) practical ("hands-on") CPR-training, before (C) and after (D) defined physical exercise and (E) under combined physical/psychological stress. Data are mean \pm sem, $p<0.05$.

Results and Discussions: The time required for carotid-pulse-check significantly decreased from solely theoretical training (A, $9.7 \pm 1.0$ seconds) to practical training $(\mathrm{B}, 7.7 \pm 0.7$ seconds). In contrast, the carotid-pulse-checktime significantly increased from rest-condition (C) to physical exercise condition ( $D, 9.3 \pm 1.2$ seconds). Surprisingly, the shortest time required for carotid-pulse-check was achieved under combined physical/psychological stress (E, $5.0 \pm 0.4$ seconds).

Conclusions: Standardized resuscitation training significantly improved practical resuscitation skills, e.g., the carotid-pulse-check to accepted performance levels [1,2]. Although we demonstrate significant improvement from solely theoretical to practical training, this benefit is merely lost under physical stress. Interestingly, the best performance was achieved under the condition of combined physical/psychological stress.

References:

1 Eberle B et al. (1996) Resusciation 33: 107-116.

2 Ochoa FJ et al. (1998) Resuscitation 37: 173-175.

\section{AP2-2}

Cardiopulmonary Resuscitation: Early Results and Success Predictors, A Single Center Registry

M. Najafi, B. Haghighat, N. Paydari, M. Khajavi, A. Karimi

Anesthesiology, Tehran Heart Center, Tehran University of Medical Sciences, Tehran, Iran

Background and Goal of Study: Cardiopulmonary resuscitation (CPR) is one of the most important and unfortunately disappointing components of the standard hospital cares. This study was designed to evaluate the early results and success predictors of the CPR.

Materials and methods: This study was performed on our center database which contains demographical and clinical data for each resuscitated patient. CPR in the operating rooms was excluded. Successful CPR was defined as survival without any residual sequel or complication. Regarding CPR results patients were in one of these groups: successful or unsuccessful CPR. Also regarding first observed rhythm (FR) patients were divided into 2 groups: Ventricular Fibrillation, Ventricular Tachycardia (VF/NT); Asystole, Bradycardia (Asys). 
Results and Discussions: Between January 2005 and March 2006, 577 patients (320 men, 257 women, Mean age: $65.24 \pm 13.7$ ) were resuscitated. The most important causes of the CPR were arrhythmia (38.4\%), respiratory failure (30.8\%), and homodynamic instability $(20.0 \%)$. Survival after resuscitation, at 24 hours and at discharge was $51.0 \%, 40.0 \%$ and $28.3 \%$, respectively. Regarding FA survival rates were $74.1 \%, 66.7 \%$ and $40 \%$ [VF-VT] vs. $43 \%, 25.8 \%$ and $14.3 \%$ [Asys] $(\mathrm{P}<0.05)$. Age, sex, concurrent diseases, and serum $\mathrm{pH}$ were similar between groups. Predictors of successful CPR are shown in table.

\begin{tabular}{lll}
\hline Predictors & OR $(95 \% \mathrm{Cl})$ & $\mathrm{P}$ value \\
\hline Admission due to unstable angina & $2.14(1.34-3.41)$ & 0.001 \\
VF/VT vs. Asystole & $3.79(2.42-5.93)$ & $<0.001$ \\
Atropine dose $<2 \mathrm{mg}$ & $1.85(1.23-2.78)$ & 0.003 \\
No need for epinephrine & $9.81(5.96-16.18)$ & $<0.001$ \\
Epinephrine dose $<3 \mathrm{mg}$ & $1.83(1.21-2.78)$ & 0.004 \\
Time to first shock $<60 \mathrm{sec}$ & $1.91(1.18-3.10)$ & 0.009 \\
Cardiac massage time $<15 \mathrm{~min}$ & $16.27(8.34-31.73)$ & $<0.001$ \\
CPR time $<30 \mathrm{~min}$ & $4.21(2.91-6.10)$ & $<0.001$ \\
Serum potassium $<6 \mathrm{mEq} / \mathrm{L}$ & $2.49(1.27-4.87)$ & 0.007 \\
GCS level $>8(6 \mathrm{~h}$ after CPR) & $22.17(5.15-95.39)$ & $<0.001$ \\
\hline
\end{tabular}

OR: Odds Ratio; Cl: Confidence Interval

Conclusion: Our results are comparable with the former studies. Further studies are required to determine other predictors of the successful CPR.

\section{AP2-3}

\section{Bispectral index (BIS) helps predict neurological outcome in} hypothermia-treated cardiac arrest patients

C. Werer, P. Stammet, L. Mertens, C. Lorang, M. Hemmer

Department of Anesthesia and Intensive Care, Centre Hospitalier de Luxembourg, Luxembourg, Luxembourg

Goal of the study: Comparison of BIS values with the neurological outcome in all patients admitted to our intensive care unit (ICU) after successful resuscitation from cardiac arrest and treated with therapeutic hypothermia.

Material and methods: After return of spontaneous circulation, 24 patients (age $53.6+/-18.7$ years) were all treated with therapeutic hypothermia $\left(33^{\circ} \mathrm{C}\right)$ for 24 hours. In all patients hypothermia was induced by cold iv fluid infusion and for maintenance we either used iv cooling devices (Alsius $\AA$ ) in $16(67 \%)$ patients or surface cooling in $8(33 \%)$ patients. BIS values were continuously recorded during the first 48 hours. To guarantee an optimal signal quality all patients were sedated (midazolam $0.2 \mathrm{mg} \cdot \mathrm{kg}^{-1} \cdot \mathrm{h}^{-1}$, fentanyl $1.5 \mu \mathrm{g} \cdot \mathrm{kg}^{-1} \cdot \mathrm{h}^{-1}$ ) and paralysed (Cisatracurium $0.1 \mathrm{mg} \cdot \mathrm{kg}^{-1} \cdot \mathrm{h}^{-1}$ ). At the end of the ICU-stay, neurological assessment was done using Cerebral Performance Category Score (CPC).

Results: Target temperature of $33^{\circ} \mathrm{C}$ was achieved in all patients within $195 \pm 94$ min. after cardiac arrest. At the end of the ICU-stay, 14 (58\%) patients survived and $10(42 \%)$ died (CPC5). All survivors had excellent (CPC1) or good (CPC2) neurological outcome, except for 2 (8\%) having severe neurological impairment (CPC3) and for $1(4 \%)$ who remained in a vegetative state (CPC4). Distribution of BIS-values, with a good signal quality, over 80 and of 0 is shown below.

Table: Best and worst BIS-values within the first 48 hours

\begin{tabular}{lllc}
\hline & $\begin{array}{l}\text { Good neuro } \\
\text { CPC 1-2 }\end{array}$ & $\begin{array}{l}\text { Bad neuro } \\
\text { CPC 3-4 }\end{array}$ & $\begin{array}{l}\text { Dead } \\
\text { CPC 5 }\end{array}$ \\
\hline Patients $($ n) & 11 & 3 & 10 \\
BIS $>80$ & 7 & 3 & 1 \\
BIS $=\mathbf{0}$ (flat EEG) & $\mathbf{0}$ & $\mathbf{1}$ & $\mathbf{8}$ \\
\hline
\end{tabular}

Conclusions: All patients having a BIS-value of 0 ( = isoelectric EEG) during the first 48 hours after cardiac arrest, either died or had bad neurological outcome. High BIS-values don't seem to have any reliable prognostic value since even patients with bad neurological outcome had values above 80 (artefacts?).

References:

1 Shibata S., J. Anesth 2005; 19: 243-6.

2 Stammet P., Rescucitation 2006; 69 Abstract P 70.

\section{AP2-4}

Revised resuscitation guidelines: epinephrine versus epinephrine/vasopressin in a pig model of cardiopulmonary resuscitation - a randomised, controlled trial

P. Meybohm, E. Cavus, V. Dörges, J. Scholz, B. Bein

Dept. of Anaesthesiology and Intensive Care Medicine, University Hospital Schleswig-Holstein, Kiel, Germany
Background and Goal of Study: Synergistic effects of epinephrine and vasopressin may be beneficial during cardiopulmonary resuscitation (1-3). However, it is unknown whether either epinephrine alone or an alternating administration of epinephrine and vasopressin is superior for restoring vital organ perfusion following basic life support (BLS) according to the revised algorithm with a compression-to-ventilation (c/v) ratio of 30:2.

Materials and Methods: After 4 minutes of ventricular fibrillation, and 6 minutes of BLS with a $\mathrm{c} / \mathrm{v}$ ratio of $30: 2$, sixteen pigs were randomised to receive either $45 \mu \mathrm{g} / \mathrm{kg}$ epinephrine alone, or alternating $45 \mu \mathrm{g} / \mathrm{kg}$ epinephrine and $0.4 \mathrm{U} / \mathrm{kg}$ vasopressin, respectively, every 5 minutes. Data were analyzed using two-way repeated measures analysis of variance factoring for time and treatment effects.

Results and Discussions: Coronary perfusion pressure (mean $\pm \mathrm{SD}) 20$ and 25 minutes after cardiac arrest was $7 \pm 4$ and $5 \pm 3 \mathrm{~mm} \mathrm{Hg}$ after epinephrine, and $25 \pm 2$ and $14 \pm 3 \mathrm{~mm} \mathrm{Hg}$ after epinephrine/vasopressin ( $p<0.001$ and $p<0.01$ versus epinephrine), respectively. Cerebral perfusion pressure was $23 \pm 7$ and $19 \pm 9 \mathrm{~mm} \mathrm{Hg}$ after epinephrine, and $40 \pm 10$ and $33 \pm 7 \mathrm{~mm} \mathrm{Hg}$ after epinephrine/vasopressin $(p<0.001$ and $p<0.01$ versus epinephrine), and cerebral laser doppler flow was $30 \pm 10$ and $27 \pm 11 \%$ of baseline after epinephrine, and $65 \pm 40$ and $50 \pm 31 \%$ of baseline after epinephrine/vasopressin ( $p<0.05$ versus epinephrine), respectively. Return of spontaneous circulation (ROSC) did not differ significantly between the epinephrine group (0/8) and the epinephrine/vasopressin group (3/8).

Conclusion: Epinephrine/vasopressin resulted in higher coronary and cerebral perfusion pressure, and cerebral laser doppler flow, while ROSC was comparable.

References:

1 Mayr VD, Wenzel V, Voelckel WG, et al. Circulation 2001; 104: 1651-6.

2 Lindner KH, Prengel AW, Pfenninger EG, et al. Circulation 1995; 91: 215-21.

3 Krismer AC, Wenzel V, Stadlbauer KH, et al. Crit Care Med 2004; 32: S432-5.

\section{AP2-5}

Effect of ventilation on resuscitation from cardiac arrest: impact of different compression-ventilation ratios during BLS-CPR

E. Cavus, P. Meybohm, V. Doerges, J. Scholz, B. Bein

Anaesthesiology and Intensive Care Medicine, University Hospital Schleswig-Holstein, Campus Kiel, Kiel, Germany

Background and Goal of Study: The 2005 revised guidelines for cardiopulmonary resuscitation (CPR) recommend a compression-to-ventilation (C/N) ratio of 30:2 $(1,2)$. The effects of this CPR mode in a realistic CPR scenario have not yet been fully elucidated.

Materials and Methods: Following approval of the Animal Investigational Committee, ventricular fibrillation (VF) was induced in 24 anaesthetised pigs. After $4 \mathrm{~min}$ of untreated VF, animals were randomly assigned to $6 \mathrm{~min}$ of basic-life support (BLS) CPR, and either (1) compressions only ("CC" group, $\mathrm{n}=8$ ), or (2) 30 compressions followed by two rescue breaths with a selfinflating bag ( $\mathrm{FiO}_{2} 0.21, \mathrm{C} /$ ratio $30: 2$; "30:2" group, $\mathrm{n}=8$ ), or (3) two breaths, followed by 15 compressions (C/ ratio 15:2; "15:2" group, $n=8$ ), followed by advanced cardiac life support.

Results: Arterial $\mathrm{Po}_{2}$ during BLS-CPR was higher in the 15:2 compared to the $30: 2$ and $C C$ group ( $74 \pm 3$ vs. $59 \pm 2$ and $31 \pm 4 \mathrm{mmHg}$, respectively; $p<.05)$. Mixed venous $\mathrm{Po}_{2}$ was comparable between groups. The 15:2 group remained normocapnic, and arterial $\mathrm{PcO}_{2}$ was lower compared to the $30: 2$ and $\mathrm{CC}$ group ( $32 \pm 2$ vs. $47 \pm 3$ and $65 \pm 3 \mathrm{mmHg}$, respectively; $p<.05$ ). In parallel, arterial pH decreased in the $30: 2$ and CC group compared to the $15: 2$ group $(7.33 \pm 0.03$ and $7.25 \pm 0.02$ vs. $7.51 \pm 0.04$, respectively; $p<.001) .4 / 8,2 / 8$, and $0 / 8$ animals in the $15: 2,30: 2$, and CC group, respectively, had return of spontaneous circulation at the end of the study period $(P=\mathrm{ns})$.

Conclusion(s): Ventilation during BLS-CPR after prolonged cardiac arrest has an important impact on both oxygenation and acid-base status; however, from our data we cannot comment on the optimal C/V ratio.

References:

1 Resuscitation 2005; 67: S39-86.

2 Circulation 2005; 112: IV1-203.

\section{AP2-8}

Perioperative cardiac arrest in 36,313 non-cardiac anesthetics in a tertiary hospital

S. El-Rouby, H. Al-Oufy, M. Shoukri

Anesthesiology, King Faisal Specialist Hospital and Research Center, Riyadh, Saudi Arabia 
Background and Goals: To determine the incidence of perioperative cardiac arrest in patients undergoing non-cardiac procedures and to study its relationship to physical status, age, and type of surgery (elective vs. emergency). Material and Methods: This prospective study is part of a quality improvement program from 1994-1999. Physical status was assessed preoperatively according to the classification of the American Society of Anesthesiologists (ASA). Data from 36,313 non-cardiac anesthetics were collected. Adverse events, including cardiac arrest (CA), were recorded intraoperatively by the attending anesthesiologist, in the post anesthetic unit (PACU) by the nurse, and postoperatively by another anesthesiologist. Data were encoded using a customized data base program, and analyzed using SAS software Results: $29.9 \%$ of the patients were ASA $(3,4,5), 39.6 \%$ were ASA(2) and $30.5 \%$ were ASA(1). Results were reported as rates per 10,000. Cardiac arrest was encountered in 5.51. Intraoperative was 3.03 and PACU was 2.48. (CA) correlation to ASA was highly significant ( $p<$ than 0.001$)$. The incidence in the age group ( $<1$ year) was 11.8 , from ( 1 to $<5$ years) was 2.8 and it was zero from (5-15 years), indicating a negative correlation in the pediatric group $(r=-0.808)$. The incidence in the adult age group from (15-20) was none, $(20$ to $<40)$ was 3.05 and from $(40$ to $>60)$ was 5.06 , indicating positive correlation in the adult group ( $r=0.927)$. In emergency procedures the incidence was (15.15), higher than in elective procedures (2.57), with $P=0.0763$.

Conclusions: Cardiac arrest has a negative correlation with age in the pediatric group, and a positive one in the adult group . Children younger than 1 year, patients with higher ASA and emergency procedures have a higher incidence of cardiac arrest. Preoperative optimization of co-morbid conditions could improve this adverse event.

References:

1 Murat, I. Pediatric Anesthesia 2004; 14:158-166.

2 Tiret, L. Can Anesth Soc 1986; 336-344.

3 Keenan, RL. JAMA 1985; 253: 2373-2377.

\section{AP3-1}

Influence of air-purifying respirators on the simulated emergency treatment of chemical, biological and radionuclear victims

A. Brinker, SA. Gray, J. Schumacher

Anaesthetics, St George's Hospital, London, United Kingdom

Background and Goal of Study: Medical first responders as well as emergency room personnel are potentially threatened by exposure to primary or secondary intoxication by chemical, biological, or radionuclear (CBRN) substances $^{1,2}$. The impact of personal respiratory protection by air-purifying respirators on the performance of resuscitation requires evaluation. This will help to improve major incident planning and measures for protecting medical pre-hospital or hospital staff.

Materials and Methods: We investigated the influence of two modern airpurifying respirator designs on the resuscitation of simulated CBRN victims. Fourteen UK paramedics followed a standardized resuscitation algorithm, either unprotected or wearing a bi-ocular and a panoramic visor respirator in a randomized crossover design. Treatment times and wearer comfort was determined and compared.

Results and Discussion: We did not find any difference in treatment times between the groups wearing respiratory protection and the controls $(189 \pm 8.3$ seconds for the controls, $191 \pm 9.5$ seconds for the panoramic visor mask and $206 \pm 9.1$ seconds for the biocular respirator [Mean \pm SEM]). Endotracheal intubation appeared to be the most time consuming task. In a questionnaire, volunteers expressed that orientation whilst wearing the respirator with the panoramic visor was better compared to the bi-ocular one (85\% versus $15 \%$ ). With respect to the fit, the majority $(79 \%)$ rated the bi-ocular respirator as more comfortable.

Conclusions: Modern personal respiratory protection has only a negligible effect in the delay of treatment of simulated CBRN casualties. Furthermore, compared to bi-ocular designs, air-purifying respirators with panoramic visors seem to allow a better orientation for medical first responders during simulated resuscitation.

References:

1 White SM. Br J Anaesth 2002; 89: 306-24.

2 Lockey D, Davies G. Resuscitation 2003; 58: 293-6.

\section{AP3-2}

Uneven distribution of ethanol in rat brain following acute administration, with highest level in striatum

M.H. Shyr, J.C. Chen, C.C. Lin, C.C. NG, T.F. Chiu

Anesthesiology, Buddhist Tzu Chi General Hospital, Hualein, Taiwan
Background and Goal of Study: Evidence exists for unequal extracellular levels of ethanol in different areas of the brain (1) after acute administration of ethanol. The aim of this study was to investigate the relative tissue levels and rates of decline of ethanol in various regions of the brain and in the plasma of rats.

Materials and Methods: Adult male Wistar rats (250-300 g) were injected intraperitoneally with a $40 \%, w / v$ ethanol/water solution at a dose of $1 \mathrm{~g} / \mathrm{kg}$ body weight. Brain were dissected into cerebral cortex (CX), diencephalons (DE), striatum (ST), hippocampus (HP), pons (PN), midbrain (MB), medulla (MD), cerebellum (CB), and spinal cord (SC) and plasma samples (BL) were analyzed thereafter at specified times for ethanol content by an enzymatic rate method (2).

Results and Discussions: The highest tissue and plasma concentrations of ethanol occurred 30 minutes after administration, with concentrations of ethanol being highest in the striatum. Data are expressed as mean \pm SD.

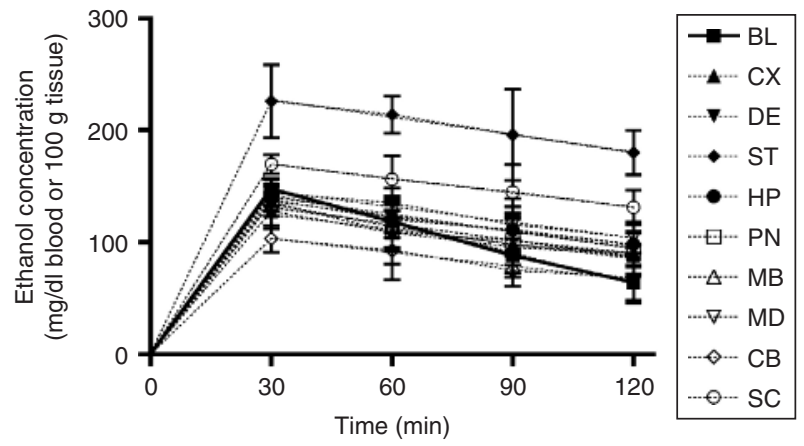

Conclusion(s): Ethanol was eliminated more slowly from the brain than from plasma. The higher level of ethanol in the striatum, an area implicated in movement regulation and substance addiction, may thus have a significant bearing on the neurological effects and addictive properties of ethanol.

References:

1 Erickson C. K. Life Sci. 1976; 19: 1439-46.

2 Hannigan J. H. Alcohol. Clin. Exp. Res. 1995: 19: 238-246.

\section{AP3-3}

\section{Evaluation of M-AID, a mobile phone first aid application}

R. Zanner, D. Wilhelm, H. Feussner, G. Schneider

Department of Anaesthesiology, Klinikum rechts der Isar der Technischen Universität, Munich, Germany

Background and Goal of Study: Cardiopulmonary resuscitation (CPR) by bystanders has been shown to reduce mortality due to sudden cardiac arrest when it is effectively performed. Telemedicine applications might support bystanders by giving specific instructions for handling an emergency situation. M-AID is a mobile phone first aid application. With an algorithm of 'yes' or 'no' questions, the software judges the current situation and instructs the user in detail what to do. The aim of this study was to evaluate the benefit of M-AID in an emergency scenario of sudden cardiac arrest.

Materials and Methods: 119 volunteers were randomly assigned either to the test or the control group. All participants had to manage the same emergency scenario - acute coronary syndrome leading to cardiac arrest. The participants were either equipped with a mobile phone running M-AID (test group) or had to handle the situation without any support (control group). The participants received a certain amount of credits for each action taken according to a pre-defined protocol. These credits were added to a score and compared between both groups. Furthermore, all participants were divided into subgroups according to their medical and technical experience.

Results and Discussions: The test group achieved a slightly higher average score that was not statistically significant ( 21.11 vs. $19.97 ; p=0.302)$. In contrast, the performance of the individuals in the control group was significantly faster (2.41 min. vs. $4.24 \mathrm{~min} ; \mathrm{p}<0.001$ ). Subgroup analysis showed that experienced mobile phone users performed significantly better than non experienced individuals, but not as good as participants with advanced first aid knowledge.

Conclusions: Experience in the use of mobile phones is a precondition for the efficient use of the tested M-AID version. Furthermore, the software cannot replace skills acquisition by practical training. In a subgroup with experience in the handling of mobile phones and basic knowledge in CPR, the device improved performance of CPR. 


\section{AP3-4}

Comparison of three techniques for emergency cricothyrotomy in a human cadaver study

P. Schober, T. Hegemann, L.A. Schwarte, S.A. Loer, P. Noetges

Department of Anaesthesiology, VU University Medical Center, Amsterdam, The Netherlands

Background and Goal of Study: Cricothyrotomy is the final lifesaving option whenever neither ventilation nor endotracheal intubation is possible [1]. Efficient management is indispensable to reestablish oxygenation and thus the quickest and safest method should be used. Therefore, this study aimed to compare three cricothyrotomy techniques.

Materials and Methods: Cricothyrotomy was performed in 61 human cadavers by 61 participants ( 57 medical students, 4 anaesthesiology residents). After theoretical instruction, participants were assigned to one of the following techniques: 1. surgical technique $\left(n=21\right.$, modified $\operatorname{ATLS}^{\circledR}$ approach), 2. catheter-over-needle technique ( $n=20$, Quicktrach, VBM-Medizintechnik), 3. wire guided cricothyrotomy (Seldinger) technique $(n=20$, Melker Cricothyrotomy Set, Cook). Time to insertion of the cannula as well as success rate and complication rate were recorded. Statistics: mean \pm SD, ANOVA and Chi-square tests, Bonferroni.

Results and Discussions: Cricothyrotomy was successful in $95 \%$ in the surgical group, in $85 \%$ in the Quicktrach group and in $75 \%$ in the Seldinger group (not significant). Speed was comparable between the surgical (106 $\pm 65 \mathrm{sec})$ and the Quicktrach technique $(114 \pm 94 \mathrm{sec})$. Seldinger-cricothyrotomy took significantly longer $(180 \pm 111 \mathrm{sec}, \mathrm{p}<0.05)$. No complications were observed in the surgical group. One or more complications were found in $55 \%$ of cadavers in the Seldinger group and in 65\% in the Quicktrach group (both groups $p<0.001$ vs. surgical).

Conclusion: Surgical cricothyrotomy had the lowest complication rate and tended to be quicker and more successful than the other techniques.

Reference:

1 Henderson et al. Anaesthesia 2004; 59: 675-694.

\section{AP3-5}

Risk Analysis for emergency missions with physician-staffed ambulances compared to rescue helicopters

J. Hinkelbein, J. Basters, T. Viergutz, H.V. Genzwuerker, J. Meinhardt Clinic for Anesthesiology and Intensive Care Medicine, University Hospital Mannheim, Mannheim, Germany

Background and Goal of Study: In Germany, annually approx. 2 million ground-rescue missions with physician-staffed ambulances (PSA) were carried out. In addition approx. 80.000 helicopter emergency medical services (HEMS) air-missions were accomplished [1]. National and international data $[2,3]$ revealed 10.8 accidents $/ 100,000$ flight-hours (0.54 accidents $/ 10,000$ air-missions). Data for the risk during PSA ground-missions are not available. The aim of the present study was to compare the accident risk of PSA to HEMS in a 10-year period.

Materials and Methods: A retrospective analysis ranging from 1996-2005 was carried out using published accident data from the BASt (Federal Highway Research Institute) for PSA and flight accident reports from the BFU (Federal Agency for Flight Accident Investigation) for HEMS. Additional data (operating hours, missions, accident characteristics) were gathered and compared to published data $[2,3,4]$. Fisher's exact test was used for statistical analysis; $\mathrm{P}<0.05$ was considered statistically significant.

Results and Discussions: In the analyzed period per year 1.245 PSA and 75 helicopters were at service ( 1.74 million vs. 36,775 operating hours). Each year 90 vs. 4 accidents occurred. On the basis of 10,000 missions data were comparable ( 0.43 vs. 0.5 accidents, n.s.). A significant difference was found per 100,000 operating hours $(5.17$ vs. $10.87, \mathrm{P}<0.05)$. International data were partly discrepant $[3,4]$.

Conclusion(s): The risk for accidents on the basis of missions is comparable for PSA and HEMS, but significantly higher for HEMS on the basis of operating hours. For Germany, published studies on this topic are not available and denominator data are not published sufficiently. Denominator data are often inhomogeneous and discrepant making direct comparisons difficult or nearly impossible.

\section{References:}

1 Behrendt $\mathrm{H}$ et al. Handbook of emergency rescue, 2003.

2 Viergutz T et al. Notarzt 2006; 22(6): 186-192.

3 Rhee KJ et al. Aviat Space Environ Med 1990; 61(8): 750-752.

4 Biggers WA Jr et al. Prehospital Disaster Med. 1996; 11: 195-201.

\section{AP3-6}

Basophil activation test in perioperative anaphylaxis.

L. Rees, O. Adekanye, G. Read, P. Williams, J. Hall

Anaesthetics and Clinical Immunology, Cardiff University and University Hospital of Wales, Cardiff, United Kingdom

Background and Goal: The basophil activation test (BAT) is a method for investigation of perioperative anaphylaxis. BAT specificity is high and correlates well with skin prick testing; however sensitivity may be low. We noticed apparent basophil cell loss in allergen-stimulated samples and postulated that this might account for false negatives. In this preliminary work, we sought to determine whether the basophil activator formyl-methionyl-leucylphenylalanine (fMLP) caused a dose-dependent decrease in basophil count and activation.

Methods: With LREC approval, whole blood was taken from 6 volunteers with no allergy history. Duplicate samples were exposed to increasing concentrations of $\mathrm{MLP}\left(10^{-8}\right.$ to $\left.10^{-4} \mathrm{M}\right)$ or controls. Flow cytometry was used to identify basophils by CD45 and IgE expression; cell activation was assessed by CD63 expression [1]. Cell count was enumerated using CellBeads [2].

Results: fMLP caused a concentration-dependent stimulation of CD63 expression between $10^{-7}$ and $10^{-4} \mathrm{M}$, maximal at $10^{-6}$ and $10^{-5} \mathrm{M}$. Basophil count appeared to decrease with increasing concentration, though this was statistically significant only at $10^{-5} \mathrm{M}$.

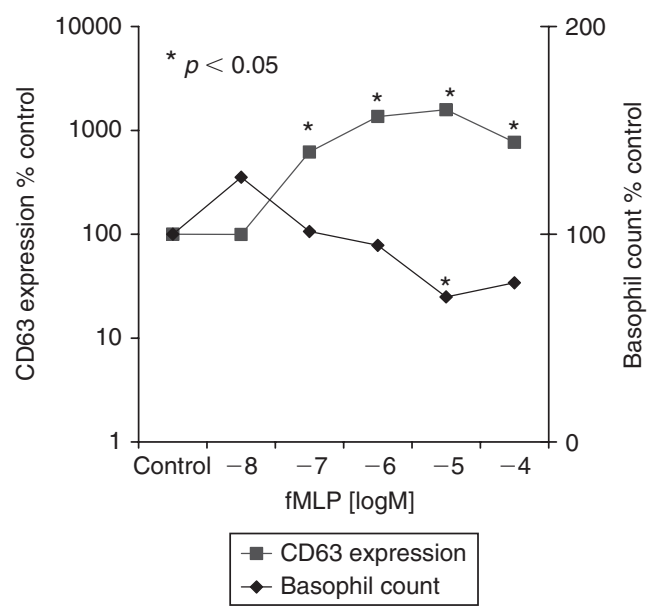

Conclusion: Basophil activation is dose-dependent. This preliminary data suggests count may be affected by fMLP concentration. Further work will investigate whether significant cell loss occurs in patients with a confirmed allergy to neuromuscular relaxants.

References:

1 Monneret $\mathrm{G}$ et al. Clin Exp Immunol 1999; 115: 393-6.

2 Harrison $G$ et al. Clin Diag Lab Immunol 2001; 8: 397-401.

\section{AP3-7}

Life guarded delight - implementation of a rapid response team in the semperoper

M. Frank, C. Hoffmann, M. Mueller, R.J. Litz, T. Koch

Anaesthesiology, University of Technology, Dresden, Germany

Background and Goal of Study: With more than 350 performances annually and an evening audience of about 1000 guests the Dresden opera house "Semperoper" is well known and highly frequented .To ensure audience' medical security and to acquaint artists as well as audience with immediate and specialized medical care in case of emergency events a medical rapid response team (RRT) was established in 2001.

Materials and Methods: For initial treatment in emergencies a physician with fundamental experience in emergency medicine as well as the Opera staff trained in logistics and medical basic emergency algorithms create a rapid response team (RRT). The team is demanded via a central alerting system operated pager. A room with complete emergency equipment such as defibrillator, ecg, oxygen and emergency bags was set up in the centre of the building. All emergency cases since the initiation in 2001 were evaluated to challenge the proceedings' benefit. Therefore emergency documentation performed was retrospectively analyzed.

Results and Discussions: Within a six year period the RRT was demanded in 291 cases of emergency. The team was on scene within a maximum of 5 minutes of call. Patients were in average 63 years of age [14-88] and $68 \%$ of the patients were female. In $56 \%$ the RRT was confronted with cardiac, 
pulmonary and metabolic emergencies. Surgical emergency was documented in $31.6 \%$, neurological emergency in $3.8 \%$ and psychiatric emergency was reported in $3.8 \%$. In $10.6 \%$ of all cases patients tripped on stairs and fell. NACA score 1 and 2 were found in $64.2 \% .20 .6 \%$ were classified with NACA 3. NACA 4 was documented for $8.2 \%$ and in $5.1 \%$ NACA 5 . Three cases of cardiac arrest occurred during this six year period, 1 person died. $23 \%$ of all patients were admitted to hospital immediately by ambulance.

Conclusion(s): The introduction of an own rapid response team with a specialized physician in highly frequented public institutions such as theaters and opera houses enables an early detection and treatment of severe emergency and ensures reliable evaluation of enhanced treatment or hospital admission.

\section{AP4-1}

\section{Whole blood viscosity in the initial phase of the acute coronary syndrome}

A. Bauer, J. Woishuber, M. Ruppert, M. Mooney, F. Christ

Clinic for Anaesthesiology, Ludwig-Maximilians University, Munich, Germany Background and Goal of Study: Elevated whole blood viscosity $(\eta)$ has been observed in atherosclerotic disease [1]. We investigated the association of whole blood viscosity with acute coronary syndrome (ACS) in an out-of-hospital setting.

Materials and Methods: 113 patients (age range: 19-96 years) treated by the emergency service were studied. We used a novel capillary viscometer (Rheologics, Exton, USA), which enables the assessment of $\eta$ over a wide range of shear rates [2].

Results and Discussions: Whilst mean $\eta$ in ACS patients $(n=33)$ was higher compared to emergency patients without atherosclerotic disease $(n=60)$ the difference did not reach significance (see figure). Viscosity was mainly determined by hematocrit $(r=0.43 ; P<0.001)$ but did not correlate with classical risk factors like arterial hypertension, smoking habits and hyperlipoproteinemia.

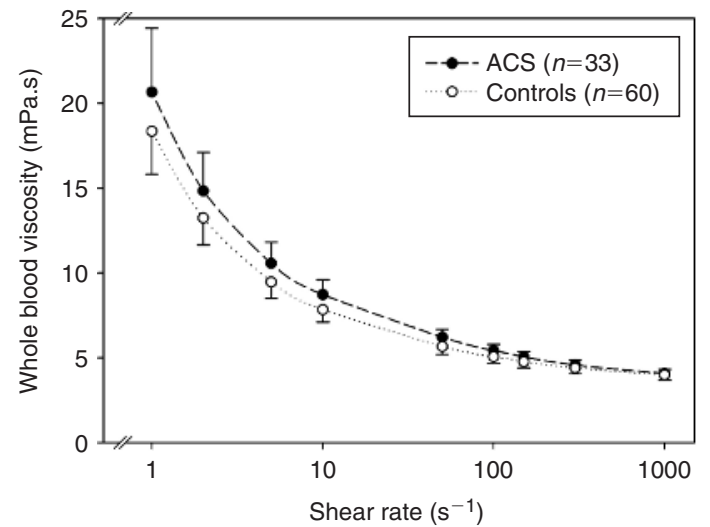

Fig. Mean and $95 \%$ confidence interval of shear rate dependent whole blood viscosity $(\eta)$.

Conclusion: In our heterogenic population of emergency patients, $\eta$ was no significantly elevated in the initial phase of ACS.

References:

1 Danesh Eur Heart J 2000; 21: 515-520

2 Wang Clin Chim Acta 2003; 332: 79-82.

\section{AP4-2}

Effect of intraoperative fluid optimisation on renal function in patients undergoing emergency abdominal surgery; a randomised controlled pilot study

J. Harten, J. Crocier, B. McCreath, D. McMillan, J. Kinsella

Department of Anaesthesia, University of Glasgow, Glasgow, United Kingdom

Back ground and Goal of the Study: This pilot study aims to examine the effect of goal directed therapy using fluid alone on postoperative renal function in patients undergoing emergency abdominal surgery.

Materials and Methods: This prospective randomized study included patients over the age of 50 undergoing emergency abdominal surgery. Patients presenting following trauma, undergoing vascular surgery or who were on lithium therapy were excluded from the study. Research ethic committee approval and informed consent was obtained. In the intervention group the Lidco monitor (Lidco plus system, Lidco Ltd., UK) was used to guide fluid boluses of $250 \mathrm{ml}$ of $6 \%$ Hydroxyethylstarch 130/0.4 (Voluven, Fresenius Ltd., UK) based on pulse pressure variation measurement (bolus given if $>10 \%)$. The control group received standard care. Serum urea, creatinine and cystatin $C$ levels were measured prior to and at the end of surgery, and postoperatively on days $1 \& 3 \& 5$. Data are median (range) and were analyzed using chi-square test or Mann Whitney test as appropriate.

Results: 30 patients were recruited. Their age was 65 yrs (51-76), 23 patients were male, ASA $=3(1-4)$, Lee risk score $=3(2-4)$. One patient died prior to surgery and was excluded from the analysis. 10 patients had upper and 19 lower abdominal surgery. Duration of surgery was 110 min (40-295). There were no demographic differences between the groups. The intervention group received a median of $750 \mathrm{ml}$ of hydroxyethylstarch. The peak values of postoperative urea were $6.9(2.7-31.8)$ vs $6.4(3.5-11.5) \mathrm{mmol} / \mathrm{l}(\mathrm{p}=0.425)$, creatinine $100(60-300)$ vs $85(65-150) \mu \mathrm{mol} / \mathrm{l}(\mathrm{p}=0.085)$ and cystatin $\mathrm{C} 1.09$ $(0.66-4.94)$ vs $1.01(0.33-2.29) \mathrm{mg} / \mathrm{dl}(\mathrm{p}=0.352)$ in the control and intervention group respectively.

Conclusions: Replacing the identified fluid deficit was not associated with a change in renal function in this study. These results do not preclude that goal directed therapy may have an effect on renal function but they would suggest that the effect size of fluid optimization alone on renal function is small.

\section{AP4-3}

Effect of intraoperative fluid optimisation on postoperative morbidity score in patients undergoing emergency abdominal surgery; a randomised controlled pilot study

J. Harten, J. Crozier, B. McCreath, A. Hay, J. Kinsella

Anaesthesia, University of Glasgow, Glasgow, United Kingdom

Back ground and Goal of the Study: This pilot study aims to examine the effect of goal directed therapy using fluid alone on postoperative morbidity score (PMS) in patients following emergency abdominal surgery [1].

Materials and Methods: This prospective randomized study included patients over the age of 50 undergoing emergency abdominal surgery. Patients presenting following trauma, undergoing vascular surgery or who were on lithium therapy were excluded from the study. Research ethic committee approval and informed consent was obtained. In the intervention group the Lidco monitor (Lidco plus system, Lidco Ltd., UK) was used to guide fluid boluses of $250 \mathrm{ml}$ of $6 \%$ Hydroxyethylstarch 130/0.4 (Voluven, Fresenius Ltd., UK) based on pulse pressure variation measurement (bolus given if $>10 \%$ ). The control group received standard care. PMS were measured postoperatively on days $5 \& 15 \& 30$. Data are median (range) and were analyzed using chisquare test or Mann Whitney test as appropriate.

Results: 30 patients were recruited. Their age was 65 yrs (51-76), 23 patients were male, ASA $=3(1-4)$, Lee risk score $=3(2-4)$. One patient died prior to surgery and was excluded from the analysis.10 patients had upper and 19 lower abdominal surgery. Duration of surgery was $110 \mathrm{~min}$ (40-295). There were no demographic differences between the groups. The intervention group received a median of $750 \mathrm{ml}$ of hydroxyethylstarch. On day 5 \& 30 there were no differences in PMS scores. Day 15 data as displayed in table.

\begin{tabular}{llll}
\hline Day 15 & Control & Intervention & $\mathrm{P}$ \\
\hline In hospital (yes/no) & $4 / 11$ & $9 / 5$ & 0.042 \\
PMS score & $0(0-5)$ & $1(0-7)$ & 0.328 \\
Complication (yes/no) & $4 / 11$ & $7 / 7$ & 0.196 \\
\hline
\end{tabular}

Conclusions: Goal directed therapy using fluid alone may be associated with an increased incidence of in hospital stay at 15 days.

Reference:

1 Bennett-Guerrero E. Anesthesia \& Analgesia 1999; 89: 514.

\section{AP4-4}

Severe perioperative allergic reactions to non-esteroidal antiinflammatory drugs

C. Rodriguez Cosmen, L. Moltó García, N. Baldomà Heras, A. Gimenez Arnau, F. Escolano Villen

Anestesiologia, reanimación y terapeutica del dolor, Hospital del MarEsperanza, Barcelona, Spain

Background and Goal of Study: There isn't any study about the incidence and severity of allergic reactions (AR) to non-steroidal anti-inflammatory drugs (NSAID) during the perioperative period. We investigated the incidence, severity, evolution and immunological mechanisms of these AR in our hospital. 
Material and Methods: A prospective study was carried between 1996 and 2005. We included all the anaesthetized patients exposed to NSAID in the operating room and in the postoperative care unit. We recorder the medical history and description of the allergy reactions. During the first 24 hours we performed the immediate immunoalergic study (IIS): histamine, serica tryptase, urine metilhistamine, complement factors, latex and other drugs and histamine release test (HRT). Posteriorly, we performed the delayed immunoalergic study (skin test, Ig E and histamine release tests specific).

Results and Discussions: 148.399 vials of NSAID were administered: 81.893 of metamizol, 44.315 of paracetamol, 12.015 of diclofenaco and 10.176 of dexketoprofeno. 10 severe AR occurred: 7 cases to metamizol and 3 cases to diclofenaco. All patients had a good evolution. The IIS was positive in all cases and the delayed immunoalergic study was positive in the patients studied. Conclusion: The incidence of severe perioperative RA was 1 in 14.840 vials of NSAID used. Metamizol is more common cause of adverse reaction than diclofenaco. Paracetamol and dexketoprofeno didn't cause RA. The immune mechanism of the reaction was confirmed by the IIS, and the delayed immunoalergic study diagnosed anaphylactic reactions mediated by $\lg \mathrm{E}$ antibody.

\section{AP4-5}

Estimation of necessary medical staffing for an efficient emergency department management

B. Bohy, C. De Deyne, G. Vundelinckx, R. Heylen

Anesthesia and Critical Care Medicine, Ziekenhuis Oost-Limburg, Genk, Belgium
Introduction: Necessary medical staffing at an emergency department (ED) is an important issue for the medical quality of care as well as for the financial implications at a large tertiary hospital. Critical factors for efficient ED management are the grade of urgency for medical assistance and the level of medical assistance needed.

Patients and Methods: We retrospectively analysed all patients identified as direct admissions (without specific referral to any medical discipline) registered at the ED of our hospital for a 3 months period. Pts were classified according to 2 variables : the time period to necessary medical intervention ( $<1 \mathrm{hr}$, between 1-12 hrs, between 12-24 hrs) and the level of medical assistance needed (general practitioner (GP), GP + technical investigations, specialized medical intervention).

Results: A total of 4.250 pts were registered in the ED. 215 pts (5\%) needed immediate medical assistance ( $<1 \mathrm{hr}), 2.728$ pts $(61 \%)$ needed assistance within a $12 \mathrm{hrs}$ period, whereas $1.307 \mathrm{pts}(33.7 \%)$ could wait for a period upto $24 \mathrm{hrs}$. A majority of pts (62\%) needed GP assistance with technical investigations, $30 \%$ of pts needed specialized assistance, whereas $8 \%$ of pts were categorized as necessitating "only" GP assistance.

Conclusion: Our ED population merely consists of pts necessitating GP medical (and technical) assistance within $12 \mathrm{hr}$ of ED admission. These results suggest that a large percentage of our ED patients may not require the extra facilities of the department, thereby decreasing the efficiency of medical staff dealing with more complicated cases.

\section{Acute and Chronic Pain Management}

\section{AP1-1}

\section{N-Methyl-D-Aspartate receptor blocking therapy and post-operative analgesia: the methadone option}

E. Tognoli, S. Sangion, M. Langer

Intensive Care Unit, Istituto Nazionale Per lo Studio E La Cura Dei Tumori, Milano, Italy

Background and Goal of Study: N-Methyl-D-Aspartate (NMDA) receptordependent enhancement of nociceptive synaptic transmission is considered a key mechanism in the development of perioperative acute opioids tolerance and hyperalgesia observed after remifentanil based anaesthesia(1,2). Methadone is both a $\mu$-opioid receptor agonist like morphine both an NMDA receptor antagonist like ketamine. Our hypothesis is that NMDA receptor antagonism activity of methadone could reduce opioids requirement after surgery. The aim of the study was to compare methadone and morphine consumption after major abdominal surgery.

Materials and Methods: We studied 10 patients undergoing major abdominal surgery. All patients received a propofol-remifentanil based anaesthesia (TCl modality) supplemented with low-dose Ketamine(2). Patients were randomly assigned to receive morphine (Mo group) or methadone (Me group) for post-operative analgesia. Opioids were administered with a loading dose of $0,15 \mathrm{mg} / \mathrm{Kg} 40$ minutes before the end of surgery and 1-2 mg/10 minutes if pain score (Numerical Rating Scale: NRS) was $>3$ in recovery room (RR). Patients were monitored for $2 \mathrm{~h}$ in $\mathrm{RR}$ : we registered vital signs and NRS every 10 minutes by a physician blind to the treatment, and a Blood Gas Analysis after 2 hours. Data are presented as mean \pm DS, comparisons between groups were performed with test One way ANOVA (sigma-stat 2.0).

Results: Methadone group presents a significantly lesser cumulative opioids consumption and a better pain scores during the first two postoperative hours without significantly breathing depression.

\begin{tabular}{llllll}
\hline & & NRS & \multicolumn{2}{c}{ Opioid $\mathrm{mg}$} & \multicolumn{2}{c}{$\mathrm{BGA}^{*}$} \\
& $\mathrm{RR}$ & $2 \mathrm{~h}$ & $2 \mathrm{~h}$ & $\mathrm{pO}_{2}$ & $\mathrm{pCO}_{2}$ \\
\hline $\mathrm{Mo}$ & $3 \pm 3$ & $2 \pm 1$ & $7 \pm 2$ & $100 \pm 16$ & $44 \pm 6$ \\
$\mathrm{Me}$ & $1 \pm 3$ & $0 \pm 0$ & $2 \pm 2$ & $106 \pm 11$ & $43 \pm 5$ \\
$\mathrm{p}$ & $<0,05$ & $<0,05$ & $<0,05$ & $\mathrm{~ns}$ & $\mathrm{~ns}$ \\
\hline
\end{tabular}

*supplemented with Venturi face mask: $\mathrm{FiO}_{2} 35 \%$.

Conclusion: Our data suggest that Methadone permits a good postoperative pain control with a reduction in opioid consumption after propofol-remifentanil based anaesthesia supplemented with Ketamine.
References:

1 Clifford J. Woolf: Science 2000; 288: 1765-68

2 Guignard B.: Anesth Analg 2002 Jul; 95(1): 103-108.

\section{AP1-2}

Fascia lliaca compartment block for pre-operative pain relief in adult fracture neck of femur: a nurse led initiative in luton and dunstable hospital UK

I. Srikantharajah, ID. Srikanthrajah, O. Ayodele, L. Grigg, A. Randall

Anaesthesia, Luton and Dunstable Hospital NHS Trust, LUTON, United Kingdom

Aim: To provide a nurse led service in our Hospital and to assess the efficacy of fascia iliaca compartment block administered by Pain Specialist Nurses for fractured neck of femur pain relief for first twenty four hours.

Methodology: Following the approval of the guideline for the management of pain relief in fractured neck of femur by the Hospital Guidelines Committee, trained the pain specialist nurses to perform this block. Patients audited were either referred by A \& E personnel or the Trauma/Ward sister. Exclusion criteria included patient's refusal, unconscious, demented and anticoagulated patients. Others include patients with known sensitivity to local anaesthetic, body mass index (BMI) more than 40 and peripheral neuropathy. After obtaining verbal consent, preceded by explanation of the procedure to the patient, fascia iliaca compartment block was instituted. Twenty to thirty $\mathrm{ml}$ of $0.25 \%$ plain bupivacaine was given. Pain score using a verbal rating score was assessed at 15 minutes, 2, 8 and 24 hours after the block.

Result: The 14 patients were made the age range is 63 to 91 years. All of them had oral paracetamol $1 \mathrm{~g}$ and variable doses of oral and parenteral opioid on admission. The pain score at presentation for all the patients was $10.71 \%$, $78 \%, 84 \%$ and $83 \%$ of the patients had a pain score of 3 or less at 15 minutes, 2,8 and 24 hours respectively after the block. There were no reported complications as result of the procedure.

Conclusion: The audit showed that fascia iliaca compartment block provided pain relief after fractured neck of femur in more than $70 \%$ of the situations. It also showed that non-anaesthetic personnel can be trained to do the block successfully. Although no complication was recorded in the series, it must be noted that the number of patients done is small. The audit is ongoing. Future Prospect: It is hoped that more personnel, as mentioned above will be trained to provide this service in our hospital.

References:

1 Lopez S. Reg Anesth. Pain Med 2003; 28: 203-207.

2 Dalens B. Anesth. Analg. 1989; 69: 705-713. 


\section{AP1-3}

Effects of perioperative i.v. infusion of low dose ketamine associated with thoracic epidural analgesia for abdominal aortic surgery on postoperative analgesia and early postoperative outcome

E. Aertgeerts, M. Senard, D. Ledoux, L. Roediger, J. Joris

Anaesthesia and Intensive Care, University Hospital, Liège, Belgium

Background and Goal of Study: Thoracic epidural analgesia (TEA) provides effective analgesia and improves outcome after abdominal surgery (1). Some peritoneal nociceptive inputs are not blocked by TEA (2). Ketamine (K.) prevents postop. hyperalgesia through central and peripheral mechanisms (3). We investigated effects of the adjunction of perioperative iv $\mathrm{K}$. to TEA, on early postop. analgesia and outcome after open abdominal aortic surgery (AAS). Materials and Methods: After approval of ethic committee and informed consent, 36 patients scheduled for open AAS were included in this study. Combined general anaesthesia with TEA were used in all patients. TEA (T9-T10) was started before surgical incision and maintained for the first $60 \mathrm{~h}$ postop (ropivacaine $0.2 \%+0.5 \mu \mathrm{g} / \mathrm{ml}$ sufentanil). After induction of anaesthesia, patients were randomly allocated in two groups $(n=18$ in each group): patients were given an iv bolus of K. $(225 \mu \mathrm{g} / \mathrm{kg})$ followed by an infusion $(100 \mu \mathrm{g} / \mathrm{kg} / \mathrm{h})$ during the first $24 \mathrm{~h}$ postop $(\mathrm{K})$ or same volume of saline (S). All patients were provided with a piritramide PCA pump for $96 \mathrm{~h}$. Pain scores (VAS), piritramide consumption, respiratory function, postop outcome (PONV, satisfaction, fatigue, time to first flatus, ambulation, hospital stay, stress response, and morbidity) were recorded. ANOVA for repeated measures, Student's t, Mann-Whitney and Fischer exact test were used with $\mathrm{p}<0.05$ as significant.

Results and Discussions: Demographic data, pain scores at rest $(p=0.1)$, coughing $(p=0.2)$ and mobilization $(p=0.24)$ and piritramide consumption $(p=0.56)$ (table) were not significantly different between the groups.

\begin{tabular}{lllll}
\hline Piritramide & Day 1 & Day 2 & Day 3 & Day 4 \\
\hline K. (mg) & $8.4 \pm 12$ & $16.6 \pm 20$ & $12.8 \pm 14$ & $8.4 \pm 9$ \\
S. (mg) & $5.8 \pm 7$ & $15.2 \pm 16$ & $14.7 \pm 13$ & $3 \pm 5.2$
\end{tabular}

There was no difference with regards to morbidity or postop. outcome between the two groups.

Conclusion(s): When associated with TEA using local anaesthetic and opioid, perioperative iv infusion of low dose ketamine does not improve analgesia nor early postop. outcome after open AAS.

References:

1 Ballantyne JC. Anesth Analg1998; 86: 598-612.

2 Aida S. Anesth Analg 1999; 89: 711-6.

3 De Kock M. Pain 2001; 92: 373-80.

\section{AP1-4}

Intraoperative ketamine does not improve postoperative analgesia after scoliosis surgery in adolescent

\section{O. Kaabachi, R. Ouezzini, W. Koubaa, B. Ghrab, R. Cherif}

anesthesiology and Intensive Care, Institut Kassab Dorthopédie, Tunis, Tunisia

Background and Goal of Study: Major surgeries with remifentanil-based anaesthesia are associated with severe postoperative pain. Large-dose remifentanil causes acute opioid tolerance and hyperalgesia. The NMDA receptor antagonist ketamine may be appropriate for preventing postoperative hyperalgesia. There are controversial results concerning the pre-emptive effect of ketamine on postoperative pain after a remifentanil-based anaesthesia in adults (1-3). We therefore tested the hypothesis that intraoperative small-dose ketamine improves postoperative analgesia after scoliosis surgery with remifentanil-based anesthesia.

Materials and Methods: after ethic committee approval and obtaining patient informed consent, 46 patients were enrolled in a prospective, double blinded, controlled study. Patients were randomly allocated to receive either $0.25 \mathrm{mg} / \mathrm{kg}$ of ketamine before anesthesia induction and $4 \mu \mathrm{g} / \mathrm{kg} / \mathrm{min}$ during the procedure or saline. Anesthesia was induced with Propofol $2.5 \mathrm{mg} / \mathrm{kg}$ and remifentanil $0.5 \mu \mathrm{g} / \mathrm{kg}$ and maintained with the same drugs. The drugs infusion rate was adjusted according to blood pressure and heart rate. All patients had intrathecal morphine $5 \mu \mathrm{g} / \mathrm{kg}$ before induction, paracetamol and ketoprofen at the end of surgery. We recorded: hemodynamic parameters, intraoperative drug consumption, blood loss, time to anesthesia recovery, pain scores, time to first analgesic rescue and morphine requirement (VA > 30).

Results and Discussions: Demographic data and mean procedure time were similar in both groups. There was a tendency for better hemodynamic stability in ketamine (K) group. But, intraoperative blood loss and drug consumption were similar in both groups. Time for extubation was longer in $\mathrm{K}$ group (30.1 min vs $20.4 \mathrm{~min}$ ). Pain scores were low in both groups. Morphine consumption was significantly lower in $\mathrm{K}$ group but only during the first postoperative hour ( $2.8 \mathrm{mg}$ vs $4.5 \mathrm{mg}$ )

Conclusion(s): intraoperative low-dose ketamine had no effect on morphine consumption after remifentanil-based anesthesia for scoliosis.

References:

1 Guignard B. Anesth Analg 2002; 95: 103-8.

2 Van Elstraete AC. Acta Anaesthesio Scand 2004; 48: 756-60.

3 Ganne O. Eur J Anaesthesiology 2005; 22: 426-30.

\section{AP1-5}

The effect of low dose magnesium sulphate on early postoperative pain after laparoscopic cholecystectomy

I. Bacak, R. Krobot, J. Premuzic, G. Kocijan

Anesthesiology and Intensive Care, General Hospital Varazdin, Varazdin,

Croatia

Background and Goal of Study: As a NMDA receptor antagonist, magnesium sulphate $\left(\mathrm{MgSO}_{4}\right)$ has analgesic properties(1). The study was designed to evaluate whether preemptive, low intravenous dose of $\mathrm{MgSO}_{4}$ affects postoperative pain after laparoscopic cholecystectomy.

Materials and Methods: 60 ASA I-II patients undergoing laparoscopic cholecystectomy were enrolled to the study. Anesthesia was performed with the same drugs in all groups. After anesthesia induction, prior to surgical incision, patients in group $C(n=20)$ received saline, group $M g 1(n=20) 5 \mathrm{mg} / \mathrm{kg}$ $\mathrm{MgSO}_{4}$ and group $\mathrm{Mg} 2(\mathrm{n}=20) 7,5 \mathrm{mg} / \mathrm{kg} \mathrm{MgSO}$. Pain intensities at rest according to visual analog scale (VAS) were evaluated at 1,3,6,9 and $24 \mathrm{~h}$ postoperatively. Postoperative analgesia included iv methamizol $2,5 \mathrm{~g}$ (VAS 2-4), diclofenac $75 \mathrm{mg}$ (VAS 5-7) or tramadol $1 \mathrm{mg} / \mathrm{kg}$ (VAS 8-10).Whole analgesic consumption in $24 \mathrm{~h}$ was documented.

Statistics: Mann-Whitney, $X^{2}$ and t-test ( $p<0.05$ significant).

Results and Discussions: Demographic data were comparable in all groups. Mean VAS pain scores \pm SD are shown in the table.

\begin{tabular}{llll}
\hline Postop. $\mathrm{h}$ & $\mathrm{C}$ & $\mathrm{Mg} 1$ & $\mathrm{Mg} 2$ \\
\hline 1st & $5.15 \pm 2.01$ & $4.70 \pm 1.78$ & $3.20 \pm 1.82^{* \#}$ \\
3rd & $3.80 \pm 2.26$ & $3.70 \pm 1.81$ & $2.40 \pm 1.54^{\$}$ \\
6th & $1.95 \pm 1.73$ & $2.05 \pm 1.00$ & $2.15 \pm 1.42$ \\
\hline${ }^{*} \mathrm{P}<0.01$ vs group C, \# $\mathrm{P}<0.05$ vs group $\mathrm{Mg} 1$ & \\
$\$ \mathrm{P}<0.05$ vs group C and vs group Mg1 & &
\end{tabular}

Within the first $24 \mathrm{~h}$ methamizol consumption was lower in $\mathrm{Mg} 2$ than in $\mathrm{C}$ and $\mathrm{Mg} 1$ group $(\mathrm{P}<0.001$ vs $\mathrm{C}, \mathrm{P}<0.05$ vs $\mathrm{Mg} 1)$. Diclofenac consumption was also lower in Mg2 group $(\mathrm{P}<0.05$ vs $\mathrm{C}$ and vs Mg1).Tramadol needed 3 patients in group $C$.

Conclusion: Preemptive dose of $7,5 \mathrm{mg} / \mathrm{kg} \mathrm{MgSO}_{4}$ given to the patients undergoing laparoscopic cholecystectomy is the lowest efficacious dose against early postoperative pain (first $3 \mathrm{~h}$ ). Analgesic consumption in the first $24 \mathrm{~h}$ is highly reduced in those patients when compared to patients who received $5 \mathrm{mg} / \mathrm{kg} \mathrm{MgSO}_{4}$ or placebo.

Reference:

1 Bhatia A. et al. Journal of Clin Anesth 2004; 16(4): 262-5.

\section{AP1-6}

Patient-controlled epidural analgesia and its influence on postoperative pain and vegetative status after major abdominal surgery

D. Uvarov, M. Orlov, N. Ovchinnikov, M. Kirov, E. Nedashkovsky Department of Anesthesiology and Intensive Care, Northern State Medical University, Arkhangelsk, Russian Federation

Background and Goal of Study: The patient controlled epidural analgesia (PCEA) is supposed to be an effective manner of postoperative analgesia after major surgery (1). However, association between intensity of pain and of patient's vegetative status remains unclear. Therefore, the main goal of our study was to compare the efficacy of epidural bupivacaine and morphine administered as a bolus vs. PCEA of these drugs and its influence on the dynamic of vegetative status of patient after laparotomy.

Materials and Methods: Sixty-five adult patients underwent major abdominal surgery were enrolled in a prospective randomized study. All patients were randomized to two groups. In the PCEA group $(n=34)$, patients have received controlled epidural analgesia with morphine $100 \mathrm{mcg} / \mathrm{ml}$ in $0.125 \%$ bupivacaine solution. In the EBI (Epidural Bolus Injection) group $(n=31)$, these analgesics were given in same concentrations via bolus injections. Pain scores were assessed during first 24 hours after surgery by $100-\mathrm{mm}$ visual analogue 
scale (VAS). Measurements were done hourly during first 3 hours postoperatively and then every 3 hours. The dynamic of the stress index of R.M. Baevsky (SI) were assessed every 3 hours postoperatively. Data were compared by using Student's t-test. $p<0.05$ was regarded as statistically significant.

Results and Discussions: At 6, 12 and $24 \mathrm{~h}$ after ICU admission, VAS was significantly lower in the PCEA group compared with the EBI group $(p<0.05)$. The dynamic of the SI have shown, that PCEA provides better stability of sympathetic nervous system of patient after laparotomy when bolus epidural injection method.

Conclusion(s): The PCEA profile of postoperative analgesia using bupivacaine and morphine in patients after major abdominal surgery allowed adequate postoperative analgesia. Moreover, PCEA provided the stability of vegetative status of patient while improving the quality of analgesia.

Reference:

1 Yavuz Let al. Eur. J. Gynaecol. Oncol. 2004; 25(2): 215-218.

\section{AP1-8}

Postoperative mapping of sensitive dysesthesia and residual pain after sternotomy for cardiac surgery

M. Momeni, Philippe. Baele, Hilde. Waterloos, Marc. De Kock, Patricia. Lavandhomme

Anesthesiology, Clin Universitaires St Luc, Brussels, Belgium

Background and Goals: Residual pain (RP) is not rare after sternotomy for cardiac surgery (1). Abnormal postoperative skin sensitivity seems associated with higher risk to develop RP after surgery. Although both hypoesthesia and hyperalgesia have been reported after heart surgery (2), their correlation with $\mathrm{RP}$ remains unknown. The study compares early postoperative dysesthesia (at day5 and day30) between patients presenting with RP (RP group) or not after sternotomy for cardiac surgery.

Material and Methods: 60 consecutive patients scheduled for CABG with IMA harvesting or valve replacement (VR) were included (redo-operation and preoperative analgesics intake were excluded). At postoperative day 5 and 30 , presence and area of both hypoesthesia (HYPO) and mechanical hyperalgesia $(\mathrm{MH})$ surrounding sternotomy incision were assessed. Two months after surgery, incidence of chest RP was questioned by mail. Statistical analysis used one way ANOVA and chi-square analysis, $\mathrm{P}<0.05$ significant.

Results: Demographic data did not differ between groups: average age $64 \pm 10 \mathrm{yrs}$, BMI $28 \pm 5$. Respectively in $\mathrm{C}$ and $\mathrm{RP}$ group, M/F ratio was $25 / 12$ vs $20 / 2$ and CABG/NR ratio was $19 / 15$ vs $19 / 7$. One patient died before postoperative day(D)5. At $D 5$, all patients were evaluated while only 34 patients were available for D30 examination. RP development was unrelated to the type of cardiac surgery. Area $\left(\mathrm{cm}^{2}\right.$; mean $\left.\pm \mathrm{SD}\right)$ and percentage (\%) of HYPO and MH are expressed in table; ${ }^{*} p<0.05$ between both groups.

\begin{tabular}{lllll}
\hline & No pain & No pain & With RP & With RP \\
\hline HYPO D5 & $6.5 \pm 16$ & $22 \%$ & $27.2 \pm 38^{*}$ & $59 \%^{*}$ \\
HYPO D30 & $52 \pm 48$ & $18 \%$ & $39 \pm 46$ & $59 \%^{*}$ \\
MH D5 & $8.3 \pm 41$ & $19 \%$ & $12.5 \pm 47$ & $32 \%$ \\
MH D30 & $50 \pm 49$ & $36 \%$ & $25 \pm 41$ & $29 \%$ \\
CABG & & $49 \%$ & & $70 \%$ \\
VR & & $51 \%$ & & $32 \%$ \\
\hline
\end{tabular}

Conclusion: The presence and area of postoperative hypoesthesia may predict the risk to develop RP at 2 months after sternotomy, independently of the type of cardiac surgical procedure (CABG or VR).

References:

1 Meyerson J et al. Acta Anaesthesiol Scand 2001; 45: 927-8.

2 Alston RP et al. Br J Anaesth 2005; 95: 153-8.

\section{AP1-9}

Analgesic potency of Parecoxib after vaginal hysterectomy. N. Rooselaer, K. Van Elsen, C. Verborgh, E. Van Droogenbroeck, F. Camu Anesthesie, UZ Brussel, Brussel, Belgium

Background and Goal of Study: Parecoxib is a selective COX-2-inhibitor with opioid-sparing effect in acute postoperative pain (1). Vaginal hysterectomy causes considerable pain in the immediate postoperative period. Analgesic effects of parecoxib after vaginal hysterectomy have not been documented so far. We therefore studied the analgesic effect of parecoxib after vaginal surgery with reference the non-selective COX-inhibitor diclofenac.
Materials and Methods: Thirty-nine patients scheduled for vaginal hysterectomy agreed to take part in this randomised double-blind study. Induction of anaesthesia consisted of propofol, $0.2 \mu \mathrm{g} / \mathrm{kg}$ sufentanil and rocuronium. Anaesthesia was maintained with sevoflurane in $\mathrm{O}_{2} / \mathrm{N}_{2} \mathrm{O}$. Parecoxib $40 \mathrm{mg}$, diclofenac $75 \mathrm{mg}$ or plain saline were given after induction. These doses were repeated after 12, 24 and 36 hours. I.v. consumption of morphine was recorded via a PCA-pump (Abbott Laboratories) with regular print-outs. Statistical analysis was done with Statistica ${ }^{\circledR}$. Data are expressed as means (s.e.m.)

Results and Discussions: The three groups were comparable with respect to age, weight, length, preoperative $\mathrm{Hb}$ value and total fluid administered. Morphine consumption is shown below.

\begin{tabular}{lcccc}
\hline & $0-12 \mathrm{~h}$ & $12-24 \mathrm{~h}$ & $24-36 \mathrm{~h}$ & $36-48$ \\
\hline Parecoxib & $18(3)^{\star}$ & $5(2)^{\star}$ & $3(1)^{\star}$ & $1(0)$ \\
Diclofenac & $23(2)$ & $6(1)^{\star}$ & $6(1)^{\star}$ & $2(1)$ \\
Placebo & $29(4)$ & $16(4)$ & $13(4)$ & $5(2)$ \\
\hline
\end{tabular}

*: significantly less than placebo with $p<0.05$. MWU-test.

Side-effects such as nausea, vomiting or stomachache were minimal and comparable between groups.

Conclusion(s): Parecoxib and diclofenac are equally effective in preventing postoperative pain after vaginal hysterectomy, but parecoxib has a faster onset. Reference

1 Beaussier M et al. Anesth Analg 2005;100:1309-15.

\section{AP1-10}

Intra-operative remifentanil might influence pain levels in the immediate post-operative period after major abdominal surgery G. Ruggiero, D. Di Gennaro, L. Vivona, G. De Simone, G. Vivona

Anaesthesia, Federico II University, Naples, Italy

Background and Goals: Remifentanil, a widely used analgesic agent in anaesthesia, has a rapid onset and short duration of action. In clinical settings, this requires an appropriate pain strategy to prevent unacceptabel pain in the post-operative period. The aim of this study was to investigate whether remifentanil had impact on post-operative pain and opioid consumption after major abdominal surgery.

Material and Methods: Fifty patients undergoing major abdominal surgery were randomized to receive either remifentanil $0.4 \mathrm{mg} / \mathrm{kg} / \mathrm{min}$ or placebo intraoperatively, in addiction to basic combined general and epidural anaesthesia, in this double-blind study. Patients received patient- controlled analgesia with morphine for $24 \mathrm{~h}$ post-operatively. Morphine consumption, assessment of pain at rest and during coughing, side-effects and levels of sensory block were recorded during the first $24 \mathrm{~h}$ post-operatively.

Results: Twenty-one patients receiving remifentanil and 18 patients receiving placebo completed the study. The median visual analogue scale (VAS) score at rest from 0 to $2 \mathrm{~h}$ was significantly increased in the remifentanil group [40 mm (27-61 mm)] vs. placebo [13 mm $(3-35 \mathrm{~mm})](\mathrm{p}<0.05)$. No significant differences in morphine consumption, VAS score during coughing or adverse effects were observed between the groups.

Conclusions: The results are weak and difficult to interpret.

They could indicate that a high dose of remifentanil added to otherwise sufficient combined general and epidural anaesthesia may induce opioid-induced hyperalgesia and/or clinically acute opioid tolerance after major abdominal surgery; however, as no significant differences could be observed between the groups after $2 \mathrm{~h}$ post-operatively, the clinical relevance of these observations is questionable.

\section{AP2-1}

\section{Interaction between serotonin and clonidine in spinally} mediated analgesia in rats

T. Nishiyama, Y. Yamada

Anesthesiology, The University of Tokyo, Byunkyo-ku, Japan

Background and Goal of Study: Serotonin receptor and $\alpha_{2}$ adrenoceptor had a great role in pain mechanism in the spinal cord. We investigated the analgesic interaction between spinally administered serotonin and $\alpha_{2}$ receptor agonist, clonidine in two different pain models in rats.

Materials and Methods: Sprague-Dawley rats with lumbar intrathecal catheters were tested for their thermal tail withdrawal response using the tail flick test and for their paw flinches by formalin injection after intrathecal administration of serotonin or clonidine. The effects of the combination of serotonin and clonidine were tested by an isobolographic analysis using $\mathrm{ED}_{50}(50 \%$ effective dose) values. Eight rats were used in each dose group. Behavioral side effects were also investigated.

Results and Discussion: $E_{50}$ values are shown as mean and $95 \%$ confidence interval (in parenthesis). 


\begin{tabular}{llll}
\hline & Tail flick & $\begin{array}{l}\text { Formalin } \\
\text { phase 1 }\end{array}$ & $\begin{array}{l}\text { Formalin } \\
\text { phase 2 }\end{array}$ \\
\hline Serotonin & 34.5 & 12.4 & 1.3 \\
$(\mu \mathrm{g})$ & $(22.5-45.6)$ & $(5.6-22.0)$ & $(0.6-7.3)$ \\
Clonidine & 0.25 & 0.12 & 0.13 \\
$(\mu \mathrm{g})$ & $(0.16-0.42)$ & $(0.07-0.20)$ & $(0.08-0.23)$ \\
Serotonin in combination & 4.6 & 0.24 & 0.26 \\
$(\mu \mathrm{g})$ & $(3.7-5.6)$ & $(0.13-0.45)$ & $(0.12-0.38)$ \\
Clonidine in combination & 0.035 & 0.024 & 0.026 \\
$(\mu \mathrm{g})$ & $(0.028-0.042)$ & $(0.013-0.045)$ & $(0.012-0.038)$ \\
\hline
\end{tabular}

(): $95 \%$ confidence interval.

Allodynia was observed in $25 \%$ rats with $0.3 \mu \mathrm{g}$ clonidine, $12.5 \%$ rats with $100 \mu \mathrm{g}$ serotonin and $12.5 \%$ rats with $1 / 2 \mathrm{ED}_{50}$ of the combination. Motor disturbance shown in $12.5 \%$ rats with $3 \mu \mathrm{g}$ clonidine was not observed in combination.

Conclusions: Intrathecal serotonin and clonidine had synergistic analgesic effects on thermal induced acute pain and inflammatory induced acute and facilitated pain.

\section{AP2-2}

The effect of dexamethasone on the spinal glutamine synthetase and glutamate dehydrogenase expression in morphine tolerant rats

C. Wong, Gong-Jhe. Wu, Zhi-Hog. Wen, Yi-Chen. Chang, Chen-Hwan. Cherng

Anesthesiology, Tri-Service General Hospital, Taipei, Taiwan

Background and Goal of Study: Excitatory amino acids (EAAs) play an important role in morphine tolerance. Recently, we demonstrated that morphine challenge induced an increase in CSF EAAs concentration in morphinetolerant rats, and dexamethasone co-infusion attenuated morphine tolerance. The present study further examined the effect of intrathecal (i.t.) dexamethasone infusion on morphine tolerance and the expression of intracellular glutamate metabolizing enzymes, glutamine synthetase (GS) and glutamate dehydrogenase (GDH) in the rat spinal cords.

Materials and Methods: Male Wistar rat tolerance was induced by morphine infusion ( $15 \mu \mathrm{g} / \mathrm{hr}$, i.t.) for 5 days. Other group of rats received either saline $(1 \mu \mathrm{l} / \mathrm{hr})$, or dexamethasone $(2 \mu \mathrm{g} / \mathrm{hr})$, or dexamethasone $(2 \mu \mathrm{g} / \mathrm{hr})$ plus morphine $(15 \mu \mathrm{g} / \mathrm{hr})$ infusion. On day 5 , the spinal cords were removed and prepared for Western blot analysis of GS and GDH.

Results and Discussions: Down-regulation of GS and GDH expression was observed in morphine-tolerant rat spinal cords. Co-infusion of dexamethasone attenuated morphine tolerance and the associated GS and GDH down-regulation.

Conclusions: Intrathecal dexamethasone attenuates morphine tolerance; the preventing of GS and GDH down-regulation also play a role.

References:

Wen ZH, Chang YC, Cherng CH, Wang JJ, Tap PL, Wong CS. Brain Res 2004; 995: 253-259.

Fonseca LL, Monteiro MA, Alves PM, Carrondo MJ, Santos H. Glia 2005; 51: 286-96. Wen ZH, Wu GJ, Chang YC, Wang JJ, Wong CS. Neuroscience 2005; 133: 807-817.

\section{AP2-3}

\section{Neuropeptide and behavioural expressions in chronic} neuropathic pain using the intercostal nerve ligation model E.C. So, C.H. Chien, P.W.F. Poon, K.L. Wong, C.H Hsing

Department of Anesthesia, Institute of Basic Medical Science, National Cheng Kung University, Chi Mei Medical Center, Yung Kang, Taiwan

Background and Goal of Study: We reported here an original animal model for study of post-thoracotomy pain. Specifically we measured the number of degenerating neurons in the dorsal root ganglion (DRG) and their relationship to pain behaviors following a intercostal nerve chronic constriction injury.

Materials and Methods: Twenty male Wistar rats $(200-250 \mathrm{~g})$ received a tie (10-0 nylon) over the right 5 th intercostal nerve. The same intercostal space on the opposite side was shame-operated as the control. Behavioral tests included cold stimulation with Acetone and pain threshold assessment with von Frey filaments (postoperatively day 1, 7, 14, 28 and 56). Animals were then sacrificed and bilateral (T4, T5 \&T6) DRG were harvested after transcardial perfusion and cut into $5 \mu \mathrm{m}$ sections for the study of S-100 stain.

Results and Discussions: We found increased S-100 positive neurons on the ligation side compared with control $(28.92 \pm 4 \%$ vs. $12.6 \pm 2 \% ; p<0.05)$. There was also a higher scratching frequency (first $3 \mathrm{~min}$ ), and a lowered pain threshold starting from post-operative day 28 till day 56. Results showed that degeneration of DRG neuron after intercostal nerve ligation may be one of the leading causes of post-thoracotomy pain.
Conclusion(s): We have developed an original animal model for the study of post-thoracotomy pain. Subjects showed abnormal behavioral reactions to mechanical and thermal stimuli, concomitant with degenerative changes in the DRG.

References:

1 A. Buvanendran, J.S. Kroin, J.M. Kerns et al, Anesth Analg 2004 Nov; 99(5): 1453-60. 2 T. Nara, S. Saito, H. Obata et al, Can J Anaesth. 2001 Jul-Aug; 48(7): 665-76.

\section{AP2-4}

Axotomy alters sodium and ATP-sensitive potassium currents elicited by action potential waveform voltage commands in mammalian primary afferent neurons

C. Sarantopoulos, J.B. McCallum, W.M. Kwok, M. Rigaud, Q.H. Hogan Departments of Anesthesiology, Pharmacology and Toxicology, Medical College of Wisconsin, Milwaukee, USA

Background and Goal of Study: Certain membrane ion currents regulate neuronal excitability and protection from cell death, functions pertinent to the pathophysiology of neuropathic pain. We investigated changes of $\mathrm{Na}^{+}$and $\mathrm{K}^{+}$ currents on DRG neurons stratified by size, after spinal nerve ligation (SNL) [1], using each individual neuron's action potential (AP) as a voltage command stimulus, in a fashion that simulates normal physiological excitation. Materials and Methods: We studied whole-cell $\mathrm{Na}^{+}$and $\mathrm{K}^{+}$currents in control DRG neurons from sham operated rats, as well as axotomized neurons from L5 DRG, or adjacent L4 DRG neurons from rats with hyperalgesia following L5 SNL. In order to record $\mathrm{K}_{\text {ATP }}$ currents and to identify changes of other currents sensitive to ATP $[2,3]$ we used internal pipette solutions with low ATP. Each neuron's AP was recorded in Tyrode's solution in current clamp, and replayed as a voltage command sequentially in voltage clamp. Thus, currents in response to APs were recorded first in Tyrode's solution, and sequentially after: 1) blocking $\mathrm{Na}^{+}$current with NMDG and TTX; 2) addition of the $\mathrm{K}_{\text {ATP }}$ opener diazoxide; 3) blocking $\mathrm{K}_{\text {ATP }}$ current with glibenclamide; and 4 ) blocking remaining $\mathrm{K}^{+}$current with the addition of 4-AP and TEA-CI. Currents were estimated by trace subtraction indicating sensitivity to modulators, and normalization for cell capacitance. Peak current density and charge transfer were estimated digitally, and analyzed by ANOVA, testing for the main effect of neuronal size (indicative of functional modality) and injury status (control versus axotomized or adjacent).

Results and Discussion: 1) Large, medium and small DRG neurons express $\mathrm{K}_{\text {ATP }}$ current, which decreases after axotomy. 2) Total $\mathrm{Na}^{+}$current decreases after SNL in axotomized neurons: this most likely reflects a decrease in the TTX-sensitive component. 3) Total $\mathrm{K}^{+}$current was less in small sized neurons, irrespective of the injury effect.

Conclusions: Recording of $\mathrm{K}_{\text {ATP }}$ current in DRG neurons is a novel finding. Decrease of $\mathrm{K}_{\text {ATP }}$ current by axotomy may explain pertinent pathophysiological changes leading to neuropathic pain. Both findings provide opportunities for selective pharmacological targeting at the DRG.

References:

1 Pain 1992; 50: 355.

2 Neuroreport 2000; 11: 1683.

3 Neuroreport 2001; 12: 3659.

\section{AP2-5}

The role of central and peripheral cannabinoid receptors for postoperative thermal hyperalgesia after incision in the rat M. Wenk, P.K. Zahn, E.M. Pogatzki-Zahn

Department of Anesthesiology and Intensive Care, University Hospital Muenster, Muenster, Germany

Background and Goal of Study: There is ample evidence that endogenous and synthetic cannabinoids reduce neuropathic, inflammatory and tumorinduced hyperalgesia. However, little is known about the effects on postoperative pain. In this animal study we investigated the role of cannabinoid receptor agonists for incision induced thermal hyperalgesia.

Materials and Methods: Withdrawal latencies (WL) for heat hyperalgesia were measured with a Hargreaves Box in separate groups of rats before and after plantar incision. Subsequently, animals received WIN552122 (CB1/CB2 agonist) either intrathecally (IT; via catheter) $(10 \mu \mathrm{g})$, systemically (i.p.; $3 \mathrm{mg} / \mathrm{kg}$ ) or intraplantar (i.pl.; $60 \mu \mathrm{g}$ ). For differentiation between CB1 and CB2 receptor effects, further groups of rats were treated with i.pl. WIN $552122(60 \mu \mathrm{g})$ and AM251 (CB2-antagonist; $30 \mu \mathrm{g})$ or WIN $552122(60 \mu \mathrm{g})$ and AM630 (CB1-anagonist; $30 \mu \mathrm{g})$. WL were measured up to $4 \mathrm{~h}$ after drug application. Results and Discussions: Systemic and local application of WIN 552122 decreased incision induced thermal hyperalgesia for 90 min after drug application. Peripheral application of CB2 antagonist AM251 but not CB1 antagonist AM630 blocked WIN552122 induced antinociception indicating an important role of peripheral CB2 receptors for incision induced thermal hyperalgesia. 
Local application of CB1 and CB2 antagonists had no effect on withdrawal latencies after incision. IT application of WIN552122 did not modify thermal hyperalgesia after incision demonstrating that spinal cannabinoid receptors are not involved in thermal nociception.

Conclusion(s): Peripheral CB2 receptors but not spinal cannabinoid receptors are involved in modulation of thermal hyperalgesia after incision. Since adverse effects of cannabinoids are mainly based on CB1-receptor activation, the peripheral application of selective CB2 agonists might prove an effective and safe therapeutical option.

\section{AP2-6}

High dose remifentanil prevents development of thermal hyperalgesia in a neuropathic pain model in rats

A. Tzabazis, N. Manering, T. Reuter, H. Schwilden

Department of Anaesthesia, University Hospital, Erlangen, Germany

Background and Goal of Study: This study investigated whether remifentanil (remi) administered intraoperatively can prevent the development of thermal hyperalgesia in a neuropathic pain model in rats.

Materials and Methods: After approval of the ethics committee, 65 male Sprague-Dawley rats were anesthetized with isoflurane, paralyzed, intubated, and the tail vein was instrumented with a catheter for drug infusion. Remi was given low-dose $(2 \mu \mathrm{g} / \mathrm{kg} / \mathrm{min}$, Id) or high-dose $(20 \mu \mathrm{g} / \mathrm{kg} / \mathrm{min}$, hd). Rats that received saline intraoperatively served as controls. 10 to 12 rats of each group (ld, hd, and saline) received a L5 spinal nerve transection (modified Chung model), another 10 to 12 rats were sham operated. A blinded experimenter tested for thermal hyperalgesia using the Hargreaves test at different time points before and after surgery. Paw withdrawal latencies (PWL) were measured, and tested for statistical significance between groups.

Results and Discussions: Rats that received saline (fig.1) or ld remi (data not shown) intraoperatively developed significant thermal hyperalgesia starting on postoperative day (pod) 3. Animals that received hd remi showed significantly longer PWL to heat as compared to saline (fig. 1, data presented as mean \pm SEM) and Id remi (data not shown) groups.

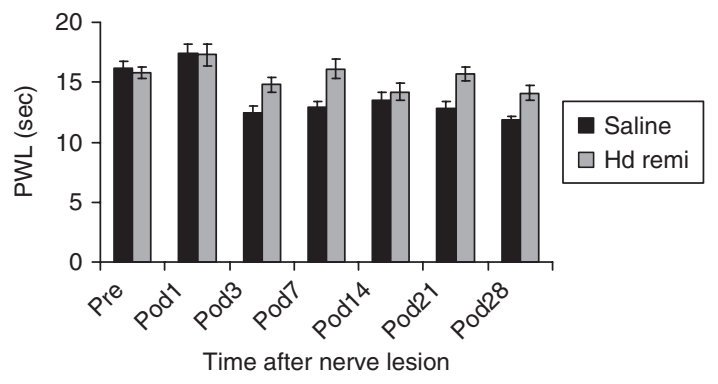

Conclusion: In this study hd remi administered intraoperatively was capable of preventing thermal hyperalgesia that normally develops after spinal nerve L5 transection.

\section{AP2-7}

\section{Effects of sodium diclofenac in spinal cords of rats}

L. Ozdogan, T. Ayerden, H. Sastim, O. Kilci, B. Dikmen

Anesthesiology and Reanimation, Numune Education and Research Hospital, Ankara, Turkey

Background and Goal of the Study: NSAIDs are widely used for patients suffering from pain. Some accidental administrations of sodium diclofenac has been reported (1). In this study we aimed to investigate the neurotoxic effects of intrathecally given $\mathrm{Na}$ diclofenac.

Material and Methods: After ethics committee approval 24 male Spraque Dawley rats weighing $250-300 \mathrm{~g}$ were randomly divided into 3 groups. Following intraperitoneal anaesthesia with ketamine $\mathrm{HCl}$ and $\mathrm{Xylasin} \mathrm{HCl}$ rats in group II received $10 \mu \mathrm{l} 200 \mu \mathrm{g} \mathrm{Na}$ diclofenac on day 7 as a sole injection; in group I received $10 \mu \mathrm{l} 0.9 \%$ saline and group III received $10 \mu \mathrm{l} 200 \mu \mathrm{g} \mathrm{Na}$ diclofenac once a day for 7 days intrathecally at the lumbar region. Rats observed for clinical neurotoxicity throughout the study period were sacrificed by formaldehyde at day 8 and medulla spinalis sections were assessed by electron microscopy. Data were analyzed by Kruskal-Wallis test. Results: Neither vital nor neurological function deficit was observed. While no ultrastructural changes were determined in group I (control); there was cytoplasmic condensation in group II (single dose) and nucleolic deformation and significant neurodegeneration in group III (repetitive dose). While histopathological assessment score was significantly higher in groups II and III compared with group I ( $p=0.002$ and $p=0.003$, respectively), it was significantly higher in group III compared with group II $(p=0.002)$.

Conclusion: Electron microscopy showed us that intrathecally given $\mathrm{Na}$ diclofenac causes significant neurodegeneration at repeated doses. Therefore the probability of neurotoxicity and undesirable effects must be remembered on clinical practice in humans.

Reference:

1 Lauretti R. Anesthesia \& Analgesia 1998; 86: 117-118.

\section{AP2-8}

Mu-opioid receptor and binding affinity to fentanyl is affected by sex but not by A118G polymorphism

R. Landau, J-L. Blouin, I. Charvet

Anesthesiology, University Hospital of Geneva, Geneva, Switzerland

Background and Goals: Opioids are widely used for management of pain, even though they display large inter-individual variability in efficacy and side effects. The $\mu$-opioid receptor ( $\mu \mathrm{OR}$ ) is the primary site of action for endogenous peptides including $\beta$-endorphin, and the major target for opioid analgesics such as fentanyl. The $\mu \mathrm{OR}$ displays genetic variability. In vitro, the G118 allele of A118G polymorphism increases the binding affinity of $\beta$-endorphin ${ }^{1}$, and a reduced ED50 of spinal fentanyl for labor analgesia in women with the G118 allele has been shown ${ }^{2}$. We examined whether subjects carrying the G118 allele have an increased binding affinity to fentanyl. Material and Methods: Blood samples were obtained from volunteers for genotyping of $\mu \mathrm{OR}^{2}$. In addition, we determined by radioligand binding assays the capacity of fentanyl to displace ${ }^{3}[\mathrm{H}]$-naloxone bound to whole, freshly isolated lymphocytes ${ }^{3}$. Displacement of ${ }^{3}[\mathrm{H}]$-naloxone $(20 \mathrm{nM})$ by fentanyl $(20 \mu \mathrm{M})$ was compared to the maximal displacement by unlabeled naloxone $(20 \mu \mathrm{M})$ itself.

Results: While the $\mathrm{A} 118 \mathrm{G}$ polymorphism of $\mu \mathrm{OR}$ did not seem to affect $\mu \mathrm{OR}$ binding affinity, we found a significant difference between $\gamma$ and less of genotype (data presented as relative 'naloxone

displacement ability' of fentanyl, in \%).

\begin{tabular}{llll}
\hline Men 2 & $\%$ & Women & $\%$ \\
\hline Total $(n=12)$ & $41.1 \pm 2.8$ & Total $(n=12)$ & $74.5 \pm 4.8^{*}$ \\
A118 $(n=9)$ & $40.1 \pm 3.5$ & A118 $(n=8)$ & $73.3 \pm 3.6$ \\
A118G $(n=3)$ & $44 \pm 1.73$ & A118G $(n=4)$ & $77.0 \pm 6.4$ \\
\hline
\end{tabular}

Mean $\pm \mathrm{SD},{ }^{*} p<0.0001$ (between 2 and

Conclusions: We found a difference between men and women with regards to fentanyl binding properties. This finding is congruent with previously described sex differences in opioid analgesia ${ }^{4}$ and may explain why women have been shown to require less opioids for management of pain. However, other pathways such as expression, transduction or receptor trafficking rather than $\mu \mathrm{OR}$ binding affinity need to be explored to investigate the potential mechanism by which A118G polymorphism appears to affect the clinical response to opioid therapy.

References:

1 Bond C. Proc Natl Acad Sci USA 1998; 95: 9608-13.

2 Landau R. http://www.asaabstracts.com. 2005; A1469.

3 Madden J, Biochem Pharmacol 1987; 36: 1403-9.

4 Fillingim R, Eur J Pain 2004; 8: 413-25.

\section{AP2-9}

Effect of superoxide on the development of complex regional pain syndrome - type I

D.G. Lim, H. Chung, C.K. Han, K.H. Kwak, Y.H. Jeon

Anesthesiology and Pain Medicine, Kyungpook National University

Hospital, Daegu, Republic of Korea

Background and Goal of Study: Reactive oxygen species (ROS) and inflammatory responses contribute to development of neuropathic pain. ${ }^{1,2)}$ Superoxide $\left(\mathrm{O}_{2}{ }^{-}\right)$serve to mediate cell signaling processes, directly induce tissue injury during inflammation. A neuropathic pain syndrome was produced in rats following prolonged hindpaw ischemia/reperfusion, creating an animal model of complex regional pain syndrome-Type I (CRPS-I). ${ }^{3)}$ This study was designed to evaluate the effects of $\mathrm{O}_{2}{ }^{-}$on the development of CRPS-I. Materials and Methods: Male adult SD rats were used for CRPS-I model. Plasma $\mathrm{O}_{2}{ }^{-}$production rate was measured via cytochrome $\mathrm{c}$ reduction in the presence xanthine (without xanthine oxidase, kinetics, $550 \mathrm{~nm}$ ). Superoxide dismutase (SOD, $4000 \mathrm{U} / \mathrm{kg}$ ) was treated before ischemia (preemptive, G1), just after reperfusion (beginning of increased $\mathrm{O}_{2}{ }^{-}$production, G2), or 3 days after reperfusion (the lowest withdrawal threshold on von Frey stimulation, G3) Mechanical and cold allodynia were measured in both hindpaws. The effects of SOD were confirmed by histologic changes of the hindpaws. 
Results and Discussions: Allopurinol-inhibitable, xanthine oxidase-mediated plasma $\mathrm{O}_{2}{ }^{--}$production was the highest at the just reperfusion and lasted at least 1 week. Mechanical and cold allodynia were present in both hindpaws as early as $4 \mathrm{hr}$ after reperfusion, and lasted at least 4 weeks. Pain behavior was significantly attenuated in G1, G2 and G3 compared with control. In G3, pain behavior was less attenuated than G1 and G2. Microscopic findings showed less inflammatory reaction in the $\mathrm{G} 1$ and $\mathrm{G} 2$.

Conclusion(s): This study suggests that the $\mathrm{O}_{2}{ }^{-}$is partly responsible for development of the CRPS-I. Even though $\mathrm{O}_{2}{ }^{--}$inhibition is less effective after CRPS-I has been already developed, $\mathrm{O}_{2}{ }^{--}$inhibition is still effective for reduce CRPS-I pain.

References:

1 White FA, Bhangoo SK, Miller RJ. Nat Rev Drug Discov 2005; 4: 834-44.

2 Kim HK, Park SK, Zhou JL, et al. Pain 2004; 111: 116-24.

3 Coderre TJ, Xanthos DN, Francis L, et al. Pain 2004; 112: 94-105.

\section{AP2-10}

Analgesic and hyperalgesic effect of single intrathecal dose of morphine under normal and neuropathic conditions

M. Docquier, V. Collet, M. De Kock, P. Lavandhomme

Anesthesiology, Université Catholique de Louvain, St Luc Hospital, UCL, Brussels, Belgium

Background: Beyond shortlasting analgesic effect, spinal opioids can also induce paradoxical pain and longlasting hyperalgesia $(1,2)$. Further, opioid effectiveness and development of tolerance remain subject to discussion in chronic pain conditions. The study compares the effect of single intrathecal (it) dose of morphine (MOR) under normal (C, controls) and NP conditions. Materials and Methods: Adult male Wistar rats, $C(n=6)$ and established $\mathrm{NP}(n=6)$ induced by partial ligation of the right sciatic nerve were implanted with lumbar it catheter. After recovery, animals received it MOR $20 \mu \mathrm{g}$ (1). Thermal and mechanical hyperalgesia were evaluated by paw withdrawal latency (PWL, in sec) from radiant heat and by paw withdrawal threshold (PWT, in g) to application of electronic von Frey filament before MOR (baseline,BL) and at day 0 ( $2 \mathrm{~h}, 4 \mathrm{~h})$, day $1,2,3$ and 7 . Statistical analysis used repeated ttest, $\mathrm{P}<0.05$ was significant.

Results: $P W L$ are expressed in table; $p<0.05\left({ }^{*}\right)$ with baseline, (\#) with $C$ rats. PWT were not affected by it MOR $20 \mu \mathrm{g}$ in any group.

\begin{tabular}{lllllll}
\hline & BL & Day0 & Day1 & Day2 & Day3 & Day7 \\
\hline C group & $12.1 \pm 1.5$ & $17.8 \pm 1.9^{\star}$ & $9.1 \pm 1^{\star}$ & $9.4 \pm 1.4^{\star}$ & $9.9 \pm 1.3^{\star}$ & $8.9 \pm 1.5^{\star}$ \\
NP group & $9.5 \pm 0.5 \#$ & $17.4 \pm 2.4^{\star}$ & $10.3 \pm 0.9$ & $11 \pm 0.8^{\star}$ & $11 \pm 0.5^{\star}$ & $11.7 \pm 2.5$
\end{tabular}

Discussion and Conclusion: As reported, after analgesia, normal rats develop delayed longlasting thermal hyperalgesia (1). In contrast, NP animals do not display hyperalgesia and show long duration analgesic effect on thermal sensitivity. The results confirm the efficacy of spinal MOR to relieve some NP components.

References:

1 Van Elstraete et al. Anesth Analg 2005; 101:1750-6.

2 Eisenberg et al. Cochrane Database Syst Rev 2006.

3 Suzuki et al. Pain 1999; 80: 215-28.

\section{AP2-11}

\section{Amitriptyline activates GABAergic neurons in the dorsal horn} of the spinal cord in rats

T. Hashimoto, Sijian. Tang, Yuji. Morimoto

Department of Anesthesiology, Hokkaido University School of Medicine,

Sapporo, Japan

Background and Goal of Study: Findings to date indicate that tricyclic antidepressants, such as amitriptyline, have an antinociceptive effect. It is supposed that descending inhibitory neurons are involved in the antinociceptive action of antidepressants. The mechanism whereby descending inhibitory neurons, including noradrenergic neurons, produce antinociception remains unclear. Recent electrophysiological studies have shown that noradrenalin activates GABAergic interneurons in the spinal cord, suggesting its role in mediating the antinociceptive effect. In the present study, we investigated whether amitriptyline activates GABAergic interneurons in the spinal cord using c-Fos protein as a marker for neuronal activation.

Materials and Methods: Adult male Wistar rats were administered 0 (control), $15,30,60$ or $90 \mu \mathrm{g}$ of amitriptyline intrathecally. After 4 hours, animals were anesthetized with intraperitoneal injection of pentobarbital and perfused with paraformaldehyde for fixation. The spinal cords were dissected at the lumbar level and frozen-sectioned. Sections were then stained for c-Fos protein using diaminobenzidine and the number of c-Fos positive cells was counted. Sections also double stained for c-Fos and glutamic acid decarboxylase (GAD; a rate-limiting enzyme for GABA synthesis) using fluorescent conjugated secondary antibodies.

Results and Discussions: Amitriptyline administration increased Fos positive cells in the dorsal horn of the spinal cord in a dose-dependent manner. The number of Fos positive cells per section was $40.8 \pm 2.3$ (mean \pm std. error) in control group, while $89.2 \pm 8.0$ in $90 \mu \mathrm{g}$ group. Amitriptyline-induced c-Fos protein expression was localized within the laminar I, II, III and IV. Analysis by double staining indicated that approximately $80 \%$ of c-Fos-positive cells were colocalized with GAD positive cells.

Conclusion(s): Results from the present study indicate that amitriptyline activates GABAergic interneurons in the dorsal horn of the spinal cord. Our finding suggests that GABAergic interneurons and their downstream effectors play at least a part in the antinociceptive action of amitriptyline in the spinal cord.

\section{AP3-1}

Distinction of pain mechanisms in pregnant women with low back pain at the end of pregnancy

D. Mayr, I. Sulyok, M. Bruckner, B. Gustorff

Vienna Human Pain Research Group, Department of Anesthesia and General Intensive Care Medicine, Medical University of Vienna, Vienna, Austria

Background and Goal of Study: Low back pain (LBP) in pregnancy is a common complaint causing substantial disability and cost (1). The etiology is not entirely clear. The aim was to differentiate pain mechanisms between LBP provokable by provocation maneuvers (i.e. musculoskeletal pain) and non-provokable pain and to compare with pain free controls.

Materials and Methods: After approval of the ethics committee 102 pregnant women (age $31.6 \pm 1.0$, 3rd trimester) were assigned to 3 groups using pain provocation maneuvers (34 provokable pain, 34 non-provokable pain, 34 pain free controls) (2). Quantitative sensory testing for heat, cold, pressure and mechanical pain thresholds was carried out by a blinded examiner in 3 dermatomes (forearm, T1; lower back, T11; pelvis, L1).

Results and Discussions:

\begin{tabular}{lllll}
\hline & Pressure T11 & Heat T11 & Pressure L1 & Heat L1 \\
\hline Provok & $2.73 \pm 0.2^{\star} \#$ & $37.66 \pm 1.2^{\star \$}$ & $2.89 \pm 0.3^{\star}$ & $40.05 \pm 1.5$ \\
NonPro & $3.39 \pm 1.5$ & $39.35 \pm 1.4$ & $3.32 \pm 0.3$ & $41.35 \pm 1.4$ \\
Control & $3.68 \pm 0.4$ & $40.30 \pm 1.4$ & $3.50 \pm 0.5$ & $41.61 \pm 1.3$ \\
\hline
\end{tabular}

Pressure pain threshold $(\mathrm{kg})$ and heat pain threshold $\left({ }^{\circ} \mathrm{C}\right)$ in $\mathrm{T} 11$ (back) and L1 (ventral), mean $\pm 95 \% \mathrm{Cl}, \mathrm{n}=34$ /group.

Provokable LBP vs pain free control ${ }^{*} \mathrm{p} \leqslant 0.05$

Provokable vs non-provokable LBP ${ }^{*} p=0.011 \$ p=0.077$

No group differences were found for mechanical and cold pain thresholds. Conclusion(s): Thresholds for pressure and heat pain are highly significantly reduced in pregnant women with provokable LBP, but remain normal in pregnant women with non-provokable LBP and pain free women. Our results allow for the first time the distinction of sensitization of nociception in an important subgroup of pregnant women suffering from pain and provides the basis for further studies on mechanism based pain therapy.

References:

1 Sydsjo A, Sydsjo G, Alexanderson K. J Womens Health Gend Based Med. 2001; 10 : 707-14.

2 Albert H, Godskesen M, Westergaard J. Eur Spine J. 2000; 9: 161-166.

\section{AP3-2}

Acute postoperative pain predicts chronic pain after breast surgery for cancer

A. Fassoulaki, A. Melemeni, C. Staikou, A. Triga, C. Sarantopoulos Department of Anesthesiology, Aretaieio Hospital, Medical School, University of Athens, Athens, Greece

Background and Goal of Study: Postoperative pain may constitute a risk factor leading to the development of chronic pain few months after surgery. We hypothesized that the intensity of acute postoperative pain, documented by actual recorded VAS scores, as well as postoperative analgesic consumption, may predict chronic pain and the need for analgesics after breast surgery for cancer.

Materials and Methods: We retrospectively examined postop. pain scores and analgesics in 98 patients who underwent breast surgery for cancer and served as controls in 4 previous studies [1-4]. We averaged VAS scores at rest 
and with movement: 1) during the first 9 postop. hrs, and 2) during postop. days 1 to 6 . We also calculated the total opioids (parenteral morphine equivalent dose) and paracetamol consumed during the acute postop. period. These data (early and late postop. VAS, and analgesics) were compared between those patients who had chronic pain or those who needed analgesia, versus those who were pain free or did not need analgesia, 3 months later.

Results and Discussions: Demographics, type of surgery, or radio- or chemotherapy did not differ. Patients with chronic pain had higher VAS scores at the first 9 postop. hours, but not from days 1-6. Patients needing analgesics at $3 \mathrm{mo}$. had higher VAS scores, as well as analgesic consumption postop. Analgesics at the acute postop. period, and prevalence of chronic pain at $3 \mathrm{mo}$. differed depending on the type of the placebo interventions used.

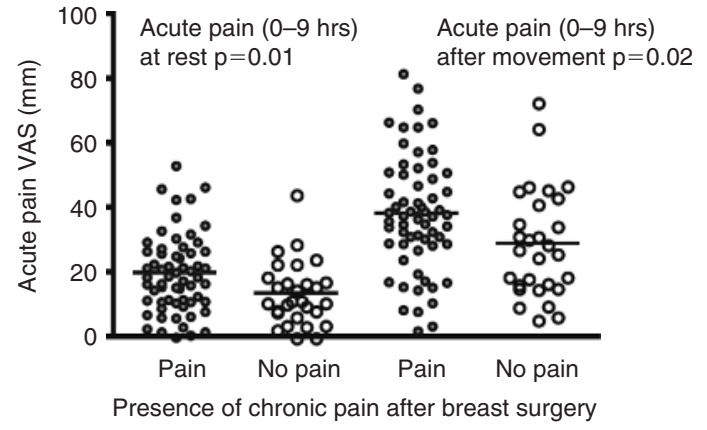

Conclusion: Early postoperative pain predicts chronic pain and need for analgesia after breast surgery for cancer.

References:

1,2 Fassoulaki A et al: Reg Anesth Pain Med 2000;25:350 and 2001; 26: 223.

3,4 Fassoulaki A et al: Anesth Analg 2002;95:985 and 2005; 101: 1427.

\section{AP3-3}

Nociceptive innervation of the sacroiliac joint-consequences for invasive treatments

K. Szadek, P. Hoogland, W. Zuurmond, J. de Lange, R. Perez

Anesthesiology, VU University Medical Center, Amsterdam, The Netherlands

Background and Goals: A positive outcome of intra-articular infiltration with local anaesthetics is used to confirm sacroiliac joint pain (1). However, current anatomical and histological knowledge concerning the nociceptive innervation of sacroiliac joint is insufficient to explain the efficacy of intra-articular application of local anaesthetics, due to the use of unspecific histochemical visualisation techniques. Use of accurate methods for detection of nociceptors, such as antigen tagging of Calcitonin Gene-Related Peptide (CGRP), should provide better understanding of the nociceptive innervation of the Sacroiliac joint, and therefore explain the analgesic mode of action of intraarticular infiltration with local anaesthetics.

Material and Methods: Anterior and interosseous sacroiliac ligaments tissue of five human cadavers ( 3 male and 2 female, aged 61-91), were used to trace nerve fibres with primary antiserum against CGRP (Chemicon International Inc., Temecula, catalog no. AB5920). Free-floating sections, $40 \mu \mathrm{m}$ thick, were stained according to the avidin-biotin method.

Results: Immunohistochemical analysis of anterior and interosseous sacroiliac ligaments showed CGRP-immunoreactive axons. Thick, wavy formed bundles, classified as myelinated fibres (Aס-type), were observed in dense and loose connective tissue. The single nerve fibres, classified as unmyelinated (C-type) were bead shaped and occasionally ramified and were more frequently observed in dense connective tissue. Additionally, Pacini corpuscles were found in the interosseous ligament, which contained immunoreactive free nerve endings. Conclusions: Presence of CGRP immunoreactive fibres in anterior and interosseous ligament, provide a base for pain originating from the sacroiliac joint. Invasive treatments for sacroiliac joint pain should reach the sacroiliac joint cavity but, target the nociceptive structures in ligaments adjacent to sacroiliac joint as well.

Reference:

1 Schwarzer AC, Aprill CN, Bogduk N. The Sacroiliac Joint in Chronic Low-Back-Pain. Spine 1995; 20(1): 31-37.

\section{AP3-4}

Influence of psychosomatic and somatic chronic pain on patient's life quality and depression

A. Macas, D. Bilskiene, A. Makunaite, L. Pieteris, A. Karbonskiene

Anesthesiology, Kaunas University of Medicine, Kaunas, Lithuania
Background and Goal of the Study: Chronic pain is recognized as a biopsychosocial phenomenon in which psychological, biological, social factors interact with each other. Therefore these aspects of chronic pain are widely recognized, but poorly understood. Goal of the study was to evaluate influence of psychosomatic and somatic chronic pain on patient's life quality and development of depression.

Methods and Materials: Interview of chronic pain patients using standard questionnaire, where answers are scored from 0 to 3 .

Results: 93 patients responded to the questionnaire. Assessment of somatic and psychosomatic pain intensity showed significant difference between groups of psychosomatic (PS) and somatic (S) pain: mean value in PS group was 7.53 according visual analog scale, while in $S$ group $-6.76(p=0.045)$. Patients of PS group indicated longer duration of pain and it's treatment. Influence of pain on daily activity was statistically significantly more expressed in $S$ group $(p<0.05)$. Evaluation of new problems in patients' families and expectations of positive results of therapy did not reveal statistically significant difference between the groups.

Assessment of quality of life did not show significant difference between the groups (65 points in PS group and 78 points in S group). Assessment of development of depression revealed that $63.3 \%$ of patients in PS group and $38.1 \%$ in S group had high depression level. $23.3 \%$ of patients in PS group and $36.5 \%$ in S group demonstrated low depression level.

Conclusions: Life quality of patients experiencing psychosomatic pain was significantly lower than of patients experiencing somatic pain. Level of depression was comparable in both groups.

\section{AP3-5}

\section{Long term follow up of impairments of Complex Regional} Pain Syndrome type I

R. Perez, S. Collins, W. Zuurmond, J. De Lange, S. Loer Anesthesiology, VU University Medical Center, Amsterdam, The Netherlands

Background and Goal of Study: Complex Regional Pain Syndrome type I (CRPS I), is characterized by sensory, autonomic, motor and thropic disturbances in an extremity A variable progression has been recognized [1], but few data are available on the course of symptoms on the long term. We performed a 6-9 years follow up measurement on CRPS I patients previously participating in a RCT [2].

Materials and Methods: 47 CRPS I patients ( 33 female, 14 male; mean age 59 years (SD: 13.9); median CRPS I duration in first study 109 days (IQR 60-168); upper (UE)/lower extremity (LE) 29/18) who participated in a RCT (1997-1999), were assessed using the Impairment level Sum Score (ISS) [3], which comprised pain (VAS, McGill), temperature (infrared thermometry), volume (water displacement volumeters) and Active Range of Motion (AROM; universal goniometers) measurements. The Wilcoxon signed ranks test was used for analyses.

Results and Discussions: ISS data are presented in Table 1:

\begin{tabular}{lllll}
\hline & UE RCT & UE follow up & LE RCT & LE follow up \\
\hline ISS & $11(9-18)$ & $17(13-23)^{\star}$ & $17.5(10.5-23)$ & $18(1-6.5)$ \\
VAS & $1(1-3.5)$ & $6(1-8)^{\star}$ & $4.5(1-8.25)$ & $3.5(1.75-8)$ \\
McGill & $3(1-5)$ & $1.5(1-7)$ & $3.5(1-6.25)$ & $3.5(1-6.25)$ \\
Temp. & $2(1-3)$ & $3(2-6.5)^{\star}$ & $2.5(1-4.75)$ & $4.5(1-6.5)$ \\
Volume & $1(1-2)$ & $3(1-3)$ & $1.5(1-2.25)$ & $1(1-1.25)$ \\
AROM & $3(2-5.5)$ & $2(2-4)$ & $3(3-4.25)$ & $1(1-3.25)^{\star}$ \\
\hline
\end{tabular}

ISS range 5-50 points; Data in medians (IQR); * $p<0.01$

A significant ISS deterioration was found for upper extremity CRPS, due higher levels of VAS-pain. Lower extremity CRPS I improved significantly on AROM.

Conclusion(s): Complaints on impairment level for upper extremity CRPS I may increase after 6-9 years, related to a relapse in pain intensity. Long term clinical follow up seems advisable for CRPS I patients in order to prevent deterioration of complaints in the course of time.

References:

1 Pain 1995; 63: 127-33.

2 Pain 2003; 102: 297-307.

3 Disabil Rehabil 2003; 25: 984-91.

\section{AP3-6}

Reliability of the TREND Symptom Inventory assessed in Complex Regional Pain Syndrome type 1 and Fibromyalgia patients

S. Collins, W. Zuurmond, S. Loer, J. de Lange, R. Perez

Anesthesiology, VU University Medical Center, Amsterdam, The Netherlands 
Background and Goal of Study: Common disease pathways based on similarities in clinical features have been suggested for Complex Regional Pain Syndrome type 1 (CRPS1), Fibromyalgia (FM), and repetitive strain injury (RSI) (1). To evaluate clinical manifestations of these disorders, the test-retest reliability of the TREND Symptom Inventory (TSI), measuring complaints on sensory, motor, autonomic, visceral and spinal domains, was assessed in CRPS1 and FM patients.

Materials and Methods: The overall and domain item reliability, calculated with Intra Class Coefficient (ICC), was calculated for 27 CRPS1 and 45 FM patients. Student-t test and Mann-Whitney $U$ test were used to compare groups.

Results and Discussions: Patient characteristics, ICC's and comparisons are shown in Table 1:

\begin{tabular}{|c|c|c|}
\hline & CRPS1 (N 5 27) & FM (N 5 45) \\
\hline Gender Male/female & $5 / 22$ & $2 / 43$ \\
\hline Age (year) ${ }^{1}$ & $54.0(16.43)^{\star}$ & $45.36(10.25)^{\star}$ \\
\hline Duration complaints (year) ${ }^{1}$ & $2.6(2.26)^{\star}$ & $8.15(8.9)^{\star}$ \\
\hline Overall ICC ${ }^{1}$ & $0.95(0.058)$ & $0.85(0.098)$ \\
\hline Domain ICC ${ }^{2}$ & $0.88-0.92$ & $0.79-0.85$ \\
\hline Sensory $^{3}$ & $0.67(0.60-0.73)^{\star}$ & $0.60(0.56-0.64)^{\star}$ \\
\hline Motor $^{3}$ & $0.64(0.52-0.72)^{\star}$ & $0.59(0.51-0.63)^{\star}$ \\
\hline Autonomic ${ }^{3}$ & $0.66(0.52-0.74)^{*}$ & $0.39(.0 .29-0.50)^{\star}$ \\
\hline Visceral $^{3}$ & $1.4(1.2-1.6)^{\star}$ & $1.6(1.4-1.8)^{\star}$ \\
\hline Spinal3 & $0.67(0.67-1.67)^{\star}$ & $2.33(2.17-2.67)^{\star}$ \\
\hline
\end{tabular}

Table 1: ${ }^{1}$ mean (SD), ${ }^{2}$ min-max domain reliability, ${ }^{3}$ median domain complaints (IQR),

higher scores correspond to higher levels of complaints ${ }^{\star} p<0.05$

Conclusion(s): The TSI questionnaire is a reliable instrument to evaluate similarities and differences between CRPS1 and FM. Although significant differences between CRPS1 and FM were found in sensory, autonomic visceral and spinal complaints, a substantial part of both patient groups reported to have complaints in each of these domains.

Reference:

1 Disabil Rehabil 2006; 28: 351-362.

\section{AP3-7}

\section{Study of cortisol and the pituitary-adrenal function in our population of fibromyalgia patients}

J. Vilaplana, Antoni. Arxer, C. Busquets, A. Villalonga, J. Fernández-Real anesthesia, Hospital Universitari de Girona "Doctor Josep Trueta", Girona, Spain

Background and Goal of Study: Current understanding of the pathophysiology of the fibromyalgia (FM) suggests that dysfunction of central pain regulation and neuroendocrine mechanisms are probably important factors $(1,2)$. We decided to evaluate the alteration of the pituitary-adrenal function in our population of FM patients.

Materials and Methods: We included all women newly referred for family physician or rheumatology consultation in a 18-month period who met the 1990 American College of Rheumatology (ACR) criteria for the diagnosis of FM. We recorded demographic data and clinical characteristics: body mass index (BMI), duration of pain, number of tender points, Lettinnen test, Fibromyalgia Impact Questionnaire (FIQ) and visual analog scales for pain, disturbed sleep, fatigue and current health statuts. Pituitary-adrenal function were evaluated with insulin-induced hypoglycemia. Blood sampling was performed for cortisol, adrenocorticotropin (ACTH), insulin and glycemia at basal, 30, 60 and 90 minutes.

Results and Discussion: We evaluated 98 patients but only $12(12,24 \%)$ were finally diagnosed of FM. The final diagnoses for these $86(87,76 \%)$ excluded patients were: low back pain (18), musculoskeletal pain no FM (33), depresion (14), arthralgia (13), shoulder pain (8). There were no significant differences between the means of demographic data and BMI. For FM patients FIQ was $77.22 \pm 10.38$, duration of pain $40.80 \pm 24.13$, number of tender points $13.81 \pm 2.96$, Lettinnen test $13.3 \pm 1.82$ and FIQ $77.22 \pm 10.38$. The area under the curve (AUC) in FM patients respect to control patients were (mean \pm SEM) for cortisol $27.005 \pm 2.45$ vs $25.74 \pm 3.06$; for ACTH $70.87 \pm 12.24$ vs $131.55 \pm 31.38$; for hypoglycemia $53.78 \pm 5.06$ vs $42.58 \pm$ 3.87 but there were not significative.

Conclusions: We have observed a disturbing inaccuracy in the diagnosis of FM by referring physicians. We have found disturbances but not significatives in pituitary-adrenal axis in FM patients.

References:

1 Pillemer SR.Conference summary: the neuroscience and endocrimology of fibromialgia. Arthritis Rheum. 1997; 40: 1928-1939.

2 Crofford J. Endocrin Metab Clin Nort Am 2002; 31(1): 1-31.

\section{AP3-8}

Effect of single high dose of spinal clonidine under normal and neuropathic conditions

M. Docquier, V. Collet, M. De Kock, P. Lavandhomme

Anesthesiology, Université catholique de Louvain, St Luc Hospital, UCL, Brussels, Belgium

Background: Spinal $\alpha 2$-adrenoceptor agonist, clonidine (CLO), is widely used for acute and chronic pain management, particularly to relieve neuropathic pain (NP). However, both continuous administration and high dose injection might induce paradoxical pain and hyperalgesia $(1,2)$. The study evaluates the effect of CLO single high dose under normal ( $C$, controls) and NP conditions.

Materials and Methods: Adult male Wistar rats, $C(n=6)$ and established $\mathrm{NP}(n=6)$ induced by partial ligation of the right sciatic nerve were implanted with lumbar intrathecal (it) catheter. After recovery, animals received it CLO $300 \mu \mathrm{g}$ (1). Thermal and mechanical hyperalgesia were evaluated by paw withdrawal latency (PWL, in sec) from radiant heat and by paw withdrawal threshold (PWT, in g) to application of electronic von Frey filament before CLO and at day $1(20,24,26 \mathrm{~h})$, day2 and day 7 . Statistical analysis used repeated t-test, $\mathrm{p}<0.05$ was significant.

Results: Baseline PWL was $12.4 \pm 0.7 \mathrm{sec}$ in $\mathrm{C}$ and $9.7 \pm 1.7 \mathrm{sec}$ in NP $(p<0.05)$. Spinal CLO injection induced significant thermal hyperalgesia, PWL $10 \pm 1.4 \mathrm{sec}$, at day1 and day2 in C group but not in NP rats. Baseline PWT was $123 \pm 19 \mathrm{~g}$ in C group and $84 \pm 40 \mathrm{~g}$ (ligated paw) $/ 141 \pm 30 \mathrm{~g}$ (contralat paw) in NP rats. Spinal CLO significantly decreased PWT at day 1 in C rats $(101 \pm 10 \mathrm{~g}$, $p<0.05$ with baseline) and decreased contralateral paw PWT only from day 1 to day7 while PWT of ligated paw were not affected in NP animals.

Discussion and Conclusion: In contrast with normal animals which display delayed thermal and mechanical hyperalgesia, NP do not develop hyperalgesia. These findings support the clinical use of spinal CLO, even at high doses, to relieve NP (3).

References:

1 Quartilho et al. Anesthesiology 2004; 100: 1538-44.

2 Takano et al. JPET 1993; 264: 327-35.

3 Ackerman et al. J Pain Symptom Manage 2003; 26: 668-77.

\section{AP3-9}

Changes in pain perception threshold and chronic pain outcome following sternotomy

M. Hogan, G. Shorten

Anaesthesia, Cork University Hospital, Cork, Ireland

Background and Goal of Study: Chronic pain has an incidence of approximately $30 \%$ in patients following cardiac surgery (1). Patients undergoing sternotomy are prone to develop thoracic neuropathic injury, and consequent areas of chest wall dysaesthesia (2). The aim of this study was to determine whether changes in chest wall sensation are associated with the development of chronic post sternotomy pain.

Materials and Methods: With institutional ethical approval, and having obtained written informed consent, patients undergoing elective coronary artery bypass graft surgery, were studied. Pre-operatively, at 5 days, and at 2 months after surgery we measured anterior chest thresholds to sensation (ST), pain perception (PPT), and pain tolerance (PTT), $2.5 \mathrm{~cm}$ both sides of midline at the level of $\mathrm{T} 4$, using electrical stimulation $(100 \mathrm{~Hz}$, square wave pulse, duration $0.2 \mathrm{msec}$ ). The presence of chronic post sternotomy pain (CPSP) at 2 months was determined by a positive response to the question; "have you had chest pain within the last 2 weeks, which is not angina, and which you believe arose as a result of your surgery?"

Results and Discussions: Seven male patients (mean age 63.3 years, SD 10.1) are included in this interim analysis. The absolute change in PPT (chPPT) from pre-operation to 2 months post-op was calculated for each patient. Patients were grouped according to the presence $(n=4)$, or absence $(n=3)$ of CPSP. The mean (SD) chPPT $(m A)$ for each group was calculated, as shown in the table:

\begin{tabular}{lcc}
\hline & No CPSP $(n=3)$ & CPSP $(n=4)$ \\
\hline chPPT Left Chest & $0.2(0.11)$ & $14.58(9.53)$ \\
chPPT Right Chest & $-1.55(0.77)$ & $2.39(4.21)$ \\
\hline
\end{tabular}

Conclusion(s): These preliminary data appear to indicate that PPT increases in patients who develop CPSP, compared with those who do not, and that this increase is most pronounced over the left chest.

References:

1 Kalso et al. Acta Anaesthesiol Scand 2001; 45: 935-39.

2 Alston RP and Pechon P, Br J Anaesth 2005; 95 (2): 153-8. 


\section{AP4-1}

Effectiveness and safety of catheter related postoperative pain management - a survey of 18925 consecutive patients between 1998 and 2006

D. Pöpping, P.K. Zahn, H.K. Van Aken, R. Boche, E.M. Pogatzki-Zahn Anaesthesiology and Intensive Care, University Hospital Münster, Münster, Germany

Background and Goal: Optimal treatment regimes in postoperative pain therapy are still a matter of debate. ${ }^{1}$ The aim of this study was to investigate the efficacy, adverse effects and safety in catheter related postoperative pain management under "real life conditions" by analysing the patients treated by an acute pain service. ${ }^{2}$

Methods: We analysed prospectively raised data documented by the acute pain service team in a computer based system concerning the quality of analgesia, adverse effects, and risks of the special pain management techniques in an university hospital. Adverse events were validated and evaluated in detail by written consultations from neurologists, surgeons and radiologists.

Results: We obtained data of 18925 patients visited in the postoperative period between 1998 and 2006. 14.223 patients received patient controlled epidural analgesia, 1.591 patient controlled intravenous analgesia, 1.737 continuous axillar or interscalene plexus blockade and 1.374 continuous femoral and sciatic nerve blockade. Mean dynamic pain scores (VAS 0-100) with patient controlled epidural analgesia were 16,4 (SEM 0,62) or less during the observation period of five postoperative days. This was significantly lower $(P<0,05)$ than dynamic pain scores of patients with patient controlled intravenous analgesia (30,4; SEM 5,06 or less) and continuous peripheral nerve blockades (24,7; SEM 0,47 or less).

In $8,3 \%$ of these 18925 patients the initial pain strategy failed because of insufficient pain relief, occlusion or dislocation of the catheter whereupon an alternative treatment was necessary. Severe complications that implies additional treatment, were observed in 26 cases $(0,14 \%)$. One patient developed an epidural hematoma, one patient suffered from epidural abscess after insertion of an epidural catheter.

Conclusion: Pain treatment with catheter related regimens, observed by an acute pain service in the early postoperative period was effective, successful and practicable. However, adverse effects and complications are not negligible supporting supervision of these techniques by an acute pain service.

References:

1 Liu SS et al.: Reg Anesth Pain Med 2006; 31: 291-3.

2 Brodner $\mathrm{G}$ et al.: Eur J Anaesthesiol 2000; 17: 566-75.

\section{AP4-2}

An effect of non-opioid analgesics in addition to continuous thoracic epidural analgesia on postoperative pain in abdominal surgery

D. Borisov, A. Levin, D. Uvarov, A. Sokolov, E. Nedashkovsky

Department of Anesthesiology, Northern State Medical University, Arkhangelsk, Russian Federation

Background and Goal of Study: Effect of non-opioid analgesics as supplement to continuous epidural analgesia (CEA) on postoperative pain relief is unclear. The goal of our study was to assess the effect of concomitant use of thoracic CEA and ketorolac or ketorolac and paracetamol on postoperative pain in abdominal surgery.

Materials and Methods: In a randomized controlled study, 75 patients underwent surgery for abdominal cancer lesions (epidural $0.75 \%$ ropivacaine and general anaesthesia). Postoperatively, all patients received thoracic CEA with the mixture of $0.2 \%$ ropivacaine and $2 \mathrm{mcg} / \mathrm{ml}$ fentanyl $(4-12 \mathrm{ml} / \mathrm{hr})$. All patients were randomized to receive either CEA alone (group CON, $54 \pm 13 \mathrm{yrs}$ ) or $30 \mathrm{mg}$ ketorolac i.m. every $8 \mathrm{hrs}$ (group KET, $59 \pm 11 \mathrm{yrs}$ ) or $30 \mathrm{mg}$ ketorolac i.m. every $8 \mathrm{hrs}$ and $1 \mathrm{~g}$ paracetamol i.v. every 6 hrs (group PAR, $53 \pm 12 \mathrm{yrs}$ ). The rescue patient-controlled analgesia was provided with morphine i.v. Visual analog scale (VAS) in coughing at 3, 6, 12, 18, and $24 \mathrm{hrs}$ of the postoperative period, the consumption of ropivacaine-fentanyl solution and morphine during $24 \mathrm{hrs}$ and the rates of pruritis, nausea, and vomiting were recorded. Data were analyzed by one-way ANOVA followed by Tukey's post hoc test and $\chi^{2}$ test accordingly.

Results and Discussions: The VAS score in the CON group was higher than in the KET group, while and VAS score in the KET group was higher than in the PAR group at all time points during the study period. However, significant differences were found only between the CON group and the PAR group at $6,12,18$, and $24 \mathrm{hrs}$. The consumption of ropivacaine and morphine in the CON group was greater compared with the KET and PAR groups $(p<0.05)$. The incidence of adverse effects did not differ between the groups.

Conclusion: The addition of both ketorolac and paracetamol to thoracic CEA with ropivacaine-fentanyl solution improves postoperative pain control and reduces the analgesic consumption after abdominal surgery.

\section{AP4-3}

\section{A comparative study on the analgesic effect of patient controlled morphine, pethidine and tramadol for postoperative pain management after abdominal hysterectomy}

H. Unlugenc, M.A. Vardar, S. Tetiker

Anaesthesiology, Cukurova University, Adana, Turkey

Background and Goal of Study: This prospective, randomized, double-blind study, was designed to compare the analgesic effectiveness and side effects of intravenous patient controlled (IV-PC) morphine, pethidine and tramadol for postoperative pain management after abdominal hysterectomy.

Materials and Methods: Following Ethics Committee approval and informed patient consent 90 ASA I or II patients undergoing abdominal hysterectomy were allocated randomly to receive IV-PC morphine $(\mathrm{M})$, pethidine $(\mathrm{P})$ or tramadol (T) for postoperative analgesia. Twenty minutes before the end of surgery, all patients received a standardised loading dose of morphine $(0.1 \mathrm{mg}$ $\left.\mathrm{kg}^{-1}\right)$, pethidine $\left(1 \mathrm{mg} \mathrm{kg}^{-1}\right)$ or tramadol $\left(1 \mathrm{mg} \mathrm{kg}^{-1}\right)$ for postoperative analgesia in a double-blind fashion. They were then allowed to use a patientcontrolled analgesia (PCA) device giving boluses of morphine $(0.02 \mathrm{mg}$ $\left.\mathrm{kg}^{-1}\right)$, pethidine $\left(0.2 \mathrm{mg} \mathrm{kg}^{-1}\right)$ or tramadol $\left(0.2 \mathrm{mg} \mathrm{kg}^{-1}\right)$. Pain, sedation and nausea scores, cumulative analgesic consumption, the number of patient requiring rescue fentanyl, time to recovery, and any side effects were recorded after recovery and at 1, 2, 6, 12 and 24 hours after the start of PCA. Results and Discussions: The total analgesic consumptions were $25.7 \pm 9.5 \mathrm{mg}$ for morphine, $266 \pm 90 \mathrm{mg}$ for pethidine and $341 \pm 111 \mathrm{mg}$ for tramadol in 24 hour. The ratio of morphine/pethidine/tramadol dose sizes, used for postoperative pain management, was a ratio 1/10/13, respectively. Twenty-four patients (26.6\%); four in group M (13.3\%), six in group P $(20 \%)$ and fourteen in group $\mathrm{T}(46.6 \%)$, complained of pain during the first twentyfour hour despite the PCA therapy. The number of patients requiring rescue fentanyl and average supplementary fentanyl dose used were significantly higher in T group than in $\mathrm{M}$ and $\mathrm{P}$ groups $(p<0.05)$. However, there was no difference between group $M$ and $P$.

Conclusion(s): In patients, who underwent abdominal hysterectomy, patient controlled morphine and pethidine has provided better pain control with similar side-effects than tramadol, perhaps making them better suited for IV-PCA. Thus, tramadol should be reserved for those patients, in whom morphine or pethidine is judged inappropriate because of high rescue fentanyl requirement.

\section{AP4-4}

\section{Opioids or NSAID in postoperative analgesia after total hip} arthroplasty? Comparison of adverse events

I.G. Cucereanu Badica, L. Badica, I. Grecu, A.A. Bradis, I.M. Grintescu Anesthesia and Intensive Care, Emergency Clinical Hospital of Bucharest, Bucharest, Romania

Background and Goal of Study: NSAID and opioid analgetics have both their own spectrum of adverse events. Paracetamol has the safest profile but is not enough for pain relieving after hip arthroplasty. This study tries to assess the best analgesic regimen for those patients.

Materials and Methods: After Hospital Ethics Committee approval 90 patients who underwent hip arthroplasty under spinal anesthesia were randomized in two groups: Group O $(n=45)$ received Tramadol and Group NSAID $(n=45)$ received iv Diclofenac or Ketorolac as supplementary analgesia (all pts. received Paracetamol iv $4 \mathrm{~g} /$ day for at least 5 days, gastric protection therapy and DVT prophylaxis with Enoxaparine). We assessed the quantity of transfused blood, the incidence of vomiting, the increase in creatinine serum level and the quality of sleep during the first five postoperative days and the incidence of digestive hemorrhage during the first 42 days after surgery. Statistics used t-test, $\chi^{2}$ test and Mann-Whitney U-test $(p<0,05)$.

Results and Discussions: The groups did not differ regarding the demographic data, blood loss during surgery and postoperative sedation and analgesia (VRS - none or mild for $\geqslant 90 \%$ of time). The NSAID group received more blood during the first 5 postoperative days $(p<0,05)$, have a lower incidence of vomiting episodes $(p<0,01)$ and an increased in creatinine levels not statistically significant. The quality of sleep was better in the group $O$ $(p<0,01)$. In group $O$ no digestive hemorrhage was recorded; in group NSAID 2 patients were readmitted with digestive hemorrhage and 2 patients experienced this during the first 10 days after surgery. 
Conclusions: We consider it is advisable to avoid NSAID in these patients because of demonstrated increase in postoperative blood loss and the increased risk of gastrointestinal bleeding. A regimen of iv. paracetamol and a weak opioid as Tramadol provides good pain relief with just one inconvenient side effect: nausea and vomiting.

Reference:

1 M. Hyllested et al, B J of Anaesth, 2002, 88: 199-214.

\section{AP4-5}

Intranasal tramadol versus intravenous tramadol in patients that have undergone a laparocopic cholecystectomy

C. Añez, A. Rodriguez, M. Rull, F. Pellicer, S. Jornet

Anesthesiology, Hospital Universitari de Tarragona Joan XXIII, Tarragona,

Spain

Introduction: The goal of the study was to compare intranasal tramadol with intravenous tramadol in patients that have undergone a laparoscopic cholecystectomy.

Materials and Methods: With the approval of the ethical committee of our hospital, the "Agencia Española del Medicamento" and the informed consent of patients, we did a randomised double-blind controlled study with 30 patients scheduled for laparoscopic cholecystectomy.

Patients were divided into two groups: group IV, which received intravenous tramadol $(100 \mathrm{mg})$ and normal saline intranasal $(2 \mathrm{ml})$ and group IN, which received intravenous normal saline $(100 \mathrm{ml})$ and tramadol intranasal $(100 \mathrm{mg})$.

Exclusion criteria were patients younger than 18 or older than 65 , with liver, kidney or respiratory chronic diseases, history of allergy to opioids, nasal mucosa disease, pregnancy, etc.

All patients were assessed by an ENT specialist to evaluate the nasal mucosa status before going to OR and the day after surgery. After surgery, patients were admitted into the post-anaesthetic care unit. Pain intensity was measured by using visual analogic scale (VAS) and categorical pain scale at rest and coughing. When patients referred VAS $>3$ or moderate pain, analgesia was started. Other variables recorded were arterial pressure, heart rate, breathing rate, Ramsay's sedation scale, nasal and systemic side-effects. The day after surgery patients answered a satisfaction questionnaire.

Statistical tests were Mann-Withney $U$ and Chi-square or Fisher's test. Statistical significance were $p<0,05$.

Results and Discussions: No demographic differences were found unless in sex. Thirteen patients in each group said that analgesia had been good or excellent. Seven patients in IV group and ten in IN group demanded rescue analgesia. However, ten in IV group and twelve in IN group had a decrease in VAS of $20 \mathrm{~mm}$ or $30 \%$ in his higher VAS value. No important systemic sideeffects were registered. Nasal side-effects detected by anamnesis or anterior rhinoscopy were not important.

We discussed efficacy, patient's satisfaction, administration system and safety of Tramadol administered by intranasal route.

Conclusion: Intranasal tramadol produces analgesia without significant sideeffects.

\section{AP4-6}

The postoperative analgesic efficacy of intraperitoneal tramadol compared to intravenous tramadol to normal saline for laparascopic cholecystectomy

S. Akinci, B. Ayhan, I. Aycan, B. Tirnaksiz, E. Basgul

Department of Anesthesiology and Reanimation, Hacettepe University Faculty of Medicine, Ankara, Turkey

Background and Goal of Study: The aim of this study was to compare the postoperative analgesic efficacy of intraperitoneal (ip) tramadol with intravenous (iv) tramadol or normal saline in patients undergoing laparascopic cholecystectomy.

Materials and Methods: 60 patients undergoing laparascopic cholecystectomy were randomized into three groups in a double-blind manner via coded syringes. The patients received either $100 \mathrm{mg}$ tramadol in $20 \mathrm{~mL}$ ip twice (predissection and postdissection) in the group ipT, or $100 \mathrm{mg}$ tramadol twice in group ivT or normal saline in group C (control group). All patients received a standard anesthetic protocol and postoperative analgesia with PCA morphine. Postoperative parietal (pNRS) and deep (dNRS) numeric rating pain scores, $1 \mathrm{~h}$ and $24 \mathrm{~h}$ morphine consumption, and side effects (opioid-related symptom distress scale, SDS score (1)) were recorded. For statistical analysis, ANOVA, Kruskal-Wallis, Chi-square, paired t test, student $t$ tests were used.
Results and Discussions:

\begin{tabular}{lccc}
\hline mean \pm SD/median (range) & Group C & Group ivT & Group ipT \\
\hline Age & $47 \pm 11$ & $43 \pm 10$ & $52 \pm 13$ \\
Weight, Female $(\mathrm{n})$ & $71 \pm 11.13$ & $72 \pm 10,13$ & $77 \pm 12,12$ \\
Time to first analgesic, min & $1(1-30)$ & $23(1-45)$ & $10(1-120)$ \\
PNRS at arrival to PACU\# & $5.5 \pm 3.4$ & $2.7 \pm 2.9^{\star}$ & $4 \pm 3$ \\
PNRS at 15th min\# & $6.2 \pm 3.3$ & $2.5 \pm 2.7^{*}$ & $3.9 \pm 3.2$ \\
PNRS at 30th min\# & $5.6 \pm 3.2$ & $2.8 \pm 2.6^{*}$ & $3.4 \pm 2.8$ \\
PNRS at 1 h & $3.9 \pm 2.7$ & $2.4 \pm 2.6$ & $3.2 \pm 2.2$ \\
PNRS at 24h $\mathrm{h}$ & $1.4 \pm 1.8$ & $1.2 \pm 1.9$ & $1.3 \pm 1.4$ \\
DNRS at arrival to PACU\# & $7.3 \pm 2.8$ & $2.9 \pm 3.6^{\star}$ & $5 \pm 3.2$ \\
DNRS at 15th min\# & $7.9 \pm 2$ & $3 \pm 2.9^{\star}$ & $5.3 \pm 3^{\star}$ \\
DNRS at 30th min\# & $7.2 \pm 2.3$ & $3.4 \pm 2.4^{\star}$ & $4.8 \pm 3$ \\
DNRS at 1 h\# & $5.1 \pm 2.2$ & $2.4 \pm 2.3^{\star}$ & $3.8 \pm 2.8$ \\
DNRS at 24h $\mathrm{h}$ & $1.9 \pm 2.4$ & $1 \pm 1.8$ & $1.6 \pm 1.5$ \\
24 $\mathrm{h}$ SDS pain score\# & $2.5 \pm 0.7$ & $1.7 \pm 0.8^{\star}$ & $2 \pm 0.7$ \\
SDS score & $0.9 \pm 0.7$ & $0.8 \pm 0.5$ & $0.7 \pm 0.6$ \\
1 $\mathrm{h}$ morphine (mg)\# & $6 \pm 2$ & $3.4 \pm 25$ & $4.4 \pm 4.3$ \\
24 $\mathrm{h}$ morphine (mg) & $24 \pm 18$ & $16 \pm 15$ & $16 \pm 15$ \\
\hline
\end{tabular}

$\# p<0.05$ 3group comparison, ${ }^{\star} \mathrm{p}<0.016$ compared to group $\mathrm{C}$

Conclusion(s): Iv tramadol provides superior postoperative analgesia after laparascopic cholecystectomy compared to ip tramadol and to normal saline. Reference:

1 Apfelbaum JL. Anesth Analg 2004; 99: 699-709.

\section{AP4-8}

Effectiveness of intraoperative bolus IV administration of three different doses of morphine for postoperative analgesia in remifentanil-based anesthesia for major surgery

B. Kiamiloglou, C. Dragoumanis, T. Vogiatzaki, V. Dimitriou, C. latrou Anaesthesiology, Medical School, Democritus University of Thrace, Alexandroupolis, Greece

Background and Goal of Study: To evaluate the postoperative analgesic efficacy of morphine after intraoperative analgesia based on remifentanil.

Materials and Methods: We studied 75 patients ASA I, II underwent major operations under general anesthesia using remifentanil for intraoperative analgesia. Patients were divided randomly into three groups with comparative demographic characteristics. A morphine bolus of $0.2 \mathrm{mg} \cdot \mathrm{kg}^{-1}$ (A Group, $\mathrm{n}=25$ ) or $0.25 \mathrm{mg} \cdot \mathrm{kg}^{-1}$ (B Group, $\mathrm{n}=25$ ) or $0.3 \mathrm{mg} \cdot \mathrm{kg}^{-1}$ (C Group, $\mathrm{n}=25$ ) was administered IV $30 \mathrm{~min}$ before the end of operation. Pain evaluation was assessed $5,10,20,30,45$ and 60 min postoperatively using the numerical pain scale scores of $0-10$. Complementary IV morphine of $2 \mathrm{mg}$ was used depending on the need. We recorded: a) the postoperative pain level, b) the need for complementary morphine, c) the modified Aldrete scale, d) the adverse reactions. Statistical analysis was done using the ANOVA test. Values of $p<0.05$ were accepted as statistically significant.

Results and Discussions: There were no demographic differences between all groups. Postoperative analgesia was ineffective in all three groups except in three patients of group $C$ who experienced adequate analgesia $(p=N S)$. Mean pain level comparison was statistically significant between all groups with the highest pain levels noted in group $A(p<0.05)$. Statistically significant differences in the mean pain level between the three groups were noted at 20 ( $p<0.001), 30(p<0.002), 45(p<0.001)$ and $60 \min (p<0.004)$. The complementary use of morphine was $10.6 \pm 2.6$ in group $A, 9.3 \pm 1.9$ in group $B$ and $7.8 \pm 3.5$ in group $C(p<0.004)$. The comparison between the values of the three groups using the modified Aldrete scale did not yield significant differences. No statistical differences were found in the side effects in each group. Conclusion: When intraoperative analgesia is based on remifentanil, administration of morphine $\left(0.2-0.3 \mathrm{mg} \mathrm{kg}^{-1}\right) 30 \mathrm{~min}$ prior the end of major surgery cannot provide adequate immediate postoperative analgesia.

\section{AP4-9}

Intra-peritoneal bupivacaine alone or in combination with morphine in patients undergoing vertical bypass gastroplasty Y. Ali, H. Negmi, N. Elmasry, M. Rabie, F. Bamehrez

Anesthesiology MBC22, King Faisal Specialist Hospital \& Research Center, Riyadh, Saudi Arabia

Background and Knowledge: Intra-peritoneal instillation of local anesthesia and morphine has been used to alleviate post-operative pain in laparoscopic surgery. Controversy exists about the efficacy of this technique. Methods: We studied 48 patients scheduled for Vertical Bypass Gastroplasy (VBG). All of them received the same technique of general anesthesia (GA). 
Patients were randomly allocated into four equal groups. They received equal volumes of the test drug instilled in the peritoneal cavity at the end of laparoscopy, $50 \mathrm{ml}$ of normal saline (Group S); $50 \mathrm{ml}$ of bupivacaine $0.25 \%$ (Group B), $50 \mathrm{ml}$ of bupivacaine $0.25 \%$, plus morphine $40 \mathrm{mcg} \cdot \mathrm{kg}^{-1}$ (maximum of $5 \mathrm{mg}$ ) Group M or (Group D) patients received the same regimen as Group $\mathrm{M}$ in addition, they received $75 \mathrm{mg}$ intra-muscular diclophenac after induction of general anesthesia. Wound edges were infiltrated with $10 \mathrm{ml}$ bupivacaine $0.25 \%$ in all patients. Morphine $25-50 \mathrm{mcg} \cdot \mathrm{kg}^{-1}$ was given intravenously every $10 \mathrm{~min}$ as a rescue analgesic to control postoperative pain in Post Anesthesia Care Unit (PACU). Post operative pain was evaluated using Visual Analogue Scale (VAS), vital signs, and morphine consumption, and time to receive rescue analgesia were measured at different intervals. The incidence of post-operative complications (respiratory depression, oxygen desaturation, and nausea and vomiting) was recorded as well as hospital stay. Results: There was significant decrease in VAS, HR, MBP and morphine consumption in Groups M \& D when compared to Groups $S$ \& B on admission and on discharge from PACU. There were significant decrease in time to receive rescue analgesia as well as significant reduction in hospital study in Groups $M$ and D when compared to Groups $S$ and B. However, there was no significant difference between group $S \& B$ regarding the same parameters. Conclusions: The presented technique is safe and easy to use with good postoperative morphine sparing analgesia, excellent patient satisfaction and short hospital stay.

\section{AP4-10}

\section{Parecoxib is equal effective to morphine for postoperative} pain management after nasal septoplasty

A. Zambouri, A. Leontidou, C. Bratzou, G. Karystianou, M. Ventouri 4th Department of Anesthesiology, "Hippokratio" General Hospital, Thessaloniki, Greece

Background and Goal of Study: Parecoxib is an injectable cyclooxygenase-2 inhibitor which has proved effective analgesic after laparotomy surgery ${ }^{1}$. The purpose of the study was to compare the analgesic efficacy of parecoxib with that of morphine in nasal septal surgery under total intravenous anesthesia with propofol and remifentanil.

Materials and Methods: Fifty ASA I patients scheduled to undergo nasal septoplasty were randomized to receive either parecoxib (P) $40 \mathrm{mg}(n=25)$ or morphine $(\mathrm{M}) 0.05 \mathrm{mg} / \mathrm{kg}(n=25)$ after induction of anesthesia with propofol $1-1.5 \mathrm{mg} / \mathrm{kg}$ and remifentanil $0.5 \mu \mathrm{g} / \mathrm{kg} / \mathrm{min}$. All patients received ondansetron $4 \mathrm{mg}$ and lidocaine $0.5 \mathrm{mg} / \mathrm{kg}$ during induction. Cis-atracurium was used for tracheal intubation. Anesthesia was maintained with a propofol and remifentanil infusion. All patients received local anesthesia at the operative site (lidocaine $2 \%$ with epinephrine).

Postoperative pain assessment was made on a visual analog scale (VAS; $0=$ no pain and $10=$ worst pain) at 0,30 and $60 \mathrm{~min}$ after emergence from anesthesia. During the postoperative period, paracetamol was administered for analgesia at the patient's request. The total paracetamol consumption by each patient within $24 \mathrm{~h}$ was determined and noted. Patients were questioned the next morning about the occurrence of any side effects. Statistical analysis was performed with unpaired Student's $t$-test and $\chi^{2}$-test.

Results: There were no significant differences between the two groups with respect to age, weight and duration of surgery. All patients in group $\mathrm{M}$ and $23 / 25$ in group $P$ had no pain (VAS score $=0$ ) during the 1 st postoperative hour. Fourteen patients $(56 \%)$ in each group requested no analgesia (they felt no pain) and 11 patients (44\%) requested additional analgesia because of moderate pain during the first 24 hours. There were no differences between the two groups in the total paracetamol consumption and in the time to first analgesic request. No side effects were observed.

Conclusion: As evaluated in this study, parecoxib $40 \mathrm{mg}$ is at least as effective as morphine $0.05 \mathrm{mg} / \mathrm{kg}$ for the management of postoperative pain after nasal septum surgery.

Reference:

1 Ng A, Smith G, Davidson AC. Br. J. Anaesth. 2003; 90: 746-749

\section{AP5-1}

\section{Preemptive Gabapentin for postoperative pain relief in gynaecological surgery}

I. Vanags, A. Sondore, I. Kokars

Anaesthesiology and Reanimatology, Riga Stradins University, Riga, Latvia Background and Goal of Study: Gabapentin (G) has been suggested to decrease acute postoperative pain. It selectively affects the nociceptive process, blocking the development of hyperalgesia, and allodynia (1). However the data about its effectiveness for improving postoperative pain control remains controversial (2). The aim of this study was to determine the preemptive use of $\mathrm{G}$ for postoperative pain relief and fentanyl $(\mathrm{F})$ consumption following hysterectomy.

Materials and Methods: The 80 patients (ASA I-II, aging 45-65) were randomized to receive either oral placebo (group $P, n=40$ ) or gabapentin (group $\mathrm{G}, \mathrm{n}=40$ ) $1200 \mathrm{mg}$ two hours prior to induction of anaesthesia. Anaesthetic technique was standardised. After surgery were given $F$ $40 \mathrm{mkg} / \mathrm{h} \mathrm{i} / \mathrm{v}$ continuously and on demand bolus dose of $\mathrm{F} 20 \mathrm{mkg}$ i/v with the lock-out time $15 \mathrm{~min}$. Assessments of postoperative pain included visual analogue scale (VAS) scoring for pain at 1, 4, 8, 12 and $24 \mathrm{hr}$ and $\mathrm{F}$ consumption during $24 \mathrm{hr}$ after surgery. Side effects were controlled.

Results: The VAS scores were lower in the G group on average: at $1 \mathrm{hr}$ by $5 \%(\mathrm{p}>0.05)$, at $4 \mathrm{hr}$ by $33 \%(\mathrm{p}<0.05)$, at $8 \mathrm{hr}$ by $16 \%(\mathrm{p}<0.05)$, at $12 \mathrm{hr}$ by $16 \%(p<0.05)$ and at $24 \mathrm{hr}$ by $5 \%(\mathrm{p}>0.05)$. F consumption was less in $\mathrm{G}$ group (by $9.5 \%, p<0.05$ ). $G$ was well tolerated. There were no differences in the incidence of side effects.

Conclusion: Gabapentine given preoperatively decreased pain scores and fentanyl consumption in the first day after abdominal hysterectomy.

References:

1 Turan A. et al., Anesth.Analg, 2006; 102(1), 175-181.

2 Fassoulaki A. et al., EJA, 2006; 23(2), 136-141.

\section{AP5-2}

Preemptive analgesia with paracetamol in postoperative analgesia for abdominal surgery

D. Borisov, A. Levin, D. Uvarov, I. Viljurov, E. Nedashkovsky

Department of Anesthesiology, Northern State Medical University, Arkhangelsk, Russian Federation

Background and Goal of Study: Balanced analgesia usually includes nonopioid analgesics. Paracetamol does not increase the risk of hemorrhagic complications, that is important in major surgery for malignancies. Thus, the goal of our study was to assess the effect of single dose of paracetamol for preemptive analgesia after abdominal surgery with perioperative epidural analgesia (EA).

Materials and Methods: In a randomized, double-blind study, 50 patients underwent surgery for abdominal cancer lesions (epidural $0.75 \%$ ropivacaine and general anaesthesia). All patients were randomized to receive either $1 \mathrm{~g}$ intravenous paracetamol (group PAR, $59 \pm 13 \mathrm{yrs}$ ) or placebo (group PLB, $61 \pm 9 \mathrm{yrs}) 30 \mathrm{~min}$ prior to incision. Postoperatively, all patients received continuous EA with $0.25 \%$ ropivacaine $(10-15 \mathrm{mg} / \mathrm{hr}$ ). In cases when visual analog scale (VAS) in coughing $\geqslant 40$, fentanyl $(0.05 \mathrm{mg})$ was added as an epidural bolus. Fentanyl consumption ( $\mathrm{mg}$ ), time of first fentanyl injection (hr), VAS at 2, 3, 6, 12, 18, and $24 \mathrm{hrs,}$ and nausea rate were recorded. Data (mean \pm SD and relative frequencies) were analyzed by Student's t-test and $\chi^{2}$ test.

Results and Discussions: Data are shown in the table:

\begin{tabular}{lccl}
\hline & Group PAR & Group PLB & $\mathrm{p}$ \\
\hline Gender (M/F) & $17 / 6$ & $11 / 13$ & 0.10 \\
Ropivacaine (mg) & $310 \pm 62$ & $304 \pm 51$ & 0.73 \\
Fentanyl (mg) & $0.13 \pm 0.06$ & $0.11 \pm 0.05$ & 0.43 \\
First fentanyl (hr) & $9.3 \pm 7.8$ & $7.8 \pm 5.6$ & 0.51 \\
Nausea (\%) & 13 & 42 & 0.05 \\
\hline
\end{tabular}

There were no significant differences in VAS score at any time-point during the $24 \mathrm{hrs}$ of the study period $(p>0.35)$. The nausea rate only had the explicit tendency to decrease in the group PAR that could partly be caused by the greater proportion of females in group PLB.

Conclusion: The use of $1 \mathrm{~g}$ of paracetamol as a single intravenous preemptive dose in abdominal surgery with perioperative epidural analgesia does not reduce the analgesic consumption and the intensity of pain in the postoperative period.

\section{AP5-3}

Safety and effectiveness of acute post-surgical pain management: observational database of $\mathbf{2 8 3 6}$ patients

M. Baena, N. Esteve, J. Mata, C. Segura, JL. Aguilar

Anesthesiology, Hospital Son Llátzer, Palma de Mallorca, Spain

Background Goal of Study: The absence of clearly, well defined, and widely accepted outcome indicators of Acute Postoperative Pain (APP) Management, makes difficult to assess the effectiveness of our clinical daily practice and impedes benchmarking and improvement (1). We defined indicators of outcome to assess the treatments employed in APP and to evaluate our results comparing them with extracted external data of the literature review (2). 
Methods: A form in the computerized Clinical Informatics Medical Chart of our Hospital (HP Doctor) was elaborated to record daily follow-up variables, previously defined, of patients controlled by the Acute Pain Unit (APU). With semester regularity the data of 4 groups of outcome indicators are analysed. Results: We classified the indicators in 4 blocks, Case - mix (5), Effectiveness (7), Safety (6) and Patient's Satisfaction (1). We present the data of 2836 patients, who were treated in the APU from May 2004 to December 2006. We present 19 indicators, among them: Average Time follow-up 3.06 days (1-12 days). Effectiveness (Patient with pain Numeric Rate Scale (NRS) $<3$ with adverse events solved or without adverse events) 91\%. Severe Pain at rest $(\mathrm{NRS}>7)$ ) 1\% (Review: $8 \%-13 \%$ ). Severe Pain at movement (NRS > 7) $8 \%$. Accidental exit of epidural catheter 6\% (Review: 8\%-15\%). Nausea/Vomiting in IV PCA 16\% (Review 33\%). Motor Blockade in Regional PCA 5\%. Serious Adverse Events: PCA Epidural Polirradiculopaty $0.08 \%$ (1 case) (Review: 0.005\%-0.03\%). IV PCA Respiratory Depression 0.4\% (3 cases) (Review: $0.1 \%-3.9 \%$ ).

Conclusions: The existence of a large sample observational database, facilitates the knowledge of treatments that really work in "a real" scene (1) in APP management.

References:

1 Liu SS, Wu ChL, Ballantyne J, Ginsberg B, Rathmell J,. Rosenquist RW, Viscusi ER. A Lovely Idea: Forming an ASRA Acute Postoperative Pain (AcutePOP) Database. Regional Anesthesia and Pain Medicine 2006; 31: 291-293.

2 S.J. Dolin JN Cashman JM Bland. Effectiveness of acute postoperative pain management: Evidence from published data. Br J Anaesth 89 (2002) 409-423.

\section{AP5-4}

The effect of patient-controlled epidural analgesia on cytokine response after gastrectomy

C.J. Chung, S.R. Choi, S.C. Lee, J.H. Lee

Anesthesiology and Pain Medicine, Donga University Hospital, Busan, Republic of Korea

Background and Goal of study: The postoperative period is associated with the increased production of cytokines, which augments the sensitivity to pain (1). Epidural analgesia might reduce the postoperative stress response and influence the immune function (2). This study evaluated the effect of patient-controlled epidural analgesia (PCEA) on the cytokine response, compared with patient-controlled intravenous analgesia ( $\mathrm{PCIA}$ ) after gastrectomy.

Materials and Methods: Twenty-nine patients undergoing gastrectomy were randomly assigned to one of two postoperative pain management techniques: PCIA (fentanyl $15 \mu \mathrm{g} / \mathrm{ml}$ ) or PCEA (fentanyl $3 \mu \mathrm{g} / \mathrm{ml}$ in $0.1 \%$ ropivacaine). Postoperative pain was assessed at rest and during coughing using the visual analog scale (VAS). The plasma concentrations of the tumor necrosis factor (TNF)- $\alpha$, interleukin (IL)- $1 \beta$, IL-6, IL-8, IL-1ra, IL-10, and IL-2 were assessed before administering anesthesia and 6,24 , and $48 \mathrm{~h}$ after surgery. Data were analyzed with t-test, repeated measures of ANOVA and Bonferroni test. Results and Discussions: The patients in the PCEA group showed lower VAS pain scores during $72 \mathrm{~h}$ after surgery $(P<0.05)$, compared with the patients in the PCIA group. There were no changes in the levels of TNF- $\alpha$ and IL-2 between the two groups at all times examined. In both groups, the levels of IL-1 $\beta$, IL-6, IL-8, IL-1ra, and IL-10 increased after surgery (each group, $\mathrm{P}<0.05$ ), but there were no significant differences between the two groups. Conclusions: These results suggest that PCEA has no added influences on the cytokine responses after gastrectomy.

References:

1 Watkins LR, Maier SF, Goehler LE. Pain 1995; 63: 289-302.

2 Beilin B, Shavit Y, Trabekin E, et al. Anesth Analg 2003; 97: 822-827.

\section{AP5-5}

\section{Esmolol decreases the postoperative morphine} consumption in septorhinoplasty

N. Celebi, O. Canbay, B. Saridemir, V. Celiker, U. Aypar

Anaesthesiology and Reanimation, Hacettepe University Faculty of Medicine, Ankara, Turkey

Background and Goal of Study: Intraoperative use of esmolol may attenue intraoperative nociceptive stimuli and influence the depth of anaesthesia. Esmolol may also have analgesic activity and reduce the analgesic consumption(1).

Materials and Methods: We studied 60 patients [30 pts receiving esmolol + remifentanil $(E+R), 30$ pts receiving placebo + remifentanil $(P+R)$ ] undergoing septorhinoplasty. Mean blood pressure (MBP), heart rate $(\mathrm{HR}), \mathrm{BIS}$ value and total remifentanil consumption were recorded. After surgery, the patients whose VAS score were $>3$ received i.v.morphine by a patient-controlled analgesia device. VAS score, effective analgesia time and total morphine consumption were recorded. T-tests and $\chi^{2}$ tests were used for statistical analyses.

Results and Discussions: Data (mean $\pm \mathrm{SD}$ ) are shown in the table.

\begin{tabular}{lcrl}
\hline & $\mathrm{E}+\mathrm{R}(\mathrm{n}: 30)$ & $\mathrm{P}+\mathrm{R}(\mathrm{n}: 30)$ & $\mathrm{p}$ value \\
\hline BIS values & $49.26 \pm 1.35$ & $54.04 \pm 0.78$ & 0.093 \\
Total remifentanil & $0.83 \pm 0.49^{\star}$ & $1.58 \pm 0.78$ & 0.004 \\
Total morphine & $7.13 \pm 8.41^{\star}$ & $12.90 \pm 8.69$ & 0.011 \\
VAS 0th minute & $2.38 \pm 1.59^{\star}$ & $4.81 \pm 2.19$ & 0.001 \\
VAS 20th minute & $3.50 \pm 1.76^{\star}$ & $4.72 \pm 2.03$ & 0.034 \\
VAS 1st hour & $2.64 \pm 1.54^{\star}$ & $3.68 \pm 1.56$ & 0.016 \\
Effective analgesia time & $108.00 \pm 81.59^{\star}$ & $43.83 \pm 60.78$ & 0.001 \\
\hline
\end{tabular}

Conclusion(s): Intraoperative use of esmolol: 1) do not influence the depth of anaesthesia; 2) decrease the total morphine consumption in the postoperative period.

\section{Reference:}

1 Y.Y. Chia. M.H. Chan. N.H. Ko. K. Liu. Role of $\beta$-blockade in anaesthesia and postoperative pain management after hyterectomy. Br. J. Anaesth. 2004 Dec; 93(6): 799-805.

\section{AP5-6}

The use of pre-emptive analgesia in major reconstructive orthopaedic surgery llizarov method:

\section{the gabapentin effect}

O. Kiskira, V. Polyzois, N. Vassilas, A. Kolotoura, E. Anastassiou

Department of Anesthesiology and Pain Clinic, Thriassio General Hospital, Elefsina, Greece, Magoula, Greece

Background and Goal of Study: Gabapentin(G) is an anticonvulsant structurally related to the neurotransmitter $\gamma$-aminobutyric acid (GABA). Preventing the initial neural cascade could lead to long-term benefits by eliminating the hypersensitivity produced by noxious stimuli. The aim of the study was to evaluate the pre-emptive effects of $\mathrm{G}$ on postoperative pain and morphine requirement in major reconstructive orthopaedic surgery (MROS). The hypothesis tested was that premedication with gabapentin diminish morphine consumption.

Materials and Methods: Forty (40) patients, ASA I-II, aged 24-56, who were scheduled to undergo MROS after traumatic injury using the Ilizarov method, were recruited in this randomized double - blind controlled trial. They were assigned into two groups. They either received $800 \mathrm{mg}$ of gabapentin per os (Group G) or placebo (Group P) 60 minutes prior to the operation. Patients were given access to a PCA device set to deliver 1-mg boluses of intravenous morphine $(\mathrm{M})$ with a lockout period of 8 minutes and a constant flow of $2 \mathrm{mg}$ per hour. The pain score was recorded at rest on a visual analogue scale (VAS; $0-10 \mathrm{~cm} ; 0=$ no pain and $10=$ worst possible pain) at the $1 \mathrm{st}$, $2 \mathrm{nd}, 4$ th, 8th, 16th, 24th hour after the end of the operation, morphine consuming was recorded at the same time.

Results and Discussions: Forty patients, twenty per group enrolled in the study. The variable in the analysis was the changes from baseline of these measures. The statistical method used was repeated measures ANOVA. The $\mathrm{p}$-value for the comparison of $\mathrm{M}$ consumption was less than 0.0001 and $\mathrm{p}$-value for the comparison of the rate of change at $\mathrm{M}$ consumption between the groups was 0.0007 , final $M$ consumption was $62.1 \pm 13.8$ for $G$ group and $92.65 \pm 30.7$ for $P$ group. Data are reported as mean \pm standard deviation (SD). The $p$-value for the comparison of VAS score was 0.0871 and $p$ value for the comparison of the rate of change of VAS score changes between the groups was 0.23 .

Conclusion(s): In conclusion, the pre-emptive use of gabapentin significantly decreases postoperative pain and analgesic requirement in MROS. Gabapentin, appears to have significant analgesic properties on post-operative pain.

\section{AP5-7}

\section{Acute postoperative pain management in Serbia-clinical audit}

\section{Schaffer}

Anesthesia and ICU, Medical Center, Kikinda, Serbia

Background and Goal of Study: The postoperative pain management is still unsatisfactory in many hospitals world wide. Adequate pain relief is integrated in many quality assurance programs in US (1) and EU countries (2). Aim of this study is to assess the postoperative pain management in Serbian hospitals and health centers.

Materials and Methods: On the sample of 200 anesthesiologists from 15 medical centers (regional hospitals and clinics) a survey was performed. Data about the acute pain services (pain protocols, evaluation and documentation 
of postoperative pain, the preferred analgesic techniques) were analyzed. The colleagues also were asked about the satisfaction with own therapy and suggestion for improving the domestic postoperative pain management. Statistical data were analyzed by hi square and Student t-tests.

Results and Discussions: Data showed that $27 \%$ of asked medical institutions have pain protocols and acute pain teams. In $18 \%$ of surgical wards the postoperative pain is documented and has been visible. For pain intensity assessment visual analogue scale (VAS), numerical rating scale (NRS) and verbal rating scale (VRS) are used. In $64 \%$ of medical centers the anesthesiologist is in charge for pain therapy. Nonsteroid anti-inflammatory drugs (NSAID), weak and strong opioids are used for pain killing in surgical patients. The application route of analgesics in $73 \%$ of hospitals mainly was intravenous. Regional analgesic techniques like epidural analgesia (EDA) and peripheral nerve blocks (PNB) are used in $27 \%$ of asked institutions. Individual analgesia is preferred in $82 \%$ of hospitals. $64 \%$ of interviewed anesthesiologists are not satisfied with the quality of postoperative pain therapy in own institution. Conclusion(s): Improvement of postoperative pain management in Serbia needs: (1) better education of medical stuff, (2) new pain protocols, guidelines and (3) reform in health policies.

References:

1 JCAHO: www.jcaho.org. Accessed April 14, 2005

2 Rawal N, Allvin Eur J Anaesthesiol 1998; 15: 354-363.

\section{AP5-8}

Clonidine improves postoperative analgesia and reduces nausea and vomiting after laparoscopic cholecystectomy

B. Ghrab, K. Khemakhem, M. Chaari, K. Kolsi, A. Karoui

Anesthesiology, Habib Bourghiba Hospital, Sfax, Tunisia

Background and Goal of Study: laparoscopic cholecystectomy is associated with some postoperative problems, especially pain and nausea or vomiting. Clonidine has been shown to reduce postoperative nausea and vomiting (PONV) after breast cancer surgery [1]. The postoperative analgesic effect of clonidine was also known in previous studies [2]. The aim of this study was to investigate the clinical efficiency of clonidine in postoperative management after laparoscopic cholecystectomy.

Materials and Methods: Following ethical committee approval and informed consent, we conducted a prospective randomized double blind study including 44 patients aged 20 to 65 years who were randomly allocated to two groups: Group C(22 patients) : received an IV perfusion of $3 \mu \mathrm{g} / \mathrm{kg}$ clonidine in $20 \mathrm{ml}$ of saline solution over $20 \mathrm{~min}$ before surgery. Group P (22 patients): received the same volume of a saline solution over $20 \mathrm{~min}$ before surgery. The anesthetic protocol was standardized.

Patients with Visual Analogic score $\geqslant 30$ were treated with nefopam ( $20 \mathrm{mg}$ IV up to six times per $24 \mathrm{~h}$ ). Pain scores, the analgesic use, occurrence of nausea-vomiting shivering and the frequency of clonidine side effects (hypotension and bradycardia) were recorded.

Results and Discussions: Demographic data and mean surgery time were similar in both groups. Pain scores were significantly lower in group $C$ in the first six postoperative hours. The main results are in the table below.

\begin{tabular}{llll}
\hline & $\begin{array}{l}\text { Group P } \\
\mathrm{n}=22\end{array}$ & $\begin{array}{l}\text { Group C } \\
\mathrm{n}=22\end{array}$ & $\mathrm{p}$ \\
\hline First analgesic requirement (min) & $677.7 \pm 460.8$ & $1096.5 \pm 416.9$ & $<0.01$ \\
Rescue analgesia (\%) & $77.2 \%$ & $45.4 \%$ & 0.03 \\
PONV (\%) & $54.5 \%$ & $18.1 \%$ & 0.01 \\
Postoperative shivering (\%) & $45.4 \%$ & $9 \%$ & $<0.01$ \\
Hypotension (\%) & $13.6 \%$ & $9 \%$ & 0.64 \\
Bradycardia (\%) & $9 \%$ & $4.5 \%$ & 0.46 \\
\hline
\end{tabular}

Conclusion(s): our findings suggest that preoperative administration of $3 \mu \mathrm{g} / \mathrm{kg}$ clonidine statistically improves postoperative analgesia, and reduces nausea-vomiting and shivering after laparoscopic cholecystectomy.

References:

1 Oddby E, et al. Anesthesiology 2002; 96: 1109-14.

2 Yu, et al. Acta Anesthesiol Scand 2003; 47: 185-90.

\section{AP5-9}

The efficacy and safety of dexmedetomidine-ketamine combination for analgesic sedation

J. Malek, L. Hess, A. Kurzova, J. Schreiberova, J. Jandova

Anaesthesiology and IC, Charles University, 3rd Medical Faculty, Praha, Czech Republic

Background and Goal of Study: The aim of our clinical randomized blinded study was to assess the efficacy and safety of the combination of dexmedetomidine and ketamine for analgesic sedation.
Materials and Methods: After ethic committee approval and written consent patients scheduled for Duputreyn's contracture repair under local anaesthesia were randomly divided in groups $C$ and $D$. D group were administered the mixture of dexmedetomidine 1 microgram and ketamine $1 \mathrm{mg}$ in $1 \mathrm{ml}$ normal saline according to the scheme: 10 minutes before surgery, bolus $0.04 \mathrm{ml} \cdot \mathrm{kg}^{-1}$ was administered and followed by infusion $2 \mathrm{ml} \cdot \mathrm{kg}^{-1} \cdot \mathrm{h}^{-1}$ for the first 10 minutes and $1 \mathrm{ml} \cdot \mathrm{kg}^{-1} \cdot \mathrm{h}^{-1}$ for the rest of surgery. Group $\mathrm{C}$ were administered normal saline in the same regimen. Vital functions, quality of analgesia and sedation using Ramsay score were measured. The patients were questioned in the afternoon and next day about side effects and amnesia. Results and Discussions: The study was early terminated because differences between $D$ and $C$ were eminent. There were 7 patients in $C$ and 8 patients in D. There were differences in C vs. $D$ in Ramsay score (all in $C$ scored 1 vs. 7 pts. scored 2 and 1 pt. scored 3 in $D, p<0.001$ ), absent reaction to painful application of local anaesthetic and tourniquet ( 0 vs. $6, p=0.02)$, amnesia (0 vs.6, $p=0.02$ ) and light-headedness 1 hour after surgery (0 vs.7, $p=0.005)$. Five patients in $D$ remembered dreams, but the difference was not significant. There was slight fall in blood pressure (25-30\% bellow base line) in 4 pts. in D (insignificant) and no effect on ventilation. The effects of combination of dexmedetomidine-ketamine except of light-headedness (lasted up to 2 hours after surgery) subsided 10 minutes after the infusion had been stopped. Conclusion(s): The combination of dexmedetomidine $1 \mu \mathrm{g}$ and ketamine $1 \mathrm{mg}$ in $1 \mathrm{ml}$ saline can be used for sedation during surgery. It provides good analgesia, amnesia and has no effect on ventilation.

\section{AP5-10}

Effects of intratecal morphine on pulmonary function and postoperative analgesia in patients undergoing coronary surgery

M.J. Carmona, L.M. Santos, J.L. Caires, L.M. Malbouisson, J.O. Auler Jr

Department of Anesthesiology, Heart Institute, São Paulo, Brazil

Background and Goal of Study: Changes on pulmonary function due to pain contributes to pulmonary morbidity in postoperative cardiac surgery. Central neuroaxial analgesia may reduce risks of pulmonary complications and improve outcome after cardiac surgery ${ }^{1}$. The purpose of this study was to evaluate the effect of intratecal morphine on pulmonary function and postoperative analgesia in patients undergoing coronary artery bypass surgery.

Materials and Methods: We studied 27 patients undergoing CABG randomized to either general anesthesia (control group, $n=13$ ) or general anesthesia plus intrathecal morphine $400 \mathrm{mcg}$ (intratecal group, $n=14$ ). Intraoperative anesthetic management was standardized and for the postoperative analgesia patients received intravenous morphine via patientcontrolled analgesia. The patients had an spirometry test and arterial blood gas analysis before surgery, in first and second postoperative days and postoperative pain during cough was assessed using a numeric pain score $(0=$ no pain, 1 = mild, 2 = moderate, 3 = strong). Intravenous morphine consumption, forced expiratory volume in first second (FEV1) and forced vital capacity (FVC), $\mathrm{FEV} 1 / \mathrm{FVC}$ ratio and $\mathrm{PO}_{2} / \mathrm{FIO}_{2}$ ratio were evaluated. Data were compared by ANOVA $\left({ }^{*} p<0,05\right)$.

Results and Disscussion: Consumption of morphine was greater in control group during the first 36 hours of postoperative. Intratecal group has reduced pain scores $(1,79$ in control group and 0,77 in intratecal group, $\mathrm{p}=0.001)$. Mean $\mathrm{PO}_{2} / \mathrm{FIO}_{2}$ ratio was 286.73 in control group and 351,65 in morphine group $(p=0.002)$.

Significative reduction in FEV1, CVF and VEF1/CVF was observed in both groups after surgery $(p<0,01)$ without difference between them.

Conclusions: Although intratecal morphine was not associated with mechanical respiratory improvement it provided reduction in dynamic pain scores and better blood oxygenation. These benefits could allow effective pulmonary toilet and better outcomes in postoperative period.

Reference:

1 Liu S, Block B, Wu Christopher L. - Anesthesiology 2004; 101: 153-61.

\section{AP5-11}

The Influence of the alpha-2 Agonist Clonidine on Perioperative Haemodynamic Stability, Sedation, Anxiety and Analgesia in Medium Amplitude Abdominal Surgery

C. Guran, A. Moise, E. Dragulin, N. Mincu, G. Stelea

Anesthesia and Intensive Care, Emergency Hospital MAI Prof. Dr. D. Gerota, Bucharest, Romania

Background and Goal of Study: Prospective, randomised, double-blind study designed to evaluate the haemodynamic pattern, pain control, the degree 
of anxiety and sedation and the incidence of side effects when Clonidine preanesthesia (the only $\alpha-2$ agonist locally available at the moment) was compared to placebo.

Materials and Methods: Two equivalent groups of 40 patients (ASA I-III) were randomised to receive Diazepam in the evening before and oral Clonidine $150 \mu \mathrm{g}$ or placebo in the morning of the surgery. They have received equivalent ballanced general anesthesia for medium amplitude abdominal procedures.

Intra- and postoperative haemodynamic was assesed, as well as opiod consumption during surgery, opioid antagonisation at emergence. Anxiety, sedation, postoperatory respiratory depression and analgesia were quantified at 5 min postoperatory, at $1 \mathrm{~h}$, then each $3 \mathrm{~h}$ until $24 \mathrm{~h}$. Patients' satisfaction (pain control, side effects) was assesed by a self-administred questionnaire in day 4 .

The statistic significance of the differences observed in the evolution of these parameters was tested by T Student test.

Results and Discussions: Haemodynamic was better in the Clonidine group (variation greater than $\pm 20 \%$ for arterial pressure and heart rate at induction and during surgery for placebo group, $\mathrm{p}<0.05$ ). This was even stronger for hypertensive patients.

Opioid consumption reported to the duration of the intervention was comparable in the two groups Sedation and respiratory depression was slightly higher in the Clonidine group during the first 12 hours $(p<0.05)$, Analgetic consumption was not statistically different in the two groups, as well as the pain control, Postoperative satisfaction (pain control, sedation, other side effects) was equivalent in the two groups.

Conclusion(s): Clonidine premedication for moderate amplitude abdominal surgery ensures a better haemodynamic both intra- and postoperatively, with minor side effects concearning sedation and respiratory depression.

References:

Bonnet F, Houhou A, Aveline C, ESA Refresher Courses 2000

Foëx P, Sear JW Continuing Education in Anaesthesia, Critical Care \& Pain 2004 4(5): 139-143.

\section{Education, Research and Presentation}

\section{AP1-1}

\section{Conflicts between anaesthetists and surgeons}

A. Pelavski, M. Rochera, M. Roca, M. De Miguel, J. Roigé Deptartment of Anaesthesia, Vall d'Hebron Hospital, Barcelona, Spain

Background and Goal of Study: This survey sought to determine the main situations that lead to conflicts between anaesthetists and surgeons and their causes.

Materials and Methods: We e-mailed a 20 question-survey to the Heads of Anaesthesia Departments of all public hospitals in Catalonia (Spain) accompanied by an explanatory letter, in which we begged them to deliver the survey among the anaesthetists of each hospital. Residents were excluded.

Results and Discussions: A total of 117 surveys were returned (about $13.76 \%$ of all specialists in Catalonia). $60.7 \%$ of respondents had arguments with the surgeons: $33.3 \%$ once a month, $21.4 \%$ once a week, and $6 \%$ more than once a week. The frequency of conflicts was neither related to the hospital size, nor to the years of experience as anaesthetist. The most frequent conflictive situation was an exceedingly long surgical program (97.5\%), which occurred sometimes (36.8\% of the respondents), very often $(48.7 \%)$ and always (12\%). 95.8\% considered that the surgeons try to operate elective cases in the emergency OT on the day they are on call $(35.4 \%$ sometimes, $45.8 \%$ very often and $14 \%$ always). The 3rd place (94\%) went for the constant complaints of the surgeons about the patients not being relaxed enough; and the 4 th $(92.3 \%)$ to the disagreement on whether the patient should or should not be operated. Of this group, $31.6 \%$ agreed that the situation was more common when dealing with general surgeons. Other conflictive situations were: the patient did not undergo a preoperative assessment $(88.9 \%)$, the surgeon only arrives when the patient is asleep (85.5\%), the surgeon informs the family before extubation (82.9\%). The main causes considered as a source of conflict were the lack of teamwork (57.3\%) and the different interests and objectives (55.7\%). Most anaesthetists agreed that more conflicts arose in the emergency context.

Conclusion(s): A harmonious operating environment, does not always exist, and conflicts touch more often on behavioural and attitude issues than on expertise ones. Therefore we agree with some respondents that common sessions between surgeons and anaesthetists could enable to discuss different points of view, fortify the feeling of a team as well as strengthen interpersonal relationships. Furthermore, a clear horizontal division of the work is capital to avoid interference between specialities.

\section{AP1-2}

Adequacy of euroanesthesia 2006 abstracts with ad hoc ethic rules prescription: still more a concept than a real fact

A. Dhollander, A. Cahana

\section{Anesthesiology, CHU- Geneva, Geneva, Switzerland}

Background and Goal of the Study: At ESA, studies involving animal or human subjects must satisfy ethical requirements of the institution or organization of the presenting authors (1). The goal of our study was to verify the application of these rules in all Euroanesthesia 2006 abstracts presented as randomized controlled trials $[R C T]$ and $C T$ designs.

Methods: Wordings concerning IRB/IEC and informed consent [IC] were systematically reviewed in the EJA 2006 suppl 37 for the concerned peer reviewed abstracts.

Results and discussion: The number of items observed are illustrated in the following table.

\begin{tabular}{lccc}
\hline Euroanesthesia 2006 Abstracts & RCT + CT & IRB or IEC & IC + Written IC \\
\hline ESA PRIZE COM & 4 & 3 & 2 \\
ESA COM 1 & 13 & 0 & 0 \\
ESA COM 2 & 17 & 6 & 4 \\
ESA COM 3 & 31 & 18 & 12 \\
ESA COM 4 & 49 & 4 & 7 \\
ESA COM 5 & 19 & 5 & 2 \\
ESA COM 6 & 15 & 7 & 8 \\
ESA COM 7 & 22 & 3 & 1 \\
ESA COM 8 & 56 & 14 & 14 \\
ESA COM 9 & 83 & 22 & 6 \\
ESA COM 10 & 28 & 11 & 8 \\
ESA COM 11 & 21 & 5 & 9 \\
ESA COM 12 & 29 & 2 & 2 \\
ESA COM 13 & 7 & 4 & 1 \\
ESA COM 14 & 68 & 18 & 17 \\
ESA COM 15 & 8 & 0 & 1 \\
ESA COM 17 & 7 & 1 & 1 \\
EAMS COM & 39 & 16 & 18
\end{tabular}

Conclusion: Rigorous application of the existing policy should be mandatory in order to avoid the problem of under declarations of the IRB/IEC and IC mentions in RCT and CT trials in Euroanesthesia meetings.

Reference:

1 http://www.euroanesthesia.org/esa abs cgi/

\section{AP1-3}

Assessment of educational objectives with problem-based learning in undergraduate students of Anaesthesiology

E. Moret, R. Garcia-Guasch, A. Escudero, R. Llasera, T. Franco Anaesthesiology, Universitat Autònoma de Barcelona, Faculty of Medicine, Hospital Germans Trias i Pujol, Badalona, Spain

Background and Goal of Study: After 5 years' experience in problem-based learning (PBL) in Anaesthesiology we consider that it helps our fifth-year undergraduate students toward better interactive learning and its acceptance is excellent. The aim of the study was to assess the accomplishment of educational objectives in these students of Anaesthesiology based on PBL. Materials and Methods: Educational objectives were defined and included in 12 PBL-cases. During each PBL session the tutor evaluated content, oral presentation and development of PBL-related competencies, such as ability to work in a team, interpersonal skills, problem solving, self-directed learning, information gathering, and task-supporting competencies. At the end of the teaching programme the students were asked to undergo a voluntary and anonymous test (100 true/false sentences) in order to analyse the understanding and assimilation levels of theoretical knowledge acquired with the PBL method. Data are presented as mean \pm standard deviation.

Results: All students $(n=24: 5$ men and 19 women; mean age: $23.5 \pm 2.2$ years) filled in the test. The mean total score was $7.2 \pm 1.5$ (minimum score, 0 ; maximum score, 10). The issues with the highest percentage of correct answers $(>70 \%)$ were preoperative assessment, cardiopulmonary resuscitation, informed consent, acute and chronic pain therapy, regional anaesthesia, airway management and postoperative care. $40 \%$ of the answers on anaesthetics and obstetric anaesthesia were wrong.

Conclusion: PBL seems to be valid for meeting educational objectives among undergraduate students of Anaesthesiology. Further studies are necessary to demonstrate whether PBL improves academic results and favours better comprehension and assimilation of knowledge as a long-term effect compared to a conventional curriculum. 
Reference:

Lucas M, Garcia-Guasch R, Moret E, et al. El aprendizaje basado en problemas aplicado a la asignatura de pregrado de Anestesiología, Reanimación y Terapéutica del Dolor. Rev. Esp. Anestesiol. Reanim. 2006; 53: 419-425.

\section{AP1-4}

Medical students perception of their capacity for reflection-in-learning

P. Chalmers, G. Shorten, M. ORourke, S. OFlynn, S. Curtin Lawton

Department of Anaesthesia and Intensive Care Medicine, Cork University Hospital, Cork, Ireland

Background and Goal of Study: At our university, reflective practice is being integrated into a competence-based curriculum for the undergraduate, medical education programme. It is reported that this practice promotes critical thinking, self-awareness and self-regulated learning.[1,2] The goal of this study was to examine the association between reflection-in-learning scores and students' self-perception of reflective capacity. This is one component of a larger evaluation of the influence of reflective practice on learning efficacy, professionalism and identity amongst final year medical students.

Materials and Methods: Following ethical approval and informed consent, 99 of a class of 110 final year medical students, at the beginning of the school year, completed a two part, previously validated, questionnaire [3]: i. a 14 item reflection-in-learning scale with a response graded on a 7-point Likert scale ranging from 1 , never to 7 , always; ii. a graded tool for self-assessment of the level of personal skill in reflective practice (null/minimal to maximal). Results:

Table 1. Reflection-in-learning (R-i-L) Scores and Self-perception of Reflective Capacity

\begin{tabular}{lll}
\hline Self-perception Category (n) & R-i-L Score median (range) & p value \\
\hline Null/Minimal (8) & $39.5(25-64)$ & \\
Restricted (29) & $55(36-83)$ & $<0.04$ \\
Partial (35) & $62(40-79)$ & $<0.01$ \\
Ample (21) & $65(37-84)$ & $<0.004$ \\
Maximal (6) & $69(57-85)$ & $<0.002$ \\
\hline
\end{tabular}

*relative to null/minimal

Conclusion(s): Final year medical students demonstrate substantial selfperception of their capacity for reflection-in-learning. The completed investigation will follow this association over the duration of the final year of the medical curriculum.

References:

1 Lachman N, Pawlina W. Clin Anat 2006; 19: 456-460.

2 Grant A, Kinnersley P, Metcalf E et al. Med Educ 2006; 40: 379-388.

3 Sobral DT. Med Educ 2000; 34: 182-187.

\section{AP1-5}

Temperament and anxiety of anaesthesiologists and comparison with other specialists

P. Hernández, S. Beltrán de Heredia, X. Santiveri, M.A. Cruz, LI. Gallart

Anaesthesiologia, Hospital del Mar, Barcelona, Spain

Background and Goal of Study: Medical staff, and particularly anaesthesiologists, are daily exposed to high levels of risk and stress, as well as to the need to take immediate reaction. Zuckerman created in the 70s the "Sensation Seeker Scale" (SSS) applied in professions such as firemen or pilots to identify temperamental personalities. The aim of the study was to know the prevalence of "sensation seeker" and to compare the degree of anxiety of anaesthesiologists to other specialists.

Materials and Methods: Cross-sectional study utilizing both "SSS" and the "State-Trait Anxiety Inventory" (STAIt) questionnaires in order to evaluate the temperament and the trait anxiety, respectively. The "SSS" is divided in four subscales that evaluate: "Thrill and Adventure-Seeking" (TAS), "ExperienceSeeking" (ES), "Disinhibition" (DIS) and "Boredom-Susceptibility" (BS). These tests were completed by a sample of medical staff from different specialities who qualified also in different years. ANOVA and Student's t-test were used. Results: Forty-one anaesthesiologists, 25 surgeons and 38 other specialists were included. The anaesthesiologists $(20 \pm 0.8)$ and surgeons $(20 \pm 1.1)$ are significantly more "novelty seeker" than other medical specialists $(13 \pm 0.8)$ $(p<0.05)$. Anaesthesiologists $(5.5 \pm 0.4)$ are prone to seek thrill and adventure (TAS) while surgeons $(5.3 \pm 0.4)$ are prone to social disinhibition (DIS). Singles/divorced people $(21 \pm 0.6)$ from the sample present more "novelty seeker" features than the married ones. STAlt questionnaire showed differences in anxiety between women $(17 \pm 8)$ and men $(14 \pm 7)(p<0.05)$ but did not show differences among specialists, although surgeons are prone to have more trait anxiety.
Conclusion: Anaesthesiologists and surgeons are more "sensation seekers" and, thus,more temperamental, than other specialists. In this sens, anaesthesiologists stand out because of their desire of unusual sensations and physically risky activities. In relation to anxiety, women are more anxious than men. A speciality-specific analysis shows that the anaesthesiologists have similar anxiety levels than other specialities, while surgeons are prone to have more trait anxiety.

References:

Kluger MT et al. Anaesthesia 1999; 54: 926-35.

Nyssen AS et al. Br J Anaesth 2003; 90: 333-7.

\section{AP1-6}

Medical students attitudes towards scientific research

L. Niemi-Murola, E. Kalso, P.H. Rosenberg

Department of Anaesthesiology and Intensive Care Medicine, Helsinki University Hospital, Helsinki, Finland

Background and Goal: Medical students have generally positive attitudes towards scientific research, but the general research activity is decreasing (1). The purpose of this study was to assess factors motivating the students to extracurricular scientific work.

Materials and Methods: An electronic questionnaire with six demographic and 31 research-related items was sent via e-mail to 680 medical students at the University of Helsinki, Finland. The students were asked to answer the questions using Likert scale ( $1=$ totally disagree, $7=$ totally disagree). Factor loading of the questionnaire consisting of 31 items was made using maximum likelihood analysis and varimax rotation. Five scales were constructed (Research, Career, No research, Who should do, Example). Statistics: Pearson Correlation, Student's t-test.

Results and Discussion: The questionnaire was returned by $63.4 \%$ of the students $(N=431)$. Eighty-seven students $(20.76 \%)$ were involved in extracurricular research, 123 respondents $(29.29 \%)$ had intention to start scientific work and $130(30.95 \%)$ students aimed to academic dissertation. Scale Research correlated with Scale Career $(p<0.01)$ and Scale Career correlated with Scale Example $(\mathrm{p}<0.01)$. Students involved with scientific work were more interested in solving problems compared to students not involved (mean 6.0 vs. $4.74,95 \% \mathrm{Cl}-1,713--0,803, \mathrm{p}<0.001)$. Students involved wished to examine mechanisms behind diseases more often compared to students not involved (mean 5.69 vs. $4.61,95 \% \mathrm{Cl}-1,689-0,680, \mathrm{p}<0.001$ ). An interesting specialty (mean 6.03 vs. $5.02,95 \% \mathrm{Cl}-1,463--0,566, \mathrm{p}<0.05$ ) and a topic (mean 5.86 vs. $4.86,95 \% \mathrm{Cl}-1,371--0,455, \mathrm{p}<0.05$ ) were important to both student groups. Great impact factor of the publishing journal was not very important for them (mean 4.84 vs. $3.33,95 \% \mathrm{Cl}-2,036-1,012$, $\mathrm{p}<0.001$ )

Conclusions: Interest of the subject and the relevance are more frequent motivating factors than competitive approach. Successful career was more important as a motivating factor to male students than to females.

\section{Reference:}

1 Clark J, Smith R. BMJ 2003; 327: 1001-1002.

\section{AP2-1}

\section{3-D visualization of cardiac murmur improves teaching of cardiac auscultation}

H. Makino, Y. Sanjo, T. Katoh, Y. Shiraishi, S. Sato

Anesthesiology and Intensive Care, Hamamatsu University School of Medicine, Hamamatsu, Japan

Background and Goal of Study: We have tried to invent a new technology for changing the breathing sounds signals into a visual form. We could visualize breathing sounds in a three-dimensional (3-D) color visual form continuously in the previous study. We applied our system (VisiStetho) to explain to patients who have cardiac disease about changes of their cardiac murmur before and after cardiac surgery. Patients were satisfied with this explanation. VisiStetho was appreciated as a good tool for explaining the characteristic of the cardiac sound for a non-physician. In this study, we tried to apply our system to cardiac auscultation training for under graduate medical students.

Materials and Methods: 11 typical cardiac murmurs were chosen from Compact Disk which is a supplement of cardiac auscultation textbook.

29 medical students were involved in the study. Students were classified into two groups: (1) a visualization group, who were lectured on the 11 cardiac murmurs with visualizing the murmurs as 3-D wave form, (2) a control group, who were lectured on the cardiac murmur with only sound without 3-D visualization. Two groups were tested using a pretest and posttest methodology. Results and Discussions: The 15 subjects in the visualization group improved from $33.9 \pm 15.4$ to $88.6 \pm 15 \%$ following the lecture (mean $\pm S D$ ). 14 students in the control group improved from $40.9 \pm 18$ to $74.9 \pm 20.5 \%$. Posttest score of the Visualization group was better than that of control group $(p<0.05$; 
by analysis of variance). Lecture with 3-D Visualization got good opinions from questionnaires.

Conclusion(s): Lecture on cardiac murmur with 3-D visualization might be effective for medical students.

\section{AP2-2}

Teaching cricothyroidotomy and retention of the skill performance

M. Arnberger, A. Vogt, C. Hunkeler, R. Greif

Anesthesiology, University Hospital Bern, Inselspital, Bern, Switzerland

Background: Cricothyroidotomy ( $\mathrm{CrT})$, a rarely used life saving emergency skill, needs special training on manikins or cadavers. Anaesthetists are thought to perform the skill on a competent level after the training. Knowledge about the retention of skill performance over time has an impact on CME-program organization. We assessed retention of skill performance 1, 3 and 6 months after a CrT-training to determine the appropriate time for refresher training. Methods: With IRB approval and informed consent anaesthesia personnel performed CrT after 1, 3 and 6 months of an airway workshop in which CrT was performed $5 x$ on prepared pig larynxes. Performance time was measured from skin palpation until tracheal ventilation.

Results: Of the 64 airway course participants only 48 participated after 1 month of the training, 40 after 3 and 6 months. Only the 36 participants who attended all the training were analyzed. See table for duration of different attempts in the original training and the follow up.

Comparison: original training vs. follow up $(n=36)$

\begin{tabular}{clll}
\hline Original Training & $\begin{array}{l}\text { Time of performance } \\
\text { (mean } \pm \text { SD, sec) }\end{array}$ & $P$ & $\begin{array}{l}\text { \% of Participants } \\
<1 \text { min }\end{array}$ \\
\hline 1st attempt & $94 \pm 48$ & & 19 \\
5th attempt & $50 \pm 19^{1}$ & 75 \\
Time after Training & & \\
1 month & $75 \pm 30^{2}$ & $0.023^{3}$ & 33 \\
3 months & $69 \pm 27^{2}$ & $0.002^{3}$ & 47 \\
6 months & $78 \pm 32^{2}$ & $0.053^{3}$ & 27 \\
\hline
\end{tabular}

1 paired T-test, $\mathrm{P}<0.001,5$ th vs. 1 st attempt

2 paired T-test, $\mathrm{P}<0.001,5$ th attempt vs. $1,3,6$ months

3 paired T-test, 1 st attempt vs. 1, 3, and 6 months

The number of participants who performed the skill in a clinically reasonable time of less than 1 minute dropped from $3 / 4$ at the end of the original training to $1 / 3$ after 1 month, and less after 6 months.

Conclusion: The high level of competence of this rarely used skill was lost within 1 month and all the competence within 6 months. This has impact on all efforts to maintain a certain competence level, on training and further teaching strategies, as well as for global costs for clinical teaching of skills that are not used on a regular basis.

\section{AP2-3}

\section{A qualitative approach to defining learning determinants of} spinal anaesthesia

Z. Kulcsar, A. Aboulafia, T. Hall, D. Sabova, G. Shorten

Department of Anaesthesia and Intensive Care Medicine, Cork University Hospital, Cork, Ireland

Background and Goal: The objective of this study was to perform a structured, prospective, qualitative analysis of the determinants of teaching and learning components of spinal anaesthetic technique in an acute teaching hospital environment.

Materials and Methods: Standard qualitative research techniques (audio taping, transcripts, "post its") were employed to extract and analyse participants' opinions, behaviors and experiences. Data were collected in three phases: 1. preliminary questionnaires (completed by 18 anaesthetic trainers and 24 trainees), 2. focused questionnaires (completed by nine trainers and 22 trainees) 3. focus group interviews (with participation of four trainers and ten trainees). (1)

Results and Discussions: Preliminary questionnaires: Although most trainees $(88 \%, 21 / 24)$ learned the technique from a trainer, only a minority of trainers $(39 \%, 7 / 18)$ had formal background in teaching methodology. The most stressful elements in both teaching and learning were related to patient safety $(43 \%, 18 / 42)$. Some trainers expressed that the tactile element of spinal anaesthesia can be acquired only by hands-on experience $(44 \%, 8 / 18)$ and that this was difficult to explain verbally to trainees $(33 \%, 6 / 18)$. Focused questionnaires: Both trainers $(89 \%, 8 / 9)$ and trainees $(95 \%, 21 / 22)$ judged the overall quality of the teaching programme to be the greatest influence on the teaching-learning continuum. Trainers considered that their characteristics (e.g. motivation, patience) $(100 \%, 9 / 9)$ and experience in education ( $89 \%$, $8 / 9)$ were important determinants. In contrast, most trainees $(77 \%, 17 / 22)$ identified the quality of trainer-trainee interaction as critical. Focus group interviews: Four important points emerged: i. need for a formal, structured training programme, ii. the importance of the visualisation, iii. the critical role of trainer-trainee interaction and iv. optimising the teaching environment (specifically the correct balance between teaching and working).

Conclusion: Our findings indicate that both trainers and trainees believe that the provision of a formal, structured training programme was an important determinant of the learning. Trainees emphasised the importance of the trainertrainee interaction and of the teaching environment.

Reference:

1 Greaves JD. British Journal of Anaesthesia. 2000; 84(4): 525-533.

\section{AP2-4}

\section{Times in learning fiberoptics}

J. Balaguer, A. Lluch, J. Soliveres, C. Sifre, C. Solaz Anesthesiology and Critical Care, Hospital Universitario Dr Peset, Valencia, Spain

Background and Goal of Study: Fiberoptic intubation (FOB) in anaesthesia is widespread used for the difficult airway management (1). Learning fiberoptics is mandatory in anaesthesia practice, and should be performed in non-difficult airway patients. Our objective is to know if learning FOB delays starting surgery.

Materials and Methods: After local ethics committee approval, and obtaining written informed consent, 60 ASA I-III patients were enrolled in the study. Patients with anticipated difficult airway were excluded. Patients were randomly allocated to FOB (FOB group) or oral intubation (O group). In the FOB group, after standard monitoring, atropine $10 \mathrm{mcg} / \mathrm{kg}$ and $6-10 \mathrm{mg} / \mathrm{kgh}$ propofol were administered to reach level 5 in the Ramsay's sedation score. $10 \%$ lidocaĺne was used in the best air-pass-through nostril and supraglotic region, and a 6,5 or $7,5 \mathrm{~mm}$ diameter nasotracheal tube was used as appropriate. Time to intubation was considered as time from atropine injection ultil capnography was present in the monitor. In the $\mathrm{O}$ group, after standard monitoring, atropine $10 \mathrm{mcg} / \mathrm{kg}$ and fentanyl $0,1 \mathrm{mg}$ were administered, followed by preoxigenation and administration of propofol $2-4 \mathrm{mg} / \mathrm{kg}$ and atracurium or cis-atracurium. When the anesthesiologist considered, the trachea was intubated an mechanical ventilation started. Time to perform oral intubation was defined as time from atropine administration until capnography was present. T-test was used at $5 \%$ significance level. Data show number or mean \pm SD.

Results and Discussions: 8 patients were excluded. FOB intubations were performed by 4 anesthesiologists with 4-20 FOB intubation experience. Oral intubations were performed by 5 blinded experienced anesthesiologists.

\begin{tabular}{llllll}
\hline & ASA (I/II/III) & Age (years) & Weight $(\mathrm{kg})$ & Height $(\mathrm{cm})$ & Time $\left(^{*}\right)(\mathrm{mmss})$ \\
\hline FOB & $12 / 08 / 7$ & $52,6 \pm 19$ & $80,3 \pm 21$ & $164,8 \pm 11$ & $9^{\prime} 55^{\prime \prime} \pm 22^{\prime} 52^{\prime \prime}$ \\
$\mathrm{O}$ & $10 / 9 / 6$ & $55,4 \pm 17$ & $81,3 \pm 22$ & $166,4 \pm 9$ & $5^{\prime} 26^{\prime \prime} \pm 1^{\prime} 43^{\prime \prime}$ \\
\hline
\end{tabular}

${ }^{*} \mathrm{p}<0,0001$

Conclusion(s): FOB under deep sedation with propofol introduces a statistically significative but not clinical relevant delay in starting surgery.

Reference:

1 Heidegger T. Curr Opin Anaesthesiol. 2004 Dec; 17(6): 483-4

\section{AP2-5}

Patients, nurses and physicians beliefs and attitudes towards pain in the surgical emergency department

L. Niemi-Murola, D. Grenman, E. Kalso

Department of Anaesthesiology and Intensive Care Medicine, Helsinki University Hospital, Helsinki, Finland

Background and Goal: Inadequate pain management appears to be related to poor staff assessment of pain (1). The aim of this study was to examine nurses', physicians' and patients' attitudes towards pain.

Materials and Methods: A questionnaire with 31 pain-related items was distributed to 50 physicians and 82 nurses at the surgical Emergency Department at Helsinki University Hospital, Finland. Six of these items were interviewed from 100 consecutive patients. The respondents were asked to answer the questions using Likert scale ( $1=$ totally disagree, $5=$ totally disagree). Factor loading of the questionnaire consisting of 31 items was made using maximum likelihood analysis and varimax rotation. Six scales were constructed (Patients and pain, Treatment, Measurement of pain, Encouragement, Problems with analgesics). Statistics: Student's t-test, 2-tailed ANOVA 
Results and Discussion: The questionnaire was returned by $57.31 \%$ of the nurses $(N=47), 58.0 \%$ of the physicians $(N=29)$ and $73.0 \%$ of the patients $(\mathrm{N}=73)$. The nurses were more positive towards scales Measurement of pain (scale mean 4.19 vs. $3.66,95 \% \mathrm{Cl} 0.02-1.04, \mathrm{p}<0.05$ ) and Encouragement (scale mean 3.80 vs. $3.06,95 \% \mathrm{Cl} 0.31-1.17, \mathrm{p}<0.001$ ) than the physicians. The physicians were less concerned about Problems with analgesics than the nurses (scale mean 3.05 vs. $2.32,95 \% \mathrm{Cl} 0.23-1.25, \mathrm{p}<0.01$ ). The patients agreed more with items "It is easier to suffer from pain than the side effects" $[F 2,133)=17.823$, pVt; 0.001$]$, "Pain complaint might distract the doctor from my real problem" [(F 2,134) $=5.505$, pltt;0.01], "Analgesics should be given only when pain is unbearable" $[F(2,134)=9.481, p<0.001]$ than the nurses and the physicians.

Conclusions: The ED personnel need education about measurement of pain and pain medication. Pain history affects the patients' willingness to report their pain and their attitudes towards pain medication. The patients should be encouraged to talk about their pain.

Reference:

1 Stalnikowicz R, Mahamid R, Kaspi S, Brezis M. Int J Qual Health Care 2005; 17: 173-176.

\section{AP2-6}

\section{A design based approach to development of a mixed} interface simulator for learning spinal anaesthesia

Z. Kulcsar, E. Lovquist, M. Fernstrom, A. Aboulafia, G. Shorten

Department of Anaesthesia and Intensive Care Medicine, Cork University Hospital, Cork, Ireland

Background and Goal: The goal of this study was to apply end-user input (both expert and naïve) to the design of a simulator for learning spinal anaesthesia. (1) This forms part of a larger project, Design Based Medical Training, which may optimise training in medical procedural skills. The specific aims were i. to provide the data necessary for programming a realistic simulator device for teaching and learning of spinal anaesthesia and ii. to test the hypothesis that a body of tacit information exists regarding tactile elements of this technique.

Materials and Methods: This study comprised two phases: 1 . The design of Tactile Landscapes 2. The testing phase. The first (design) phase was informed by: i. analysis of video material acquired in the operating room, ii. previously identified determinants of teaching and learning efficacy of spinal anaesthesia, and iii. input on tactile sensation relevant to the procedure from practicing anaesthetists. The second phase involved 24 experts (anaesthetic doctors) and 12 non-experts (medical students) who were invited to test three basic sensations - individually and combined - which typically occur during the performance of spinal anaesthesia with a $25 \mathrm{G}$ pencil point needle: 1 . touching different surfaces (skin, bone), 2. the "pop" sensations when the needle passes through skin and dura mater, 3 . the sensations of advancing the spinal needle through particular tissues (subcutaneous tissue, ligaments, intrathecal space). This study was performed using a haptic device simulating the above sensations. (2) The perception of each participant was recorded, and analysed for "best fit" consistency and expert/non-expert comparison using one way ANOVA statistical analysis.

Results: 1. Great variation within i. experts and ii. non-experts. 2. At haptic values close to "best fit", expert and non-expert perceptions were similar (within 20\%) for most sensations, but different for dura puncture $(59 \%$ less for experts), intrathecal space and subcutaneous tissue (50\% and 30\% less for non-experts, respectively).

Conclusion: Experts appear to possess a "tacit knowledge" regarding some sensations (e.g. dura puncture). This is not true for some other sensations relevant to the performance of spinal anaesthesia.

References:

1 Educational Researcher, 2003; 32(1): 5-8.

2 Medical Education Online, 2005; www.med-ed-online.org/f0000053.htm.

\section{AP2-7}

\section{Patient feedback on preoperative assessment}

K. Chelliah, C. Janakiraman, I. Hodzovic

Anaesthetics, Royal Gwent Hospital, Newport, United Kingdom

Background: Doctor patient interaction is an essential part of medical practice. Preoperative assessment has been used as a tool to evaluate anesthetist's communication skills (1). This survey was designed to record the patient's assessment of anesthetist's ability to communicate.

Materials and Methods: Four hundred unpremedicated adult patients scheduled for elective surgery in four hospitals in South Wales (UK) were asked to fill the consultation and relational empathy questionnaire (CARE) (1). The patients scored the anesthetists on a five point scoring system (poor to excellent) preoperatively.

Results: The findings are presented in the table. Values are number (proportion out of 400 presented as \%).

\begin{tabular}{|c|c|c|c|c|c|}
\hline $\begin{array}{l}\text { How good was the } \\
\text { doctor at.... }\end{array}$ & Poor & Fair & Good & Very good & Excellent \\
\hline $\begin{array}{l}\text { Making you feel } \\
\text { at ease }\end{array}$ & $0(0)$ & $4(1)$ & $63(15)$ & $163(40)$ & $170(42)$ \\
\hline $\begin{array}{l}\text { Letting you tell } \\
\text { your story }\end{array}$ & $4(1)$ & $14(3)$ & $55(13)$ & $170(42)$ & $142(35)$ \\
\hline Really listening & $1(0.3)$ & $8(2)$ & $66(16)$ & $153(38)$ & $155(38)$ \\
\hline $\begin{array}{l}\text { Treating you as a } \\
\text { whole person }\end{array}$ & $2(0.5)$ & $20(5)$ & $83(20)$ & $160(40)$ & $124(31)$ \\
\hline $\begin{array}{l}\text { Understanding } \\
\text { your concerns }\end{array}$ & $3(0.7)$ & $16(4)$ & $60(15)$ & $171(42)$ & $146(36)$ \\
\hline $\begin{array}{l}\text { Showing care } \\
\text { and compassion }\end{array}$ & $1(0.3)$ & $5(1.2)$ & $66(16)$ & $160(40)$ & $155(37)$ \\
\hline Being positive & $0(0)$ & $4(1)$ & $72(18)$ & $167(41)$ & 157 (39) \\
\hline $\begin{array}{l}\text { Explaining things } \\
\text { clearly }\end{array}$ & $0(0)$ & $12(3)$ & $52(13)$ & $157(39)$ & $175(43)$ \\
\hline
\end{tabular}

Conclusion: Our survey shows that patients are satisfied with the communication skills of anesthetists, with $95 \%$ of patients scoring anesthetists as good or excellent. As specific patient comment plays an important role in delivery of anesthetic service (2), we believe 'CARE' scoring system would be invaluable in appraisal of anesthetists.

References:

1 Hatch D. BJA. Bulletin 36: 1804

2 Carnie J. Anesthesia: 2002, 57: 697

\section{AP2-8}

Postoperative intake of oral paracetamol: an evaluation of ward based educational strategiesto change administration practice G. Mathew, K. Grimsehl, I. Christie

\section{Anaesthetics, Derriford Hospital, Plymouth, United Kingdom}

Background and Goal of Study: Paracetamol is widely used as part of a multi-modal approach to analgesia [1]. Although it is recommended that paracetamol should be taken regularly for maximum analgesic effect [2], our experience is that paracetamol administration in the post operative period is variable. We assessed whether educational interventions were effective in changing this practice.

Materials and Methods: A prospective audit was performed on 50 neurosurgical patients with GCS 14-15 and ASA I-III undergoing either spinal or intracranial procedures. The intake of oral paracetamol in the post operative period was obtained from the prescription chart. The reason for omission of doses was noted. Based on the initial results the acute pain team undertook an intensive program of ward staff education. This included lectures, presentations, and workshops stressing the effectiveness of regular simple analgesia. Posters of the analgesic ladder were prominently displayed on all wards. A re-audit was done a year later on a further 50 patients meeting the same criteria. The data were compared using $\chi^{2}$-test

Results and Discussions: 45 patients were prescribed regular paracetamol in the first audit. Despite this, in the first $24 \mathrm{hrs}$ after surgery, only 24 patients received paracetamol regularly. In 19 patients this was due to patient refusal. In the re-audit that followed the educational programme, 38 of the 45 patients prescribed regular paracetamol received it $(p<0.01)$; and patient refusal had decreased to $6(p<0.01)$.

Conclusion(s): We have shown that a targeted educational strategy can significantly improve regular administration of paracetamol, an effective analgesic with minimal adverse effects. However, patients still need encouragement to take this analgesic regularly.

References:

1 Barden J, Edwards J, Moore A, McQuay H Single oral dose of paracetamol for postoperative pain; Cochrane Review, Cochrane Library, Issue 3, 2006.

2 Acute pain in the surgical patient; Medical Information Leaflet Vol. 19, December 1998.

\section{AP2-9}

\section{Development of a curriculum for catastrophe preparedness}

\section{E. Pfenninger, S. Himmelseher}

\section{Anesthesiology, University of UIm, UIm, Germany}

Background and Goal of Study: The new German Federal Regulations on Educational Issues for Medical Schools (in operation since 2003) outline that medical students need to prove knowledge in catastrophe and disaster medicine in board examinations, and the American Society of Anesthesiology has initiated activities for catastrophe preparedness in 2004. Because anesthesiologists play a special role in providing trauma and catastrophe victim care, we have created a model curriculum for catastrophe medicine (amended and now accredited by the German Interior Federal Ministry). 
Materials and Methods: Educational objectives were the provision of core components of catastrophe medicine; the conceptual construction was based on a modular approach incorporating experiential training. The exploration of international principles on education for major community threatening events (1), and analysis of Public Health Physician Competencies for Disaster Preparedness as demanded by German Authorities (2) formed a foundation for content development.

Results and Discussions: In a step-by-step design of 28 units, catastrophe (medicine) terminology, legal basics of catastrophe management, psychological and ethical issues relevant in disaster situations, and preparedness planning are taught. Lectures on management of mass casualties and for care in case of release of radioactive, biological, and chemical agents with special attention on infectious diseases and terrorist attacks follow. To provide primary care, fundamentals of shock therapy, analgesia, sedation, and anesthesia as well as trauma care in the field are taught. Limitations of individual patient medicine in disaster are discussed. At least $25 \%$ of the course consist of practical training experiences, such as emergency room simulations or exposure to agency-led activities.

Conclusions: Because of its focussed applicability and readiness, the curriculum met with enthusiastic response from participants, and has been established as an option of the 4th year student obligatory program at the University of Ulm. It is taught by anesthesiologists, who fulfil historical tasks by preparing for effective medical disaster response.

References:

1 Seynaeve et al. Prehosp Disaster Med 2004; 19 Suppl 2.

2 Pfenninger E, Himmelseher S: Publications 2005 http://www.bva.bund.de/zivilschutz/

\section{Patient Safety}

\section{AP1-1}

The Anaesthetic Room - Friend or Foe? An audit to assess the frequency of incidents relating to patient safety, occurring on transfer of anaesthetised patients from the anaesthetic room to the operating theatre

T. Hinde, T. Gale

Anesthetic, Derriford Hospital, Plymouth, United Kingdom

Background and Goal of Study: In the USA, anaesthetic rooms have all but disappeared [1]. At a time of proposed refurbishment in our hospital, an audit was carried out to assess the frequency of incidents relating to safety, on transfer of anaesthetised patients from the anaesthetic room to the operating theatre. This transfer process was once described by a barrister prosecuting in an anaesthetic death as "clumsy and ill conceived" [2].

Materials and Methods: The audit, in our General Emergency Theatre, looked at 101 patients in a 1 month period. An anonymous questionnaire was completed which included: patient demographics, whether the anaesthetic was performed in the anaesthetic room (AR) or operating theatre (OT), and any incidents occurring on transfer. The response rate was $78 \%$.

Results and Discussions: 91/101 (90\%) of patients were anaesthetised in the AR. $71 / 101(70 \%)$ of all patients were ASA 1 or $2.10 \%$ of patients were anaesthetised in theatre, of whom $6 / 10(60 \%)$ were ASA 3 or more. $10 / 101(10 \%)$ of all patients weighed $\geqslant 100 \mathrm{~kg}$.

At least one incident was described in 17/91(18\%) of cases anaesthetised in the AR. Of 21 incidents described, 19/21(90\%) were considered to have been avoidable had the patient been anaesthetised in the OT.

Table 1 Summary of incidents on patient transfer:

\begin{tabular}{ll}
\hline Incident & Frequency \\
\hline Patient 'light' & $6 / 21(29 \%)$ \\
Lines tangled & $3 / 21(14 \%)$ \\
Missing part of circuit & $3 / 21(14 \%)$ \\
Monitoring delay in OR & $3 / 21(14 \%)$ \\
Hypoxia $-\mathrm{SpO}_{2}<93 \%$ & $2 / 21(10 \%)$ \\
Manual handling issue & $2 / 21(10 \%)$ \\
Lines not working & $1 / 21(0.5 \%)$ \\
Delay in transfer assistance & $1 / 21(0.5 \%)$ \\
\hline
\end{tabular}

Conclusion(s): We are more likely to anaesthetise ASA 3 and 4 patients in the OT. Of those patients anaesthetised in the AR, incidents relating to safety occurred in $18 \%$ of patients in our emergency theatre. $90 \%$ of these incidents were felt to be avoidable had the patients been anaesthetised in the OT.

This has implications in both patient and staff safety.

References:

1 Meyer-Wittington. Anaesthesia 1992; 47: 1011-1012

2 Brahams.D. Anaesthesia 1990; 45: 332-333

\section{AP1-2}

Post anaesthesia care units are lacking in most italian hospitals: a survey in the Veneto region

P. Trevisan

Anaesthesia and Intensive Care, Ospedale S. Martino, Belluno, Italy

Background and Goal of Study: International guidelines state that the Post Anaesthesia Care Unit (PACU) is an essential facility to guarantee patients' safety (1). In Italy only a few hospitals are equipped with a PACU, but there are no published data. It was conducted a survey to find out how many PACUs are present in the Veneto region (Italy; north-east).
Materials and Methods: on the 18th and 19th October 2006 the author contacted by phone all public hospitals in Veneto undertaking surgical activity $=n^{\circ} 49$. An anaesthetist from every department of anaesthesia was asked: 1) $n^{\circ}$ of operating rooms (ORs) 2) presence/absence of PACU 3) if PACU present, $n^{\circ}$ of beds. The reply rate was $100 \%$.

Results and Discussions: in Veneto there are 49 public hospitals where surgical activities are undertaken; 6 hospitals (12\%) are equipped with a PACU. The PACU beds/ORs ratio is shown in the table:

\begin{tabular}{llllllc}
\hline Hospital & 1 & 2 & 3 & 4 & 5 & 6 \\
\hline $\mathrm{N}^{\circ}$ PACU beds & 2 & 4 & 4 & 6 & 6 & 12 \\
$\mathrm{~N}^{\circ}$ ORs & 5 & 6 & 7 & 8 & 6 & 13 \\
PACU beds/ORs & 0.4 & 0.66 & 0.6 & 0.75 & 1 & 0.9 \\
\hline
\end{tabular}

This survey shows that in Veneto only $12 \%$ of public hospitals are equipped with a PACU. The optimal PACU beds/ORs ratio is thought to be 1.5-2 (2). In Veneto the ratio is far less than optimal, therefore PACU beds are insufficient even where a PACU is present. A previous study demonstrated that is very difficult to guarantee a proper and safe post-anaesthesia care without a PACU (3). Conclusion(s): In Veneto only 6 out of 49 public hospitals are equipped with a PACU. As it is very difficult to guarantee post-anaesthesia safety and care without a PACU, it is mandatory for every hospital in Veneto to open a PACU with a sufficient number of beds.

References:

1 Leykin Y. Minerva Anestesiol 2001; 67: 563-71.

2 Leykin Y. Minerva Anestesiol 2001; 67: 539-54.

3 Trevisan P. Minerva Anestesiol 2004; 70: 631-42.

\section{AP1-3}

Should patients be anaesthetized in a dedicated anaesthetic room? A survey of attitude of anaesthetists and patients in a District General Hospital in UK

A. Obideyi

Anaesthesia, Peterborough \& Stamford Foundation Hospitals NHS Trust, Peterborough, United Kingdom

Background and Goals: $94 \%$ of UK hospitals have anaesthetic rooms. The usefulness of these rooms is controversial. A survey of the attitude of Anaesthetists and that of patients in a District General Hospital in the UK on their preferred site of induction of anaesthesia was conducted between February and April 2006.

Methodology: Two questionnaires were designed. The first questionnaire was completed by the anaesthetists of all grades working in Luton \& Dunstable Hospital, UK. The second set was completed by patients scheduled for operation in the same hospital.

Results:

Table I (Anaesthetists)

\begin{tabular}{|c|c|c|c|c|c|c|}
\hline \multirow[b]{2}{*}{ Grades } & \multicolumn{2}{|c|}{$\begin{array}{l}\text { Where do you } \\
\text { anaesthetize } \\
\text { elective patients? }\end{array}$} & \multicolumn{2}{|c|}{$\begin{array}{l}\text { Where do you } \\
\text { anaesthetize high } \\
\text { risk patient? }\end{array}$} & \multicolumn{2}{|c|}{$\begin{array}{l}\text { Will you give up } \\
\text { the AR? }\end{array}$} \\
\hline & $A R$ & OT & $\mathrm{AR}$ & OT & Yes & Never \\
\hline Cons & 15 & 2 & 6 & 11 & 10 & 7 \\
\hline TDs & 2 & 2 & 2 & 2 & 4 & 0 \\
\hline SpRs & 4 & 0 & 2 & 2 & 2 & 2 \\
\hline SHOs & 5 & 1 & 2 & 4 & 1 & 5 \\
\hline Total & $26(84 \%)$ & $5(6 \%)$ & 12 (38.7\%) & 19 (61.3\%) & 17 (54.8\%) & $14(45.2 \%)$ \\
\hline
\end{tabular}


Table II Patients' preferential site for induction

\begin{tabular}{lllc}
\hline & No previous operations & 1 or $>1$ operations & Total \\
\hline AR & 5 & 19 & $24(60 \%)$ \\
OR & 2 & 3 & $5(12.5 \%)$ \\
No preference & 4 & 7 & $11(27.5 \%)$ \\
\hline
\end{tabular}

AR - Anaesthetic room, OT - Operating theatre, Cons - Consultants, TDs - Trust

Doctors, SpRs - Specialist Registrars, SHOs - Senior House Officers

Discussion: Although anaesthetic rooms have been in existence in the UK for decades, a robust argument for their continuous use is largely lacking from the literature. Patient safety, medico legal liabilities and the prevailing financial situations of most hospitals may lead to their disappearance as is the practice in most developed countries around the world. Educating the surgical patients may alter their preferential site for induction of anaesthesia.

References:

Bromhead HJ, Jones NA. The use of anaesthetic rooms for induction of anaesthesia. Anaesthesia 2002; 57: 850-854.

O'Connor D, Dobson A, Smith S: Inducing anaesthesia in the operating theatre: Anaesthesia 2003; 58: 912-13.

\section{AP1-4}

\section{Full-scale simulator training rapidly improves crisis resource} management skills

\section{E. Michael, R. Krage, O. Picker}

Klinik fuer Anaesthesiologie, Universitaetsklinikum Duesseldorf,

Duesseldorf, Germany

Introduction: Health care is not as safe as it should be. Deaths in hospitals due to preventable adverse events still remain in the top 10 of the leading cause of death (1). Roughly $80 \%$ of these events are related to the so called human factor due to a lack of knowledge of proper Crisis Resource Management (2). Patient simulators are receiving increasing support as an educational tool. Therefore, we studied whether frequent simulation sessions during an anesthesia residency programme increase knowledge of CRM and thus patient safety.

Methods: All members of our department $(n=62)$ participated in simulation sessions on a regular basis (residents twice a year, board-certified anesthesiologists once a year) to teach both, technical abilities (hard skills) as well as Crisis Resource Management (soft skills). All scenarios were developed using a full-scale-simulator (HPS, METI). After 18 months the quality of the simulation sessions was evaluated using a standardized questionnaire (20 questions). All questions could be answered by using standard school grades (1-6). Grades were then divided in three groups (1-2: agree, 3-4: intermediate, 5-6: disagree).

Results: Simulation sessions on a regular basis improved hard skills (68\%) and soft skills (81\%). After 18 months of simulation training, $53 \%$ of the participants reported that simulations sessions influenced their every day practise. $51 \%$ stated that they altered their behaviour in an OR setting in terms of communication and team organisation. All scenarios were evaluated as being very realistic (92\%) and appropriate in terms of skill level (95\%). 52\% of the questionnaires were answered.

Conclusion: 18 months after establishing a full scale simulation programme modulation of the individual performance can clearly be attributed to our programme. Therefore, consequent and frequent simulation sessions increase knowledge of CRM and may thus contribute to the improvement of patient safety.

\section{References:}

1 Kohn, LT et al. (2000): "To err is human", National Academy of Science, Washington (http:/www.nap.edu/books/0309068371/html/;21.07.2005).

2 Flin R et al.: "Anaesthetists Non-Technical Skills System (ANTS) Handbook V1.0", Aberdeen.

\section{AP1-5}

Quantitative comparison of weight-bearing areas on the body surfaces before and after the induction of general anesthesia H. Kang, S. Kim, S. Ryu

Department of Anesthesiology and Pain Medicine, College of Medicine, Chungbuk National University, Chungju, Republic of Korea

Background and Goal of Study: While surgical position is very important for the prevention of excessive pressure on the specific body surfaces during anesthesia, resulting in pressure sores and peripheral nerve damage, we quantitatively determined weight-bearing areas by measuring pressure on the body surface areas of patients before and after the induction of general anesthesia. Materials and Methods: Fifteen men patients (aged over 20 and ASA class 1 ) scheduled for elective surgery were selected for this study. A mattress equipped with a force sensing resistor (FSR) placed on the operating table was used to determine pressure on the body surfaces in the supine, lateral and prone position. The pressure signal generated by this mattress was processed using an analogue-digital converter (ADC), and then quantitatively displayed as pressure distribution (\%) on a computer screen (pressure points before and after general anesthesia were compared qualitatively, and, for quantitative comparison, were digitally expressed as pressure distribution).

Results and Discussions: Pressure was concentrated on the shoulder and sacral areas in the supine position before and after the induction of general anesthesia. There was significant difference $(p<0.05$, paired t-test) in pressure distribution on the shoulder before (29.8\%) and after (20.3\%) the induction of anesthesia. In the lateral position, the shoulder and trochanteric areas were identified as the main weight-bearing areas. Pressure distribution on the shoulder $(21.6 \%)$ and trochanteric areas $(15.3 \%)$ after anesthesia was significantly higher $(p<0.05)$ than before anesthesia $(11.2 \%$ and $8.1 \%$, respectively). In the prone position, the chest was the main weight-bearing area. Pressure distribution in the chest area was $30.8 \%$ and $24.4 \%$ before and after the induction of anesthesia, respectively. Tilting the operating table in each surgical position also produced marked changes in pressure distribution on the weight-bearing areas before and after anesthesia was induced. Conclusion(s): In each surgical position, weight-bearing areas before anesthesia did not migrate to other areas after anesthesia, but there was change in pressure distribution on some specific weight-bearing areas after the induction of anesthesia.

\section{AP2-1}

\section{Does sleep deprivation cause cognitive dysfunction in residents in training? \\ M. Vila, A. Garrido, P. Vila, J. Canet \\ Anaesthesiology, Hospital Germans Trias i Pujol, Badalona, Spain}

Background and Goal of Study: Sleep deprivation and night work have been identified as causes of cognitive dysfunction and technical errors. By applying the principles of chronobiology to resident work schedules, the effect of sleep deprivation on cognitive function was assessed in trainees of a University Hospital after they were 24 hours on call.

Materials and Methods: Prospective, controlled, single-blind study. Thirty residents from first to fifth year of academic training in different medical specialties were studied. Residents were randomized to answer a battery of neuropsychological tests either after 24 hours on call when they had slept no more than three continuous hours $(A)$ or after a normal day and sleep hours (B). The test battery consisted of the Visual Learning Test, Letter Digit Coding Test and Stroop Colour Interference Test. The tests were presented in random order to compensate for a learning effect. Time used and number of errors were recorded. Vital signs (blood pressure, heart rate, pulse oximetry and core body temperature) were also recorded. ANOVA, $\chi^{2}$ tests and linear regression analysis were performed.

Results and Discussion: Stroop test performance was significantly different after sleep deprivation (A: $31.3 \pm 6.2 \mathrm{~s}$ ) than after a normal shift (B: $29.1 \pm 6.6 \mathrm{~s} ; \mathrm{p}<0.005$ ); no significant differences were observed on the other neuropsychological tests. There was also a significant negative correlation between time on the Stroop test and sleep deprivation (hours slept) $(r=-0.395 ; p<0.03)$. All vital signs were similar at the different moments studied.

Conclusion: Specific neurocognitive performances (i.e.: attention measured by the Stroop test) are vulnerable to sleep loss. The controversy over resident work hours and the effect on education, professionalism, wellness and safety should be further reviewed.

References:

Durmer JS. Seminars in Neurology 2005; 25: 117-129.

Klara K. Sleep Medicine reviews 2006; 10: 339-345.

\section{AP2-2}

\section{High incidence of distracting events and diverted time during} induction of general anesthesia for urgent surgical cases

J. Thieblemont, Georges L. Savoldelli, Philippe. Garnerin, Alain. Forster, François. Clergue

Anesthesia, Geneva University Hospital, Geneva 14, Switzerland

Background and Goal of Study: Distractions and interruptions in the workplace have been shown to decrease vigilance and increase errors. ${ }^{1,2}$ Although sources of distraction appear to be common in the operating room, little is known about their origin and their effect on anesthesia providers. The goal of this pilot study was to determine the origin, the frequency and the impact of distracting events (DE) on anesthesia tasks during the induction phase of general anesthesia (IGA) for urgent surgical cases.

Materials and Methods: Thirteen IGAs were videotaped. The videotapes were reviewed and analyzed using an original scoring system developed by 
expert consensus in order to identify and characterize DEs. The duration, the origin (whether internal, i.e. related to anesthesia tasks - e.g. informationsharing dysfunction, equipment problem - or external, i.e. all other events - e.g. pager) and the impact of DEs on team activities were categorized.

Results: On average we observed 5.2 DEs per IGA. This represented $25.3 \%$ of total IGA time. DEs directly impacted on the activity of the "airway manager" and the "operator injecting the drugs" in $51.5 \%$ and $61.8 \%$ of instances respectively (e.g. task switching, multitasking, break in task).

\begin{tabular}{lllll}
\hline & IGA & DE & Int DEs & Ext DEs \\
\hline Number & 13 & 68 & 43 & 25 \\
Total time (min) & 106 & 26.8 & 17.4 & 9.4 \\
\% of IGA time & NA & 25.3 & 16.4 & 8.8 \\
Mean[sd](min) & $8.2[2.6]$ & $0.4[0.4]$ & $0.4[0.5]$ & $0.4[0.3]$ \\
\hline
\end{tabular}

Conclusions: During IGAs, DEs are frequent and significantly divert anesthesia-related tasks. This pilot work will help us to further characterize how DEs are generated, how they affect performance and patient care. In the future, this may lead to the design and implementation of preventive and/or coping strategies which could be incorporated in a team-oriented training approach to improve patient safety.

References:

1 Chisholm CD. Ann Emerg Med 2001;38(2):146-51.

2 Brixey JJ. AMIA Annu Symp Proc 2005:86-90.

\section{AP2-3}

\section{"PAPEROS" : A new meta-reporting model to categorise} critical incident reports: first results

A. Dhollander, H. Weibel, A. Cahana, D. Tassaux

\section{Anesthesiology, CHU-Geneva, Geneve, Switzerland}

Background and Goal of the Study: Faced to the reporting problem of the numerous critical incident reports [CIR] locally collected, a practical multidisciplinary classification model appeared needed. The design of this new reporting frame was derived from the historical DEPOSE model used, in many industrial domains, for accident analysis ${ }^{1}$. The goal of this study was to test the applicability of this new meta-reporting system on CIRs issued from a 15 beds PACU in a tertiary care university hospital.

Methods: The first $350 \mathrm{CIRS}$ collected were categorized according the 7 meta-categories composing the present model [Patient, Actors, care Process, Equipment, care pro-cess Room, Organisation, Supplies-PAPEROS]. The CIRs classification was performed by iterative sessions of 50 successive CIRs. Classification reproducibility was control-led after each batch completion. The rules used for this classification were elaborated gradually batch after batch. Results and Discussion: PAPEROS classification showed that voluntary CIRs reporting in PACU is essentially concentrated on care Process [45\%] and Organisation [50\%] issues. The importance of Actors [3\%] and care process Room [1\%] was presently minimal. The frequency of Patient, Equipment and Supplies reports were less than $1 \%$. These results reflect the interface characteristics of a PACU, whose activities must overall match the "internal" care Process non conformities and the patient flux with the different hospital units: the Organisation. The classification reproducibility was well above the threshold limit of $95 \%$. The present PACU metareporting pattern obtained was different from the CIRs collected from other sectors of the department were Patient, Equipment and Supplies categories were quite more frequently reported

Conclusion: The feasibility of this new meta-reporting PAPEROS model appears very attractive for internal qua-lity control and for launching relevant information about the CIRs content collected in larger entities.

Reference:

1 Living with high-risk technologies. C Perrow, (Ed.) Princeton University Press, 1999.

\section{AP2-4}

\section{Etiology and incidence of perioperative severe allergic reaction}

S. Sanchez-Navas, L. Moltó, A. Gimenez, C. Rodriguez, F. Escolano Anaesthesia, Hospital del Mar-Esperanza, Barcelona, Spain

Goal of Study: 1) To determine the incidence and symptoms of perioperative severe allergic reactions (SAR) in our hospital. 2) To identify the drugs suspected of causing SAR. 3) To evaluate the immediate and delayed allergic study in our hospital.

Material and Methods: A prospective study was carried between 1996 and 2006. All the patients who presented SAR were included in our study. We recorder the medical history and description of the allergy reactions. During the first 24 hours we performed the immediate inmmunoalergic study
(IIS) (histamine, serica tryptase, urine metilhistamine, complement factors, latex and other drugs and histamine release test (HRT). Posteriorly, we did the delayed inmmunoalergic study (cutaneus test, Ig $E$ and histamine release tests specific).

Results: SAR occurred in 30 of 139.075 anaesthesia. The SAR incidence was 1 in 4.635 cases of anesthesia. The manifestations were cardiovascular shock, tachycardia, and wheezing in 80,75 and 50 percent of the overall symptoms. $83 \%$ were drugs (non steroidal anti-inflamatory drugs (NSAIDS), 33.3\%; antibiotics, $30 \%$; muscle relaxants, $13.3 \%$; protamine, $3.3 \%$ ). There were 2 cases due to dextrano, 1 latex, 1 gelafundine and another due to a hidatide cyst. One patient died. In all patients the IIS was positive in one or several tests (sensitivity 100\%) and the delayed inmunoalergic study was positive in $94.4 \%$. Conclusions: The incidence of perioperative allergic reactions during anaesthesia was 1 in 4.635 cases. The drugs most suspected of causing an severe allergic reaction were NSAIDS. Our protocol of immediate study is highly effective and has a global sensitivity of $100 \%$. The most frequent symptom was cardiovascular collapse.

\section{AP2-5}

The value of a voluntary reporting system of peroperative complications as quantitative key performance indicator K. Soufidi, T. Schouten, T. Kappen, J. Bijker, C. Kalkman

Division of Perioperative Care and Emergency Medicine, University Medical Center Utrecht, Utrecht, The Netherlands

Background and Goal of Study: Since 2004 the University Medical Centre Utrecht participates in a national anaesthetic quality assurance programme for reporting perioperative incidents. Following each anaesthetic a standardized computerized audit form is completed by the anaesthesiologist. Perioperative incidents are defined according to a categorized list of the 20 most frequent perioperative adverse events, including process outcomes such as hypotension, hypertension and hypoxemia. Goal of the study is to evaluate the accuracy of complication reports and completeness of the list of categories.

Materials and Methods: The computerized voluntary record system is a module within the hospital anaesthesia information management system. The audit form can be amended up to 24 hours after the anaesthetic. An automatic reminder is sent by e-mail if the audit form is not completed. All surgical procedures in 2005 were selected, excluding cardiothoracic surgery and patients younger than 18 years. Reported incidents were checked by two independent reviewers and judged on misclassification.

Results: In 13,346 cases 634 (4.8\%) adverse events were reported, spread over 618 (4.6\%) procedures. There were 579 cases in which the audit form was not filled in, resulting in a reporting rate of $95.7 \%$. Misclassification was noticed in $119(18.8 \%)$ of the reported events. Reclassification in the appropriate category was possible in $91(76.5 \%)$ reports. Altogether $92(14.5 \%)$ reports did not correspond with the list of 20 most frequent perioperative adverse events. Conclusion: Voluntary reporting in our hospital shows a $4.6 \%$ incidence of adverse events with a compliance of $95.7 \%$. Because of a misclassification of $18.8 \%$, we consider the reliability of the complication registration system to be low. The large number of unclassifiable events urges to reassess the list of definitions. Based on these findings the current voluntary reporting system should not be used as a quantitative key performance indicator.

\section{AP2-6}

\section{Hypotension as peroperative complication: self-reported} records versus computerized analysis of the anesthesia information management system

T. Kappen, T. Schouten, K. Soufidi, J. Bijker, C. Kalkman

Division of Perioperative Care and Emergency Medicine, University Medical Center Utrecht, Utrecht, The Netherlands

Background and Goal of Study: Since 2004 the University Medical Centre Utrecht participates in a national anaesthetic quality assurance programme. Following each anaesthetic a standardized computerized audit form is completed by the anaesthesiologist, based on the 20 most frequent perioperative adverse events, including process outcomes such as hypotension. Hypotension is defined as a mean arterial pressure below $40 \mathrm{~mm} \mathrm{Hg}$ longer than 5 minutes, which requires intervention. In this study the accuracy of reporting of hypotension by anaesthesiologists consistent with the definition is verified. Materials and Methods: The surgical procedures in 2005 were reviewed, excluding cardiothoracic surgery and patients younger than 18 years. Using computer software (LabView, National Instruments) the hemodynamic variables of the anaesthesia information management system were analyzed for hypotension according to the aforementioned definition, without the requirement of intervention. The number of analyzed cases with hypotension was compared with the number of reported cases. 
Results and Discussions: Of the 13.346 anaesthetics the audit form was not filled in 597 cases, resulting in a reporting rate of $95.7 \%$. Of $131(1.0 \%)$ reported hypotension cases, 15 were confirmed by analysis (positive predictive value $11.5 \%$; sensitivity $8.9 \%$ ). In 154 anaesthetics no hypotension was reported in contrast with the analysis (negative predictive value $98.8 \%$, specificity $99.1 \%)$

Conclusion(s): Although the negative predictive value and specificity are high, the positive predictive value and sensitivity are very low. Apparently anaesthesiologists use a different definition of hypotension. Moreover the low sensitivity can be explained by a low compliance. Therefore the current definition of hypotension in our voluntary reporting system is inappropriate as a key performance indicator.

\section{AP2-7}

\section{Analysis of incidents using a system approach}

J.M. Marzal, J.A. Santa-Ursula, G. Puebla, D. Arnal, A. Bartolome Unidad de Anestesia y Reanimacion, Fundacion Hospital Alcorcon, Alcorcon, Spain

Background and Goal of Study: A critical component of a comprehensive strategy to improve patient safety is to establish an incident reporting system. The goal of this study is to describe incidents reported last year and analyzed using a system approach.

Materials and Methods: Anaesthetists were invited to report, on an anonymous and voluntary basis, any unintended incident which reduced, or could have reduced, the safety margin for a patient. Any incident could be reported, not only those which were deemed preventable or were thought to involve human error. All the incidents were reported using a computerised intranet accessible form. A five anaesthetists committee analyzed every incident using a system approach to determine why it happened (active errors and latent factors in the system) and what should be done to prevent this type of incident from recurring.

Results and Discussions: Between October 1st 2005 and October 1st 2006 12692 anaesthetic procedures were performed and 93 incidents were reported $(0,73 \%)$. The three most frequent types of incident reported were equipment $(n=25)$, communication $(n=21)$ and drug related $(n=16)$. In 63 incidents (67.7\%) 66 active errors were identified: 24 skill-based slips, 5 skill-based lapses, 21 rule-based mistakes, 11 knowledge-based mistakes and 5 violations of protocols. 201 latent factors were identified: 24 patient factors ( 21 of them complexity), 18 work environment factors ( 7 unavailable protocols), 49 individual factors (20 lack of knowledge), 40 team factors (19 communication problems), 37 task factors (12 poor equipment design) and 33 organisational factors (19 deficient safety culture). Main implications and action points were 24 meetings, 18 scientific sessions, 12 e-mail alerts, 8 formative actions and 6 equipment acquisition. There were no effect on patient or minor morbidity in 78 incidents, whereas intermediate morbidity, major morbidity and death occurred in 10, 3 and 1.

Conclusion(s): Analysis of incidents using a system approach identified latent factors. Remedial actions were designed to prevent incidents from recurring.

\section{AP3-1}

\section{Comparison of different positions of the safety frog on the ventilator in case of technical failure}

J.P. Mulier, G. Van Droogenbroek, M. Weiss

Anaesthesiology, az St Jan AV Brugge, Brugge, Belgium

Background and Goal of Study: The safety frog device from MedecBenelux $\mathrm{nv}$ is an intelligent pressure release valve opening in case of a continuous elevated airway pressure. Goal of this study was to analyze what the best position is in case of a blocked inspiratory or expiratory valve and in case of an occluded inspiratory or expiratory tubing line.

Materials and Methods: An artificial lung (no patient or animal) with a compliance of $12 \mathrm{ml} / \mathrm{mmHg}$ is connected to an aespire $\mathrm{S} / 5$ ventilator from DatexOhmeda. The active safety frog is put in four positions while the different defects are mechanical simulated during spontaneous (simulated with the artificial lung) and during controlled volume ventilation. The APL valve is open. If a continuous pressures above $20 \mathrm{cmH} 20$ is measured in the artificial lung in one of the simulated technical failures the position is noted as dangerous. Results and Discussions:

\begin{tabular}{|c|c|c|c|c|}
\hline $\begin{array}{l}\text { Position of } \\
\text { safety frog }\end{array}$ & $\begin{array}{l}\text { Insp valve } \\
\text { occlusion }\end{array}$ & $\begin{array}{l}\text { Insp tube } \\
\text { occlusion }\end{array}$ & $\begin{array}{l}\text { Exp valve } \\
\text { occlusion }\end{array}$ & $\begin{array}{l}\text { Exp tube } \\
\text { occlusion }\end{array}$ \\
\hline Inspir line & no risk & no risk & protected & protected \\
\hline Expir line & no risk & no risk & protected & danger \\
\hline Patient connection & no risk & no risk & protected & protected \\
\hline Man line & no risk & no risk & danger & danger \\
\hline
\end{tabular}

Table gives the different results. During spontaneous breathing with an open APL valve high airway pressure is not possible in any simulated failure on this ventilator. With an occluded tubing however the patient can only inspire and not expire and will finally get a volutrauma if no safety frog is positioned on the inspiratory line. An occluded inspiratory valve or inspiratory tubing does not give volutrauma in contrary to an occluded expiratory valve or tubing during mechanical ventilation.

A position in the manual breathing bag line fails to protect during mechanical ventilation because of exclusion. The expiratory line position at the ventilator fails to protect during occlusion of the expiratory tubing. The inspiratory line position is simpler than the patient's connection position. Both protect the lungs in all investigated failures.

Conclusion: The position at the inspiratory line of the ventilator is mandatory to obtain the best safety protection.

\section{AP3-2}

The carina as a landmark for evaluation of adequate central catheter tip position with computerized tomography

I.W. Shin, J.T. Sohn, H.K. Lee, Y.K. Chung

Anesthesiology and Pain Medicine, Gyeongsang National University Hospital, Jinju, Republic of Korea

Background and Goal of Study: The aim of this study was to determine whether the carina can be used as a landmark for evaluation of adequate central catheter tip position, and to examine the relationship between easily measurable body size and variable anatomical parameter.

Materials and Methods: The SVC dimensions and relationship to radiographic landmarks were retrospectively determined from computerized tomography (CT) scans of 200 patients. The CT findings were assessed in terms of SCV length (SCVL), the distance between the carina and the right atrium inlet (CAL), and the sternal length (STL). Pearson's correlation and a regression test for height versus SCVL, STL versus SCVL and CAL were performed.

Results: The median length of the SVC was $4.2 \mathrm{~cm}$ (range; 1.6 to $7.2 \mathrm{~cm}$ ) and the distance between the carina and the right atrium inlet was $2.4 \mathrm{~cm}$ (range; 0.8 to $5.6 \mathrm{~cm}$ ). With the regression test, height was correlated with SVCL $(r 2=0.09)$, and STL was correlated with both SVCL $(r 2=0.12)$ and STL $(\mathrm{r} 2=0.04)$.

Conclusion: The carina was located always above the right atrium inlet. The carina was a reliable, simple anatomical landmark for the determination of correct placement with computerized tomography.

References:

1 Aslamy Z, Dewald CL, Heffner JE: MRI of central venous anatomy; implications for central venous catheter insertion. Chest 1998; 114: 820-6.

2 Dunbar RD, Mitchell R, Lavine M: Aberrant locations of central venous catheters. Lancet 1981; 28: 711-5.

Table 1. Means of measured SVCL and CAL

\begin{tabular}{llllll}
\hline & & All patients $(\mathrm{n}=200)$ & Men $(\mathrm{n}=114)$ & Women $(\mathrm{n}=86)$ & $\mathrm{p}$ \\
\hline $\mathrm{SVCL}(\mathrm{cm})$ & Mean & $4.2 \pm 1.1$ & $4.5 \pm 1.0$ & $3.9 \pm 1.1^{\star}$ & 0.005 \\
& Range & 1.6 to 7.2 & 1.6 to 7.2 & 2.4 to 7.2 & \\
$\mathrm{CAL}(\mathrm{cm})$ & Mean & $2.4 \pm 1.1$ & $2.5 \pm 1.0$ & $2.4 \pm 1.0$ & 0.385 \\
& Range & 0.8 to 5.6 & 0.8 to 5.6 & 0.8 to 5.6 & \\
\hline
\end{tabular}

Data are expressed as mean $\pm \mathrm{SD} . \mathrm{SVCL}=\mathrm{SVC}$ length, $\mathrm{CAL}=$ the distance between the carina and the right atrium inlet. ${ }^{*} \mathrm{P}<0.05$ compared to Men.

\section{AP3-3}

Safe placement of central venous catheter tips:-an analysis N. Smart, R. Sundaram, S. Koteeswaran

Department of Anaesthesia and Pain, Victoria Infirmary, Glasgow, United Kingdom

Background and Goal of Study: Optimal positioning of central venous catheters (CVC) is contentious. While recommendations for correct CVC placement usually include placing the tip of the catheter in the SVC, above the atrium, and parallel to the vessel wall, numerous publications draw attention to the association between catheter related thrombosis and proximal positioning $(1,2)$. The purpose of this study was to identify catheter tip position on post procedure radiograph and determine if the guideline requirements for safe placement had been met.

Materials and Methods: Over a 6 month period, 134 consecutive intensive care patients with $139 \mathrm{CVCs}$ (internal jugular or subclavian) were included. Using the carina as a surrogate marker for the pericardial reflection, tip position was identified independently by both an anaesthetist and radiologist. The angle of the tip to the vertical was used as a proxy for the angle of incidence with the superior vena cava. Finally, the original radiology report was compared with each finding. Results calculated as percentages. 
Results and Discussions: Catheter placement below the carina was common with 50(35.9\%) right sided and $8(5.7 \%)$ left sided so sited. All right sided catheters above the carina had a shallow angle of incidence $\left(<30^{\circ}\right)$ to the vertical whereas more than $75 \%$ (24) of left sided lines had a steep angle of incidence $\left(>30^{\circ}\right)$. Differences in perception of tip position were common with $25(18 \%)$ of radiology reports failing to match study findings. The SVC is relatively short and patient rotation about the axial plane associated with the use of semi-erect and kyphotic projections may explain this apparent anomaly (3). Conclusions: This study demonstrates a high proportion of CVCs suboptimally positioned, despite current guidelines. However, for left sided catheters, these results also suggest that satisfying all criteria for safe placement is difficult.

References:

1 Cadman AClin Radiol 2004; 54: 349-55.

2 Fletcher S, Bodenham A. BJA 2000; 85: 188-91.

3 Sundaram R, Koteeswaran S, Smart N.BJA 2006; 97 : 574

\section{AP3-4}

\section{Risk mortality in surgical patients: two years analysis}

J. Gil Bona, A. Sabaté, R. Adroer, E. Jaurrieta

\section{Anestesiology, Bellvitge Hospital, Hospitalet de Llobregat, Spain}

Objective: Risk mortality factors for surgical patients in a university tertiary hospital

Material and Method: We performed a cross-sectional study. Data from surgical death patients at the first 30 days after surgery for two years (2004-05) period were collected. Pre, intra and postoperative data were analysed. A bivariate and multivariate analysis was performed relating death cause with pre and postoperative observations. Chi square of Pearson and regression analysis were made.

Results: 4559 and 5044 patients under ambulatory surgery for 2004-05 period were excluded of this study.

\begin{tabular}{llllll}
\hline Year & \multicolumn{2}{l}{ Number of operations } & & & Number of deaths \\
\cline { 2 - 3 } \cline { 5 - 6 } & Emergency & Elective & & Emergency & Elective \\
\hline 2004 & 1883 & 10489 & & 105 & 58 \\
2005 & 1998 & 10500 & & 107 & 30 \\
\hline
\end{tabular}

Mortality-relationship with preoperative risk factors, only arterial hypertension, diabetes mellitus and cancer diagnosis had signification with mortality in the postoperative period.

Age was related to associated diseases, need for ICU, and death at the first 48h. Men have a tendency to toxics habits and kidney diseases as preoperative risk factors, while women have it to neurological and psychical diseases.

Patients with cancer or hepatics diseases need more for ICU. The most aggressive surgery has more intra and postoperative complications, including sepsis. To admit in ICU is an independent risk factor for sepsis and death for sepsis.

Cardiac death (ischemic events, heart failure, arrhythmias) was related with reoperations, postoperative sepsis, and postoperative bleeding). Sepsis related death was associated to admittance in ICU and reoperations. Respiratory death was related to bronco aspiration, pneumonia, and acute respiratory failure. Neurological death was related with stroke.

Conclusion(s): Surgical patients continue to account for a large number of dead in the hospitals.

A more exhaustive control of cardiac complications and sepsis should improve the outcome. More studies should be necessaries to confirm if admission of these patients in ICU could meliorate results.

\section{AP3-5}

\section{Family member presence during induction of anesthesia in} elderly patients - a feasibility study

A. Perel, Y. Mishuk, I. Matot

Anesthesiology and Intensive Care, Sheba Medical Center, Tel Aviv University, Israel, Tel Aviv, Israel

Background and Goal of Study: Elderly patients who undergo anesthesia often present with varying degrees of dementia, disorientation and sensory impairment which may cause communication problems and increase anxiety before anesthesia. We have therefore explored the feasibility of the presence of a family member (FM) during induction of anesthesia in elderly patients by recording its acceptability and its effects on the patient, the FM and the staff.

Materials and Methods: Patients $>70$ years old who were escorted by a FM on arrival to the OR were included in the study. Following informed consent, the FM's escorted the patients into the OR, and stayed throughout the induction period. Separate questionnaires were filled by patients and FM's before and after anesthesia, and by the anesthesiologist and OR nurse postoperatively.

Results and Discussions: Out of the 33 (out of $40,82.5 \%$ ) that gave consent (age $76.8 \pm 4.9$, range $70-89$ ), $67 \%$ spoke Hebrew well, and $21 \%, 70 \%$ and $88 \%$ reported symptoms of mental, hearing and sight impairments, respectively. Their reported anxiety from anesthesia (VAS score $10=$ not anxious; $0=$ very anxious) was $6.7 \pm 3.4$ pre- and $8.8 \pm 2.2$ post-operatively $(\mathrm{p}<.006)$. FM's overestimated patients' anxiety both pre- $(5.4 \pm 3.3, p<.05)$ and postanesthesia $(6.0 \pm 3.2, p<.0001)$. Anesthesiologists and nurses also overestimated the patient's anxiety from anesthesia $(7.1 \pm 2.6, p<.001,7.0 \pm 2.7$, $\mathrm{p}<.002$, respectively). Patients had 21 general, 10 regional and 2 combined anesthetic procedures. IV lines were inserted in 19 and regional anesthesia performed in 5 patients in the FM's presence. Post- operatively, 85\% of the patients reported that the FM's presence improved their feeling in the OR and $76 \%$ would like to be escorted again if needed. Of the FM's, $97 \%$ enjoyed the experience, $91 \%$ felt that their presence was helpful, and $85 \%$ would prefer to do it again if needed. Of the anesthesiologists and the nurses $44 \%$ and $79 \%$, respectively, thought that the FM presence was helpful, and $50 \%$ and $18 \%$, respectively, that it made no change.

Conclusions: The presence of family member during induction of anesthesia in elderly patients reduces patients' anxiety, is well accepted by patients, FM's, and most of the OR staff, and should be considered as an integral part of the anesthetic management of elderly patients.

\section{AP3-6}

\section{Unexpected postoperative hypoxia during patients transfer} from operating theatre to the recovery room

A. Dylczyk-Sommer, M. Sidorowicz, M. Wujtewicz, A. Piankowski,

R. Skowroñski

Anaesthesiology and Intensive Therapy, Medical University of Gdañsk, Gdañsk, Poland

Background and Goal of Study: Transport of postoperative patients from the operating theatre $(\mathrm{OP})$ to the recovery room $(\mathrm{RR})$ without any monitoring and oxygenation $(\mathrm{O})$ may lead to the unexpected ventilatory disturbances.

Materials and Methods: 90 ASA II-III patients, mean age 58.8 ( \pm 15.3$)$ years, after general anaesthesia were studied during transfer from OT to the RR. During the transport no oxygen and monitoring were used. Last $\mathrm{SpO}_{2}$ measurement in the OT and the first in the RR were estimated.To evaluate the breathing improvement while oxygenation the acid-based balance (ABB) were performed 10 minutes after admission to the RR. Mean postoperative times of: T1 - transfer , T2 - without monitoring, T3 - without oxygenation were measured.

Results and Discussions: Mean $\mathrm{SpO}_{2}$ in the OT was $96.9 \%( \pm 10.55)$, in the $R R-94 \%( \pm 6.35)$.

After 10 min of oxygenation in the ABB test acidosis was observed with mean values: $\mathrm{pH} 7.31( \pm 0.054), \mathrm{pCO}_{2} 48.01( \pm 6.39) \mathrm{mmHg}$, the $\mathrm{pO}_{2}$ was normal $-126( \pm 44.45) \mathrm{mmHg}$. The mean T values were: $\mathrm{T} 1-90( \pm 94.2) \mathrm{s}$, T2 - $152.63( \pm 86.63) \mathrm{s}, \mathrm{T} 3-122.9( \pm 86.8) \mathrm{s}$.

Conclusion(s): 1. Although transfer time takes only about $90 \mathrm{~s}$, decrease in $\mathrm{SpO}_{2}$ was observed in every patient. 2. The transfer time takes $90 \mathrm{~s}$, but the time without monitoring and oxygenation is longer. 3 . In $73 \%$ of studied patients after $10 \mathrm{~min}$ of oxygenation there was acidosis with hypercapnia observed in the ABB. 4. Right after admission to the RR hipoksemia was observed in every patient, but after 10 min oxygenation the $\mathrm{pO}_{2}$ was normal. 5. After general anaesthesia patient should be oxygenated during the transfer because of hypoxemia and admitted to the RR because of the risk of hypoventilation.

\section{AP3-7}

Automated anesthesia medication dispensing systems: single-issue, limited-access drawers may reduce medication errors

L. Cooper, M. Barron, C. Gallagher, J. Sciarra

Anesthesiology, University of Miami Miller School of Medicine, Miami, USA

Background and Goal of Study: Although automated medication dispensing systems (AMDS) have been shown to reduce medication errors in the nursing environment, the same is not true in the anesthesia environment. Previous reports have suggested that anesthesia medication error is not eliminated with AMDS. However, due to lack of pharmacist review prior to administration of anesthetics, and the element of human error that exists in the anesthesia realm, AMDS have gained widespread use throughout the United States. We present the first report that suggests a reduction in anesthesia medication error when an AMDS is used. 
Materials and Methods: Following a medication error that occurred when a look-alike vial of norepinephrine (norepi) was mistaken for a vial of dexamethasone, a root-cause analysis was performed. This error occurred despite original configuration of the AMDS drawers with no two vials similar in appearance being located in close proximity. An in-depth review showed that the wholesaler did not have the usual vials of norepi in stock and substituted the same drug in the same concentration in a different vial, which was nearly identical to our stock of dexamethasone. Both of these drugs were located in close proximity to one another in the AMDS drawers. A reconfiguration of the drawers was undertaken by pharmacy and anesthesiology, noting the extreme danger of undiluted norepi and redefining it as a "non-emergency" drug.
Norepi was placed in a single-issue, limited-access mini-drawer, thus preventing an anesthesia provider from obtaining the drug inadvertently, and limiting the access to only the specific drug requested.

Results and Discussions: After reconfiguration of the AMDS drawers, there have been no further medication incidents in over 2 years with norepi, or any drug that was assigned and controlled via the single-issue, limited-access drawers.

Conclusion(s): A simple reconfiguration of the AMDS, by locating high-risk and high-alert medications in single-issue, limited-access drawers, may result in reduced medication errors. Further prospective trials should be undertaken to confirm this suggestion.

\section{EAMS (European Airway Management Society) - Airway Management}

\section{AP1-1}

\section{Comparison of three video laryngoscopes with Macintosh} laryngoscope in simulated difficult intubation: a manikin study M. Sandby-Thomas, E. Duff, I. Hodzovic

Anaesthesia and Intensive Care, Wales College of Medicine, Cardiff, United Kingdom

Background and Goal of Study: Tracheal intubation is difficult in 1\% - 4\% of patients who have seemingly normal airways [1]. The GlideScope (GS), Fibreoptic Macintosh (FM), and McGrath (MG) video laryngoscopes are designed to assist with difficult intubation. Previous studies comparing GlideScope and Macintosh laryngoscope were inconclusive [2, 3]. We used an airway manikin to compare the three video laryngoscopes with a standard Macintosh (Mac) laryngoscope.

Materials and Methods: Fifty two anaesthetists were invited to take part in this randomized crossover study. After standardized practice, anaesthetists were asked to place a tracheal introducer in the trachea of a manikin with a grade 3 laryngoscopy view, using each of the four laryngoscopes. Our outcome parameters were time to and success rate of tracheal introducer placement (TIP), vertical force exerted during laryngoscopy and preference in an airway emergency.

Results and Discussions: Study findings are presented in the table (Mean (SD) and number (proportion)). In an unexpected difficult airway $63 \%$ of participants stated a preference for the Fibreoptic Macintosh laryngoscope $(p<0.0001)$

\begin{tabular}{llllll}
\hline & Mac & GS & MG & FM & p value \\
\hline TIP Time (s) & $42(29)$ & $55(43)$ & $51(33)$ & $32(32)$ & $0.001^{1}$ \\
TIP Success & $46 / 52(88 \%)$ & $45 / 52(86 \%)$ & $36 / 52(69 \%)$ & $51 / 52(98 \%)$ & $0.0003^{2}$ \\
Force (N) & $3.9(1.5)$ & $3.5(1.7)$ & $3.2(1.7)$ & $2.9(1.7)$ & $0.0003^{1}$ \\
\hline
\end{tabular}

${ }^{1}$ Repeated measures ANOVA; ${ }^{2}$ Cochran's $Q$ test

Conclusion(s): The Fibreoptic Macintosh laryngoscope had a significantly shorter time and higher success rate for tracheal introducer placement than any of the three laryngoscopes. Further clinical studies are necessary to evaluate its role in the management of patients with difficult airways.

References:

1 Benumof JL Anesthesiology 1991; 75: 1087-110.

2 Sun DA et al. BJA 2005; 94: 381-4.

3 Lim TJ, Lim Y, Liu EH. Anaesthesia 2005; 60: 180-3.

\section{AP1-2}

Clinical Evaluation of a New Video Laryngoscope (Airwayscope) for Tracheal Intubation

H. Saijo, S. Sugino, N. Kanaya, H. Watanabe, A. Namiki

Department of Anesthesiology, Sapporo Medical University School of Medicine, Sapporo, Japan

Background and Goals: A new video laryngoscope (Airwayscope ${ }^{\mathrm{TM}}$, PENTAX ${ }^{T M}$ CO, Japan) (AWS) has recently been developed. The AWS is designed to provide a view of the glottis without requiring alignment of the oral, pharyngeal, and tracheal axes. Although this novel device may have advantages over conventional direct laryngoscopes (DL), its performance in a clinical setting has not been elucidated. The aim of this study was to compare the performance of an AWS and that of a DL for tracheal intubation.

Materials and Methods: After approval of the protocol of the present study by the Ethics Committee of our institution and obtaining informed consent, 36 patients (ASA I, II) who were scheduled to undergo surgery were randomly assigned to an AWS group $(n=18)$ or a DL group $(n=18)$. After general anesthesia had been induced with propofol and vecuronium IV, the lungs were ventilated with $100 \%$ oxygen and $5 \%$ sevoflurane via a facemask. All tracheal intubations were performed after ventilating for five minute. Number of attempts, time to successful intubation, and the changes in hemodynamics after $1 \mathrm{~min}$ of intubation were measured. Cormack grades, the use of sniffing position, and the use of the BURP maneuver were also evaluated. The differences were analyzed using unpaired Student t-tests and chi-square tests. $P$ values of $<0.05$ were considered statistically significant.

Results: Data (means $\pm \mathrm{SD}$ ) are shown in the table:

\begin{tabular}{lcc}
\hline & AWS group $(\mathrm{n}=18)$ & DL group $(\mathrm{n}=18)$ \\
\hline Attempts & $1.4 \pm 0.8$ & $1.3 \pm 0.5$ \\
Time $(\mathrm{sec})$ & $40.8 \pm 24.5$ & $35.1 \pm 12.3$ \\
Increase of BP $(\%)$ & $140 \pm 30.8^{\star}$ & $161 \pm 34.0$ \\
Increase of HR (\%) & $115 \pm 14.0^{\star}$ & $136 \pm 31.1$ \\
Cormack & $1.2 \pm 0.5$ & $1.4 \pm 0.5$ \\
Sniffing & $2 / 18^{\star}$ & $10 / 18$ \\
BURP & $0 / 18^{\star}$ & $8 / 18$ \\
\hline
\end{tabular}

${ }^{\star} P<0.05$ vs. LS group.

Conclusions: The AWS is less invasive than a conventional DL for tracheal intubation in clinical situations.

\section{AP1-3}

Anesthetic implications of Morquio syndrome: impossible intubation though fiberoptic endoscopy

J. Mourão, E. Chow, J. Tavares

\section{Anestesiologia, Hospital São João, Porto, Portugal}

Background: Morquio syndrome is a subtype of the mucopolysacharidoses characterized by the intracellular accumulation of keratin sulphate. The prevalence of this disease is 1:100000. Infiltration of tissues leading to distortion of upper airway anatomy with difficult or impossible endotraqueal intubation, infiltration of the cervical spine with odontoid hipoplasia places these patients at risk of atlantoaxial subluxation.

Case Report: A 16 years old, 22-kg girl, with Morquio syndrome was propose for a bilateral cornea transplant. Physical examination revealed broadened face, short and wide nose, macroglossia amygdaline hypertrophy, short neck, and Mallampati III classification. Ten years before the patient was submitted to general anesthesia for implantofix implantation and due to postoperative respiratory difficulties was transfer to an intensive care unit. Inhalation induction was performed, but assisted ventilation was ineffective. Intubation with a bronchofiberscope was attempted, but also failed due to mucous membrane hypertrophy. Thus, intubation with a laryngeal mask was performed with adequate ventilation. Maintenance and recovery of anaesthesia were unremarkable.

Clinical Implications: Spontaneous respiration is recommended in the induction of general anesthesia until the patient is intube, as airway anomalies, bleeding and salivation may make intubation extremely difficult. To prevent cervical spine movement we choose intubate the patient with a bronchofiberscope. The exuberant hypertrophy of the oropharynx mucous membrane and of the hipopharynx of this patient prevented the visualization of the laryngeal structures, which made intubation impossible. Confronted with the need to ventilate patient, it was then advanced to the intubation with a laryngeal mask with success.

Conclusion: The laryngeal mask proved to be an efficient gadget in the patency of the airway where the fiberoptic endoscopy attempted by an expert did not permit intubation.

References:

1 Tobias JD. J Clin Anesth 1999; 11(3): 242-6.

2 Moya J, Tortosa JA, Ros M, Hernandez-Palazon J, Sanchez-Ortega JL, Castano MI. Rev Esp Anestesiol Reanim 1996 May; 43(5): 189-90. 


\section{AP1-4}

\section{Prospective clinical trial comparing Airway Scope with Macintosh laryngoscope in $\mathbf{3 0 6}$ patients}

J. Koyama, K. Hongo

Neurosurgery, Shinshu University School of Medicine, Matsumoto, Japan Background and Goal of Study: The AirWay Scope was developed expecting to alleviate the difficult intubation, and to reduce the invasiveness for patients (1). The purpose of this study was to describe basic usability of the AirWay Scope in comparison with Macintosh laryngoscope for tracheal intubation.

Materials and Methods: Three hundred and six patients were randomly assigned to intubation using Macintosh laryngoscope (DLS, $n=153$ ) or intubation using the AirWay Scope (AWS, $n=153$ ). A Cormack and Lehane (C\&L) grade, time taken for intubation ( $\mathrm{TI})$, optional techniques necessary to intubate and complications was recorded.

Results and Discussion: Concerning C\&L grade, 149 patients (97.4\%) showed grade 1, and remaining 4 patients (2.6\%) in grade 2 in the AWS group. This is significantly low in compared with 89 patients $(58.2 \%)$ in grade 1, 59 patients $(38.5 \%)$ in grade 2 , and remaining 5 patients $(3.3 \%)$ in grade 3 in the DLS group ( $p<0.0001)$. TTI was $28.9 \pm 15.7 \mathrm{~s}$ in the AWS group. It was significantly longer than $21.6 \pm 9.4 \mathrm{~s}$ in the DLS group for $7.3 \mathrm{~s}(\mathrm{p}<0.0001)$. Optional techniques such as neck extension and BURP maneuver were necessary less frequently in the AWS group significantly $(p=0.0056$ and $\mathrm{p}<0.0001)$.

Conclusion: The AirWay Scope provided a better laryngeal view than that of direct laryngoscopy with few opportunities for optional techniques. Although it took an additional $7.3 \mathrm{~s}$ to intubate the trachea, the AirWay Scope is the device having equal to or higher potential than the Macintosh laryngoscope for ordinary and difficult tracheal intubation.

Reference:

1 Koyama J. J Neurosurg Anesthesiol 2006; 18: 247-50.

\section{AP1-5}

\section{Evaluation of airways in patients with Benign Symmetrical} Lipomatosis of the neck

S. Sandru, V. Topalo, B. Pirgari, S. Cobiletchi, R. Baltaga

Anesthesiology Reanimatology, State Medical and Pharmaceutical

University, Chisinau, Republic of Moldova

Background and Goal of Study: Possible anaestheszologic problems related to Benign Symmetrical Lipomatosis of the Neck (BSLN) - Syndrome Madelung Lanois and Bensaude are difficult airways, insufficiency of peripheral veins, polyneuropathy and delayed drug metabolisation, and related to this delayed weaning. The goal of our study was to describe particularities of anaesthesiology assistance in patients with BSLN in comparison to general population. Materials and Methods: Were investigated 81 patients: 51 with BSLN and 30 surgical patients non-BSLN from general population (control) comparable for age, weight, duration of anesthesia (DA). Was compared risk of difficult airways: Mallampaty, Wilson, Bellhouse-Dore, spyrography -VC and $\mathrm{FEV}_{1}$, recovery of spontaneous respiration (RSR) and time of extubation (TE). In both group was performed TIVA.

Results and Discussions:

\begin{tabular}{|c|c|c|}
\hline Criteria & $\begin{array}{l}\text { BSLN } \\
(\mathrm{n}=51)\end{array}$ & $\begin{array}{l}\text { Control } \\
(n=30)\end{array}$ \\
\hline Age, years & $47 \pm 0.9$ & $46 \pm 0.8$ \\
\hline $\mathrm{BW}, \mathrm{kg}$ & $78 \pm 0.4$ & $77 \pm 0.5$ \\
\hline Mallampati I & $6(12 \%)$ & 12 p-nts (40\%) \\
\hline Mallampati II & 24 p-nts (47\%) & 11 p-nts (37\%) \\
\hline Mallampati III & 18 p-nts (35\%) & 6 p-nts $(20 \%)$ \\
\hline Mallampati IV & 3 p-nts (6\%) & $1 \mathrm{p}-\mathrm{nt}(3 \%)$ \\
\hline Wilson: 0-2 & 12 p-nts (24\%) & 16 p-nts (53\%) \\
\hline Wilson: 3-4 & 31 p-nts $(61 \%)$ & 12 p-nts (40\%) \\
\hline Wilson: 5-6 & 8 p-nts $(15 \%)$ & 2 p-nts $(7 \%)$ \\
\hline Bellhouse-Dore: I & 5 p-nts (10\%) & 13 p-nts (43\%) \\
\hline Bellhouse-Dore: II & 29 p-nts (57\%) & 15 p-nts (50\%) \\
\hline Bellhouse-Dore: III & 17 p-nts (33\%) & 2 p-nts $(7 \%)$ \\
\hline VC, \% & $80.4 \pm 4.7^{\star \star}$ & $112.5 \pm 6.2$ \\
\hline $\mathrm{FEV}_{1}$ & $64.5 \pm 4.3^{\star \star \star}$ & $88.2 \pm 5.6$ \\
\hline $\mathrm{DA}, \mathrm{h}$. & $3.1 \pm 0.3$ & $3 \pm 0.33$ \\
\hline RSR, h & $10.5 \pm 0.62^{\star \star}$ & $0.13 \pm 0.09$ \\
\hline $\mathrm{ET}, \mathrm{h}$. & $2.75 \pm 0.86^{*}$ & $0.65 \pm 0.31$ \\
\hline
\end{tabular}

Conclusions: Patients with BSLN in comparison to general population present higher risk of difficult airway, and delayed time of restoration of spontaneous respiration and extubation.

\section{AP1-6}

Postoperative Stridor resulting from paradoxical vocal cord motion

D. Varveris, F. Henderson, NG. Smart

Department of Anaesthesia, Southern General Hospital, Glasgow, United Kingdom

Background and Goal of Study: Upper airway obstruction following general anaesthesia requires prompt diagnosis and treatment. We report a case of stridor following laryngeal mask anaesthesia caused by paradoxical vocal cord motion(PVCM). PVCM is very rare following anaesthesia and all previous reported cases occurred after endotracheal intubation (1).

Materials and Methods: A case report

Results: A 44 year old woman presented for PIP joint replacement. Past history included asthma, psoriatic arthropathy, and breathing difficulties following a previous anaesthetic which had been diagnosed as hyperventilation. A regional block was declined. After an uneventful general anaesthesic, she had no respiratory difficulty for the first 45 minutes and then started retching. Immediately thereafter, she developed difficulty in breathing, inspiratory stridor, and increasing anxiety. Nebulised salbutamol $5 \mathrm{mg}$, nebulised epinephrine $1 \mathrm{mg}$, hydrocortisone $100 \mathrm{mg}$, and midazolam $5 \mathrm{mg}$ all failed to improve the stridor. Although tachycardic $(120 \mathrm{bpm}), \mathrm{S}_{\mathrm{p}} \mathrm{O}_{2}$ and arterial blood gases remained within normal limits, and there was no hypotension, rash or bronchospasm. An ENT consult was obtained and a decision to proceed to endoscopy made. On passing the scope through the nostril, stridor disappeared. Pharynx and larynx were normal with no blood, edema or trauma evident. Recovery was thereafter uneventful, the whole episode lasting 2 hours. Conclusions: Resulting from intrinsic laryngeal muscle dysfunction, PVCM is more common in females and may be associated with anxiety states. As a cause of postoperative stridor, it should be considered only after elimination of life threatening conditions such as airway or pulmonary edema, foreign body aspiration, and bronchospasm. Diagnosis is by laryngoscopic evidence of vocal cord adduction in inspiration and abduction in expiration. Management should concentrate on ensuring oxygenation. Sedation with benzodiazepines (2) and reassurance can be successful but CPAP, bag and mask ventilation, or tracheal intubation may be required.

References:

1 Arndt $\mathrm{G}$ et al.Can J Anaes 1996; 43: 249-51.

2 Robert K et al. Anesthesiol 1998; 89: 517-9.

\section{AP1-8}

A comparison of hemodynamic response during anesthetic induction between AirWay Scope ${ }^{\mathrm{TM}}$ and Macintosh laryngoscope

H. Tokoroyama, T. Yokoyama, R. Yokoyama, K. Yamashita, M. Manabe Department of Anesthesiology and Critical Care Medicine, Kochi Medical School, Nankoku, Japan

Background and Goal of Study: AirWay Scope ${ }^{\mathrm{TM}}$ (AWS, Pentax Co. Tokyo) is a newly developed fiberoptic laryngoscope with fiber optic light source and a rigid disposable polycarbonate blade (INTLOCK). This device does not require direct laryngoscopy, thus it may be less effective on hemodynamic response. In this study, we examined the effect of orotracheal intubation using AWS on the hemodynamic response and compared to Macintosh laryngoscope.

Materials and Methods: After institutional approval and written consent, 20 patients undergoing elective surgery were randomly assigned to receive orotracheal intubation with the AWS or the Macintosh laryngoscope. Anesthesia was induced with fentanyl $2 \mu \mathrm{g} / \mathrm{kg}$ and Midazolam $0.1 \mathrm{mg} / \mathrm{kg}$, and lung were ventilated manually with $2 \%$ sevoflurane in oxygen. Vecuronium $0.1 \mathrm{mg} / \mathrm{kg}$ was administered when BIS decreased to 60 . Endotracheal intubation was performed after muscular relaxation. Heart rate and blood pressure were measured continuously during induction, and blood samples were collected before and 2 min after the orotracheal intubation.

Results and Discussions: There were no significant differences in patients' background between the AWS group and the Macintosh group. Systolic BP decreased to $73.1 \%$ (AWS) and $73.7 \%$ (Macintosh) to the baseline before intubation, and recovered to $96.1 \%$ (AWS) and $97.0 \%$ (Macintosh) after intubation. Orotracheal intubation with the AWS required $31 \pm 22$ seconds for intubation, while the Macintosh laryngoscope required $23 \pm 7$ seconds. Serum noradrenaline after intubation was $196 \pm 87 \mathrm{pg} / \mathrm{ml}$ (AWS) and $192 \pm 34 \mathrm{pg} / \mathrm{ml}$ (Macintosh). There were no significant differences in HR, BP, intubation time and serum noradrenaline concentration.

Conclusion: The AWS has a wide monitor with a good view and easy to operate, but the hemodynamic response to orotracheal intubation was not significantly different from that with the Macintosh laryngoscope. 


\section{AP1-9}

The temperature if laryngoscope light bulbs compared to light emitting diodes

E. Lewis, T.E. Sheraton, M.A. Hampson, A.R. Wilkes, J.E. Hall

Department of Anaesthetics, University Hospital of Wales, Cardiff, United Kingdom

Background and Goal of Study: Oropharyngeal burns due to laryngoscope light bulbs reaching high temperatures have been documented. ${ }^{1}$ This is a particular hazard in neonates where the thin immature surface mucosa is at increased risk. Light emitting diodes (LEDs) are thought to emit less heat. The aim of this study was to measure the temperature of incandescent laryngoscope bulbs, currently used in the UK in laryngoscope blades, and an LED in a custom-made laryngoscope blade.

Materials and Methods: The temperature of a xenon bulb (A), a vacuum bulb (B) and an LED (C) contained in different laryngoscopes blades was measured using a K-type thermocouple under standardized conditions. The current used by the laryngoscopes was also documented.

Results and Discussions: The temperature of the LED was much lower than either of the incandescent bulbs. The current recorded for the LED was $40 \mathrm{~mA}$ compared to that recorded for the incandescent bulbs at $750 \mathrm{~mA}$. The lower temperature of the LED bulb will reduce the risk of oropharyngeal burns particularly if laryngoscopy is prolonged. The much lower current requirements of the LED reflect its greater efficiency with less energy being lost as heat.

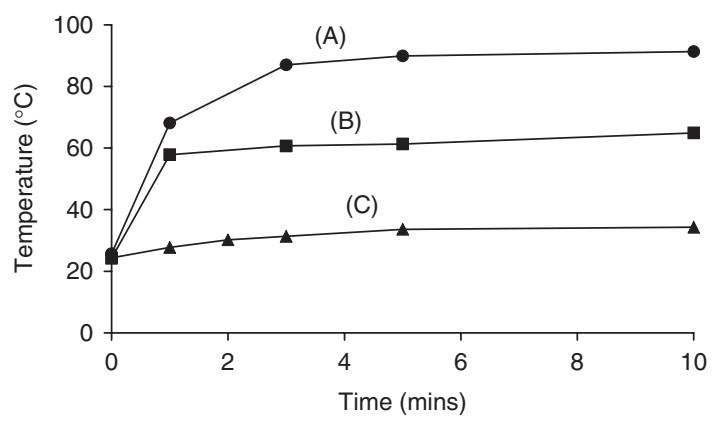

Conclusion(s): The LED bulb is less likely to cause thermal injury at laryngoscopy. This may be particularly important in neonates or where difficult and prolonged laryngoscopy is anticipated.

Reference:

1 Koh THHG, Coleman R. Anesthesiology 2000: 92; 277.

\section{AP1-10}

Radiographic evaluation of cervical spine motion during airway management comparing flexible bronchoscopy (FB) with the Lo-Pro GlideScope (LP-G)

A. Prabhu, D. Wong, E. Massicotte, G. Tan, R. Cooper Department of Anesthesia, Toronto Western Hospital, University Health Network, University of Toronto, Toronto, Canada

Background and Goal of Study: It is essential to prevent or minimize cervical spine movement in patients with c-spine injury. Fibreoptic bronchospcopy (FB) is widely regarded as the gold standard for intubating patients with airway instability. The Lo-Pro Glidescope (LP-G) is a new improved video laryngoscope and may be associated with less c-spine movement. We aimed to compare c-spine movement during intubation with FB versus LP-G.

Materials and Methods: After ethics board approval 28 consenting adults with no cervical spine abnormality were randomized to be intubated with either a FB or LP-G. Demographic data was collected. During intubation continuous video fluroscopy of the cervical spine was performed. A neuroradiologist and a spine surgeon assessed c-spine movement independently. The primary endpoint was maximum angulations during the intubation sequence (Occ- $\mathrm{C}$, C1-2, C2-4, and C4-5). Power of the study was determined by considering a 1-degree change in angulations as significant. Mann Whitney $U$ test was used to compare maximum range of motion and Wilcoxin signed rank test for interobserver differences.

Results and Discussions: No significant differences between FB and the LP-G groups in demographic data. Intubation times were comparable between LP-G $(31 \pm 2 \mathrm{sec})$ and FB $(31 \pm 3$ secs $)$.
Maximum angulation during intubation (measured by radiologist)

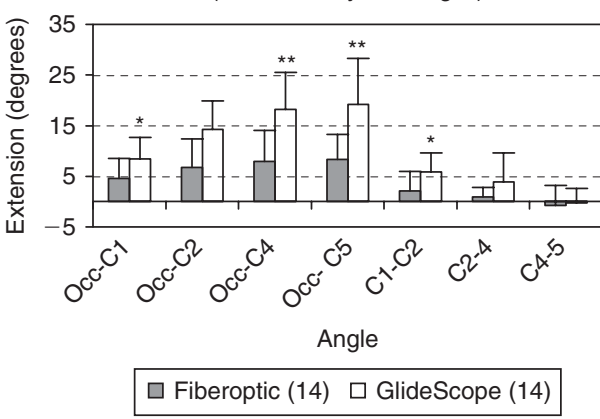

Max. change mean $\pm \mathrm{SD},{ }^{\star} \mathrm{p}<0.05,{ }^{\star \star} \mathrm{p}<0.01$

Conclusion(s): The Lo-Pro Glidescope has significantly greater movement that the Fibreoptic bronchoscopy but this study has shown that there is some movement associated even with this technique.

References:

1 Turkstra TP. Crane RA. Pelz DM. Gelb AW. Anesth Analg. 2005: 101: 910-5.

2 Cooper RM. Can J Anesth 2003; 50: 611-3.

\section{AP2-1}

Preoperative Lornoxicam attenuates the haemodynamic response to intubation in elderly

W.R. Soliman, A.A. Moussa, M.A. Nassef

Anesthesia, King Khaled Eye Specialist Hospital, Riyadh, Saudi Arabia

Background and Goal of Study: Lornoxicam is a novel non-steroidal antiinflammatory drug (NSAID) with marked analgesic properties. Laryngoscopy and endotracheal intubation cause a reflex increase in sympathetic activity that results in hypertension, tachycardia, arrhythmia and may cause myocardial ischemia in susceptible patients. Minimizing perioperative adverse events especially in elderly patients is of utmost importance. The aim of this study is to demonstrate the effect of preoperative administration of Lornoxicam on haemodynamic changes during laryngoscopy and tracheal intubation in elderly. Materials and Methods: After obtaining approval from the Hospital Ethics Committee and patients' informed consent, 50 elderly patients, aged between 65-75 year, ASA class I and II enrolled in this study. All patients received no premedication. Patients randomly assigned into two groups using sealed enveloped technique, to received either Lornoxicam $8 \mathrm{mg}$ or placebo in $5 \mathrm{ml}$ covered syringe half hour before surgery. anaesthesia was induced with fentanyl, propofol and atracurium to facilitate tracheal intubation. Systolic, diastolic, mean arterial blood pressure and heart rate were recorded before and after administration of the intravenous anaesthetic, also at 1, 3, 5 and $10 \mathrm{~min}$ after intubation.

Results and Discussions: Demographic and clinical characteristics of the study patients were statistically similar between the two treatment groups. In the control group, a significant increase in the heart rate, systolic, diastolic and mean blood pressure were observed $1,3,5$ and $10 \mathrm{~min}$ following intubation ( $P$ value $<0.05$ ).

Conclusion(s): Preoperative administration of Lornoxicam attenuates the haemodynamic response to laryngoscopy and endotracheal intubation in elderly population.

\section{AP2-2}

\section{Effects of low dose ketamine on intubation conditions with} rocuronium

P. Topcuoglu, S. Uzun, O. Canbay, G. Pamuk, S. Ozgen

Anaesthesiology and Reanimation Department, Hacettepe University Faculty of Medicine, Ankara, Turkey

Background and Goal of Study: Ketamine due to its sympatomimetic effects would reduce the onset time of rocuronium and would be beneficial for better intubation conditions $(1,2)$. This study investigated the effects of low dose ketamine and priming on onset time of rocuronium and intubation conditions. Materials and Methods: After ethics committee approval and informed consent, thi prospective, randomized, double blind study was planned. ASA I-II, 120 patients were recruited for the study and they were randomly allocated into four groups (Priming, Ketamine-priming, Ketamine and Control groups) and anaesthesia was induced with $2.5 \mathrm{mg} \cdot \mathrm{kg}^{-1}$ propofol. Additional ketamine dose was $0.5 \mathrm{mg} \cdot \mathrm{kg}^{-1}$. Rocuronium at a priming dose $0.06 \mathrm{mg} \cdot \mathrm{kg}^{-1}$ followed by $0.54 \mathrm{mg} \cdot \mathrm{kg}^{-1}$ after $2 \mathrm{~min}$ was used. In Ketamine and Control groups, 
rocuronium dose was $0,6 \mathrm{mg} \cdot \mathrm{kg}^{-1} . \mathrm{S} / 5 \mathrm{M}-\mathrm{NMT}$ neuromuscular transmission module (Datex-Ohmeda, Madison, WI) was used for neuromuscular transmission monitorisation. One-way Annova, Kruskal-Wallis, Mann-Whitney U and Bonferroni tests were used for statistical analysis. The results are given as percentages, $95 \%$ confidence interval.

Results and Discussions: In groups with ketamine, intubation conditions were better compared to priming and control groups $\left({ }^{*} p=0.001,{ }^{* *} p=0.001\right)$ (Table 1). Onset time of rocuronium in ketamine receiving groups was not shorter after post-hoc correction (ketamine; $162.7 \mathrm{sec}$, ketamine-priming; $168.8 \mathrm{sec}$, priming; 212.8 second control; $216.8 \mathrm{sec})(p>0.05)$. Priming hadn't any effects on intubation conditions and on onset time $(p>0.05)$.

Table 1

\begin{tabular}{llll}
\hline & Excellent & Good & Poor \\
\hline Ketamine & $14^{\star}$ & 15 & $1^{\star \star}$ \\
Priming & 9 & 11 & 10 \\
Ketamine-priming & $17^{\star}$ & 10 & $3^{\star \star}$ \\
Control & 6 & 10 & 14 \\
\hline
\end{tabular}

Conclusion(s): Addition of low dose ketamine $\left(0.5 \mathrm{mg} \cdot \mathrm{kg}^{-1}\right)$ with or without priming ameliorated the intubation conditions. It can be used in cases where smooth intubation is desired.

References:

1 Leykin Y, Pellis T, Lucca M, Gullo A. Acta Anaesthesiol Scand 2005; 49: 792-97.

2 Viby_Mogensen J, Engbaek J, Eriksson LI et al. Acta Anaesthesiol Scand 1996; 40: 59-74.

\section{AP2-3}

\section{LMA ProSeal ${ }^{\mathrm{TM}}$ in the veterinary anaesthesia - the animal is} patient as well

M. Boldizar, K. Pelikan, D. Repel, P. Vidrickova, A. Sevcik

Clinic of Surgery, Orthopedic \& Radiology, University of Veterinary

Medicine, Kosice, Slovakia

Background and Goal of Study: Airway management during general anaesthesia [GA] is a cardinal problem in the veterinary anaesthesia as well. Open airways can be achieved by a change of a patient's head position, a face mask [FM] or by an insertion of an endotracheal tube [ETT]. These techniques have advantages and disadvantages. The introduction of the laryngeal mask [LMA] into human anaesthesia was a revolutionary breakthrough at the end of the 20th century which solved problems connected with the FM and ETT. The first use of LMA Classic ${ }^{T M}$ in the veterinary anaesthesia dates back to 1991 and LMA ProSealTM[PLMA] to 2005.

Materials and Methods: The use of PLMA on animals $n=49$ in three species [dogs 35 , sheep 7 , swines 7] was studied. In all the animals Propofol without myorelaxants for the induction into $\mathrm{GA}$, the cuff pressure $40 \mathrm{~cm} \mathrm{H}_{2} \mathrm{O}$, lubricant jelly without a local anaesthetic were used. Ventilation time [VT] through the PLMA, the signs of the gastric reflux [R], successful PLMA insertion on the first attempt [SI] and the malposition [MP] were followed up. The proper position of PLMA cuff in the hypopharynx was held in check by X Ray. The proper position of the distal aperture of the drain tube [DT] was confirmed by insertion of a gastric tube into oesophagus via DT. Three insertion techniques recommended for human anaesthesia as well as bubble test were tested.

Results: The three insertion techniques recommended for human anaesthesia are unsuitable in a veterinary anaesthesia. The bubble test is necessary to be modified because performing a suprasternal test in animals is impossible. Statistical data are shown in the table:

\begin{tabular}{lccrcl}
\hline & $\mathrm{n}$ & $\mathrm{VT} \varnothing \mathrm{min}$ & $\mathrm{R} \%$ & $\mathrm{SI} \%$ & $\mathrm{MP} \%$ \\
\hline DOG & 35 & $76,43 \pm 45,56 \mathrm{SD}$ & 8,6 & 91,4 & 5,7 \\
SHEEP & 7 & $50,71 \pm 30,99 \mathrm{SD}$ & 71,4 & 100 & 0 \\
SWINE & 7 & $115,71 \pm 41,98 \mathrm{SD}$ & 0,0 & 100 & 0 \\
\hline
\end{tabular}

Conclusion: The use of PLMA in the veterinary anaesthetic practice significantly improves airway management in animals during GA.

\section{A92-4}

Awake nasotracheal retrograde intubation using the subcricoid region: A comparison of two techniques

S.K. Nadarajan, D. Sethuraman, M. Ravishankar

Anaesthesiology and Critical Care, Jipmer, Pondicherry, India

Background and Goal of Study: An important drawback of the retrograde tracheal intubation $(\mathrm{RI})$ is inadvertent oesophageal intubation. The subcricoid approach (1) and the use of fibrescope as antegrade intubation guide before the removal of the guidewire have been advocated to prevent oesophageal intubation (2). In this study we compared two RI techniques using the subcricoid approach.
Materials and Methods: After obtaining the hospital ethics committee's approval, 40 adult patients (ASA I-III) with anticipated difficult intubation scheduled for elective maxillofacial surgery, were recruited. Awake nasotracheal Rl using the sub-cricoid approach was performed under topical anaesthesia and sedation with the Cook's RI kit (C-retro-14.0-70-38J-110-CAE, Cook Critical Care Inc. Bloomington, IND). After subcricoid positioning of the antegrade intubation guide and the endotracheal tube (ETT), patients were randomized into two groups. In group $1(n=20)$ the intubation guide was advanced into the mid-trachea after removing the GW and the ETT was advanced over it. In group2 $(n=20)$, before removing the guidewire (GW), the intubation guide was withdrawn antegradely and passed through the ETT by the side of the GW into the trachea.

Results and Discussions: Nasotracheal intubation was successful in 38 patients (95\%). Oesophageal intubation occurred in 1 patient in each group. Achieving intubation in Group1 was easier and less time consuming although the mean time for intubation was not statistically significant between the groups (241.7 $\pm 76.4 \mathrm{sec}$ vs. $281.1 \pm 64.1$. . Complications were minor and self-limiting. Conclusion(s): We conclude that nasotracheal RI using the cook's RI kit, with the subcricoid approach is a safe and effective way of securing the airway in patients with anticipated difficult intubation. Both the retrograde techniques are simple and can be performed without significant patient discomfort under topical airway anesthesia and sedation.

References:

1 Shantha TR: Retrograde intubation using the subcricoid region. Br J Anaesth 1992; 68: 109-112.

2 Tobias R: Increased success with retrograde guide for endotracheal intubation. Anesth Analg 1983; 62: 366-367.

\section{AP2-5}

\section{Transtracheal high-frequency jet ventilation using a two-lumen central venous catheter for laryngomicrosurgery}

K. Lee, B. Choi

Department of Anesthesiology and Pain Medicine, Yonsei University

Medical College, Seoul, Republic of Korea

Background: Transtracheal jet ventilation can be used for endoscopic laryngeal surgery and patients with acute airway problems. We report a case of transtracheal high-frequency jet ventilation (HFJV) using a two-lumen central venous $(\mathrm{CV})$ catheter for laser laryngomicrosurgery (LMS) to remove a vocal cord polyp.

Case: A 33 years old female, $54 \mathrm{~kg}$ and $167 \mathrm{~cm}$, was scheduled for laser LMS to remove a polyp arising from posterior $1 / 3$ of vocal cord. Anesthesia was induced with intravenous alfentanil $10 \mu \mathrm{g} / \mathrm{kg}$, propofol $1.5 \mathrm{mg} / \mathrm{kg}$, and atracurium $0.5 \mathrm{mg} / \mathrm{kg}$, then a laryngeal mask airway (LMA) was inserted and maintained with $3 \%$ sevoflurane in $\mathrm{O}_{2}$. After confirming that the patient was ventilated appropriately at the extended neck position, a 7 Fr., $20 \mathrm{~cm}$ twolumen $\mathrm{CV}$ catheter, containing a $14 \mathrm{G}$ distal lumen and an $18 \mathrm{G}$ proximal lumen, inserted into the trachea caudad via cricothyroid membrane using Seldinger's technique. Then, $2 \mathrm{mg}$ of midazolam and $10 \mu \mathrm{g} / \mathrm{kg}$ of additive alfentanil were administered intravenously and the LMA was removed, then the surgeon confirmed that the catheter tip was positioned about $2 \mathrm{~cm}$ above the carina via a direct bronchoscope. Jet ventilation was started via the distal tubing of $\mathrm{CV}$ catheter using the following settings: rate $2 \mathrm{~Hz}, \mathrm{I} / \mathrm{E}$ ratio 0.5 , driving pressure 2.5 bar, $\mathrm{FiO}_{2}$ 0.3. A carbon-coated spatula was used by the surgeon for protection of $\mathrm{CV}$ catheter from laser beam during the surgery. The vocal polyp was removed and bleeding was controlled, and $1 \mathrm{~mL}$ of arterial blood was sampled for gas analysis. After ending of the surgery, jet ventilation was stopped, and the spontaneous respiration was recovered with ventilatory support via a face mask with $100 \% \mathrm{O}_{2}$. The $\mathrm{CV}$ catheter was removed and the patient was transported to the PACU.

Conclusion: We could supply an unobstructed and good operation field with less vibration to the surgeon by transtracheal HFJV using a two-lumen CV catheter for laser LMS to remove a vocal cord polyp. And we suggest that capnography and airway pressure monitorings during jet ventilation are possible using the proximal tubing of CV catheter, and also barotrauma can be prevented by supplying another gas outlet via the proximal tubing of $\mathrm{CV}$ catheter.

\section{AP2-6}

\section{Comparison of different doses of remifentanil for insertion of IMA using etomidate}

N. Ediz, N.M. Mutlu, M. Baydar, E. Karabulut, N. Gogus

Anesthesiology and Reanimation, Ankara Numune Training and Research Hospital, Ankara, Turkey

Background and Goal of Study: Concurrent use of opioids or succinylcholine with etomidate, the induction agent, can reduce the occurrence of airway 
reflexes and increase the success rate of LMA. We aimed to compare effectiveness of different doses of remifentanil on inserting LMA with etomidate. Materials and Methods: 120 adult patients, ASA I-II, scheduled for urologic or orthopaedic procedures under general anesthesia, were randomly allocated into four groups: remifentanil was applied at $1 \mu \mathrm{g} \mathrm{kg}^{-1}$ in group Rl, $0.75 \mu \mathrm{g} \mathrm{kg}^{-1}$ in group RII and $0.5 \mu \mathrm{g} \mathrm{kg}^{-1}$ in group RIII. Then $0.3 \mathrm{mg} \mathrm{kg}^{-1}$ etomidate was applied. In group L, after isotonic saline, $0.3 \mathrm{mg} \mathrm{kg}^{-1}$ etomidate and $1 \mathrm{mg} \mathrm{kg}^{-1}$ succinylcholine were applied. LMA was inserted by a blinded anaesthesist. All patients were assesed with a 4 point scale (1:easy, 2:moderate, 3:difficult, 4:impossible). We compared the time for LMA placement, haemodynamic changes and additional doses of remifentanil (ADR), succinylcholine (ADS), and conditions for LMA placement. Data were analysed using Annova, Krusskall-Wallis, Mann Whitney $\mathrm{U}$ and Chi-square tests, with $\mathrm{p}<0.05$ considered statistically significant (mean $\pm \mathrm{SD}$ ).

Results and Discussions: Though there was no difference in ease of LMA insertion between the groups $(p=0.065)$ LMA was inserted within the first attempt and less than 10 seconds (easy) in $90 \%$ of the patients in group RI and RII, while $82 \%$ in group RIII and $77 \%$ in group L. ADR were less needed in group $\mathrm{Rl}$ and $\mathrm{RIl}$ compared to groups $\mathrm{L}$ and RIII while, (ADS) was less needed in groups $\mathrm{RI}$ and $\mathrm{L}$ compared to group RII and RIII $(p<0.05)$. Hiccuping, involuntay muscle movements were less common in groups $\mathrm{L}, \mathrm{RI}$ and RII compared to group RIII $(p=0.011, p=0.007)$. Though significant fall in systolic blood pressure (SAP) in groups RI, RII and RIII, the mean of lowest SAP values of groups RI, RII and RIII were all above the $100 \mathrm{mmHg}$.

Conclusion(s): $1 \mu \mathrm{g} \mathrm{kg}^{-1}$ dose of remifentanil with etomidate would be a good alternative for LMA insertion with the less additional doses of succinylcholine and remifentanil.

Reference:

1 P.L. Lee Monica, Kua J.S.W., et al. Anesth Analg 2001; 93: 359-62.

\section{AP2-7}

\section{Haemodynamic response to fiberoptic nasotracheal intubation in deeply sedatet patients}

\author{
J. Balaguer, J. Martín, M. Estruch, J. Soliveres, C. Solaz
}

Anaesthesia and Critical Care, Hospital Universitario Dr Peset, Valencia, Spain

Background and Goal of Study: Fiberoptic intubation (FOB) in anaesthesia is widespread used for the difficult airway management (1). Although haemodynamic response to awake fiberoptic intubation uses to be a rise in blood pressure, haemodynamic response in deeply sedated patients is not well studied yet $(2,3)$. The objective of this paper is to study the haemodynamic response to fiberoptic nasotrachaeal intubation in the patient deeply sedated. Materials and Methods: After local ethics committee aproval, and obtaining written informed consent, 30 consecutive ASA I-III patients were enrolled in the study. Hypertense patients were excluded. Patients with basal SBP (systolic blood pressure) over $150 \mathrm{mmHg}$ also were excluded. After standard monitoring, atropine sulfate $\left(10 \mu \mathrm{gkg}^{-1}\right)$ was given i.v. and sedation was started with propofol at $6-10 \mathrm{mgkg}^{-1} \mathrm{~h}^{-1}$ until Ramsay 5 sedation score was achieved. $2 \%$ lidocaine with epinephrin $1 \%$ was used for nasal mucosa; lidocaine $2 \%(2 \mathrm{cc})$ was sprayed on the supraglotic region. Paired samples t-test was used at $5 \%$ significance level. Data shows mean \pm SD.

Results and Discussions: 9 patients were excluded because of basal SBP $>150 \mathrm{mmHg}$. 21 patients were analyzed (ASA I/II/III: 10/5/6. Height: $166.4 \pm 9.9 \mathrm{~cm}$. Weight: $81.3 \pm 20.2 \mathrm{~kg}$. Gender M/F: $12 / 9)$. Table shows basal SBP, basal diastolic blood pressure (basal DBP), mean blood pressure (basal MBP), and inmediately after FOB (post SBP, post DBP and post MBP).

\begin{tabular}{lllll}
\hline & $\mathrm{SBP}\left({ }^{*}\right)$ & $\mathrm{MAP}\left(^{*}\right)$ & $\mathrm{DBP}\left(^{*}\right)$ & $\mathrm{FC}$ \\
\hline Basal & $133,9 \pm 18,5$ & $90,5 \pm 9,3$ & $68,7 \pm 11,0$ & $87,5 \pm 13,4$ \\
Post & $115,5 \pm 18,5$ & $79,2 \pm 12,4$ & $58,6 \pm 11,1$ & $89,4 \pm 18,1$ \\
\hline
\end{tabular}

$\left.{ }^{*}\right) \mathrm{p}<0,05$

Conclusion(s): FOB under deep sedation with propofol perfusion produces a statistically significative but not clinically significant disminution in blood presure, while FC is not affected.

References:

1 Heidegger T. Curr Opin Anaesthesiol. 2004 Dec; 17(6): 483-4.

2 Xue FS. Anaesthesia 2006; 61: 444-448.

3 Machata AM. Anesth Analg 2003; 97: 904-8.

\section{AP2-8}

Utility of HFJV in management of upper airway complication after thyroid surgery - a case report

D. Janjevic, R. Jovic, D. Jevtic

Department of Anaesthesiology, Clinicaj Centre Novi SAd, Novi Sad, Serbia
Background and Goal of Study: High frequency jet ventilation (HFJV) has been an established anaesthetic technique of ventilation for laryngomicro surgery (1). Bilateral cord palsy due to lesion recurrent laryngeal nerves is a serious upper airway complication of thyroid surgery. The aim of the study was to evaluate the efficiency of HFJV during the surgical treatment of bilateral vocal cord paralysis after thyroidectomy.

Materials and Methods: We report successful use of HFJV, infraglottic technique, with the patient with serious breathing problems.

Results and Discussions: A 36 -year-old female $\left(\mathrm{BMI} 23 \mathrm{~kg} / \mathrm{m}^{2}\right)$ was admitted with noisy difficult breathing.The thyroidectomy was performed 10 months before. Preoperative examination revealed inspiratory stridor with chest wall retraction. Arterial blood gas on the room air showed $\mathrm{pH} 7,34, \mathrm{PaO}_{2}$ $71,5 \mathrm{mmHg}, \mathrm{PaCO}_{2} 39,8 \mathrm{mmHg}$ and $\mathrm{SaO}_{2} 94 \%$. Preoperative pulmonary function test showed FEV 70\%, FVC 75\%, PEF 58\%. A flow-volume loop showed a flattering of inspiratory curve.On airway evaluation, Mallampati Class II, hyoid-mental distance of $3 \mathrm{~cm}$ with no limited neck extension. Anaesthesia was induced and maintained with continuous intravenous propofol $(8-10 \mathrm{mg} / \mathrm{kg} / \mathrm{h})$, remifentanil $(0.2-0.5 \mathrm{mg} / \mathrm{kg} / \mathrm{min})$ and paralysis was obtained with rocuronium $(0.5 \mathrm{mg} / \mathrm{kg})$. After bag-and-mask ventilation with $100 \%$ oxygen the jet catheter (Accucath, Acutronic) was inserted through the vocal cords into tracheae. HFJV was applied with the commercial jet ventilator (Minstral,Acutronic Switzerland) using mix air-oxygen (40\%, 60\%), driving pressure 1,6 bar,frequency $160 / \mathrm{min}$ and inspiratory time $40 \%$. Monitoring included EKG, NIBP.SaO, $\mathrm{EtCO}_{2}$. The laryngomicrosurgical procedure was completed in $40 \mathrm{~min}$. Return to spontaneous breathing was established. Haemodynamic variables were satisfactory. The surgical access and viewing of the larynx was excellent.

Conclusion: HFJV for laryngomicro surgery, offers:optimal visibility, immobility of vocal cords, accessibility and exposure of the operation field with a patient with bilateral vocal cord paralysis after thyroidectomy.

Reference:

1 Ihra G. at all.EJA 2000; 17: 418-430.

\section{AP3-1}

\section{Airway device preference in difficult airway scenarios}

\section{N.G. Smart, S. Mohammed}

Department of Anaesthesia and Pain Management, Victoria Infirmary, Glasgow, United Kingdom

Background and Goal of Study: There are few randomized clinical trials evaluating airway devices and little consensus regarding optimal equipment use in various situations(1). The purpose of this study was to determine anaesthetist preference in difficult intubation and can't intubate can't ventilate $(\mathrm{CICV})$ situations.

Materials and Methods: Using a piloted questionnaire, 77 anaesthetists at 2 UK hospitals were asked their preferences for alternative airway devices in a difficult intubation scenario, and infraglottic airways in a CICV scenario. Previous use and comfort level in use were assessed on a 5 point Likert scale. Awareness of the Difficult Airway Society CICV guideline and previous attendance at an airway workshop were also assessed. Likert scale data were converted to a binary variable: comfortable (score 4 or 5) and uncomfortable (score 1 or 2). Equivocal data (score 3) were excluded. Results calculated as percentages and compared by Chi square analysis $(p<0.05$ significant).

Results and Discussions: Response rate was $87 \%$. In the difficult intubation scenario the preferred first choices were alternative blade (63\%), ILMA(18\%), and fibreoptic laryngoscope(15\%). Over $56 \%$ had experienced a CICV situation in practice. For this, the preferred infraglottic device was Melker(29\%) followed by Quicktrach(26\%), cricothyroidotomy by IV catheter(16\%), surgical tracheostomy(13\%), open surgical cricothyroidotomy (OSC)(8\%), and Minitrach 2(5\%) Comfort levels were significantly lower than those for equipment used in the difficult airway scenario $(p<0.05)$, and those for OSC the final step in the DAS CICV guideline, were very low. In both scenarios, previous use of an airway device was associated with significantly higher comfort levels $(p<0.05)$. Only $62 \%$ had attended an airway workshop while $68 \%$ were familiar with the DAS guideline.

Conclusion(s): Comfort levels for devices chosen in the difficult airway scenario were significantly higher than for infraglottic devices. This may reflect previous use and experience. Training on models has been shown to improve manual skills(2). These results suggest that airway training may be underused.

References:

1 Wong D et al Anes Analg 2005; 100: 1439-46.

2 Schwid HA et al Anesthesi 2002; 97: 1434-4. 


\section{AP3-2}

Ultrasound can be a useful tool for predicting of the diameter of a left mainstem bronchus: tracheal width measured by ultrasonography vs. tracheal and bronchial width measured by computed tomography

A. Sustic, A. Protic, D. Miletic

Department of Anesthesiology and ICU, University Hospital Rijeka, Rijeka, Croatia

Background and Goal: Left double-lumen tubes (LDLT) are used to isolate and/or collapse the lungs selectively during thoracic procedures in over $98 \%$ of patients. One of the most important steps to avoid the complications associated with tube is appropriate selection of the size of the LDLT. There is direct correlation between tracheal and left mainstem bronchial (LMB) width and measurement of the tracheal width can be used as a guide to help predict diameter of LMB and the size of LDLT. ${ }^{1}$ Our hypothesis is that tracheal width measured by ultrasound correlate with tracheal and bronchial width measured by computed tomography (CT), and that US can be a useful additional tool for predicting of the size of a LMB and LDLT.

Materials and Methods: Twenty-five adult patients (14 male; 11 female) aged 23-80 yr who required a LDLT during anesthesia for elective thoracic surgery were entered into this study. The inclusion criteria included patients who already had a preoperative CT scan performed in our institution. Measurements of the internal tracheal diameter were made by radiologist at the coronary plane $0.5 \mathrm{~cm}$ above sterno-clavicles joints. Measurements of the internal diameter of the LMB were made $1 \mathrm{~cm}$ below carina, in coronary (transversal) plate, too. Ultrasound measurement of outer diameter of the trachea was performed on the level just above sterno-clavicles joints in transversal section.

Results: There was strong and statistically significant correlation between outer tracheal width measured by US (TWUS) and inner tracheal (TWCT) and LMB width (LMBW) measured by MSCT, as well as strong correlation between tracheal and LMB width, itself.

\begin{tabular}{llll}
\hline$x$ & TWUS & TWCT & LMBW \\
\hline TWUS & $x$ & $r=0.882 ; p<0.001$ & $r=0.832 ; p<0.001$ \\
TWCT & $r=0.882 ; p<0.001$ & $x$ & $r=0.878 ; p<0.001$ \\
LBW & $r=0.832 ; p<0.001$ & $r=0.878 ; p<0.001$ & $x$ \\
\hline
\end{tabular}

Conclusion: This study shows that measurement of outer tracheal width by ultrasound can be an useful method for predicting of the diameter of a left mainstem bronchus.

Reference:

1 Olivier P, et al. Chest 2006; 130: 101-7.

\section{AP3-4}

\section{Sniffing position and three axis theory: evaluation by MRI in} Japanese subjects

H. Dohgomori, K. Takeuchi, T. Matsumoto, T. Fujimoto, K. Okamoto

Division of Emergency Medicine, Shinshu University Hospital, Matsumoto, Japan

Background and Goal of Study: A previous study has already reported measurement values of some anatomical positions using MRI, and discussed the sniffing position is not useful for tracheal intubation (R-1). Our purpose was to investigate the relationship between the sniffing position and three axis theory in Japanese using MRI when tracheal intubation was performed.

Materials and Methods: Using MRI apparatus (Siemens, Germany), two T1 weighted images (i.e., in neutral $(\mathrm{N})$ and sniffing $(\mathrm{S})$ positions) were obtained in each volunteer. The sniffing position was obtained by placing a $5-\mathrm{cm}$ pillow underneath the head. Four lines were drawn on the MRI films, and then three angles were measured by following the method described in R-1. Shortly, (1) the axis of the mouth (MA); (2) the pharyngeal axis (PA); (3) the laryngeal axis (LA); and (4) the line of vision were drawn on each film. Alpha angle (A) was defined as the one obtained between MA and PA; beta angle (B) between PA and LA, and delta angles (D) between line of vision and LA. When the intersection of the axes was located under the Th-2 level, B was regarded to be unmeasurable in the present study although all the images could show above Th-2 level in R-1. Data were expressed as mean (SD) and statistical analysis were performed using parried t-test.

Results and Discussions: Six male volunteers with no history of diseases in their upper airways were enrolled. The age was 38.4 years $(10,20-49)$. Height was $165 \mathrm{~cm}(8,160-175)$. Body Materials Index (BMI) was 23.2 (2, 22-25). B in four volunteers could not be measured since the intersection of the axes was under the Th-2 level. A was 71.5 degrees in N, 51 degrees in S, respectively
$(P=0.03)$. $D$ was 43.5 in $\mathrm{N}$ and 35 in $\mathrm{P}(\mathrm{P}=0.02)$. Our results indicated the sniffing position provided smaller $D$ values, which suggested the better vision for tracheal intubation.

Conclusions: One of the reasons for the discrepancy between our study result and R-1 result might be the racial difference. Further studies would be necessary to determine an influence of the sniffing position on the tracheal intubation.

Reference:

Adnet $\mathrm{F}$ et al. Study of the sniffing position by Magnetic Resonance Imaging. Anesthesiology 2001; 94: 83-6.

\section{AP3-5}

Does cigarette smoking affect the haemodynamic response after tracheal intubation?

O. Cuvas, A. Er, O. Ikeda, B. Dýkmen, H. Basar

Department of Anaesthesiology and Reanimation, Ankara Training and Research Hospital, Ankara, Turkey

Background and Goal of Study: Tracheal intubation results in marked sympathetic overactivity. Smokers are more prone to coronary vessel disease than nonsmokers and an exaggerated increase in rate-pressure product may cause critical myocardial ischemia (1). In our study we aimed to investigate heart rate, blood pressure and rate-pressure product changes in smokers and nonsmokers after tracheal intubation.

Materials and Methods: In a prospective, blinded study, 72 ASA I patients, aged 20-49 year, who were scheduled for elective surgery requiring tracheal intubation were studied. Patients were stratified into four groups $(n=18$ in each group): Group FN (Female, nonsmokers), Group MN (Male, nonsmokers), Group FS (Female, smokers), Group MS (Male, smokers). After the stabilization period, baseline measurements were recorded. Time was started after administration of neuromusculer bloking drug and haemodynamic values were measured at every minute during a period of $3 \mathrm{~min}$ before intubation, at the completion of intubation and $60 \mathrm{~s}$ intervals thereafter for a period of $5 \mathrm{~min}$. Results: After tracheal intubation, maximum positive percentage change in heart rate $(30 \pm 18 \%)$ and rate-pressure product $(40 \pm 29 \%)$ was seen in Group MS at the completion of intubation. The rise in heart rate and ratepressure product in Group MS was different from group FN until 4 and 3 min after intubation, respectively $(p<0.05)$. The rise in rate-pressure product was significantly different between Group MS and MN at the completion of intubation $(\mathrm{p}=0.022)$.

Conclusion: We concluded that blunting the rise in haemodynamic response after intubation are important in respect to the prognosis of patients, especially in male smokers with preexisting coroner heart disease.

Reference:

1 McBride PE. Med Clin North Am 1992; 76: 333-353.

\section{AP3-7}

\section{Post-operative tracheal extubation practices at a district general hospital}

H. Sadia, K. Srinivas, S. Gupta

Anaesthetics, Queen Elizabeth Hospital, London, United Kingdom

Background and Goals: Previous studies have shown a correlation between extubation related complications and extubation techniques (1-2). The aims of this study were to establish current practices and investigate the problems associated with extubation.

Materials and Methods: Anaesthetists of all grades completed questionnaires, which detailed their extubation practices following non-obstetric elective and emergency cases. The tick-box questionnaires provided information about the type of surgery, patient profile, extubation techniques and complications encountered.

Results: In total, 100 questionnaires were analysed, out of which 56 were elective cases and 44 emergency cases. Most patients were extubated awake (98\%). The position during extubation included 53 in supine position, 27 in left lateral position and 20 in head up position. Actual management of extubation is illustrated by the table below

\begin{tabular}{lc}
\hline Management of tracheal extubation & $\mathrm{n \&} \%$ \\
\hline $100 \%$ oxygen for 2-5 mins before extubation & 100 \\
Reversal agents & 69 \\
Direct laryngoscopy and suction & 64 \\
Extubated at the end of inspiration & 18 \\
Use of any Airway Devices & 20 \\
Transfer with oxygen to recovery & 97 \\
\hline
\end{tabular}

Complications included apnoea (4\%), desaturation (4\%), coughing (4\%), laryngospasm (2\%), breath holding (2\%) and aspiration (2\%). Majority of 
complications were managed with oxygen, jaw support and suctioning if needed and none required re-intubation.

Conclusion: This survey shows marked differences in practice amongst a small group of anaesthetists in our hospital. About $70 \%$ used reversal agents to reverse neuromuscular blockade and only $20 \%$ used airway adjuncts in the peri-extubation period. The complications associated with extubation were also quite common, as shown by our survey. Guidelines have been suggested in the past (2), but there is still a huge variation in the practice of extubation. References:

1 Rassam S, SandbyThomas M, Vaughan RS, et al. Anaesthesia 2005; 60: 995-1001.

2 Hartley M. Difficulties in tracheal intubation, 2nd edn. London: W.B Saunders, 1997: 347-70.

\section{AP3-8}

\section{Is nasotracheal intubation an "at risk" manipulation of bacteraemia?}

J.S. Medina, Concepcion. Rodríguez Valdés, Maximilian. Alvarez, Inmaculada. Tomás, Jacobo. Limeres

Anaesthesia, Hospital de Conxo. CHUS, Santiago de Compostela, Spain

Background and Goal of Study: Different Expert Committees on prevention of Bacterial Endocarditis have shown heterogeneous attitudes on the importance of the intubation technique as "at risk" manipulation. This lack of consensus could be justify because the prevalence of bacteraemia related to endotracheal intubation found in the literature varies between $0 \%$ and $33 \%$. The present study aims to investigate the prevalence of bacteraemia following orotracheal intubation (B-OTI) and nasotracheal intubation (B-NTI). Materials and Methods: The study group was formed of 110 patients who underwent elective surgery under general anaesthesia in the Santiago de Compostela University Hospital. Venous blood samples were collected from each patient at baseline (before performing any manipulation) and 30 seconds after completing the orotracheal intubation (OTI) or the nasotracheal intubation (NTI). Samples were inoculated in BACTEC plus aerobic and anaerobic blood culture bottles, and were processed in the Bactec 9240. The subculture and further identification of the isolated bacteria were performed by conventional microbiological techniques. Level of difficulty of endotracheal intubation and oral health status were recorded in all patients.

Results: At baseline, the percentage of positive blood cultures detected was $2 \%$. The prevalence of bacteraemia at 30 seconds after finishing the endotracheal intubation was $11.8 \%$ (13 cases). OTI provoked bacteraemia in $12 \%$ of patients (6 cases) and NTI in the $11.7 \%$ (7 cases). Staphylococcus spp. and Streptococcus spp. were the most commonly isolated genera. Level of difficulty of endotracheal intubation and oral health status didn't conditioned the development of bacteraemia.

Conclusion: Endotracheal intubation is associated to a low percentage of bacteraemia, this being similar following OTI and NTI. In consequence, the inclusion of nasotracheal intubation as "at risk" manipulation needing antibiotic prophylaxis of bacterial endocarditis recently suggested by the British Society of Antimicrobial Chemotherapy needs further discussion.

Reference:

Gould FK, Elliott TS, Foweraker J et al. J Antimicrob Chemother 2006; 57: 1035-42.

\section{AP3-9}

\section{A simplified risk score to predict difficult endotracheal intubation}

L. Eberhart, C. Arndt, H. Lange, T. Koch, H. Wulf

Department Anaesthesiology \& Critical Care Medicine, Philipps-University Marburg, Marburg, Germany

Background and Goal of Study: Despite the presence of numerous predictive tests for the difficult airway and substantive criticism concerning the usefulness of predictive tools in this setting [1] most anaesthesiologists feel that there is still a tool missing that allows an easy bedside and reliable estimation of the likelihood that an individual patient will present difficult intubation (DI: need for technical or personal support, more than three attempts or duration of $>10$ minutes).

A simplified risk model can facilitate the use and the clinical acceptance of a composite predictive tool since it allows a rough calculation of the expected risk without using equations or other estimations difficult to perform in real clinical situations.

Materials and Methods: A total of 3763 patients were screened for a number of potential risk factors for DI. A random sample $(n=2509)$ was subjected to a multivariate stepwise logistic regression analysis. The remaining risk factors were used to build a simplified model. The latter was validated in a validation dataset $(n=1254)$ by calculating the AUC under a ROC-curve and by analysing the calibration characteristics of each model.
Results and Discussions: The following factors (odds-ratio) were associated with DI: presence of upper front teeth (3.61), a history of difficult intubation (2.88), Mallampati status higher than "1" (2.55) and equal to "4" (1.91), and mouth opening $<4 \mathrm{~cm}(1.80)$. The validation in the data of the 1254 patients not used for the creation of the model suggests that the predictive power of both scores is acceptable. For DI it was 0.72 (95\%-confidence interval: $0.63-0.81$ ). The likelihood for $\mathrm{DL}$ increases from $0 \%$ (when no risk factor is present) to $2 \%, 4 \%, 8 \%$, and $17 \%$ when $1,2,3$, and more than 3 factors are present.

Conclusion: The present simplified composite risk score that can be viewed as a modification of the Mallampati-scoring system might facilitate the prediction of a difficult intubation.

Reference:

1 Yentis SM: Predicting difficult intubation-worthwhile exercise or pointless ritual? Anaesthesia 2002; 57: 105-9

\section{AP3-10}

\section{A manikin study into the effect of handedness on the ability to bag-mask ventilate \\ M. Sandby-Thomas, M. Alqatrani, M. Stacey, J. Hall, A. Wilkes \\ Anaesthetics, Cardiff University, Cardiff, United Kingdom}

Background and Goal of Study: Bag-Mask Ventilation (BMV) is a core medical skill requiring a good seal between mask and patient to ensure effectiveness. Operator technique is central to its success. In our study we have looked at whether which hand is used to hold the mask, dominant (DH) or nondominant (NDH), confers any advantage when performing BMV.

Materials and Methods: This was a prospective paired crossover manikin study. We recruited 57 anaesthetists and 54 non-anaesthetist healthcare workers from our Hospital. Personal details and grip strengths were recorded. The Equipment used was the Basic Airway Manikin (BAM) (which measures volumes expelled from the bag and volumes delivered to the manikin's lungs during BMV) and a standard bag and mask. Volunteers were asked to BMV the manikin at a rate of 10 breaths per minute over 2 minutes using their $\mathrm{DH}$ and $\mathrm{NDH}$ at both easy and difficult settings. We measured the effectiveness and efficiency of BMV. Effectiveness was a measure of the lung volumes delivered ( $>400 \mathrm{mls}$ per breath was considered successful). Efficiency was a measure of the leak produced (proportion of lung volume to bag volume).

Results and Discussions: $84 \%$ of anaesthetists used their NDH for BMV which equated to the left hand in $91 \% .59 \%$ of non-anaesthetists used their $\mathrm{NDH}$ for BMV which equated to the left hand in 59\%. Anaesthetists were significantly more effective and efficient with their NDH in all settings. Nonanaesthetists were significantly more effective with their $\mathrm{DH}$ at the easier setting. Hand size, grip strength and gender had no effect on ability to BMV. Anaesthetists were, as expected, significantly better at BMV than nonanaesthetists although the standard was generally disappointing.

Conclusion(s): Anaesthetists were better at BMV with their NDH which probably reflects training and the need to simultaneously use their $\mathrm{DH}$ for fine motor skills whilst performing BMV. Non-anaesthetists were naturally better with their $\mathrm{DH}$. This suggests that occasional practitioners of BMV should be trained to use their $\mathrm{DH}$ to maximize their effectiveness. The overall standard was poor and we would recommend that more training is required for all in this vital skill.

There are no references or acknowledgements.

\section{AP4-1}

\section{Intubation time with and without inflatable intubation device}

A. Mathijs, J.P. Mulier

Anaesthesiology, AZ St jan AV Brugge, Brugge, Belgium

Background and Goal of Study: A special inflatable pillow was made to elevate the thoracic column in obese patients to improve the intubating conditions.(1) This lengthens the sterno mandibular distance and seems to improve the vocal cord visualization. The goal of this study was to evaluate its impact on the intubation condition by measuring the intubation time. Materials and Methods: 43 Obese patients with a BMI above 40 and scheduled for a bariatric operation get an inflatable pillow under their thorax. A resident in training and an anesthetist with more than 10 years experience intubate patients with measurement of the time in seconds between introduction of the laryngoscope till inflation of the cuff. The pillow is inflated at random. A boogie was used in every intubation. Two attempts with oxygenation in between are given before an other intubation technique or anesthetist is chosen. Time count is halted then and the case is noted as a failed intubation. 
Results and Discussions:

Table 1

\begin{tabular}{lcccc}
\hline & $\begin{array}{l}\text { sec with } \\
\text { by R }\end{array}$ & $\begin{array}{l}\text { sec without } \\
\text { by R }\end{array}$ & $\begin{array}{l}\text { sec with } \\
\text { by A }\end{array}$ & $\begin{array}{l}\text { sec without } \\
\text { by A }\end{array}$ \\
\hline Number of patients & 6 & 7 & 14 & 16 \\
Number of failures & 1 & 2 & 0 & 1 \\
Mean & 40 & 48 & 26 & 35 \\
Standard deviation & 24 & 42 & 12 & 32 \\
$t$ test & 0.173 & & 0.046 & \\
\hline
\end{tabular}

Table 1 gives the mean, standard deviation and $t$ test between inflated and non inflated pillow for trainees $(\mathrm{R})$ and experienced anesthetists (A). The number of failed intubations is given too. The time was only significant longer in an experienced anesthetist probably because the number of patients was larger. Failures could not be analyzed because of the small number of patients. The intubation time has some outliners in one direction what would require a non parametric test. The shorter intubation time could also be explained by the easier introduction of the laryngoscope with a longer sterno mandibular distance. Conclusion: More patients are needed to confirm the shorter intubation time when the inflatable positioning device is used.

Reference:

1 J P Mulier Anesthesiology 2006; 105: A873.

\section{AP4-2}

Airway management during major intranasal surgeryA study of practices among different anaesthetists

H. Krovvidi, C. Parcha, G. Jeskins

Anaesthetics, Queen Elizabeth Hospital, Birmingham, United Kingdom

Background and Goal of Study: Reinforced Laryngeal Mask Airway (LMA) forms a reliable seal around laryngeal inlet and protects the airway from blood and other debris ${ }^{1}$. It also forms an unobstructed airway for surgeon produces minimal hemodynamic changes compared to endotracheal tube, we audited the practices among different anaesthetists for maintaining airway during intranasal surgeries and incidence of complications associated with various airway adjuncts used.

Materials and Methods: We collected data for 5 weeks in all elective major intranasal surgeries.

Results and Discussions: A total of 47 patients were audited during a period of 5 weeks. Consultants and trainee registrars shared equally the case load (51\%: $49 \%)$. Anaesthetic technique most commonly used was an intravenous bolus of anaesthetic agent followed by maintenance with inhalational agent $(72 \%)$, followed by remifentanil infusion + inhalational agent technique (23\%) and Total intravenous Anaesthesia was used in $5 \%$ cases. Airway was maintained in $68 \%$ cases with endotracheal tube (standard tube $10 \%$ and preformed RAE tube 58\%), Reinforced LMA was used to maintain airway in $32 \%$ patients. Patients who had endotracheal tube to maintain their airway had more postoperative complications than patients who had LMA to maintain their airway $(46.8 \%$ vs $13.3 \%)$. Blood in upper airway, upper airway obstruction, laryngospasm, aspiration of blood and reintubation were complications noted.

Conclusion(s): In our prospective audit endotracheal tube is the popular choice of maintaining airway compared to LMA. Postoperative complications were more common in patients who had endotracheal tube for their airway protection than LMA. Despite its remarkable safety, LMA was not the first line of choice to maintain the airway in nasal surgery in our institute.

Reference:

1 John RE, Hill S, Hughes TJ. Airway protection by the laryngeal mask. A barrier to dye placed in the pharynx. Anaesthesia 1991; 42: 366-7.

\section{AP4-3}

Algorithm of action in the case of acute intraoperative airway obstruction in a patient with a large goiter

M. Moguilevitch

Anesthesiology, Montefiore Medical Center, Bronx, USA

Background and Goal of Study: Intraoperative airway obstruction in the patient with a large goiter is a challenging situation and requires prompt and coordinated response (1). We are introducing stepwise approach in the management of this anesthetic emergency.

Case Report: 46 years old male, $146 \mathrm{~kg}, 173 \mathrm{~cm}$ with past medical history of hypertension, morbid obesity, obstructive sleep apnea, large goiter. He complained of difficulty breathing lying flat. On physical exam patient had Mallampati class 3 airway and prominent neck mass. Remaining physical exam is noncontributory. X-ray of the neck showed right sided tracheal deviation. Anesthesia was induced safely after awake fiberoptic intubation. During surgical manipulation of the trachea and one of the lobes of the goiter, sudden decrease of the tidal volume and increase of peak airway pressure noticed. Oxygen saturation decreased to $93 \%$.

Algorithm: 1. Hand ventilate patient with $100 \%$ oxygen to assess compliance and chest excursion. 2. Auscultate chest for bilateral breath sounds and exclude wheezing. 3 . Examine surgical field for possible external compression. 4. Suction endotracheal tube to rule out mucous plug or secretions. 5. Fiberoptic bronchoscopy to exclude tube malpositioning and possible foreign body. In the case described above fiberoptic examination revealed endotracheal tube pressed against the tracheal wall. Position of the tube corrected and ventilation restored.

Conclusion(s): Development of the structured algorithm of action leading through possible differential diagnosis in the case of intraoperative airway obstruction is very powerful tool in management of this critical anesthetic problem.

Reference:

1 Farling, P.A Thyroid disease. British Journal of Anesthesia. July 2000, vol. 85. 1991-2004.

\section{AP4-4}

\section{Fiberbronchoscopic intubation for difficult airways in emergency situations}

N. Jakushenko, U. Kopeika, D. Nagobade, M. Mihelsons, I. Taivans

Department of Anesthesiology, Emergency Care Hospital, Riga, Latvia

Background and Goal of Study: Unexpected difficult airways (DA) are one of the most critical situations in anaesthesia, intensive care practice. The goal of this study was to detect the early and late complications of fiberbronchoscopic intubations (FBI) in patients in emergency situations with: a) previously not predicted and unexpected DA; b) maxillo-facial and cervical injuries (MF/CSI); c) for the predicted DA in the acute abdominal surgery.

Materials and Methods: The study was conducted in 46 patients (pts). Among them 19 pts were with previously unpredicted difficult tracheal intubations, that was unsuccessful; 15 pts with MF/SCl and 12 pts with acute abdominal pathology and risk of aspiration. In 7 pts nasotracheal and in 39 pts orotracheal intubations were performed under the local anaesthesia with Sol. Lidocaini $2 \%$ and sedation with Midasolam in doses 1-5 mg. Early complications during FBI procedures were recorded.

Results and Discussions: There was no difference between patients groups as related to demographic factors. The average age was $49 \pm 14$ years. There was no unsuccessful FBI. The mean time for performing of FBI was statistically significant longer in group $a-142 \mathrm{sec}(55-62)$, versus group $b-67 \mathrm{sec}$ $(32-182)$ and group $c-43 \mathrm{sec}(17-136)$; $(p<0.05)$. The early detected complications after FBI in all patients' groups were: laryngospasms in 7 pts (15.2\%), epistaxis in 6 pts (13\%), cough in 9 pts $(19.5 \%)$, regurgitation in 1 pts $(2.2 \%)$ and aspiration in $1 \mathrm{pts}(2.2 \%)$. The late complications were: sore throat in $15 \mathrm{pts}$ with unexpected $\mathrm{TI}$ under usage of conventional method $(79 \%)$, in both other groups sore throat was statistically significant $(p<0.05)$ lower - only 9 pts $(30 \%)$ had sore throat. Pneumonia, verified as complication after aspiration on prehospital stage was detected in 1 pts $(2.2 \%)$. Mortality was $8.7 \%$ due to multiple organ dysfunction and acute respiratory syndrome.

Conclusion(s): 1. the duration of $\mathrm{FBI}$ depends on the concrete emergency situation. In unexpected cases there is need for longer procedure. 2. The complications of FBI are epistaxis, laryngospasm and cough, sore throat was observed more frequently after unsuccessful conventional intubation. 3. $\mathrm{FBI}$ is the effective method of management of difficult airways and prevention of severe hypoxia in emergency situations.

\section{AP4-5}

\section{Using video feedback to train anesthesia residents in fiberoptic intubation}

A. Apostol, N.A. Aranha, D. Kuzmin, B. Hub

Anesthesia, State University of New York Downstate, Brooklyn, USA

Background and Goal of study: FOI requires practice to establish competency. We explored the feasibility of DVD recording of performed FOI by anesthesia residents as a tool for feedback.

Materials and Methods: This is a prospective study using DVD recorded real time video to enhance FOI training by anesthesia residents. Clinical Anesthesia (CA) 1-3 residents were randomly picked to do FOI. Seven residents from each level of training were chosen. FOI is performed after IV induction of anesthesia with muscle relaxant, regardless of airway class (AWC). Hemodynamic changes (HR, BP, etc.) are also recorded. Recorded DVD video is edited into time-framed intervals. Time-framed video segments were compared with consecutive FOI attempts by the same operator with different patients. A scoring system is used in order to establish competency. 
Results and Discussion: 152 fiberoptic intubations were performed; surgeon satisfaction regarding the fiberoptic intubations was $100 \%, 90 \%$ among attending anesthesiologists. There was no significant difference between the difficulty of airway and penalty score. There were no significant differences between the three levels of anesthesia residency training. Significantly less hemodynamic changes during fiberoptic intubation when compared to classical laryngoscopy. The most common mistakes made by the residents were secondary to over-estimation of fiberoptic movements.

Conclusion: The study had a tremendous positive feedback from surgeons, anesthesiologists, and residents. As a result, this study evolved to a training rotation for all 70 residents in the program with daily evaluations of personal performance using a DVD recorder.

Reference:

Cole AFD, Mallon JS, Rolbin SH, Ananthanarayan C: Fiberoptic intubation using anesthetized paralyzed, apneic patients. Anesthesiology 1996, 84: 1101-6.

\section{AP4-6}

Laryngoscopy versus fiberoptic intubation: cardiovascular and catecolamines level changes

E. Congedo, P. Aceto, R. Sicuranza, R. Petrucci, G. De Cosmo

Anaesthesiology and Intensive Care, Catholic University of Sacred Heart, Rome, Italy

Introduction: The aim of this study was to compare stress response degree during intubation with direct laryngoscopy and fiberoptic-facilitated oral intubation.

Materials and Methods: After obtaining informed consent, 40 patients, aged 25-70 years, ASA I-II, undergoing minor abdominal surgery, were enrolled in the study. At the induction of anaesthesia, propofol $2 \mathrm{mg} / \mathrm{kg}$, alfentanil $20 \mathrm{mcg} / \mathrm{kg}$ and cisatracurium $0.25 \mathrm{mg} / \mathrm{kg}$ were administered to patients. They were randomly assigned to be intubated with two different methods: laryngoscopy (A group) and fibroscopy (B group). Cardiovascular parameters (arterial blood pressure and heart rate) were recorded at the following times: before induction (T1), 1 minute after intubation (T2) and 5 minutes after intubation (T3). Blood samples were also taken at the same times to measure plasmatic catecholamines level. MANOVA test was used for statistical analysis.

Results: Heart rate values increased at $\mathrm{T} 2$ compared with $\mathrm{T} 1$ in both groups. At T3, heart rate values remain invariate in B group and decreased in A group with a significant gap $(p<0.01)$. Arterial blood pressure values raised at T2 and decreased at T3 in a comparable way in the two groups. Catecholamines levels followed the same trend as arterial pressure whitout differences between the groups.

Conclusions: Fibroscopy does not protect from stress response compared with laryngoscopy.

Reference:

Barak M. J Clin Anesth 2003; 15: 132-6.

\section{AP4-7}

\section{Effect of blade angulation on field of vision in rigid optical laryngoscopes}

S. Darshane, P. Charters

Anaesthesia, University Hospital Aintree, Liverpool, United Kingdom

Background and Goal of Study: We previously defined a 2-dimensional "field of vision" as the measure of angle from the distal straight segment of a laryngoscope blade to the maximum view away from it [1]. We planned to compare EVO2, Glidescope and Airtraq laryngoscopes. Direct comparison is otherwise difficult because the viewing systems are quite different.

Materials and Methods: We constructed a bench model to test image linearity using a $5 \times 5 \mathrm{~mm}$ graph-paper fastened to a rigid board. The blade tips were positioned in contact with the board and the apparatus allowed alteration of the angle between the distal straight segment of the blades and the board. Each blade was rotated 30 degrees either side of the $(90 \mathrm{deg})$ normal position in 10 deg. intervals steps. Airtraq and EVO2 were connected to monitor stack systems for image recording to digital outputs. Glidescope has its own monitor which was photographed for subsequent analysis. Based on an assumption of linearity and the starting normal position, a mathematical model was used to predict the expected cell sizes.

Results and Discussions: Simple linear modelling gave good representation of the imaging systems. It was less good for Airtraq where distal straight segment and field of vision were difficult to measure accurately. Squares were only represented as squares when the object was at 90 degrees to the blade tip. At more acute angles the field of vision increased with the cells coming vertically closer and horizontally more compact as distance from the blade tip increased (i.e. they became more rhomboid). The opposite was the case with more obtuse angles. Any increase in the field of vision is at a cost of greater image distortion.

Conclusion(s): Ideally the anaesthetist wants to have a perfect image projection (i.e. squares represented as squares). In our view this is unlikely to be true for most clinical settings with these blades. Further clinical trials will determine whether or not this is the case in practice.

Reference:

1 Sethuraman D, Darshane S, Charters P. Br J of Anaes 2006; 97: 434P.

\section{AP4-8}

Use of a new tapered endotracheal tube guide to facilitate rapid intubation by novice laryngoscopists

L. Nelson, K. Foreman, D. McCann, G. Heimburger, S. Lisco

Anesthesiology, University of Cincinnati College of Medicine, Cincinnati, USA Background and Goal of Study: Prehospital personnel have poor success with intubation in non-obtunded patients. Lack of experience and limited availability of airway adjuncts contribute to the poor success rates. ${ }^{1}$ A readilyavailable, simple device to facilitate passage of an endotracheal tube (ETT) would be an advantage for field intubations. The RADLyn endotracheal tube guide (ETT-R) has a malleable tip and tapered balloon to facilitate ETT placement in spontaneously breathing patients. This study compares use of the ETT-R, an ETT with stylet (ETT-S), and an ETT with Eschmann tracheal tube introducer (ETT-E) by novice laryngoscopists.

Materials and Methods: Protocol was institutionally reviewed and monitored. 110 novice laryngoscopists intubated a mannequin (Airway Management Trainer, Laerdal Medical, USA) using an ETT-S, ETT-R and ETT-E in randomized order after instruction / practice on all methods.

Results and Discussions:

\begin{tabular}{lll}
\hline Device & Mean Time (seconds) & Mean Attempts \\
\hline ETT-S & $28.3 \pm 1.8 \#$ & $1.05 \pm 0.03^{\star}$ \\
ETT-R & $47.7 \pm 4.5 \#$ & $1.09 \pm 0.03^{\star}$ \\
ETT-E & $86.0 \pm 8.5 \#$ & $1.21 \pm 0.06^{\star}$ \\
\hline
\end{tabular}

$\# P<0.001{ }^{*} \mathrm{P}=\mathrm{NS}$

Time/attempts (Mean \pm SEM) shown in the table. On a 5-point Likert scale, $68 \%$ of subjects report ETT-R use as easy/easier than ETT-S $(p=0.003)$. $84 \%$ report ETT-R use as easy/easier than ETT-E $(p<0.001)$.

Conclusion(s): For the novice laryngoscopists intubation with ETT-S was performed most quickly; despite this, ETT-R was reported to be as easy/easier than intubation with both ETT-S and ETT-R. For prehospital providers who rarely intubate non-obtunded patients the RADLyn ETG may be an important yet simple adjunct to facilitate rapid and correct placement of an ETT.

Reference:

1 Deakin CD, et al. Emerg Med J 2005; 22: 64-67.

\section{AP4-9}

\section{Use of the UNIBLOCKER-bronchial blocker tube to facilitate one-lung ventilation during video-assisted thoracoscopic surgery}

T. lizuka, M. Tanno, Y. Hamada, T. Shiga, Y. Ohe

Anaesthesia, Toho University Medical center Ohashi Hospital, Meguro, Japan

Background and Goal of This Study: In this study, we evaluated the efficacy of a wire-guided bronchial blocker, UNIBLOCKER ${ }^{\text {TM }}$ (Fuji Systems Corporation, Tokyo, Japan) for achieving one-lung ventilation (OLV) during a video-assisted thoracoscopic surgery (VATS).

Materials and Methods: 16 patients undergoing a VATS approach with the new device, a bronchial blocker tube, UNIBLOCKER ${ }^{\text {TM }}$ to establish OLV were studied. The time to place the UNIBLOCKER ${ }^{\text {TM }}$ to the right or the left mainstem bronchus, the quality of lung deflation was rated by the surgeon under direct visualization as excellent, good, fair, or poor.

Results and Discussions: In all 16 patients, placement of the UNIBLOCKER ${ }^{\mathrm{TM}}$ was easily with fiberoptic aided technique. Speed of insertion increasing as experience improved. One-lung ventilation was well tolerated in all. The quality of lung deflation was judged as being excellent or good in all patients, and the surgical field was excellent in all cases. 
Data (mean $\pm \mathrm{SD})$ are shown in the table:

\begin{tabular}{lll}
\hline & right VATS & left VATS \\
\hline $\mathrm{N}$ & 12 & 4 \\
time (sec) & $40.1 \pm 11.6$ & $58.0 \pm 24.5$ \\
lung deflation & excellent: $3 / 12$ & excellent: $2 / 4$ \\
& good: $9 / 12$ & good: $2 / 4$ \\
\hline
\end{tabular}

Conclusions: Lung isolation with the new device, UNIBLOCKERTM is both safe and very effective in VATS. In this study, UNIBLOCKER ${ }^{\mathrm{TM}}$ showed ease to placement to the right main bronchus, but a better quality of lung collapse showed left $>$ right. The development and clinical use of UNIBLOCKER ${ }^{\text {TM }}$ proved to be effective and easy to use for establishing OLV.

\section{AP4-10}

\section{Comparison of two different techniques to prevent loss of} airway control during percutaneous tracheostomy

R. Badaev, M. Croitoru, A. Pirogov, N. Davidova, E. Altman

Cardiothoracic Surgery Department, Carmel Hospital, Haifa, Israel

Background and Goal of Study: Loss of airway control during percutaneous tracheostomy (PCT) is one of the serious complications. It may happen due to unstable position of the endotracheal tube (ETT) with its tip in the larynx and cuff above the vocal cords. This position of ETT is the main request for PCT performance. We retrospectively reviewed our experience with additional use of fiberoptic bronchoscope (FOB) and tube exchanger (TE) for stabilization of ETT during PCT.

Materials and Methods: From the 160 adult critically ill patients that underwent PCT by Griggs technique between January 2000 and August 2001, we selected 33 patients receiving anesthesia from the same anesthetist. From this group 12 patients were ventilated through ETT by standard technique; in 11 patients pediatric $\mathrm{FOB}$ was used to control and stabilize the position of ETT during PCT, and in the rest 10 patients, $15 \mathrm{~F} \mathrm{TE}$ was used with the same aim instead of pediatric FOB. The optimal diameters of FOB and TE suitable for ETT $(7.5 \mathrm{~mm}, 8 \mathrm{~mm})$ were found in our previous experiments, using mechanical lung simulator.

Results and Discussions: Loss of airway control during PCT has happened in 3 pts, where ventilation through ETT was performed by standard technique. This complication was corrected by expeditious actions of anesthetist and surgeon. In the other patients, additional use of pediatric FOB or TE has created secure and proper position of ETT and PCT passed smoothly without complications. Moreover, we could not register negative influence of pediatric $\mathrm{FOB}$ and $15 \mathrm{~F} \mathrm{TE}$ presence in $\mathrm{ETT}$ on ventilation parameters during PCT performance.

Conclusion(s): Stabilization of ETT position and prevention of airway control loss during PCT performance can be reached by the use of pediatric FOB or by $15 \mathrm{~F} \mathrm{TE}$ with the same reliable results. Employment of pediatric FOB is more expensive than TE.

\section{AP5-2}

\section{A comparison of the truview-EV02 blade with the Macintosh blade for tracheal intubation \\ W. Mao, L. Zhang, M. Tian}

Department of Anesthesiology, Beijing Friendship Hospital, Beijing, China

Background and Goals: The optical system of new Truview-EVO2 blade (Truphatek, Israel), offers the possible advantage of improved laryngeal view(1). The goal of this study was to compare the use of Truview-EVO2 and Macintosh blades in humans and in order to assess the effectiveness of the Truview-EVO2 in providing glottic exposure.

Material and Methods: We recruited 70 ASA I and II patients to our randomized controlled trial. Group $M(n=35)$ had tracheal intubation performed using the Macintosh blade (size 3). Group T $(n=35)$ were intubated using the Truview-EVO2 blade. Under full monitoring, anesthesia was induced with fentanyl $4 \mu \mathrm{g} / \mathrm{kg}$ IV, propofol $1.5 \mathrm{mg} / \mathrm{kg} \mathrm{IV}$, followed by rocuronium $0.6 \mathrm{mg} / \mathrm{kg} \mathrm{IV}$ for muscle relaxation. We recorded the follows: Mallampati score, thromental distance $(\mathrm{cm})$, max mouth opeing $(\mathrm{cm})$, best laryngeal view (assessed by Cormack and Lehane grade), time taken for successful tracheal intubation, grading of ease of intubation (I: difficult, II: common, III: easy), hemodynamics and signs of trauma (24 hours after the intubation). Data were analyzed using the two-tails t-test and Chi-square test, with a p-value $<0.05$ considered statistically-significant (mean $\pm \mathrm{SD}$ ).

Result: The Cormack and Lehane grade was significantly better in Group $T$ than Group M (I/II/III/IV M: 16/11/8/0; T: 30/2/2/1, P = 0.002). The grading of ease of intubation was significantly improved in Group T than Group M (P = 0.003 ). Group $T$ had longer intubation time than group $M$ (mean $18 \pm 7 \mathrm{~s}$ vs. mean $14 \pm 6 \mathrm{~s}, \mathrm{P}=0.016)$. After the intubation, there was one case of visible bloodstain on the blade in the two groups respectively. There was no difference in hemodynamics and signs of trauma between two groups.

Conclusions: Compared to the Macintosh blade, The Truview-EVO2 blade significantly improved glottic exposure and easily intubate as well as no more trauma.

Reference:

1 Leung YY, Hung CT, Tan ST. Acta Anaesthesiol Scand. 2006;50(5):562-7.

\section{AP5-3}

\section{Comparison of Truview ${ }^{\circledR}$ Laryngoscope Blade with Mackintosh Blade in Normal and Anticipated Difficult Intubation Adult Patients}

Y. Katz, M. Barak, P. Philipchuck, P. Abecassis

Anesthesiology, Rambam Health Care Campus, Haifa, Israel

Background and Goal: Difficult intubation may lead to grave morbidity and mortality (1). Several airway devices are used to assist intubation when the vocal cords are not visualized by direct laryngoscopy (2). Truview ${ }^{\circledR} \mathrm{EVO}^{\mathrm{TM}}$ laryngoscope blade facilitates indirect view of the vocal cords by optical lenses and prisms. We prospectively compared intubating parameters of Truview ${ }^{\circledR}$ blade with Macintosh blade in adult patients with normal and anticipated difficult airway.

Materials and Methods: One hundred and seventy patients who were scheduled to have general anesthesia were randomly allocated into two: Group 1 patients were intubated with Truview ${ }^{\circledR}$ blade and group 2 patients were intubated with Macintosh blade. The following parameters were recorded: pre-operative airway evaluation, laryngoscopic view, duration of intubation, maximal force applied during intubation, anesthesiologist's estimation of intubation effort on 1 to 3 scale, and post-operative teeth and soft tissue damage, stridor and hoarseness.

Results and Discussion: Truview ${ }^{\circledR}$ blade enabled better laryngoscopic view than Macintosh blade $(p=0.002)$. Mean duration of intubation using Truview $^{\circledR}$ blade was longer (33 $\pm 12 \mathrm{sec}$ ) compared with Macintosh blade (24 $\pm 13, p=0.0001)$. Maximal force applied during intubation was lower using Truview $^{\circledR}$ blade $(7.2 \pm 2.9 \mathrm{~kg})$ compared with Mackintosh blade $(13.5 \pm 4.5$, $p=0.002)$. No significant difference was found in the anesthesiologist's estimation of intubation effort, teeth damage, stridor and hoarseness. Significantly fewer patients suffered soft tissue damage following intubation with Truview $^{\circledR}$ blade than with Macintosh blade $(p=0.0004)$.

Conclusion: Truview ${ }^{\circledR}$ laryngoscope blade may be beneficial in difficult intubation when direct laryngoscopy with Macintosh blade does not allow viewing of the vocal cords.

References:

1 Peterson GN, Domino KB, Caplan RA et al. Anesthesiology 2005;103:33-9.

2 Henderson JJ, Popat MT, Latto IP et al. Anaesthesia 2004;59:675-94.

\section{AP5-4}

Importance of direct visualization in preglottic larynx tumors: glidescope + cook intubation guide

O. Valencia, R. Lopez-Vicente, MA. Escudero

Anesthesiology, Hospital 12 de Octubre, Madrid, Spain

Background and Goal of Study: The anesthetic management of patients with critical upper airway obstruction from tumours involving the area around the larynx can be a challenging problem.

We present a technique which allows to avoid local anaesthetic tracheostomy with the patient awake, and also allows to direct see the entering of the endotracheal tube into the larynx, avoiding any impact in tumours' area which can result in dangerous bleeding, and airway obstruction.

Materials and Methods: We present the case of a 45 years old man, who presented into the operating room for making a biopsy of a scamous cells cancer. The patient was very nervous and rejected the possibility of a tracheostomy with local anesthesia and sedation. We made a probe of deep inspiration with capnography registry and flow/volume curve which showed a moderate obstructive pattern. We decided to induce general anesthesia under spontaneous ventilation, used a intravenous technique with propofol $1 \mathrm{mg} / \mathrm{kg}$ due to the negative of the patient to breathe throw a conventional mask. After that a direct laryngoscopy was made using a glidescope ${ }^{\circledR}$ video laryngoscope, in order to obtain the better and bigger image of vocal cords possible. We obtained a I/IV Cormack-Lehane grade vision and a tumourlike mass which occupied a $75 \%$ of glottic space.

With a "spray as you go" technique we avoided upper airway reflex and used a cook intubation catheter to pass through vocal cords. The angulation of the tip of the intubation catheter was used to avoid the impact on tumourlike mass. 
After that and always under direct visualization we railroaded an endotraqueal tube and turned the bevel of the tube in order to avoid the impact on the tumour mass.

Conclusion(s): The use of glidescope video laryngoscope plus a cook intubation catheter allows to avoid the impact in supraglottic tumoral masses and direct the bevel of the endotracheal tube in order to avoid this impact, because of direct visualization along the whole process.

References:

1 Rees L, Mason RA. Advanced upper airway obstruction in ENT surgery. BJA. 2002; 2(5): 134-138.

2 Mason RA, Fielder CP. The obstructed airway in head and neck surgery. Anaesthesia 1999; 54: 625-628.

\section{AP5-5}

\section{Fiberoptic laryngo/bronchoscope as the flexible lighted}

\section{stylet}

H. Mashio, H. Kawahigashi, Y. Ito, F. Sakuraya, M. Takase

Anaesthesiology, Sapporo City General Hospital, Sapporo, Japan

Background and Goal of Study: Lightwand (Trachlight ${ }^{\mathrm{TM}}$, Laerdal) is useful for the cases of difficult intubation. But the use is limited because it cannot mold easily to every patient's anatomy. In contrast, flexible fibreoptic broncho/laryngoscope can mold easily to any patient's anatomy. So we devised a new intubating method using fibrescope as flexible lightwand named "Firefly Intubation". The aim of our study is to evaluate the efficacy of this method comparing with Trachlight ${ }^{\mathrm{TM}}$.

Materials and Methods: The technique of "Firefly Intubation": Attach endotracheal tube to proximal site of fibrescope (LF2 ${ }^{\mathrm{TM}}$, Olympus). Introduce fibrescope to patient orally through Ovassapian airway or mouthpiece with jaw lift. Dim room light. Operate fibrescope until distal tip shows well-defined glow transilluminated through anterior neck of patient just below thyroid prominence. Advance fibrescope with flexing distal tip slightly posterior until glow begins to disappear at sternal notch. Pass endotracheal tube over fibrescope into trachea. 78 adult patients undergoing elective surgery were investigated. After induction of general anaesthesia with propofol, fentanyl and vecuronium, 34 patients (Group A) were intubated with "Firefly Intubation". 44 patients (Group B) were intubated with Trachlight ${ }^{\mathrm{TM}}$. The success or failure and the time for intubation were recorded in each patient. Success rate and time for intubation were compared in the groups. Data were analysed using unpaired t-test and $\chi$-square test. $P<0.05$ was considered significant.

Results and Discussions: Height, age, M/F ratio showed no differences in the two groups. Body weight is heavier in group $B(P<0.05)$. Success rate and time for intubation showed no differences in the two groups $(P=0.26$ and $\mathrm{P}=0.57$ ).

\begin{tabular}{lllll}
\hline Group & Success & Failure & Succ. (\%) & Time (sec) \\
\hline Group A & 30 & 4 & 88.2 & $18.9 \pm 7.9$ \\
Group B & 39 & 5 & 88.6 & $17.8 \pm 7.7$ \\
\hline
\end{tabular}

Time was shown in mean \pm SD

Conclusions: This method can be applied for the case of difficult intubation, as same as Trachlight ${ }^{\mathrm{TM}}$. It may be more widely applicable. And it could be an alternative method to fibreoptic intubation in the case of copious oral secretions or bleeding.

\section{AP5-6}

\section{Comparison of the shikani optical stylet and the bougie in} simulated difficult intubation

J. Butcher, A. Scholz, A. Evans, J. Hall, A. Wilkes

Anaesthetics and Intensive Care Medicine, Cardiff University, Cardiff, United Kingdom

Background and Goal of Study: Direct laryngoscopy remains the standard method for tracheal intubation (1). However difficulty occurs in 1-3\% of the population (2). The bougie remains popular but is not infallible (3). The Shikani Optical Stylet ${ }^{\mathrm{TM}}$ is a rigid fibreoptic endoscope and may provide an alternative in difficult intubation. In this study we compared the Shikani Optical Stylet ${ }^{T M}$ with a bougie in a simulated Cormack and Lehane grade 3 laryngoscopy in patients.

Materials and Methods: We studied 25 adults undergoing elective surgery in a randomized cross over study. 17 were female and 8 male. The mean age was 48 years (range: 24 to 81 ). Anaesthesia was induced with propofol and paralysis achieved with a non-depolarizing muscle relaxant. Direct laryngoscopy was performed to ensure no unanticipated airway abnormalities and the epiglottis was allowed to fall back to simulate a grade 3 view. Each patient was then intubated twice using a bougie (Frova, Cook Medical) and the Shikani Optical
Stylet ${ }^{\mathrm{TM}}$ (Clarus Medical). Time in seconds was recorded from laryngoscopy to confirmation of tracheal intubation by capnography. Any oesophageal intubations that occurred were also recorded.

Results and Discussions: The Median [IQR] time (s) taken to detect successful tracheal intubation was analyzed using Wilcoxon signed rank test. The time taken was significantly longer for the stylet than for the bougie: 41[34 to 81] and $37[32$ to 40$]$ respectively $(p=0.001)$. The stylet took longer in 21 patients, the bougie took longer in 3 patients and there was one tie. The number of oesophageal intubations was analyzed using McNemar's test. Oesophageal intubation occurred on 6 occasions (24\%) using the stylet and 2 occasions $(8 \%)$ for the bougie $(p=0.22)$

Conclusion(s): The Shikani Optical Stylet ${ }^{\mathrm{TM}}$ offers the advantage of being able to visualize the vocal cords. Despite this we found that tracheal intubation was achieved quicker using a bougie.

References:

1 Nolan JP. Anaesthesia 1992; 47: 878-881.

2 Agro F. Can J Anaesth 2001; 48: 592-599.

3 Gataure PS. Anaesthesia 1996; 51: 935-938.

\section{AP5-7}

\section{Optimal spectral irradiance for laryngoscopy}

E. Lewis, MA. Hampson, AR. Wilkes, S. Wentworth, JE. Hall Department of Anaesthetics, University Hospital of Wales, Cardiff, United Kingdom

Background and Goal of Study: Changing the colour of light produced by laryngoscopes may improve the view at laryngoscopy. This may be crucial when presented with a difficult airway. Anaesthetists have expressed a preference for blue/white light to facilitate laryngoscopy. ${ }^{1}$ Infra red light has been shown to be detrimental to an optimum view. ${ }^{2}$ Light emitting diodes (LEDs) have the advantage of producing light in a more appropriate part of the spectrum. The aim of this study was to compare the spectral irradiance of laryngoscopes currently used in the UK (which contain incandescent bulbs) with a custom made laryngoscope containing an LED bulb.

Materials and Methods: The spectral irradiance from laryngoscope blades was measured under standardized conditions, using the Bentham spectroradiometer. The tip of each laryngoscope blade was placed in contact with the sample port of the spectroradiometer. The mains voltage supply was set at 2, 2.5 and $3 \mathrm{~V}$ for the incandescent bulbs and 3, 3.3 and 3.6 V for the LED. The maximum voltages are those specified by the manufacturers.

Results and Discussions:
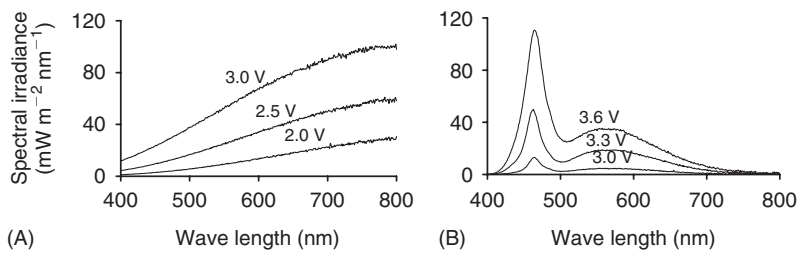

Figure 1. Spectral irradiance from incandescent bulbs (A) and LED (B).

The output from laryngoscopes currently available in the UK is mainly at the infrared $(>600 \mathrm{~nm})$ end of the spectrum. Laryngoscopes fitted with LEDs produced most output in the blue/green $(400-550 \mathrm{~nm})$ part of the spectrum. Enhanced blue/green light output with minimal infrared output is thought to optimize the view at laryngoscopy.

Conclusion(s): Laryngoscopes with LED bulbs may provide superior intubating conditions than those currently available with incandescent light sources. References:

1 Scholz A, Farnum N, Wilkes AR, Hampson MA, et al. Anaesthesia: article in press.

2 Crosby E, Cleland M. Can J Anesth 1999;46:492-6.

\section{AP5-8}

\section{Use of Truview EVO 2 optical laryngoscope system in anticipated difficult airway situation}

\section{Z. Alanoglu, O. SelviCan, F. Alver, F. Okten, O. Ozatamer}

Department of Anesthesiology and ICM, Medical School of Ankara University, Ankara, Turkey

Background and Goal of Study: Detecting patients at risk of difficult endotracheal intubation and adequate preparation of additional airway devices are crucial (1). The new "Truview EVO 2" system (Truphatek, Natanya, Israel) 
a Macintosh-type blade with an optical lens attached is designed to improve the glottic view during intubation (2). The estimated potential advantages of Truview EVO 2 system are diminished force applied during intubation, reduced trauma, facilitated insertion of an endotracheal tube into the trachea, shortened "time to intubation" and decreased incidence of "blind intubation" in patients with difficult airway and enhanced opportunities for teaching, data collecting and archiving. We presented our experience with Truview EVO 2 system in a number of patients with anticipated difficult airway.

Material and Methods: All of the patients were know as difficult intubation (Cervical stabilization $n=3$, Ancylosing Spondilitis $n=2$, short neck $n=1$ ) as they experienced a previous "can ventilate, can't intubate" situation. Anesthesia was induced with $7 \mathrm{mg} / \mathrm{kg}$ thiopental and succinylcholine $2 \%$ was initiated after ensuring the adequate mask ventilation. All standard laryngoscopy and intubation attempts were failed. However, all endotracheal intubations were successfully performed with Truview EVO 2 system.

Results:

Table 1: Review of the cases

\begin{tabular}{lllllll}
\hline & $\# 1$ & $\# 2$ & $\# 3$ & $\# 4$ & $\# 5$ & $\# 6$ \\
\hline Age (y) & 48 & 52 & 50 & 45 & 47 & 61 \\
Gender (M/F) & $\mathrm{M}$ & $\mathrm{M}$ & $\mathrm{F}$ & $\mathrm{F}$ & $\mathrm{M}$ & $\mathrm{M}$ \\
Pathology & 1 & 1 & 1 & 2 & 2 & 3 \\
Mallampati Score & 4 & 3 & 4 & 4 & 3 & 4 \\
Neck Extension-Mob. (degree) & $0^{\circ}$ & $0^{\circ}$ & $14^{\circ}$ & $15^{\circ}$ & $0^{\circ}$ & $35^{\circ}$ \\
$\begin{array}{l}\text { Cormack-Lehane Score } \\
\text { (Machintosh/Truview) }\end{array}$ & $4 / 2$ & $4 / 2$ & $3 / 1$ & $4 / 2$ & $4 / 1$ & $4 / 2$ \\
Time to Intubation (sec) & 23 & 21 & 28 & 15 & 17 & 20 \\
\hline
\end{tabular}

Conclusion: Truview EVO 2 system provides better glottic view and laryngoscopy condition which leads successful endotracheal intubation in patients with anticipated difficult intubation.

References:

1 Türkan S. et al Anesth. Analg 2002; 94: 1340-4.

2 Matsumoto $S$ et al. Anesth Analg 2006; 103: 492.

\section{AP5-9}

\section{The Truview EVO2: Initial clinical evaluation of a new optical} laryngoscope system

Z. Alanoglu, O. SelviCan, F. Alver, K. Gokcan, Y. Ates

Department of Anesthesiology and ICM, Medical School of Ankara University, Ankara, Turkey

Background and Goal of Study: The availability of different laryngoscope blades loaded with optic devices improves the view of glottic structures during tracheal intubation (1). Truview optical laryngoscope system (Truphatek, Natanya, Israel) is a Macintosh-type blade with an optical lens attached, providing the magnified image of the glottis near the tip of the laryngoscope blade (2). The aim of this randomized, prospective trial was to investigate the intubation conditions and intubation time of Truview EVO2 system, and compare them with the Macintosh blade in patients with estimated normal airway.

Material and Methods: With IRB approval and informed consent 75 ASA I-II adult patients were allocated to two groups at random via sealed envelope technique. Thyromental distance, hyomental distance, maximal voluntary mouth opening distance were recorded and upper lip bite test and Mallampati tests were performed before the initiation of general anesthesia. Laryngoscopy was performed twice in random order once first using a regular Macintosh 3 blade (Group M [ $\mathrm{n}=38]$ ) and once first using a Truview Evo2 blade (Group $\mathrm{T}[\mathrm{n}=37])$. Cormack and Lehane score was used to assess the laryngeal view during intubation. The laryngoscopy time was recorded consecutively for both blades. The ease of laryngoscopy score was assessed via verbal rating score from 0 (difficult) to 100 (easy). Mann-Whitney $U$ and Chi-Square test were used and $p<0.05$ was considered as significant. Values are expressed as median and quartiles.

Results: Patients characteristics and demographic data were similar among groups. The study parameters among groups were comparable for the same type blades. The Median Cormack-Lehane score with Macintosh blade (2, $1-2)$ was higher than TruView EVO2 $(1,1-1)(p=0.023)$. The laryngoscopy time for Macintosh blade $(13.8,11.6-16.7 \mathrm{sec})$ was comparable with TruView EVO2 $(13.5,11-16 \mathrm{sec})(p=0.73)$. The ease of laryngoscopy score was higher with TruView EVO2 $(30,10-50)$ compared to Macintosh blade $(10,10-15)$ $(p=0.30)$.

Conclusion: Truview EVO2 system compared to Macintosh blade provides better glottic view and laryngoscopy conditions with comparable laryngoscopy times.

References:

1 Anesthesiology 2003; 98: 1269-77

2 Matsumoto S. et al. Anesth Analg 2006; 103: 492

\section{AP5-10}

The performance of senior vs junior anesthesiology residents on airway mannequin intubation with Truview EVO2 system and Macintosh blade

Z. Alanoglu, O. SelviCan, F. Alver, Y. Ates, Y. Kecik

Department of Anesthesiology and ICM, Medical School of Ankara University, Ankara, Turkey

Background and Goal of Study: The airway management training via airway mannequin is much safer than patient based training and also provides extra features for both the trainees and trainers. Truview optical laryngoscope system (Truphatek, Natanya, Israel) is a Macintosh-type blade with an optical lens attached, providing the magnified image of the glottis near the tip of the laryngoscope blade (1). The aim of this study was to investigate direct laryngoscopy features of Macintosh blade and Truview EVO2 system of inexperienced and experienced anesthesia residents.

Material and Methods: With IRB approval, 14 anesthesia resident (Group S, $n=7$, Group J, $n=7$ ) were enrolled to the study. A standard airway mannequin was used. Standard teaching tools such as video tapes and drawings were used to teach Cormach Lehane score and the use of Truview EVO2 system and standard Macintosh blade. Each resident had five intubation attempts with each system. Cormach Lehane score (I-IV) and ease of intubation [0 (difficult) to 100 (easy)] were assessed by the residents for all individual intubation attempts. The time to intubation (sec) was recorded by an independent observer. The residents were also rated their impression ( 0 = dissatisfied, $4=$ Fully satisfied $)$ for all individual intubation attempts. Mann-Whitney $U$ and Wilcoxon Rank sum test were used and $p<0.05$ was considered as significant. Values are expressed as median and quartiles.

Results: Residents in Group S [35 mon. (28-49)] were more experienced than Group J [0,75 mon. $(0,5-11)](p=0.002)$. The total intubation (5 attempts) time for Truview EVO2 in Group S [49 sec. (41-57)] was shorter than Group J [74 sec. $(66-76)](p=0.002)$, however it was similar for Macintosh blade (Group S $47 \mathrm{sec}$ (42-63) and Group R [46 sec (44-69)]. The Median CormackLehane score for all attempts in both groups were lower with TruView EVO2 compared to Macintosh blade. The impression scores (mostly 4) were comparable among groups and also similar for both systems.

Conclusion: Senior residents perform quicker intubations than junior residents with Truview EVO2 system but the performance is similar for Macintosh blade. Additionally, Truview EVO2 system provides better glottic view.

\section{Reference:}

1 Matsumoto S. et al. Anesth Analg 2006; 103: 492

\section{AP6-1}

\section{Re-evaluation of the Ambu AuraOnce laryngeal mask after modification of the distal cuff reinforcement}

H.V. Genzwuerker, M. Keller, J. Hinkelbein, O. Jandewerth

Clinic of Anaesthesiology and Intensive Care Medicine, University Hospital Mannheim, Mannheim, Germany

Background and Goal of Study: Due to the modification of the distal cuff reinforcement by the manufacturer in September 2006, the single-use Ambu laryngeal mask "AuraOnce" underwent reinvestigation in our institution following participation in an international multicenter trial (1) and conduction of an own comparative trial (2) with the original device.

Materials and Methods: After approval of the local ethics committee and written consent, 50 ASA I to III patients, 18 to 75 years, scheduled for elective ambulatory interventions were ventilated with the redesigned AuraOnce. Following standardized induction of general anaesthesia, the completely deflated airway device was placed and cuffs were inflated according to manufacturer's instructions. Number of attempts (maximum 2), time until first tidal volume, initial cuff pressure, airway leak pressure with cuff pressure adjusted to $60 \mathrm{cmH}_{2} \mathrm{O}$, intraoperative airway pressures and tidal volumes (goal: petCO $\mathrm{O}_{2}$ of $35 \mathrm{mmHg}$ ) were recorded. Devices were inspected for traces of blood after removal. Patients were questioned for postoperative complaints. Results and Discussions: The device was successfully inserted in all 50 patients after a maximum of two attempts (first attempt: 96\%). 28 patients were male, 22 female. Size 4 was used in 20 patients, size 5 in 30 patients. Average age was $43.5 \pm 16.5$ years, weight $81.0 \pm 14.2 \mathrm{~kg}$, BMl $27.5 \pm 4.8 \mathrm{~kg} \mathrm{~m}^{-2}$. Time until first tidal volume was $22.5 \pm 6.0$ seconds. Initial cuff pressure with recommended inflation volumes was $70.8 \pm 19.6 \mathrm{cmH}_{2} \mathrm{O}$, airway leak pressure was $25.7 \pm 5.3 \mathrm{cmH}_{2} \mathrm{O}$ with cuff pressure adjusted to $60 \mathrm{cmH}_{2} \mathrm{O}$. Peak airway pressure was $14.1 \pm 3.2 \mathrm{cmH}_{2} \mathrm{O}$ with tidal volumes of $7.2 \pm 0.9 \mathrm{ml} \mathrm{kg}^{-1}$. No intraoperative dislocation of the device occurred, no traces of blood attachments were found after removal. In the recovery area, 6 patients complained of sore throat (visual analogue scale 1-10: 2 patients VAS 1, 3 patients VAS 2, 1 patient VAS 3); no complaints were stated after 24 hours. 
Conclusion(s): After redesign of the distal cuff portion, the performance of the Ambu AuraOnce remains unimpaired with a high insertion success rate and airway leak pressures comparable to those described in earlier trials. Postoperative complaints are infrequent and minor.

References:

1 Anesth Analg 2005; 101: 1862-1866.

2 Anaesthesist 2006; 55: 263-269.

\section{AP6-3}

Laryngeal Tube Suction II versus the Proseal laryngeal mask in anesthetized children with spontaneous ventilation

L. Gaitini, B. Yanovski, R. Toame, N. Carmi, M. Somri

Anesthesiology, Bnai Zion Med.Center, The B. Rappaport Faculty of

Medicine, Institute of Technology, Haifa, Israel, Haifa, Israel

Background and Goal of Study: The Laryngeal Tube Suction II is a new supraglottic device with a channel for the gastrointestinal tube. Preliminary studies in anesthetized adult patients have proved its role in mechanical ventilation (1). Recently, size 1, 2 and 2.5 LTS II have been introduced for use in pediatric anesthesia. This study compared the LTS II and Proseal Laryngeal Mask Airway (PLMA) with respect to: 1) insertion success rate and times; 2) efficacy of seal; 3) fiberoptical view; 4) oxygenation and ventilation; 5) orogastric tube insertion; 6) intraoperative complications.

Materials and Methods: Informed consent was obtained from the parents.

Fifty pediatric patients (ASA I) undergoing general anesthesia for routine minor procedures with spontaneous ventilation were randomly assigned to either the LTS II or PLMA group for airway management.

Unpremeditated children were induced with Sevofluorane up to $8 \%$ in Oxygen $100 \%$.

Anesthesia was maintained with Sevofluorane in 33\% oxygen and fentanyl 2-3 $\mu \mathrm{gr} \cdot \mathrm{Kg}$. The devices were inserted according to the manufacturer's recommendation.

Results and Discussions: First attempt insertion success rate was higher with the LTS II $-98 \%$, PLMA 95\%. Median seal leak pressure was similar in both groups; LTS II $34.5 \pm 3 \mathrm{cmH}_{2} \mathrm{O}$ and $35.2 \pm 2 \mathrm{cmH}_{2} \mathrm{O}$ with the PLMA.

Oxygen saturation and end-tidal $\mathrm{CO}_{2}$ were $99 \pm 0.6 \%$ and $41.8 \pm 3 \mathrm{mmHg}$ respectively for the LTS II and $98.5 \pm 1 \%$ and $40.3 \pm 2 \mathrm{mmHg}$ respectively for the PLMA.

The median fiberoptic score was 2.9 for the LTS II and 3.8 for the PLMA.

Successful insertion of the orogastric tube was achieved in $100 \%$ of both groups.

Blood staining was detected in 2 patients with LTS II and 2 patients with PLMA.

Conclusion(s): This study suggests effectiveness of the LTS II for spontaneous ventilation in pediatric patients under minor elective surgery.

Reference:

1 Gaitini L et al. Anesthesiology. 2004 Aug; 101(2): 316-20.

\section{AP6-4}

Comparison of placement of the disposable Laryngeal tube suction and the reusable ProSeal Laryngeal mask airway

M. Wrobel, Ulrich. Bauer, Sascha. Kreuer, Clemens. Bauer, Ulrich. Grundmann

Department of Anesthesiology, Intensive Care Medicine and Pain Therapy, University of Saarland, Homburg, Germany

Goal of Study: Because of possible infection with prions or other pathogens disposable extraglottic airway devices became much more common in the last few years. We compared the reusable ProSeal laryngeal mask airway (PLMA, Intavent, UK) and the laryngeal tube suction disposable (LTS-D, VBM, Germany) with respect to ease of insertion and time to achieve sufficient ventilation in a standardised clinical setting.

Methods: With institutional review board approval and written informed consent 40 patients scheduled for minor elective gynecological surgery were randomly allocated to receive either a PLMA or the LTS-D. Anesthesia was induced and maintained with remifentanil and propofol. No neuromuscular blocker was given. After completion of induction, loss of eyelash reflex and jaw relaxation, insertion of the LTS-D or PLMA was done as recommended by the manufacturer. 3 attempts for insertion of the airway device were allowed. The time between removal of the facemask for manual ventilation and sufficient ventilation either through LTS-D or PLMA was recorded. Ease of insertion was graded as easy (1 attempt), difficult (2 or 3 attempts) or impossible. A gastric drain tube was placed after sufficient ventilation and the number of attempts of insertion were recorded.

Statistics: Mann-Whitney-U-test, data are mean \pm SD.
Results: The time for insertion was shorter, but not significantly, for the PLMA compared to the LTS-D $(29.8 \pm 23.3 \mathrm{sec}$ versus $49.9 \pm 42.6 \mathrm{sec}, \mathrm{p}>0.05)$. Ease of insertion was also not significantly better for any device (easy $85 \%$ (LTS-D) versus $80 \%$ (PLMA), difficult $15 \%$ versus $15 \%$, impossible $0 \%$ (LTSD) versus $5 \%$ (PLMA) respectively, $p>0.05$ ). With both devices placement of a gastric drain tube was always possible at the first attempt after sufficient ventilation was confirmed.

Conclusion: Concerning rapidity and ease of insertion the laryngeal tube suction disposable compares similar to the ProSeal laryngeal mask airway and can be used for elective surgery without any risk of infection.

\section{AP6-5}

\section{Laryngeal Mask ProSeal ${ }^{\mathrm{TM}}$ in prolonged anaesthesia}

K. Pelikan, M. Boldizar

Department of Anaesthesiology and Intensive Care Medicine, University Hospital Saint Anne, Brno, Czech Republic

Background and Goal of Study: Tracheal intubation (TI) has been traditionally used in airway management for prolonged anaesthesia for two reasons: facilitation of positive pressure ventilation and protection of the airway due to an increase in aspiration risk with time ${ }^{1}$. The LMA ProSeal (PLMA), with its unique anti-aspiration strategy ${ }^{2}$, seems to be an ideal airway protection in prolonged anesthesia.

Materials and Methods: We studied adult female patients $(n=41)$ undergoing microsurgical breast replacement using abdominal wall tissue free flaps. Age range: 28-63 years, average: 45,8 \pm SD 11,61; BMI: 17,9-33,9; average: $24,1 \pm S D$ 3,9. Induction of anesthesia with midasolam, sufentanil, propofol, maintenance with isofluran, sufentanil, cisatracurium. Pressure controlled ventilation, FGF 02/AIR $200 \mathrm{ml} / \mathrm{min}$. PLMA size $4(85,7 \%)$, size 3 $(14,3 \%)$ was used to obtain a clear airway. Midline approach placement first attempt in $80,5 \%$. Bougie guided technique was used in 2nd $(14,6 \%)$ and 3 rd $(4,9 \%)$ placement attempts. The cuff was well lubricated with water spray and neutral jelly.

Results and Discussions: The PLMA was successfully used in all cases $(n=41)$. Ventilation time with PLMA: total: 19908 min., average $485,6 \mathrm{~min}$. \pm SD 87,58 ; max: $689 \mathrm{~min}$. There was no perioperative regurgitation or aspiration. Smooth emergence with PLMA: removal in OR in only $9,8 \%$ cases; $91,2 \%$ in PACU: $80,4 \%$ removal by trained nurse, $9,8 \%$ by patient. PLMA cuffs were observed after use - in all cases internal and external parts were clean. There were no major postoperative complications and morbidity: mild sore throat in $9,8 \%$.

Conclusion: PLMA is suitable and safe alternative to $\mathrm{Tl}$ in prolonged anesthesia. According to our experience there is no increase in aspiration risk with prolonged ventilation time.

References:

1 Blitt CD, et al. Anesth Analg 1970; 49: 707-713.

2 Brain AlJ, et al. Br J Anaesth 2000; 84: 650-654.

\section{AP6-6}

\section{A comparison of Easytube and standard endotracheal tube} for intubation during routine surgical procedures

H. Francksen, B. Bein, W. Deitmer, J. Scholz, V. Doerges

Department of Anaesthesiology and Intensive Care Medicine, University Hospital Schleswig-Holstein, Campus Kiel, Kiel, Germany

Background and Goal of Study: The Easytube ${ }^{\circledR}$ Rüsch $(E z T)$ is a relatively new disposable airway device developed for both endotracheal intubation and as a supraglottic airway device (1). The purpose of this prospective, randomized, controlled trial was to assess endotracheal intubation with the Easytube ${ }^{\circledR}$ $(E z T)$ and a standard endotracheal Tube (ETT) in routine clinical practice. Materials and Methods: After IRB approval and written informed consent 40 patients (ASA 1-3), were randomly allocated to endotracheal intubation with the EzT (size $4 / n=20$ ) or ETT (ID $7.5 / n=20$ ) followed by controlled ventilation. Anesthesia was induced with sufentanyl and propofol. Neuromuscular blockade was provided with rocuronium $0.6 \mathrm{mg} \cdot \mathrm{kg}^{-1}$. Both devices were inserted by a single experienced anesthesiologist; cuff was inflated with $10 \mathrm{ml}$ of air (EzT; ETT). Capillary blood gas samples were taken before induction of anesthesia, and after 10 minutes of ventilation. After five and 10 minutes of ventilation $\mathrm{SpO}_{2}$, etCO $\mathrm{CVT}_{\mathrm{ex}}$ and $\mathrm{P}_{\mathrm{aw}}$ were recorded. Time of insertion and failure rate were measured. Patients were asked about sore throat, dysphonia, and dysphagia 24 hours after surgery (post-operative airway morbidity).

Results and Discussions: There were no differences in demographic data between groups at baseline. Time of insertion and failure rate were significantly higher with the EzT vs. ETT (median) 80 sec; range, 36-240 sec. vs. 23; 10-70; $P<0.0001$; failure rate (intubation impossible): EzT $11 / 20$ vs. ETT 1/20). Blood 
gas samples and ventilation variables revealed sufficient ventilation and oxygenation in patients successfully intubated with either device. Post-operative airway morbidity was significantly higher with the EzT and subjective assessment of handling was significantly inferior $(p<0.0001)$.

Conclusion(s): The complex handling, resulting in a significant higher insertion time, failure rate and post-operative patient discomfort compared to a standard endotracheal tube suggest that endotracheal intubation with the Easytube may not be the best choice.

Reference:

1 Luis A. Gaitini. ASA 2004; A-517.

\section{AP6-7}

\section{Comparison of two differrent endotracheal tubes through LMA-fastrach ${ }^{\mathrm{TM}}$ for intubation}

Y. Gürkan, S. San, K. Toker, T. Hosten, M. Solak

Anesthesiology and Reanimation, Kocaeli University, Kocaeli, Turkey

LMA-Fastrach ${ }^{\mathrm{TM}}$ or Intubating Laryngeal Mask (ILMA) which provides ventilation and endotracheal intubation through plays an important role in managing difficult airway. In this study, we compared the success rate of blind endotracheal intubation through the ILMA by using the specific spiraled endotracheal tube (ST) and polyvinyl chloride endotracheal tube (PVC-ET).

One hundred twenty, ASA physical status I-II patients, aged 18 to 60 years were enrolled into this prospective study. Patients were randomly divided into two groups. In Group I (ILMA-ST), ST and in Group II (ILMA-PVC) PVC-ET were used for intubating through ILMA, respectively. Ventilation was successful in all cases in both groups. Three maneuvers were attempted if resistance was felt while endotracheal tube was passed through ILMA. Intubation success, number of the maneuvers attempted, esophageal intubation, oxygen saturation values were recorded. Sore throat and hoarseness were assessed at the postoperative 24th hour.

Although intubation success rate was higher in ILMA-ST group than ILMAPVC group (98.3\% vs $88.3 \%$, respectively) this difference did not reach statistical significance. However the intubation success rate at first attempt in ILMA-ST group was statistically different when compared with ILMA-PVC group $(57.6 \%$ vs $41.5 \% \mathrm{p}<0.05$, respectively). Esophageal intubation was significantly more frequent in ILMA-ST group compared to ILMA-PVC group $(p<0.05)$. Hoarseness was statistically more significant in ILMA-PVC group compared to ILMA-ST group ( $p<0.05)$.

For intubation through ILMA ST was more successful at first attempt. With the aid of different maneuvers success rate of intubation using PVC-ET through ILMA could be considered acceptable. Considering the wide availability and low cost, PVC-ET could be an alternative to the ST.

\section{AP6-8}

\section{Evaluation of a new disposable supra-glottic airway device} with an esophageal vent: the i-gel

B. Richez, F. Banchereau, L. Saltel, B. Julliac, A.M. Cros

Departement danesthésie-réanimationIV, maternité, Hôpital Pellegrin, Bordeaux, France

Background: The i-gel is a new single-use supra-glottic airway device with a non-inflatable cuff. It is made up of an airway tube and a drain tube in thermoplastic elastomer and a soft gel like cuff. This is the first clinical study evaluating the i-gel.

Methods: After ethic committee approval and written informed consent, 69 patients undergoing gynaecological surgery, ASA I-II, were included in this prospective, observational study. Standard monitoring was performed. Anaesthesia was not standardized. The size of the device was determined according the manufacturer's recommendations. Ventilatory parameters were set to obtain $8 \mathrm{ml} / \mathrm{kg}$ end tidal volume and $\mathrm{Pet} \mathrm{CO}_{2}$ of $40 \pm 5 \mathrm{cmH}_{2} \mathrm{O}$. We have recorded ease of insertion and number of attempt, leak pressure, peak pressure, complications after insertion and removal, ventilatory parameters and ease of nasogastric tube insertion.

Résults and Discussions: 50 size 4 and 19 size 5 i-gel were inserted. The first-attempt success rate was $97 \%$. All insertions were successful at the first attempt. Two failures occurred. In these cases, proseal insertion failed too and tracheal intubation was performed. The devices were very easy to insert in 63 cases and easy in 4 cases. The mean seal pressure was $30.2 \pm 6.8 \mathrm{cmH}_{2} \mathrm{O}$. The mean peak pressure was $11.5 \pm 2.6 \mathrm{cmH}_{2} \mathrm{O}$. Neither airway obstruction nor displacement occurred. Well lubricated nasogastric tubes were easy to insert in $100 \%$ of cases. Morbidity was very low. No regurgitation occured. No blood staining was found. There were only 2 minor complications: a transient moderate sore throat and an episode of cough.

Conclusion: These first clinical results confirm those obtain on cadavers ${ }^{1}$ and are comparable with proseal performances ${ }^{2}$. High leak pressure and low peak pressure assure safe ventilation and suggest correct positioning of the device. l-gel has some advantages: high insertion success rate, ease of insertion, low morbidity rate, disposability.

References:

1 Levitan, RM. et al. Anaesthesia 2005; 60: 1022-1026.

2 Cook, TM. et al. Can J Anesth 2005 (52); 7: 739-760.

\section{AP6-9}

Evaluation in children of a new disposable supra-glottic airway device: the i-gel

L. Beylac, B. Richez, M. Bordes, S. Français, A.M. Cros

Département danesthésie réanimation IV, Hôpital Pellegrin, Bordeaux, France

Background: Use of supra-glottic airway devices in children has increased during last decade and demands for safe and disposable products are required. The i-gel is a new single-use supra-glottic airway device with a non-inflatable cuff. It is made up of an airway tube and a drain tube in thermoplastic elastomer and a soft gel like cuff. The aim of this study is to evaluate the i-gel.

Methods: 50 children above $30 \mathrm{~kg}$, undergoing short duration surgery, classified as ASA I or II, were included in this prospective, observational study. Standard monitoring was performed. Anaesthesia was not standardized. The size of the device was determined according the manufacturer's recommendations. Ventilatory parameters were set to obtain $8 \mathrm{ml} / \mathrm{kg}$ end tidal volume and Pet $\mathrm{CO}_{2}$ of $40 \pm 5 \mathrm{cmH}_{2} \mathrm{O}$. We have recorded ease of insertion and number of attempt, leak pressure, peak pressure, complications after insertion and removal, ventilatory parameters and ease of nasogastric tube insertion. Leak pressure was not allowed to exceed $30 \mathrm{cmH}_{2} \mathrm{O}$. Results were mean and standard deviation.

Résults and discussion: Age range from 8 to 17 years and weight from 30 to $76 \mathrm{~kg}$. 45 size 3 and 5 size $4 \mathrm{i}$-gel were inserted. The first-attempt success rate was $100 \%$. The mean seal pressure was $24.9 \pm 5.8 \mathrm{cmH}_{2} \mathrm{O}$. The peak pressure was $13.5 \pm 2.7 \mathrm{cmH}_{2} \mathrm{O}$. Neither airway obstruction nor displacement occurred. Well lubricated nasogastric tubes were easy to insert in $100 \%$ of cases. Morbidity was very low. No regurgitation occured. Blood staining was found in one case. There were 2 episodes of desaturation and 4 episodes of transient cough.

Conclusion: This was the first clinical study in children evaluating the i-gel. The results appear similar to previous study concerning laryngeal mask airway in terms of leak pressure and rate of complications. The insertion success rate is excellent. I-gel is safe for paediatric airway management.

\section{AP6-10}

\section{Ima proseal management in body contouring surgery after} bariatric surgery in morbidly obese patients

\section{J.M. Nieto, I. Vives, MA. Gonzalez, JA. Jimenez, J. Roige}

Anesthesia, Hospital General Universitari Vall d Hebron, barcelona, Spain

Background and Goal of Study: Body contouring surgery is done between 18-24 months after bariatric surgery in morbidly obese patients ${ }^{(1)}$. Recent advances in bariatric surgery have improved the safety and efficacy of weightloss operations ${ }^{(2)}$. The goal of our study is to show the efficacy of LMA ProSeal (PLMA) in airway management in these patients.

Materials and Methods: 62 patients were scheduled for elective body contouring surgery from January 2005 to December 2006, TIVA anesthesia was performed with a standardized application of propofol, fentanil and rocuronium. PLMA was used. Gastric tube through PLMA and 2 intravenous lines $16 \mathrm{G}$ were placed. Were measured heart rate, arterial blood pressure, end tidal $\mathrm{CO}_{2}$, pulse oximetry, anesthetic gas analysis, urine output.

Results: Abdominoplasty vertical modify procedures were performed in all patients. Airway management was done with PLMA n ${ }^{\circ}$. Placement of PLMA and gastric tube was done at first attempt. Not one patients needed intubation. PLMA allowed airway control in 15 patients that needed fiberscope for intubation in previous gastric by pass surgery.

Conclusion: PLMA showed her efficacy in airway control in patients operated of body contouring surgery after gastric bypass in morbidly obese patients. PLMA allowed airway control in those patients that previously needed fiberscopy intubation. It gives a tighter seal against glottic opening, separates the digestive tube from the respiratory tract and allows gastric tube placement against possible regurgitation.

References:

1 Hanad G.G. Clinics in Plastic Surgery. 2004; 31: 591-600.

2 Strauch B, Herman C, Rohde C. Plast Reconst Surg. 2006; 117(7): 2200-11. 


\section{SUBJECT INDEX}

Acid-base equilibrium, metabolic acidosis 5AP2-6

Acid-base equilibrium, respiratory acidosis 5AP2-6

Acid-base equilibrium, respiratory alkalosis 7AP3-8, 19AP1-6

Acupuncture 9AP6-6

Age factors 3AP3-10

Airway 2AP1-3, 2AP1-9, 12AP6-6, 19AP1-4, 19AP1-5, 19AP1-8,

19AP3-1, 19AP4-2, 19AP5-10, 19AP6-4, 19AP6-8, 19AP6-9

Airway, anatomy 19AP1-3

Airway, complications 10AP2-5, 19AP2-8, 19AP4-10, 19AP5-8

Airway, deadspace 12AP1-1, 12AP1-2

Airway, elastance 3AP1-1, 12AP1-6

Airway, impedance 5AP3-1, 5AP4-5

Airway, infections 12AP1-4, 19AP6-4

Airway, obstruction 5AP1-5, 19AP1-6, 19AP4-3

Airway, pharynx 2AP1-3

Airway, pressure 3AP1-1, 3AP3-6, 3AP8-4, 5AP1-2, 5AP1-4, 5AP1-8, 10AP2-6

Airway, reflexes 19AP2-6

Airway, resistance 5AP3-1, 5AP4-5

Alcohol BAPC1-1, 9AP1-4, 9AP4-2

Allergy 3AP2-4, 3AP4-4, 13AP3-6, 14AP1-1

Anaesthesia, audit 1AP1-1, 1AP2-1, 8AP1-5, 8AP7-3, 9AP7-9, 11AP2-4, 17AP1-1

Anaesthesia, day-case 2AP2-4

Anaesthesia, dental 2AP1-2

Anaesthesia, depth 3AP2-3, 3AP2-7, 3AP2-10, 3AP6-6, 3AP9-2, 7AP1-5, 7AP1-8, 7AP4-7, 9AP3-1, 9AP3-10, 9AP5-4, 10AP1-3, 10AP1-7

Anaesthesia, emergency service 1AP1-4, 11AP2-6

Anaesthesia, general 3AP2-9, 3AP4-5, 3AP9-5, 4AP2-9, 5AP3-1, 7AP1-1, 7AP3-6, 7AP3-7, 8AP10-6

Anaesthesia, geriatric 2AP1-6, 8AP4-7

Anaesthesia, journals 1AP2-6

Anaesthesia, neurosurgical 3AP1-5, 7AP3-6

Anaesthesia, obstetric 11AP1-1, 11AP1-2, 11AP1-3, 11AP1-5

Anaesthesia, otolaryngological 10AP6-5

Anaesthesia, paediatric 3AP5-2, 10AP3-4, 10AP3-7

Anaesthetic techniques, bronchoscopy 12AP1-4, 19AP1-10

Anaesthetic techniques, conduction 3AP3-4

Anaesthetic techniques, endobronchial 3AP2-3

Anaesthetic techniques, extradural 8AP1-5

Anaesthetic techniques, fibreoptic 19AP1-1

Anaesthetic techniques, hypotensive 6AP3-1

Anaesthetic techniques, hypothermia 3AP1-8, 3AP2-2

Anaesthetic techniques, i.m. 9AP6-4

Anaesthetic techniques, induction 1AP1-8, 3AP8-11, 7AP1-8, 9AP5-9, 17AP1-5, 17AP2-2, 17AP3-5, 19AP1-8

Anaesthetic techniques, inhalation 3AP5-6, 9AP6-4

Anaesthetic techniques, insufflation 4AP6-4

Anaesthetic techniques, i.v. 3AP2-1, 10AP3-5, 14AP1-3

Anaesthetic techniques, i.v. regional 8AP9-8

Anaesthetic techniques, laryngoscopy 19AP4-6, 19AP5-10

Anaesthetic techniques, neuroleptanaesthesia 3AP5-6

Anaesthetic techniques, preoxygenation 11AP1-5

Anaesthetic techniques, regional 2AP1-7, 3AP3-3, 8AP4-8, 8AP9-3

Anaesthetic techniques, subarachnoid 11AP2-2, 11AP2-8

Anaesthetic techniques, topical 8AP5-8

Anaesthetic techniques, transdermal 3AP3-5, 8AP5-1

Anaesthetics gases 9AP3-1, 9AP3-2, 10AP1-2

Anaesthetics gases, cyclopropane 9AP6-4

Anaesthetics gases, nitrogen 12AP4-5

Anaesthetics gases, nitrous oxide 4AP9-3, 9AP3-1, 9AP3-2, 10AP1-2

Anaesthetics gases, trace concentrations 9AP6-5

Anaesthetics i.v., Althesin 19AP2-1

Anaesthetics i.v., etomidate 9AP3-9, 9AP5-5, 19AP2-6

Anaesthetics i.v., ketamine 8AP9-8, 12AP9-1

Anaesthetics i.v., methohexitone 19AP2-1

Anaesthetics i.v., metomidate

Anaesthetics i.v., pregnanolone

Anaesthetics i.v., propofol 3AP1-5, 9AP3-1, 9AP5-9

Anaesthetics i.v., stereoisomers 4AP6-7, 9AP3-7

Anaesthetics i.v., steroid 10AP6-4

Anaesthetics i.v., thiopentone 3AP3-8

Anaesthetics local 8AP5-1, 8AP9-2, 11AP2-2

Anaesthetics local, stereoisomers 8AP9-2
Anaesthetics volatile 3AP1-7

Anaesthetics volatile, atmospheric pollution 3AP8-3

Anaesthetics volatile, trace concentrations 9AP6-5

Anaesthetist, activity 19AP4-2

Anaesthetist, risks 1AP2-5

Analeptics 2AP1-3, 8AP2-2, 8AP9-2, 8AP9-3, 8AP9-10, 9AP2-9

Analgesia, obstetric 11AP1-3, 11AP2-4

Analgesia, paediatric 10AP6-1, 10AP6-7

Analgesia, patient-controlled 14AP5-4

Analgesia, postoperative 3AP6-5, 8AP1-8, 8AP5-3, 8AP5-5, 8AP9-9, 10AP6-2, 10AP6-6, 10AP6-9, 11AP2-10,14AP1-1, 14AP1-3, 14AP1-4, 14AP1-6, 14AP4-2, 14AP4-4, 14AP4-8, 14AP5-8, 14AP5-10, 15AP2-8

Analgesia, pre-emptive 14AP5-2, 14AP5-6

Analgesic techniques, buccal 4AP7-7

Analgesic techniques, extradural 11AP2-5

Analgesic techniques, i.m. 11AP2-8

Analgesic techniques, infiltration 8AP5-1

Analgesic techniques, infusion 2AP2-3, 14AP1-3

Analgesic techniques, intra-articular 14AP3-3

Analgesic techniques, nasal 14AP4-5, 14AP4-10

Analgesic techniques, neurolysis 3AP6-8

Analgesic techniques, regional, i.a. 2AP1-7

Analgesic techniques, subarachnoid 7AP2-7

Analgesic techniques, topical 8AP5-8

Analgesics anti-inflammatory, steroid 10AP6-2

Analgesics, non-opioid 14AP4-2

Analgesics opioid 3AP5-1, 14AP4-4

Analgesics opioid, addiction 14AP1-10

Analgesics opioid, morphine 9AP4-1, 14AP2-10, 14AP4-3, 14AP4-8, 14AP4-10, 14AP5-5, 14AP5-10

Analgesics, postoperative 10AP6-8, 11AP2-10, 14AP1-9, 14AP4-6

Anatomy, airway 19AP1-3

Anatomy, axilla 8AP3-8

Anatomy, brain 7AP4-5, 7AP5-2, 7AP5-9, 7AP6-1

Anatomy, jugular vein 7AP4-5

Anatomy, larynx 19AP5-4

Antagonists, benzodiazepine 1AP3-7

Antagonists, neuromuscular block 9AP2-6

Antagonists, opioid 9AP4-1

Antibiotics 7AP1-6, 12AP6-1, 12AP6-2, 12AP9-6, 17AP2-4

Anticonvulsants 9AP4-4, 14AP5-6

Arterial pressure 4AP9-2

Arterial pressure, drug effects 12AP5-2

Arterial pressure, hypertension 8AP6-5, 9AP8-5

Arterial pressure, hypotension 4AP9-2, 17AP2-6

Arterial pressure, measurement 8AP1-8

Arterial pressure, regulation automatic 3AP5-7, 3AP8-11

Arteries, aorta 4AP2-4

Arteries, cannulation 8AP10-5

Arteries, cerebral 7AP2-5

Assessment, preanaesthetic 1AP1-1, 8AP5-1, 8AP9-1, 17AP1-1, 17AP1-3

Asthma 19AP1-6

Blood, coagulation 10AP5-1

Blood, colloid osmotic pressure 6AP1-5

Blood, erythrocytes 8AP10-9, 9AP1-10

Blood, flow 12AP4-6

Blood, glucose 3AP5-3, 4AP5-6, 10AP5-4

Blood, haemodilution 7AP2-10

Blood, haemofiltration 12AP3-4

Blood, haemoglobin 3AP7-5, 4AP3-3, 4AP6-3, 6AP1-1, 6AP1-8, 6AP2-3, 6AP2-6, 6AP2-9, 6AP3-4, 6AP3-5, 6AP3-9, 12AP7-2, 12AP7-8

Blood, leucocytes 4AP4-5, 6AP3-6

Blood, loss 6AP3-4, 6AP3-9, 7AP1-7

Blood, lymphocytes 9AP4-6, 12AP5-6, ABT:12AP6-3, 14AP2-8

Blood, neutrophils 9AP1-10

Blood, PCV 5AP1-2, 5AP1-7

Blood, platelets 6AP1-7, 6AP1-8

Blood, salvage 6AP3-5, 7AP1-7

Blood, transfusion 6AP2-1, 6AP2-5, 6AP3-1, 6AP3-4, 6AP3-6, 6AP3-7, 6AP3-9, 6AP3-10, 10AP5-7

Blood, volume 4AP9-2, 12AP7-2

Brain, anaesthesia, molecular effects 5AP3-5, 7AP1-8, 7AP6-5 
Brain, anatomy 13AP3-2

Brain, blood-brain barrier 12AP4-6

Brain, blood flow 7AP4-5

Brain, carbon dioxide tension 3AP5-1

Brain, cerebellum 13AP3-2

Brain, cerebral cortex 13AP3-2

Brain, electroencephalography 3AP2-7, 3AP3-9, 3AP9-10, 3AP9-10,

7AP3-3, 9AP5-4, 10AP1-1, 10AP1-2, 10AP1-3

Brain, evoked potentials 3AP6-4, 3AP6-8, 7AP4-1, 7AP5-1

Brain, GABA 14AP2-11

Brain, hippocampus 7AP6-5

Brain, injury 7AP2-9, 7AP4-6, 7AP6-1

Brain, intracranial haemorrhage 7AP5-10, 7AP6-8

Brain, intracranial neoplasm 7AP1-7

Brain, intracranial pressure 7AP5-9

Brain, ischaemia 3AP5-7

Brain, magnetic resonance imaging 7AP2-6, 10AP3-4, 19AP3-4

Brain, metabolism 7AP3-4

Brain, oxygen consumption 3AP5-2

Brain, saccadic eye movements $3 A P 6-3$

British Journal of Anaesthesia 3AP1-8, 6AP1-9, 8AP7-3, 8AP8-5, 9AP4-10, 15AP2-3

\section{Burns 9AP2-2}

Calcium channel block 4AP5-2, 4AP5-3, 8AP2-4, 9AP8-3

Cancer 6AP1-9, 12AP2-1, 12AP6-8, 14AP3-2

Carbon dioxide, hypocapnia 7AP4-5

Carbon dioxide, measurement 12AP1-2

Carbon dioxide, partial pressure 3AP5-1

Carbon dioxide, ventilatory response 5AP1-2

Cardiorespiratory system 4AP6-7

Cerebrospinal fluid 8AP1-6, 8AP4-5

Chemotherapy, cancer 12AP8-8

Clinical trials 3AP8-3

Complications, abscess 14AP4-1

Complications, accidents 3AP5-7

Complications, acidosis 7AP4-3

Complications, adult respiratory distress syndrome 5AP2-1

Complications, airway obstruction 19AP2-8, 19AP4-2, 12AP1-1

Complications, alcohol abuse 3AP2-4

Complications, anaemia 6AP1-1

Complications, anaphylaxis 13AP3-6

Complications, anastomotic dehiscence 3AP8-7, 12AP7-1

Complications, anatomy 1AP2-3

Complications, aneurysm 7AP2-3

Complications, anoxia 4AP4-8

Complications, aortic coarctation 4AP2-4

Complications, aortic compression 5AP4-4

Complications, aortic rupture 7AP1-9

Complications, aortic valve disease 6AP1-7

Complications, apnoea 19AP3-7

Complications, arrhythmia 4AP4-2

Complications, arteriovenous malformation 7AP2-5, 7AP2-5, 7AP4-4

Complications, arthritis 8AP5-7

Complications, arytenoid dislocation 3AP8-10

Complications, asphyxia 11AP1-9

Complications, aspiration 8AP1-3

Complications, atelectasis 5AP4-2

Complications, backache 2AP1-4

Complications, barotrauma 12AP1-1, 19AP2-5

Complications, breath-holding 19AP3-7

Complications, bronchospasm 19AP4-4

Complications, burns 9AP2-2

Complications, carboxyhaemoglobinaemia 8AP5-8

Complications, carcinoid syndrome 6AP1-8

Complications, cardiac arrest 13AP2-3, 13AP2-5, 13AP2-8

Complications, catheter migration

Complications, catheter misplacement 3AP1-9

Complications, cerebral ischaemia 7AP6-8

Complications, chylothorax 1AP2-9

Complications, coagulopathy 7AP2-4

Complications, colitis 4AP6-3

Complications, coma 7AP1-9, 9AP5-9

Complications, compartment syndrome 12AP7-7

Complications, convulsions 9AP4-4

Complications, coronary vasospasm 4AP1-4, 4AP6-1

Complications, Crohn's disease 10AP3-6

Complications, death 4AP5-8
Complications, diabetes 3AP2-5

Complications, disseminated intravascular coagulation 12AP8-8

Complications, drug resistance 12AP6-2

Complications, drug tolerance 14AP1-1, 14AP1-4, 14AP2-2

Complications, dural puncture 8AP1-2

Complications, eclampsia 11AP1-9, 11AP1-10, 11AP2-9

Complications, embolism 4AP2-4

Complications, emphysema 5AP3-7

Complications, endotoxaemia 4AP9-2, 12AP5-2, 12AP5-5

Complications, extradural abscess 14AP4-1

Complications, extubation tracheal 9AP7-9, 12AP6-6, 19AP3-7

Complications, fistula 5AP4-2, 8AP2-7, 8AP5-7

Complications, gastric dilatation 19AP6-3, 19AP6-8

Complications, haematoma 7AP1-9, 7AP2-9, 8AP1-5, 8AP9-10, 10AP5-1

Complications, haemorrhage 3AP1-9, 4AP1-4, 14AP5-2

Complications, haemorrhagic disorder 7AP2-9, 7AP6-8, 7AP6-8

Complications, head injury 7AP2-9, 7AP3-8

Complications, headache 2AP1-4, 2AP1-4, 2AP1-6, 7AP2-4, 8AP1-2, 8AP4-6, 8AP6-3, 11AP1-2

Complications, heat stroke 9AP6-1, 9AP8-6

Complications, HELLP syndrome 11AP1-1, 11AP1-10

Complications, hepatic 3AP1-9, 17AP3-4

Complications, hyperalgesia 2AP2-1

Complications, hyperkalaemia 4AP1-3, 4AP1-5, 4AP1-7, 12AP7-2

Complications, hypertension 17AP2-6

Complications, hyperthermia 9AP6-1, 9AP8-6

Complications, hypervolaemia 8AP4-2, 8AP6-5

Complications, hypoglycemia 10AP5-4, 14AP3-7, 14AP3-7

Complications, hyponatraemia 10AP5-2

Complications, hypotension 1AP3-2, 1AP3-2, 3AP8-5, 4AP1-2, 4AP2-9, 4AP9-2, 6AP3-1, 7AP2-3, 7AP6-8, 8AP2-6, 8AP4-1, 8AP4-1

Complications, hypothermia 4AP9-6, 9AP7-9, 9AP7-9, 13AP2-3, 13AP2-3, 13AP2-3

Complications, hypoventilation 3AP5-1, 5AP1-7, 17AP3-6

Complications, hypovolaemia 3AP4-1, 4AP6-8, 12AP7-3

Complications, hypoxia BAPC1-4, 4AP5-7, 4AP9-8, 5AP2-1, 5AP2-2, 5AP2-4, 10AP2-6, 12AP1-7, 12AP1-9, 17AP3-6, 19AP4-4

Complications, infections 6AP3-6, 8AP3-2, 10AP4-5, 12AP6-8

Complications, instrumental delivery 12AP8-4

Complications, intubation endobronchial 3AP2-3

Complications, intubation nasotracheal 19A92-4, 19AP2-7, 19AP3-8

Complications, intubation tracheal 19AP1-1, 19AP1-2, 19AP3-5, 19AP5-2

Complications, laryngospasm 10AP2-5

Complications, liver disease 3AP5-9, 12AP4-7

Complications, malignant hyperthermia 9AP6-1, 9AP8-6

Complications, masseter spasm 8AP1-8

Complications, meningitis 7AP1-2

Complications, methaemoglobinaemia 8AP5-8

Complications, morbidity 3AP1-9, 19AP6-5

Complications, mucosal damage 4AP2-3

Complications, multiple organ dysfunction syndrome 12AP3-5, 12AP7-2, 19AP4-4

Complications, muscular dystrophy 9AP5-3

Complications, myalgia 14AP3-6, 14AP3-7

Complications, myasthenia gravis 3AP2-4

Complications, myasthenic syndrome 12AP9-5

Complications, myocardial infarction 4AP5-6

Complications, myotonic dystrophy 9AP5-3

Complications, neurological 13AP2-3, 4AP5-8, 6AP2-4, 6AP2-4, 7AP1-2, 8AP6-3, 8AP6-8

Complications, neuromuscular disease 12AP9-5

Complications, neuropathy 14AP1-2

Complications, obesity 5AP1-2

Complications, obstructive airways disease 5AP1-5

Complications, obstructive sleep apnoea 19AP4-3

Complications, oedema 9AP4-4, 11AP1-10

Complications, pacemakers 4AP7-6

Complications, pharyngeal obstruction 3AP3-6

Complications, pneumothorax 1AP2-3, 12AP8-8

Complications, postoperative desaturation 1AP2-2

Complications, prolonged QT syndrome 4AP3-8, 4AP6-7, 8AP8-1

Complications, pruritus 2AP1-6, 8AP6-3, 8AP7-2

Complications, pulmonary 8AP7-2, 14AP5-10

Complications, pulmonary hypertension BAPC1-4, 5AP2-4, 12AP5-2

Complications, pulmonary oedema 11AP1-10

Complications, regurgitation 3AP7-4, 10AP2-6, 19AP4-4, 19AP6-5, 19AP6-8, 19AP6-9, 19AP6-10

Complications, renal 1AP2-9, 4AP5-5, 12AP7-7 
Complications, respiratory 8AP8-2, 12AP7-7

Complications, shivering 8AP1-5, 14AP5-8, 14AP5-8

Complications, smokers 1AP3-1

Complications, sore throat 19AP6-8

Complications, spinal injury 7AP2-1

Complications, spontaneous excitatory movements 2AP2-3

Complications, stenosis 4AP5-7, 8AP1-6, 8AP5-7

Complications, strabismus 10AP4-4, 10AP4-6, 10AP5-4

Complications, stridor 19AP1-6, 19AP2-8, 19AP5-3

Complications, tension pneumothorax 1AP2-3, 12AP8-8

Complications, thrombosis 6AP2-9

Complications, torticollis

Complications, tracheal compression 19AP4-3

Complications, trauma 7AP2-9, 7AP4-6, 7AP5-10

Complications, ultrasound 8AP2-2, 8AP3-4, 8AP8-7, 8AP9-2, 10AP6-3, 12AP7-6

Complications, vasoconstriction 4AP9-8, 5AP2-1, 5AP2-2, 9AP8-5, 9AP8-5

Complications, venous admixture 5AP3-5

Crohn's disease 10AP3-6

Diabetes 4AP9-4

Donors, organ transplantation 9AP8-9

Drug delivery 10AP2-2

Drug delivery, bolus 10AP3-5, 11AP2-8, 13AP4-2

Drug delivery, buccal 4AP7-7

Drug delivery, computerized 4AP6-7

Drug delivery, infusion 6AP1-2, 9AP5-5, 14AP1-4, 14AP2-6

Drug delivery, nasal 3AP2-4

Drug delivery, oral 14AP1-2, 15AP2-8

Drug delivery, priming 19AP2-2

Drug delivery, rectal 1AP3-7

Drug delivery, subcutaneous 12AP5-5

Drug delivery, sublingual 2AP1-3

Drug delivery, temperature 3AP2-2

Drug delivery, transdermal 3AP3-5, 8AP5-1

Drug delivery, volume 8AP9-8

Education 1AP3-8, 2AP2-4, 6AP3-3, 8AP3-3, 11AP1-6, 14AP5-7, 15AP1-4, 15AP2-3, 15AP2-5, 15AP2-8, 15AP2-9, 17AP2-1

Education, ambulance personnel 15AP2-8

Education, continuing 15AP1-2

Education, medical students 15AP1-4, 15AP1-6, 15AP2-1, 15AP2-1

Education, untrained personnel 14AP1-2

Electrical system, burns 19AP1-9

Embolism, air 8AP7-3

Embolism, fat 11AP1-9

Enzymes, acetylcholinesterase 7AP6-5, 9AP7-4

Enzymes, angiotensin converting, inhibition 4AP5-2, 4AP5-3

Enzymes, plasma cholinesterase 11AP2-10

Enzymes, creatine kinase 4AP3-6, 9AP8-1

Enzymes, cyclo-oxygenase BAPC1-3, 5AP2-3

Enzymes, cytochrome b5 reductase 4AP8-3, 14AP2-9

Enzymes, cytochrome P450 4AP8-3, 14AP2-9

Enzymes, glutathione S-transferase BAPC1-6, 12AP5-8

Enzymes, hyaluronidase 8AP7-7

Enzymes, methionine synthase BAPC1-1, 4AP4-2, 9AP1-4

Enzymes, phospholipase C 5AP4-7

Enzymes, tryptase 14AP1-1, 17AP2-4

Epilepsy 7AP3-4, 7AP4-3, 7AP4-4

Equipment, airway 19AP3-1

Equipment, alarms 3AP4-1

Equipment, anaesthesia machines 3AP8-11, 3AP9-2

Equipment, breathing systems $3 \mathrm{AP} 1-7$

Equipment, bronchoscopes 19AP2-5, 19AP4-10, 19AP5-5

Equipment, calibration 3AP7-8, 4AP2-8, 12AP8-3, 19AP3-9

Equipment, cannulae intravascular 9AP3-4

Equipment, cannulae nasal 3AP8-10

Equipment, catheter guides 14AP2-6

Equipment, catheters gas injection 19AP2-5

Equipment, catheters subarachnoid 9AP1-6

Equipment, catheters suction 3AP1-4

Equipment, circulatory support devices 4AP7-5

Equipment, collars 13AP1-5

Equipment, Combitube 13AP1-5

Equipment, computers 1AP1-6, 1AP2-8, 3AP2-2, 3AP6-10, 3AP8-10

Equipment, cuffs endobronchial 3AP2-3

Equipment, cuffs tracheal 10AP2-1
Equipment, Dinamap

Equipment, electrodes 3AP6-10, 3AP8-11, 9AP2-3, 9AP7-7

Equipment, Engstrom Metabolic Computer

Equipment, extracorporeal circulation 6AP1-7, 12AP9-3

Equipment, filters 3AP1-7, 3AP8-2, 3AP8-6

Equipment, gas analysers 10AP3-3, 12AP7-8

Equipment, gas exchangers, i.v. $12 \mathrm{AP} 1-3$

Equipment, hearts artificial 9AP7-6

Equipment, heat-moisture exchangers 14AP2-5

Equipment, helicopters 13AP3-5

Equipment, infusion systems 9AP5-6

Equipment, infusion systems, patient-controlled

Equipment, laryngoscopes 19AP1-2, 19AP1-4, 19AP1-8, 19AP1-9, 19AP5-3, 19AP5-8, 19AP5-9

Equipment, laryngoscopes fibreoptic 19AP1-1

Equipment, lasers 4AP7-8, 19AP2-5

Equipment, masks anaesthesia 3AP9-2

Equipment, masks surgical 2AP1-9

Equipment, mass spectrometers 8AP10-1

Equipment, models 3AP8-11

Equipment, monitors 2AP1-2, 3AP4-1, 3AP4-2, 3AP5-1, 3AP5-3, 3AP5-7, 7AP3-4, 12AP7-9, 12AP7-10

Equipment, myographs 4AP9-3

Equipment, needles 8AP7-1, 11AP2-2

Equipment, oesophageal balloon catheters 5AP3-4

Equipment, pacemakers 4AP7-6

Equipment, pulse oximeters 3AP5-8, 4AP2-7, 7AP4-8, 10AP1-5, 10AP1-6, 10AP3-3

Equipment, scavenging devices BAPC1-5, 3AP8-3, 4AP3-6, 4AP4-4, 7AP5-6, 7AP6-6

Equipment, sensors 3AP6-2, 3AP8-10

Equipment, sensors fibreoptic 19AP5-6

Equipment, standardization 12AP8-5

Equipment, stimulators 8AP9-3

Equipment, temperature probes $3 A P 1-8$

Equipment, tourniquets 8AP4-1, 8AP9-8, 14AP5-9

Equipment, transducers 3AP1-2, 3AP3-6, 8AP3-4, 8AP8-7, 8AP10-5, 9AP7-9, 9AP8-2

Equipment, tubes double-lumen 3AP2-3, 5AP4-5, 19AP3-2

Equipment, tubes endobronchial $3 \mathrm{AP} 2-3$

Equipment, tubes nasopharyngeal 19AP6-8, 19AP6-9

Equipment, tubes suction 3AP1-4, 13AP1-5, 19AP4-3, 19AP6-3, 19AP6-4, 19AP6-4, 19AP6-4

Equipment, tubes tracheal 3AP3-6, 10AP2-5, 10AP2-7

Equipment, tubes tracheostomy $12 \mathrm{AP} 6-6$

Equipment, ultrasound machines 12AP7-6

Equipment, valves 3AP8-3

Equipment, ventilators BAPC1-3, 5AP2-3, 12AP1-3, 12AP6-7, 12AP9-7, 17AP3-1

Equipment, warming devices $3 \mathrm{AP} 1-6$

Ethanol 13AP3-2

Eye, cataract $8 \mathrm{AP} 7-1$

Eye, orbicularis oculi 11AP1-1

Facioscapulohumeral muscolar dystrophy 9AP5-3

Fatigue 5AP2-7, 5AP2-8

Fetus 7AP6-6

Fluids, i.v. 10AP5-2, 10AP6-5

Fluids, oral 6AP3-9

Forensic medicine

Formulations, bupivacaine 3AP2-3, 3AP5-1, 4AP3-5, 8AP1-7, 8AP2-4, 8AP4-3, 8AP5-5, 8AP6-5, 8AP6-7, 8AP9-9, 8AP10-1, 8AP10-4, 10AP6-4, 11AP2-5, 11AP2-11, 14AP4-9

Formulations, diazepam 9AP6-4, 14AP5-11

Formulations, diluents 8AP6-7

Formulations, etomidate 9AP3-9, 9AP5-5, 19AP2-6

Formulations, liposomes 6AP1-2

Formulations, osmolality 7AP5-9, 7AP5-10

Formulations, $\mathrm{pH}$ 5AP2-6, 5AP2-8, 6AP2-6, 8AP4-8, 9AP1-2, 11AP2-6, 13AP2-5, 17AP3-6, 19AP2-8

Formulations, stability $8 \mathrm{AP} 4-3$

Formulations, temazepam 1AP3-7, 3AP2-4, 9AP3-3, 9AP7-4, 19AP1-6

Gastrointestinal tract 10AP2-6

Gastrointestinal tract, emptying 10AP2-6

Gastrointestinal tract, endoscopy 3AP9-1

Gastrointestinal tract, lower oesophageal sphincter 10AP3-5, 10AP6-8

Gastrointestinal tract, mucosal perfusion 8AP8-9 
Gastrointestinal tract, oesophageal contractility 10AP3-5, 10AP6-8 Gastrointestinal tract, pH 6AP2-6

Gastrointestinal tract, reflux 10AP3-5, 10AP6-8, 19AP2-3

Genetic factors 4AP4-8

Genetic factors, hyperthermia 9AP6-1

Genetic factors, plasma/cholinesterase 9AP2-2

Head, injury 7AP2-9, 7AP3-8

Heart, angiography $1 \mathrm{AP} 3-5$

Heart, arrhythmia 4AP4-2

Heart, arrhythmia, electroversion 4AP4-2

Heart block, calcium channel blockers 4AP5-2, 4AP5-3

Heart, blood flow, myocardial 4AP9-4

Heart, cardiac massage 13AP2-2

Heart, cardiac output 3AP7-9, 3AP7-10, 4AP2-8, 10AP1-5, 10AP1-6

Heart, cardiopulmonary bypass 4AP2-7, 4AP4-5, 4AP5-1, 4AP5-4, 4AP8-5, 4AP8-6

Heart, catheterization $1 \mathrm{AP} 2-3$

Heart, conduction 4AP4-2

Heart, coronary artery bypass 3AP2-5, 3AP8-7, 4AP2-8, 4AP3-7, 4AP7-9, 8AP8-2, 12AP9-3

Heart, coronary occlusion 4AP4-6, 4AP9-4

Heart, dobutamine 4AP8-6, 4AP9-6

Heart, dopamine 7AP5-8, 9AP4-2

Heart, enoximone 12AP6-2

Heart, esmolol 14AP5-5

Heart, heart rate 4AP2-9, 4AP3-8, 4AP7-6, 9AP5-10

Heart, ischaemia 1AP2-2, 3AP4-2, 4AP3-5, 4AP6-6, 8AP8-8

Heart, isolated preparation 4AP4-1, 4AP9-8

Heart, isoprenaline 9AP2-6

Heart, metabolism 4AP4-2, 12AP1-7

Heart, milrinone 4AP7-2

Heart, myocardial function 4AP6-1

Heart, myocardial preservation technique 4AP4-8

Heart, myocytes BAPC1-2, 12AP4-2

Heart, myopathy 4AP9-7, 12AP9-4

Heart, nitroglycerin 4AP7-5

Heart, pacemakers artificial 4AP7-6

Heart, pindolol 4AP9-7

Heart, preservation 4AP4-8

Heart, propranolol 9AP2-6

Heart, resuscitation 13AP2-2, 13AP2-4, 13AP3-3

Heart, sinoatrial node 4AP4-5, 12AP8-2

Heart, sodium nitroprusside 4AP9-8

Heart, tamponade 3AP8-5

Heart, transplantation 4AP2-5

Heart, ventricles BAPC1-2, 4AP6-1, 12AP4-2

HELLP syndrome 11AP1-1, 11AP1-10

Histamine 9AP7-6

History, anaesthesia 8AP4-6, 9AP6-6

History, anaesthesia, local 8AP5-1, 8AP5-7, 8AP6-1, 8AP7-7, 8AP9-2, 11AP2-2

Hormones, adrenal 14AP3-7

Hormones, adrenocorticotrophic 14AP3-7

Hormones, antidiuretic 13AP2-4

Hormones, atrial natriuretic peptide 4AP8-7

Hormones, corticosteroid 7AP1-6

Hormones, glucocorticoid 9AP6-7, 12AP5-1

Hormones, insulin BAPC1-2, 3AP4-8, 8AP4-4, 12AP4-2, 12AP4-3

Hormones, somatostatin 10AP3-6

Humidification 3AP8-6

Hyperthermia, postoperative 9AP6-1, 9AP8-6

Hypnotics barbiturate, pentobarbitone 9AP2-6

Hypnotics benzodiazepine 19AP1-6

Hypothermia 3AP2-2, 13AP2-3

Hypoxaemia 11AP1-5

Hypoxia BAPC1-4, 4AP5-7, 5AP2-1, 5AP2-4, 12AP1-7, 12AP1-9, 17AP3-6

Immune response 9AP4-6, 12AP5-4

Induction, anaesthesia 1AP1-8, 3AP1-1, 3AP2-10, 3AP3-8, 3AP4-3, 3AP4-7, 3AP5-4, 3AP6-8, 3AP7-1, 3AP8-11, 3AP9-3, 3AP9-10, 4AP3-8, 4AP7-4, 5AP1-3, 7AP1-8, 7AP5-10, 9AP2-10, 9AP3-4 9AP3-6, 9AP3-9, 9AP5-5, 9AP5-6, 9AP5-9, 9AP7-6, 10AP3-4, 10AP5-4, 10AP6-3, 11AP1-5, 17AP1-3, 17AP1-5, 17AP2-2,

17AP3-5, 19AP1-8, 19AP2-6, 19AP4-5

Induction, enzymes 4AP8-6

Infants 8AP7-8, 9AP7-5, 10AP1-3, 10AP2-3

Infection, bacterial 12AP9-5
Infection, central nervous system 8AP8-5, 8AP9-9, 8AP10-11, 8AP3-2 Infection, nosocomial 6AP3-7

Infection, pulmonary 8AP10-11, ABT:12AP6-3

Infection, tetanus 12AP9-5

Infection, upper respiratory tract 12AP8-9

Infusions 4AP7-9, 12AP2-3, 12AP3-8, 12AP6-4

Infusions, i.v. 12AP5-3, 14AP1-3

Intensive care BAPC1-6, 1AP2-8, 5AP2-3, 7AP1-6, 12AP2-4, 12AP2-5, 12AP2-7, 12AP5-8, 12AP8-1, 12AP8-3, 12AP8-4

Intensive care, infections 12AP3-7

Intensive care, sedation 12AP9-1

Interactions (drug) 9AP1-5, 9AP4-4, 9AP4-9, 9AP8-3

Intubation endobronchial 3AP2-3

Intubation endobronchial, complications 19AP4-4

Intubation nasotracheal, complications 19AP2-4

Intubation nasotracheal, technique 19AP2-4, 19AP2-7, 19AP3-8

Intubation tracheal 3AP3-8, 3AP5-4, 4AP7-2, 9AP2-10, 9AP3-6, 13AP1-5, 19AP2-4, 19AP5-2, 19AP5-6

Intubation tracheal, complications 19AP4-4

Intubation tracheal, difficult 19AP1-1, 19AP3-9

Intubation tracheal, extubation 12AP6-6, 19AP3-7

Intubation tracheal, technique 13AP3-4, 19AP1-2, 19AP6-5

Intubation tracheal, training

lons, calcium 8AP10-3 , 9AP1-9, 9AP8-6

lons, chloride BAPC1-1, 9AP1-4

lons, intracellular 9AP1-10, 9AP8-5

lons, ion channels BAPC1-5, 4AP3-6, 4AP5-2, 4AP5-3, 7AP5-6, 8AP10-3

lons, ion channels, ligand-gated 9AP8-2

lons, ion channels, voltage-gated 5AP2-2

Ions, lithium 13AP4-2, 13AP4-3

lons, magnesium 7AP2-8, 7AP5-8, 14AP1-5

lons, potassium BAPC1-5, 4AP8-3, 5AP2-2, 7AP5-6, 14AP2-4

lons, sodium 8AP10-3, 10AP4-4, 12AP4-7, 12AP5-1, 14AP2-4, 14AP2-7

Kidney, anephric patient 1AP2-9, 12AP9-6

Kidney, blood flow 3AP7-5

Kidney, diuretics 4AP5-2, 12AP7-3

Kidney, failure 4AP1-3, 4AP1-5, 4AP1-8, 4AP6-6, 6AP3-9, 9AP7-7

Kidney, filtration, glomerular 3AP7-5

Kidney, function 6AP3-9, 9AP7-1, 12AP5-1, 12AP5-3, 12AP5-4, 13AP4-2

Kidney, lithotripsy 1AP2-9

Kidney, metabolism 3AP5-2

Kidney, transplantation 1AP2-9, 12AP9-6

Kidney, urine 10AP6-7, 12AP5-1

Larynx, cricoid pressure 3AP8-10, 19AP2-4

Larynx, damage 19AP5-3

Larynx, laryngoscopy 19AP1-8, 19AP1-9, 19AP1-10, 19AP2-1, 19AP2-5 19AP4-6, 19AP4-7, 19AP4-8, 19AP5-2, 19AP5-3 19AP5-4, 19AP5-7, 19AP5-8, 19AP5-9, 19AP5-10

Larynx, laryngoscopy fibreoptic 19AP1-8, 19AP3-1, 19AP5-5

Larynx, vocal cords 19AP5-4, 19AP5-6

Liver, bile 12AP7-10

Liver, blood flow 3AP5-2, 4AP8-5, 4AP8-6

Liver, cirrhosis 12AP4-7

Liver, damage 3AP1-9, 4AP8-2

Liver, disease 3AP5-9, 3AP7-6, 3AP7-10, 4AP8-2, 12AP4-7, 12AP7-10, 12AP8-8

Liver, function 12AP4-1

Liver, hepatitis 12AP3-6, 17AP3-4

Liver, metabolism 3AP1-9, 12AP9-6

Liver, oxygen consumption 3AP1-9

Liver, transplantation 3AP1-8, 3AP5-9, 4AP6-6, 6AP2-10, 12AP4-7, 12AP9-6

Lung, adult respiratory distress syndrome 5AP2-1, 5AP2-2, 12AP1-1

Lung, atelectasis 5AP4-2, 12AP1-4

Lung, bronchus 19AP3-2, 19AP4-9

Lung, damage 12AP1-6

Lung, deadspace 12AP1-1, 12AP1-2

Lung, function 8AP7-2

Lung, functional residual capacity 5AP3-6

Lung, gas exchange 5AP1-3, 5AP4-2, 12AP1-2, 12AP1-3

Lung, hyperoxia 5AP3-2

Lung, hypoxia BAPC1-4, 5AP2-1, 5AP2-2

Lung, interpleural space 8AP3-1, 8AP8-2

Lung, lavage BAPC1-3, 5AP2-3, 12AP1-6

Lung, mechanics 12AP1-6

Lung, model 5AP1-7 
Lung, oedema 11AP1-10

Lung, pathophysiology BAPC1-4, 5AP2-4, 5AP4-1

Lung, pneumothorax 12AP8-8

Lung, respiratory distress syndrome 5AP2-1, 5AP2-2, 12AP1-1

Lung, respiratory resistance $5 \mathrm{AP} 3-1$

Lung, shunting 12AP1-3

Lung, surfactant $5 A P 1-1$

Lung, tidal volume BAPC1-3, 5AP1-7, 5AP2-3, 5AP4-5, 12AP1-6 12AP2-8

Lung, transplantation 5AP2-5

Lung, vasculature 5AP4-1, 12AP7-2

Lung, volume 5AP1-3, 19AP3-10

Malignant hyperthermia 9AP6-1, 9AP8-6

Measurement techniques, accelography 9AP2-1, 9AP2-5, 9AP2-7, 9AP2-8, 9AP7-9

Measurement techniques, airway obstruction 19AP1-6, 19AP4-3, 19AP5-4

Measurement techniques, airway reflexes 19AP2-6, 19AP5-4

Measurement techniques, airway resistance 5AP4-5, 19AP2-6

Measurement techniques, autoradiography 13AP1-1

Measurement techniques, calorimetry 3AP5-2, 5AP3-2

Measurement techniques, cardiac output 3AP8-7, 4AP2-8, 4AP2-8, 8AP6-5 10AP1-5, 10AP1-6

Measurement techniques, chemoluminescence 12AP9-8

Measurement techniques, chromatography 3AP1-5, 9AP1-2

Measurement techniques, coagulation 3AP5-9, 10AP5-1

Measurement techniques, contracture test for MH 14AP5-9

Measurement techniques, Doppler echocardiography BAPC1-4, 5AP2-4

Measurement techniques, drug concentration 3AP4-5, 9AP1-1

Measurement techniques, elastance 3AP1-1, 3AP1-2, 4AP9-5

Measurement techniques, electrodes, impedance 3AP6-10

Measurement techniques, electromyography 3AP6-8

Measurement techniques, electrophysiology 4AP3-1, 14AP2-11

Measurement techniques, endoscopy 3AP9-1, 9AP3-3, 19AP1-3

Measurement techniques, energy expenditure 5AP3-2

Measurement techniques, fibreoptic 19AP1-3, 19AP1-10, 19AP3-1, 19AP5-5

Measurement techniques, Fick principle 10AP1-5, 10AP1-6

Measurement techniques, flow velocity waveform analysis 12AP3-3

Measurement techniques, gas exchange 8AP7-2, 12AP1-6

Measurement techniques, ions, calcium 8AP10-3

Measurement techniques, lung shunting 12AP1-3

Measurement techniques, mass spectroscopy 3AP1-5, 9AP2-7

Measurement techniques, mechanomygraphy 12AP9-5

Measurement techniques, microdialysis 7AP3-4, 7AP3-5, 7AP5-8, 12AP7-1

Measurement techniques, neuromuscular block 3AP3-4, 3AP6-6, 9AP2-1, 9AP2-3, 9AP2-4, 9AP2-5, 9AP2-7

Measurement techniques, nuclear magnetic resonance 10AP3-4

Measurement techniques, oximeters 3AP5-2, 3AP5-8, 3AP6-7, 3AP8-7, 4AP2-7, 7AP4-8, 10AP1-6, 10AP3-3

Measurement techniques, partition coefficients 9AP1-3

Measurement techniques, plethysmography 3AP5-4, 3AP6-5, 3AP7-1

Measurement techniques, pleural pressure 8AP3-1, 8AP8-2

Measurement techniques, pneumotachography 3AP8-10

Measurement techniques, radioimmunoassay BAPC1-4, 5AP2-4

Measurement techniques, respiratory acoustical impedance 5AP3-1

Measurement techniques, respiratory compliance 12AP1-1

Measurement techniques, skin conductance 3AP3-5

Measurement techniques, spectral analysis 9AP3-1

Measurement techniques, thermodilution 3AP7-10, 4AP2-5, 5AP4-1, 8AP3-5

Measurement techniques, thoracic impedance cardiograph 4AP4-2, 4AP7-6

Measurement techniques, thrombelastography 6AP1-8

Measurement techniques, transthoracic electrical impedance 4AP4-2

Measurement techniques, ultrasound 1AP2-7, 7AP5-10, 8AP2-2, 8AP3-4, 8AP3-8, 8AP8-7, 8AP9-2, 10AP6-3, 12AP7-6, 19AP3-2

Measurement techniques, ventilation volumes 12AP6-6

Measurement techniques, visual analogue scale 2AP2-1, 8AP4-4, 8AP8-8, 10AP6-5, 14AP1-6, 14AP1-10, 14AP5-7

Medico-legal 17AP1-3

Membrane, cell 9AP1-1, 14AP2-4

Membrane, erythrocyte 4AP7-8, 6AP1-8

Membrane, nerve 14AP2-4

Memory 3AP6-6, 7AP2-6, 7AP6-4

Metabolism, DNA 9AP1-5, 9AP8-1
Metabolism, endocrine 8AP7-9

Metabolism, energy expenditure 5AP3-2

Metabolism, fasting 6AP3-9, 8AP4-4, 8AP6-6

Metabolism, folic acid 12AP2-5

Metabolism, free radicals BAPC1-5, 4AP4-4, 4AP8-3

Metabolism, glucose 7AP3-4, 7AP3-5

Metabolism, glycine BAPC1-5, 9AP1-4

Metabolism, hyperglycaemia 1AP3-4, 4AP5-8

Metabolism, lactate 7AP3-4, 7AP3-5

Metabolism, leucine 9AP1-4

Metabolism, metabolites 10AP3-5

Metabolism, methionine 4AP4-2

Metabolism, oxygen consumption 3AP5-2

Metabolism, respiratory quotient 5AP3-2

Metabolism, second messengers 4AP3-6, 4AP9-7

Metabolism, thymidine 9AP8-1

Methaemoglobinaemia 8AP5-8

Microcirculation 4AP6-2, 4AP6-9, 4AP8-8, 8AP8-9, 12AP3-3

Model, acute pain service 14AP4-1

Model, animal BAPC1-2, 4AP2-5, 4AP2-6, 5AP2-1, 8AP10-5, 8AP10-6, 8AP10-11, 12AP4-2, 14AP2-3

Model, blood-brain barrier permeability 8AP10-9, 9AP1-10

Model, brainstem-spinal cord preparation 7AP1-8

Model, breathing systems 3AP8-6

Model, cardiomyopathy 4AP9-7, 12AP9-4

Model, computer simulation 9AP5-8, 13AP1-5, 17AP1-4

Model, heart 4AP4-1, 4AP9-8

Model, jet ventilation 19AP2-5, 19AP2-8

Model, liver 4AP8-3, 12AP4-7

Model, lung 4AP8-9, 5AP1-7

Model, mathematical 3AP4-8, 19AP4-7

Model, neuropathy 9AP4-3, 14AP2-6

Model, pharmacodynamic 3AP9-2

Model, pharmacokinetic 3AP8-11, 3AP9-2, 3AP9-3, 9AP5-4

Model, pharmaco-physiological 9AP5-8

Model, pulmonary aspiration 8AP8-7, 17AP3-4

Model, respiratory failure $3 \mathrm{AP} 6-2$

Model, sodium channel 8AP10-3, 12AP4-7

Model, trachea 3AP3-6

Monitoring, anaesthetist activity 1AP1-1, 1AP3-8, 17AP1-3

Monitoring, angiography 1AP3-5, 7AP1-9

Monitoring, apnoea 19AP3-7

Monitoring, arterial pressure 3AP2-1, 3AP5-7, 3AP8-11, 8AP4-1

Monitoring, carbon dioxide 3AP5-1, 4AP3-8

Monitoring, cardiopulmonary 4AP2-7

Monitoring, computerized 3AP5-7, 4AP6-7

Monitoring, depth of anaesthesia 3AP6-8, 3AP8-11, 5AP3-5, 10AP1-4 10AP1-7

Monitoring, echocardiography 12AP7-9

Monitoring, electrocardiography 3AP4-2

Monitoring, electroencephalography 3AP3-7, 3AP8-11

Monitoring, evoked potentials 3AP6-1, 7AP5-1

Monitoring, extracorporeal circulation 6AP1-7, 12AP3-4

Monitoring, interstitial perfusion pressure 5AP2-2, 5AP4-1

Monitoring, intracranial pressure 7AP1-6, 7AP3-8

Monitoring, intraoperative 7AP5-1

Monitoring, motion artefact 3AP4-1

Monitoring, neuromuscular function 3AP6-9

Monitoring, oxygen 4AP2-7, 7AP4-8

Monitoring, radiological 10AP2-4

Monitoring, respiratory sinus arrhythmia 8AP8-1

Monitoring, spirometry 5AP3-8

Monitoring, sympathetic block $3 \mathrm{AP} 3-4$

Monitoring, temperature 3AP1-8, 7AP6-9

Monitoring, ultrasound 19AP3-2

Monitoring, vapours 3AP9-10

Monitoring, ventilation 5AP1-3

Muscle cardiac 4AP3-8, 4AP9-7

Muscle cardiac, contractility BAPC1-2, 3AP7-2, 5AP2-8, 12AP4-2

Muscle respiratory 12AP1-9

Muscle respiratory, fatigue 5AP2-6, 5AP2-7, 5AP2-8

Muscle skeletal 8AP10-4, 9AP6-1, 9AP8-6

Muscle skeletal, blood flow 6AP1-2

Muscle skeletal, contractility 5AP2-6, 5AP2-7

Muscle skeletal, diaphragm 5AP2-6

Muscle skeletal, relaxation 3AP1-4, 3AP3-4, 9AP3-7, 19AP5-2

Muscle vascular, pharmacology 9AP8-5

Muscle vascular, responses 4AP7-4, 7AP4-8, 9AP3-4 
Myasthenia gravis 3AP2-4

Myasthenic syndrome 3AP2-4

Myotonia dystrophica 9AP8-6

Neonates 8AP5-8, 9AP5-1, 10AP2-3

Nerve, damage (postoperative) 17AP1-5

Nerve-muscle preparations 9AP8-2

Nerve, neurotransmitters 7AP5-8, 14AP5-6

Nerve, regeneration 8AP10-10

Nerve, somatic 8AP3-4

Nerve, synaptosome 9AP1-9

Nerve, transmission 7AP5-2, 14AP1-1

Nerve, trigeminal 3AP6-8

Neuromuscular block, antagonism 14AP1-1

Neuromuscular block, atracurium 3AP3-4, 9AP2-3

Neuromuscular block, mivacurium 9AP2-2

Neuromuscular block, pancuronium 9AP2-1

Neuromuscular block, propofol 3AP6-6

Neuromuscular block, recovery 9AP2-3

Neuromuscular block, resistance 9AP2-2

Neuromuscular block, rocuronium 3AP6-6, 8AP7-8, 9AP2-4, 9AP2-5, 9AP2-6, 9AP2-7, 9AP7-2, 9AP7-3, 9AP7-5, 9AP7-10, 9AP8-2

Neuromuscular block, suxamethonium 9AP2-7

Neuromuscular block, vecuronium 9AP2-1, 9AP2-5, 9AP2-6, 9AP2-7, 9AP7-3, 9AP7-8

Neuromuscular function, synapses 7AP5-2

Neuromuscular relaxants, dantrolene 3AP6-1, 3AP6-9

Neuromuscular transmission 3AP6-9, 9AP8-3, 12AP9-5, 19AP2-2

Non-steroidal anti-inflammatory drugs 10AP6-2, 11AP2-1, 13AP4-4

Operating rooms, contamination 12AP6-4

Operating rooms, exhaust systems 17AP3-6

Operating rooms, personnel 1AP1-9

Organizations, European Community 1AP2-6

Organizations, Royal College of Anaesthetists 10AP5-2

Oxygen, consumption 3AP5-2, 4AP7-2, 5AP3-2, 12AP1-7

Oxygen, delivery systems 4AP3-3, 6AP1-2, 6AP1-4, 9AP2-4

Oxygen, inspired concentration 5AP3-6

Oxygen, measurement 3AP6-2

Oxygen, saturation 3AP5-1, 3AP6-2, 3AP6-7, 3AP8-7, 4AP2-7, 7AP2-10, 7AP4-8, 12AP7-8

Oxygen, therapy 12AP9-8

Oxygen, tissue 6AP1-2

Oxygen, transport 6AP1-2, 6AP1-4, 8AP8-6, 9AP2-4, 12AP7-8

Pain 2AP2-1, 2AP2-2, 2AP2-7, 3AP5-5, 3AP6-5, 3AP9-10, 8AP1-8, 8AP5-18AP5-2, 8AP9-9, 10AP4-7, 10AP6-4, 11AP1-4,

11AP2-1, 14AP1-10, 14AP3-1, 14AP3-9, 14AP5-4, 15AP2-5

Pain, acute 14AP2-1, 14AP3-2, 14AP4-1

Pain, cannulation 1AP2-3

Pain, chronic 3AP5-5, 14AP2-10, 14AP3-2, 14AP3-4, 14AP3-9

Pain, injection 9AP3-9, 9AP5-6

Pain, mechanism 14AP2-1, 14AP3-1

Pain, neuropathic 9AP4-1, 9AP4-3, 14AP2-3, 14AP2-4, 14AP2-6 14AP2-9, 14AP3-8

Pain, paediatric 10AP6-7

Pain, postoperative 2AP2-1, 2AP2-2, 2AP2-3, 6AP3-2, 8AP4-9, 8AP7-8 8AP9-9, 8AP10-1, 9AP4-6, 9AP6-8, 10AP6-1, 10AP6-4, 10AP6-9, 11AP2-8 14AP1-4, 14AP1-5, 14AP1-6, 14AP1-9, 14AP2-5, 14AP3-2, 14AP4-1, 14AP4-2, 14AP4-3, 14AP4-8, 14AP4-10, 14AP5-1, 14AP5-6, 14AP5-10

Pain, tourniquet 8AP9-8

Pain, visceral 14AP3-6

Parasympathetic nervous system 7AP1-8

Parasympathetic nervous system, atropine 3AP6-6

Parasympathetic nervous system, glycopyrronium 9AP7-2

Parasympathetic nervous system, vagus 3AP6-1

Parkinsonism 9AP1-5

Partial pressure 3AP5-1, 5AP3-5

Partial pressure, alveolar-arterial 5AP1-7, 5AP3-5

Partial pressure, arterial 5AP3-5

Partial pressure, arterial-end-tidal 5AP3-5

Partial pressure, carbon dioxide 3AP5-1

Partial pressure, end-tidal AP3-5

Partial pressure, nitrous oxide 9AP3-2

Pharmacodynamics 9AP2-2, 9AP5-5

Pharmacokinetics 8AP10-6, 9AP1-3, 9AP2-7, 9AP5-1, 9AP5-3, 9AP5-5, 9AP5-8, 10AP3-5, 10AP3-7, 10AP6-2
Pharmacokinetics, kidney 9AP7-1, 12AP5-3, 12AP5-4, 12AP9-6

Pharmacokinetics, liver 4AP3-3, 6AP2-10, 12AP4-1, 12AP9-6

Pharmacokinetics, lung 5AP4-2, 8AP7-2, 12AP5-5, 12AP5-8

Pharmacokinetics, models 3AP8-11, 9AP5-8, 10AP3-7

Pharmacology, agonists adrenergic 5AP2-8, 9AP1-5, 9AP2-6

Pharmacology, agonists opioid 9AP4-1

Pharmacology, analgesics opioid 9AP4-1

Pharmacology, benzodiazepines 1AP3-7, 3AP2-4, 9AP7-4, 19AP1-6

Pharmacology, dose-response 9AP2-5, 9AP7-3

Pharmacology, second messenger effects 4AP3-6, 4AP9-7

Pharmacology, sodium bicarbonate 7AP4-3

Pharmacology, synergism 8AP4-3, 9AP4-8, 9AP8-3, 13AP2-4, 14AP2-1

Physics, chaos 3AP3-9, 3AP4-3, 12AP1-6

Polypeptides, aprotinin 4AP5-1, 4AP5-5, 6AP2-4, 6AP2-7, 6AP2-8, 6AP2-10

Polypeptides, cytokines 4AP4-5, 7AP1-4, 12AP3-4, 12AP5-1, 14AP5-4

Polypeptides, endorphins 14AP2-8

Polypeptides, endothelin BAPC1-4, 5AP2-4, 9AP8-1

Polypeptides, enkephalins 4AP4-6

Position, head 19AP2-3

Position, jaw 19AP2-5

Position, lateral decubitus 2AP1-4, 5AP4-5, 8AP3-4, 8AP4-7, 8AP4-9

Position, neck 19AP2-5

Position, prone 12AP1-3, 17AP1-5

Position, sitting 7AP1-3, 8AP2-3, 8AP2-6

Position, supine 3AP3-10, 3AP7-2, 5AP3-3

Position, Trendelenburg 3AP7-5, 4AP6-4, 5AP4-6, 8AP4-5

Potency, anaesthetic 7AP6-5, 8AP10-3

Potency, anaesthetic, age factors 7AP6-5

Potency, anaesthetic, ED $\{50\}$ 10AP4-6

Potency, analgesic 14AP1-9

Potency, drug 7AP6-5, 8AP10-3, 14AP1-9

Potency, drug, age factors 7AP6-5

Potency, drug, tolerance 6AP1-4

Pregnancy 11AP1-1, 11AP1-5, 11AP1-10, 14AP3-1

Pregnancy, abortion 11AP1-5

Pregnancy, aspirin therapy 6AP1-10

Premedication 2AP1-1, 3AP2-4, 14AP5-6, 15AP1-2

Protein, albumin 4AP6-6, 12AP1-5

Protein, amino acids 7AP5-4, 7AP6-2, 9AP4-1, 14AP2-2

Protein, binding 6AP3-10

Protein, plasma BAPC1-2, 12AP3-6

Protein, transmembrane 9AP1-4

Psychological responses 13AP2-1

Radiotherapy 9AP6-3

Receptors, adrenergic 9AP1-5

Receptors, amino acid BAPC1-1, 9AP1-4

Receptors, cholinergic 7AP6-5

Receptors, opioid 4AP4-6, 9AP4-7, 14AP1-1, 14AP2-8

Receptors, transmembrane BAPC1-1, 9AP1-4, 12AP3-5

Records, anaesthesia 1AP1-4, 4AP5-6

Records, medical 7AP1-6

Recovery 1AP2-10, 1AP3-3, 2AP1-7, 2AP2-6, 2AP2-7, 3AP3-3 7AP3-2, 8AP9-5, 9AP2-3, 9AP2-5, 9AP3-5, 9AP7-4, 12AP4-5, 12AP9-5, 17AP3-6

Recovery, cognitive 4AP1-7, 9AP3-10

Recovery, neurological 7AP1-9, 7AP1-9

Recovery, postoperative 7AP2-3, 12AP4-5

Recovery, psychomotor 1AP1-6

Reflexes, baroreceptor 7AP1-8, 8AP4-2

Reflexes, spinal 7AP1-8, 8AP4-2

Research, anaesthesia 15AP2-3, 15AP2-6

Research, animal 4AP2-6

Risk 1AP2-5, 1AP3-1, 2AP1-2, 4AP1-4, 4AP4-2, 4AP5-7, 4AP5-8, 6AP2-8, 10AP5-7, 11AP2-6, 12AP7-7, 12AP8-9, 13АР3-5, 17AP3-4, 19AP3-8, 19AP3-9

Safety 12AP2-3, 17AP1-1, 17AP1-2, 17AP1-4, 17AP2-2, 17AP2-7, 17AP3-1

Safety, drug 2AP1-2, 3AP5-6, 6AP2-9, 8AP4-8, 8AP8-5, 9AP2-1 9AP2-5, 9AP6-9, 10AP3-4, 11AP2-8, 14AP5-9

Safety, equipment 3AP5-5, 12AP6-6

Safety, techniques 1AP2-7, 14AP4-1, 14AP5-3

Scoliosis 6AP2-1, 14AP1-4 
Sedation 1AP3-5, 2AP1-2, 3AP6-3, 3AP9-1, 3AP9-9, 7AP1-1, 7AP2-5, 7AP4-1, 8AP5-7, 8AP6-6, 9AP4-9, 10AP2-2, 12AP2-9, 12AP9-1, 14AP5-9

Serotonin(5-hydroxytryptamine) 9AP4-2, 9AP8-2, 9AP8-5

Serotonin(5-hydroxytryptamine), antagonism 14AP2-1

Sleep 1AP1-6, 7AP6-9, 9AP3-3, 17AP2-1

Sleep apnoea 19AP3-7

Solubility 3AP2-3

Solubility, partition coefficients 9AP1-3

Solubility, temperature 3AP1-5

Spinal cord, cranial nerve palsy 2AP2-3

Spinal cord, evoked potentials 3AP6-4, 3AP6-8, 7AP5-1

Spinal cord, extradural pressure 8AP1-6

Spinal cord, extradural space 7AP2-4, 8AP1-2, 8AP1-3, 8AP1-6

Spinal cord, GABA 14AP2-11

Spinal cord, metabolism 7AP3-4, 7AP3-5

Spinal cord, reflexes 7AP1-8, 8AP4-2

Spinal cord, sensory block 8AP6-2, 8AP6-3, 8AP6-8

Spinal cord, vertebral interspace 8AP1-10

Statistics 2AP1-1, 3AP3-8, 3AP5-9, 4AP4-6, 4AP5-6, 5AP3-8, 7AP2-1, 7AP4-6, 9AP5-10, 9AP6-8, 15AP1-6

Strabismus 10AP4-4, 10AP4-6, 10AP5-4

Stress 4AP8-9, 7AP4-2, 9AP3-7, 13AP2-1, 14AP5-4, 19AP4-6

Surgery, abdominal 1AP1-7, 1AP2-10, 2AP1-4, 3AP1-6, 3AP7-7, 3AP9-6, 4AP1-2, 4AP6-3, 6AP1-8, 6AP3-6, 8AP10-2, 12AP7-7, 13AP4-2, 13AP4-3, 13AP4-3, 14AP1-10, 14AP4-2, 14AP5-2, 14AP5-11

Surgery, aneurysm 3AP6-4, 7AP3-2, 7AP3-5

Surgery, autonomic response 4AP6-7

Surgery, cardiovascular 3AP4-8, 4AP1-5, 4AP2-3, 4AP2-4, 4AP2-7, 4AP3-2, 4AP3-7, 4AP5-1, 4AP5-5, 4AP5-7, 4AP7-1, 4AP7-2, 4AP7-8, 6AP1-7, 6AP2-4, 6AP3-7, 6AP3-10, 8AP8-3, 9AP7-6, 14AP1-8

Surgery, cataract $8 \mathrm{AP} 7-1$

Surgery, cleft palate 8AP7-8

Surgery, craniotomy 7AP3-4, 7AP3-5, 7AP4-7

Surgery, day-case 2AP2-4

Surgery, dental 2AP1-2

Surgery, endarterectomy 4AP5-7

Surgery, endoscopy 3AP9-1, 19AP1-3

Surgery, gastrointestinal 3AP9-1, 4AP6-3, 19AP1-3

Surgery, gynaecological 3AP2-1, 14AP5-1

Surgery, hepatic 1AP2-10, 4AP2-2, 6AP3-4

Surgery, laparoscopy 1AP3-4, 4AP3-4, 4AP6-4

Surgery, laparotomy 3AP6-5, 8AP10-5, 9AP1-6, 14AP1-6, 14AP4-10

Surgery, laser 4AP7-8, 19AP2-5

Surgery, maxillofacial 19A92-4

Surgery, non-cardiac 1AP3-7, 8AP8-8, 9AP8-10

Surgery, ophthalmological $8 \mathrm{AP} 7-1$

Surgery, orthopaedic 3AP3-3, 6AP1-1, 8AP6-6, 10AP3-3, 10AP6-2, 14AP5-6

Surgery, paediatric 10AP3-3, 10AP4-2, 10AP4-6

Surgery, plastic 1AP1-4

Surgery, postoperative period 4AP5-8, 6AP2-7, 8AP7-8, 8AP8-2

Surgery, post-transplantation 4AP8-6

Surgery, preoperative period 1AP3-9, 4AP2-5, 4AP3-8, 5AP3-7, 6AP2-7

Surgery, spinal 8AP1-6, 8AP6-8, 12AP8-1

Surgery, thoracic 1AP2-8, 3AP2-3, 5AP4-6, 8AP3-4, 8AP3-5, 8AP7-2

Surgery, thyroidectomy 1AP3-3, 3AP6-1, 19AP2-8

Surgery, tracheotomy 12AP9-7

Surgery, transplantation 3AP1-8, 4AP2-5, 4AP6-6

Surgery, urological 8AP4-3, 8AP6-1, 8AP6-3, 10AP6-6

Surgery, vascular 1AP2-2, 4AP1-6, 5AP4-4, 8AP6-5, 9AP8-10

Sympathetic nervous system 14AP1-6

Sympathetic nervous system, adrenaline BAPC1-4, 8AP9-6

Sympathetic nervous system, adrenergic block 8AP8-1

Sympathetic nervous system, clonidine 14AP2-1, 14AP3-8, 14AP5-8

Sympathetic nervous system, dexmedetomidine 7AP2-5, 9AP3-5

Sympathetic nervous system, dobutamine 4AP8-6, 4AP9-6

Sympathetic nervous system, dopamine 7AP5-8, 9AP4-2

Sympathetic nervous system, ephedrine 8AP4-7, 9AP2-8, 11AP2-6

Sympathetic nervous system, esmolol 14AP5-5

Sympathetic nervous system, ganglia 8AP1-4

Sympathetic nervous system, ganglion block 8AP1-4

Sympathetic nervous system, isoprenaline 9AP2-6

Sympathetic nervous system, noradrenaline BAPC1-4, 5AP2-4, 7AP6-8
Sympathetic nervous system, ornithine-8-vasopressin 12AP3-3, 13AP2-4 Sympathetic nervous system, phenylephrine 4AP9-3, 11AP2-6

Sympathetic nervous system, propranolol 9AP2-6

Sympathetic nervous system, salbutamol 19AP1-6

Sympathetic nervous system, sympathoadrenal responses 8AP4-4, 8AP9-2, 9AP3-2

Sympathetic nervous system, xylazine 4AP9-1

Temperature 3AP1-8, 3AP8-1, 4AP9-6, 7AP6-9, 19AP1-9

Temperature, body 3AP1-8, 4AP9-6, 17AP2-1

Temperature, metabolism 12AP4-5

Temperature, monitoring 3AP1-8, 7AP6-9

Temperature, regulation 7AP6-9

Temperature, skin 3AP1-8, 4AP9-6, 17AP2-1

Theories of anaesthetic action, neuromuscular block 9AP1-9

Theories of surfactant function 5AP1-1

Thromboxane 9AP8-5

Toxicity 8AP8-5, 8AP10-4, 8AP10-10, 8AP10-11, 8AP10-10, 8AP10-11, 9AP6-5

Toxicity, fetal 11AP1-9

Toxicity, hepatic 12AP3-6

Toxicity, local anaesthetics 8AP10-7

Toxicity, metabolites 10AP3-5

Toxicity, neurotoxicity 8AP9-10, 8AP10-3, 8AP10-7, 8AP10-8, 8AP10-8, 9AP1-6, 9AP1-8, 14AP2-7

Transfusion 4AP1-8, 6AP1-2, 6AP2-1, 6AP3-1, 6AP3-3, 6AP3-5, 6AP3-7, 6AP3-9, 6AP3-10

Transfusion, autotransfusion 6AP2-5

Transfusion, complications 4AP5-1, 6AP2-4, 6AP2-5, 10AP5-7

Transfusion, stored blood 6AP2-1, 6AP3-1

Trigeminal neuralgia 3AP6-8

Veins, cannulation 1AP2-3, 8AP10-5

Veins, complications 1AP2-3, 3AP1-9

Veins, jugular 1AP2-3, 7AP4-5

Veins, subclavian 12AP6-8, 17AP3-3

Veins, venepuncture 3AP2-4

Veins, veno-venous bypass 12AP3-4

Ventilation, airway muscles 5AP3-1

Ventilation, airway pressure 3AP1-1, 5AP4-5

Ventilation, airway resistance 5AP4-5

Ventilation, anaesthetics 4AP3-3

Ventilation, analgesics 14AP5-9

Ventilation, artificial 7AP3-8, 9AP2-6

Ventilation, carbon dioxide response $3 A P 1-3$

Ventilation, continuous positive pressure 10AP2-3, 19AP6-5

Ventilation, deadspace 12AP1-1

Ventilation, failure 8AP7-2

Ventilation, fresh gas flow 19AP6-6

Ventilation, high frequency 19AP2-5, 19AP2-8

Ventilation, high frequency jet 19AP2-5, 19AP2-8

Ventilation, hyperventilation 7AP3-8

Ventilation, hypoventilation 3AP5-1, 5AP1-7, 7AP2-6, 17AP3-6

Ventilation, hypoxic response 5AP2-2

Ventilation, intermittent positive pressure 10AP3-1, 12AP6-6

Ventilation, mechanical BAPC1-3, 3AP5-6, 3AP6-2, 3AP8-2, 5AP1-2, 5AP2-3

Ventilation, mechanics 5AP1-2

Ventilation, muscle, airway 5AP3-1

Ventilation, muscle, respiratory 5AP3-1

Ventilation, obstruction 7AP4-8

Ventilation, one-lung 3AP2-3, 5AP4-5, 5AP4-6, 8AP3-5, 19AP4-9

Ventilation, pattern 19AP5-4

Ventilation, positive end-expiratory pressure 5AP4-7

Ventilation, postoperative 19AP6-6

Ventilation, pressure support 5AP1-1

Ventilation, spontaneous 5AP1-1, 19AP5-4, 19AP6-3

Ventilation, transtracheal 19AP2-5

Ventilation, ventilation-perfusion 5AP3-5, 5AP4-6

Vomiting, antiemetics 1AP3-3

Vomiting, nausea 2AP2-6, 9AP6-2, 9AP6-3, 9AP6-6, 14AP5-8

Vomiting, nausea, anaesthetic factors 10AP6-5

Vomiting, nausea, patient factors 8AP4-9, 9AP6-3

Vomiting, nausea, surgical factors 8AP3-6, 9AP6-2, 9AP6-3 
Aarts, L.P. $\quad 6 \mathrm{AP} 3-10$

Aarts, L.P.H.J. $\quad$ 4AP7-8, 4AP9-3, 8AP6-4

Aas-Eng, K. 1AP2-6, 1AP2-7

Abad-Gurumeta, A. 3AP5-2, 3AP8-4, 3AP9-10

Abecassis, P. 19AP5-3

Abelha, F. 12AP8-4

Aboulafia, A. 15AP2-3, 15AP2-6

Abreu, M. 5AP1-8

Absalom, A.R. 9AP5-8

Aceto, P. 9AP6-6, 19AP4-6

Adachi, Y. 7AP5-8

Adamanti, S. 8AP9-5

Adanir, T. 2AP1-5

Adekanye, O. 2AP1-7, 3AP6-9, 3AP8-8, 8AP3-7, 13AP1-3, 13AP3-6

Adroer, R. 17AP3-4

Aertgeerts, E. 14AP1-3

Agalar, F. 12AP9-7

Agreda, G. 2AP2-3

Aguilar, G. 7AP4-6, 12AP7-10

Aguilar, J.L. 3AP4-4, 14AP5-3

Aguilera, L. 9AP7-2

Aguilera, L.G. 5AP3-4

Agustin, E. 12AP8-7

Ahmed, N. 8AP7-1

Ahn, D. 4AP3-1

Ahrens, J. BAPC1-1, 9AP1-4

Aikele, P. 5AP1-1

Aikele, S. 8AP3-1

Ainslie, P. 7AP4-5

Akin, F. 3AP1-4

Akinci, S. 14AP4-6

Al, M. 12AP2-9

Al-Hassani, A. 3AP4-2

Al-Jumaily, A. 3AP4-1

Al-Oufy, H. 13AP2-8

Alagna, G. 3AP2-1

Alanoglu, Z. 3AP2-7, 19AP5-8, 19AP5-9, 19AP5-10

Albani, M. 8AP10-10

Albertin, A. 6AP3-2, 9AP3-2

Ali, Y. 14AP4-9

Allam, S. 2AP1-2

Allard, M. 3AP5-8, 3AP8-10

Allegaert, K. 9AP5-1

Allegri, M. 8AP8-5

Alonso, $\mathrm{H}$. 3AP9-3

Alqatrani, M. 19AP3-10

Alsina, E. 11AP2-7

Altman, E. 19AP4-10

Altunbay, K.U. 2AP1-5

Alvarez, J.C. $5 A$ AP3-4

Álvarez, J.C. 3AP2-10

Alvarez, M. 19AP3-8

Alvarez-Gómez, J.A. 9AP7-8

Alver, F. 19AP5-8, 19AP5-9, 19AP5-10

Alves, H.C. $9 A \mathrm{AP}-10$

Amadeu, E. 3AP2-9, 3AP9-4, 3AP9-5

Amadeu, M.E. 8AP4-3

Amaniti, E. 7AP6-1, 8AP10-10, 9AP8-3

Amanor-Boadu, S. 8AP6-2

Ament, Ch. 7AP5-9, 7AP5-10

Amorim, P. 3AP2-9, 3AP8-11, 3AP9-3, 7AP3-6, 9AP5-9, 9AP5-10

Amour, J. 4AP9-7, 5AP2-8

Amouri, F. 8AP4-7, 11AP2-11

Anastassiou, E. 14AP5-6

Anderson, K. 1AP2-2, 4AP7-6

Anderson, K.J. 2AP1-2

Andrade, J. 9AP4-10

Andreu, E. 10AP4-2

Andrews, P. 12AP7-1

Añez, C. 14AP4-5

Anfiloquio, C. 3AP3-10
Anguseva, T. 8AP8-2

Annecke, T. 5AP4-4

Antkowiak, B. 7AP3-3

Antonopoulou, E. 2AP1-6, 8AP9-10

Antunes, L. 3AP8-11, 9AP5-4, 9AP5-10

Antunes, L.M. 9AP3-10

Antypa, E. 6AP1-5, 9AP3-6

Aparicio, R. 3AP9-1

Apostol, A. 19AP4-5

Applegate II, R. 3AP5-8, 3AP8-10

Aran, G. 2AP1-5

Aranha, N.A. 19AP4-5

Arikan, O. 9AP6-4

Ariyama, J. 4AP6-5

Armstrong, M. 12AP8-2

Arnal, D. 6AP2-7, 17AP2-7

Arnberger, M. 1AP2-10, 15AP2-2

Arndt, C. 12AP5-7, 19AP3-9

Arpino, I. 7AP2-4

Arslan, M. 8AP4-5, 8AP9-8

Artés, D. 10AP2-4

Artigas, A. 10AP2-5

Arxer, A. 8AP4-1, 14AP3-7

Ashrafi, L. 10AP6-5

Askar, F.Z. 9AP7-7

Askitopoulou, H. 8AP1-10, 8AP4-2

Asouhidou, I. 1AP2-3

Asteri, Th. 1AP2-3

Astyrakaki, E. 8AP4-2

Ates, Y. 3AP2-7, 19AP5-9, 19AP5-10

Attof, Y. 1AP2-8, 3AP7-1, 4AP2-8, 6AP2-8

Augutiene, A. 8AP5-5

Auler Jr, J.O. 14AP5-10

Autschbach, R. 4AP9-6

Avidan, M. 3AP4-8

Aycan, I. 14AP4-6

Aydinuraz, K. 12AP9-7

Ayedi, M. 8AP4-7

Ayerden, T. 9AP1-8, 14AP2-7

Aygün, A. 4AP7-9

Ayhan, B. 14AP4-6

Ayodele, O. 14AP1-2

Aypar, U. 14AP5-5

Azevedo, P. 3AP3-10

Aziz, T. 12AP7-1

Azizi, Sh. 10AP6-7

Azzari, S. 12AP1-1

Bacak, I. 8AP6-3, 14AP1-5

Baciarello, M. 8AP2-2, 8AP9-2

Badaev, R. 19AP4-10

Badenes, R. 7AP4-6

Badica, L. 14AP4-4

Bae, H. 12AP1-5, 12AP5-5

Baele, P. 14AP1-8

Baena, M. 14AP5-3

Baftiu, N. 7AP2-9, 10AP6-7

Bagka, D. 8AP4-8, 8AP4-9

Bagó, J. 7AP2-1

Bahar, M. 8AP10-5, 9AP1-6

Bakker, J. 12AP2-9

Balaguer, J. 15AP2-4, 19AP2-7

Balaji, P. 11AP2-3

Balcan, A. 2AP1-4, 6AP3-6

Baldassarri, R. 9AP8-7

Baldi, C. 8AP8-5

Ballester, M. 12AP7-10

Baltaga, R. 19AP1-5

Bamadhaj, M. 12AP6-2

Bamehrez, F. 14AP4-9

Banchereau, F. 19AP6-8

Banevicius, G. 7AP4-7

Barak, M. 19AP5-3

Barbagallo, M. 5AP4-2
Barcia, A. 12AP7-3

Barcin, S. 8AP1-7

Barcýn, S. 8AP1-9

Barreiro, M. 4AP6-3

Barron, M. 17AP3-7

Barros, F. 2AP2-6, 2AP2-7

Barthelaix, A. 9AP8-8

Bartolome, A. 17AP2-7

Bärtsch, P. BAPC1-4, 5AP2-4

Barvais, L. 12AP5-4

Barwise, J. 3AP8-3

Basar, H. 4AP3-8, 19AP3-5

Basciani, R.M. 4AP5-6

Basgul, E. 14AP4-6

Basora, M. 6AP2-9

Basters, J. 13AP3-5

Bastien, O. 3AP8-2

Baud, A. 6AP2-5

Bauer, A. 4AP2-5, 13AP4-1

Bauer, C. 19AP6-4

Bauer, I. 12AP4-1

Bauer, U. 19AP6-4

Bauernschmitt, R. 6AP2-4

Bauersfeld, U. 10AP1-5, 10AP1-6

Bauhofer, A. 12AP5-7

Baxarias, P. 1AP2-9, 3AP7-5

Baydar, M. 19AP2-6

Beards, S. 12AP8-8

Bein, B. 4AP2-6, 4AP2-9, 7AP4-1, 7AP6-8, 9AP1-5, 9AP4-9, 13AP2-4, 13AP2-5, 19AP6-6

Beiras, A. 9AP5-9

Bekos, V. 12AP6-6

Belbachir, A. 8AP3-3

Belda, F.J. 7AP4-6

Belhaj, S. 8AP7-8

Belinchon, E. 1AP3-2, 6AP3-1

Bell, S. 8AP1-5

Bellamy, M. 4AP6-6

Belostotskii, V. 8AP8-2

Belov, A. 7AP1-7

Beltrán De Heredia, S. 6AP2-6

Beltrán de Heredia, S. 15AP1-5

Bencsik, G. 8AP9-3

Bentley, A. 12AP4-1

Berends, N. 11AP1-2

Berg, S. 7AP2-6

Berger, M.M. BAPC1-4, 5AP2-4

Bergonzi, P.C. 9AP2-3

Berkel, B. 3AP2-7

Bernet, V. 3AP3-6

Berritta, C. 11AP1-4, 11AP1-6

Berry, C. 4AP7-6

Berry, J. 3AP8-3

Berry, M. 1AP1-3, 1AP1-9

Berry-Stoelzle, T. 1AP1-3

Berta, E. 10AP6-6

Berteanu, C. 8AP8-8

Berteanu, M. 8AP8-8

Berti, M. 8AP5-2

Bestas, A. 9AP7-4

Beylac, L. 19AP6-9

Biais, M. 3AP7-10, 12AP7-9

Biazotto, C. 9AP7-9

Biesemans, J. 7AP3-4

Bignami, E. 4AP3-2

Bijker, J. 17AP2-5, 17AP2-6

Bilskiene, D. 7AP4-7, 14AP3-4

Bingham, J. 5AP1-4

Binning, S. 3AP5-1

Birenbaum, A. 4AP9-7

Bisbe, E. 6AP2-6

Bissonnette, B. 8AP10-1, 10AP3-1, 10AP6-4 
Bjertnaes, L. 5AP4-1

Blanco, R. 2AP2-2

Blanloeil, Y. 3AP5-7

Blasco, L. 12AP7-10

Blaszyk, M. 9AP2-5

Blobner, M. 9AP7-10, 12AP9-5

Bloch, K.D. 5AP2-2

Bloos, F. 12AP2-8

Blouin, J-L. 14AP2-8

Bobbio, A. 5AP4-2

Boche, R. 14AP4-1

Boddu, K. 8AP1-2

Boehm, C. 3AP9-2

Boer, C. 4AP3-6

Boer, F. 3AP7-9

Bogar, L. 12AP7-2

Bogdanets, A. 5AP3-1

Bohy, B. 3AP4-6, 13AP4-5

Bojahr, H. 8AP5-7

Boldizar, M. 19AP2-3, 19AP6-5

Boldrini, A. 4AP8-7

Bom, A. 9AP2-6

Bonnet, F. 8AP3-3

Bonofiglio, F. 9AP4-10

Boogaerts, J. 4AP8-3

Bordes, M. 19AP6-9

Borisov, D. 14AP4-2, 14AP5-2

Bosch, C. 12AP4-7

Bosomworth, M. 4AP6-6

Botana, C. 2AP2-3

Bouattour, L. 8AP2-8, 8AP7-8

Bourgain, J.L. 12AP2-1

Bouwman, R.A. 4AP3-5, 4AP3-6

Bove, T. 4AP1-5

Boylan, G. 3AP6-3

Brãdi ${ }^{\circ}$, A.A. 2AP1-1

Bradis, A.A. 14AP4-4

Brand, P. 9AP4-9

Brandl, U. 4AP2-5

Brandon Bravo Bruinsma, G.J. 4AP2-3

Braschi, A. 8AP8-5

Bratzou, C. 14AP4-10

Braun, G. 12AP2-7

Breitbarth, J. 8AP3-2

Bressan, N. 3AP8-11, 9AP5-4

Bretschneider, M. 7AP3-3

Breuer, T. 6AP2-4, 10AP5-7

Brimioulle, S. 4AP9-5

Brinker, A. 13AP3-1

Briones, Z. 1AP3-6, 5AP4-5

Bröking, K. 12AP5-2

Broscheit, J. 1AP3-7

Brown, S.C. 10AP2-2

Bruckner, M. 14AP3-1

Bruder, N. BAPC1-5, 7AP3-8, 7AP5-6, 7AP5-7

Brugger, J. 1AP3-7

Bruhn, J. 3AP3-3, 3AP9-2

Bruno, G. 4AP1-3, 4AP1-5, 6AP3-2, 9AP3-2

Bruqi, B. 12AP6-3

Bucher, M. 12AP5-1

Buchet, S. 8AP5-4

Budic, I. 10AP3-6, 10AP6-9

Buettner, J. 8AP3-2

Buhre, W.F. 4AP2-3

Buikema, H. 4AP9-3

Bukoshi, Z. 12AP6-3

Bulo-Kasneci, A. 7AP1-4

Bunchungmongkol, N. 10AP5-3

Burchman, C. 8AP5-1

Busch, C.J. 5AP2-1, 5AP2-2

Busquets, C. 14AP3-7

Butcher, J. 19AP5-6

Buyukkocak, Ü. 9AP1-1, 9AP6-4, 12AP9-7

Bystritski, D. 4AP6-8

Cabral, L. 1AP3-9

Cabre, P. 2AP2-3
Cabrera Schulmeyer, C. $\quad 3$ AP8-5

Caelen, O. 3AP2-8

Cafiero, T. 4AP6-7

Caglayan, O. 9AP1-1

Cahana, A. 15AP1-2, 17AP2-3

Caires, J.L. 14AP5-10

Cairns, C. 1AP3-1

Calabrò, M.G. 4AP5-4

Calatzis, A. 6AP1-10

Calvo-Vecino, J.M. 3AP5-2, 3AP8-4, 3AP9-10

Cammu, G. 9AP2-1, 9AP2-5

Camorcia, M. 11AP1-4, 11AP1-6

Campos, J.M. 3AP4-3

Camu, F. 3AP8-7, 4AP9-1, 14AP1-9

Canas, P. BAPC1-5, 7AP5-6

Canbay, O. 14AP5-5, 19AP2-2

Canet, J. 1AP3-6, 5AP3-7, 17AP2-1

Canik, S. 4AP7-9

Cann, C. 3AP1-7, 3AP8-6

Cannesson, M. 1AP2-8, 3AP7-1, 3AP8-2, 4AP2-1, 4AP2-8, 6AP2-8

Cano, J. 3AP9-1

Canturk, M. 8AP4-5, 8AP9-8

Capdevila, LI. 4AP8-5

Capdevila, X. 10AP3-5, 10AP6-8

Capogna, G. 11AP1-4, 11AP1-6

Carazo, J. 7AP3-2

Cardenas, E. 3AP9-1

Cardone, A. 9AP6-6

Cardoso, G. 8AP4-3

Carev, M. 1AP1-6, 3AP2-5

Carey, M. 11AP2-4

Cariello, C. 9AP8-7

Carlsson, P. 8AP3-6

Carmi, N. 19AP6-3

Carmona, M. 9AP7-9

Carmona, M.J. 14AP5-10

Carrel, T. 4AP5-6

Carrero, E.J. 7AP3-2

Carter, R. 5AP3-3

Carvalho, A. 5AP1-8

Casal, M. 3AP4-7

Casalino, S. 6AP3-7

Casas, J.I. 5AP1-2

Casati, A. 3AP6-2, 5AP4-2, 8AP2-2, 8AP5-2, 8AP9-2

Casellas, J.A. 3AP9-1

Cassel, J.C. 7AP6-5

Castillo, J. 8AP4-1

Castro E Melo, A.M. 2AP1-3

Castro, A. 2AP2-6, 2AP2-7, 3AP2-9, 3AP3-10, 3AP4-7, 3AP9-3, 3AP9-4, 3AP9-5, 7AP3-6, 8AP9-6, 9AP5-4

Castro, M. 12AP8-4

Castro, R. 8AP9-6

Cavus, E. 4AP2-6, 7AP6-8, 13AP2-4, 13AP2-5

Cazzato, M.T. 3AP5-9

Celebi, N. 14AP5-5

Celi, L. 7AP4-5

Celiker, V. 14AP5-5

Cernea, D. 8AP8-8

Cerny, V. 4AP6-9

Cetinkaya, Z. BAPC1-6, 12AP5-8

Cevik, B. 8AP4-6

Chaari, M. 14AP5-8

Chae, J. 4AP3-1

Chafii, D. 6AP2-1

Chaitov, Z. 8AP10-5

Challet, E. 9AP3-3

Chalmers, P. 15AP1-4

Chalo, D. 8AP4-3

Chambrier, C. 1AP2-8

Chamogeorgakis, T. 5AP2-5

Chan, K. 7AP6-2

Chang, Yi-Chen. 14AP2-2

Chanimov, M. 8AP10-5, 9AP1-6
Chapman, G. 7AP3-7

Charters, P. 19AP4-7

Charvet, I. 14AP2-8

Chastain, D. 10AP2-2

Chatzimichali, A. 8AP4-2

Cheah, C. 1AP1-4

Chelliah, K. 15AP2-7

Chen, C. 3AP7-4

Chen, C.C. 8AP10-3

Chen, J.C. 13AP3-2

Chen, L. 3AP5-4

Cheng, T.H. 9AP8-1

Cheong, S.H. 9AP5-2

Cherekaev, V. 7AP1-7

Cherif, A. 12AP6-2

Cherif, R. 14AP1-4

Cherng, C. 8AP10-4

Cherng, C-H. 14AP2-2

Chethan, D. 3AP1-7, 3AP8-6

Chettaoui, O. 10AP6-2

Chevanne, F. 8AP10-6

Chiari, P. 4AP2-8

Chien, C.H. 14AP2-3

Chien, I. 8AP1-1

Chierichetti, M. 12AP1-1, 12AP1-2

Chikhrouhou, H. 8AP7-8

Chikrouhou, $\mathrm{H}$. 11AP2-11

Chin, Y.J. 10AP4-6

Chinè, E. 9AP6-6

Chiu, T.F. 13AP3-2

Chiumello, D. 12AP1-1, 12AP1-2

Cho, S. 8AP2-6

Choi, A.M.K. BAPC1-3, 5AP2-3

Choi, B. 9AP3-4, 19AP2-5

Choi, S. 10AP5-4

Choi, S.R. 10AP4-6, 14AP5-4

Choi, W.C. 9AP8-2

Choi, Y. 5AP4-6

Choi, Y.G. 9AP5-2

Chow, E. 19AP1-3

Christ, F. 4AP2-5, 13AP4-1

Christie, I. 15AP2-8

Christodoulaki, K. 5AP4-7

Chu, J. 3AP7-4

Chu, K.S. 3AP6-1

Chu, K-S. 8AP2-1

Chui, K. 11AP2-6

Chumpathong, S. 10AP5-3

Chung, C.J. 10AP4-6, 14AP5-4

Chung, $H$. 14AP2-9

Chung, J. 8AP2-6

Chung, S. 12AP1-5, 12AP5-5

Chung, Y.K. 17AP3-2

Cifarelli, C. 11AP1-4, 11AP1-6

Cimic, A. 12AP9-3

Cindea, I. 2AP1-4, 6AP3-6

Ciszewski, P. 3AP2-3

Citterio, A. 7AP2-4

Clergue, F. 17AP2-2

Cobiletchi, S. 19AP1-5

Cocu, S. 12AP3-7

Codaccioni, J.L. 7AP5-7

Colakoglu, S. 8AP4-6

Coll Rosell, I. 12AP8-9

Collange, O. 6AP2-10, 12AP9-6

Collet, V. 4AP9-8, 14AP2-10, 14AP3-8

Collins, G. 3AP4-8

Collins, S. 14AP3-5, 14AP3-6

Colomina, M.J. 6AP2-3, 7AP2-1

Columb, M. 8AP2-3

Columb, M.O. 8AP6-7

Colvin, H.P. $\quad$ 8AP10-8

Company, R. 5AP1-7

Congedo, E. 9AP6-6, 19AP4-6

Constant, I. 10AP1-4

Conzen, P. 5AP4-4

Cooper, L. 17AP3-7 
Cooper, R. 19AP1-10

Cooreman, H. 11AP1-3

Coppola, P. 3AP7-2, 8AP6-5

Coriat, P. 3AP7-3, 4AP1-6, 4AP9-7, 9AP8-10

Cortés, C. 12AP4-7

Cortínez, L. 10AP1-7

Costa, G. 7AP3-6

Coste, O. 9AP3-3

Cotena, S. 7AP1-2

Cottenceau, V. 3AP7-10, 12AP7-9

Couvret, C. 6AP2-5

Crescenzi, G. 4AP1-3

Cressoni, M. 12AP1-2

Cristallo, E. 4AP5-4

Cristofaro, A. 3AP7-7

Crocier, J. 13AP4-2

Croitoru, M. 19AP4-10

Cromheecke, S. 4AP6-1, 4AP7-2

Cros, A.M. 19AP6-8, 19AP6-9

Crozier, J. 13AP4-3

Cruz, M.A. 15AP1-5

Cserép, Zs. 10AP5-7

Csontos, C. 12AP7-2

Cucereanu Badica, I.G. 14AP4-4

Cucereanu Bãdicã, I.G. 2AP1-1

Curtin Lawton, S. 15AP1-4

Cusato, M. 8AP8-5

Cuvas, O. 19AP3-5

Czaja, E. 6AP1-7

Czikajló, Z. 12AP2-3

Czuczwar, K. 9AP4-4

Czuczwar, M. 9AP4-3, 9AP4-4

Dabbabi, M. 8AP2-8

Dabo, B. 8AP5-4

Dadure, Ch. 10AP3-5, 10AP6-8

Daflou, K. 8AP1-4

Daghfous, M. 12AP6-2

Dailler, F. 7AP1-9

Dalsasso, M. 9AP2-8

Dambrosio, M. 10AP6-1

Dancey, F.M.L. 1AP3-5

Danella, A. 9AP8-7

Danelli, G. 8AP2-2, 8AP5-2, 8AP9-2, 8AP9-5

Daniels, D. 12AP2-6

Daoud, A. 12AP6-2

Daper, A. 4AP5-1

Daphan, C. 12AP9-7

Darshane, S. 19AP4-7

Darvas, K. 3AP7-6

Dashti, S. C. 8AP9-3

Datli, F. 9AP6-4

Davidova, N. 8AP10-7, 19AP4-10

Davies, R. 13AP1-3

De Cosmo, G. 9AP6-6, 19AP4-6

De Deyne, C. 3AP4-6, 7AP1-6, 7AP3-4, 7AP3-5, 13AP4-5

De Groote, F. 10AP1-3

de Haes, $H$. 2AP2-5

De Hert, S. 4AP5-2, 4AP5-3, 4AP6-1, 4AP7-2, 10AP1-1, 10AP1-2, 10AP1-3

de Hert, S. 4AP5-1

de Hoon, J. 9AP5-1

DE Kock, M. 4AP9-8

De Kock, M. 14AP1-8, 14AP2-10, 14AP3-8

de la Fuente, E. 8AP3-5

De la Maza, J. 3AP8-5

de Lange, J. 14AP3-3, 14AP3-5, 14AP3-6

de Lange, J.J. 4AP3-6

De Lorenzo, B. 4AP8-7

De Miguel, M. 10AP4-2, 15AP1-1

De Pouvourville, G. 12AP2-1

De Robertis, E. 7AP1-2, 12AP3-6

De Simone, G. 14AP1-10

De Villé, A. 10AP1-1, 10AP1-3

De Voss, R. BAPC1-2, 12AP4-2
De Wit, E. $\quad$ 4AP5-2, 4AP5-3

Debaene, B. 9AP7-3

Debaveye, Y. BAPC1-2, 12AP4-2

Declercq, T. 3AP1-1

Decoopman, M. 9AP2-1

Decoster, J. 11AP1-3

Dedola, E. 4AP1-3, 4AP1-5, 4AP3-2, 4AP5-4 6AP3-2, 9AP3-2

Dehnert, C. BAPC1-4, 5AP2-4

Deitmer, W. 19AP6-6

Dejonckheere, M. 4AP2-7

Del Fresno, J. 5AP1-7

Delgado, P. 7AP4-8

Deliaslani, D. 4AP1-7

Della Rocca, G. 3AP7-7

Dellagrammaticas, D. 7AP3-7

DeMello, W. 8AP6-7

Demeyer, I. 9AP2-1

Demneri, M. 7AP1-3, 7AP1-4, 7AP2-8

Dempsey, G. 12AP6-1

Denner, A.M. 2AP2-4

Depka, M.v. 6AP1-7

Dereeper, E. 8AP9-7

DeRoover, A. 1AP3-4

Desebbe, O. 3AP7-1, 3AP8-2, 4AP2-1

Deshko, Y. 9AP2-10

deVilliers, F. J. 12AP6-4

Devlieger, R. 11AP1-2

Dewachter, C. 4AP9-5

Dewachter, L. 4AP9-5

Dhillon, P. 11AP2-3

Dhollander, A. 3AP1-6, 15AP1-2, 17AP2-3

Di Cianni, S. 8AP2-2

Di Gennaro, D. 14AP1-10

Di lorio, C. 4AP6-7

Di Marco, L. 8AP9-7

Di Minno, R.M. 4AP6-7

Di Roio, C. 7AP1-9

Diaz, D. 4AP6-3

Díaz, J. 11AP2-7

Dibbelt, L. 3AP8-9

Dieterich, H.-J. 12AP5-3

Dikmen, B. 8AP2-7, 9AP1-8, 14AP2-7

Dillemans, B. 3AP1-1, 3AP1-2, 3AP1-3, 3AP1-4

Dimitriou, V. 14AP4-8

Dingler, S. 6AP3-9

Dispersyn, G. 7AP6-9, 9AP3-3, 9AP6-7

Dix, P. 10AP5-2

Djordjevic, V. 10AP3-6, 10AP6-9

Dmitriiev, D. 12AP1-4

Dmytriev, D. 12AP1-3

Dmytriiev, D. 10AP2-3

Docquier, M. 14AP2-10, 14AP3-8

Doerges, V. 7AP6-8, 13AP2-5, 19AP6-6

Dogas, Z. 1AP1-6, 3AP2-5

Dogru, T. 4AP3-8

Dohgomori, $H$. 7AP4-2, 19AP3-4

Doi, K. 8AP3-4

Doi, M. 7AP5-8

Dolch, M. 3AP1-5

Dollo, G. 8AP10-6

Domínguez, A. 11AP2-7

Dominguez, $X$. 8AP9-6

Donmez, F. 8AP1-9

Doolke, A. 2AP1-9

Dorairaj, I.L. 5AP1-4, 5AP3-5

Dörges, V. 13AP2-4

Dostal, P. 4AP6-9

Douvantzi, A. 10AP4-7

Drago, R. 8AP6-5

Dragoumanis, C. 14AP4-8

Dragulin, E. 14AP5-11

Draisci, G. 11AP2-8

Drew, D. 12AP9-1

Drexler, B. 7AP3-3

Driessen, J.J. 9AP7-1

Driesz, L. 8AP9-3
Droc, G. 12AP2-4

Dubniks, M. 4AP8-4

Dubost, A.F. $\quad$ 8AP5-6

Ducart, A. 4AP2-7

Ducombs, O. 3AP7-3

Duda, I. 3AP3-4

Duff, E. 1AP2-1, 19AP1-1

Durak, P. 4AP3-7

Durieux, M.E. 8AP10-9, 9AP1-10

Dutta, A. 10AP4-1

Duvaldestin, P. 9AP7-3

Dýkmen, B. 19AP3-5

Dylczyk-Sommer, A. 12AP8-6, 17AP3-6

Dýncer, E. 9AP3-5

Eberhart, L. 19AP3-9

Eberle, B. 4AP5-6

Eden, A. 4AP6-8

Ediz, N. 9AP3-9, 19AP2-6

Edward, G.M. 2AP2-5

Egi, A. 4AP9-2

Egilmez, H. 10AP3-4

Ehrmann, S. 8AP5-6

Eichler, W. 5AP3-6

Ekti, Y. 8AP4-6

El Beheiry, H. 9AP1-9

El-Beheiry, H. 7AP2-7

El Kady, N. 10AP5-1

El Mekawi, S. 10AP5-1

El-Rouby, S. 13AP2-8

Elamin, E. 12AP9-1

Elia, N. 8AP7-2

Ellerkmann, R. 3AP9-2

Ellerkmann, R.K. 3AP3-3

Ellger, B. BAPC1-2, 12AP4-2, 12AP4-3

Elliott, R. 1AP1-1

Elmasry, N. 14AP4-9

Engelborghs, K. 7AP3-5

Engelhardt, T. 10AP3-1

Engelman, E. 3AP2-8

Enselmann, P. 5AP3-2

Epema, A.H. $\quad$ 4AP7-8, 4AP9-3, 6AP3-10

Er, A. 19AP3-5

Erdemli, O. 4AP3-7

Erhan, O.L. 9AP7-4

Eriksson, L. 9AP7-10

Ermolenko, A. 9AP7-6

Erol, M. 8AP1-7

Ertmer, C. 12AP5-2

Eschertzhuber, S. 10AP2-7

Escolano Villen, F. 13AP4-4

Escolano, F. 6AP2-6, 13AP4-4, 17AP2-4

Escudero, A. 4AP1-8, 5AP4-5, 15AP1-3

Escudero, M.A. 19AP5-4

Escudero, R. 1AP3-2, 6AP3-1

Esper, T. 9AP1-3

Espinal, V. 8AP10-1, 10AP6-4

Espitalier, F. 8AP5-6

Estebe, J.P. 8AP10-6

Esteve, N. 14AP5-3

Estrada, V.M. 3AP4-4

Estruch, M. 19AP2-7

Estruch-Pérez, M.J. 3AP2-4

Evans, A. 19AP5-6

Eyelade, R. 8AP6-2

Eyer, J. 9AP8-8

Eyrolle, L. 8AP3-3

Fabregas, N. 7AP3-2

Fàbregas, N. 7AP4-3

Factor, D. 2AP2-2

Fafoulakis, F. 8AP4-8

Fanelli, G. 3AP6-2

Fang, $H$. 4AP4-4, 4AP4-5, 7AP5-1, 7AP5-4

Fanning, R. 11AP2-4

Fanous, Z. 9AP1-6

Fanus, Z. 8AP10-5 
Farbo, M. 3AP2-1

Fassoulaki, A. 14AP3-2

Favel, C. 6AP2-10

Fawcett, W.C. $\quad$ 4AP2-2, 6AP3-4

Federico, A. 10AP6-1

Fernández, S. 7AP4-3

Fernandez-Candil, J. 1AP1-8

Fernández-Real, J. 14AP3-7

Fernstrom, M. 15AP2-6

Ferrante, E. 7AP2-4

Ferreira, D. 3AP8-11

Ferreira, D.A. 9AP5-10

Ferreira, L. 3AP4-7

Feussner, $\mathrm{H}$. 13AP3-3

Fidan, $H$. BAPC1-6, 12AP5-8

Figueiredo Lima, J. 1AP3-9

Filimonovic, J. 8AP9-4

Filipovic-Grcic, I. 12AP7-7

Filoni, G. 8AP9-9

Finteis, F. 13AP1-5

Fischer, L.G. 8AP8-9

Fischer, S. 3AP7-6

Fischer, T. 12AP7-2

Fleron, M.H. 4AP1-6

Flo, A. 4AP1-8

Flockton, E. 9AP7-1, 9AP7-2

Flonta, M. 12AP3-7

Florquin, S. 12AP5-4

Fochi, O. 4AP1-3, 4AP1-5, 4AP3-2, 4AP5-4

Foex, P. 1AP2-2

Foldi, V. 12AP7-2

Fontes, S. 8AP4-3

Foreman, K. 19AP4-8

Foremny, J. 4AP5-8

Forster, A. 17AP2-2

Foulidou, A. 10AP4-7

Fournell, A. 4AP3-3, 12AP1-7

Franca, R. 3AP6-6

Français, S. 19AP6-9

Francksen, H. 19AP6-6

Franco, T. 15AP1-3

Frank, M. 8AP3-8, 13AP3-7

Franken, P. 4AP9-1

Fräßdorf, J. 4AP4-6

Freise, $\mathrm{H}$. 8AP8-9

Freyburger, G. 6AP1-9

Freys, G. 6AP2-10

Frick, C. 12AP9-5

Friedl, S. 12AP2-7

Frkovic, V. 4AP4-8

Fudickar, A. 7AP4-1

Fuentes, L. 6AP2-7

Fuentes-Pradera, M.A. 9AP1-2

Fujimoto, T. 19AP3-4

Furuya, $\mathrm{H}$. 3AP6-4

Fusciardi, J. 6AP2-5, 8AP5-4, 8AP5-6

Gabrhelik, T. 10AP6-6

Gairola, R. 7AP1-5

Gaitini, L. 19AP6-3

Gal, J. 4AP5-5

Galante, D. 10AP6-1

Gale, T. 17AP1-1

Gallagher, C. 17AP3-7

Gallart, L. 5AP3-4

Gallart, LI. 1AP1-8, 15AP1-5

Galletti, C. 8AP9-9

Gama De Abreu, M. 5AP1-1

Gama de Abreu, M. 12AP1-6

Gamli, M. 8AP2-7, 8AP9-8

Gamsjager, T. 12AP2-5

Gandolfi, A. 6AP3-2, 9AP3-2

Gao, F. 12AP2-6

Garcés, A. 10AP2-4

Garcia, J.G.N. 9AP4-8

García, M. 3AP2-10
Garcia-Guasch, R. 5AP4-5, 15AP1-3

García-Pérez, M.L. 7AP4-6

García-Torrado, F. 9AP1-2

García-Valdecasas, J.C. 4AP8-5, 4AP8-6

Garnerin, P. 17AP2-2

Garozzo, A.F. 9AP2-3

Garrido, A. 17AP2-1

Garutti, I. 8AP3-5

Gaszynski, T. 4AP3-4

Gaszynski, W. 4AP3-4

Gath, V. 4AP7-7

Gatti, M. 8AP9-5

Gaudio, M. 8AP9-5

Gaupp, R. 4AP2-9

Gehring, H. 3AP8-9

Geier, D. 9AP1-3, 9AP8-5

Gekiere, J.P. 6AP1-9

Gelbrich, G. 9AP6-9

Gelmanas, A. 8AP5-5

Genel, N. 4AP3-7

Genzwuerker, H.V. 10AP2-1, 13AP1-5, 13AP3-5, 19AP6-1

Georgiadou, Th. 6AP1-5, 9AP3-6, 9AP3-7

Georgiou, L. 8AP8-6

Georgiou, M. 9AP3-7

Georgopoulou, S. 8AP4-8, 8AP4-9

Gerber, A. 10AP2-7

Gerber, S. 3AP3-6

Gerin, M. 10AP1-2

Gerner, P. 8AP10-3, 8AP10-8

Geros, D. 3AP9-8

Gherghina, V. 2AP1-4, 6AP3-6

Ghisi, D. 8AP5-2

Gholam-Hossieni, H. 3AP4-1

Ghrab, B. 8AP5-3, 10AP6-2, 14AP1-4, 14AP5-8

Giala, M. 4AP1-7

Giambartino, F. 3AP2-1

Giannecchini, L. 9AP8-7

Giannella-Neto, A. 5AP1-8

Giaourakis, M. 12AP6-6

Gibson, S. 4AP6-2

Gil Bona, J. 17AP3-4

Giles, J. 1AP2-2

Gillon, M.C. 12AP9-6

Gilsanz, F. 11AP2-7

Gimenez Arnau, A. 13AP4-4,

Gimenez, A. 17AP2-4

Giovanardi, C. 3AP6-2

Girard, T. 9AP8-11

Glen, J.B. 9AP5-7

Glumcher, F. 12AP1-3, 12AP1-4

Goarin, J.P. 3AP7-3, 4AP1-6

Gocmen, J.S. 9AP1-1

Godet, G. 4AP1-4, 4AP1-6, 9AP8-10

Gogus, N. 9AP3-9, 19AP2-6

Gohil, B. 3AP4-1

Gokcan, K. 19AP5-9

Goksu, H. 9AP7-4

Goldman, M. 12AP5-4

Gomar, C. 9AP7-2

Gombotz, H. 6AP3-3

Gomez-Arnau, J.I. 6AP2-7

González, I. 3AP2-10

Gonzalez, M.A. 19AP6-10

González, S. 6AP2-3, 8AP2-5, 12AP4-7

Gonzalez-Rodriguez, R. 1AP1-8

Gordon, M. 9AP2-7

Gorgias, N. 9AP8-3

Goueffic, Y. 3AP5-7

Gough, M. 7AP3-7

Gouin, F. 7AP3-8

Gourdin, M. 4AP9-8

Goutziomitrou, E. 10AP4-7

Gramowski, A. 3AP4-9

Grande, P.O. 4AP8-4

Granry, J.C. 9AP8-8
Gratchev, S.J. 3AP8-1

Gratchev, S.S. 3AP8-1

Gray, S.A. 13AP3-1

Grebenik, C. 4AP5-5

Grecu, I. 9AP6-8, 14AP4-4

Greif, R., 1AP2-10, 3AP7-8, 15AP2-2

Grenman, D. 15AP2-5

Griffiths, R. 8AP7-3

Grigg, L. 14AP1-2

Grigoriev, S.V. 12AP4-5

Grimsehl, K. 15AP2-8

Grinpescu, I.M. 2AP1-1

Grintescu, I. 9AP6-8

Grintescu, I.M. 14AP4-4

Groessle, R.M. 6AP1-8

Gromova, V. 7AP1-7

Gronwald, C.A. 9AP4-7

Grossherr, M. 3AP8-9, 5AP1-3, 5AP3-6

Gruber, M. 9AP6-5

Gruenewald, M. 4AP2-6

Grundmann, U. 19AP6-4

Grzybowska, K. 3AP3-4

Grzybowski, A. 10AP4-5

$\mathrm{Gu}$, W. 4AP9-4

Guarnerio, C. 7AP2-4

Guarracino, F. 4AP8-7, 9AP8-7

Guasch, E. 11AP2-7

Guerra, D. 8AP1-2

Guerrero, E. 6AP2-3, 7AP2-1

Guerricchio, A. 3AP7-2

Guerricchio, R. 3AP7-2

Guillet, B. BAPC1-5, 7AP5-6, 7AP5-7

Gulati, A. 8AP7-3

Gulec, H. 8AP4-4

Guler, T. 11AP2-5

Gunal, S. 8AP4-4

Gunastý, S. 11AP2-5

Guntz, E. 8AP9-7

Gupta, S. 13AP1-3, 19AP3-7

Guran, C. 14AP5-11

Gurkan, Y. 9AP2-9

Gürkan, Y. 19AP6-7

Gursoy, S. 10AP3-4

Gustorff, B. 14AP3-1

Gutierrez, B. 8AP3-5

H, Y. 4AP8-2,

Habler, O. 5AP3-2, 6AP1-2, 6AP1-4, 9AP2-4

Hachemi, M. 1AP2-8, 4AP2-1, 6AP2-8, 7AP1-9

Haeseler, G. BAPC1-1, 9AP1-4

Hagau, N. 12AP3-7

Haghighat, B. 13AP2-2

Hagihira, S. 3AP2-2, 9AP5-6

Hagmajer, E. 12AP3-8

Hahnenkamp, K. 9AP4-7

Haitov, Z. 9AP1-6

Hakenberg, O.W. 6AP3-9

Hakkaart, M. Al, L. 12AP2-9

Hakobyan, R. 8AP1-8

Hall, J. 3AP8-6, 8AP9-1, 13AP3-6, 19AP3-10, 19AP5-6

Hall, J.E. 3AP1-7, 3AP6-9, 3AP8-8, 19AP1-9, 19AP5-7

Hall, T. 15AP2-3

Haller, I. 8AP10-8

Hamada, Y. 19AP4-9

Hampson, M.A. 3AP1-7, 19AP1-9, 19AP5-7

Han, A. 9AP2-2

Han, C.K. 14AP2-9

Han, J.I. 4AP7-4

Han, T.H. 9AP5-5

Hanart, C. 10AP1-1, 10AP1-2

Hansen, S. 7AP1-8

Hanss, R. 4AP2-9

Hanssens, M. 11AP1-3 
Hara, K. 8AP3-4

Harasawa, K. 3AP6-7

Harbers, J.B.M. 1AP2-4

Hardman, J.G. 1AP3-8, 5AP1-5, 5AP3-5, 11AP1-5

Hardy, E. 11AP2-10

Hargitai, B. 3AP7-6

Harrison, M. 3AP4-1

Hart, G. 10AP2-2

Harten, J. 4AP7-6, 5AP3-3, 13AP4-2, 13AP4-3

Hartwich, V. 3AP1-9

Harutyunyan, G. 8AP1-8

Harz, A. 12AP2-8

Hasan, S. 3AP8-7

Hasani, A. 10AP6-7

Hasehnoune, K. 12AP9-6

Haseneder, R. 7AP5-2

Hashimoto, T. 14AP2-11

Hauck, N.C. 4AP4-1

Hawe, C. 1AP3-1

Hay, A. 13AP4-3

Hayashi, H. 3AP6-4

Hayek, J. El. 9AP1-9

Hayes, J. 8AP10-1, 10AP6-4

Heeringa, M. 9AP2-1, 9AP2-5, 9AP7-1

Hegemann, T. 13AP3-4

Heimburger, G. 19AP4-8

Heinen, A. 4AP4-1

Heinke, W. 7AP2-6

Heinze, $H$. 5AP3-6

Heller, A.R. 8AP10-11

Hemmer, M. 13AP2-3

Henderson, F. 19AP1-6

Hengstenberg, A. 3AP8-9

Henning, R.H. 4AP9-3

Hentschke, $H$. 7AP5-5

Heras, N.B. 13AP4-4

Heremans, L. 3AP1-2

Hernández, A. 11AP2-1

Hernández, P. 15AP1-5

Hernández, R. 3AP8-5

Hernando, D. 1AP2-9

Hess, L. 7AP2-5, 14AP5-9

Heylen, R. 3AP4-6, 7AP1-6, 7AP3-4, 7AP3-5, 13AP4-5

Higgins, D. 12AP8-2

Hilberath, J. 5AP4-4

Hilbert, P. 13AP1-1

Hillebrand, $H$. 9AP7-10

Himmelseher, S. 7AP5-9, 7AP5-10, 15AP2-9

Hinde, T. 17AP1-1

Hinkelbein, J. 10AP2-1, 13AP1-5, 13AP3-5, 19AP6-1

Hisano, K. 7AP6-6

Hitosugi, T. 12AP4-6

Ho, C. 3AP7-4

Hock, A. 3AP3-9

Hodzovic, I. 3AP6-9, 15AP2-7, 19AP1-1

Hoecherl, K. 12AP5-1

Hoefer, S. 1AP3-7

Hoeft, A. 3AP3-3, 3AP9-2, 12AP5-6

Hoekstra, F. 6AP3-10

Hoetzel, A. BAPC1-3, 5AP2-3

Hoffmann, C. 8AP3-8, 13AP3-7

Hoffmann, C.F. 6AP1-8

Hoffmann, R. 5AP1-2

Hofmann, A. 6AP3-3

Hofmockel, R. 3AP4-9

Hogan, M. 14AP3-9

Hogan, Q.H. 14AP2-4

Hohl, E. Ch. 10AP2-1

Homer-Vanniasinkam, S. 4AP6-6

Hong, Ki-Hyuk. 6AP1-6

Hong, Y. 5AP4-6

Hongo, K. 19AP1-4

Hoogland, P. 14AP3-3
Hope, F. 9AP2-6

Hoppe, C. 8AP5-8

Horasanli, E. 8AP2-7

Horisberger, T. 3AP3-6

Horiuchi, T. 3AP6-4

Horn, B. 3AP3-7

Horn, O. 6AP1-2

Hornuss, C. 3AP1-5

Hosten, T. 19AP6-7

Houweling, P.L. 1AP2-4

Howard-Alpe, G. 1AP2-2

Howell, S. 4AP6-6, 7AP3-7

Hoxha, A. 7AP1-3, 7AP1-4, 7AP2-8, 7AP2-9

Hristov, N. 8AP8-2, 8AP8-3, 12AP9-4

Hsieh, Y. 7AP6-2

Hsing, C.H. 14AP2-3

Huang, P.K. 9AP6-3

Hub, B. 19AP4-5

Huggins, N. 7AP1-1

Huhn, R. 4AP4-1

Huiku, M. 3AP3-8, 3AP6-5

Huismann, S. 5AP3-2

Hung, Y.C. 8AP10-3

Hunkeler, C. 15AP2-2

Hupuczi, P. 11AP1-10

Husedzinovic, I. 3AP2-5

Hwang, K. 2AP2-1

latrou, C. 14AP4-8

lckx, B. 3AP2-8

Igarashi, $H$. 3AP5-5

Igl, B.W. 3AP8-9

Igropoulou, O. 8AP1-4

Ihmsen, $\mathrm{H}$. 9AP5-3

lizuka, T. 19AP4-9

Ikeda, O. 19AP3-5

Ilie, A. 11AP2-9

Ilnitsky, V. 9AP7-6

Imaev, A. 7AP1-7

Imanishi, H. 4AP6-5

Immer, F.F. 4AP5-6

Imoto, A. 8AP1-6

Ince, C. 12AP5-3

Inderbitzin, D. 1AP2-10, 3AP1-9, 3AP7-8

Ingimarsson, J. 9AP2-7

Inoue, S. 3AP6-4

Inoue, T. 8AP1-3

lonescu, D. 8AP8-8

Israelyan, L. 7AP2-10

Ito, Y. 19AP5-5

Ivanova, V. 12AP9-2

Jaber, S. 5AP2-6

Jack, E. 5AP3-3

Jacobsen, J.H. 7AP4-1

Jacobsohn, E. 3AP4-8

Jahromi, S.S. 9AP1-9

Jakob, S. 1AP2-10, 3AP1-9, 3AP7-8

Jakobsson, J. 2AP1-8, 2AP1-9

Jakushenko, N. 19AP4-4

Jamart, J. 4AP9-8

Jamjoom, A. 1AP3-8

Janakiraman, C. 2AP1-7, 8AP3-7, 15AP2-7

Jandewerth, O. 10AP2-1, 19AP6-1

Jandova, J. 14AP5-9

Jandre, F. 5AP1-8

Janin, P. 3AP9-6, 8AP10-2

Janjevic, D. 19AP2-8

Jaunalksne, I. 12AP3-2, 12AP9-8

Jaurrieta, E. 17AP3-4

Jedmowski, A. 5AP1-3

Jee, D.L. 10AP4-4

Jeon, Y.H. 14AP2-9

Jeskins, G. 19AP4-2

Jevtic, D. 19AP2-8

Jimenez, J.A. 19AP6-10

Johannes, T. 12AP5-3
Jones, C. 12AP2-6

Jordan, D. 3AP3-9

Joris, J. 1AP3-3, 1AP3-4, 3AP9-6, 8AP10-2, 14AP1-3

Jornet, S. 14AP4-5

Jouffroy, L. 7AP6-5

Jourdan, C. 7AP1-9

Jovic, R. 19AP2-8

Judy, K. 8AP5-1

Julliac, B. 19AP6-8

Julu, P.O.O. 7AP1-8

Jummani, Z. 12AP8-5

Kaabachi, O. 6AP2-1, 8AP5-3, 10AP6-2, 14AP1-4

$\mathrm{Kaba}, \mathrm{A} . \quad$ AP3-3, 1AP3-4, 3AP9-6, 8AP10-2

Kabbani, Y. 6AP1-9

Kacila, M. 12AP9-3

Kahr, S. 5AP4-4

Kalimeris, K. 5AP2-5, 5AP4-7

Kalkman, C. 17AP2-5, 17AP2-6

Kallel, S. 11AP2-11

Kalpidis, I. 8AP10-10

Kalso, E. 15AP1-6, 15AP2-5

Kamin, G. 11AP1-9

Kampouroglou, G. 5AP2-5

Kanakoudis, F. 6AP1-5, 9AP3-6, 9AP3-7

Kanaya, N. 3AP6-10, 10AP6-3, 19AP1-2

Kanemaru, R. 7AP4-2

Kang, $H$. 17AP1-5

Kanus, I. 3AP8-1

Kapoukranidou, D. 7AP6-1, 8AP10-10

Kappen, T. 17AP2-5, 17AP2-6

Karabulut, E. 9AP3-9, 19AP2-6

Karahan, N. 2AP1-5

Karakoulas, K. 9AP8-3

Karaman, S. 8AP4-6

Karamoulas, V. 8AP9-10

Karanovic, N. 1AP1-6, 3AP2-5

Karathanos, A. 11AP2-2

Karbonskiene, A. 7AP4-7, 8AP5-5, 14AP3-4

Kardum, G. 1AP1-6

Karimi, A. 13AP2-2

Kärkäs, P. 3AP4-5, 3AP6-5

Karoly, O. 11AP2-9

Karoui, A. 8AP2-8, 8AP4-7, 11AP2-11, 14AP5-8

Karpel, E. 3AP3-4

Karsli, C. 10AP3-1

Karsten, J. 5AP1-3

Karta, M. 3AP9-8

Karystianou, G. 14AP4-10

Kasper, M. 12AP1-6

Katoh, T. 15AP2-1

Katonis, P. 8AP1-10

Katsaragakis, S. 8AP8-6

Katz,Y. 19AP5-3

Kaufmann, M. 4AP2-9

Kaufmann, T. 1AP1-4, 1AP2-5

Kawae, T. 3AP6-10

Kawaguchi, M. 3AP6-4

Kawahigashi, H. 19AP5-5

Kawamoto, M. 4AP9-2, 9AP6-1, 9AP8-6

Kawanishi, A. 7AP4-2

Kaygusuz, K. 10AP3-4

Kaymak, C. 4AP3-8

Kazemi, M. 8AP2-4

Kecik, Y. 19AP5-10

Keijzer, Ch. 1AP2-4

Keller, M. 19AP6-1

Kendrisic, M. 8AP7-7

Kenny, G.N. 2AP1-2

Keraitiene, G. 8AP5-5

Kerbaul, F. 4AP9-5

Kerci, M. 7AP1-3, 7AP2-8

Kërçi, M. 7AP2-9 
Kersten, J. 4AP9-4

Kertai, M. 4AP5-5

Kertscho, H. 6AP1-2, 6AP1-4, 9AP2-4

Khajavi, M. 13AP2-2

Khan, E. 3AP9-9

Kharkar, Y. 3AP6-9

Khaw, K. 11AP2-6

Khemakhem, K. 8AP7-8, 14AP5-8

Khünl-Brady, K.S. 9AP7-8

Kiamiloglou, B. 14AP4-8

Kiefer, R.T. 8AP10-9, 9AP1-10

Kilbas, A. BAPC1-6, 12AP5-8

Kilci, O. 8AP4-5, 14AP2-7

Kilic, R. 9AP6-4

Kim, B. 8AP2-6

Kim, D. 7AP2-3, 12AP8-3

Kim, J.H. 8AP6-8

Kim, K. 7AP2-3

Kim, K-M. 6AP1-6

Kim, M. 4AP3-1

Kim, O. 6AP1-6

Kim, S. 8AP6-8, 12AP1-5, 12AP5-5, 17AP1-5

Kim, Y.J. 9AP5-2

Kimball-Jones, P. 3AP5-8, 3AP8-10

Kinnear, J. 12AP8-2

Kinsella, J. 4AP7-6, 5AP3-3, 13AP4-2, 13AP4-3

Kirov, M. 5AP4-1, 14AP1-6

Kis, J. 9AP4-3, 9AP4-4

Kiskira, O. 14AP5-6

Kitamura, A. 4AP6-5

Kjaer, C. 9AP2-9

Kjaer, C.C. 9AP7-3

Klarin, B. 9AP2-7

Klein, G.E. 7AP4-8

Klepstad, P. 3AP3-5

Kletzin, F. 3AP4-9

Kligman, M. 4AP6-8

Klimi, P. 11AP2-2

Klimaschewski, L. 8AP10-8

Klinzing, S. 4AP7-7, 12AP3-3

Klopfenstein, C-E. 3AP1-6

Knapik, P. 4AP5-8, 10AP4-5

Knirsch, W. 10AP1-5, 10AP1-6, 10AP3-3

Kobayashi, E. 9AP8-9

Kobayashi, M. 9AP6-1, 9AP8-6

Kocabas, S. 9AP7-7

Koch, A. 4AP4-7

Koch, M. 4AP2-7

Koch, T. 5AP1-1, 6AP1-8, 6AP3-9, 8AP3-8, 8AP8-7, 8AP10-11 11AP1-9, 12AP1-6, 13AP3-7, 19AP3-9

Kochhar, P. 12AP8-8

Kochs, E. 7AP5-2, 7AP5-9, 7AP5-10

Kochs, E. F. 3AP3-7, 3AP3-9

Kochs, E.F. 7AP3-3, 7AP5-5

Kocijan, G. 14AP1-5

Koenig, F. 9AP1-3, 9AP8-5

Kokars, I. 14AP5-1

Kolacz, A. 9AP4-4

Kolotoura, A. 14AP5-6

Kolsi, K. 14AP5-8

Konova, E. 9AP4-6

Konstadinidis, K. 12AP6-6

Konstantinou, G. 2AP1-6

Koo, A. 2AP2-2

Kopeika, U. 19AP4-4

Kopka, A. 3AP5-1

Korolija, D. 1AP1-7

Kostopanagiotou, G. 5AP2-5, 5AP4-7

Koteeswaran, S. 17AP3-3

Koubaa, W. 6AP2-1, 8AP5-3, 10AP6-2, 14AP1-4

Kouvelas, D. 7AP6-1

Kowalski, R. 3AP6-3, 3AP9-9

Koyama, J. 19AP1-4
Kozhikunnath, A. 12AP8-1

Kozlov, I. 9AP7-6

Krage, R. 17AP1-4

Krahl, S. 13AP1-5

Krajina, A. 7AP2-5

Krana, A. 8AP8-6

Kratzer, S. 7AP5-2

Kretschmar, O. 10AP1-5, 10AP1-6, 10AP3-3

Kreuer, S. 19AP6-4

Kreuzer, M. 3AP3-7, 7AP5-5

Krid, K. 8AP4-7

Krist, K. 9AP8-5

Krivic, B. 8AP9-4

Krobot, R. 8AP6-3, 14AP1-5

Krokowicz, L. 4AP6-2

Krovvidi, H. 7AP1-1, 19AP4-2

Krul-Sterk, A. 8AP6-4

Kucewicz-Czech, E. 4AP5-8

Kuchyn, I. 10AP2-3, 12AP1-3, 12AP1-4

Kuczkowski, K. 11AP1-1

Kuebler, W.M. 5AP2-1, 5AP2-2

Kuizenga, K. 8AP6-4, 9AP7-3

Kulcsar, Z. 15AP2-3, 15AP2-6

Kulic, M. 12AP9-3

Kumar, D. 10AP4-1

Kumar, G. 12AP7-4

Kume, Y. 3AP5-5

Kunkele, S. 12AP3-5

Kuo, Y-W. 8AP2-1

Kupcsulik, P. 3AP7-6

Kurian, J. 12AP7-6

Kurita, S. 4AP9-2

Kurtz, A. 12AP5-1

Kurzova, A. 14AP5-9

Kusza, K. 4AP6-2

Kutschker, S. 6AP1-4

Kuzkov, V. 5AP4-1

Kuzmin, D. 19AP4-5

Kwak, K.H. 14AP2-9

Kwak, S. 12AP1-5, 12AP5-5

Kwak, Y. 5AP4-6

Kwok, W.M. 14AP2-4

Kyi, M. 6AP3-5

Kymalainen, M. 3AP3-8

Kymäläinen, M. 3AP4-5

Kyriakides, C. 8AP5-7

Laalou, F. 7AP6-5

Lacasta Fornells, A. 12AP8-9

Lacasta, A. 8AP2-5

Ladjevic, N. 8AP9-4

Laffon, M. 8AP5-6

Lagadec, $\mathrm{H}$. 3AP5-7

Lages, N. 2AP1-3

Lagoudianakis, E. 8AP7-9

Lahiji, F. 8AP2-4

Lahoute, T. 4AP9-1

Lai, H.Y. 9AP6-3

Lambert, J. BAPC1-1, 9AP1-4

Lamberts, R.R. 4AP3-5, 4AP3-6

Lamy, M. 1AP3-3, 1AP3-4, 8AP10-2

Lancaster, L. 3AP8-3

Landau, R. 14AP2-8

Landoni, G. 4AP1-5, 4AP3-2

Lange, $H$. 19AP3-9

Lange, M. 4AP8-9, 12AP5-2

Langer, K. 5AP4-4

Langer, M. 14AP1-1

Langeron, O. 5AP2-6, 5AP2-7, 5AP2-8, 12AP1-9

Langford, L. $\quad 6 A P 3-4$

Langham, B. 1AP1-1

Langouche, L. 12AP4-3

Lanzillo, G. 6AP3-7

Lau, T. 11AP2-6

Laurentie, M. 8AP10-6

Lauwers, R. 3AP8-7, 4AP9-1
Lauwick, S. 1AP3-3, 3AP9-6, 8AP10-2

Lavandhomme, P. 11AP1-7, 11AP2-10, 14AP1-8, 14AP2-10, 14AP3-8

Le Manach, Y. $\quad$ 4AP1-4, 4AP1-6, 9AP8-10

Le Saché, F. 4AP1-4

Lebedinskiy, K. 4AP7-5

Leclercq, S. 10AP1-1, 10AP1-2, 10AP1-3

Ledoux, D. 14AP1-3

Lee, C. 3AP7-4

Lee, D.H. 10AP4-4

Lee, H.K. 17AP3-2

Lee, J. 10AP4-3

Lee, J.H. 14AP5-4

Lee, K. 9AP3-4, 10AP4-3, 10AP5-4, 19AP2-5

Lee, P. 11AP2-4

Lee, S. 2AP2-1

Lee, S.C. 10AP4-6, 14AP5-4,

Lee, S.H. $\quad$ 4AP7-4

Lee, S.I. 10AP4-6

Lee, S-S. 6AP1-6

Lee, S.J. 8AP6-8

Lee, Y. 9AP6-3

Lee, Y.K. 9AP8-2

Lefebvre, F. 12AP2-1

Lehmann, C. 4AP8-8

Lehmann, L.E. 12AP5-6

Lehot, J.J. 1AP2-8, 3AP7-1, 3AP8-2, 4AP2-1, 4AP2-8, 6AP2-8

Lemaire, L.C.J.M. 2AP2-5

Lemos, P. 2AP2-6, 2AP2-7

León, P. 10AP1-7

Leone, S. 8AP2-2, 8AP9-2

Leonhardt, S. 5AP1-3

Leontidou, A. 14AP4-10

Leontopoulou, S. 11AP2-2

Lerch, M.M. 8AP8-9

Lerintiu, M. 11AP2-9

Lestrat, S. 10AP1-4

Leteurnier, Y. 3AP5-7

Letournel, F. 9AP8-8

Leuwer, M. BAPC1-1, 9AP1-4

Levano, S. 9AP8-11

Levin, A. 14AP4-2, 14AP5-2

Lewis, E. 19AP1-9, 19AP5-7

Leykin, Y. 9AP2-8

Leyvraz, P-F. 6AP1-1

Li, C.X. 4AP4-4, 4AP4-5, 7AP5-1

Li, E. 4AP8-2

Li, P. 4AP8-2

Li, T. 9AP3-1

$\mathrm{Li}, \mathrm{X}$. 9AP3-1

Likic Ladjevic, I. 8AP9-4

Lim, D.G. 14AP2-9

Lim, S.H. 9AP5-2

Limeres, J. 19AP3-8

Lin, B.C. 3AP1-8

Lin, C.C. 13AP3-2

Lindner, R. 9AP6-5

Linhartova, L. 12AP2-6

Lirk, P. 8AP10-8

Lisagors, I. 12AP9-8

Lisco, S. 19AP4-8

Litvan, H. 3AP4-3

Litz, R.J. $\quad$ 6AP1-8, 6AP3-9, 8AP3-8, 8AP8-7, 8AP10-11, 11AP1-9, 13AP3-7

Liu, J. 3AP3-2, 4AP4-4, 4AP4-5, 7AP5-1, 7AP5-4

Liu, S. 4AP8-2

Liu, S.K. 3AP1-8

Llasera, R. 15AP1-3

LLeu, J. 9AP3-3

Lleu, J.C. 7AP6-4

Llorens, J. 12AP7-10

Lluch, A. 15AP2-4

Lobo, F. 3AP4-7, 9AP5-9, 9AP5-10

Loef, B.G. $\quad 4 A P 7-8,6 A P 3-10$

Loer, S. 14AP3-5, 14AP3-6 
Loer, S.A. $\quad$ 4AP3-5, 4AP3-6, 4AP4-7, 13AP3-4

Loizou, C. 8AP8-6

Loizou, Ch. 8AP7-9

Lonardo, M. 7AP1-2, 12AP3-4

Longmate, $A$. 1AP3-1

López, M. 6AP2-6

López, R. 11AP2-1

López, S. 10AP2-5

Lopez-Quesada, T. 3AP5-2, 3AP8-4, 3AP9-10

López-Romero, J.L. 9AP1-2

Lopez-Vicente, R. 19AP5-4

Lopez-Zayas, E. 12AP8-7

Lora-Tamayo, J.I. 9AP7-8

Lorang, C. 13AP2-3

Lorsomradee, S. 4AP6-1, 4AP7-2

Lossen, M. 9AP2-4

Loureiro, R. 1AP1-2

Lourenço, A.P. 4AP5-7

Lovquist, E. 15AP2-6

Low, J. 1AP1-1

Lowe, A. 3AP4-1

Lozano, M. 6AP2-9

Lu, I-Cheng. 8AP1-1

Lu, I. 3AP6-1, 8AP2-1

Lubnin, A. 7AP1-7, 7AP2-10, 7AP4-4

Luginbuehl, I. 10AP3-1

Luijten, A. 3AP1-3

Lukaszewska, A 12AP3-8, 12AP6-8

Lum Hee, W. 12AP8-5

Lynch III, C. 4AP3-1

Mabrock, M. 10AP2-4

Macas, A. 14AP3-4

MacGregor, M. 12AP7-6

Macknet, M. 3AP5-8, 3AP8-10

Macpherson, A. 2AP1-2

Macule, F. 6AP2-9

Mademli, A. 9AP3-6

Madzar, I. 3AP1-6

Maestre, M.L. 3AP4-3

Maguire, S.L. $\quad$ 8AP6-7, 12AP8-8

Mahajan, R.P. 5AP1-4

Mahon, P. 3AP6-3, 3AP9-9

Maia, P. 4AP5-7, 12AP8-4

Mairbäurl, H. BAPC1-4, 5AP2-4

Majeric Kogler, V. 12AP7-7

Majeric-Kogler, V. 1AP1-7

Makino, H. 15AP2-1

Makita, T. 9AP8-4

Makunaite, A. 14AP3-4

Malbouisson, L.M. 14AP5-10

Malchow, R. 8AP3-1

Maldonado-Campos, A. 1AP3-2, 6AP3-1

Maldonado-Contreras, J. 1AP3-2, 6AP3-1

Malek, J. 14AP5-9

Malhotra, S.K. 10AP4-1

Mamatha, J. 5AP3-5

Mamaya, B. 3AP6-8, 10AP2-6

Manabe, M. 3AP5-3, 19AP1-8

Manailova, T. 8AP8-3

Manataki, A. 8AP1-4

Mandersloot, G. 1AP3-5

Mandolfino, T. 8AP9-9

Manering, N. 14AP2-6

Mangia, F. 6AP3-7

Maniadakis, M. 8AP4-2

Manninen, P. 7AP2-7

Manrique, S. 10AP4-2

Mantz, J. 12AP9-6

Mao, Ch. 6AP1-3

Mao, W. 19AP5-2

Marchetti, C. 4AP1-3

Marco, J. 10AP2-5

Marino, G. 4AP5-4

Marinou, S. 6AP1-5

Marjanski, T. 8AP8-1
Marret, E. 8AP7-2

Martani, C. 9AP2-3

Martella, N. 3AP5-9

Martí, A. 5AP3-7

Martí, I. 1AP3-6

Martín, J. 19AP2-7

Martin, K. 6AP2-4

Martin, R. 3AP5-8, 3AP8-10

Martínez Silva, O. 12AP8-9

Martinez, R. 8AP4-1

Martins, C. 3AP6-6

Martins, F. 3AP2-9, 3AP6-6, 3AP9-4, 3AP9-5

Martorell, M. 6AP2-3

Martyn, J.A.J. 9AP2-2, 12AP9-5

Maruenda, A. 7AP4-6

Marx, G. $\quad$ 4AP7-7, 12AP2-8, 12AP3-3

Marzal, J.M. 6AP2-7, 17AP2-7

Mashimo, T. 3AP2-2, 9AP5-6

Mashio, H. 19AP5-5

Masoni, E. 5AP4-2

Massicotte, E. 19AP1-10

Massó, E. 4AP1-8

Mata, J. 3AP4-4, 14AP5-3

Mathes, A. 12AP4-1

Mathew, G. 15AP2-8

Mathijs, A. 19AP4-1

Matot, I. 17AP3-5

Matsaridou, E. 9AP8-3

Matsota, P. 5AP2-5

Matsui, H. 3AP5-5

Matsumoto, M. 3AP5-5

Matsumoto, T. 19AP3-4

Mavridou, P. 8AP1-4

Mayr, D. 14AP3-1

Mayumi, T. 3AP6-7

Mazzeo, G. 8AP9-9

Mazzola, A. 12AP7-3

McCallum, J.B. 14AP2-4

McCann, D. 19AP4-8

McCheyne, A.J. 10AP3-1

McClelland, S.H. 11AP1-5

McConnachie, A. 4AP7-6

Mccormack, J. 12AP7-1

McCreath, B. 13AP4-2, 13AP4-3

McGrath, P.A. 10AP2-2

McGrattan, K. 1AP2-6, 1AP2-7, 7AP4-5

McMillan, D. 13AP4-2

McNamara, B. 3AP6-3, 3AP9-9

McPherson, D. 2AP1-7, 3AP8-8, 8AP3-7

Medina, J.S. 19AP3-8

Meier, J. 5AP3-2

Meier, T. 5AP1-3

Meinhardt, J. 13AP3-5

Meissner, C. 12AP1-6

Mele, R. 8AP5-2

Melemeni, A. 14AP3-2

Melo, M. 5AP1-8

Mendonca, T. 3AP9-3

Meretoja, O. 9AP7-5

Merino, I. 6AP2-9

Merlan, V. 9AP6-8

Mertens, L. 13AP2-3

Messada, K. 3AP9-6

Meybohm, P. 7AP6-8, 9AP4-9, 13AP2-4, 13AP2-5

Michael, E. 17AP1-4

Michelet, P. 4AP9-7, 5AP2-6, 5AP2-7, 5AP2-8, 12AP1-9

Mielniczuk, M. 4AP6-2

Migita, T. 9AP6-1, 9AP8-6

Mihelsons, M. 19AP4-4

Mik, E.G. 12AP5-3

Mikstacki, A. 3AP2-3, 12AP6-7

Miletic, D. 19AP3-2

Mimaroglu, C. 10AP3-4

Mimuro, S. 7AP5-8

Minas, G. 10AP4-7
Mincu, N. 14AP5-11

Mirakhur, R.K. 9AP7-5

Mirkheshti, A.R. 8AP2-4

Mishra, L.D. 7AP1-5

Mishuk, Y. 17AP3-5

Misiak, M. 12AP6-8

Mitrev, Z. 8AP8-2, 8AP8-3, 12AP9-4

Mizikov, V. 9AP2-10

Mkhoyan, G. 8AP1-8

Modesti, C. 3AP5-9

Moeller, J.F. 8AP3-6

Moguilevitch, M. 19AP4-3

Mohabir, A. 4AP2-2

Mohammed, S. 19AP3-1

Moise, A. 14AP5-11

Molies, D. 10AP2-5

Moltó García, L. 13AP4-4,

Moltó, L. 17AP2-4

Momeni, M. 14AP1-8

Monclus, E. 10AP2-4

Monerris, M. 5AP4-5

Monfort, E. 1AP3-3

Monnin, D. 6AP1-9

Montazeri, K. 10AP6-5

Montferrer, N. 10AP4-2

Moody, C. 7AP1-1

Mooney, M. 13AP4-1

Moons, K.G.M. 4AP2-3, 4AP2-4

Mooren, R.A.G. 3AP7-9

Moppett, I.K. 1AP3-8, 5AP1-5

Mora, A. 12AP4-7

Mora, L. $\quad$ 6AP2-3, 7AP2-1, 8AP2-5

Moral, M.V. 5AP1-2

Moral, V. 3AP4-3

Morand, A. 6AP2-8

Moreira, D. 2AP2-6, 2AP2-7

Moret, E. 4AP1-8, 15AP1-3

Mori, T. 3AP2-2

Morimoto, Y. 3AP6-7, 7AP6-6, 14AP2-11

Morina, A. 12AP6-3

Morina, Q. 12AP6-3

Mosaffa, F. 8AP2-4

Moshchev, D. 7AP4-4

Moss, J. 9AP4-8

Mourão, J. 19AP1-3

Moussa, A. 9AP6-2

Moussa, A.A. 19AP2-1

Moustafa, M.K. 5AP3-8

Mueller, M. 13AP3-7

Mueller, S. 12AP2-8

Mukaida, K. 9AP6-1, 9AP8-6

Mulder, P. 12AP2-9

Mulier, J.P. $\quad 3 A P 1-1,3 A P 1-2,3 A P 1-3,3 A P 1-4$, 17AP3-1, 19AP4-1

Munar, F. 10AP4-2

Mungroop, $\mathrm{H}$. 4AP7-8

Mungroop, H.E. 6AP3-10

Muñoz, H. 10AP1-7

Munster, T. 9AP5-3, 12AP3-5

Murat, I. 9AP5-1

Murray, M. 4AP1-2

Murrell, C. 7AP4-5

Musayeva, T.S. 12AP4-5

Musiol, E. 3AP3-4

Mustola, S. 3AP3-8

Mutlu, N.M. 9AP3-9, 19AP2-6

Muzha, D. 7AP2-9

Nadarajan, S.K. $\quad$ 19A92-4

Nadolski, J. 3AP2-3

Nadziakiewicz, P. 4AP5-8

Nagata, O. 9AP5-6

Nagata, S. 7AP4-2

Nagobade, D. 19AP4-4

Najafi, M. 13AP2-2

Nakos, G. 5AP4-7

Namiki, A. 3AP6-10, 10AP6-3, 19AP1-2

Narcisse, F. 6AP2-5 
Nassef, M.A. 19AP2-1

Natsis, K. 1AP2-3

Naulaers, G. 9AP5-1

Navarro-Martinez, J. 5AP1-7

Nedashkovskiy, E. 12AP9-2

Nedashkovsky, E. 5AP4-1, 14AP1-6, 14AP4-2, 14AP5-2

Negmi, H. 14AP4-9

Nelson, L. 19AP4-8

Net, M. 4AP8-5, 4AP8-6

Neuburger, M. 8AP3-2

NG, C.C. 13AP3-2

$\mathrm{Ng}, \mathrm{K} . \quad 11 \mathrm{AP} 2-6$

Ngan Kee, W. 11AP2-6

Nicolae, G. 2AP1-4, 6AP3-6

Nicolau, A.E. 9AP6-8

Nicolau, L. 1AP3-9

Nicolau, M. 9AP6-8

Niemi-Murola, L. 15AP1-6, 15AP2-5

Nierich, A.P. $\quad 4$ AP2-3, 4AP2-4

Nieto, J.M. 19AP6-10

Nieuwenhuizen, R. 3AP1-3

Nikic, N. 3AP2-5

Nikitina-Zake, L. 12AP3-2

Nikolajsen, L. 8AP3-6

Nikouli, E. 2AP1-6

Nimmo, A.F. 9AP5-7

Nishioka, K. 9AP8-4

Nishiyama, T. 14AP2-1

Noble, J.S. 4AP1-2

Noeldge-Schomburg, G.F.E. 3AP4-9

Noetges, P. 13AP3-4

Nomura, T. 8AP3-4

Nouette-Gaulain, K. 3AP7-10, 12AP7-9

Novakovic, D. 10AP3-6, 10AP6-9

Novalija, E. 4AP4-8

Novalija, J. 4AP4-8

Novelli, E. 6AP3-7

Nunes, C.S. 3AP3-10, 3AP9-3, 3AP9-4, 3AP9-5, 7AP3-6, 9AP5-4, 9AP5-10

Nuyens, V. 4AP8-3

Nyktari, V. 8AP1-10

Oberling, P. 7AP6-4

Oberreiter, M. 4AP6-9

Obideyi, A. 17AP1-3

Obrien, C. 2AP1-2

Oda, F. 9AP7-9

OFlynn, S. 15AP1-4

Ogica, A. 12AP2-4

Ogura, A. 8AP1-3

Ogura, J. 8AP1-3

Ogurtsova, A. 7AP4-4

Ohe, Y. 19AP4-9

Oi, Y. 12AP4-6

Okamoto, K. 19AP3-4

Okhovat, A. 10AP6-5

Okten, F. 19AP5-8

Okur, S. 8AP1-9

Oliveira, D. 3AP3-10

Oliveira, S. 7AP3-6

Olsson, I.A.S. 9AP3-10

Olthoff, D. 9AP6-9

Omerbasic, E. 12AP9-3

Onaka, T. 9AP8-9

Oort, F. 2AP2-5

Opramolla, G. 12AP3-6

Oregan, P. 9AP6-2

Oremus, K. 1AP1-7

Orliaguet, G. 5AP2-6, 5AP2-7, 5AP2-8, 12AP1-9

Orlov, M. 14AP1-6

Ormonde, L. 1AP3-9

Ornek, D. 8AP2-7

ORourke, M. 15AP1-4

Orskiran, A. 8AP4-6

Ortega-Urbaneja, M. 3AP5-2, 3AP8-4, 3AP9-10
Ortiz, J.C. $\quad$ 1AP2-9, 3AP7-5

Osinaike, B. 8AP6-2

Oss, P. 12AP3-2, 12AP9-8

Oswald, D. 5AP2-1

Ota, J. 8AP3-4

Oti, C. 12AP7-6

Ouaki, J. 10AP3-5, 10AP6-8

Ouezzini, R. 6AP2-1, 8AP5-3, 10AP6-2, 14AP1-4

Ovchinnikov, N. 14AP1-6

Owczuk, R. 8AP8-1, 12AP8-6

Ozaki, M. 9AP5-6

Ozalevli, M. 11AP2-5

Ozatamer, O. 19AP5-8

Ozdemir KOI, I. 10AP3-4

Ozdogan, L. 8AP1-7, 8AP4-5, 9AP1-8, 14AP2-7

Ozgen, S. 19AP2-2

Ozgultekin, A. 9AP3-5

Ozgurbuz, U. 2AP1-5

Ozier, Y. 8AP3-3

Ozolins, J. 3AP6-8

Oztekin, D. 4AP7-9

Oztekin, I. 4AP7-9

Pagan, F. 12AP1-2

Pahaut, J. 1AP3-4

Pahl, A. 12AP3-5

Pain, L. 7AP6-4, 7AP6-5, 7AP6-9, 9AP3-3, 9AP6-7

Pala, Y. 8AP1-9, 8AP4-5, 8AP9-8

Palas, T. 8AP6-1

Palud, M. 8AP5-4

Paluzie, G. 5AP3-7

Pamuk, G. 19AP2-2

Panchagnula, U. 8AP2-3

Panteleou, K. 8AP1-4

Papadima, A. 8AP7-9

Papadopoulos, D. 8AP9-10

Papaioannou, K. 2AP1-6, 8AP9-10

Pape, A. 6AP1-2, 6AP1-4, 9AP2-4

Papoutsidakis, N. 5AP4-7

Papp, Z. 11AP1-10

Pappin, D. 10AP5-2

Paprotny, S. 3AP3-7

Parcha, C. 19AP4-2

Pardina, B. 8AP4-1

Parente, S. 1AP1-2

Parera, A. 5AP1-2

Parise, M. 8AP8-5

Parizkova, R. 4AP6-9

Park, C. 8AP2-6

Park, W. 4AP3-1

Parker, M. 8AP7-3

Parkkari, T. 3AP3-8

Parvanian, S. 4AP7-5

Paschalidis, I. 3AP9-8

Pasquarelli, L. 8AP6-5

Pasquini, C. 4AP8-7

Patel, A. 4AP2-2, 6AP3-4

Pavlovic, D. 4AP8-8

Paydari, N. 13AP2-2

Peebles, K. 7AP4-5

Peidro, L. 6AP2-9

Pekel, M. 8AP2-7, 8AP9-8

Pelavski, A. 15AP1-1

Pelikan, K. 19AP2-3, 19AP6-5

Pellicer, F. 14AP4-5

Pellico, G. 10AP6-1

Pellis, T. 9AP2-8

Pellisé, F. 7AP2-1

Pelosi, P. 5AP1-1

Peramo, F. 1AP3-2, 6AP3-1

Perbet, S. 3AP7-3, 4AP1-4, 9AP8-10

Pereira, T. 2AP1-3

Perel, A. 17AP3-5

Perez, R. 14AP3-3, 14AP3-5, 14AP3-6

Pérez, S. 8AP9-6, 9AP4-10
Pérez-Gallardo, A. 3AP9-10

Perrin, L. 4AP2-7

Peters, M. 12AP8-1

Petropoulou, P. 10AP4-7

Petropoulou, O. 11AP2-2

Petrovski, V. 8AP8-2, 8AP8-3, 12AP9-4

Petrucci, R. 19AP4-6

Peuskens, D. 7AP1-6

Pfenninger, E. 7AP5-9, 7AP5-10, 15AP2-9

Philipchuck, P. 19AP5-3

Piana, F. 10AP1-4

Piankowski, A. 17AP3-6

Piazza, O. 7AP1-2, 12AP3-4, 12AP3-6

Piccardo, A. 3AP7-2, 8AP6-5

Picker, O. 4AP3-3, 4AP8-1, 12AP1-7, 17AP1-4

Pico, S. 12AP8-7

Piepenbrock, S. 4AP7-1, 8AP5-8

Pieteris, L. 14AP3-4

Pietrin, D. 9AP8-8

Pilika, K. 7AP1-3, 7AP1-4, 7AP2-8

Piñeiro, P. 8AP3-5

Pinto, B. 8AP4-3,

Pinto, R. 11AP2-8

Pirgari, B. 19AP1-5

Pirogov, A. 19AP4-10

Pirri, S. 3AP2-1, 12AP7-3

Pisano, P. BAPC1-5, 7AP5-6, 7AP5-7

Pitsis, A. 4AP1-7

Pizov, R. 4AP6-8

Plaud, B. 9AP7-5

Ploppa, A. 8AP10-9, 9AP1-10

Pogatzki-Zahn, E.M. 14AP2-5, 14AP4-1

Pohl, B. 9AP7-5

Poli, D. 4AP3-2, 6AP3-2

Polli, F. 12AP1-1

Polyzois, V. 14AP5-6

Pompei, L. 3AP7-7, 9AP7-10

Poon, P.W.F. 14AP2-3

Pooni, J. 12AP2-6

Popescu, H. 12AP2-4

Popov, J. 9AP4-6

Popovic, N. 8AP9-4

Pöpping, D. 14AP4-1

Pöpping, D.M. 8AP7-2

Postaci, A. 9AP1-8

Pottecher, T. 6AP2-10

Pourzitaki, C. 7AP6-1

Prabhu, A. 7AP2-7, 19AP1-10

Pradarutti, S. 12AP4-1

Pratsas, K. 8AP4-8, 8AP4-9

Pratt, G. 8AP1-2

Praun, S. 3AP1-5

Preckel, B. 2AP2-5, 4AP4-1, 4AP4-6

Premuzic, J. 8AP6-3, 14AP1-5

Priston, M.J. 10AP3-7

Protic, A. 8AP6-6, 19AP3-2

Przesmycki, K. 4AP6-4, 9AP4-3, 9AP4-4

Puebla, G. 17AP2-7

Pühringer, F. 9AP2-5

Pulver, C. 3AP1-9

Punjasawadwong, Y. 10AP5-3

Puzanova, I. 4AP7-5

Quasim, S. 6AP3-5

Quattrone, D. 8AP9-9

Quesada, P. 8AP3-5

Quiney, N. 6AP3-4

Quiney, N.F. 4AP2-2

Rabie, M. 14AP4-9

Ræder, J. 3AP3-5

Radke, R.M. 9AP4-7

Raft, J. 9AP7-5

Raghavan, K. 2AP2-2

Rahe-Meyer, N. 4AP7-1, 6AP1-7, 6AP1-10, 8AP5-8 
Raines, D. 9AP4-2

Rajkumar, N. 7AP1-5

Ramaiah, R. 12AP8-5

Ramelli, A. 5AP4-2

Ramirez, S. 6AP2-7

Rammes, G. 7AP5-2

Ramos, I. 5AP3-4

Rancurel, E. 13AP1-6

Randall, A. 14AP1-2

Rashid, A. 12AP7-6

Ratajczak, M. 8AP10-6

Raux, O. 10AP3-5, 10AP6-8

Ravishankar, M. 19A92-4

Read, G. 13AP3-6

Reekers, M. 3AP7-9

Rees, L. 13AP3-6

Rehnig, R. 11AP1-9

Reich, C. 5AP2-1

Reid, A.W. 4AP1-2

Reinhard, C. 12AP3-3

Reinhart, K. 12AP2-8, 12AP3-3

Reisig, F. 8AP3-2

Reitsma, J.B. 4AP2-4

Remerand, F. 6AP2-5, 8AP5-4

Remy, C. 8AP7-2

Renfrew, I. 1AP3-5

Renner, J. 4AP2-6

Rensing, $\mathrm{H}$. 12AP4-1

Renz, V. 4AP2-5

Repel, D. 19AP2-3

Repin, K. 8AP10-7

Reuter, T. 14AP2-6

Revel, P. 3AP7-10, 12AP7-9

Revuelta, M. 3AP4-3

Rex, C. 9AP2-7

Ribeiro, L. 3AP8-11

Richard, A 11AP1-7

Richart, M.T. 3AP2-4

Richez, B. 6AP1-9, 19AP6-8, 19AP6-9

Richir, M. 12AP4-3

Rico-Feijoo, J. 12AP8-7

Riedel, B. 8AP1-2

Rieg, A. 4AP9-6

Riese, G. 3AP3-3

Rigaud, M. 14AP2-4

Rigby-Jones, A. 9AP5-8, 10AP3-7

Rigó, B. 11AP1-10

Rigouzzo, A. 10AP1-4

Riou, B. 4AP9-7, 5AP2-6, 5AP2-7, 5AP2-8, 12AP1-9, 13AP1-6

Roca, M. 15AP1-1

Roca, R. 5AP1-7

Rocha, F. 4AP6-3

Rochera Villach, M.I. 12AP8-9

Rochera, M. 15AP1-1

Rochera, M.I. 8AP2-5, 12AP8-9

Rochette, A. 10AP3-5, 10AP6-8

Rodrigues, T. 1AP3-9

Rodriguez Cosmen, C. 13AP4-4

Rodríguez Valdés, C. 19AP3-8

Rodriguez, A. 14AP4-5

Rodríguez, B. 3AP2-10

Rodriguez, C. 17AP2-4

Rodriguez, L. 7AP4-3

Rodriguez, M. 5AP3-7, 12AP8-7

Rodriguez, W. 4AP4-2

Rodriguez-Laiz, G. 4AP8-6

Rodt, S.A. 8AP3-6

Roediger, L. 14AP1-3

Røeggen, I. 3AP3-5

Roelants, F. 11AP1-7, 11AP2-10

Roenning, $\mathrm{H}$. 8AP3-6

Roessel, T. 8AP8-7, 8AP10-11

Roewer, N. 1AP3-7

Roh, W. 8AP2-6

Roige Sole, J. 12AP8-9

Roige, J. 19AP6-10

Roigé, J. 8AP2-5, 15AP1-1
Roiu, C. 8AP8-8

Rojo, A. 6AP2-6

Romero, F. 3AP6-6

Rondelet, B. 4AP9-5

Rooselaer, N. 3AP8-7, 14AP1-9

Rosamel, P. 3AP7-1, 4AP2-8

Rosenberg, P.H. 15AP1-6

Rosenberger, D. 4AP4-2

Rosencher, N. 8AP3-3

Rossaint, R. 4AP9-6

Rossi, M. 8AP9-2

Roth, W. 10AP2-7

Royston, D. 4AP5-5

Rozec, B. 3AP5-7

Rudolph, M. 9AP6-9

Rueffert, H. 9AP1-3, 9AP8-5

Ruesch, D. 9AP4-2

Ruggieri, F. 9AP2-3

Ruggiero, G. 14AP1-10

Rühlmann, S. 12AP5-7

Rull, M. 14AP4-5

Ruppert, M. 13AP4-1

Russell, I.F. 11AP2-3

Ryu, S. 17AP1-5

Sa Couto, P. 3AP2-9, 3AP9-4, 3AP9-5

Sá Monteiro, M. 2AP2-6, 2AP2-7

Saager, L. 3AP4-8

Sabaté, A. 17AP3-4

Sabaté, S. 3AP7-5

Sabelnikovs, O. 12AP3-2

Sablon, T. 3AP1-1

Sabova, D. 15AP2-3

Sada, F. ABT:12AP6-3

Sadia, H. 19AP3-7

Sadis, C. 12AP5-4

Sagadai, S. 8AP2-3, 8AP6-7

Sagadei, S. 12AP8-8

Sahin, F. 8AP1-7

Sahin, O. BAPC1-6, 12AP5-8

Saidi, Y. 12AP6-2

Saijo, H. 19AP1-2

Saito, T. 8AP1-3, 12AP4-6

Sakamoto, A. 8AP1-3

Sakamoto, H. 3AP6-7

Sakka, S. 12AP7-8

Sakr, Y. 4AP7-7

Sakura, S. 8AP3-4

Sakuraya, F. 19AP5-5

Saldien, V. 9AP7-3

Salem, M. 5AP3-8

Salemi, S. 12AP7-3

Salgado, I. 2AP2-3

Salgado, S. 9AP4-10

Salgo, B. 10AP2-7

Salhi, M. 8AP2-8

Salimi, A.R. $\quad$ 8AP2-4

Saltel, L. 19AP6-8

Salvy, N. 5AP2-7, 12AP1-9

Samanta, E. 8AP7-9, 8AP8-6

Samarska, I. 4AP9-3

Sammartino, M. 10AP1-4

San, S. 19AP6-7

Sánchez, J. 3AP2-4

Sanchez-Navas, S. 17AP2-4

Sandby-Thomas, M. 8AP9-1, 19AP1-1, 19AP3-10

Sandru, S. 19AP1-5

Sanfilippo, M. 3AP7-2, 8AP6-5

Sangion, S. 14AP1-1

Sanjo, Y. 15AP2-1

Sanjoaquín, T. 11AP2-1

Santa-Ursula, J.A. 17AP2-7

Santiveri, X. 8AP4-1, 15AP1-5

Santos, C. 12AP8-4

Santos, I. 3AP4-7

Santos, L.M. 14AP5-10

Santos-Cidón, P. 4AP8-5, 4AP8-6
Sanusi, A. 8AP6-2

Sápi, E. 10AP5-7

Saraci, M. 7AP1-3, 7AP1-4, 7AP2-8

Sarantopoulos, C. 14AP2-4, 14AP3-2

Saridemir, B. 14AP5-5

Sariñena, M. 4AP1-8, 5AP3-7

Saros, G.B. 2AP1-9

Sartorius, D. 13AP1-6

Sastim, H. 9AP1-8, 14AP2-7

Sato, S. 3AP5-5, 7AP5-8, 15AP2-1

Sato, Y. 9AP8-9

Saunders, F. 4AP8-9

Savolainen, T. 3AP6-5

Savoldelli, G.L. 17AP2-2

Sawicka, W. 12AP8-6

Sazonova, O. 7AP4-4

Scanni, E. 9AP7-2

Scarpati, G. 7AP1-2

Schade, M. 4AP8-8

Schaden, E. 12AP2-5

Schaedeli, C. 7AP5-9, 7AP5-10

Schaffer, I. 14AP5-7

Schälte, G. 4AP9-6

Schaper, C. 9AP1-5

Scheeren, T.W.L. 12AP1-7

Scheibe, R. 4AP8-8

Scheit, M. 8AP8-7

Schelenz, C. 12AP7-8

Schelling, G. 3AP1-5

Schember, C. 3AP7-7

Schepers, R. 11AP1-2

Schewe, J.C. 12AP5-6

Schier, R. 8AP1-2

Schiere, S. 8AP6-4

Schiewe-Langgartner, F. 9AP6-5

Schiffer, E. 3AP1-6

Schlack, W. 4AP4-6

Schleppers, A. 1AP1-3, 1AP1-9

Schmartz, D. 4AP2-7

Schmidt, C. 12AP5-1

Schmied, S. 3AP8-5

Schmitt, H. 9AP5-3

Schmitz, A. 3AP3-6, 10AP1-5, 10AP1-6, 10AP3-3

Schmoeckel, M. 4AP2-5

Schmucker, P. 5AP3-6

Schneeweiss, S. 10AP2-2

Schneider, G. 3AP3-7, 3AP3-9, 7AP3-3, 7AP5-5, 13AP3-3

Schneppenheim, S. 6AP1-7

Schober, P. 4AP4-7, 13AP2-1, 13AP3-4

Schobinger, E. 9AP8-11

Scholz, A. 19AP5-6

Scholz, J. 4AP2-6, 4AP2-9, 7AP4-1, 9AP1-5, 9AP4-9, 9AP7-10, 13AP2-4 13AP2-5, 19AP6-6

Schouten, T. 17AP2-5, 17AP2-6

Schouteten, L. 7AP1-6

Schreiber, M. 8AP7-1

Schreiberova, J. 7AP2-5, 14AP5-9

Schroth, S. 4AP9-6

Schuepfer, G. 1AP1-4, 1AP2-5

Schuler, U. 6AP1-8

Schumacher, J. 13AP3-1

Schumacher, P. 3AP7-8

Schuster, A. 9AP4-2

Schuurhuis, A. 1AP2-4

Schwarte, L.A. 4AP3-3, 4AP8-1, 12AP1-7, 13AP2-1, 13AP3-4

Schwartges, I. 4AP3-3, 4AP8-1, 12AP1-7

Schwarz, C. 7AP5-5

Schwarz, G. 7AP4-8

Schwilden, H. 12AP2-7, 14AP2-6

Sciarra, J. 17AP3-7

Scimeca, L. 3AP2-1

Sear, J.W. 1AP2-2

Sebastian, S. 12AP8-1

Segura, C. 14AP5-3 
Seifert, B. 3AP3-4

SelviCan, O. 3AP2-7, 19AP5-8, 19AP5-9, 19AP5-10

Senard, M. 14AP1-3

Seo, I.S. 10AP4-4

Seo, N. 9AP8-9

Seong, C.L. 10AP4-4

Serrano, H. 12AP4-7

Serrano, S. 10AP2-4

Sert, O. 9AP6-4

Servillo, G. 12AP3-4, 12AP3-6

Sethuraman, D. 19A92-4

Setti, T. 9AP2-8

Sevcik, A. 19AP2-3

Shah, A. 5AP1-5

Shen, C.H. 9AP4-1

Sheraton, T. 8AP1-5

Sheraton, T.E. 19AP1-9

Shibata, O. 9AP8-4

Shields, M. 9AP7-2

Shifman, E. 3AP5-6

Shiga, T. 19AP4-9

Shim, J. 5AP4-6, 7AP2-3, 12AP8-3

Shimizu, T. 12AP4-6

Shin, B. 5AP4-6

Shin, C.M. 9AP5-2

Shin, I.W. 17AP3-2

Shin, S. 9AP3-4

Shin, Y.S. 8AP6-8

Shiraishi, Y. 15AP2-1

Shishkin, O. 5AP3-1

Shivji, F. 5AP1-4

Shkesters, A. 12AP9-8

Shorten, G. 3AP6-3, 3AP9-9, 14AP3-9 15AP1-4, 15AP2-3, 15AP2-6

Shoukri, M. 13AP2-8

Shu, J. 7AP2-3

Shyr, M.H. 13AP3-2

Siakavaras, V. 3AP9-8

Sicuranza, R. 19AP4-6

Siddiqui, Q. 5AP1-5

Sidorowicz, M. 17AP3-6

Sielenkaemper, A.W. 8AP8-9

Siemionow, M. 4AP6-2

Sierra, P. 1AP2-9, 3AP7-5

Sifre, C. 15AP2-4

Simic, D. 10AP3-6, 10AP6-9

Simon, M.J.G. 3AP7-9

Simon, S. 4AP7-1

Simon, T. 4AP7-7

Simon, T.P. 12AP3-3

Singleton, P.A. 9AP4-8

Sinkowitsch, J. 10AP2-1

Sitzwohl, C. 12AP2-5

Skalski, J. 10AP4-5

Skegro, M. 1AP1-7

Skorzynski, W. 4AP6-4

Skovgaard, L.T. 9AP2-9

Skowroñski, R. 17AP3-6

Skrzypczak, M. 3AP2-3

Skudre, A. 3AP6-8

Slavcovici, A. 12AP3-7

Slavevski, D. 12AP9-4

Slavkovic, A. 10AP3-6, 10AP6-9

Slieker, J. 3AP8-2, 4AP2-1

Sluka, K.A. 8AP1-6

Smakal, O. 10AP6-6

Smaoui, M. 8AP2-8, 8AP7-8

Smart, N. 17AP3-3

Smart, N.G. 19AP1-6, 19AP3-1

Smeets, J. 9AP2-7

Smith, A.F. 1AP2-6, 1AP2-7

Smith, M.D. 8AP10-9, 9AP1-10

Smith, S. 5AP3-3

Smolka, V. 10AP6-6

Smorodnikov, A. 3AP5-6

Sneyd, J.R. 9AP5-8, 10AP3-7

Snoeck, M.M.J. 9AP7-1
So, E.C. 9AP8-1, 14AP2-3

Soares, C. 4AP5-7

Soehle, M. 3AP9-2

Söhle, M. 3AP3-3

Sohn, J.T. 17AP3-2

Sokolov, A. 14AP4-2

Solak, M. 19AP6-7

Solaz, C. 3AP2-4, 15AP2-4, 19AP2-7

Soler, E. 3AP2-10

Soler-Cantos, F. 9AP1-2

Soler-Mándoli, E. 1AP1-8

Soliman, W.R. 8AP7-1, 19AP2-1

Soliveres, J. 3AP2-4, 15AP2-4, 19AP2-7

Sollazzi, L. 3AP5-9

Solomon, C. 6AP1-7

Somboonviboon, W. 10AP5-3

Somri, M. 19AP6-3

Sondore, A. 12AP9-8, 14AP5-1

Song, $X$. 9AP3-1

Sood, J. 10AP4-1

Sosnowski, M. 8AP9-7

Soufidi, K. 17AP2-5, 17AP2-6

Soummer, A. 9AP8-10

Soumpasis, I. 6AP1-5, 9AP3-6, 9AP3-7

Sountoulides, P. 1AP2-3

Souvatzis, $X$. 8AP1-10

Sowinski, P. 12AP3-8, 12AP6-8

Spadini, E. 3AP6-2

Spahn, D.R. 6AP1-1, 6AP3-3

Spak, F. 9AP4-10

Spanhel, J. 10AP6-6

Spannagl, M. 6AP1-10

Sparr, H. 9AP2-5

Spieth, P.M. 5AP1-1, 12AP1-6

Spiridonov, S. 12AP9-2

Spodeck, J. 9AP4-2

Spoehr, F. 5AP2-2

Squicciarini, G. 3AP6-2

Srikantharajah, I. 14AP1-2

Srikanthrajah, I.D. 14AP1-2

Srinivas, J. 13AP1-3

Srinivas, K. 19AP3-7

Staals, L. 9AP7-1

Staber, M. 7AP1-8, 12AP6-4

Stacey, M. 19AP3-10

Stadler, M. 4AP8-3

Staikou, C. 14AP3-2

Stamatian, F. 11AP2-9

Stamatiou, G. 8AP4-9

Stammet, P. 13AP2-3

Stamov, V. 9AP2-10

Stavreva, G. 9AP4-6

Steed, M. 4AP4-2

Steffek, M. 8AP8-1

Stegmann, I. 4AP4-6

Stehr, S. 8AP10-11, 11AP1-9

Steinfath, M. 7AP6-8

Stelea, G. 14AP5-11

Stelian, E. 6AP3-7

Stepanovs, J. 3AP6-8, 10AP2-6

Stephan, M. 4AP3-3, 4AP8-1

Sterzi, R. 7AP2-4

Stirparo, S. 11AP1-4

Stockmanns, G. 3AP3-9

Støen, R. 3AP3-5

Stoicovski, E. 8AP8-3, 12AP9-4

Stoleru, S. 8AP10-5, 9AP1-6

Stone, A. 12AP8-2

Storm, H. 3AP3-5

Straus, S. 12AP9-3

Strüber, M. 4AP7-1

Struck, M. 13AP1-1

Studnicska, D. 12AP3-7

Stueber, F. 12AP5-6

Stuttmann, R. 13AP1-1

Stutz, K. 10AP3-3

Sugimoto, Y. 4AP6-5

Sugino, S. 10AP6-3, 19AP1-2
Suh, J. 12AP8-3

Sullivan, G. 8AP9-1

Sulyok, I. 14AP3-1

Sumikawa, K. 9AP8-4

Sundaram, R. 4AP1-2, 17AP3-3

Suppa, E. 11AP2-8

Suraseranivongse, S. 10AP5-3

Sustic, A. 8AP6-6, 19AP3-2

Suszyñska-Mosiewicz, A. 12AP8-6

Suy, K. 9AP2-1

Suzuki, S. 12AP4-6

Swenson, E.R. BAPC1-4, 5AP2-4

Symonides, M. 12AP3-8, 12AP6-8

Szabo, C. 4AP8-9

Szadek, K. 14AP3-3

Szatmári, A. 10AP5-7

Székely, A. 10AP5-7

Szewczyk, T. 4AP3-4

Szijarto, A. 3AP7-6

Sztark, F. 3AP7-10, 12AP7-9

Szymanowicz, W. 8AP8-1

Tablov, B. 9AP4-6

Tablov, V. 9AP4-6

Tai, Y.H. 9AP4-1

Taivans, I. 19AP4-4

Takase, M. 19AP5-5

Takashina, M. 3AP2-2

Takeuchi, K. 19AP3-4

Tallarini, F. 12AP1-1, 12AP1-2

Tamosiunas, R. 8AP5-5

Tamowicz, B. 12AP6-7

Tan, G. 19AP1-10

Tan, S.S. 12AP2-9

Tang, C-S. 8AP1-1

Tang, S. 14AP2-11

Tangwiwat, S. 7AP2-7

Tanioka, K. 7AP4-2

Tanno, M. 19AP4-9

Tansey, D. 12AP8-8

Tardeli, M. 3AP6-6

Taslýpýnar, M. 4AP3-7

Taspinar, V. 8AP1-7, 8AP1-9

Tassani, P. 6AP2-4

Tassaux, D. 17AP2-3

Tataraki, K. 2AP1-6, 8AP9-10

Tavares, J. 2AP1-3, 19AP1-3

Teixeira, C. 11AP2-1

Ten Broecke, P. 4AP6-1

Terbille, $\mathrm{H}$. 9AP4-7

Tercero, F.J. 7AP3-2

Tercero, J. 7AP4-3

Teske, G. 12AP5-4

Tessmer, J. 4AP9-4

Tetiker, S. 14AP4-3

Tezcan, B. 4AP3-7

Theisen, F. 6AP1-10

Theusinger, O.M. 6AP1-1, 6AP3-3

Thicoïpé, M. 13AP1-6

Thieblemont, J. 17AP2-2

Thomaidis, G. 12AP6-6

Thomson, A.J. 9AP5-7

Thuemer, O. 12AP7-8

Tian, M. 19AP5-2

Tirnaksiz, B. 14AP4-6

Tiwari, A. 7AP1-5

To-oyama, Y. 4AP6-5

Toame, R. 19AP6-3

Todd, M. 9AP5-5

Tognoli, E. 14AP1-1

Toker, K. 19AP6-7

Tokoroyama, H. 3AP5-3, 19AP1-8

Tölgyes, A. 8AP9-3

Tolksdorf, B. 3AP4-4

Tomanovic, N. 8AP7-7

Tomás, I. 19AP3-8

Tomasino, S. 3AP7-7

Tomescu, D. 12AP2-4 
Tonkovic, D. 1AP1-7, 12AP7-7

Topalo, V. 19AP1-5

Topcu, G. 4AP7-9

Topcuoglu, P. 19AP2-2

Topkaya, C. 8AP4-4

Torossian, A. 12AP5-7

Torrallardona, J. 10AP2-5

Touitou, Y. 7AP6-9, 9AP6-7

Townley, S.A. 2AP2-4

Traber, D.L. 4AP8-9

Tramèr, M.R. 8AP7-2

Trenchant, A. 4AP5-1

Trevisan, P. 17AP1-2

Triantyfyllou, C. 4AP1-2

Triga, A. 14AP3-2

Triki, Z. $\quad$ 8AP4-7, 11AP2-11

Tsai, R. 9AP4-1

Tsaousi, G. 4AP1-7

Tseng, C.J. 3AP6-1

Tsikaras, P. 1AP2-3

Tsirtsiridou, V. 8AP7-9

Tsukigase, N. 10AP6-3

Tufano, R. 12AP3-4, 12AP3-6

Tulbure, D. 12AP2-4

Turan, C. 9AP3-5

Turan, G. 9AP3-5

Turan, I. 2AP1-8

Turan, Z. 2AP1-8, 2AP1-9

Turek, $Z$. 4AP6-9

Turene, $\mathrm{H}$. 8AP9-6

Turi, S. 9AP3-2

Turina, D. 8AP6-6

Turjman, F. 7AP1-9

Turner, D. 3AP4-2

Turner, S. 4AP6-6

Tuzuner, F. 3AP2-7

Tyagi, N. 4AP4-2

Tyczka, J. 3AP2-3, 12AP6-7

Tymkew, H. 3AP4-8

Tzabazis, A. 14AP2-6

Tzanakis, N. 8AP1-10

Tzovaras, G. 8AP4-8, 8AP4-9

Uddin, S. 1AP3-5

Ujevic, A. 1AP1-6

Unertl, K.E. 8AP10-9, 9AP1-10, 12AP5-3

Unlugenc, H. 11AP2-5, 14AP4-3

Urbanska, E. 4AP5-8, 10AP4-5

Urunsak, I. 11AP2-5

Urwyler, A. 9AP8-11

Ushida, T. 8AP1-6

Uutela, K. 3AP3-8, 3AP4-5, 3AP6-5

Uvarov, D. 5AP4-1, 14AP1-6, 14AP4-2, 14AP5-2

Uzun, S. 19AP2-2

Valencia, O. 19AP5-4

Valente, A. 11AP2-8

Valentim, A.M. 9AP3-10

Valeri, R. 9AP2-3

Valero, R. 4AP8-5, 4AP8-6, 7AP3-2, 7AP4-3

Vallaud, D. 12AP9-6

Valldeperas, I. 3AP4-4

Vallès, J. 1AP1-8, 1AP3-6, 5AP3-4

Valsamidis, D. 11AP2-2

Van Aken, H. 8AP8-9, 12AP5-2

Van Aken, H.K. 14AP4-1

van Barneveld, T.A. 1AP2-4

Van de Velde, M. 11AP1-2, 11AP1-3

Van den Berghe, G. BAPC1-2, 12AP4-2, 12AP4-3

Van Der Goot, L. $\quad 4 A P 8-3$

Van der Linden, P. 10AP1-1, 10AP1-2, 10AP1-3

Van der Linden, P.J. 4AP7-2

Van Der Linden, Ph. 4AP5-1, 4AP5-2, 4AP5-3, 4AP6-1

Van Doninck, D. 4AP5-1
Van Droogenbroek, G. 17AP3-1

Van Leeuwen, P. 12AP4-3

van Oeveren, W. 4AP7-8

Van Rompaey, N. 3AP2-8

van Zaane, B. 4AP2-3, 4AP2-4

Vanacker, B. 9AP7-8

Vanags, I. 3AP6-8, 12AP3-2, 14AP5-1

Vanden Eede, $\mathrm{H}$. 4AP7-2

Vander Laenen, M. 3AP4-6

Vandermeersch, E. 11AP1-2, 11AP1-3

Vanhorebeek, I. BAPC1-2, 12AP4-2

Vanlander, A. 3AP1-2

Vardar, M.A. 14AP4-3

Varga, K. 4AP5-5

Varosyan, A. 8AP1-8

Varveris, D. 19AP1-6

Vasilakos, D. 4AP1-7, 7AP6-1, 8AP10-10, 9AP8-3

Vassilas, N. 14AP5-6

Vassiliadis, M. 3AP9-8

Vega, R. 3AP8-5

Vegh, V. 9AP4-7

Velly, L. BAPC1-5, 7AP3-8, 7AP5-6, 7AP5-7

Ventouri, M. 14AP4-10

Verbeke, K. 3AP1-4

Verbesselt, R. 9AP5-1

Verborgh, C. 3AP8-7, 4AP9-1, 14AP1-9

Verdonck, P. 4AP5-2, 4AP5-3

Verleije, A. 3AP2-8

Vernetta, D. 1AP2-9, 3AP7-5

Vershuta, D. 9AP7-6

Vescovo, A. 8AP9-6

Videira, R. 9AP7-9

Vidrickova, P. 19AP2-3

Vieira, F. 4AP5-7

Vieira, J. 9AP7-9

Viergutz, T. 13AP3-5

Vieri, J. 3AP4-5

Viethen, V. 9AP5-3

Vilà, E. 1AP3-6

vila, E. 5AP4-5

Vila, M. 17AP2-1

Vila, P. 17AP2-1

Vilaplana, J. 14AP3-7

Viljurov, I. 14AP5-2

Villalonga, A. 14AP3-7

Villegas-Duque, D. 9AP1-2

Villinger, J. 3AP1-5

Vimláti, L. 12AP2-3

Vitale, F. 3AP5-9

Viterbo, J. 4AP5-7

Vives, I. 2AP2-3, 19AP6-10

Vivona, G. 14AP1-10

Vivona, L. 14AP1-10

Voets, E. 7AP3-5

Vogiatzaki, T. 14AP4-8

Vogt, A. 1AP2-10, 3AP1-9, 3AP7-8 15AP2-2

Vreden, M.J.A. 4AP3-5

Vullo, C. 4AP8-7

Vundelinckx, G. 3AP4-6, 13AP4-5

Vuyk, J. 3AP7-9

Wakisaka, $\mathrm{H}$. 3AP6-7

Walker, K. 1AP2-6, 1AP2-7

Wallace, E. 3AP5-1

Wallenborn, J. 9AP6-9

Wallet, F. 3AP7-3

Wang, C-J. 8AP1-1

Wang, F-Y. 8AP2-1

Wang, G.K. $\quad$ 8AP10-3

Wang, J.J. 9AP6-3

Wang, Q. 7AP5-4

Wang, Q.Y. $\quad$ 4AP4-4, 4AP4-5, 7AP5-1

Wang,Y. 7AP6-2

Warltier, D. 4AP9-4

Wassenaar, L.E.J. 4AP3-5
Wassermann, K. 1AP2-5

Watanabe, H. 3AP6-10, 19AP1-2

Waterloos, $H$. 14AP1-8

Wattwill, M. 9AP7-8

Weber, B. 9AP1-5

Weber, N.C. 4AP4-6

Weber, S. 12AP5-6

Wehner, M. 9AP1-3, 9AP8-5

Weibel, $H$. 17AP2-3

Weilbach, C. 8AP5-8

Weiler, N. 7AP4-1

Weimann, J. 4AP4-7, 5AP2-1, 5AP2-2

Weinstabl, C. 12AP2-5

Weiß, D.G. 3AP4-9

Weiss, M. 3AP3-6, 10AP1-5, 10AP1-6, 10AP2-7, 10AP3-3, 17AP3-1

Wen, Z-H. 14AP2-2

Wendt, M. 4AP8-8

Wenk, M. 14AP2-5

Wens, J. 4AP5-2, 4AP5-3

Wentworth, S. 19AP5-7

Werer, C. 13AP2-3

Werner, C. 9AP4-9

Westphal, M. 4AP8-9, 12AP5-2

Weyns, F. 7AP3-4

Whitelaw, M. 12AP6-4

Wiel, E. 13AP1-6

Wiesner,G. 9AP6-5

Wiessner, D. 6AP3-9, 8AP3-8, 8AP8-7

Wijayatilake, D. 7AP2-7

Wilhelm, D. 13AP3-3

Wilkes, A. 3AP8-6, 19AP5-6, 19AP3-10

Wilkes, A.R. 2AP1-7, 3AP1-7, 3AP6-9, 3AP8-8, 8AP3-7, 19AP1-9, 19AP5-7

Williams, P. 13AP3-6

Wilson, G. 12AP7-1

Wilson, L. 12AP6-1

Wiltzinski, V. 12AP6-6

Winklmaier, T. 1AP3-7

Winterhalter, M. 4AP7-1, 8AP5-8

Wittenberg, T. 12AP2-7

Woishuber, J. 13AP4-1

Wolf, A.R. 10AP3-7

Wolf, P. 6AP2-10

Wong, C. 7AP6-2, 8AP10-4, 14AP2-2

Wong, C.S. 9AP4-1

Wong, D. 19AP1-10

Wong, K.L. 9AP8-1, 14AP2-3

Wrobel, M. 19AP6-4

Wronka, K. 12AP6-7

Wu, G-J. 14AP2-2

Wu, K.C. 9AP8-1

Wu, R.S.C. 3AP1-8, 9AP8-1

Wu, X. 7AP6-7

Wujtewicz, M. 17AP3-6

Wujtewicz, M.A. 8AP8-1, 12AP8-6

Wulf, H. 9AP4-2, 12AP5-7, 19AP3-9

Wuyts, J. 7AP1-6, 7AP3-4, 7AP3-5

Xu, G. 4AP8-2

Xue, Q. 3AP5-4, 7AP6-7

Yagmurdur, H. 8AP4-4

Yamada, Y. 14AP2-1

Yamaguchi, M. 9AP8-4

Yamanaka, H. 9AP5-6

Yamashita, K. 3AP5-3, 19AP1-8

Yamauchi, M. 10AP6-3

Yang, C.P. 9AP4-1

Yang, H.S. 9AP8-2

Yang, M. 3AP7-4

Yang, Y.L. 9AP6-3

Yanovski, B. 19AP6-3

Yatabe, T. 3AP5-3

Yavuz, Y. BAPC1-6, 12AP5-8

Yedicocuklu, D. 9AP7-7

Yi, H. 7AP2-3, 12AP8-3

Yildiz, $\mathrm{H}$. 8AP4-4 
Yli-Hankala, A. $\quad 3 A P 4-5,3 A P 6-5$ Yokoyama, R. 8AP1-6, 19AP1-8 Yokoyama, T. 3AP5-3, 8AP1-6, 19AP1-8

Yon, J-H. 6AP1-6

Yosry, M. 10AP5-1

Yu, B. 3AP5-4, 4AP9-4, 7AP6-7

Yue, Y. 3AP3-2

Yuge, O. 4AP9-2, 9AP6-1, 9AP8-6

Yuksel, G. 9AP3-5

Zabolotskikh, I.B. 12AP4-5

Zacharowski, K. 4AP4-7

Zahn, P.K. 14AP2-5, 14AP4-1
Zambouri, A. 14AP4-10

Zamparelli, E. 12AP3-4

Zanfini, B.A. 11AP2-8

Zanner, R. 13AP3-3

Zarguouni, A. 8AP5-3

Zasa, M. 8AP9-5

Zembala, M. 10AP4-5

Zhang, B. 9AP3-1

Zhang, L. 19AP5-2

Zhang, Y. BAPC1-3, 5AP2-3

Zhang, Y. I. 7AP5-4

Zhou, B. 9AP3-1

Zhu, X. 3AP5-4

Zhurda, T. 7AP2-9
Ziaga, P. 9AP3-7

Zieglgaensberger, W. 7AP5-2

Zielinski, R. 12AP6-8

Zizzo, C. 3AP4-2

Zobrist, C. 4AP5-6

Zoumprouli, A. 8AP4-2

Zuithoff, N.P.A. 4AP2-4

Zuo, Y.X. 4AP4-4, 4AP4-5, 7AP5-1, 7AP5-4

Zuurbier, C.J. $\quad$ 4AP4-1

Zuurmond, W. 14AP3-3, 14AP3-5, 14AP3-6

Zwissler, B. 5AP3-2, 6AP1-2, 6AP1-4, 9AP2-4

Zysset, S. 7AP2-6 